atremote sensing

Volume 2

Advances in.

Quantitative

Remote Sensing

in China -

In Memory of Prof.

Xiaowen Li

Edited by

Shunlin Liang, Guangjian Yan and Jiancheng Shi Printed Edition of the Special Issue Published in Remote Sensing 



\section{Advances in Quantitative Remote Sensing in China-In Memory of Prof. Xiaowen Li}





\section{Advances in Quantitative Remote Sensing in China-In Memory of Prof. Xiaowen Li}

\section{Volume 2}

Special Issue Editors

Shunlin Liang

Guangjian Yan

Jiancheng Shi 
Special Issue Editors

Shunlin Liang

Guangjian Yan

University of Maryland

Beijing Normal University

USA

China

Jiancheng Shi

Institute of Remote Sensing and Digital Earth

China

\section{Editorial Office}

MDPI

St. Alban-Anlage 66

Basel, Switzerland

This is a reprint of articles from the Special Issue published online in the open access journal Remote Sensing (ISSN 2072-4292) from 2017 to 2018 (available at: https://www.mdpi.com/journal/ remotesensing/special_issues/quantitative_rs)

For citation purposes, cite each article independently as indicated on the article page online and as indicated below:

LastName, A.A.; LastName, B.B.; LastName, C.C. Article Title. Journal Name Year, Article Number, Page Range.

Volume 2

ISBN 978-3-03897-276-1 (Pbk)

ISBN 978-3-03897-277-8 (PDF)
Volume 1-2

ISBN 978-3-03897-278-5 (Pbk)

ISBN 978-3-03897-279-2 (PDF)

Articles in this volume are Open Access and distributed under the Creative Commons Attribution (CC BY) license, which allows users to download, copy and build upon published articles even for commercial purposes, as long as the author and publisher are properly credited, which ensures maximum dissemination and a wider impact of our publications. The book taken as a whole is (C) 2019 MDPI, Basel, Switzerland, distributed under the terms and conditions of the Creative Commons license CC BY-NC-ND (http:/ / creativecommons.org/licenses/by-nc-nd/4.0/). 


\section{Contents}

About the Special Issue Editors $\ldots \ldots \ldots \ldots \ldots \ldots \ldots \ldots \ldots \ldots$

Mengjia Wang, Rui Sun and Zhiqiang Xiao

Estimation of Forest Canopy Height and Aboveground Biomass from Spaceborne LiDAR and Landsat Imageries in Maryland

Reprinted from: Remote Sens. 2018, 10, 344, doi: 10.3390/rs10020344 . . . . . . . . . . . . . . 1

Qiaolin Zeng, Yongqian Wang, Liangfu Chen, Zifeng Wang, Hao Zhu and Bin Li

Inter-Comparison and Evaluation of Remote Sensing Precipitation Products over China from 2005 to 2013

Reprinted from: Remote Sens. 2018, 10, 168, doi: 10.3390/rs10020168 . . . . . . . . . . . . . 23

Xiaojun Li, Xiaozhou Xin, Zhiqing Peng, Hailong Zhang, Chuanxiang Yi and Bin Li

Analysis of the Spatial Variability of Land Surface Variables for ET Estimation: Case Study in HiWATER Campaign

Reprinted from: Remote Sens. 2018, 10, 91, doi: 10.3390/rs10010091 _ . . . . . . . . . . . . . . 41

Lilin Zhang, Yunjun Yao, Zhiqiang Wang, Kun Jia, Xiaotong Zhang, Yuhu Zhang, Xuanyu Wang, Jia $\mathrm{Xu}$ and Xiaowei Chen

Satellite-Derived Spatiotemporal Variations in Evapotranspiration over Northeast China during 1982-2010

Reprinted from: Remote Sens. 2017, 9, 1140, doi: 10.3390/rs9111140

Xuanyu Wang, Yunjun Yao, Shaohua Zhao, Kun Jia, Xiaotong Zhang, Yuhu Zhang, Lilin Zhang, Jia $\mathrm{Xu}$ and Xiaowei Chen

MODIS-Based Estimation of Terrestrial Latent Heat Flux over North America Using Three Machine Learning Algorithms

Reprinted from: Remote Sens. 2017, 9, 1326, doi: 10.3390/rs9121326

Tongxi Hu, Tianjie Zhao, Jiancheng Shi, Shengli Wu, Dan Liu, Haiming Qin and Kaiguang Zhao

High-Resolution Mapping of Freeze/Thaw Status in China via Fusion of MODIS and AMSR2 Data

Reprinted from: Remote Sens. 2017, 9, 1339, doi: 10.3390/rs9121339

Xiaojing Liu, Lingmei Jiang, Shengli Wu, Shirui Hao, Gongxue Wang and Jianwei Yang Assessment of Methods for Passive Microwave Snow Cover Mapping Using FY-3C/MWRI Data in China

Reprinted from: Remote Sens. 2018, 10, 524, doi: 10.3390/rs10040524 . . . . . . . . . . . . . . 12

Tao Yu, Rui Sun, Zhiqiang Xiao, Qiang Zhang, Gang Liu, Tianxiang Cui and Juanmin Wang Estimation of Global Vegetation Productivity from Global LAnd Surface Satellite Data Reprinted from: Remote Sens. 2018, 10, 327, doi: 10.3390/rs10020327 . . . . . . . . . . . . . . . 142

Ling Hu, Wenjie Fan, Huazhong Ren, Suhong Liu, Yaokui Cui and Peng Zhao

Spatiotemporal Dynamics in Vegetation GPP over the Great Khingan Mountains Using GLASS Products from 1982 to 2015

Reprinted from: Remote Sens. 2018, 10, 488, doi: 10.3390/rs10030488 . . . . . . . . . . . . . . 162 
Xinyao Xie, Ainong Li, Huaan Jin, Gaofei Yin and Jinhu Bian

Spatial Downscaling of Gross Primary Productivity Using Topographic and Vegetation Heterogeneity Information: A Case Study in the Gongga Mountain Region of China

Reprinted from: Remote Sens. 2018, 10, 647, doi: 10.3390/rs10040647 . . . . . . . . . . . . . . . 184

Shangrong Lin, Jing Li, Qinhuo Liu, Alfredo Huete and Longhui Li

Effects of Forest Canopy Vertical Stratification on the Estimation of Gross Primary Production by Remote Sensing

Reprinted from: Remote Sens. 2018, 10, 1329, doi: 10.3390/rs10091329 _ . . . . . . . . . . . . 199

Tianxiang Cui, Rui Sun, Chen Qiao, Qiang Zhang, Tao Yu, Gang Liu and Zhigang Liu

Estimating Diurnal Courses of Gross Primary Production for Maize: A Comparison of Sun-Induced Chlorophyll Fluorescence, Light-Use Efficiency and Process-Based Models

Reprinted from: Remote Sens. 2017, 9, 1267, doi: 10.3390/rs9121267 . . . . . . . . . . . . . . . .

\section{Ze He, Shihua Li, Yong Wang, Leiyu Dai and Sen Lin}

Monitoring Rice Phenology Based on Backscattering Characteristics of Multi-Temporal RADARSAT-2 Datasets

Reprinted from: Remote Sens. 2018, 10, 340, doi: 10.3390/rs10020340 . . . . . . . . . . . . . . . 247

\section{Zhoutao Zheng and Wenquan Zhu}

Uncertainty of Remote Sensing Data in Monitoring Vegetation Phenology: A Comparison of MODIS C5 and C6 Vegetation Index Products on the Tibetan Plateau

Reprinted from: Remote Sens. 2017, 9, 1288, doi: 10.3390/rs9121288

Jinhui Wu and Shunlin Liang

Developing an Integrated Remote Sensing Based Biodiversity Index for Predicting Animal Species Richness

Reprinted from: Remote Sens. 2018, 10, 739, doi: 10.3390/rs10050739 _ . . . . . . . . . . . . . 281

Lang Xia, Fen Zhao, Kebiao Mao, Zijin Yuan, Zhiyuan Zuo and Tongren Xu

SPI-Based Analyses of Drought Changes over the Past 60 Years in China's Major Crop-Growing Areas

Reprinted from: Remote Sens. 2018, 10, 171, doi: 10.3390/rs10020171 . . . . . . . . . . . . . 301

Guoliang Yun, Shudi Zuo, Shaoqing Dai, Xiaodong Song, Chengdong Xu, Yilan Liao, Peiqiang Zhao, Weiyin Chang, Qi Chen, Yaying Li, Jianfeng Tang, Man Wang and Yin Ren Individual and Interactive Influences of Anthropogenic and Ecological Factors on Forest $\mathrm{PM}_{2.5}$ Concentrations at an Urban Scale Reprinted from: Remote Sens. 2018, 10, 521, doi: 10.3390/rs10040521 . . . . . . . . . . . . . . 316

\section{Hongmin Zhou, Jindi Wang and Shunlin Liang}

Design of a Novel Spectral Albedometer for Validating the MODerate Resolution Imaging Spectroradiometer Spectral Albedo Product

Reprinted from: Remote Sens. 2018, 10, 101, doi: 10.3390/rs10010101 . . . . . . . . . . . . . . 333

Gaofei Yin, Ainong Li and Aleixandre Verger

Spatiotemporally Representative and Cost-Efficient Sampling Design for Validation Activities in Wanglang Experimental Site

Reprinted from: Remote Sens. 2017, 9, 1217, doi: 10.3390/rs9121217 . . . . . . . . . . . . . . 349

Donghui Xie, Xiangyu Wang, Jianbo Qi, Yiming Chen, Xihan Mu, Wuming Zhang and Guangjian Yan

Reconstruction of Single Tree with Leaves Based on Terrestrial LiDAR Point Cloud Data Reprinted from: Remote Sens. 2018, 10, 686, doi: 10.3390/rs10050686 . . . . . . . . . . . . . . 363 
Qinhuo Liu, Guangjian Yan, Ziti Jiao, Qing Xiao, Jianguang Wen, Shunlin Liang, Jindi Wang, Crystal Schaaf and Alan Strahler

From Geometric-Optical Remote Sensing Modeling to Quantitative Remote Sensing Science-In Memory of Academician Xiaowen Li

Reprinted from: Remote Sens. 2018, 10, 1764, doi: 10.3390/rs10111764 . . . . . . . . . . . . . 381 



\section{About the Special Issue Editors}

Shunlin Liang earned a Ph.D. from Boston University in 1993. His main research interests focus on the estimation of land surface variables from satellite data, earth energy balance, and the assessment of environmental changes. He authored the book "Quantitative Remote Sensing of Land Surfaces" (Wiley, 2004), co-authored the book "Global LAnd Surface Satellite (GLASS) Products: Algorithms, Validation and Analysis"(Springer, 2013), edited the book "Advances in Land Remote Sensing: System, Modeling, Inversion and Application" (Springer, 2008), and co-edited the books "Advanced Remote Sensing: Terrestrial Information Extraction and Applications" (Academic Press, 2012) and "Land Surface Observation, Modeling, Data Assimilation" (World Scientific, 2013). He was also the Editor-in-Chief of the nine-volume book series "Comprehensive Remote Sensing". He has published over 320 SCI-indexed papers and 32 book chapters. According to Google Scholar, his H-index is 64. He is an IEEE fellow.

Guangjian Yan is a professor and vice director of the State Key Laboratory of Remote Sensing Science. He received his B.A. and M.A. degrees from the Beijing Institute of Technology in 1993 and 1996, respectively. He earned a Ph.D. degree from the Institute of Remote Sensing Applications, Chinese Academy of Sciences in 1999. He was selected as the New Century Excellent Talent at the University of China in 2005. He has been the principle investigator of national programs (numbers 973 and 863) and the key program of NSFC. His main research interests include multi-angular remote sensing, vegetation remote sensing, and radiation budget. He invented a series of multi-angle platforms for ground-based and airborne remote sensing measurement. He proposed a path length distribution model which can improve the accuracy of LAI indirect measurement more than one time. He has published more than 200 papers.

Jiancheng Shi received his B.A. in Hydrogeology and Engineering Geology from the University of Lanzhou in China in 1982. He earned his M.A. and Ph.D. degrees in Geography from the University of California, Santa Barbara (UCSB) in 1987 and 1991, respectively. He then worked at the Institute for Computational Earth System Sciences (later the Earth Research Institute) at UCSB as a research professor. In 2010, he joined the Institute of Remote Sensing and Digital Earth, Chinese Academy of Sciences as director and worked as a senior research scientist at the State Key Laboratory of Remote Sensing Science in Beijing, China. His research interests include microwave remote sensing of water cycle-related components. He has published more than 300 journal and conference papers. He is a Fellow of IEEE and SPIE. 

Article

\title{
Estimation of Forest Canopy Height and Aboveground Biomass from Spaceborne LiDAR and Landsat Imageries in Maryland
}

\author{
Mengjia Wang ${ }^{1,2}$, Rui Sun ${ }^{1,2, *}$ and Zhiqiang Xiao ${ }^{1,2}$ \\ 1 State Key Laboratory of Remote Sensing Science, Jointly Sponsored by Beijing Normal University and the \\ Institute of Remote Sensing and Digital Earth, CAS, Beijing 100875, China; wmeng_jia@163.com (M.W.); \\ zhqxiao@bnu.edu.cn (Z.X.) \\ 2 Beijing Engineering Research Center for Global Land Remote Sensing Products, Institute of Remote Sensing \\ Science and Engineering, Faculty of Geographical Science, Beijing Normal University, Beijing 100875, China \\ * Correspondence: sunrui@bnu.edu.cn; Tel.: +86-10-58805457
}

Received: 24 November 2017; Accepted: 18 February 2018; Published: 23 February 2018

\begin{abstract}
Mapping the regional distribution of forest canopy height and aboveground biomass is worthwhile and necessary for estimating the carbon stocks on Earth and assessing the terrestrial carbon flux. In this study, we produced maps of forest canopy height and the aboveground biomass at a $30 \mathrm{~m}$ spatial resolution in Maryland by combining Geoscience Laser Altimeter System (GLAS) data and Landsat spectral imageries. The processes for calculating the forest biomass included the following: (i) processing the GLAS waveform and calculating spatially discrete forest canopy heights; (ii) developing canopy height models from Landsat imagery and extrapolating them to spatially contiguous canopy heights in Maryland; and, (iii) estimating forest aboveground biomass according to the relationship between canopy height and biomass. In our study, we explore the ability to use the GLAS waveform to calculate canopy height without ground-measured forest metrics $\left(R^{2}=0.669\right.$, $\mathrm{RMSE}=4.82 \mathrm{~m}, \mathrm{MRE}=15.4 \%$ ). The machine learning models performed better than the principal component model when mapping the regional forest canopy height and aboveground biomass. The total forest aboveground biomass in Maryland reached approximately $160 \mathrm{Tg}$. When compared with the existing Biomass_CMS map, our biomass estimates presented a similar distribution where higher values were in the Western Shore Uplands region and Folded Application Mountain section, while lower values were located in the Delmarva Peninsula and Allegheny Mountain regions.
\end{abstract}

Keywords: forest canopy height; aboveground biomass; ICESat GLAS; Landsat; random forest model

\section{Introduction}

Increasing concerns regarding global climatic changes have emphasized the urgency of finding efficient ways to quantify terrestrial carbon stocks at regional, continental, and global scales [1]. Forest biomass is of primary importance for the assessment and management of carbon resources on Earth. At the same time, changes in forest biomass can be a good proxy for the analysis of the global carbon cycle and a valid resource for the estimation of sequestration and carbon sources/sinks [2,3]. Scientific researchers also use forest biomass to study ecosystem biodiversity [4,5]. In recent years, efforts have been made to mitigate the deforestation and emissions of greenhouse gases. Such forest biomass studies will record valuable information that can be used to evaluate the effects of these efforts. Furthermore, it is helpful to provide governments with constructive suggestions for biodiversity conservation and ecosystem management. Hence, it is strongly necessary to explore an efficient approach to assess forest biomass at regional, continental, and even global scales.

Field forest measurements provide a straightforward, accurate method in order to calculate the amount of terrestrial aboveground forest resources and evaluate the global carbon cycle [6]. Tree metrics, 
such as height, DBH (diameter at breast height), tree age, tree species, and LAI (leaf area index) can link forest biomass estimates with allometric relationships [7-10]. Nevertheless, ground inventory campaigns require huge investments of labor, time, and money, which result in inconsistency over large regions and the lack of ground-based inventories in remote areas.

LiDAR is another method to link forest height and other biophysical characteristics. It can be used to make digital three-dimensional (3D)-representations of the target and high-resolution maps with wide applications in forestry [11-13]. Tree heights obtained by airborne LiDAR have proven to be a good predictor of forest biomass [14]. Naesset and Goabakken explained 88\% and 85\% of the variability in aboveground and belowground biomass, respectively, using airborne LiDAR [15]. Drake et al. explored the sensitivity of LiDAR to differences in canopy structure and aboveground biomass in a dense, neotropical rainforest [16]. However, the application of airborne LiDAR to forest biomass also has challenges as airborne LiDAR relies heavily on financial support and does not cover the global surface. Thus, researchers have turned to Earth observation satellites to obtain nearly real-time overviews of the terrestrial carbon stocks [17]. The NASA (National Aeronautics and Space Administration) GLAS (Geoscience Laser Altimeter System) instrument, on board the ICESat (Ice, Cloud and Land Elevation Satellite) records the full waveforms of detected footprints ( $\sim 65 \mathrm{~m}$ on the surface of Earth), which provide vertical information of the surface of the Earth, including vegetation $[18,19]$. The GLAS waveform has demonstrated tremendous potential in calculating forest canopy heights and biomasses even though it was designed with the primary objective of monitoring polar ice sheets. One of the distinct superiorities of GLAS data is that we are able to acquire tree heights within a single footprint, which makes it a valuable supplement to field inventories. Therefore, the ICESat potentially supports the study of terrestrial carbon resources, especially in remote areas that humans are unable to reach. However, GLAS data, which are distributed as scattered points, cannot provide spatially contiguous records. Thus, interpolation processes, such as the kriging method [20-22] or spatially continuous optical imagery, are included to produce regional forest biomass estimates [23-31].

Multispectral sensors record the information from visible light to short wave infrared light, and red and near-infrared light are sensitive to vegetation. Therefore, spectral imagery offers another resource for estimating forest aboveground biomass. Landsat sensors provide 16-day repeat coverage of the surface of the Earth with a spatial resolution of $30 \mathrm{~m}$. Numerous studies have proven the feasibility of using Landsat data to survey forest resources and monitor the changes in terrestrial carbon stocks [32-38].

NASA is planning to launch ICESat-2 and GEDI (The Global Ecosystem Dynamics Investigation) in 2018; ESA (The European Space Agency) will launch BIOMASS in 2020. These missions will provide great supports for the research of forest biomass and carbon cycle. Therefore, it is necessary to study efficient methods of evaluating forests to service further research. The main purpose of this paper was to analyze and compare the performances of the principal components model and three machine learning models, including BPANN (backpropagation artificial neuronal network), SVR (support vector regression), and RF (random forest) for estimating the forest biomass. Our basic processes were (i) to compute the forest canopy height within discrete GLAS footprints using the full waveform parameter; (ii) to link the canopy height produced by GLAS with the vegetation indexes and then establish the principal component model and machine learning models to map spatially contiguous canopy heights; and, (iii) to develop a power equation from the forest canopy heights to estimate the aboveground biomass. Finally, we compare our estimates to other existing forest aboveground biomass values in Maryland and explain why our method has potential at larger scales. 


\section{Study Area and Datasets}

\subsection{Study Area}

Maryland $\left(37.8^{\circ} \mathrm{N}-39.8^{\circ} \mathrm{N}, 75.0^{\circ} \mathrm{W}-79.5^{\circ} \mathrm{W}\right)$ lies in the mid-Atlantic region of the United States, bordering Virginia and Washington to the south and west and covering a land area of $\sim 25,600 \mathrm{~km}^{2}$ [39]. The east of Maryland is relatively flat with elevations from 0-200 $\mathrm{m}$ and has a humid subtropical climate, with hot, humid summers and short, cool winters, while the opposite region around the Appalachian Mountains is higher and more complicated with elevations from 150-900 m and has a humid continental climate. The rainfall in Maryland ranges from 890-1140 mm and is even greater at higher elevations. Maryland is divided into 24 counties when considering the political functions, and it can also be separated into nine physical regions when natural elements, such as location, topography, and climate are concerned (Figure 1a). Overall, Maryland has a wide array of climates due to the variances in the elevation and proximity to water.

Maryland possesses abundant forest resources, and the forested lands account for $42.8 \%$ of the total land cover in spite of the urbanization that occurred by 2000 [40]. From the forest distribution map produced by the MRLC (Multi-Resolution Land Characteristics Consortium (Figure 1b)), it is apparent that Maryland is dominated by deciduous forest apart from the evergreen forests in the southeast and there are only minimal mixed forests in the western shore upland region. It is the wide variabilities in the topography and natural environment and the abundant forest resources that make Maryland a suitable place to study forest biomass.
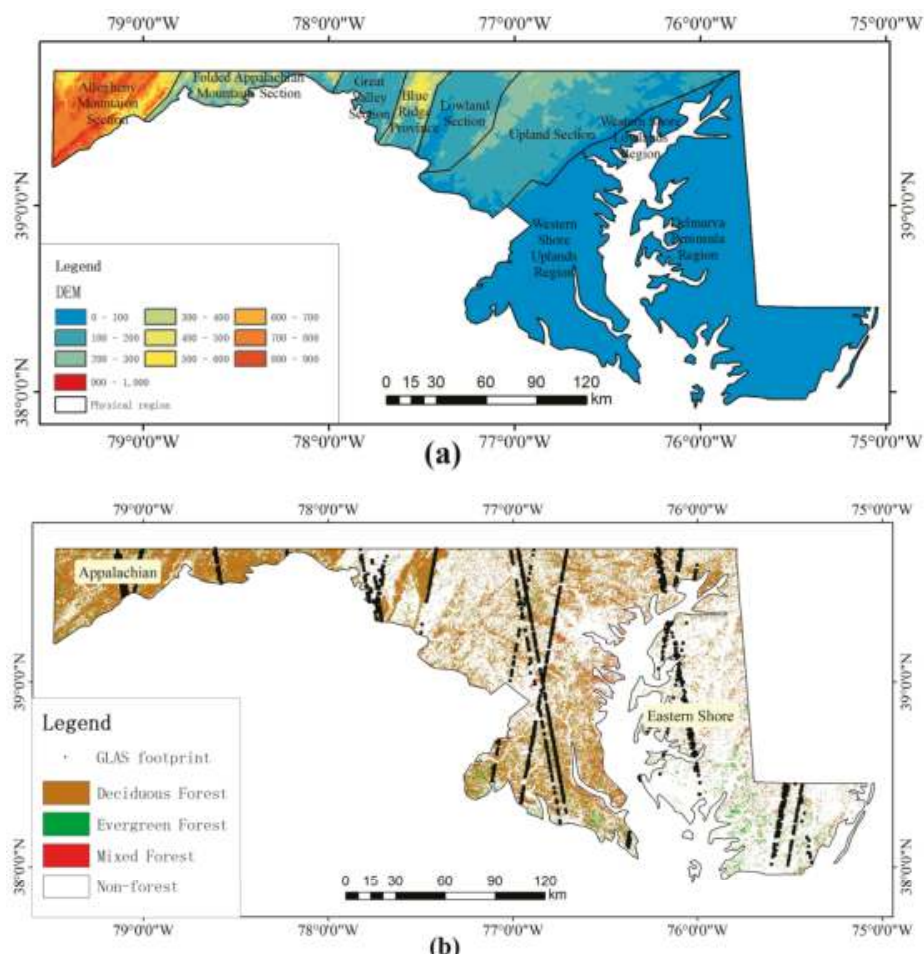

(b)

Figure 1. Overall introduction to the study area. (a) The elevation map and the distribution of the physical regions. (b) The distribution of the forest in Maryland and Geoscience Laser Altimeter System (GLAS) footprints located in forest areas. 


\subsection{ICESat GLAS Laser Altimetry Data}

The GLAS instrument was mounted on the Ice, Cloud and Land Elevation Satellite (ICESat) and was the first satellite-based earth orbiting laser altimeter that was part of the Earth Observing System (EOS) of NASA and was operational from January 2003 to October 2009 [41]. Even though the primary purpose of the ICESat mission was to take ice sheet elevation measurements in the polar regions [42], GLAS data have been widely applied to accurately map regional, continental, and global vegetation heights and carbon volumes with active pulse emission/reception [43]. The laser altimeters provide the range between the spacecraft and the illuminated spot on the surface of the Earth by measuring the round-trip travel time of the laser pulse. The GLAS sensor acquires altimetry information at $1064 \mathrm{~nm}$ with $40 \mathrm{~Hz}$ lasers, which consequently leave nominal $\sim 65$ m diameter footprints and $\sim 170$ m intervals between spots [44].

GLAS laser altimeter data can be downloaded from the National Snow and Ice Data Center (NSIDC) website (https://nsidc.org/data/icesat). The database provides 15 Level-1 and Level-2 data products (GLA01 to GLA15), and the GLA01 and GLA14 products were used in this study. The instrument determines the range by measuring the time between the emission of the laser pulse and the detection of the photons reflected from the surface of the Earth. The detected pulse is digitized in $1 \mathrm{~ns}(15 \mathrm{~cm})$ range bins. From a total of 1000 selected range bins, a smaller number 544 over land and ice sheets and 200 over oceans and sea ice regions was selected for transmission. The corresponding range widths of the transmissions were $81.5 \mathrm{~m}$ and $30 \mathrm{~m}$. What requires more attention is that the size from 1 to 151 bins is prolonged to $4 \mathrm{~ns}(60 \mathrm{~cm})$, starting from L3A; therefore, the length of the corresponding waveform increases to $150 \mathrm{~m}[45,46]$. GLA01 is a level 1A product and records the actual waveform. GLA14 is a level 2 land product and contains other crucial information on the footprints, such as the elevation (i_elev), latitude (i_lat), longitude (i_lon), transmission time of the first shot in the frame in J2000 (i_UTMTime), start and end range increment of the signal (i_SigBegOff, i_SigEndOff), signal saturation index (i_satNdx), standard deviation of the background noise (i_sDevNsObl), max amplitude of the received echo (i_maxRecAmp), likely presence of clouds (i_Frir_qaFlag), amplitude of the Gaussian distribution (i_Gamp), sigma of the Gaussian distribution (i_Gsigma), and the centroid range increment for the Gaussian fits (gpCntRngOFF). Sun et al. demonstrate that the six Gaussian distributions have a strong relationship with the vertical features of the forest and the underlying topography [47].

In this study, we downloaded all year round GLAS data in Maryland from 2005-2008 to maintain consistency with the validation data. Then, we extracted the data from the raw GLAS file using the IDL tools provided by the NSIDC. Next, it was necessary to filter out invalid data according to the parameters of GLA14. We deleted all of the contaminated points when i_satNdx exceeded 2 or the SNR (signal-to-noise ratio $=$ i_maxRecAmp $/ \mathrm{i} \_s D e v N s O b l$ ) was less than 60 . At the same time, we only retained the cloud-free footprint when the i_Frir_qaFlag was equal to 15. We also used NLCD 2011 (National Land Cover Database 2011) to select points, which were within forest areas in Maryland. Finally, the selected dataset contained a total of 2720 valid GLAS records (Figure 1b). The laser campaigns we used in this study included 3B, 3C, 3D, 3E, 3G, 3H, 3I, 3J, and 3K. The ICESat elevation corresponded to the TOPEX/Poseidon ellipsoid; so, we converted it into WGS84 to maintain consistency with other data. After that, the GLAS waveforms needed to be filtered using wavelet transformation to eliminate sharp noise. Furthermore, a critical step was to decompose the GLAS waveform into multiple Gaussian distribution curves, as described in previous studies $[17,47,48]$. Finally, we quantified the background noise by calculating the mean value and standard deviation of the background signal, and then we set thresholds to identify the start and end of the signal. The start noise and end noise presented different characteristics, so we dealt with them separately. Usually, the threshold is defined as the mean value plus $\mathrm{n}$ times of the standard deviation. However, the value of $\mathrm{n}$ varies in different studies $(\mathrm{n}=3$ [47], $n=3.5$ [49], $n=4$ [50], $n=4.5$ [51]). In this study, $n=3$ was used. Furthermore, we also calculated the slope index [52] within each footprint to reduce the impact of terrain. 


\subsection{CMS_RF Forest Canopy Height and Aboveground Biomass for Maryland}

The CMS (Carbon Monitoring System) forest canopy height and aboveground biomass data, as part of the NASA CMS plan, are accessible from the ORNL DAAC (The Oak Ridge National Laboratory Distributed Active Archive Center) CMS website (https:/ / daac.ornl.gov / cgi-bin/dsviewer. pl?ds_id=1320). The NASA CMS is designed to make significant contributions to characterizing, quantifying, and predicting the evolution of global carbon sources and sinks by improving the monitoring of carbon stocks and fluxes. This dataset provided a 30-m gridded estimation of canopy height and aboveground biomass for the state of Maryland in 2011 [53]. The tree canopy height was modeled by high-resolution (one meter) airborne LiDAR, which is then aggregated to a height distribution of $30 \mathrm{~m}$ [54]. The aboveground biomass map was produced by combining the leaf-off LiDAR data with file allometric estimates from 848 variable plots across Maryland. Field-based estimates were then related to the LiDAR height and volume metrics using random forest regression models to generate a statewide biomass map. The aboveground biomass map explained $\sim 50 \%$ of the variability when compared with the field plots $\left(\mathrm{R}^{2}=0.49, \mathrm{RMSE}=89.3 \mathrm{Mg} \mathrm{ha}^{-1}, \mathrm{n}=848\right)$, and was then cross validated with FIA (forest inventory and national analysis program) data that showed better agreement $\left(\mathrm{R}^{2}=0.69, \mathrm{RMSE}=58.2 \mathrm{Mg} \mathrm{ha}^{-1}, \mathrm{n}=1055\right)$. More details of the canopy height and aboveground biomass are available in $[39,55]$.

\subsection{Landsat Data}

The Landsat program is co-managed by NASA and USGS (United States Geological Survey) and provides the longest continuous records to help policymakers and land managers to make wise and farsighted decisions regarding global resources and the environment. Multi-temporal data ensures the possibility of downloading cloud-free, high quality, and atmospherically uncontaminated imageries covering the entire state [56]. The TM (Thematic Mapper) and ETM+ (Enhanced Thematic Mapper Plus) data record information from visible to infrared light, which has been proven in previous studies as qualified to distinguish different land cover types or even vegetation types with different volumes [57-61]. Powell et al. modeled live aboveground biomass using Landsat satellite imagery and derived the trajectories of the biomass dynamics in Arizona and Minnesota [62]. Liu et al. built an algorithm to estimate the forest aboveground biomass using TM data, which provided a reliable estimate $\left(\mathrm{R}^{2}=0.71, \mathrm{RMSE}=39.60 \mathrm{Mg} \mathrm{ha}^{-1}\right)$ in the Changbai Mountains [24].

We downloaded surface reflectance data products that had been atmospherically corrected, from the USGS website (https:earthexplorer.usgs.gov). In this study, the data included six scenes of TM imagery (P14R33_20090521, P14R34_20090521, P15R32_20110603, P15R33_20100429, P16R32_20090519, and P16R33_20090519) and two scenes of ETM+ imagery (P17R32_20090518, and P17R33_20090518), which were acquired during the vegetation growing period in this study. The products were mapped in World Geodetic System (WGS) 84 datum and the Universal Transverse Mercator (UTM) projection.

\subsection{National Land Cover Database 2011}

We downloaded the NLCD 2011 from https:/ / www.mrlc.gov.nlcd2011.php. The data are the most recent national land cover product created by the MRLC Consortium. The product uses Landsat imagery as the primary data resource and has a spatial resolution of $30 \mathrm{~m}$. The database classifies the national surface into 20 land cover types, and deciduous forest, evergreen forest, and mixed forest constitute the American forest. The overall accuracies of NLCD 2011 was 88\% [63]. The NLCD 2011 has been recognized as an important source to analyze carbon cycle problems within certain land cover types at both the regional and national scales [64]. The CMS_RF forest canopy height and the aboveground biomass introduced in Section 2.3 were also calculated with the help of the NLCD. 


\section{Methods}

\subsection{Estimating Forest Canopy Height from the GLAS Waveform}

After the preprocessing steps described in Section 2.2, we extracted several key variables (Figure 2) needed to calculate the forest canopy height and biomass from the GLAS waveforms. Even though the GLA14 product contains information that can be used to identify these critical parameters, we chose to define the key positions through a series of algorithms when considering the underlying noise in the GLA14 data [49]. The total waveform length, which is the distance from signal start to signal end, contains all of the vertical height information in the vegetation region and even includes some topographic slope noise [18]. The signal start and end were determined using the start and end thresholds acquired by the preprocessing steps. The last peak was regarded as the ground peak only if the amplitude was higher than those of the nearby peaks and the distance between the selected peak and the signal end exceeded the half width of the laser pulse $(60 \mathrm{~cm})[18,65,66]$. The centroid of the waveform records the half energy from the signal start to the ground peak. The trailing edge extent was determined as the difference between the signal end and the last bin where the signal intensity of the waveform is half the maximum intensity. In this study, we calculated the distance from the signal start to the ground peak as the canopy height, and then added the terrain index to optimize the canopy height within GLAS footprint.

An approach developed by Mahoney [52] for calculating the slope from GLAS was applied for terrain correction. We used a simple way to calculate the slope $(\theta)$ within each GLAS footprint with Equation (1).

$$
\theta=\operatorname{atan}\left(\frac{2 * \operatorname{Trail}_{\text {ext }}}{d}\right)
$$

where Trail ext $_{\text {is }}$ the trailing edge extent; and $d$ is the mean diameter for the GLAS footprint.

In this process, Trail $l_{\text {ext }}$ was directly used as the terrain index. We built a linear formula to combine the maximum canopy height and terrain index to improve the accuracy of the estimated canopy height.

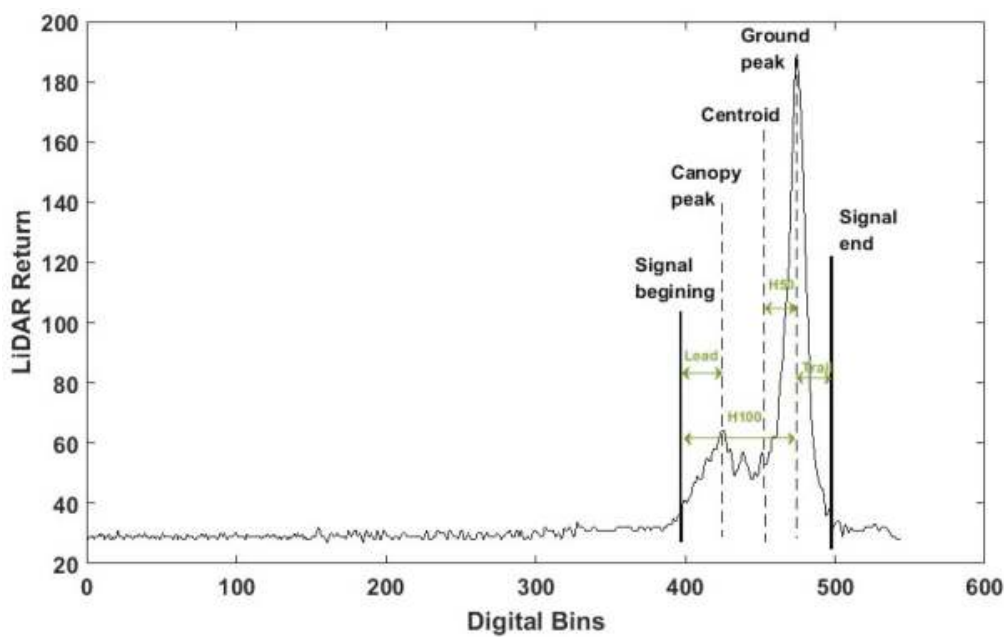

Figure 2. A typical waveform profile of a GLAS shot in Maryland.

\subsection{Extrapolating Canopy Height to the Study Area}

Using the GLAS waveform to supplement field inventory data is a significant method to evaluate the forest volume and carbon stocks at regional and global scales. However, the GLAS data are scattered over the study area, so it is necessary to combine these data with spectral imageries (Landsat 
imageries) to produce a spatially contiguous canopy height map. The size of a GLAS footprint is approximately $65 \mathrm{~m}$, while the resolution of Landsat imagery is $30 \mathrm{~m}$. Thus, we upscaled the spectral data to $60 \mathrm{~m}$, and then built relationships between the forest canopy height and the vegetation indexes. Next, we applied the relationships to the non-upscaled imageries. In total, we selected five vegetation indexes, the NDVI (normalized difference vegetation index) [67-69], EVI (enhanced vegetation index) [68], RVI (ratio vegetation index) [70], DVI (difference vegetation index) [69], and the VARI (visible atmospherically resistant index) [71].

In order to take into account all the vegetation indexes, we conducted PCA (principal components analysis), machine learning models (BPANN, SVR, and RF) to estimate the spatially contiguous canopy height.

PCA is a statistical procedure that uses an orthogonal transformation to convert a set of observations of possibly correlated variables into a set of values of linearly uncorrelated variables called principal components. These new components are linear combinations of the original variables [72-74]. The first principal component has the largest possible variance (that is, accounts for as much of the variability in the data as possible), and each succeeding component in turn has the highest variance possible under the constraint that it is orthogonal to the preceding components. The resulting vectors are an uncorrelated orthogonal basis set. Thus, PCA is often used in data reduction to identify a small number of factors that can explain most of the variances.

The BPANN algorithm consists of forward-propagation of the data stream and back-propagation of the error signal. For the forward propagation, the direction is input layer $\rightarrow$ hidden layer $\rightarrow$ output layer, and each layer determines the next layer. If the results fail to meet the expected output, the error signal helps adjust the data stream in reverse [75]. BPANN is sensitive to the number of neurons in hidden layers and the type of activation functions for the layers [76]. In this process, Matlab toolboxes were used to train the models. We chose three categories of transfer functions: the tansig (hyperbolic tangent sigmoid), logsig (log-sigmoid), and purelin (linear) transfer function. The tansig transfer function can produce both positive and negative values, which tends to yield faster training than the logsig transfer function, which produces only positive values. In terms of training algorithms, we chose traingd, traingdx, trainingda, trainrp, trainlm, trainbfg, trainscg, trainoss, traincgf, and traincgp. More detailed information of these training algorithms is available in [77].

The SVR is a version of SVM (support vector machine) for regression. Its basic idea is to minimize the structural risk and keep the target as flat as possible [78]. The main design of a SVR model is the kernel, which is an inner product in the SVR feature space. Since inner products induce distance metrics and vice versa, the basic goal in SVR kernel design is to find an appropriate metric in the SVR feature space relevant to the regression problem [79]. The Libsvm toolbox supports two kinds of formulations for regression. In this study, we trained $v$-SVR [80] and $\varepsilon$-SVR [81], and chose linear, polynomial, and RBF (Radial Basis Function) kernels.

Random forest is an ensemble learning method that constructs a multitude of decision trees and then outputs a mean prediction regression of the individual trees [82]. RF takes advantage of two powerful machine learning techniques: bagging and random feature selection. In bagging, each tree is trained on a bootstrap sample of the training data, and predictions are determined by the majority vote of the trees. It performs a type of cross-validation in parallel using the so-called out-of-bag samples to assess the predictions [83]. In this research, we changed the number of trees and the features randomly selected in each node of each tree in order to optimize the model.

Furthermore, to avoid overfitting, we conducted 10-fold cross validation to analyze the result of each model.

\subsection{Estimating Forest Aboveground Biomass in Maryland}

Forest canopy height provides a crucial indicator to calculate the forest aboveground biomass. Several studies have studied the relationships between canopy height and biomass $[1,51,84]$. In this section, we extracted forest canopy height and biomass from CMS maps within GLAS shot locations, 
and then developed a power model from forest canopy height to biomass. Similarly, 1800 points were used to establish the model and 920 points were used to validate the model.

\section{Results}

\subsection{GLAS Waveform to Forest Canopy Height}

After preprocessing the GLAS waveform data and identifying the key parameters, we obtained 2720 forest canopy heights in Maryland. Figure 3a shows the results of the simple canopy height method $\left(\mathrm{R}^{2}=0.606, \mathrm{RMSE}=4.78 \mathrm{~m}, \mathrm{MRE}=15.9 \%\right)$.

Furthermore, we also used 1820 points to establish a linear empirical relationship (Equation (2)) in order to optimize canopy height $(\mathrm{H})$.

$$
\mathrm{H}=H_{\max }-0.73 * \text { Trail }_{\text {ext }}
$$

where $H_{\text {max }}$ presents the distance between the signal start and ground peak.

Figure $3 \mathrm{~b}$ illustrates the evaluation result of the terrain corrected canopy height by the remaining 900 points $\left(\mathrm{R}^{2}=0.669, \mathrm{RMSE}=4.82 \mathrm{~m}, \mathrm{MRE}=15.4 \%\right)$. This result indicates that the method in this study was qualified and reliable, and terrain correction could improve the estimation accuracy. In previous studies, Lee et al. [85] and Cao et al. [86] conducted slope correction by $\mathrm{H}=H_{\max }-0.5 * d * \tan (\theta)$, which was similar to ours. Therefore, we considered the equation to be reliable for the slope correction.

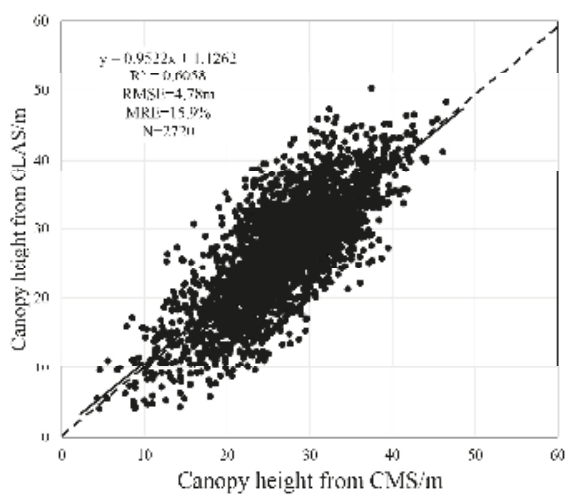

(a)

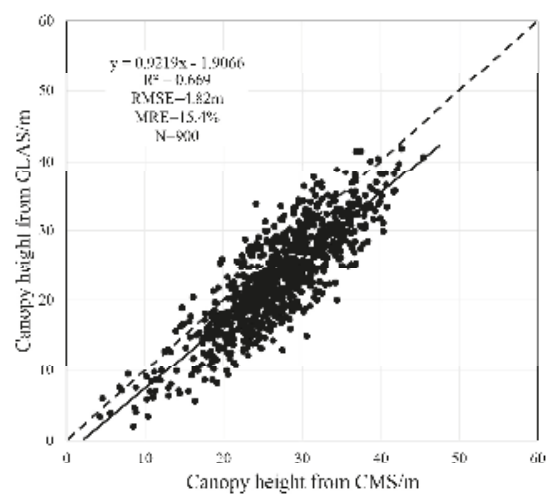

(b)

Figure 3. The evaluation of estimated canopy height by the GLAS waveform. (a) The evaluation of canopy height without slope correction; and, (b) The evaluation of canopy height with slope correction.

\subsection{Extrapolating Canopy Height to the Study Area}

When considering the high correlations between vegetation indexes, PCA was first conducted to reduce the number of variances. We extracted the principal components if the corresponding eigenvalue exceeded 1 . Finally, only the first principal component was selected. Figure 4 shows the relationship between the first principal component and canopy height. 


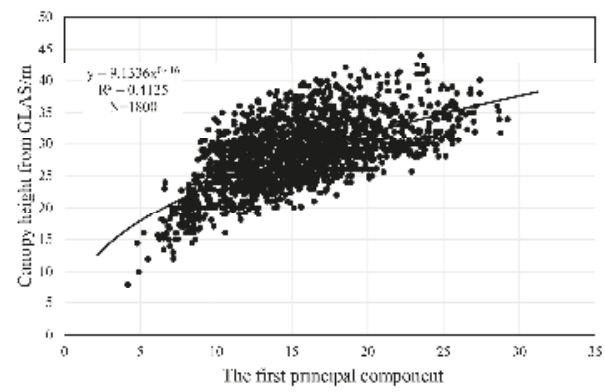

(a)

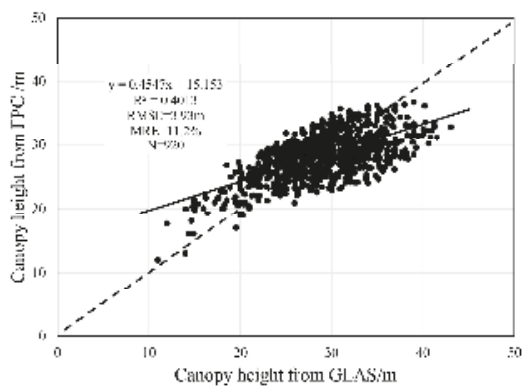

(b)

Figure 4. PCA analysis results. (a) Power model; and, (b) Evaluation of this model.

We also trained 22 BPANN models, four SVR models, and 11 RF models to estimate the forest canopy height. Appendix A Table A1 summarizes the BPANN performances $\left(R^{2}\right)$ using different numbers of neurons, transfer functions, and training algorithms. Generally, all of the BPANN models obtained similar performance results. It can be seen that eight neurons-model performed best, while 50 neurons-model gained a poor result. The different transfer functions and training algorithms seemingly had few effects on the results, except for the training algorithm of traingd. The best BPANN model was constructed by eight neurons, logsig, and purelin transfer functions, and a trainlm training algorithm. Appendix A Table A2 illustrates the SVR performances using different SVR formulations and kernels. This showed that these SVR models obtained nearly the same performances, and the model with $\varepsilon$-SVR, RBF kernel performed better than the others. Similarly, few variations were seen in different RF models. Appendix A Table A3 demonstrates that the more trees gained better results, and when the number of features randomly selected in each node of each tree was 2, the result was better.

Next, we respectively chose the best model to analyze the performances of three machine learning models. The evaluation results (Figure 5) indicated that machine learning models could potentially be used to estimate forest canopy heights from spectral imagery. The three methods performed similarly with $R^{2}$ values from 0.42 to 0.46 , RMSE values from $3.71 \mathrm{~m}$ to $4.11 \mathrm{~m}$, and MRE values from 10.6 to $11.96 \%$, and the RF model reached the most acceptable agreement with the validated canopy height from GLAS $\left(R^{2}=0.46\right.$, RMSE $\left.=3.71 \mathrm{~m}, \mathrm{MRE}=10.6 \%\right)$. However, there was a common limitation where the canopy height estimates were lower than the validated values in the high canopy height areas (greater than $35 \mathrm{~m}$ ), while the canopy height estimates were higher in the low canopy height areas (less than $15 \mathrm{~m}$ ). Namely, most estimates were inclined to fall in a median area, which usually resulted from the machine learning model.

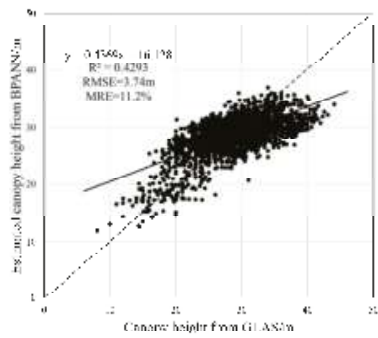

(n)

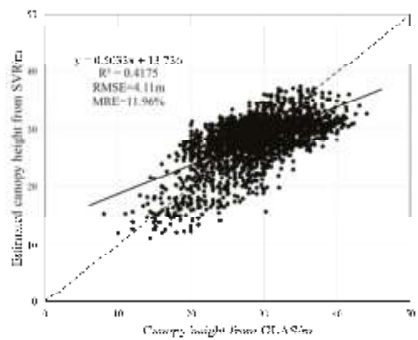

(b)

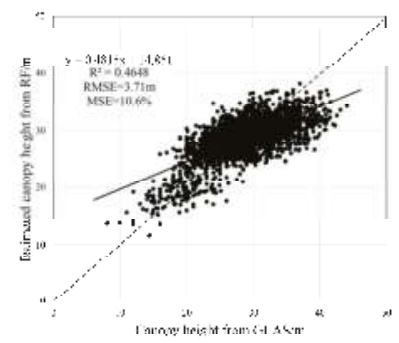

(c)

Figure 5. The evaluation results of the machine learning models. (a) Evaluation result of the BPANN model; (b) evaluation result of the SVR model; and, (c) evaluation result of the RF power model. 
Next, we applied the first principal component power model and three machine learning models to the entirety of Maryland. Figure 6 demonstrates that these four models performed similarly even though there were subtle differences. Overall, the forest canopy height was relatively high at 30-50 m within the Western Shore Uplands region and Folded Application Mountain section, while it was lower at 10-20 $\mathrm{m}$ in the Blue Ridge province and Allegheny Mountain section. The trees with extremely low canopy heights $(0-10 \mathrm{~m})$ were scattered at the edges of the forest instead of being aggregating into blocks. The Lowland and Upland sections were made up of median forest heights $(10-30 \mathrm{~m})$. From the distribution of these models, machine learning models performed better than the first principal component power model that produced canopy height concentrated at 20-40 m and showed few local variations. However, machine learning models require significant additional costs in terms of time, effort, and computational resources.

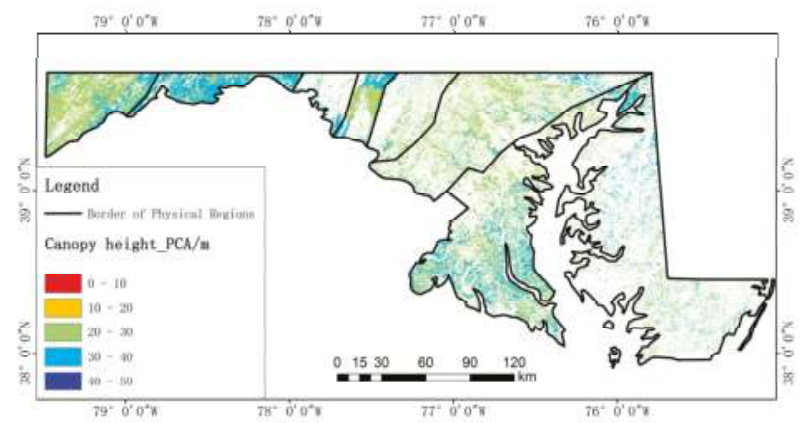

(a)

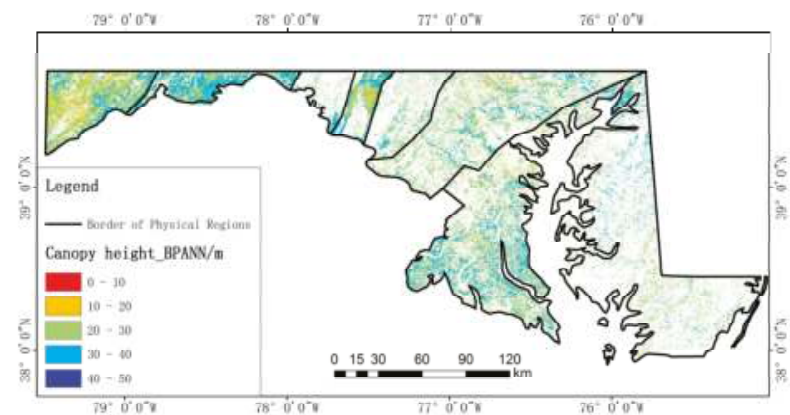

(b)

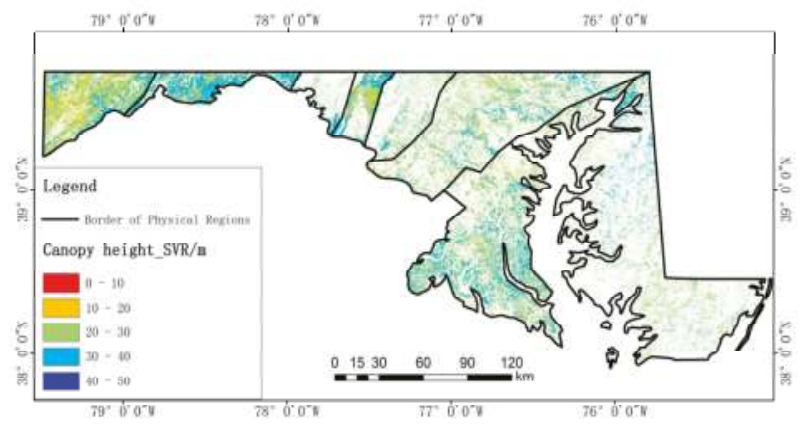

(c)

Figure 6. Cont. 


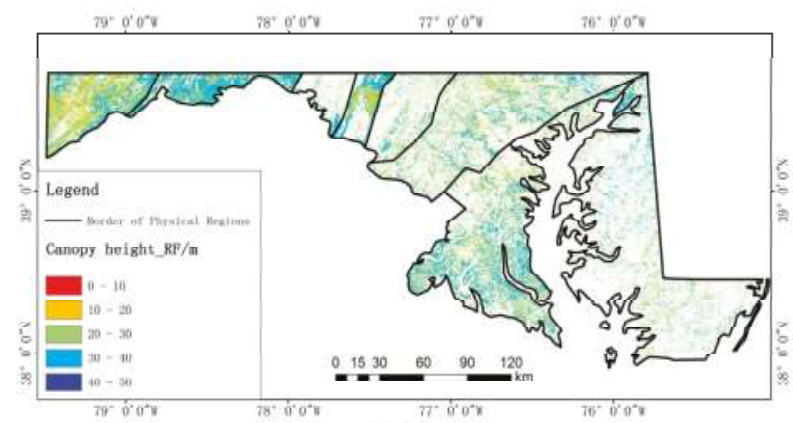

(d)

Figure 6. The distribution of forest canopy height in Maryland. (a) Forest canopy height estimated by the first principal component power model; (b) Forest canopy height estimated by the BPANN model; (c) Forest canopy height estimated by the SVR model; and (d) Forest canopy height estimated by the RF model.

\subsection{Forest Canopy Height to Aboveground Biomass}

In this section, we developed a general model to estimate forest biomass from the spatially contiguous canopy height data throughout Maryland. Figure 7 demonstrates that the biomass and canopy height in Maryland are closely linked, and the evaluation results were satisfactory $\left(R^{2}=0.70\right.$, RMSE $=35.81 \mathrm{Mg} / \mathrm{ha}, \mathrm{MRE}=17.0 \%$ ). Cao [86] and Lefsky [50] discussed biomass estimation models in their studies, and our equation was similar to those in the previous studies. Therefore, we considered the equation to be valuable and reliable for biomass estimation in Maryland.

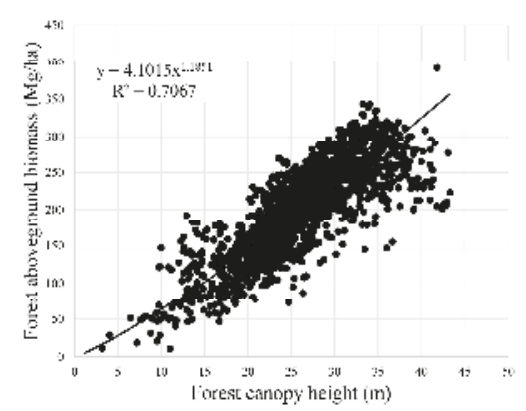

(a)

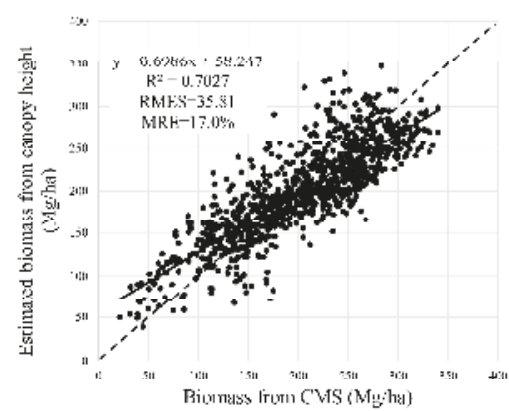

(b)

Figure 7. Forest aboveground biomass model and the evaluation results. (a) Power model to estimate forest aboveground biomass; and, (b) Evaluation result of the biomass estimation model.

As shown in Figure 8, the forest aboveground biomass ranged from $0-400 \mathrm{Mg} / \mathrm{ha}$. As we estimated the forest aboveground biomass using a direct, univariate power model, the distribution of the biomass resembled the canopy heights. The forest biomass in the Western Shore Uplands region and Folded Application Mountain section was higher. In contrast, the lower biomass forests were distributed in the Blue Ridge province and Allegheny Mountain regions. Similarly, the first principal component model failed to distinguish the high biomass and low biomass areas. After taking the model evaluation results described in Section 3.2 and the forest biomass distribution described in this section into comprehensive consideration, we suggest that the RF estimate was the best. 


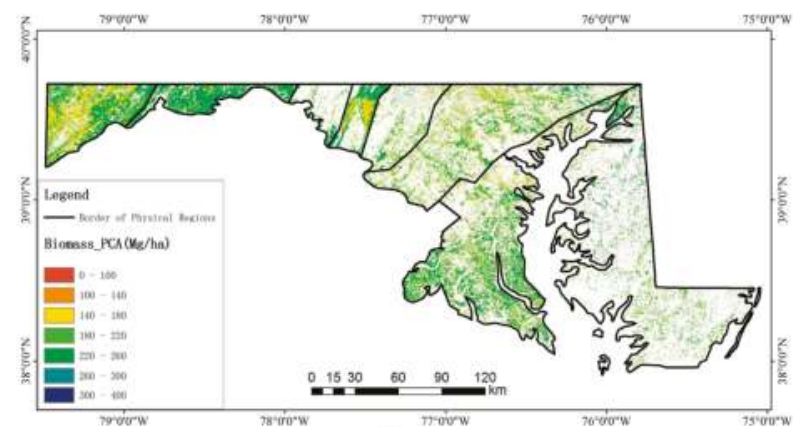

(a)

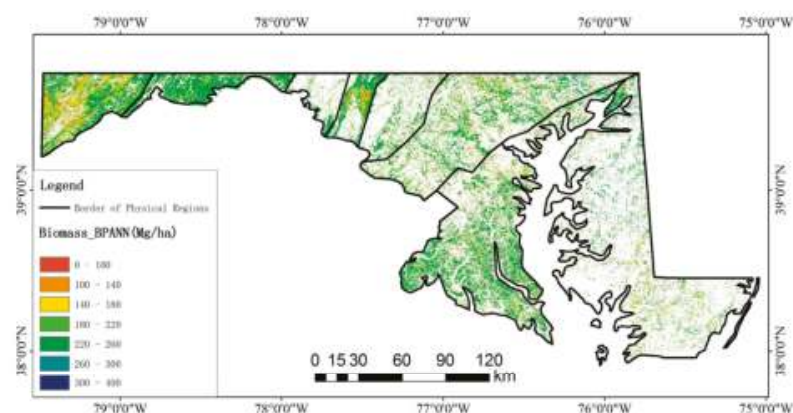

(b)

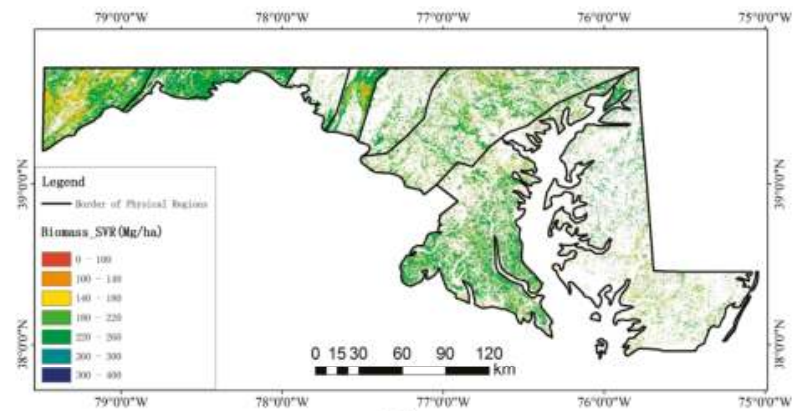

(c)

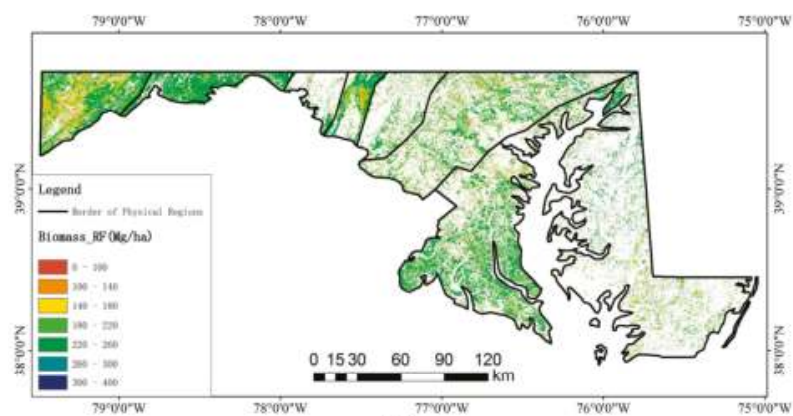

(d)

Figure 8. Cont. 


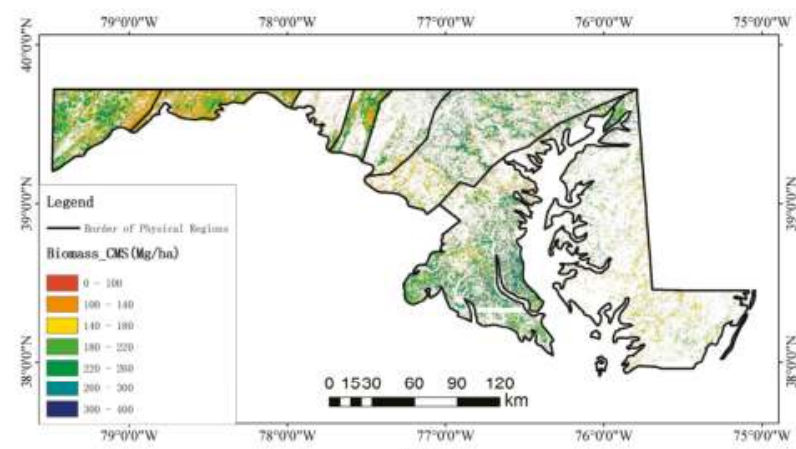

(e)

Figure 8. The distribution of forest aboveground biomass in Maryland. (a) Forest aboveground biomass estimated by the PCA power model; (b) Forest aboveground biomass estimated by the BP-ANN model; (c) Forest aboveground biomass estimated by the SVR model; (d) Forest aboveground biomass estimated by the RF model; and, (e) Forest aboveground biomass estimated by the CMS.

\subsection{Comparing Biomass Estimates to Other Data Resources}

In this section, we compared our forest biomass maps with Biomass_CMS in terms of the distribution and quantity. As introduced in Section 2.3, the forest Biomass_CMS in Maryland was produced by the NASA CMS using LiDAR and allometric estimates with small footprints. Overall, our biomass estimates shared a similar distribution with Biomass_CMS, where higher values were obtained in the Western Shore Uplands region, while lower values were located in Blue Ridge province and Allegheny Mountain region. However, the Biomass_CMS appeared more scattered with low biomass flecks due to the land cover errors. The non-forest, which was mistakenly regarded as forest, may be identified as high biomass, according to the spectral information (the biomass data source in this study), while the Biomass_CMS was small when considering the lower airborne LiDAR values (the Biomass_CMS data source). Figure 9 demonstrates the distribution of forest biomass difference between the biomass estimated by RF and Biomass_CMS in Maryland. It can be seen that 53\% of the absolute difference of forest biomass were less than $50 \mathrm{Mg} / \mathrm{ha}$, and $85 \%$ for $100 \mathrm{Mg} / \mathrm{ha}$. In this map, positive values represent that the Biomass_RF exceeded the Biomass_CMS. Our method overvalued the forest biomass in the Folded Application Mountain section and southern areas in the Upland section. The areas where the difference exceeded $100 \mathrm{Mg} /$ ha took up around $10 \%$ of the total forest in Maryland. On the other hand, our model underestimated the forest biomass in some small areas of the Allegheny Mountain region, Upland section, and Western Shore Upland region.

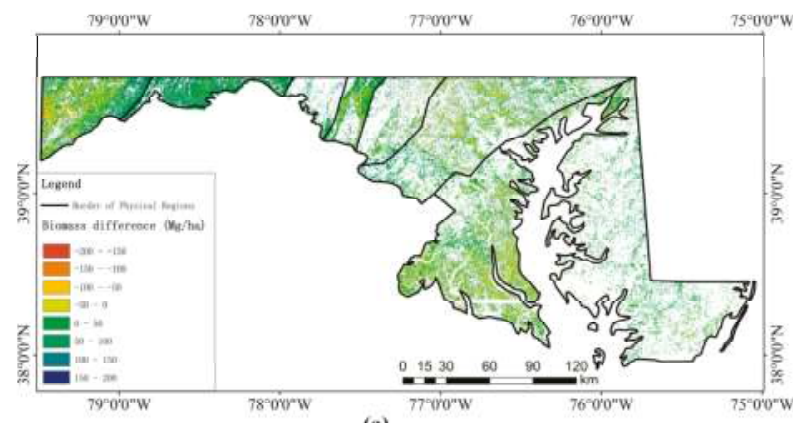

(a)

Figure 9. Cont. 


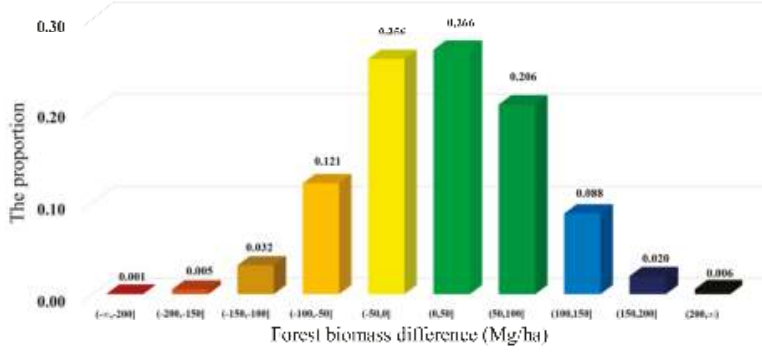

(b)

Figure 9. The results of forest biomass difference. (a) The map of biomass difference in Maryland; (b) The statistical result of biomass difference.

Next, the forest biomass in each physical region and political county were statistically compared to quantitatively analyze the biomass distribution. Figure 10 presents the forest aboveground biomass $(\mathrm{Tg})$ in each county and physical region and the total values throughout Maryland. The estimates from all of the models had parallel tendencies. Garrett and Allegany counties had the most abundant forest resources (more than $15 \mathrm{Tg}$ ) due to the large forest areas, followed by Charles, Baltimore, and Frederick counties (more than $10 \mathrm{Tg}$ ). In contrast, there were five counties (Worcester, Talbot, Dorchester, Somerset, and Baltimore City) where the forest aboveground biomass was less than $3 \mathrm{Tg}$. When analyzing the biomass based on the physical regions, distinct characteristics were recognized. The forest biomass in the Western Shore Uplands region (approximately $45 \mathrm{Tg}$ ) constituted $30 \%$ of the total forest aboveground biomass in Maryland, which was far more than that in other regions. In contrast, the Great Valley section and the Western Shore Lowlands region contained the least forest, around 3Tg. Following the Western Shore Uplands region, the upland section had the second highest forest biomass (nearly $35 \mathrm{Tg}$ ). Then, the Allegheny Mountain section, Delmarva Lowlands region, and Folded Appalachian Mountains section followed, where the forest resources were similarly rich (approximately $20 \mathrm{Tg}$ ). In addition, the Blue Ridge Province and the Lowland section made petty contributions to the forest biomass in Maryland.

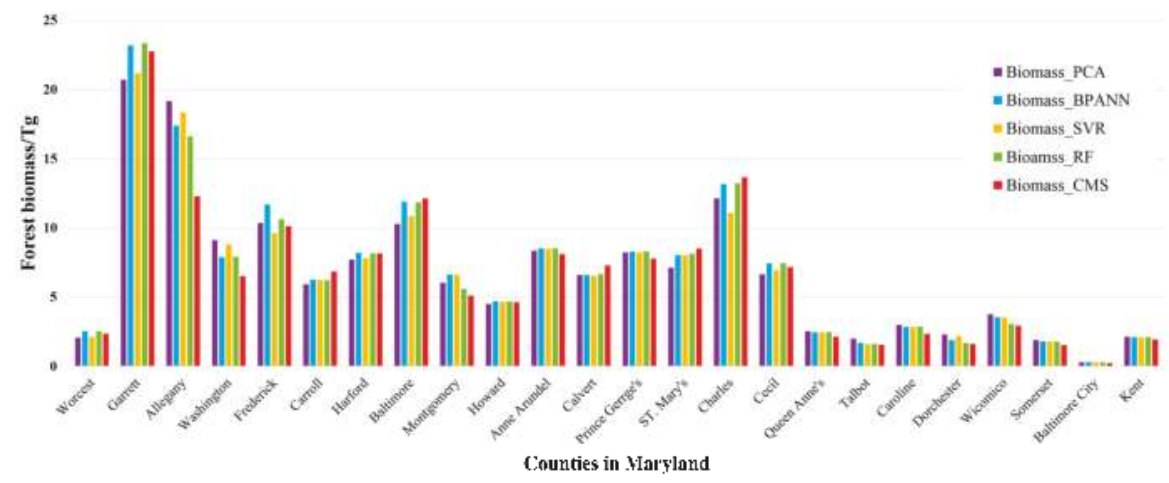

(a)

Figure 10. Cont. 


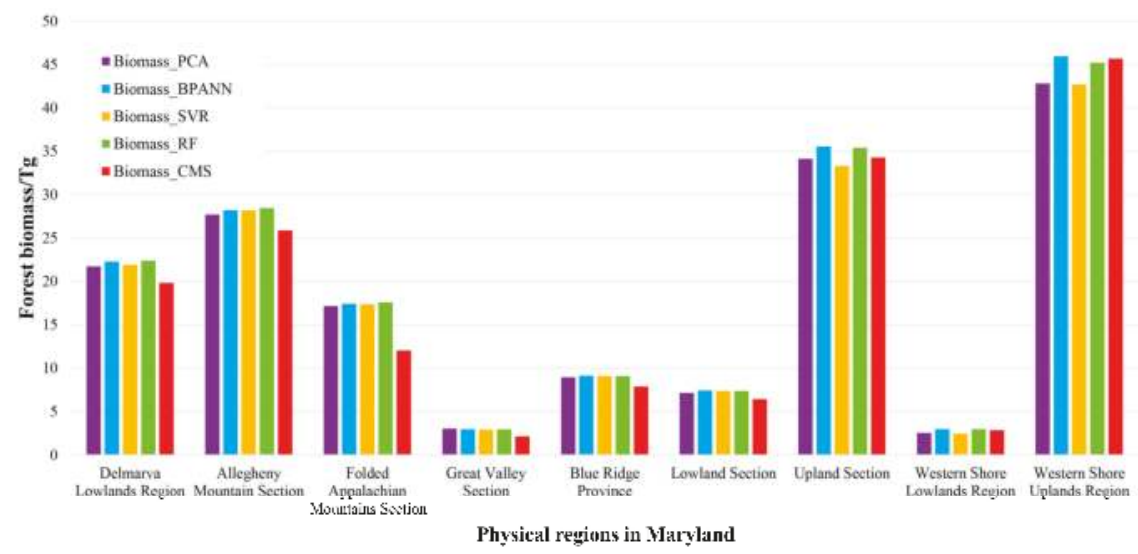

(b)

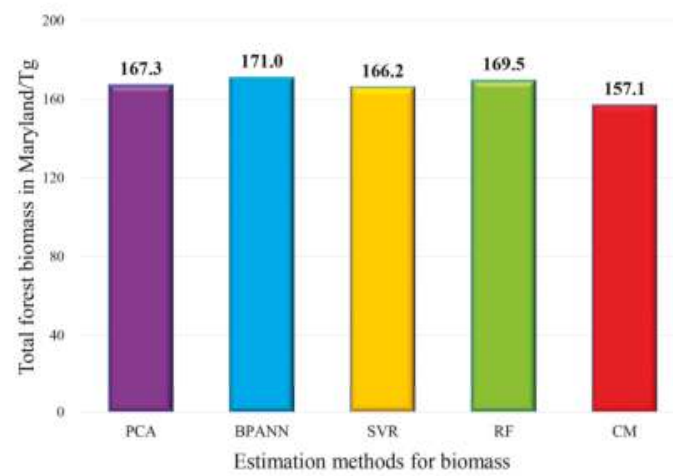

(c)

Figure 10. The forest aboveground biomass in Maryland. (a) Statistical forest biomass values of each county; (b) statistical forest biomass values of each physical region; and (c) the total biomass estimated by all models.

The total biomass in the state of Maryland reached approximately $160 \mathrm{Tg}$ (Figure 10). Generally, our models all overvalued the total forest biomass in Maryland. The BPANN method gained the highest estimate with $171 \mathrm{Tg}$, while the SVR estimate was comparatively lower with $166.2 \mathrm{Tg}$, which most likely resulted from the underestimation of some areas.

\section{Discussion}

We estimated the forest canopy height and aboveground biomass in the state of Maryland by combining spaceborne LiDAR, spectral imageries, and forest resource data produced by NASA. The results showed the capacity of our approach for regional-scale forest biomass estimation and allowed us to evaluate the various sources of error and uncertainty.

\subsection{Processing the GLAS Waveform}

The GLAS waveform provides a crucial approach to calculate forest parameters, such as canopy height, especially without any tree metrics. Even though the GLAS records the waveform along only the narrow flight lines, it still presented a strong ability to extract valuable forest parameters, which 
has also been proven in a large number of studies. We estimated the canopy height from GLAS by calculating the distance from the start of the signal to the ground peak and added the terrain index to optimize the canopy height. Then, a regression was run between the estimated canopy height from the GLAS waveform and the canopy height from the NASA CMS with an acceptable result $\left(R^{2}=0.669\right.$, RMSE $=4.82 \mathrm{~m}, \mathrm{MRE}=15.4 \%$ ). Nevertheless, the result also suggests that future efforts need to focus on forming a better relationship.

In Section 3.1, we introduced a series of Gaussian parameters within the GLA14 product that could be used to estimate the canopy height. Gaussian parameters always include mass noise, so they may fail to reliably estimate the canopy height. Thus, we utilized a different method in this study. The procedure comprised preprocessing the GLAS waveform, identifying critical parameters, and, adding the terrain index to optimize canopy height. Even though we filtered out invalid GLAS points, some still failed to produce the desired canopy height. The error may be caused by the fact that each GLAS laser had different pulse intensities. If we deal with all of the GLAS data using the same method, some may lead to errors. We can separately process waveform according to GLAS laser in future study. Furthermore, to gain a large number of the GLAS data, we did not filter out the points that were recorded within leaf off periods. This may be another error source.

\subsection{Extrapolating to A Larger Spatial Scale with Spectral Images}

The extrapolation process described in this article was used to establish the first component model and machine learning models between the canopy height estimated from GLAS and the vegetation indexes from spectral imageries. Therefore, the quality of the spatially contiguous canopy height heavily depended on the spectral imageries. In this study, we first upscaled the Landsat data to $60 \mathrm{~m}$ to keep it spatially consistent with the GLAS waveform data, and then the model that was established at the upscaled resolution was used to estimate the spatially continuous canopy height at the original resolution of $30 \mathrm{~m}$. A scale effect may exist between the remote sensing data of different spatial resolutions, which might cause an error when applying the relationship built from $60 \mathrm{~m}$ resolution data to $30 \mathrm{~m}$ resolution data. The study area was covered by eight Landsat imagery scenes. Even though we selected images that were as close as possible during the vegetation growing season, there were still variations in the acquisition time. Due to the different phenophases of the forest in different images, the vegetation index that corresponded to the same biomass value might present different records in different images. That is, using the same formula to estimate biomass over multiple scene images is likely to introduce an error. Furthermore, the difference in the spectral bandpass of Landsat 5 and Landsat7 may introduce errors, as it leads to a difference performance between the indices that we used to estimate forest biomass. Landsat data also contains the information of SWIR (short wave infrared), which supports the detection of vegetation sensitivity, so it is worthwhile adding SWIR data into the estimation models in further study.

\subsection{Linking Forest Canopy Height to Biomass}

In this study, the forest aboveground biomass was calculated through a power equation that is associated with canopy height. The evaluation result $\left(R^{2}=0.70, \mathrm{RMSE}=35.81 \mathrm{Mg} / \mathrm{ha}, \mathrm{MRE}=17.0 \%\right)$ proved the equation to be reliable when producing a biomass map in Maryland. Our biomass estimation formula agreed well with previous studies [50,86]. However, the CMS maps error, as described in Section 2.3, will reduce the accuracy of our model. Apart from the forest canopy height, the forest aboveground biomass also maintained a close relationship with the DBH [9]. We can improve the accuracy by adding other remote sensing parameters that are related to $\mathrm{DBH}$, such as LAI in future research.

\subsection{Future Development}

The GLAS instrument offers an unprecedented opportunity to estimate canopy height and biomass without field-measured trees metrics. Even though its principal objective was to measure ice 
sheet elevation change and sea ice thickness, the GLAS products were also widely applied to monitor land and vegetation. In this article, we only focused on only the vegetation within Maryland where the forest is relatively homogeneous. In future studies, an improved method will be put forward to compute the forest biomass at the continental or even global scales. Fortunately, NASA is planning to launch ICESat-2 and GEDI in 2018, and ESA will launch BIOMASS in 2020. With these data, researchers will have free access to the latest valuable data for assessing terrestrial forest changes.

\section{Conclusions}

This study demonstrated that the combination of the GLAS waveform and Landsat imagery could be used to monitor the forest aboveground biomass in Maryland. The total forest aboveground biomass in Maryland reached approximately $160 \mathrm{Tg}$. As the GLAS and Landsat data both covered the global surface, this method has the potential to evaluate forest resources at a larger scale. In this study, we conducted four different methods to estimate forest biomass and compared their performances. From the distribution of the estimates, machine learning models perform better than the first principal component power model, which produced canopy height concentrated in $20-40 \mathrm{~m}$ and showed few local variations. However, machine learning models require significant additional costs in terms of time, effort, and computational resources.

Acknowledgments: This work was funded by the National Key R\&D Program of China (2016YFB0501502) and the National Natural Science Foundation of China $(41471349,61661136006001,41531174)$. The authors wish to thank the NASA Carbon Monitoring System (CMS) project for providing free access to the canopy height and aboveground biomass dataset. The authors also acknowledge the National Snow and Ice Data Center (NSDIC) for the ICESat/GLAS data, and the Multi-Resolution Land Characteristics (MRLC) Consortium for the NLCD2011.

Author Contributions: R.S., Z.X. and M.W. and had the original idea for the study, with all co-authors carrying out the design. M.W. performed the experiments, and the results were interpreted by all co-authors. M.W. and R.S. drafted the manuscript, which was then improved and revised by all authors. All authors read and approved the final manuscript.

Conflicts of Interest: The authors declare no conflict of interest.

\section{Appendix A}

Table A1. Results $\left(\mathrm{R}^{2}\right)$ using different parameters in the BP-ANN model.

\begin{tabular}{|c|c|c|c|c|c|c|c|c|c|c|c|}
\hline Cross Validation & no.1 & no.2 & no.3 & no.4 & no.5 & no.6 & no.7 & no.8 & no.9 & no.10 & Average Value \\
\hline $5, \mathrm{t}, 1, \mathrm{tlm}$ & 0.441 & 0.328 & 0.439 & 0.422 & 0.443 & 0.322 & 0.423 & 0.479 & 0.340 & 0.289 & 0.392 \\
\hline $8, \mathrm{t}, 1, \mathrm{tlm}$ & 0.427 & 0.420 & 0.453 & 0.399 & 0.438 & 0.406 & 0.396 & 0.411 & 0.341 & 0.299 & 0.399 \\
\hline $20, \mathrm{t}, 1, \mathrm{tlm}$ & 0.234 & 0.362 & 0.497 & 0.430 & 0.435 & 0.411 & 0.270 & 0.426 & 0.326 & 0.265 & 0.366 \\
\hline $50, \mathrm{t}, 1, \mathrm{tlm}$ & 0.308 & 0.246 & 0.563 & 0.239 & 0.240 & 0.377 & 0.207 & 0.330 & 0.238 & 0.251 & 0.300 \\
\hline $8, t, t, t \operatorname{lm}$ & 0.418 & 0.383 & 0.473 & 0.435 & 0.452 & 0.438 & 0.402 & 0.408 & 0.330 & 0.310 & 0.405 \\
\hline $8, t, 1, t \operatorname{lm}$ & 0.461 & 0.409 & 0.454 & 0.446 & 0.409 & 0.414 & 0.385 & 0.451 & 0.286 & 0.337 & 0.405 \\
\hline $8,1, t, t \operatorname{lm}$ & 0.413 & 0.398 & 0.464 & 0.424 & 0.437 & 0.418 & 0.381 & 0.268 & 0.345 & 0.290 & 0.384 \\
\hline $8,1,1, \mathrm{tlm}$ & 0.468 & 0.368 & 0.457 & 0.402 & 0.458 & 0.419 & 0.376 & 0.393 & 0.328 & 0.307 & 0.398 \\
\hline $8,1, \mathrm{p}, \mathrm{tlm}$ & 0.481 & 0.434 & 0.425 & 0.439 & 0.440 & 0.433 & 0.409 & 0.500 & 0.373 & 0.352 & 0.429 \\
\hline $8, \mathrm{p}, \mathrm{t}, \mathrm{tlm}$ & 0.461 & 0.403 & 0.430 & 0.438 & 0.442 & 0.436 & 0.383 & 0.485 & 0.339 & 0.326 & 0.414 \\
\hline 8,p,l,tlm & 0.214 & 0.436 & 0.420 & 0.413 & 0.434 & 0.438 & 0.417 & 0.430 & 0.321 & 0.339 & 0.389 \\
\hline 8,p,p,tlm & 0.468 & 0.413 & 0.391 & 0.401 & 0.403 & 0.414 & 0.418 & 0.457 & 0.350 & 0.328 & 0.404 \\
\hline $8, \mathrm{t}, 1, \operatorname{tgd}$ & 0.408 & 0.051 & 0.287 & 0.217 & 0.206 & 0.321 & 0.216 & 0.301 & 0.013 & 0.256 & 0.228 \\
\hline $8, t, 1, \operatorname{tgdx}$ & 0.448 & 0.412 & 0.382 & 0.396 & 0.408 & 0.400 & 0.408 & 0.469 & 0.344 & 0.352 & 0.402 \\
\hline $8, \mathrm{t}, 1, \mathrm{tgda}$ & 0.462 & 0.407 & 0.372 & 0.373 & 0.370 & 0.424 & 0.397 & 0.423 & 0.339 & 0.306 & 0.387 \\
\hline $8, t, 1, \operatorname{trp}$ & 0.477 & 0.426 & 0.422 & 0.445 & 0.432 & 0.436 & 0.421 & 0.481 & 0.357 & 0.345 & 0.424 \\
\hline $8, \mathrm{t}, 1, \mathrm{tlm}$ & 0.449 & 0.402 & 0.446 & 0.439 & 0.422 & 0.408 & 0.381 & 0.454 & 0.338 & 0.335 & 0.407 \\
\hline $8, \mathrm{t}, \mathrm{l}, \mathrm{tfg}$ & 0.463 & 0.429 & 0.435 & 0.455 & 0.436 & 0.442 & 0.423 & 0.470 & 0.375 & 0.351 & 0.428 \\
\hline $8, t, 1, t c g$ & 0.478 & 0.427 & 0.427 & 0.443 & 0.419 & 0.436 & 0.418 & 0.469 & 0.345 & 0.364 & 0.423 \\
\hline $8, \mathrm{t}, \mathrm{l}, \mathrm{tss}$ & 0.480 & 0.425 & 0.422 & 0.439 & 0.421 & 0.444 & 0.414 & 0.452 & 0.363 & 0.357 & 0.422 \\
\hline $8, t, 1, \operatorname{tgf}$ & 0.470 & 0.365 & 0.425 & 0.347 & 0.427 & 0.443 & 0.417 & 0.478 & 0.352 & 0.352 & 0.408 \\
\hline $8, t, 1, \operatorname{tgp}$ & 0.401 & 0.433 & 0.406 & 0.412 & 0.433 & 0.434 & 0.409 & 0.476 & 0.359 & 0.355 & 0.412 \\
\hline
\end{tabular}

$5,8,20,50$ represent the the number of neurons in hidden layers; $t, 1, p$ represent the tansig, logsig, and purelin transfer function; tgd, tgdx, tgda, trp, tlm, tfg, tcg, tss, tgf, tgp represent traingd, traingdx, trainingda, trainrp, trainlm, trainbfg, trainscg, trainoss, traincgf, and traincgp training algorithms, respectively. The bold were the experiments with the best validation results. 
Table A2. Results $\left(\mathrm{R}^{2}\right)$ using different parameters in the SVR model.

\begin{tabular}{cccccccccccc}
\hline Cross Validation & no.1 & no.2 & no.3 & no.4 & no.5 & no.6 & no.7 & no.8 & no.9 & no.10 & Average Value \\
\hline -s 4 -t 2 -c 1 -g 1 -n 0.5 & $\mathbf{0 . 4 7 7}$ & 0.418 & 0.405 & 0.416 & 0.383 & 0.414 & 0.406 & 0.466 & 0.336 & 0.332 & 0.405 \\
-s 3 t 0 -c 1 -g 1 -p 0.1 & $\mathbf{0 . 4 6 8}$ & 0.410 & 0.389 & 0.400 & 0.401 & 0.413 & 0.419 & 0.452 & 0.350 & 0.328 & 0.403 \\
-s 3 -t 1 -c 1 -g 1 -p 0.1 & $\mathbf{0 . 4 5 5}$ & 0.378 & 0.379 & 0.394 & 0.418 & 0.406 & 0.379 & 0.427 & 0.363 & 0.339 & 0.394 \\
-s 3 -t 2 -c 1 -g 1 -p 0.1 & $\mathbf{0 . 4 7 1}$ & $\mathbf{0 . 4 2 5}$ & $\mathbf{0 . 4 1 0}$ & $\mathbf{0 . 4 2 2}$ & $\mathbf{0 . 3 8 9}$ & $\mathbf{0 . 4 2 6}$ & $\mathbf{0 . 4 1 2}$ & $\mathbf{0 . 4 6 6}$ & $\mathbf{0 . 3 3 0}$ & $\mathbf{0 . 3 4 4}$ & $\mathbf{0 . 4 0 9}$ \\
\hline
\end{tabular}

-s 3, -s 4 represent $\varepsilon$-SVR and $v$-SVR; -t 0 , -t 1 , -t 2 represent the linear, polynomial and RBF kernels; -c represents the cost function; - $g$ represents the gamma function; and $-\mathrm{p}$ and $-\mathrm{n}$ are the parameters in cost function for $\varepsilon-S V R$ and $v$-SVR respectively. We used defaults for $-c,-g,-p$ and $-n$. The bold were the experiments with the best validation results.

Table A3. Results using different parameters in the RF model.

\begin{tabular}{cccccccccccc}
\hline Cross Validation & no.1 & no.2 & no.3 & no.4 & no.5 & no.6 & no.7 & no.8 & no.9 & no.10 & Average Value \\
\hline 10_2_1 & 0.407 & 0.376 & $\mathbf{0 . 6 5 5}$ & 0.432 & 0.416 & 0.402 & 0.389 & 0.435 & 0.409 & 0.396 & 0.432 \\
50_2_1 & 0.480 & 0.410 & $\mathbf{0 . 6 8 9}$ & 0.459 & 0.439 & 0.418 & 0.393 & 0.468 & 0.456 & 0.409 & 0.462 \\
100_2_1 & 0.482 & 0.419 & $\mathbf{0 . 7 0 0}$ & 0.469 & 0.442 & 0.417 & 0.398 & 0.471 & 0.452 & 0.420 & 0.467 \\
200_2_1 & 0.478 & 0.424 & $\mathbf{0 . 7 0 4}$ & 0.475 & 0.442 & 0.424 & 0.395 & 0.476 & 0.449 & 0.418 & 0.468 \\
500_2_1 & 0.478 & 0.423 & $\mathbf{0 . 7 0 7}$ & 0.473 & 0.446 & 0.428 & 0.396 & 0.482 & 0.445 & 0.423 & 0.470 \\
1000_2_1 & $\mathbf{0 . 4 8 0}$ & $\mathbf{0 . 4 2 3}$ & $\mathbf{0 . 7 0 6}$ & $\mathbf{0 . 4 7 5}$ & $\mathbf{0 . 4 4 5}$ & $\mathbf{0 . 4 3 0}$ & $\mathbf{0 . 3 9 6}$ & $\mathbf{0 . 4 8 2}$ & $\mathbf{0 . 4 4 3}$ & $\mathbf{0 . 4 2 3}$ & $\mathbf{0 . 4 7 0}$ \\
100_1_1 & 0.481 & 0.419 & $\mathbf{0 . 6 9 3}$ & 0.470 & 0.451 & 0.424 & 0.392 & 0.474 & 0.440 & 0.412 & 0.466 \\
100_3_1 & 0.472 & 0.414 & $\mathbf{0 . 7 0 8}$ & 0.465 & 0.439 & 0.414 & 0.393 & 0.487 & 0.438 & 0.427 & 0.466 \\
100_4_1 & 0.461 & 0.415 & $\mathbf{0 . 7 1 2}$ & 0.447 & 0.447 & 0.413 & 0.399 & 0.476 & 0.432 & 0.429 & 0.463 \\
100_5_1 & 0.457 & 0.421 & $\mathbf{0 . 7 1 3}$ & 0.445 & 0.429 & 0.411 & 0.388 & 0.474 & 0.434 & 0.424 & 0.460 \\
100_2_0 & 0.472 & 0.414 & $\mathbf{0 . 7 0 7}$ & 0.465 & 0.429 & 0.411 & 0.395 & 0.486 & 0.440 & 0.428 & 0.465 \\
\hline
\end{tabular}

10,50,100, 200,500, 1000 represent the number of trees; 1, 2, 3, 4, 5 in the second parameter represent the features randomly selected in each node of each tree; and 1, 0 in the third parameter represent calculating the importance or not. The bold were the experiments with the best validation results.

\section{References}

1. Boudreau, J.; Nelson, R.F.; Margolis, H.A.; Beaudoin, A.; Guindon, L.; Kimes, D.S. Regional aboveground forest biomass using airborne and spaceborne LiDAR in Québec. Remote Sens. Environ. 2008, 112, 3876-3890. [CrossRef]

2. Hamburg, S.P.; Zamolodchikov, D.G.; Korovin, G.N.; Nefedjev, V.V.; Utkin, A.I.; Gulbe, J.I.; Gulbe, T.A. Estimating the carbon content of Russian forests; a comparison of phytomass/volume and allometric projections. Mitig. Adapt. Strateg. Glob. Chang. 1997, 2, 247-265. [CrossRef]

3. Ding, H.; Nunes, P.A.; Teelucksingh, S.S. European forests and carbon sequestration services: An economic assessment of climate change impacts. SSRN Electron. J. 2010. [CrossRef]

4. Lasky, J.R.; Uriarte, M.; Boukili, V.K.; Erickson, D.L.; John Kress, W.; Chazdon, R.L. The relationship between tree biodiversity and biomass dynamics changes with tropical forest succession. Ecol. Lett. 2014, 17, 1158-1167. [CrossRef] [PubMed]

5. Vance-Chalcraft, H.D.; Willig, M.R.; Cox, S.B.; Lugo, A.E.; Scatena, F.N. Relationship between aboveground biomass and multiple measures of biodiversity in subtropical forest of Puerto Rico. Biotropica 2010, 42, 290-299. [CrossRef]

6. Nelson, R.; Parker, G.; Hom, M. A portable airborne laser system for forest inventory. Photogramm. Eng. Remote Sens. 2003, 69, 267-273. [CrossRef]

7. Botkin, D.B.; Simpson, L.G. Biomass of the North American boreal forest: A step toward accurate global measures. Biogeochemistry 1990, 9, 161-174.

8. Botkin, D.B.; Simpson, L.G.; Nisbet, R.A. Biomass and carbon storage of the North American deciduous forest. Biogeochemistry 1993, 20, 1-17. [CrossRef]

9. Jenkins, J.C.; Chojnacky, D.C.; Heath, L.S.; Birdsey, R.A. National-scale biomass estimators for United States tree species. For. Sci. 2003, 49, 12-35.

10. Penner, M.; Power, K.; Muhairwe, C.; Tellier, R.; Wang, Y. Canada's Forest Biomass Resources: Deriving Estimates from Canada Forest Inventory; Information Report BC-X-370; Pacific Forestry Centre: Victoria, BC, Canada, 1997.

11. Naesset, E. Airborne laser scanning as a method in operational forest inventory: Status of accuracy assessments accomplished in scandinavia. Scand. J. For. Res. 2007, 22, 433-442. [CrossRef] 
12. Næsset, E. Practical large-scale forest stand inventory using a small-footprint airborne scanning laser. Scand. J. For. Res. 2004, 19, 164-179. [CrossRef]

13. Thomas, V.; Treitz, P.; Mccaughey, J.H.; Morrison, I. Mapping stand-level forest biophysical variables for a mixedwood boreal forest using lidar: An examination of scanning density. Can. J. For. Res. 2006, 36, 34-47. [CrossRef]

14. Nelson, R. Measuring biomass and carbon in delaware using an airborne profiling lidar. Scand. J. For. Res. 2004, 19, 500-511. [CrossRef]

15. Næsset, E.; Gobakken, T. Estimation of above- and below-ground biomass across regions of the boreal forest zone using airborne laser. Remote Sens. Environ. 2008, 112, 3079-3090. [CrossRef]

16. Drake, J.B.; Dubayah, R.O.; Knox, R.G.; Clark, D.B.; Blair, J.B. Sensitivity of large-footprint lidar to canopy structure and biomass in a neotropical rainforest. Remote Sens. Environ. 2002, 81, 378-392. [CrossRef]

17. Castro, K.L.; Sanchez-Azofeifa, G.A.; Rivard, B. Monitoring secondary tropical forests using space-borne data: Implications for Central America. Int. J. Remote Sens. 2003, 24, 1853-1894. [CrossRef]

18. Harding, D.J.; Carabajal, C.C. ICESat waveform measurements of within-footprint topographic relief and vegetation vertical structure. Geophys. Res. Lett. 2005, 32, 741-746. [CrossRef]

19. Brenner, A.; Zwally, H.; Bentley, C.; Csathó, B.; Harding, D.; Hofton, M.; Minster, J.; Roberts, L.; Saba, J.; Thomas, R.; et al. Derivation of Range and Range Distributions From Laser Pulse Waveform Analysis for Surface Elevations, Roughness, Slope, and Vegetation Heights. In Algorithm Theoretical Basis Document V4. 1; Goddard Space Flight Center: Greenbelt, MD, USA, 2003; p. 92.

20. Fayad, I.; Baghdadi, N.; Bailly, J.S.; Barbier, N.; Gond, V.; Hérault, B.; El Hajj, M.; Lochard, J.; Perrin, J. Regional scale rain-forest height mapping using regression-kriging of spaceborne and airborne LiDAR data: Application on French Guiana. In Proceedings of the 2015 IEEE International Geoscience and Remote Sensing Symposium (IGARSS), Milan, Italy, 26-31 July 2015; pp. 4109-4112.

21. Park, H.J.; Shin, H.S.; Roh, Y.H.; Kim, K.M.; Park, K.H. Estimating forest carbon stocks in Danyang using Kriging methods for aboveground biomass. J. Korean Assoc. Geogr. Inf. Stud. 2012, 15, 16-33. [CrossRef]

22. Hajj, M.E.; Baghdadi, N.; Fayad, I.; Vieilledent, G.; Bailly, J.S.; Minh, D.H.T. Interest of integrating spaceborne LiDAR data to improve the estimation of biomass in high biomass forested areas. Remote Sens. 2017, 9, 213. [CrossRef]

23. Chi, H.; Sun, G.; Huang, J.; Li, R.; Ren, X.; Ni, W.; Fu, A. Estimation of Forest Aboveground Biomass in Changbai Mountain Region Using ICESat/GLAS and Landsat/TM Data. Remote Sens. 2017, 9, 707. [CrossRef]

24. Liu, K.; Wang, J.; Zeng, W.; Song, J. Comparison and Evaluation of Three Methods for Estimating Forest above Ground Biomass Using TM and GLAS Data. Remote Sens. 2017, 9, 341. [CrossRef]

25. Dhanda, P.; Nandy, S.; Kushwaha, S.P.S.; Ghosh, S.; Murthy, Y.K.; Dadhwal, V.K. Optimizing spaceborne LiDAR and very high resolution optical sensor parameters for biomass estimation at ICESat/GLAS footprint level using regression algorithms. Prog. Phys. Geogr. 2017, 2, 1-21. [CrossRef]

26. Nelson, R.; Margolis, H.; Montesano, P.; Sun, G.; Cook, B.; Corp, L.; Andersen, H.; Jong, B.; Pellat, F.P.; Fickl, T.; et al. Lidar-based estimates of aboveground biomass in the continental US and Mexico using ground, airborne, and satellite observations. Remote Sens. Environ. 2017, 188, 127-140. [CrossRef]

27. Helmer, E.H.; Lefsky, M.A.; Roberts, D.A. Biomass accumulation rates of amazonian secondary forest and biomass of old-growth forests from landsat time series and the geoscience laser altimeter system. J. Appl. Remote Sens. 2009, 3, 201-210.

28. Zhang, Y.; Liang, S.; Sun, G. Forest biomass mapping of northeastern China using GLAS and MODIS data. IEEE J. Sel. Top. Appl. Earth Obs. Remote Sens. 2014, 7, 140-152. [CrossRef]

29. Fu, A.; Sun, G.; Guo, Z. Estimating forest biomass with GLAS samples and MODIS imagery in northeastern China. Proc. SPIE 2009, 7498, 1-8.

30. Huang, K.; Pang, Y.; Shu, Q.; Fu, T. Aboveground forest biomass estimation using ICESat GLAS in Yunnan, China. Yaogan Xuebao-J. Remote Sens. 2013, 17, 165-179.

31. Yavaşl1, D.D. Estimation of above ground forest biomass at Muğla using ICESat/GLAS and Landsat data. Remote Sens. Appl. Soc. Environ. 2016, 4, 211-218. [CrossRef]

32. Hermosilla, T.; Wulder, M.A.; White, J.C.; Coops, N.C.; Hobart, G.W. An integrated Landsat time series protocol for change detection and generation of annual gap-free surface reflectance composites. Remote Sens. Environ. 2015, 158, 220-234. [CrossRef] 
33. Armston, J.D.; Denham, R.J.; Danaher, T.J.; Scarth, P.F.; Moffiet, T.N. Prediction and validation of foliage projective cover from Landsat-5 TM and Landsat-7 ETM+ imagery. J. Appl. Remote Sens. 2009, 3, 033540. [CrossRef]

34. Yan, L.; Roy, D.P. Conterminous United States crop field size quantification from multi-temporal Landsat data. Remote Sens. Environ. 2016, 172, 67-86. [CrossRef]

35. Sesnie, S.E.; Gessler, P.E.; Finegan, B.; Thessler, S. Integrating Landsat TM and SRTM-DEM derived variables with decision trees for habitat classification and change detection in complex neotropical environments. Remote Sens. Environ. 2008, 112, 2145-2159. [CrossRef]

36. Oyama, Y.; Matsushita, B.; Fukushima, T. Distinguishing surface cyanobacterial blooms and aquatic macrophytes using Landsat/TM and ETM+ shortwave infrared bands. Remote Sens. Environ. 2015, 157, 35-47. [CrossRef]

37. Kovalskyy, V.; Roy, D.P. The global availability of Landsat 5 TM and Landsat 7 ETM+ land surface observations and implications for global $30 \mathrm{~m}$ Landsat data product generation. Remote Sens. Environ. 2013, 130, 280-293. [CrossRef]

38. Feng, M.; Sexton, J.O.; Huang, C.; Masek, J.G.; Vermote, E.F.; Gao, F.; Narasimhan, R.; Channan, S.; Wolfe, R.E.; Townshend, J.R. Global surface reflectance products from Landsat: Assessment using coincident MODIS observations. Remote Sens. Environ. 2013, 134, 276-293. [CrossRef]

39. Huang, W.; Swatantran, A.; Johnson, K.; Duncanson, L.; Tang, H.; Dunne, J.O.N.; Hurtt, G.; Dubayah, R. Local discrepancies in continental scale biomass maps: A case study over forested and non-forested landscapes in Maryland, USA. Carbon Balance Manag. 2015, 10, 19. [CrossRef] [PubMed]

40. Weber, T.; Sloan, A.; Wolf, J. Maryland's Green Infrastructure Assessment: Development of a comprehensive approach to land conservation. Landsc. Urban Plan. 2006, 77, 94-110. [CrossRef]

41. O'Loughlin, F.E.; Paiva, R.C.D.; Durand, M.; Alsdorf, D.E.; Bates, P.D. A multi-sensor approach towards a global vegetation corrected SRTM DEM product. Remote Sens. Environ. 2016, 182, 49-59. [CrossRef]

42. Schutz, B.E. Spaceborne laser altimetry: 2001 and beyond. In Book of Extended Abstracts WEGENER-98; Plag, H.P., Ed.; Norwegian Mapping Authority: Honefoss, Norway, 1998.

43. Bye, I.J.; North, P.R.J.; Los, S.O.; Kljun, N.; Rosette, J.A.B.; Hopkinson, C.; Chasmer, L.; Mahoney, C. Estimating forest canopy parameters from satellite waveform LiDAR by inversion of the FLIGHT three-dimensional radiative transfer model. Remote Sens. Environ. 2017, 188, 177-189. [CrossRef]

44. Schutz, B.E.; Zwally, H.J.; Shuman, C.A.; Hancock, D.; DiMarzio, J.P. Overview of the ICESat mission. Geophys. Res. Lett. 2005, 32, L21S01. [CrossRef]

45. Chi, H.; Sun, G.; Huang, J.; Guo, Z.; Ni, W.; Fu, A. National forest aboveground biomass mapping from ICESat/GLAS data and MODIS imagery in China. Remote Sens. 2015, 7, 5534-5564. [CrossRef]

46. Su, Y.; Guo, Q.; Xue, B.; Hu, T.; Alvarez, O.; Tao, S.; Fang, J. Spatial distribution of forest aboveground biomass in China: Estimation through combination of spaceborne lidar, optical imagery, and forest inventory data. Remote Sens. Environ. 2016, 173, 187-199. [CrossRef]

47. Sun, G.; Ranson, K.J.; Kimes, D.S.; Blair, J.B.; Kovacs, K. Forest vertical structure from GLAS: An evaluation using LVIS and SRTM data. Remote Sens. Environ. 2008, 112, 107-117. [CrossRef]

48. Neuenschwander, A.L.; Urban, T.J.; Gutierrez, R.; Schutz, B.E. Characterization of ICESat/GLAS waveforms over terrestrial ecosystems: Implications for vegetation mapping. J. Geophys. Res. Biogeosci. 2008, 113, 1032. [CrossRef]

49. Hilbert, C.; Schmullius, C. Influence of surface topography on ICESat/GLAS forest height estimation and waveform shape. Remote Sens. 2012, 4, 2210-2235. [CrossRef]

50. Lefsky, M.A.; Harding, D.J.; Keller, M.; Cohen, W.B.; Carabajal, C.C.; Del Bom Espirito-Santo, F.; Hunter, M.O.; de Oliveira, R., Jr.; de Camargo, P.B. Estimates of forest canopy height and aboveground biomass using ICESat. Geophys. Res. Lett. 2006, 33, L05501. [CrossRef]

51. Fayad, I.; Baghdadi, N.; Gond, V.; Bailly, J.S.; Barbier, N.; El Hajj, M.; Fabre, F. Coupling potential of ICESat/GLAS and SRTM for the discrimination of forest landscape types in French Guiana. Int. J. Appl. Earth Obs. Geoinf. 2014, 33, 21-31. [CrossRef]

52. Mahoney, C.; Kljun, N.; Los, S.O.; Chasmer, L.; Hacker, J.M.; Hopkinson, C.; North, P.; Rosette, J.; van Gorsel, E. Slope estimation from ICESat/GLAS. Remote Sens. 2014, 6, 10051-10069. [CrossRef]

53. Dubayah, R.O.; Swatantran, A.; Huang, W.; Duncanson, L.; Johnson, K.; Tang, H.; Dunne, J.O.; Hurtt, G.C. CMS: LiDAR-Derived Aboveground Biomass, Canopy Height and Cover for Maryland, 2011; ORNL DAAC: Oak Ridge, TN, USA, 2016. 
54. O'Neil-Dunne, J.P.; MacFaden, S.W.; Royar, A.R.; Pelletier, K.C. An object-based system for LiDAR data fusion and feature extraction. Geocarto Int. 2014, 28, 227-242. [CrossRef]

55. Dubayah, R. County-Scale Carbon Estimation in NASA's Carbon Monitoring System. Biomass Carbon Storage 2012. Available online: https://www.researchgate.net/profile/Ross_Nelson/publication/258459476_CountyScale_Carbon_Estimation_in_NASA's_Carbon_Monitoring_System/links/564e2fe508ae1ef9296_c6779.pdf (accessed on 1 November 2017).

56. Yan, L.; Roy, D.P. Automated crop field extraction from multi-temporal Web Enabled Landsat Data. Remote Sens. Environ. 2014, 144, 42-64. [CrossRef]

57. Baumann, M.; Ozdogan, M.; Kuemmerle, T.; Wendland, K.J.; Esipova, E.; Radeloff, V.C. Using the Landsat record to detect forest-cover changes during and after the collapse of the Soviet Union in the temperate zone of European Russia. Remote Sens. Environ. 2012, 124, 174-184. [CrossRef]

58. Melaas, E.K.; Friedl, M.A.; Zhu, Z. Detecting interannual variation in deciduous broadleaf forest phenology using Landsat TM/ETM + data. Remote Sens. Environ. 2013, 132, 176-185. [CrossRef]

59. Lasanta, T.; Vicente-Serrano, S.M. Complex land cover change processes in semiarid Mediterranean regions: An approach using Landsat images in northeast Spain. Remote Sens. Environ. 2012, 124, 1-14. [CrossRef]

60. Solomon, D.S.; Hosmer, R.A.; Hayslett, H.T., Jr. A two-stage matrix model for predicting growth of forest stands in the Northeast. Can. J. For. Res. 1986, 16, 521-528. [CrossRef]

61. Clewley, D.; Lucas, R.; Accad, A.; Armston, J.; Bowen, M.; Dwyer, J.; Pollock, S.; Bunting, P.; McAlpine, C.; Kelly, A.; et al. An approach to mapping forest growth stages in Queensland, Australia through integration of ALOS PALSAR and Landsat sensor data. Remote Sens. 2012, 4, 2236-2255. [CrossRef]

62. Powell, S.L.; Cohen, W.B.; Healey, S.P.; Kennedy, R.E.; Moisen, G.G.; Pierce, K.B.; Ohmann, J.L. Quantification of live aboveground forest biomass dynamics with Landsat time-series and field inventory data: A comparison of empirical modeling approaches. Remote Sens. Environ. 2010, 114, 1053-1068. [CrossRef]

63. Wickham, J.; Stehman, S.V.; Gass, L.; Dewitz, J.A.; Sorenson, D.G.; Granneman, B.J.; Poss, R.V.; Baer, L.A. Thematic accuracy assessment of the 2011 national land cover database (NLCD). Remote Sens. Environ. 2017, 191, 328-341. [CrossRef]

64. Xian, G.; Homer, C.; Dewitz, J.; Fry, J.; Hossain, N.; Wickham, J. Change of impervious surface area between 2001 and 2006 in the conterminous United States. Photogramm. Eng. Remote Sens. 2011, 77, 758-762.

65. Sun, G.; Masek, J.; Guo, Z.; Pang, Y.; Fu, A.; Wang, D. Estimation of tree height and forest biomass from GLAS data. J. For. Plan. 2008, 13, 157-164.

66. Chen, Q. Retrieving vegetation height of forests and woodlands over mountainous areas in the pacific coast region using satellite laser altimetry. Remote Sens. Environ. 2010, 114, 1610-1627. [CrossRef]

67. Valor, E.; Caselles, V. Mapping land surface emissivity from NDVI: Application to European, African, and South American areas. Remote Sens. Environ. 1996, 57, 167-184. [CrossRef]

68. Matsushita, B.; Yang, W.; Chen, J.; Onda, Y.; Qiu, G. Sensitivity of the enhanced vegetation index (EVI) and normalized difference vegetation index (NDVI) to topographic effects: A case study in high-density cypress forest. Sensors 2007, 7, 2636-2651. [CrossRef] [PubMed]

69. Tucker, C.J. Red and photographic infrared linear combinations for monitoring vegetation. Remote Sens. Environ. 1979, 8, 127-150. [CrossRef]

70. Gupta, R.K. Comparative study of AVHRR ratio vegetation index and normalized difference vegetation index in district level agricultural monitoring. Int. J. Remote Sens. 1993, 14, 53-73. [CrossRef]

71. Kaiser, J. MODIS-derived visible atmospherically resistant index for monitoring chaparral moisture content. Int. J. Remote Sens. 2005, 26, 3867-3873.

72. Hammer, O.; Harper, D.A.; Ryan, P.D. Past: Paleontological statistics software package for education and data analysis. Palaeontol. Electron. 2001, 4,1-9.

73. Roweis, S. EM algorithms for PCA and SPCA. Adv. Neural Inf. Process. Syst. 1998, 10, 626-632.

74. Mika, S.; Schölkopf, B.; Smola, A.J.; Müller, K.R.; Scholz, M.; Rätsch, G. Kernel PCA and de-noising in feature spaces. In Advances in Neural Information Processing Systems; The MIT Press: Cambridge, MA, USA, 1999; pp. 536-542.

75. Rumelhart, D.E.; Hinton, G.E.; Williams, R.J. Learning representations by back-propagating errors. Nature 1986, 323, 533-538. [CrossRef] 
76. Sun, G.; Hoff, S.J.; Zelle, B.C.; Smith, M.A. Development and comparison of backpropagation and generalized regression neural network models to predict diurnal and seasonal gas and PM10 concentrations and emissions from swine buildings. Trans. Asabe 2008, 51, 685-694. [CrossRef]

77. Tamilarasan, A.; Mukkamala, S.; Sung, A.H.; Yendrapalli, K. Feature ranking and selection for intrusion detection using artificial neural networks and statistical methods. In Proceedings of the International Joint Conference on Neural Networks, Vancouver, BC, Canada, 16-21 July 2006; pp. 4754-4761.

78. Chen, P.H.; Lin, C.J.; Schölkopf, B. A tutorial on v-support vector machines. Appl. Stoch. Models Bus. Ind. 2005, 21, 111-136. [CrossRef]

79. Campbell, W.M.; Sturim, D.E.; Reynolds, D.A.; Solomonoff, A. SVM Based Speaker Verification using a GMM Supervector Kernel and NAP Variability Compensation. In Proceedings of the IEEE International Conference on Acoustics, Speech, and Signal Processing, Toulouse, France, 14-19 May 2006; Volume 1, pp. 97-100.

80. Chang, C.C.; Lin, C.J. Training nu-support vector regression: Theory and algorithms. Neural Comput. 2002, 14, 1959-1977. [CrossRef] [PubMed]

81. Ma, J.; Song, A.; Xiao, J. A robust static decoupling algorithm for 3-axis force sensors based on coupling error model and $\varepsilon$-SVR. Sensors 2012, 12, 14537-14555. [CrossRef] [PubMed]

82. Breiman, L. Random forests. Mach. Learn. 2001, 45, 5-32. [CrossRef]

83. Jiang, P.; Wu, H.; Wei, J.; Sang, F.; Sun, X.; Lu, Z. RF-DYMHC: Detecting the yeast meiotic recombination hotspots and coldspots by random forest model using gapped dinucleotide composition features. Nucleic Acids Res. 2007, 35, W47-W51. [CrossRef] [PubMed]

84. Saatchi, S.S.; Harris, N.L.; Brown, S.; Lefsky, M.; Mitchard, E.T.; Salas, W. Benchmark map of forest carbon stocks in tropical regions across three continents. Proc. Natl. Acad. Sci. USA 2011, 108, 9899-9904. [CrossRef] [PubMed]

85. Lee, S.; Ni-Meister, W.; Yang, W.; Chen, Q. Physically based vertical vegetation structure retrieval from ICESat data: Validation using LVIS in White Mountain National Forest, New Hampshire, USA. Remote Sens. Environ. 2011, 115, 2776-2785. [CrossRef]

86. Cao, C.; Ni, X.; Wang, X.; Lu, S.; Zhang, Y.; Dang, Y.; Singh, R.P. Allometric scaling theory-based maximum forest tree height and biomass estimation in the Three Gorges reservoir region using multi-source remote-sensing data. Int. J. Remote Sens. 2016, 37, 1210-1222. [CrossRef]

(c) 2018 by the authors. Licensee MDPI, Basel, Switzerland. This article is an open access article distributed under the terms and conditions of the Creative Commons Attribution (CC BY) license (http:/ / creativecommons.org/licenses/by/4.0/). 
Article

\title{
Inter-Comparison and Evaluation of Remote Sensing Precipitation Products over China from 2005 to 2013
}

\author{
Qiaolin Zeng ${ }^{1}$, Yongqian Wang ${ }^{1,2,3}$, Liangfu Chen ${ }^{1,4, *}$, Zifeng Wang ${ }^{1}$, Hao Zhu ${ }^{1,2}$ and Bin $\mathrm{Li}^{5}$ \\ 1 State Key Laboratory of Remote Sensing Science, Jointly Sponsored by Institute of Remote Sensing and \\ Digital Earth of Chinese Academy of Sciences and Beijing Normal University, Beijing 100101, China; \\ zengql@radi.ac.cn (Q.Z.); wyqq@cuit.edu.cn (Y.W.); wangzf@radi.ac.cn (Z.W.); zhuh1993@yeah.net (H.Z.) \\ 2 College of Environmental and Resource Science, Chengdu University of Information Technology, \\ Chengdu 610225, China \\ 3 Environmental Meteorological and 3S Application Technology Laboratory, Chongqing Institute of \\ Meteorological Sciences, Chongqing 401147, China \\ 4 University of Chinese Academy of Sciences, Beijing 100049, China \\ 5 Beijing Huayun Shinetek Science and Technology Co., Ltd., Beijing 100081, China; libin033@163.com \\ * Correspondence: chenlf@radi.ac.cn
}

Received: 7 December 2017; Accepted: 19 January 2018; Published: 25 January 2018

\begin{abstract}
Precipitation is a key aspect of the climate system. In this paper, the dependability of five satellite precipitation products (TRMM [Tropical Rainfall Measuring Mission] 3BV42, PERSIANN [Precipitation Estimation from Remotely Sensed Information using Artificial Neural Networks] CDR, GSMaP [Global Satellite Mapping of Precipitation] RENALYSIS, CMORPH [Climate Prediction Center's morphing technique] BLD and CMORPH_RAW) were compared with in situ measurements over China for the period of 2005 to 2013. To completely evaluate these precipitation products, the annual, seasonal and monthly precipitation averages were calculated. Overall, the Huaihe River and Qinlin mountains are shown to have heavy precipitation to the southeast and lighter precipitation to the northwest. The comparison results indicate that Gauge correction (CMORPH_BLD) improves the quality of the original satellite products (CMORPH_RAW), resulting in the higher correlation coefficient (CC), the low relative bias (BIAS) and root mean square error (RMSE). Over China, the GSMaP_RENALYSIS outperforms other products and shows the highest CC (0.91) and lowest RMSE $(0.85 \mathrm{~mm} /$ day $)$ and all products except for PERSIANN_CDR exhibit underestimation. GSMaP_RENALYSIS gives the highest of probability of detection (81\%), critical success index $(63 \%)$ and lowest false alarm ratio (36\%) while TRMM3BV42 gives the highest of frequency bias index (1.00). Over Tibetan Plateau, CMORPH_RAW demonstrates the poorest performance with the biggest BIAS (4.2 mm/month) and lowest CC (0.22) in December 2013. GSMaP_RENALYSIS displays quite consistent with in situ measurements in summer. However, GSMaP_RENALYSIS and CMORPH_RAW underestimate precipitation over South China. CMORPH_BLD and TRMM3BV42 show consistent with high CC $(>0.8)$ but relatively large RMSE in summer.
\end{abstract}

Keywords: precipitation; statistics methods; China; Tibetan Plateau; South China's

\section{Introduction}

Precipitation is one of important factors in the global water cycle and plays a key role in the global energy system. Accurate precipitation data is helpful for short-term and long-term weather forecasts, which allow proactive measures to prevent natural hazards, such as floods, landslide and debris flow, using rainfall models. Measurements of precipitation can be made using rain gauge stations, ground radar, or remote sensing technology. Rain gauge stations are traditional point measurements and have higher accuracy but it is difficult to obtain such precipitation measurements over oceanic and 
high-altitude areas where only sparse rain gauge stations are available. Ground radar is considered to more accurate for retrieving regional rainfall estimates but ground radar is aimed at monitoring extreme events over limited time spans [1]. Additionally, ground radar is limited over relatively complex terrains, where radar signals maybe be affect by beam blockages and vertical reflectivity profiles [2]. The development of satellite remote sensing has provided several advantages, including its broad spatial coverage, automatic data acquisition and long-term continuity but the signals themselves are affected by the atmospheric and the surface, which will lead to retrieval uncertainties [3]. However, remote sensing is an important method for retrieving high spatial and temporal resolution rainfall measurements over complex terrains and mountainous areas, where both rain gauge stations and ground radar are very limited or unavailable. The satellite retrieval methodologies fall primarily into three categories, which there are the visible and infrared (VIS/IR) methods, microwave (MW) methods and multi-sensor methods.

The VIS precipitation retrieval method mainly utilizes the empirical relationship between cloud brightness and rainfall because precipitation is considered to occur under highly bright clouds, which can be associated with cloud types, cloud areas and cloud growth rates [4-6]. IR imagery is associated with cloud top temperatures and cloud growth rates can be obtained via the thermal emissions during both night and day; generally, heavier rainfall tends to be associated with larger, taller clouds with colder cloud tops [5]. Many researchers have developed different methods to retrieve precipitation based on VIR/IR data from geosynchronous earth orbit (GEO) and low-earth orbit (LEO) satellites, including the 3-hourly and monthly mean rainfalls [6-10]. MW sensors can detect rain clouds directly and can provide information about the atmospheric constituents and hydro-meteorological profiles, which are more directly related to the ground precipitation rate [11,12]. Many previous works have developed a number of MW methods to retrieve precipitation [13-15]. However, precipitation is not always associated with clouds and the VIS/IR methods cannot detect information below clouds [16]. Meanwhile, MW instruments are restricted to polar-orbiting platforms, meaning that they obtain only a small amount of data and have the disadvantages of low spatial and temporal resolutions [17]. The idea of combining VIS/IR and MW observations to retrieve precipitation from multiple satellite sensors was proposed to compensate for the weaknesses of the two methods [18]. Many products were developed by blending the MW and IR data, such as CMORPH (Climate Prediction Center's morphing technique), PERSIANN (Precipitation Estimation from Remotely Sensed Information using Artificial Neural Networks), TRMM (Tropical Rainfall Measuring Mission), GSMaP (Global Satellite Mapping of Precipitation). These precipitation products can help climate and hydrological research. Many researchers have compared different satellite precipitation products with in situ measurements over different areas and have proved that satellite estimates showed non-negligible biases. For example, Hirpa et al. evaluated the accuracies of three products (CMORPH, PERSIANN and TRMM3BV42) and found that both CMORPH and TRMM underestimated precipitation over higher elevations [19]. Guo Hao et al. evaluated four products (TRMM, CMORPH, PERSIANN, GSMaP) using in situ measurements over Central Asia from 2004 to 2006 and most of the products overestimated the precipitation [20]. Awange et al. used a "three-cornered-hat" method to assess six precipitation products and indicated that the RG-merged products had higher accuracies than the satellite-only products [21]. It is necessary to discuss the performances of satellite precipitation products with the aim of determining whether a certain product is appropriate for a specific region. However, there are few researches among multi-satellite precipitation products evaluation based on long time series over China.

In this study, considering the study region and the availability of period for all satellite precipitation products, we will mainly assess the strengths and weaknesses of a few widely used rainfall products (CMORPH_CDR, CMORPH_RAW, TRMM3BV42, GSMaP_gauge_renalysis and PERSIANN_CDR) over China from 2005 to 2013. The spatial distributions, temporal variations and rainfall intensities were analyzed and compared with in situ measurements. Precipitation has a particular spatiotemporal distribution over China and thus, we discuss the precipitation over the Tibetan Plateau and South China in 2013. This paper aims to lead the reader to select more accurate 
satellite precipitation products over China and this research can help to improve the corresponding precipitation retrieval algorithms. This paper is structured as follows: the first part is an introduction. The rainfall products will be present in Section 2. Section 3 expounds on an integrated analytical approach and debates the performances of the space-time variations and errors of different datasets. Finally, the comparative result will be discussed.

\section{Satellite and Rain Gauge Precipitation Datasets}

\subsection{Satellite-Based Precipitation Products}

The TRMM satellite was launched in 1997 and was first used to measure precipitation. The National Aeronautics and Space Administration (NASA) Goddard Space Flight Center (GSFC) developed the Multisatellite Precipitation analysis (TMPA) algorithm. The TMPA algorithm combined multiple pass microwave (PMW) datasets, including the Microwave Imager (TMI), Special Sensor Microwave Imager (SSM/I), Advanced Microwave Scanning Radiometer-Earth Observing System (AMSR-E), Advanced Microwave sounding Unit-B (AMSU-B) and IR data being obtained by the international constellation of GEOs satellites [22]. The algorithm first calibrated the PMW data, which were used to create infrared precipitation data. Then, both datasets were combined and the rain gauge data were incorporated. TRMM has different spatial and temporal resolutions to satisfy the demands of its users. The real-time and post-real-time versions have the same spatial resolutions of $0.25^{\circ}$ but the data in the post-real-time version is not corrected by the rain gauge data and has a relatively narrow coverage area. The TRMM products have three different temporal resolutions, including three hourly, daily and monthly. This study obtained a TRMM3BV42 with a daily $/ 0.25^{\circ}$ resolution, which was gauge-corrected by the Global Precipitation Climatology Center (GPCC) and the Climate Assessment and Monitoring System (CAMS).

CMORPH is produced based on PMW and IR measurements from the National Oceanic and Atmospheric Administration (NOAA)/Climate Prediction Center (CPC) and has a relatively high spatiotemporal resolution [11,23]. IR images (including Meteosat-5/7, Geostationary Meteorological Satellite-5 [GMS-5] and Geostationary Operational Environmental Satellite [GOES-8/10]) are obtained every $30 \mathrm{~min}$ at large zenith angles [23] and PMW (including TMI, SSM/I and AMSU-B) can be used to retrieve rain rate relations to the IR images. Joyce et al. exploited IR data to deduce motion fields and spread the PMW rain domains across space and time via a time-weighting interpolation algorithm [11]. There are three different spatial and temporal resolution CMORPH products: $8 \mathrm{~km}-30 \mathrm{~min}, 0.25^{\circ}-3$ hourly and $0.25^{\circ}$-daily. These products can be freely downloaded from NOAA for all dates since 2002. This paper employed two types of CMORPH data (CMORPH_RAW and CMORPH_BLD) to analyze the precipitation characteristics from 2005 to 2013 with a spatiotemporal resolution of $0.25^{\circ} / \mathrm{h}$. CMORPH_BLD indicates that the satellite data have been calibrated by CPC unified daily gauge analysis methods over land [24].

PERSIANN is a pure satellite precipitation product, which is produced based on an artificial neural network (ANN) model by the Center for Hydrometeorology and Remote Sensing (CHRS) of the University of California [25,26]. The PERSIANN algorithm combines IR images of the Geostationary Environmental satellite and TMI on TRMM. The algorithm mainly depends on the statistical relationship between IR and the precipitation rate and the retrieved precipitation displays considerable uncertainty [26]. Therefore, when PMW data are available, studies adopt a training technique to revise the PERSIANN products with the PMW data. PERSIANN products have three different temporal resolutions $\left(3 \mathrm{~h}, 6 \mathrm{~h}\right.$ and daily) and a spatial resolution of $0.25^{\circ}$ and the all products can be freely downloaded for dates since 2000. This paper employed the PERSIANN_CDR products with a spatiotemporal resolution of $0.25^{\circ}$ /daily; these are calibrated by the Global Precipitation Climatology Project (GPCP) monthly gauge analysis [27].

GSMaP is a multi-satellite precipitation data source developed by the Japan Science and Technology Agency (JST) and Japan Aerospace Exploration Agency (JAXA) in November 2002 [28,29]. 
The algorithms adopt a Kalman filtering technique to estimate the hourly global precipitation, wherein the highest spatiotemporal resolution of GSMaP is $30 \mathrm{~min} / 0.0365^{\circ}$ over the equator and $0.1^{\circ}$-daily other area, as well as merged PMW (including TMI, AMSR-E and SSM/I) data and IR images from GEO satellite [28]. GSMaP is researched to achieve three mainly goals: first, the project uses the current PMW data to produce high spatiotemporal resolution global precipitation maps. Second, the algorithm of the physical model of the precipitation and rainfall rate can constantly be improved. Third, evaluating the accuracy of the precipitation products can aid the Global Precipitation Measurement (GPM) projection, which launched in 2014. The GSMaP team has achieved different standard products according to diverse algorithms, including the GSMaP_NRT, GSMaP_MVK, GSMaP_Gauge and GSMaP_RENALYSIS. The study selected the GSMaP_RENALYSIS merged with the CPC global rain gauge data to analyze the precipitation over China after considering the features of the study period [30]. Information concerning all the products is listed in Table 1.

Table 1. Description of the precipitation datasets utilized in this study.

\begin{tabular}{ccccc}
\hline Product & Temporal Resolution & Spatial Resolution & Domain & Yes or No Added Gauges \\
\hline CMORPH_RAW & 1 day & $0.25^{\circ}$ & $60^{\circ} \mathrm{S}-60^{\circ} \mathrm{N}$ & No \\
CMORPH_BLD & 1 day & $0.25^{\circ}$ & $60^{\circ} \mathrm{S}-60^{\circ} \mathrm{N}$ & Yes \\
PERSIANN_CDR & 1 day & $0.25^{\circ}$ & $60^{\circ} \mathrm{S}-60^{\circ} \mathrm{N}$ & Yes \\
GSMaP-RENALYSIS & 1 day & $0.1^{\circ}$ & $60^{\circ} \mathrm{S}-60^{\circ} \mathrm{N}$ & Yes \\
TRMM3BV42 & 1 day & $0.25^{\circ}$ & $50^{\circ} \mathrm{S}-50^{\circ} \mathrm{N}$ & Yes \\
\hline
\end{tabular}

\subsection{Gauge Stations}

This study adopted 830 rain gauge stations from the China Meteorological Data Sharing Service System (http: / / data.cma.cn/) and nine years of daily data were obtained from 2005 to 2013 to assess the distribution of precipitation over a long time. Meanwhile, we used data from 2280 rain gauge stations in 2013 to completely analyze the accuracies of the satellite precipitation products over the Tibetan Plateau and South China areas. Rain gauge stations are sparsely distributed across the Tibetan Plateau because of its extreme terrain and climatic conditions and some rain gauge stations are closed in the winter to protect their instruments, so some data is unavailable. There are more stations over the Yangtze River, South China and North China, which are used to accurately analyze the distributions of the rainfall. The western Northwest region also has relatively few stations. The elevation data of the study area were obtained from Shuttle Radar Topographic Mission (SRTM on http:/ / srtm.csi.cgiar.org/), of which the spatial resolution is about $90 \mathrm{~m}$. The rain gauge station distributions and the topography of China are shown Figure 1.

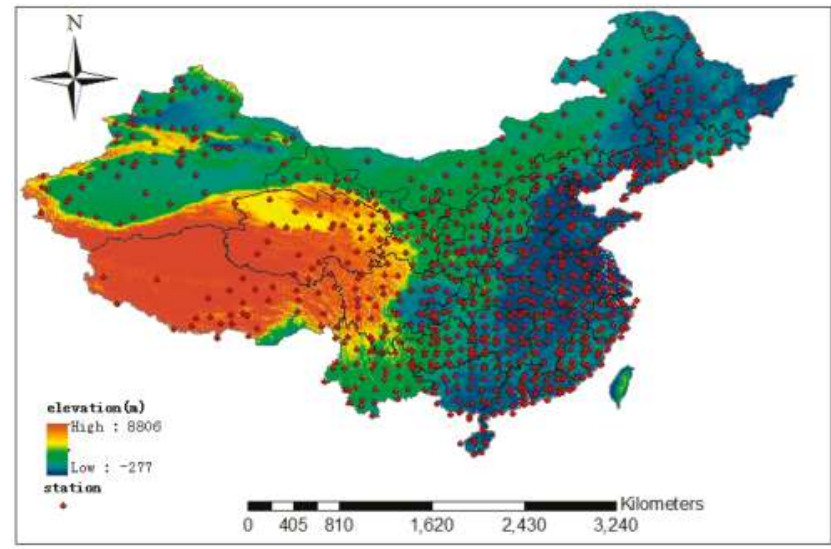

Figure 1. Site distribution and topography. 


\section{Statistical Evaluation Methods}

In this study, five products are compared with the data from rain gauge stations, which are often viewed as true values. We will discuss the characteristics of the precipitation on the yearly, seasonally, monthly and daily time scales. We use statistical analysis methods, including the linear correlation coefficient (CC), relative bias (BIAS), root mean square error (RMSE), the frequency bias index (FBI), probability of detection (POD), false alarm ratio (FAR) and critical success index (CSI) to compare the differences from pixel to point $[31,32]$. There is a high correlation between the satellite products and in situ measurements if the CC is greater than 0.7 [33]. The result is considered underestimation (overestimation) of the precipitation when the BIAS is less than (more than) zero [34]. POD and FAR express the ratios of the rainfall events, which are accurately measured and provide error warnings. The CSI denotes the overall ratio of the rainfall events that are accurately estimated by the satellite. The best comparison result occurs when the FBI, POD and CSI are equal to one and when FAR equals zero [35]. All indices are defined as follows.

$$
\begin{aligned}
& \mathrm{CC}=\frac{\operatorname{cov}(S-G)}{\sigma_{s} \sigma_{v}}= \frac{\sum_{i=1}^{N}\left(G_{i}-\bar{G}\right)\left(S_{i}-\bar{S}\right)}{\sqrt{\sum_{i=1}^{N}\left(G_{i}-\bar{G}\right)^{2}} \sqrt{\sum_{i=1}^{N}\left(S_{i}-\bar{S}\right)^{2}}} \\
& \text { BIAS }=\frac{\sum_{i=1}^{N}\left(S_{i}-G_{i}\right)}{\sum_{i=1}^{N} G_{i}} \\
& \text { RMSE }=\sqrt{\frac{1}{N} \sum_{i=1}^{N}\left(S_{i}-G_{i}\right)^{2}} \\
& \text { FBI }=\frac{H+F}{H+M} \\
& \text { POD }=\frac{H}{H+M} \\
& \text { FAR }=\frac{F}{H+F} \\
& \text { CSI }=\frac{H}{H+M+F}
\end{aligned}
$$

where $S$ represents the satellite-retrieved precipitation and $G$ represents the in situ-measured precipitation. $\bar{S}$ and $\bar{G}$ indicate the mean satellite precipitation and in situ-measured precipitation, respectively. $N$ is the number of rainfall pairs between the gauge and satellite data. $\sigma_{s}$ and $\sigma_{v}$ are the standard deviations of the satellite and gauge. $H, F$ and $M$ denote the amounts of hits, false alarms and misses, which are calculated as described in Table 2 [32]. The threshold was set $0.1 \mathrm{~mm} /$ day considering the minimum of the situ measurements.

Table 2. Contingency table comparing rainfall retrievals by satellites and gauges.

\begin{tabular}{ccc}
\hline & Gauge $\geq$ Threshold & Gauge $<$ Threshold \\
\hline Satellites $\geq$ threshold & $H$ & $F$ \\
Satellites $<$ threshold & $M$ & $Z$ \\
\hline
\end{tabular}




\section{Results and Discussion}

\subsection{Evaluation on the Regional Scale}

\subsubsection{Nine-Year Daily Mean Precipitation}

China has special topographic and geomorphological features and its precipitation shows different distributions. Overall, all satellite-based products and in situ measurements have the same trends, wherein the precipitation distribution decreases from the southeast coast to the northwest inland. Figure 2 shows the nine-year daily mean precipitation of 2005 to 2013 . The southeast has a higher mean daily precipitation than that in other areas, which some areas are as high as to $12 \mathrm{~mm} / \mathrm{day}$. The northwest has a typical semi-arid and arid climate with little precipitation, which most of areas are less than $2 \mathrm{~mm} /$ day. The phenomenon is mainly determined by climate and topography. CMORPH_BLD, CMORPH_RAW and TRMM3BV42 have clearly underestimated precipitation compared with the in-situ measurements in the south. This can be explained that there is more shallow and warm rains in the south, while the microwave-based algorithms can more easily obtain data for heavy rainfall events than weak rainfall events [36,37]. PERSIANN-CDR show a remarkable overestimation of precipitation in most areas, such as southern Tibet and regions of South China (such as Guangzhou and Fujian), wherein PERSIANN mainly adopts the IR brightness temperature data from geostationary satellites to estimate rainfall rates. MW is used only to adjust the model parameters, which differs from other satellite retrieval algorithms $[25,27]$. CMORPH_BLD, CMORPH_RAW, TRMM3BV42 and PERSIANN underestimated in north China, in where the precipitation mainly occurs in July and August. The precipitation is characterized by strong rainfall but short duration, so it is possible that sensors missed precipitation during the period. GSMaP_RENALYSIS is most consistent with the in-situ measurements but also overestimated values in some regions in the south (such as the south of Sichuan, Guangdong, Jiangxi) and northeast of China. All satellite products showed overestimations over the south of Tibet, in where are no-rain gauge stations (Figure 1). The precipitation was obtained by interpolation technique based on surrounding stations located semi-humid region. The south of Tibet belongs to humid region and has heavy precipitation. Therefore, the precipitation by interpolation is less than satellite retrieval. However, all satellite products underestimated precipitation over the most of arid region, the passive microwave retrieval of precipitation might be hampered by the snow and ice surface [38,39]. The satellite bias-corrected product (CMORPH_BLD) is more consistent with the in-situ measurements than the original satellite product (CMORPH_RAW) according to the precipitation distribution. Therefore, the bias-correction using the in-situ measurements greatly improved the product accuracy.

The density-colored scatter plots are shown in Figure 3, which provides a quantitative comparison among the satellite products and in situ measurements for the nine-year mean daily precipitation values over China (including 830 stations). The bias-corrected products, except for that of PERSIANN_CDR, have greater $\mathrm{R}$ and lower RMSE than CMORPH_RAW. The $\mathrm{R}$ of CMORPH_BLD is 0.8 higher than CMORPH_RAW (0.73) and the slope of CMORPH_BLD and CMORPH_RAW are 0.58 and 0.32, respectively. The result suggest that the bias-corrected products have improved the accuracy greatly (Figure 3a,b). TRMM3BV42 and CMORPH_BLD have similar distributions of density scatter and the differences of $R$ and RMSE are small (Figure 3b,c). The PERSIANN_CDR has a high R (0.78) but also yields high RMSE (2.93 mm/day) and the discrete degree of point is larger than other products, which PERSIANN uses only IR data to estimate rainfall rate (Figure $3 \mathrm{~d}$ ). The highest $R$ and lowest RMSE of GSMaP_RENALYSIS are 0.91 and $0.85 \mathrm{~mm}$ /day and these points are uniform distributed, because GSMaP_RENALYSIS has inherited CMORPH's morphing algorithm and employs a new Kalman filter approach to assimilate IR-derived rain rates, which it can help to reduce the total errors (Figure 3e) [11,39,40].

To analyze the continuous variations, the CC, RMSE and BIAS are calculated for each year from 2005 to 2013, as is shown Table 3. GSMaP_RENALYSIS has the highest CC (more than 0.8) and the 
lowest RMSE and BIAS (about $\pm 0.1 \mathrm{~mm}$ /day). GSMaP_RENALYSIS underestimated the precipitation in both 2005 and 2006 according to its BIAS, which is less than zero but it slightly overestimated the precipitation during 2007 to 2013. The PERSIANN_CDR overestimated the precipitation in each year and CMORPH_BLD,CMORPH_RAW and TRMM3BV42 underestimated the precipitation, which is similar to the nine-year daily mean precipitation. Note that all products have greater CC in 2007 than in 2008 but the RMSE are also higher, which is attributed to the occurrence of the decadal maximum of precipitation, such that the national mean precipitation was approximately $654.8 \mathrm{~mm}$. In 2012, the CC of CMORPH_RAW and CMORPH_BLD were 0.37 and 0.76 , respectively, which shows that the gauge correction greatly improved the precipitation estimation accuracies. Overall, GSMaP_RENALYSIS's performance is comparable to other satellite-based products over China.

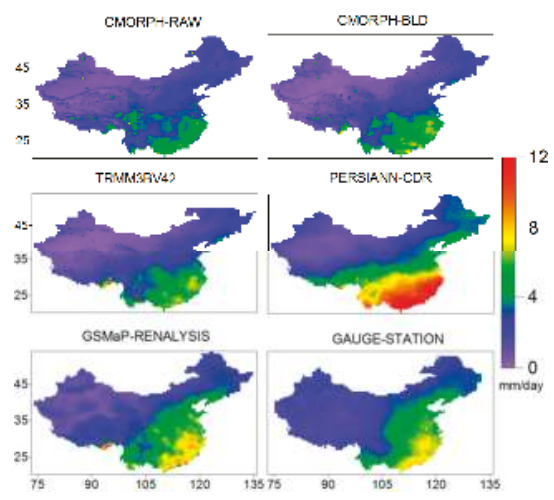

Figure 2. Spatial features of the nine-year mean daily rainfall obtained from CMOPRH_BLD, CMORPH_RAW, TRMM3BV42, PERSIANN_CDR, GSMaP_RENALYSIS and GAUGE_STATION.

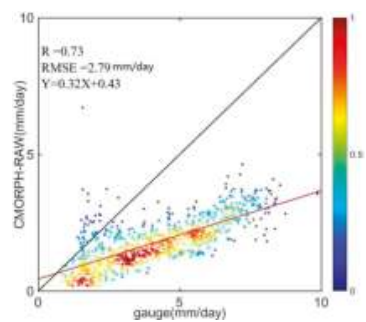

(a)

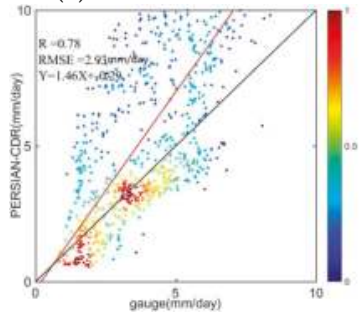

(d)

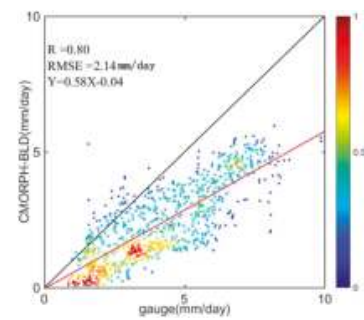

(b)

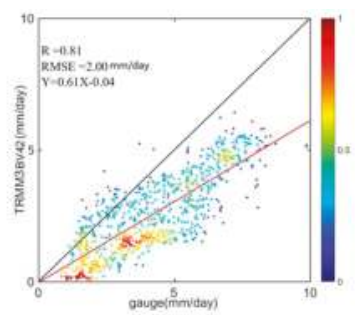

(c)

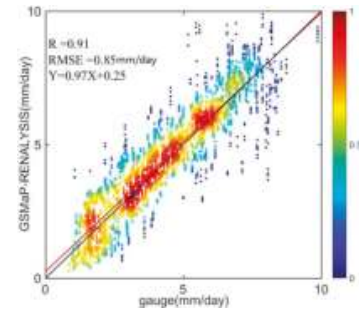

(e)

Figure 3. Density-colored scatterplots of the different products against the in-situ measurements for the nine-year mean daily precipitation. The red line is the fit and the black is 1:1. (a) denotes CMOPRH_RAW \&gauge, (b) denotes CMOPRH_BLD \&gauge, (c) denotes TRMM3BV42 \&gauge, (d) denotes PERSIANN_CDR \&gauge, (e) denotes GSMaP_RENALYSIS \&gauge. 
Table 3. The CC, BIAS and RMSE values for a variety products and the gauge station data for a year of the daily mean precipitation values over China.

\begin{tabular}{ccccccccccc}
\hline Products & Factors & $\mathbf{2 0 0 5}$ & $\mathbf{2 0 0 6}$ & $\mathbf{2 0 0 7}$ & $\mathbf{2 0 0 8}$ & $\mathbf{2 0 0 9}$ & $\mathbf{2 0 1 0}$ & $\mathbf{2 0 1 1}$ & $\mathbf{2 0 1 2}$ & $\mathbf{2 0 1 3}$ \\
\hline \multirow{4}{*}{ CMORPH_BLD } & CC & 0.74 & 0.82 & 0.70 & 0.75 & 0.76 & 0.77 & 0.74 & 0.76 & 0.76 \\
& RMSE & 1.45 & 1.34 & 1.46 & 1.50 & 1.41 & 1.36 & 1.30 & 1.38 & 1.50 \\
& BIAS & -0.42 & -0.46 & -0.47 & -0.45 & -0.46 & -0.39 & -0.47 & -0.39 & -0.46 \\
\hline \multirow{4}{*}{ CMORPH_RAW } & CC & 0.68 & 0.71 & 0.60 & 0.67 & 0.63 & 0.71 & 0.61 & 0.37 & 0.66 \\
& RMSE & 1.57 & 1.63 & 1.65 & 1.69 & 1.70 & 1.53 & 1.56 & 1.61 & 1.77 \\
& BIAS & -0.58 & -0.57 & -0.56 & -0.58 & -0.62 & -0.54 & -0.59 & -0.55 & -0.57 \\
\hline & CC & 0.74 & 0.83 & 0.70 & 0.76 & 0.76 & 0.78 & 0.76 & 0.78 & 0.79 \\
TRMM3BV42 & RMSE & 1.45 & 1.31 & 1.48 & 1.48 & 1.42 & 1.35 & 1.30 & 1.35 & 1.47 \\
& BIAS & -0.38 & -0.42 & -0.43 & -0.41 & -0.42 & -0.36 & -0.44 & -0.35 & -0.43 \\
\hline \multirow{5}{*}{ PERSIANN_CDR } & CC & 0.73 & 0.80 & 0.67 & 0.72 & 0.67 & 0.73 & 0.71 & 0.73 & 0.80 \\
& RMSE & 1.48 & 1.41 & 1.54 & 1.56 & 1.63 & 1.47 & 1.40 & 1.50 & 1.42 \\
& BIAS & 0.47 & 0.31 & 0.30 & 0.34 & 0.42 & 0.34 & 0.41 & 0.54 & 0.26 \\
\hline \multirow{3}{*}{ GSMaP_RENALYSIS } & CC & 0.82 & 0.87 & 0.81 & 0.82 & 0.84 & 0.84 & 0.83 & 0.85 & 0.84 \\
& RMSE & 1.28 & 1.21 & 1.28 & 1.42 & 1.23 & 1.36 & 1.16 & 1.19 & 1.27 \\
& BIAS & -0.05 & -0.11 & 0.04 & 0.03 & 0.04 & 0.07 & 0.02 & 0.05 & 0.01 \\
\hline
\end{tabular}

\subsubsection{Seasonal Daily Mean Precipitation}

Figure 4 shows the distribution of the seasonal daily mean precipitation values interpolated from the rain gauge stations and the multiple satellite-based precipitation products over China. The CC, BIAS and RMSE statistical values are plotted in Figure 5.

As shown in the seasonal precipitation distribution interpolated from the rain gauge stations (GAUGE_STATION), the summer and spring experience more precipitation (the maximum amounts are $15 \mathrm{~mm} /$ day and $10 \mathrm{~mm} /$ day, respectively) because both are part of the "rainy" season in China influenced by the southwest monsoon and southeast monsoon. The winter and autumn experienced less precipitation (with maxima of $5 \mathrm{~mm} /$ day and $8 \mathrm{~mm} /$ day, respectively). This phenomenon is related to the passage of rain over China. It should be noted that there have more precipitation in summer and spring than in autumn and winter because the rainfall events begin in southern China from February to May every year. The precipitation then moves north with the heat of the seasons. Rainfall begins to fall rapidly when the cold air comes from the north at the end of August or the beginning of September. There is greater rainfall in the summer than in the autumn over the south because the period of the "plum rains" season occurs from April to June in China. The northwest experiences little precipitation in all four seasons as it belongs to the arid and semi-arid region of China, in where is far from the sea and is blocked by mountains.

Generally, the distributions of the satellite precipitation products are similar to that of the rain gauge stations, except for CMORPH_RAW, which is not bias corrected. The GSMaP_RENALYSIS are the most similar to the rain gauge data (CC more than 0.8 and BIAS less than $\pm 0.1 \mathrm{~mm} /$ day, respectively). All satellite products underestimated the precipitation in the winter over some regions, such as northeast of Neimeng and northwest of Tibet. This might be attributed to the following reasons: The little precipitation in this area is mainly produced from the Atlantic Ocean through the western wind belt and it is difficult to capture low-level precipitation by PMW sensor. Meanwhile, it might be caused the more snow and ice cover on the ground in winter. All products overestimated the precipitation in the summer over the south of Tibet, in where have heavy precipitation. The phenomenon might be related to the sparsely and unevenly rain gauges. The interpolation technique is used to spread nonzero precipitation into zero and lead to obtain less precipitation. CMORPH_RAW is poorly consistent with all other products and with the in-situ measurements over all four seasons. CMORPH_RAW and CMORPH_BLD show some abnormal zero precipitation over Tibetan Plateau in winter. It is possible that the ice and snow surface tend to influence PWM-based accurate retrieval over land [37]. PERSIANN_CDR exhibited serious overestimations of precipitation over southern China. This result may be attributed to the lack of training of the ANN parameters over China because PERSIANN_CDR is only adequately trained over the United 
States [41]. We calculated the quality analysis of the satellite products and in situ measurements, as shown in Figure 5. All products provide the highest CC and lowest RMSE (greater than 0.8 and less than $1 \mathrm{~mm} /$ day, respectively) in the winter but the highest BIAS also occurs in the winter. This might be related to the following reasons: the rainfall events are less than other seasons due to climate and the rainfall intensity is usually small in winter. Next, the ice and snow surface might to influence satellite retrievals over land and the gauge stations might measure abnormal data over the high-latitude areas and high-altitude areas, which the data were considered abnormal data during dealing. This should be partially responsible for the high CC, BIAS and low RMSE in winter. There are more strong convective precipitation events well detected by microwave-base algorithms during the summer [36,37]. However, the satellite might miss some precipitation events because the duration of precipitation is short, which may cause some errors. All products show the lowest BIAS (less than $0.5 \mathrm{~mm} /$ day) and highest RMSE (about $2 \mathrm{~mm} /$ day) in summer, meanwhile the CC (more than 0.7) is relative high.

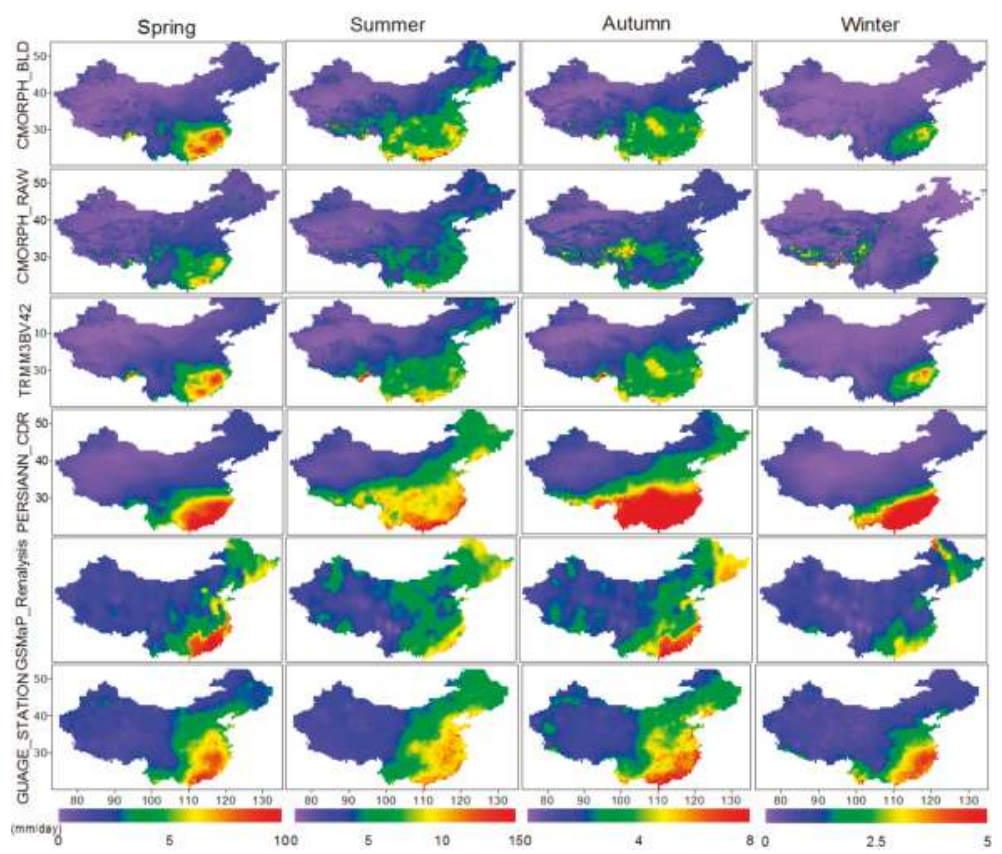

Figure 4. Seasonal nine-year daily mean rainfall features of CMOPRH_BLD, CMORPH_RAW, TRMM3BV42, PERSIANN_CDR, GSMaP_RENALYSIS and GAUGE_STATION.
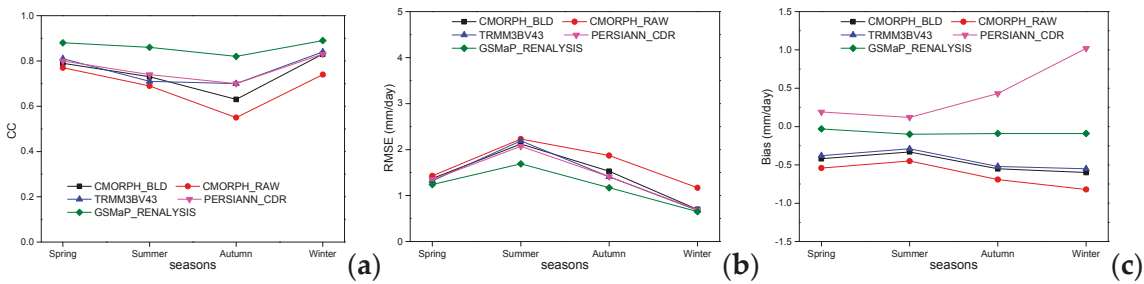

Figure 5. (a-c) denote the CC, BIAS and RMSE values of the satellite products and rain gauge stations for the seasonal daily mean precipitation values, respectively. 


\subsubsection{Monthly Daily Mean Precipitation}

Statistical information (e.g., CC, BIAS and RMSE) is computed to study the monthly daily mean precipitation from 2005 to 2013, as shown in Figure 6. Some previous works have suggested that the biases and errors could be magnified over regions with no gauge stations [42]. Therefore, this study calculated only the monthly mean daily data to guarantee more than one gauge station per pixel.

The CC, RMSE and BIAS of the products exhibit similar fluctuates, except for those of CMORPH_RAW, as shown in Figure 6. The higher CC generally occurs in May and December, while lower CC occur in September and October. Overall, CMORPH_BLD and GSMaP_RENALYSIS have higher CC than the other products but the CC of GSMaP_RENALYSIS is also occasionally low, such as in February 2008. When CMORPH_BLD and TRMM3BV42 are greater than 0.7, the GSMaP_RENALYSIS $\mathrm{CC}$ is approximately 0.4 . The trends of the RMSE distributions for several satellite-based precipitation time series are the same, showing a w-style trend. The maximum is in July and both January and December are low because there is a higher daily mean rainfall the in summer and a lower daily mean rainfall in the winter. The BIAS trends have the following characteristics: GSMaP_RENALYSIS and CMORPH_BLD BIAS present stable and relative low values ( $\pm 0.5 \mathrm{~mm} /$ day). CMORPH_RAW and TRMM_3BV42 have the same regular biases, while PERSIANN_CDR presents a large fluctuating trend and positive deviation with a high CC in the winter (December, January and February). This can be put down to the inaccurate input of the IR data in the winter, which can bring about inferior data from the IR-based PERSIANN retrieval method.
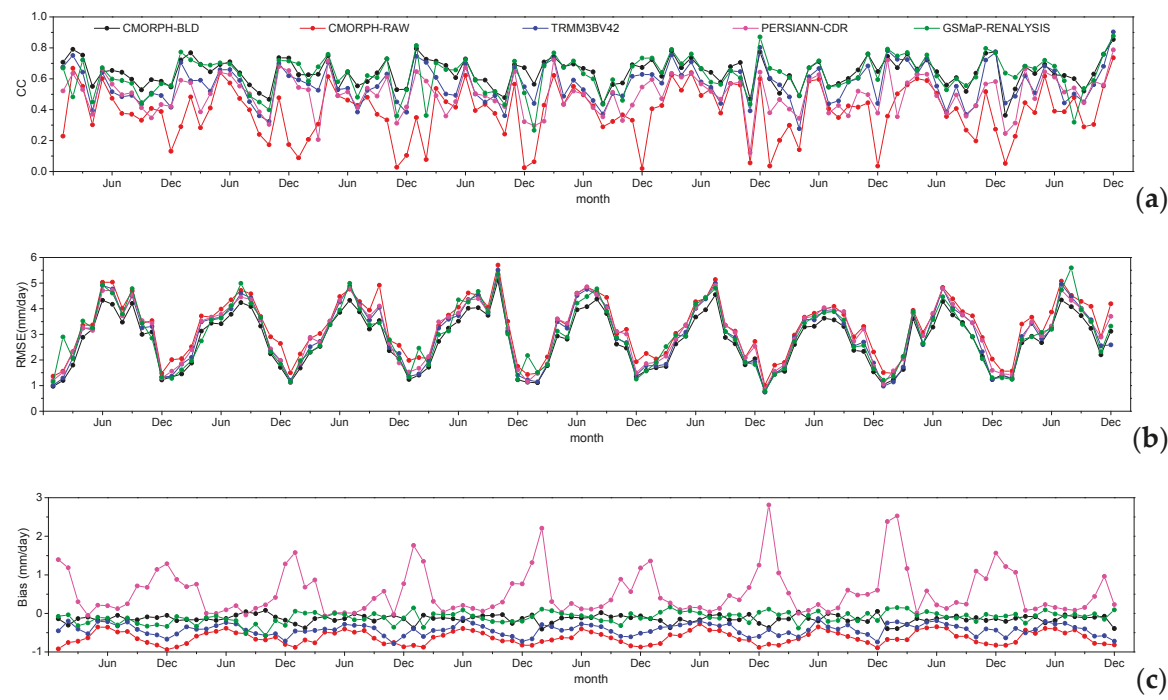

Figure 6. (a-c) denote the time series of the CC, RMSE and BIAS from a variety products spanning 2005 to 2013 over China, respectively.

\subsection{Probability Distribution and Contingency Statistics}

To determine the universal characteristics of precipitation, it is important to evaluate the precipitation frequencies of different intensities as well as their means and spatiotemporal variation distributions [37]. Different precipitation intensities may lead to different natural disasters, such as floods and mudslides [41,43]. Therefore, the PDF (probability distribution function), which offers more information about the frequencies of different intensities of precipitation events, is used to analysis more detailed rainfall characteristics. 
In this paragraph, the satellite precipitation products are compared with in situ measurements for detecting the precipitation events and intensities over China during the nine years from 2005 to 2013. The PDF is the proportion of the number of times that rainfall events from each bin occurs divided by the total number of rainfall events. To considering the rainfall intensity $(R)$, this study has divided rainfall into eight bins [20]: (1) no rain $(R=0),(2) 0<R \leq 0.5 \mathrm{~mm} /$ day, (3) $0.5 \mathrm{~mm} /$ day $<R$ $\leq 1 \mathrm{~mm} /$ day, (4) $1 \mathrm{~mm} /$ day $<R \leq 2 \mathrm{~mm} /$ day, (5) $2 \mathrm{~mm} /$ day $<R \leq 5 \mathrm{~mm} /$ day, (6) $5 \mathrm{~mm} /$ day $<R \leq$ $10 \mathrm{~mm} /$ day, (7) $10 \mathrm{~mm} /$ day $<R \leq 20 \mathrm{~mm} /$ day and (8) $R>20 \mathrm{~mm} /$ day. The pixels nearest the gauge stations were selected to calculate the rainfall intensities. Approximately $60 \%$ of the values computed using the rain gauge stations show no-rain events, as shown in Figures 7 and 8. CMORPH_RAW, CMORPH_BLD and TRMM3BV42 can be inclined to detect more no-rain events than the gauge stations (around 79\%, 65.4\% and 64.3\%, respectively), which suggests that satellite measurements may miss some rain events. CMORPH_RAW detected more no-rain events than CMORPH_BLD, which showed that the bias-corrected satellite data tend to record more rainfall events. GSMaP_RENALYSIS and CMORPH_BLD record more light rainfall events $(0<R \leq 0.5 \mathrm{~mm} /$ day, around $8.5 \%$ and $8.8 \%$, respectively) than the other datasets. CMORPH_BLD, GSMaP_RENALYSIS and PERSIANN_CDR can record more rainfall events than the other products when the rainfall intensities are between 0.5 $\mathrm{mm} /$ day and $5 \mathrm{~mm} /$ day, where they may have an $R$ value as high as $2<R \leq 5 \mathrm{~mm} /$ day (around $7.7 \%, 9.3 \%$ and $8.1 \%$, respectively). These results can be explained follows: (1) the satellites can easily detect strong, convective rainfall events but are inclined to miss shallow and warm rains; (2) the bias-corrected methods can boost the amplitudes of the detected events to compensate for the missed events [37]. This study analyzed FBI, POD, CSI and FAR to evaluate the qualities of the errors between the satellite products and the gauge station data. The results of the statistical analyses are shown in Table 4. CMORPH_RAW FBI is better than CMORPH_BLD, such that the POD and CSI are low and FAR is high. TRMM3BV42 FBI is the closest to the gauge data of all the products but the POD and CSI are lower and the FAR is higher than for GSMaP_RENALYSIS. Therefore, GSMaP_RENALYSIS can more accurately detect rain events than the other products.

Table 4. The results of the statistics of FBI, POD, CSI and FAR.

\begin{tabular}{cccccc}
\hline & CMORPH_BLD & CMORPH_RAW & TRMM3B42 V & PERSIANN & GSMaP_RENALYSIS \\
\hline FBI & 1.27 & 1.13 & 1.00 & 1.03 & 1.01 \\
POD & 0.76 & 0.57 & 0.60 & 0.57 & 0.81 \\
CSI & 0.50 & 0.37 & 0.57 & 0.46 & 0.63 \\
FAR & 0.40 & 0.49 & 0.45 & 0.42 & 0.36 \\
\hline
\end{tabular}

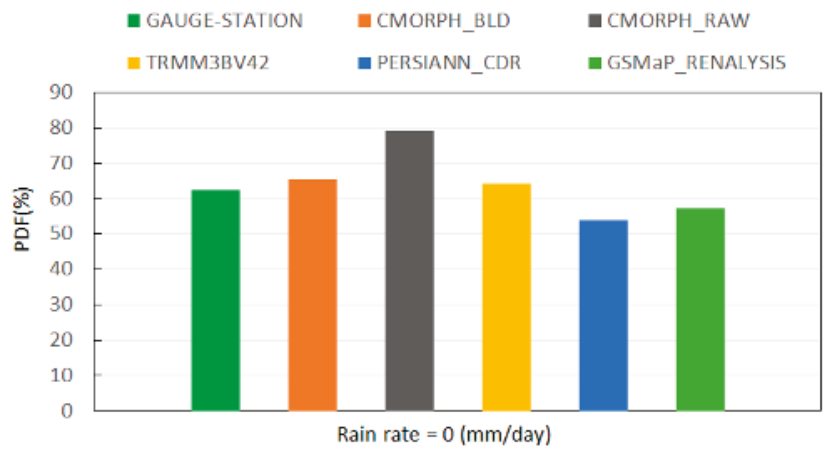

Figure 7. Probability density function of daily rainfall for the no-rain case. 


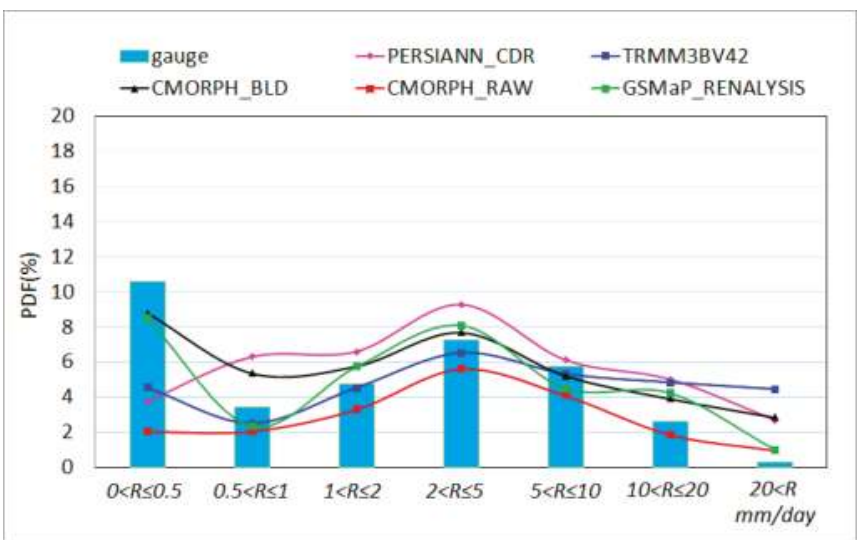

Figure 8. Probability density function of the daily rainfall events with different intensities.

\subsection{Typical Regional Analysis}

In this study, we collected data from 2280 rain gauge stations to analyze the precipitation in 2013 over the Tibetan Plateau and South China. The total precipitation was mapped in all four seasons and the CC, RMSE and BIAS were calculated in every month, which are shown in Figures 9-12. Tibetan Plateau is a typical of rainfall distribution including humid regions, semi-humid regions and semi-arid regions, which the distribution is influenced by atmospheric circulation and topography. Rain gauge stations are sparsely distributed over the Tibetan Plateau and the elevation ranges from $86 \mathrm{~m}$ to $8755 \mathrm{~m}$ and there are exceedingly few gauge stations built in areas with high elevation (Figure 9a). Figure 9b shows the spatial distribution of the total precipitation in four seasons. The total precipitation is relatively low and the spatial distribution trend of precipitation is decreasing from southeast to northwest. The satellite precipitation distribution is similar to the gauge stations but some regions show considerable differences. All products exhibited overestimation in the winter over the western regions, which the result related to the no-gauge stations and interpolated technique. All products overestimated the precipitation in the summer, excepting CMORPH_BLD. CMORPH_RAW overestimated the precipitation and its RMSE and BIAS showed large fluctuating patterns, demonstrating that uncorrected satellite products perform poorly over semi-arid and arid areas. CMORPH_RAW and CMORPH_BLD show some abnormal zero precipitation over Tibetan Plateau. It is possible that the ice and snow surface tend to influence PWM-based accurate retrieval over land [37]. The RMSE of GSMaP_RENALYSIS showed large differences in four seasons and GSMaP_RENALYSIS produces underestimation in the spring and overestimation in the summer and autumn.

To evaluate the performances of the satellite-based precipitation measurements, we calculated the CC, RMSE and BIAS values for each month, as shown in Figure 10. The CC of all the products are high from April to October (more than 0.6) and are relatively low in those months when the climate is dry. This result can be explained by the following reasons: (1) compared with weak precipitation, satellite sensors are more able to obtain heavy rainfall events; (2) Over the higher elevation mountainous area of eastern China, it is difficult to obtain accurate precipitation values via remote sensing methods due to the snow and ice on the surface in the winter; (3) Because of the complicated land surface characteristics and their effects on the upward microwave radiation, impacting SSM/I, AMSR-E and AMSU-B, it is difficult for the satellites to resolve rainfall over areas with low rainfall amounts [44]. PERSIANN_CDR has the worst CC among the satellite products and its RMSE and BIAS values show the largest errors, which can be explained by the poor input IR data over Tibetan Plateau. Overall, GSMaP_RENALYSIS 
has the best performance of all the products over the Tibetan Plateau and PERSIANN_CDR has the worst performance, both seasonally and monthly.

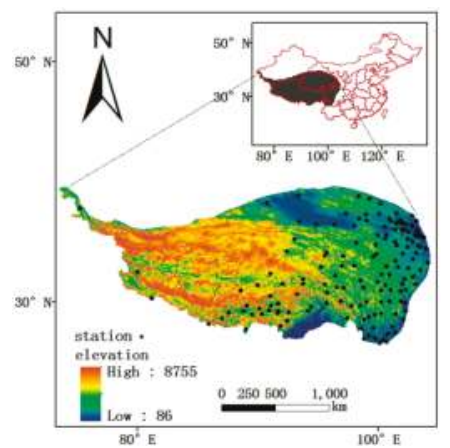

(a)

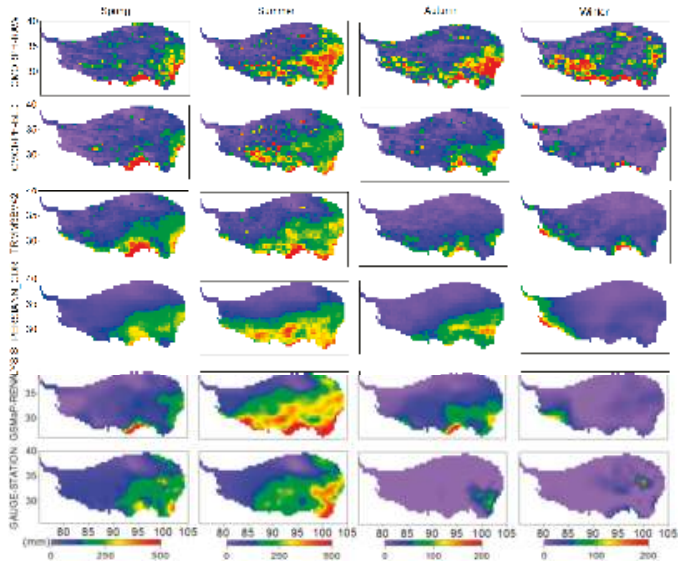

(b)

Figure 9. The precipitation distributions of the rain gauge stations (a) and multiple satellite (b) in 2013 over southern China.

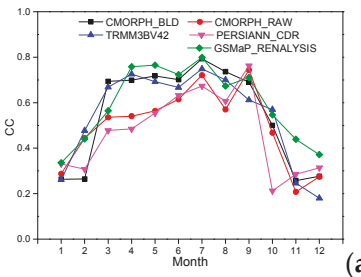

(a)

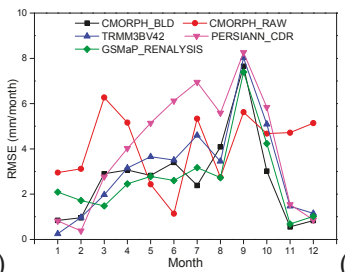

(b)

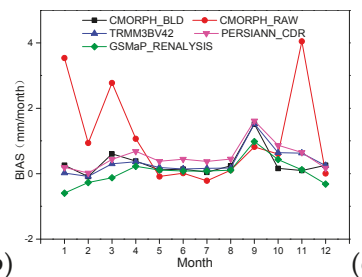

(c)

Figure 10. (a-c) denote the CC, RMSE and BIAS among a variety of products for each month in 2013 over the Tibetan Plateau, respectively.

There are more rain gauge stations and precipitation events in southern China than over the Tibetan Plateau, so we researched the regions including Fujian, Guangdong, Jiangxi, Hunan and Guangxi. The area of study and the elevation data are shown in Figure 11. The seasonal precipitation events detected by the satellites are similar to the in-situ measurements and the max annual precipitation is approximately $1500 \mathrm{~mm}$ in the spring and summer over Guangdong as well as over the south of Guangxi. This might be related to the period of precipitation: the study area is mainly affected by the southeast monsoon from the Pacific Ocean and the southwest monsoon from the India Ocean, which the period of precipitation is from May to September. The north of Hunan and Guangdong had more precipitation events than the other regions in all seasons except winter. Jiangxi and Fujian had more precipitation in the winter. This regional precipitation is mainly affected by the cold and warm air in the south. Note that little precipitation was measured over Hunan because of the effects of summer winds leading the precipitation northward in the summer. The CC, RMSE and BIAS values were calculated for each month and were compared with in situ measurements, as shown in Figure 12. The CC of all products are low in January, October and December and the RMSEs are high in the summer. The BIAS is near zero, except in CMORPH_RAW. CMORPH_RAW shows poor CC values, with a low near zero occurring in January. CMORPH_BLD and TRMM3BV42 show highly consistent precipitation distributions and both have higher CC than the other products. However, the CC of CMORPH_BDL 
is higher than that of TRMM3BV42 in January and November and its BIAS is lower. According to the precipitation distribution and the calculated statistical correlations, the CMORPH_BDL product is consistent with the in-situ measurements.

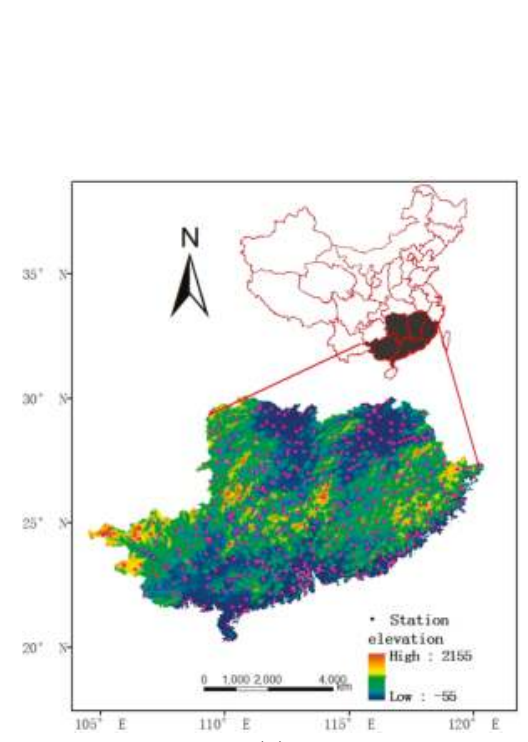

(a)

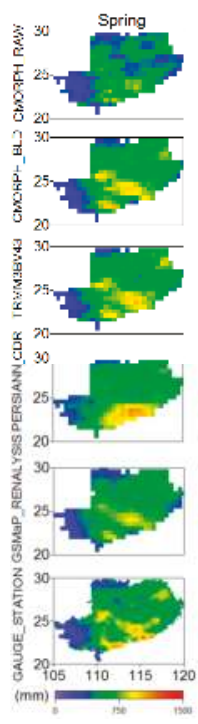

(mm) $\prod_{100}^{10}$
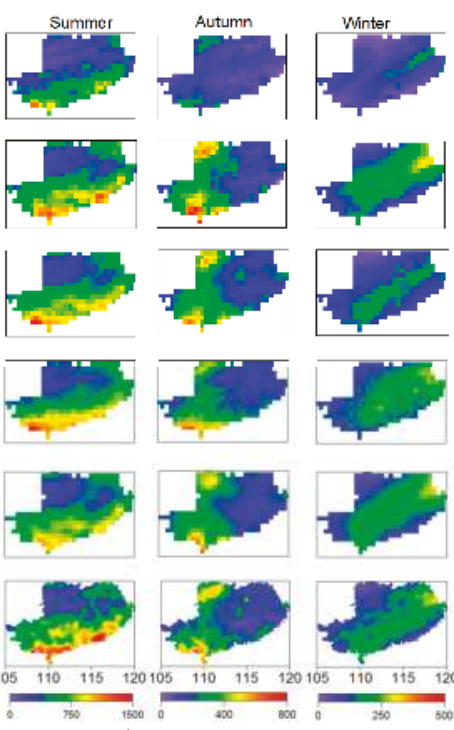

(b)

Figure 11. The precipitation distributions from the rain gauge stations (a) and multiple satellites (b) in 2013 over southern China.

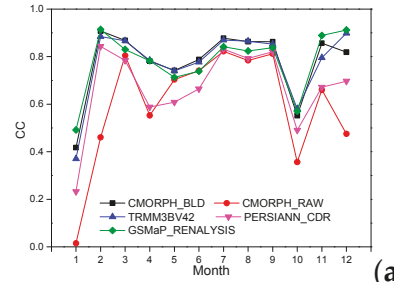

(a)

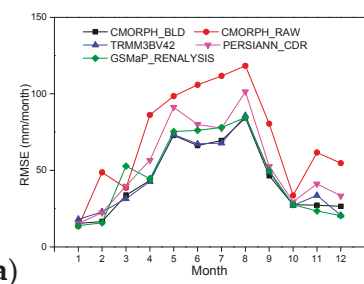

(b)

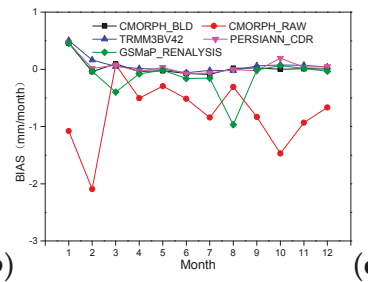

Figure 12. (a-c) denote the CC, RMSE and BIAS values of the studied products for each month in 2013 over southern China, respectively.

\section{Conclusions}

This paper mainly discusses the CMORPH_BLD, CMORPH_RAW, TRMM3BV42, PERSIANN_CDR and GSMaP_RENALYSIS precipitation products and their spatiotemporal precipitation distributions based on daily, monthly, seasonal and annual values. We compared the satellite products with data from rain gauge stations using statistical tools, such as their CC, BIAS and RMSE, for the period of 2005 to 2013. The five satellite rainfall products based on four different methods were analyzed via a quantitative study of their error features and the following points were concluded:

(1) According to the nine-year daily mean precipitation over China (Figures 2 and 3), the characteristics of precipitation is clearly gradually increasing in the south and decreasing in the northern regions of the Qinling Mountain and Huaihe River. There is more than $10 \mathrm{~mm} /$ day in the south and less than $5 \mathrm{~mm} /$ day in the northwest. Among the five satellite products, 
GSMaP_RENALYSIS suggests the best performance with the highest of $R(0.91)$ and the lowest RMSE $(0.85 \mathrm{~mm} /$ day). CMORPH_RAW demonstrates the poorest capability with the lowest of $R$ (0.73) and higher RMSE ( $2.79 \mathrm{~mm} /$ day). For the CC, RMSE and BIAS each annual daily mean precipitation from 2005 to 2013 (Table 3), GSMaP_RENALYSIS gives the highest CC $(>0.8)$ and smallest RMSE $(\sim 1.2 \mathrm{~mm} /$ day) and the low BIAS $( \pm 0.1)$ in every year. GSMaP_RENALYSIS underestimates precipitation from 2005 to 2006 but it overestimates precipitation from 2007 to 2013. CMORPH_BLD, CMORPH_RAW and TRMM3BV42 exhibit underestimation precipitation but PERSIANN_CDR shows overestimation precipitation.

(2) For the seasonal daily mean precipitation, the maximum precipitation is more than $15 \mathrm{~mm} /$ day in summer and about $5 \mathrm{~mm} /$ day in winter. Five satellite products show the best capability with the highest CC $(\sim 0.8)$ and the smallest RMSE ( 1 mm/day) but relatively the biggest BIAS in winter. In summer, all products give the smallest BIAS but the biggest RMSE ( $2 \mathrm{~mm} /$ day $)$ and high CC. GSMaP_RENALYSIS remains CC more than 0.8 and BIAS less than 0.2 , as well as low RMSE ( $<2 \mathrm{~mm} /$ day). For the monthly daily mean precipitation, CMORPH_RAW gives the lowest CC $(<0.2)$ in December and January and PERSIANN_CDR exhibits the biggest BIAS in January.

(3) The PDFs reveal that CMORPH_RAW, CMORPH_BLD and TRMM3BV42 detected more no-rainfall events than other products $(79 \%, 65.4 \%$ and $64.3 \%$, respectively) and GSMaP_RENALYSIS is consistent with gauge stations. All the precipitation products detected heavier rainfall events than the rain gauge stations measured. According to the statistical parameters of the rainfall events (e.g., FBI, POD, CSI and FAR), TRMM3B42V exhibits the relative perfect performance with FBI but the low POD and high FAR. CMORPH_BLD gives the higher POD but relative high FBI and FAR. GSMaP_RENALYSIS outperforms other products with the highest POD and CSI and lowest FAR.

(4) We analyzed two typical regions (the Tibetan Plateau and South China) in 2013. Over Tibetan Plateau, all products show the highest CC $(\sim 0.7)$ and low BIAS $( \pm 0.5 \mathrm{~mm} / \mathrm{month})$ and relative low RMSE $(\sim 3 \mathrm{~mm} /$ month) in July. The lowest CC $(<0.3)$ and biggest RMSE were calculated in December and September, respectively. GSMaP_RENALYSIS exhibits the best consistency with gauge stations. Over south China, the maximum total monthly precipitation is more than $1500 \mathrm{~mm} /$ month. All products (except PERSIANN_CDR) give the highest CC $(>0.8)$ and smallest BIAS in February. According to the precipitation distribution and the calculated statistical correlations, the CMORPH_BLD product outperforms other products with reference to the in situ measurements.

(5) The gauge-corrected products outperform the CMORPH_RAW. CMORPH_BLD compared with CMORPH_RAW performs better with increase in CC (from 0.73 to 0.80 ) and the reduction of RMSE (from $2.79 \mathrm{~mm} /$ day to $2.14 \mathrm{~mm} /$ day) in nine-year mean daily precipitation. The CC of CMORPH_BLD is from 0.70 to 0.82 during 2005-2013, while the CC of CMORPH_RAW is from 0.36 to 0.71 .

This paper analyzed the CMORPH_BLD, CMORPH_RAW, TRMM3BV42, PERSIANN_CDR and GSMaP_RENALYSIS precipitation products over China. The results are useful for selecting suitable satellite precipitation products for users with a variety of research interests. Because the differences in the algorithms may lead to varying degrees of error, it is necessary to adapt the method of retrieving precipitation information. This study evaluated only the study area of the Tibetan Plateau and southern China, so further precipitation research focusing on different terrains, such as high altitudes, basins and southern rainy areas, is necessary. These studies are useful for researching on atmospheric compositions and other fields and can help to improve GPM algorithms and comparisons.

Acknowledgments: This study was supported by the National Key Research and Development Program of China (Grant No. 2016YFC0200404), the National Natural Science Foundation of China (Grant No. 41571347), the Open Foundation of Chongqing Meteorology (KFJJ201402) and the Education Department Innovation team in Sichuan (16TD0024). The authors are grateful to China Meteorological Data Sharing Service System (http://data.cma. cn/), NASA (https://pmm.nasa.gov/), Japan Aerospace Exploration Agency (http://sharaku.eorc.jaxa.jp/), 
Shuttle Radar Topographic Mission (SRTM on http:/ / srtm.csi.cgiar.org/) and NOAA (https:/ / rda.ucar.edu/) scientific team for the provision of satellite data and situ measurement data utilized in this study. The authors acknowledge the China Meteorological Service Network and European Center, which made gauge station and satellite data available to us.

Author Contributions: Qiaolin Zeng proposed the method, collected data and wrote this paper; Yongqian Wang and Liangfu Chen conceived of the experiment and revised the paper; Zifeng Wang, Hao Zhu and Bin Li provided technical guidance and analyzed the data.

Conflicts of Interest: The authors declare no conflict of interest.

\section{References}

1. Immerzeel, W.W.; Rutten, M.M.; Droogers, P. Spatial downscaling of trmm precipitation using vegetative response on the Iberian Peninsula. Remote Sens. Environ. 2009, 113, 362-370. [CrossRef]

2. Maddox, R.A.; Zhang, J.; Gourley, J.J.; Howard, K.W. Weather radar coverage over the contiguous united states. Weather Forecast. 2001, 17, 927-934. [CrossRef]

3. Tang, L.; Hossain, F. Investigating the similarity of satellite rainfall error metrics as a function of köppen climate classification. Atmos. Res. 2012, 104-105, 182-192. [CrossRef]

4. Barrett, E.C.; Martin, D.W. Use of Satellite Data in Rainfall Monitoring; Academic Press: Cambridge, MA, USA, 1981.

5. Kidd, C.; Levizanni, V. Status of satellite precipitation retrievals. In Proceedings of the Earth Observation and Water Cycle Science, Frascati, Italy, 18-20 November 2009.

6. Levizzani, V.; Schmetz, J.; Lutz, H.J.; Kerkmann, J.; Alberoni, P.P.; Cervino, M. Precipitation estimations from geostationary orbit and prospects for meteosat second generation. Meteorol. Appl. 2001, 8, 23-41. [CrossRef]

7. Atlas, D.; Thiele, O.W. Precipitation Measurements from Space: Workshop Report. An Element of the Climate Observing System Study; NASA Tech. Memo. NASA-TM-85329431; NASA Goddard Space Flight Center: Greenbelt, MD, USA, 1981.

8. Behrangi, A.; Hsu, K.L.; Imam, B.; Sorooshian, S.; Kuligowski, R.J. Evaluating the utility of multispectral information in delineating the areal extent of precipitation. J. Hydrometeorol. 2009, 10, 684-700. [CrossRef]

9. Kühnlein, M.; Appelhans, T.; Thies, B.; Nauss, T. Improving the accuracy of rainfall rates from optical satellite sensors with machine learning-A random forests-based approach applied to msg seviri. Remote Sens. Environ. 2014, 141, 129-143. [CrossRef]

10. Stenz, R.; Dong, X.; Xi, B.; Feng, Z.; Kuligowski, R.J. Improving satellite quantitative precipitation estimation using goes-retrieved cloud optical depth. J. Hydrometeorol. 2015, 17. [CrossRef]

11. Joyce, R.J.; Janowiak, J.E.; Arkin, P.A.; Xie, P. Cmorph: A method that produces global precipitation estimates from passive microwave and infrared data at high spatial and temporal resolution. J. Hydrometeorol. 2003, 5, 287-296. [CrossRef]

12. Kidd, C.; Kniveton, D.R.; Todd, M.C.; Bellerby, T.J. Satellite rainfall estimation using combined passive microwave and infrared algorithms. J. Hydrometeorol. 2003, 4, 1088. [CrossRef]

13. Wilheit, T.T.; Chang, A.T.C.; Rao, M.S.V.; Rodgers, E.B.; Theon, J.S. A satellite technique for quantitatively mapping rainfall rates over the oceans. J. Appl. Meteorol. 1977, 16. [CrossRef]

14. Wilheit, T.; Kummerow, C.D.; Ferraro, R. Nasdarainfall algorithms for amsr-e. IEEE Trans. Geosci. Remote Sens. 2003, 41, 204-214. [CrossRef]

15. Stephens, G.L.; Vane, D.G.; Tanelli, S.; Im, E.; Durden, S.; Rokey, M.; Reinke, D.; Partain, P.; Mace, G.G.; Austin, R. Cloudsat mission: Performance and early science after the first year of operation. J. Geophys. Res. Atmos. 2008, 113, 2036-2044. [CrossRef]

16. Kalinga, O.A.; Gan, T.Y. Estimation of rainfall from infrared-microwave satellite data for basin-scale hydrologic modelling. Hydrol. Process. 2010, 24, 2068-2086. [CrossRef]

17. Pan, M.; Li, H.; Wood, E. Assessing the skill of satellite-based precipitation estimates in hydrologic applications. Water Resour. Res. 2010, 46, 201-210. [CrossRef]

18. Boushaki, F.I.; Hsu, K.L.; Sorooshian, S.; Park, G.H.; Mahani, S.; Shi, W. Bias adjustment of satellite precipitation estimation using ground-based measurement: A case study evaluation over the southwestern united states. J. Hydrometeorol. 2008, 10, 1231-1242. [CrossRef]

19. Hirpa, F.A.; Gebremichael, M.; Hopson, T. Evaluation of high-resolution satellite precipitation products over very complex terrain in Ethiopia. J. Appl. Meteorol. Climatol. 2010, 49, 1044-1051. [CrossRef] 
20. Guo, H.; Chen, S.; Bao, A.; Hu, J.; Gebregiorgis, A.; Xue, X.; Zhang, X. Inter-comparison of high-resolution satellite precipitation products over central Asia. Remote Sens. 2015, 7, 7181-7211. [CrossRef]

21. Awange, J.L.; Ferreira, V.G.; Forootan, E.; Andam-Akorful, S.A.; Agutu, N.O.; He, X.F. Uncertainties in remotely sensed precipitation data over Africa. Int. J. Climatol. 2016, 36, 303-323. [CrossRef]

22. Huffman, G.J.; Adler, R.F.; Bolvin, D.T.; Gu, G.; Nelkin, E.J.; Bowman, K.P.; Hong, Y.; Stocker, E.F.; Wolff, D.B. The TRMM Multisatellite Precipitation Analysis (TMPA): Quasi-Global, Multiyear, Combined-Sensor Precipitation Estimates at Fine Scales. J. Hydrometeorol. 2007, 8, 38-55. [CrossRef]

23. Joyce, R.; Janowiak, J.; Huffman, G. Latitudinally and seasonally dependent zenith-angle corrections for geostationary satellite ir brightness temperatures. J. Appl. Meteorol. 2001, 40, 689-703. [CrossRef]

24. Xie, P.; Joyce, R.; Wu, S.; Yoo, S.H.; Yarosh, Y.; Sun, F.; Lin, R. Reprocessed, bias-corrected cmorph global high-resolution precipitation estimates from 1998. J. Hydrometeorol. 2017, 18. [CrossRef]

25. Sorooshian, S.; Hsu, K.L.; Gao, X.; Gupta, H.V.; Imam, B.; Dan, B. Evaluation of PERSIANN system satellite-based estimates of tropical rainfall. Bull. Am. Meteorol. Soc. 2000, 81, 2035-2046. [CrossRef]

26. Hsu, K.; Gao, X.; Sorooshian, S.; Gupta, H.V. Precipitation estimation from remotely sensed information using artificial neural networks. J. Appl. Meteorol. 2004, 36, 1176-1190. [CrossRef]

27. Ashouri, H.; Hsu, K.L.; Sorooshian, S.; Braithwaite, D.K.; Knapp, K.R.; Cecil, L.D.; Nelson, B.R.; Prat, O.P. Persiann-cdr: Daily precipitation climate data record from multisatellite observations for hydrological and climate studies. Bull. Am. Meteorol. Soc. 2014, 96, 197-210. [CrossRef]

28. Okamoto, K.I.; Ushio, T.; Iguchi, T.; Takahashi, N.; Iwanami, K. The global satellite mapping of precipitation (gsmap) project. In Proceedings of the 2005 IEEE International Geoscience and Remote Sensing Symposium-2005. IGARSS '05, Seoul, Korea, 29-29 July 2005; pp. 3414-3416.

29. Kubota, T.; Shige, S.; Hashizume, H.; Aonashi, K.; Takahashi, N.; Seto, S.; Takayabu, Y.N.; Ushio, T.; Nakagawa, K.; Iwanami, K. Global precipitation map using satellite-borne microwave radiometers by the gsmap project: Production and validation. IEEE Trans. Geosci. Remote Sens. 2007, 45, 2259-2275. [CrossRef]

30. Mega, T.; Ushio, T.; Kubota, T.; Kachi, M.; Aonashi, K.; Shige, S. Gauge adjusted global satellite mapping of precipitation (gsmap_gauge). In Proceedings of the 2014 XXXIth URSI General Assembly and Scientific Symposium (URSI GASS), Beijing, China, 16-23 August 2014; pp. 1-4.

31. Vila, D.A.; Goncalves, L.G.G.D.; Toll, D.L.; Rozante, J.R. Statistical evaluation of combined daily gauge observations and rainfall satellite estimates over continental south America. J. Hydrometeorol. 2009, 10, 533-543. [CrossRef]

32. Wilks, D.S. Statistical Methods in the Atmospheric Sciences; Elsevier: San Diego, CA, USA, 2011.

33. Condom, T.; Rau, P.; Espinoza, J.C. Correction of trmm $3 \mathrm{~b} 43$ monthly precipitation data over the mountainous areas of Peru during the period 1998-2007. Hydrol. Process. 2011, 25, 1924-1933. [CrossRef]

34. Brown, J.E.M. An analysis of the performance of hybrid infrared and microwave satellite precipitation algorithms over India and adjacent regions. Remote Sens. Environ. 2006, 101, 63-81. [CrossRef]

35. Su, F.; Gao, H.; Huffman, G.J.; Lettenmaier, D.P. Potential utility of the real-time tmpa-rt precipitation estimates in streamflow prediction. J. Hydrometeorol. 2011, 12, 444-455. [CrossRef]

36. Chen, S.; Hong, Y.; Cao, Q.; Tian, Y.; Hu, J.; Zhang, X.; Li, W.; Carr, N.; Shen, X.; Qiao, L. Intercomparison of precipitation estimates from wsr-88d radar and trmm measurement over continental united states. IEEE Trans. Geosci. Remote Sens. 2015, 53, 4444-4456. [CrossRef]

37. Tian, Y.; Peterslidard, C.D.; Choudhury, B.J.; Garcia, M. Multitemporal analysis of trmm-based satellite precipitation products for land data assimilation applications. J. Hydrometeorol. 2007, 8, 1165-1183. [CrossRef]

38. Chen, S.; Hong, Y.; Cao, Q.; Gourley, J.J.; Kirstetter, P.E.; Yong, B.; Tian, Y.; Zhang, Z.; Shen, Y.; Hu, J. Similarity and difference of the two successive $\mathrm{v} 6$ and $\mathrm{v} 7 \mathrm{trmm}$ multisatellite precipitation analysis performance over china. J. Geophys. Res. Atmos. 2013, 118, 13060-13074. [CrossRef]

39. Tian, Y.D.; Peterslidard, C.D.; Adler, R.F.; Kubota, T.; Ushio, T. Evaluation of gsmap precipitation estimates over the contiguous united states. J. Hydrometeorol. 2010, 11, 566-574. [CrossRef]

40. Ushio, T.; Sasashige, K.; Kubota, T.; Shige, S.; Okamoto, K.; Aonashi, K.; Inoue, T.; Takahashi, N.; Iguchi, T.; Kachi, M.; et al. A kalman filter approach to the global satellite mapping of precipitation (gsmap) from combined passive microwave and infrared radiometric data. J. Meteorol. Soc. Jpn. 2009, 87A, 137-151. [CrossRef]

41. Li, Z.; Yang, D.; Hong, Y. Multi-scale evaluation of high-resolution multi-sensor blended global precipitation products over the Yangtze River. J. Hydrol. 2013, 500, 157-169. [CrossRef] 
42. Chen, M.; Shi, W.; Xie, P.; Silva, V.B.S.; Kousky, V.E.; Wayne Higgins, R.; Janowiak, J.E. Assessing objective techniques for gauge-based analyses of global daily precipitation. J. Geophys. Res. Atmos. 2008, 113, D04110. [CrossRef]

43. Shen, Y.; Xiong, A.; Wang, Y.; Xie, P. Performance of high-resolution satellite precipitation products over china. J. Geophys. Res. Atmos. 2010, 115, 355-365. [CrossRef]

44. Grody, N.C.; Weng, F. Microwave emission and scattering from deserts: Theory compared with satellite measurements. IEEE Trans. Geosci. Remote Sens. 2008, 46, 361-375. [CrossRef]

(C) 2018 by the authors. Licensee MDPI, Basel, Switzerland. This article is an open access article distributed under the terms and conditions of the Creative Commons Attribution (CC BY) license (http://creativecommons.org/licenses/by/4.0/). 
Article

\title{
Analysis of the Spatial Variability of Land Surface Variables for ET Estimation: Case Study in HiWATER Campaign
}

\author{
Xiaojun Li ${ }^{1,2,3}$, Xiaozhou Xin ${ }^{1,2, *}$, Zhiqing Peng ${ }^{1,3}$, Hailong Zhang ${ }^{1,2}$, Chuanxiang $\mathrm{Yi}^{4}$ \\ and $\mathrm{Bin} \mathrm{Li}^{5}$ \\ 1 State Key Laboratory of Remote Sensing Science, Jointly Sponsored by Institute of Remote Sensing and \\ Digital Earth of Chinese Academy of Sciences and Beijing Normal University, Beijing 100101, China; \\ kdxiaojun@126.com (X.L.); pengzhiqing1991@126.com (Z.P.); zhlnjnu@163.com (H.Z.) \\ 2 Joint Center for Global Change Studies (JCGCS), Beijing 100875, China \\ 3 University of Chinese Academy of Sciences, Beijing 100049, China \\ 4 College of Applied Meteorology, Nanjing University of Information Science \& Technology, \\ Nanjing 210044, China; 18761808890@163.com \\ 5 Inner Mongolia Ecological and Agricultural Meteorological Center, Hohhot 010051, China; \\ 15632470343@163.com \\ * Correspondence: xin_xzh@163.com; Tel.: +86-10-6487-9382
}

Received: 13 December 2017; Accepted: 10 January 2018; Published: 11 January 2018

\begin{abstract}
Heterogeneity, including the inhomogeneity of landscapes and surface variables, significantly affects the accuracy of evapotranspiration (ET) (or latent heat flux, LE) estimated from remote sensing satellite data. However, most of the current research uses statistical methods in the mixed pixel to correct the ET or LE estimation error, and there is a lack of research from the perspective of the remote sensing model. The method of using frequency distributions or generalized probability density functions (PDFs), which is called the "statistical-dynamical" approach to describe the heterogeneity of land surface characteristics, is a good way to solve the problem. However, in attempting to produce an efficient PDF-based parameterization of remotely sensed ET or LE, first and foremost, it is necessary to systematically understand the variables that are most consistent with the heterogeneity (i.e., variability for a fixed target area or landscape, where the variation in the surface parameter value is primarily concerned with the PDF-based model) of surface turbulence flux. However, the use of PDF alone does not facilitate direct comparisons of the spatial variability of surface variables. To address this issue, the objective of this study is to find an indicator based on PDF to express variability of surface variables. We select the dimensionless or dimensional consistent coefficient of variation (CV), Gini coefficient and entropy to express variability. Based on the analysis of simulated data and field experimental data, we find that entropy is more stable and accurate than the $\mathrm{CV}$ and Gini coefficient for expressing the variability of surface variables. In addition, the results of the three methods show that the variability of the leaf area index (LAI) is greater than that of the land surface temperature (LST). Our results provide a suitable method for comparing the variability of different variables.
\end{abstract}

Keywords: spatial heterogeneity; variability; evapotranspiration; land surface variables; probability density function; HiWATER

\section{Introduction}

Evapotranspiration plays a key role in the Earth's surface energy and water balances, and it has substantial effects on global climate change, water management and crop yield [1-3]. Satellite-based remote sensing has been identified as a suitable means of mapping the spatial distribution of ET or LE. 
Because of technical and methodological limitations, many remote sensing-based ET or LE models have been conducted under quite homogeneous and flat conditions, with the use of decametric to kilometric spatial resolution sensors [4,5]. However, the Earth's surface geometry, physical processes and associated variables are inherently heterogeneous. Due to the nonlinear nature of many land surface processes, heterogeneity can profoundly affect the result of estimating ET or LE $[1,6]$.

The effect of surface heterogeneity on remote sensing-based ET or LE estimation is mainly caused by two aspects: the heterogeneity produced by the landscape and the heterogeneity produced by surface variables [1]. Three basic types of methods have been proposed to compensate for the errors caused by surface heterogeneity or considering subpixel scale land surface heterogeneities when estimating surface ET or LE [7-12]. The error caused by the landscape heterogeneity is usually corrected using the mosaic or area weighting method $[1,11]$ and the correction factor compensation method [13]. The mosaic or area weighting method neglects all small-scale interactions by dividing each grid cell into several homogeneous patches according to the different land-use types, assuming that the various patches do not interact with each other but interact vertically with the atmosphere directly above them [14]. The grid fluxes are the averages of the patch fluxes weighted by their fractional area no matter where within the grid cell the individual patches are located $[9,15]$. Therefore, in the same landscape, the errors caused by the heterogeneity of spatial patterns change of the surface variable are negligible for the ET or LE calculation. The error caused by the heterogeneity of the surface variables (i.e., land surface temperature (LST)) is usually corrected by the temperature-sharpening method [1,16], and it is capable of decreasing the influences of the heterogeneity of the LST [17,18]. The core of these two methods is to combine the high-resolution satellite data with the low-resolution satellite data and using statistical methods to describe and express the heterogeneity of the landscape composition or the spatial distribution of the variables in the mixed pixel to correct the ET or LE estimation error. In addition, the physical mechanisms of some methods (e.g., the correction factor compensation method) are not clear, leading to low portability $[13,19]$.

Therefore, the study of surface heterogeneity in ET or LE estimation from the perspective of remote sensing model is still needed. The method of using frequency distributions or generalized probability density functions (PDF), which is called the "statistical-dynamical" approach, to describe the variability of land surface characteristics is a good way to solve the problem [10,20-22]. It is based on the assumption that climate forcing (temperature, precipitation, humidity, etc.) and land surface characteristics (i.e., soil, vegetation, topography, etc.) vary according to the distributions which can be approximated by continuous analytical PDFs rather than a single representative value. The grid fluxes are calculated by statistical-dynamical method using numerical or analytical integration over appropriate PDFs [23]. Li and Avissar [10] demonstrated that the results of determined fluxes depend on the spatial distribution of the land surface parameters, and the more skewed the distribution within the range of values, the larger the error (for nonlinear relationships) of the flux calculated using the mean instead of the distribution. Tittebrand and Berger [14] confirmed the possible application of the PDF-approach for the determination of LE with Penman-Monteith using Normalized Difference Vegetation Index (NDVI), albedo, relative humidity and wind speed for grassland and coniferous forest. The PDF-based flux estimation model can be used to describe spatial variability of specific surface parameters for a model grid or a satellite pixel with coarser spatial resolution that is described by a higher resolved dataset $[10,14,20-23]$.

The appropriate choice of spatial heterogeneity description method based on different usages is the key to eliminate ET or LE estimation error. The heterogeneity produced by landscape is often expressed by a landscape pattern index including Moran's I spatial autocorrelation index [24], Getis statistics [25], porosity indices, etc. The heterogeneities of surface variables are regarded as two different aspects with or without considering the spatial patterns of surface variables [26,27]. By considering spatial patterns or spatial arrangement as well as the value of the gray scale of the pixels, the changes in spatial patterns with more or less chaotic spatial arrangements correspond to greater or smaller spatial heterogeneity $[2,10]$. In the other way, only the heterogeneity caused by the change in the 
variable value or gray value (i.e., spatial variability of the surface property over the observed scene) is considered [26]. Correspondingly, the description of spatial heterogeneity contains two groups of methods. One group considers both the spatial patterns and spatial variability of the variable, including empirical, probabilistic and other methods. Empirical approaches such as local variance [28], Haralick indices [29] and ANOVA-quadtree analysis [30] may be limited in charactering the image spatial heterogeneity because of the lack of an underlying theoretical framework [26]. Probabilistic approaches (e.g., spatial entropy, multifractal, fractal, variogram [31], and q-statistic method [27]), which consider the image as a realization of the stochastic process called random function [26], provide more efficient tools to model the spatial heterogeneity of components. Other methods involve mathematical models such as wavelet analysis [19] and Fourier transform [32]; moreover, geographically weighted regression has become popular to explore spatial heterogeneity [33]. These methods are mostly used to analyze the heterogeneity of land surface solely, so the spatial patterns of pixels and spatial variability of the variable values both need to be considered [26,34]. The other group focuses only on spatial variability of the variable values and does not consider the spatial patterns. The commonly used methods include the quantile, range, deviation, variance/standard deviation, moment, coefficient of variation (CV) [25], Gini coefficient and entropy [34,35], and these methods are often used for specific purposes (e.g., building models) [36].

Though most PDFs are described by only a few parameters, implementing a PDF for each land-surface characteristic would greatly increase the complexity of this type of parameterization, as well as the computational burden of the model [10]. Therefore, in attempting to produce efficient PDF-based parameterization of remotely sensed ET or LE, one needs to solve a basic question: which surface variables' heterogeneity must be considered when establishing an ET or LE estimation model, i.e., which variability of surface variables varies similarly or consistently with the variability of the turbulent flux in time. Since the use of PDF alone does not facilitate direct comparisons of the spatial variability of surface variables, in order to answer the question, an index that quantitatively describes the overall variability of the PDF is still required [37]. The main objective of this study is to find an index to describe the heterogeneity caused by the fluctuation of the surface variable values, i.e., the spatial variability of the surface variables over the observed scene. Based on simulation data and Heihe Watershed Allied Telemetry Experimental Research (HiWATER) data, we used the PDF as a starting point to explore the spatial variability expression method of surface variables, and this approach could be used to systematically analyze the spatial variability of surface variables and compare the variability of different variables over the same observed scene.

\section{Study Area and Datasets}

\subsection{Study Area}

The study area (Figure 1b), which includes the Yingke and Daman irrigation districts in the Zhangye oasis and adjacent Gobi Desert, is located at $100.10^{\circ} \mathrm{E}-100.66^{\circ} \mathrm{E}, 38.68^{\circ} \mathrm{N}-39.15^{\circ} \mathrm{N}$ in the central reaches of the Heihe River Basin (HRB) and near the city of Zhangye in the arid region of Gansu Province in northwestern China (Figure 1a). The climate is cold and arid, with a mean annual precipitation, temperature, and potential evapotranspiration of 100-250 $\mathrm{mm}$, approximately $7{ }^{\circ} \mathrm{C}$, and $1200-1800 \mathrm{~mm}$, respectively. Its terrain is flat, and its elevation is approximately $1480 \mathrm{~m}$. The two irrigation districts are key experimental areas of the HiWATER project (see Li et al. [38] and Liu et al. [39] for details). One major objective of the HiWATER project, namely, the Multi-Scale Observation Experiment on Evapotranspiration (HiWATER-MUSOEXE), is to capture the strong land surface heterogeneities and associated uncertainties within a watershed $[3,38,39]$. The core experimental area is heterogeneous and dominated by maize, spring wheat, vegetables, orchards, and residential areas (Figure 1c). 


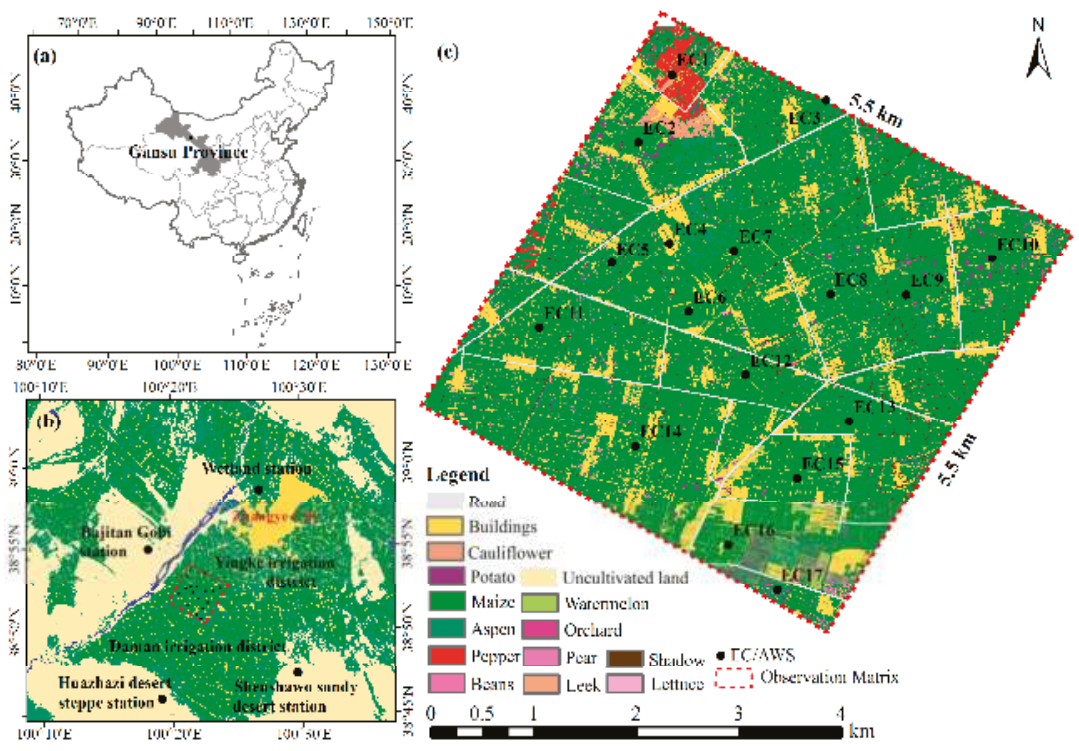

Figure 1. Geographical information of the study area: (a) schematic of Zhangye city and middle Heihe River Basin; (b) locations of HiWATER-MUSOEXE observation matrix; (c) detailed locations of the 17 EC systems and AWSs inside the Zhangye oasis. EC/AWS is the abbreviation for eddy covariance/automatic weather station.

\subsection{Datasets}

\subsubsection{Ground Observation Data}

Several ground observation datasets from HiWATER were employed in this study. HiWATER was designed as a comprehensive ecohydrological experiment to address problems that include heterogeneity, uncertainty, scaling, and closing the water cycle at the watershed scale implemented in the HRB, an inland river watershed [2]. MUSOEXE, a component of HiWATER, was implemented to reveal the spatial heterogeneities of heat and water fluxes by constructing a flux matrix (Figure 1c) from May to September 2012. The matrix includes 17 eddy covariance (EC) systems together with automatic weather stations (AWSs) that were installed in the $5.5 \times 5.5 \mathrm{~km}^{2}$ kernel experimental area (Figure 1c) according to the distribution of crops, shelterbelts, roads, residential areas and canals, as well as according to soil moisture and irrigation status [39]. Among the 17 EC systems, 14 were installed in maize fields, and the other three were located in a residential area (EC4 in Figure 1c), a vegetable field (EC1 in Figure 1c) and an orchard (EC17 in Figure 1c). The EC station mainly provides flux data (including carbon dioxide flux, sensible heat flux and latent heat flux), friction wind speed, etc. Meteorological data, including air temperature and humidity, wind speed and direction, solar radiation and net radiation, layers of soil temperature and humidity, etc., were recorded at the AWSs.

The consistency of all of the EC systems and the quality of the data were ensured using the following steps. First, the consistency of the EC instruments was tested during an inter-comparison campaign in the flat, open Gobi Desert, over a surface covered by coarse grain sand and small pebbles with withered sparse scrub vegetation. This campaign was completed before HiWATER-MUSOEXE was conducted [40]. Second, all of the raw data were acquired at $10 \mathrm{HZ}$ and processed using the Edire software package developed by the University of Edinburgh (Edinburgh, UK). The processing steps included spike removal, lag time correction, coordinate rotation (2D rotation), frequency response correction, corrections for density fluctuations (WPL-correction), and averaging to half-hourly 
fluxes $[39,41]$. Third, a four-step procedure was performed to control the quality of the EC data; for more details, see [1,2]. Because night turbulence is weak and considerable data are either missing or of poor quality, this study used data from 10:00 to 18:00 Beijing time to ensure the quality of turbulent flux observation data. The crop growth season is from June to September, and good quality data for 5 days were selected for each month, including 25, 26, 28-30 June; 23, 26, 27, 30 and 31 July; 4, 5, 8, 22 and 25 August; and 2, 6, 8, 12 and 14 September, for a total of 20 days.

\subsubsection{Airborne Data}

The remote sensing data used in this study included multiangular and multispectral images that were collected by an airborne wide-angle infrared dual-model line/area array scanner (WiDAS) and high-resolution land cover maps derived from an airborne compact airborne spectrographic imager (CASI). Land cover maps with 1-m resolution were produced by an object-oriented workflow using CASI images [42]. The landscape was classified into fourteen detailed land cover types (Figure 1c), and the results were validated using ground survey points. The overall accuracy was $88.48 \%$, and the Kappa coefficient was 0.87 [42]. WiDAS is one of the major instruments used in the HiWATER-MUSOEXE airborne missions to acquire multiangular observation data. It acquires images using four CCD cameras in the visible near-infrared (VNIR) bands and two thermal cameras in the mid-infrared (MIR) and thermal infrared (TIR) bands. The specification payloads of WiDAS can be seen in Liu et al. [43]. For the flight data collected on 3 August 2012, 15 tracks are included in total. After calibration, lens distortion correction, geometric correction, viewing angle retrieval, and atmospheric correction, the resolutions of the visible infrared band and thermal infrared band are $0.5 \mathrm{~m}$ and $5 \mathrm{~m}$, respectively. To ensure that each track has 750 rows $\times 250$ columns $\left(3750 \times 1250 \mathrm{~m}^{2}\right)$ as a prerequisite, six tracks were selected from 15 tracks. Each track is divided into three regions, each with a size of $1250 \times 1250 \mathrm{~m}^{2}$ as the test area. We used pre-processed WiDAS data to calculate the LAI [1] and LST [43], and resampled the surface variable data to $5 \mathrm{~m}$.

\section{Methodology}

The remote sensor above the land surface receives not only the signal of the mean and variance but also the signal of all values present on the surface. Thus, modelers are especially interested in the PDF of the surface variable $[14,34]$, which tells how frequent a certain parameter value occurs. For the same variable, if the PDF is narrow, then the surface is more homogeneous, and if the PDF is broad, the surface is more heterogeneous [34]. PDF can describe heterogeneity in a qualitative manner, however, it is unable to quantify the information content and cannot be used to compare the variability of different variables over the same observed scene [34]. As we mentioned in the introduction, the commonly used methods that express the spatial variability of surface variables include the quantile, range, deviation, variance/standard deviation, moment, CV, Gini coefficient and entropy. Because the magnitude and dimension of each surface variable vary, a dimensionless or dimensional consistent indicator is required to compare the variability between variables directly. After comparing the aforementioned methods, the indicators that can satisfy the above demand and better represent the variability are the $\mathrm{CV}$, Gini coefficient and entropy. The first two are dimensionless. For a fixed scale, the amount of information can be quantified via the entropy and its dimension is consistent; the unit in this paper is nats. We illustrate the characteristics of the three methods in expressing spatial variability from both aspects of simulated data and experimental data. The framework and flowchart of this paper is shown in Figure 2. PDF is the starting point for our research of surface spatial variability. The framework in Figure 2 is as we reviewed in the introduction, and for the flowchart (i.e., the main purpose of this paper), it is the first step to answer the basic question, and also the first step to build a more efficient PDF-based ET or LE estimation model. The details of the three methods are described in the following paragraphs.

$\mathrm{CV}$ is dimensionless and often used for auxiliary modeling, such as the use of $\mathrm{CV}$ to characterize surface variability when establishing a temperature-sharpening method, and lower $\mathrm{CV}$ values 
correspond to more homogeneous land surface values $[1,3,36]$. CV can evaluate the variability of a sample in a population as follows [25]:

$$
C V=\frac{\operatorname{std}(x)}{u(x)}=\frac{\sqrt{\frac{1}{n-1} \sum_{i=1}^{n}\left(x_{i}-u\right)^{2}}}{\frac{1}{n} \sum_{i=1}^{n} x_{i}}
$$

where $\operatorname{std}(\mathrm{x})$ and $\mathrm{u}(\mathrm{x})$ are the standard deviation and the average value of the sampling point, respectively. $\mathrm{u}(\mathrm{x})$ can be negative, then the above equation is also negative. However, if $\mathrm{CV}$ is negative, its absolute value should be used to measure the degree of variability (i.e., the greater the absolute value, the stronger the degree of variability, and vice versa). Generally, a value of $\mathrm{CV}<0.15$ indicates a variable with low variability; $0.15 \leq \mathrm{CV} \leq 1$ indicates a variable with moderate variability; and CV $>1$ indicates a variable with a high degree of variability $[1,36]$.

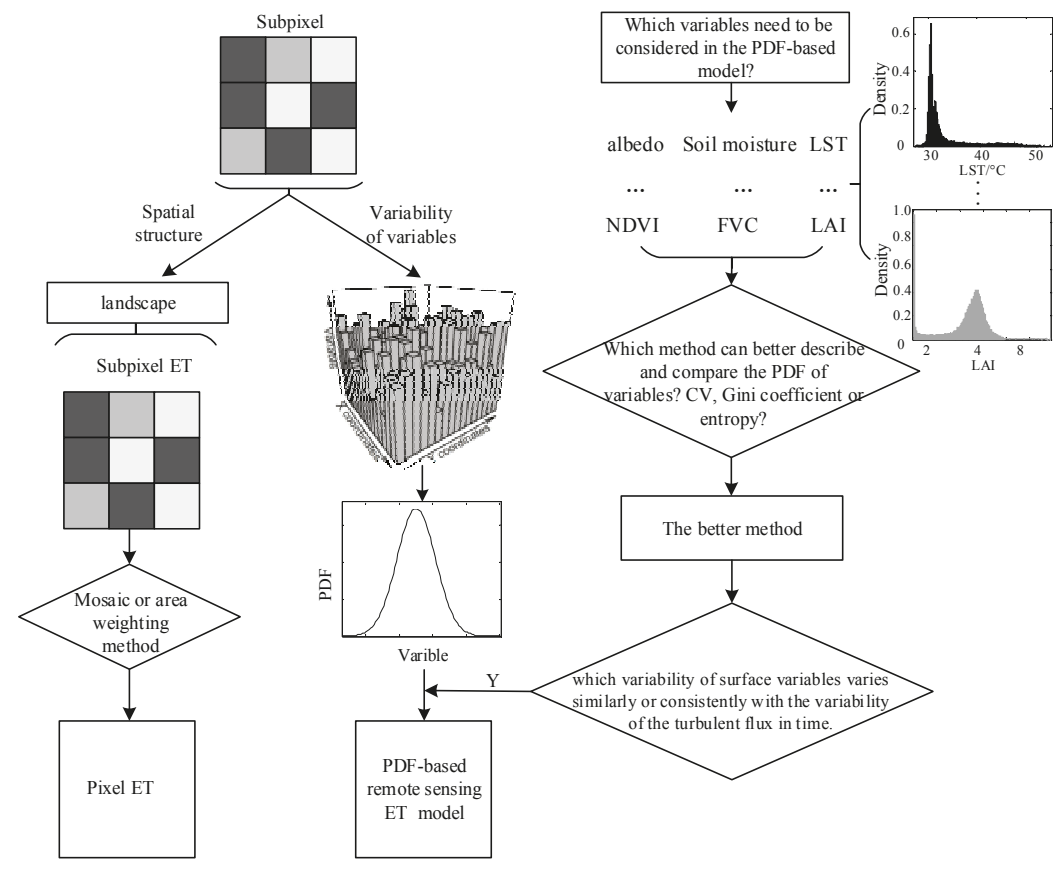

(a) framework

(b) flowchart

Figure 2. Framework and flowchart of this paper.

Compared to $\mathrm{CV}$, the Gini coefficient is also dimensionless and is more likely to represent the ratio of deviations between data [44]. The Gini coefficient is an index of the fairness of the income distribution in the field of economics, and it is in the range of $[0,1]$. For $n$ values of the variable $x$, a number of methods are available to calculate the Gini coefficient. The calculation method used in this study is as follows [44]:

$$
\text { Gini }=\frac{\sum_{\mathrm{i}} \sum_{\mathrm{j}}\left|\mathrm{x}_{\mathrm{i}}-\mathrm{x}_{\mathrm{j}}\right|}{2 \sum_{\mathrm{i}} \sum_{\mathrm{j}} \mathrm{x}_{\mathrm{i}}}=\frac{\sum_{\mathrm{i}} \sum_{\mathrm{j}}\left|\mathrm{x}_{\mathrm{i}}-\mathrm{x}_{\mathrm{j}}\right|}{2 \mathrm{n}^{2} \overline{\mathrm{x}}}
$$


Information entropy is a state variable in thermodynamics that indicates the degree of chaos, and it was introduced into information theory by Shannon (sometimes called Shannon entropy) and now represents a measure of the uncertainty of a random variable. The random variable is divided into several intervals, each of which represents a state of the variable, and entropy is the average uncertainty of all possible states of the variable. It is also a common tool to quantify the information included in the PDF [34,45]. For discrete data, the formula is as follows [46]:

$$
\mathrm{S}=-\sum_{i}^{n} \mathrm{p}_{\mathrm{i}} \ln \mathrm{p}_{\mathrm{i}}
$$

where $\mathrm{p}_{\mathrm{i}}$ is the probabilistic mass function (PMS) of the variable that falls within the $i$ th interval after dividing the set of points into $\mathrm{n}$ intervals, and it satisfies the normalization condition $\sum_{i}^{n} \mathrm{p}_{\mathrm{i}}=1$. The calculation of entropy is related to the size and the start of the probability density interval segmentation, and Brunsell, Ham and Owensby [35] suggested using the kernel density estimation method to determine the interval size and the starting value. In this paper, we used the Gaussian kernel density estimation method to divide the interval into 512 aliquots and calculate the probability [47]. When the variable has only one state or a single value distribution, the entropy value is 0 ; and when the variable is evenly distributed, the entropy value of the variable is the largest, which is $\ln n$.

Because the number of observations involved in the calculation of each variable varies, in order to make the variability of each variable comparable, the entropy of all variables must be normalized, and the formula is as follows:

$$
\mathrm{S}^{\prime}=-\sum_{i}^{n} \mathrm{p}_{\mathrm{i}} \ln \mathrm{p}_{\mathrm{i}} / \operatorname{lnm}
$$

where $m$ is the number of observations.

The above three methods are often used in remote sensing-assisted modeling $[1,3,34,44]$. Therefore, compared with other methods (i.e., quantile, range, deviation, variance/standard deviation and moment) the three methods are not only dimensionless or dimensional consistent, but also have representativeness. In this paper, we analyze the three methods from different type of data (e.g., remote sensing airborne data) to express variability.

\section{Results and Analysis}

\subsection{Analysis Based on Simulation Data}

As we mentioned before, PDF is the starting point for our research of surface spatial variability. Therefore, the variability of the value of remote sensing variables is the object of this paper. The variability of different surface variables (such as LST, soil moisture, and stomatal conductance) is commonly studied using the following distribution models: normal distribution, beta distribution, gamma distribution, Weibull distribution, and exponential distribution [12,14]. To better compare the ability of the Gini coefficient, CV and entropy to express variability, we have designed three schemes from the perspective of simulation data. In Scheme 1 (see Figure 3 for details), we simulated 18 groups of random numbers with six types of distribution, all of which passed the Kolmogorov-Smirnov test at $p>0.05$. The design of Scheme 1 is mainly used for the comparison of the methods within the distribution, and each group yields 10,000 random numbers to calculate the aforementioned methods. The Schemes 2 and 3 are designed to compare the three methods in describing the variability between the different distributions. However, even for the same remote sensing variable that satisfies the same distribution, the range of its value may also be different for various reasons (e.g., different acquisition times). Scheme 3 is designed to reflect this difference and is therefore more specific. The calculation results of the three methods for the simulated data in Scheme 1 are shown in Table 1. 

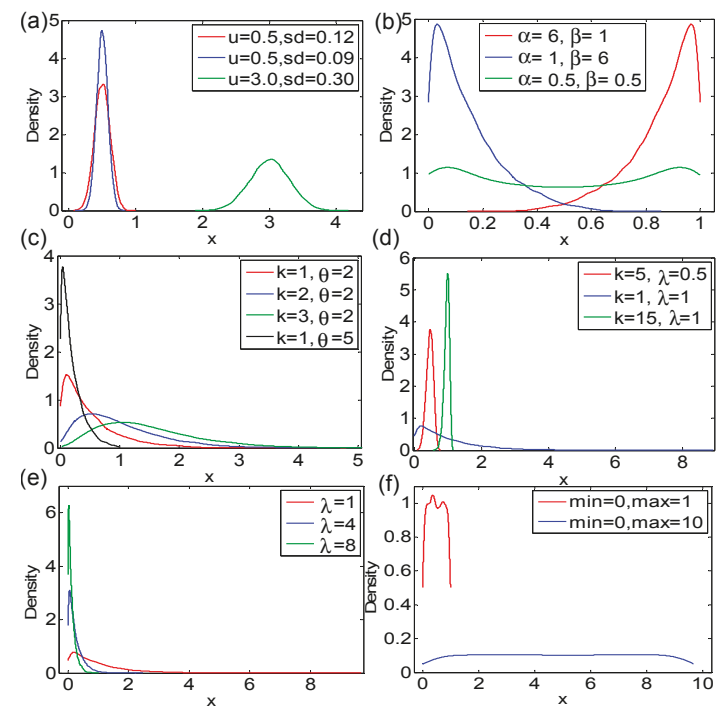

Figure 3. The PDF distribution of simulated data in Scheme 1. (a-f) refer to Gaussian distribution, beta distribution, gamma distribution, weibull distribution, exponential distribution and uniform distribution, respectively.

Table 1. Calculation results of the three methods for the simulated data in Scheme 1.

\begin{tabular}{ccccc}
\hline Distribution Type & Specific Parameters & S & CV & Gini \\
\hline \multirow{3}{*}{ Normal } & $\mathrm{N}(1) \mathrm{u}=0.5, \mathrm{sd}=0.12$ & 3.8596 & 0.2311 & 0.1305 \\
& $\mathrm{~N}(2) \mathrm{u}=0.5, \mathrm{sd}=0.09$ & 3.5437 & 0.1685 & 0.0950 \\
& $\mathrm{~N}(3) \mathrm{u}=3, \mathrm{sd}=0.30$ & 4.7925 & 0.0986 & 0.0557 \\
\hline \multirow{3}{*}{ Beta } & $\mathrm{B}(1) \alpha=6, \beta=1$ & 5.0479 & 0.1445 & 0.0771 \\
& $\mathrm{~B}(2) \alpha=1, \beta=6$ & 5.0478 & 0.8697 & 0.4638 \\
& $\mathrm{~B}(3) \alpha=0.5, \beta=0.5$ & 5.7996 & 0.7081 & 0.4057 \\
\hline \multirow{5}{*}{ Gamma } & $\mathrm{G}(1) \mathrm{k}=1, \theta=2$ & 4.3049 & 0.9901 & 0.4965 \\
& $\mathrm{G}(2) \mathrm{k}=2, \theta=2$ & 4.8676 & 0.7066 & 0.3756 \\
& $\mathrm{G}(3) \mathrm{k}=3, \theta=2$ & 5.1169 & 0.5777 & 0.3126 \\
Weibull & $\mathrm{G}(4) \mathrm{k}=1, \theta=5$ & 3.3721 & 0.9291 & 0.4848 \\
\hline \multirow{3}{*}{ Exponential } & $\mathrm{W}(1) \mathrm{k}=5, \lambda=0.5$ & 2.9733 & 0.2262 & 0.1278 \\
& $\mathrm{~W}(2) \mathrm{k}=1, \lambda=1$ & 4.8165 & 0.9824 & 0.4947 \\
& $\mathrm{~W}(3) \mathrm{k}=15, \lambda=1$ & 2.6494 & 0.0807 & 0.0446 \\
\hline \multirow{2}{*}{ Uniform } & $\mathrm{E}(1) \lambda=1$ & 4.7244 & 1.0090 & 0.5011 \\
& $\mathrm{E}(2) \lambda=4$ & 3.3821 & 0.9968 & 0.4975 \\
& $\mathrm{E}(3) \lambda=8$ & 2.7327 & 0.9941 & 0.5005 \\
\hline & $\mathrm{U}(1) \min =0, \max =1$ & 3.7550 & 0.5779 & 0.3336 \\
& $\mathrm{U}(2)=0, \max =10$ & 5.9966 & 0.5734 & 0.3310 \\
\hline
\end{tabular}

Note: $\mathrm{u}$ represents the mean; sd represents the standard deviation; $\mathrm{s}$ represents the entropy value; in the same distribution, the interval division of entropy is consistent.

Table 1 shows that there are some differences between the three methods (i.e., entropy, CV and Gini coefficient) in expressing spatial variability, and the trends of CV and Gini coefficient are basically the same. Through the analysis, we can find that the difference between the three methods basically occurs in the range of data values that vary greatly or with extreme data value distribution. For example, the data range of the $\mathrm{N}(3)$ group is larger than the other two groups in the normal distribution or three 
group data in the beta distribution. However, the N(3) group of PDF is broader than the other two groups, so it should be more heterogeneous, while the $C V$ and Gini coefficient are not very stable. In addition, from the exponential distribution, the $\mathrm{CV}$ of the $\mathrm{E}(2)$ group is larger than the $\mathrm{CV}$ of the $\mathrm{E}(3)$ group, whereas the Gini coefficient is the opposite. Combined with Figure 3, it can be found that the PDF distribution of $\mathrm{E}(3)$ group is more concentrated, which indicates that it is more homogeneous. Gini coefficient is more likely to represent the ratio of deviations between data [44], so it is worse than the $\mathrm{CV}$ when expressing the variability.

To compare the three methods in describing the variability between the different distributions, we designed Scheme 2 . The data of Scheme 2 are the nine groups of data that are in the same interval (i.e., $[0,1])$ selected in Scheme 1. These nine sets of data also cover six distributions, and their PDF distributions are shown in Figure 4. The nine groups of data in Scheme 2 represent the different variability distributions: the variability represented by (a) is stronger than that represented by (b); (c) and (d) represent a large number of uniform data mixed with a small amount of non-uniform data, but their spatial variability is basically the same; (e) represents a bimodal distribution; (f) and (h) are similar to (d), and (g) is similar to (a); (i) is an extreme case with a maximum variability, where the uniform distribution of (i) refers to the probability that the spatial distribution of the data is uniform, which is completely opposite to the uniform distribution of surface variables in the study of remote sensing (the uniform distribution in remote sensing usually refers to a single value distribution, that is, the entire study area has only one value). Combined with Figure $4 a$, the variability of these data is roughly sorted as follows: (i) $>$ (e) $>$ (f) $>$ (c) $\approx($ d $)>($ h) $;$ (a) $>($ g $)>($ b). The entropy is calculated in the same way as the interval division of the data in group (i), and the calculation results of the three methods are shown in Table 2. We can find that the results of the entropy can better reflect the variability of the data in Scheme 2, while the results of CV and Gini coefficient are not very stable. For example, the variability of (c) and (d) is basically the same, whereas the results of the CV and the Gini coefficient vary considerably. The reason is that $\mathrm{CV}$ and Gini coefficient are affected by the values of the variable. To confirm this, we designed Scheme 3.

In Scheme 3, we add 1 to the original random number generated in Scheme 2 so that the interval range becomes [1, 2], as shown in Figure $4 \mathrm{~b}$. The variability of each simulated data in Scheme 3 is also calculated using three methods, and the results are shown in Table 3. The data in Scheme 3 have the same variability as in Scheme 2, and the standard deviation is also the same. However, from the calculation results, only the value of entropy is unchanged, and the CV and Gini coefficient results undergo great changes. Thus, the $\mathrm{CV}$ and Gini coefficient are influenced by the variable values, specifically the mean of the variables, which can be seen via the calculation equations of the two $[25,44]$.

Table 2. Calculation results of the three methods for the simulated data in Scheme 2.

\begin{tabular}{ccccc}
\hline Distribution Type & Specific Parameters & S & CV & Gini \\
\hline \multirow{2}{*}{ Normal } & (a) $\mathrm{u}=0.5, \mathrm{sd}=0.12$ & 5.2604 & 0.2311 & 0.1305 \\
& (b) $\mathrm{u}=0.5, \mathrm{sd}=0.09$ & 4.9459 & 0.1685 & 0.0950 \\
\hline \multirow{2}{*}{ Beta } & (c) $\alpha=6, \beta=1$ & 5.0479 & 0.1445 & 0.0771 \\
& (d) $\alpha=1, \beta=6$ & 5.0478 & 0.8697 & 0.4638 \\
Gamma & (e) $\alpha=0.5, \beta=0.5$ & 5.8003 & 0.7081 & 0.4057 \\
\hline Weibull & (f) $\mathrm{k}=1, \theta=5$ & 5.3579 & 0.9291 & 0.4848 \\
\hline Exponential & (g) $\mathrm{k}=5, \lambda=0.5$ & 5.1505 & 0.2262 & 0.1278 \\
\hline Uniform & (h) $\lambda=8$ & 4.9230 & 0.9941 & 0.5005 \\
\hline
\end{tabular}

Note: $u$ represents the mean; sd represents the standard deviation; s represents the entropy value. 

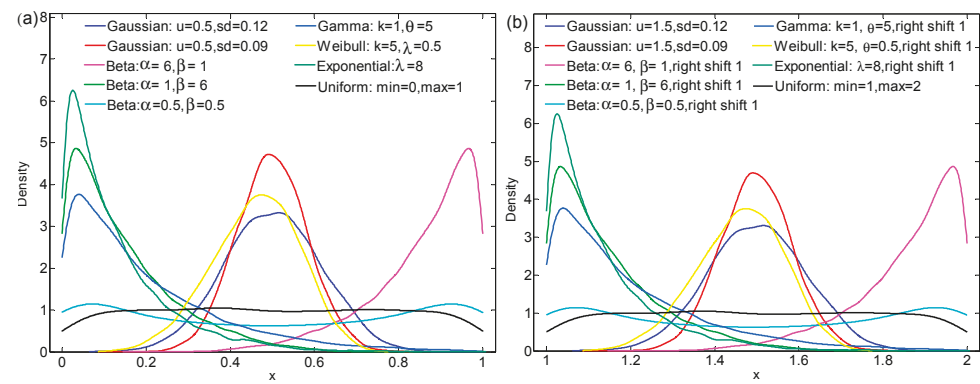

Figure 4. The PDF distribution of simulated data: (a) Scheme 2; (b) Scheme 3.

Table 3. Calculation results of the three methods for the simulated data in Scheme 3.

\begin{tabular}{ccccc}
\hline Distribution Type & Specific Parameters & S & CV & Gini \\
\hline \multirow{2}{*}{ Normal } & (a) $\mathrm{u}=1.5, \mathrm{sd}=0.12$ & 5.2604 & 0.0771 & 0.0435 \\
& (b) $\mathrm{u}=1.5, \mathrm{sd}=0.09$ & 4.9459 & 0.0561 & 0.0316 \\
\hline \multirow{2}{*}{ Beta } & (c) $\alpha=6, \beta=1$ & 5.0479 & 0.0667 & 0.0356 \\
& (d) $\alpha=1, \beta=6$ & 5.0478 & 0.1085 & 0.0578 \\
Gamma & (e) $\alpha=0.5, \beta=0.5$ & 5.8003 & 0.2361 & 0.1353 \\
\hline Weibull & (f) $\mathrm{k}=1, \theta=5$ & 5.3579 & 0.1516 & 0.0791 \\
\hline Exponential & (g) $\mathrm{k}=5, \lambda=0.5$ & 5.1505 & 0.0713 & 0.0403 \\
\hline Uniform & (h) $\lambda=8$ & 4.9230 & 0.1103 & 0.0556 \\
\hline
\end{tabular}

Note: $\mathrm{u}$ represents the mean; sd represents the standard deviation; s represents the entropy value; $(\mathrm{c}-\mathrm{h})$ are obtained by shifting one unit to the right on the basis of the Scheme 2 .

From a comprehensive comparison of Tables 1-3, we can find that: (1) the variation range of entropy is larger than that of the CV and Gini coefficient, although the magnitude fluctuation of entropy is smaller than that of the other two; (2) the variation trend of the CV is consistent with that of the Gini coefficient; (3) for non-extreme cases, the entropy, CV and the Gini coefficient can be a good measure of the degree of discretization, such as (a) and (b) in Schemes 2 and 3; (4) however, for extreme cases, the $\mathrm{CV}$ and the Gini coefficient are less effective than the entropy value for measuring spatial fluctuations, for example, the fluctuation degree of (i) is the largest but the CV and Gini coefficient values are smaller than (d) and (e) in Scheme 2; moreover, the variability of (c) and (d) is basically the same, whereas the results of the CV and Gini coefficient vary considerably in both Schemes 2 and 3 ; and (5) the entropy values are relatively stable and do not change with the value of the variable given for the same distribution, whereas the CV and Gini coefficient values are easily affected by the variable mean value. Specifically, when the range of variable values in Scheme 3 changes, the entropy values remain unchanged, while the $\mathrm{CV}$ and the Gini coefficient change greatly. From the analysis of the simulated data, we can draw the preliminary conclusion that entropy can better describe the spatial fluctuation than the other two methods, and it is more suitable for the comparison of variability among different variables.

\subsection{Analysis Based on Field Experimental Data}

\subsubsection{Analysis Based on Airborne Data}

As mentioned in Section 2.2.2, we divided the selected tracks into three equal areas, with each containing $250 \times 250$ pixels. The LAI and LST of each track are retrieved respectively. The CV, Gini coefficient and entropy of each variable in each region were calculated, and the calculation results are shown in Table 4. In addition, the corresponding land cover maps for each region is obtained. 
Taking track 5-1 as an example, the experimental area corresponds to Area NO. 1-Area NO. 3. The spatial distribution of the variables and the surface types of the three study areas are shown in Figure 5. The area (pixel number) proportion of vegetated, shadow and non-vegetated areas were 0.858:0.016:0.126 (53,634:1018:7848); 0.825:0.01:0.165 (51,554:633:10,313) and 0.784:0.01:0.206 $(49,001: 559: 12,900)$, respectively, which resulted in a larger variation of the variable range. Figure 6 is the probability density distribution of the surface variables (i.e., LAI and LST) and the surface types. The proportion of vegetated area (especially corn area) is large; therefore, the distribution of variables is concentrated. Combined with Table 4, the following conclusions can be drawn.

(1) Figure $6 \mathrm{~b}$ shows that the LAI values of the three study areas are mainly distributed near 0 and high value (between 4.0 and 7.0), thus showing a bimodal distribution. For the LAI, the CV and Gini coefficient calculation results show that Area NO. 2 has more variation than Area NO. 1, although the entropy results show that Area NO. 1 is more variable. A comparison of the frequency distribution histograms (bandwidth $=1$ ) of LAI in the two study areas (Figure 6c) shows that the LAI peak width of Area NO. 2 is narrower, meaning that the probability distribution of LAI in Area NO. 2 is more concentrated; therefore, the entropy of Area NO. 2 is smaller than that of Area NO. 1. Moreover, the proportion of non-vegetated area (LAI =0) in Area NO. 2 is larger, which results in a widened standard deviation and a lower mean. Therefore, the calculated CV and Gini coefficient results are relatively large. However, the spatial variability of LAI in Area NO. 1 is greater overall than that in Area NO. 2.

(2) The LST range of Area NO. 1 Area NO. 3 is nearly $30{ }^{\circ} \mathrm{C}$, and the distribution of the values is mainly between $30 \sim 35^{\circ} \mathrm{C}$ (Figure 6d). For the LST, the entropy, CV and Gini coefficient calculation results are consistent and all are incremental; thus, the LST variability of the three study areas is as follows: Area NO. $3>$ Area NO. $2>$ Area NO. 1.

(3) For the variability of variables, LAI > LST. Although the value range of LST is larger than that of LAI, the variability of LAI is larger than that of LST, which can be directly obtained via calculations using the three methods.

(4) The variability of LST seems to have a certain relationship with the variability of LAI, in this paper, the LST-LAI variability is negative. Because of the complex relationship between vegetation information and LST (e.g., NDVI-LST) [48], the correlation between the two variabilities needs to be further studied.

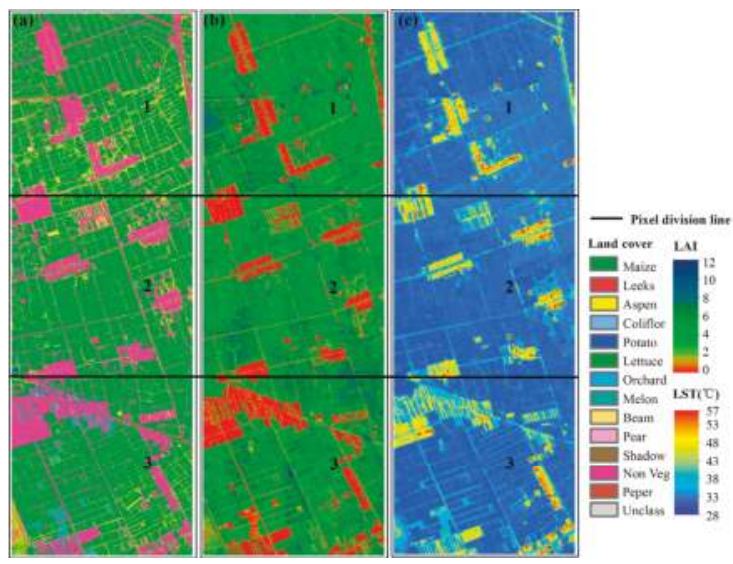

Figure 5. Data for each region on track 5-1: (a) 1-m classification data derived from CASI and (b) LAI and (c) LST retrieved by WiDAS. 

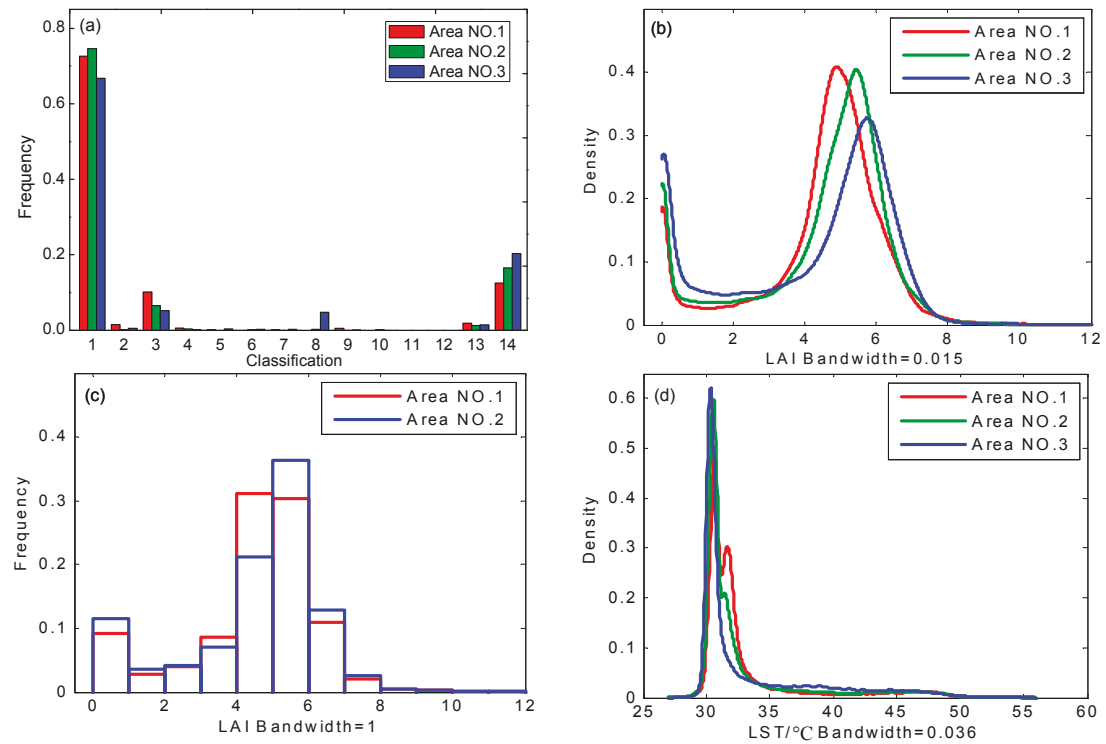

Figure 6. Probability density distribution and histogram of the surface variables and the surface types in three study areas: (a) histogram of 1-m classification data; (b) probability density distribution of LAI; (c) frequency distribution histograms of LAI (bandwidth $=1$ ); and (d) probability density distribution of LST. Note: in the classification histogram, the values 1-14 represent maize, leeks, aspen, cauliflower, potato, lettuce, orchard, melon, beam, pear, peper, unclass, shadow and non-veg, respectively.

Figure 7 shows the variation of the three methods as the range of the LST increases (i.e., the number of pixels increases gradually). The trend of the three methods is similar. In the range of 5-300 m, the curve changes steeply, and the slope is large, which is due to additional points caused by the new mixed other types of land or the growth of large differences in vegetation, so the variability in the study area increases rapidly. In the range of $300-800 \mathrm{~m}$, the variability of the variable tends to be stable, which may be related to the optimal scale [19]. From the perspective of probability density distribution, the scale range increases, which leads to more occurrences of the variable in a certain range (state), that is, the probability is greater, indicating that the value of the variable is more concentrated and more dominant than other states, so its spatial variability remains stable or even reduces.
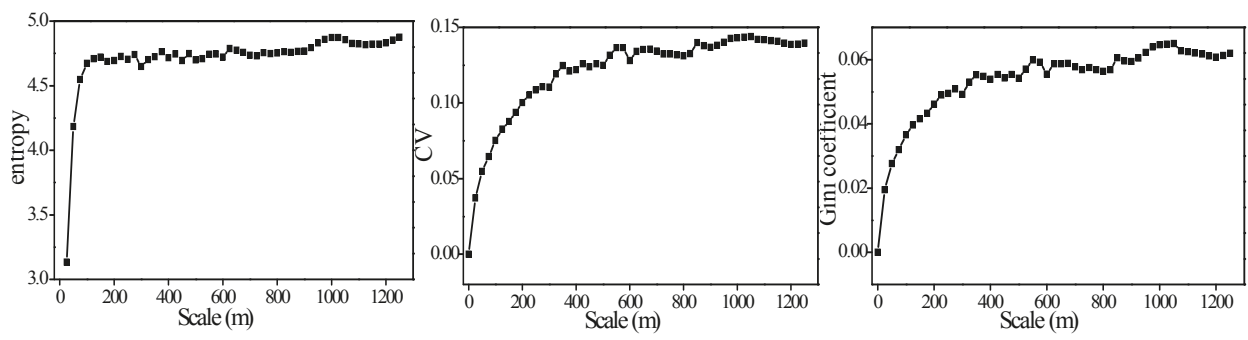

Figure 7. Variation of the three methods when the LST scale is expanded. Note: The abscissa indicates the length of the study area that gradually increases from the upper left corner of Figure $5 \mathrm{c}$ with a gradient of pixels $5 i \times 5 i(i=0,1,2 \ldots 50)$. 
Table 4. Calculation results of the three methods for each variable in different areas.

\begin{tabular}{|c|c|c|c|c|c|c|c|c|c|c|c|c|c|c|c|}
\hline \multirow{2}{*}{ Track NO. } & \multirow{2}{*}{ Area NO. } & \multicolumn{2}{|c|}{$\mathrm{S}$} & \multicolumn{2}{|c|}{$\mathrm{CV}$} & \multicolumn{2}{|c|}{ Gini } & \multirow{2}{*}{ Track NO. } & \multirow{2}{*}{ Area NO. } & \multicolumn{2}{|c|}{$\mathrm{S}$} & \multicolumn{2}{|c|}{$\mathrm{CV}$} & \multicolumn{2}{|c|}{ Gini } \\
\hline & & LAI & LST & LAI & LST & LAI & LST & & & LAI & LST & LAI & LST & LAI & LST \\
\hline \multirow{3}{*}{ track 5-1 } & 1 & 5.3350 & 4.4594 & 0.4050 & 0.1303 & 0.2114 & 0.0545 & \multirow{3}{*}{ track 10-1 } & 10 & 5.3660 & 4.2306 & 0.4715 & 0.1329 & 0.2478 & 0.0574 \\
\hline & 2 & 5.3209 & 4.5029 & 0.4427 & 0.1389 & 0.2334 & 0.0604 & & 11 & 5.3818 & 4.0387 & 0.4022 & 0.1023 & 0.2093 & 0.0401 \\
\hline & 3 & 5.3494 & 4.6007 & 0.5692 & 0.1494 & 0.3128 & 0.0708 & & 12 & 5.5580 & 4.3174 & 0.4653 & 0.1106 & 0.2528 & 0.0473 \\
\hline \multirow{3}{*}{ track 5-2 } & 4 & 5.3034 & 4.5020 & 0.4014 & 1288 & 0.2080 & 0.0543 & \multirow{3}{*}{ track $11-1$} & 1 & 5.5324 & 4.3848 & 0.5158 & 0.1292 & 0.2814 & 0.05 \\
\hline & 5 & 5.3407 & 4.5748 & 0.4309 & 0.1347 & 0.2268 & 0.0591 & & 14 & 5.5262 & 3.9989 & 0.3937 & 0.0968 & 0.2065 & 0.0383 \\
\hline & 6 & 5.3939 & 4.5033 & 0.5083 & 0.1410 & 0.2750 & 0.0632 & & 15 & 5.5739 & 4.1628 & 0.4020 & 0.0997 & 0.2120 & 0.0402 \\
\hline \multirow{3}{*}{ track $7-2$} & 7 & 5.3480 & 4.1616 & 0.3737 & 0.1140 & 0.1925 & 0.0434 & \multirow{3}{*}{ track $12-1$} & 1 & 4.8 & 4.4 & 0.7941 & 0.1176 & 0.4462 & 0.05 \\
\hline & 8 & 5.3573 & 4.3790 & 0.4258 & 0.1196 & 0.2240 & 0.0508 & & 17 & 4.7221 & 4.1904 & 0.7181 & 0.0868 & 0.3955 & 0.0362 \\
\hline & 9 & 5.4848 & 4.5773 & 0.5030 & 0.1357 & 0.2751 & 0.0667 & & 18 & 4.8323 & 4.3546 & 0.6973 & 0.1084 & 0.3830 & 0.0480 \\
\hline
\end{tabular}

For the LAI in our study area, as the scale range increases, the following occurs: (1) when the dominant land cover of the pixel is covered by buildings or bare soil and only a very small number of pixels or individual pixels are vegetation cover, the value of LAI is largely zero and only a small number of non-zero values are observed; or (2) only a small amount of the pixel is covered with buildings or bare soil and these land types are mixed with a large number of vegetation area; thus, the value of LAI is largely non-zero. These two cases often occur with mixed pixels, such as at the junction of farms and villages. In such situations, the pixels are obviously relatively pure, the spatial variability is relatively weak, but the CV values in case (1) are much larger than those in case (2) and the Gini coefficient values are close to 1 and 0 in both cases. However, the entropy values are consistent in both cases, which is similar to cases (c) and (d) in Schemes 2 and 3 of the simulated data described in Section 4.1. These findings show that the CV and the Gini coefficient are insufficient for measuring spatial variability. Because the Gini coefficient is not ideal, the calculation is large and the trend is similar to that of the $\mathrm{CV}$; thus, the following analyses will no longer consider the Gini coefficient.

\subsubsection{Analysis Based on Ground Observation Data}

The variables that can be analyzed using airborne data are limited, and ground observation data (i.e., AWSs and ECs) can provide multivariate continuous observation data, which are beneficial for analyzing the overall variability of each variable and its temporal variation. Nine surface variables are analyzed in this section, and the number of observations for each variable is shown in Table 5.

Table 5. The number of observations in the calculation of each variable.

\begin{tabular}{cccccc}
\hline Variable & Number of Observation & Variable & Number of Observation & Variable & Number of Observation \\
\hline H & 17 & RH_5m & 17 & albedo & 16 \\
LE & 17 & Ta_5m & 17 & Rn & 16 \\
Ustar & 17 & Ms_2cm & 17 & Ts_0cm & 17 \\
\hline
\end{tabular}

Note: $\mathrm{H}$ represents sensible heat flux; LE represents latent heat flux; Ustar represents the friction wind speed which is observed by an ultrasonic anemometer; RH_5m represents air humidity at $5 \mathrm{~m}$; Ta_ $5 \mathrm{~m}$ represents air temperature at $5 \mathrm{~m}$; Ms_2cm represents soil moisture at a depth of $2 \mathrm{~cm}$; albedo represents the surface albedo, which is obtained by the ratio of the upward shortwave radiation to the downward shortwave radiation; $\mathrm{Rn}$ represents net radiation; Ts_0 $\mathrm{cm}$ represents soil temperature at a depth of $0 \mathrm{~cm}$ (i.e., the temperature measured by the detector exposed to the Earth's surface).

Among them, H, LE, and Ustar were observed by the EC systems and RH_5m, Ta_5m, Ms_2cm, Rn and Ts_0cm were observed by the AWS stations. Because one of the stations (i.e., EC16) lacked radiation observation data, the albedo and $\mathrm{Rn}$ have only 16 observation data. To make the variability of each variable comparable, the entropy of each variable needs to be normalized according to Equation (4). The normalized entropy and CV are calculated from the half-hourly of ground observation data processed in Section 2.2.1. Taking the surface variables (i.e., H, LE and Ts_0cm) from June 2012 as an example, the variation in entropy and CV with time is shown in Figure 8. The evaporation from crops is greater during the growing season, and the value of the sensible heat flux $(\mathrm{H})$ is much smaller than that of the latent heat flux (LE). Studies have shown that at an oasis in the summer, a temperature 
inversion occurs [1-3], and $\mathrm{H}$ is sometimes less than 0 and its mean is close to 0 or even negative. In addition, the $C V$ values are large and the variation is intense; therefore, the $C V$ values of $\mathrm{H}$ are unstable. This situation is similar to that described in Section 4.1, meaning that a large number of zero values are mixed with non-zero values. In contrast, entropy values do not change dramatically because entropy does not consider the specific size of the variable value and is only related to the frequency of the variable value within a certain interval. In addition, the $\mathrm{CV}$ values of $\mathrm{H}$ show that the turbulence flux spatial fluctuation is relatively small at approximately 11:00 a.m. because with solar radiation enhancement, the surface begins to heat up, evapotranspiration increases, and the $\mathrm{H}$ spatial variation changes are stable. However, in the afternoon, the oasis effect appears, and $\mathrm{H}$ is negative for the observation site and gradually increases, resulting in CV values that begin to fluctuate violently. Figure 8 shows that the $C V$ values and entropy values of the LE variations are also more intense. Furthermore, the trend of $\mathrm{H}$ and LE seems to show a certain diurnal variation and this requires further detailed study.
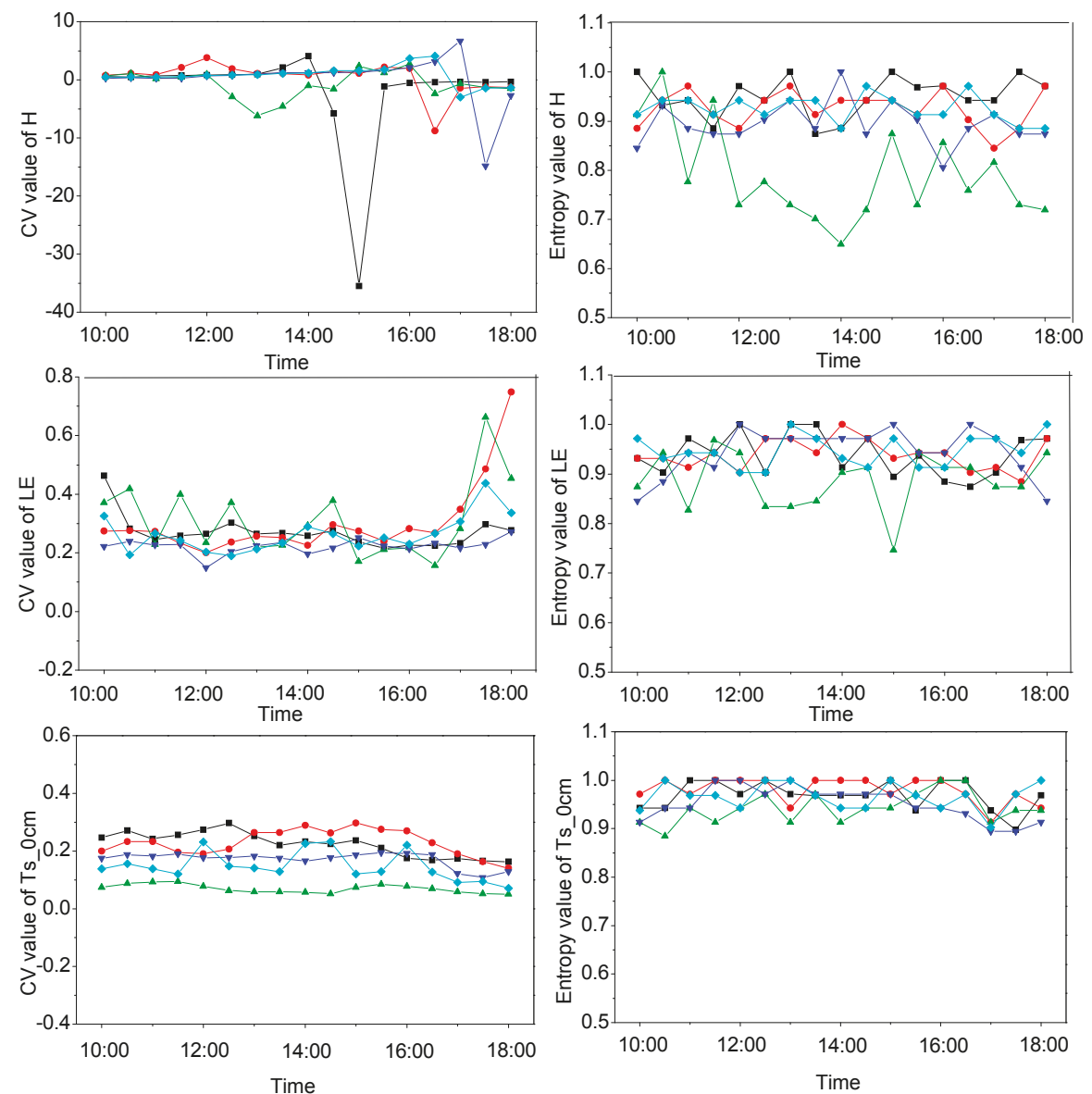

-2012-6-25 - 2012-6-26 $\triangle$ 2012-6-28 $>-2012-6-29-2012-6-30$

Figure 8. Temporal variation of the entropy and CV of surface variables (i.e., H, LE and Ts_0cm). 
Table 6 shows the statistics of entropy calculated for the nine variables over 20 days. It can be seen that the entropy of Ms_2cm is the largest. The reason may be due to that the LE has a strong variability and is relatively discrete (STD $=0.048)$, coupled with other factors such as wind speed, resulting in significant differences in evapotranspiration [2]. Thus, the distribution of surface soil moisture is quite different. Followed by Ustar and Ts_0cm, they also have strong variability. The reason for the entropy of Ustar being higher may be related to the influence of surface geometry and atmospheric conditions on wind speed; the HiWATER-MUSOEXE test area is surrounded by HRB and the Gobi Desert (Figure 1b), so the spatial variation of the wind speed is large. For the soil temperature at $0 \mathrm{~cm}$ (Ts_0 cm), there is a high degree of spatial variability because of the influence of surface humidity and the shadow of each observation site. In general, the evaluation of entropy as a representation of variability is more credible than that of the CV.

Table 6. Statistics of the entropy for each variable.

\begin{tabular}{ccccc}
\hline Variable & MIN & MAX & MEAN & STD \\
\hline H & 0.649 & 1.000 & 0.913 & 0.061 \\
LE & 0.747 & 1.000 & 0.929 & 0.048 \\
Ustar & 0.798 & 1.000 & 0.946 & 0.036 \\
RH_5m & 0.748 & 1.000 & 0.923 & 0.041 \\
Ta_5m & 0.693 & 1.000 & 0.875 & 0.050 \\
Ms_2cm & 0.863 & 1.000 & 0.982 & 0.024 \\
albedo & 0.670 & 1.000 & 0.919 & 0.049 \\
Rn & 0.423 & 1.000 & 0.918 & 0.056 \\
Ts_0cm & 0.769 & 1.000 & 0.932 & 0.048 \\
\hline
\end{tabular}

\section{Discussion}

The entropy describes the spatial variability much better than CV and Gini coefficient. Generally, the CV and Gini coefficient have similar characteristics when expressing spatial variability, but the Gini coefficient is more focused on the deviation ratio between data. Therefore, in some cases, the Gini coefficient is worse than the CV in expressing spatial variability, and the Gini coefficient has a large computational effort. In summary, the advantages of entropy are described as follows:

1. Entropy is more consistent with PDF when describing spatial variability, and in some extreme distributions, entropy can express spatial variability more accurately, while CV and Gini coefficients cannot.

2. In this paper, the unit of entropy is nats, which can be used directly to compare the spatial variability of variables. While the $\mathrm{CV}$ and Gini coefficients cannot, they are affected by the specific value (i.e., the average) of the variable.

Nevertheless, it is worth mentioning that PDF is the starting point for our research of surface spatial variability. If we do not use this as a prerequisite to analyze the heterogeneity of surface variables separately, the spatial patterns (also known as spatial structure) or spatial autocorrelation of the surface must be considered [26,34]. Entropy calculates the variability of variables in their own range. In Table 1, if we compare the three methods in describing the variability between the different distributions, the result is still reliable. However, we must consider the actual range of variables. For example, if variable A satisfies a uniform distribution, its value can range from 0 to 10 , and in some cases (e.g., an observation scene of a remote sensing image or the target area that we are interested in) its value falls in the range of $0-1$. If there is no value in the 1-10 range, similar to $\mathrm{U}$ (1) in Table 1, then its variability is not necessarily stronger than variable B which belongs to other distributions (e.g., the beta distribution in Table 1) in the actual sense. In this article, the design and arrangement of the scheme is to gradually illustrate the characteristics of the three methods in expressing spatial variability. In addition, entropy takes into account the distribution of variable values, 
and its calculation has a certain dependence on the number of samples. Considering the high cost of the experiments, the datasets of the seventeen EC systems and AWS stations in the $5.5 \times 5.5 \mathrm{~km}^{2} \mathrm{kernel}$ experimental area are very valuable [39]. Moreover, the conclusions drawn from the analysis based on the spatial data may be quite preliminary but are important and valuable for future exploration in this area. More data from wireless sensor network [49] will be added and used for more sophisticated analysis. More reliable and interesting findings will be discovered with increased number of sites and observations. Detailed information on the spatial variability of variables derived from the ground observation network and the time variation of the variability of surface variables will be introduced in another paper.

\section{Conclusions}

The estimation of ET or LE using remote sensing data does not usually consider the influence of advection or interaction between subpixels; hence, the distribution of surface variable values (i.e., spatial variability) is usually the focus for building a model that considers the heterogeneity produced by surface variables for a target area or landscape. PDF is the most promising way to describe subpixel variability for given data. In attempting to produce efficient PDF-based parameterization of remotely sensed ET or LE, it is important to determine which variables are similar or consistent with the variability of turbulent flux in time. However, the use of PDF alone does not facilitate direct comparisons of the spatial variability of surface variables. To address this, we chose three dimensionless or dimensional consistent indicators, i.e., CV, Gini coefficient and entropy values, to express the variability in surface variables. Based on the analysis of simulated data and the field experiment data, we found the following: (1) Entropy has a high consistency with the PDF of surface variables, which is more stable and efficient in expressing the variability of surface variables. The entropy of the airborne data shows that the variability of LAI is greater than that of LST; (2) Regardless of whether it is from the analysis of simulated data or field experimental data, the CV and the Gini coefficient are insufficient for measuring spatial variability. They are susceptible to the mixing of special values, such as the inversion of temperature at the oasis in the summer; (3) The results of normalized entropy of different variables observed by EC systems and AWS stations show that the variability of soil moisture at depth of $2 \mathrm{~cm}\left(\mathrm{MS} \_2 \mathrm{~cm}\right)$ is the strongest, and the variability of friction wind speed (Ustar) is relatively strong, which is related to the special geographical environment of the study area. Furthermore, the trend of sensible heat flux $(\mathrm{H})$ and latent heat flux (LE) seems to show a certain diurnal variation.

The findings of this study provide a reference for expressing the spatial variability of surface variables and illustrate a suitable method for comparing the variability of different variables. How to combine it with the analysis in which surface variables vary similarly or consistently with the variability of the turbulent flux in time and to establish a more efficient PDF-based remote sensing ET or LE model will be the next research goal.

Acknowledgments: This study was jointly supported by the Chinese Natural Science Foundation Project (grant No. 41371360) and the Special Fund of the Chinese Academy of Sciences (grant No. KZZD-EW-TZ-18). We are grateful to Prof. Qing Xiao of Institute of Remote Sensing and Digital Earth Chinese Academy of Sciences, Beijing, China for providing the WiDAS data. In addition, we thank all of the scientists and engineers who took part in the HiWATER experiment. We also thank the reviewers and editors for their insightful and constructive comments.

Author Contributions: Xiaozhou Xin provided the ideas for this work; Xiaozhou Xin, Xiaojun Li and Zhiqing Peng prepared the manuscript and designed the experiments; Xiaojun Li and Hailong Zhang performed the experiments; Chuanxiang Yi, and Bin Li collected and processed the data. Xiaozhou Xin participated in discussions and revising the manuscript; Xiaojun Li wrote the paper. These authors contributed equally.

Conflicts of Interest: The authors declare no conflict of interest. 


\section{References}

1. Peng, Z.Q.; Xin, X.Z.; Jiao, J.J.; Zhou, T.; Liu, Q.H. Remote sensing algorithm for surface evapotranspiration considering landscape and statistical effects on mixed pixels. Hydrol. Earth Syst. Sci. 2016, 20, 4409-4438. [CrossRef]

2. Wang, Y.Q.; Xiong, Y.J.; Qiu, G.Y.; Zhang, Q.T. Is scale really a challenge in evapotranspiration estimation? A multi-scale study in the Heihe oasis using thermal remote sensing and the three-temperature model. Agric. For. Meteorol. 2016, 230-231, 128-141. [CrossRef]

3. Li, X.J.; Xin, X.Z.; Jiao, J.J.; Peng, Z.Q.; Zhang, H.L.; Shao, S.S.; Liu, Q.H. Estimating subpixel surface heat fluxes through applying temperature-sharpening methods to MODIS data. Remote Sens. 2017, 9, 836. [CrossRef]

4. Jacob, F.; Weiss, M. Mapping biophysical variables from solar and thermal infrared remote sensing: Focus on agricultural landscapes with spatial heterogeneity. IEEE Geosci. Remote Sens. Lett. 2016, 11, 1844-1848. [CrossRef]

5. Kustas, W.; Li, F.; Jackson, T.; Prueger, J.; MacPherson, J.; Wolde, M. Effects of remote sensing pixel resolution on modeled energy flux variability of croplands in Iowa. Remote Sens. Environ. 2004, 92, 535-547. [CrossRef]

6. Kustas, W.P.; Norman, J.M. Evaluating the effects of subpixel heterogeneity on pixel average fluxes. Remote Sens. Environ. 2000, 74, 327-342. [CrossRef]

7. Arain, A.M.; Michaud, J.; Shuttleworth, W.J.; Dolman, A.J. Testing of vegetation parameter aggregation rules applicable to the Biosphere Atmosphere Transfer Scheme (BATS) and the FIFE site. J. Hydrol. 1996, 177, 1-22. [CrossRef]

8. Chehbouni, A.; Njoku, E.; Lhomme, J.; Kerr, Y. Approach for averaging surface parameters and fluxes over heterogeneous terrain. J. Clim. 1995, 8, 1386-1393. [CrossRef]

9. Koster, R.D.; Suarez, M.J. A comparative analysis of two land surface heterogeneity representations. J. Clim. 1992, 5, 1379-1390. [CrossRef]

10. Li, B.; Avissar, R. The impact of spatial variability of land-surface characteristics on land-surface heat fluxes. J. Clim. 1994, 7, 527-537. [CrossRef]

11. Blyth, E.M.; Harding, R.J. Application of aggregation models to surface heat flux from the Sahelian tiger bush. Agric. For. Meteorol. 1995, 72, 213-235. [CrossRef]

12. Avissar, R. Conceptual aspects of a statistical-dynamical approach to represent landscape subgrid-scale heterogeneities in atmospheric models. J. Geophys. Res. Atmos. 1992, 97, 2729-2742. [CrossRef]

13. Maayar, M.E.; Chen, J.M. Spatial scaling of evapotranspiration as affected by heterogeneities in vegetation, topography, and soil texture. Remote Sens. Environ. 2006, 102, 33-51. [CrossRef]

14. Tittebrand, A.; Berger, F. Spatial heterogeneity of satellite derived land surface parameters and energy flux densities for LITFASS-area. Atmos. Chem. Phys. 2009, 9, 16219-16254. [CrossRef]

15. Mölders, N.; Raabe, A. Numerical investigations on the influence of subgrid-scale surface heterogeneity on evapotranspiration and cloud processes. J. Appl. Meteorol. 1996, 35, 782-795. [CrossRef]

16. Norman, J.M.; Anderson, M.C.; Kustas, W.P.; French, A.N.; Mecikalski, J.; Torn, R.; Diak, G.R.; Schmugge, T.J.; Tanner, B.C.W. Remote sensing of surface energy fluxes at $10^{1}-\mathrm{m}$ pixel resolutions. Water Resour. Res. 2003, 39. [CrossRef]

17. Bayala, M.I.; Rivas, R.E. Enhanced sharpening procedures on edge difference and water stress index basis over heterogeneous landscape of sub-humid region. Egypt. J. Remote Sens. Space Sci. 2014, 17, 17-27. [CrossRef]

18. Mukherjee, S.; Joshi, P.K.; Garg, R.D. A comparison of different regression models for downscaling Landsat and MODIS land surface temperature images over heterogeneous landscape. Adv. Space Res. 2014, 54, 655-669. [CrossRef]

19. Wu, H.; Tang, B.H.; Li, Z.L. Impact of nonlinearity and discontinuity on the spatial scaling effects of the leaf area index retrieved from remotely sensed data. Int. J. Remote Sens. 2013, 34, 3503-3519. [CrossRef]

20. Zeng, X.M.; Zhao, M.; Su, B.K.; Tang, J.P.; Zheng, Y.Q.; Zhang, Y.J.; Chen, J. Effects of the land-surface heterogeneities in temperature and moisture from the "combined approach" on regional climate: A sensitivity study. Glob. Planet. Chang. 2003, 37, 247-263. [CrossRef]

21. Giorgi, F. An approach for the representation of surface heterogeneity in land surface models. Part II: Validation and sensitivity experiments. Mon. Weather Rev. 1997, 125, 1900-1919. [CrossRef] 
22. Yeh, P.J.F.; Eltahir, E.A.B. Representation of water table dynamics in a land surface scheme. Part I: Model development. J. Clim. 2005, 18, 1861-1880. [CrossRef]

23. Giorgi, F. An approach for the representation of surface heterogeneity in land surface models. Part I: Theoretical framework. Mon. Weather Rev. 1997, 125, 1885-1899. [CrossRef]

24. Wang, Q.; Ni, J.; Tenhunen, J. Application of a geographically-weighted regression analysis to estimate net primary production of Chinese forest ecosystems. Glob. Ecol. Biogeogr. 2010, 14, 379-393. [CrossRef]

25. Odongo, V.; Hamm, N.; Milton, E. Spatio-temporal assessment of Tuz Gölü, Turkey as a potential radiometric vicarious calibration site. Remote Sens. 2014, 6, 2494-2513. [CrossRef]

26. Garrigues, S.; Allard, D.; Baret, F.; Weiss, M. Quantifying spatial heterogeneity at the landscape scale using variogram models. Remote Sens. Environ. 2006, 103, 81-96. [CrossRef]

27. Wang, J.F.; Zhang, T.L.; Fu, B.J. A measure of spatial stratified heterogeneity. Ecol. Indic. 2016, 67, $250-256$. [CrossRef]

28. Rahman, A.F.; Gamon, J.A.; Sims, D.A.; Schmidts, M. Optimum pixel size for hyperspectral studies of ecosystem function in southern California chaparral and grassland. Remote Sens. Environ. 2003, 84, 192-207. [CrossRef]

29. Haralick, R.M.; Shanmugam, K.S. Combined spectral and spatial processing of ERTS imagery data. Remote Sens. Environ. 1974, 3, 3-13. [CrossRef]

30. Csillag, F.; Kabos, S. Wavelets, boundaries, and the spatial analysis of landscape pattern. Écoscience 2002, 9, 177-190. [CrossRef]

31. Qiu, B.W.; Zeng, C.Y.; Cheng, C.C.; Tang, Z.H.; Gao, J.Y.; Sui, Y.P. Characterizing landscape spatial heterogeneity in multisensor images with variogram models. Chin. Geogr. Sci. 2014, 24, 317-327. [CrossRef]

32. Cosh, M.H.; Brutsaert, W. Microscale structural aspects of vegetation density variability. J. Hydrol. 2003, 276, 128-136. [CrossRef]

33. Zhang, L.J.; Ma, Z.H.; Luo, G. An evaluation of spatial autocorrelation and heterogeneity in the residuals of six regression models. For. Sci. 2009, 55, 533-548.

34. Hintz, M.; Lennartz-Sassinek, S.; Liu, S.F.; Shao, Y.P. Quantification of land-surface heterogeneity via entropy spectrum method. J. Geophys. Res. Atmos. 2014, 119, 8764-8777. [CrossRef]

35. Brunsell, N.A.; Ham, J.M.; Owensby, C.E. Assessing the multi-resolution information content of remotely sensed variables and elevation for evapotranspiration in a tall-grass prairie environment. Remote Sens. Environ. 2008, 112, 2977-2987. [CrossRef]

36. Kustas, W.P.; Norman, J.M.; Anderson, M.C.; French, A.N. Estimating subpixel surface temperatures and energy fluxes from the vegetation index-radiometric temperature relationship. Remote Sens. Environ. 2003, 85, 429-440. [CrossRef]

37. Wang, D.; Wang, G.; Anagnostou, E.N. Use of satellite-based precipitation observation in improving the parameterization of canopy hydrological processes in land surface models. J. Hydrometeorol. 2005, 6, 745-763. [CrossRef]

38. Li, X.; Cheng, G.; Liu, S.; Xiao, Q.; Ma, M.; Jin, R.; Che, T.; Liu, Q.; Wang, W.; Qi, Y. Heihe watershed allied telemetry experimental research (HiWATER): Scientific objectives and experimental design. Bull. Am. Meteorol. Soc. 2013, 94, 1145-1160. [CrossRef]

39. Liu, S.M.; Xu, Z.W.; Song, L.S.; Zhao, Q.Y.; Ge, Y.; Xu, T.R.; Ma, Y.F.; Zhu, Z.L.; Jia, Z.Z.; Zhang, F. Upscaling evapotranspiration measurements from multi-site to the satellite pixel scale over heterogeneous land surfaces. Agric. For. Meteorol. 2016, 230, 97-113. [CrossRef]

40. Xu, Z.W.; Liu, S.M.; Li, X.; Shi, S.J.; Wang, J.M.; Zhu, Z.L.; Xu, T.R.; Wang, W.Z.; Ma, M.G. Intercomparison of surface energy flux measurement systems used during the HiWATER-MUSOEXE. J. Geophys. Res. Atmos. 2013, 118, 13140-13157. [CrossRef]

41. Ran, Y.H.; Li, X.; Sun, R.; Kljun, N.; Zhang, L.; Wang, X.F.; Zhu, G.F. Spatial representativeness and uncertainty of eddy covariance carbon flux measurements for upscaling net ecosystem productivity to the grid scale. Agric. For. Meteorol. 2016, 230-231, 114-124. [CrossRef]

42. Li, W.; Niu, Z.; Huang, N.; Wang, C.; Gao, S.; Wu, C. Airborne LiDAR technique for estimating biomass components of maize: A case study in Zhangye City, Northwest China. Ecol. Indic. 2015, 57, 486-496. [CrossRef]

43. Liu, Q.; Yan, C.Y.; Xiao, Q.; Yan, G.J.; Fang, L. Separating vegetation and soil temperature using airborne multiangular remote sensing image data. Int. J. Appl. Earth Obs. Geoinf. 2012, 17, 66-75. [CrossRef] 
44. Valbuena, R.; Maltamo, M.; Mehtätalo, L.; Packalen, P. Key structural features of boreal forests may be detected directly using L-moments from airborne lidar data. Remote Sens. Environ. 2017, 194, 437-446. [CrossRef]

45. Journel, A.G.; Deutsch, C.V. Entropy and spatial disorder. Math. Geol. 1993, 25, 329-355. [CrossRef]

46. Shannon, C.E. A mathematical theory of communication. Bell Syst. Tech. J. 1948, 27, 379-423. [CrossRef]

47. Silverman, B.W. Density estimation in the exploration and presentation of data. In Density Estimation for Statistics and Data Analysis, 1st ed.; CRC Press: Boca Raton, FL, USA, 1986; pp. 2-4.

48. Raynolds, M.K.; Comiso, J.C.; Walker, D.A.; Verbyla, D. Relationship between satellite-derived land surface temperatures, arctic vegetation types, and NDVI. Remote Sens. Environ. 2008, 112, 1884-1894. [CrossRef]

49. Jin, R.; Li, X.; Yan, B.; Li, X.; Luo, W.; Ma, M.; Guo, J.; Kang, J.; Zhu, Z.; Zhao, S. A nested ecohydrological wireless sensor network for capturing the surface heterogeneity in the midstream areas of the Heihe River Basin, China. IEEE Geosci. Remote Sens. Lett. 2014, 11, 2015-2019. [CrossRef]

(C) 2018 by the authors. Licensee MDPI, Basel, Switzerland. This article is an open access article distributed under the terms and conditions of the Creative Commons Attribution (CC BY) license (http:/ / creativecommons.org/licenses/by/4.0/). 
Article

\title{
Satellite-Derived Spatiotemporal Variations in Evapotranspiration over Northeast China during 1982-2010
}

\author{
Lilin Zhang ${ }^{1,2}$, Yunjun Yao ${ }^{1,2, *}$, Zhiqiang Wang ${ }^{3}$, Kun Jia ${ }^{1,2}$, Xiaotong Zhang ${ }^{1,2}$, Yuhu Zhang ${ }^{4}$, \\ Xuanyu Wang ${ }^{1,2}$, Jia Xu ${ }^{1,2}$ and Xiaowei Chen ${ }^{1,2}$ \\ 1 State Key Laboratory of Remote Sensing Science, Faculty of Geographical Science, Beijing Normal \\ University, Beijing 100875, China; 201521170059@mail.bnu.edu.cn (L.Z.); jiakun@bnu.edu.cn (K.J.); \\ xtngzhang@bnu.edu.cn (X.Z.); skywalkerwxy@163.com (X.W.); diamond1201@sina.cn (J.X.); \\ 18368091857@163.com (X.C.) \\ 2 Beijing Engineering Research Center for Global Land Remote Sensing Products, Institute of Remote Sensing \\ Science and Engineering, Faculty of Geographical Science, Beijing Normal University, Beijing 100875, China \\ 3 National Disaster Reduction Center/Satellite Application Center for Disaster Reduction of the Ministry of \\ Civil Affairs, Beijing 100124, China; wzqbnu@163.com \\ 4 College of Resource Environment and Tourism, Capital Normal University, Beijing 100048, China; \\ zhang_yuhu@163.com \\ * Correspondence: boyyunjun@163.com; Tel.: +86-10-5880-3002
}

Received: 25 September 2017; Accepted: 2 November 2017; Published: 7 November 2017

\begin{abstract}
Evapotranspiration (ET) is a critical process for the climate system and water cycles. However, the spatiotemporal variations in terrestrial ET over Northeast China over the past three decades calculated from sparse meteorological point-based data remain large uncertain. In this paper, a recently proposed modified satellite-based Priestley-Taylor (MS-PT) algorithm was applied to estimate ET of Northeast China during 1982-2010. Validation results show that the square of the correlation coefficients $\left(\mathrm{R}^{2}\right)$ for the six flux tower sites varies from 0.55 to $0.88(p<0.01)$, and the mean root mean square error (RMSE) is $0.92 \mathrm{~mm} / \mathrm{d}$. The ET estimated by MS-PT has an annual mean of $441.14 \pm 18 \mathrm{~mm} /$ year in Northeast China, with a decreasing trend from southeast coast to northwest inland. The ET also shows in both annual and seasonal linear trends over Northeast China during 1982-2010, although this trend seems to have ceased after 1998, which increased on average by $12.3 \mathrm{~mm}$ per decade pre-1998 $(p<0.1)$ and decreased with large interannual fluctuations post-1998. Importantly, our analysis on ET trends highlights a large difference from previous studies that the change of potential evapotranspiration (PET) plays a key role for the change of ET over Northeast China. Only in the western part of Northeast China does precipitation appear to be a major controlling influence on ET.
\end{abstract}

Keywords: evapotranspiration; Northeast China; MS-PT algorithm; spatial-temporal variations; controlling factors; potential evapotranspiration

\section{Introduction}

Over the past three decades, global warming has influenced the climate condition and the hydrological cycle of Northeast China [1,2]. Especially for evapotranspiration (ET), it is an important process in an ecosystem water budget, energy balance, and carbon cycles, and strongly affects surface dry conditions and climate change [3-5]. Numerous studies have reported that significant changes in ET are influencing ecosystem processes, surface drought conditions, and the consequent local climate feedback over Northeast China [6-11]. Based on the meteorological measurements, the annual reference $\mathrm{ET}\left(\mathrm{ET}_{0}\right)$ of Northeast China has increased at a rate of $3.89 \mathrm{~mm} /$ decade during 1961-2007, 
with large interannual fluctuations [12]. However, due to a lack of direct measurements, there are still large biases in estimating the actual ET for the detection of variations in water budget and surface drought in Northeast China. Therefore, it is urgent to improve ET estimation and evaluate the long-term variation of ET for understanding the hydrologic changes and solving problems in water resource management.

Based on the large ground-measurements, several traditional approaches have been widely used to estimate ET in Northeast China. Among them, eddy covariance data can be considered to be reliable for quantifying ET at the local scale $[13,14]$. However, since their measurements are of short duration and sparse spatial coverage, they cannot provide long-term regional estimates of ET [15]. Similarly, the application of traditional ET estimation models, whose parameters derived mostly from field observations, is greatly restricted in Northeast China [16]. For example, Gao et al. [7] used the water balance methodology to estimate monthly ET for 686 stations over China and found the actual ET tends to decrease in most areas of Northeast China during 1960-2002. Nevertheless, due to the problem of scale conversion, such ET methods require a very large amount of ground-observation data and can only be accurate at the local scale [3].

Currently, remote sensing has provided real-time and dynamic information for terrestrial energy systems, facilitating effective regional ET estimation $[17,18]$. Based on satellite-based observations, several approaches have been used to estimate the spatial and temporal variation in ET over Northeast China. For instance, Tian et al. [19] combined water balance components and surface energy balance components by Noah land surface model and found decreasing changes in ET over Northeast China during 1986-2008. Based on global Moderate Resolution Imaging Spectroradiometer (MODIS) ET products, Tian et al. [20] reported that ET shows a positive trend in the Northeast Plain during 2001-2010 and attributed this trend to the change of vegetation condition. Yang et al. [21] using the GLEAM (global land-surface evaporation: the Amsterdam methodology) model, documented a negative trend in the western part of Northeastern China during the 1981 to 2010 period and pointed out that the annual trend shows a remarkable correlation with the trend in summer. Although these studies have focused on the detection of variation in ET over Northeast China, due to the difference in model structures and dominant variables, the interannual variability of ET vary significantly between models [22]. Large uncertainties in the spatial-temporal variation of ET over long time periods still remain in Northeast China. A recently modified satellite-based Priestley-Taylor (MS-PT) algorithm proposed by Yao et al. [23] has been used to estimate ET in mainland China. According to validation for 16 EC flux tower sites throughout China, the average RMSE between measured and predicted site-averaged daily ET was approximately $5 \mathrm{~W} / \mathrm{m}^{2}$ lower (99\% confidence) for the MS-PT compared to the Priestley-Taylor-based ET (PT-JPL) algorithm. It is physically based, requiring no subjective parameter calibrations as employed by many other traditional methods, which exactly solved the problem of insufficient input data. Importantly, this method is also satisfactory in detecting the interannual variability in China [24]. However, there is a lack of similar studies that simulate regional terrestrial ET over Northeast China by using the MS-PT algorithm and satellite datasets. As a result, little is accurately understood regarding spatiotemporal characterization of the response of regional terrestrial ET to climate change for long periods.

In this study, we used the MS-PT algorithm driven by remote sensing data and meteorological reanalysis data to estimate ET in Northeast China. We had two major objectives. First, we evaluated the performance of the MS-PT algorithm using ground-measured flux data collected from six flux towers in Northeast China. Second, we analyzed the spatiotemporal variation in ET from 1982 to 2010 and detected the issue of what factors contribute to the variability and trends of ET in Northeast China. 


\section{Materials and Methods}

\subsection{Study Area}

Northeast China $\left(38^{\circ} 42^{\prime}-53^{\circ} 55^{\prime} \mathrm{N}, 115^{\circ} 32^{\prime}-135^{\circ} 09^{\prime} \mathrm{E}\right)$ is located in a transition region between the subarctic region and temperate region [25]. The total area is approximately $1.24 \times 10^{6} \mathrm{~km}^{2}$, covering four provinces, including Heilongjiang, Jilin, and Liaoning, and some areas of the Inner Mongolia Autonomous Region: Hulun Buir, Xingan Meng, Tongliao, and Chifeng. The study area has a typical continental monsoon climate with cold, dry winters and warm, wet summers. According to a previous study, the annual precipitation of Northeast China is about $489 \mathrm{~mm}$, of which $75 \%$ falls in the period of June to September [26]. The annual average temperature is about $1.9{ }^{\circ} \mathrm{C}-5.3{ }^{\circ} \mathrm{C}$, and has increased at a rate of 0.34/decade during 1959-2002 [1]. Due to the suitable plant growth environment, Northeast China not only produces a large quantity of grain, but also has abundant forest reserves, which accounts for more than $1 / 3$ of total in China [27]. The natural vegetation of Northeast China includes the forest steppe or meadow steppe, typical steppe, and desert steppe, rendering the ecological environment sensitive and fragile. A previous study illustrates that extreme precipitation and dryness events have become more and more frequent and intensified [6].

The study area can be divided into two major basins: Liaohe (LH) basin and Songjiang (SJ) basin (Figure 1). These two basins have different climatic and hydrological characteristics. For instance, the land use type of the LH basin is mainly cropland and desert land [28], which has severe water environment problems. Although the utilization rate of water resources is high, the water resources of the LH basin are still quite limited [29]. On the contrary, the land use type of the SJ basin is mainly forestland and cropland [30]. Since the annual precipitation of the SJ basin varies significantly, droughts and floods occurred frequently [31]. Separating Northeast China into these two basins will be helpful to evaluate the ET variability.

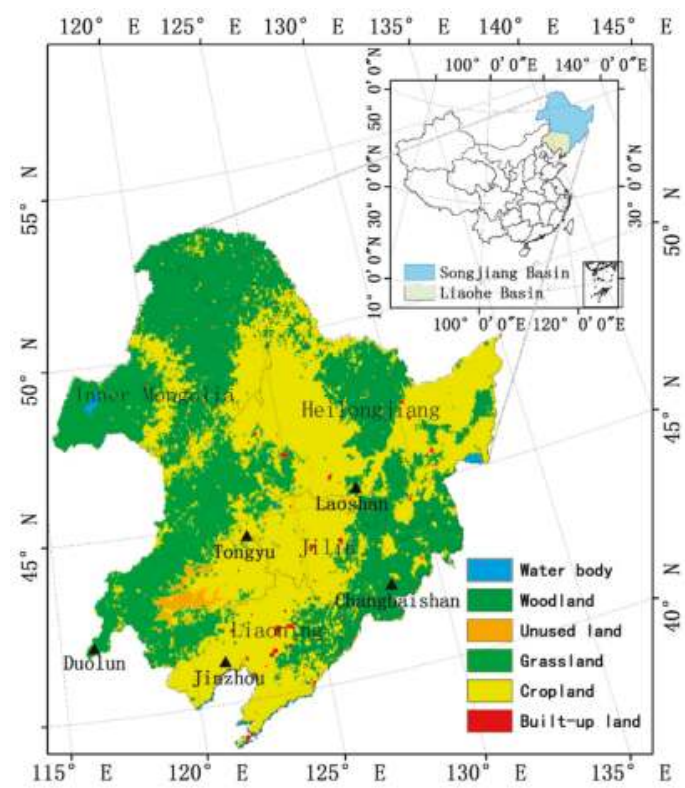

Figure 1. Map of the land cover type and the location of flux towers (Jinzhou, Duolun, Changbaishan, Tongyu, Laoshan) in Northeast China. The two main basins are also shown in inset panel: Songjiang (SJ) basin and Liaohe (LH) basin. 


\subsection{Data}

\subsubsection{Remote Sensing Data}

To estimate long-term ET over Northeast China, we use the Global Inventory Modeling and Mapping Studies (GIMMS) NDVI3g data (1982-2010) generated from the Advanced Very High Resolution Radiometer (AVHRR). The GIMMS NDVI3g product contains global bimonthly NDVI at $8 \mathrm{~km}$ spatial resolution and can be obtained from National Oceanic and Atmospheric Administration (NOAA) [32]. In our study, the GIMMS NDVI3g data was converted to a resolution of $0.1^{\circ} \times 0.1^{\circ}$ using bilinear interpolation and temporally interpolated from the bimonthly averages to a daily value using linear interpolation.

To evaluate the performance of the MS-PT algorithm, the MOD16 eight-day ET product with $1 \mathrm{~km}$ spatial resolution is used to compare with the estimated ET. The MOD16 product is available from the National Aeronautics and Space Administration (NASA) Land Processes Distributed Active Archive Center (LP DAAC) [33]. The MOD16 product is based on the Penman-Monteith equation and uses daily meteorological reanalysis data and eight-day vegetation dynamics as inputs [34,35].

We also use the Climate Prediction Center soil moisture dataset (1982-2010) and MODIS land cover type product (MCD12C1). The soil moisture dataset provided by the NOAA [36] contains monthly averaged soil moisture at 0.5 degree and can be used to analyze the relationship with ET. The MCD12C1 data from 2001 to 2010 can be obtained online [33] and the spatial resolution of the data is 0.05 degrees.

\subsubsection{Meteorological Reanalysis Data}

The China Meteorological Forcing Dataset was produced by merging a variety of surface meteorological and environmental data sources, including instantaneous near surface air temperature, pressure, relative humidity, wind speed, precipitation, and surface downward shortwave radiation. To estimate the spatiotemporal variations in ET over Northeast China, we use the daily gridded meteorological reanalysis dataset as input parameters (except precipitation) to calculate ET during 1982-2010 [37]. The independent precipitation data is used to analyze the relationship with ET. This daily meteorological reanalysis dataset covers the period 1981-2010, with a resolution of $0.1 \mathrm{deg}$ and $3 \mathrm{~h}$. This dataset can be used for hydrological modeling, land surface modeling, land data assimilation, and other terrestrial modeling. Previous studies demonstrated that this dataset provides more accurate meteorological parameters than other reanalysis and satellite products, with less RMSE and higher correlation coefficients [38,39].

\subsubsection{Ground-Based Observations}

To evaluate the performance of both MS-PT algorithm on the site scale, we use the ground-observed data from six flux towers, which are distributed all around Northeast China (Figure 1). The data are provided by ChinaFLUX, LathuileFlux, and the Coordinated Enhanced Observation Network of China (CEOP) [40]. The basic information of flux observation site is shown in Table 1. All sites are based on the eddy correlation (EC) method [41,42]. Although the EC method has been widely used in the global measurement experiment, this method does not conserve energy. Therefore, in this study, we have selected the method developed by Twine et al. [43] to correct the ET from all flux towers. 
Table 1. Location of the six flux towers used in this study.

\begin{tabular}{cccccc}
\hline Site Name & Lat, Lon & Elevation(m) & Land Cover Type & Time Period & Network \\
\hline $\begin{array}{c}\text { Changbaishan, } \\
\text { Jilin }\end{array}$ & $\begin{array}{l}42.40^{\circ} \mathrm{N}, \\
128.10^{\circ} \mathrm{E}\end{array}$ & 761 & Mixed forest & 2002-2007 & China Flux \\
\hline $\begin{array}{c}\text { Laoshan, } \\
\text { Heilongjiang }\end{array}$ & $\begin{array}{l}45.28^{\circ} \mathrm{N}, \\
127.58^{\circ} \mathrm{E}\end{array}$ & 340 & Larch forest & 2001-2007 & China Flux \\
\hline $\begin{array}{c}\text { Duolun1, Inner } \\
\text { Mongolia }\end{array}$ & $\begin{array}{l}42.045^{\circ} \mathrm{N}, \\
116.671^{\circ} \mathrm{E}\end{array}$ & 1350 & Cropland & 2006 & Lathuile-Flux \\
\hline $\begin{array}{c}\text { Jinzhou, } \\
\text { Liaoning }\end{array}$ & $\begin{array}{l}41.18^{\circ} \mathrm{N}, \\
121.21^{\circ} \mathrm{E}\end{array}$ & 22.3 & Cropland (maize) & $2008-2009$ & CEOP \\
\hline $\begin{array}{c}\text { Duolun2, Inner } \\
\text { Mongolia }\end{array}$ & $\begin{array}{l}42.047^{\circ} \mathrm{N}, \\
116.284^{\circ} \mathrm{E}\end{array}$ & 1350 & Grassland & 2006 & Lathuile-Flux \\
\hline $\begin{array}{c}44.57^{\circ} \mathrm{N}, \\
\text { Tongyu, Jilin }\end{array}$ & $122.88^{\circ} \mathrm{E}$ & 184 & Grassland & 2008 & CEOP \\
\hline
\end{tabular}

\subsection{Methods}

\subsubsection{MS-PT Algorithm}

The value of ET can be influenced by numerous factors, including surface moisture, stomatal conductance, air temperature (Tair), surface radiation energy [44]. Priestley and Taylor [45] combined theoretical and practical experiences and proposed a simple ET equation:

$$
\mathrm{ET}=\alpha \frac{\Delta}{\Delta+\gamma}\left(R_{n}-G\right)
$$

where $\alpha$ is the PT coefficient and $\Delta$ is the slope of the saturated vapor pressure curve $\left(\mathrm{kPa} /{ }^{\circ} \mathrm{C}\right) . \gamma$ is the psychometric constant $\left(\mathrm{kPa} /{ }^{\circ} \mathrm{C}\right) . R_{n}$ is the surface net radiation $\left(\mathrm{W} / \mathrm{m}^{2}\right)$. G represents the soil heat flux $\left(\mathrm{W} / \mathrm{m}^{2}\right)$. The Priestley-Taylor algorithm solve the uncertainty of the resistances in the Penman-Monteith equation and can be accurate where aerodynamic and surface resistance is not available [44,46]. Based on the Priestley-Taylor algorithm, many revised approaches to estimate ET have been built. However, these methods require a continuum of soil moisture and vegetation status to acquire the surface conditions [46].

To minimize the need for ancillary meteorological data while maintaining a physically-realistic representation of evapotranspiration process, Yao et al. [23] modified the Priestley-Taylor [45] algorithm by using vegetation indices (VIs) and apparent thermal inertia (ATI) as the primary parameters and propose the MS-PT algorithm:

$$
\mathrm{ET}=E T_{s}+E T_{c}+E T_{w s}+E T_{i c}
$$

The MS-PT algorithm separates the total ET into the unsaturated soil evaporation $\left(E T_{S}\right)$, the canopy transpiration $\left(E T_{C}\right)$, the saturated wet soil surface evaporation $\left(E T_{w s}\right)$, and the canopy interception evaporation $\left(E T_{i c}\right)$. Unsaturated soil evaporation can be expressed as

$$
E T_{s}=\left(1-f_{\text {wet }}\right) f_{s m} \alpha \frac{\Delta}{\Delta+\gamma}\left(R_{n s}-G\right)
$$

where $f_{\text {wet }}$ is the wet surface fraction $\left(f_{s m}{ }^{4}\right), f_{s m}$ can be derived from ATI (ATI $=(1 / \mathrm{DT})^{\mathrm{DT} / \mathrm{DTmax}}$ ). DT is the diurnal air temperature range $\left(\mathrm{DT}_{\max }=40^{\circ} \mathrm{C}\right) \cdot R_{n s}$ is the surface net radiation to the soil $\left(R_{n s}=R_{n}\left(1-f_{c}\right)\right), f_{c}$ is the vegetation cover fraction $\left(f_{c}=\left(\mathrm{NDVI}-\mathrm{NDVI}_{\min }\right) /\left(\mathrm{NDVI}_{\max }-\mathrm{NDVI}_{\min }\right)\right)$. $\mathrm{NDVI}_{\min }$ and $\mathrm{NDVI}_{\text {max }}$ are the minimum and maximum NDVI during the study period, respectively. 
$R_{n}$ is the surface net radiation and can be calculated using the method of Wang et al. [47]. G is the sum of the soil heat flux and can be calculated using a simple empirical $R_{n}$-based algorithm $\left(0.18\left(1-f_{c}\right) R_{n}\right)$ [24].

Canopy transpiration can be calculated using a modified linear two-source model:

$$
E T_{c}=\left(1-f_{w e t}\right) f_{c} f_{T} \alpha \frac{\Delta}{\Delta+\gamma} R_{n v}
$$

where $f_{T}$ represents plant temperature constraint $\left(\exp \left(-\left(\mathrm{T}_{\max }-\mathrm{T}_{\mathrm{opt}}\right) / \mathrm{T}_{\mathrm{opt}}\right) 2\right), \mathrm{T}_{\mathrm{opt}}$ is an optimum temperature $\left(25^{\circ} \mathrm{C}\right) . R_{n v}$ represents the surface net radiation to the vegetation $\left(R_{n v}=R_{n} f_{c}\right)$.

Saturated wet soil surface evaporation can be described as

$$
E T_{\text {ws }}=f_{\text {wet }} \alpha \frac{\Delta}{\Delta+\gamma}\left(R_{n s}-G\right)
$$

Vegetation interception evaporation can be calculated from the following equation:

$$
E T_{i c}=f_{w e t} \alpha \frac{\Delta}{\Delta+\gamma} R_{n v}
$$

\subsubsection{Potential ET Estimation}

Potential evapotranspiration (PET) is an important indicator of atmospheric evaporation demand and refers to ideal evaporation rate when soil moisture is abundant. In this study, the FAO Penman-Monteith (PM) method driven by meteorological reanalysis data and NDVI3g data was used to estimate PET of Northeast China, which was validated to all basins of China in previous studies $[48,49]$. The PET estimation method can be expressed as

$$
\mathrm{PET}=\frac{\left.0.408 \Delta\left(R_{n}-G\right)+\gamma\left(\frac{900}{T_{\text {air }}+273}\right) W S\left(e_{S}-e\right)\right)}{\Delta+\gamma(1+0.34 W S)}
$$

where WS is the wind speed, $e_{S}$ is the saturated vapor pressure, and $e$ refers to air water vapor pressure. VPD is vapor pressure deficit and equals to $e_{S}-e$.

\subsubsection{Statistical Analysis}

The Mann-Kendall test, proposed by Mann [50] and improved by Kendall [51], is a nonparametric method for testing trends in hydrological processes and other related physical variables [52]. The null hypothesis $\mathrm{H}_{0}$ suggests that the data are a sample of independent variables and the hypothesis $\mathrm{H}_{1}$ states that the distribution of $x_{k}$ and $x_{j}$ are not identical for all $k, j \leq n$ with $k \neq j$. The test statistic is given as follows:

$$
S=\sum_{j=1}^{n-1} \sum_{k=j+1}^{n} \operatorname{sgn}\left(x_{k}-x_{j}\right)
$$

where $x$ is the individual data values, $n$ is the total number of data, and

$$
\operatorname{sgn}\left(x_{k}-x_{j}\right)=\left\{\begin{array}{cr}
1 & x_{k}-x_{j}>0 \\
0 & x_{k}-x_{j}=0 \\
-0 & x_{k}-x_{j}<0
\end{array}\right.
$$


The statistic $S$ is nearly normally distributed, and the statistic $\mathrm{Z}$ is a standard normal variable:

$$
\begin{gathered}
\mathrm{Z}=\left\{\begin{array}{c}
\frac{s-1}{\sqrt{\operatorname{Var}(S)}} S>0 \\
0 \quad S=0 \\
\frac{s+1}{\sqrt{\operatorname{Var}(S)}} S<0
\end{array}\right. \\
\operatorname{Var}(S)=\frac{1}{18}\left[n(n+1)(2 n+5)-\sum_{i=1}^{n} t_{i}\left(t_{i}-1\right)\left(2 t_{i}+5\right)\right]
\end{gathered}
$$

where $i$ is the number of tied groups, and $t_{i}$ is the number of data values in the group. The presence of a statistically significant trend is evaluated using the $Z$ value: positive (negative) value of $Z$ indicates an upward (downward) trend. The null hypothesis $\mathrm{H}_{0}$ that $\mathrm{Z}$ is statistically significant or not is determined by whether $-Z_{1-\alpha / 2} \leq Z \leq Z_{1-\alpha / 2}$, where $\pm Z_{1-\alpha / 2}$ are the standard normal deviates obtained from the standard normal cumulative distribution tables, and $\alpha$ is the significance level for the test.

The Mann-Kendall test is also satisfactory for estimating the magnitude of the trend. The Kendall slope can be obtained by an unbiased estimator of trend magnitude:

$$
\beta=\operatorname{Median}\left(\frac{x_{i}-x_{j}}{i-j}\right)
$$

where $x_{i}$ is the annual value of ET or other meteorological elements.

\section{Results}

\subsection{Evaluation of MS-PT Performance in Estimating ET}

To evaluate the ability of the MS-PT algorithm to estimate ET over Northeast China, we have validated the MS-PT algorithm at daily timescales based on the EC observations. Figure 2 shows that the bias of the estimated daily ET using the MS-PT algorithm varies from 0.02 to $0.85 \mathrm{~mm} / \mathrm{d}$ with a mean value of $0.33 \mathrm{~mm} / \mathrm{d}$, the root mean square error (RMSE) varies from 0.69 to $1.43 \mathrm{~mm} / \mathrm{d}$ with a mean value of $0.92 \mathrm{~mm} / \mathrm{d}$, and the square of correlation coefficients $\left(\mathrm{R}^{2}\right)$ varies from 0.55 to $0.88(p<0.01)$ with a mean value of 0.68 . Since the sites have different biomes, a comparison of the site-averaged ET demonstrates the ability of MS-PT algorithm to estimate spatial variation in ET, as shown in Figure 3. The RMSE of the comparison is $0.43 \mathrm{~mm} / \mathrm{d}$ and the $\mathrm{R}^{2}$ is 0.93 . Batra et al. [53] showed that ET was estimated with an RMSE of 1.85, 1.78, and 1.97 from MODIS, NOAA16, and NOAA14 sensors, respectively. Seguin et al. [54] proposed that the majority of published remote sensing methods for estimating ET had an accuracy of $\pm 1.5 \mathrm{~mm} / \mathrm{d}$. Kalma et al. [55] summarized most remote sensing ET models have an average RMSE just over $1.75 \mathrm{~mm} / \mathrm{d}$. Therefore, the good accuracy of the MS-PT algorithm indicates that the model has a potential to be used for analyzing ET patterns in Northeast China.

A statistical comparison of MS-PT monthly estimations and MOD16 monthly products illustrates that the MS-PT algorithm provides a more favorable agreement with the ground-measured data at most sites (except Laoshan station). The MODIS monthly ET are composed of MOD16 eight-day products. As shown in Table 2, the average monthly $\mathrm{R}^{2}$ of MOD16 products for all sites is 0.67 and the average RMSE is $21.02 \mathrm{~mm} / \mathrm{month}$. In contrast, the ET simulations using the MS-PT algorithm has a higher $\mathrm{R}^{2}(0.8)$ and lower RMSE (19.68 mm/month). The negative bias of both MOD16 products (-22.4 mm/month) and MS-PT model (-12.5 mm/month) may be owing to the unclosed energy balance problem in the eddy covariance datasets [42]. One should note that the $\mathrm{R}^{2}$ of MOD16 is higher than it of MS-PT in the Laoshan station, which may be related to the quality of the observation data. To further illustrate the performance of the MS-PT algorithm to detect the interannual variability in ET, we map the monthly variations of ET over Northeast China from 2001 to 2010 (Figure 4). The flux 
tower site Changbaishan was selected, where at least five years of observation are available. As shown in Figure 4, the monthly variations of ET are well captured by MS-PT-estimated ET, with a lower RMSE and a higher $\mathrm{R}^{2}$. Thus, we can know clearly that the MS-PT algorithm improves the ET estimates at most flux towers sites and shows a better ability to detect the interannual variability in ET at different sites.

Table 2. Statistics of estimated eight-day evapotranspiration (ET) against the eddy-flux tower observations. All $\mathrm{R}^{2}$ values are significant with a $99 \%$ confidence.

\begin{tabular}{ccccccc}
\hline \multirow{2}{*}{ Site Name } & \multicolumn{2}{c}{ Bias (mm/Month) } & \multicolumn{2}{c}{$\begin{array}{c}\text { RMSE } \\
\text { (mm/Month) }\end{array}$} & \multicolumn{2}{c}{$\mathbf{R}^{2}$} \\
\cline { 2 - 7 } & MS-PT & MOD16 & MS-PT & MOD16 & MS-PT & MOD16 \\
\hline Changbaishan & -11.04 & -13.14 & 18.68 & 22.13 & 0.94 & 0.92 \\
Laoshan & -7.35 & -13.54 & 25.39 & 25.5 & 0.76 & 0.79 \\
Duolun1 & -7.93 & -18.37 & 15.71 & 32.24 & 0.91 & 0.63 \\
Jinzhou & -27.78 & -42.13 & 30.15 & 47.09 & 0.46 & 0.46 \\
Duolun2 & -9.52 & -11.26 & 13.86 & 20.58 & 0.9 & 0.65 \\
Tongyu & -11.38 & -35.96 & 14.3 & 38.55 & 0.85 & 0.56 \\
ALL & -12.5 & -22.4 & 19.68 & 21.02 & 0.8 & 0.67 \\
\hline
\end{tabular}
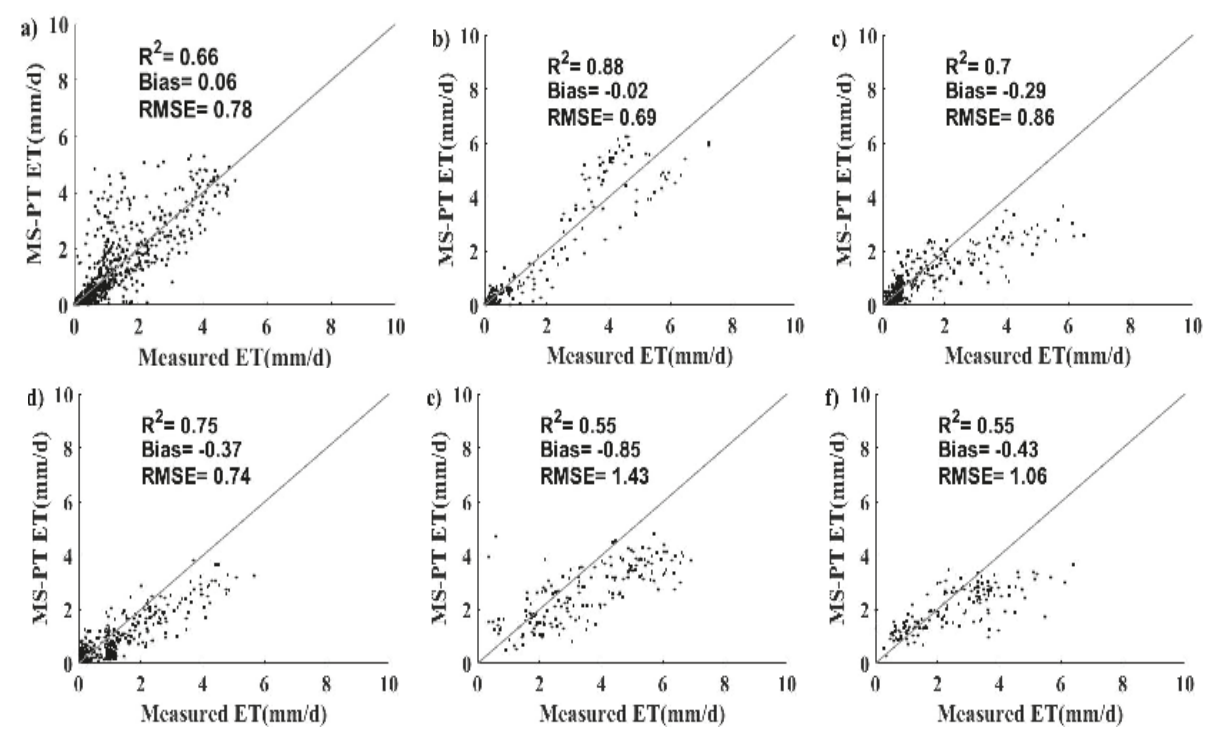

Figure 2. The scatterplots between flux tower observations and estimated ET at daily scale: (a) Changbaishan; (b) Laoshan; (c) Duolun1; (d) Duolun2; (e) Jinzhou; (f) Tongyu. The bias and RMSE are in units of $\mathrm{mm} / \mathrm{d}$. The solid line is the 1:1 line. 


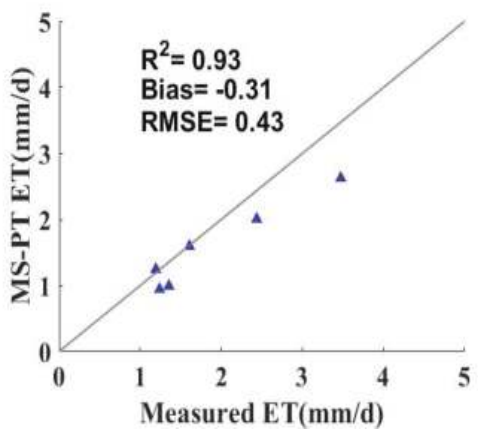

Figure 3. Comparisons of the predicted and measured site-averaged ET at six sites. The bias and RMSE are in units of $\mathrm{mm} / \mathrm{d}$. The solid line is the $1: 1$ line.

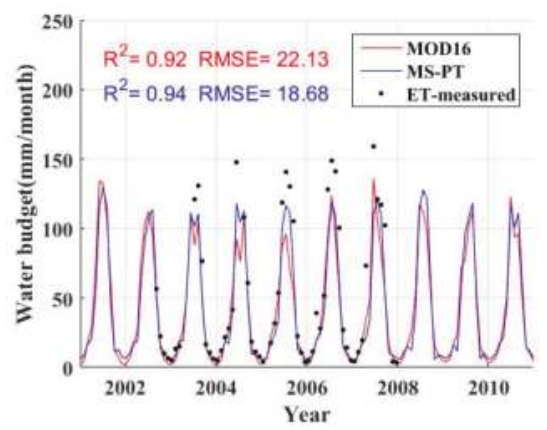

Figure 4. The monthly time series comparison of MS-PT-based ET and MOD16 ET in the Changbaishan station. RMSE is in units of $\mathrm{mm} / \mathrm{month}$.

\subsection{Mean Spatial Pattern of ET in Northeast China}

\subsubsection{Annual}

The multiyear (1982-2010) average annual ET spatial distribution is shown in Figure 5a. The annual mean ET estimated by the MS-PT algorithm during 1982-2010 is $441.14 \pm 18 \mathrm{~mm} /$ year in Northeast China. As shown in Figure 5a, the area with lower ET (300-400 mm/year) is mainly distributed in Inner Mongolia, where the land use type is mainly desert and grassland. We also found that the regions with larger ET (500-600 mm/year) were mainly distributed in the eastern part of the LH basin, where croplands and forests were densely distributed. More importantly, this area was also characterized by a relatively abundant precipitation. Moreover, a decreasing trend of ET from the southeast coast to northwest inland is noticeable in Figure 5a. Since Northeast China is located in East Asian monsoon climate zone influenced by the ocean monsoon, the precipitation can directly affect the surface soil moisture, further controlling ET of this region. Thus, compared with the average precipitation and soil moisture pattern (Figure 5b,c), we can clearly find that the annual average ET spatial pattern illustrates the strong regional variation corresponding to the surface water supply. 


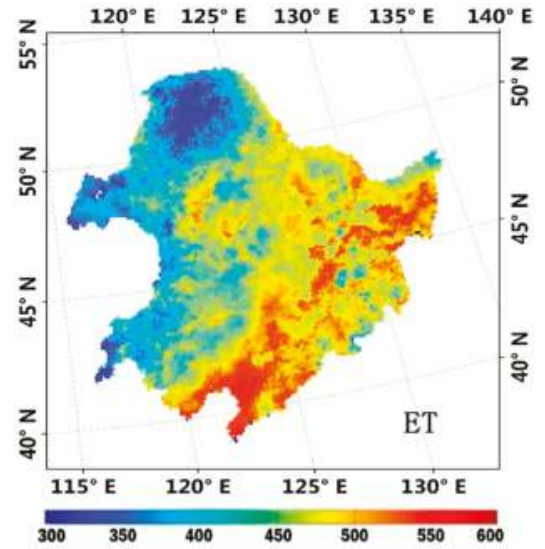

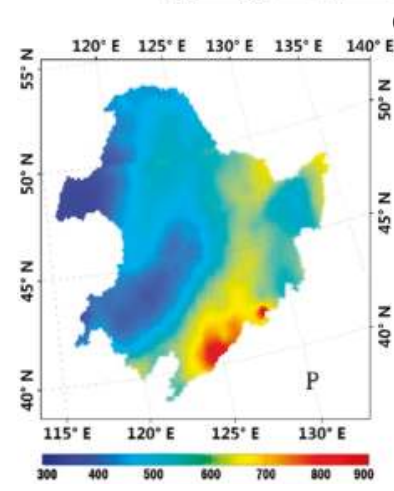

(b)

(a)

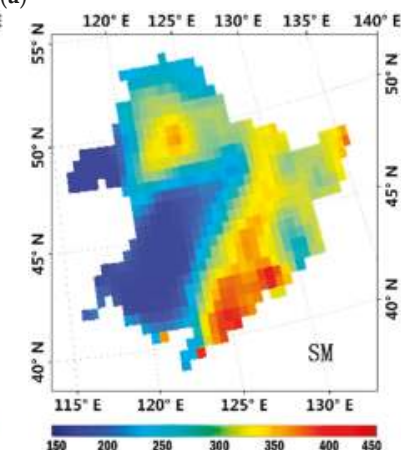

(c)

Figure 5. Spatial patterns of (a) multiyear average ET, (b) multiyear average precipitation, and (c) multiyear average soil moisture in Northeast China. All are in units of $\mathrm{mm} /$ year.

\subsubsection{Seasonal}

Multiyear (1982-2010) seasonal patterns of ET over Northeast China are shown in Figure 6. Distinct seasonal cycles of ET can be detected over Northeast China, which corresponds to the East Asian monsoon climate characterized by a wet summer and a dry winter. As shown in Figure 6a, the seasonal mean ET of MAM (March, April, and May) is 20-50 mm/month because of the lower temperature and precipitation. In contrast, during JJA (June, July, and August), when the air temperature and precipitation reach the maximum for the whole year, the monthly ET of most areas in Northeast China exceeds $60 \mathrm{~mm} / \mathrm{month}$ and accounts for over half of the annual ET (see Figure 6b). After summer, ET has significantly decreased over Northeast China (see Figure 6c,d).

In addition, we also found that the regional distribution characteristic of ET over Northeast China differs among the four seasons. For example, during MAM, peak ET occurs in the southeastern part of the region, which has plentiful solar radiation and rising temperature. However, when summer comes, peak ET expands and moves northward (Figure 6b). Previous study states that, accompanied by plentiful solar radiation, rising air temperature, and fierce vegetation transpiration, the ET of the forest ecosystem is stronger than other ecosystems in the summer [56]. As seen from Figure 6b, the region with high ET is mainly distributed in the north and east regions of Northeast China, where forests were densely distributed. In other words, the distribution of forest determines the distribution characteristic of ET in JJA. When the rainy season has gone and the air temperature is declining, ET has decreased 
noticeably from SON (September, October, and November) to DJF (December, January, and February). As a result, the ET of the southern part of the study area is higher than the northern part, which is different from the distribution feature of the ET in JJA.

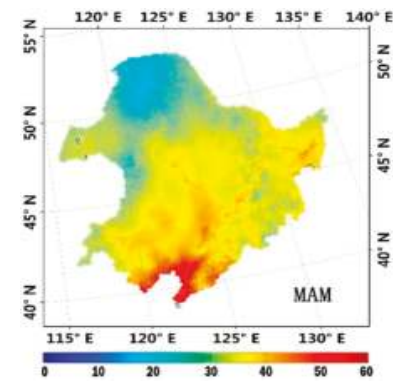

(a)

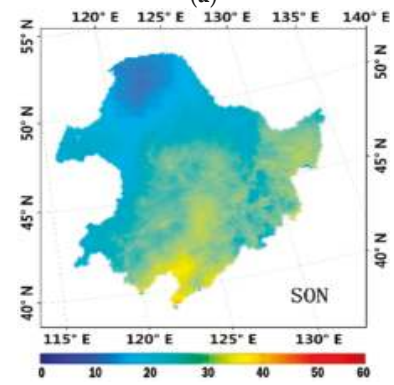

(c)

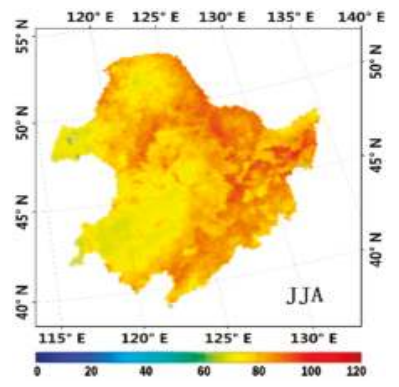

(b)

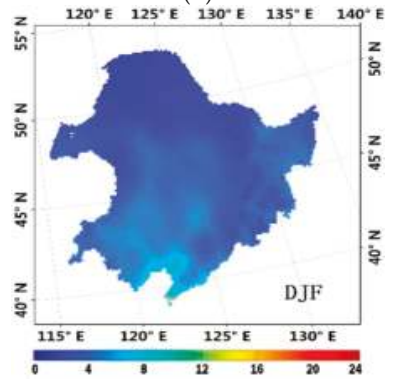

(d)

Figure 6. Multiyear seasonal patterns of ET in Northeast China: (a) MAM (March, April, and May); (b) JJA (June, July, and August); (c) SON (September, October, and November); (d) DJF (December, January, and February). The ET are in units of $\mathrm{mm} / \mathrm{month}$.

\subsection{Characteristics of ET Trends}

\subsubsection{Annual}

The ET of Northeast China estimated by MS-PT has increased by $4.5 \mathrm{~mm} /$ decade during 1982-2010 (Figure 7a). This increase in annual ET mainly occurred in the period from 1982 to 1998, with a linear trend of $12.3 \mathrm{~mm} /$ decade $(p<0.1)$, which is consistent with the expected acceleration in response to global warming [9]. After that, coincident with the last major El Nino event in 1998, the positive trend in ET seems to have ceased, with a non-significant trend of $-13.0 \mathrm{~mm} /$ decade $(p=0.42)$. The estimates of the trend statistics $Z$ values and the Mann-Kendall slope for annual ET are given in Table 3.

Considering the difference in climatic and hydrological condition between the LH basin and the SJ basin, we further calculated the trend of ET in the SJ basin and the LH basin, respectively. The difference in the ET trends between the pre-1998 period and the post-1998 period became more obvious. As seen from Figure 7b, in the years between 1982 and 1998, the annual land ET of the LH basin increased at a rate of $16.4 \mathrm{~mm} /$ decade $(p<0.01)$. After 1998, the annual land ET of the LH basin decreased rapidly with a linear trend of $-45.1 \mathrm{~mm} /$ decade $(p<0.01)$. This enormous decrease in ET may be driven by the human activity, such as the reclamation of grassland into cropland or the exploitation of mineral resources [20]. Similarly, the annual land ET of the SJ basin increased from 1982 to 1998 with a linear fit having a slope of $11.4 \mathrm{~mm} /$ decade. After that, the annual land ET of the SJ basin also decreased by $-4.2 \mathrm{~mm} /$ decade. 

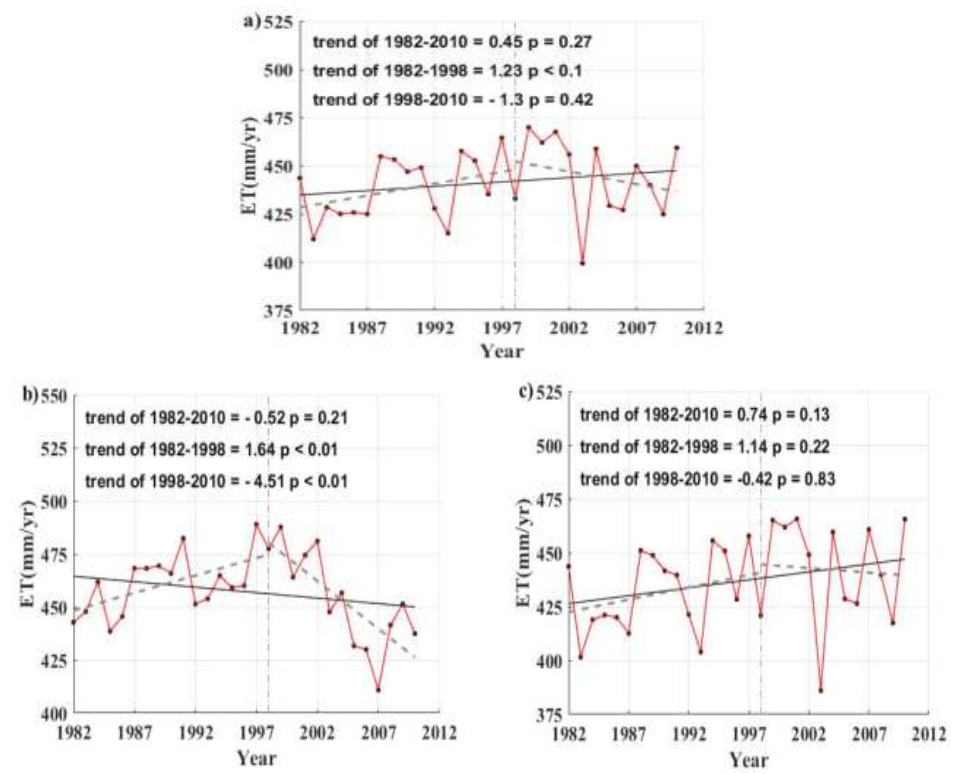

Figure 7. (a) Interannual variability of ET from 1982 to 2010 in Northeast China; partly interannual variability of ET: (b) the LH basin; and (c) the SJ basin.

Table 3. Mann-Kendall test results for the ET trend.

\begin{tabular}{ccccc}
\hline Region & Season & $\mathbf{Z}$ & $\boldsymbol{\beta}$ & $\mathbf{R} / \mathbf{A}$ \\
\hline \multirow{4}{*}{ Northeast China } & All & 1.46 & 0.45 & $\mathrm{R}$ \\
& MAM & -0.14 & -0.04 & $\mathrm{R}$ \\
& JJA & 0.82 & 0.26 & $\mathrm{R}$ \\
& SON & 3.24 & 0.19 & $\mathrm{~A}$ \\
& DJF & 2.42 & 0.05 & $\mathrm{~A}$ \\
\hline \multirow{5}{*}{ The LH Basin } & All & -0.78 & -0.52 & $\mathrm{R}$ \\
& MAM & -0.25 & -0.13 & $\mathrm{R}$ \\
& JJA & -1.57 & -0.51 & $\mathrm{R}$ \\
& SON & 1.07 & 0.04 & $\mathrm{R}$ \\
& DJF & 2.71 & 0.08 & $\mathrm{~A}$ \\
\hline \multirow{5}{*}{ The SJ Basin } & All & 2.07 & 0.74 & $\mathrm{~A}$ \\
& MAM & 0.03 & -0.02 & $\mathrm{R}$ \\
& JJA & 1.74 & 0.48 & $\mathrm{R}$ \\
& SON & 3.85 & 0.24 & $\mathrm{~A}$ \\
& DJF & 2.03 & 0.04 & $\mathrm{~A}$ \\
\hline
\end{tabular}

R: reject hypothesis $\mathrm{H}_{0}$; A: accept hypothesis $\mathrm{H}_{0}$.

\subsubsection{Seasonal}

Figure 8 shows interannual land ET variations of Northeast China, the SJ basin, and the LH basin for the four seasons. Except for summer, we found ET increases significantly $(p<0.1)$ from 1982 to 1998 for all periods. However, after 1988, these positive trends appear to have stalled, which is similar to the trend of interannual ET variations. For example, in MAM, ET increased significantly at a rate of 0.4, 0.36, and $0.58 \mathrm{~mm} \cdot \mathrm{month}^{-1}$ per year from 1982 to 1998 for the whole study area, the SJ basin, and the LH basin, respectively (Figure 8(a-a2)). After 1998, ET decreased year by year, with a rate of $-1.05,-0.91$, and $-1.55 \mathrm{~mm} \cdot \mathrm{month}^{-1}$ per year for the whole study area, the SJ basin, and the LH basin. The estimates of the trend statistics $Z$ values and the Mann-Kendall slope for seasonal ET are also given in Table 3. 

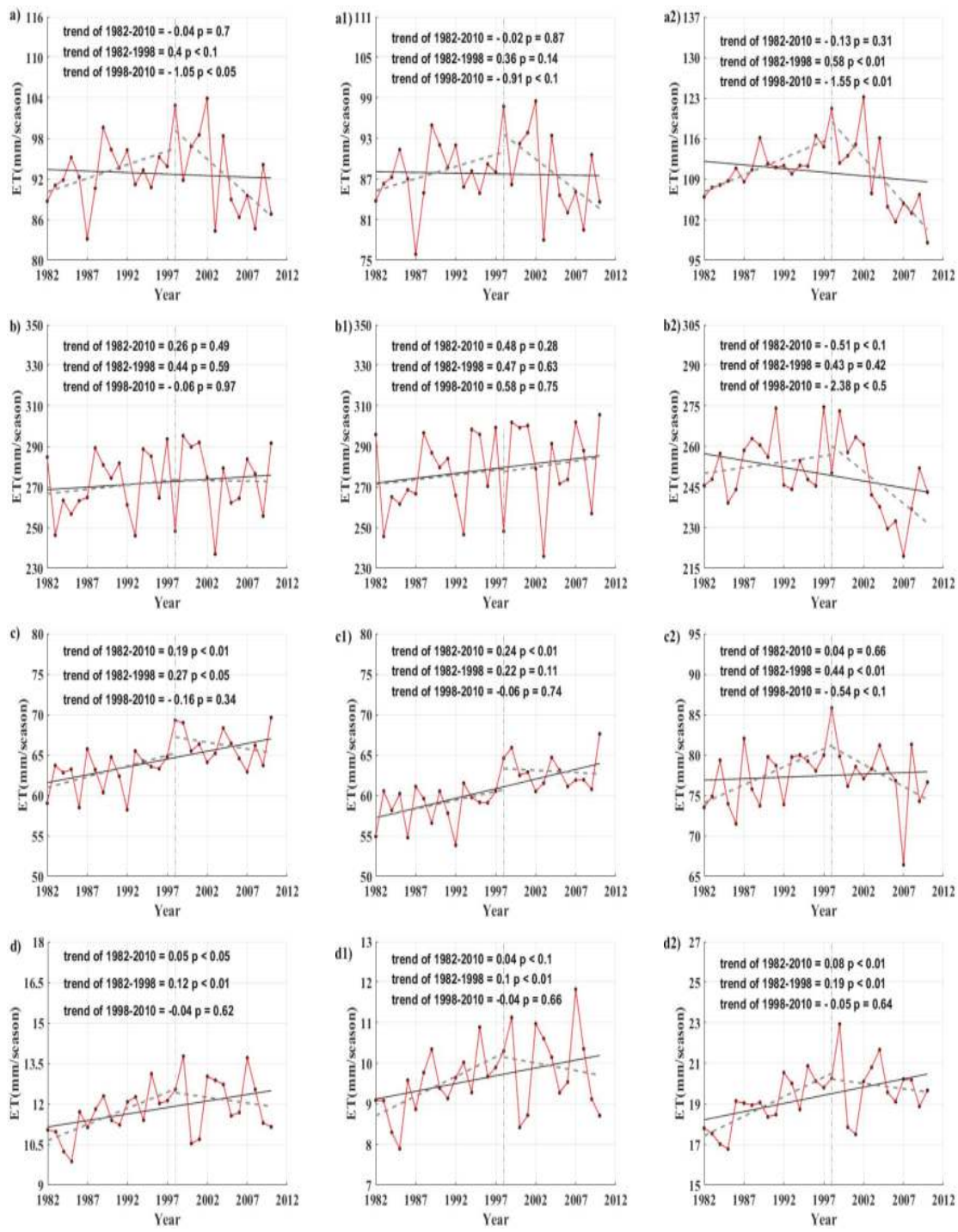

Figure 8. First column: interannual variability of ET from 1982-2010 in Northeast China: (a) MAM; (b) JJA; (c) SON; (d) DJF. Second column: interannual variability of ET in the SJ basin: (a1) MAM; (b1) JJA; (c1) SON; (d1) DJF. Third column: interannual variability of ET in the LH basin: (a2) MAM; (b2) JJA; (c2) SON; (d2) DJF.

We also found the variation trend of interannual land ET has obvious seasonal cycles (Figure 8). The trends of seasonal ET for MAM, SON, and DJF are similar to the annual ET trend. However, for JJA, the annual ET changes irregularly. During 1982-2010, the annual ET of Northeast China increases at a rate of $0.26 \mathrm{~mm} \mathrm{month}{ }^{-1}$ per year in summer $(p=0.46)$. Such a non-significant increment mainly occurred in the SJ basin with $0.48 \mathrm{~mm} \cdot \mathrm{month}^{-1}$ per year. For the LH basin, the annual ET decrease at a rate of $-0.51 \mathrm{~mm} \cdot$ month $^{-1}$ per year in summer $(p<0.1)$. Moreover, the magnitudes of the ET trends changes during JJA are higher than the other three seasons. For example, the annual ET increased at 
a rate of 0.47 and $0.43 \mathrm{~mm}$ month $^{-1}$ per year in JJA before 1998 over the SJ basin and the LH basin. After 1998, ET still increased $0.58 \mathrm{~mm}^{\text {month }}{ }^{-1}$ per year in the SJ basin. For the LH basin, the trend of ET becomes negative and significant $\left(-2.38 \mathrm{~mm} \cdot\right.$ month $^{-1}$ per year, $\left.p<0.05\right)$. On the contrary, as seen from Figure $8(\mathrm{~d} 1, \mathrm{~d} 2)$, the trend of ET is nearly zero for both the SJ basin and the LH basin during 1982-2010 (0.04 mm month ${ }^{-1}$ per year, $p<0.1 ; 0.08 \mathrm{~mm} \cdot \mathrm{month}^{-1}$ per decade, $\left.p<0.01\right)$, which is far lower than the trend in JJA. This is because low temperature and limited solar energy restrain soil surface evaporation and vegetation transpiration.

\subsection{Spatial Patterns of ET Trend Changes in Northeast China}

\subsubsection{Annual}

Figure 9a shows the spatial distribution of ET trends over Northeast China from 1982 to 2010. Our results illustrate that the ET of Northeast China has increased on average by $6.8 \mathrm{~mm} /$ decade during 1982-2010. Fifty-nine percent of the pixels in the study area show an increasing trend in ET and $23.6 \%$ of the pixels have a significant increasing trend $(p<0.05)$. Among them, the positive ET trends mainly occur in the north part and east part of the SJ basin, where exactly is vegetated land surface [28]. Only $9.8 \%$ of the pixels show a significant decreasing trend and mainly located in the southern part of Inner Mongolia and Western Jilin, which mainly consists of unused land. The reclamation of desert land for cropland has influenced the original ecosystem of these regions and may result in this negative trend of ET [20].

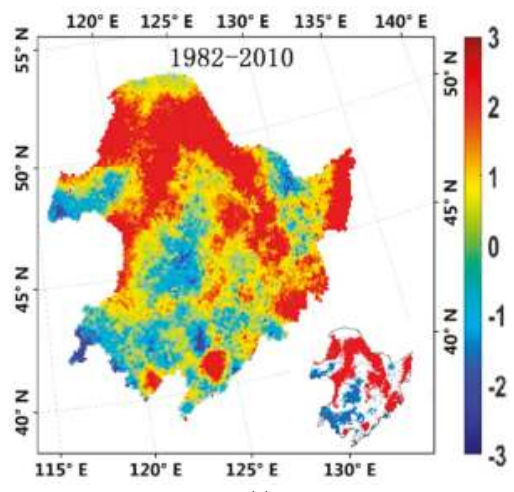

(a)

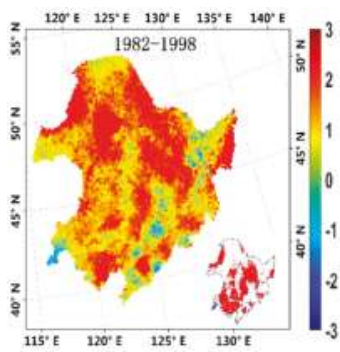

(b)

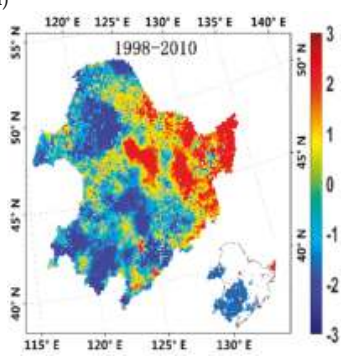

(c)

Figure 9. Spatial distributions of ET trends in Northeast China: (a) during 1982-2010; (b) during 1982-1998; (c) during 1998-2010. The ET trend is in units of $\mathrm{mm} /$ year. The inset panels show the area where the ET trend is statistically significant $(p<0.05)$. Red represents a significant increase and blue represents a significant decrease. 
We also analyzed changes in annual ET trends before and after 1998. We found that, for most regions of Northeast China, the overall increment in annual ET from 1982 to 2010 can largely be attributed to the upward trend of 1982-1998. As shown in Figure 9b, 86.5\% of the regions show an upward trend in ET from 1982 to 1998, which is relative to the remarkable warming phenomenon over Northeast China [1]. After 1998, most parts of Northeast China show a negative part, only a few regions of Songjiang basin (32.5\%) keep rising in ET (Figure 9c). The difference in ET trends between the two periods indicates that the positive trend of annual ET has ceased, or even reversed.

\subsubsection{Seasonal}

Figure 10a-d shows the spatial distributions of four seasonal ET trends over Northeast China. From our results, the distribution of interannual land ET trend has obvious seasonal characteristics. In MAM, areas with positive ET trends are mostly located in the LH basin, with its vegetated land surface (Figure 10a). In contrast, for DJF, positive ET trends are mostly located in the SJ basin, with a lower latitude (Figure 10d). As shown in Figure 10b, the spatial distribution of ET trends in JJA is consistent with the annual spatial distribution (Figure 9a), which coincides with the finding by Yang et al. [21]. In summer, ET is the highest among the four seasons. As a result, the ET trend change of JJA can account for most of the total annual ET trend change. We also notice that the spatial distribution of significant trends also coincides with annual significant trends.

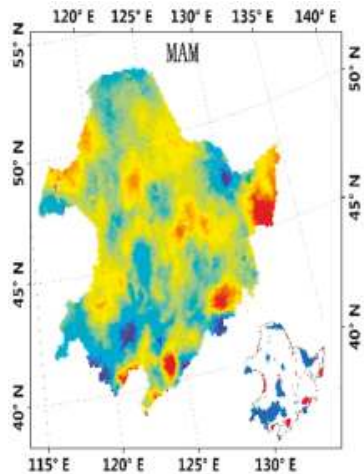

(a)

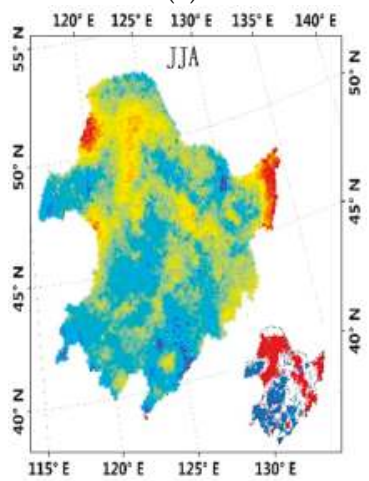

(b)

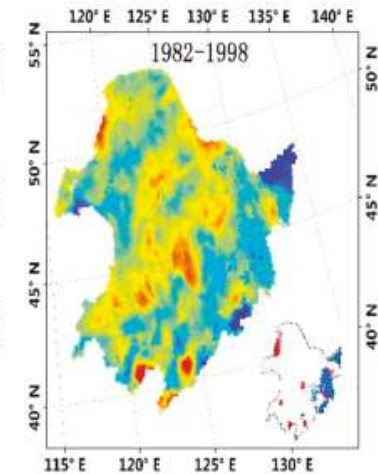

(a1)

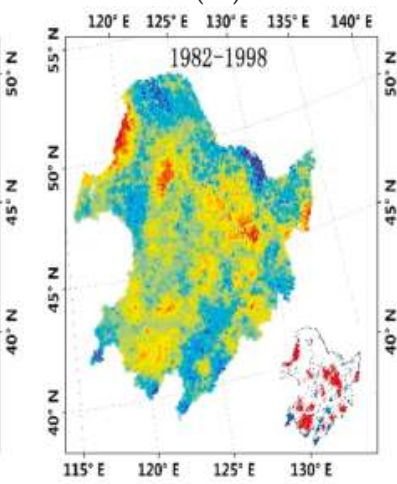

(b1)

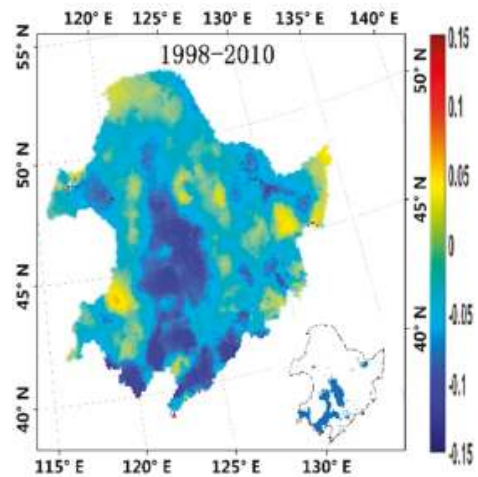

(a2)

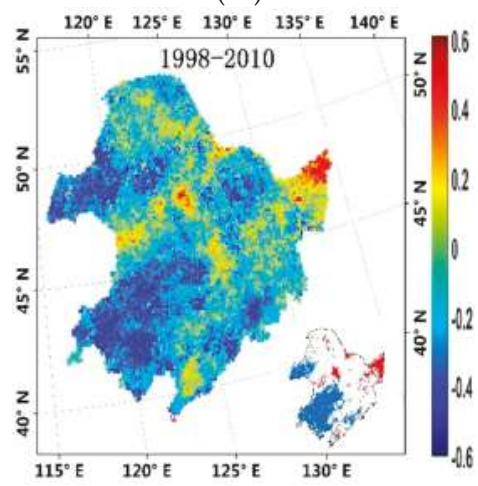

(b2)

Figure 10. Cont. 


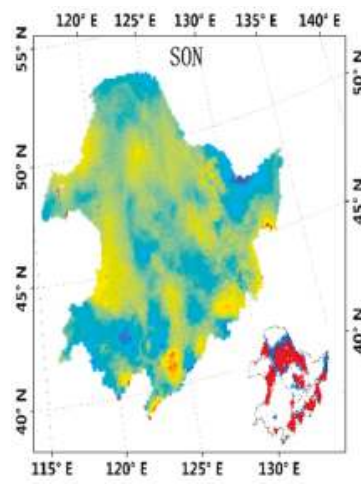

(c)

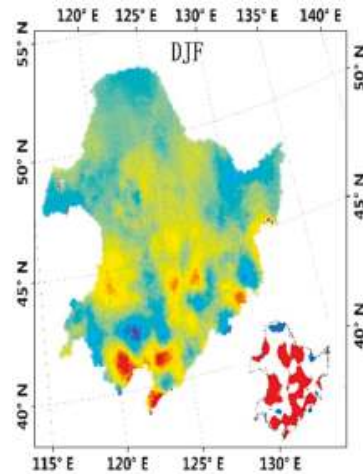

(d)

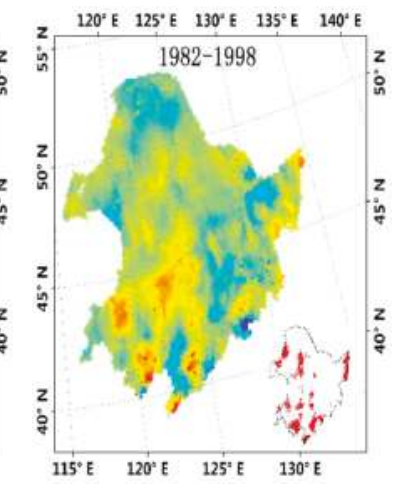

(c1)

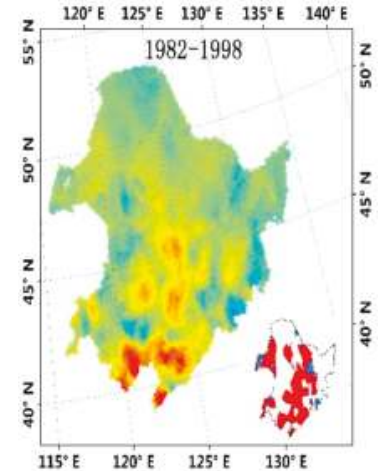

(d1)

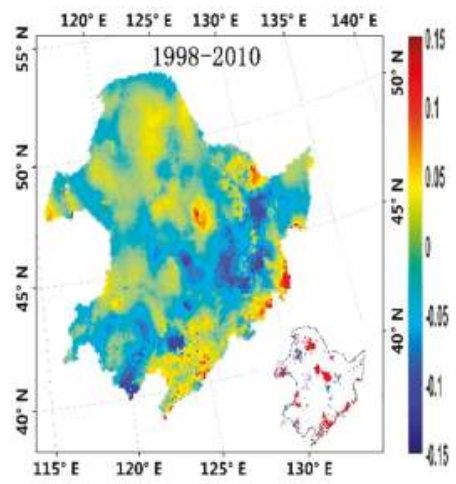

(c2)

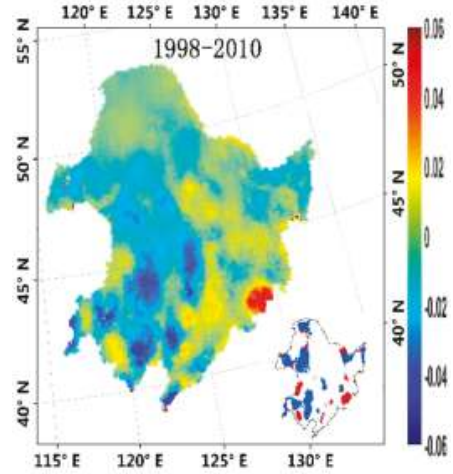

(d2)

Figure 10. (First column) Spatial distributions of ET trends in Northeast China during 1982-2010: (a) MAM; (b) JJA; (c) SON; (d) DJF; (second column) during 1982-1998: (a1) MAM; (b1) JJA; (c1) SON; (d1) DJF; (third column) during 1998-2010: (a2) MAM; (b2) JJA; (c2) SON; (d2) DJF. The inset panels show the area where the ET trend is statistically significant $(p<0.1)$.

Then, we analyzed changes in seasonal ET trends before and after 1998. The trend of ET is dominated by that in summer during both the pre-1998 period and the post-1998 period. In other words, the influence of the other three seasons on the trend of annual ET is weaker than JJA. Additionally, the positive annual ET trend change during the four seasons mainly originates from that influence during 1982-1998. Comparing annual ET trends for all seasons before and after 1998, more and more pixels experienced a decreasing trend during the post-1998 period. This is particularly evident for the first half of year, when more pixels of Northeast China show a negative trend change from 1988 to 2010 .

\section{Discussion}

\subsection{The Performance of the MS-PT Algorithm in Estimating ET}

Model validation at six EC flux tower sites illustrates that the MS-PT algorithm for estimating ET was reliable and robust across multiple biomes in Northeast China (Figure 2). Due to the difference in the water cycle and land surface characteristics, biases exist in the application of global ET products in specific areas [57]. Yao et al. [24] compared the MS-PT algorithm with the PT-JPL algorithm using ground observations collected from 40 flux towers distributed around the world and found MS-PT 
algorithm improved ET estimates at most flux towers sites, with a higher $\mathrm{R}^{2}(0.41-0.89)$ and a lower RMSE (23.7-48.6 W/m²). Compared with MOD16 product, our results illustrate that the MS-PT algorithm improves the accuracy of ET quantification at most flux tower sites in Northeast China, indicating this method may be a better tool for analyzing the variation of ET over Northeast China. However, one should note that the MS-PT algorithm has a relatively poor performance at flux sites from the CEOP network. Perhaps complex terrain and instrument calibration contribute to large biases.

We also found that the MS-PT algorithm show inter-biome differences and performs better for forest land sites (Figure 2). The seasonality for vegetation indices and canopy structure may be critical in determining satellite-based ET model performance [58]. Previous studies have revealed that some satellite-based ET algorithms can estimate ET more accurately for deciduous broadleaf forest [46,59]. Since the major seasonal vegetation types of the forest region in Northeast China are deciduous broadleaf forest and coniferous forest $[25,60]$, our validation results are similar with previous findings. In contrast, for some cropland and grassland sites, such as the Jinzhou flux site, the MS-PT algorithm still has relatively low $\mathrm{R}^{2}$ and high RMSE. The limitation of the MS-PT algorithm, such as neglecting of the differences in parameters from different biome types, may cause this problem [24].

We should note that the accuracy of MS-PT algorithm depends on the errors for EC ET observations because we consider ET observations as true values in calculating the weights for the MS-PT algorithm. Although we correct ET by Twine et al. [43] method, the inaccuracy in the measured data still exists, such as the energy imbalance issue, with $\mathrm{H}+\mathrm{LE}<R_{n}-G$ (H: sensible heat flux; LE: latent heat flux) [61]. The mismatches in scale among different datasets may also lead to differences between the simulated and measured values. For example, the original spatial resolutions of meteorological reanalysis data and the NDVI3g data are 0.1 degree and $8 \mathrm{~km}$, respectively. While the NDVI3g data was converted to a resolution of $0.1^{\circ} \times 0.1^{\circ}$ using bilinear interpolation, the resample process may also result in biases. Additionally, EC observations represent a point-based value with merely hundreds of meters in diameter, which may also cause differences between ET observations and the true ET [62]. If the input data has a higher spatial resolution, the performance of MS-PT can be greatly improved.

\subsection{Climate Change Controls on Land ET Trends in Northeast China}

Major global climatic perturbations and influences, such as the last major El Niño event in 1998, have caused alterations in the global water cycle and land-atmosphere water flux [46]. Our findings confirm that ET of Northeast China has increased significantly $(p<0.1)$ over the last two decades of the twentieth century [19] and decreased with large interannual fluctuations $(p=0.42)$ after 1998 (Figure 6a) [20]. Most previous studies suggest that the limitation by surface water supply is the dominant factor in determining this ET trend change $[7,22,63,64]$, because of the relatively sparse precipitation in some regions of Northern China. However, some studies propose that the changes in the ET trend may be the result of the variation in air temperature, vegetation condition, or surface energy [56,65-68]. Additionally, due to the nonlinear dependence of ET on related climate factors, their relative contributions to the ET trend are not obvious. Thus, it is difficult to assess climate change controls on ET trends in Northeast China.

In this study, based on the interannual variations of ET calculated by MS-PT algorithm, we find moderate spatial correlation between the trend of ET and precipitation in the west part of Northeast China (Figure 11a). Only in the western region of Northeast China, where annual rainfall is infrequent, the trend of precipitation shows a positive correlation with the variation of ET. Similarly, the trend of soil moisture also shows a correlation with ET trends in this area (Figure 11b). These positive correlations can be explained by the fact that, under dry condition (Figure $5 b$ ), less precipitation leads to lower soil moisture, which, in turn, limits ET [69]. Furthermore, a reduced ET would decrease the precipitation and further reduce soil moisture $[70,71]$. Therefore, precipitation can play a key role for the annual variation of ET. However, in other areas of Northeast China, weak spatial consistency is found between ET trend changes and precipitation trend changes, which is also documented by 
Tian et al. [19]. It is possible that different surface vegetation and water supply cause the consistency difference. For instance, there is more deep-rooted vegetation in the northern part of Northeast China, where roots of the vegetation can reach groundwater and extract water from the whole layer of soil [72]. As a result, precipitation is no longer a good indication of ET in these areas, which is different from previous studies. In a word, only under dry conditions, such as the western region of Northeast China, is precipitation is a critical meteorological factor influencing ET trend.

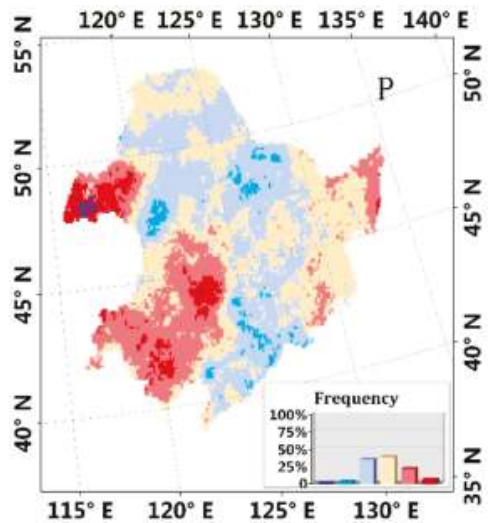

(a)

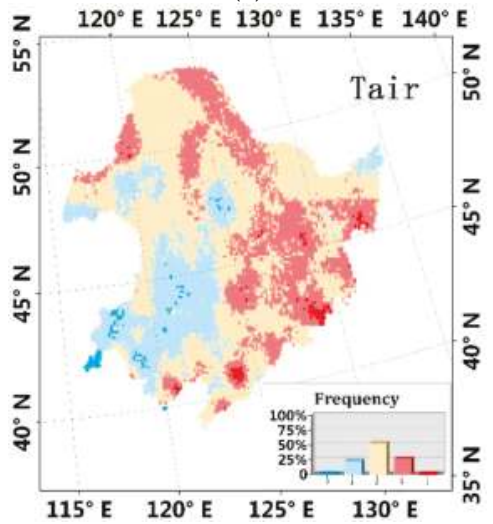

(c)

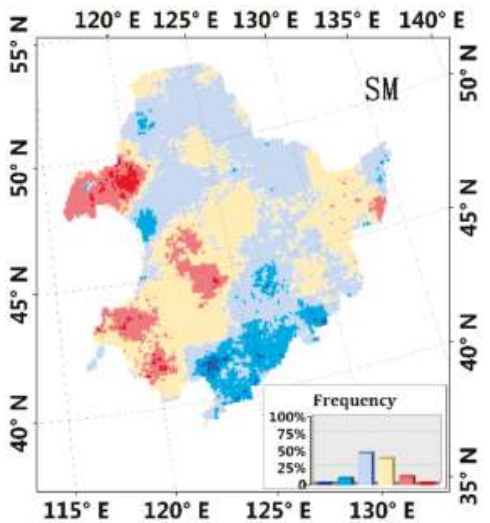

(b)

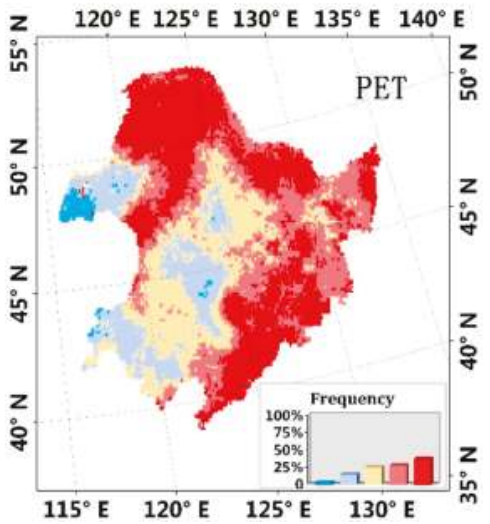

(d)

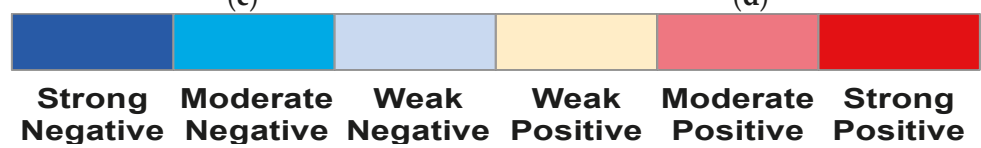

Figure 11. Map of the correlation coefficient between the annual ET trend (1982-2010) and the trend of (a) precipitation, (b) soil moisture, (c) Tair, and (d) PET. The inset panels show the frequency of various change trends. The degree of correlation is classified into three ranks according to the correlation coefficient: strong $\left(\mathrm{R}^{2} \geq 0.7\right)$; moderate $\left(0.3<\mathrm{R}^{2}<0.7\right)$; weak $\left(\mathrm{R}^{2} \leq 0.3\right)$.

As shown in Figure 5c, in the humid areas, such as the southeast coast and north part of Northeast China, the changes in ET are unrestricted by water supply because of relatively abundant soil moisture. PET, an important indicator of atmospheric evaporation demand, represents the maximum ET rate when given an unlimited water supply and may influence the trends of ET in these areas $[73,74]$. Mo et al. [56] suggested that there exists an opposite trend of ET and PET in China, which reveals 
a complementary relationship between ET and PET. Gao et al. [7] found that the decreasing PET largely influences the decreasing trends of ET in Southeast China and proposed that the change of PET appeared to be the major factor determining the change of actual ET in subtropical humid area. However, we found a strong spatial correlation between PET trend changes and ET trend changes in wet regions of Northeast China, which demonstrates that PET may be the dominant factor determining the trend changes in ET (Figure 11d). Moreover, previous substantial studies suggest that Tair is a key controlling factor influencing ET in high latitude region [75], because it can be recognized as a surrogate for atmospheric demand [76]. Our results illustrate that the trend of Tair is positively related to ET trends in these areas (Figure 11c), but the correlation is weaker than PET. This can be explained that PET is influenced by a number of climate elements, including Tair, net radiation, wind speed, and vapor pressure, and ET is limited by freezing temperatures, wind speed, and surface energy in Northeast China [77]. Integrated with different climatic factors, PET appears to better control the trend of ET. Overall, in high latitude and wet canopy conditions, PET tends to exert the most significant control over ET.

\section{Conclusions}

The goal of this study was to estimate terrestrial ET of Northeast China during 1982-2010 using the MS-PT algorithm. This approach in modeling ET is physically based, requiring no subjective parameter calibration as employed by many other traditional ET methods. Using the ET measurements of the six eddy covariance sites, our validation showed MS-PT algorithm provided the reliable ET estimations at site scale. We also used meteorological reanalysis data and remote sensing data to analyze the spatiotemporal variation in ET during 1982-2010. The results demonstrated that MS-PT algorithm has a good performance in estimating regional ET over Northeast China.

In this paper, we found the annual mean ET during 1982-2010 is $440.68 \pm 18 \mathrm{~mm} /$ year in Northeast China. For the spatial pattern of annual averaged ET over Northeast China, we found a decreasing trend from southeast coast to northwest inland, which is influenced by the surface water resource. Over the entire study area, ET has increased on average by $5 \mathrm{~mm}_{\text {year }}{ }^{-1}$ per decade over Northeast China during 1982-2010, and the pixels with a positive trend were mainly distributed in the SJ basin. We found an especially obvious difference before and after 1998 and concluded that the increase in annual ET mainly occurred in the period from 1982 to 1998. Further, we evaluate what have caused any spatial-temporal variation in ET. Our findings illustrate that PET is the most important factor influencing ET trend in most parts of Northeast China. Over semiarid and arid areas, such as the west of Northeast China, precipitation can also affect ET variation. Thus, future studies are needed to clarify the relative contributions of the different climatic variables and understand the contributing process of different climatic variables in ET.

Acknowledgments: The authors thank Meng Liu from Geographic Sciences and Natural Resources Research, CAS, and Jianmin Zhou and Aolin Jia from Beijing Normal University, for their helpful suggestions. The authors thank Jie He and Kun Yang from the Cold and Arid Regions Science Data Center at Lanzhou (http:/ / westdc.westgis.ac. $\mathrm{cn} /$ ) for providing the China Meteorological Forcing Dataset. The authors thank Dr. Shaomin Liu from Beijing Normal University, and Guirui Yu from the Institute of Geographic Sciences and Natural Resources Research, CAS, for providing flux observation data. Other ground-measured data were obtained from the Chinaflux network (http:/ / 159.226.111.42/pingtai/LoginRe/opendata.jsp) and the Coordinated Enhanced Observation Project (CEOP) in arid and semi-arid regions of Northern China (http:/ / observation.tea.ac.cn/). GIMMIS NDVI products were obtained from NOAA (http:/ / islscp2.sesda.com/ISLSCP21/data), MODIS ET, and land cover-type products were obtained online (http://reverb.echo.nasa.gov/reverb). The Climate Prediction Center soil moisture dataset was obtained online (http://www.esrl.noaa.gov/psd/). This work was partially supported by the National Key Research and Development Program of China (No. 2016YFA0600102) and the Natural Science Fund of China (No. 61661136006, and No. 41671331).

Author Contributions: Lilin Zhang, Yunjun Yao, and Zhiqiang Wang prepared the manuscript. Xuanyu Wang, Jia $\mathrm{Xu}$, and Xiaowei Chen conducted the remote sensing image processing. Kun Jia, Xiaotong Zhang, and Yuhu Zhang contributed to the discussion.

Conflicts of Interest: The authors declare no conflict of interest. 


\section{References}

1. Sun, F.H.; Yang, X.Q.; Lu, S.; Yang, S.Y. The contrast analysis on the average and extreme temperature trend in northeast china. Sci. Meteorol. Sin. 2006, 26, 157-163.

2. Mao, D.; Wang, Z.; Luo, L.; Yang, G. Correlation analysis between ndvi and climate in northeast china based on avhrr and gimms data sources. Remote Sens. Technol. Appl. 2012, 27, 81-89.

3. Wang, K.; Dickinson, R.E. A review of global terrestrial evapotranspiration: Observation, modeling, climatology, and climatic variability. Rev. Geophys. 2012, 50. [CrossRef]

4. Yao, Y.; Liang, S.; Li, X.; Chen, J.; Wang, K.; Jia, K.; Cheng, J.; Jiang, B.; Fisher, J.B.; Mu, Q. A satellite-based hybrid algorithm to determine the priestley-taylor parameter for global terrestrial latent heat flux estimation across multiple biomes. Remote Sens. Environ. 2015, 165, 216-233. [CrossRef]

5. Yao, Y.; Liang, S.; Yu, J.; Chen, J.; Liu, S.; Lin, Y.; Fisher, J.B.; Mcvicar, T.R.; Cheng, J.; Jia, K. A simple temperature domain two-source model for estimating agricultural field surface energy fluxes from Landsat images. J. Geophys. Res. Atmos. 2017. [CrossRef]

6. Sun, F.; Wu, Z.; Yang, S. Temporal and spatial variations of extreme precipitation and dryness events in northeast china in last 50 years. Chin. J. Ecol. 2006, 25, 779-784.

7. Gao, G.; Chen, D.; Xu, C.Y.; Simelton, E. Trend of estimated actual evapotranspiration over china during 1960-2002. J. Geophys. Res. Atmos. 2007, 112, 71-81. [CrossRef]

8. Zhang, S.J.; Zhang, Y.S.; Sui, D.; Cai, F.; Fu, C.; Wu, J.-W.; Chen, P.S.; Liu, Q.W. Changes in reference evapotranspiration and its causes in northeast china. J. Nat. Resour. 2010, 25, 1750-1761.

9. Jung, M.; Reichstein, M.; Ciais, P.; Seneviratne, S.I.; Sheffield, J.; Goulden, M.L.; Bonan, G.; Cescatti, A.; Chen, J.; De, J.R. Recent decline in the global land evapotranspiration trend due to limited moisture supply. Nature 2010, 467, 951-954. [CrossRef] [PubMed]

10. Wang, K.; Dickinson, R.E.; Wild, M.; Liang, S. Evidence for decadal variation in global terrestrial evapotranspiration between 1982 and 2002. J. Geophys. Res. Atmos. 2010, 115, 898-907. [CrossRef]

11. Shukla, J.; Mintz, Y. Influence of land-surface evapotranspiration on the earth's climate. Science 1982, 215, 1498-1501. [CrossRef] [PubMed]

12. Zeng, L.H.; Song, K.S.; Zhang, B.; Wang, Z.M.; Du, J. Spatiotemporal variability of reference evapotranspiration over the northeast region of china in the last 60 years. Adv. Water Sci. 2010, 21, 194-200.

13. Zhang, B.; Kang, S.; Li, F.; Zhang, L. Comparison of three evapotranspiration models to bowen ratio-energy balance method for a vineyard in an arid desert region of northwest china. Agric. For. Meteorol. 2008, 148, 1629-1640. [CrossRef]

14. Liu, S.M.; Xu, Z.W.; Wang, W.Z.; Jia, Z.Z.; Zhu, M.J.; Bai, J.; Wang, J.M. A comparison of eddy-covariance and large aperture scintillometer measurements with respect to the energy balance closure problem. Hydrol. Earth Syst. Sci. 2011, 15, 1291-1306. [CrossRef]

15. Allen, R.G.; Pereira, L.S.; Raes, D.; Smith, M. Crop Evapotranspiration-Guidelines for Computing Crop Water Requirements. Available online: https://appgeodb.nancy.inra.fr/biljou/pdf/Allen_FAO1998.pdf (accessed on 1 November 2017).

16. Yang, Y. Remote sensing temporal and spatial patterns of evapotranspiration and the responses to water management in a large irrigation district of north china. Agric. For. Meteorol. 2012, 164, 112-122. [CrossRef]

17. Zhang, K.; Kimball, J.S.; Nemani, R.R.; Running, S.W. A continuous satellite-derived global record of land surface evapotranspiration from 1983 to 2006. Water Resour. Res. 2010, 46, 109-118. [CrossRef]

18. Mueller, B.; Hirschi, M.; Jimenez, C.; Ciais, P. Benchmark products for land evapotranspiration: Landflux-eval multi-dataset synthesis. Hydrol. Earth Syst. Sci. 2013, 17, 3707-3720. [CrossRef]

19. Tian, J.; Hongbo, S.U.; Chen, S.; Sun, X.; Chen, Q. Spatial-temporal variations of evapotranspiration in china mainland in recent 20 years. Resour. Sci. 2012, 34, 1277-1286.

20. Tian, H.E.; Shao, Q. Spatial-temporal variation of terrestrial evapotranspiration in china from 2001 to 2010 using mod16 products. J. Geo-Inf. Sci. 2014, 16, 979-988.

21. Yang, X.; Wang, G.; Pan, X.; Zhang, Y. Spatio-temporal variability of terrestrial evapotranspiration in china from 1980 to 2011 based on gleam data. Trans. Chin. Soc. Agric. Eng. 2015, 31, 132-141.

22. Chen, Y.; Xia, J.; Liang, S.; Feng, J.; Fisher, J.B.; Li, X.; Li, X.; Liu, S.; Ma, Z.; Miyata, A. Comparison of satellite-based evapotranspiration models over terrestrial ecosystems in china. Remote Sens. Environ. 2014, 140, 279-293. [CrossRef] 
23. Yao, Y.; Liang, S.; Cheng, J.; Liu, S.; Fisher, J.B.; Zhang, X.; Jia, K.; Zhao, X.; Qin, Q.; Zhao, B. Modis-driven estimation of terrestrial latent heat flux in china based on a modified priestley-taylor algorithm. Agric. For. Meteorol. 2013, 171-172, 187-202. [CrossRef]

24. Yao, Y.; Liang, S.; Zhao, S.; Zhang, Y.; Qin, Q.; Cheng, J.; Jia, K.; Xie, X.; Zhang, N.; Liu, M. Validation and application of the modified satellite-based priestley-taylor algorithm for mapping? Terrestrial evapotranspiration. Remote Sens. 2014, 6, 880-904. [CrossRef]

25. Mao, D.H.; Wang, Z.M.; Han, J.X.; Ren, C.Y. Spatio-temporal Pattern of Net Primary Productivity and Its Driven Factors in Northeast China in 1982-2010. Sci. Geogr. Sin. 2012. [CrossRef]

26. Tang, Y.; Wang, H.; Yan, D.H.; Wang, S.S. Research on the spatial-temporal differentiation of precipitation in northeast China in recent 50 years. Sci. Geogr. Sin. 2005, 18, 901-906.

27. Qian, Z. Strategic concern to land and water resources allocation, ecology and environment protection and sustainable development of northeast China. Eng. Sci. 2006, 8, 1-24.

28. Liu, J.Y.; Kuang, W.H.; Zhang, Z.X.; Xu, X.L.; Qin, Y.W.; Ning, J.; Zhou, W.C.; Zhang, S.W.; Li, R.D.; Yan, C.Z.; et al. Spatiotemporal characteristics, patterns, and causes of land-use changes in China since the late 1980s. J. Geogr. Sci. 2014, 24, 195-210. [CrossRef]

29. Wang, X.Q.; Zhang, Y.; Liu, C.M. Estimation of eco-water requirement in the liaohe river basin. Geogr. Res. 2007, 26, 22-28.

30. Han, J.X.; Wang, Z.M.; Mao, D.H.; Song, K.S.; Ren, C.Y. Dynamic change of vegetation coverage in the songhua river basin and itscorrelation analysis with climatic factor from 1982 to 2010. Chin. J. Agrometeorol. 2011, 32, 430-436.

31. Liu, Q. A study on flood and drought change and its long-term prediction in the songhua river basin. Adv. Water Sci. 1994, 4, 319-327.

32. National Oceanic and Atmospheric Administration, Avhrr. Available online: http://islscp2.sesda.com/ ISLSCP21/data (accessed on 24 November 2016).

33. National Aeronautics and Space Administration, lp Daac. Available online: http://reverb.echo.nasa.gov/ reverb (accessed on 16 October 2016).

34. Mu, Q.; Heinsch, F.A.; Zhao, M.; Running, S.W. Development of a global evapotranspiration algorithm based on modis and global meteorology data. Remote Sens. Environ. 2007, 111, 519-536. [CrossRef]

35. Mu, Q.; Zhao, M.; Running, S.W. Improvements to a modis global terrestrial evapotranspiration algorithm. Remote Sens. Environ. 2011, 115, 1781-1800. [CrossRef]

36. National Oceanic and Atmospheric Administration/oar/esrl psd, Boulder, Colorado, USA. Available online: http:/ / www.esrl.noaa.gov/psd/ (accessed on 19 September 2016).

37. He, J.; Yang, K. China meteorological forcing dataset. In Cold and Arid Regions Science Data Center at Lanzhou . Available online: http:/ / westdc.westgis.ac.cn/ (accessed on 26 December 2016).

38. Yang, K.; Jie, H.; Tang, W.; Qin, J.; Cheng, C.C.K. On downward shortwave and longwave radiations over high altitude regions: Observation and modeling in the tibetan plateau. Agric. For. Meteorol. 2010, 150, 38-46. [CrossRef]

39. Chen, Y.; Yang, K.; Jie, H.; Qin, J.; Shi, J.; Du, J.; He, Q. Improving land surface temperature modeling for dry land of china. J. Geophys. Res. Atmos. 2011, 116, D20104. [CrossRef]

40. Guirui, Y.U.; Zhang, L.; Sun, X. Progresses and prospects of chinese terrestrial ecosystem flux observation and research network (chinaflux). Prog. Geogr. 2014, 33, 903-917.

41. Jia, Z.; Liu, S.; Xu, Z.; Chen, Y.; Zhu, M. Validation of remotely sensed evapotranspiration over the hai river basin, china. J. Geophys. Res. Atmos. 2012, 117, 13113. [CrossRef]

42. Yao, Y.; Liang, S.; Li, X.; Liu, S.; Chen, J.; Zhang, X.; Jia, K.; Jiang, B.; Xie, X.; Munier, S. Assessment and simulation of global terrestrial latent heat flux by synthesis of cmip5 climate models and surface eddy covariance observations. Agric. For. Meteorol. 2016, 223, 151-167. [CrossRef]

43. Twine, T.E.; Kustas, W.P.; Norman, J.M.; Cook, D.R.; Houser, P.R.; Teyers, T.P.; Prueger, J.H.; Starks, P.J.; Wesely, M.L. Correcting eddy-covariance flux underestimates over a grassland. Agric. For. Meteorol. 2000, 103, 279-300. [CrossRef]

44. Monteith, J.L. Evaporation and environment. Symp. Soc. Exp. Biol. 1965, 19, 205. [PubMed]

45. Priestley, C.H.B.; Taylor, R.J. On the assessment of surface heat flux and evaporation using large-scale parameters. Mon. Weather Rev. 2009, 100, 81-92. [CrossRef] 
46. Fisher, J.B.; Tu, K.P.; Baldocchi, D.D. Global estimates of the land-atmosphere water flux based on monthly avhrr and islscp-ii data, validated at 16 fluxnet sites. Remote Sens. Environ. 2008, 112, 901-919. [CrossRef]

47. Wang, K.; Liang, S. Estimation of surface net radiation from solar shortwave radiation measurements. In Proceedings of the IEEE International Geoscience and Remote Sensing, Boston, MA, USA, 7-11 July 2008; pp. 483-486.

48. Chen, S.; Liu, Y.; Thomas, A. Climatic change on the tibetan plateau: Potential evapotranspiration trends from 1961-2000. Clim. Chang. 2006, 76, 291-319.

49. Yao, Y.; Zhao, S.; Zhang, Y.; Jia, K.; Liu, M. Spatial and decadal variations in potential evapotranspiration of china based on reanalysis datasets during 1982-2010. Atmosphere 2014, 5, 737-754. [CrossRef]

50. Mann, H.B. Nonparametric test against trend. Econometrica 1945, 13, 245-259. [CrossRef]

51. Kendall, M.G. Rank Correlation Methods; University of Michigan: Ann Arbor, MI, USA, 1948.

52. Rahman, A.; Dawood, M. Spatio-statistical analysis of temperature fluctuation using Mann-Kendall and Sen's slope approach. Clim. Dyn. 2017, 48, 1-15.

53. Batra, N.; Islam, S.; Venturini, V.; Bisht, G.; Jiang, L. Estimation and comparison of evapotranspiration from modis and avhrr sensors for clear sky days over the southern great plains. Remote Sens. Environ. 2006, 103, 1-15. [CrossRef]

54. Seguin, B.; Becker, F.; Phulpin, T.; Gu, X.F.; Guyot, G.; Kerr, Y.; King, C.; Lagouarde, J.P.; Ottlé, C.; Stoll, M.P. Irsute: A minisatellite project for land surface heat flux estimation from field to regional scale. Remote Sens. Environ. 1999, 68, 357-369. [CrossRef]

55. Kalma, J.D.; Mcvicar, T.R.; Mccabe, M.F. Estimating land surface evaporation: A review of methods using remotely sensed surface temperature data. Surv. Geophys. 2008, 29, 421-469. [CrossRef]

56. Lin, Z. Trends in land surface evapotranspiration across china with remotely sensed ndvi and climatological data for 1981-2010. Hydrol. Sci. J. 2015, 60, 2163-2177.

57. Li, Z.L.; Tang, R.; Wan, Z.; Bi, Y.; Zhou, C.; Tang, B.; Yan, G.; Zhang, X. A review of current methodologies for regional evapotranspiration estimation from remotely sensed data. Sensors 2009, 9, 3801. [CrossRef] [PubMed]

58. Yebra, M.; Dijk, A.V.; Leuning, R.; Huete, A.; Guerschman, J.P. Evaluation of optical remote sensing to estimate actual evapotranspiration and canopy conductance. Remote Sens. Environ. 2013, 129, $250-261$. [CrossRef]

59. Vinukollu, R.K.; Meynadier, R.; Sheffield, J.; Wood, E.F. Multi-model, multi-sensor estimates of global evapotranspiration: Climatology, uncertainties and trends. Hydrol. Process. 2011, 25, 3993-4010. [CrossRef]

60. Wang, Z.M.; Guo, Z.X.; Song, K.S.; Ling, L.; Bai, Z.; Liu, D.W.; Ni, H.; Ren, C.Y. Responses of vegetation ndvi in northeast china to climate change. Chin. J. Ecol. 2009, 28, 1041-1048.

61. Yao, Y.; Liang, S.; Li, X.; Hong, Y.; Fisher, J.B.; Zhang, N.; Chen, J.; Cheng, J.; Zhao, S.; Zhang, X. Bayesian multimodel estimation of global terrestrial latent heat flux from eddy covariance, meteorological, and satellite observations. J. Geophys. Res. Atmos. 2014, 119, 4521-4545. [CrossRef]

62. Liang, S.; Wang, K.; Zhang, X.; Wild, M. Review on estimation of land surface radiation and energy budgets from ground measurement, remote sensing and model simulations. IEEE J. Sel. Top. Appl. Earth Obs. Remote Sens. 2010, 3, 225-240. [CrossRef]

63. Zhou, L.; Wang, S.; Cheng, J.; Feng, X.; Ju, W.; Wu, W. The spatial-temporal characteristics of evapotranspiration of China's terrestrial ecosystems during 1991-2000. Resour. Sci. 2009, 31. [CrossRef]

64. Mcvicar, T.R.; Roderick, M.L.; Donohue, R.J.; Li, L.T.; Niel, T.G.V.; Thomas, A.; Grieser, J.; Jhajharia, D.; Himri, Y.; Mahowald, N.M. Global review and synthesis of trends in observed terrestrial near-surface wind speeds: Implications for evaporation. J. Hydrol. 2012, 416-417, 182-205. [CrossRef]

65. Liu, M.; Tian, H.; Chen, G.; Ren, W.; Zhang, C.; Liu, J. Effects of land-use and land-cover change on evapotranspiration and water yield in China during 1900-2000. JAWRA J. Am. Water Resour. Assoc. 2008, 44, 1193-1207. [CrossRef]

66. Zhou, L.; Zhou, G. Measurement and modelling of evapotranspiration over a reed (phragmites australis) marsh in northeast China. J. Hydrol. 2009, 372, 41-47. [CrossRef]

67. Han, Z.; Guirui, Y.U.; Wang, Q.; Zhu, X.; Honglin, H.E.; Wang, Y.; Zhang, J.; Yingnian, L.I.; Liang, Z.; Zhao, F. Spatial variation in annual actual evapotranspiration of terrestrial ecosystems in china: Results from eddy covariance measurements. J. Geogr. Sci. 2016, 26, 1391-1411. 
68. Penman, H.L. Natural evaporation from open water, hare soil and grass. Proc. R. Soc. Lond. A Math. Phys. 1948, 193, 120. [CrossRef]

69. Zeng, Z.; Wang, T.; Zhou, F.; Ciais, P.; Mao, J.; Shi, X.; Piao, S. A worldwide analysis of spatiotemporal changes in water balance-based evapotranspiration from 1982 to 2009. J. Geophys. Res. Atmos. 2014, 119, 1186-1202. [CrossRef]

70. Koster, R.D.; Suarez, M.J. A simple framework for examining the interannual variability of land surface moisture fluxes. J. Clim. 1999, 12, 1911-1917. [CrossRef]

71. Koster, R.D.; Dirmeyer, P.A.; Guo, Z.; Bonan, G.; Chan, E.; Cox, P.; Gordon, C.T.; Kanae, S.; Kowalczyk, E.; Lawrence, D. Regions of strong coupling between soil moisture and precipitation. Science 2004, 305, 1138. [CrossRef] [PubMed]

72. Federer, C.A.; Vörösmarty, C.; Fekete, B. Sensitivity of annual evaporation to soil and root properties in two models of contrasting complexity. J. Hydrometeorol. 2003, 4, 1276-1290. [CrossRef]

73. Thomas, A. Spatial and temporal characteristics of potential evapotranspiration trends over China. Int. J. Climatol. 2015, 20, 381-396. [CrossRef]

74. Zhao, S.H.; Yang, Y.H.; Zhang, F.; Sui, X.X.; Yao, Y.J.; Zhao, N.; Zhao, Q.; Li, C.Q. Rapid evaluation of reference evapotranspiration in northern China. Arab. J. Geosci. 2015, 8, 647-657. [CrossRef]

75. Iwasaki, H.; Saito, H.; Kuwao, K.; Maximov, T.C.; Hasegawa, S. Forest decline caused by high soil water conditions in a permafrost region. Hydrol. Earth Syst. Sci. Discuss. 2010, 14, 301-307. [CrossRef]

76. Wang, K.; Dickinson, R.E.; Liang, S. Global atmospheric evaporative demand over land from 1973 to 2008. J. Clim. 2012, 25, 8353-8361. [CrossRef]

77. Gao, G.; Chen, D.; Ren, G.; Chen, Y.; Liao, Y. Spatial and temporal variations and controlling factors of potential et in China: 1956-2000. J. Geogr. Sci. 2006, 16, 3-12. [CrossRef]

(C) 2017 by the authors. Licensee MDPI, Basel, Switzerland. This article is an open access article distributed under the terms and conditions of the Creative Commons Attribution (CC BY) license (http:/ / creativecommons.org/licenses/by/4.0/). 


\title{
Article \\ MODIS-Based Estimation of Terrestrial Latent Heat Flux over North America Using Three Machine Learning Algorithms
}

\author{
Xuanyu Wang ${ }^{1,2}$, Yunjun Yao ${ }^{1,2, *}$, Shaohua Zhao ${ }^{3}$, Kun Jia ${ }^{1,2}$, Xiaotong Zhang ${ }^{1,2}$, \\ Yuhu Zhang ${ }^{4}$, Lilin Zhang ${ }^{1,2}$, Jia $\mathrm{Xu}^{1,2}$ and Xiaowei Chen ${ }^{1,2}$ \\ 1 State Key Laboratory of Remote Sensing Science, Faculty of Geographical Science, \\ Beijing Normal University, Beijing 100875, China; skywalkerwxy@163.com (X.W.); jiakun@bnu.edu.cn (K.J.); \\ xtngzhang@bnu.edu.cn (X.Z.); 201521170059@mail.bnu.edu.cn (L.Z.); diamond1201@sina.cn (J.X.); \\ 18368091857@163.com (X.C.) \\ 2 Beijing Engineering Research Center for Global Land Remote Sensing Products, Institute of Remote Sensing \\ Science and Engineering, Faculty of Geographical Science, Beijing Normal University, Beijing 100875, China \\ 3 Satellite Environment Center, Ministry of Environmental Protection, Beijing 100094, China; zshyytt@126.com \\ 4 College of Resource Environment and Tourism, Capital Normal University, Beijing 100048, China; \\ zhang_yuhu@163.com \\ * Correspondence: boyyunjun@163.com; Tel.: +86-10-5880-3002
}

Received: 3 October 2017; Accepted: 14 December 2017; Published: 16 December 2017

\begin{abstract}
Terrestrial latent heat flux (LE) is a key component of the global terrestrial water, energy, and carbon exchanges. Accurate estimation of LE from moderate resolution imaging spectroradiometer (MODIS) data remains a major challenge. In this study, we estimated the daily LE for different plant functional types (PFTs) across North America using three machine learning algorithms: artificial neural network (ANN); support vector machines (SVM); and, multivariate adaptive regression spline (MARS) driven by MODIS and Modern Era Retrospective Analysis for Research and Applications (MERRA) meteorology data. These three predictive algorithms, which were trained and validated using observed LE over the period 2000-2007, all proved to be accurate. However, ANN outperformed the other two algorithms for the majority of the tested configurations for most PFTs and was the only method that arrived at $80 \%$ precision for LE estimation. We also applied three machine learning algorithms for MODIS data and MERRA meteorology to map the average annual terrestrial LE of North America during 2002-2004 using a spatial resolution of $0.05^{\circ}$, which proved to be useful for estimating the long-term LE over North America.
\end{abstract}

Keywords: latent heat; machine learning algorithms; plant functional type

\section{Introduction}

Terrestrial latent heat flux (LE) plays an important role in the global water cycle, carbon, and surface energy exchanges [1-3]. LE can be measured directly by using lysimeter and eddy covariance (EC) flux towers, but the former method is time-consuming, expensive, and needs carefully planned experiments [4]. Although the FLUXNET project has provided EC flux towers to measure LE, it is inherently difficult to accurately estimate LE, especially at large spatial scales due to the heterogeneity in the terrestrial landscape. Moreover, the sparse observations from EC flux towers limit the accurate characterization of spatiotemporal LE patterns over large spatial scales.

Remote sensing, especially from the Moderate Resolution Imaging Spectroradiometer (MODIS) satellite, can provide temporally and spatially continuous information for estimating LE, such as: land surface temperature (LST); the fraction of absorbed photosynthetically active radiation (FPAR); the normalized difference vegetation index (NDVI); the enhanced vegetation index (EVI); leaf area 
index (LAI); and, albedo [5-7]. Currently, there are numerous satellite-based algorithms available to estimate LE, e.g., surface energy balance (SEB) models [8,9] use remotely sensed products and meteorological data driving a residual SEB equation to estimate LE. Penman-Monteith (PM) and Priestley-Taylor (PT) approaches used PM and PT equations to calculate LE, respectively [10-13]. Statistical and empirical methods calculated LE using empirical equations, which include vegetation parameters and LE observations [6,14-17]. Data assimilate methods improved LE estimation by assimilating satellite land-surface variables into land surface models [18,19]. However, these algorithms show substantial differences in LE estimation [20]. Additionally, the existing global LE products, such as MOD16 and the EUMETSAT Satellite Application Facility on Land Surface Analysis (LSA-SAF) product (LSA-SAF MSG), have a $5 \mathrm{~km}$ spatial resolution and daily temporal resolution [7], but validation results have revealed that these products often overestimate LE at most AsiaFlux sites [21].

Satellite-based empirical and semi-empirical LE algorithms have been scaled up from site to regional scales by relating observed LE to satellite-based vegetation parameters and other key meteorological variables [14]. As the most viable empirical algorithms, machine learning algorithms have the potential to generate robust relationships, and once trained, they are very fast to apply [22]. These algorithms can provide accurate LE estimation as long as enough training datasets are representative of all the behaviors in the systems. However, there is still some controversy about which machine learning algorithm is best in LE estimates. Different input variables with different plant functional types (PFTs) over different study regions may lead to different result. For example, Bruton et al. [23] employed precipitation (P), air temperature (Ta), solar radiation (Rs), wind speed (Ws), and relative humidity $(\mathrm{RH})$ as input data for the artificial neural network (ANN) algorithms to estimate LE. The validation results showed that ANN algorithm was able to perform reliable LE estimation with an $R^{2}$ of 0.717 and a root mean square error of $1.11 \mathrm{~mm}$. Compared with multiple linear regression and the Priestley-Taylor method, the ANN method estimated the most accurate LE with all of the available variables. El-Shafie et al. [24] used ANN models for prediction evapotranspiration in Iran. The results demonstrated that an ANN model predicted daily E with a significant level of accuracy using only the maximum and minimum temperatures successfully. Shrestha and Shukla [25] used SVM for predicting generic crop coefficient $(\mathrm{Kc})$ and crop evapotranspiration $(\mathrm{ETc})$, using a uniquely large dataset from lysimeters for multiple crop-season combinations under plastic mulch conditions. The results showed that the SVM model was superior to the Artificial Neural Network and Relevance Vector Machine models, two data-driven models used in hydrology. Deo et al. [26] used three machine learning algorithms, including Relevance Vector Machine (RVM), Extreme Learning Machine (ELM), and MARS for the prediction of monthly evaporative loss using limited meteorological data from the Amberley weather station in Australia. All three machine learning models performed well, with RVM proving to be the best. They also found that incident solar radiation and air temperatures are the two most influential factors in determining the performance of machine learning algorithms.

The observation data from EC flux towers are more adequate and accurate over North America, so there already exist some studies of machine learning algorithms for LE estimation over North America. For example, Yang et al. [27] used SVM combined with ground-measured ET from 25 AmeriFlux sites and remotely sensed inputs (land surface temperature, enhanced vegetation index, and land cover) to predict ET over the United States (U.S.) with an average test error of $0.62 \mathrm{~mm} /$ day and an $R^{2}$ of 0.75 . When compared to neural network and multiple regressions, SVM performed better. Adnan et al. [28] developed a model to estimate evapotranspiration with meteorological parameters (temperature, relative humidity, wind speed, and precipitation) using different machine learning techniques. They found that ANN performed better than LLSVM, MARS, and M5Tree models and gave the nearest values as compared with the actual value. Therefore, substantial differences still exist in simulating LE for different machine learning algorithms. Moreover, there is a lack of validating and evaluating different machine learning algorithms for LE estimation at different PFTs over North America. 
In this study, we evaluated and applied three machine learning algorithms to estimate terrestrial LE over North America. These machine learning algorithms included artificial neural network (ANN), support vector machines (SVM), and multivariate adaptive regression spline (MARS), which were using air temperature $(\mathrm{T})$, relative humidity (RH), wind speed (Ws), and MODIS-based NDVI as input data. The objectives of this study were as follows: (1) to assess the performance of the three machine learning algorithms for different PFTs based on a series of cross-validations using long-term Ameriflux EC observations from 2000 to 2007, and (2) to generate a daily LE product during 2002-2004 over North America using three machine learning algorithms with well-quantified accuracy based on MODIS data and Modern Era Retrospective Analysis for Research and Applications (MERRA) meteorological data.

\section{Materials and Methods}

\subsection{Machine Learning Algorithms}

\subsubsection{Artificial Neural Network}

An Artificial neural network (ANN) consists of a set of processing elements, including input, hidden, and output layers. Each layer includes an array of processing elements. There is a connection between each of the neurons in any given layer with each of the neurons in the next layer [4]. This connection between neurons can be described as following function:

$$
y_{i}=f_{i}\left(\sum_{j=1}^{n} w_{i j} x_{j}-\theta_{i}\right)
$$

where $y_{i}$ is the output of the neuron $i, f_{i}$ is nonlinear, such as Gaussian function, etc. $w_{i j}$ is the connection weight between neuron $i$ and $j$. $x_{j}$ is the $j$ th input to the neuron and $\theta_{i}$ is the bias of the neuron.

The processing steps require aggregating the weighted inputs and the activation function, such as the Sigmoid function and Tanh function. The activation function limits the output values between two asymptotic values, typically 0 and 1 or -1 and +1 . In feed-forward artificial neural networks (FFNN), each neuron at input and inner layers receives input data then processes and passes to the next layer. The output result is carried out layer by layer in the forward direction. The weights are adjusted through reverse pass, which calculated error signals propagate backward through the network. The weights of the output neuron layer are adjusted first because the target value of output neuron layer can guide the adjustment of the associated weights. The numbers of input and output parameters determine the numbers of neurons in the input layer and the output layer, respectively [29]. The model of FFNN is shown in Figure 1.

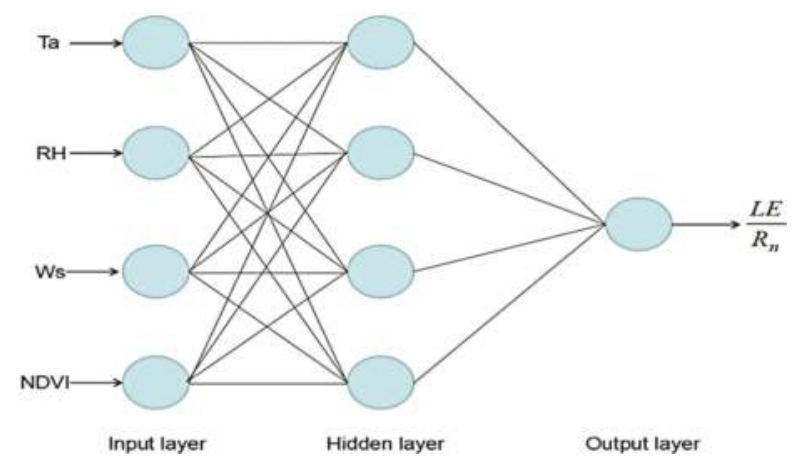

Figure 1. Architecture of the neural network model used in this study. 


\subsubsection{Support Vector Machine}

Support vector machine (SVM) were developed by Cortes and Vapnik (1995) to separate the sample data through some nonlinear mapping from the input vectors into the high dimensional feature space, and can find an optimal separating hyper plane between data points of different classes in a high dimension space $[27,30,31]$. When using SVM classification, we seek the optimal classification plane to maximize the distance between the two classes, with the points falling within the boundary, called the support vector [30]. Mapping technology is implemented through the kernel function. We used the radial basis function (RBF) kernel function type in this study because former studies shows that the RBF kernel performs better than any other kernel functions [32], it can be expressed as:

$$
K\left(x_{i}, x_{j}\right)=\exp \left(-\frac{1}{2 \sigma^{2}}\left\|x_{i}-x_{j}\right\|^{2}\right)
$$

Here, $\sigma$ is variance. The complexity of SVM does not depend on the dimensions of the feature space, but rather on the number of support vectors (Figure 2).

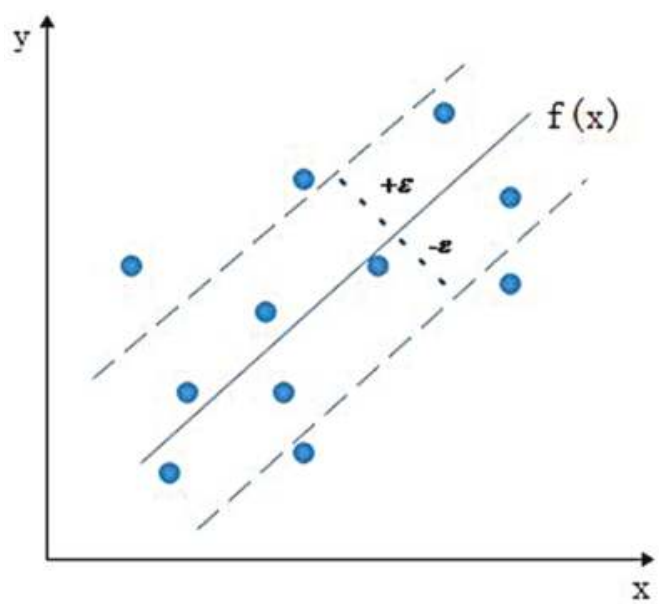

Figure 2. One-dimensional linear regression with $\varepsilon$-insensitive band for the support vector machine (SVM) algorithm.

The regression estimation with support vector is to estimate a function according to a given data set $\left\{\left(x_{i}, y_{i}\right)\right\}_{n}$. Herein, the input vectors $\left(x_{i}\right)$ refer to T, RH, Ws, and NDVI, whereas the target values $\left(y_{i}\right)$ refer to the ratio of LE values as determined by the ground-measured data and surface net radiation $\left(R_{n}\right)$, and $n$ is the total number of data sets.

The linear regression function uses the following function:

$$
f(x)^{\prime}=w \cdot f(x)+b
$$

Here, $f(x)$ is a nonlinear function, by which $x$ is mapped into a feature space, $b$ is a weight vector, and $w$ denotes a coefficient that has to be estimated from the data. A nonlinear regression function is given by minimizing the sum of the empirical risk and a complexity term, as in the following expression:

$$
f(x)=\sum_{i=1}^{n}\left(a_{i}^{*}-a_{i}\right) K\left(x_{i}, x_{j}\right)+b
$$

With $a_{i} a_{i}^{*} \geq 0$, ( $a_{i}$ and $a_{i}^{*}$ are Lagrange multipliers). $i=1, \ldots, N, K\left(x_{i}, x_{j}\right)$ is the kernel function and $b$ can be calculated as following function: 


$$
b=\frac{1}{N_{S V}}\left\{\sum_{0<a_{i}<C}\left[y_{i}-\sum_{x_{j} \in S V}\left(a_{j}-a_{j}^{*}\right) K\left(x_{j}, x_{i}\right)-\varepsilon\right]+\sum_{0<a_{i}^{*}<C}\left[y_{i}-\sum_{x_{j} \in S V}\left(a_{j}-a_{j}^{*}\right) K\left(x_{j}, x_{i}\right)+\varepsilon\right]\right\}
$$

where $N_{S V}$ is the number of support vectors.

\subsubsection{Multivariate Adaptive Regression Spline}

Multivariate adaptive regression spline (MARS) is a flexible nonparametric modeling tool that was popularized by Friedman (1991) for solving regression-type problems [33]. It does not require an assumption about the particular type of relationship between outcome variable and predictor variables $[34,35]$. Instead, MARS use coefficients and basic functions that are decided entirely by regression data to construct this relation. The MARS model can be described as follows:

$$
f(x)=\beta_{0}+\sum_{i=1}^{k} c_{i} B_{i}(x)
$$

Here, the summation $f(x)$ is over the k non-constant terms in the model. $\beta_{0}$ is the intercept parameter. Each $c_{i}$ is a constant coefficient, $B_{i}(x)$ is a basic function, which takes one of following forms: a constant 1, a hinge function or two or more hinge functions. A hinge function (Figure 3 ) has the form $\max (0, x-c)$ or $\max (0, c-x), c$ is a constant, called the knot.

The first step to build a MARS model is partitioning the training data set into several splines in equivalent interval basis [33]. Most importantly, the model ensures that there is adequate data in a subgroup in order to avoid over-fitting using the shortest distance between neighboring knots $[36,37]$. The forward pass and the backward pass are two important phases in building a MARS model. To find the best subset, we use generalized cross validation (GCV) to compare the performance of sub-models. Better sub-model has lower GCV values. GCV can be calculated as follows:

$$
G C V(N)=\left(\frac{1}{n} \frac{\sum_{m=1}^{N}\left(y_{i}-\hat{y}_{i}\right)^{2}}{\left(1-\frac{C(M)}{n}\right)^{2}}\right)
$$

Here, the $n$ was the number of observations, $y_{i}$ was the response value of object $i, \hat{y}_{i}$ was the predicted response value of object $i$, and $C(M)$ was the penalty factor.

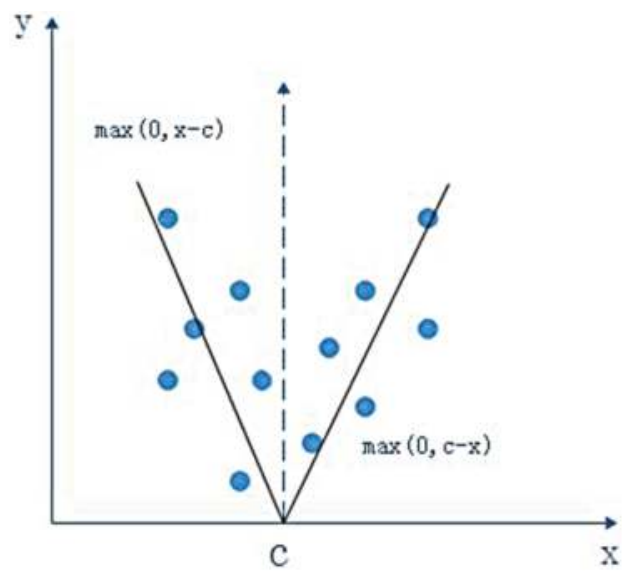

Figure 3. The hinge functions and knot location in the multivariate adaptive regression spline (MARS) model. 


\subsection{Experimental and Simulated Data}

\subsubsection{Eddy Covariance Observations}

The machine learning algorithms were validated and evaluated using ground-measured flux data. All of the turbulent flux observations were measured by the EC method and the data cover the period from 2000 to 2007. The data were collected from $85 \mathrm{EC}$ flux tower sites and were provided by AmeriFlux. These flux towers are located mainly in North America $\left(50^{\circ}-170^{\circ} \mathrm{W}, 10^{\circ}-70^{\circ} \mathrm{N}\right.$, Figure 4$)$. The flux tower sites cover five major global land-surface biomes: cropland (CRO; 7 sites), deciduous broadleaf forest (DBF; 11 sites), evergreen needleleaf forest (ENF; 41 sites), grass and other types (GRA; 17 sites), and shrubland (SHR; 9 sites). These data sets include half-hourly or hourly ground-measured incident Rs, RH, Ta, diurnal air temperature range (DT), Ws, vapor pressure (e), sensible heat flux (H), surface net radiation $\left(R_{n}\right)$, ground heat flux $(G)$, and LE. We acquired daily data from aggregated half-hourly or hourly data without using additional quality control [38-40] and removed the zero values. For the unclosed energy problem, we used the following method that was developed by Twine et al. [41] to correct the LE for all flux towers:

$$
L E=\left(R_{n}-G\right) /\left(L E_{o r i}+H_{o r i}\right) \times L E_{o r i}
$$

where $\mathrm{LE}$ is the corrected latent heat, $H_{\text {ori }}$ and $L E_{\text {ori }}$ are the uncorrected sensible heat flux and latent heat, respectively.

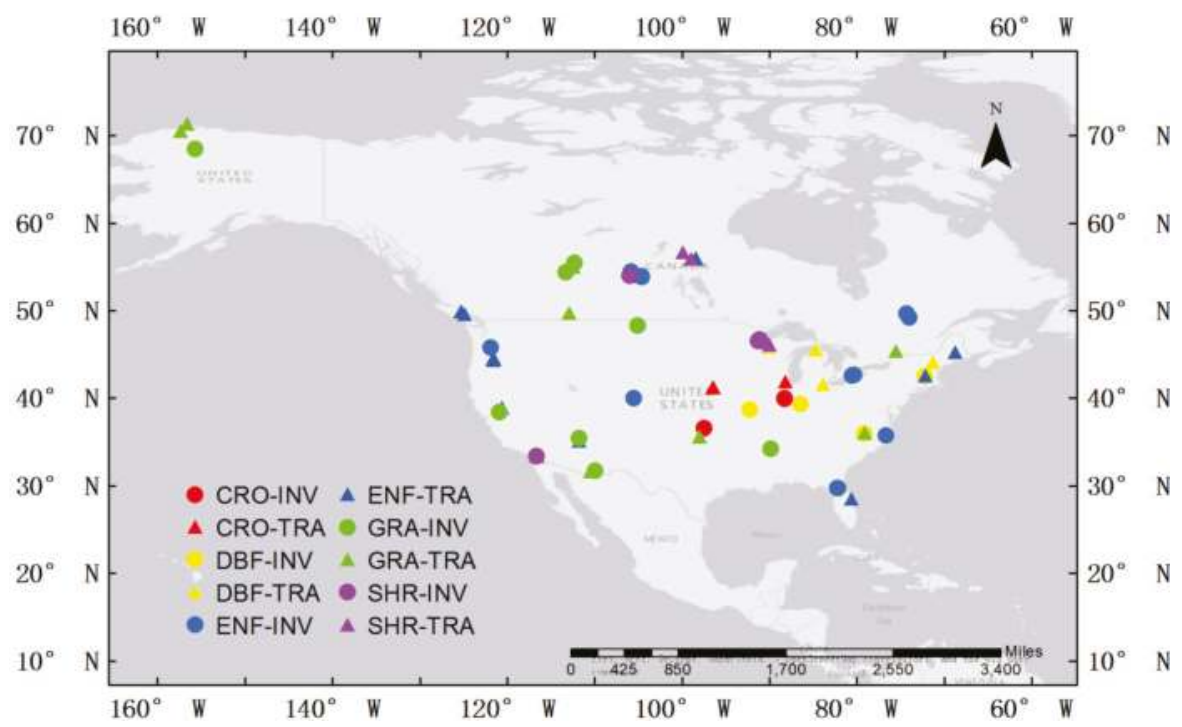

Figure 4. Location of the 85 eddy covariance flux towers used in this study. INV means the data of this site were used to inverse, TRA means that the data of this site were used for training.

\subsubsection{MODIS and MERRA Data}

We used the NDVI values as the input variables and the daily NDVI values were temporally interpolated from the 16 day MODIS NDVI (MOD13A2) product [42] at 1-km spatial resolution using linear interpolation [43]. When 16 day NDVI data are missing or not available, we used the closest reliable 16 day values to replace the missing or original data.

We used the daily $R_{n}, R H, T a$, and Ws products with a spatial resolution of $1 / 2^{\circ} \times 2 / 3^{\circ}$ from MERRA data provided by National Aeronautics and Space Administration (NASA) to evaluate the 
performance of all the LE algorithms for all the flux tower sites in this study. To match MODIS pixels, we used the method proposed by Zhao et al. [43] which is a spatial interpolation method using a cosine function to interpolate coarse-resolution MERRA data to $1 \mathrm{~km}^{2}$ pixels. Theoretically, this method uses the four MERRA cells surrounding a given pixel to remove sharp changes from one side of a MERRA boundary to the other to improve the accuracy of MERRA data for each $1 \mathrm{~km}$ pixel.

To map the final LE product with $0.05^{\circ}$ spatial resolution using three machine learning algorithms, we used Collection 5 MODIS NDVI (MOD13C1: CMG, $0.05^{\circ}$ ) [44] and the daily $0.05^{\circ}$ MERRA data interpolated using the method proposed by Zhao et al. [43] from the original MERRA data. To evaluate the performance of three machine learning algorithms, we used MODIS LE product (MOD16A2) [7] with $0.05^{\circ}$ spatial resolution.

\subsubsection{Criteria of Evaluation}

We used three different statistical criteria to evaluate the performance of the machine learning algorithms that were used in this study: coefficient of determination $\left(R^{2}\right)$; the average deviation of ground-measured LE value and estimated LE value (Bias); and, root mean square error (RMSE) $[45,46]$.

$R^{2}$ can be defined as the square of correlation coefficient $R$. The difference between it and the correlation coefficient is to remove $|R|=0$ and 1 , as it can prevent an exaggerated interpretation of the correlation coefficient. The closer that $R^{2}$ is to 1 , the more relevant the observations and estimates. The following equation was used to calculated coefficient of determination:

$$
R^{2}=\left(\frac{\sum_{i=1}^{n}\left(X_{i}-\bar{X}\right)\left(Y_{i}-\bar{Y}\right)}{\sqrt{\sum_{i=1}^{n}\left(X_{i}-\bar{X}\right)^{2}\left(Y_{i}-\bar{Y}\right)^{2}}}\right)^{2}
$$

where $X_{i}$ and $Y_{i}$ are the observed and estimated values, respectively, $\bar{X}$ and $\bar{Y}$ are the average of $X_{i}$ and $Y_{i}$, and $n$ is the total number of the data.

Bias is the average value of the absolute of differences between the predicted and observed LE values. A low bias indicates a good model performance. The bias can be calculated from the following equation:

$$
\text { Bias }=\frac{\sum_{i=1}^{n}\left(X_{i}-Y_{i}\right)}{n}
$$

where $X_{i}$ and $Y_{i}$ are the observed and estimated values, respectively, and $n$ is the total number of the data.

RMSE can be defined as the square root of the average value of the differences between the predicted and observed LE values. A perfect match between the two values would yield RMSE $=0$, and it implies the best performance model. The larger RMSE is, the lower model performance. RMSE can be calculated from the following function:

$$
R M S E=\sqrt{\frac{1}{n} \sum_{i=1}^{n}\left(X_{i}-Y_{i}\right)^{2}}
$$

where $X_{i}$ and $Y_{i}$ are the observed and estimated values, respectively, and $\mathrm{n}$ is the total number of the data.

\subsubsection{Experimental Setup}

For each PFT, we chose half of the sites to train, the remaining sites were used to reverse. As for PFT $\mathrm{CRO}$, three sites were for training and four for reversing. For DBF, five sites were for training and six for reversing. ENF/GRA/SHR has 21/9/5 sites for training and 20/8/4 for reversing, respectively. We put the same PFT data together for the training and validation of three machine learning algorithms. For the training step, we chose Ta, RH, Ws, and NDVI as input variables and the ratio of ground-measured LE and $R_{n}$ as output variables, and built the ANN/SVM/MARS model. Then, we put the input variables 
of testing data to the model and got the predicted $L E / R_{n}$. To get the predicted $L E$, we multiplied the results by the $R_{n}$ of the testing data. Finally, we evaluated the predicted LE with the ground-measured $\mathrm{LE}$ of the testing data. Meteorology data including $\mathrm{Ta}, \mathrm{RH}, \mathrm{Ws}$, and $\mathrm{R}_{\mathrm{n}}$ were acquired from EC flux tower sites and MERRA data, respectively.

For the ANN algorithm, we trained, validated, and tested the data by using feed-forward artificial neural networks (FFNN), which is the most commonly used ANN model. Levenberg-Marquardt algorithms were applied to train the network and a Sigmoid function was used since it gave the best results of the model. The number of hidden layers and neurons in hidden layers was determined by trial and error, and we finally decided that the number of hidden layers was 10. For the SVM algorithm, we implement the SVM on R platform using R language with package e1071, which provided an interface connects libsvm that was based on the $\mathrm{C}++$ language, developed by Chih Chung-Chang and Chih-Jen Lin $[47,48]$. The process of modeling training and inversion was similar to the ANN process. The present study built the MARS model and estimated testing data LE using ARESlab (Adaptive Regression Splines toolbox) in MATLAB, and set the maxfuncs and maxinteractions to 15 and 4 , respectively.

\section{Results}

\subsection{Algorithms Evaluation Based on Specific Site Data}

At the flux tower site scale, the three machine learning algorithms exhibited substantial differences in LE modeling for different PFTs. The statistical parameters of training and validation of each model are provided in Table 1.

Table 1. Training and validation statistics for the different algorithms based on specific site. All of the coefficient of determination $\left(R^{2}\right)$ values are significant with a $99 \%$ confidence.

\begin{tabular}{cccccccc}
\hline & \multicolumn{2}{c}{$R^{2}$} & \multicolumn{2}{c}{ Bias $\left(\mathbf{W} / \mathbf{m}^{2}\right)$} & \multicolumn{2}{c}{ RMSE $\left(\mathbf{W} / \mathbf{m}^{2}\right)$} \\
\hline \multirow{2}{*}{ PFT } & Algorithms & Train & Test & Train & Test & Train & Test \\
\hline \multirow{3}{*}{ CRO } & ANN & 0.83 & 0.81 & 0.4 & 0.6 & 16.47 & 21.86 \\
& SVM & 0.83 & 0.8 & -0.54 & 1.1 & 17.92 & 24.37 \\
& MARS & 0.81 & 0.78 & 1.15 & 2.7 & 19.23 & 25.38 \\
\hline \multirow{3}{*}{ DBF } & ANN & 0.89 & 0.88 & -0.08 & 0.7 & 14.48 & 17.17 \\
& SVM & 0.89 & 0.84 & 1.19 & 1.6 & 17.25 & 17.91 \\
& MARS & 0.88 & 0.85 & -0.8 & -1.6 & 17.15 & 18.19 \\
\hline \multirow{5}{*}{ ENF } & ANN & 0.82 & 0.77 & 0.55 & 1.05 & 12.15 & 17.74 \\
& SVM & 0.83 & 0.72 & -1.35 & -3.47 & 13.69 & 20.02 \\
GRA & MARS & 0.81 & 0.72 & -1.88 & -2.36 & 14.63 & 20.05 \\
\hline \multirow{3}{*}{ SHR } & ANN & 0.83 & 0.8 & 1.1 & 3.55 & 15.05 & 16.14 \\
& SVM & 0.84 & 0.62 & 5.3 & 8.37 & 15.81 & 24.76 \\
& MARS & 0.83 & 0.6 & 4.12 & 6.35 & 16.45 & 25.39 \\
\hline
\end{tabular}

Figure 5 shows that the ANN algorithm performed best among the three algorithms for each PFT, while SVM performed better than MARS for CRO, GRA, and SHR, and made a poor performance for DBF land cover type. For CRO, the validation result showed that the $R^{2}$ was very close among the three algorithms, the highest $R^{2}$ of ANN was 0.81 , while the lowest $R^{2}$ was 0.03 lower than that and each of the three algorithms had a large RMSE greater than $20 \mathrm{~W} / \mathrm{m}^{2}$. Meanwhile, ANN had a lower bias than SVM and MARS. ANN for DBF had the best performance with the highest $R^{2}(0.89 / 0.88)(p<0.01)$ and lower RMSE $\left(14.48 / 17.17 \mathrm{~W} / \mathrm{m}^{2}\right)$ in comparison to other PFTs for training and validation, respectively. 
This may be caused by the characteristic of the DBF. Although the $R^{2}$ of SHR was the lowest among the five PFTs for validation results, the bias and the RMSE of SHR were lower than any other PFTs, with the lowest bias and RMSE reaches of -0.17 and $13.35 \mathrm{~W} / \mathrm{m}^{2}$. For ENF and GRA sites, the lowest RMSE of the estimated LE versus ground observations was approximately 17.74 and $16.14 \mathrm{~W} / \mathrm{m}^{2}$, respectively, and the $R^{2}$ was approximately less than 0.8. Especially, for GRA sites, the ANN algorithm that was driven by tower-specific meteorological variables and satellite-based NDVI had an absolutely higher $R^{2}$ and a lower bias and RMSE than the other machine learning algorithms tested.
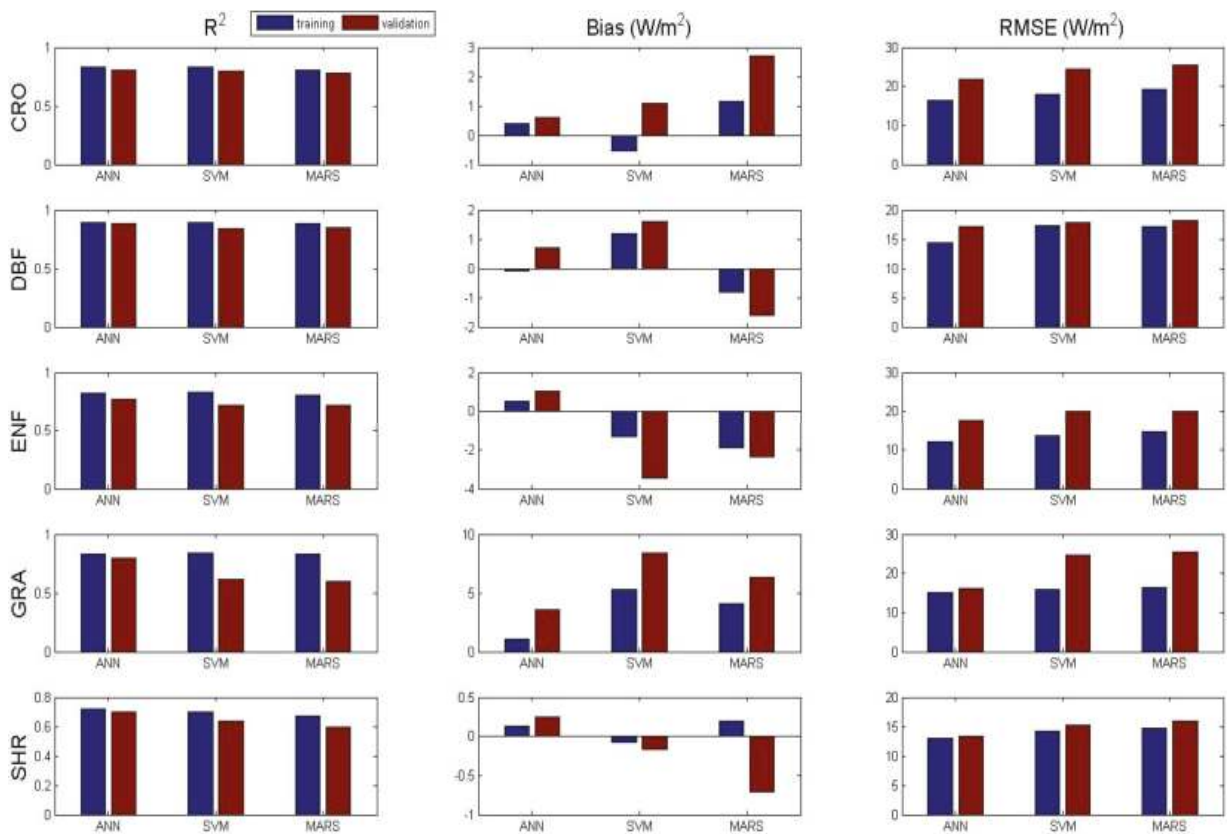

Figure 5. Bar graphs of the training and validation statistics $\left(R^{2}\right.$, Bias and root mean square error (RMSE)) of three algorithms driven by tower-specific meteorology for five PFTs at the 85 flux tower site. All $R^{2}$ values are significant with a $99 \%$ confidence.

Figure 6 shows that the three algorithms exhibited most features of measured LE seasonality in the observed inversion data for different PFTs. We randomly selected an inversion site that has at least three years of complete ground-measured data for each PFT, and then chose three years of complete ground-measured data and the corresponding estimated LE data using three machine learning algorithms. We plotted the eight-day average LE to replace daily LE data. Five sites were US-Ne3 (CRO), US-WCr (DBF), CA-Qcu (ENF), US-FPe (GRA), and US-SO4 (SHR). We compared the estimated LE with the observed LE in 2002-2004 except at US-SO4, for which we used the period 2004-2006. In comparison to the other two algorithms, the ANN algorithm produced seasonal LE variations that were closest to the observed LE. Estimated LE showed great consistency, with observed LE in lower values for all of the PFTs in Figure 6, but for GRA land cover type, the ANN and the SVM kept the same trend, while the results of the MARS were absolutely lower than result of the ANN, the SVM and observed LE in high value area. We also found some abnormal observed LE value in 2004 for SHR, which may lead to the inaccuracy of estimation results. 

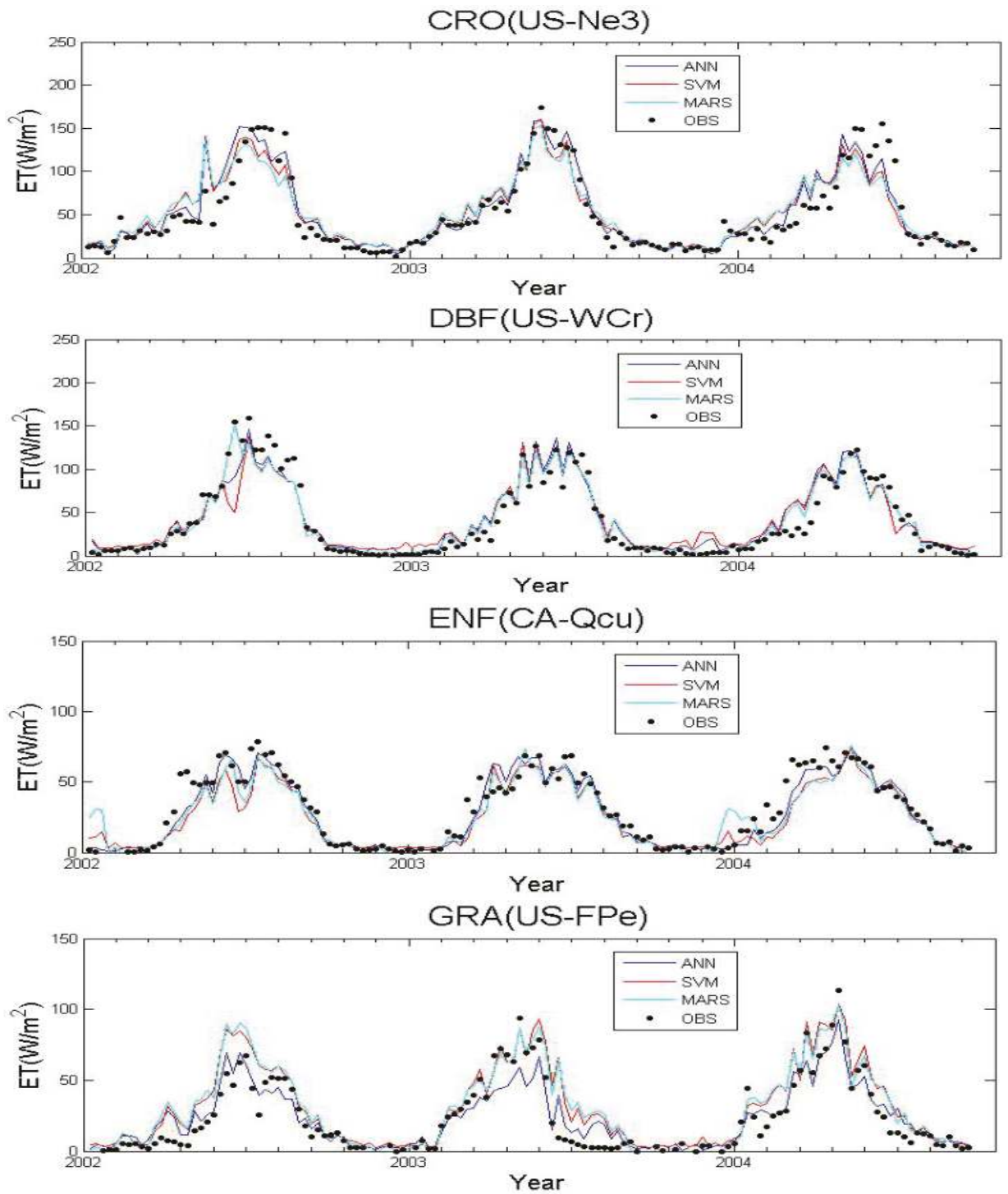

SHR(US-SO4)

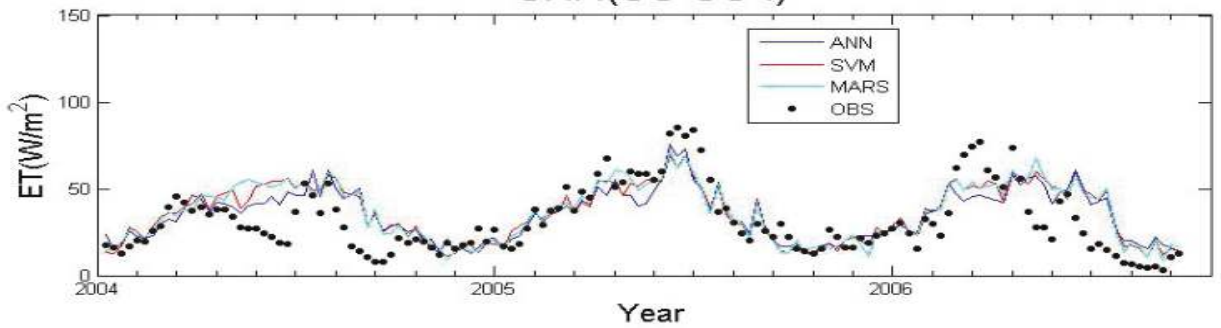

Figure 6. Examples of the eight-day terrestrial latent heat flux (LE) average as measured and estimated using different machine learning algorithms for the different PFTs. 


\subsection{Algorithms Evaluation Based on MERRA Data}

Table 2 shows the statistical parameters of training and validation of each model that is driven by MERRA meteorology. When compared to the results that are driven by tower-specific meteorology, results driven by MERRA meteorology showed poor performance in all PFTs. This may be related to the uncertainty of MERRA data.

Figure 7 shows the training and validation statistics $R^{2}$, bias, and RMSE of the three algorithms that are driven by MERRA meteorology for five PFTs. The value of $R^{2}$ was lower and RMSE was higher when compared to the results that are driven by tower-specific meteorology. With similar results being shown in Figure 5, the ANN demonstrated the best performance among the three machine learning algorithms at both the training and validation stages. The biggest difference, however, was that the ANN performed absolutely higher $R^{2}$ than any of the other algorithms in Figure 7, while the $R^{2}$ is very close among the three algorithms in Figure 5 for $\mathrm{CRO}$ and DBF. Since the only difference in input was meteorology data, the most likely reason that the ANN performed better than than SVM and MARS when using inaccurate input variables (besides MERRA meteorology data being more inaccurate than the tower-specific meteorology data) has to do with the ANN's own characteristics. As for bias, ANN performed much better than the others, while SVM showed the poorest performance for most PFTs. As for RMSE, results of SVM may be a little better than results of MARS for some PFTs, but on the whole, their performances were similar.
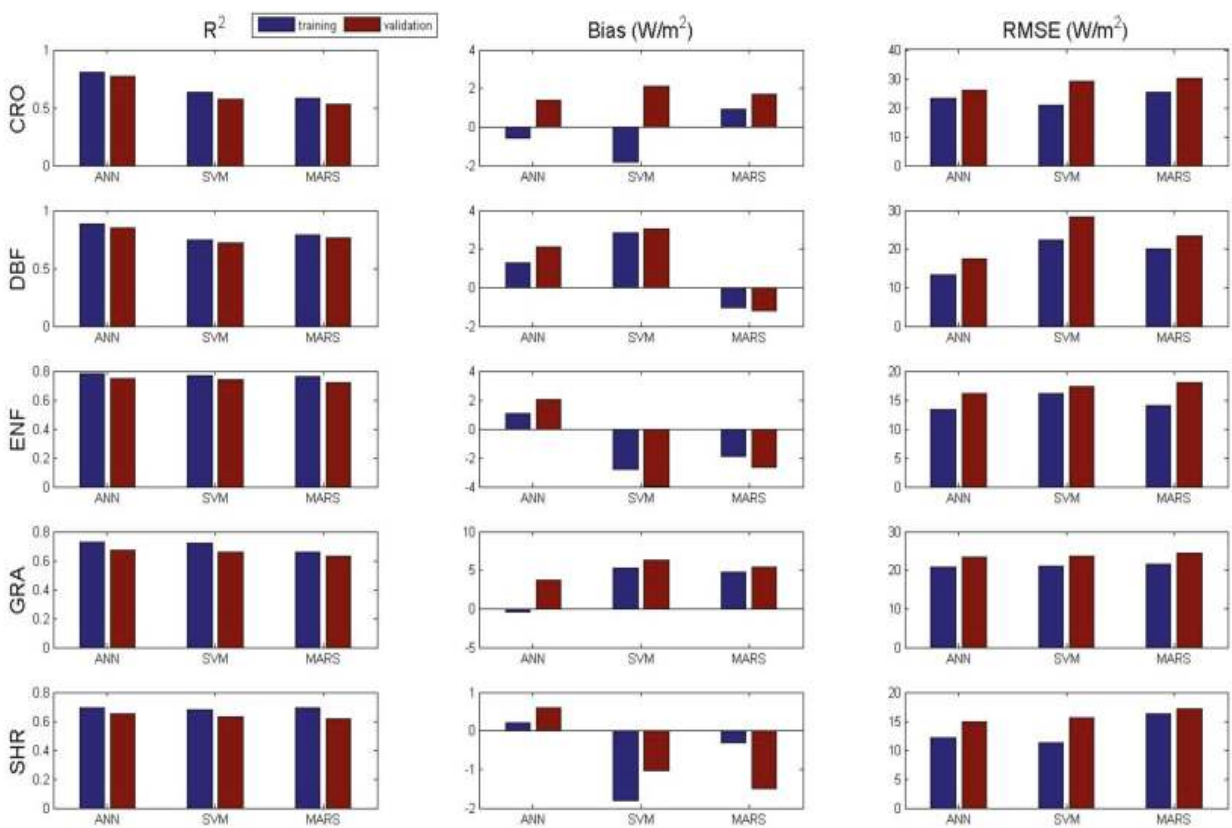

Figure 7. Bar graphs of the training and validation statistics $\left(R^{2}\right.$, Bias and RMSE) of three algorithms driven by MERRA meteorology for five PFTs at the 85 flux tower sites. All of the $R^{2}$ values are significant with a $99 \%$ confidence. 
Table 2. Training and validation statistics for the different algorithms based on MERRA data. All $R^{2}$ values are significant with a $99 \%$ confidence.

\begin{tabular}{|c|c|c|c|c|c|c|c|}
\hline \multirow[b]{2}{*}{ PFT } & \multirow[b]{2}{*}{ Algorithms } & \multicolumn{2}{|c|}{$R^{2}$} & \multicolumn{2}{|c|}{ Bias $\left(W / m^{2}\right)$} & \multicolumn{2}{|c|}{$\operatorname{RMSE}\left(\mathrm{W} / \mathrm{m}^{2}\right)$} \\
\hline & & Train & Test & Train & Test & Train & Test \\
\hline \multirow{4}{*}{ CRO } & ANN & 0.81 & 0.77 & -0.6 & 1.4 & 23.2 & 25.9 \\
\hline & $S V M$ & 0.63 & 0.57 & -1.8 & 2.1 & 20.83 & 29.22 \\
\hline & MARS & 0.58 & 0.53 & 0.9 & 1.7 & 25.35 & 30.33 \\
\hline & $A N N$ & 0.88 & 0.85 & 1.3 & 2.1 & 13.25 & 17.45 \\
\hline \multirow[t]{3}{*}{ DBF } & SVM & 0.75 & 0.72 & 2.82 & 3.06 & 22.42 & 28.39 \\
\hline & MARS & 0.79 & 0.76 & -1.03 & -1.19 & 19.92 & 23.44 \\
\hline & $A N N$ & 0.78 & 0.75 & 1.06 & 2.05 & 13.34 & 16.18 \\
\hline \multirow[t]{3}{*}{ ENF } & SVM & 0.77 & 0.74 & -2.81 & -3.95 & 16.13 & 17.31 \\
\hline & MARS & 0.76 & 0.72 & -1.9 & -2.65 & 14.12 & 18 \\
\hline & $A N N$ & 0.73 & 0.67 & -0.35 & 3.75 & 20.85 & 23.3 \\
\hline \multirow[t]{3}{*}{ GRA } & $S V M$ & 0.72 & 0.66 & 5.27 & 6.37 & 21.01 & 23.68 \\
\hline & MARS & 0.66 & 0.63 & 4.8 & 5.35 & 21.68 & 24.43 \\
\hline & $A N N$ & 0.69 & 0.65 & 0.22 & 0.61 & 12.08 & 14.85 \\
\hline \multirow[t]{2}{*}{ SHR } & $S V M$ & 0.68 & 0.63 & -1.81 & -1.04 & 11.22 & 15.63 \\
\hline & MARS & 0.69 & 0.62 & -0.31 & -1.5 & 16.21 & 17.1 \\
\hline
\end{tabular}

\subsection{Mapping of Terrestrial LE Using Three Machine Learning Algorithms}

We applied the ANN algorithm, the SVM algorithm, and the MARS algorithm with MERRA meteorological data and MODIS product to estimate annual LE at a $0.05^{\circ}$ spatial resolution from 2002-2004 over North America. Figure 8 shows maps of annual terrestrial LE averaged for 2002-2004 over North America.
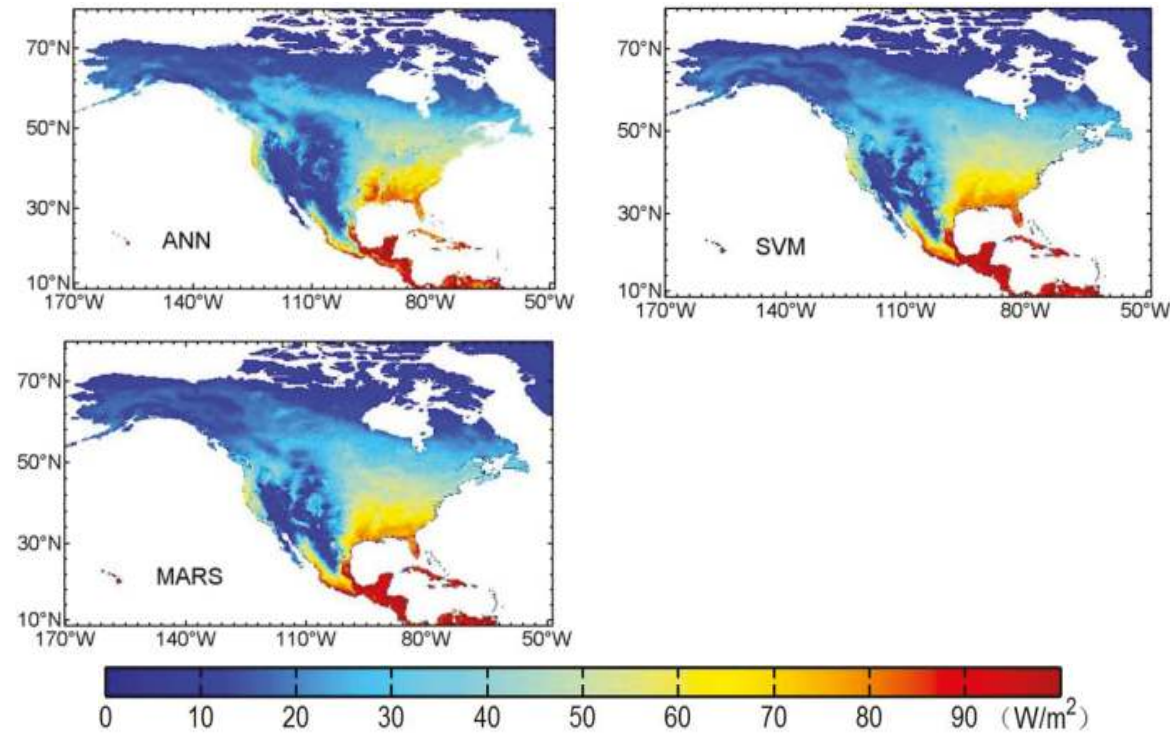

Figure 8. The map of mean annual terrestrial LE from 2002 to 2004 at a spatial resolution of $0.05^{\circ}$ using three machine learning algorithms driven by MERRA meteorology over North America.

All of the algorithms yielded high annual LE over the east and west coasts of the United States. In the low latitudes, the highest annual LE was approximately larger than $90 \mathrm{~W} / \mathrm{m}^{2}$, appearing on the area with latitudes lower than $20^{\circ} \mathrm{N}$. The biggest difference between the three algorithms was the 
ANN estimated higher LE values on the east coast of the United States and lower LE values in areas of the western United States when compared to the SVM and the MARS, which returned similar results.

\section{Discussion}

\subsection{Performance of the Machine Learning Algorithms}

Our study, based on the estimated daily LE at 85 EC flux tower sites using different machine learning algorithms that were driven by tower-specific and MERRA meteorology, illustrated that the ANN algorithm yielded the best LE estimate. The ANN had the highest $R^{2}(0.81$ and 0.70$)(p<0.01)$ and lowest RMSE (17.33 and $\left.20.22 \mathrm{~W} / \mathrm{m}^{2}\right)$ in comparison to the SVM and MARS algorithms for tower-specific and MERRA meteorological data, respectively (Figures 9 and 10). The results were consistent with the findings of Lu and Zhuang, 2010, which used remote sensing data from the MODIS, meteorological and eddy flux data, and an ANN technique to develop a daily product for the period of 2004-2005 for the conterminous U.S. [49]. They found that the ANN predicted daily LE well $\left(R^{2}=0.52-0.86\right)$.
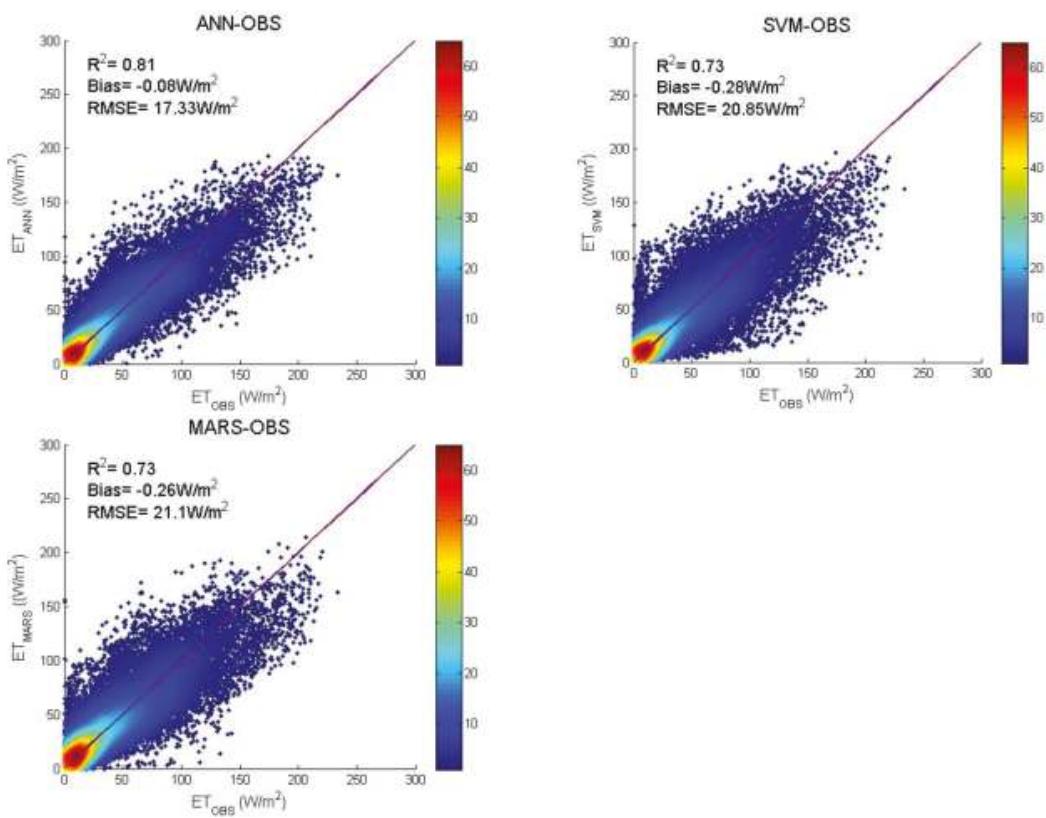

Figure 9. Comparison of daily LE observations for all 85 flux tower sites and LE estimates using different machine learning algorithms driven by tower-specific meteorology.

Validation for $85 \mathrm{EC}$ flux tower sites also indicated that the three machine learning algorithms used were reliable and robust for major land cover types in North America. Figures 9 and 10 both show that the ANN algorithm had no significant LE bias and yielded the closest LE to tower flux data relative to the SVM and MARS algorithms. We also found large inter-biome differences, with better performance at DBF and CRO sites. Several studies have revealed that some algorithms that exhibit strong seasonality for vegetation indices, such as NDVI for accurate capture of information on seasonal changes in vegetation can yield considerably better LE estimates for seasonal vegetation types such as DBF [10,50-54]. Thus, NDVI as an input variable determined the accuracy of LE quantification. All of the machine learning algorithms yielded poor LE estimation for GRA and SHR sites, which may be attributable in part to the fact that seasonal GRA and SHR variation was less evident when 
satellite-derived vegetation indices (e.g., NDVI) saturate asymptotically. Performance differences among machine learning algorithms may be caused by the different scope for each algorithm, as well as the quality and quantity of input variables. The SVM and MARS highlight global rather than local optima, while the ANN may ensure local optimization and leads to a better performance when compared to SVM and MARS [24,27,30,55]. When using MERRA meteorology data as input variables, SVM and MARS showed even worse results, while ANN was slightly worse as compared with results using tower-specific meteorology data as input variables. On the other hand, some studies have indicated that ANN can be considered more suitable to serve as a tool to estimate LE when input meteorological variables are insufficient [29]. Thus, we may conclude that ANN performs better than SVM and MARS, especially when there are insufficient input variables or the accuracy of them was not very high.
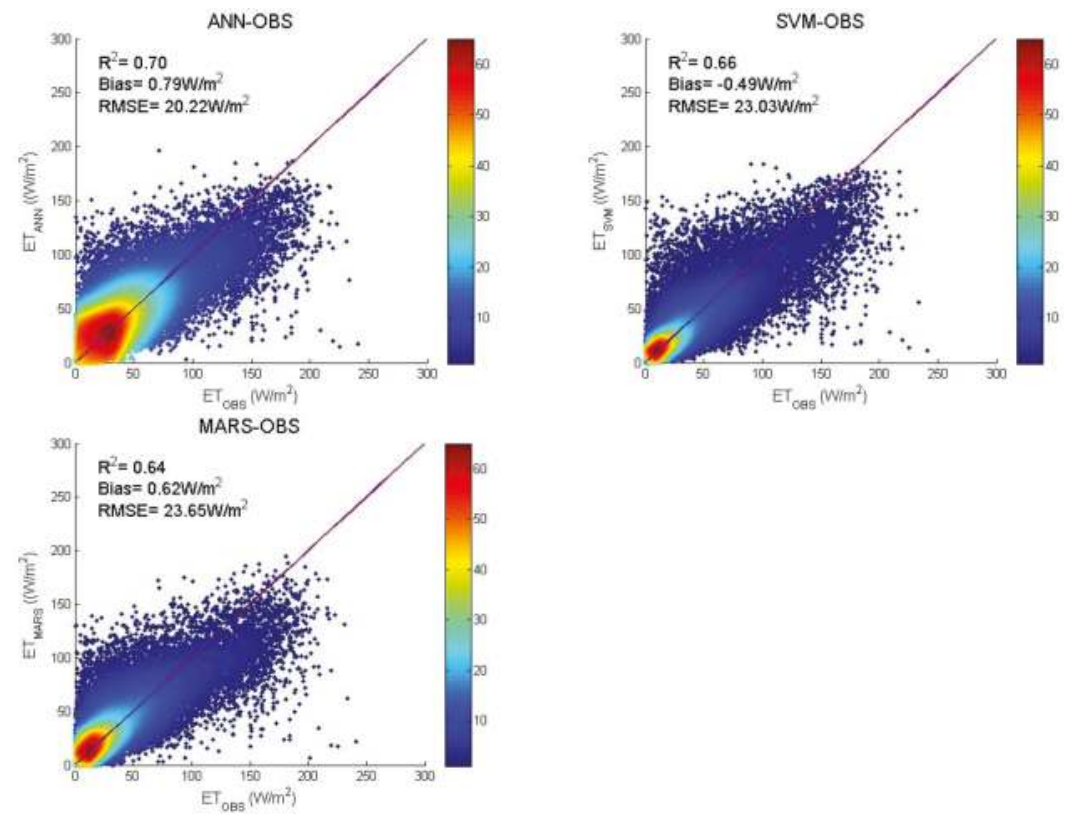

Figure 10. Comparison of daily LE observations for all 85 flux tower sites and LE estimates using different machine learning algorithms driven by MERRA meteorology.

Several previous studies have shown that spatial scale mismatch among different data sources, model input errors, and the limitations of the machine learning algorithms itself all affect the accuracy of LE estimation [7,10,56]. We used MERRA products with a spatial resolution of $1 / 2^{\circ} \times 1 / 3^{\circ}$ and MODIS products with a resolution of $1 \mathrm{~km}$, their resolution being greater than the footprint for field measurements, which is usually several hundred meters [57,58]. This is one of the reasons that estimated LE using tower meteorology as input data performs better than the estimated LE using MERRA meteorology as input data. Such representation of the field measurement footprint may also lead to bias in the machine learning algorithms. In addition, the accuracy of model input variables can also influence the accuracy of the LE estimates. Many studies have demonstrated that there are large errors in the MERRA meteorology and MERRA data tend to underestimate $R_{n}$ at high values when compared with ground measurements [49,58]. Recent studies have also revealed errors in MODIS NDVI when compared with ground measurements [49,57]. 


\subsection{Comparison between Different LE Products}

When compared with the SVM and the MARS algorithms, the ANN algorithm yielded lower annual terrestrial LE in central and southern North America and higher LE in the northern and eastern portions of this study area (Figure 11a,b). The LE values that were estimated using MARS algorithm were lower than the LE values estimated using the SVM algorithm in most areas, but overall, the difference between them was small and less than $10 \mathrm{~W} / \mathrm{m}^{2}$ (Figure 11c). The relevant differences between the flux estimations at the Mexican highlands, which are surrounded by Sierra Madre, may be mostly caused by the land surface heterogeneity and the different plant functional types. The PFT of the highlands is mainly grassland, while coastal and southeastern parts are covered with rainforest. The spatial differences among three machine learning algorithms may be mostly caused by different PFTs. The ANN showed higher LE for ENF and DBF, which are mostly located at the coasts of North America. In the center and west of the study area, due to the relatively high elevation and its own geographic and geomorphic conditions, the PFTs mostly contain SHR or GRA, so the ANN yielded lower LE than SVM and MARS. However, the results that are presented by SVM and MARS show no obvious difference for different PFTs.

Figure 12 shows spatial differences in annual terrestrial LE (2002-2004) between the MODIS LE product and the LE product using three machine learning algorithms. Relative to MODIS LE product, the ANN, SVM, and MARS results yielded lower LE in the north and central study area, and higher LE on the east coast and in the northeast of North America. This may be caused by the precision and quantity of the test data sample, as well as the characteristics of the machine learning algorithms, so the simulation value of machine learning LE is lower than the value of the MODIS LE product over a high LE area. This finding is consistent with other studies that showed that the MODIS LE product overestimated LE at high LE areas [23]. The difference between them was less than $20 \mathrm{~W} / \mathrm{m}^{2}$ in most areas. Given the accuracy of MERRA meteorology data and the MODIS LE product, we can conclude that machine learning algorithms are applicable for terrestrial LE mapping and the inversion results have a relatively small gap when compared to the MODIS LE product.
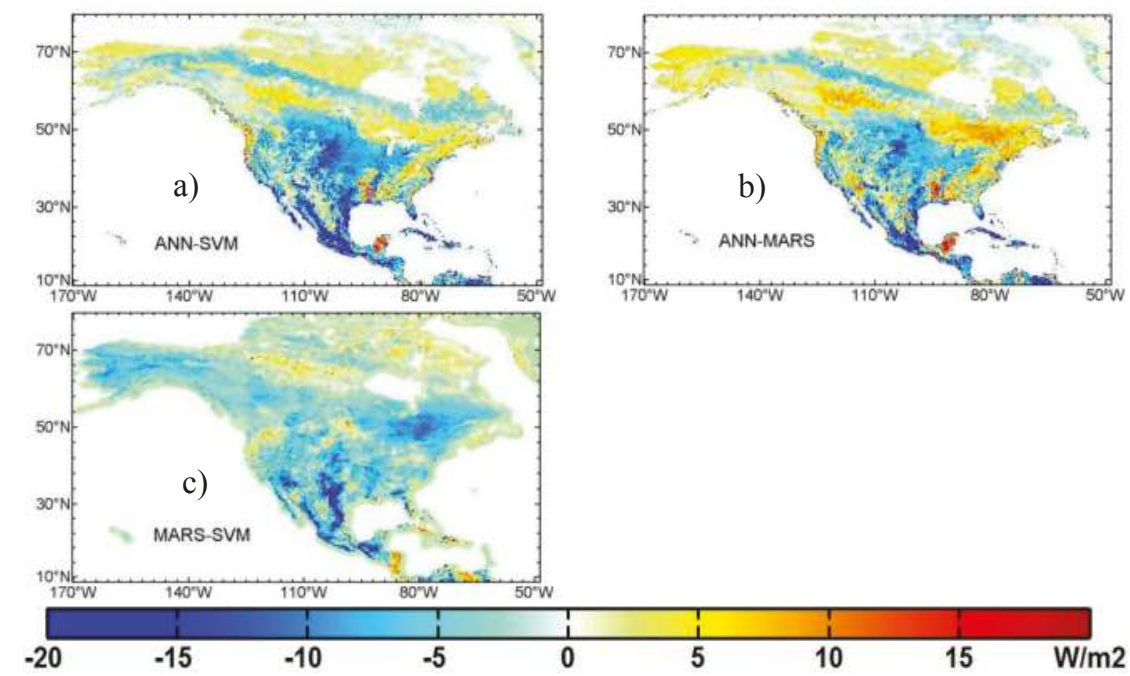

Figure 11. Spatial differences in the average annual terrestrial LE (2002-2004) between three machine learning algorithms. . 

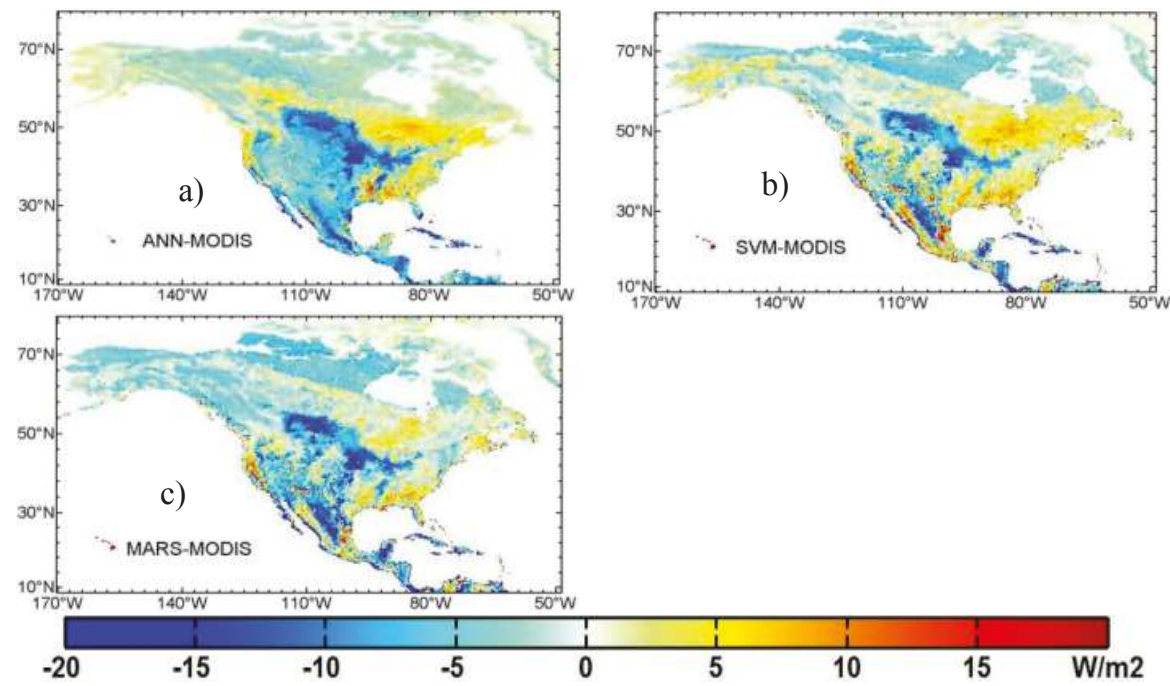

Figure 12. Spatial differences in the average annual terrestrial LE (2002-2004) between MODIS LE product and LE product using three machine learning algorithms.

\subsection{Limitations and Recommendations for Future Research}

Although the ANN leads to better performance when compared to other machine learning algorithms, it has several limitations. First, ANN requires a relatively long processing time to train a model, especially for ENF and GRA, the input data of which are inaccurate, and thus require more learning cycles. Second, the ANN model may trap in local optimization, leading to worse generalization performance. Further, all machine learning algorithms behave relatively unpredictably when used with input ground-measured LE deviating from those presented during the training stage $[24,59]$. Most importantly, we evaluated and validated LE performance using the same ground-measure LE data from EC sites. The machine learning algorithms do not possess the useful information to directly deliver additional confidence LE maps, and we cannot ensure the accuracy of these ground-measure LE data [60].

To make the training samples more applicable over North America, we should add samples from other PFTs, such as evergreen broadleaf forest and deciduous needleleaf forest [59]. The EC data of these specific PFTs is few over North America, however. We may develop the machine learning algorithms coupled with semi-empirical and physical methods that do not require training samples in the future to improve the terrestrial LE at more different PFTs.

\section{Conclusions}

The primary objective of this paper was to demonstrate the usage of three machine learning algorithms to estimate terrestrial LE for different PFTs over North America. We used tower-specific and MERRA meteorology data and MODIS NDVI product as input data and the ratio of observed LE and meteorology parameter $R_{n}$ as output data to build training models. All three machine learning algorithms proved to be reliable and robust for major land cover types in North America but the ANN algorithm performed better than the SVM and MARS algorithms based on specific site and MERRA data.

The results revealed that the ANN algorithm was more efficient in accounting for the nonlinear relationship between climatic variables, vegetation indices, and the corresponding LE. We found that ANN performed better in most land cover types, for except ENF and GRA, when using MERRA 
meteorology data as input data in the study area. The validation results for machine learning algorithms driven by tower-specific meteorology and MERRA meteorology showed that the results of SVM were similar to the results of MARS. For DBF sites, the MARS has a higher $R^{2}$ and a lower RMSE than SVM, for other sites, SVM perform a little better than MARS.

We concluded that it is reasonable to use machine learning algorithms to estimate LE by building relationships between input variables and outputs (LE). The accuracy of input variables, such as meteorology data and MODIS NDVI, can also influence the accuracy of the LE estimates. It is also applicable to map the terrestrial LE in different regions for different PFTs using any of these three machine learning algorithms; although, the performance of ANN was the best, the differences among them were small. The inversion results have a relatively small gap compared with MODIS LE product, with the difference between them being less than $20 \mathrm{~W} / \mathrm{m}^{2}$ in most areas, and machine learning algorithms performing better at the area where MODIS LE product overestimated LE.

Acknowledgments: Authors thank Meng Liu from Geographic Sciences and Natural Resources Research, CAS, Fei Feng, Shijie Chen, Aolin Jia, Jianmin Zhou, Jingcan Liu from Beijing Normal University, Xueni Pan from Capital Normal University and Dezhao Zhang, Dezhi Yang from China University of Geosciences (Beijing) for their helpful suggestions. MODIS NDVI and ET products were obtained online (http:/ / reverb.echo.nasa.gov/reverb). MERRA data were obtained online (http://gmao.gsfc.nasa.gov/merra/news/merra-land_release.php). All turbulent flux observations were downloaded from Ameriflux website (http://ameriflux.lbl.gov/). This work was partially supported by the National Key Research and Development Program of China (No. 2016YFA0600102) and the Natural Science Fund of China (No. 41671331).

Author Contributions: Xuanyu Wang and Yunjun Yao prepared the manuscript. Shaohua Zhao, Kun Jia, Xiaotong Zhang, Yuhu Zhang, Lilin Zhang, Jia Xu and Xiaowei Chen contributed to the discussion.

Conflicts of Interest: The authors declare no conflict of interest.

\section{References}

1. Allen, R.G.; Pereira, L.S.; Raes, D.; Smith, M. Crop Evapotranspiration: Guidelines for Computing Crop Water Requirements; Irrigation and Drainage Paper No 56; Food and Agriculture Organization of the United Nations (FAO): Rome, Italy, 1998.

2. Liang, S.; Wang, K.; Zhang, X.; Wild, M. Review on estimation of land surface radiation and energy budgets from ground measurement, remote sensing and model simulations. IEEE J. Sel. Top. Appl. Earth Obs. Remote Sens. 2010, 3, 225-240. [CrossRef]

3. Wang, K.; Dickinson, R.E. A review of global terrestrial evapotranspiration: Observation, modeling, climatology, and climatic variability. Rev. Geophys. 2012, 50, RG2005. [CrossRef]

4. Kumar, M.; Raghuwanshi, N.S.; Singh, R.; Wallender, W.W.; Pruitt, W.O. Estimating evapotranspiration using artificial neural network. J. Irrig. Drain. Eng. 2002, 128, 224-233. [CrossRef]

5. Los, S.O.; Collatz, G.J.; Sellers, P.J.; Malmström, C.M.; Pollack, N.H.; Defries, R.S.; Bounoua, L.; Parris, M.T.; Tucker, C.J.; Dazlich, D.A. A global 9-yr biophysical land surface dataset from noaa avhrr data. J. Hydrometeorol. 2000, 1, 183-199. [CrossRef]

6. Jin, Y.; Randerson, J.T.; Goulden, M.L. Continental-scale net radiation and evapotranspiration estimated using modis satellite observations. Remote Sens. Environ. 2011, 115, 2302-2319. [CrossRef]

7. Mu, Q.; Zhao, M.; Running, S.W. Improvements to a modis global terrestrial evapotranspiration algorithm. Remote Sens. Environ. 2011, 115, 1781-1800. [CrossRef]

8. Norman, J.M.; Kustas, W.P.; Humes, K.S. Source approach for estimating soil and vegetation energy fluxes in observations of directional radiometric surface temperature. Agric. For. Meteorol. 1995, 77, 263-293. [CrossRef]

9. Kustas, W.P.; Norman, J.M. Use of remote sensing for evapotranspiration monitoring over land surfaces. Hydrol. Sci. J. 1996, 41, 495-516. [CrossRef]

10. Mu, Q.; Heinsch, F.A.; Zhao, M.; Running, S.W. Development of a global evapotranspiration algorithm based on modis and global meteorology data. Remote Sens. Environ. 2007, 111, 519-536. [CrossRef]

11. Monteith, J.L. Evaporation and environment. Symp. Soc. Exp. Biol. 1965, 19, 205. [PubMed]

12. Priestley, C.H.B.; Taylor, R.J. On the assessment of surface heat flux and evaporation using large-scale parameters. Mon. Weather Rev. 2009, 100, 81-92. [CrossRef] 
13. Cleugh, H.A.; Leuning, R.; Mu, Q.; Running, S.W. Regional evaporation estimates from flux tower and modis satellite data. Remote Sens. Environ. 2007, 106, 285-304. [CrossRef]

14. Wang, K.; Wang, P.; Li, Z.; Cribb, M.; Sparrow, M. A simple method to estimate actual evapotranspiration from a combination of net radiation, vegetation index, and temperature. J. Geophys. Res. Atmos. 2007, 112, D15107. [CrossRef]

15. Jung, M.; Reichstein, M.; Ciais, P.; Seneviratne, S.I.; Sheffield, J.; Goulden, M.L.; Bonan, G.; Cescatti, A.; Chen, J.Q.; Jeu, R.D. Recent decline in the global land evapotranspiration trend due to limited moisture supply. Nature 2010, 467, 951-954. [CrossRef] [PubMed]

16. Yao, Y.; Liang, S.; Qin, Q.; Wang, K.; Zhao, S. Monitoring global land surface drought based on a hybrid evapotranspiration model. Int. J. Appl. Earth Obs. Geoinf. 2011, 13, 447-457. [CrossRef]

17. Qin, Q. Simple method to determine the priestley-taylor parameter for evapotranspiration estimation using albedo-vi triangular space from modis data. J. Appl. Remote Sens. 2011, 5, 3505.

18. Xu, T.; Liang, S.; Liu, S. Estimating turbulent fluxes through assimilation of geostationary operational environmental satellites data using ensemble kalman filter. J. Geophys. Res. Atmos. 2011, 116, 644. [CrossRef]

19. Xu, T.; Liu, S.; Liang, S.; Qin, J. Improving predictions of water and heat fluxes by assimilating modis land surface temperature products into the common land model. J. Hydrometeorol. 2010, 12, 227-244. [CrossRef]

20. Jiménez, C.; Prigent, C.; Mueller, B.; Seneviratne, S.I.; Mccabe, M.F.; Wood, E.F.; Rossow, W.B.; Balsamo, G.; Betts, A.K.; Dirmeyer, P.A. Global intercomparison of 12 land surface heat flux estimates. J. Geophys. Res. Atmos. 2011, 116, 3-25. [CrossRef]

21. Kim, H.W.; Hwang, K.; Mu, Q.; Lee, S.O.; Choi, M. Validation of modis 16 global terrestrial evapotranspiration products in various climates and land cover types in asia. KSCE J. Civ. Eng. 2012, 16, 229-238. [CrossRef]

22. Sheffield, J.; Wood, E.F. Projected changes in drought occurrence under future global warming from multi-model, multi-scenario, ipcc ar4 simulations. Clim. Dyn. 2008, 31, 79-105. [CrossRef]

23. Bruton, J.M.; Mcclendon, R.W.; Hoogenboom, G. Estimating daily pan evaporation with artificial neural networks. Am. Soc. Agric. Biol. Eng. 2000, 43, 491-496. [CrossRef]

24. El-Shafie, A.; Alsulami, H.M.; Jahanbani, H.; Najah, A. Multi-lead ahead prediction model of reference evapotranspiration utilizing ann with ensemble procedure. Stoch. Environ. Res. Risk Assess. 2013, 27, 1423-1440. [CrossRef]

25. Shrestha, N.K.; Shukla, S. Support vector machine based modeling of evapotranspiration using hydro-climatic variables in a sub-tropical environment. Bioresour. Technol. 2013, 128, 351. [CrossRef]

26. Deo, R.C.; Samui, P.; Kim, D. Estimation of monthly evaporative loss using relevance vector machine, extreme learning machine and multivariate adaptive regression spline models. Stoch. Environ. Res. Risk Assess. 2016, 30, 1769-1784. [CrossRef]

27. Yang, F.; White, M.A.; Michaelis, A.R.; Ichii, K.; Hashimoto, H.; Votava, P.; Zhu, A.X.; Nemani, R.R. Prediction of continental-scale evapotranspiration by combining modis and ameriflux data through support vector machine. IEEE Trans. Geosci. Remote Sens. 2006, 44, 3452-3461. [CrossRef]

28. Adnan, M.; Latif, M.A.; Abaid-ur-Rehman; Nazir, M. Estimating evapotranspiration using machine learning techniques. Int. J. Adv. Comput. Sci. Appl. 2017, 8, 108-113.

29. Tabari, H.; Marofi, S.; Sabziparvar, A.A. Estimation of daily pan evaporation using artificial neural network and multivariate non-linear regression. Irrig. Sci. 2010, 28, 399-406. [CrossRef]

30. Vapnik, V.N. The Nature of Statistical Learning Theory; Springer: New York, NY, USA, 1995; pp. 988-999.

31. Nurmemet, I.; Ghulam, A.; Tiyip, T.; Elkadiri, R.; Ding, J.L.; Maimaitiyiming, M.; Abliz, A.; Sawut, M.; Zhang, F.; Abliz, A. Monitoring soil salinization in keriya river basin, northwestern China using passive reflective and active microwave remote sensing data. Remote Sens. 2015, 7, 8803-8829. [CrossRef]

32. Dibike, Y.B.; Velickov, S.; Solomatine, D.; Abbott, M.B. Model induction with support vector machines: Introduction and applications. J. Comput. Civ. Eng. 2001, 15, 208-216. [CrossRef]

33. Friedman, J.H. Multivariate adaptive regression splines. Ann. Stat. 1991, 19, 1-67. [CrossRef]

34. Butte, N.F.; Wong, W.W.; Adolph, A.L.; Puyau, M.R.; Vohra, F.A.; Zakeri, I.F. Validation of cross-sectional time series and multivariate adaptive regression splines models for the prediction of energy expenditure in children and adolescents using doubly labeled water. J. Nutr. 2010, 140, 1516. [CrossRef] [PubMed]

35. Veaux, R.D.D.; Ungar, L.H. Multicollinearity: A Tale of Two Nonparametric Regressions; Springer: New York, NY, USA, 2007; pp. 393-402. 
36. Adamowski, J.; Chan, H.; Prasher, S.; Ozga-Zielinski, B.; Sliusarieva, A. Comparison of multiple linear and nonlinear regression, autoregressive integrated moving average, artificial neural network, and wavelet artificial neural network methods for urban water demand forecasting in montreal, Canada. Water Resour. Res. 2012, 48, 1528. [CrossRef]

37. Sephton, P. Forecasting Recessions: Can We Do Better on Mars? Federal Reserve Bank of St. Louis: Washington, DC, USA, 2001; Volume 83.

38. Liu, S.M.; Xu, Z.W.; Wang, W.Z.; Jia, Z.Z.; Zhu, M.J.; Bai, J.; Wang, J.M. A comparison of eddy-covariance and large aperture scintillometer measurements with respect to the energy balance closure problem. Hydrol. Earth Syst. Sci. 2011, 15, 1291-1306. [CrossRef]

39. Jia, Z.; Liu, S.; Xu, Z.; Chen, Y.; Zhu, M. Validation of remotely sensed evapotranspiration over the hai river basin, China. J. Geophys. Res. Atmos. 2012, 117, 13113. [CrossRef]

40. Xu, Z.; Liu, S.; Li, X.; Shi, S.; Wang, J.; Zhu, Z.; Xu, T.; Wang, W.; Ma, M. Intercomparison of surface energy flux measurement systems used during the HiWATER-MUSOEXE. J. Geophys. Res. Atmos. 2013, 118, 13140-13157. [CrossRef]

41. Twine, T.E.; Kustas, W.P.; Norman, J.M.; Cook, D.R.; Houser, P.R.; Teyers, T.P.; Prueger, J.H.; Starks, P.J.; Wesely, M.L. Correcting eddy-covariance flux underestimates over a grassland. Agric. For. Meteorol. 2000, 103, 279-300. [CrossRef]

42. Huete, A.; Didan, K.; Miura, T.; Rodriguez, E.P.; Gao, X.; Ferreira, L.G. Overview of the radiometric and biophysical performance of the modis vegetation indices. Remote Sens. Environ. 2002, 83, 195-213. [CrossRef]

43. Zhao, M.; Heinsch, F.A.; Nemani, R.R.; Running, S.W. Improvements of the modis terrestrial gross and net primary production global data set. Remote Sens. Environ. 2005, 95, 164-176. [CrossRef]

44. Friedl, M.A.; Mciver, D.K.; Hodges, J.C.F.; Zhang, X.Y.; Muchoney, D.; Strahler, A.H.; Woodcock, C.E.; Gopal, S.; Schneider, A.; Cooper, A. Global land cover mapping from modis: Algorithms and early results. Remote Sens. Environ. 2002, 83, 287-302. [CrossRef]

45. Gandomi, A.H.; Roke, D.A. Intelligent formulation of structural engineering systems. In Proceedings of the Seventh MIT Conference on Computational Fluid and Solid Mechanics-Focus: Multipgysics \& Multiscale, Cambridge, MA, USA, 12-14 June 2013.

46. Cristau, H.-J.; Cellier, P.P.; Spindler, J.F.; Taillefer, M. Highly efficient and mild copper-catalyzed N- and C-arylations with aryl bromides and iodides. Chemistry 2004, 10, 5607-5622. [CrossRef] [PubMed]

47. Chang, C.C.; Lin, C.J. Libsum: A Library for Support Vector Machines; ACM: New York, NY, USA, 2011; pp. 1-27.

48. Meyer, D. Support vector machines the interface to libsvm in package e1071. R News 2013, 1, 1-3.

49. Lu, X.L.; Zhuang, Q.L. Evaluating evapotranspiration and water-use efficiency of terrestrial ecosystems in the conterminous united states using modis and ameriflux data. Remote Sens. Environ. 2010, 114, 1924-1939. [CrossRef]

50. Fisher, J.B.; Tu, K.P.; Baldocchi, D.D. Global estimates of the land-atmosphere water flux based on monthly avhrr and islscp-ii data, validated at 16 fluxnet sites. Remote Sens. Environ. 2008, 112, 901-919. [CrossRef]

51. Vinukollu, R.K.; Wood, E.F.; Ferguson, C.R.; Fisher, J.B. Global estimates of evapotranspiration for climate studies using multi-sensor remote sensing data: Evaluation of three process-based approaches. Remote Sens. Environ. 2011, 115, 801-823. [CrossRef]

52. Vinukollu, R.K.; Meynadier, R.; Sheffield, J.; Wood, E.F. Multi-model, multi-sensor estimates of global evapotranspiration: Climatology, uncertainties and trends. Hydrol. Process. 2011, 25, 3993-4010. [CrossRef]

53. Yebra, M.; Dijk, A.V.; Leuning, R.; Huete, A.; Guerschman, J.P. Evaluation of optical remote sensing to estimate actual evapotranspiration and canopy conductance. Remote Sens. Environ. 2013, 129, 250-261. [CrossRef]

54. Demarty, J.; Chevallier, F.; Friend, A.; Viovy, N.; Piao, S.; Ciais, P. Assimilation of Global Modis Leaf Area Index Retrievals within a Terrestrial Biosphere Model. Geophys. Res. Lett. 2007, 34. [CrossRef]

55. Specht, D.F. A general regression neural network. IEEE Trans. Neural Netw. 1991, 2, 568. [CrossRef] [PubMed]

56. Zhang, L.; Yao, Y.; Wang, Z.; Jia, K.; Zhang, X.; Zhang, Y.; Wang, X.; Xu, J.; Chen, X. Satellite-derived spatiotemporal variations in evapotranspiration over northeast China during 1982-2010. Remote Sens. 2017, 9, 1140. [CrossRef]

57. Zhao, M.; Running, S.W.; Nemani, R.R. Sensitivity of moderate resolution imaging spectroradiometer (modis) terrestrial primary production to the accuracy of meteorological reanalyses. J. Geophys. Res. Biogeosci. 2006, 111, 338-356. [CrossRef] 
58. Baldocchi, D. Breathing of the terrestrial biosphere: Lessons learned from a global network of carbon dioxide flux measurement systems. Aust. J. Bot. 2008, 56, 1-26. [CrossRef]

59. Yao, Y.; Liang, S.; Li, X.; Chen, J.; Liu, S.; Jia, K.; Zhang, X.; Xiao, Z.; Fisher, J.B.; Mu, Q. Improving global terrestrial evapotranspiration estimation using support vector machine by integrating three process-based algorithms. Agric. For. Meteorol. 2017, 242, 55-74. [CrossRef]

60. Yao, Y.; Liang, S.; Yu, J.; Zhao, S.; Lin, Y.; Jia, K.; Zhang, X.; Cheng, J.; Xie, X.; Sun, L. Differences in estimating terrestrial water flux from three satellite-based priestley-taylor algorithms. Int. J. Appl. Earth Obs. Geoinf. 2017, 56, 1-12. [CrossRef]

(c) 2017 by the authors. Licensee MDPI, Basel, Switzerland. This article is an open access article distributed under the terms and conditions of the Creative Commons Attribution (CC BY) license (http:/ / creativecommons.org/licenses/by/4.0/). 


\title{
Article \\ High-Resolution Mapping of Freeze/Thaw Status in China via Fusion of MODIS and AMSR2 Data
}

\author{
Tongxi Hu ${ }^{1,2}$, Tianjie Zhao ${ }^{2, *}$, Jiancheng Shi ${ }^{2}$, Shengli Wu ${ }^{3}$, Dan Liu ${ }^{1}$, Haiming Qin ${ }^{2,4}$ and \\ Kaiguang Zhao ${ }^{1,4, *}$ \\ 1 Environmental Science Graduate Program, The Ohio State University, Columbus, OH 43210, USA; \\ hu.1555@osu.edu (T.H.); liu.6253@osu.edu (D.L.) \\ 2 State Key Laboratory of Remote Sensing Science, Institute of Remote Sensing and Digital Earth, \\ Chinese Academy of Sciences, No. 20 Datun Road, Beijing 100101, China; shijc@radi.ac.cn (J.S.); \\ qin.434@osu.edu (H.Q.) \\ 3 National Satellite Meteorological Center, Beijing 100081, China; wusl@cma.gov.cn \\ 4 School of Environment and Natural Resources, OARDC, The Ohio State University, Columbus, OH 43210, USA \\ * Correspondence: zhaotj@radi.ac.cn (T.Z.); zhao.1423@osu.edu (K.Z.); Tel.: +1-330-263-3722 (K.Z.)
}

Received: 30 October 2017; Accepted: 13 December 2017; Published: 20 December 2017

\begin{abstract}
Transition of freeze/thaw (F/T) affects land-atmospheric interactions and other biospheric dynamics. Global F/T statuses are normally monitored using microwave remote sensing, but at coarse resolutions (e.g., $25 \mathrm{~km}$ ). Integration of coarse microwave remote sensing data with finer satellite products represents an opportunity to further enhance our ability to map F/T statuses regionally and globally. Here, we implemented and tested an approach to generate daily F/T status maps at a 5-km spatial resolution through the fusion of passive microwave data from AMSR2 and land surface temperature products from MODIS, using China as our study area for the year 2013 and 2014. Moreover, possible influences from elevation, vegetation, seasonality, etc., were also analyzed, as such analysis provides a direction to improve the approach. Overall, our freeze/thaw maps agreed well with ground reference observations, with an accuracy of $\sim 86.6 \%$. The new F/T maps helped to identify regions subject to frequent $\mathrm{F} / \mathrm{T}$ transitions through the year, such as the Qinghai-Tibetan Plateau, Xinjiang, Gansu, Heilongjiang, Jilin, and Liaoning Province. This study indicates that the combination of AMSR2 and MODIS observations provides an effective method to obtain finer F/T maps (5-km or lower) for extensive regions. The finer F/T maps improve our knowledge of the F/T state detected by satellite remote sensing, and have a wide range of applications in regional studies considering land surface heterogeneity and models (e.g., community land models).
\end{abstract}

Keywords: high-resolution freeze/thaw; AMSR2; MODIS

\section{Introduction}

About $55 \%$ of the exposed land surface in the northern hemisphere experiences freeze/thaw (F/T) transitions annually [1]. These transitions occur within the upper permafrost layers and frozen grounds that react sensitively to climate and environmental changes. By controlling water and heat flux exchange at the land-atmosphere interface, $\mathrm{F} / \mathrm{T}$ status imposes a significant constraint on ecosystem processes [2,3] and vegetation growth [4,5], resulting in important impacts on surface water, and the nitrogen [6] and carbon cycle [7]. To evaluate these ecological or hydrological impacts accurately, data of the F/T status with high spatial and temporal resolutions are essential [8]. However, observations of the F/T status with high spatial and temporal resolution in a global or regional scale are still unavailable. Traditional in situ observations or numerical modeling could provide accurate point measurements for the investigation of $\mathrm{F} / \mathrm{T}$ transitions [9], but these conventional methods are limited to regional areas with continuous coverage. Instead, remote sensing data, especially microwave remote 
sensing, has provided a chance to detect the F/T status in recent years. The foundation of detecting the $\mathrm{F} / \mathrm{T}$ status by microwave remote sensing is the distinct contrast between dielectric properties that occurs when water transitions between solid and liquid [10], which means the phase of soil water during the $\mathrm{F} / \mathrm{T}$ transitions will severely affect the sensitivity of radiometer brightness temperature $(\mathrm{Tb})$ and radar backscatter signatures.

Based on the dramatic changes of the signal on the F/T status observed by passive microwave sensors, many approaches have been developed. Most of these approaches, such as dual-index [9], decision tree [11], seasonal threshold [4,12], and discriminant function algorithm (DFA) [13], combine observations from multiple channels from a specific sensor, for example, SSM/I, AMSR-E, AMSR2, SMMIS, etc., to capture the changes of soil temperature and moisture during F/T transitions. However, the $\mathrm{F} / \mathrm{T}$ status derived using passive microwave remote sensing suffers from a coarse spatial resolution of 0.25 degrees (about $25 \mathrm{~km}$ at the equator). Data or products with such a coarse resolution are no longer applicable for hydro-meteorological and agricultural studies [14]. High-resolution data are needed for a deeper understanding of processes that link to the terrestrial water, energy, and carbon cycles, as well as for significant improvement in numerical models, such as weather prediction and seasonal climate, flood and drought forecasting, crop yield estimating models, etc., that relate to land surface heterogeneity $[8,14,15]$.

Backscatter signatures from active remote sensors, such as satellite- or aircraft-based radar and scatterometers, are also able to capture signal changes during F/T transitions. Daily frozen and non-frozen statuses of Alaska were determined by Kimball et al. [5] with temporal change detection analysis relying on NASA scatterometer observations. A similar method was also used by Du et al. [15] to classify F/T status over Alaska based on Polarimetric SAR data with a spatial resolution of about $100 \mathrm{~m}$. Zwieback et al. [16] developed a statistical model to detect frozen soils using Advanced Scatterometer data and ERA Interim temperatures. Usually, observations acquired from active microwave remote sensing have a high spatial resolution (several kilometers or less). However, these observations are still limited to regional areas, as well as long revisit periods that are not feasible to monitor $\mathrm{F} / \mathrm{T}$ transitions.

To overcome the limitations introduced by purely relying on passive or active remote sensing, a combination of multi-source remote sensing data for $\mathrm{F} / \mathrm{T}$ retrieval has been recently studied for two satellite missions. One is the Soil Moisture Active Passive (SMAP) mission initiated to map the F/T status with a high spatial resolution of $2-3 \mathrm{~km}$ covering high northern latitude $\left(>45^{\circ} \mathrm{N}\right)$ areas and a short revisit period of 2-3 days by combining its active and passive measurements [8]. Unfortunately, the radar equipped on the satellite failed to work after operations of about three months, so the F/T maps are produced at a resolution of about 25 miles with just its radiometer now. The other satellite mission, Water Cycle Observation Mission (WCOM), aims to obtain the F/T status globally with a resolution of less than $5 \mathrm{~km}[17,18]$ relying on both innovative theory and techniques for retrieval and new sensing payloads. The payload configuration of the WCOM is a combination of active and passive, wide frequency coverage, microwave remote sensors. Although the combination of passive and active microwave remote sensing is promising to provide high-resolution $\mathrm{F} / \mathrm{T}$ maps covering a large area, such a map is still presently unavailable.

Instead of combining active and passive microwave observations, a recent experiment of fusing thermal and passive microwave remote sensing shows potential to obtain the $\mathrm{F} / \mathrm{T}$ status at a fine spatial resolution [17]. Classification of the F/T status by remote sensing is based on changes in two main aspects of the soil: water content and temperature. The changes of the soil water content can be captured by passive microwave bands. And the soil temperature can be measured by both thermal and microwave bands. The overlapping temperature information makes it possible to fuse observations from sensors of these two bands. Both thermal and microwave sensors have their own advantages. Thermal remote sensing data may suffer from many limitations, such as long revisit time periods (8-16 days) in boreal regions, but they have higher spatial resolutions compared with data from passive microwave remote sensing, for instance, the resolution of land surface temperature 
products derived from MODerate-resolution Imaging Spectroradiometer (MODIS) thermal bands can be as high as $1 \mathrm{~km}$. Passive microwave observations, such as brightness temperatures from AMSR2, have a coarse spatial resolution of $25 \mathrm{~km}$ at the equator, but they can capture dynamics during the $\mathrm{F} / \mathrm{T}$ transition process in a short revisit period (2-3 days) over large areas. The fusion of thermal and passive remote sensing combines the relative strengths of thermal and passive microwave remote sensing for enhanced soil F/T mapping.

The overall objective of this article was to evaluate a method to obtain high-resolution F/T dataset (here "high-resolution" is compared to the spatial resolution of passive microwave observations) by taking advantage of current thermal and passive microwave remote sensing data in large regions, such as China. An algorithm developed for AMSR sensors, DFA, was applied to observations from AMSR2 to acquire coarse resolution maps of $F / T$. These $F / T$ maps have a spatial resolution of 0.25 degree, the same to that of AMSR2 observations. Two variables, $F$ and $T$ score $\left(D_{F}\right.$ and $\left.D_{T}\right)$, were generated by the DFA for each pixel, and they were the criteria to determine if a pixel is frozen or thawing. The specific objectives of this study were to: (1) analyze the relationship between land surface temperature from MODIS and the two variables $\left(\mathrm{D}_{\mathrm{F}}\right.$ and $\left.\mathrm{D}_{\mathrm{T}}\right)$ calculated by DFA; $(2)$ investigate the usage of this relationship over China; and (3) combine the MODIS land surface temperature with coarse $\mathrm{F} / \mathrm{T}$ datasets obtained from passive microwave observation (AMSR2) to generate the high-resolution F/T map of China ( 0.05 degree, around 5-km at the equator). Finally, the derived high-resolution F/T maps were verified by in situ soil temperatures at 0 -cm depth across China.

\section{Materials and Methods}

\subsection{Remote Sensing Observations}

Remote sensing observations used here are acquired from passive microwave and thermal sensors. The passive microwave remote sensing data are obtained from the Advanced Microwave Scanning Radiometer-2 (AMSR2) onboard the Global Change Observation Mission 1st-Water (GCOM-W1) satellite [18] which was launched on 18 May 2012. AMSR2 is equipped with the world's largest revolving space antenna to detect radiometry from the Earth with an appropriate 1450-km width in one scan, so its observation can cover almost $99 \%$ of the globe in two days. The observations are obtained in six different channels $(6.9,10.7,18.7,23.8,36.5$, and $89.0 \mathrm{GHz})$ with horizontal and vertical polarization, and twice daily at local times around 13:30 (ascending) and 1:30 (descending) at the equator. The detailed characteristics of AMSR2 were summarized in Table 1. Daily brightness temperatures $(\mathrm{Tb})$ can be obtained from both the National Snow and Ice Data Center (NSIDC) and the Japan Aerospace Exploration Agency (JAXA). The Tb used here were from GCOM-W1 Data Providing Service [19] and they are daily quarter-degree gridded (version 02) data in global coverage. In addition to $\mathrm{Tb}$, precipitation data are also used here to exclude raining areas because of their significant influence on microwave emission. The precipitation data are the Level 3 standard products (version 02) of AMSR2, which can also be obtained from the GCOM-W1 Data Providing Service.

Table 1. The characteristics of AMSR2.

\begin{tabular}{|c|c|c|c|c|c|c|c|}
\hline Central Frequency (GHz) & 6.925 & 10.65 & 18.7 & 23.8 & 36.5 & \multicolumn{2}{|c|}{89.0} \\
\hline IFOV (km) & $43 \times 75$ & $29 \times 51$ & $16 \times 27$ & $18 \times 32$ & $8.2 \times 14.4$ & $3.7 \times 6.5$ & $3.5 \times 5.9$ \\
\hline Polarization & & & Ho & ontal and & ertical & & \\
\hline Incidence Angle & & & & $55^{\circ}$ & & & \\
\hline Swath & & & & $1445 \mathrm{~km}$ & & & \\
\hline Dynamic Range (K) & & & & $2.7-340$ & & & \\
\hline
\end{tabular}

Thermal observations are daily land surface temperature (LST) products (MYD11C1, version 5) from MODIS, a key instrument aboard the Terra and Aqua satellites. MODIS carried on the Aqua satellite was selected for this study [20]. Since the orbit of MODIS on Aqua shares numerous characteristics, for instance, the equator crossing time, revisit period, etc., with that of AMSR2, 
these characteristics provide a unique opportunity to combine observations of these two sensors with less systematic differences. MODIS can provide observations in as many as 36 spectral bands to improve our understanding of global dynamics and processes occurring on the land, in the atmosphere, and in the ocean. The data from MODIS are processed at various levels ranging from level 0 to level 4 . Products at level 3 of two years $(2013,2014)$ are used here, which includes both LST and quality assessment values at 0.05 degree latitude/longitude climate modelling grids (CMG, 7200 columns $\times 3600$ rows) covering the global area [20]. The LST of two years $(2013,2014)$ was selected to investigate its potential relationship with $\mathrm{D}_{\mathrm{F}}$ and $\mathrm{D}_{\mathrm{T}}$ that are used to classify the $\mathrm{F} / \mathrm{T}$ status. In addition to the land surface temperature data, ancillary data, such as the MODIS IGBP (International Geosphere-Biosphere Program) map, are also included to identify the areas with permanent snow.

\subsection{Meteorological Data}

Assessment of F/T maps usually relies on in situ measurements. Soil temperatures at $0-\mathrm{cm}$ depth were collected from 2425 stations across China to evaluate the high-resolution F/T maps here. The temperature measurements are extracted from the datasets (SURF_CLI_CHN_MUL_DAY_V3.0) archived by China Meteorological Administration (CMA) [21]. Most of these meteorological stations have data records for more than one year, but not all of them have continuous records for every day of the year. Moreover, the temperatures are only measured by sensors on the ground four times (2:00, 8:00, 14:00, 20:00) daily. The records at 2:00 and 14:00 were chosen to compare with the F/T maps generated by satellite observations at 1:30 and 13:30, respectively. The comparison is based on our assumption that the $\mathrm{F} / \mathrm{T}$ status when the soil temperature measurements are conducted is approximate to that when the satellites pass over. Similar assumptions have also been made to verify accuracy of the $\mathrm{F} / \mathrm{T}$ status derived from satellite observations $[4,12,16]$. To make the assessment convincing, in situ temperature measurements in 2013 were chosen to evaluate the agreement with the F/T maps.

\subsection{Methods}

Our methodological framework (Figure 1) includes combining thermal and passive microwave remote sensing data to generate $\mathrm{F} / \mathrm{T}$ maps in China and evaluating the accuracy of this approach using in situ ground temperatures.

\subsubsection{Discriminant Function Algorithm (DFA)}

DFA is developed specifically for AMSR-E and performs well to determine the land surface F/T status. This algorithm has been evaluated by Zhao et al. [13] using the 4-cm soil temperature on Qinghai-Tibetan Plateau and the result shows an overall classification accuracy of $86 \%$. It also has the highest accuracy compared with two other algorithms, dual index [9] and decision tree algorithm [11], when applied to AMSR-E data [22]. In addition, further validation of this algorithm was conducted in our previous work using in situ air temperature measurements from the World Meteorological Organization (WMO, $88.37 \%$ and $82.76 \%$ for observations at 1:30 and 13:30, respectively), $0-5 \mathrm{~cm}$ soil temperature from International Soil Moisture Network (ISMN, $86.63 \%$ ), as well as modelled soil temperature by the Global Land Data Assimilation System (GLDAS, 89.74\% and 87.6\% for observations at 1:30 and 13:30, respectively) [23]. 


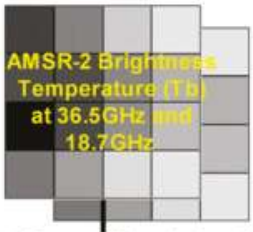

Calculate Freeze/Thaw Index (FTI) with Tb
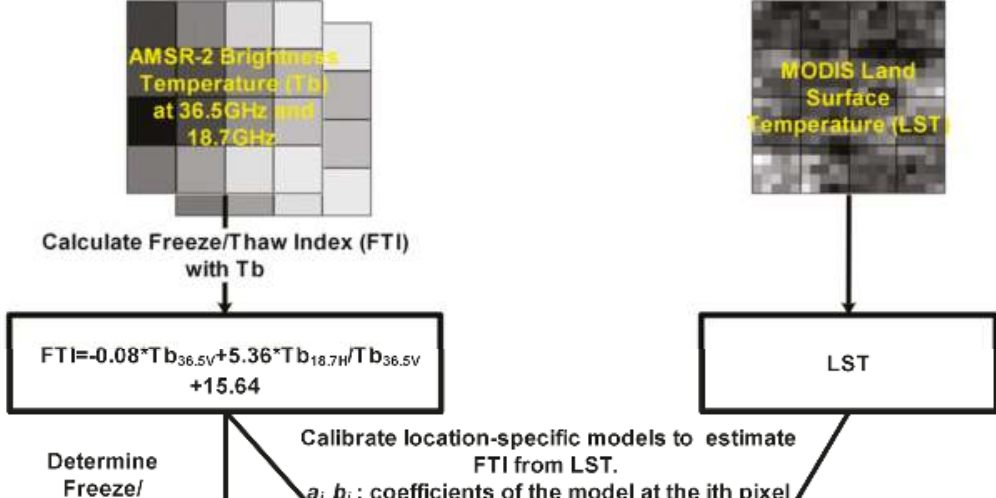

Thaw based

on FTI

calcuated

by AMSR-2

data directly

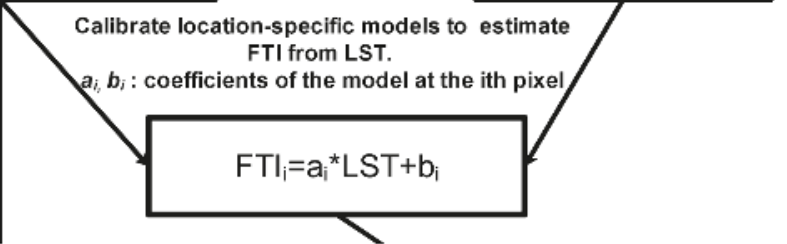

Discrimination Algorithm Function (DFA):

Freeze: if $\mathrm{FTI}>0$
Thaw: if $\mathrm{FTI}<0$

Determine Freeze/Thaw

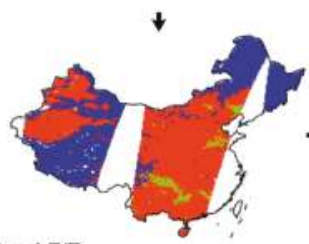

AMSR2-derived F/T map at about 25-km resolution

\section{Gap-fill MODIS map with} AMSR-2 results for regions with no valid MODIS data

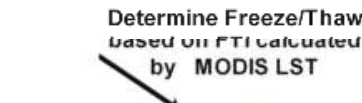

by MODIS LST

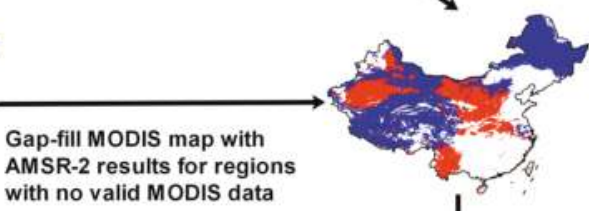

MODIS-derived F/T map at about 5-km resolution

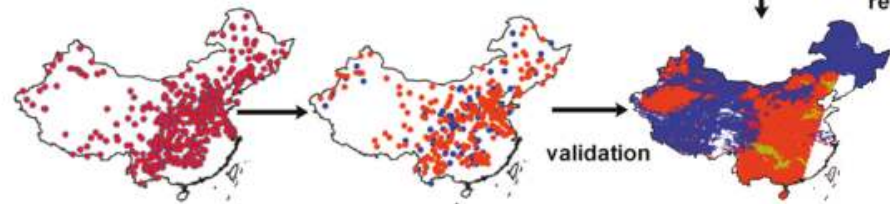

Ground surface temperature measurments at the depth of $0 \mathrm{~cm}$ from meteorological stations

\section{Ground-thruhing $F / T$ status determined by temperature $(\mathrm{T})$ : Freeze: if $T>0^{\circ} \mathrm{C}$} Thaw: if $\mathrm{T} \angle 0^{\circ} \mathrm{C}$

Figure 1. Flowchart of generating high-resolution F/T maps.

A brief introduction of DFA is presented here. DFA was developed based on observations from a truck-mounted multi-frequency microwave radiometer and simulated data from a set of combined models related to vegetation, snow, and soil [13]. The principle of the algorithm is to determine the status of soil in each pixel using two criteria: one is the brightness temperature observed by radiometers at $36.5 \mathrm{GHz}$ in vertical polarization, which is closely related to surface temperature; the other one is called quasi-emissivity and it is the ratio of the brightness temperature in the horizontal polarization at low frequency, such as $6.9,10.65$, and $18.7 \mathrm{GHz}$, to that in vertical polarization at 
36.5 GHz. The quasi-emissivity is related to soil water content. These two criteria are used to construct the discriminant functions based on Fisher linear discriminant analysis [24]:

$$
\begin{gathered}
D_{F}=1.47 * T b_{36.5 \mathrm{~V}}+91.69 * Q e_{18.7 \mathrm{H}}-226.7 \\
D_{T}=1.55 * T b_{36.5 \mathrm{~V}}+86.33 * Q e_{18.7 \mathrm{H}}-242.41 \\
Q e_{18.7 \mathrm{H}}=\frac{T b_{18.7 H}}{T b_{36.5 \mathrm{~V}}} \\
F T I=D_{F}-D_{T}
\end{gathered}
$$

where $T b_{36.5 \mathrm{~V}}$ is the vertically-polarized brightness temperature at $36.5 \mathrm{GHz}$ and $Q_{e 18.7 \mathrm{H}}$ is the quasi-emissivity calculated by the low frequency of $18.7 \mathrm{GHz} . D_{F}$ and $D_{T}$ are calculated for each pixel and used to classify a pixel as frozen or thawing. If $D_{F}$ is greater than $D_{T}$, the pixel will be classified as frozen; otherwise it will be classified as thawing. The difference of $D_{F}$ and $D_{T}$ is called $\mathrm{F} / \mathrm{T}$ index (FTI) [17].

\subsubsection{Fusion of F/T Dataset with LST}

The F/T index has shown a linear relationship with $L S T$ in previous studies conducted on the Qinghai Tibetan Plateau (QTP). The regression model is in the following form:

$$
F T I=a * L S T+b
$$

where $a$ and $b$ are parameters in the model. The main problem of determining $a$ and $b$ is the unmatched spatial resolutions of LST and FTI.

The approach to matching LST and FTI is by upscaling the spatial resolution of LST to that of FTI. LST is a product of MODIS with 0.05-degree spatial resolution. However, FTI has the same resolution as the passive microwave observations ( 0.25 degree). For each grid of FTI, $5 \times 5$ grids are needed to cover the same area in the LST. If more than 12 of the $5 \times 5$ grids have valid records (determined by quality control data), the average of these valid values will be used to match the value in an FTI grid. Then, a simple linear regression analysis is conducted to determine the relationship based on the matched data pairs between FTI and LST. Once the two parameters $a$ and $b$ are obtained, the linear model can be applied to each pixel of the MODIS LST products to acquire a new FTI, which we called LST-based FTI because it has the same spatial resolution as the LST. For pixels in the LST-based FTI, those with values greater than 0 are classified as frozen, otherwise, they are classified as thawing. If pixels in the LST-based FTI have no values because of gaps or invalid data in the LST, they will be determined using microwave observations alone. Those pixels which are detected as raining will not be determined and just labelled as rain. The new F/T map based on the LST-based FTI is called the high-resolution $\mathrm{F} / \mathrm{T}$ map because its spatial resolution ( 0.05 degree) is higher than F/T maps derived from FTI just using microwave observations ( 0.25 degree). Permanent snow areas are also marked according to the MODIS-International Geosphere-Biosphere Programme (IGBP) land cover classifications.

\subsubsection{Evaluation of the High-Resolution F/T Maps}

A comparison of high-resolution F/T maps and 0-cm soil temperatures is divided into two aspects: temporal and spatial agreement. Temporal agreement is calculated daily and it is the ratio of the number of stations whose observations are consistent with the classification result of F/T maps and the total number of stations; spatial agreement is calculated by dividing the total observations in a year with the observations that are consistent with the F/T maps for each station. Both the temporal and spatial agreement can be calculated by the equation below:

$$
A=\frac{N_{F F}+N_{T T}}{N_{F F}+N_{F T}+N_{T F}+N_{T T}}
$$


where $N_{F F}$ means the number of pixels where a station is located are classified as frozen, while the ground temperatures are equal, or less than, $0{ }^{\circ} \mathrm{C} ; N_{F T}$ represents the numbers of pixels when they are classified as frozen, while the ground temperatures are above $0{ }^{\circ} \mathrm{C} . N_{T T}$ and $N_{T F}$ follow similar meanings.

\section{Results}

\subsection{Relationship of MODIS LST and Freeze/Thaw Index}

MODIS LST and FTI determined by DFA (Equation (4)) show a high negative correlation in most areas of China (Figure 2). Areas with values below -0.8 take up about $95.4 \%$ and $92.6 \%$ of the domain for observations at 13:30 and 1:30, respectively. The correlation values for both passes are not homogeneously distributed. For ascending and descending time, low correlation less than 0.7 occurs on the QTP, in Central Northeast China and northeast of Xinjiang Province; and most of these three areas also have correlation values around -0.85 . Other parts of the domain have a high negative correlation above 0.9 . Correlation for observations at 1:30 has many more values lower than 0.85. Especially in Southeast of China, in the Hengduan Mountains, correlations are below 0.6. Low correlation values will introduce some inconsistencies between coarse-resolution F/T maps and high-resolution F/T maps, and affect the accuracy of F/T classification. The low correlations may result from many factors, for instance, frequent F/T transitions [25], elevation, land cover, as well as the accuracy of observations [26,27].

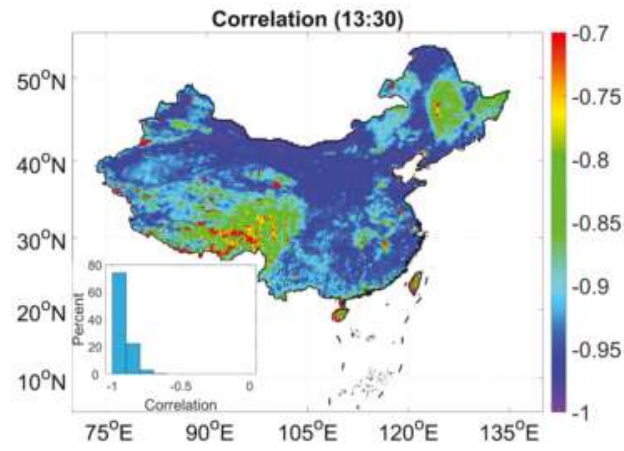

(a)

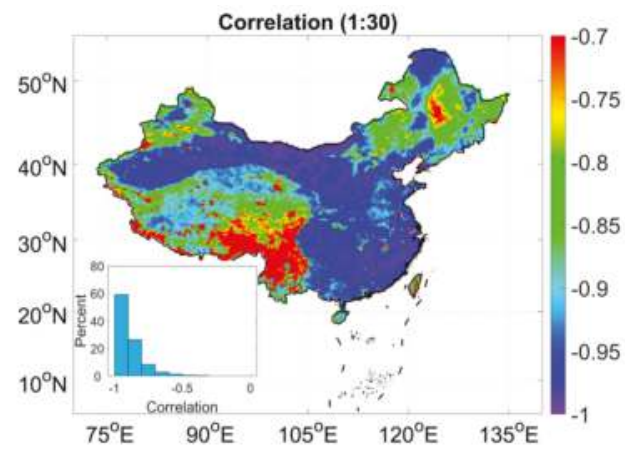

(b)

Figure 2. (a,b) are correlations of the MODIS LST and freeze/thaw index (FTI) for observations at 13:30 and $1: 30$, respectively.

The parameters "a" and "b" in Equation (5) for each pixel also vary spatially (Figure 3). The low values $(<-0.1)$ of "a", the slope of regression models, exists in the northeast and parts of Xinjiang province in China. In southeast and central parts, the slope is above -0.1 . About $70 \%$ of the pixels have a value between -0.1 and -0.06 for "a" observations at both 13:30 and 1:30. However, observations at 1:30 have more low values than that at 13:30. The slope of a model, as well as the intercept, are adjustments made to match the emissivity of MODIS in thermal bands to that of AMSR2 in the $36.5 \mathrm{GHz}$ band (Equations (4) and (5)). These two parameters depend on how many observations are used and at what time observations are acquired. Here, observations of only two years (2013 and 2014) were used to fit linear models for each pixel. Taking a pixel $\left(31^{\circ} \mathrm{N}, 91^{\circ} \mathrm{E}\right)$ as an example, 455 effective data pairs are selected for 13:30, while only 350 data pairs are used for observations at 1:30 to fit a model. In addition, the pattern of data pairs is not the same (subplots in Figure 3a,b). This pixel experiences both freezing and thawing at 13:30 during the two years, so some values of FTI are greater 
than 0 and some are less than 0 . However, for observations at 1:30, the status of this pixel is frozen most of the time, so many values of FTI are less than 0 .

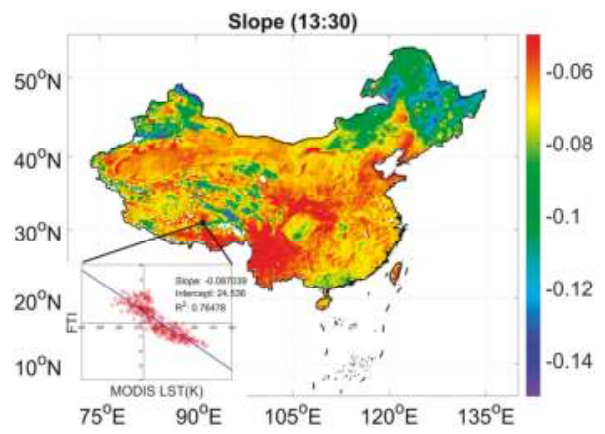

(a)

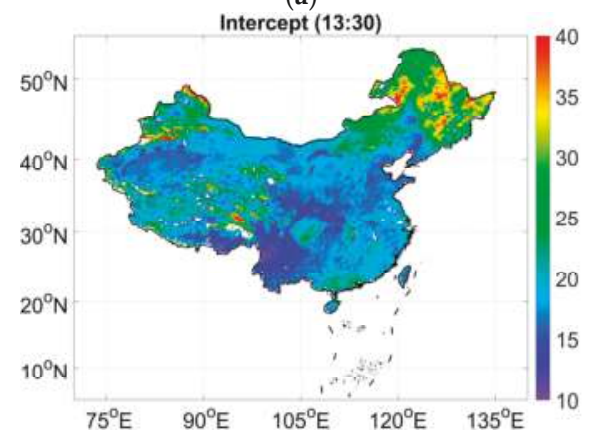

(c)

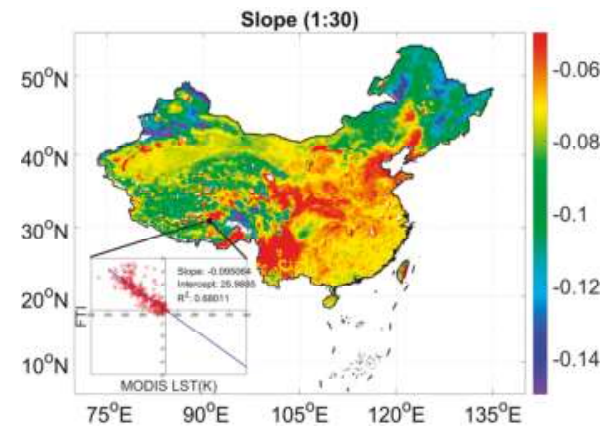

(b)

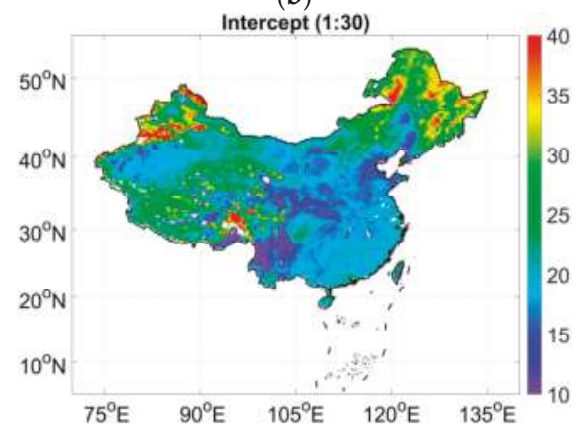

(d)

Figure 3. Spatial distribution of parameters " $a$ " and " $b$ " in regression models for observations at 13:30 and 1:30, respectively. $(\mathbf{a}, \mathbf{b})$ are for "a"; $(\mathbf{c}, \mathbf{d})$ are for " $b$ ".

\subsection{Daily High-Resolution F/T Map of China}

Daily high-resolution F/T maps are a combination of AMSR2-based and MODIS LST-based F/T maps (Figure 1). AMSR2-based F/T maps are determined by FTI, which is calculated by applying DFA to AMSR2 observations directly. Such a map has a coarse resolution of 0.25 degree, so we use "coarse-resolution" here to describe F/T maps based on passive microwave observations. MODIS LST-based FTI is calculated by applying Equation (5) to daily MODIS LST maps. Once the LST-based FTI was acquired, discrimination analysis is applied to each pixel to acquire high-resolution F/T maps. Such an F/T map has a resolution of 0.05 degree. Although 0.05 degrees is not actually high, we use "high-resolution" to represent the F/T map generated by the MODIS LST compared with that by passive microwave observations. The high-resolution F/T maps cover all land surfaces in China where F/T transitions probably occur and consist of five values, namely: frozen (0), thawing (1), no data (2), rain (3), and permanent snow (15). The rain information comes from coarse-resolution F/T maps. Permanent snow areas are labeled as 15 because they are determined by the MODIS-IGBP land cover classifications.

Coarse-resolution F/T maps not only provide information about precipitation, but also act as supplements where gaps or invalid data appear in MODIS observations. The gaps in high-resolution maps means observations are invalid for both MODIS and AMSR2, so they are smaller than that from either sensor. Since parts of gaps are filled, daily high-resolution F/T maps can provide much more information in a finer resolution than coarse-resolution F/T maps. As the high-resolution F/T maps 
are combined by information from both high-resolution and coarse-resolution, we estimated how much information from MODIS (actual high resolution) can be used for each day. Figure 4a shows that an average of $58.5 \%$ and $65.4 \%$ of the whole area can have actual high-resolution information at ascending and descending times, respectively. Moreover, the frequency of each pixel with actual high resolution in the year of 2013 varies spatially (Figure 4b,c). Areas in Western China have more days of high-resolution observations. Some areas, such as Sichuan, Guizhou, and Guangxi Provinces have fewer days of high-resolution observations. This pattern is much more obvious at the descending time.

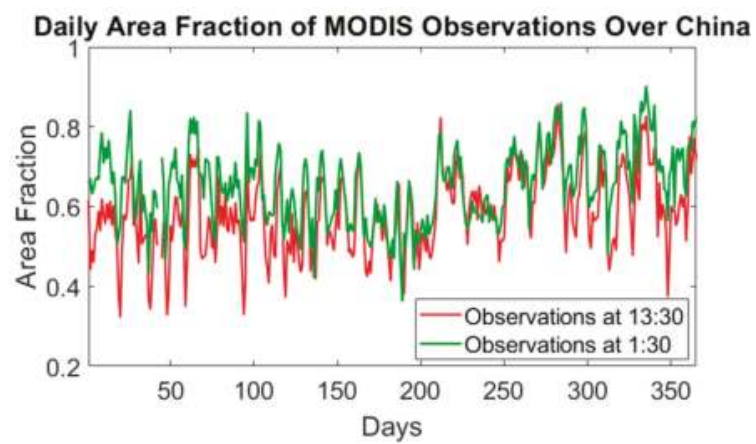

(a)

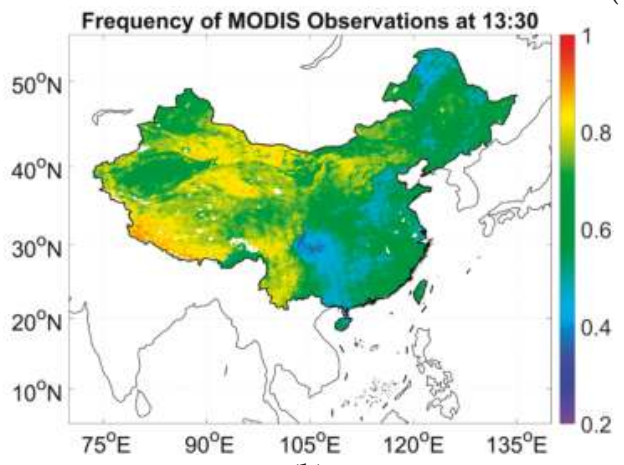

(b)

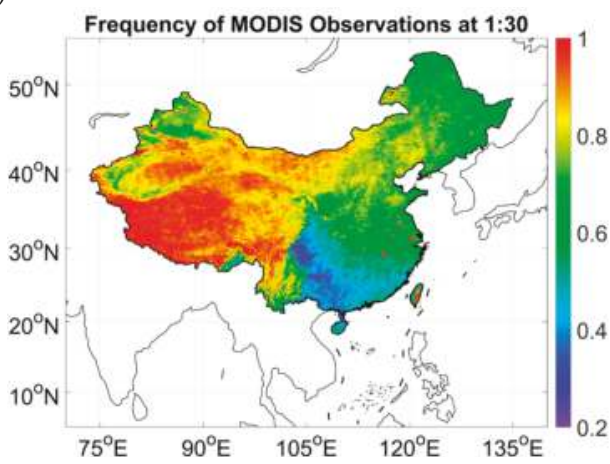

(c)

Figure 4. Coverage of MODIS observations over China in 2013 (a) and frequency of valid MODIS observations for each pixel at 13:30 (b) and 1:30 (c) in 2013.

\subsection{Agreement between the Ground Temperature and High-Resolution F/T Maps}

Agreements between high-resolution F/T maps and ground temperatures are calculated temporally and spatially. The temporal agreement was calculated separately at ascending (Figure 5a) and descending times (Figure 5b). Overall temporal agreements for ascending and descending times are $95.37 \%$ and $86.6 \%$, respectively. The high temporal agreements come from thaw status. $\mathrm{N}_{\text {TT }}$, the number of stations where the ground temperature is above 0 degrees and the pixels where stations are located are classified as thaw, take up more than $90 \%$ of the four components $\left(\mathrm{N}_{\mathrm{TT}}, \mathrm{N}_{\mathrm{TF}}, \mathrm{N}_{\mathrm{FF}}, \mathrm{N}_{\mathrm{FT}}\right)$ through the whole year, which means that many days in 2013 are thawing at around 13:30. The overall low agreement at 13:30 appears in late fall, winter, and early spring (the average accuracy is $93 \%$ in winter and $91.01 \%$ in spring) during which the $\mathrm{F} / \mathrm{T}$ transitions are occurring in most areas of the north of the Yangzi River in China. Low agreement in similar periods also appears for observations at $1: 30(82.1 \%$ in spring and $82.5 \%$ in winter), but the pattern of time-series agreement at $1: 30$ is slightly different from that at 13:30. Thawing components detected by both stations and satellite $\left(\mathrm{N}_{\mathrm{TT}}\right)$ 
are lower than frozen components $\left(\mathrm{N}_{\mathrm{FF}}\right)$ in winter and early spring. This is reasonable because the descending time is around 1:30 a.m., when the minimum temperature of a day is usually observed.

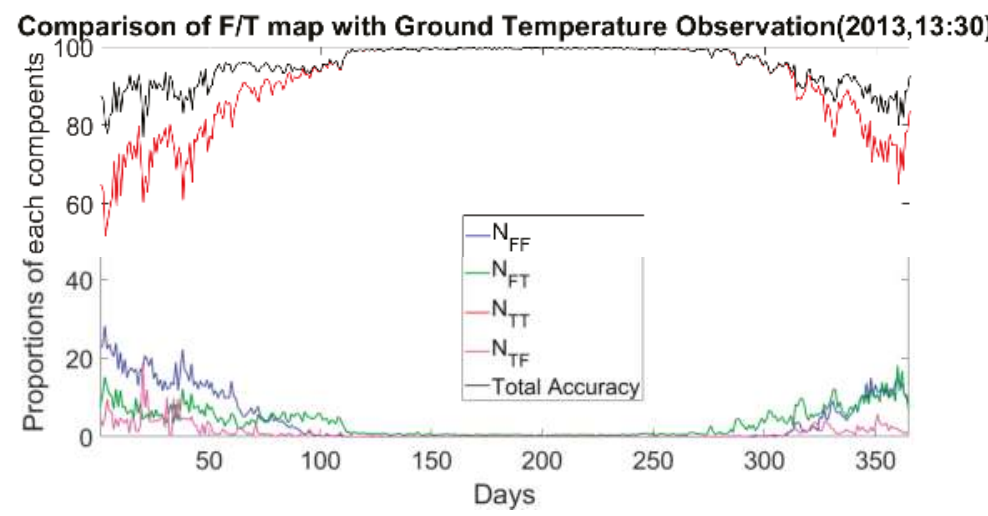

(a)

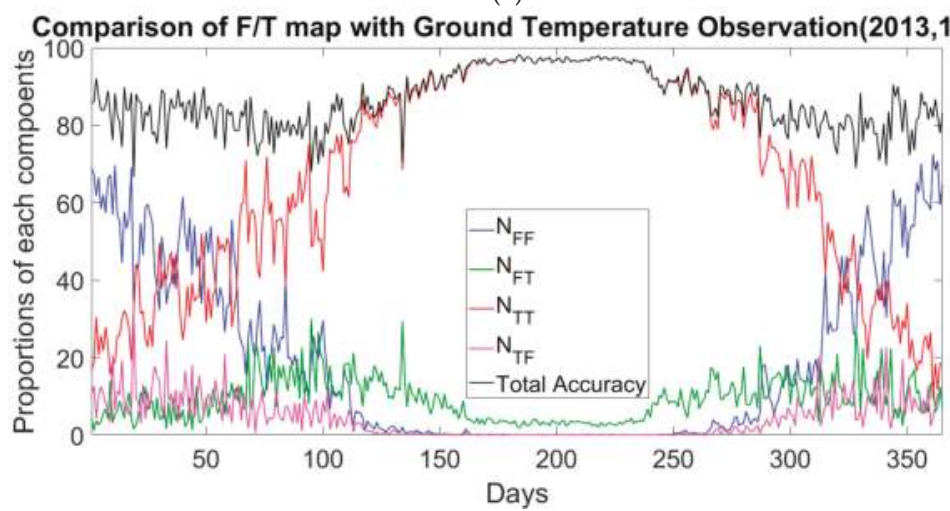

(b)

Figure 5. Temporal agreement between in situ measurement and F/T maps for observations at 13:30 (a) and 1:30 (b).

Figure 6 shows that high-resolution F/T maps have high agreement with in situ ground temperature (overall agreement: $91.25 \%$ ). The agreement is not distributed homogeneously across China, but the pattern is similar to that of correlation mentioned in Section 3.1. Areas with low agreement below $80 \%$ are mainly located in Western and Northeast China, the Yangzi River Delta, and other areas near the sea. The reasons for low agreement are various. In Western China, the meteorological stations are distributed sparsely, and not enough measurements may account for the low agreement. In addition, both the northwest and northeast are cold and arid zones, such as the Qinghai-Tibetan Plateau, Xinjiang, Gansu, Heilongjiang, Jilin, and Liaoning Province. These areas experience low mean annual temperature and large F/T dynamics. Other areas, for example, the Yangzi River Delta, Liaoning, and Shandong Peninsula, are near rivers or the sea. Locations near water may be an important factor leading to low accuracy [28]. 


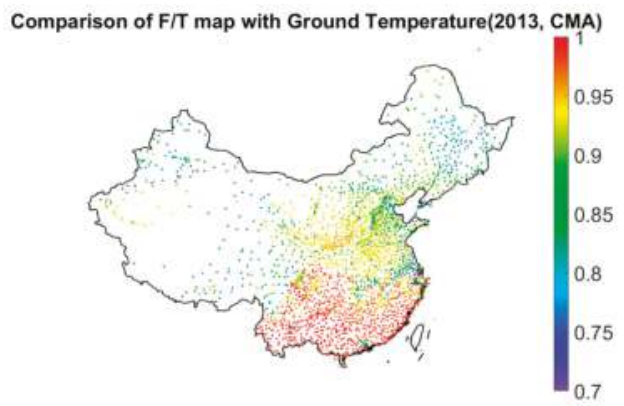

Figure 6. Accuracy assessment of F/T maps at individual meteorological stations in China.

\section{Discussion}

\subsection{High-Resolution F/T Maps}

Fusion of passive microwave and thermal observations to generate high-resolution $\mathrm{F} / \mathrm{T}$ maps is proved possible. However, fused F/T maps depend greatly on DFA, an algorithm derived for observations of AMSR-E. Although AMSR-E and AMSR2 have been reported to have slight differences [29,30], eliminating the difference is still necessary through inter-calibration before applying the same algorithm (e.g., DFA) to observations of these two sensors [31]. The data of AMSR2 used in this study have been calibrated with AMSR-E without many details being obtained [23]. The high-resolution F/T maps also depend significantly on thermal observations, especially the spatial resolution. Theoretically, the higher the resolution of thermal observations, the finer the fused F/T maps will be. For example, F/T maps with 1-km resolution are also possible to acquire if the LST products with 1-km resolution are used.

Increasing the resolution of land surface parameters (LST, albedo, soil moisture, etc.) has been of considerable interest for the land surface community as the improved knowledge of land surface parameters can enhance a wide range of applications and scientific studies, such as the community land model [32-36]. F/T maps derived by DFA or other algorithms [12] based on passive microwave observations usually have a spatial resolution of 0.25 degree. Such a coarse resolution limits the application of $\mathrm{F} / \mathrm{T}$ data in climate or hydrological models that consider land surface heterogeneity [14]. Finer resolution of $\mathrm{F} / \mathrm{T}$ maps are needed to meet the current scientific research requirements [15]. High-resolution F/T status detections by radar or scatterometer are considerably mature [37], but F/T maps with high resolution in large scales and short revisit times are still unavailable. The fusion of microwave observation-based F/T maps and thermal observations has been proved promising on QTP areas [17]. The similar approach has been tested in China and performs well. Theoretically, the study areas can be extended to the globe, and a long-time series F/T record can also be built using observations within the life span of AMSR2 and MODIS. However, impacts on F/T transitions from factors, for instance, snow, vegetation, topography, etc., are challenges faced by remote sensing observations [38]. The influence of land cover is weakness of the approach proposed here. More work is needed to tackle the influence the F/T dynamics caused by snow, vegetation, elevation, etc., at a finer scale.

\subsection{Strengths and Weaknesses of Fused F/T Maps}

The fused F/T maps are superior to those derived directly based on passive microwave or thermal observations alone in at least two aspects: more information and finer spatial resolution (at least part of the map actually has high resolution). Compared with F/T maps derived directly from passive microwave observations, much finer information was introduced to the $\mathrm{F} / \mathrm{T}$ maps, which is more promising for future use in many regional studies [33] or land models [14,32]. Compared with the F/T status determined by the MODIS LST threshold (we use $0{ }^{\circ} \mathrm{C}$ as a cutoff: frozen if a pixel lower or equal 
0 , otherwise thawing), fused maps provide more effective observations and without losing accuracy. Table 2 is a case study using several randomly-selected meteorological stations for different land cover types. MODIS LST has many observations of barren areas, such as Qinghai Province (Figure $4 b, c$ ), with high agreement with station measurements, but it has fewer observations in areas with a large amount of vegetation and high elevation (e.g., station 56649). For fused F/T maps, both accuracy and effective observations are very stable in different kinds of areas and land cover types.

Table 2. Comparision of F/T status determined by the MODIS LST threshod $\left(0^{\circ} \mathrm{C}\right)$ and fused F/T maps.

\begin{tabular}{cccccccc}
\hline Station ID & Land Cover & $\begin{array}{c}\text { Latitude } \\
\left({ }^{\circ} \mathbf{N}\right)\end{array}$ & $\begin{array}{c}\text { Lontitude } \\
\left({ }^{\circ} \mathbf{E}\right)\end{array}$ & Accuracy_MOD & $\begin{array}{c}\text { Effective } \\
\text { Observations (MOD) }\end{array}$ & $\begin{array}{c}\text { Effective } \\
\text { Accuracy_F } \\
\text { Observations (F) }\end{array}$ \\
\hline 51777 & Barren areas & 39.033 & 88.167 & $93.27 \%$ & 271 & $89.63 \%$ & 363 \\
56067 & Gras land (1) ${ }^{1}$ & 33.433 & 101.48 & $80.76 \%$ & 260 & $86.96 \%$ & 363 \\
54012 & Grassland (2) & & & & \\
50434 & Forest (1) & 44.567 & 117.63 & $89.91 \%$ & 238 & $87.68 \%$ & 363 \\
50349 & Forest (2) & 50.483 & 121.68 & $93.47 \%$ & 230 & $90.51 \%$ & 363 \\
56649 & Forest (3) & 51.667 & 124.4 & $90.91 \%$ & 231 & $36.33 \%$ & $36.80 \%$ \\
58011 & Cropland & 21.6 & 99.967 & $77.38 \%$ & 183 & $90.89 \%$ & 363 \\
57343 & Deciduous Forest & 34.8 & 116.07 & $86.34 \%$ & 201 & $93.36 \%$ & 363 \\
59205 & Savanna & 23.65 & 109.53 & $86.07 \%$ & 119 & $99.42 \%$ & 363 \\
\hline
\end{tabular}

1: Grassland (1) is located on the QTP; Grassland (2): Inner Monngolia; Forest (1): Daxinganling Forest; Forest (2): Xiaoxinganling Forest; Forest (3): the Hengduan Mountains.

The fused F/T maps also have some limitations because they depended on the match of MODIS LST with microwave observations. The correlation of and coefficents ("a" and "b" in Equation (5)) of the linear regression model will be influenced by many factors, for instance, land cover types, vegetaion, elevation, seasonality, etc. Figure 7 shows how correlation varies with different elevations between the MODIS LST and FTI derived by microwave observations. For both observations at 13:30 and 1:30, the rise in elevation is companied with the decrease in correlation, and correlation remains high and stable when elevations are below $2000 \mathrm{~m}$. We also investigated how correlations vary with Normalized Difference Vegetation Index (NDVI) at different sites with different land cover (Figure 8). NDVI also shows a negative influence on correlation, especially for observations at 1:30 with extreme high NDVI values. We guess that MODIS measures the temperature of vegetation while AMSR2 can penetrate vegetation to measure near surface temperature of the ground in forest areas. Another limitation for the regression models is that parameters " $a$ " and " $\mathrm{b}$ " for each pixel are fixed at different seasons through the year. However, as they are adjusted to approximate the emissivity of MODIS at thermal bands to AMSR2 in the $36.5 \mathrm{GHz}$ band, they may vary at different times of the year, especially as land cover changes with time, for example, vegetation (Figure A1) and snow cover. This problem is not solved in this study, but the analysis provides directions on how to improve our regression model; for example, we can let "a" and "b" vary with NDVI.

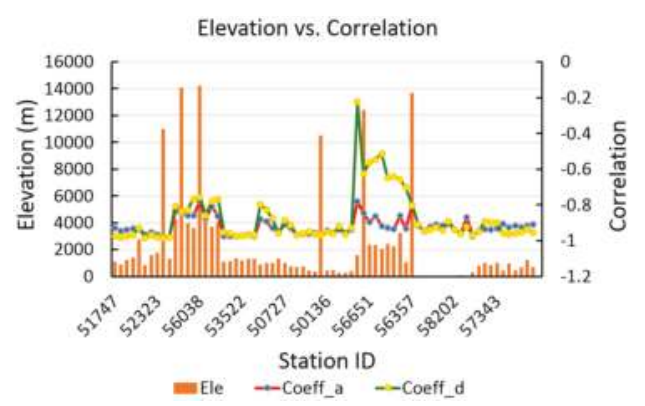

Figure 7. Correlation of the MODIS LST and FTI derived by microwave observations from 70 randomly-selected stations at different elevations. Ele is the elevation of the stations; Coeff_a is the correaltion at 13:30; Coeff_d is the correlation at 1:30. 


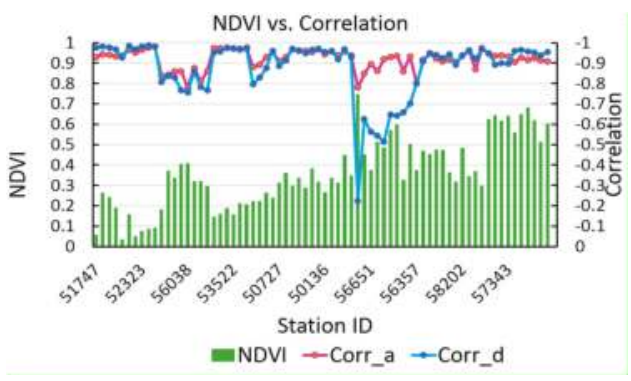

Figure 8. Correlation of the MODIS LST and FTI derived by microwave observations from 70 randomly-selected stations with different annual average NDVI. Corr_a is the correaltion at 13:30; Corr_d is the correlation at 1:30.

\subsection{Accuracy Estimate by Ground Temperature}

The accuracy estimation shows that the high-resolution F/T maps have high agreement with ground temperature. However, the main concern is, and has been argued for a long time, that the 0 -cm temperature on the ground is explicit to define the $\mathrm{F} / \mathrm{T}$ state of the land surface. The ground may be thawing when the 0 -cm temperature is equal, or less than, $0^{\circ} \mathrm{C}$, and factors, such as wind or land cover (snow, vegetation, etc.) may also lead to the thaw status. However, until now, there has been no better criteria to define the absolute real boundary of the temperature when the ground is frozen or thawing. Compared with other choices, for example, air temperature [4] and soil temperatures at different depths or from models [16], 0-cm ground temperature may be a better choice because it reflects the surface state of the ground. This "surface " is probably what remote sensing is detecting.

\section{Conclusions}

$\mathrm{F} / \mathrm{T}$ transitions of near surface soil have been investigated by microwave remote sensing, but most of them are in a coarse resolution or in a small area. Fine-resolution F/T status data records at a large scale and short revisit time are still unavailable presently. Thus, a new approach to detecting $\mathrm{F} / \mathrm{T}$ status was tested and evaluated in a large area (China) in this study. This approach takes advantage of passive microwave and thermal remote sensing observations to map F/T status in a high spatial resolution ( 0.05 degree). This high-resolution $\mathrm{F} / \mathrm{T}$ map is a fusion of the coarse-resolution F/T maps from AMSR2 and MODIS LST-based FTI. Then the high-resolution F/T map was validated using 0-cm ground temperatures across China. The following conclusions can be drawn from this paper:

(1) The determination function algorithm (DFA) provides an interface between passive microwave and thermal observations. Their relationship can be described using a linear regression model through the parameter FTI.

(2) The regression model applied to MODIS temperature can provide high-resolution F/T maps which are promising to fill the gap when no other high-resolution F/T maps can cover the globe or a large area.

(3) The high-resolution F/T maps show high agreement with 0 -cm ground temperatures with spatial agreement above $90 \%$ and temporal agreement around $95.37 \%$ and $86.62 \%$ for ascending and descending passes, respectively. The high agreement enhances the reliability of the derived high-resolution F/T maps which can be used for further research in Earth sciences.

High-resolution $\mathrm{F} / \mathrm{T}$ maps by fusing multi-source remote sensing data have several advantages: providing much more $\mathrm{F} / \mathrm{T}$ information than those relying purely on passive microwave remote sensing; and having fewer gaps in the image than that from either AMSR2 or MODIS. Both microwave and thermal sensors have their own advantages, fused F/T maps reach a balance between temporal and 
spatial resolution of remote sensing observations. Although the approach used here is proved to be promising, it still has many limitations because of the influence of topography, seasonality, vegetation cover, etc. Future studies of incorporating NDIV, emissivity, and flexible coefficients varying with time are highly recommended to improve the approach.

Acknowledgments: This study was jointly supported by the National Key Basic Research Program of China (2015CB953701), the National Natural Science Foundation of China (41671355), the Chinese Academy of Sciences "Light of West China" Program, and the Youth Innovation Promotion Association (no. 2016061). K.Z. acknowledges the supports from the Open Research Fund from the State Key Laboratory of Digital Earth Science, Institute of Remote Sensing and Digital Earth, Chinese Academy of Sciences(OFSLRSS201604) and a Microsoft Azure Research Award (CRM:0518513). All data of AMSR-E and MODIS are obtained from the National Snow and Ice Data Center (NSIDC). The ground temperature data are from the China Meteorological Administration.

Author Contributions: T.H., T.Z., J.S., and K.Z. contributed to intellectual design and initial drafting. T.H. compiled and processed data. T.H., T.Z., S.W. and K.Z. contributed to data analysis and interpretation. D.L. and H.Q. assisted with graphing and the manuscript revision. All the authors contributed to the writing of the final draft.

Conflicts of Interest: The authors declare no conflict of interest.

\section{Appendix A}

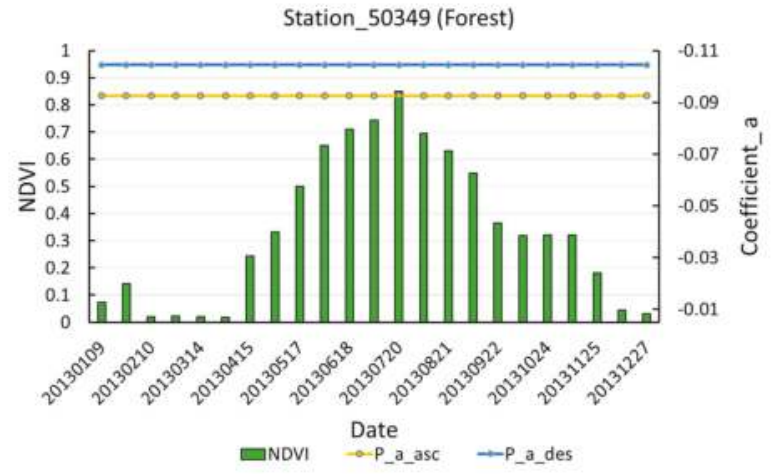

(a)

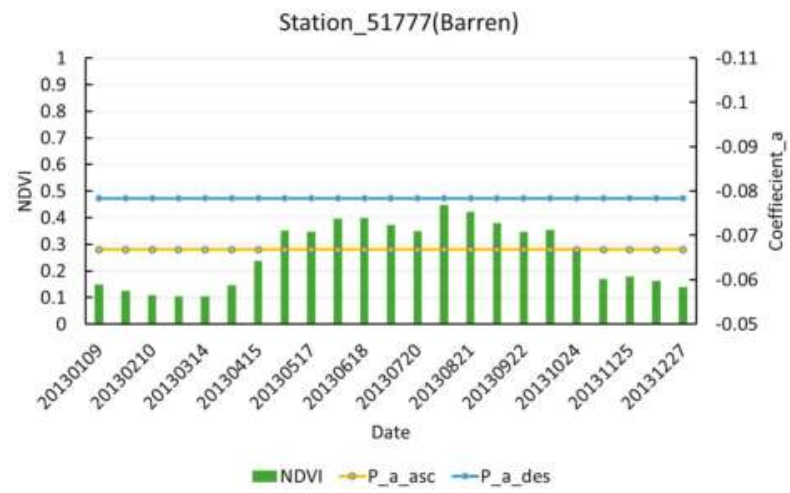

(b)

Figure A1. Cont. 


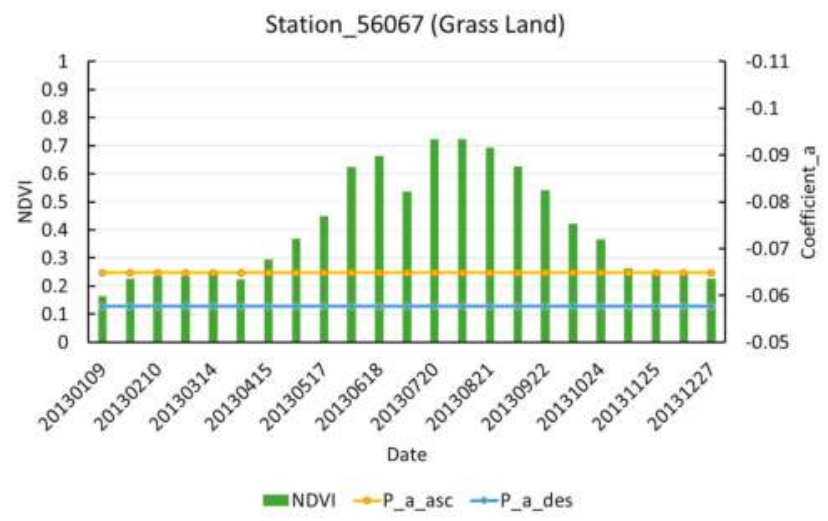

(c)

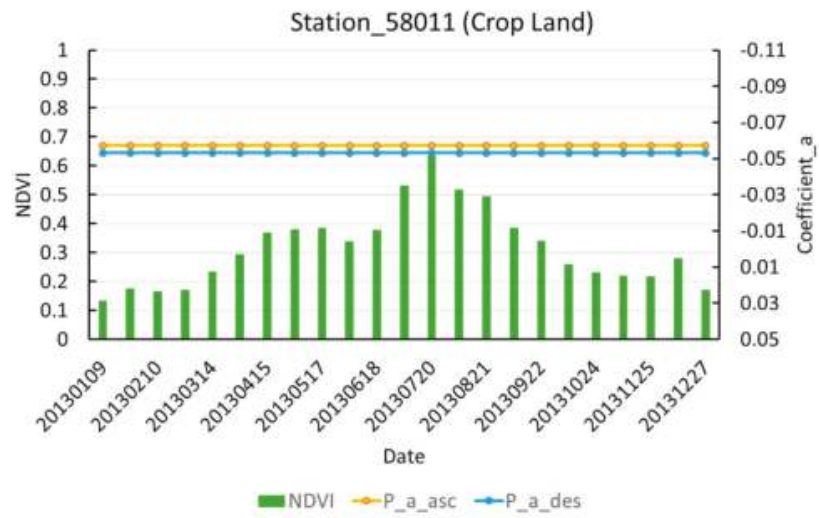

(d)

Figure A1. Parameter "a" and NDVI on forest (a), barren (b), grass land (c) and crop land (d). P_a_asc and P_a_des are for observations at 13:30 and 1:30, respectively.

\section{References}

1. Zhang, T.; Barry, R.; Knowles, K.; Ling, F.; Armstrong, R. Distribution of seasonally and perennially frozen ground in the Northern Hemisphere. In Proceedings of the 8th International Conference on Permafrost, Zurich, Switzerland, 21-25 July 2003; AA Balkema Publishers: Amsterdam, The Netherlands, 2003; pp. 1289-1294.

2. McDonald, K.C.; Kimball, J.S. Estimation of surface freeze-thaw states using microwave sensors. In Encyclopedia of Hydrological Sciences; John Wiley \& Sons, Ltd.: Hoboken, NJ, USA, 2005.

3. Intergovernmental Panel on Climate Change (IPCC). Climate Change 2013: The Physical Science Basis. Contribution of Working Group I to the Fifth Assessment Report of the Intergovernmental Panel on Climate Change; Stocker, T.F., Qin, D., Plattner, G.-K., Tignor, M., Allen, S.K., Boschung, J., Nauels, A., Xia, Y., Bex, V., Midgley, P.M., Eds.; Cambridge University Press: Cambridge, UK; New York, NY, USA, 2013; p. 1535.

4. Kim, Y.; Kimball, J.S.; McDonald, K.C.; Glassy, J. Developing a global data record of daily landscape freeze/thaw status using satellite passive microwave remote sensing. IEEE Trans. Geosci. Remote Sens. 2011, 49, 949-960. [CrossRef] 
5. Kimball, J.S.; McDonald, K.C.; Keyser, A.R.; Frolking, S.; Running, S.W. Application of the NASA scatterometer (NSCAT) for determining the daily frozen and nonfrozen landscape of Alaska. Remote Sens. Environ. 2001, 75, 113-126. [CrossRef]

6. Wagner-Riddle, C.; Congreves, K.A.; Abalos, D.; Berg, A.A.; Brown, S.E.; Ambadan, J.T.; Gao, X.; Tenuta, M. Globally important nitrous oxide emissions from croplands induced by freeze-thaw cycles. Nat. Geosci. 2017, 10, 279-283. [CrossRef]

7. Global Climate Observing System. The Global Observing System for Climate: Implementation Needs; Global Climate Observing System: Guayaquil, Ecuador, 2016.

8. Entekhabi, D.; Njoku, E.G.; O’Neill, P.E.; Kellogg, K.H.; Crow, W.T.; Edelstein, W.N.; Entin, J.K.; Goodman, S.D.; Jackson, T.J.; Johnson, J. The soil moisture active passive (SMAP) mission. Proc. IEEE 2010, 98, 704-716. [CrossRef]

9. Zhang, T.; Armstrong, R. Soil freeze/thaw cycles over snow-free land detected by passive microwave remote sensing. Geophys. Res. Lett. 2001, 28, 763-766. [CrossRef]

10. Ulaby, F.T.; Moore, R.K.; Fung, A.K. Microwave Remote Sensing Active and Passive-Volume III: From Theory to Applications; Artech House, Inc.: Norwood, MA, USA, 1986.

11. Jin, R.; Li, X.; Che, T. A decision tree algorithm for surface soil freeze/thaw classification over China using SSM/I brightness temperature. Remote Sens. Environ. 2009, 113, 2651-2660. [CrossRef]

12. Kim, Y.; Kimball, J.S.; Glassy, J.; Du, J. An extended global earth system data record on daily landscape freeze-thaw status determined from satellite passive microwave remote sensing. Earth Syst. Sci. Data 2017, 9 , 133-147. [CrossRef]

13. Zhao, T.; Zhang, L.; Jiang, L.; Zhao, S.; Chai, L.; Jin, R. A new soil freeze/thaw discriminant algorithm using AMSR-E passive microwave imagery. Hydrol. Process. 2011, 25, 1704-1716. [CrossRef]

14. Chakrabarti, S.; Bongiovanni, T.; Judge, J.; Nagarajan, K.; Principe, J.C. Downscaling satellite-based soil moisture in heterogeneous regions using high-resolution remote sensing products and information theory: A synthetic study. IEEE Trans. Geosci. Remote Sens. 2015, 53, 85-101. [CrossRef]

15. Shi, J.; Dong, X.; Zhao, T.; Du, J.; Jiang, L.; Du, Y.; Liu, H.; Wang, Z.; Ji, D.; Xiong, C. WCOM: The science scenario and objectives of a global water cycle observation mission. In Proceedings of the 2014 IEEE International Geoscience and Remote Sensing Symposium (IGARSS), Quebec City, QC, Canada, 13-18 July 2014; pp. 3646-3649.

16. Zwieback, S.; Paulik, C.; Wagner, W. Frozen soil detection based on advanced scatterometer observations and air temperature data as part of soil moisture retrieval. Remote Sens. 2015, 7, 3206-3231. [CrossRef]

17. Zhao, T.; Shi, J.; Hu, T.; Zhao, L.; Zou, D.; Wang, T.; Ji, D.; Li, R.; Wang, P. Estimation of high-resolution near-surface freeze/ thaw state by the integration of microwave and thermal infrared remote sensing data on the Tibetan Plateau. In Earth and Space Science; Wiley: Hoboken, NJ, USA, 2017.

18. Wentz, F.; Meissner, T.; Gentemann, C.; Hilburn, K.; Scott, J. Remote Sensing Systems GCOM-W1 AMSR2 Daily Environmental Suite on 0.25 Deg Grid Version 7.2; Remote Sensing Systems: Santa Rosa, CA, USA, 2014.

19. Gcom-W1 Data Providing Service. Available online: https://gcom-w1.jaxa.jp/auth.html (accessed on 23 Octorber 2016).

20. Wan, Z. New refinements and validation of the modis land-surface temperature/emissivity products. Remote Sens. Environ. 2008, 112, 59-74. [CrossRef]

21. China Meteorological Data Service Center (CMDC). Available online: http://data.cma.cn/en/?r=data/ detail\&dataCode=A.0029.0004 (accessed on 12 December 2016).

22. Chai, L.N.; Zhang, L.X.; Zhang, Y.Y.; Hao, Z.G.; Jiang, L.M.; Zhao, S.J. Comparison of the classification accuracy of three soil freeze-thaw discrimination algorithms in China using SSMIS and AMSR-E passive microwave imagery. Int. Remote. Sens. 2014, 35, 7631-7649. [CrossRef]

23. Hu, T.; Zhao, T.; Shi, J.; Wang, T.; Ji, D.; Al Bitar, A.; Peng, B.; Cui, Y. Development and analysis of a continuous record of global near-surface soil freeze/thaw patterns from AMSR-E and AMSR2 data. Cryosphere Discuss. 2016. [CrossRef]

24. Welling, M. Fisher Linear Discriminant Analysis; Department of Computer Science, University of Toronto: Toronto, ON, Canada, 2005; Volume 3.

25. Ran, Y.; Li, X.; Cheng, G.; Zhang, T.; Wu, Q.; Jin, H.; Jin, R. Distribution of permafrost in China: An overview of existing permafrost maps. Permafr. Periglac. Process. 2012, 23, 322-333. [CrossRef] 
26. Shi, Q.; Liang, S. Characterizing the surface radiation budget over the Tibetan Plateau with ground-measured, reanalysis, and remote sensing data sets: 1. Methodology. J. Geophys. Res. Atmos. 2013, 118, 9642-9657. [CrossRef]

27. Chen, Y.; Yang, K.; Qin, J.; Zhao, L.; Tang, W.; Han, M. Evaluation of AMSR-E retrievals and GLDAS simulations against observations of a soil moisture network on the central Tibetan Plateau. J. Geophys. Res. Atmos. 2013, 118, 4466-4475. [CrossRef]

28. Kim, Y.; Kimball, J.S.; Du, J.; Glassy, J.M. Improvements and Extension to a Global Earth System Data Record of Daily Landscape Freeze-Thaw Status Determined from Satellite Microwave Remote Sensing; University of Montana: Missoula, MT, USA, 2014.

29. Imaoka, K. Jaxa Agency Report; Earth Observation Research Center (EORC) Japan Aerospace Exploration Agency (JAXA): Tokyo, Japan, 2016.

30. Parinussa, R.M.; Holmes, T.R.; Wanders, N.; Dorigo, W.A.; de Jeu, R.A. A preliminary study toward consistent soil moisture from AMSR2. J. Hydrometeorol. 2015, 16, 932-947. [CrossRef]

31. Du, J.; Kimball, J.S.; Shi, J.; Jones, L.A.; Wu, S.; Sun, R.; Yang, H. Inter-calibration of satellite passive microwave land observations from AMSR-E and AMSR2 using overlapping FY3B-MWRI sensor measurements. Remote Sens. 2014, 6, 8594-8616. [CrossRef]

32. Ke, Y.; Leung, L.; Huang, M.; Coleman, A.M.; Li, H.; Wigmosta, M.S. Development of high resolution land surface parameters for the community land model. Geosci. Model Dev. 2012, 5, 1341-1362. [CrossRef]

33. Song, C.; Jia, L.; Menenti, M. Retrieving high-resolution surface soil moisture by downscaling AMSR-E brightness temperature using MODIS LST and NDVI data. IEEE J. Sel. Top. Appl. Earth Obs. Remote Sens. 2014, 7, 935-942. [CrossRef]

34. Xue, H.; Wang, J.; Xiao, Z.; Chen, P.; Liu, Y. Combining modis and AMSR-E observations to improve MCD43A3 short-time snow-covered albedo estimation. Hydrol. Process. 2014, 28, 570-580. [CrossRef]

35. Parinussa, R.M.; Lakshmi, V.; Johnson, F.; Sharma, A. Comparing and combining remotely sensed land surface temperature products for improved hydrological applications. Remote Sens. 2016, 8, 162. [CrossRef]

36. Shwetha, H.; Kumar, D.N. Prediction of high spatio-temporal resolution land surface temperature under cloudy conditions using microwave vegetation index and ANN. ISPRS J. Photogramm. Remote Sens. 2016, 117, 40-55. [CrossRef]

37. Du, J.; Kimball, J.S.; Azarderakhsh, M.; Dunbar, R.S.; Moghaddam, M.; McDonald, K.C. Classification of Alaska spring thaw characteristics using satellite L-band radar remote sensing. IEEE Trans. Geosci. Remote Sens. 2015, 53, 542-556.

38. Roy, A.; Toose, P.; Williamson, M.; Rowlandson, T.; Derksen, C.; Royer, A.; Berg, A.A.; Lemmetyinen, J.; Arnold, L. Response of L-band brightness temperatures to freeze/thaw and snow dynamics in a prairie environment from ground-based radiometer measurements. Remote Sens. Environ. 2017, 191, 67-80. [CrossRef]

(C) 2017 by the authors. Licensee MDPI, Basel, Switzerland. This article is an open access article distributed under the terms and conditions of the Creative Commons Attribution (CC BY) license (http:/ / creativecommons.org/licenses/by/4.0/). 
Article

\title{
Assessment of Methods for Passive Microwave Snow Cover Mapping Using FY-3C/MWRI Data in China
}

\author{
Xiaojing Liu ${ }^{1}$, Lingmei Jiang ${ }^{1, *}$, Shengli $\mathrm{Wu}^{2}$, Shirui Hao ${ }^{1}$, Gongxue Wang ${ }^{1}$ and Jianwei Yang ${ }^{1}$ \\ 1 State Key Laboratory of Remote Sensing Science, Jointly Sponsored by Beijing Normal University and \\ Institute of Remote Sensing and Digital Earth of Chinese Academy of Sciences, Faculty of Geographical \\ Science, Beijing Normal University and Joint Center for Global Change Studies, Beijing 100875, China; \\ lxjing@mail.bnu.edu.cn (X.L.); kydhao@126.com (S.H.); wanggx@mail.bnu.edu.cn (G.W.); \\ stu_yangjiangwei@163.com (J.Y.) \\ 2 National Satellite Meteorological Center, China Meteorological Administration, Beijing 100081, China; \\ wusl@cma.gov.cn \\ * Correspondence: jiang@bnu.edu.cn; Tel.: +86-10-5880-5042
}

Received: 10 January 2018; Accepted: 23 March 2018; Published: 27 March 2018

\begin{abstract}
Ongoing information on snow and its extent is critical for understanding global water and energy cycles. Passive microwave data have been widely used in snow cover mapping given their long-time observation capabilities under all-weather conditions. However, assessments of different passive microwave (PMW) snow cover area (SCA) mapping algorithms have rarely been reported, especially in China. In this study, the performances of seven PMW SCA mapping algorithms were tested using in situ snow depth measurements and a one-kilometer Interactive Multisensor Snow and Ice Mapping System (IMS) snow cover product over China. The selected algorithms are the FY3 algorithm, Grody's algorithm, the South China algorithm, Kelly's algorithm, Singh's algorithm, Hall's algorithm and Neal's algorithm. During the test period, most algorithms performed reasonably well. The overall accuracy of all algorithms is higher than 0.895 against in situ observations and higher than 0.713 against the IMS product. In general, Singh's algorithm, Hall's algorithm and Neal's algorithm had poor performance during the test. Their misclassification errors were larger than those of the remaining algorithms. Grody's algorithm, the South China algorithm and Kelly's algorithm had higher positive predictive values and lower omission errors than those of the others. The errors of these three algorithms were mainly caused by variations in commission errors. Comparing to Grody's algorithm, the South China algorithm and Kelly's algorithm, the FY3 algorithm presented a conservative snow cover estimation to balance the problem between snow identification and overestimation. As a result, the overall accuracy of the FY3 algorithm was the highest of all the tested algorithms. The accuracy of all algorithms tended to decline with a decreased snow cover fraction as well as $\mathrm{SD}<5 \mathrm{~cm}$. All tested algorithms have severe omission errors over barren land and grasslands. The results shown in this study contribute to ongoing efforts to improve the performance and applicability of PMW SCA algorithms.
\end{abstract}

Keywords: snow cover; passive microwave; FY-3C/MWRI; algorithmic assessment; China

\section{Introduction}

Snow cover is an important geophysical parameter for understanding global climate change, the radiation budget and the water cycle [1,2]. Given the importance of snow, snow cover extent has been a key observation target since the beginning of the satellite remote-sensing era dating to the mid-1960s [3]. Snow cover area (SCA) monitoring using optical and microwave sensors has been reported for decades [4]. A number of snow cover detection algorithms using optical sensors have been developed since the 1980s [5-9]. However, snow cover maps derived from optical sensors are strongly 
influenced by observation conditions such as cloud obscuration and solar illumination. Inaccessibility in cloud cover and weak sun exposure regions greatly limit the applicability of optical SCA products in regional and global applications [4].

Passive microwave (PMW) observation is another data source for SCA detection [10-12]. Dry snow is a type of microwave scattering material and can be identified by its volume scattering signature. The positive brightness temperature $(\mathrm{Tb})$ gradient between low and high frequencies is a crucial criterion for snow cover identification [13]. Most PMW SCA detection algorithms are based on a decision tree classification approach. Snow can be distinguished from other scattering or non-scattering surfaces via various filters. These algorithms can be divided into three groups: (1) identify snow with detailed types of snow [14,15]; (2) identify snow and non-snow types simultaneously [16,17]; and (3) simply identify snow without any in-depth information [18-20].

The primary advantage of using PMW data is the ability of microwaves to observe land surface conditions through clouds during day and night. At present, the PMW SCA products have been mainly used to fill the cloud gap of long-term optical SCA products [21-24] or act as a preprocessing step for producing PMW snow water equivalent (SWE) and snow depth (SD) products [25-28]. Errors inherent to PMW SCA products propagate into and corrupt the combined products. The false snow and snow-free identifications affect the accuracy of the associated SD, SWE and SCA products. Thus, an in-depth evaluation is needed to understand the uncertainty of PMW SCA mapping methods as well as to develop new methods.

In this study, seven PMW SCA mapping methods were tested, including Kelly's algorithm [14], the FY3 algorithm [15], Grody's algorithm [16], the South China algorithm [17], Singh's algorithm [18], and Hall's algorithm [19], Neal's algorithm [20]. These algorithms were selected because they are well-documented, have been successfully applied and have an indicative effect on later research. Common approaches for evaluating satellite-derived SCA would be to compare it to in situ measurements $[11,29,30]$ or satellite images with higher spatial resolutions $[8,31-34]$. We used similar strategies in this assessment. The performance of different PMW SCA mapping algorithms was evaluated against in situ snow depth measurements along with the Multisensor Snow and Ice Mapping System (IMS) snow cover product at a one-kilometer resolution. The one-kilometer IMS snow cover product was taken as a validation dataset because it is cloud-free and of high spatial resolution (compared to PMW observations) and is a high-quality SCA product.

This paper is organized as follows. Section 2 describes the data used and the PMW SCA algorithms. Section 3 presents the evaluation results and the effects of land cover, snow cover fraction (SCF), and snow depth on SCA mapping accuracy. Section 4 is dedicated to the discussion of the tested algorithms. Finally, a conclusion has been presented in Section 5 for the whole work of this paper.

\section{Data and Methodology}

In this study, FY3C-MWRI data were used for snow cover mapping. In situ snow depth observations together with the IMS snow cover product were used to evaluate different PMW SCA mapping algorithms. The data and algorithms are described in detail in the following subsections.

\subsection{FY-3C/MWRI Data}

The FY-3C satellite is one of the second generation polar-orbit meteorological satellite series of China. The FY-3C satellite was launched on 23 September 2013 with the goal of observing global atmospheric and geophysical features around the clock. The Microwave Radiation Imager (MWRI) is one of the 13 remote-sensing instruments onboard the FY-3C satellite. MWRI is a ten-channel, five-frequency, PMW radiometer system. It measures horizontally and vertically polarized brightness temperatures ranging from $10.65 \mathrm{GHz}$ to $89 \mathrm{GHz}$. The local time on the descending node (LTDN) is near 10:00 a.m. Spatial resolution of the individual measurements varies from $7.5 \mathrm{~km} \times 12 \mathrm{~km}$ at $89 \mathrm{GHz}$ to $51 \mathrm{~km} \times 85 \mathrm{~km}$ at $10.65 \mathrm{GHz}$. The FY3C-MWRI L1 swath data are 
available from the China Meteorological Administration/National Satellite Meteorological Center website (http:/ / www.nsmc.org.cn/).

\subsection{In Situ Measurements}

Daily meteorological data are provided by the National Meteorological Information Center, China Meteorological Administration. Daily SD observations from 753 stations (Figure 1) were used to evaluate the PMW snow cover detection algorithms during the snow season. Recorded variables of weather stations include site name, observation time, site location (latitude and longitude in degrees), geodetic elevation $(\mathrm{m})$, surface temperature and snow depth $(\mathrm{cm})$. The records were selected only if the surface temperature was less than $0{ }^{\circ} \mathrm{C}$ to avoid the impact of wet snow.

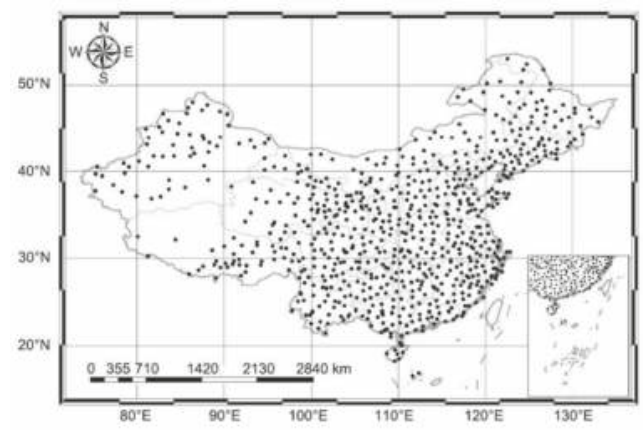

Figure 1. Chinese meteorological stations used in this work.

\subsection{IMS Data}

The IMS snow cover product of the National Ice Center combines multiple data sources to map daily cloud-free snow extent of the Northern Hemisphere at three different resolutions: $1 \mathrm{~km}, 4 \mathrm{~km}$ and $24 \mathrm{~km}$. The 1-km IMS data became available during December of 2014. In this study, 1-km IMS data were used as the validation dataset. The data were obtained from the National Snow and Ice Data Center (http://nsidc.org/).

\subsection{PMW Snow Cover Mapping Algorithms}

Dry snow is a type of strong scattering material. Snow cover produces a positive brightness temperature gradient between low- and high-frequency channels [13]. Although this characteristic of scattering materials identifies snow, it identifies other scattering materials such as precipitation, deserts, and frozen ground [10] because these non-snow types may produce a spectral response in the microwave similar to that of snow. To objectively detect snow cover, various filters are used to separate scattering signals of snow cover from other scattering and non-scattering surfaces [35].

Seven PMW SCA mapping algorithms were selected for evaluation in this paper. Classification criteria of these algorithms are shown in Table 1 . The frequencies listed in Table 1 are subject to FY-3C/MWRI. Grody's algorithm and the South China algorithm identify snow and the non-snow types (precipitation, cold deserts and frozen soil) simultaneously. The FY3 algorithm and Kelly's algorithm not only identify snow-covered areas but also divide snow into detailed categories. The last three algorithms are simply designed to detect snow. 


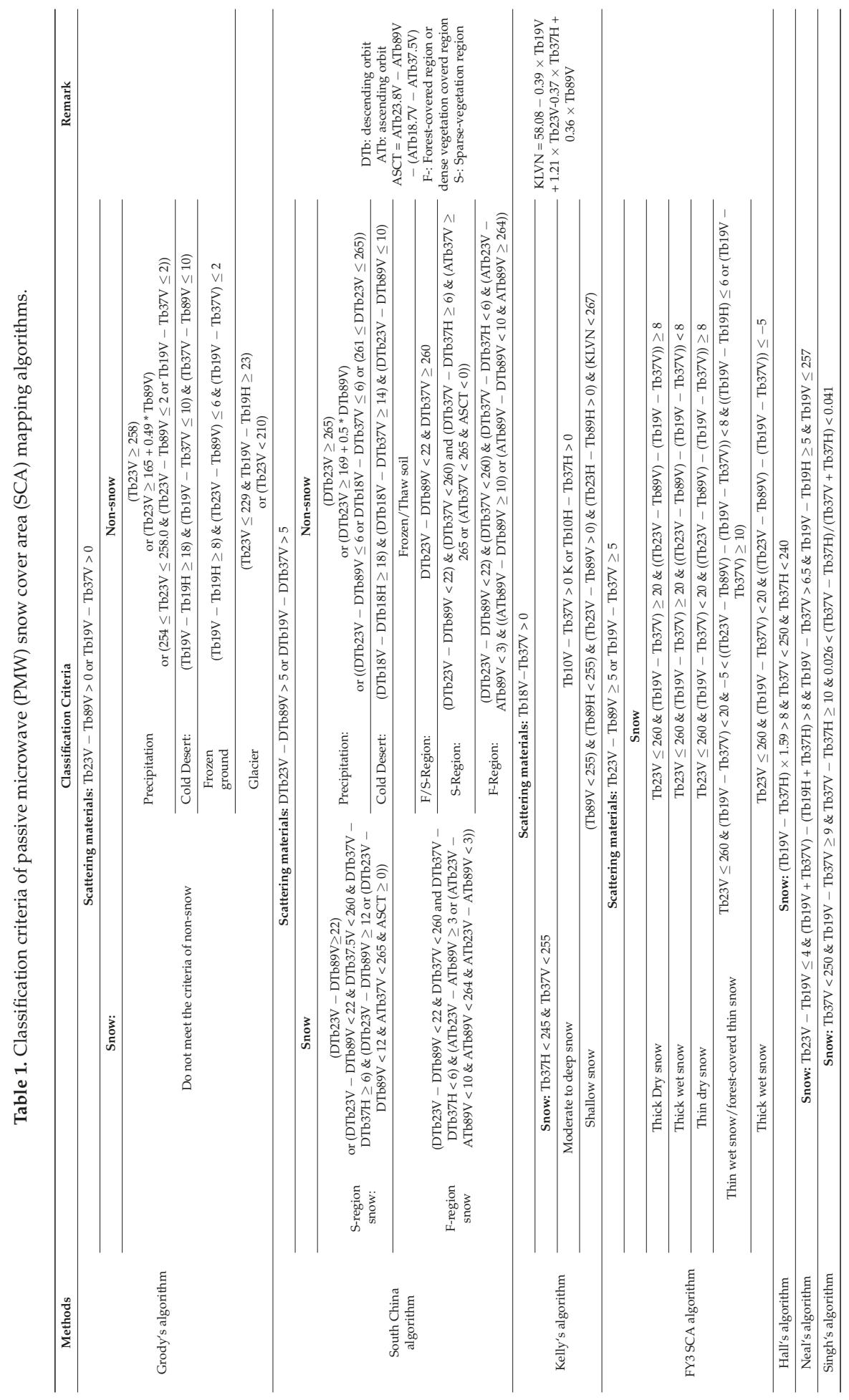




\section{Results and Analysis}

To test and compare the performance of the seven algorithms, the SCAs derived from PMW SCA mapping algorithms were quantitatively evaluated using in situ SD observations and the one-kilometer IMS snow cover product. Only dry snow records of in situ observations were used for analysis because the seven tested algorithms are mostly for dry snow discrimination. The one-kilometer IMS data were reprojected to the projection of the PMW SCA maps. Then, the snow cover fraction in each PMW pixel was calculated. We set the threshold of a snow cover fraction value to $50 \%$ to determine whether pixels have snow or do not. All pixels with SCF less than the threshold were labelled snow-free.

Four assessment indexes, overall accuracy (OA), omission error (OE), commission error (CE), and positive predictive value (PPV), were used for the analysis. OA describes the percentage of the correct classifications including inerrant snow-covered and snow-free identifications. PPV describes the probability that a pixel identified with snow indeed has snow [31]. OE and CE are both related to false classification. OE indicates PMW snow map misclassifications as snow-free instead of snow-covered and $\mathrm{CE}$ as snow-covered instead of snow-free. Given the available data, the testing period using in situ measurements was from October 2013 to December 2015, and that using IMS data was from December 2014 to December 2015. Table 2 shows the normal metrics used to evaluate the PMW SCA mapping algorithms.

Table 2. Classification error matrix.

\begin{tabular}{lll}
\hline & \multicolumn{1}{c}{ Reference SCA: Snow } & Reference SCA: Snow Free \\
\hline PMW SCA: snow & true positive $(\mathrm{TP})$ & false negative $(\mathrm{FN})$ \\
PMW SCA: snow free & false positive $(\mathrm{FP})$ & \\
Overall accuracy $(\mathrm{OA}):(\mathrm{TP}+\mathrm{TN}) /(\mathrm{TP}+\mathrm{TN}+\mathrm{FN}+\mathrm{FP})$ & \\
$\begin{array}{l}\text { Omission error }(\mathrm{OE}): \mathrm{FP} /(\mathrm{FP}+\mathrm{TP}) \\
\text { Commission error }(\mathrm{CE}): \mathrm{FN} /(\mathrm{FN}+\mathrm{TP})\end{array}$ \\
$\begin{array}{l}\text { Positive predictive value }(\mathrm{PPV}): \mathrm{TP} /(\mathrm{TP}+\mathrm{FP}) \\
\text { Reference SCA: Ice mapping system }(\mathrm{IMS}) \text { SCA data and in situ measurements }\end{array}$ \\
\hline
\end{tabular}

Tests were conducted for both ascending and descending data. The FY-3C/MWRI L1 swath data were resampled to a global equidistant cylindrical projection at $0.25^{\circ}$ resolution for snow mapping. It should be noted that the South China algorithm identified snow using both ascending and descending data, resulting in a blended product for testing. The performances of the different PMW SCA maps for 7 January 2014 are shown in Figure 2. Snow extent determined using the seven methods clearly indicates the geographical distribution of snow over the three main seasonal snow-covered regions (Northwest, Northeast, and Tibetan Plateau). All methods achieved similar SCA estimations in the Northwest and the Northeast except for Singh's algorithm, which missed significant snow. Over the Tibetan Plateau, the Kelly SCA algorithm tended to identify more snow. The FY3 algorithm and Singh algorithm, in contrast, estimated less snow.

For each algorithm, OA, PPV, OE and CE were calculated for the testing periods. Table 3 summarizes the evaluation results. For each algorithm, 276,946 station records were used for evaluating the ascending PMW SAC mapping results, and 275,774 station records were used for the descending data. Compared to in situ SD observations, all methods achieved a high OA ranging from 0.895 to 0.950. Grody's algorithm, the South China algorithm and Kelly's algorithm have higher PPV values (from 0.656 to 0.827 ) and lower OE values (from 0.173 to 0.344 ). Singh's algorithm, Hall's algorithm and Neal's algorithm, with higher OE (from 0.492 to 0.860 ) and lower PPV (from 0.140 to 0.508 ) values, severely underestimated SCA during the testing periods. The descending orbit shows lower PPV and $\mathrm{CE}$ but higher $\mathrm{OE}$ than that of the ascending orbit. Because the local times on the ascending node and descending node are near 10:00 p.m. and 10:00 a.m., respectively, microwave brightness temperature at a high frequency may be more affected by atmospheric conditions during the descending orbits than during ascending orbits $[33,36]$. In addition, snow would melt during the day (descending orbit), 
and moist or wet snow is difficult to separate from land, which may lead to an increasing OE and decreasing PPV. Soil tends to be frozen during cold nights (ascending orbit), and it is difficult to separate frozen soil from dry snow, resulting in a higher CE. Evaluation results using IMS snow cover data as a reference are similar to the case using station observations. Whereas compared to evaluation results based on in situ observations, the results based on IMS snow cover product show higher classification error. The possible reasons for this difference include (i) the differences in the resolution of the evaluation datasets (i.e., point scale vs. image scale); (ii) the different snow types of the two datasets: only dry snow records of in situ observations were used for analysis, but IMS maps show both dry and wet snow; and (iii) errors in the IMS snow cover product. The performance of the IMS snow cover product deteriorates when identifying snow-free areas [37]. IMS is likely to overestimate snow cover in rugged terrain and tends to map more snow when the snow cover is patchy [31].
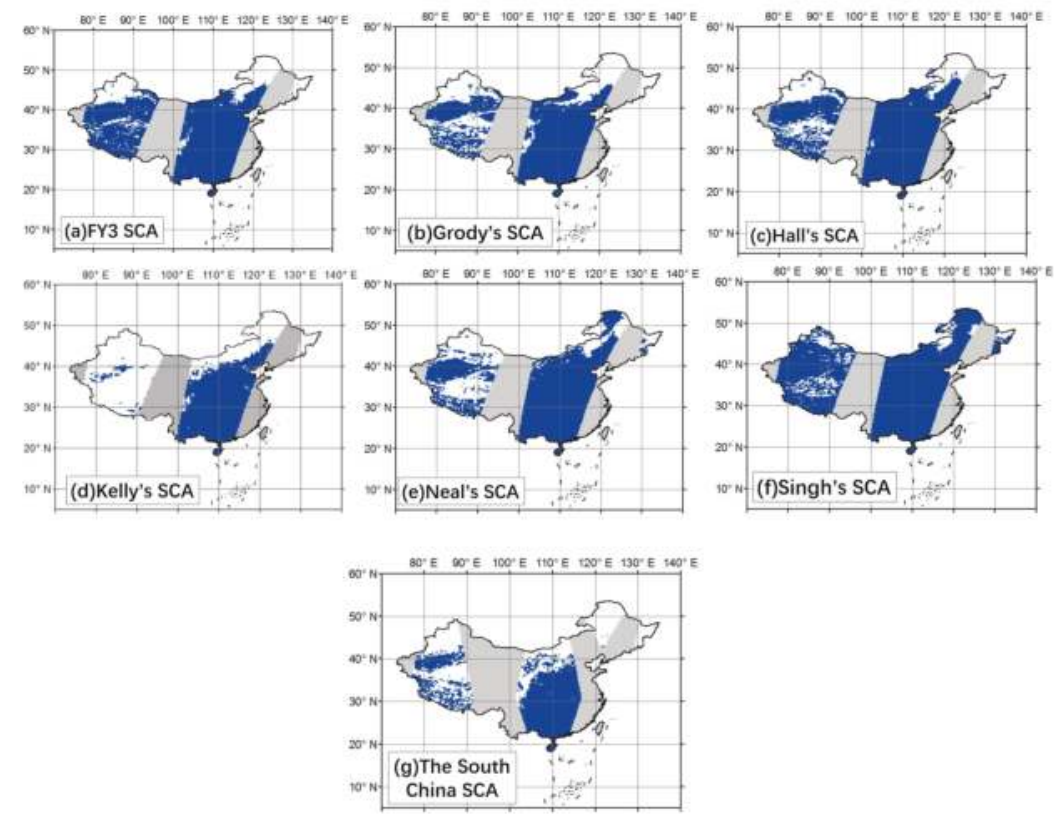

Figure 2. Comparison of PMW SCA maps for 7 January 2014: (a) FY SCA map, (b) Grody's SCA map, (c) Hall's SCA map, (d) Kelly's SCA map, (e) Neal's SCA map, (f) Singh's SCA map, (g) the South China SCA map. (blended SCA image (g) using both ascending and descending data for the South China algorithm and descending SCA images(a-f) for the remaining PMW SCA mapping algorithms).

Monthly OA, OE, CE and PPV present a clear seasonal pattern as shown in Figures 3 and 4. The OA of all algorithms is mostly high throughout the testing periods, and it improves to nearly 1.0 during the summer months when most regions are snow-free. Snow cover is small and patchy during the shoulder seasons (i.e., autumn, spring, and summer) leading to deteriorating capability of all PMW SCA mapping algorithms to detect snow. The OE and CE for all methods increase during the shoulder seasons when snow is accumulating or melting. During winter, the OE and CE of the seven algorithms mostly decrease to less than 0.5 , and the PPV exceeds 0.5 . Overall, the performance of the seven algorithms is comparable except for that of Singh's algorithm, which exhibits larger errors than the others. 
Table 3. OA, OE, CE and PPV of the PMW SCA mapping algorithms.

\begin{tabular}{|c|c|c|c|c|c|c|c|c|c|}
\hline \multirow[b]{3}{*}{ Algorithm } & \multicolumn{9}{|c|}{ SCA Reference } \\
\hline & \multicolumn{4}{|c|}{ In Situ Measurements } & \multicolumn{4}{|c|}{ IMS SCA $(\mathrm{SCF}>\mathbf{5 0} \%)$} & \multirow[b]{2}{*}{ Node } \\
\hline & OA & $\mathrm{OE}$ & CE & PPV & OA & $\mathrm{OE}$ & CE & PPV & \\
\hline FY3 SCA & 0.950 & 0.384 & 0.210 & 0.616 & 0.902 & 0.289 & 0.102 & 0.711 & $\mathrm{~A}^{1}$ \\
\hline Algorithm & 0.950 & 0.399 & 0.201 & 0.601 & 0.894 & 0.319 & 0.105 & 0.681 & $\mathrm{D}^{1}$ \\
\hline Grody's & 0.921 & 0.268 & 0.453 & 0.732 & 0.853 & 0.155 & 0.319 & 0.845 & $\mathrm{~A}^{1}$ \\
\hline Algorithm & 0.945 & 0.329 & 0.291 & 0.671 & 0.899 & 0.207 & 0.179 & 0.793 & $\mathrm{D}^{1}$ \\
\hline $\begin{array}{l}\text { South China } \\
\text { Algorithm }\end{array}$ & 0.919 & 0.173 & 0.498 & 0.827 & 0.958 & 0.164 & 0.420 & 0.836 & $\mathrm{~A}^{1}$ and $\mathrm{D}^{1}$ \\
\hline \multirow{2}{*}{ Neal's Algorithm } & 0.926 & 0.606 & 0.348 & 0.394 & 0.782 & 0.487 & 0.395 & 0.513 & $\mathrm{~A}^{1}$ \\
\hline & 0.934 & 0.645 & 0.187 & 0.355 & 0.820 & 0.531 & 0.239 & 0.469 & $\mathrm{D}^{1}$ \\
\hline \multirow{2}{*}{$\begin{array}{l}\text { Singh's } \\
\text { Algorithm }\end{array}$} & 0.920 & 0.855 & 0.178 & 0.145 & 0.745 & 0.831 & 0.431 & 0.169 & $\mathrm{~A}^{1}$ \\
\hline & 0.921 & 0.860 & 0.040 & 0.140 & 0.764 & 0.860 & 0.160 & 0.140 & $\mathrm{D}^{1}$ \\
\hline \multirow{2}{*}{ Hall's Algorithm } & 0.931 & 0.492 & 0.347 & 0.508 & 0.848 & 0.305 & 0.276 & 0.695 & $\mathrm{~A}^{1}$ \\
\hline & 0.941 & 0.534 & 0.197 & 0.466 & 0.874 & 0.382 & 0.128 & 0.618 & $\mathrm{D}^{1}$ \\
\hline Kelly's & 0.895 & 0.280 & 0.549 & 0.720 & 0.713 & 0.299 & 0.526 & 0.701 & $\mathrm{~A}^{1}$ \\
\hline Algorithm & 0.931 & 0.344 & 0.387 & 0.656 & 0.754 & 0.347 & 0.469 & 0.653 & $\mathrm{D}^{1}$ \\
\hline
\end{tabular}

${ }^{1} \mathrm{~A}=$ ascending data, $\mathrm{D}=$ descending data.
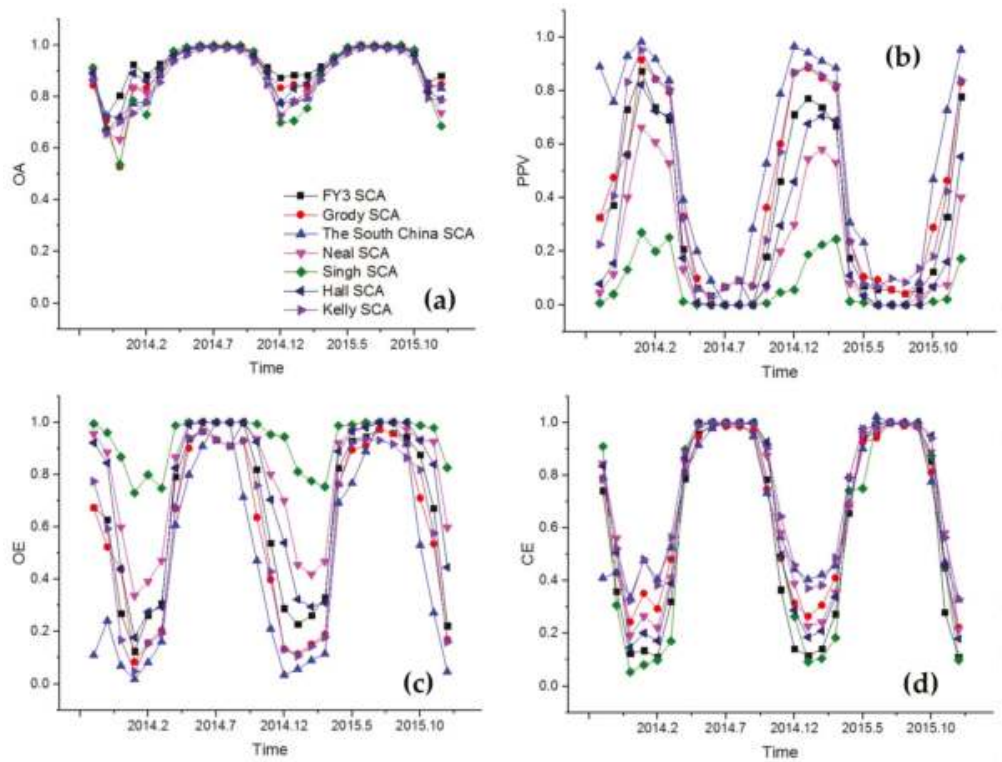

Figure 3. Monthly OA (a), PPV (b), OE (c) and CE (d) of the seven PMW SCA maps based on in situ SD observations. 

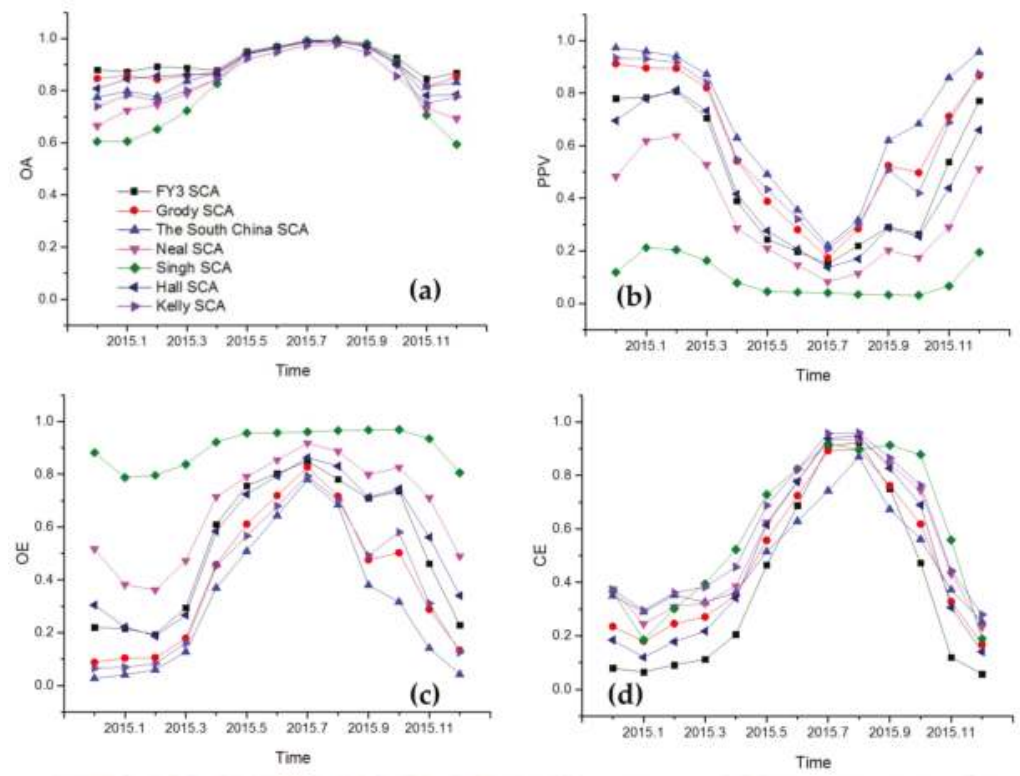

Figure 4. Monthly OA (a), PPV (b), OE (c) and CE (d) of the seven PMW SCA maps based on the IMS snow cover product.

\subsection{Effect of Land-Cover Types on Snow Cover Mapping Accuracy}

The seven SCA identification algorithms presented in this study are all based on the decision tree approach with fixed threshold filters. The thresholds remain constant within different land-cover types. Because microwave radiation characteristics are related to land-cover types [13], the constant thresholds may introduce errors into classification. Therefore, it is necessary to identify the effects of land cover on PMW SCA mapping accuracy. Cropland, forest, grassland, and barren are the main land-cover types of seasonal-snow-dominated regions in China [38]. Their influences on snow mapping accuracy were analyzed using the land-cover map and in situ observational data.

The land-cover map (Figure 5) used in this study is a resampled product from the Globeland30 land-cover map. The Globeland30 land-cover map was projected and resampled to the same projection as that of the PMW SCA maps using the majority method. The majority algorithm assigns the most popular values within the filter window as the label of a pixel. This provides a more interpretable sense of the majority of land-cover types within each filter window. Pixels with a major land-cover fraction less than $70 \%$ have been removed from analysis because they are dominated by more than one land-cover type.

Figure 6 shows the OA, CE, PPV and OE of seven PMW SCA maps against station observations over barren, grassland, cropland and forests. As seen in Figure 6, the OA of all algorithms is not sensitive to land-cover type. Except for the South China algorithm, the CE of all algorithms over barren land and grasslands is higher than over the other land-cover types. The misclassification over barren land and grasslands may be a result of the difficulty in separating snow from frozen ground. Snow and frozen ground are scattering materials and have similar microwave radiation characteristics, making them difficult to distinguish. For the South China algorithm, severe omission error occurred over barren land versus other types of land cover. The OE in the FY algorithm and Grody's algorithm is not sensitive to land-cover type. For Hall's algorithm, Neal's algorithm, Singh's algorithm and Kelly's algorithm, the $\mathrm{OE}$ is slightly higher over forested areas than over other land-cover types. 


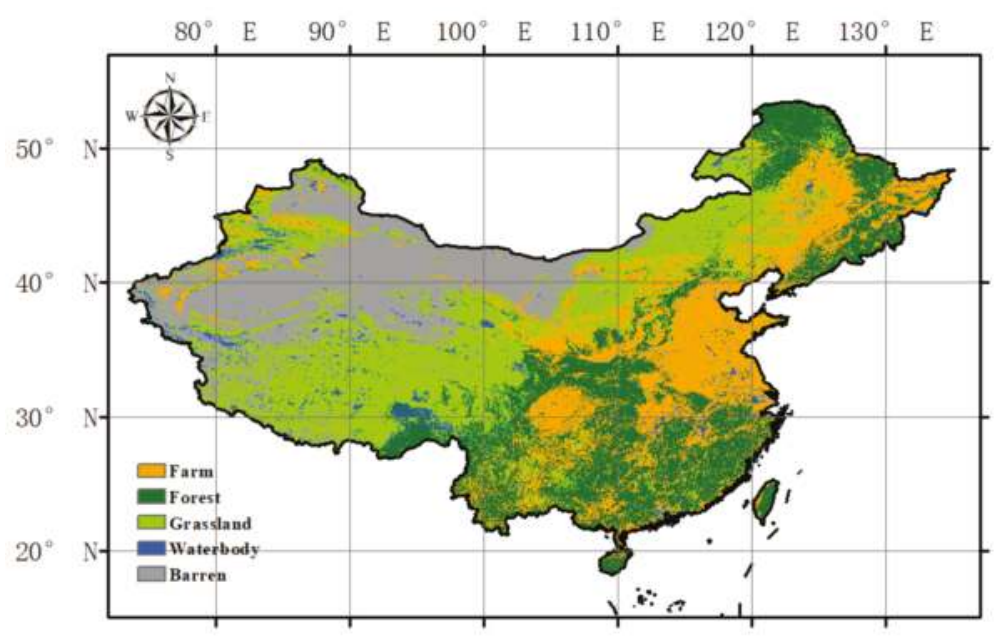

Figure 5. Globeland30 land-cover map.
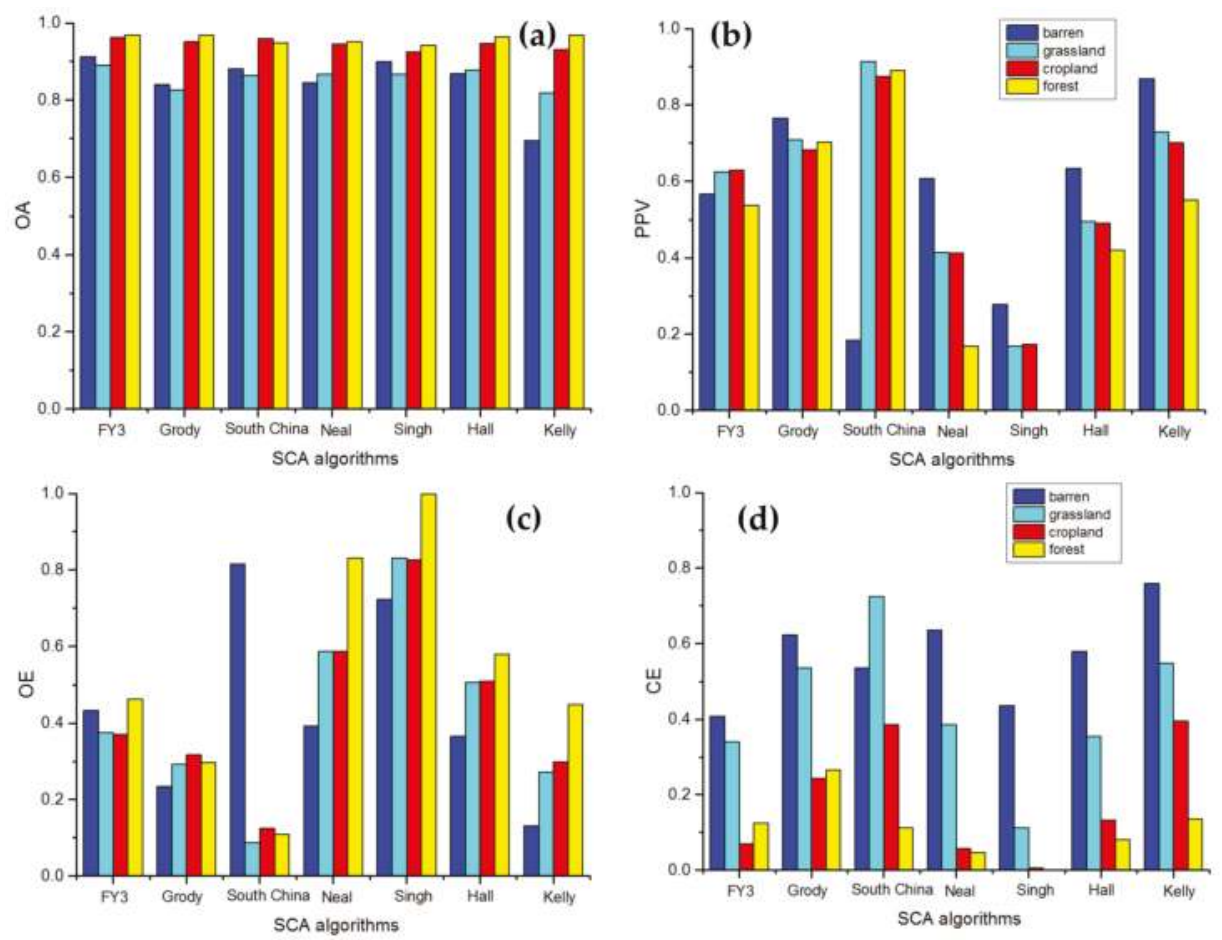

Figure 6. OA (a), PPV (b), OE (c) and CE (d) of the seven PMW SCA maps compared to station observations in different land-cover types.

\subsection{Effect of SCF on Snow-Cover Mapping Accuracy}

PMW SCA detection methods provide a binary snow classification that is sensitive to SCF [7,32]. Pixels with low SCF would be hard to detect using binary methods as the lack of snow signals 
can be captured by satellite sensors. The SCF values at $0.25^{\circ}$ resolution were calculated using the one-kilometer IMS snow-cover product. Because only the snow-covered pixels (SCF $>0$ ) were part of the analysis, the indexes OE and PPV were used for assessment. Figure 7 shows the statistics of OE and PPV associated with SCF. An increase in SCF from 0 to $100 \%$ results in a linear decline in underestimation of snow cover. In contrast to OE, values of PPV increase with increased SCF. Generally, the binary SCA product derived from optical remote sensing intends to indicate snow when the pixel's snow cover exceeds 50\% [7,32]. The PMW binary SCA does have apparent omission errors of less than 50\% snow cover as shown in Figure 7. Though the IMS product has the problem of overestimating snow cover [31], it is still reasonable to believe that PMW binary SCA algorithms are sensitive to SCF, and their capability to estimate snow cover improves with higher SCF.
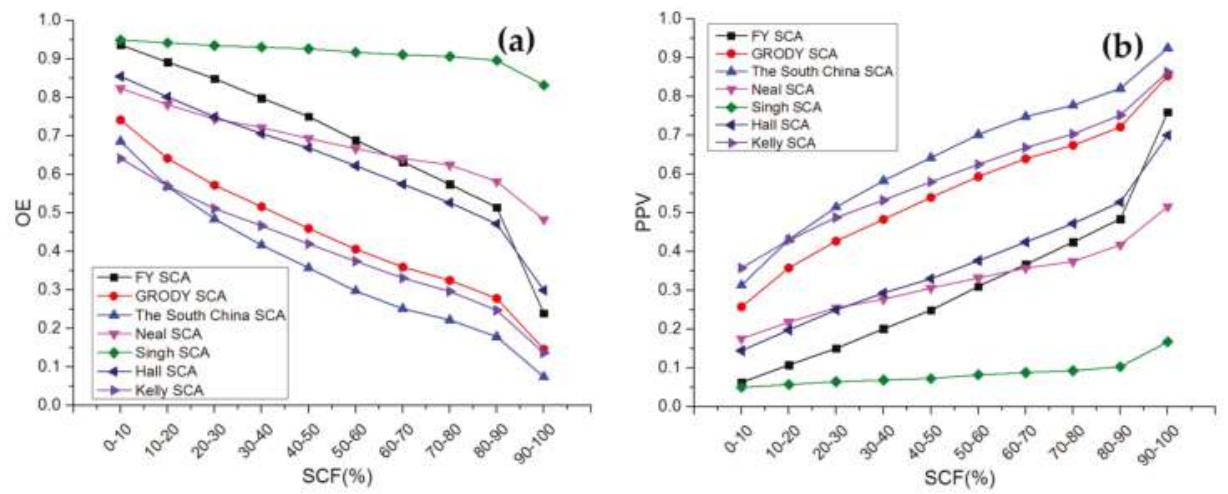

Figure 7. OE (a) and PPV (b) of the PMW SCA mapping algorithms associated with different SCFs.

\subsection{Effect of SD on Snow Cover Mapping Accuracy}

Previous studies have shown the relationship between SD and the accuracy of SCA products: the accuracy of SCA decreases with decreasing SD $[39,40]$. In this study, we found a similar relationship between SD and PMW SCA. In addition, we found different effects of SD on the seven PMW SCA mapping methods.

Figure 8 presents the PPV and OE of the snow-cover maps for different SD ranges. Observed SD data were divided into six categories: $\mathrm{SD}<5 \mathrm{~cm}, \mathrm{SD}=5-15 \mathrm{~cm}, \mathrm{SD}=15-25 \mathrm{~cm}, \mathrm{SD}=25-35 \mathrm{~cm}$, $\mathrm{SD}=35-45 \mathrm{~cm}$ and SD $>45 \mathrm{~cm}$. The omission errors of the seven PMW SC maps show similar responses to SD ranges. The OE for all algorithms was highest when SD is less than five centimeters and tended to decline as SD exceeded five centimeters. This can be explained by the positive relation between SD (or SWE) and snow-cover fractions [41,42]. When snow surrounding the station is shallow, snowfall events are more likely to occur within a small area of the sensor's field of view. Under this situation, pixels tend to be classified as snow-free generating increased OE. In contrast, when SD observed by station is quite high, pixels tend to be entirely covered by snow resulting in a decreased possibility of underestimation. PPV for all algorithms was lowest when SD is less than five centimeters. PPV values were nearly greater than 0.7 for the FY3 algorithm, Grody's algorithm, the South China algorithm and Kelly's algorithm when SD is greater than five centimeters. Variations in PPV of Grody's algorithm and Kelly's algorithm were less affected by SD than that of the other algorithms. These results indicate that Grody's algorithm and Kelly's algorithm are less sensitive to SD than the other algorithms. 

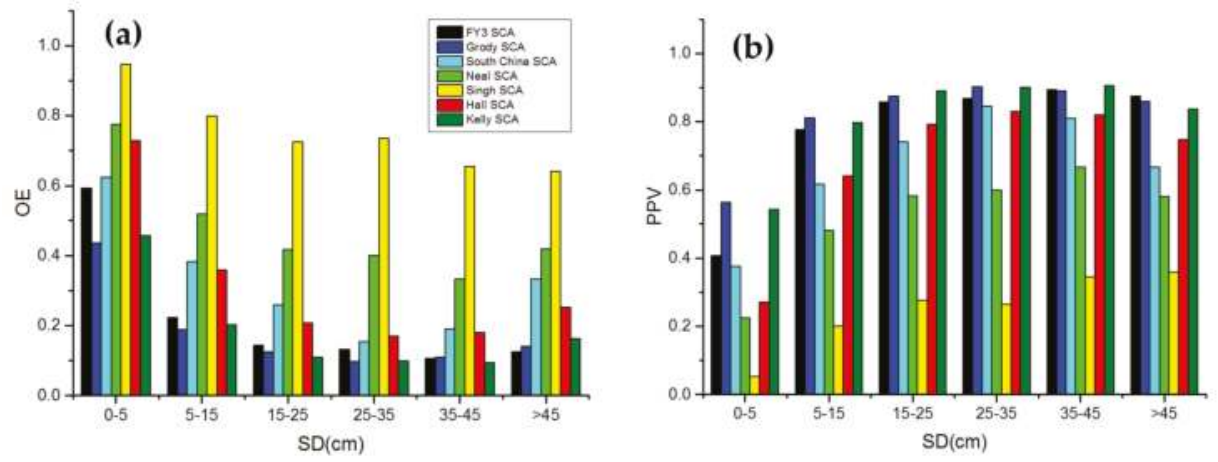

Figure 8. OE (a) and PPV (b) of the seven PMW SCA maps compared to ground observations at different snow depths.

\section{Discussion}

In this study, the performances of seven PMW SCA mapping methods in China were evaluated using in situ snow depth measurements and the one-kilometer IMS snow-cover product. The purpose of the study was to evaluate the differences among the PMW SCA mapping algorithms and to identify the influencing factors on the algorithms. Previous studies have demonstrated several difficulties in evaluating satellite-derived snow cover [30-32,39]. The use of point data and high-resolution SCA products for evaluation are both problematic [43]. Which data source is better for validation needs further study. However, to date, they are still the most reliable and useful validation datasets for evaluating satellite-derived SCA products $[8,11,29,32]$.

All tested methods are based on the decision tree approach. The different SCA estimations are attributed to various classification criteria. Criteria for identifying various types of snow may lead to different positive predictive values and commission errors. Criteria for non-snow type identification such as precipitation and cold desert may introduce omission errors into snow identification. To determine whether these criteria can separate snow from other features accurately, the performances of the criteria in each algorithm as listed in Table 1 were tested using in situ data as described in Section 4.1. In addition, a classification structure of a decision tree was made of various nodes [44]. For PMW SCA detection, the nodes of the decision tree were mostly constructed by a selected multi-band combination of $\mathrm{Tb}$ or single-band $\mathrm{Tb}$ (hereafter termed $\mathrm{Tb}$ index) and the given thresholds. Proper Tb indexes and thresholds would exactly and accurately separate snow cover from other features. The performances and effects of Tb indexes and thresholds used in the seven PMW SCA mapping algorithms were analyzed and are described in Sections 4.2-4.4.

It should be noted that this study tended to evaluate the capability of each tested algorithm on snow identification, rather than on other feature classification. Thus, we only tested and assessed the performance of each criterion on snow identification. The classification accuracy of non-snow features such as precipitation, frozen ground, etc. are not discussed in this paper.

\subsection{Criteria of PMW SCA Algorithms}

Criteria of the testing PMW SCA algorithms for assessment are listed in Table 1. Evaluation results are shown in Figure 9. For the FY3 algorithm, the criterion for thin dry snow had a much higher PPV and slightly higher CE than that of the other four criteria. Therefore, the thin dry snow criterion could be regarded as a more effective criterion than the other four criteria for snow identification in China. The criteria of Grody's algorithm for snow identification and Kelly's algorithm for deep snow identification had higher PPV values $(>0.65)$ but introduced large commission errors. For Grody's algorithm, the $\mathrm{OE}$ caused by the non-snow identification criteria was very small $(<0.023)$ except 
for the precipitation filter, which is the major OE source. Both the PPV and CE of the shallow snow criterion of Kelly's algorithm were near zero. These results indicated that the shallow snow criterion was essentially useless for snow detection in China. The South China algorithm designed two sets of criteria for identifying snow in forest- or dense-vegetation-covered regions (F-region) and sparse-vegetation regions (S-region), respectively. Testing results showed that the F-region snow criteria, with a higher PPV $(>0.67)$ and lower CE $(<0.18)$, performed much better than the S-region snow criteria. The commission errors of the South China algorithm were mainly attributed to the S-region snow criterion. The omission errors caused by each non-snow criterion were small $(<0.14)$. The frozen ground filters were the major OE source of the South China algorithm, and this could be an important reason why severe omission errors occurred over barren rather than other types of land cover. Singh's algorithm, Hall's algorithm and Neal's algorithm have shown apparent underestimation issues as shown in Figure 9, especially for Singh's algorithm. The OE of Singh's algorithm reached 0.86 , the highest of the seven algorithms. Otherwise, the differences in error characteristics between ascending and descending data are identical to the conclusion described in Section 3.
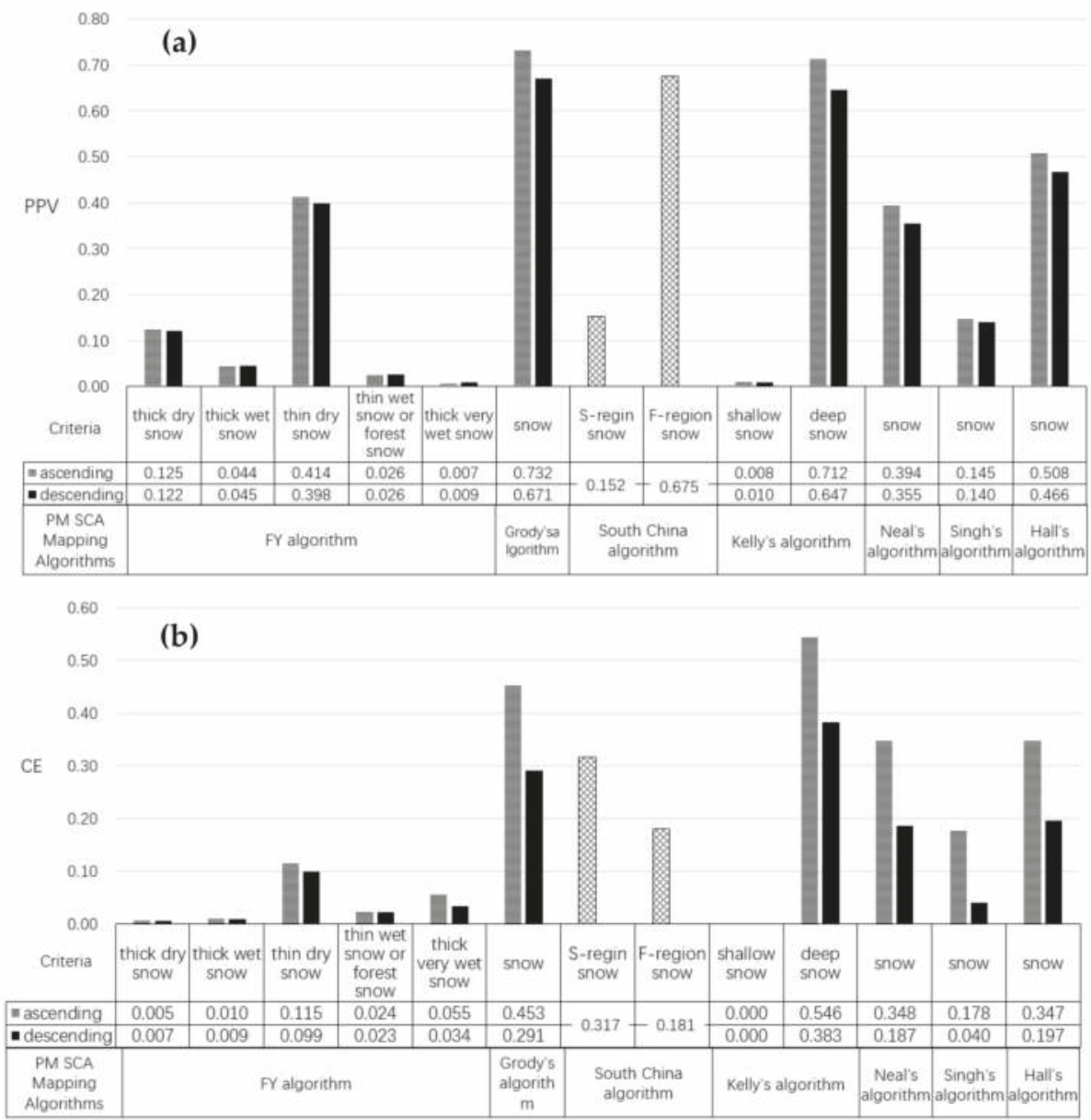

Figure 9. Cont. 


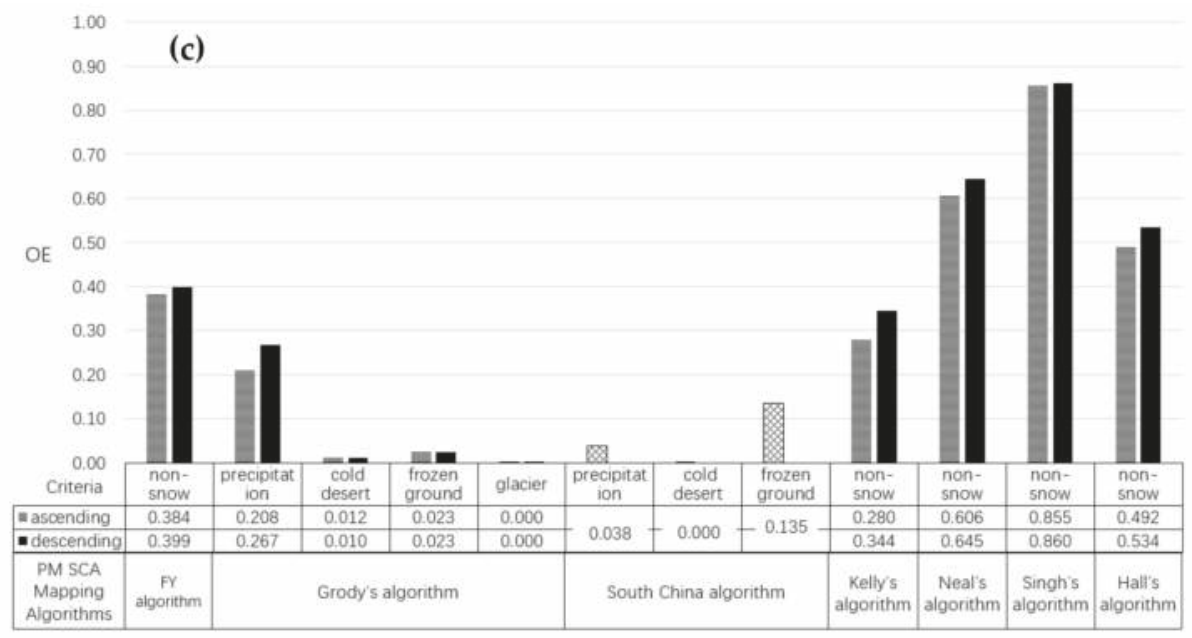

Figure 9. The evaluation results of classification criteria for each algorithm: PPV (a), CE (b), OE (c).

\subsection{Tb Indexes of PMW SCA Algorithms}

The ideal $\mathrm{Tb}$ index should exactly separate snow cover from snow-free land. This means the $\mathrm{Tb}$ index values for snow and non-snow should be completely different. The decision tree would have better classification if its $\mathrm{Tb}$ indexes had significantly different values for snow and non-snow.

The $\mathrm{Tb}$ indexes used in the seven tested algorithms can be divided into five classes: single-band $\mathrm{Tb}, \mathrm{Tb}$ gradient, polarization difference, polarization ratio and day-night $\mathrm{Tb}$ difference indexes. The five classes of $\mathrm{Tb}$ indexes and their values for snow and non-snow are shown in Figure 10. Snow, as a typical cold scattering material, has lower Tb values than the absorbing material (moist soil, vegetation, etc.). The $\mathrm{Tb}$ of snow decreases with increasing frequency. Thus, single-band $\mathrm{Tb}$ indexes and $\mathrm{Tb}$ gradient indexes are mainly used to identify snow from other absorbing or warm materials $[16,35]$. As expected, values of the single-band $\mathrm{Tb}$ indexes for snow and non-snow are largely different (Figure 10). These results demonstrate the availability of the single-band $\mathrm{Tb}$ indexes for snow discrimination. Most of the $\mathrm{Tb}$ gradient indexes have similar snow discrimination ability, except for $(\mathrm{Tb} 23 \mathrm{~V}-0.49 \times \mathrm{Tb} 89 \mathrm{~V})$. The index $(\mathrm{Tb} 23 \mathrm{~V}-0.49 \times \mathrm{Tb} 89 \mathrm{~V})$ has been used for filtering precipitation $[16,17]$. In this study, precipitation is considered non-snow. The range of $(\mathrm{Tb} 23 \mathrm{~V}-0.49 \times \mathrm{Tb} 89 \mathrm{~V})$ for snow entirely contains non-snow. This means snow would be barely separable from any other features (including precipitation) using this index. The failure of $(\mathrm{Tb} 23 \mathrm{~V}-0.49 \times \mathrm{Tb} 89 \mathrm{~V})$ in this study may have been caused by the insufficient training and analysis data for a global algorithm development $[16,35]$. Previous studies have proven that the polarization difference at $19 \mathrm{GHz}$ for cold desert was greater than that of snow $[16,18,35]$. In this study, there is no observation station in desert. The index (Tb19V-Tb19H) presents little effect on snow discrimination. This is reasonable because the polarization difference for various absorbing materials and scattering materials could be the same, although their scattering characteristics are different [16,45]. The capability of the polarization difference index at $19 \mathrm{GHz}$ should be investigated based on reliable data in further studies. The day-night $\mathrm{Tb}$ difference index, the polarization difference and the polarization ratio index at $37 \mathrm{GHz}$ compromised with the $\mathrm{Tb}$ gradient indexes can be used to filter wet snow $[17,18,45,46]$. Only dry snow station records have been used in this study. Therefore, we just tested their effect on dry snow detection. As seen in Figure 10, the three types of indexes previously mentioned do not divide snow from non-snow effectively because the index values for snow and non-snow are very similar. Further research needs to be completed to understand their effects on wet snow detection. 

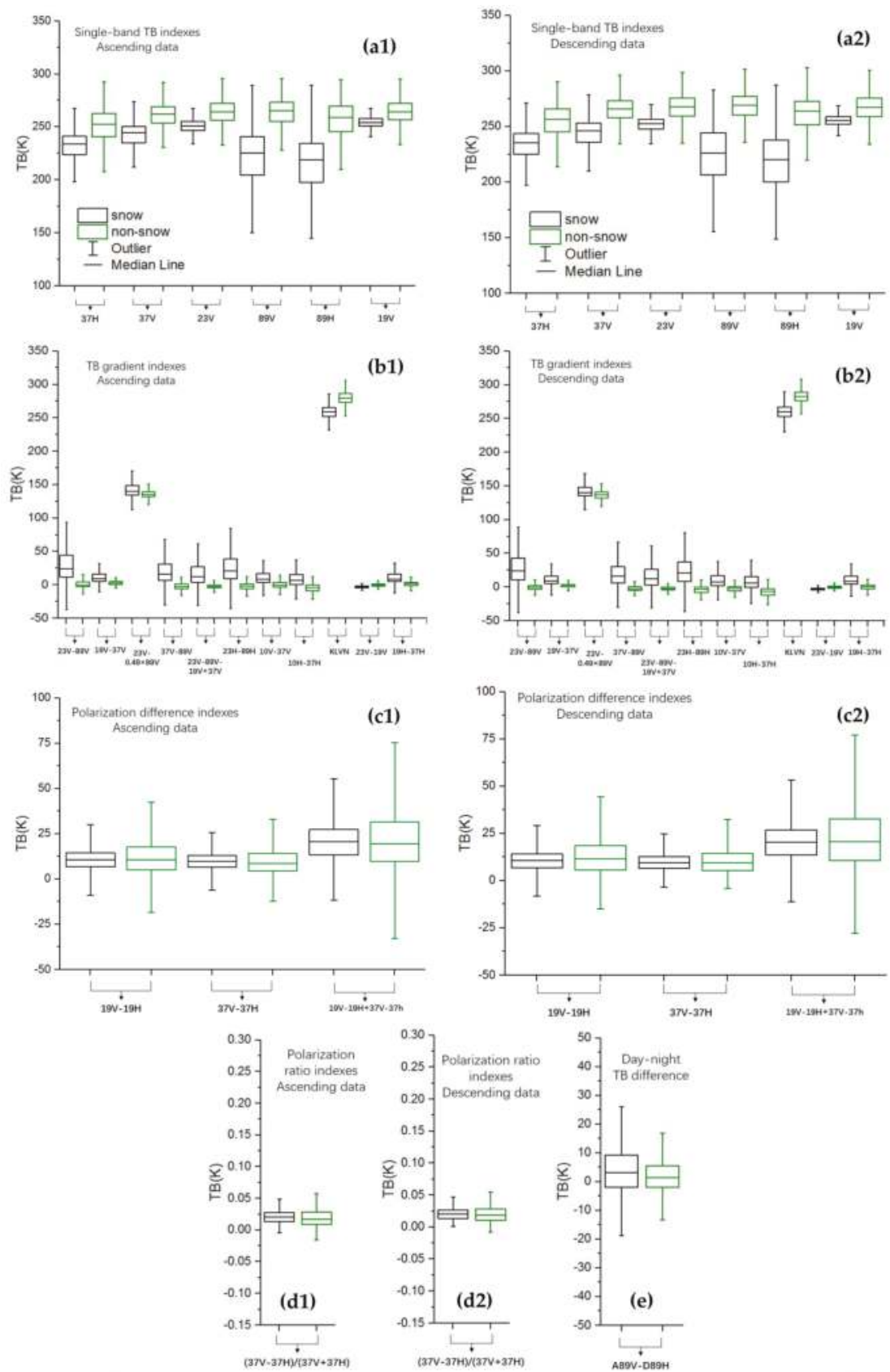

Figure 10. Box plots of the single-band indexes $(\mathbf{a} 1, \mathbf{a} 2)$, the Tb gradient indexes $(\mathbf{b} 1, \mathbf{b} 2)$, the polarization difference indexes $(\mathbf{c} \mathbf{1}, \mathbf{c} 2)$, the polarization ratio indexes $(\mathbf{d} \mathbf{1}, \mathbf{d} \mathbf{2})$ and the day-night $\mathrm{Tb}$ difference indexes (e). 


\subsection{Thresholds of PMW SCA Algorithms}

Thresholds are the other important factor in decision trees. Figure 11 summarizes the $\mathrm{Tb}$ indexes and thresholds used in the seven PMW SCA algorithms. Thresholds defined the upper bounds and lower bounds of the $\mathrm{Tb}$ index values for classification. The upper bounds for snow identification were mostly near the upper quartile of the snow box plots and lower quartile of the non-snow box plots. In contrast, the lower bounds for snow identification were mostly near the lower quartile of the snow box plots and the upper quartile of the non-snow box plots. These results mean nearly $75 \%$ of the snow could be detected and nearly $25 \%$ of the non-snow would be misclassified as snow. Similarly, the upper (lower) bounds for non-snow identification were mostly near the upper (lower) quartile of the non-snow box plots and the lower (upper) quartile of the snow box plots. These results mean nearly $75 \%$ of the non-snow could be successfully filtered and nearly $25 \%$ of the snow would be misclassified as non-snow.

\subsection{Effects of $T b$ Indexes and Thresholds on PMW SCA Algorithms}

The criteria for snow identification are essentially a compromising set of various filtering conditions [16]. The classification results are the compromising product of multiple conditions. The SCA mapping accuracy is attributed to all of the Tb indexes and thresholds used in the algorithm. The assessment results are described in Section 3 and can be explained based on the analysis of $\mathrm{Tb}$ indexes and thresholds.

Grody's algorithm, the South China algorithm and Kelly's algorithm have a higher PPV and a lower OE than the other algorithms. As seen in Figures 10 and 11, it was found that most of the $\mathrm{Tb}$ indexes used in the Grody's algorithm, the South China algorithm and Kelly's algorithm have significantly different values for snow and non-snow. The proper $\mathrm{Tb}$ indexes provide a higher possibility for snow discrimination. In contrast, the relatively poorer discrimination ability of the $\mathrm{Tb}$ indexes for snow discrimination lead to misclassification, such as index $(\mathrm{Tb} 23 \mathrm{~V}-0.49 \times \mathrm{Tb} 89 \mathrm{~V})$. The performances of $(\mathrm{Tb} 23 \mathrm{~V}-0.49 \times \mathrm{Tb} 89 \mathrm{~V})$ used in Grody's algorithm and the South China algorithm are unsatisfactory in this study. The index (Tb23V $-0.49 \times \mathrm{Tb} 89 \mathrm{~V})$ should be an error source for the two algorithms. The thresholds are the other important error source of the algorithms. For example, most of the thresholds used in Kelly's algorithm tended to identify more snow, resulting in higher PPV and CE. Similarly, the thresholds of Tb89V and (Tb23V - Tb89V - Tb19V + Tb37V) used in the South China algorithm were likely to overestimate SCA, leading to a higher PPV and CE. Compared to Grody's algorithm, the South China algorithm and Kelly's algorithm, the compromising set of criteria used in the FY3 algorithm is conservative for snow discrimination. It balances the problem between snow identification and overestimation. Though the conservative criteria for snow identification increases the $\mathrm{OE}$, the FY3 algorithm still balances misclassification errors and snow identification well. As a result, the overall accuracy of the FY3 algorithm was the highest of all the tested algorithms.

The misclassification problem is severe for Neal's algorithm, Singh's algorithm and Hall's algorithm. The three algorithms do not have typical criteria for identifying snow from other scattering materials, and they build conservative condition criteria for snow detection. The lack of filters and the strict criteria for snow are important error sources. In addition, there are some other triggers for misclassification. For Neal's algorithm, its $\mathrm{Tb}$ indexes have a poor ability for snow discrimination (as seen in Figure 11). The threshold for the polarization ratio index $(\mathrm{Tb} 37 \mathrm{~V}-\mathrm{Tb} 37 \mathrm{H}) /(\mathrm{Tb} 37 \mathrm{~V}+\mathrm{Tb} 37 \mathrm{H})$ used in Singh's algorithm seems too high and tends to misclassify snow as non-snow, leading to a high $\mathrm{OE}$. 
Remote Sens. 2018, 10, 524
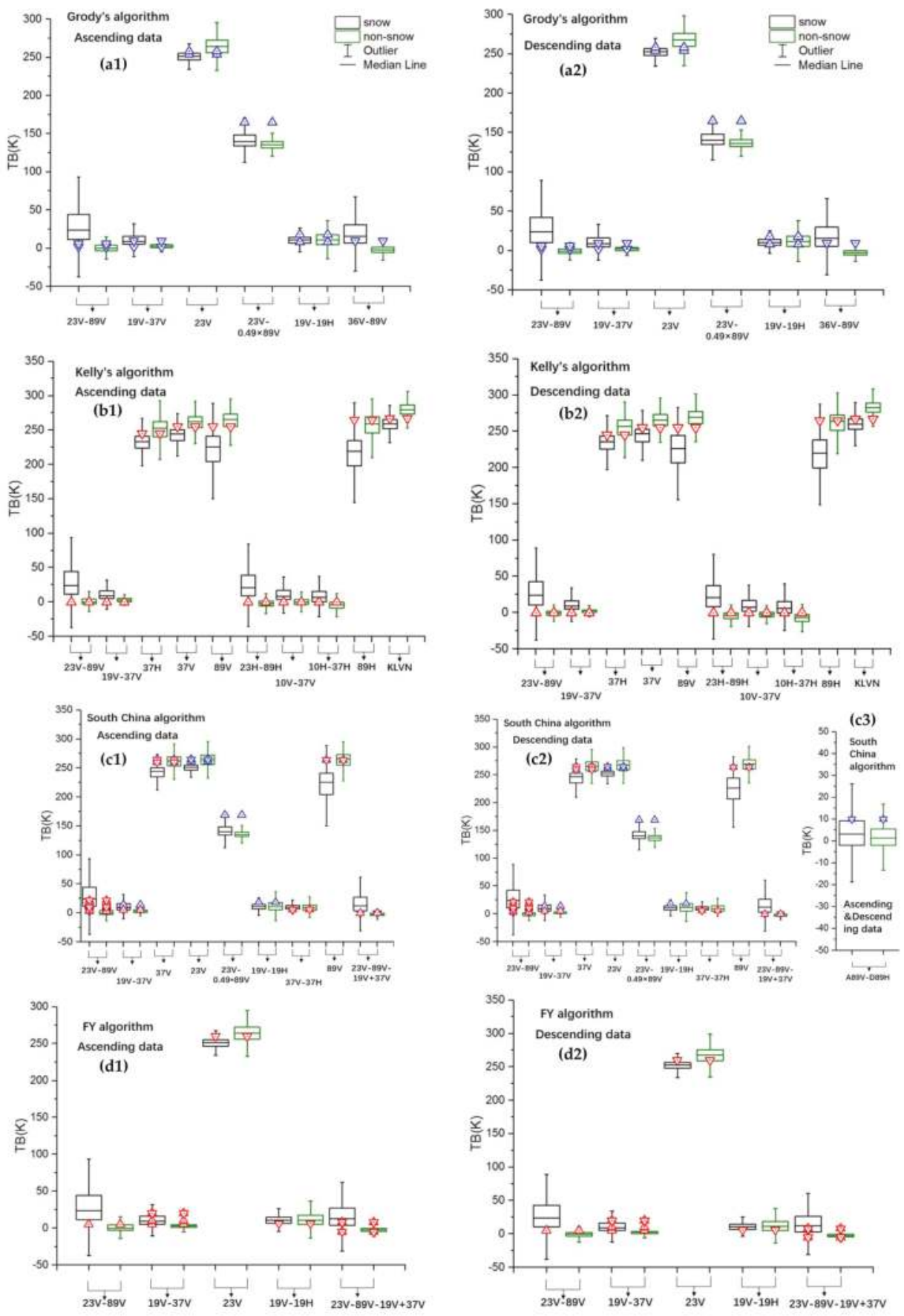

Figure 11. Cont.

135 

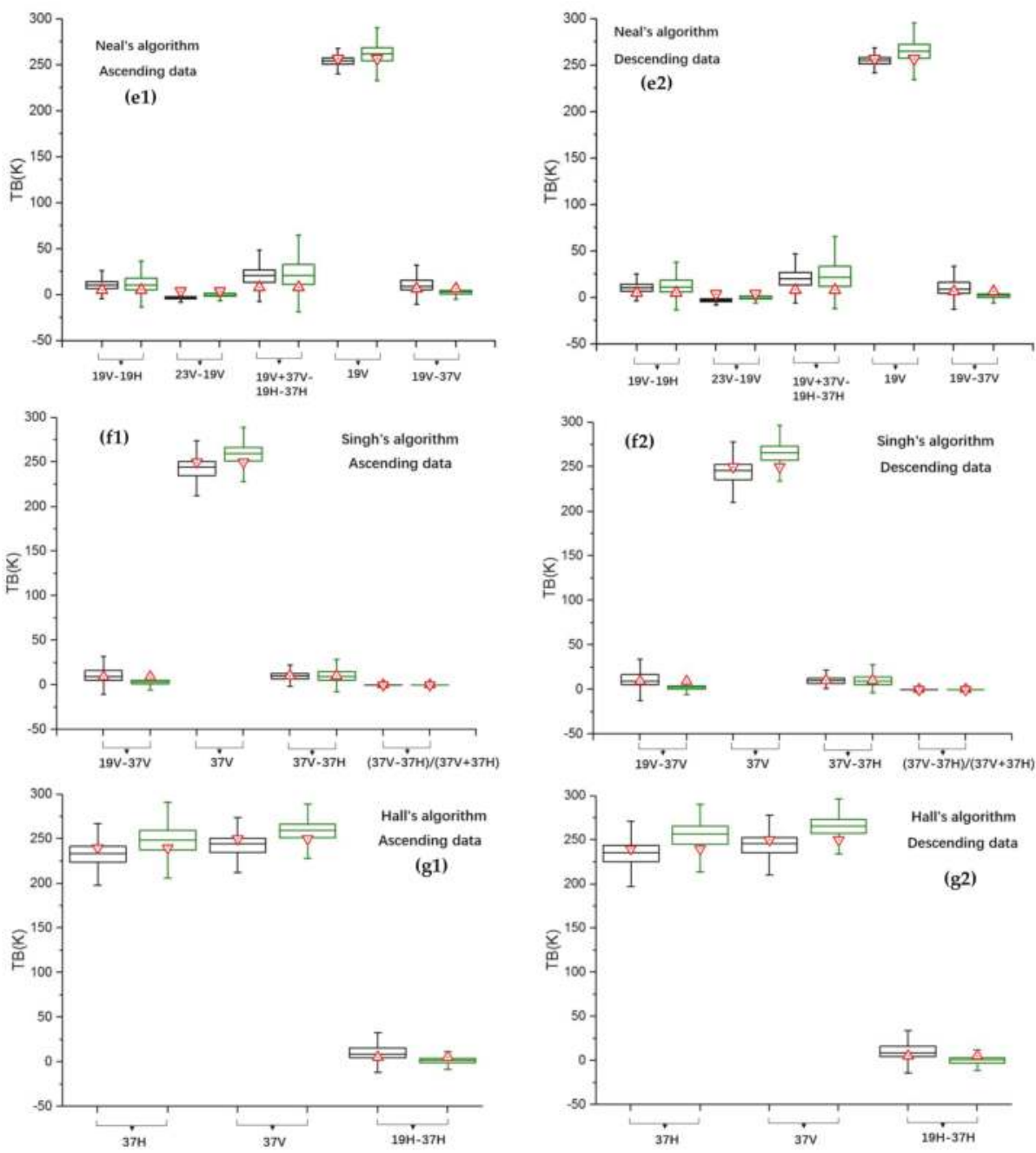

Figure 11. Box plots of $\mathrm{Tb}$ index values and thresholds of the seven PMW SCA mapping algorithms: Grody's algorithm (a1,a2), Kelly's SCA algorithm (b1,b2), the South China algorithm (c1-c3), FY SCA algorithm (d1,d2), Neal's algorithm (e1,e2), Singh's algorithm (f1,f2), Hall's algorithm (g1,g2). (The up and down arrows indicate the lower bounds and upper bounds of the thresholds, respectively. Arrows in red and in blue indicate thresholds for snow identification and non-snow identification, respectively.)

It was found that the thresholds and $\mathrm{Tb}$ indexes were identical for both ascending data and descending data as shown in Figures 10 and 11. Though the difference between ascending data and descending data for most $\mathrm{Tb}$ indexes was small, the identical thresholds used in the algorithms still possibly generate false classification. Thus, the identical thresholds for ascending data and descending data may be a reason why ascending data and descending data resulted in a different performance.

As shown in Table 4, data for algorithm training and for the presented assessment work are from various satellite datasets, ground observation datasets and auxiliary datasets spanning different regions and years. A rich data source for algorithm development as for Grody's algorithm can improve the stability of the algorithm. The South China algorithm and the FY3 algorithm were trained 
using Chinese local ground data. As a result, they were more likely to identify the land surface characteristics as compared to the remaining algorithms over China. This should be a reason for their good performance in the assessment.

The satellite data for developing the seven tested algorithms include AMSR-E data and SSM/I data. The $\mathrm{Tb}$ data used for evaluating the algorithms are from FY-3C/MWRI. The frequencies for MWRI are in accord with AMSR-E, and similar to SSM/I. MWRI, SSMI and AMSR-E have similar configurations. The difference between MWRI, SSMI and AMSR-E observations approximates to $0-2 \mathrm{~K}[47,48]$. The impact of inter-sensor calibration issue is around $-0.064-0.056$ for OE and CE calculation, which has rarely affected the assessment results and conclusions shown in this paper. If the difference between various satellite measurements is obvious, an assessment of the impact of inter-sensor calibration issues should be carefully considered.

Table 4. Training dataset information for the tested algorithms.

\begin{tabular}{|c|c|c|c|c|}
\hline \multirow{2}{*}{ Methods } & \multicolumn{4}{|c|}{ Information of the Training Datasets } \\
\hline & Spatial Coverage & $\begin{array}{l}\text { Temporal } \\
\text { Coverage }\end{array}$ & $\begin{array}{l}\text { Satellite } \\
\text { Data }\end{array}$ & Ground Truth Data and Auxiliary Data \\
\hline $\begin{array}{l}\text { Grody's } \\
\text { algorithm }\end{array}$ & $\begin{array}{l}\text { Various regions in the world } \\
\text { including the USA, Canada, } \\
\text { Africa, Australia, etc. }\end{array}$ & $\begin{array}{l}\text { Various days in } \\
1987 \text { and } 1993\end{array}$ & SSM/I & $\begin{array}{l}\text { Surface survey reports including } \\
\text { precipitation, snow cover, etc. }\end{array}$ \\
\hline $\begin{array}{l}\text { South China } \\
\text { algorithm }\end{array}$ & $\begin{array}{l}\text { North China Plain, the South } \\
\text { China and the southern part } \\
\text { of Inner Mongolia }\end{array}$ & $\begin{array}{l}1 \text { Jaunary 2008-20 } \\
\text { February } 2008\end{array}$ & AMSR-E & $\begin{array}{l}\text { Chinese Meteorological observations, } \\
\text { one-kilometer Chinese land-use map }\end{array}$ \\
\hline $\begin{array}{l}\text { Kelly's } \\
\text { algorithm }\end{array}$ & Northern hemisphere & $\begin{array}{l}\text { 2007-2008 winter } \\
\text { seasons }\end{array}$ & AMSR-E & $\begin{array}{l}\text { Snow climatology dataset } \\
\text { Land, ocean, coasts and ice product, } \\
\text { MODIS land cover product } \\
\text { Forest characterization map }\end{array}$ \\
\hline FY3 algorithm & $\begin{array}{l}\text { North-east Inner Mongolia } \\
\text { Autonomous Region and } \\
\text { Taklamakan Desert }\end{array}$ & $\begin{array}{l}\text { October } \\
\text { 1998-March } 2003 \\
\text { winter seasons }\end{array}$ & $\mathrm{SSM} / \mathrm{I}$ & $\begin{array}{l}\text { Chinese Meteorological observations, } \\
\text { NOAA/AVHRR snow cover map }\end{array}$ \\
\hline $\begin{array}{l}\text { Neal's } \\
\text { algorithm }\end{array}$ & USA & $\begin{array}{l}\text { Several days } \\
\text { during 1987-1988 }\end{array}$ & $\mathrm{SSM} / \mathrm{I}$ & $\begin{array}{l}\text { Major Land Resource Region classification } \\
\text { of the Soil Conservation Service; NOAA } \\
\text { cooperative network of stations }\end{array}$ \\
\hline $\begin{array}{l}\text { Singh's } \\
\text { algorithm }\end{array}$ & Red River basin in USA & $\begin{array}{l}1988,1989 \text { and } 1997 \\
\text { winter seasons }\end{array}$ & $\mathrm{SSM} / \mathrm{I}$ & $\begin{array}{l}\text { Airborne gamma radiation survey dataset } \\
\text { of NWS-USA }\end{array}$ \\
\hline $\begin{array}{l}\text { Hall's } \\
\text { algorithm }\end{array}$ & \multicolumn{4}{|c|}{ A modified version of Chang snow depth algorithm $(\mathrm{SD}=1.59 \times(\mathrm{Tb} 19 \mathrm{H}-\mathrm{Tb} 37 \mathrm{H}))$ for dry snow identification } \\
\hline
\end{tabular}

\section{Conclusions}

PMW SCA products are an important data source for snow cover monitoring. However, to date, uncertainty analysis for current PMW SCA mapping methods is rarely reported, especially in China. To investigate the performances of existing PMW SCA mapping algorithms in China, a thorough quantitative assessment of seven PMW SCA mapping algorithms in China was conducted in this study. Taking in situ SD observations and IMS snow cover as "ground truth" references, we compared the results of the PMW SCA maps derived from the seven algorithms.

Evaluation results for the PMW SCA maps showed the OA to be generally greater than 0.713 and 0.895 with respect to IMS and in situ observations, respectively. Grody's algorithm, the South China algorithm and Kelly's algorithm had higher positive predictive values and lower omission errors than the remaining studied algorithms. Their major error sources were the commission errors, which means they tended to estimate more snow. The FY3 algorithm estimated snow conservatively to reduce these commission issues. The overall accuracy of the FY3 algorithm was the highest of all the tested algorithms. Because of their conservative criteria for snow detection and the lack of filters for eliminating non-snow scattering materials, Singh's algorithm, Hall's algorithm and Neal's algorithm had greater misclassification errors. The descending orbit exhibited a larger PPV, OA, and CE but a smaller OE for all of the algorithms. The difference in SCA accuracy between the descending and ascending orbits may be driven by the different atmospheric conditions and daily freeze/thaw cycles. The OA, OE and CE of 
each algorithm showed a clear seasonal pattern, decreasing at the onset of winter and increasing as the shoulder seasons progressed, while the PPV had the opposite seasonal trend.

The effects of land-cover type, SCF and SD were also analyzed in this study. The results indicated that serious underestimation of snow occurred over barren land and grasslands using the tested algorithms. All algorithms are sensitive to SCF and SD. However, Grody's algorithm and Kelly's algorithm are less sensitive to SD than the other algorithms. The capability of the PMW SCA mapping methods improved as SCF increased as well as when the SD exceeded five centimeters.

In addition, we analyzed each criterion used in the seven PMW SCA mapping algorithms. The characteristics of the Tb indexes and thresholds used in the tested PMW SCA algorithms were dissected. We found that some criteria used in the algorithms did not work effectively as designed. The failure criteria had a slight contribution to snow identification or introduced large classification errors. Revising the failure $\mathrm{Tb}$ indexes and thresholds would be a means to improve the algorithms. For example, the omission error is the main error course of FY algorithm. Extending the snow criteria of FY algorithm is the proposed method to reduce its omission error. For Kelly's algorithm, Grody's algorithm and the South China algorithm, their main error source is the commission error. It means these three algorithms tend to overestimate snow. Tightening their criteria for snow may reduce their omission error and improve their accuracy. Our analysis can be useful in understanding the uncertainties and the weaknesses of different PMW SCA mapping algorithms. The results shown in this study can be useful in developing new methods and can be taken as a reference when using PMW SCA products in different applications.

Acknowledgments: This study was supported by the National Natural Science Foundation of China (41671334), the Science \& Technology Basic Resources Investigation Program of China (2017FY10052), the National Basic Research Program of China (2015CB953701) and the Strategic Pionner Program on Space Science, Chinese Academy of Sciences (XDA15052300). The authors would like to thank the China Meteorological Administration, National Geomatics Center of China, National Snow and Ice Data Center and US Geological Survey for providing meteorological station data, land-cover products and satellite data.

Author Contributions: Lingmei Jiang conceived and designed the study; Shengli Wu provided the FY-3C/MWRI data; Xiaojing Liu analyzed the data and wrote the results; Shirui Hao, Gongxue Wang and Jianwei Yang contributed analysis tools and methods.

Conflicts of Interest: The authors declare no conflict of interest.

$\begin{array}{ll}\text { Abbreviations } & \\ \text { PMW } & \text { Passive microwave } \\ \text { SCA } & \text { Snow cover area } \\ \text { SCF } & \text { Snow cover fraction } \\ \text { SD } & \text { Snow depth } \\ \text { SWE } & \text { Snow water equivalent } \\ \text { Tb } & \text { Brightness temperature } \\ \text { DTb } & \text { Brightness temperature of descending orbit } \\ \text { ATb } & \text { Brightness temperature of ascending orbit } \\ 10 \mathrm{H}, 18 \mathrm{H}, 23 \mathrm{H}, 37 \mathrm{H}, 89 \mathrm{H} & \text { Brightness temperatures at the frequencies of } 10.65 \mathrm{GHz}, 18.7 \mathrm{GHz}, 23.8 \mathrm{GHz}, \\ & 36.5 \mathrm{GHz} \text { and } 89.0 \mathrm{GHz} \text { for horizontal polarization } \\ 10 \mathrm{~V}, 18 \mathrm{~V}, 23 \mathrm{~V}, 37 \mathrm{~V}, 89 \mathrm{~V} & \text { Brightness temperatures at the frequencies of } 10.65 \mathrm{GHz}, 18.7 \mathrm{GHz}, 23.8 \mathrm{GHz}, \\ \text { ASCT = ATb23.8V }-\mathrm{ATb} 89 \mathrm{~V}-(\text { ATb18.7V }- \text { ATb37.5V) } \\ \text { KLVN = 58.08 }-0.39 \times \text { Tb19V }+1.21 \times \text { Tb23V }-0.37 \times \text { Tb37H }+0.36 \times \text { Tb89V } \\ \text { F-region } & \text { Forest-covered region or dense-vegetation-covered region } \\ \text { S-region } & \text { Sparse-vegetation-covered region } \\ \text { OA } & \text { Overall accuracy } \\ \text { OE } & \text { Omission error } \\ \text { CE } & \text { Commission error } \\ \text { PPV } & \text { Positive predictive value } \\ & \end{array}$




\section{References}

1. Liang, S. Advances in Land Remote Sensing: System, Modeling, Inversion and Application; Springer Science \& Business Media: Houten, The Netherlands, 2008.

2. AghaKouchak, A.; Farahmand, A.; Melton, F.S.; Teixeira, J.; Anderson, M.C.; Wardlow, B.D.; Hain, C.R. Remote sensing of drought: Progress, challenges and opportunities. Geophys. Res. 2015, 53, 452-480. [CrossRef]

3. Frei, A.; Tedesco, M.; Lee, S.; Foster, J.; Hall, D.K.; Kelly, R.; Robinson, D.A. A review of global satellite-derived snow products. Adv. Space Res. 2012, 50, 1007-1029. [CrossRef]

4. Dietz, A.J.; Kuenzer, C.; Gessner, U.; Dech, S. Remote sensing of snow—A review of available methods. Int. J. Remote Sens. 2012, 33, 4094-4134. [CrossRef]

5. Frei, A.; Robinson, D.A. Northern hemisphere snow extent: Regional variability 1972-1994. J. Clim. 1999, 19, 1535-1560. [CrossRef]

6. Dozier, J. Spectral signature of alpine snow cover from the landsat thematic mapper. Remote Sens. Environ. 1989, 28, 9-22. [CrossRef]

7. Hall, D.K.; Riggs, G.A.; Salomonson, V.V.; DiGirolamo, N.E.; Bayr, K.J. MODIS snow-cover products. Remote Sens. Environ. 2002, 83, 181-194. [CrossRef]

8. Painter, T.H.; Rittger, K.; McKenzie, C.; Slaughter, P.; Davis, R.E.; Dozier, J. Retrieval of subpixel snow covered area, grain size, and albedo from MODIS. Remote Sens. Environ. 2009, 113, 868-879. [CrossRef]

9. Shi, J. An automatic algorithm on estimating sub-pixel snow cover from MODIS. Quat. Sci. 2012, 32, 6-15.

10. Grody, N.C. Surface identification using satellite microwave radiometers. IEEE Trans. Geosci. Remote Sens. 1988, 26, 850-859. [CrossRef]

11. Xu, X.; Liu, X.; Li, X.; Xin, Q.; Chen, Y.; Shi, Q.; Ai, B. Global snow cover estimation with microwave brightness temperature measurements and one-class in situ observations. Remote Sens. Environ. 2016, 182, 227-251. [CrossRef]

12. Kongoli, C.; Romanov, P.; Ferraro, R. Snow cover monitoring from remote sensing satellites: Possibilities for drought assessment. In Remote Sensing of Drought: Innovative Monitoring Approaches; CRC Press: Boca Raton, FL, USA, 2012; pp. 359-386.

13. Shi, J.C.; Xiong, C.; Jiang, L.M. Review of snow water equivalent microwave remote sensing. Sci. China Earth Sci. 2016, 59, 731-745. [CrossRef]

14. Kelly, R. The AMSR-E snow depth algorithm: Description and initial results. J. Remote Sens. 2009, 29, 307-317.

15. Li, X.; Liu, Y.; Zhu, X.; Zheng, Z.; Chen, A. Snow cover identification with SSM/I data in china. J. Appl. Meteorol. Sci. 2007, 18, 12-20.

16. Grody, N.C.; Basist, A.N. Global identification of snowcover using SSM/I measurements. IEEE Trans. Geosci. Remote Sens. 1996, 34, 237-249. [CrossRef]

17. Pan, J.; Jiang, L.; Zhang, L. In Wet snow detection in the south of china by passive microwave remote sensing. In Proceedings of the 2012 32nd IEEE International Geoscience and Remote Sensing Symposium, Munich, Germany, 22-27 July 2012; Institute of Electrical and Electronics Engineers Inc.: Munich, Germany, 2012; pp. 4863-4866.

18. Singh, P.R.; Gan, T.Y. Retrieval of snow water equivalent using passive microwave brightness temperature data. Remote Sens. Environ. 2000, 74, 275-286. [CrossRef]

19. Hall, D.K.; Kelly, R.E.; Riggs, G.A.; Chang, A.T.; Foster, J.L. Assessment of the relative accuracy of hemispheric-scale snow-cover maps. Ann. Glaciol. 2002, 34, 24-30. [CrossRef]

20. Neale, C.M.U.; Mcfarland, M.J.; Chang, K. Land-surface-type classification using microwave brightness temperatures from the special sensor microwave/imager. IEEE Trans. Geosci. Remote Sens. 1990, 28, 829-838. [CrossRef]

21. Liang, T.; Zhang, X.; Xie, H.; Wu, C.; Feng, Q.; Huang, X.; Chen, Q. Toward improved daily snow cover mapping with advanced combination of MODIS and AMSR-E measurements. Remote Sens. Environ. 2008, 112, 3750-3761. [CrossRef]

22. Ramsay, B.H. The interactive multisensor snow and ice mapping system. Hydrol. Process. 1998, 12, 1537-1546. [CrossRef] 
23. Helfrich, S.R.; McNamara, D.; Ramsay, B.H.; Baldwin, T.; Kasheta, T. Enhancements to, and Forthcoming Developments in the Interactive Multisensor Snow and Ice Mapping System (IMS). Hydrol. Process. 2007, 21, 1576-1586. [CrossRef]

24. Romanov, P. Global multisensor automated satellite-based snow and ice mapping system (GMASI) for cryosphere monitoring. Remote Sens. Environ. 2017, 196, 42-55. [CrossRef]

25. Luojus, K.; Pulliainen, J.; Takala, M.; Kangwa, M.; Smolander, T.; Wiesmann, A.; Derksen, C.; Metsamaki, S.; Salminen, M.; Solberg, R.; et al. Globsnow-2 Product User Guide Version 1.0; European Space Agency Study Contract Report, ESRIN Contract 21703/08/I-EC; ESA/ESRIN: Frascati, Italy, 2013.

26. Jiang, L.; Wang, P.; Zhang, L.; Yang, H.; Yang, J. Improvement of snow depth retrieval for fy3b-mwri in china. Sci. China Earth Sci. 2014, 57, 1278-1292. [CrossRef]

27. Maeda, T.; Taniguchi, Y. Descriptions of GCOM-W1 AMSR-2 Level 1R and Level 2 Algorithms; Japan Aerospace Exploration Agency Earth Observation Research Center: Ibaraki, Japan, 2013.

28. Chang, A.T.; Rango, A. Algorithm Theoretical Basis Document (ATBD) for the AMSR-E Snow Water Equivalent Algorithm; NASA/GSFC: Greenbelt, MD, USA, 2000.

29. Hori, M.; Sugiura, K.; Kobayashi, K.; Aoki, T.; Tanikawa, T.; Kuchiki, K.; Niwano, M.; Enomoto, H. A 38-year (1978-2015) northern hemisphere daily snow cover extent product derived using consistent objective criteria from satellite-borne optical sensors. Remote Sens. Environ. 2017, 191, 402-418. [CrossRef]

30. Romanov, P.; Gutman, G.; Csiszar, I. Automated monitoring of snow cover over North America with multispectral satellite data. J. Appl. Meteorol. 2000, 39, 1866-1880. [CrossRef]

31. Chen, X.; Jiang, L.; Yang, J.; Pan, J. Validation of ice mapping system snow cover over southern china based on landsat enhanced thematic mapper plus imagery. J. Appl. Remote Sens. 2014, 8. [CrossRef]

32. Rittger, K.; Painter, T.H.; Dozier, J. Assessment of methods for mapping snow cover from MODIS. Adv. Water Resour. 2013, 51, 367-380. [CrossRef]

33. Lee, Y.K.; Kongoli, C.; Key, J. An in-depth evaluation of heritage algorithms for snow cover and snow depth using AMSR-E and AMSR2 measurements. J. Atmos. Ocean. Technol. 2015, 32, 2319-2336. [CrossRef]

34. Siljamo, N.; Hyvärinen, O. New geostationary satellite-based snow-cover algorithm. J. Appl. Meteorol. Clim. 2011, 50, 1275-1290. [CrossRef]

35. Grody, N.C. Classification of snow cover and precipitation using the special sensor microwave imager. J. Geophys. Res. Atmos. 1991, 96, 7423-7435. [CrossRef]

36. Foster, J.L.; Hall, D.K.; Chang, A.T.C.; Rango, A. An overview of passive microwave snow research and results. Rev. Geophys. Space Phys. 1984, 22, 195-208. [CrossRef]

37. Brubaker, K.L.; Pinker, R.T.; Deviatova, E. Evaluation and comparison of MODIS and IMS snow-cover estimates for the continental united states using station data. J. Hydrometeorol. 2005, 6, 1002-1017. [CrossRef]

38. Wang, J.; Li, H.; Hao, X.; Huang, X.; Hou, J.; Che, T.; Dai, L.; Liang, T.; Huang, C.; Li, H.; et al. Remote sensing for snow hydrology in China: Challenges and perspectives. J. Appl. Remote Sens. 2014, 8. [CrossRef]

39. Yang, J.; Jiang, L.; Menard, C.B.; Luojus, K.; Lemmetyinen, J.; Pulliainen, J. Evaluation of Snow Products over the Tibetan Plateau. Hydrol. Process. 2015, 29, 3247-3260. [CrossRef]

40. Klein, A.G.; Barnett, A.C. Validation of daily MODIS snow cover maps of the Upper Rio Grande River Basin for the 2000-2001 snow year. Remote Sens. Environ. 2003, 86, 162-176. [CrossRef]

41. Rittger, K.; Bair, E.H.; Kahl, A.; Dozier, J. Spatial estimates of snow water equivalent from reconstruction. Adv. Water Resour. 2016, 94, 345-363. [CrossRef]

42. Romanov, P.; Dan, T.; Gutman, G.; Carroll, T. Mapping and monitoring of the snow cover fraction over North America. J. Geophys. Res. 2003, 108, 137-141. [CrossRef]

43. Bitner, D.; Carroll, T.; Cline, D.; Romanov, P. An assessment of the differences between three satellite snow cover mapping techniques. Hydrol. Process. 2002, 16, 3723-3733. [CrossRef]

44. Sharma, R.; Ghosh, A.; Joshi, P.K. Decision tree approach for classification of remotely sensed satellite data using open source support. J. Erath Syst. Sci. 2013, 122, 1237-1247. [CrossRef]

45. Pan, J. Study of the Snow Detection Decision Tree Algorithm Using Passive Microwave Remote Sensing Technology in the South of China. Master's Thesis, Beijing Normal University, Beijing, China, 2012.

46. Walker, A.E.; Goodison, B.E. Discrimination of a wet snowcover using passive microwave satellite data. Ann. Glaclol. 1993, 17. [CrossRef] 
47. Wu, S.; Chen, J. Instrument performance and cross calibration of FY-3C MWRI. In Proceedings of the 36th IEEE International Geoscience and Remote Sensing Symposium, IGARSS 2016, Beijing, China, 10-15 July 2016; pp. 388-391.

48. Yang, J.; Luojus, K.; Lemmetyinen, J.; Jiang, L.; Pulliainen, J. Comparison of SSMIS, AMSR-E and MWRI brightness temperature data. In Proceedings of the Joint 2014 IEEE International Geoscience and Remote Sensing Symposium, IGARSS 2014 and the 35th Canadian Symposium on Remote Sensing, CSRS 2014, Quebec City, QC, Canada, 13-18 July 2014; pp. 2574-2577.

(C) 2018 by the authors. Licensee MDPI, Basel, Switzerland. This article is an open access article distributed under the terms and conditions of the Creative Commons Attribution (CC BY) license (http:/ / creativecommons.org/licenses/by/4.0/). 
Article

\title{
Estimation of Global Vegetation Productivity from Global LAnd Surface Satellite Data
}

\author{
Tao Yu ${ }^{1,2}$, Rui Sun ${ }^{1,2, *}$, Zhiqiang Xiao ${ }^{1,2, *}$, Qiang Zhang ${ }^{1,2}$, Gang Liu ${ }^{1,2}$, Tianxiang Cui ${ }^{1,2}$ and \\ Juanmin Wang ${ }^{1,2}$ \\ 1 State Key Laboratory of Remote Sensing Science, Jointly Sponsored by Beijing Normal University \\ and Institute of Remote Sensing and Digital Earth of Chinese Academy of Sciences, Beijing 100875, China; \\ yutaogis@mail.bnu.edu.cn (T.Y.); zhangqiang1228@mail.bnu.edu.cn (Q.Z.); \\ gang4.liu@changhong.com (G.L.); txiang.c@gmail.com (T.C.); 201631170025@mail.bnu.edu.cn (J.W.) \\ 2 Beijing Engineering Research Center for Global Land Remote Sensing Products, Institute of Remote Sensing \\ Science and Engineering, Faculty of Geographical Science, Beijing Normal University, Beijing 100875, China \\ * Correspondence: sunrui@bnu.edu.cn (R.S.); zhqxiao@bnu.edu.cn (Z.X.); \\ Tel.: +86-10-5880-5457 (R.S.); +86-10-5880-7698 (Z.X.)
}

Received: 28 December 2017; Accepted: 18 February 2018; Published: 22 February 2018

\begin{abstract}
Accurately estimating vegetation productivity is important in research on terrestrial ecosystems, carbon cycles and climate change. Eight-day gross primary production (GPP) and annual net primary production (NPP) are contained in MODerate Resolution Imaging Spectroradiometer (MODIS) products (MOD17), which are considered the first operational datasets for monitoring global vegetation productivity. However, the cloud-contaminated MODIS leaf area index (LAI) and Fraction of Photosynthetically Active Radiation (FPAR) retrievals may introduce some considerable errors to MODIS GPP and NPP products. In this paper, global eight-day GPP and eight-day NPP were first estimated based on Global LAnd Surface Satellite (GLASS) LAI and FPAR products. Then, GPP and NPP estimates were validated by FLUXNET GPP data and BigFoot NPP data and were compared with MODIS GPP and NPP products. Compared with MODIS GPP, a time series showed that estimated GLASS GPP in our study was more temporally continuous and spatially complete with smoother trajectories. Validated with FLUXNET GPP and BigFoot NPP, we demonstrated that estimated GLASS GPP and NPP achieved higher precision for most vegetation types.
\end{abstract}

Keywords: GPP; NPP; MODIS; validation

\section{Introduction}

As vegetation productivity is one of the most variable components of the terrestrial carbon cycle, accurately estimating this component is important in research on terrestrial ecosystems, carbon cycles and climate change [1]. On a global scale, MOD17 products are the first operational data sets to regularly monitor global vegetation productivity from MODerate Resolution Imaging Spectroradiometer (MODIS). Eight-day gross primary production (GPP) and annual net primary production (NPP) with $1 \mathrm{~km}$ spatial resolution are components of the MOD17 product. MOD17 is derived from a light use efficiency (LUE) model [2,3] based on the MOD12 land cover product, Data Assimilation Office meteorological datasets, and the MOD15 Leaf Area Index (LAI) and Fraction of Photosynthetically Active Radiation (FPAR) products. Consequently, the uncertainties of these input data will influence the accuracy of the MOD17 product [1]. Therefore, estimating global vegetation productivity products from datasets with a higher precision is imperative for improving the quality of vegetation productivity products.

Studies have shown that the contaminated or missing MOD15 LAI and FPAR products may introduce some considerable errors to MODIS GPP and NPP products [4]. In addition, MOD15 LAI 
and FPAR products generated from individual satellite data have been demonstrated to be spatially and temporally discontinuous on regional and global scales, and are less representative in some vegetation types [5-8]. Thus, to generate more accurate and consistent GPP and NPP products, more consistent and higher quality LAI and FPAR datasets should be used. Global LAnd Surface Satellite (GLASS) LAI products were developed based on time-series MODIS and Advanced Very High Resolution Radiometer reflectance data using General Regression Neural Networks (GRNNs) trained with fused time-series LAI from MODIS and Carbon CYcle and Change in Land Observational Products from an Ensemble of Satellites (CYCLOPES) [9-11]. Studies [9,10,12] have shown that the GLASS LAI product achieves a higher precision than MODIS LAI and is more temporally continuous and spatially complete with smoother trajectories. Moreover, Xiao et al. [13] developed the GLASS FPAR product with continuous trajectories and high accuracy based on the GLASS LAI product. Therefore, more continuous results with higher precision may be obtained when estimating global vegetation productivity using GLASS LAI and FPAR products.

For the estimation of vegetation productivity, many models have been developed on both regional and global scales. Climate productivity models, such as the Miami model, Thornthwaite Memorial model [14], and Chikugo model [15], are based on the relationships between GPP/NPP and climate factors. LUE models have great potential to address the spatial and temporal dynamics of GPP/NPP [16], such as the Carnegie, Ames, Stanford Approach (CASA) [17] and the GLObal Production Efficiency Model (GLO-PEM) [18]. The basis of eco-physiological process models is ecological and biophysical process theories, such as the CENTURY [19], Terrestrial Ecosystem Model (TEM) [20], and Biome Bio-Geochemical Cycle (Biome-BGC) model [21]. MOD17 daily GPP and annual NPP products are derived from the LUE model. FPAR is related to GPP assimilation, and LAI is related to respiration when estimating NPP. However, daily MODIS Net Photosynthesis (PSNnet) does not include the calculation of growth respiration and maintenance respiration associated with living wood when compared with NPP. In this case, developing a more reliable algorithm to estimate global daily GPP and NPP by integrating high quality GLASS data and eco-physiological processes is of great significance.

The aims of this paper are (i) to generate more accurate and continuous global GPP and NPP products with GLASS data; (ii) to validate the GPP and NPP estimates, and to compare the results with products; and (iii) to analyze the temporal and spatial variation of global GPP and NPP from 2004 to 2012.

\section{Materials and Methods}

\subsection{Method}

\subsubsection{Estimation of GPP and NPP}

A flowchart of GPP and NPP estimation and validation is shown in Figure 1. To estimate global daily GPP/NPP by integrating remote sensing data and eco-physiological processes, the Multi-source data Synergized Quantitative (MuSyQ) NPP algorithm [22] was adopted in this paper. The MuSyQ-NPP algorithm is essentially a LUE model; thus, daily GPP could be described as:

$$
\mathrm{GPP}=\varepsilon \times \mathrm{FPAR} \times \mathrm{PAR}
$$

where $\varepsilon$ is the LUE, and PAR is the photosynthetically active radiation (PAR), which could be obtained from Global Land Data Assimilation System (GLDAS) incident shortwave radiation $\mathrm{R}$ using the following equation:

$$
\mathrm{PAR}=0.5 \times \mathrm{R}
$$

LUE may be influenced by many environmental stressors, such as low temperature and water shortage. In this condition, $\varepsilon$ could be described as:

$$
\varepsilon=\varepsilon_{\max } \times f_{1}(T) \times f_{2}(\beta)
$$


where $\varepsilon_{\max }$ is the biome-specific potential of LUE, and $f_{1}(T)$ and $f_{2}(\beta)$ are the down-regulation effects of temperature and water conditions on $\varepsilon_{\max }$, respectively. A biome-specific potential $\varepsilon_{\max }$ Look-Up Table (LUT) was established by referring to land cover categories according to the Biome Properties Look-Up Table (BPLUT) of the MODIS GPP/NPP algorithm [23]. It is noted that a potential $\varepsilon_{\text {max }}$ value of $1.5 \mathrm{~g} \mathrm{C} \mathrm{MJ}^{-1}$ for crops was used in the algorithm. $f_{1}(T)$ was calculated according to the CASA model [17]. The effect of water conditions on plant photosynthesis $f_{2}(\beta)$ could be described as:

$$
f_{2}(\beta)=0.5+0.5 E T / E T_{P}
$$

where ET represents actual evapotranspiration, which is estimated from a modified P-M approach, LAI and Fcover products [22,24,25]. ET $P$ represents potential evapotranspiration, which is estimated using the Priestley and Taylor (PT) equation [26].

NPP is the net flow of carbon from the atmosphere to plants, which is defined as the balance between GPP and autotrophic respiration.

$$
N P P=G P P-R_{a}
$$

where $R_{a}$ is autotrophic respiration. Autotrophic respiration $\left(R_{a}\right)$ could be separated into two parts: maintenance respiration $R_{m}$, which refers to the energy necessary to maintain biomass, and growth respiration $R_{g}$ which refers to the energy needed for converting assimilates into new structural plant constituents.

$$
R_{a}=R_{m}+R_{g}
$$

When estimating $R_{m}$, non-forest and forest lands were estimated separately. For non-forest land, $R_{m}$ was estimated with LAI and specific leaf area (SLA).

$$
R_{m}=L A I / S L A \times 0.5 \times 2^{\left(T-T_{b}\right) / 10}
$$

where SLA was obtained from the BPLUT of MODIS GPP/NPP algorithm [23] in our model.

For forest land, $R_{m}$ was obtained by summing the maintenance respiration of leaves, stems, and roots [22].

$$
R_{m, i}=M_{i} r_{m, i} Q_{10, i}^{T-T_{b} / 10}
$$

where $M_{i}$ is the biomass of plant component $i, r_{m, i}$ is maintenance respiration coefficient for component $i, Q_{10, i}$ is the temperature sensitivity factor, $T$ is the daily average temperature and $T_{b}$ is the base temperature, $r_{m, i}$ is the maintenance respiration coefficient. Forest lands are classified into four classes: needle-leaf forests, broadleaved forests, mixed forests and others, and $Q_{10, i}$ and $r_{m, i}$ for each class are obtained according to the BPLUT of MODIS GPP/NPP algorithm [23]. Specifically, leaf mass is estimated from LAI and SLA:

$$
M_{1}=L A I / S L A
$$

Stem and root mass are obtained using:

$$
\begin{gathered}
M_{2}=\text { biomass } /(1+y) \\
M_{3}=y \times M_{2}
\end{gathered}
$$

where $y$ is the ecophysiological biome-specific constant obtained from the Boreal Ecosystem Productivity Simulator (BEPS) model [27]. The leaf, stem, fine root and coarse root respiration items were calculated separately, according to the BEPS model.

Growth respiration $\left(R_{g}\right)$ was considered to be proportional to the difference between GPP and maintenance respiration $\left(R_{m}\right)$ :

$$
R_{g}=\gamma\left(G P P-R_{m}\right)
$$


where $\gamma$ is the growth respiration coefficient and is defined as 0.25 .

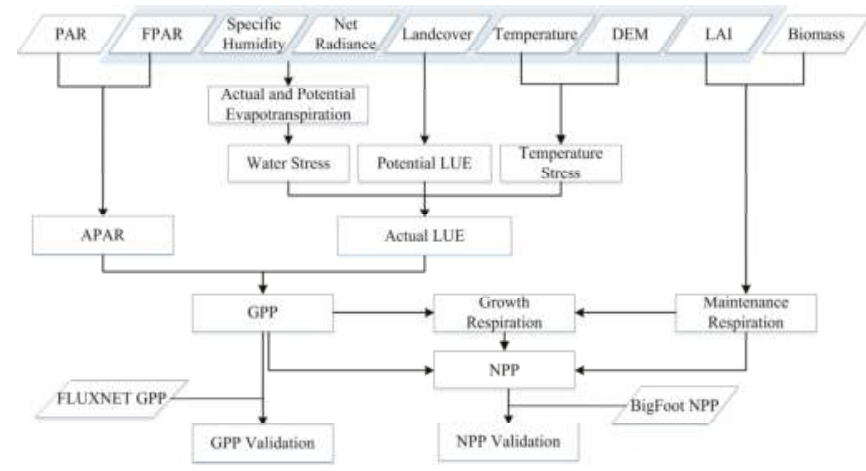

Figure 1. Flowchart of gross primary production (GPP) and net primary production (NPP) estimation and validation. FPAR: Fraction of Photosynthetically Active Radiation; LAI: Leaf Area Index; LUE: Light Use Efficiency; PAR: Photosynthetically Active Radiation; APAR: Absorbed Photosynthetically Active Radiation; DEM: Digital Elevation Model.

\subsubsection{Validation of Estimated GLASS GPP and NPP}

To assess the performance of the MuSyQ-NPP algorithm, FLUXNET GPP and BigFoot NPP were used to validate the GLASS GPP and NPP estimates. Specifically, eight-day GLASS GPP estimates were compared with ground level eight-day FLUXNET GPP, and the average GLASS NPP estimates in 2004, 2008 and 2012 at each Bigfoot site were compared with ground level yearly BigFoot NPP for 2000-2004.

\subsection{Data and Data Processing}

\subsubsection{Remote Sensing Data}

GLASS LAI/FPAR Products: The GLASS LAI/FPAR products, with a temporal resolution of eight days and a spatial resolution of $1 \mathrm{~km}$, were generated and released by the Center for Global Change Data Processing and Analysis of Beijing Normal University [28]. They are also available from the Global Land Cover Facility [29]. The GLASS LAI product was estimated from time-series MODIS and AVHRR reflectance data using GRNNs, which were trained for each biome type by fusing MODIS and CYCLOPES LAI. The GLASS LAI product is believed to be more temporally continuous and spatially complete compared to the MODIS LAI product [11]. The GLASS FPAR product was generated from the GLASS LAI product; thus, it shows the same properties as the GLASS LAI product. Validation against the VAlidation of Land European Remote sensing Instrument (VALERI) ground-based estimates has shown that both GLASS LAI and FPAR products had high accuracy (RMSE $=0.7848$ and $R^{2}=0.8095$ for the LAI product, $\mathrm{RMSE}=0.1276$ and $\mathrm{R}^{2}=0.8048$ for the FPAR product) $[10,13]$.

MODIS land cover product: The MODIS Land Cover Dynamics product (MCD12Q2) provided estimates of yearly vegetation phenology on a global scale [30]. The product identified 17 land cover classes defined by the International Geosphere Biosphere Program (IGBP) with a spatial resolution of $1 \mathrm{~km}$, which includes 11 natural vegetation classes, three developed and mosaicked land classes, and three non-vegetated land classes [31].

TRAGL fractional vegetation cover (FCover) product: The TRAGL FCover product, with a temporal resolution of eight days and a spatial resolution of $1 \mathrm{~km}$, was derived from the GLASS LAI product based on the method proposed by Xiao et al. [32]. The product is believed to be more spatially and temporally complete with continuous trajectories compared with the GEOV1 product. Direct validation 
with ground-based FCover estimates demonstrated that the TRAGL FCover product was generated with high accuracy (RMSE $=0.0865$ and $\mathrm{R}^{2}=0.8848$ ) [26].

Global Biomass Map: A global biomass map with a spatial resolution of $1 \mathrm{~km}$ was generated by the Carbon Dioxide Information Analysis Center (CDIAC) [33] using International Panel on Climate Change (IPCC) methods $[34,35]$. The global biomass carbon stocks were firstly estimated using the globally consistent default values provided for aboveground biomass [35], then belowground biomass (root) was added using the IPCC root to shoot ratios for each vegetation type.

\subsubsection{Meteorological Data}

Meteorological data were derived from Global Land Data Assimilation System (GLDAS) products [36]. GLDAS products were generated using four land surface models and data assimilation techniques with both satellite data and ground based observational data [37,38]. Daily near surface air temperature, near surface specific humidity, and net shortwave radiation were obtained in our study by averaging the GLDAS three-hour products. As the spatial resolution of this product was $0.25^{\circ}$, bilinear interpolation was adopted to generate the $1 \mathrm{~km}$ meteorological datasets.

\subsubsection{Field Data}

FLUXNET GPP data: FLUXNET is a global network of micrometeorological tower sites used to measure the exchanges of carbon dioxide, water vapor, and energy between the biosphere and atmosphere. This global network includes more than eight hundred active and historic flux sites, which are dispersed across most of the world's climate space and across representative biomes. FLUXNET data are most commonly used in validation and calibration of ecosystem models, parameter estimation and optimization procedures in data assimilation systems [39,40]. In our study, daily GPP derived by the daytime partitioning method from 59 FLUXNET sites (See Table S1 in Supplementary Materials) was firstly collected, and then eight-Day GPP were obtained by averaging the daily GPP to validate our GPP estimates and MODIS GPP product [41]. Vegetation types in these FLUXNET sites include deciduous broadleaved forest (DBF), evergreen broadleaved forest (EBF), evergreen needle-leaf forest (ENF), cropland (CRO), grassland (GRA), and mixed forest (MF). The locations of FLUXNET sites used in this study, and the IGBP landcover classes are shown in Figure 2.

BigFoot NPP data: The overall objective of BigFoot was to provide ground validation for MODIS land cover, LAI, FPAR and NPP products [42]. Nine BigFoot sites were established from Alaska to Brazil. The BigFoot NPP product was generated using the Biome-BGC model [21], based on Landsat ETM+ derived land cover and LAI, tower measured meteorological data and eco-physical parameters. Each BigFoot NPP product covered a $7 \times 7 \mathrm{~km}$ area with a temporal resolution of one year and a spatial resolution of $25 \mathrm{~m}$. In our study, BigFoot NPP data were used to validate NPP estimates and the MODIS NPP product. Basic information on BigFoot sites is summarized in Table 1.

Table 1. Basic information of BigFoot sites.

\begin{tabular}{ccccc}
\hline Site Name & Latitude & Longitude & Biome Type & Year \\
\hline NOBS & 55.8853 & -98.4773 & Boreal forest & $2001-2003$ \\
AGRO & 40.0067 & -88.2915 & Crop & 2000 \\
HARV & 42.5285 & -72.1729 & Temperate mixed forest & 2003 \\
CHEQ & 45.9453 & -90.2731 & Temperate mixed forest & $2000-2002$ \\
METL & 44.4508 & -121.5733 & Temperate needleleaf forest & 2002 \\
TAPA & -2.86957 & -54.9497 & Tropical broadleaf evergreen forest & 2004 \\
KONZ & 39.0890 & -96.5714 & Tallgrass prairie & $2000-2002$ \\
SEVI & 34.3509 & -106.6900 & Desert & 2003 \\
TUND & 71.2719 & -156.6130 & Arctic tundra & 2002 \\
\hline
\end{tabular}




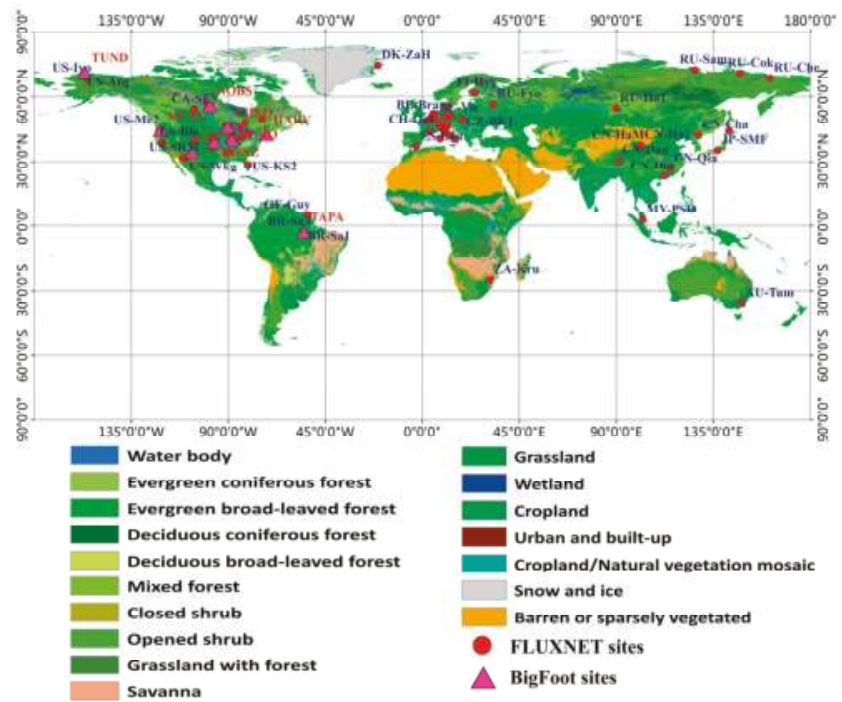

Figure 2. MODerate Resolution Imaging Spectroradiometer (MODIS) International Geosphere Biosphere Program (IGBP) land-cover and location of FLUXNET sites and BigFoot sites.

\section{Results}

\subsection{Temporal and Spatial Variation of Global GPP and NPP}

Using the MuSyQ-NPP algorithm, maps of global estimated $1 \mathrm{~km}$ GLASS GPP and NPP in 2004, 2008, and 2012 were obtained, as shown in Figure 3. The highest vegetation productivity $\left(\mathrm{GPP}>2000 \mathrm{~g} \mathrm{C} \cdot \mathrm{m}^{-2}, \mathrm{NPP}>1000 \mathrm{~g} \mathrm{C} \cdot \mathrm{m}^{-2}\right)$ was found in the humid tropic areas, such as Amazonia, Central Africa and South-east Asia, where both temperature and moisture requirements are fully satisfied for photosynthesis. Temperate regions have an intermediate vegetation productivity $\left(\mathrm{GPP} \approx 1000-1400 \mathrm{~g} \mathrm{C} \cdot \mathrm{m}^{-2}, \mathrm{NPP} \approx 500-800 \mathrm{~g} \mathrm{C}^{-\mathrm{m}^{-2}}\right.$ ), and the lowest vegetation productivity (GPP $<400 \mathrm{~g} \mathrm{C} \cdot \mathrm{m}^{-2}$, NPP $<200 \mathrm{~g} \mathrm{C} \cdot \mathrm{m}^{-2}$ ) was found in either cold or arid regions, where either temperature or precipitation are limiting factors. Variations in GPP and NPP are demonstrated in Figure 4. In general, from 2004 to 2008, few changes occurred in most areas. The largest GPP increases, about $100 \mathrm{~g} \mathrm{C} \cdot \mathrm{m}^{-2}$, were in Central China and Eastern Russia. In addition, the largest decreases, about $150 \mathrm{~g} \mathrm{C} \cdot \mathrm{m}^{-2}$, occurred in Amazonia and South-eastern Asia. From 2008 to 2012, GPP and NPP increased in most areas. Specifically, the largest increasing trends occurred in tropic areas, such as Amazonia and South-eastern Asia, in which GPP increased by approximately $400 \mathrm{~g} \mathrm{C} \cdot \mathrm{m}^{-2}$ and NPP increased approximately by $200 \mathrm{~g} \mathrm{C} \cdot \mathrm{m}^{-2}$. The next largest increases were seen in areas in Russia, Northern America, and Northeastern China, in which GPP increased by about $100 \mathrm{~g} \mathrm{C} \cdot \mathrm{m}^{-2}$ and NPP increased by about $50 \mathrm{~g}$ $\mathrm{C} \cdot \mathrm{m}^{-2}$. The largest decreasing trends occurred in areas of Eastern Brazil, where GPP decreased by about $300 \mathrm{~g} \mathrm{C} \cdot \mathrm{m}^{-2}$ and NPP decreased by about $150 \mathrm{~g} \mathrm{C} \cdot \mathrm{m}^{-2}$.

Global total GPP in 2004, 2008 and 2012 was 108.84 Pg C, 107.30 Pg C and 119.73 Pg C, respectively. Global total GPP estimates are comparable with values of 109.29 Pg C [4], $110.5 \pm 21.3 \mathrm{Pg}$ C [43] and $132 \pm 22 \mathrm{Pg} \mathrm{C}$ [1]. Global total GPP decreased 1.41\% from 2004 to 2008 and increased 11.58\% from 2008 to 2012. Global NPP in 2004, 2008 and 2012 was 61.15 Pg C, 60.15 Pg C and 69.53 Pg C, respectively, which was comparable to previously reported values of 56.40 Pg C [44], 56.02 Pg C [4] and values between $39.9 \mathrm{Pg} \mathrm{C}$ and $80.5 \mathrm{Pg} \mathrm{C}$ with a mean of 54.9 Pg C [45]. Global total NPP decreased by $1.64 \%$ from 2004 to 2008 and increased by $15.59 \%$ from 2008 to 2012 according to our study (Figure 5). 
The mean and standard deviation of GPP and NPP for different land cover types are shown in Figure 6. GPP and NPP values were highest in EBF. GPP could be as high as $2799.90 \mathrm{~g} \mathrm{C} \cdot \mathrm{m}^{-2} \cdot \mathrm{yr}^{-1}$, and NPP was approximately $1311.29 \mathrm{~g} \mathrm{C} \cdot \mathrm{m}^{-2} \cdot \mathrm{yr}^{-1}$. GPP and NPP values were lowest in Bare Land: GPP was only $47.25 \mathrm{~g} \mathrm{C} \cdot \mathrm{m}^{-2} \cdot \mathrm{yr}^{-1}$, and NPP was only $25.71 \mathrm{~g} \mathrm{C} \cdot \mathrm{m}^{-2} \cdot \mathrm{yr}^{-1}$. Generally, GPP and NPP in 2012 were higher than these in 2004 and 2008 at all land cover types. GPP and NPP in 2008 were at their minimums among these three years. This is more obvious in EBF. GPP was $3097.35 \mathrm{~g} \mathrm{C} \cdot \mathrm{m}^{-2} \cdot \mathrm{yr}^{-1}$ in 2012, $389.76 \mathrm{~g} \mathrm{C} \cdot \mathrm{m}^{-2} \cdot \mathrm{yr}^{-1}$ more than in 2004 and $502.61 \mathrm{~g} \mathrm{C} \cdot \mathrm{m}^{-2} \cdot \mathrm{yr}^{-1}$ more than in 2008. NPP in 2012 could be as high as $1571.14 \mathrm{~g} \mathrm{C} \cdot \mathrm{m}^{-2} \cdot \mathrm{yr}^{-1}, 354.75 \mathrm{~g} \mathrm{C} \cdot \mathrm{m}^{-2} \cdot \mathrm{yr}^{-1}$ more than in 2004 and $424.82 \mathrm{~g} \mathrm{C} \cdot \mathrm{m}^{-2} \cdot \mathrm{yr}^{-1}$ more than in 2008. However, in DNF, MF, open shrubland (OSH) and bare land (BL), GPP kept increasing from 2004 to 2012. Variation in global total GPP and NPP for all vegetated land cover types is demonstrated in Figure 7. Generally, variations in GPP and NPP from 2004 to 2008 were less than those from 2008 to 2012. The largest variation occurred in the EBF, in which GPP decreased $1.22 \mathrm{Pg} C$ from 2004 to 2008 and increased $3.24 \mathrm{Pg} C$ from 2008 to 2012. The next largest increases were seen in MF, OSH, woody savannas (WSAV), savannas (SAV) and GRA, GPP increased about $1 \mathrm{Pg}$ C, and NPP increased about $0.6 \mathrm{Pg}$ C from 2008 to 2012. The lowest variations were in DNF, DBF, close shrub-land (CSH), CRO, artificial land (AL), cropland/natural vegetation mosaic $(\mathrm{C} / \mathrm{NV})$ and $\mathrm{BL}$, in which variations of GPP and NPP were less than $0.2 \mathrm{Pg}$ C. Considering that some of that variability can be explained by regional patterns, we also studied the mean GPP and NPP for different biomes, as well as the variation in GPP and NPP in different biomes (See Text S1 in Supplementary Materials).
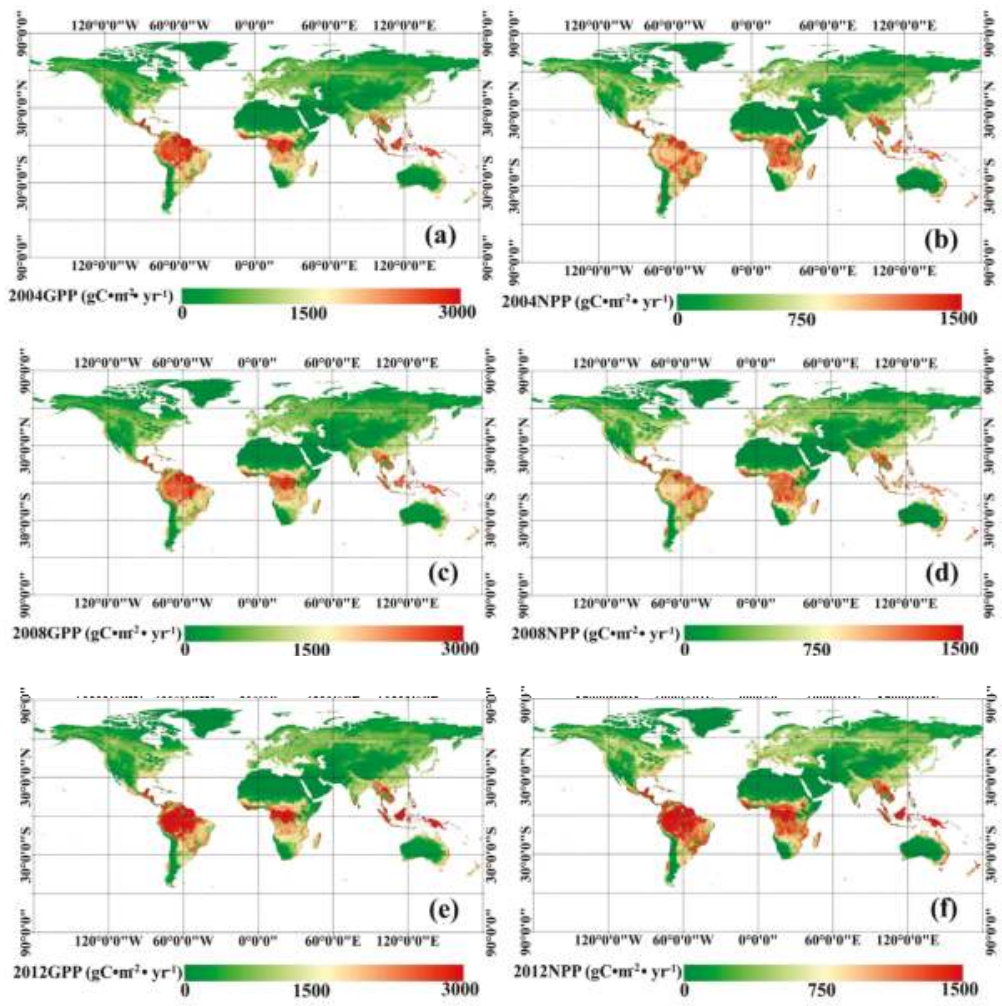

Figure 3. Global $1 \mathrm{~km}$ GPP and NPP in 2004, 2008 and 2012: (a) global GPP in 2004; (b) global NPP in 2004; (c) global GPP in 2008; (d) global NPP in 2008; (e) global GPP in 2012; (f) global NPP in 2012. 

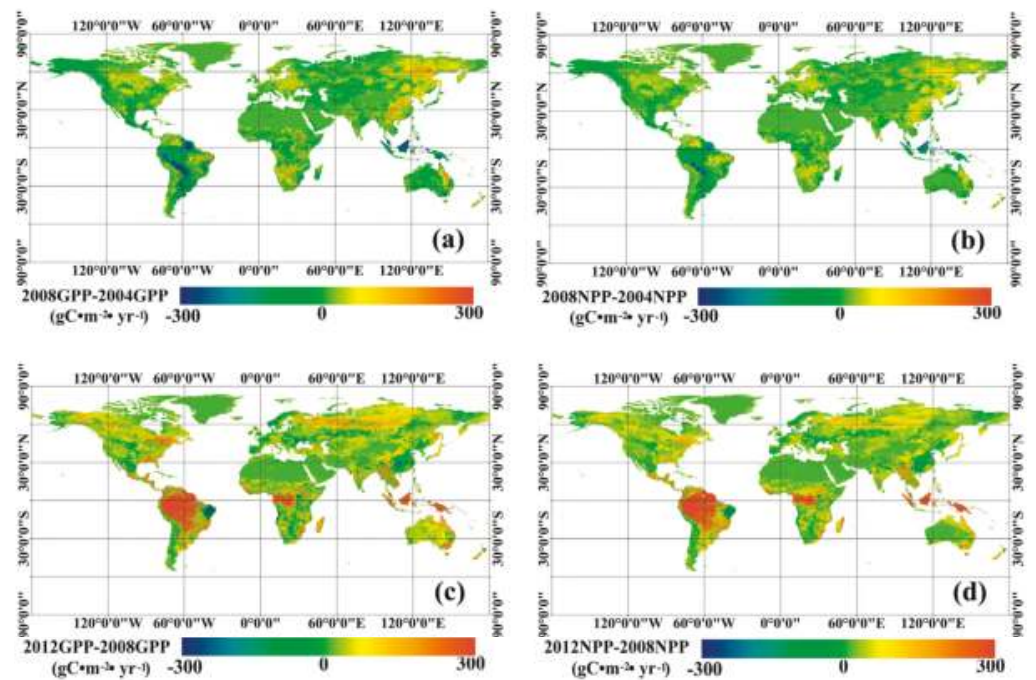

Figure 4. Variations in global GPP and NPP from 2004 to 2012: (a) variation in GPP from 2004 to 2008; (b) variation in NPP from 2004 to 2008; (c) variation in GPP from 2008 to 2012; (d) variation in NPP from 2008 to 2012.

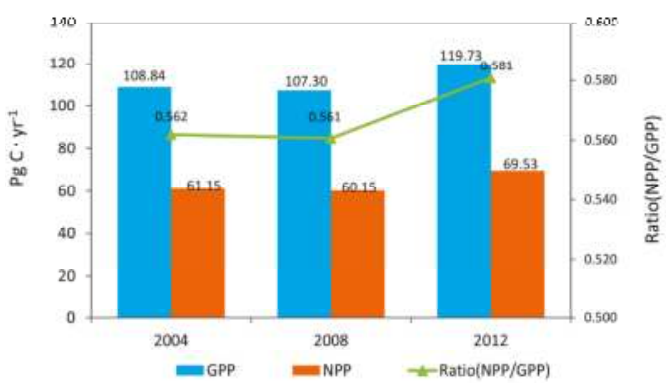

Figure 5. Global GPP and NPP in 2004, 2008 and 2012 estimated using Global LAnd Surface Satellite (GLASS) data.
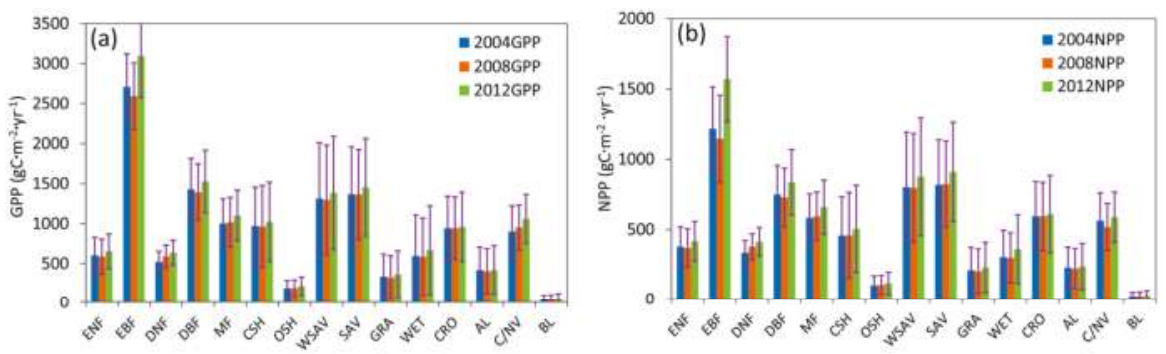

Figure 6. Global mean and standard deviations of GPP and NPP for all vegetated land cover types: (a) global mean and standard deviations of GPP; (b) global mean and standard deviations of NPP. 

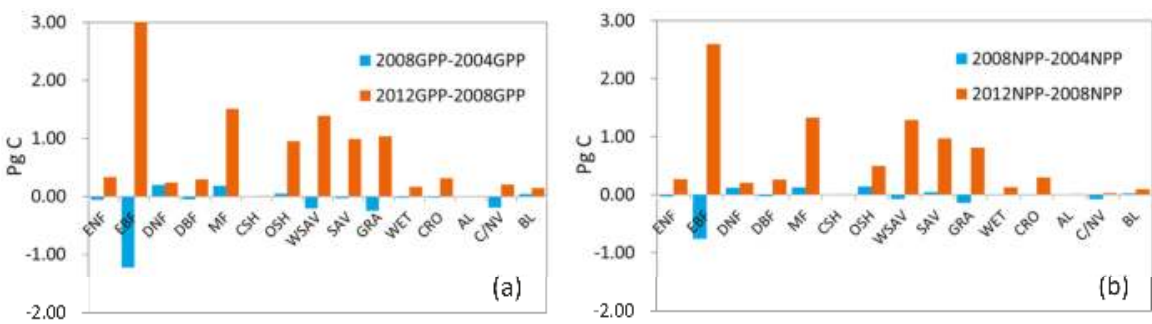

Figure 7. Variation in global total GPP and NPP for all vegetated land cover types: (a) variation in global GPP; (b) variation in global NPP.

\subsection{Validation of Estimated GLASS GPP}

\subsubsection{Seasonal Variation in GPP}

For various vegetated land cover types, we compared our GPP estimates with eight-day MODIS GPP product and FLUXNET GPP, as shown in Figure 8. With the application of temporal infilling of cloud-contaminated pixels and a consistent forcing meteorology in MODIS revised GPP product (MOD17 C55), we found that the time series of MOD17 C55 GPP was more continuous than the MODIS standard GPP product (MOD17 C05). But, the time series of estimated GLASS GPP in 2004, 2008 and 2012 are clearly more continuous with smoother trajectories than MODIS GPP (both MOD17 C05 GPP and MOD17 C55 GPP), and they agree well with the variations in FLUXNET GPP.

More specifically, the time series of estimated GLASS GPP in deciduous broadleaved forests (DBF) are shown in Figure 8a. It can be clearly seen that estimated GLASS GPP agrees better with FLUXNET GPP than MODIS GPP does and is more continuous, especially during the growing season. At US-Ha1 and De-Hai, estimated GLASS GPP matches much better with the FLUXNET GPP and reflects the peak of the growing season, but MODIS GPP is somewhat underestimated at the peak of the growing season, especially MOD17 C05 GPP in 2004. FLUXNET GPP and estimated GLASS GPP could be as high as $14 \mathrm{~g} \mathrm{C} \cdot \mathrm{m}^{-2} \cdot \mathrm{d}^{-1}$ in the peak of the growing season, but MODIS GPP is much lower, only approximately $8 \mathrm{~g} \mathrm{C} \cdot \mathrm{m}^{-2} \cdot \mathrm{d}^{-1}$.

In evergreen broadleaved forests (EBF), estimated GLASS GPP is obviously more continuous and generally showed better agreement with the FLUXNET GPP, as shown in Figure 8b. FLUXNET GPP showed little seasonal variation at the GF-Guy site, which is located in South America and near the equatorial region. But, at this site, MODIS GPP varied significantly, especially the MOD17 C05 GPP, and was underestimated in the first half of 2004, 2008 and 2012. In 2008 and 2012, MODIS GPP was underestimated most of the time and failed to catch the changes in FLUXNET GPP.

Figure $8 \mathrm{c}$ shows the GPP time series in evergreen needle-leaf forests (ENF). Good agreement was achieved between estimated GLASS GPP and FLUXNET GPP, in terms of the absolute values and seasonal trends. Overall, the time series of estimated GLASS GPP was more continuous than MODIS GPP. But at DE-Tha, estimated and MODIS GPP were a little underestimated. In 2008, the estimated growing season was delayed a little compared to the FLUXNET growing season.

Figure 8d demonstrates the GPP time series in croplands (CRO) in 2004, 2008 and 2012. Both MODIS GPP and estimated GLASS GPP significantly underestimated the FLUXNET GPP. Particularly during the growing seasons, estimated GLASS GPP and MODIS GPP were much lower than the FLUXNET GPP. The FLUXNET GPP could be as high as $16 \mathrm{~g} \mathrm{C} \cdot \mathrm{m}^{-2} \cdot \mathrm{d}^{-1}$ at the peak of the growing season, but our estimated GLASS GPP was only approximately $8 \mathrm{~g} \mathrm{C} \cdot \mathrm{m}^{-2} \cdot \mathrm{d}^{-1}$. The MODIS GPP was much less, just about $6 \mathrm{~g} \mathrm{C} \cdot \mathrm{m}^{-2} \cdot \mathrm{d}^{-1}$. But in the fallow season, estimated GLASS GPP and MODIS GPP were higher than the FLUXNET GPP. FLUXNET GPP started to increase rapidly in early June, but the estimated GLASS GPP and MODIS GPP started to increase in March. 

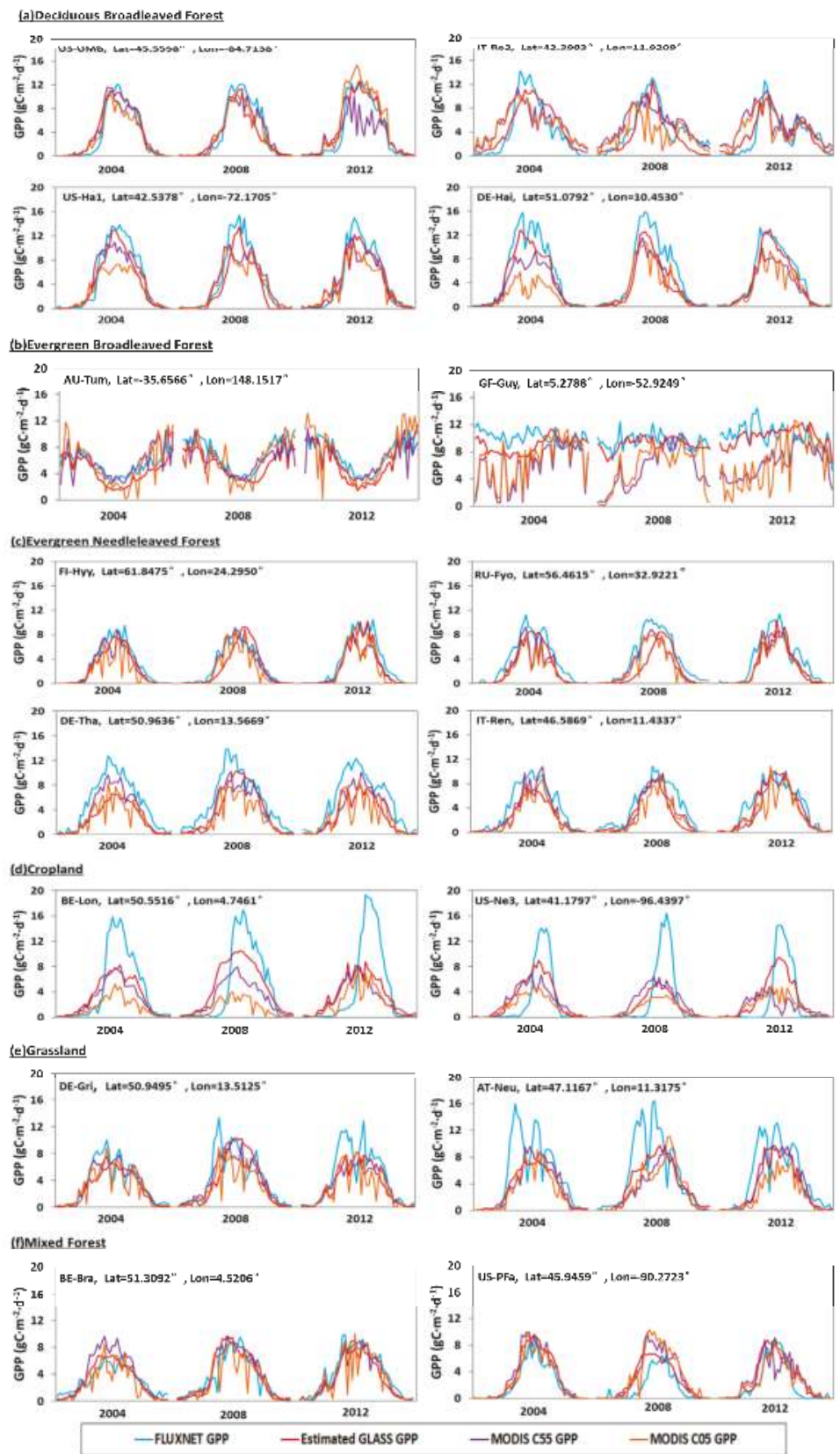

Figure 8. Seasonal variation in the estimated GLASS GPP, FLUXNET GPP, MOD17 C05 GPP and MOD17 C55 GPP for several sites with different vegetation types.

In grasslands (GRA), as shown in Figure 8e, peak profiles of FLUXNET GPP appeared in May and July at the DE-Gri and AT-Neu sites. MODIS GPP and estimated GLASS GPP did not reflect these 
high values. But the matches between the estimated GLASS GPP, MODIS GPP and FLUXNET GPP were very good at other times.

The time series for the estimated GLASS GPP, MODIS GPP and FLUXNET GPP in mixed forests (MF) are displayed in Figure 8f. Generally, estimated GLASS GPP and MODIS GPP could capture the seasonal dynamics of FLUXNET GPP. But estimated and MODIS GPP were a little overestimated during the growing seasons in 2008 and 2012.

In conclusion, estimated GLASS GPP in DBF, EBF, ENF and MF could capture the variations of FLUXNET GPP, and their time series are obviously more continuous with smoother trajectories than MOD17 C05 GPP and MOD17 C55 GPP time series. But in CRO and GRA, estimated GLASS GPP, MOD17 C05 GPP and MOD17 C55 GPP underestimated the FLUXNET GPP during the growing season.

\subsubsection{Validation against FLUXNET GPP}

Scatter plots between estimated GLASS GPP and FLUXNET GPP, and scatter plots between MODIS GPP (MOD17 C05 GPP and MOD17 C55 GPP) and FLUXNET GPP over different vegetative land cover types were analyzed, as shown in Figures 9-11.

$\mathrm{R}^{2}$ between estimated GLASS GPP and FLUXNET GPP in DBF could be as high as 0.86 in 2004, 0.81 in 2008 and 0.79 in 2012, and RMSE was $1.74 \mathrm{~g} \mathrm{C} \cdot \mathrm{m}^{-2} \cdot \mathrm{d}^{-1}$ in 2004, $2.22 \mathrm{~g} \mathrm{C} \cdot \mathrm{m}^{-2} \cdot \mathrm{d}^{-1}$ in 2008 and $2.12 \mathrm{~g} \mathrm{C} \cdot \mathrm{m}^{-2} \cdot \mathrm{d}^{-1}$ in 2012. As for the MOD17 C55 GPP, RMSE was $2.80 \mathrm{~g} \mathrm{C} \cdot \mathrm{m}^{-2} \cdot \mathrm{d}^{-1}$ in 2004, $2.23 \mathrm{~g} \mathrm{C} \cdot \mathrm{m}^{-2} \cdot \mathrm{d}^{-1}$ in 2008 and $3.19 \mathrm{~g} \mathrm{C} \cdot \mathrm{m}^{-2} \cdot \mathrm{d}^{-1}$ in 2012. For the MOD15 C05 GPP, RMSE was

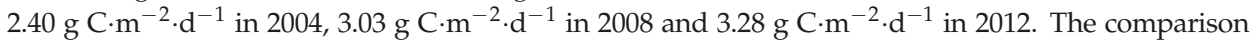
demonstrated that estimated GLASS GPP was more accurate, compared to MODIS GPP (MOD17 C05 GPP and MOD17 C55 GPP) in DBF. In EBF, RMSE between our estimated GLASS GPP and FLUXNET GPP in 2004, 2008 and 2012 were much lower than RMSE between MODIS GPP and FLUXNET GPP. Scatter plots of estimated GLASS GPP were distributed more closely around the 1:1 line, but many MODIS GPP scatter plots are decentralized and are distributed below the 1:1 line. $R^{2}$ between estimated GLASS GPP and FLUXNET GPP could be as high as 0.63, 0.65 and 0.64 in 2004, 2008 and 2012, respectively. But $\mathrm{R}^{2}$ between MODIS GPP (MOD17 C05 GPP and MOD17 C55 GPP) and FLUXNET GPP were relative smaller. In ENF, we found that RMSEs between our estimated GLASS GPP and FLUXNET GPP in 2008 and in 2012 are marginally higher than RMSE between MODIS GPP and FLUXNET GPP in 2008 and 2012. In CRO, estimated GLASS GPP and MODIS GPP were much lower than FLUXNET GPP within growing seasons. Therefore, most of the scatter plots were below the 1:1 line, which indicates that both our estimated GLASS GPP and MODIS GPP were underestimated. In GRA, we found that the RMSE was lower between estimated GLASS GPP and FLUXNET GPP than that between MODIS GPP and FLUXNET GPP in 2004. Most scatter plots of MODIS GPP were located under the 1:1 line, which demonstrates that MODIS GPP is underestimated most of the time. In MF, RMSE was lower between estimated GLASS GPP and FLUXNET GPP in 2004 and in 2008. MODIS GPP was overestimated in 2008 at BE-Bra and US-PFa.

In conclusion, we found that RMSE between estimated GLASS GPP and FLUXNET GPP was the smallest in DBF, EBF, CRO, GRA and MF. But in ENF, RMSE between MOD17 C55 GPP and FLUXNET GPP was the smallest. Therefore, estimated GPP achieved the highest precision in DBF, EBF, CRO, GRA and MF. 

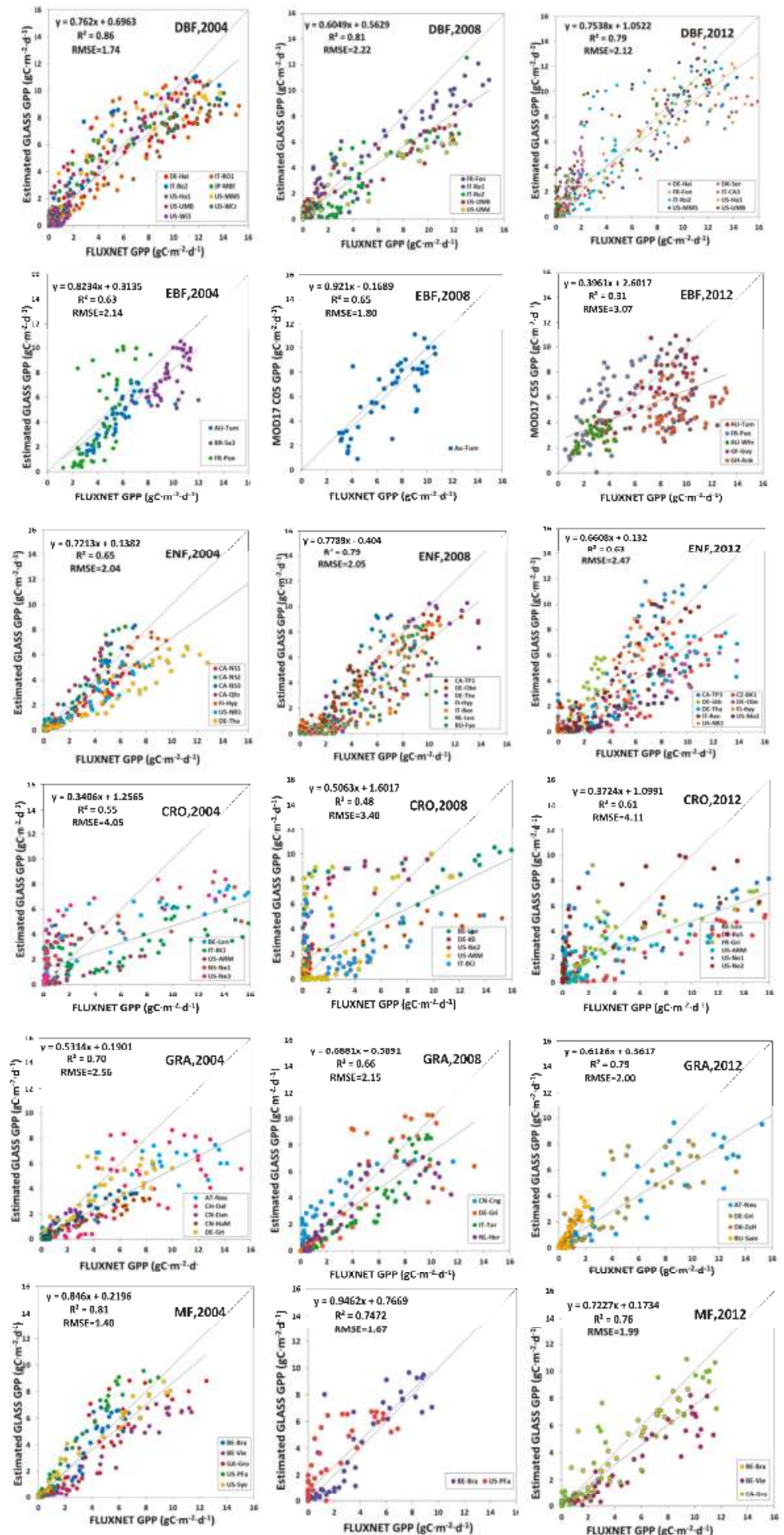

Figure 9. Validation of estimated $15 \mathrm{E}$ ASS GPP against FLUXNET GPP. 

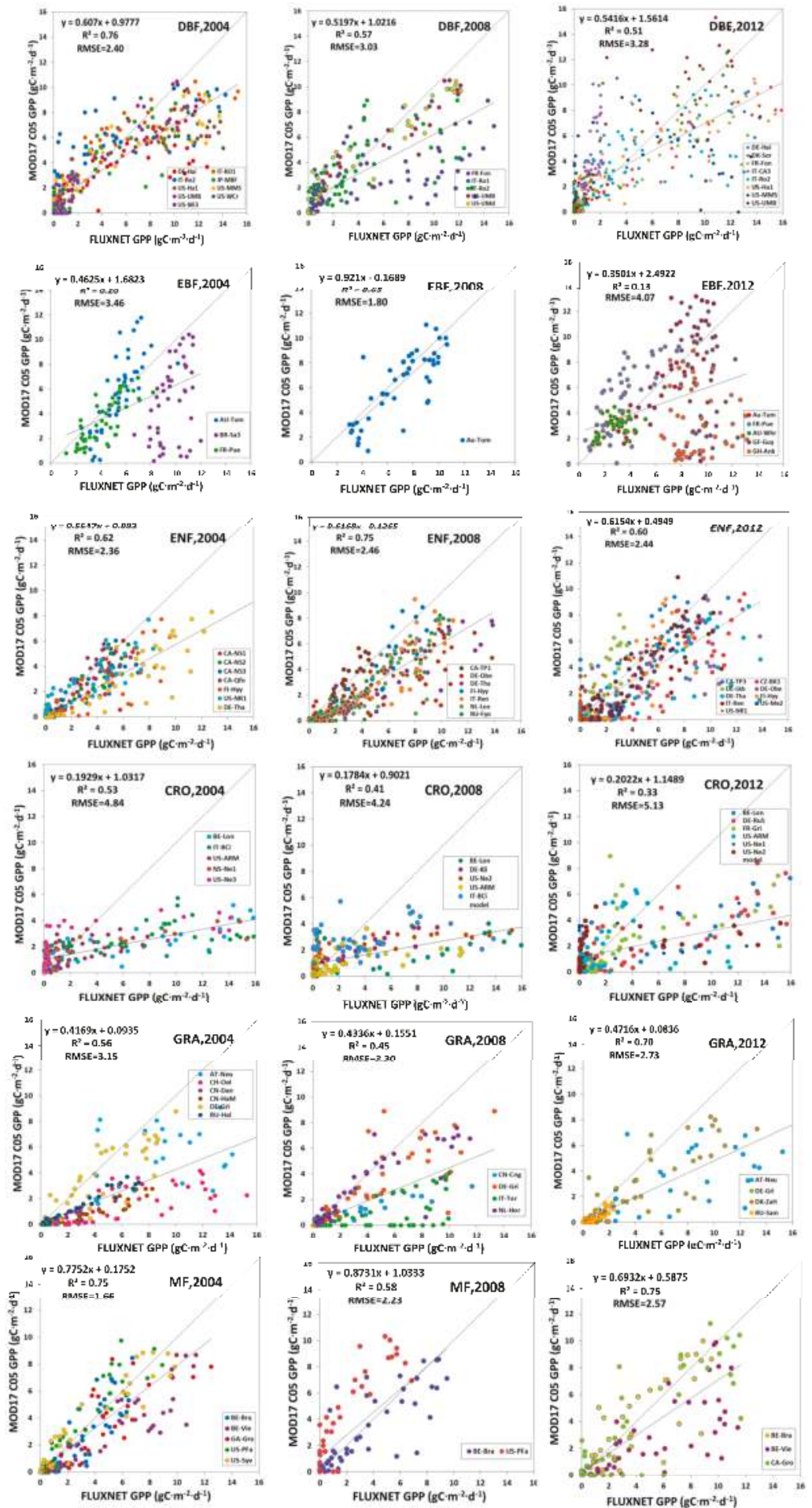

Figure 10. MODIS C05 GPP validation against FLUXNET GPP. 

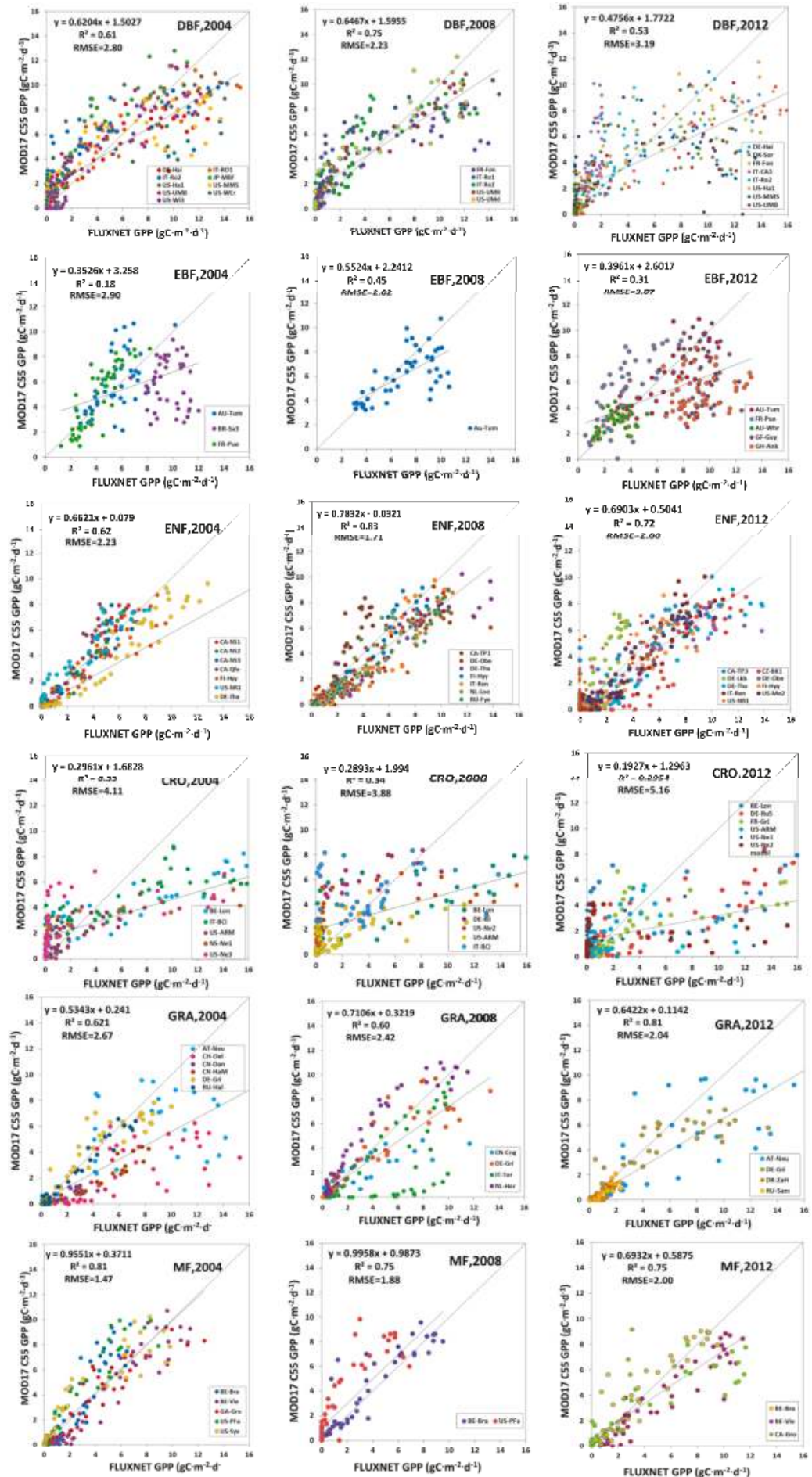

Figure 11. MODIS C55 GPP validation against FLUXNET GPP. 


\subsection{Validation of Estimated NPP}

Time-inconsistencies were found between Bigfoot NPP and our estimation. The Bigfoot NPP data were acquired from 2000 to 2004, but our estimation results are from 2004, 2008 and 2012. To preliminarily validate the model, we calculated the average NPP in 2004, 2008 and 2012 at each Bigfoot site and compared this to the average BigFoot NPP for 2000-2004. In general, a good linear relationship exists between estimated NPP and BigFoot NPP (Figure 12). The $\mathrm{R}^{2}$ between estimated NPP and BigFoot NPP was as high as 0.84, and RMSE was $160.16 \mathrm{~g} \mathrm{C} \cdot \mathrm{m}^{-2} \cdot \mathrm{yr}^{-1} \cdot \mathrm{R}^{2}$ between MODIS NPP and BigFoot NPP was 0.55, and RMSE was $219.26 \mathrm{~g} \mathrm{C} \cdot \mathrm{m}^{-2} \cdot \mathrm{yr}^{-1}$. A higher $\mathrm{R}^{2}$ and lower RMSE demonstrate that the estimated NPP achieved a better precision than the MODIS NPP product and indicate the applicability and reliability of the MuSyQ-NPP algorithm in estimating global NPP.

Some studies demonstrate that MODIS products tend towards overestimation of NPP at low productivity sites (such as SEVI) and underestimation at high productivity sites (such as TAPA) $[46,47]$. Generally, the overestimation appears to be primarily a problem with high MODIS FPAR, and the underestimations are primarily a function of low values for the maximum light use efficiency $[46,47]$. Scatter plots of estimated NPP in our study were located closer to the 1:1 line (Figure 5), especially at high productivity sites (such as TAPA, KONZ). Overestimates of FPAR at SEVI, METL and CHEQ in midseason contributed to the high MODIS NPP estimates at those sites [46]. Problems with cloud effects on FPAR and LAI estimates also lead to some artificial variation in the MODIS products [46], but the data assimilation framework to generate the GLASS FPAR and LAI products can estimate temporally complete land-surface parameter profiles from time-series reflectance data even if some of the reflectance data are contaminated by residual clouds or missing data [13]. Therefore, NPP estimates at TAPA are more accurate than MODIS NPP.
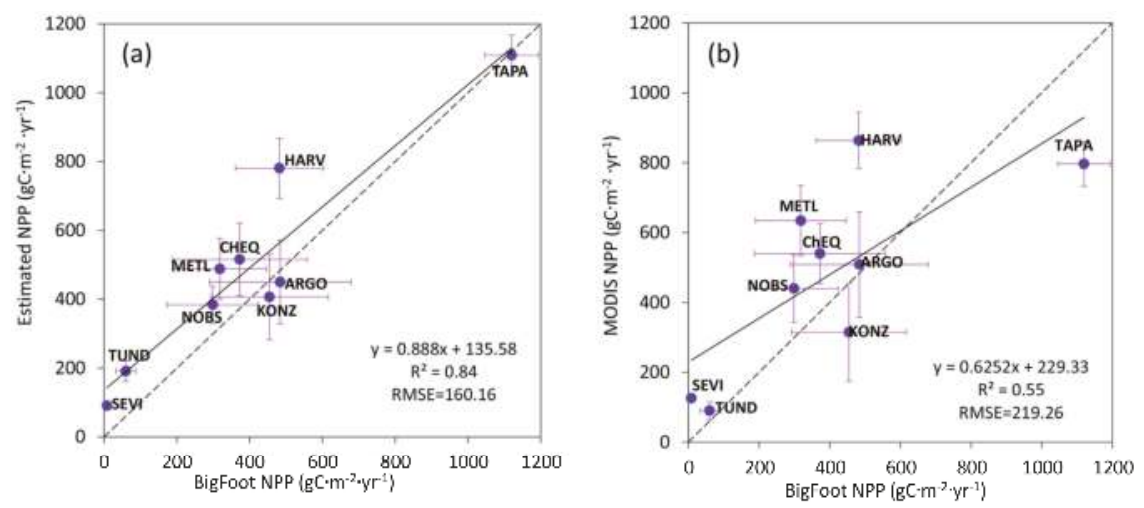

Figure 12. Validation of estimated GLASS NPP and MODIS NPP against BigFoot NPP: (a) validation of estimated GLASS NPP against BigFoot NPP; (b) validation of MODIS NPP against BigFoot NPP. $x$ is the average of BigFoot NPP, the years being averaged are shown in Table 1; $y$ is the average of NPP in 2004, 2008 and 2012.

\section{Discussion}

\subsection{Reasons for GPP Underestimation in Croplands}

Estimated GLASS GPP and the MODIS GPP product underestimated and poorly represented FLUXNET GPP, especially in croplands. Estimated GLASS GPP and MODIS GPP were much lower than FLUXNET GPP within growing seasons, but estimated GLASS GPP and MODIS GPP were higher than FLUXNET GPP in non-growing seasons. One of the reasons for these results may be that the potential LUE for crops shows no difference between C3 and C4 species in the algorithm. The C4 crop species have a larger photosynthetic capacity compared to the C 3 species; thus, it is necessary 
to have accurate species-distribution potentials for LUE products in order to determine regional and global estimates of GPP [48]. Second, the height of the eddy covariance instruments, related to the spatial representativeness of the observed carbon flux data, is usually low in croplands, such as BE-Lon and US-Ne3 (about $3.5 \mathrm{~m}$ ). Scale mismatch exists between ground observed footprint size and satellite-derived footprint size. Thirdly, the problem of mixed pixels may also have an influence on the accuracy of estimated GLASS GPP. Some other types of land cover (such as bare land, grassland and artificial land) may exist in a MODIS $1 \mathrm{~km}$ cropland pixel, which may lead to overestimation of FPAR and LAI in the non-growing season, and underestimation of FPAR and LAI in the growing season $[49,50]$. This might also be the reason for the overestimation of GPP in the non-crop-growth season and underestimation in the crop growth season.

\subsection{Limitations in NPP Validation}

Site-level validation of MODIS NPP has been more limited because of the logistical constraints of measuring NPP and scaling it to the size of a MODIS grid cell [43,51]. The BigFoot project was designed to provide ground validation data for MODIS land products, including NPP. Thus, the BigFoot data used in this paper to validate GLASS NPP results, varies widely, according site characteristics, i.e., different climate and vegetation types. As BigFoot data are only generated from 2000 to 2004 (Table 1), the problem of time-inconsistency exists when validating the GLASS NPP estimates. To reduce the bias, mean BigFoot NPP in different years (Table 1) were used to validate the estimated mean NPP in 2004, 2008 and 2012. In conclusion, BigFoot NPP could be only used to validate the GLASS NPP estimates preliminarily.

\subsection{Future Work}

Satellite derived GPP and NPP are difficult to validate, due to the differences in spatial scale between the ground and satellite data. First, the height of the sensors at FLUXNET sites, which is related to the representativeness of the observed data, depends on the type of the vegetation, the extent of fetch, the range of wind velocity and the frequency response of the instrument [51]. Second, the land surface at the site should ideally be flat, with an extensive fetch of uniform vegetation. But in the real environment, many flux tower sites are on undulating or gently sloping terrain, as this is where native vegetation exists [52]. Moreover, some sites consist of mono-specific vegetation (such as cropland and grassland), and others contain a mixture of species. Spatial heterogeneity may lead to some mixed pixels in remote sensing data, therefore, leading to some bias in GPP and NPP estimation and validation. In the future, we may study the spatial representativeness of ground observation data through footprint analysis [53-55] and validate the remotely sensed vegetation productivity through the upscaling of ground observation and fine spatial resolution remote sensing data.

Producing accurate estimates of predictions of LUE is another issue that urgently needs to be addressed when estimating vegetation productivity. In this study, the biome-specific potential LUE $\varepsilon_{\max }$ was set at a certain value for each vegetation type. In addition, in this algorithm, actual LUE was calculated based on the assumption that LUE was influenced only by low or high temperatures and water shortages. However, in the real environment, LUE could also be influenced by many other factors, such as the eco-physiological processes in the vegetation, light intensity, and concentrations of $\mathrm{CO}_{2}$ and $\mathrm{O}_{3}$ in the atmosphere [56,57]. In addition, LUE shows obvious spatial heterogeneity and temporal variation under the combined influence of vegetation distribution and climate features [58-60]. Therefore, accurate predictions of LUE are an important issue should be solved. In the future, we may obtain the spatial distribution of LUE by regression or machine learning methods, such as random forest and support vector machine. Parameter optimization based on process models (such as the Biome-BGC) may be another way to estimate the spatial patterns of LUE. Moreover, relationships between LUE and the fraction of PAR absorbed by chlorophyll (FPARchl) [61] could also be used in LUE estimation. 


\section{Conclusions}

In this paper, global GPP and NPP were firstly estimated with GLASS data using the MuSyQ-NPP algorithm, and an eight-day global GPP and NPP product with a resolution of $1 \mathrm{~km}$ was developed. Secondly, GLASS GPP/NPP estimates were validated against FLUXNET GPP data and BigFoot NPP data and were compared with MODIS GPP/NPP products. Then, the temporal and spatial variation in global GPP and NPP from 2004 to 2012 was analyzed. Lastly, the advantages of the MuSyQ-NPP algorithm and limitations of this study were discussed. The results of this study indicate that GLASS data and the MuSyQ-NPP algorithm have great potential in regional and global GPP and NPP estimates.

Generally, time series of estimated GLASS GPP in 2004, 2008 and 2012 agreed well with the time series of FLUXNET GPP and MODIS GPP. In addition, the time series of estimated GLASS GPP in our study was more temporally continuous and spatially complete with smoother trajectories compared to MODIS time series. Estimated GLASS GPP matched better with FLUXNET GPP in DBF, EBF, ENF and MF. But in CRO, estimated GLASS GPP and MODIS GPP were lower than FLUXNET GPP within the growing season. Second, estimates of global GPP/NPP in our study achieved higher precision than MODIS GPP/NPP. In DBF, EBF, CRO, GRA and MF, RMSEs were lower between our estimated GLASS GPP and FLUXNET GPP than those between MODIS GPP and FLUXNET GPP. As for NPP, the RMSE between estimated GLASS NPP and BigFoot NPP was only $160.16 \mathrm{~g} \mathrm{C} \cdot \mathrm{m}^{-2} \cdot \mathrm{yr}^{-1}$, much lower than the RMSE between MODIS NPP and BigFoot NPP. Thirdly, the global total GPP decreased by $1.41 \%$ from 2004 to 2008 and increased $11.58 \%$ from 2008 to 2012. In addition, global NPP decreased by $1.64 \%$ from 2004 to 2008 and increased by 15.59\% from 2008 to 2012. GPP and NPP in 2012 were higher than in 2004 and 2008 for almost all land cover types. Finally in 2008, GLASS GPP and NPP reached the minimum value of the period analyzed.

Supplementary Materials: The following are available online at www.mdpi.com/2072-4292/10/2/327/s1.

Acknowledgments: This work was supported by the National Key R\&D Program of China (2016YFB0501502), the National Natural Science Foundation of China (41531174, 41471349 and 61661136006001) and the National Key R\&D Program of China (2017YFA0603002).

Author Contributions: Tao Yu, Rui Sun and Zhiqiang Xiao proposed the ideas; Tao Yu, Zhiqiang Xiao, Gang Liu, Qiang Zhang, Tianxiang Cui and Juanmin Wang preprocessed and analyzed the data; Tao Yu and Rui Sun prepared the paper.

Conflicts of Interest: The authors declare no conflict of interest.

\section{References}

1. Chen, J.M.; Mo, G.; Pisek, J.; Liu, J.; Deng, F.; Ishizawa, M.; Chan, D. Effects of foliage clumping on the estimation of global terrestrial gross primary productivity. Glob. Biogeochem. Cycles 2012, 26, GB1019. [CrossRef]

2. Running, S.W.; Nemani, R.R.; Heinsch, F.A.; Zhao, M.; Reeves, M.; Hashimoto, H. A continuous satellite-derived measure of global terrestrial primary production. AIBS Bull. 2004, 54, 547-560. [CrossRef]

3. Heinsch, F.A.; Reeves, M.; Bowker, C.F. User's Guide, GPP and NPP (MOD 17A2/A3) Products, NASA MODIS Land Algorithm. Available online: https:/ /www.researchgate.net/publication/242118371_User T1 textquoterights_guide_GPP_and_NPP_MOD17A2A3_products_NASA_MODIS_land_algorithm (accessed on 5 December 2017).

4. Zhao, M.; Heinsch, F.A.; Nemani, R.R.; Running, S.W. Improvements of the MODIS terrestrial gross and net primary production global data set. Remote Sens. Environ. 2005, 95, 164-176. [CrossRef]

5. Martínez, B.; Camacho, F.; Verger, A.; García-Haro, F.J.; Gilabert, M.A. Intercomparison and quality assessment of MERIS, MODIS and SEVIRI FAPAR products over the Iberian Peninsula. Int. J. Appl. Earth Obs. 2013, 21, 463-476. [CrossRef]

6. McCallum, I.; Wagner, W.; Schmullius, C.; Shvidenko, A.; Obersteiner, M.; Fritz, S.; Nilsson, S. Comparison of four global FAPAR datasets over Northern Eurasia for the year 2000. Remote Sens. Environ. 2010, 114, 941-949. [CrossRef] 
7. Tao, X.; Liang, S.; Wang, D. Assessment of five global satellite products of fraction of absorbed photosynthetically active radiation: Intercomparison and direct validation against ground-based data. Remote Sens. Environ. 2015, 163, 270-285. [CrossRef]

8. Ma, H.; Liu, Q.; Liang, S.; Xiao, Z. Simultaneous estimation of leaf area index, fraction of absorbed photosynthetically active radiation, and surface albedo from multiple-satellite data. IEEE Trans. Geosci. Remote Sens. 2017, 55, 4334-4354. [CrossRef]

9. Xiao, Z.; Liang, S.; Wang, J.; Chen, P.; Yin, X.; Zhang, L.; Song, J. Use of general regression neural networks for generating the GLASS leaf area index product from time series MODIS surface reflectance. IEEE Trans. Geosci. Remote Sens. 2014, 52, 209-223. [CrossRef]

10. Xiao, Z.; Liang, S.; Wang, J.; Xiang, Y.; Zhao, X.; Song, J. Long-time-series global land surface satellite leaf area index product derived from MODIS and AVHRR surface reflectance. IEEE Trans. Geosci. Remote Sens. 2016, 54, 5301-5318. [CrossRef]

11. Liang, S.; Zhao, X.; Liu, S.; Yuan, W.; Cheng, X.; Xiao, Z.; Zhang, X.; Liu, Q.; Cheng, J.; Tang, H.; et al. A long-term Global LAnd Surface Satellite (GLASS) data-set for environmental studies. Int. J. Digit. Earth 2013, 6 (Suppl. S1), 5-33. [CrossRef]

12. Fang, H.; Jiang, C.; Li, W.; Wei, S.; Baret, F.; Chen, J.; Garcia-Haro, J.; Liang, S.; Liu, R.; Myneni, R.; et al. Characterization and intercomparison of global moderate resolution leaf area index (LAI) products: Analysis of climatologies and theoretical uncertainties. J. Geophys. Res. Biogeogr. 2013, 118, 529-548. [CrossRef]

13. Xiao, Z.; Liang, S.; Sun, R.; Wang, J.; Jiang, B. Estimating the fraction of absorbed photosynthetically active radiation from the MODIS data based GLASS leaf area index product. Remote Sens. Environ. 2015, 171, 105-117. [CrossRef]

14. Lieth, H. Modeling the primary productivity of the world. In Primary Productivity of the Biosphere; Springer: Berlin/ Heidelberg, Germany, 1975; pp. 237-263.

15. Uchijima, Z; Seino, H. Agroclimatic evaluation of net primary productivity of natural vegetations. J. Agric. Meteorol. 1985, 40, 343-352. [CrossRef]

16. Running, S.W.; Thornton, P.E.; Nemani, R.; Glassy, J.M. Global terrestrial gross and net primary productivity from the Earth Observing System. In Methods in Ecosystem Science; Springer: Berlin/ Heidelberg, Germany, 2000; Volume 3, pp. 44-45.

17. Potter, C.S.; Randerson, J.T.; Field, C.B.; Matson, P.A.; Vitousek, P.M.; Mooney, H.A.; Klooster, S.A. Terrestrial ecosystem production: A process model based on global satellite and surface data. Glob. Biogeochem. Cycles 1993, 7, 811-841. [CrossRef]

18. Prince, S.D.; Goward, S.N. Global primary production: A remote sensing approach. J. Biogeogr. 1995, 22, 815-835. [CrossRef]

19. Parton, W.J.; Scurlock, J.M.O.; Ojima, D.S.; Gilmanov, T.G.; Scholes, R.J.; Schimel, D.S.; Kirchner, T.; Menaut, J.-C.; Garcia, M.E.; Kamnalrut, A.; et al. Observations and modeling of biomass and soil organic matter dynamics for the grassland biome worldwide. Glob. Biogeochem. Cycles 1993, 7, 785-809. [CrossRef]

20. McGuire, A.D.; Melillo, J.M.; Kicklighter, D.W.; Joyce, L.A. Equilibrium responses of soil carbon to climate change: Empirical and process-based estimates. J. Biogeogr. 1995, 22, 785-796. [CrossRef]

21. Running, S.W.; Hunt, E.R., Jr. Generalization of a forest ecosystem process model for other biomes, BIOME-BCG, and an application for global-scale models. In Scaling Physiological Processes; Academic Press: San Diego, CA, USA, 1993; pp. 141-158.

22. Cui, T.; Wang, Y.; Sun, R.; Qiao, C.; Fan, W.; Jiang, G.; Hao, L.; Zhang, L. Estimating vegetation primary production in the Heihe River Basin of China with multi-source and multi-scale data. PLoS ONE 2016, 11, e0153971. [CrossRef] [PubMed]

23. Running, S.W.; Zhao, M.S. User's Guide. Daily GPP and Annual NPP (MOD17A2/A3) Products NASA Earth Observing System MODIS Land Algorithm. Version 3.0 for Collection 6. 2015. Available online: https:/ /pdaac.usgs. gov/sites/default/files/public/product_documentation/mod17_user_guide.pdf (accessed on 5 December 2017).

24. Qiao, C.; Sun, R.; Xu, Z.; Zhang, L.; Liu, L.; Hao, L.; Jiang, G. A study of shelterbelt transpiration and cropland evapotranspiration in an irrigated area in the middle reaches of the Heihe River in northwestern China. IEEE Geosci. Remote Sens. 2015, 12, 369-373. [CrossRef] 
25. Zhang, K.; Kimball, J.S.; Mu, Q.; Jones, L.A.; Goetz, S.J.; Running, S.W. Satellite based analysis of northern ET trends and associated changes in the regional water balance from 1983 to 2005. J. Hydrol. 2009, 379, 92-110. [CrossRef]

26. Priestley, C.H.B.; Taylor, R.J. On the assessment of surface heat flux and evaporation using large-scale parameters. Mon. Weather Rev. 1972, 100, 81-92. [CrossRef]

27. Liu, J.; Chen, J.M.; Cihlar, J.; Park, W.M. A process-based boreal ecosystem productivity simulator using remote sensing inputs. Remote Sens. Environ. 1997, 62, 158-175. [CrossRef]

28. The GLASS LAI Product at Beijing Normal University. Available online: http:/ / www.bnu-datacenter.com/ en (accessed on 5 December 2017).

29. The GLASS LAI Product at the Global Land Cover Facility. Available online: http://glcf.umd.edu (accessed on 5 December 2017).

30. MODIS Land Cover Type/Dynamics. Available online: https://modis.gsfc.nasa.gov/data/dataprod/ mod12.php (accessed on 5 December 2017).

31. Channan, S.; Collins, K.; Emanuel, W.R. Global Mosaics of The Standard MODIS Land Cover Type Data; University of Maryland and the Pacific Northwest National Laboratory: College Park, MD, USA, 2014.

32. Xiao, Z.; Wang, T.; Liang, S.; Sun, R. Estimating the fractional vegetation cover from GLASS leaf area index product. Remote Sens. 2016, 8, 337. [CrossRef]

33. Carbon Dioxide Information Analysis Center. Available online: http://cdiac.ornl.go (accessed on 5 December 2017).

34. Ruesch, A.; Gibbs, H.K. New IPCC Tier1 Global Biomass Carbon Map for the Year 2000; Carbon Dioxide Information Analysis Center, Oak Ridge National Laboratory: Oak Ridge, TN, USA, 2008.

35. Eggleston, H.S.; Buendia, L.; Miwa, K.; Ngara, T.; Tanabe, K. IPCC Guidelines for National Greenhouse Gas Inventories; Institute for Global Environmental Strategies: Hayama, Japan, 2006.

36. Global Land Data Assimilation System. Available online: http://ldas.gsfc.nasa.gov/gldas/GLDASgoals.php (accessed on 5 December 2017).

37. Rodell, M.; Houser, P.R.; Jambor, U.E.A.; Gottschalck, J.; Mitchell, K.; Meng, C.J.; Arenault, K.; Cosgrove, B.; Radakovich, J.; Bosilovich, M.; et al. The global land data assimilation system. Bull. Am. Meteorol. Soc. 2004, 85, 381-394. [CrossRef]

38. Fang, H.; Beaudoing, H.K.; Teng, W.L.; Vollmer, B.E. Global Land data assimilation system (GLDAS) products, services and application from NASA hydrology data and information services center (HDISC). In Proceedings of the ASPRS 2009 Annual Conference, Baltimore, MD, USA, 8-13 March 2009.

39. Friend, A.D.; Arneth, A.; Kiang, N.Y.; Lomas, M.; Ogee, J.; RÖDENBECK, C.; Running, S.W.; Santaren, J.; Sitch, S.; Viovy, N.; et al. FLUXNET and modelling the global carbon cycle. Glob. Chang. Biol. 2007, 13, 610-633. [CrossRef]

40. Knorr, W.; Kattge, J. Inversion of terrestrial ecosystem model parameter values against eddy covariance measurements by Monte Carlo sampling. Glob. Chang. Biol. 2005, 11, 1333-1351. [CrossRef]

41. Fluxdata. Available online: http:/ / fluxnet.fluxdata.org (accessed on 5 December 2017).

42. Cohen, W.B.; Turner, D.P.; Gower, S.T.; Running, S.W. Linking In Situ Measurements, Remote Sensing, and Models to Validate MODIS Products Related to the Terrestrial Carbon Cycle. NASA Terrestrial Ecology Program. 2009. Available online: http://www.fsl.orst.edu/larse/bigfoot/index.html (accessed on 5 December 2017).

43. Yuan, W.; Liu, S.; Yu, G.; Bonnefond, J.M.; Chen, J.; Davis, K.; Desai, A.R.; Goldstein, A.H.; Gianelle, D.; Rossi, F; et al. Global estimates of evapotranspiration and gross primary production based on MODIS and global meteorology data. Remote Sens. Environ. 2010, 114, 1416-1431. [CrossRef]

44. Field, C.B.; Behrenfeld, M.J.; Randerson, J.T.; Falkowski, P. Primary production of the biosphere: Integrating terrestrial and oceanic components. Science 1998, 281, 237-240. [CrossRef] [PubMed]

45. Cramer, W.; Kicklighter, D.W.; Bondeau, A.; Moore, B., III; Churkina, G.; Nemry, B.; Ruimy, A.; Schloss, A.L.; Model, P.O.T.P.N. Comparing global models of terrestrial net primary productivity (NPP): Overview and key results. Glob. Chang. Boil. 1999, 5, 1-15. [CrossRef]

46. Turner, D.P.; Ritts, W.D.; Cohen, W.B.; Maeirsperger, T.K.; Gower, S.T.; Kirschbaum, A.A.; Running, S.W.; Zhao, M.; Wofsy, S.C.; Dunn, A.L.; et al. Site-level evaluation of satellite-based global terrestrial gross primary production and net primary production monitoring. Glob. Chang. Biol. 2005, 11, 666-684. [CrossRef] 
47. Turner, D.P.; Ritts, W.D.; Cohen, W.B.; Gower, S.T.; Running, S.W.; Zhao, M.; Costa, M.H.; Kirschbaum, A.A.; Ham, J.M.; Saleska, S.R.; et al. Evaluation of MODIS NPP and GPP products across multiple biomes. Remote Sens. Environ. 2006, 102, 282-292. [CrossRef]

48. Yuan, W.; Cai, W.; Nguy-Robertson, A.L.; Fang, H.; Suyker, A.E.; Chen, Y.; Dong, W.; Liu, S.; Zhang, H. Uncertainty in simulating gross primary production of cropland ecosystem from satellite-based models. Agric. For. Meteorol. 2015, 207, 48-57. [CrossRef]

49. Zhang, Y.; Yu, Q.; Jiang, J.I.E.; Tang, Y. Calibration of Terra/MODIS gross primary production over an irrigated cropland on the North China Plain and an alpine meadow on the Tibetan Plateau. Glob. Chang. Biol. 2008, 14, 757-767. [CrossRef]

50. Zhang, Q.; Cheng, Y.B.; Lyapustin, A.I.; Wang, Y.; Gao, F.; Suyker, A.; Verma, S.; Middleton, E.M. Estimation of crop gross primary production (GPP): FAPAR chl versus MOD15A2 FPAR. Remote Sens. Environ. 2014, 153, 1-6. [CrossRef]

51. Turner, D.P.; Ollinger, S.; Smith, M.L.; Krankina, O.; Gregory, M. Scaling net primary production to a MODIS footprint in support of earth observing system product validation. Int. J. Remote Sens. 2004, 25, 1961-1979. [CrossRef]

52. Olson, R.J.; Holladay, S.K.; Cook, R.B.; Falge, E.; Baldocchi, D.; Gu, L. FLUXNET. Database of Fluxes, Site Characteristics, and Flux-Community Information; Oak Ridge National Laboratory (ORNL): Oak Ridge, TN, USA, 2004.

53. Schuepp, P.H.; Leclerc, M.Y.; Macpherson, J.I.; Desjardins, R.L. Footprint prediction of scalar fluxes from analytical solutions of the diffusion equation. Bound. Layer Meteorol. 1990, 50, 353-373. [CrossRef]

54. Sogachev, A.; Menzhulin, G.V.; Heimann, M.; Lloyd, J. A simple three-dimensional canopy-Planetary boundary layer simulation model for scalar concentrations and fluxes. Tellus $B$ 2002, 54, 784-819.

55. Chen, B.; Ge, Q.; Fu, D.; Yu, G.; Sun, X.; Wang, S.; Wang, H. Data-model fusion approach for upscaling gross ecosystem productivity to the landscape scale based on remote sensing and flux footprint modeling. Biogeosciences 2010, 7, 2943-2958. [CrossRef]

56. Green, D.S.; Erickson, J.E.; Kruger, E.L. Foliar morphology and canopy nitrogen as predictors of light-use efficiency in terrestrial vegetation. Agric. For. Meteorol. 2003, 115, 163-171. [CrossRef]

57. Van Oijen, M.; Dreccer, M.F.; Firsching, K.H.; Schnieders, B.J. Simple equations for dynamic models of the effects of $\mathrm{CO}_{2}$ and $\mathrm{O}_{3}$ on light-use efficiency and growth of crops. Ecol. Model. 2004, 179, 39-60. [CrossRef]

58. Turner, D.P.; Gower, S.T.; Cohen, W.B.; Gregory, M.; Maiersperger, T.K. Effects of spatial variability in light use efficiency on satellite-based NPP monitoring. Remote Sens. Environ. 2002, 80, 397-405. [CrossRef]

59. Ito, A.; Oikawa, T. Global Mapping of Terrestrial Primary Productivity and Light-Use Efficiency with a Process-Based Model; Global Environmental Change in the Ocean and on Land, Terrapub: Tokyo, Japan, 2004; pp. 343-358.

60. Still, C.J.; Randerson, J.T.; Fung, I.Y. Large-scale plant light-use efficiency inferred from the seasonal cycle of atmospheric $\mathrm{CO}_{2}$. Glob. Chang. Biol. 2004, 10, 1240-1252. [CrossRef]

61. Xiao, X. Light absorption by leaf chlorophyll and maximum light use efficiency. IEEE Trans. Geosci. Remote Sens. 2006, 44, 1933-1935. [CrossRef]

(C) 2018 by the authors. Licensee MDPI, Basel, Switzerland. This article is an open access article distributed under the terms and conditions of the Creative Commons Attribution (CC BY) license (http:/ / creativecommons.org/licenses/by/4.0/). 
Article

\title{
Spatiotemporal Dynamics in Vegetation GPP over the Great Khingan Mountains Using GLASS Products from 1982 to 2015
}

\author{
Ling Hu ${ }^{1,2}$, Wenjie Fan ${ }^{1,2, *}$, Huazhong Ren ${ }^{1,2}$, Suhong Liu ${ }^{3}$, Yaokui Cui ${ }^{1,2}$ and Peng Zhao ${ }^{1,2}$ \\ 1 Institute of Remote Sensing and Geographic Information System, School of Earth and Space Sciences, \\ Peking University, Beijing 100871, China; hul@pku.edu.cn (L.H.); renhuazhong@pku.edu.cn (H.R.); \\ yaokuicui@pku.edu.cn (Y.C.); pengzhao@pku.edu.cn (P.Z.) \\ 2 Beijing Key Lab of Spatial Information Integration and 3S Application, Peking University, \\ Beijing 100871, China \\ 3 Faculty of Geographical Science, Beijing Normal University, Beijing 100875, China; liush@bnu.edu.cn \\ * Correspondence: fanwj@pku.edu.cn; Tel.: +86-10-6275-5085
}

Received: 21 January 2018; Accepted: 19 March 2018; Published: 20 March 2018

\begin{abstract}
Gross primary productivity (GPP) is an important parameter that represents the productivity of vegetation and responses to various ecological environments. The Greater Khingan Mountain (GKM) is one of the most important state-owned forest bases, and boreal forests, including the largest primeval cold-temperature bright coniferous forest in China, are widely distributed in the GKM. This study aimed to reveal spatiotemporal vegetation variations in the GKM on the basis of GPP products that were generated by the Global LAnd Surface Satellite (GLASS) program from 1982 to 2015. First, we explored the spatiotemporal distribution of vegetation across the GKM. Then we analyzed the relationships between GPP variation and driving factors, including meteorological elements, growing season length (GSL), and Fraction of Photosynthetically Active Radiation (FPAR), to investigate the dominant factor for GPP dynamics. Results demonstrated that (1) the spatial distribution of accumulated GPP (AG) in spring, summer, autumn, and the growing season varied due to three main reasons: understory vegetation, altitude, and land cover; (2) interannual AG in summer, autumn, and the growing season significantly increased at the regional scale during the past 34 years under climate warming and drying; (3) interannual changes of accumulated GPP in the growing season (AGG) at the pixel scale displayed a rapid expansion in areas with a significant increasing trend $(p<0.05)$ during the period of 1982-2015 and this trend was caused by the natural forest protection project launched in 1998; and finally, (4) an analysis of driving factors showed that daily sunshine duration in summer was the most important factor for GPP in the GKM and this is different from previous studies, which reported that the GSL plays a crucial role in other areas.
\end{abstract}

Keywords: boreal forest; GPP; spatiotemporal distribution and variation; meteorological factors; phenological parameters

\section{Introduction}

The importance of vegetation in the global carbon cycle is well known [1-3]. Understanding the response of vegetation growth to climate variations in recent decades is critical for projecting future ecosystem dynamics. Although relatively simple in vegetation structure, boreal forests and woodlands cover nearly $14.5 \%$ of the land surface and play an important role in the global carbon budget [4-6]. They are extremely sensitive to climate change and numerous studies have documented the enhanced terrestrial vegetation growth in the middle and high latitudes of the Northern Hemisphere over the past three decades $[3,7,8]$. 
The gross primary productivity (GPP) of a terrestrial ecosystem refers to the organic compounds that are accumulated by green plants on land through assimilating carbon dioxide in the atmosphere by photosynthesis and a series of physiological processes in plants [9-11]. Terrestrial GPP is the major driver of land carbon sequestration, and it plays a pivotal role in the global carbon balance, providing terrestrial ecosystems the capacity to partly offset anthropogenic $\mathrm{CO}_{2}$ emissions [11-14]. GPP varies diurnally and seasonally in response to both changes in climate (e.g., light, precipitation, temperature, and humidity) and nutrient availability, whereas the spatial distribution is determined primarily by climatic conditions [11]. To date, only a few studies [10] have reported on how the GPP of boreal forests vary with climate.

The increasing availability of remote sensing measurements provides complete global coverage with a high revisit frequency for vegetation monitoring [15]. The popular data-driven approach to quantify vegetation variations at the global scales or regional scale is based on vegetation index, such as the NDVI (normalized difference vegetation index) and EVI (enhanced vegetation index), which are directly related to vegetation activity [16]. However, the vegetation index can be affected by many factors that are unrelated to ecosystem structure or function, such as satellite drift, calibration uncertainties, inter-satellite sensor differences, and bidirectional and atmospheric effects [3,17-22].

Another method of vegetation monitoring is based on remotely sensed GPP models. The major approach to this method is to employ the maximum light use efficiency (LUE) [23-28]. LUE is the core of physical models of global GPP products [29], including the Carnegie-Ames-Stanford Approach (CASA) [30,31], Global Production Efficiency Model (GLOPEM) [28], Eddy Covariance-Light Use Efficiency (EC-LUE) [32,33], C-Fix [34], MOD17 algorithm [35], Vegetation Photosynthesis Model (VPM) $[6,36]$, and C-Flux [37,38]. Although the formulas and parameterization schemes vary among these models, the key idea is still the same, which is to combine all of those factors affecting GPP in a relatively simple linear or nonlinear way. The EC-LUE model is one of the most powerful GPP models and has been verified at 54 eddy covariance sites, suggesting that the EC-LUE model is robust and reliable [32,33]. In addition, and the Global LAnd Surface Satellite (GLASS) GPP data using the EC-LUE model can provide a good data source for the study of GPP.

Apart from modeling research, studies about spatiotemporal distribution and variation in GPP, as well as its influence mechanism, are also hot topics [11,39,40]. GPP can be affected by many factors, such as fire, logging, harvesting, insect outbreaks, and ecosystem dynamics [11]. Temperature was reported as the main factor of GPP variability in the middle and high latitudes of the Northern Hemisphere [7,41,42]. Phenological parameters can also result in large changes in annual GPP [43].

The forest ecosystems in northeast China play an important role in the national carbon budget because they comprise more than 30\% of the total forest area in China [44]. Located in northeastern China, the Greater Khingan Mountains (GKM) is an important state-owned forestry base; also, it is a unique bright coniferous forest of the cool temperate zone in China, making up the southern boundary of the boreal forests in Eurasia. The GKM is said to be especially sensitive to climate change [45,46], and usually precedes low elevation regions in climate change. Over the past 70 years, this area has been increasingly affected by humans; many of these forests have been subjected to fire disasters and excessive deforestation. Monitoring and understanding how much the major factors contribute to GPP variation across the GKM and estimating the spatial patterns of these possible mechanisms are critical. However, studies on the vegetation of this region are rare or only consider a relatively short period. No thorough discussion has been carried out on the main factors influencing vegetation variation in the GKM.

From this point of view, the main objectives of this study are to: (1) reveal the distribution and dynamics of GPP across the boreal forest, especially the bright coniferous forest; and, (2) understand the way GPP responses to driving factors and identify the dominant driver to the variations of GPP. As a result, this paper is organized as follows: Section 2 will introduce the study area and data as well as methods; Sections 3 and 4 will present spatiotemporal dynamics of GPP and its response to driving factors respectively; finally, discussions and conclusions are presented in the last two sections. 


\section{Materials and Methods}

\subsection{Study Area}

As shown in Figure $1 \mathrm{a}$, the GKM region $\left(50^{\circ} 10^{\prime} \sim 53^{\circ} 33^{\prime} \mathrm{N}, 121^{\circ} 12^{\prime} \sim 127^{\circ} 0^{\prime} \mathrm{E}\right)$ is located in the northernmost area of China. This study area is adjacent to the Lesser Khingan Mountains in the east, near the vast Hulunbeir Prairie in the west, bordering the fertile Songnen plain in the south, and separated from Siberia in Russia by the Heilongjiang River. Lying in the east slope of the Greater Khingan Mountains ridges (GKMR), the study area has an obvious decrease in elevation from southwest to northeast (Figure 1b). The GKM belongs to the frigid temperature monsoon climate zone, which has an annual average temperature of $-2.6^{\circ} \mathrm{C}$. The annual average precipitation is $400-600 \mathrm{~mm}$, and the majority of the precipitation is concentrated in July to September, providing a relatively humid environment for vegetation growing. The soils are mainly dark brown coniferous forest soils. The GKM is an important state-owned natural forest region that serves as a biological protective screen for northeastern and northern China. Also, it includes a first-grade National Nature Ecological Reserve with abundant forest resources whose area covers about $80 \%$ of this region (Figure 1c), including the largest and best preserved primeval cold-temperature bright coniferous forest in China (Figure 1a). However, its forest structure is relatively simple due to the cold weather. The dominant species are Larix gmelinii and Mongolian pine [45].

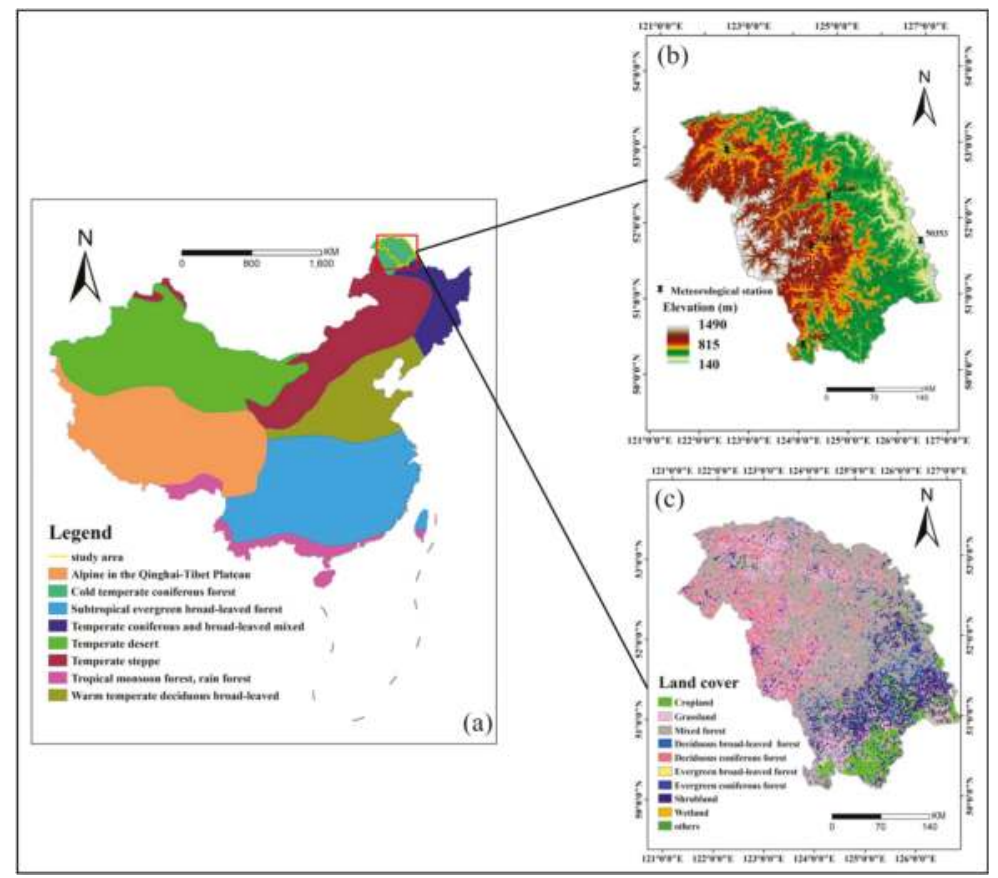

Figure 1. (a) Distribution of vegetation type and location of the Greater Khingan Mountains (GKM) in China, based on the Vegetation Regionalization Map of China (1:6,000,000) [47]; (b) DEM(Digital Elevation Model) and the meteorological station of the GKM; and, (c) Land cover of the GKM. The land cover data is from the MODIS dataset of 2001. 


\subsection{Data}

\subsubsection{GLASS GPP Dataset}

GPP data was provided by the Global Land Surface Satellite (GLASS) program [48]. This GLASS-GPP product was estimated by an improved EC-LUE model, which was derived from only four variables: normalized different vegetation index (NDVI), photosynthetically active radiation (PAR), air temperature, and the Bowen ratio of sensible-to-latent heat flux. Given the invariant parameters (i.e., the potential LUE and optimal plant growth temperature) across the various land cover types [33], GPP can address the spatial and temporal dynamics of the carbon cycle. When compared with the MODIS-GPP product, the GLASS-GPP product exhibited a longer temporal cover, which includes data every eight days starting from 1982 to the present. Also, it can provide information of vegetation before and after 1987, when a disastrous fire occurred in the northern part of the GKM. In conclusion, the GLASS-GPP product demonstrated a high reliability with the revised EC-LUE model and had a long time series dataset [33], so it can be used to analyze the spatiotemporal distribution and variation of GPP in the GKM.

A total of 1564 scenes from 1982 to 2015 with 46 scenes per year were analyzed. The original eight-day GPP product exhibited a spatial resolution of $0.05^{\circ}$ with WGS1984 coordinate. We extracted the GPP data using the GKM's boundary and then projected the data to UTM. The Savitzky-Golay (S-G) filter [49] in the software TIMESAT [50] was used to smooth some outliers caused by the environment noise and system error of sensor in the original GPP time series. The S-G filter is widely used because it can remodel the raw time series dataset to closely capture the sudden change in original values. After the repeating test, we used a $4 \times 4$ filter window to rebuild the GPP time series data that was required in the following step. We finally calculated the accumulated GPP (AG) over a certain period (i.e., total GPP during the growing season) within one year.

\subsubsection{Datasets for Auxiliary Analysis}

Meteorological data from five field observation stations in the GKM (Figure 1b) were available from China's meteorological data web [51]. Several daily meteorological parameters, including sunshine duration, average temperature, maximum temperature, minimum temperature, and precipitation, were used from 1982 to 2015, and then daily parameters were aggregated to determine seasonal averages for spring (March-May), summer (June-August), autumn (September-November), winter (December-February), and the growing season (April-October). Furthermore, with the inverse distance weighting (IDW) interpolation method, we obtained spatially distributed meteorological data that has the same spatial resolution as the GLASS-GPP products.

Phenological parameters were acquired from the 8-km AVHRR NDVI-3g product with a 15-day interval from 1982 to 2015 that was produced by the Global Inventory Modeling and Mapping Studies (GIMMS) project [52]. The AVHRR NDVI-3g dataset was generated with a maximum value composite (MVC) per half-month and then corrected to minimize the influence of calibration loss, volcanic eruptions, and orbit drift [26]. To eliminate bare soil and sparsely vegetated pixels on the NDVI image, we excluded pixels with a mean NDVI $<0.05$ in the growing season. Similarly, the S-G filter was used to smooth the original NDVI data. Finally, the dynamic threshold method [53] was used to obtain the phenological parameters, and the results were consistent with previous studies [45,54].

Similar to the GPP product, the Fraction of Photosynthetically Active Radiation (FPAR) dataset of GLASS was used. This kind of product was derived from leaf area index (LAI) via the LUT method [55] and had the same spatiotemporal resolution as the GPP product. 


\subsection{Methods}

\subsubsection{Unary Linear Regression}

On the basis of filtered GPP time series, we used unary linear regression [56] to acquire the interannual variation characteristics of GPP in the GKM during 1982-2015 at both the regional and pixel scale. Here, we assumed time $t$ as an independent variable and GPP as a dependent variable to establish the unary linear regression equation. The principal of unary linear regression can be generalized by the following equation:

$$
\theta=\frac{n \times \sum_{j=1}^{n} j \times G P P_{j}-\sum_{j=1}^{n} j \sum_{j=1}^{n} G P P_{j}}{n \times \sum_{j=1}^{n} j^{2}-\left(\sum_{j=1}^{n} j\right)^{2}}
$$

where $n$ is the length of the study period (year of monitoring interval); $G P P_{j}$ is the accumulated GPP of $j$ year; and, $\theta$ is the regression slope (annual change ratio of accumulated GPP) acquired by the least square method. $\theta>0$ indicates that the accumulated GPP increased over $n$ years and vice versa.

\subsubsection{Pearson Correlation}

Pearson correlation is considered a standard method to analyze the relationship between two variables. It can be expressed as follows:

$$
r=\frac{\sum_{i=1}^{n}\left(x_{i}-\bar{x}\right)\left(y_{i}-\bar{y}\right)}{\sqrt{\sum_{i=1}^{n}\left(x_{i}-\bar{x}\right)^{2}} \sqrt{\sum_{j=1}^{n}\left(y_{j}-\bar{y}\right)^{2}}}
$$

where $x_{i}$ and $y_{i}(i=1,2, \ldots \ldots, n)$ are the time series for two variables; $\bar{x}$ and $\bar{y}$ are the multi-year average values of two variables; and, $r$ is the correlation coefficient. $r>0$ indicates a positive correlation between two variables, whereas $r<0$ suggests a negative correlation. The absolute value of $r$ can represent the closeness of two variables; the larger the $r$, the closer the two variables are and vice versa.

Several factors affect the distribution and variation of GPP in both temporal and spatial dimensions. Meteorological elements and phenological parameters are widely considered as influencing factors and verified in many regions [40,57-59]. Apart from meteorological and phenological factors, we also considered FPAR, representing the structure and the adaptive capacity of vegetation, as a factor. Pearson Correlation analysis was conducted between the above factors and GPP during the period of 1982-2015 at a pixel resolution to assess the influence of individual environment drivers on vegetation dynamics.

\subsubsection{Standardized Multivariate Linear Regression (SMLR) Model}

The SMLR model combined several driving factors to explain the dynamics of GPP. To separate the relative contribution of each driving factor, the fraction in variation partitioning of the multiple standardized regression was applied. The SMLR model is expressed as follows:

$$
\frac{G P P-\overline{G P P}}{\sigma_{G P P}}=\beta_{0}+\beta_{1} \frac{E D_{1}-\overline{E D_{1}}}{\sigma_{1}}+\ldots+\beta_{n} \frac{E D_{n}-\overline{E D_{n}}}{\sigma_{n}},
$$

where GPP represents a time series of accumulated GPP in the growing season (AGG); $E D_{i}(i=1,2$, $\ldots \ldots, n ; n$ is the total of driving factors) is a time series of a certain influencing factor covering the whole study period at the pixel scale, including daily sunshine duration in summer, daily average temperature in summer, daily precipitation in summer, growing season length (GSL), and FPAR; $\overline{G P P}$ and $\overline{E D_{1}}$ are the multi-year mean values of AGG and influencing factors, respectively. $\delta_{G P P}$ and $\delta_{E D}$ are standard deviations of AGG and influencing factors. Each time series was normalized by subtracting its mean value and then dividing it by its standard deviation. Thus, any unit change in each variable has the same statistical meaning and the effects of changes in different factors can be 
compared. $\beta_{1}, \beta_{2} \ldots \beta_{n}$ are regression coefficients that represent the importance of each driving factors to the GPP. Factors with a large coefficient were assumed to contribute more to the variation in GPP, and the largest coefficient was identified as the dominant factor to GPP in our study area during the period of 1982-2015. Also, we used the Variance Inflation Factor (VIF) to examine the multicollinearity of our driving factors before conducting the SMLR model. Significant multicollinearity exists when the VIF $\geq 10$. Each SMLR model and their coefficients were verified by the $F$-test and $t$-test, respectively.

\section{Results}

Given that the GPP was nearly zero during winter in the GKM, only the AG in spring, summer, autumn, and the growing season (GS) were considered for analysis.

\subsection{Spatial Distribution of GPP}

We acquired the multi-year mean AG in spring, summer, autumn, and the growing season to analyze the spatial distribution of GPP in the GKM over the past 34 years (1982-2015), and the results are shown in Figure 2. The spatial distribution of AG in the three seasons and the growing season differed considerably; in spring, the AG decreased from the northeast $\left(134 \mathrm{~g} \mathrm{C} / \mathrm{m}^{2}\right)$ to southwest $\left(73 \mathrm{~g} \mathrm{C} / \mathrm{m}^{2}\right)$. In summer, the northern extreme and southern extreme had large GPP values $\left(>755 \mathrm{~g} \mathrm{C} / \mathrm{m}^{2}\right)$, while the west central GKM had the smallest GPP values $\left(<665 \mathrm{~g} \mathrm{C} / \mathrm{m}^{2}\right)$. Different from spring and summer, high values began to concentrate in the southern GKM in autumn, whereas the middle part still had the lowest values. The spatial pattern of the AG during the growing season (AGG) showed great similarities to the AG in summer, but had larger spatial heterogeneity. The highest AGG value over the whole region reached $1070 \mathrm{~g} \mathrm{C} / \mathrm{m}^{2}$, and the lowest AGG value was about $680 \mathrm{~g} \mathrm{C} / \mathrm{m}^{2}$.

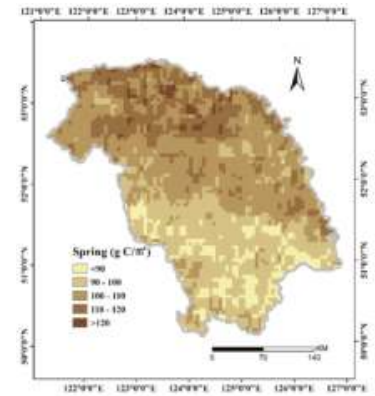

(a)

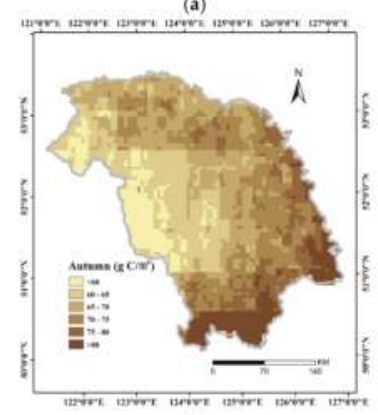

(c)

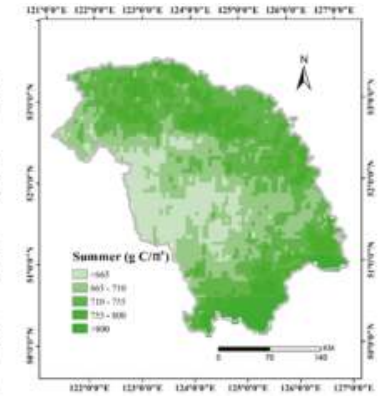

(b)

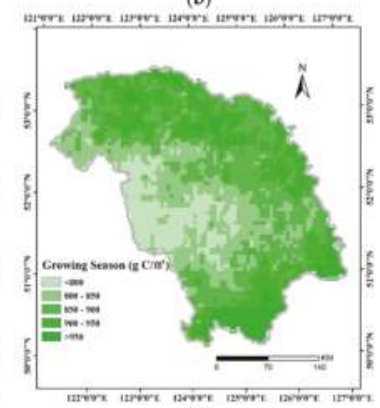

(d)

Figure 2. Spatial distribution of (a) spring-average accumulated gross primary productivity (AG); (b) summer-average AG; (c) autumn-average AG; and, (d) growing season (GS)-average AG during 1982-2015. 


\subsection{Changes in GPP at a Regional Scale}

Temporal variation of GPP at the regional scale can be investigated from two perspectives: intraand interannual variation.

Results in Figure 3 showed a significant annual unimodal variation in GPP across the GKM from 1982 to 2015. GPP values began to progressively increase in mid-April, rapidly rose during mid-May to late-June, and peaked in mid-July. In October, GPP values fell back to a relatively low level. June to August was the vigorous growth period with high GPP values in a year whose daily GPP was usually greater than $6 \mathrm{~g} \mathrm{C} / \mathrm{m}^{2}$.

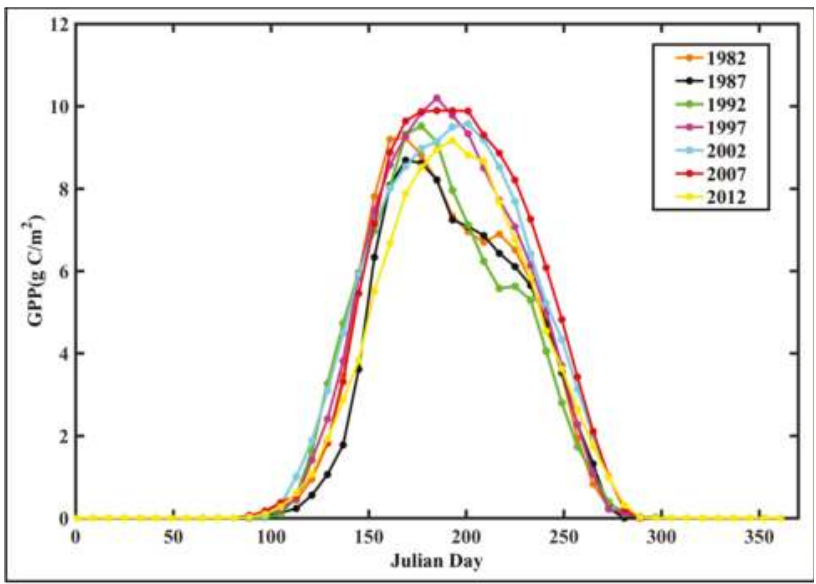

Figure 3. Intra-annual variation characteristic of gross primary productivity (GPP) values during 1982-2015.

Apart from the spatial distribution of AG in different seasons, interannual AG trends in three seasons and the growing season in the GKM during 1982-2015 were calculated and are shown in Figure 4. Except for spring, the other two seasons and the growing season exhibited a significant increase in AG with fluctuations from 1982 to 2015 (Figure 4). However, the increasing levels varied. Among the three seasons, the $\mathrm{AG}$ in summer presented the biggest enhancement (ratio $=2.669 \mathrm{~g} \mathrm{C} / \mathrm{m}^{2} \mathrm{yr}^{-1}, p<0.01$ ), followed by the autumn (ratio $=0.89546 \mathrm{~g} \mathrm{C} / \mathrm{m}^{2} \mathrm{yr}^{-1}, p<0.01$ ). Statistics showed that the $\mathrm{AG}$ in summer accounted for $80 \%$ of the AGG of a year and thus could explain the majority increment in AGG.

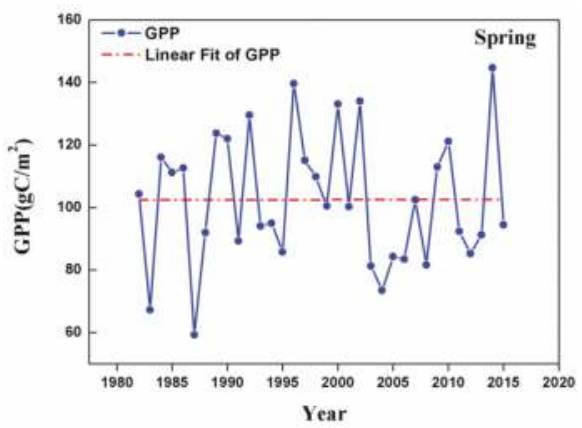

(a)

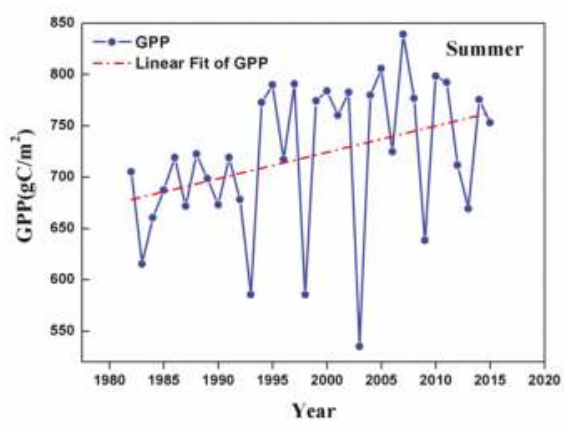

(b)

Figure 4. Cont. 


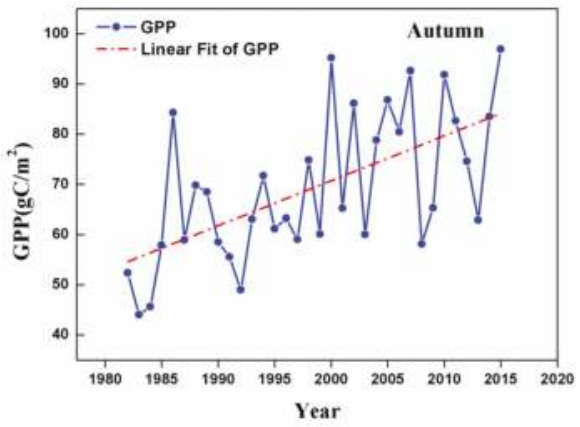

(c)

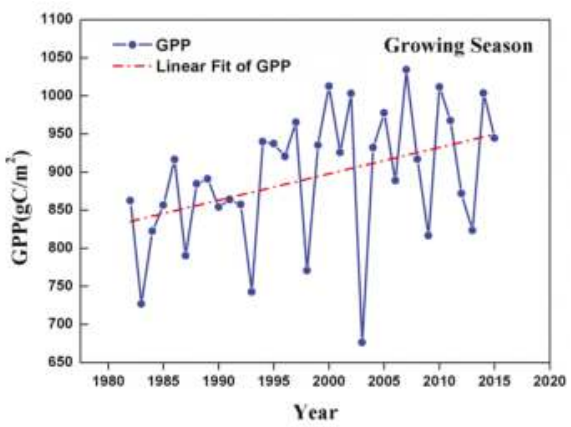

(d)

Figure 4. Interannual variation trends of AG in the GKM from 1982 to 2015.

In addition to the difference in variation trends, years with extreme AG values in different seasons also varied. In spring, the minimum AG occurred in 1987 and the maximum was in 2014. In summer, the minimum GPP occurred in 2003 and the maximum was in 2007; and, in autumn, the minimum and maximum AG occurred in 1982 and 2015, respectively. Also, extreme values in the growing season showed great similarities to those in summer.

\subsection{Spatial Patterns of GPP Trend}

Apart from interannual change at the regional scale, we also analyzed variation at the pixel scale. Because the AGG variation trend was not obvious before 1999, we only displayed linear trends of the AGG over progressively longer periods from 18 (1982-1999) to 34 (1982-2015) years, starting in 1982.

The amounts of pixels that are characterized by significantly increased AGG were greatly enhanced after 1999 (Figure 5). Two periods exhibited a notable increase: 1999-2002 and 2003-2008. A minor dip was observed in 2003 because of the combined effect of low temperature and adequate precipitation. After 2008, variations in the AGG became flat and the percentage of pixels with a significant increasing trend was maintained at about $57 \%$.

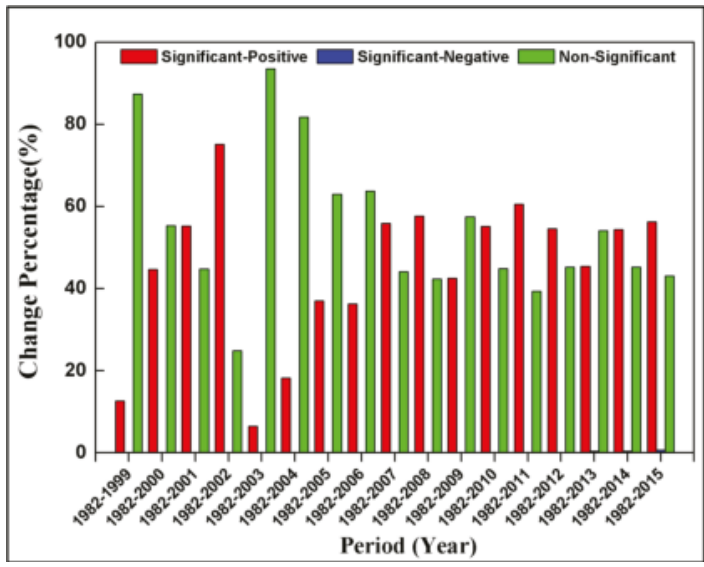

Figure 5. Area fraction of the AGG displaying different statistical significance levels with progressively longer time series since 1982 (\%). Significant positive stands for the increasing of GPP and significant negative refers to the reduction of GPP; both indicate a statistical significance of the linear regression with a Pearson correlation less than 0.05. 
Further exploration (Figure 6) found that pixels characterized by significantly increased AGG mainly concentrated in the central and southern GKM, while most of the pixels in the north still showed non-significant trends during the study period. After 2010, though areas with increasing AGG were still expanding, the variation ratio of some areas, especially the middle part, decreased (comparing Figure 6c,d).

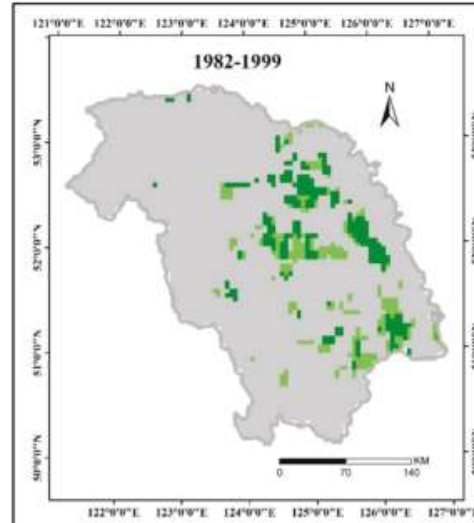

(a)

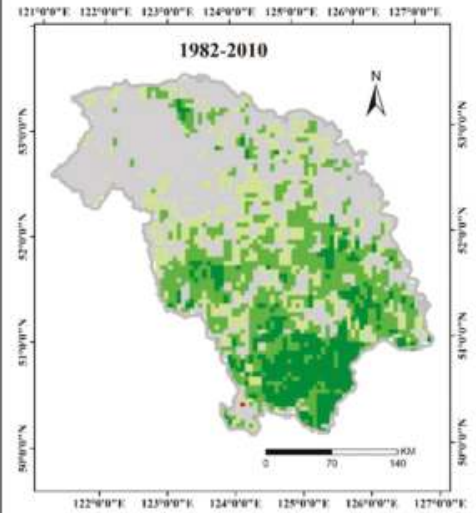

(c)

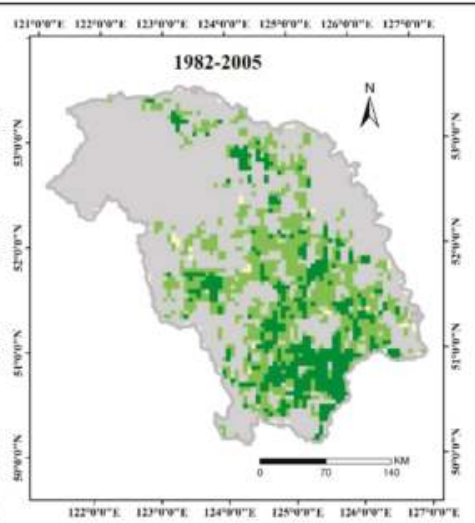

(b)

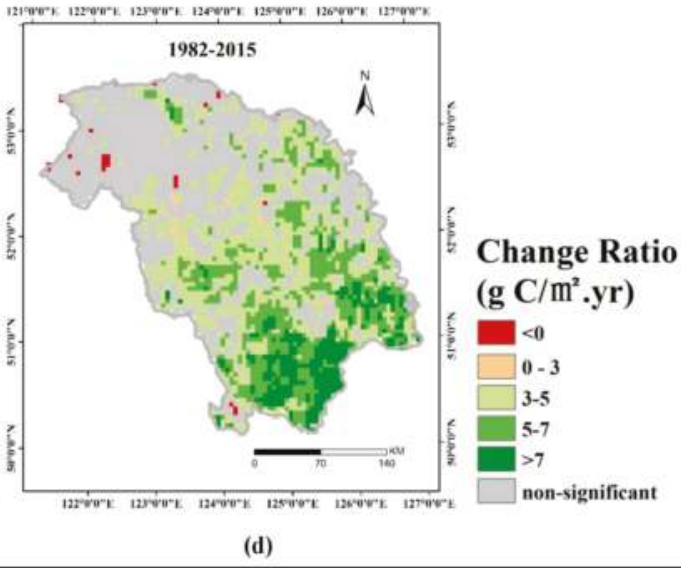

Figure 6. Spatial distribution of AGG trends across the GKM during the periods of (a) 1982-1999; (b) 1982-2005; (c) 1982-2010; and, (d) 1982-2015. The linear trends of the AGG were calculated at the $95 \%$ confidence level.

\subsection{Correlations between GPP and Driving Factors}

\subsubsection{Effects of Local Meteorological Factors on GPP}

In order to compare meteorological variables acquired from the meteorological stations with GPP values, the time series of GPP in the buffer distance of $25 \mathrm{~km} \mathrm{[60]} \mathrm{around} \mathrm{each} \mathrm{meteorological}$ station were extracted for 1982-2015. A Pearson correlation method was then performed between the extracted time-series and meteorological data to analyze the relationship between the GPP and meteorological factors. Because the effect of meteorological factors differed under varying seasons during the year, we calculated the correlation coefficient in different seasons, and the results are listed in Table 1. 
Table 1. Correlation coefficients of the AG in three seasons and the growing season versus meteorological factors obtained from five different meteorological stations over the GKM from 1982 to 2015.

\begin{tabular}{|c|c|c|c|c|c|c|}
\hline & Station & SD & DAT & DMAT & DMIT & DP \\
\hline \multirow{5}{*}{ Spring } & 50,136 & $0.419 * *$ & $0.335^{*}$ & $0.398^{* *}$ & 0.257 & -0.153 \\
\hline & 50,246 & 0.279 & $0.435^{* *}$ & $0.495^{* *}$ & 0.279 & -0.179 \\
\hline & 50,349 & 0.397 * & $0.448 * *$ & $0.482 * *$ & 0.292 * & -0.310 * \\
\hline & 50,353 & 0.390 * & 0.381 * & $0.493^{* *}$ & 0.200 & -0.150 \\
\hline & 50,425 & 0.295 * & $0.445^{* *}$ & $0.419 * *$ & $0.407^{* *}$ & -0.070 \\
\hline \multirow{5}{*}{ Summer } & 50,136 & $0.631^{* *}$ & 0.339 * & $0.459 * *$ & -0.144 & -0.324 * \\
\hline & 50,246 & $0.614^{* *}$ & $0.669^{* * *}$ & $0.811^{* *}$ & -0.155 & $-0.580^{* *}$ \\
\hline & 50,349 & $0.814^{* *}$ & $0.717^{* * *}$ & $0.810^{* *}$ & -0.017 & -0.482 ** \\
\hline & 50,353 & $0.835^{* *}$ & $0.665^{* *}$ & $0.753 * *$ & 0.247 & $-0.640^{* *}$ \\
\hline & 50,425 & $0.565^{* *}$ & 0.590 ** & $0.642^{* *}$ & 0.304 * & $-0.470^{* *}$ \\
\hline \multirow{5}{*}{ Autumn } & 50,136 & $0.474^{* *}$ & -0.041 & 0.349 * & -0.255 & $-0.608^{* *}$ \\
\hline & 50,246 & $0.491^{* *}$ & -0.026 & $0.410^{* *}$ & -0.328 * & $-0.484^{* *}$ \\
\hline & 50,349 & $0.677^{* *}$ & -0.041 & 0.314 * & -0.335 * & $-0.441^{* *}$ \\
\hline & 50,353 & 0.134 & 0.176 & 0.317 * & -0.051 & $-0.429 * *$ \\
\hline & 50,425 & $0.466^{* *}$ & 0.304 * & 0.333 * & 0.206 & $-0.447^{* *}$ \\
\hline \multirow{5}{*}{ Growing Season } & 50,136 & 0.620 ** & $0.385^{*}$ & $0.50,2 * *$ & -0.055 & -0.371 * \\
\hline & 50,246 & 0.587 ** & 0.602 ** & $0.789 * *$ & -0.137 & $-0.544^{* *}$ \\
\hline & 50,349 & $0.753 * *$ & $0.626^{* *}$ & $0.773 * *$ & 0.005 & $-0.513^{* *}$ \\
\hline & 50,353 & $0.659 * *$ & $0.604^{* *}$ & $0.704^{* *}$ & 0.294 * & $-0.623^{* *}$ \\
\hline & 50,425 & $0.577 * *$ & $0.572 * *$ & $0.603^{* *}$ & 0.367 * & -0.470 ** \\
\hline
\end{tabular}

SD refers to sunshine duration, DAT refers to daily average temperature, DMAT represents daily maximum temperature, DMIT represents daily minimum temperature, and DP represents daily precipitation; ${ }^{*} p<0.05$, statistical significance of Pearson correlation; ${ }^{* *} p<0.01$, statistical significance of Pearson correlation.

In spring, sunshine duration and temperature played key roles in AG, while precipitation had little influence on AG during the study period. In summer, vegetation grew rapidly and AG was high. All of the factors had significant impacts on AG. Sunshine duration and temperature showed nearly the same positive effects on AG, while precipitation was negatively correlated with AG. AG in autumn and summer were alike in correlation with meteorological factors, but the effect of precipitation exceeded that of temperature in autumn and demonstrated an equally significant influence on AG as sunshine duration. As for AG in the growing season, the relationship between the AG and meteorological factors were basically the same as that in summer.

\subsubsection{Effects of Phenological Parameters and FPAR on GPP}

Based on the phenological parameters acquired as shown in Figure 7, we explored the relationship between the phenological parameters and GPP.

In the GKM, an advance in SOG and delay in EOG leaded to an obvious extension of GSL over the last 34 years. This result was consistent with previous findings, demonstrating that climate warming causes an extension of GSL [46,61].

According to many studies $[59,62,63]$ that were conducted in other regions, the extension of GSL is an important factor for the increasing GPP. We also conducted correlation analysis between GPP and GSL per pixel along the temporal dimension and obtained the spatial distribution of the correlation coefficient; the result is displayed in Figure 8.

Results (Figure 8) showed that in the central and southern GKM, there was a significant positive correlation between GSL and AGG, as was the case in abovementioned areas. The extension of GSL could explain the increase of AGG in some areas belonging to the GKM over the past 34 years, and this was consistent with the results of a previous study [59]. The majority of regions in the north and along the GKMR did not show a significant correlation between AGG and GSL, therefore some other elements should be considered as driving factors in those areas. 


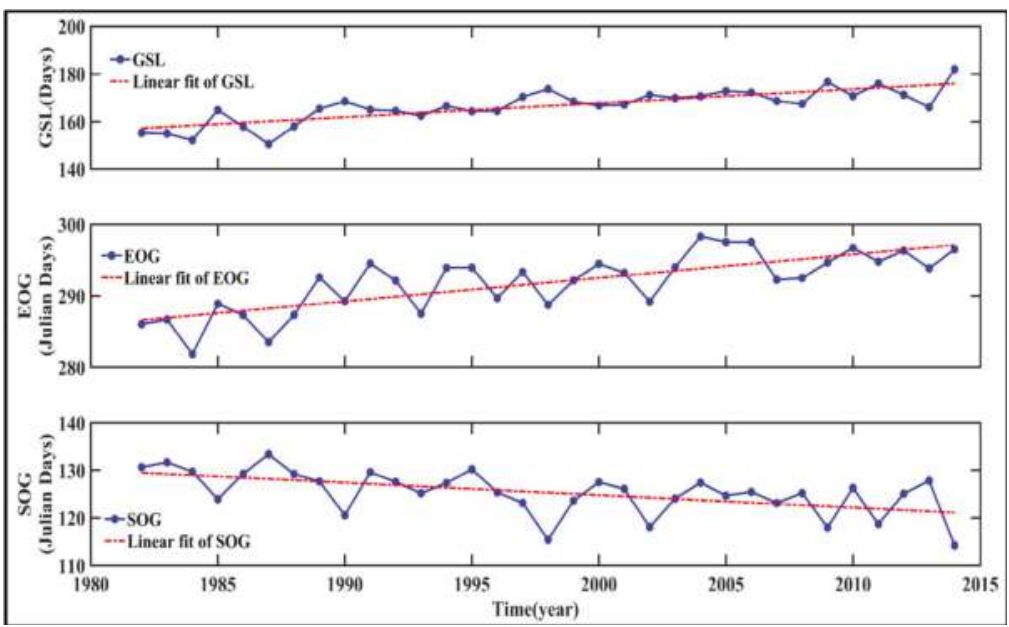

Figure 7. Variation in phenological parameters in the GKM from 1982 to 2014 (SOG means starting day of growing season, EOG means ending day of growing season, and GSL means growing season length).

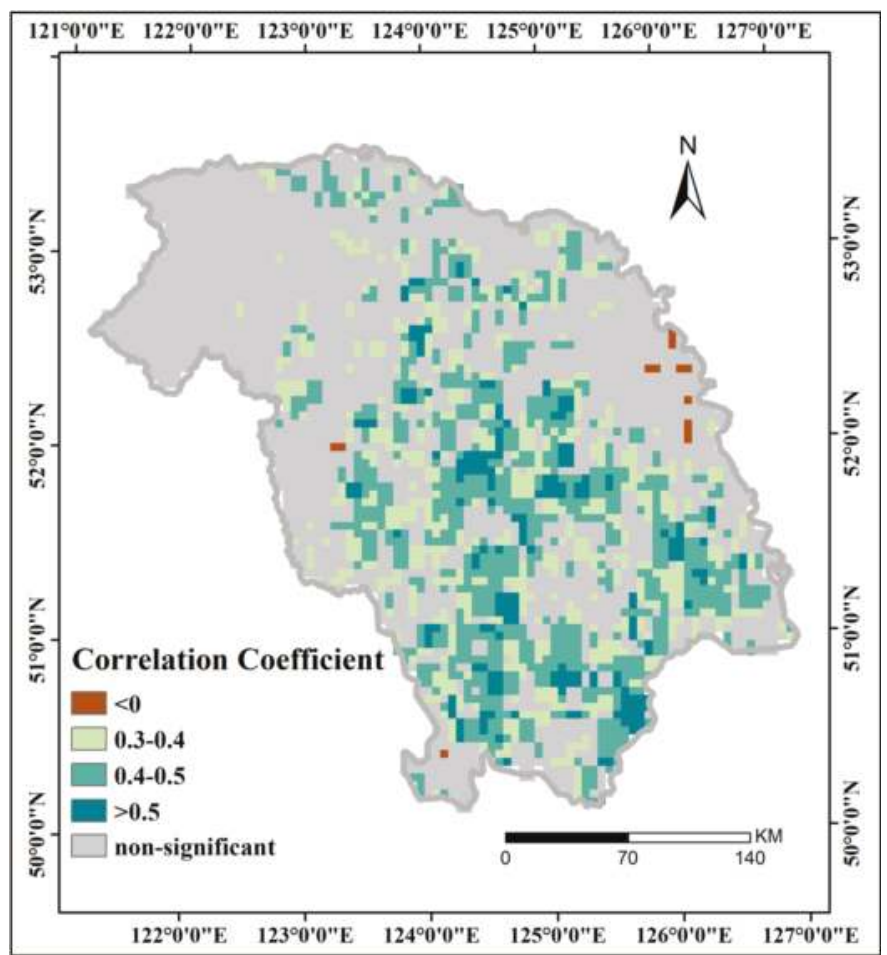

Figure 8. Correlation coefficient between GSL and AGG in the GKM during 1982-2014. (Green indicates significant positive correlation $(\mathrm{r}>0, p<0.05)$, brown indicates significant negative correlation $(\mathrm{r}<0$, $p<0.05)$, and gray indicates non-significant correlation $(p>0.05))$. 
Apart from phenological parameters, FPAR, which refers to the fraction of absorbed PAR in vegetation photosynthesis, may also be an important driving factor for GPP, because it can directly impact the total vegetation productivity during photosynthesis. Correlation analysis, as depicted in Figure 9, showed a significant positive correlation between GPP and FPAR in the Mohe County, along the GKMR, and in part of the southern GKM, meaning that FPAR in the areas mention above was an important factor for AGG; however, little significant correlation was found in the central GKM.

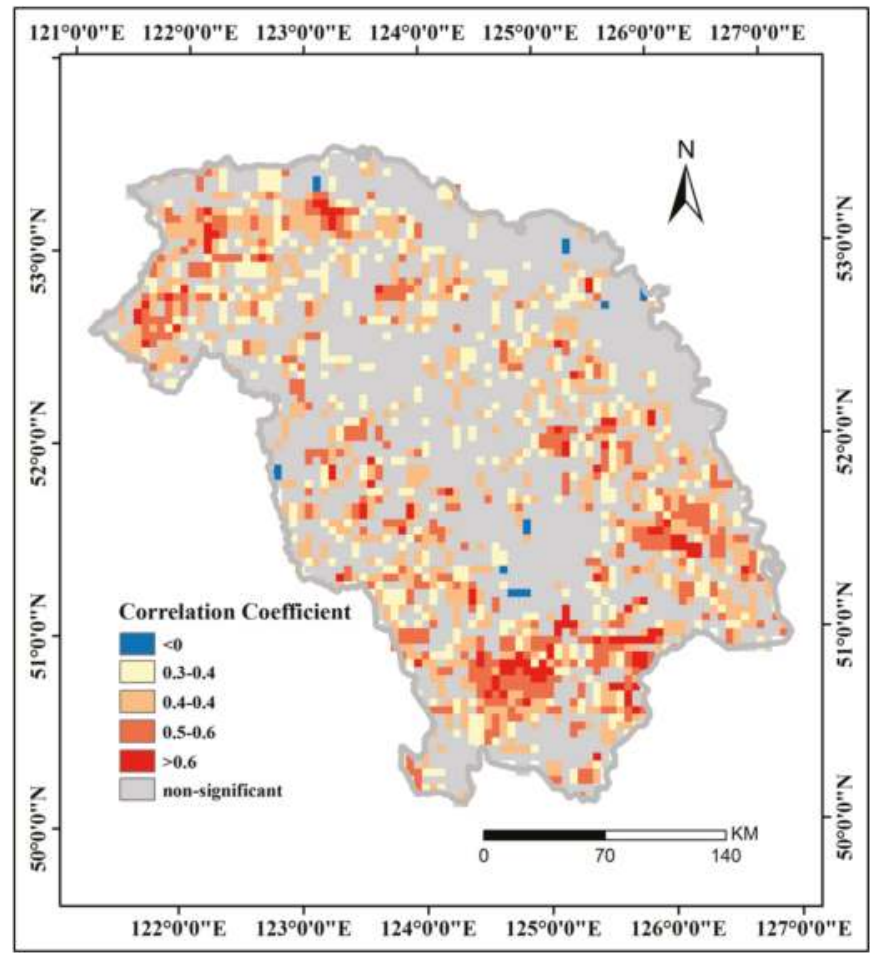

Figure 9. Correlation coefficient between Fraction of Photosynthetically Active Radiation (FPAR) and AGG in the GKM during 1982-2014. (Yellow and red indicate significant positive correlation $(\mathrm{r}>0, p<0.05)$, blue indicates significant negative correlation $(\mathrm{r}<0, p<0.05)$, and gray indicates non-significant correlation $(p>0.05))$.

A comparison between Figures 8 and 9 showed a great difference of driving factors in different regions. It is necessary to combine all of the factors mentioned above to explore the spatial distribution of the dominant factor affecting GPP across the GKM; this will help us to further understand the underlying mechanism of vegetation dynamics in our study area over the past 34 years.

\subsubsection{Dominant Factor for GPP Variation}

The above-mentioned five factors (daily sunshine duration of summer, daily mean temperature of summer, daily precipitation of summer, GSL, and FPAR) all had a direct or indirect impact on GPP. In this section, we determined the regression between AGG and these five factors with an SMLR model at the pixel level, and thus found the dominant factor for the GPP variation.

The result of the regression was displayed in Figure 10. It showed that, except for some areas in the northern GKM, most parts of the GKM were highly correlated with daily sunshine duration and mean temperature in summer, so these two factors acted as dominant or subdominant factors in 
our study area. Observations in an area called Mohe County in the northern GKM differed where most parts were closely related to FPAR instead of daily sunshine duration and mean temperature of summer. Furthermore, GSL and daily precipitation did not exert key effects on GPP when compared with the other three factors.

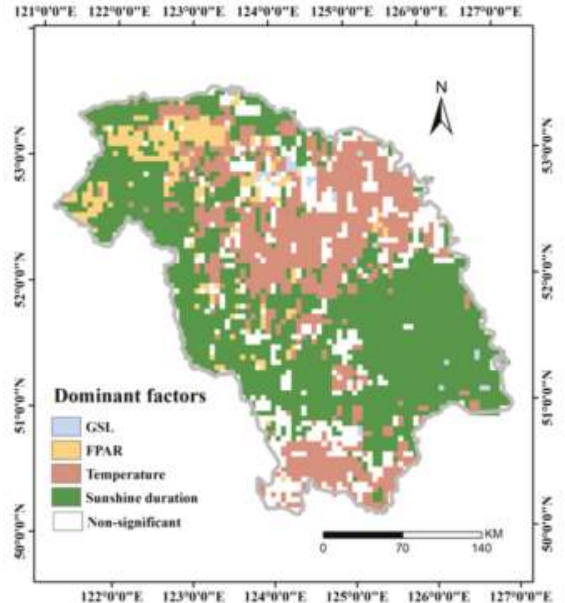

(a)

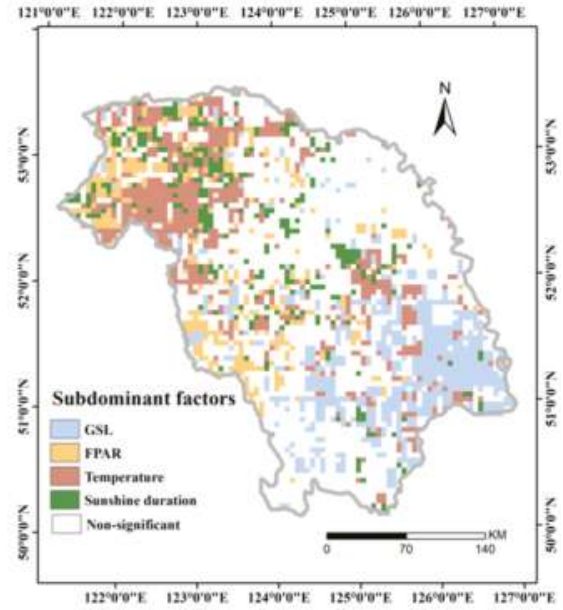

(b)

Figure 10. Spatial distribution of (a) dominant and (b) subdominant factors for AGG in the GKM from 1982 to 2015 (Non-significant means that there is no significantly effective factor for AGG among these five driving factors according to the $F$-test and $t$-test. A significant $F$-test was under $p<0.05$, and a significant $t$-test was under the absolute value of $t \geq 1$.96).

According to the coefficients before each independent variable derived from the SMLR model at the pixel level, we calculated the zonal mean value of coefficients for each dominant factor and the results are shown in Table 2. Taking sunshine duration as an example, when sunshine duration is a dominant factor in our study area, each unit increase in sunshine duration causes a 0.519 unit increase in AGG.

Table 2. Zonal mean value of the coefficient for each dominant factor.

\begin{tabular}{ccccc}
\hline Dominant Factor & GSL & FPAR & Temperature & Sunshine Duration \\
\hline Zonal mean coefficients & 0.399134 & 0.453251 & 0.448733 & 0.518619 \\
\hline
\end{tabular}

\section{Discussions}

\subsection{Analysis of GPP Spatiotemporal Dynamics}

In the GKM, different seasons displayed different patterns of spatial distribution and we considered three reasons for these phenomena according to our analysis. Understory vegetation, such as dwarf shrubs, mosses, and reindeer lichens, played an important role. Studies found that the productivity of understory vegetation is probably comparable with that of the trees [64], and this is especially obvious in spring when understory vegetation grew more lushly, while most of the trees were just starting to sprout new leaves, providing a bright environment in which understory vegetation can acquire more sunshine. The northern GKM had the largest AG values (Figure 2a) in spring, which can be attributed to understory vegetation. We found much evidence demonstrating that understory vegetation in the northern GKM was much lusher than that in other parts of the GKM. On one hand, a majority of bright coniferous forest, which is usually sparser than mixed broadleaf-conifer forest or 
broadleaf forest, mainly concentrated in the northern GKM (Figure 11 shows the contrast of spring AG in the bright coniferous zone and other areas), so understory vegetation flourished here [65]. On the other hand, fire is also a primary determinant for understory vegetation $[64,66]$, so the disastrous fire that occurred in the northern GKM in 1987 may have contributed to the luxuriance of the understory vegetation in that region.

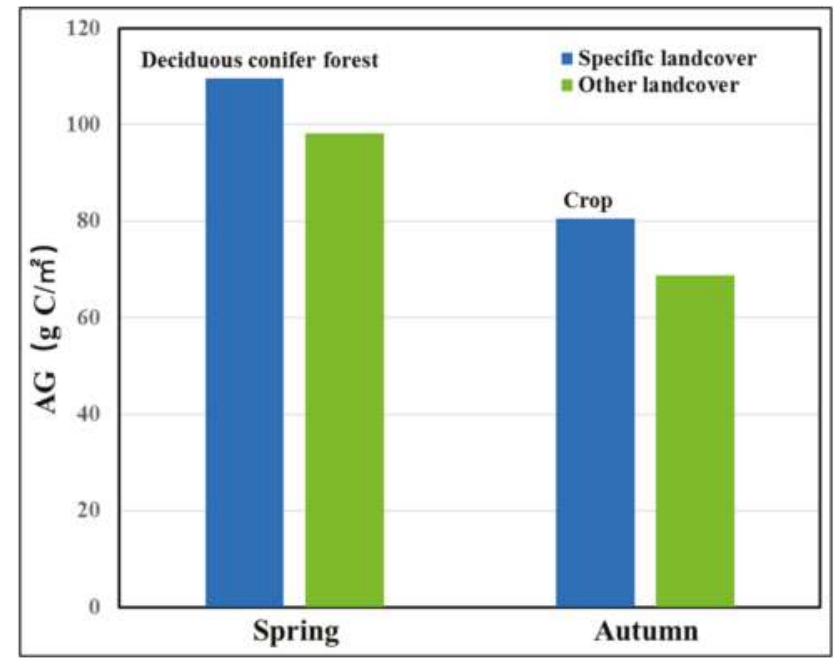

Figure 11. Contrast of AG in specific land cover and other areas in the GKM.

Altitude was another crucial factor for the spatial distribution of AG in our study area. GPP in areas lying along the Greater Khingan Mountain Ridges (GKMR) was always smaller than that in other parts (Figure 12 shows the variation of AGG with elevation, ratio $=-0.18 \mathrm{~g} \mathrm{C} / \mathrm{m}^{2} \mathrm{~m}^{-1}, p<0.05$ ); this was especially obvious in summer (as shown in Figure 3b). As we all know, temperature decreases with the increase in elevation, so the GKMR is a low-temperature zone with low GPP values by nature. In summer, vegetation in the high-temperature zone grew rapidly, while growth remained slow along the GKMR, so the gap in productivity increased further.

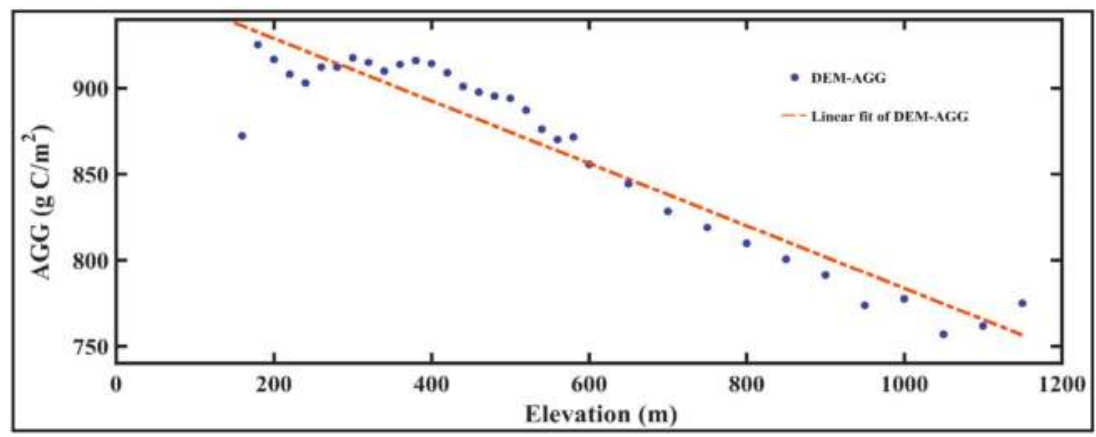

Figure 12. Variation of AGG with elevation.

Land cover also influenced the spatial distribution of AG. When combining with land cover data of the GKM (Figure 1c), we found cropland, instead of forest, being widely distributed in the southern GKM (Figure 11 shows the contrast of spring AG in the cropland zone and other areas). 
Because of human intervention, cropland can grow well in a relatively low temperature and thus the southern GKM had the largest GPP values across the GKM in autumn when the temperature has greatly decreased.

Apart from spatial distribution, reasons for the temporal variation of AG were also analyzed. Annual variation in GPP displayed an obviously unimodal seasonal cycle because Larix gmelinii, a kind of deciduous coniferous forest, is the dominant species in the GKM. Due to the low temperature and frozen soil before the 100th day (mid-April) and after the 270th day (early-October), photosynthesis is weak and thus GPP is mainly produced from mid-May to mid-September, so there is a shorter growing period than in other regions.

The interannual change of AG at the regional scale displayed significant increasing trends in summer, autumn, and the growing season; this observation was consistent with findings in a previous study [67]. Moreover, according to Figure 13 and previous studies [68,69], there was a significant warming and drying trend of the climate (daily average temperature: ratio $=0.0391{ }^{\circ} \mathrm{C} / \mathrm{yr}^{-1}, p<0.01$; daily precipitation: ratio $\left.=-0.0138 \mathrm{~mm} / \mathrm{yr}^{-1}, p=0.146\right)$ in the GKM. What is more, our study area aggregates a number of heliophilous larch, which is suited to warm and dry environments, so the climate in the GKM benefitted vegetation growth and resulted in an obvious increase in the AG over the past 34 years.

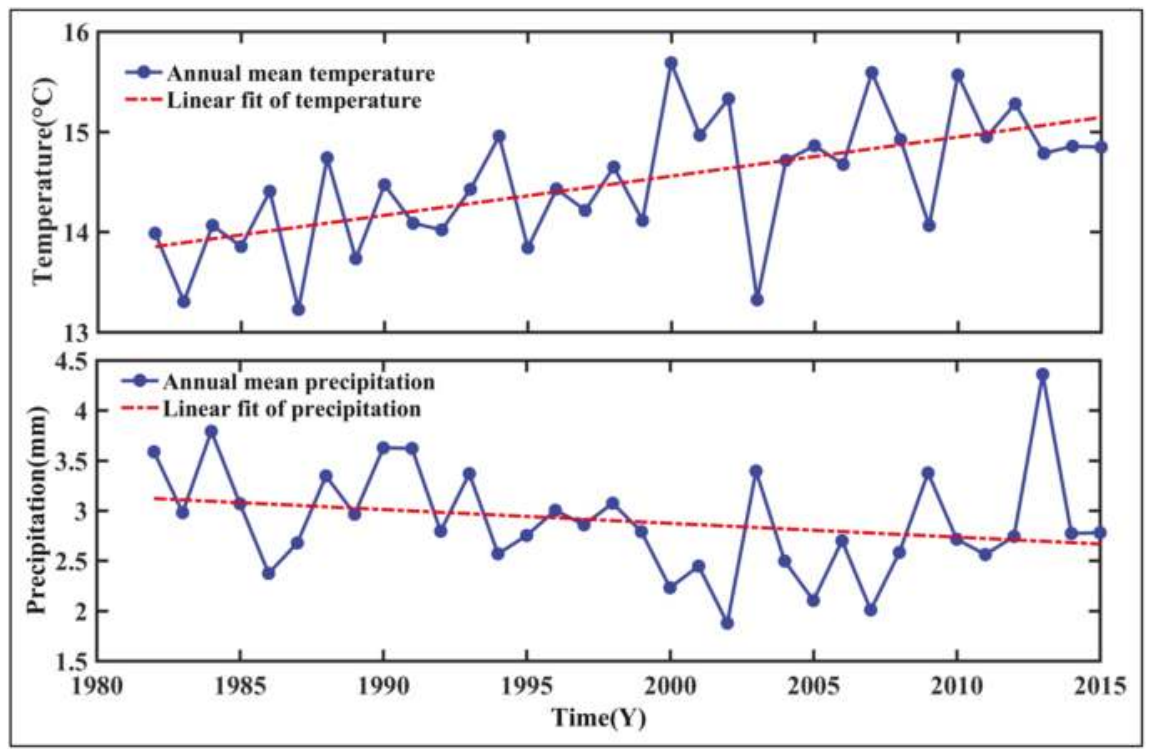

Figure 13. Variation in annual mean daily temperature and annual mean daily precipitation in the growing season over the GKM from 1982 to 2015.

Further studies on interannual variation trends at the pixel scale did not find significant variation during 1982-1999. As an important source of commercial timbers in China, the effects of the GKM on maintaining ecological balance and improving the ecological environment have always been overlooked; for instance, intense anthropogenic activity intervention, such as reclamation and excessive deforestation, occurred frequently, which led to a dramatic drop in its forest areas. After the natural forest protection project launched in 1998, timber yield dramatically decreased (Figure 14). So, there was an obvious increment of AGG in the central and southern GKM entering the 21st century. Furthermore, the variation of AGG after 2010 was slowed down because of the formation of a relatively stable vegetation community in the GKM a decade after the natural forest protection project in 1998. 
Nevertheless, some regions in the northern GKM still showed little variation trend, which could partly be explained by the severe fire in 1987 (Figure 15). In severely burnt area, there was nearly no natural forestry resources left. A secondary succession procedure occurred, which had dynamic land cover [70-73] and led to the non-significant changing ratio of AGG.

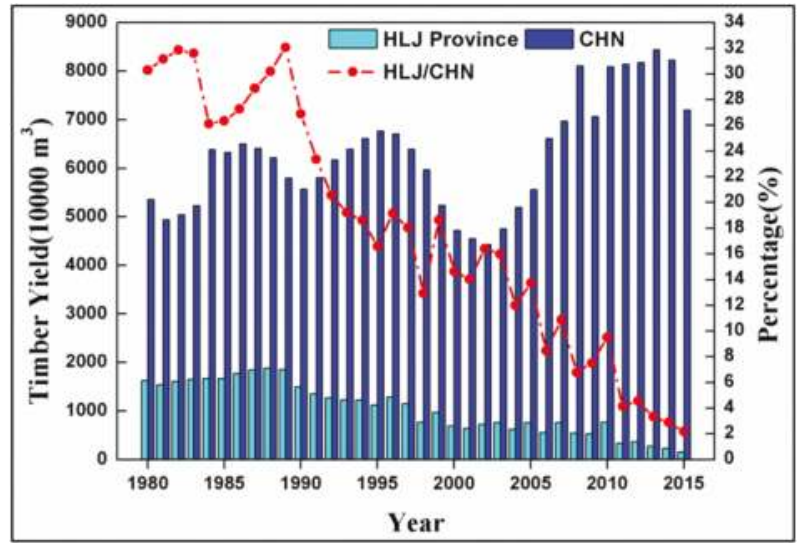

Figure 14. Variation in timber yield in Heilongjiang Province since 1980.

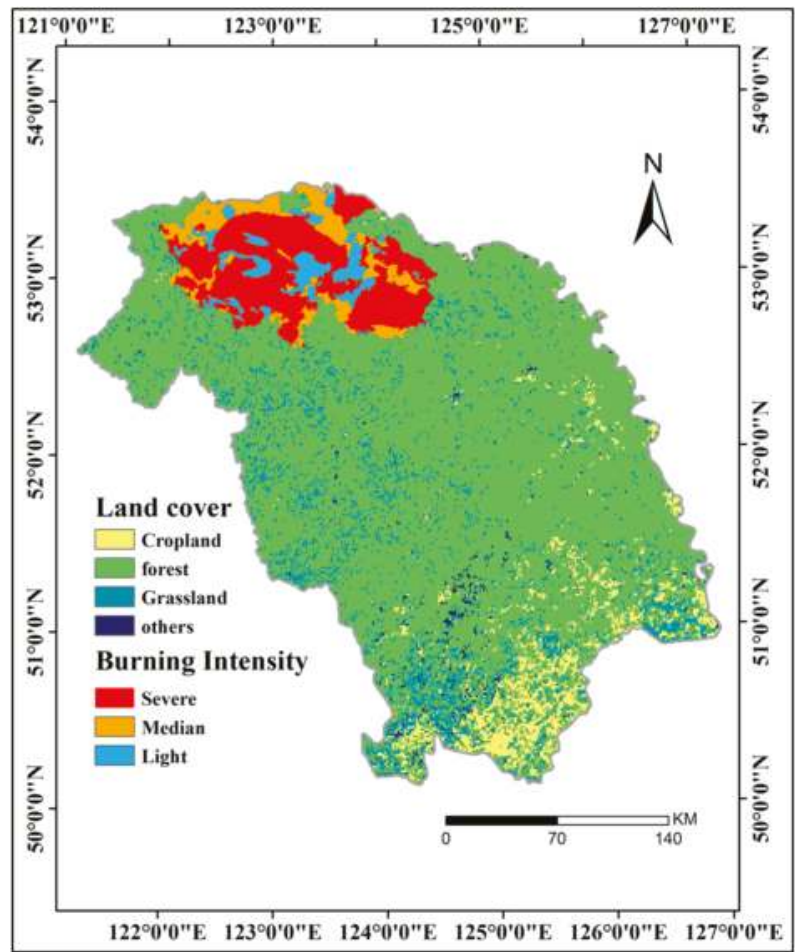

Figure 15. Burnt zone of the fire in 1987 and land cover in the GKM. 


\subsection{The Response of GPP to Driving Factors}

Daily sunshine duration in summer acted as a dominant factor for most regions in the GKM. The sunshine duration in meteorological observation is defined as the period during which direct solar irradiance exceeds a threshold value of $120 \mathrm{~W} / \mathrm{m}^{2}$ [74]. It is a general indicator of the cloudiness of a location [75]. According to Figure 13, a warming and drying trend has occurred in the GKM, so daily sunshine duration increased simultaneously. When compared with the impact of GSL on AGG by extending photosynthesis time in spring (advance of SOG) and autumn (delay of EOG), the daily sunshine duration in summer prolonged the photosynthesis time in summer when photosynthesis efficiency is high, and a slight extension of daily sunshine duration contributed to a large enhancement in AGG. Also, vegetation can absorb more solar radiation over a long sunshine duration, which is directly related to photosynthesis and was confirmed to be a limited factor in high latitude regions [76].

Temperature had positive effects on GPP, while precipitation had negative effects on GPP, which were in agreement with relevant study results [40,59-61]. GKM vegetation system was strongly constrained by low temperature, so the warming trend that is reported above was bound to increase GPP. Precipitation inhibited GPP across the GKM in most periods when precipitation was adequate, illumination time and temperature decreased with the thick cloud cover that was brought by precipitation, which weakened photosynthesis and reduced vegetation GPP; thus, the precipitation was not a direct factor for GPP. However, in spring when precipitation was rare, no obvious correlation between GPP and precipitation was found, due to the alimentation of snow.

FPAR, instead of sunshine duration and temperature, was a dominant factor in part of northern GKM. Overlay analysis revealed a high correlation of the above-mentioned areas with the severely burnt zone caused by the fire in 1987 (Figure 15). Previous studies [63-65] demonstrated a secondary succession process in burnt areas. Variation in vegetation type and tree age during the succession process may have contributed to a non-significant correlation between climate changes and GPP interannual variation in the northern GKM. On the other hand, FPAR is an important parameter for photosynthesis, and one previous study [77] revealed a close relationship between FPAR and vegetation type. Therefore, GPP in the severely burnt area in the northern GKM was closely related with FPAR.

\subsection{Study Limitations}

When compared with previous studies that used MODIS datasets after 2000 to analyze vegetation variation [78,79], this study used the GLASS GPP product and revealed the spatiotemporal characteristics of GPP in the GKM from 1982 to 2015. Obviously, an extension of the study period could provide much more information about the dynamic change of vegetation and the carbon cycle. However, some problems still existed. The precision of the GLASS-GPP time series in our study area needs to be verified with data observed in the eddy covariance flux tower, however, because of poor management, data acquired from the tower cannot be used in the verification, so supplementary analysis using the mainstream MODIS dataset is necessary for making a comparison. On the other hand, when compared with the distribution area of boreal forest (especially the bright coniferous forest), the spatial resolution of GLASS-GPP data that we used is a little coarser, and therefore details may be overlooked. For this reason, further studies combining with spatially finer TM datasets have been undertaken.

Uncertainty in our correlation results may be caused by the interpolated meteorological data, the calculated phenology parameters and pixel mismatches (use of remotely sensed data from different sources). The meteorological observation station are sparsely distributed in the GKM which is the northernmost part of China, there are only five stations in our study area and the remaining stations that are distributed around the GKM are far away. Moreover, there are no stations in the surrounding northern and eastern direction. All of these factors may increase the uncertainty of the interpolation. There are also some limitations that are caused by the phenological parameters. Although the phenology parameters we used have been verified by many relative studies $[54,80]$, 
field observation data is still necessary for authenticity verification. Unfortunately, no field observation data is available for the GKM in the study period, so our work should be improved by further field observations. Pixel mismatches also caused some errors. Since we used remotely sensed data from different source, pixel mismatches were inevitable. For example, GLASS-GPP had a 5-km resolution, while NDVI had an 8-km resolution, so we resampled NDVI to match GPP data, which caused error in NDVI.

This paper only considered five factors affecting the spatiotemporal dynamics of GPP; other factors are also important, especially anthropogenic activities and fire in the GKM. For example, the natural forest protection project launched in 1998 played an important role in our study area, as mentioned above. However, because such roles hard to quantify, it is difficult for us to know their specific effect on GPP. What is more, we cannot entirely separate the effect that is brought by anthropogenic activities and the quantified environment factors, which added some uncertainty to our study. Therefore, further studies should be concentrated on finding alternatives and analyzing effects brought by anthropogenic activities and fire. Further study is needed before we can thoroughly understand the potential mechanisms affecting the spatiotemporal pattern of GPP in the GKM.

\section{Conclusions}

Based on an analysis of vegetation spatiotemporal dynamics as well as the correlation between GPP and its driving factors, we drew the following conclusions:

1. In the GKM, different seasons displayed different patterns of spatial distribution because of the difference in understory vegetation, altitude and land cover. Areas with bright conifer forest always have a larger GPP because the understory vegetation contributes a great deal to vegetation total productivities. Altitude impacts GPP by changing temperature. Land cover with intense human intervention showed different seasonal changes in AGG.

2. Temporal trends of AG at the regional scale showed a dramatic increase from 1982 to 2015 in summer, autumn, and the growing season. This observation could be considered to be a result of the warming and drying trend that is seen in the GKM over the past 34 years.

3. Interannual GPP trends at the pixel scale showed that the areas with significant AGG variation (mostly significant increasing trends) were strikingly expanded after 2000 because of the natural forest protection project launched in 1998, but the AGG variation displayed a flattened trend over the last five years due to the formation of a relatively stable vegetation community.

4. Upon aggregating all of the above factors, daily sunshine duration in summer, instead of GSL, acted as the dominant factor in most of the areas, whereas daily mean temperature in summer was the dominant factor in a fraction of the GKM. FPAR also acted as the dominant factor in part of the northern GKM and was closely related to vegetation change that was caused by the fire in 1987.

Acknowledgments: This research was supported by the Major State Basic Research Development Program of China (2013CB733402), the National Natural Science Foundation of China (grant Nos. 41271346, 91425301). We appreciate the provision of GPP and FPAR products by GLASS Team.

Author Contributions: All authors contributed significantly to this manuscript. Specific contributions include data collection (Ling Hu, Wenjie Fan), data analyses (Ling Hu, Wenjie Fan), methodology (Ling Hu, Wenjie Fan), and manuscript preparation (Ling Hu, Wenjie Fan, Huazhong Ren, Suhong Liu, Yaokui Cui, Peng Zhao).

Conflicts of Interest: The authors declare no conflict of interest.

\section{References}

1. Tans, P.P.; Fung, I.Y.; Takahashi, T. Observational constraints on the global atmospheric $\mathrm{CO}_{2}$ budget. Science 1990, 247, 1431-1438. [CrossRef] [PubMed]

2. Schimel, D.S.; Braswell, B.H.; McKeown, R.; Ojima, D.S.; Parton, W.J.; Pulliam, W. Climate and nitrogen controls on the geography and timescales of terrestrial biogeochemical cycling. Glob. Biogeochem. Cycles 1996, 10, 677-692. [CrossRef] 
3. Zhou, L.M.; Tucker, C.J.; Kaufmann, R.K.; Slayback, D.; Shabanov, N.V.; Myneni, R.B. Variations in northern vegetation activity inferred from satellite data of vegetation index during 1981 to 1999. J. Geophys. Res. 2001, 106, 20069-20083. [CrossRef]

4. Melillo, J.M.; Mcguire, A.D.; Kicklighter, D.W.; Moore, B.; Vorosmarty, C.J.; Schloss, A.L. Global climate-change and terrestrial net primary production. Nature 1993, 363, 234-240. [CrossRef]

5. $\quad$ Fang, J.Y.; Chen, A.P.; Peng, C.H.; Zhao, S.Q.; Ci, L.J. Changes in forest biomass carbon storage in China between 1949 and 1998. Science 2001, 292, 2320-2322. [CrossRef] [PubMed]

6. Xiao, X.M.; Hollinger, D.; Aber, J.; Goltz, M.; Davidson, E.A.; Zhang, Q.Y.; Moore, B. Satellite-based modeling of gross primary production in an evergreen needleleaf forest. Remote Sens. Environ. 2004, 89, 519-534. [CrossRef]

7. Nemani, R.; Keeling, C.; Hashimoto, H.; Jolly, W.; Piper, S.; Tucker, C.; Myneni, R.; Running, S. Climate-driving increases in global terrestrial net primary production from 1982 to 1999. Science 2003, 300, 1560-1563. [CrossRef] [PubMed]

8. Zhu, Z.C.; Piao, S.L.; Myneni, R.B.; Huang, M.T.; Zeng, Z.Z.; Canadell, J.G.; Ciais, P.; Sitch, S.; Friedlingstein, P.; Arneth, A.; et al. Greening of the Earth and its drivers. Nat. Clim. Chang. 2016, 6, 791-795. [CrossRef]

9. Chapin, M.C.; Matson, P.A.; Mooney, H.A. Principles of Terrestrial Ecosystem Ecology, 2nd ed.; Spring: New York, NY, USA, 2002; p. 102.

10. Beer, C.; Reichstein, M.; Tomelleri, E.; Ciais, P.; Jung, M.; Carvalhais, N.; Rodenbeck, C.; Arain, M.A.; Baldocchi, D.; Bonan, G.B.; et al. Terrestrial gross carbon dioxide uptake: Global distribution and covariation with climate. Science 2010, 329, 834-838. [CrossRef] [PubMed]

11. Anav, A.; Friedlingstein, P.; Beer, C.; Ciais, P.; Harper, A.; Jones, C.; Murray-Tortarolo, G.; Papale, D.; Parazoo, N.C.; Peylin, P.; et al. Spatiotemporal patterns of terrestrial gross primary production: A review. Rev. Geophys. 2015, 53, 785-818. [CrossRef]

12. Jensen, M.N. Consensus on ecological impacts remains elusive. Science 2003, 299, 38. [CrossRef] [PubMed]

13. Cox, P.; Jones, C. Illuminating the modern dance of climate and $\mathrm{CO}_{2}$. Science 2008, 321, 1642-1644. [CrossRef] [PubMed]

14. Battin, T.J.; Kaplan, L.A.; Findlay, S.; Hopkinson, C.S.; Marti, E.; Packman, A.I.; Newbold, J.D.; Sabater, F. The boundless carbon cycle. Nat. Geosci. 2009, 2, 598-600. [CrossRef]

15. NASA. Earth System Science: A Closer View; NASA: Washington, DC, USA, 1988; p. 208.

16. Myneni, R.; Hoffman, R.; Knyazikhin, Y.; Privette, J.; Glassy, J.; Tian, H. Global products of vegetation leaf area and fraction absorbed PAR from one year of MODIS data. Remote Sens. Environ. 2002, 83, 214-231. [CrossRef]

17. Gutman, G.; Ignatov, A. Global land monitoring from AVHRR: Potential and limitations. Int. J. Remote Sens. 1995, 16, 2301-2309. [CrossRef]

18. Privette, J.L.; Fowler, C.; Wick, G.A.; Baldwin, D.; Emery, W.J. Effect of orbital drift on advanced very high resolution radiometer products Normalized difference vegetation index and sea surface temperature. Remote Sens. Environ. 1995, 53, 164-171. [CrossRef]

19. Rao, C.R.N.; Chen, J. Inter-satellite calibration linkages for the visible and near-infrared channels of the Advanced Very High Resolution Radiometer on the NOAA-7,-9, and-11 spacecraft. Int. J. Remote Sens. 1995, 16, 1931-1942.

20. Rao, C.R.N.; Chen, J. Post-launch calibration of the visible and near-infrared channels of the Advanced Very High Resolution Radiometer (AVHRR) on the NOAA-14 spacecraft. Int. J. Remote Sens. 1996, 17, 2743-2747. [CrossRef]

21. Myneni, R.B.; Tucker, C.J.; Asrar, G.; Keeling, C.D. Interannual variations in satellite-sensed vegetation index data from 1981 to 1991. J. Geophys. Res. 1998, 103, 6145-6160. [CrossRef]

22. Gutman, G.G. On the use of long-term global data of land reflectances and vegetation indices derived from the advanced very high resolution radiometer. J. Geophys. Res. 1999, 104, 6241-6255. [CrossRef]

23. Monteith, J.L. Solar radiation and productivity in tropical ecosystems. J. Appl. Ecol. 1972, 9, 747-766. [CrossRef]

24. Monteith, J.L. Climate and the efficiency of crop production in Britain. Phil. Trans. R. Soc. B. 1977, 281, 277-294. [CrossRef]

25. Goetz, S.J.; Prince, S.D. Modelling terrestrial carbon exchange and storage: Evidence and implications of functional convergence in light-use efficien Cycles. Adv. Ecol. Res. 1999, 28, 57-92. 
26. Tucker, C.J.; Pinzon, J.E.; Brown, M.E.; Slayback, D.A.; Pak, E.W.; Mahoney, R.; Vermote, E.F.; Saleous, N.E. An extended AVHRR 8-km NDVI dataset compatible with MODIS and SPOT vegetation NDVI data. Int. J. Remote Sens. 2005, 26, 4485-4498. [CrossRef]

27. Hilker, T.; Coops, N.C.; Hall, F.G.; Black, T.A.; Wulder, M.A.; Nesic, Z.; Krishnan, P. Separating physiologically and directionally induced changes in PRI using BRDF models. Rem. Sens. Environ. 2008, 112, 2777-2788. [CrossRef]

28. Prince, S.D.; Goward, S.N. Global net primary production: A remote sensing approach. J. Biogeogr. 1995, 22, 815-835. [CrossRef]

29. Wei, S.H.; Yi, C.X.; Fang, W.; Hendrey, G. A global study of GPP focusing on light-use efficiency in a random forest regression model. Ecosphere 2017, 8, e01724. [CrossRef]

30. Potter, C.S.; Randers, J.T.; Field, C.B.; Matson, P.A.; Vitousek, P.M.; Mooney, H.A.; Klooster, S.A. Terrestrial ecosystem production: A process model based on global satellite and surface data. Glob. Biogeochem. Cycles 1993, 7, 811-841. [CrossRef]

31. Field, C.B.; Randerson, J.T.; Malmström, C.M. Global net primary production: Combining ecology and remote sensing. Remote Sens. Environ. 1995, 51, 74-88. [CrossRef]

32. Yuan, W.P.; Liu, S.G.; Zhou, G.S.; Zhou, G.Y.; Tieszen, L.L.; Baldocchi, D.; Bernhofer, C.; Gholz, H.; Goldstein, A.H.; Goulden, M.L.; et al. Deriving a light use efficiency model from eddy covariance flux data for predicting daily gross primary production across biomes. Agric. For. Meteorol. 2007, 143, 189-207. [CrossRef]

33. Yuan, W.P.; Liu, S.G.; Yu, G.R.; Bonnefond, J.M.; Chen, J.Q.; Davis, K.; Desai, A.R.; Goldstein, A.H.; Gianelle, D.; Rossi, F.; et al. Global estimates of evapotranspiration and gross primary production based on MODIS and global meteorology data. Remote Sens. Environ. 2010, 114, 1416-1431. [CrossRef]

34. Veroustraete, F.; Sabbe, H.; Eerens, H. Estimation of carbon mass fluxes over Europe using the C-Fix model and Euroflux data. Remote Sens. Environ. 2002, 83, 376-399. [CrossRef]

35. Running, S.W.; Nemani, R.R.; Heinsch, F.A.; Zhao, M.S.; Reeves, M.; Hashimoto, H. A continuous satellite-derived measure of global terrestrial primary production. Bioscience 2004, 54, 547-560. [CrossRef]

36. Xiao, X.M.; Zhang, Q.Y.; Braswell, B.; Urbanski, S.; Boles, S.; Wofsy, S.; Moore, B., III; Ojima, D. Modeling gross primary production of temperate deciduous broadleaf forest using satellite images and climate data. Remote Sens. Environ. 2004, 91, 256-270. [CrossRef]

37. Turner, D.P.; Ritts, W.D.; Styles, J.M.; Yang, Z.; Cohen, W.B.; Law, B.E.; Thornton, P.E. A diagnostic carbon flux model to monitor the effects of disturbance and interannual variation in climate on regional NEP. Tellus $B$ 2006, 58, 476-490. [CrossRef]

38. King, D.A.; Turner, D.P.; Ritts, W.D. Parameterization of a diagnostic carbon cycle model for continental scale application. Remote Sens. Environ. 2011, 115, 1653-1664. [CrossRef]

39. Yao, Y.T.; Wang, X.H.; Li, Y.; Wang, T.; Shen, M.G.; Du, M.Y.; He, H.L.; Li, Y.; Luo, W.; Ma, M.; et al. Spatiotemporal pattern of gross primary productivity and its covariation with climate in China over the last thirty years. Glob. Change Biol. 2017, 24, 184-196. [CrossRef] [PubMed]

40. Zscheischler, J.; Mahecha, M.D.; Buttlar, J.; Harmeling, S.; Jung, M.; Rammig, A.; Randerson, J.T.; Schölkopf, B.; Seneviratne, S.; Tomelleri, E.; et al. A few extreme events dominate global interannual variability in gross primary production. Environ. Res. Lett. 2014, 9, 035001. [CrossRef]

41. Churkina, G.; Running, S.W. Contrasting climatic controls on the estimated productivity of global terrestrial biomes. Ecosystems 1998, 1, 206-215. [CrossRef]

42. Piao, S.L.; Ciais, P.; Friedlingstein, P.; Noblet-Ducoudré, N.; Cadule, P.; Viovy, N.; Wang, T. Spatiotemporal patterns of terrestrial carbon cycle during the 20th century. Glob. Biogeochem. Cycles 2009, 23, GB4026. [CrossRef]

43. Goulden, M.L.; Munger, J.W.; Fan, S.M.; Daube, B.C.; Wofsy, S.C. Exchange of carbon dioxide by a deciduous forest: Response to interannual climate variability. Science 1996, 271, 1576-1578. [CrossRef]

44. Fang, J.Y.; Chen, A.P. Dynamic forest biomass carbon pools in China and their significance. Acta Bot. Sin. 2001, 43, 967-973.

45. Zhao, H.Y.; Gong, L.J.; Qu, H.H.; Zhu, H.X.; Li, X.F.; Zhao, F. The climate change variations in the northern Greater Khingan Mountains during the past centuries. J. Geogr. Sci. 2016, 26, 585-602. [CrossRef] 
46. Cai, H.Y.; Zhang, S.W.; Yang, X.H. Forest Dynamics and Their Phenological Response to Climate Warming in the Khingan Mountains, Northeastern China. Int. J. Environ. Res. Pub. Health 2012, 9, 3943-3953. [CrossRef] [PubMed]

47. Editorial Board of Vegetation Map of China. Chinese Academy of Science. In Vegetation Atlas of China (1:1,000,000); Geological Publishing House: Beijing, China, 2007.

48. Global Land Surface Satellite (GLASS) Products Download and Service. Available online: http://glassproduct.bnu.edu.cn/ (accessed on 1 December 2016).

49. Savitzky, A.; Golay, M. Smoothing and differentiation of data by simplified least squares procedures. Anal. Chem. 1964, 36, 1627-1638. [CrossRef]

50. Eklundh, L.; Jonsson, P. TIMESAT 3.0 Software Manual; Malmö University: Malmö, Sweden, 2010.

51. Chinese Meteorological Data Service Center (CMDC). Available online: http://data.cma.cn/ (accessed on 1 October 2016).

52. NASA Ames Ecological Forecasting Lab. Available online: https://ecocast.arc.nasa.gov/data/pub/gimms / 3g.v1/ (accessed on 20 December 2016).

53. You, X.Z.; Meng, J.H.; Zhang, M.; Dong, T.F. Remote Sensing Based Detection of Crop Phenology for Agricultural Zones in China Using a New Threshold Method. Remote Sens. 2013, 5, 3190-3211. [CrossRef]

54. Zhao, J.J.; Wang, Y.Y.; Zhang, Z.X.; Zhang, H.Y.; Hong, Y.; Guo, X.Y.; Yu, S.; Du, W.L.; Huang, F. The Variations of Land Surface Phenology in Northeast China and Its Responses to Climate Change from 1982 to 2013. Remote Sens. 2016, 8, 400. [CrossRef]

55. Xiao, Z.Q.; Liang, S.L.; Sun, R.; Wang, J.D.; Jiang, B. Estimating the fraction of absorbed photosynthetically active radiation from the MODIS data based GLASS leaf area index product. Remote Sens. Environ. 2015, 171, 105-117. [CrossRef]

56. Huang, K.; Zhang, Y.J.; Zhu, J.T.; Liu, Y.J.; Zu, J.X.; Zhang, J. The Influences of Climate Change and Human Activities on Vegetation Dynamics in the Qinghai-Tibet Plateau. Remote Sens. 2016, 8, 876. [CrossRef]

57. Weber, U.; Jung, M.; Reichstein, M.; Beer, C.; Braakhekke, M.C.; Lehsten, V.; Ghent, D.; Kaduk, J.; Viovy, N.; Ciais, P.; et al. The interannual variability of Africa's ecosystem productivity: A multi-model analysis. Biogeosciences 2009, 6, 285-295. [CrossRef]

58. Peng, S.S.; Piao, S.L.; Ciais, P.; Myneni, R.B.; Chen, A.; Chevallier, F.; Dolman, A.J.; Janssens, I.A.; Peñuelas, J.; Zhang, G.X.; et al. Asymmetric effects of daytime and night-time warming on Northern Hemisphere vegetation. Nature 2013, 501, 88-92. [CrossRef] [PubMed]

59. Zhao, M.; Running, S.W. Drought-induced reduction in global terrestrial net primary production from 2000 through 2009. Science 2010, 329, 940-943. [CrossRef] [PubMed]

60. Davis, C.L.; Hoffman, M.T.; Roberts, W. Long-term trends in vegetation phenology and productivity over Namaqualand using the GIMMS AVHRR NDVI3g data from 1982 to 2011. S. Afr. J. Bot. 2017, 111, 76-85. [CrossRef]

61. Tang, H.; Li, Z.W.; Zhu, Z.L.; Chen, B.; Zhang, B.H.; Xin, X.P. Variability and Climate Change Trend in Vegetation Phenology of Recent Decades in the Greater Khingan Mountain Area, Northeastern China. Remote Sens. 2015, 7, 11914-11932. [CrossRef]

62. Piao, S.L.; Friedlingstein, P.; Ciais, P.; Viovy, N.; Demarty, J. Growing season extension and its impact on terrestrial carbon cycle in the Northern Hemisphere over the past 2 decades. Global Biogeochem. Cycles 2007, 21, GB3018. [CrossRef]

63. Murray-Tortarolo, G.; Anav, A.; Friedlingstein, P.; Sitch, S.; Piao, S.L.; Zhu, Z.; Poulter, B.; Zaehle, S.; Ahlström, A.; Lomas, M.; et al. Evaluation of land surface models in reproducing satellite-derived LAI over the high-latitude Northern Hemisphere. Part I: Uncoupled DGVMs. Remote Sens. 2013, 5, 4819-4838. [CrossRef]

64. Nilsson, M.C.; Wardle, D.A. Understory vegetation as a forest ecosystem driver: Evidence from the northern Swedish boreal forest. Front Ecol. Environ. 2005, 3, 421-428. [CrossRef]

65. Liu, Y.; Liu, R.G.; Pisek, J.; Chen, J.M. Separating overstory and understory leaf area indices for global needleleaf and deciduous broadleaf forests by fusion of MODIS and MISR data. Biogeosciences 2017, 14, 1093-1110. [CrossRef]

66. Liu, Z.; Yang, J. Quantifying ecological drivers of ecosystem productivity of the early successional boreal Larix gmelinii forest. Ecosphere 2014, 5, 1-16. [CrossRef] 
67. Myneni, R.B.; Keeling, C.; Tucker, C.; Asrar, G.; Nemani, R. Increased plant growth in the northern high latitudes from 1981 to 1991. Nature 1997, 386, 698-702. [CrossRef]

68. Zhang, Y.P.; Hu, H.Q. Climatic Change and Its Impact on Forest Fire in Daxing' anling Mountains. J. North-East For. Univ. 2008, 36, 29-31.

69. Gao, Y.G.; Zhao, H.Y.; Gao, F.; Zhu, H.X.; Qu, H.H.; Zhao, F. Climate change trend in future and its influence on wetlands in the Greater Khingan Mountains. J. Glaciol. Geocryol. 2016, 38, 47-56.

70. Han, X.C.; Zhao, Y.S.; Xin, Y. Succession process of Larix gmelinii forest with artificial restoration after fire in Daxing' anling. Sci. Soil Water Conserv. 2015, 13, 70-76.

71. Rull, V. A palynological record of a secondary succession after fire in the Gran Sabana, Venezuela. J. Quat. Sci. 1999, 14, 137-152. [CrossRef]

72. Wang, C.K.; Gower, S.T.; Wang, Y.H.; Zhao, H.X.; Yan, P.; Bond-Lamberty, B.P. The influence of fire on carbon distribution and net primary production of boreal Larix gmelinii forests in north-eastern China. Glob. Chang. Biol. 2001, 7, 719-730. [CrossRef]

73. Wang, X.G.; Li, X.Z.; He, H.S.; Leng, W.F.; Wen, Q.C. Postfire succession of larch forest on the northern slope of Daxinganling. Chin. J. Ecol. 2004, 23, 35-41.

74. Artz, R.; Ball, G.; Behrens, K.; Bonnin, G.M.; Bower, C.A.; Canterford, R.; Childs, B.; Claude, H.; Crum, T.; Dombrowsky, R.; et al. Measurement of Sunshine Duration. In Guide to Meteorological Instruments and Methods of Observation, 7th ed.; 7 bis, avenue de la Paix P.O. Box No. 2300 CH-1211 Geneva 2; WMO: Geneva, Switzerland, 2008; Volume 8, p. 199. ISBN 978-92-63-10008-5.

75. Wikipedia. Available online: https://en.wikipedia.org/wiki/Sunshine_duration (accessed on 1 May 2017).

76. Piao, S.L.; Nan, H.J.; Huntingford, C.; Ciais, P.; Friedlingstein, P.; Sitch, S.; Peng, S.S.; Ahlström, A.; Canadell, J.G.; Cong, N.; et al. Evidence for a weakening relationship between inter-annual temperature variability and northern vegetation activity. Nat. Commun. 2014, 5, 5018. [CrossRef] [PubMed]

77. Peng, D.L.; Zhang, B.; Liu, L.Y.; Fang, H.L.; Chen, D.M.; Hu, Y.; Liu, L.L. Characteristics and drivers of global NDVI-based FPAR from 1982 to 2006. Global Biogeochem. Cycles 2012, 26, GB3015. [CrossRef]

78. Karami, M.; Hansen, B.U.; Nielsen, A.W.; Abermann, J.; Lund, M.; Schmidt, N.M.; Elberling, B. Vegetation phenology gradients along the west and east coasts of Greenland from 2001 to 2015. Ambio 2017, 46 (Suppl. 1), S94-S105. [CrossRef] [PubMed]

79. Qiu, B.W.; Zeng, C.Y.; Tang, Z.H.; Chen, C.C. Characterizing spatiotemporal non-stationarity in vegetation dynamics in China using MODIS EVI dataset. Environ. Monit. Assess. 2013, 185, 9019-9035. [CrossRef] [PubMed]

80. Hou, X.H.; Niu, Z.; Gao, S. Phenology of Forest Vegetation in Northeast of China in Ten Years Using Remote Sensing. Spectrosc. Spectr. Anal. 2013, 34, 515-519.

(C) 2018 by the authors. Licensee MDPI, Basel, Switzerland. This article is an open access article distributed under the terms and conditions of the Creative Commons Attribution (CC BY) license (http:/ / creativecommons.org/licenses/by/4.0/). 
Article

\title{
Spatial Downscaling of Gross Primary Productivity Using Topographic and Vegetation Heterogeneity Information: A Case Study in the Gongga Mountain Region of China
}

\author{
Xinyao Xie ${ }^{1,2}$, Ainong $\mathrm{Li}^{1, *}$, Huaan Jin ${ }^{1}$, Gaofei Yin ${ }^{1}$ and Jinhu Bian ${ }^{1}$ \\ 1 Research Center for Digital Mountain and Remote Sensing Application, Institute of Mountain Hazards and \\ Environment, Chinese Academy of Sciences, Chengdu 610041, China; xiexinyao15@mails.ucas.ac.cn (X.X.); \\ jinhuaan@imde.ac.cn (H.J.); gaofeiyin@imde.ac.cn (G.Y.); bianjinhu@imde.ac.cn (J.B.) \\ 2 University of Chinese Academy of Sciences, Beijing 100049, China \\ * Correspondence: ainongli@imde.ac.cn
}

Received: 14 March 2018; Accepted: 20 April 2018; Published: 22 April 2018

\begin{abstract}
Due to the spatial heterogeneity of land surfaces, downscaling is an important issue in the development of carbon cycle models when evaluating the role of ecosystems in the global carbon cycle. In this study, a downscaling algorithm was developed to model gross primary productivity (GPP) at $500 \mathrm{~m}$ in a time series over rugged terrain, which considered the effects of spatial heterogeneity on carbon flux simulations. This work was carried out for a mountainous area with an altitude ranging from 2606 to $4744 \mathrm{~m}$ over the Gongga Mountain (Sichuan Province, China). In addition, the Moderate Resolution Imaging Spectroradiometer (MODIS) GPP product at $1 \mathrm{~km}$ served as the primary dataset for the downscaling algorithm, and the $500 \mathrm{~m}$ MODIS GPP product was used as the reference dataset to evaluate the downscaled GPP results. Moreover, in order to illustrate the advantages and benefits of the proposed downscaling method, the downscaled results in this work, along with ordinary kriging downscaled results, spline downscaled results and inverse distance weighted (IDW) downscaled results, were compared to the MODIS GPP at $500 \mathrm{~m}$. The results showed that (1) the GPP difference between the $500 \mathrm{~m}$ MODIS GPP and the proposed downscaled GPP results was primarily in the range of $[-1,1]$, showing that both vegetation heterogeneity factors (i.e., LAI) and topographic factors (i.e., altitude, slope and aspect) were useful for GPP downscaling; (2) the proposed downscaled results $\left(R^{2}=0.89\right.$, RMSE $=1.03$ ) had a stronger consistency with the $500 \mathrm{~m}$ MODIS GPP than those of the ordinary kriging downscaled results $\left(\mathrm{R}^{2}=0.43\right.$, RMSE $\left.=1.36\right)$, the spline downscaled results $\left(R^{2}=0.40\right.$, RMSE $\left.=1.50\right)$ and the IDW downscaled results $\left(R^{2}=0.42\right.$, RMSE $=1.10$ ) for all Julian days; and (3) the inconsistency between MODIS GPP at $500 \mathrm{~m}$ and $1 \mathrm{~km}$ increased with the increase in altitude and slope. The proposed downscaling algorithm could provide a reference when considering the effects of spatial heterogeneity on carbon flux simulations and retrieving other fine resolution ecological-physiology parameters (e.g., net primary productivity and evaporation) over topographically complex terrains.
\end{abstract}

Keywords: downscaling; GPP; spatial heterogeneity; remote sensing; subpixel information

\section{Introduction}

The gross primary productivity (GPP), which corresponds to carbon fixation by vegetation at the ecosystem level, is an important indicator to assess the photosynthetic capacity of vegetation and the function of the ecosystem. The accurate estimation of regional, continental and global GPP plays an important role when monitoring vegetation growth conditions [1], the terrestrial carbon budget [2] and the interactions in the soil-vegetation-atmosphere continuum [3]. 
Carbon cycle models have been developed over many years to predict carbon-climate feedbacks at multiple scale levels [4-6], which can be used to estimate spatially and temporally continuous GPP. However, most carbon cycle models are generated from the site scale, without considering the spatial heterogeneity within each modeling grid [7]. Moreover, due to the paucity of the data and computing complexities, the carbon cycle model is usually executed at coarse resolutions, which are based on the simplification of landscape complexities. Such a simplification results in large uncertainties due to spatial heterogeneity, especially in topographically complex terrains. Some researchers have shown that the oversimplification of landscape complexities may inevitably cause model simulations to be considerably biased [8-10]. Landscape complexities typically present high spatial heterogeneity, and spatial heterogeneity is scaled by multiple factors, including endogenous and exogenous factors $[11,12]$. Endogenous heterogeneity refers to the spatial variability of vegetation types and density (i.e., land cover and leaf area index), while exogenous heterogeneity is associated with surface topography (i.e., altitude, slope and aspect). Therefore, it is crucial to consider the effects of spatial heterogeneity on carbon flux simulation by models.

The carbon flux simulation through carbon cycle models usually requires massive forcing data. Atmospheric data are one of the most important inputs for carbon cycle model simulation. Theoretically, atmospheric forcing data, such as air temperature, air humidity and solar radiation, describe the external environment for the carbon cycle process and exhibit strong spatial variation characteristics over complex terrain [13-15]. Dense field observations are required to determine the spatial patterns of atmospheric forcing data, especially for rugged regions. However, due to insufficient meteorological stations located in mountainous area, the atmospheric forcing data could not be provided accurately at fine resolution [12]. To overcome the limitation of field meteorological observations for running the carbon cycle model, downscaling is a potential method to obtain high resolution carbon flux simulation in topographically complex terrains. Using the relationships between carbon flux estimation and the surrounding landscape, the disaggregation of low spatial resolution carbon flux estimation and the information from ancillary data could be introduced in the downscaling scheme.

Spatial scaling is a process of using information available at one scale to derive processes at another scale, including upscaling and downscaling. Downscaling refers to an increase in spatial scale following disaggregation of the coarse-resolution dataset and information from ancillary data at a finer resolution, which restores the variations at a finer scale by assuming the values of the coarser are the mean values at the finer scale. In the last decade, the downscaling of the carbon cycle process has been one of the most challenging issues in environmental science, especially over mountainous areas [16-18]. In the literature, some studies have been carried out to examine the effects of spatial heterogeneity on the upscaling process of carbon fluxes $[8,19]$, while little attention has been shown towards the issue of downscaling carbon fluxes over complex terrains. Therefore, it is necessary to downscale carbon fluxes using the subpixel information of topography and vegetation heterogeneity, which is an important issue when evaluating the role of ecosystems in the global carbon cycle.

Vegetation heterogeneity and surface topography are important factors that introduce biases into carbon modeling and water fluxes. Vegetation heterogeneity directly influences the photosynthetic capacity per unit of surface area. Topography affects the redistribution of water, radiation and heat, which have significant influences on the process of carbon assimilation. In this paper, the main purposes are (1) to develop a GPP downscaling algorithm using the subpixel information of topography and vegetation heterogeneity and (2) to assess the GPP inconsistency at $1 \mathrm{~km}$ and $500 \mathrm{~m}$, to analyze the relationship between spatial heterogeneity and the GPP difference at two scales.

\section{Materials and Methods}

\subsection{Study Area}

As shown in Figure 1, the experimental region is northwest of Gongga Mountain on the quaternary sections of the Tibetan plateau. The summit of Gongga Mountain is $7556 \mathrm{~m}$ above sea level, which is 
the highest mountain in the Sichuan Province of China. Mt. Gongga has a mountainous climate that ranges from a cool plateau climate to a subtropical lowland climate [20]. The annual precipitation is approximately $3000 \mathrm{~mm}$ at the summit and declines to $1300 \mathrm{~mm}$ at lower altitudes [21]. The average annual air temperature decrease with altitude, from approximately $11{ }^{\circ} \mathrm{C}$ at lower altitudes to $-1{ }^{\circ} \mathrm{C}$ at higher altitudes [21]. This experimental region, with an area of $2584 \mathrm{~km}^{2}$, has an altitude ranging from 2606 to $4744 \mathrm{~m}$ above sea level, and the dominant types of land cover are forestlands (i.e., evergreen forests and mixed forests), grasslands and shrublands.

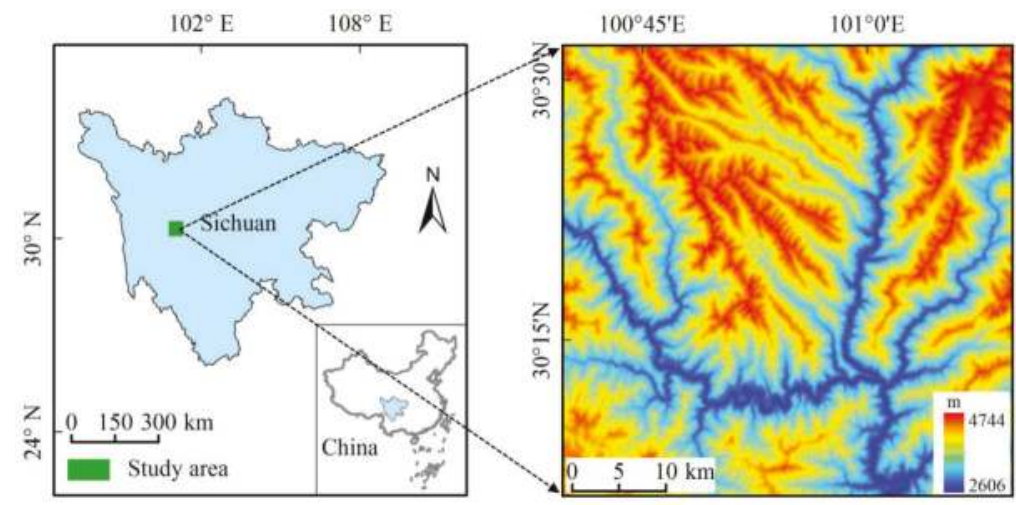

Figure 1. Study area.

\subsection{Data and Processing}

\subsubsection{GPP Images at $500 \mathrm{~m}$ and $1 \mathrm{~km}$}

The Moderate Resolution Imaging Spectroradiometer (MODIS) has produced a regular estimation of GPP since 2000, including the MOD17A2 (8-day, $1 \mathrm{~km}$ ) and MOD17A2H (8-day, $500 \mathrm{~m}$ ) products. The algorithm for the MODIS GPP $\left(\mathrm{gC} \mathrm{m}^{-2} \mathrm{day}^{-1}\right)$ is based on the concept of radiation conversion efficiency and can be described as follows:

$$
\mathrm{GPP}=\varepsilon_{\max } \times \text { TMIN_scalar } \times \text { VPD_scalar } \times \text { SWRad } \times 0.45 \times F P A R
$$

where $\varepsilon_{\max }$ represents the maximum light use efficiency $\left(\mathrm{gC} \mathrm{MJ}^{-1}\right)$ related to the plant functional type (PFT), which can be obtained from the Biome Properties Look-Up Table (BPLUT); TMIN_scalar and $V P D \_s c a l a r$ are the scalars for minimum air temperature and vapor pressure deficits (VPD), respectively, ranging from 0 to 1 ; SWRad represents the incoming short wave radiation $\left(\mathrm{MJ} \mathrm{m}^{-2} \mathrm{day}^{-1}\right)$, and FPAR represents the fraction of absorbed photosynthetic active radiation, which can be obtained from MODIS product. The MODIS GPP were scaled by multiple environmental factors, and each environmental factor has a comparable level of influence on the final simulated GPP. More details regarding the MODIS GPP algorithm can be found in the literature [22,23]. In addition, some research has been done to evaluate MODIS GPP products across multiple biomes, suggesting that the MODIS GPP works effectively for the majority of PFTs [23-25].

The MODIS GPP products MOD17A2 (version 5.0) and MOD17A2H (version 6.0) used in our study were obtained from Julian day 169, 2010 to Julian day 209, 2010, which can be freely acquired at National Aeronautics and Space Administration Distributed Active Archive Center (http://www.modis.ornl.gov/modis/index.cfm). Using spatial heterogeneity information within each $1 \mathrm{~km}$ modeling grid, our study was designed to estimate downscaled GPP from a $1 \mathrm{~km}$ resolution to a $500 \mathrm{~m}$ resolution. Because the $1 \mathrm{~km}$ GPP product is executed at a coarse resolution without considering the spatial heterogeneity within each $1 \mathrm{~km}$ modeling grid, the GPP product at $1 \mathrm{~km}$ served as the 
primary dataset for the downscaling algorithm in this work. The $500 \mathrm{~m}$ GPP product was used as a reference dataset to evaluate the downscaled GPP results at $500 \mathrm{~m}$.

\subsubsection{LAI Data}

The MODIS LAI products MCD15A2 (version 5.0) at $1 \mathrm{~km}$ and MCD15A2H (version 6.0) at $500 \mathrm{~m}$, were selected to describe the vegetation density in this study. The 8-day MODIS LAI product is available at http: / / wist.echo.nasa.gov in the sinusoidal projection. According to the quality control layer (FparLai-QC) involved in this product, the MODIS LAI consists of a main look-up-table (LUT) algorithm $(\mathrm{QC}<64)$ and a back-up algorithm $(64 \leq \mathrm{QC}<128)$. The LUT algorithm exploits surface reflectance information in the red and near-infrared bands based on a 3-D radiative transfer equation. When the LUT algorithm fails, the back-up algorithm adopts NDVI-LAI empirical relationships to estimate the LAI [26]. The LUT algorithm usually has a higher reliability than that of the back-up algorithm [26]; the clumping effects at the canopy scale are considered in the LUT algorithm [27].

\subsubsection{DEM Data}

Some researches have illustrated that the Advanced Spaceborne Thermal Emission and Reflection Radiometer (ASTER) global digital elevation model (GDEM) data have a good elevation accuracy over mountainous area [28-30], ASTER GDEM were adopted to describe the complex terrain in this work. GDEM data available at a $30 \mathrm{~m}$ were averaged to obtain the values at $500 \mathrm{~m}$ and $1 \mathrm{~km}$ resolutions [7]. The surface slope and aspect were derived from the GDEM data, according to a fast algorithm based on a $3 \times 3$ pixels window [31].

\subsection{Algorithm for Downscaling}

As shown in Figure 2, the proposed downscaling algorithm contains two phases: the regression process and area-to-point kriging (ATPK) process. It firstly conducts regression analysis between GPP and heterogeneity factors (i.e., altitude, slope, aspect and LAI), and then ATPK is performed on the downscaling residuals from the regression process.

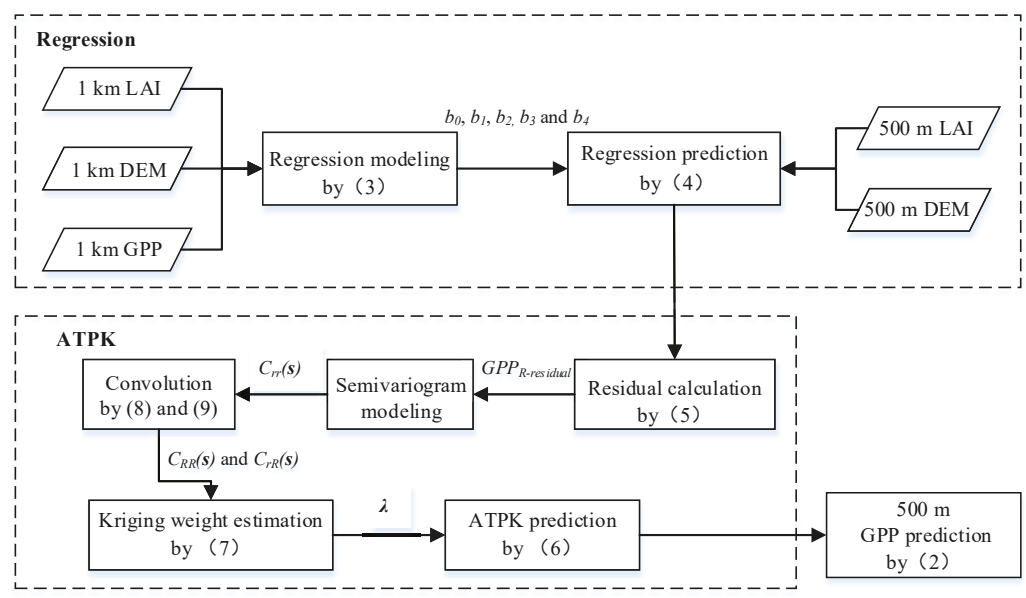

Figure 2. Flow chart of the proposed downscaling algorithm.

\subsubsection{Problem Formulation}

Let $G P P_{R}\left(x_{j}\right)$ represent the random variable of pixel $R$ centered at $x_{j}(\mathrm{j}=1,2, \ldots, \mathrm{N}$, where $\mathrm{N}$ is the number of pixels) in $1 \mathrm{~km}$ resolution image of GPP, whereas GPP $P_{r}\left(x_{i}\right)$ represents the random variable of pixel $r$ centered at $x_{i}(i=1,2, \ldots, \mathrm{M}$, where $\mathrm{M}$ is the number of pixels) in the $500 \mathrm{~m}$ resolution 
image of GPP. The notations $R$ and $r$ represent the $1 \mathrm{~km}$ and $500 \mathrm{~m}$ pixels, respectively. The purpose of the downscaling method based on topographic factors and the LAI is to predict $G P P_{r}(\mathbf{x})$ at a $500 \mathrm{~m}$ resolution. $G P P_{r}(\mathbf{x})$ can be described as

$$
G P P_{r}(\mathbf{x})=G P P_{r 1}(\mathbf{x})+G P P_{r 2}(\mathbf{x})
$$

where $G P P_{r 1}(\mathbf{x})$ and $G P P_{r 2}(\mathbf{x})$ represent the predictions of the regression and ATPK processes, respectively. More descriptions about their calculation can be found in the following sections.

\subsubsection{Regression between GPP and Topographic Factors}

The regression takes advantage of valuable spatial heterogeneity information from the ancillary data at $500 \mathrm{~m}$ spatial resolution. The relationship between topographic factors and MODIS GPP at $1 \mathrm{~km}$ is modeled by multiple linear regression:

$$
G P P_{R_{\_} o b s}(\mathbf{x})=b_{0}+b_{1} H_{R}+b_{2} \operatorname{COS}\left(S_{R}\right)+b_{3} \operatorname{COS}\left(A_{R}\right)+b_{4} L A I_{R}
$$

where GPPR_obs $(\mathbf{x}), H_{R}, S_{R}, A_{R}$ and $L A I_{R}$ represent the MODIS GPP $\left(\mathrm{gC} \mathrm{d}^{-1}\right)$, altitude (m), slope (deg), aspect (deg) and LAI $\left(\mathrm{m}^{2} \mathrm{~m}^{-2}\right)$ at a $1 \mathrm{~km}$ resolution, respectively. Since GPPR_obs $(\mathbf{x}), H_{R}, S_{R}, A_{R}$ and $\mathrm{LAI}_{\mathrm{R}}$ in Equation (3) are known, $b_{0}, b_{1}, b_{2}, b_{3}$ and $b_{4}$ can be estimated. Currently, a wide range of fitting methods has been applied in the theoretical framework of regression, such as generalized least squares (GLS) and ordinary least squares (OLS). In this paper, the OLS algorithm was adopted to estimate the regression coefficients.

The relationship in Equation (3) is assumed to be universal at different resolutions [32,33], and thus the relationship modeled at a $1 \mathrm{~km}$ resolution can be implemented at a $500 \mathrm{~m}$ resolution. Based on this assumption, the regression prediction $\operatorname{GPP}_{r 1}(\mathbf{x})$ is calculated as

$$
G P P_{r 1}(\mathbf{x})=b_{0}+b_{1} H_{r}+b_{2} \operatorname{COS}\left(S_{r}\right)+b_{3} \operatorname{COS}\left(A_{r}\right)+b_{4} L A I_{r}
$$

where $H_{r}, S_{r}, A_{r}$ and $L A I_{r}$ represent the altitude (m), slope (deg), aspect (deg) and LAI $\left(\mathrm{m}^{2} \mathrm{~m}^{-2}\right)$ at a $500 \mathrm{~m}$ resolution, respectively. Variables $b_{0}, b_{1}, b_{2}, b_{3}$ and $b_{4}$ are regression coefficients.

\subsubsection{ATPK for Downscaling Residuals}

If the result of the first phase is perfect, there should be no bias between it and the original GPP at a $1 \mathrm{~km}$ resolution. However, it is unrealistic to obtain such an ideal result, and there are inevitable residuals from the first process. The residuals at a $1 \mathrm{~km}$ resolution are calculated as:

$$
G P P_{R_{-} \text {residual }}(\mathbf{x})=G P P_{R_{-} o b s}(\mathbf{x})-\left(b_{0}+b_{1} H_{R}+b_{2} \operatorname{COS}\left(S_{R}\right)+b_{3} \operatorname{COS}\left(A_{R}\right)+b_{4} L A I_{R}\right)
$$

In this work, the residuals at a $1 \mathrm{~km}$ resolution were considered for downscaling, and the ATPK process served as the second phase when downscaling the residuals, $G P P_{R_{-} \text {residual }}(\mathbf{x})$, at $1 \mathrm{~km}$ to those at $500 \mathrm{~m}, G P P_{r_{r} \text { residual }}(\mathbf{x})$. In the ATPK approach, $G P P_{r 2}(\mathbf{x})$ represents a linear combination of residuals from $\mathrm{N} 1 \mathrm{~km}$ pixel, which can be described as:

$$
G P P_{\mathrm{r} 2}(\mathbf{x})=\sum_{\mathrm{i}=1}^{\mathrm{N}} \lambda_{\mathrm{i}} G P P_{\mathrm{R}_{\_} \text {residual }}\left(\mathbf{x}_{\mathbf{i}}\right), \text { s.t. } \quad \sum_{\mathrm{i}}^{\mathrm{N}} \lambda_{\mathrm{i}}=1
$$

where $\lambda_{i}$ represents the weight for the residual of the $i$ th $1 \mathrm{~km}$ pixel, which is centered at $\mathbf{x}_{\mathbf{i}}$. As shown in Equation (6), the ATPK phase takes the spatial correlation among pixels into account, which is not considered in the above equations. 
The main issue of the ATPK phase is obtaining the weights, $\lambda$, which are estimated by minimizing prediction error spatial variations at a $1 \mathrm{~km}$ resolution. The kriging system can be expressed as:

$$
\left[\begin{array}{cccc}
\mathrm{C}_{\mathrm{RR}}\left(x_{1}, x_{1}\right) & \ldots & \mathrm{C}_{\mathrm{RR}}\left(x_{1}, x_{N}\right) & 1 \\
\ldots & \ldots & \ldots & \ldots \\
\ldots & \ldots & \ldots & \ldots \\
\ldots & \ldots & \ldots & \ldots \\
\mathrm{C}_{\mathrm{RR}}\left(x_{N}, x_{1}\right) & \ldots & \mathrm{C}_{\mathrm{RR}}\left(x_{N}, x_{N}\right) & 1 \\
1 & \ldots & 1 & 0
\end{array}\right]\left[\begin{array}{c}
\lambda_{1} \\
\ldots \\
\ldots \\
\ldots \\
\lambda_{N} \\
\mu
\end{array}\right]=\left[\begin{array}{c}
\mathrm{C}_{r R}\left(x, x_{1}\right) \\
\ldots \\
\ldots \\
\ldots \\
\mathrm{C}_{r R}\left(x, x_{N}\right) \\
1
\end{array}\right]
$$

where $C_{R R}\left(x_{i}, x_{j}\right)$ is the residual covariance between a $1 \mathrm{~km}$ pixel centered at $x_{i}$ and a $1 \mathrm{~km}$ pixel centered at $x_{j}$, and $C_{r R}\left(x, x_{j}\right)$ is the residual covariance between a $500 \mathrm{~m}$ pixel centered at $x$ and a $1 \mathrm{~km}$ pixel centered at $x_{j}$. Variable $\mu$ represents the range multiplier. To estimate weight $\lambda$ according to Equation(7), these two types of residual covariance need to be calculated in advance.

Assuming each pixel as a point, $s$ is intended to represent the Euclidean distance between two pixels. The $1 \mathrm{~km}$ to $1 \mathrm{~km}$ residual covariance, $C_{R R}(s)$, and the $500 \mathrm{~m}$ to $1 \mathrm{~km}$ residual covariance, $C_{r R}(s)$, can be estimated as:

$$
\begin{gathered}
\mathrm{C}_{R R}(s)=\mathrm{C}_{r r}(s) * h_{R}(s) * h_{R}(-s) \\
\mathrm{C}_{r R}(s)=\mathrm{C}_{r r}(s) * h_{R}(s)
\end{gathered}
$$

where $C_{r r}(s)$ represents the $500 \mathrm{~m}$ to $500 \mathrm{~m}$ residual covariance, and $\mathrm{h}_{\mathrm{R}}(*)$ represents the point spread function (PSF). Variable $-s$ indicates that the distance from a $500 \mathrm{~m}$ pixel (A) to another $500 \mathrm{~m}$ pixel (B) within a $1 \mathrm{~km}$ pixel is opposite to the distance from a $500 \mathrm{~m}$ pixel (B) to a $500 \mathrm{~m}$ pixel (A) (i.e., s).

Supposing the value of the coarse pixel is the mean of the $500 \mathrm{~m}$ pixels within it, and $\mathrm{F}$ is the ratio between the resolutions of $1 \mathrm{~km}$ pixel and $500 \mathrm{~m}$ pixel, then, PSF can be described as:

$$
h_{R}(x)=\left\{\begin{array}{cl}
\frac{1}{F^{2}} & x \in R(x) \\
0 & \text { otherwise }
\end{array}\right.
$$

Given Equations (8)-(10), the calculation of $C_{R R}\left(x_{i}, x_{j}\right)$ and $C_{r R}\left(x, x_{j}\right)$ can be simplified as:

$$
\begin{gathered}
\mathrm{C}_{R R}\left(x_{i}, x_{j}\right)=\frac{1}{F^{4}} \sum_{m=1}^{F^{2}} \sum_{m \prime}^{F^{2}} C_{r r}\left(s_{m m \prime}\right) \\
\mathrm{C}_{r R}\left(x, x_{j}\right)=\frac{1}{F^{2}} \sum_{m=1}^{F^{2}} C_{r r}\left(s_{m}\right)
\end{gathered}
$$

where $S_{\mathrm{mm}}$ ' is the distance between each pair of $500 \mathrm{~m}$ pixels from within two $1 \mathrm{~km}$ pixels centered at $x_{i}$ and at $x_{j}$, respectively, and $s_{m}$ is the distance between $500 \mathrm{~m}$ pixels centered at $x$ and any $500 \mathrm{~m}$ pixels within the $1 \mathrm{~km}$ pixel centered at $x_{j}$. Therefore, the critical issue when estimating the weight is the estimation of the $500 \mathrm{~m}$ to $500 \mathrm{~m}$ residual covariance, $C_{r r}(s)$, which is essentially semivariogram modeling [32,34].

\subsection{Result Validation and Method Evaluation}

In this study, the performance of the proposed downscaling method was evaluated by MODIS GPP at a $500 \mathrm{~m}$ resolution. The coefficient of determination $\left(\mathrm{R}^{2}\right)$ and the root mean square error (RMSE) between the downscaled results and the $500 \mathrm{~m}$ MODIS GPP were adopted as indicators of validation. In addition to the proposed downscaling approach, other three downscaling methods, including ordinary kriging (OK) [35], spline [36] and inverse distance weighting (IDW) [37], were used to provide a systematic comparison and illustrate the advantages of the proposed downscaling method. 


\section{Results and Analyses}

\subsection{Topographic and Vegetation Heterogeneities at Two Scales}

Figure 3 shows the spatial distributions of topographic factors and the LAI on Julian day 193 at $500 \mathrm{~m}$ and $1 \mathrm{~km}$ resolutions. Although the values of these variables at two resolutions presented similarity on the whole distributions, the spatial variations at a $500 \mathrm{~m}$ resolution are more pronounced than those at a $1 \mathrm{~km}$ resolution.

(a) Altitude
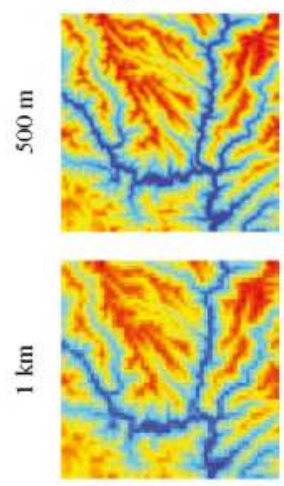

$\mathrm{m}$ (b) Slope
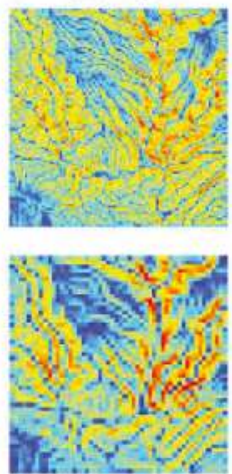

$\operatorname{deg}$ (c) Aspect
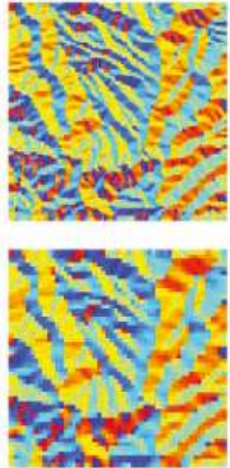

$\operatorname{deg}$

2600
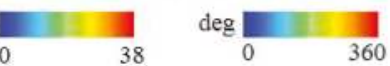

(d) LAI
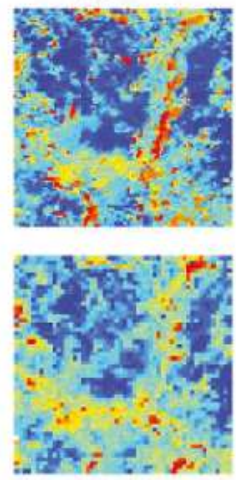

$\mathrm{m}^{2} \mathrm{~m}^{-2}$

Figure 3. Spatial distributions of altitude (a); slope (b); aspect (c) and LAI (d) at $500 \mathrm{~m}$ and $1 \mathrm{~km}$.

The statistics of the topographic factors and the LAI on Julian day 193 at $500 \mathrm{~m}$ and $1 \mathrm{~km}$ are listed in Table 1. The altitude at a $500 \mathrm{~m}$ resolution varied between a minimum of $2606 \mathrm{~m}$ and a maximum of $4744 \mathrm{~m}$; at a $1 \mathrm{~km}$ resolution, the altitude varied between a minimum of $2647 \mathrm{~m}$ and a maximum of $4671 \mathrm{~m}$. The mean altitudes at the two resolutions are similar, while the range and deviation in altitude at $500 \mathrm{~m}$ is larger than that at a $1 \mathrm{~km}$ resolution. The slope had a maximum of $38^{\circ}$ at a $500 \mathrm{~m}$ resolution, which varied from the $28^{\circ}$ maximum at a $1 \mathrm{~km}$ resolution. The mean slope value at $500 \mathrm{~m}\left(18^{\circ}\right)$ was greater than that at a $1 \mathrm{~km}$ resolution $\left(12^{\circ}\right)$, showing that there was a considerable loss of topographic information at a coarse resolution. The statistical results also show that the LAI on Julian day 193 at $1 \mathrm{~km}$ had a smaller deviation than that at $500 \mathrm{~m}$, and the mean value of the LAI at $500 \mathrm{~m}\left(7.0 \mathrm{~m}^{2} \mathrm{~m}^{-2}\right)$ was greater than that at a $1 \mathrm{~km}$ resolution $\left(5.7 \mathrm{~m}^{2} \mathrm{~m}^{-2}\right)$. In addition, the aspect at $500 \mathrm{~m}$ had a similar range, mean value and deviation to those at $1 \mathrm{~km}$.

Table 1. Comparisons of topographic factors and at $500 \mathrm{~m}$ and $1 \mathrm{~km}$.

\begin{tabular}{ccccccccc}
\hline \multirow{2}{*}{$\begin{array}{c}\text { Pixel } \\
\text { Resolution (m) }\end{array}$} & \multicolumn{2}{c}{ Min } & \multicolumn{2}{c}{ Max } & \multicolumn{2}{c}{ Mean } & \multicolumn{2}{c}{ STD } \\
\cline { 2 - 9 } & $\mathbf{5 0 0}$ & $\mathbf{1 0 0 0}$ & $\mathbf{5 0 0}$ & $\mathbf{1 0 0 0}$ & $\mathbf{5 0 0}$ & $\mathbf{1 0 0 0}$ & $\mathbf{5 0 0}$ & $\mathbf{1 0 0 0}$ \\
\hline Altitude (m) & 2606 & 2647 & 4744 & 4671 & 3784 & 3787 & 501 & 489 \\
Slope (deg) & 0 & 0 & 38 & 28 & 18 & 12 & 8 & 6 \\
Aspect (deg) & 0 & 0 & 360 & 360 & 178 & 177 & 101 & 100 \\
LAI $\left(\mathrm{m}^{2} \mathrm{~m}^{-2}\right)$ & 0 & 0 & 7.0 & 5.7 & 1.88 & 1.58 & 1.93 & 1.61 \\
\hline
\end{tabular}

\subsection{GPP Difference of the Two MODIS Products}

To assess the GPP inconsistency at $1 \mathrm{~km}$ and $500 \mathrm{~m}$, a pixel-by-pixel comparison between the MODIS GPP at two resolutions was implemented with a time interval of 8 days during Julian days 
169-209. Figure 4a presents the relationships between MODIS products at $500 \mathrm{~m}$ and $1 \mathrm{~km}$ during the study period. From Julian day 169 to Julian day 209, the $1 \mathrm{~km}$ MODIS GPP explained between $24 \%$ and $47 \%$ of MODIS GPP at $500 \mathrm{~m}$, with RMSEs varying between 1.34 and $2.14 \mathrm{gC} \mathrm{m}^{-2} \mathrm{~d}^{-1}$. In general, the MODIS GPP at $1 \mathrm{~km}$ had a significant inconsistency with MODIS GPP at $500 \mathrm{~m}$ $\left(\mathrm{R}^{2}=0.36, \mathrm{RMSE}=1.62\right)$ during the whole study period, showing that the simplification of complex terrain within each modeling pixel might cause the model results to be considerably biased.

$\begin{array}{ll}\text { (a) } 1 \mathrm{~km} \text { MODIS GPP } & \text { (b) The proposed method }\end{array}$
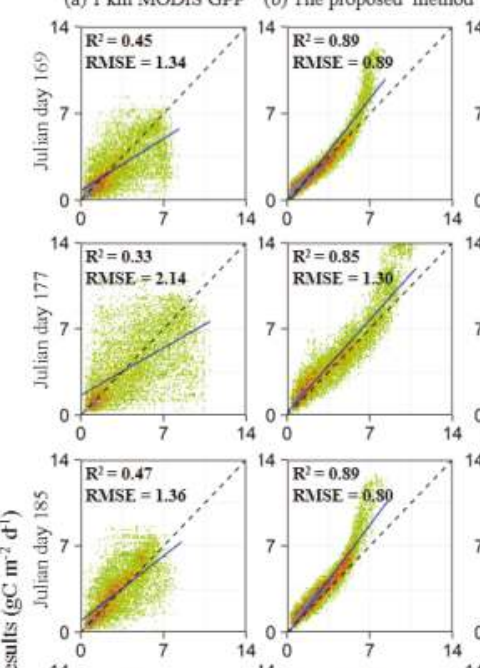

40

(c) Kriging

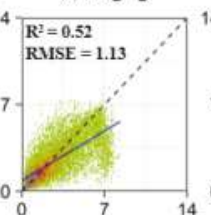

(d) Spline

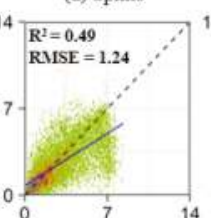

(e) IDW
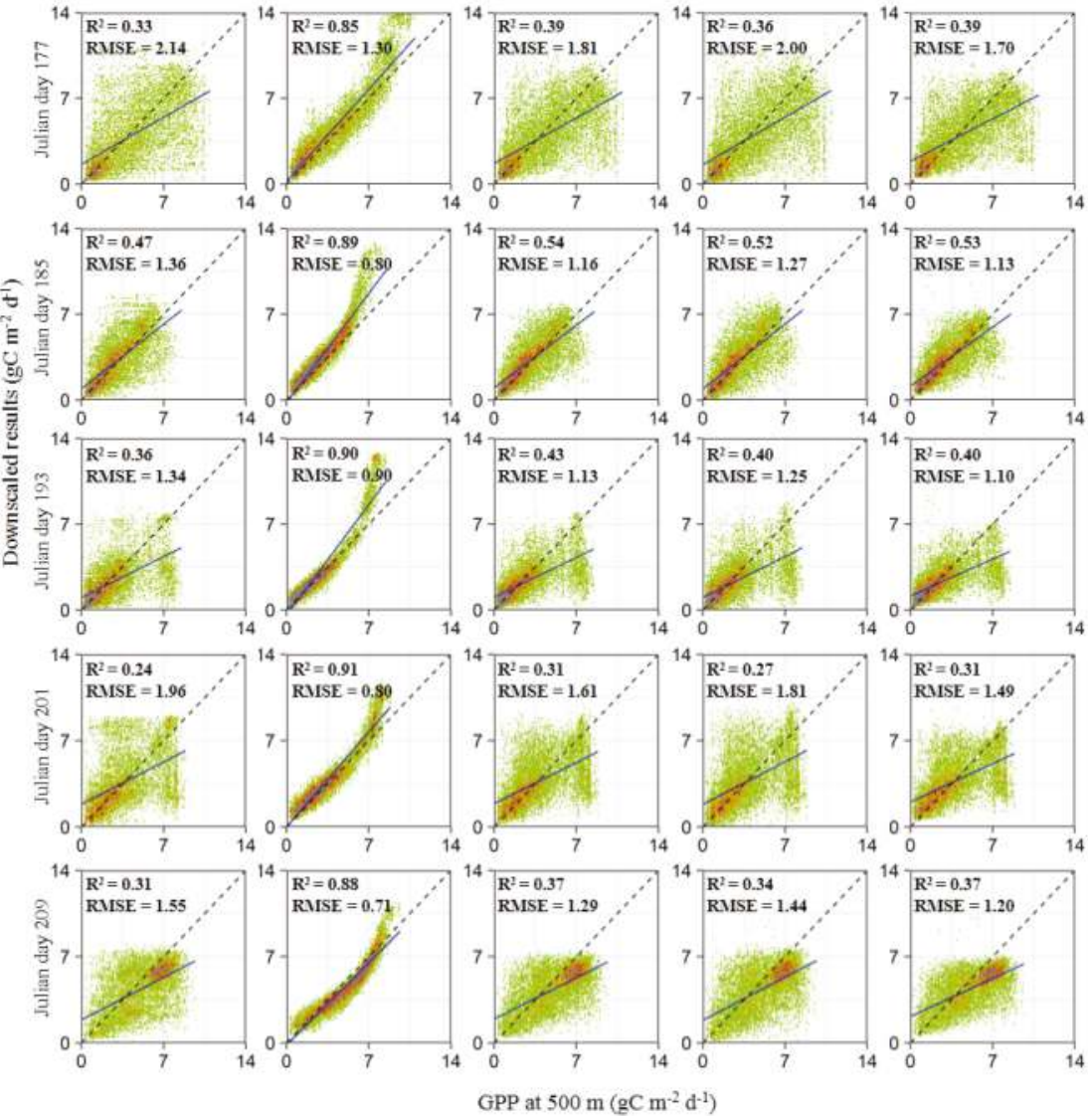

Figure 4. Density scatterplot between MODIS GPP and downscaled GPP at $500 \mathrm{~m}$ during Julian days 169-209. (a) represents the comparison between MODIS GPP products; and (b-e) represent the comparison between downscaled GPP and MODIS GPP at $500 \mathrm{~m}$. The solid lines are the regression lines, while the dashed lines are the 1:1 lines. The green and red represent the low-density and high-density areas, respectively. 
To investigate the effects of topography on GPP, a mismatched MODIS GPP between a $1 \mathrm{~km}$ resolution and a $500 \mathrm{~m}$ resolution was analyzed. Figure 5 illustrates the relationships between topographic factors (i.e., altitude, slope) and the inconsistency in the MODIS GPP at $500 \mathrm{~m}$ and $1 \mathrm{~km}$ during Julian days 169-209. Altitude and slope were divided by $500 \mathrm{~m}$ and $10^{\circ}$ intervals, respectively, and the relative error (RE) was adopted to evaluate the inconsistency in the MODIS GPP at $500 \mathrm{~m}$ and $1 \mathrm{~km}$. It was found that the inconsistency in MODIS GPP at two resolutions increased with the increase of slope and altitude. In addition, there was not an obvious relationship between the aspect and the inconsistency in MODIS GPP at two resolutions.

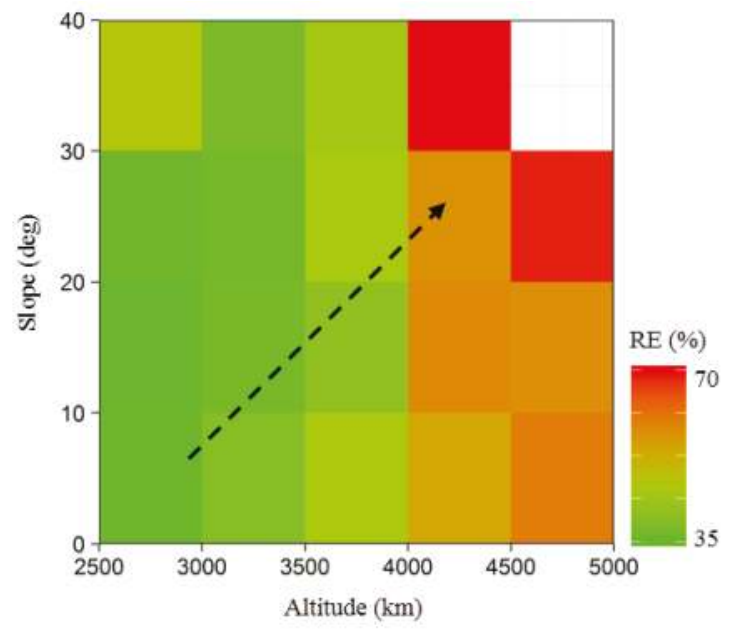

Figure 5. Relationships between topographic factors and inconsistency in the MODIS GPP at $500 \mathrm{~m}$ and $1 \mathrm{~km}$ during Julian days 169-209.

\subsection{Downscaled Results}

To evaluate the quality of the downscaled GPP, a pixel-by-pixel comparison between the MODIS GPP at $500 \mathrm{~m}$ and the downscaled GPP results was implemented during Julian days 169-209 (Figure 4b). From Julian day 169 to 209, the downscaled GPP for all pixels could be explained between $85 \%$ and $91 \%$ of $500 \mathrm{~m}$ MODIS GPP, with RMSEs varying between 0.71 and $1.30 \mathrm{gC} \mathrm{m}^{-2} \mathrm{~d}^{-1}$. The points in the scatterplots clustered around the $1: 1$ lines $\left(\mathrm{R}^{2}=0.89, \mathrm{RMSE}=1.03\right)$, and the downscaled GPP were slightly higher than MODIS GPP at high GPP values. In general, the downscaled GPP showed a strong consistency with the MODIS GPP at $500 \mathrm{~m}$.

Figure 6 illustrates the absolute differences of GPP values between the $500 \mathrm{~m}$ MODIS GPP and the downscaled GPP results. During Julian days 169-209, the percentages of absolute differences in ranges of $[0,1],[1,2],[2,3],[3,4],[4,5]$ and $[5,6]$ were $70.78 \%, 23.02 \%, 3.47 \%, 1.61 \%, 0.89 \%$ and $0.23 \%$, respectively. The pixels whose absolute GPP differences between the $500 \mathrm{~m}$ MODIS GPP and the downscaled GPP results were in the range of [0, 2], accounting for $93.80 \%$ of the total pixels in the study area, showing that the downscaling method proposed in this study could be effectively used to obtain the GPP at $500 \mathrm{~m}$ resolution. Figure 7 shows the density distributions concerning the GPP differences between the $500 \mathrm{~m}$ MODIS GPP and the downscaled GPP results. The mean values of differences between downscaled GPP and $500 \mathrm{~m}$ MODIS GPP varied between 0.60 and $1.03 \mathrm{gC}$ $\mathrm{m}^{-2} \mathrm{~d}^{-1}$, with standard deviations changing between 0.47 and $1.10 \mathrm{gC} \mathrm{m}^{-2} \mathrm{~d}^{-1}$, which were smaller than those between MODIS GPP at $500 \mathrm{~m}$ and $1 \mathrm{~km}$ (mean = 1.36, std. =1.34). It was worthwhile to note that, the proposed downscaling method could effectively solve the GPP underestimation of $1 \mathrm{~km}$ MODIS GPP. 


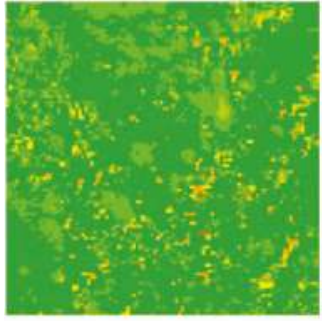

(a) Julian day 169

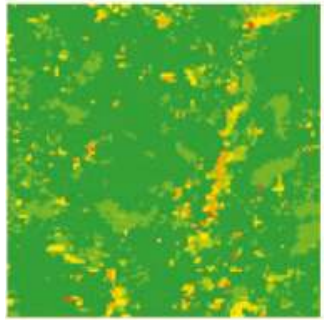

(d) Julian day 193

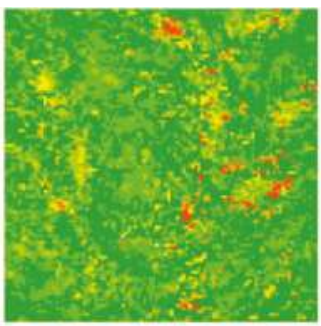

(b) Julian day 177

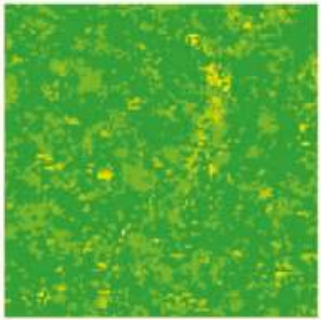

(e) Julian day 201

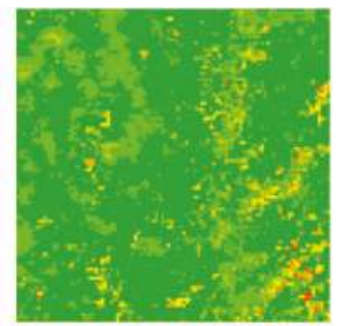

(c) Julian day 185

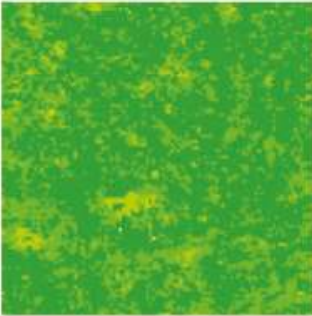

(f) Julian day 209

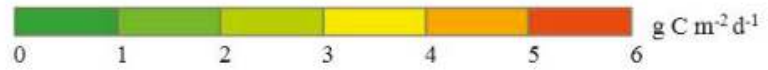

Figure 6. The spatial distribution of absolute GPP differences between the $500 \mathrm{~m}$ MODIS GPP and the downscaled GPP results for Julian days 169-209. Figures (a-f) indicate Julian days 169-209, respectively.
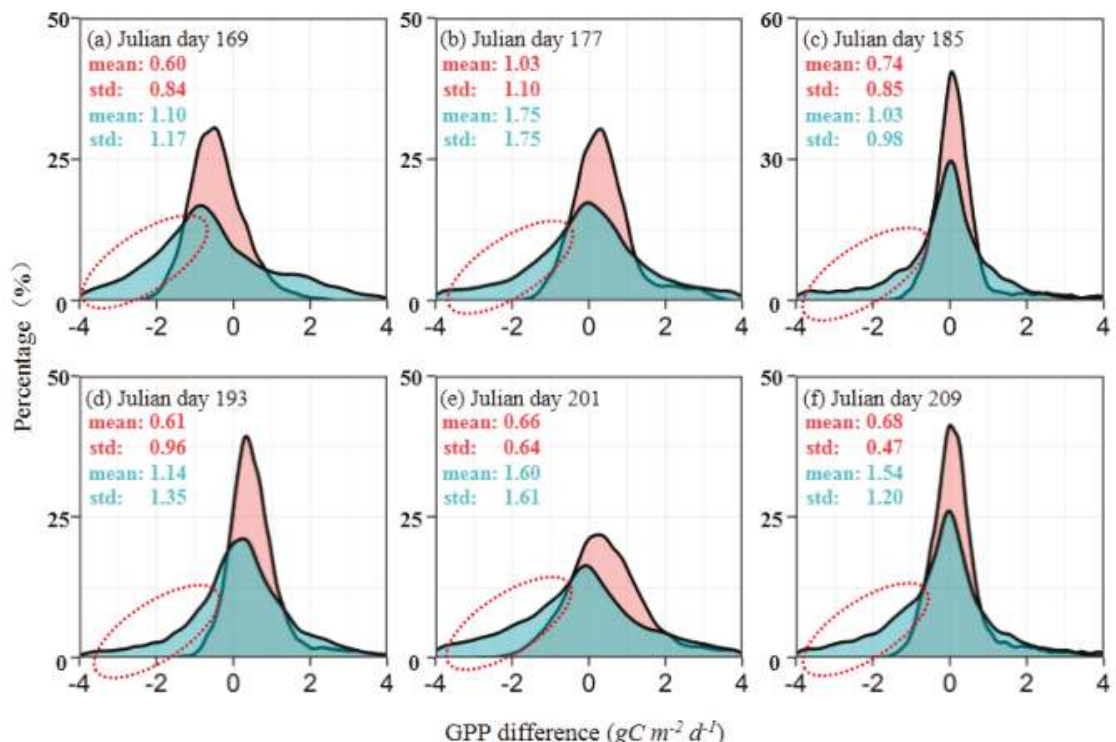

The proposed method

$1 \mathrm{~km}$ MODIS GPP

Figure 7. The density distributions concerning the GPP differences between the $500 \mathrm{~m}$ MODIS GPP and the downscaled GPP results for Julian days 169-209. Figures (a-f) indicate Julian days 169-209, respectively. 
To illustrate the advantages and benefits of the proposed downscaling method, the downscaled results in this work, along with the kriging downscaled results, spline downscaled results and IDW downscaled results, were compared against the MODIS GPP at $500 \mathrm{~m}$ (Figure 4). In general, the proposed downscaled results $\left(R^{2}=0.89\right.$, RMSE $\left.=1.03\right)$ had a stronger consistency with the $500 \mathrm{~m}$ MODIS GPP than the kriging downscaled results $\left(\mathrm{R}^{2}=0.43\right.$, RMSE $\left.=1.36\right)$, the spline downscaled results $\left(R^{2}=0.40, R M S E=1.50\right)$ and the IDW downscaled results $\left(R^{2}=0.42\right.$, RMSE $\left.=1.10\right)$ for all Julian days. Hence, it can be concluded that the proposed downscaling method has a potential application for GPP downscaling to obtain $500 \mathrm{~m}$ GPP distributions, especially in mountainous areas. The results also illustrated that vegetation heterogeneity factors and topographic factors were effective for downscaling land surface fluxes, showing good matches with other related studies $[7,8,38]$.

\section{Discussions}

\subsection{The Topographic Effects on GPP Inconsistency at Two Resolutions}

The time series GPP estimation is very important for monitoring vegetation growth conditions. Mountainous regions typically present high spatial heterogeneity, which is characteristic of steep slopes, altitude variations and notably biological diversity $[11,39,40]$. The results in this study showed the topography had a significant relationship with MODIS GPP inconsistency at two resolutions, as the topography influences the surrounding environment of the carbon assimilation procedure, such as air humidity, air temperature, water redistribution and solar radiation. Generally, air temperature increases linearly with the decrease of altitude. However, in most carbon cycle models, air temperature is assumed to be homogeneous within each modeling grid. This assumption neglects the impact of inner altitude variations in each grid, bringing uncertainties to the simulation over complex terrain. Besides air temperature, solar radiation, air humidity and precipitation also face the same problems. Related studies have illustrated that the spatial variations of altitude and slope greatly influenced hydrologic procedure [13,14,41], and hence affect the carbon assimilation procedure [42-44]. In general, the patchy ecosystem structures in mountain areas usually follow topographic and climatic gradients, and the remarkably spatial heterogeneity has considerable effects on the spatial distributions of GPP estimation.

\subsection{Evaluations of the Proposed Downscaling Method}

Serving as an effective tool in the estimation of GPP, the carbon cycle model is typically executed at coarse resolutions due to the paucity of the data and computing complexities. Landscape complexities are assumed to be simplified in a carbon cycle model by ignoring spatial heterogeneities within each modeling grid. It has been illustrated that the oversimplification of landscape complexities may inevitably cause model simulations to become considerably biased [8-10]. In this approach, massive forcing data were required to run the carbon cycle model, including the spatial distributions of temperature, radiation, vapor pressure deficits and so on. In mountainous areas, the regional estimation of the meteorological element was a challenge, especially at fine resolution $[12,13,45]$.

In this study, a downscaling algorithm of GPP was developed to obtain the GPP distribution at $500 \mathrm{~m}$ resolution. The proposed downscaling method contains two phases: a regression process and an ATPK process. The first phase was used to reduce uncertainties caused by topographic effects and vegetation heterogeneities. A regression analysis was first conducted between the GPP estimation and spatial heterogeneity factors; then, the ATPK was performed to downscale residuals from the regression process.

This study tested the thesis that the $500 \mathrm{~m}$ GPP could be obtained from $1 \mathrm{~km}$ GPP and spatial heterogeneity information, which avoided complex calculation and the limit of forcing data through the carbon cycle models. It was found that the proposed method could be effectively used to obtain the GPP at $500 \mathrm{~m}$ resolution, showing significant advantages over the kriging method, spline method and IDW method. Our results also illustrated that both endogenous factors (i.e., LAI) and exogenous 
factors (i.e., altitude, slope and aspect) were useful for GPP downscaling. Exogenous factors have significant effects on the various instantaneous land surface fluxes due to the redistribution of water and their interactions with atmospheric elements $[43,46,47]$. The endogenous factors can be regarded as the accumulated outcomes of the surrounding environmental conditions [48], such as topography, climate and soil property. Therefore, both exogenous and endogenous factors should be considered in the downscaling scheme to remove biases within the coarse modeling grid.

\subsection{Limitations of the Current Work and Prospects for Future Studies}

The proposed downscaling method in this work fully utilized valuable topographic and vegetation information from ancillary data at a $500 \mathrm{~m}$ spatial resolution to reduce uncertainties in spatial heterogeneities over rugged terrain. However, due to practical constraints, there were also several limitations in this study. First, the proposed downscaling algorithm was based on the MODIS GPP, DEM and LAI data, and any errors in the source data would propagate into the final downscaled results $[49,50]$. Second, the resampling method for $30 \mathrm{~m}$ GDEM data influenced the quality of DEM data at $500 \mathrm{~m}$ and $1 \mathrm{~km}$ resolutions, and hence affected the accuracy of the topographic information [51,52]. In addition, the choice of algorithm for slope and aspect also resulted in uncertainty [53-55], besides slightly affecting the downscaling procedure. Third, although our algorithm considered the main factors (i.e., vegetation density, altitude, slope and aspect) for GPP downscaling, several other factors (e.g., land cover, soil texture, and soil depth) were not included in the algorithm, which might result in slight uncertainties. Finally, due to the lack of reference data for validation at smaller resolution, MODIS GPP at $1 \mathrm{~km}$ resolution were scaled only down to a $500 \mathrm{~m}$ resolution. This study tested the thesis that the $500 \mathrm{~m}$ GPP could be obtained from $1 \mathrm{~km}$ GPP and spatial heterogeneity information. Based on this thesis, the relevant work of obtaining the GPP at a finer resolution (e.g., $30 \mathrm{~m}$ ) will be presented in an additional study.

To improve the performance of the proposed downscaling method, future studies should consider more factors in the downscaling algorithm, and attention should be given to the effects of accurate ancillary data (i.e., DEM and LAI data) on the final GPP downscaled results. Moreover, the proposed downscaling method should be applied in more heterogeneous areas to test the feasibility and stability of the algorithm.

\section{Conclusions}

In this work, a downscaling algorithm was developed to retrieve the GPP at $500 \mathrm{~m}$ resolution in a time series over rugged terrain, which considered the effects of spatial heterogeneity on carbon flux simulations. The GPP product at $1 \mathrm{~km}$ served as the primary dataset of the downscaling algorithm, and the $500 \mathrm{~m}$ GPP product was used as the reference dataset to evaluate the downscaled GPP results. It was found that the proposed downscaling method had advantages over the kriging method, spline method and IDW method. Our results also showed that vegetation heterogeneity factors (i.e., LAI) and topographic factors (i.e., altitude, slope and aspect) were useful for GPP downscaling.

Our study developed a reliable downscaling method to derive a $500 \mathrm{~m}$ resolution GPP for time series over mountainous areas, which is an important issue when evaluating the role of ecosystems in the global carbon cycle. Furthermore, our proposed downscaling algorithm could provide a reference when considering the effects of spatial heterogeneity on carbon flux simulations and retrieving other fine resolution parameters (e.g., net primary productivity and evaporation) in topographically complex terrains.

Acknowledgments: The research was supported from the National Key Research and Development Program of China (Grant No. 2016YFA0600103), the National Natural Science Foundation of China (Grant No. 41631180, 41571373, 41671376). We are grateful to all the data providers and the anonymous reviewers for their valuable suggestions. 
Author Contributions: Xinyao Xie, Ainong Li and Huaan Jin conceived and designed experiments; Xinyao Xie performed the experiments, analyzed the data and wrote the paper; Gaofei Yin provided guidance and helped write the codes of algorithm; Jinhu Bian downloaded the data.

Conflicts of Interest: The authors declare no conflict of interest.

\section{References}

1. Li, A.; Bian, J.; Lei, G.; Huang, C. Estimating the maximal light use efficiency for different vegetation through the casa model combined with time-series remote sensing data and ground measurements. Remote Sens. 2012, 4, 3857-3876. [CrossRef]

2. Zhou, Y.; Hilker, T.; Ju, W.; Coops, N.C.; Black, T.A.; Chen, J.M.; Wu, X. Modeling gross primary production for sunlit and shaded canopies across an evergreen and a deciduous site in canada. IEEE Trans. Geosci. Remote Sens. 2017, 55, 1859-1873. [CrossRef]

3. Liu, L.; Guan, L.; Liu, X. Directly estimating diurnal changes in gpp for c3 and c4 crops using far-red sun-induced chlorophyll fluorescence. Agric. For. Meteorol. 2017, 232, 1-9. [CrossRef]

4. Ju, W.; Chen, J.M.; Black, T.A.; Barr, A.G.; Liu, J.; Chen, B. Modelling multi-year coupled carbon and water fluxes in a boreal aspen forest. Agric. For. Meteorol. 2006, 140, 136-151. [CrossRef]

5. Alemu, G.; Chen, J.M.; Price, D.T.; Kurz, W.A.; Liu, J.; Céline, B.; Hember, R.A.; Wu, C.; Chang, K.H. Improved assessment of gross and net primary productivity of canada's landmass. J. Geophys. Res. Atmos. 2013, 99, 1089-1104.

6. Xie, X.; Li, A.; Jin, H. The simulation models of the forest carbon cycle on a large scale : A review. Acta Ecol. Sin. 2018, 38, 1-14.

7. Chen, J.M.; Chen, X.; Ju, W. Effects of vegetation heterogeneity and surface topography on spatial scaling of net primary productivity. Biogeosciences 2013, 10, 4879-4896. [CrossRef]

8. El Maayar, M.; Chen, J.M. Spatial scaling of evapotranspiration as affected by heterogeneities in vegetation, topography, and soil texture. Remote Sens. Environ. 2006, 102, 33-51. [CrossRef]

9. Mitchell, S.W.; Csillag, F.; Tague, C. Impacts of spatial partitioning in hydroecological models: Predicting grassland productivity with rhessys. Trans. GIS 2005, 9, 421-442. [CrossRef]

10. MacKay, D.S.; Ahl, D.E.; Ewers, B.E.; Gower, S.T.; Burrows, S.N.; Samanta, S.; Davis, K.J. Effects of aggregated classifications of forest composition on estimates of evapotranspiration in a northern wisconsin forest. Glob. Chang. Biol. 2002, 8, 1253-1265. [CrossRef]

11. Jin, H.; Li, A.; Bian, J.; Nan, X.; Zhao, W.; Zhang, Z.; Yin, G. Intercomparison and validation of modis and glass leaf area index (lai) products over mountain areas: A case study in southwestern china. Int. J. Appl. Earth Observ. Geoinf. 2017, 55, 52-67. [CrossRef]

12. Zhao, W.; Li, A.N. A review on land surface processes modelling over complex terrain. Adv. Meteorol. 2015, 2015, 607181. [CrossRef]

13. Immerzeel, W.W.; Petersen, L.; Ragettli, S.; Pellicciotti, F. The importance of observed gradients of air temperature and precipitation for modeling runoff from a glacierized watershed in the nepalese himalayas. Water Resour. Res. 2014, 50, 2212-2226. [CrossRef]

14. Mizukami, N.; Clark, M.P.; Slater, A.G.; Brekke, L.D.; Elsner, M.M.; Arnold, J.R.; Gangopadhyay, S. Hydrologic implications of different large-scale meteorological model forcing datasets in mountainous regions. J. Hydrometeorol. 2014, 15, 474-488. [CrossRef]

15. Helbig, N.; Loewe, H. Shortwave radiation parameterization scheme for subgrid topography. J. Geophys. Res. Atmos. 2012, 117. [CrossRef]

16. Li, A.; Bian, J.; Jin, H.; Zhao, W.; Zhang, Z.; Nan, X.; Yin, G.; Lei, G. Mountain Remote Sensing; Science Press: Beijing, China, 2016.

17. Chen, J.M. Spatial scaling of a remotely sensed surface parameter by contexture. Remote Sens. Environ. 1999, 69, 30-42. [CrossRef]

18. Liang, S.; Li, X.; Wang, J. Advanced Remote Sensing; Academic Press: Cambridge, MA, USA, 2012

19. Hong, S.-H.; Hendrickx, J.M.H.; Borchers, B. Up-scaling of sebal derived evapotranspiration maps from landsat (30 m) to modis (250 m) scale. J. Hydrol. 2009, 370, 122-138. [CrossRef]

20. Thomas, A. Overview of the geoecology of the gongga shan range, sichuan province, china. Mt. Res. Dev. 1999, 19, 17-30. [CrossRef] 
21. Zhu, W.; Fu, X.; Feng, X.; Lu, J.Y. Annual time-series analyses of total gaseous mercury measurement and its impact factors on the gongga mountains in the southeastern fringe of the qinghai-tibetan plateau. J. Mt. Sci. Engl. 2008, 5, 17-31. [CrossRef]

22. Running, S.W.; Nemani, R.R.; Heinsch, F.A.; Zhao, M.S.; Reeves, M.; Hashimoto, H. A continuous satellite-derived measure of global terrestrial primary production. Bioscience 2004, 54, 547-560. [CrossRef]

23. Heinsch, F.A.; Zhao, M.; Running, S.W.; Kimball, J.S.; Nemani, R.R.; Davis, K.J.; Bolstad, P.V.; Cook, B.D.; Desai, A.R.; Ricciuto, D.M.; et al. Evaluation of remote sensing based terrestrial productivity from modis using regional tower eddy flux network observations. IEEE Trans. Geosci. Remote Sens. 2006, 44, 1908-1925. [CrossRef]

24. Jung, M.; Reichstein, M.; Margolis, H.A.; Cescatti, A.; Richardson, A.D.; Arain, M.A.; Arneth, A.; Bernhofer, C.; Bonal, D.; Chen, J.; et al. Global patterns of land-atmosphere fluxes of carbon dioxide, latent heat, and sensible heat derived from eddy covariance, satellite, and meteorological observations. J. Geophys. Res. Biogeo 2011, 116. [CrossRef]

25. Turner, D.P.; Ritts, W.D.; Cohen, W.B.; Gower, S.T.; Running, S.W.; Zhao, M.S.; Costa, M.H.; Kirschbaum, A.A.; Ham, J.M.; Saleska, S.R.; et al. Evaluation of modis npp and gpp products across multiple biomes. Remote Sens. Environ. 2006, 102, 282-292. [CrossRef]

26. Myneni, R.B.; Hoffman, S.; Knyazikhin, Y.; Privette, J.L.; Glassy, J.; Tian, Y.; Wang, Y.; Song, X.; Zhang, Y.; Smith, G.R.; et al. Global products of vegetation leaf area and fraction absorbed par from year one of modis data. Remote Sens. Environ. 2002, 83, 214-231. [CrossRef]

27. Knyazikhin, Y.; Martonchik, J.V.; Myneni, R.B.; Diner, D.J.; Running, S.W. Synergistic algorithm for estimating vegetation canopy leaf area index and fraction of absorbed photosynthetically active radiation from modis and misr data. J. Geophys. Res. Atmos. 1998, 103, 32257-32275. [CrossRef]

28. Zhang, C.; Liu, Q.; Liu, G.; Ding, S.; Li, H.; Dong, J. Elevation quality evaluation of aster gdem data in china's eastern region. J. Geomat. Sci. Technol. 2014, 31, 67-72.

29. Zhang, C.; Liu, Q.; Liu, G.; Ding, S.; Wang, P.; Dong, J. Data processing and application progress of srtm3 and aster gdem. Geogr. Geo-inf. Sci. 2012, 28, 29-34.

30. Frey, H.; Paul, F. On the suitability of the srtm dem and aster gdem for the compilation of topographic parameters in glacier inventories. Int. J. Appl. Earth Obs. Geoinf. 2012, 18, 480-490. [CrossRef]

31. Dozier, J.; Frew, J. Rapid calculation of terrain parameters for radiation modeling from digital elevation data. IEEE Trans. Geosci. Remote Sens. 1990, 28, 963-969. [CrossRef]

32. Wang, Q.; Shi, W.; Atkinson, P.M.; Zhao, Y. Downscaling modis images with area-to-point regression kriging. Remote Sens. Environ. 2015, 166, 191-204. [CrossRef]

33. Gao, F.; Kustas, W.P.; Anderson, M.C. A data mining approach for sharpening thermal satellite imagery over land. Remote Sens. 2012, 4, 3287-3319. [CrossRef]

34. Wang, Q.; Shi, W.; Atkinson, P.M. Area-to-point regression kriging for pan-sharpening. ISPRS J. Photogramm. Remote Sens. 2016, 114, 151-165. [CrossRef]

35. Knotters, M.; Brus, D.J.; Voshaar, J.H.O. A comparison of kriging, co-kriging and kriging combined with regression for spatial interpolation of horizon depth with censored observations. Geoderma 1995, 67, 227-246. [CrossRef]

36. Tait, A.; Henderson, R.; Turner, R.; Zheng, X. Thin plate smoothing spline interpolation of daily rainfall for new zealand using a climatological rainfall surface. Int. J. Climatol. 2006, 26, 2097-2115. [CrossRef]

37. Watson, D.F.; Philip, G.M. A refinement of inverse distance weighted interpolation. Geo-Processing 1985, 2, 315-327.

38. Simic, A.; Chen, J.M.; Liu, J.; Csillag, F. Spatial scaling of net primary productivity using subpixel information. Remote Sens. Environ. 2004, 93, 246-258. [CrossRef]

39. Jin, H.; Li, A.; Bian, J.; Zhang, Z.; Huang, C.; Li, M. Validation of global land surface satellite (glass) downward shortwave radiation product in the rugged surface. J. Mt. Sci. Engl. 2013, 10, 812-823. [CrossRef]

40. Jin, H.A.; Li, A.N.; Bian, J.H. Comparative analysis of hj-1, spot, and tm data for leaf area index estimation in a mountainous area. In Proceedings of the 2013 IEEE International Geoscience and Remote Sensing Symposium, Melbourne, Australia, 21-26 July 2013; pp. 2782-2785.

41. Bonan, G.B. The land surface climatology of the ncar land surface model coupled to the ncar community climate model. J. Clim. 1998, 11, 1307-1326. [CrossRef] 
42. Qiu, Y.; Fu, B.J.; Wang, J.; Chen, L.D. Soil moisture variation in relation to topography and land use in a hillslope catchment of the loess plateau, china. J. Hydrol. 2001, 240, 243-263. [CrossRef]

43. Govind, A.; Chen, J.M.; Margolis, H.; Ju, W.; Sonnentag, O.; Giasson, M.-A. A spatially explicit hydro-ecological modeling framework (beps-terrainlab v2.0): Model description and test in a boreal ecosystem in eastern north america. J. Hydrol. 2009, 367, 200-216. [CrossRef]

44. Sabetraftar, K.; Mackey, B.; Croke, B. Sensitivity of modelled gross primary productivity to topographic effects on surface radiation: A case study in the cotter river catchment, australia. Ecol. Model. 2011, 222, 795-803. [CrossRef]

45. Konzelmann, T.; Calanca, P.; Muller, G.; Menzel, L.; Lang, H. Energy balance and evapotranspiration in a high mountain area during summer. J. Appl. Meteorol. 1997, 36, 966-973. [CrossRef]

46. Huang, W.; Zhang, L.; Furumi, S.; Muramatsu, K.; Daigo, M.; Li, P. Topographic effects on estimating net primary productivity of green coniferous forest in complex terrain using landsat data: A case study of yoshino mountain, japan. Int. J. Remote Sens. 2010, 31, 2941-2957. [CrossRef]

47. Gelybo, G.; Barcza, Z.; Kern, A.; Kljun, N. Effect of spatial heterogeneity on the validation of remote sensing based gpp estimations. Agric. For. Meteorol. 2013, 174, 43-53. [CrossRef]

48. Chen, J.M.; Mo, G.; Pisek, J.; Liu, J.; Deng, F.; Ishizawa, M.; Chan, D. Effects of foliage clumping on the estimation of global terrestrial gross primary productivity. Glob. Biogeochem. Cycle 2012, 26. [CrossRef]

49. Lin, S.; Jing, C.; Coles, N.A.; Chaplot, V.; Moore, N.J.; Wu, J. Evaluating dem source and resolution uncertainties in the soil and water assessment tool. Stoch. Environ. Res. Risk Assess. 2013, 27, $209-221$. [CrossRef]

50. Xu, F.; Dong, G.; Wang, Q.; Liu, L.; Yu, W.; Men, C.; Liu, R. Impacts of dem uncertainties on critical source areas identification for non-point source pollution control based on swat model. J. Hydrol. 2016, 540, 355-367. [CrossRef]

51. Grohmann, C.H. Effects of spatial resolution on slope and aspect derivation for regional-scale analysis. Comput. Geosci. 2015, 77, 111-117. [CrossRef]

52. Wechsler, S.P.; Kroll, C.N. Quantifying dem uncertainty and its effect on topographic parameters. Photogramm. Eng. Remote Sens. 2006, 72, 1081-1090. [CrossRef]

53. Oksanen, J.; Sarjakoski, T. Error propagation of dem-based surface derivatives. Comput. Geosci. 2005, 31, 1015-1027. [CrossRef]

54. Zhou, Q.M.; Liu, X.J. Analysis of errors of derived slope and aspect related to dem data properties. Comput. Geosci. 2004, 30, 369-378. [CrossRef]

55. Liu, H.H.; Kiesel, J.; Hormann, G.; Fohrer, N. Effects of dem horizontal resolution and methods on calculating the slope length factor in gently rolling landscapes. Catena 2011, 87, 368-375. [CrossRef]

(C) 2018 by the authors. Licensee MDPI, Basel, Switzerland. This article is an open access article distributed under the terms and conditions of the Creative Commons Attribution (CC BY) license (http:/ / creativecommons.org/licenses/by/4.0/). 


\title{
Article \\ Effects of Forest Canopy Vertical Stratification on the Estimation of Gross Primary Production by Remote Sensing
}

\author{
Shangrong Lin ${ }^{1,2}$, Jing $\mathrm{Li}^{1, *}$, Qinhuo Liu ${ }^{1,2,3, *}$, Alfredo Huete ${ }^{4}$ and Longhui $\mathrm{Li}^{5,6,7}$ \\ 1 State Key Laboratory of Remote Sensing Science, Institute of Remote Sensing and Digital Earth, \\ Chinese Academy of Sciences, Beijing 100101, China; shangrong_edu@outlook.com \\ 2 College of Resources and Environment, University of Chinese Academy of Sciences, Beijing 100049, China \\ 3 Joint Center for Global Change Studies (JCGCS), Beijing 100875, China \\ 4 Plant Functional Biology and Climate Change Cluster, University of Technology Sydney, \\ Ultimo, NSW 2007, Australia; Alfredo.Huete@uts.edu.au \\ 5 Jiangsu Center for Collaborative Innovation in Geographical Information Resource Development \\ and Application, Nanjing Normal University, Nanjing 210023, China; Longhui.Li@njnu.edu.cn \\ 6 Key Laboratory of Virtual Geographic Environment (Nanjing Normal University), Ministry of Education, \\ Nanjing 210023, China \\ 7 School of Geographical Sciences, Nanjing Normal University, Nanjing 210023, China \\ * Correspondence: lijing01@radi.ac.cn (J.L.); liuqh@radi.ac.cn (Q.L.); Tel.: +86-10-6485-1880 (J.L.)
}

Received: 21 June 2018; Accepted: 17 August 2018; Published: 21 August 2018

\begin{abstract}
Gross primary production (GPP) in forests is the most important carbon flux in terrestrial ecosystems. Forest ecosystems with high leaf area index (LAI) values have diverse species or complex forest structures with vertical stratifications that influence the carbon-water-energy cycles. In this study, we used three light use efficiency (LUE) GPP models and site-level experiment data to analyze the effects of the vertical stratification of dense forest vegetation on the estimates of remotely sensed GPP during the growing season of two forest sites in East Asia: Dinghushan (DHS) and Tomakomai (TMK). The results showed that different controlling environmental factors of the vertical layers, such as temperature and vapor pressure deficit (VPD), produce different responses for the same LUE value in the different sub-ecosystems (defined as the tree, shrub, and grass layers), which influences the GPP estimation. Air temperature and VPD play important roles in the effects of vertical stratification on the GPP estimates in dense forests, which led to differences in GPP uncertainties from $-50 \%$ to $30 \%$ because of the distinct temperature responses in TMK. The unequal vertical LAI distributions in the different sub-ecosystems led to GPP variations of $1-2 \mathrm{gC} / \mathrm{m}^{2} /$ day with uncertainties of approximately $-30 \%$ to $20 \%$ because sub-ecosystems have unique absorbed fractions of photosynthetically active radiation (APAR) and LUE. A comparison with the flux tower-based GPP data indicated that the GPP estimations from the LUE and APAR values from separate vertical layers exhibited better model performance than those calculated using the single-layer method, with 10\% less bias in DHS and more than $70 \%$ less bias in TMK. The precision of the estimated GPP in regions with thick understory vegetation could be effectively improved by considering the vertical variations in environmental parameters and the LAI values of different sub-ecosystems as separate factors when calculating the GPP of different components. Our results provide useful insight that can be used to improve the accuracy of remote sensing GPP estimations by considering vertical stratification parameters along with the LAI of sub-ecosystems in dense forests.
\end{abstract}

Keywords: vertical vegetation stratification; gross primary production (GPP); light use efficiency; dense forest; MODIS; VPM; temperature profiles; humidity profiles 


\section{Introduction}

As a key component of the global carbon cycle, the terrestrial gross primary production (GPP) in forests plays an important role in the global carbon, water, and energy cycles [1-3]. GPP is the amount of illumination energy from the sun that is transformed into biomass energy, and is dependent on the illumination, heat, and water in terrestrial ecosystems [4,5]. Precisely estimating GPP is essential for evaluating terrestrial carbon cycles, which also influence climate change [6]. Approximately $40 \%$ of GPP is directly or indirectly used by humans [7]. Vegetation is the key component in the ability of humans to adjust the climate and mitigate increasing atmospheric $\mathrm{CO}_{2}[8]$. Currently, several GPP remote sensing products, such as MPI-BGC [9], MODIS [10], JULES [11,12], BESS [13], and BEPS [14,15], which estimate GPP over large regions, exhibit different estimation accuracies for different vegetation types.

Forest ecosystems cover more than $30 \%$ of the land surface and represent $80 \%$ of the aboveground biomass on Earth $[16,17]$. In primeval forest ecosystems, the aboveground parts of the forest system can be divided into two components, the overstory and understory. The overstory component is the tree layer that intercepts direct solar radiation. The understory is defined as the vegetation structures under the upper layer of the canopy, and it is mainly composed of species of vegetation that prefer shaded conditions. In tropical and subtropical dense forests, the understory component can be divided into shrub and grass layers, but in temperate forests, this component consists of mostly grasses and herbs [18,19].

Both overstory and understory species are important contributors to the carbon uptake in terrestrial ecosystems $[20,21]$. Some studies have shown that understory vegetation contributes $32 \%$ of the total GPP in tropical forests [22,23], but this contribution may be $25 \%$ in temperate forests [24]. A recent study showed that the shaded GPP has increased by $1.1 \%$ over the past three decades [25]. This variability is due to variations in the light, temperature, and humidity conditions in the understory and overstory. Shaded leaves also have unique characteristics, with understory and overstory vegetation exhibiting different responses to the environment and various photosynthetic traits [26-30]. However, in regions with high biodiversity and a high leaf area index (LAI) values in the forest zone, such as evergreen broadleaf forests (EBF) and artificial forests, the estimation of GPP exhibits high variance [31,32]. Furthermore, GPP estimations by remote sensing methods combined with canopy photosynthesis models do not consider the understory incident radiation, and thus may underestimate the total GPP [33-35].

In dense forest ecosystems, canopy species show multiple leaf functional traits that are important for GPP estimation. Leaf traits vary greatly among overstory and understory species. When scaling up different leaf traits to whole ecosystems [36,37], the structural parameters of ecosystems are some of the most important controlling factors $[38,39]$. However, species in the same vertical stratification, such as the tree and grass layers in temperate forests and the trees, shrubs, and grasses in tropical rainforests, have similar living environments, and thus similar leaf traits [40-48].

Remote sensing is a useful approach for estimating ecosystem GPP at larger spatial scales $[8,49]$. In forest sites, many studies have demonstrated that micrometeorological parameters such as temperature, humidity, photosynthetically active radiation (PAR), and longwave incident radiation exhibit vertical gradients [50-54]. Moreover, the physiological parameters of vegetation such as chlorophyll contents also exhibit vertical gradients $[42,55,56]$. However, it is difficult to capture these signals from deep in the canopy. Many scientists have used models and methods to retrieve vertical environmental and physiological parameters such as temperature, humidity, PAR, the fraction of absorbed PAR (FPAR), the LAI, and the light use efficiency (LUE) [57-62]. Wu et al. and Kosugi et al. [63,64] also determined the rules of the leaf-scale maximum carboxylation rates (Vcmax25) at different heights. All of these models or methods were developed in specific regions; therefore, it may be difficult to determine the parameters on a global scale. In this article, we introduce three GPP models based on LUE models that are based on the Monteith [65] methods. Monteith's function is calculated as:

$$
\mathrm{GPP}=\mathrm{LUE} \times \mathrm{PAR} \times \text { FPAR }
$$


where LUE is the daily LUE, and FPAR is the fraction of the absorbed PAR estimated from the LAI and zenith angle. The daily LUE is calculated by different factors that are used to determine LUEmax, and the numerical exercises have specific input parameters.

Different models have various parameterizations of environmental stress for LUE; for example, the vegetation photosynthesis model (VPM; Xiao et al. [66]) assumes that temperature is of low importance to LUE at optimal growing temperatures, but it is of high importance at high $\left(50{ }^{\circ} \mathrm{C}\right)$ and low temperatures $\left(0^{\circ} \mathrm{C}\right)$. The MOD17 (MOD, Running et al. [4,67]) model was designed to use remote sensing data for GPP estimation, and this model utilizes a higher stress factor (less than $10 \%$ of the LUE for optimal growth) at low daily minimal temperatures $\left(-8^{\circ} \mathrm{C}\right)$ and a low stress factor $\left(100 \%\right.$ of the LUE for optimal growth) at high daily minimal temperatures $\left(9^{\circ} \mathrm{C}\right)$. The model developed by $\mathrm{Wu}$ et al. [63] assumed that high temperatures were important for LUE (more detailed information can be found in the Supplementary Material).

However, the models mentioned above did not consider the effect of vegetation stratification on the forest canopy. The goal of this study was to investigate the effect of vertical stratification on remotely sensed GPP estimations in selected dense forest. We raised the following two questions: (a) how do environmental drivers vary with height in multilayer dense forests; and (b) does adding vertical stratification parameters to remotely sensed GPP algorithms improve the performance of the estimations in forest canopies? To address these questions, we used eddy covariance flux data from two sites, including one EBF and one artificial Japanese larch forest, in three remotely sensed LUE models to evaluate the responses of GPP to different factors during the growing season. These sites have meteorological data measurements at multiple heights combined with vertical stratification information, such as temperature, vapor pressure deficit (VPD), and PAR.

\section{Materials}

\subsection{Study Sites}

In this research, we selected Dinghushan (DHS) and Tomakomai (TMK) as the research areas. Both of these areas have dense forests and a conspicuous vertically stratified vegetation structure during the growing season from April to October. DHS, which is located in a monsoon subtropical forest climate zone in the southeast part of China, has a $15 \mathrm{~m}$-high broadleaved forest canopy, while TMK, which is located in the northeast part of Japan, has a 15 m-high deciduous needle leaf (DNF) canopy. Vertical meteorological and canopy eddy covariance (EC) data were measured at both sites. A certain amount of understory LAI $\left(>0.9 \mathrm{~m}^{2} / \mathrm{m}^{2}\right)$ was found in these two sites during the growing season. Two selected sites' detailed information is showed in Figure 1 and Table 1. 


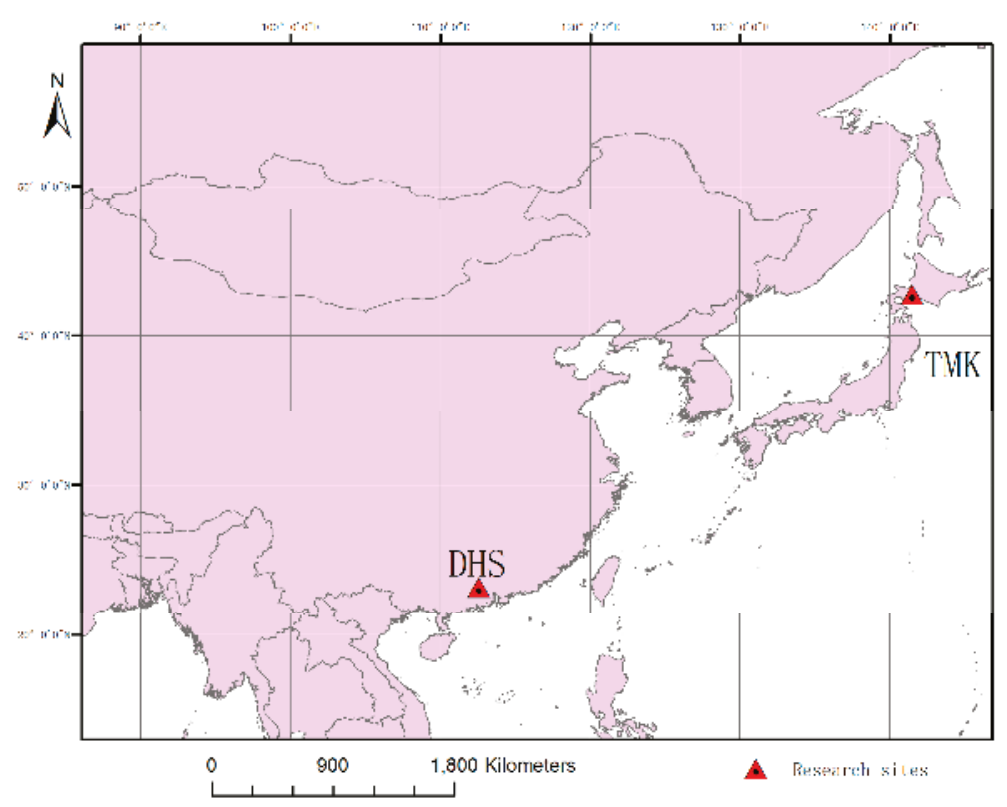

Figure 1. Locations of the two study sites in East Asia.

\subsubsection{Dinghushan (DHS)}

The study area in the DHS Nature Reserve (DNR) is located in the west-central part of Guangdong Province in southeast China. This study area is located in a southern subtropical monsoon humid climate; the average annual temperature of the DNR is $21.8^{\circ} \mathrm{C}$, and the mean annual rainfall is $1927 \mathrm{~mm}$. The period from April through September represents the rainy season, and November to January represents the dry season $[68,69]$.

The flux tower located in the DNR study area $\left(23^{\circ} 10^{\prime} \mathrm{N}, 112^{\circ} 32^{\prime} \mathrm{E}, 240\right.$ masl) is surrounded by more than $70 \%$ of $\mathrm{EBFs}$, and the remaining proportion is evergreen coniferous forest. The canopy height near the flux tower is approximately $17 \mathrm{~m}$. Vertically, the forest structure can be divided into four layers: two tree layers, one shrub layer, and one herb and grass layer. The dominant species in the canopy layers are Schima superba, Castanopsis chinensis, and Pinus massoniana. Near the flux tower, the LAI values for the tree, shrub, and grass layers were $2.6 \mathrm{~m}^{2} / \mathrm{m}^{2}, 0.26 \mathrm{~m}^{2} / \mathrm{m}^{2}$, and $0.92 \mathrm{~m}^{2} / \mathrm{m}^{2}$, respectively, on 6 August 2005.

The flux tower contained seven levels of meteorology instrumentation for measuring different variables above or within the canopy, such as temperature and humidity, with shielded and aspirated probes (HMP45C, Campbell Scientific, Inc., Logan, UT, USA) at 4 m, 9 m, 15 m, 21 m, 27 m, 31 m, and $36 \mathrm{~m}$, and photosynthetic photon flux density (PPFD) instruments were installed at $36 \mathrm{~m}, 21 \mathrm{~m}$, $9 \mathrm{~m}$, and $2 \mathrm{~m}$. For the above-canopy heights, the PPFD was measured by an LI190SB quantum sensor (LI-COR Inc., Lincoln, NE, USA). An LQS7010-SUN sensor (APOGEE, Logan, UT, USA) was used to measure the PPFD at heights under the overstory layer.

At $27 \mathrm{~m}$, an open-path $\mathrm{CO}_{2} / \mathrm{H}_{2} \mathrm{O}$ gas analyzer (model LI-7500, LI-COR Inc.) and a 3D sonic anemometer/thermometer (model CSAT3, Campbell Scientific Inc., Logan, UT, USA) were installed on the tower at approximately two times the average canopy height $(15 \mathrm{~m})$ near the tower. These instruments measure carbon and water flux exchange between terrestrial ecosystems and the atmosphere [70,71]. All of the data were recorded at 30-min intervals. At this site, the LAI values of the different layers were measured every three months from 2004 to 2005. 


\subsubsection{Tomakomai (TMK)}

The TMK study area is in the Tomakomai National Forest, which is approximately $15 \mathrm{~km}$ northwest of Tomakomai, Hokkaido, in northern Japan. The area of larch forest is approximately 100 ha. From 2001-2003, the average temperature was $6.5^{\circ} \mathrm{C}$, and the site was warm in summer and snowy in winter. The mean annual rainfall was $1055 \mathrm{~mm}$. The growing season extends from April to October, while there is little vegetation cover in other months.

The flux tower was located in the center of the larch forest $\left(42^{\circ} 44^{\prime} 13.1^{\prime \prime} \mathrm{N}, 141^{\circ} 31^{\prime} 7.1^{\prime \prime} \mathrm{E}, 115\right.$ masl), and the surrounding trees were approximately 45 years old at the time of this study. The height of the vegetation canopy was $18 \mathrm{~m}$ in 2003. The dominant species near the tower were classified as either overstory or understory species. The overstory species included Japanese larch, birch, spruce, and Japanese elm, and the understory species were ferns and other herbs. The LAI values near the flux tower in the overstory and understory layers were $5.4 \mathrm{~m}^{2} / \mathrm{m}^{2}$ and $3 \mathrm{~m}^{2} / \mathrm{m}^{2}$, respectively, in August 2005 [72].

The flux tower contained eight levels of meteorology instrumentation for measuring different variables such as temperature and humidity above and within the canopy, which were measured with a platinum resistance thermometer and a capacitive hygrometer (HMP-45D, Inc., Logan, UT, USA) at $2 \mathrm{~m}, 5 \mathrm{~m}, 8 \mathrm{~m}, 14 \mathrm{~m}, 18 \mathrm{~m}, 22 \mathrm{~m}, 27 \mathrm{~m}$, and $40 \mathrm{~m}$. The PPFD was measured at $2 \mathrm{~m}, 5 \mathrm{~m}, 14 \mathrm{~m}, 18 \mathrm{~m}$, and $40 \mathrm{~m}$ by a quantum sensor (LI-190S, LI-COR Inc., Lincoln, NE, USA) in the tower and on the forest floor. At $27 \mathrm{~m}$, the $\mathrm{CO}_{2}$ flux between this ecosystem and the atmosphere was measured by open and closed-path EC instruments (LI-7500 and LI-6262, LI-COR Inc., Lincoln, NE, USA). The tower was destroyed by a typhoon in 2004, and monitoring therefore ceased. All of these data were recorded at 30-min intervals. LAI was measured every month from July to December 2003.

Table 1. General characteristics of the two flux towers. DHS: Dinghushan, EC: eddy covariance, LAI: lead area index, PPFD: photosynthetic photon flux density, TMK: Tomakomai.

\begin{tabular}{|c|c|c|}
\hline & DHS & TMK \\
\hline Location & $23.1733 \mathrm{~N}, 112.5361 \mathrm{E}$ & $42.7333 \mathrm{~N}, 141.5167 \mathrm{E}$ \\
\hline Elevation ( $\mathrm{m}$, above sea level) & 240 & $115-140$ \\
\hline Period of data collection & July to December 2005 & July to December 2003 \\
\hline LAI overstory $\left(\mathrm{m}^{2} / \mathrm{m}^{2}\right)^{1}$ & August 2.5 & July 5.6 \\
\hline \multirow{3}{*}{ LAI understory $\left(\mathrm{m}^{2} / \mathrm{m}^{2}\right)$} & August 1.25 & July 3.6 \\
\hline & \multirow{2}{*}{ November 1.25} & September 3.0 \\
\hline & & November 0.2 \\
\hline KGCC (Köppen-Geiger climate classification [73]) & $\begin{array}{l}\text { Warm temperate, fully humid } \\
\text { with hot summer }\end{array}$ & Snowy, fully humid warm summer \\
\hline Heights of temperature and humidity measurements (m) & $4,9,15,21,27,31,36$ & $2,5,8,14,18,22,27,40$ \\
\hline Heights of PPFD measurements (m) & $2,9,21,36$ & $2,5,14,18,40$ \\
\hline Dominant species & $\begin{array}{l}\text { Cleistocalyx operculatus, } \\
\text { Syzygium jambos, } \\
\text { Castanopsis chinensis, etc. } \\
\text { Many understory herb species }\end{array}$ & $\begin{array}{l}\text { Overstory: } \\
\text { Japanese larch (Larix kaempferi Sarg.) } \\
\text { Birch (Betula ermanii and Betula platyphylla) } \\
\text { Japanese elm (Ulmus japonica) } \\
\text { Spruce (Picea jezoensis) } \\
\text { Understory: } \\
\text { Fern (Dryopteris crassirhizoma, Dryopteris austriaca) } \\
\text { and Pachysandra terminalis }\end{array}$ \\
\hline
\end{tabular}




\subsection{Vertical Data Measurements in the Forest}

\subsubsection{Temperature and VPD Data Preprocessing}

During the study period from 1 July 2005 to 31 December 2005, in DHS, the meteorological instruments on the sixth and seventh levels malfunctioned, so all of the data from $31 \mathrm{~m}$ and $36 \mathrm{~m}$ were missing for this period. Therefore, we did not compare the meteorology data from 1 July 2005 to 4 December 2005. For the other data during the study period at the two sites, the temperature and VPD data were gap-filled by the data processor, and low-quality and missing data were removed $[68,69,71]$.

\subsubsection{PPFD Data Preprocessing}

The PPFD data had some missing entries during the study period that were gap-filled by the moving average method at 30-min intervals, and the PPFD data for different vertical layers in the DHS site were provided [71]. To convert the mean PPFD data from $\mu \mathrm{mol} / \mathrm{m}^{2} / \mathrm{s}$ to W $/ \mathrm{m}^{2} / \mathrm{s}$ or MJ/day for the PAR input for each layer, we used a linear regression between shortwave radiation at the top of the tower and the PPFD of the top layer. These two factors were very highly correlated ( $y=1.4907 \mathrm{x}-0.2526, \mathrm{x}$ is the PPFD, unit in $\mu \mathrm{mol} / \mathrm{m}^{2} / \mathrm{s} ; \mathrm{y}$ is the shortwave radiation, unit in $\mathrm{W} / \mathrm{m}^{2} / \mathrm{s}$; $\mathrm{r}^{2}=0.9951$, root mean square error $(\mathrm{RMSE})=16.37 \mathrm{~W} / \mathrm{m}^{2}$, bias (Bias is defined as the predicted result minus the observational data.) $<2.5 \mathrm{~W} / \mathrm{m}^{2}$ ). In TMK, the PPFD was also calculated using this method.

\subsection{GPP Data Processing}

EC instruments directly measure the carbon exchange between terrestrial ecosystems and the atmosphere, but the data must be transformed to determine the GPP. The net ecosystem exchange $(\mathrm{NEE})$ in these two sites included exchange items $\left(\mathrm{FC}_{\mathrm{C}}\right)$ and storage items $(\mathrm{Fs})$, so these values would be more accurate at these two sites under high LAI.

In this article, we converted the FLUXNET2015 flux GPP data at DHS and the gap-filled Asia Flux NEE at TMK to GPP using the flux analysis tool [74]. After obtaining the quality-controlled NEE, we partitioned the NEE into GPP and RE. For unreliable data, we used the harmonic analysis of time series (HANTS) algorithm [75] to gap-fill the GPP data at 30-min intervals. The 30-min GPP data were excluded when the solar radiation in the PPFD was less than $10 \mu \mathrm{mol} / \mathrm{m}^{2} / \mathrm{s}$. All of the data, including the carbon flux data, micrometeorological data, and LAI, were from the same source to ensure accurate representation of the carbon exchange near the flux tower.

\section{Methods}

In this section, we used three LUE-based GPP models and parameterization methods, which applied unequal temperature and water scalars to LUE. These weights can be applied to remote sensing methods and site-level GPP modeling.

Diverse species inhabit the overstory and understory layers of the forest canopy, which have unique ecophysiological parameters. The controlling environmental factors, such as temperature, humidity, and light radiation, have vertical gradients corresponding to different heights in the canopy. However, for certain vegetation groups, such as trees, shrubs, and grasses, and their living heights in the forest canopy, the diverse ecophysiological properties of individual species are much more likely to be affected by vertical gradients than the whole forest ecosystem $[43,44]$. To simplify the photosynthetic processes of multiple species in this study, we defined the concept of sub-ecosystems, which divided the entire terrestrial ecosystem into two or three parts. Examples include the overstory and understory sub-ecosystems in TMK and the arbor, shrub, and grass sub-ecosystems in DHS. Different sub-ecosystem LAI values can be used as the criteria to separate ecosystems into two or three individual types, where GPP would be the aggregation of three different types of photosynthetic processes, because leaves are the only photosynthetic organs in a plant species. 


\subsection{Theoretical Background of the GPP Model}

In this research, we used three LUE-based GPP models, namely, VPM [66], MOD17 [10,67], and $\mathrm{Wu}$ [63], to model the GPP according to the following formula:

$$
\text { GPP }=\text { FPAR } \times \text { PAR } \times \text { LUEmax } \times f(T) \times g(V P D)
$$

In Equation (2), PAR is the photosynthetically active radiation, and FPAR is the fraction of the absorbed PAR, which is calculated as:

$$
F P A R=1-e^{-k \times L A I}
$$

where $k$ is the extinction coefficient, and LAI is the leaf area index in the selected area at a specific canopy height. LUEmax is the maximum LUE, which is scaled by temperature and the VPD response under diverse situations. Therefore, LUE can be expressed as:

$$
\text { LUE }=\text { LUEmax } \times f(T) \times g(V P D)
$$

Detailed information on the three GPP models can be found in the Supplementary Material. The models have different parameterizations for $\mathrm{f}(\mathrm{T})$ and $\mathrm{g}(\mathrm{VPD})$.

\subsection{GPP Estimation Considering the Vertical Structure}

In situ meteorology data for the height of each sub-ecosystem are available for the DHS and TMK sites. Thus, the GPP of every sub-ecosystem can be described by the GPP model with the meteorology data and model parameters from each height. In this research, we assumed that each sub-ecosystem would have a different LUEi, which is defined as the LUE of the sub-ecosystem. LUEi is affected by only certain environmental factors, such as temperature and the VPD at the corresponding height. LUEi was calculated using Equation (4), and the LUEmax of the sub-ecosystems (such as those of shrubs and grasses), temperature, and the VPD stress functions are provided in the Supplementary Material.

At the ecosystem scale, using temperature and the VPD scalar produces the LUEmax, which characterizes carbon sequestration from the LUE. Moreover, different vegetation types, such as the tree, shrub, and grass layers, have various LUEmax values. Table 2 presents the various LUEmax inputs for the GPP estimation results from four different articles. The results of Zhou and Madani stated that overstory layers, such as the overstory tree layer, have an average LUEmax of $0.28 \mathrm{gC} / \mathrm{m}^{2} / \mathrm{MJ}$, which is higher than the LUE of the understory layer (grass).

Table 2. Tree, shrub, and grass maximum light use efficiency (LUEmax) parameterization values (units: $\mathrm{gC} / \mathrm{m}^{2} /$ day) (EBF and DNF were defined as the LUEmax values of the tree layer in the GPP simulations).

\begin{tabular}{ccccc}
\hline & Tree (DHS) & Overstory (TMK) & Shrub & Grass \\
\hline Zhou [32] & 1.120 & 1.180 & 0.930 & 1.520 \\
MODIS [67] & 1.268 & 1.051 & 0.841 & 0.860 \\
Yuan [31] & 1.680 & 1.640 & 0.660 & 1.520 \\
Madani [76] & 0.980 & 1.171 & 0.631 & 1.294 \\
\hline
\end{tabular}

To analyze the effect of vertical structure on GPP estimation, we calculated GPP considering vertical layers using the following function:

$$
\mathrm{GPPm}=\sum_{i=\text { sub }- \text { ecosystem }} \mathrm{LUE}_{i} \times \mathrm{APAR}_{i}
$$


where GPPm is defined as the modeled sum of GPP in two (overstory and understory) or three (tree, shrub, and grass) sub-ecosystems. The ecosystems were divided into overstory and understory groups at TMK and into tree, shrub, and grass groups at DHS using in situ LAI measurements. The meteorology data can provide in situ air temperature and VPD stress values for each sub-ecosystem. Temperature sensors at different heights measured the air temperature, while the VPD was calculated from the relationship between VPD and both temperature and humidity [77,78]. APARi is the absorbed PAR for the LUE of a specified height, and was calculated from FPAR and PAR. At the DHS site, the whole ecosystem can be divided into three sub-ecosystems, namely, the tree, shrub, and grass layers; thus, the GPP was calculated for the EBF, shrub, and grass layers (sub-ecosystems) using the in situ temperature, VPD, PAR, and long-term LAI data for each layer, and specific LUEmax input values for each sub-ecosystem. At the TMK site, we divided the ecosystem into DNF and grass sub-ecosystems, and used the GPP input parameters for each layer, as used in the approach for DHS:

$$
\text { GPPs }=\text { LUEs } \times \text { APARs }
$$

GPPs represents the GPP of a specific ecosystem that was estimated using the big-leaf model, which assumes that the entire ecosystem is a single leaf. At the DHS site, the LUE was calculated as an EBF ecosystem with an EBF-based LUEmax (Table 3), and the temperature and VPD stress were given by the mean canopy temperature and VPD. At TMK, the DNF-based LUEmax (Table 3) was used, and the temperature and VPD stress were given by the mean canopy temperature and VPD.

$$
\text { GPPdif }=\text { GPPm }- \text { GPPs }
$$

The GPP bias (GPPdif in Equation (7)) is the multilayer GPP result (GPPm) minus the single-layer GPP result (GPPs), which represents the difference between the two models. Therefore, GPPdif is the difference between considering and not considering vertical stratification. TMK and DHS have overstory and understory vegetation, so using representative GPPs for the whole canopy would lead to estimation uncertainties. The modeled GPP values produced by a variety of models were given their own terms. For example, if a GPP result was produced without considering the vertical structure in a VPM, this value was represented as GPP_s_VPM. If a GPP result was produced based on a MODIS model that considered the vertical structure, it was represented as GPP_m_MOD.

Table 3. Understory photosynthetically active radiation (PAR) simulation: parameter value inputs and results.

\begin{tabular}{ccc}
\hline & TMK & DHS \\
\hline Input parameters & & \\
\hline Overstory LAI & 5.4 & 2.6 \\
X (leaf angle distribution in spherical distribution) & 1 & 1 \\
Daytime zenith angle (mean) & 31 & 45 \\
\hline Output results & & \\
\hline Direct PAR $\tau_{b} \%$ & 4.2 & 15.9 \\
Diffuse PAR $\tau_{d} \%$ & 1.6 & 9.3 \\
Understory simulation \% & 5.6 & 25.2 \\
Understory in situ \% & 8.2 & 27.5 \\
\hline
\end{tabular}

\subsection{Modeling Vertical PAR with a Radiative Transfer Model}

In this study, we also compared the measured PAR and modeled PAR values to simulate PAR in the vertical direction. According to the light transfer function in the vegetation canopy [58], the vertical PAR at different heights impacts the LAI through light absorption, reflectance, and transmittance. In the vertical direction, incident PAR for direct light follows the Beer-Lambert law:

$$
P A R(z)_{d i r}=P A R_{0} \times e^{\tau_{b}}
$$


where $P A R_{0}$ is the incident $P A R$ at the top of the canopy, $z$ is the length of light transmission through the canopy, and the $\tau_{b}$ is the transmitted ratio, which is calculated as:

$$
\tau_{b}=\exp \left(-K_{b} \times L A I\right)
$$

where $K_{b}$ is the extinction coefficient, which is affected by the zenith angle $\phi$ [79]

$$
\begin{aligned}
K_{b} & =\frac{\sqrt{x^{2}+\tan ^{2} \phi}}{x+1.774(x+1.182)^{-0.733}} \\
\tau_{d} & =2 \int_{0}^{\pi / 2} \tau_{b}(\phi) \sin \phi \cos \phi d \phi
\end{aligned}
$$

Therefore, $\operatorname{PAR}(z)$ is the understory incident PAR, which can be expressed as follows:

$$
\operatorname{PAR}(z)=P A R_{0} \times e^{\tau_{b}}+P A R_{\text {skylight }} \times e^{\tau_{d}}
$$

To test the proportional impact of the LAI in different sub-ecosystems on the GPP estimation in dense forests, we used the discrete anisotropic radiative transfer (DART [80,81]) model to simulate APAR based on clear sky conditions in the tree, shrub, and grass layers under different vertical LAI distributions.

\subsection{Statistical Indicators}

At the same time, the maximal air temperature in the canopy was usually found at the top of the canopy, while the minimal value was often found at the ground layer. We evaluated the largest variance in the vertical temperature and VPD profiles using two parameters: Tdif and VPDdif. Tdif is defined as the air temperature at the top of the canopy minus the temperature at the grass layer. VPDdif is the VPD of the top layer of the canopy minus the VPD of the grass layer.

\section{Results and Analysis}

\subsection{Analysis of Variance in Forest Canopy Vertical Stratification}

In forest canopies, vertical temperature and VPD stratification vary with forest type and season. Figure 2A,B show the differences in the daily average vertical temperature from day of year (DOY) 210 to 365 at the DHS site (EBF) in 2005, and from DOY 182 to 365 in 2003 at the TMK site (DNF).

Variances in air temperature occur in forest canopies during the growing season. In most cases, the air temperature in the tree layer is higher than that in the grass layer, with an average temperature difference of approximately $0.4{ }^{\circ} \mathrm{C}$ at the DHS site. In the DNF vegetation type at the TMK site, the mean temperature difference was approximately $0{ }^{\circ} \mathrm{C}$ before DOY 240 . From DOY 240 to 280, the temperature of the tree layer was $0.5^{\circ} \mathrm{C}$ higher than that of the grass layer. However, during the non-growing season after DOY 290 , the temperature differences of the forest layer ranged from $0.5^{\circ} \mathrm{C}$ to $1.5^{\circ} \mathrm{C}$, which means that the temperatures in the tree layer were higher than those in the grass layer.

Similarly, the VPD of the tree layer in the forest canopy was higher than that of the grass layer. VPD varies with air temperature. During the summer to autumn period (DOY 180 to 300), the daily average VPD of the tree layer was approximately 120 Pa higher than that of the grass layer at the DHS site, and approximately $70 \mathrm{~Pa}$ higher than that at the TMK site. With the decrease in temperature during the winter period (DOY 300 to 365), the VPD of the tree layer was 50 Pa higher than that of the grass layer at the DHS site, and 25 Pa higher than that at the TMK site, which was not as great as the difference during the summer period. Figure 2B,D shows that Tdif and VPDdif have a robust linear relationship in months during the growing season. 

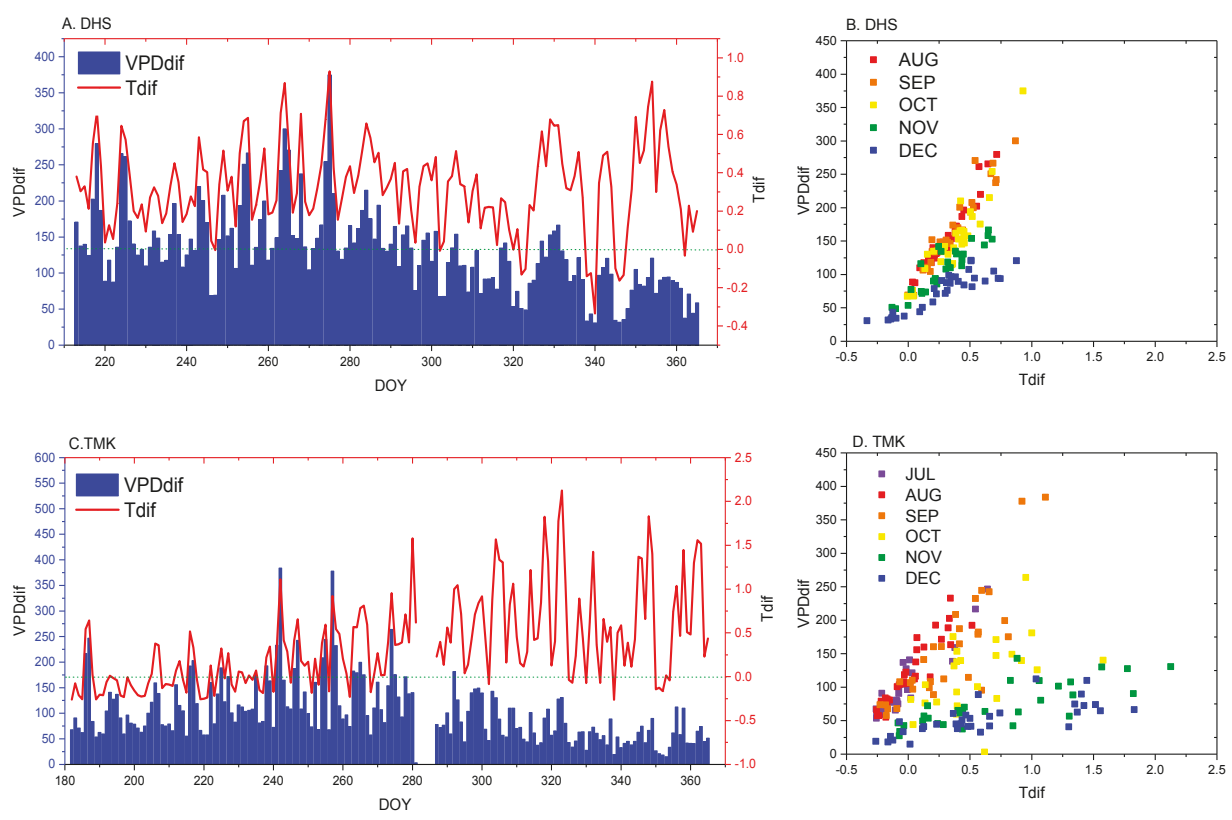

Figure 2. Seasonal patterns in the vertical variance in temperature and vapor pressure deficit (VPD) at the DHS and TMK sites. In the subplots $(\mathbf{A}, \mathbf{C})$, the blue bars (VPDdif in Pa) are the biases between the tree layer VPD and the grass layer VPD. The red line (Tdif in ${ }^{\circ} \mathrm{C}$ ) is the bias between the air temperatures of the top and grass layers. The green dotted line is the $0{ }^{\circ} \mathrm{C}$ line, indicating no difference between the temperatures of the tree and grass layers. Subplots $(\mathbf{B}, \mathbf{D})$ show the relationships between Tdif and VPDdif at the two sites. The dot colors are clustered by month.

In a forest canopy, overstory incident PAR is much higher than that in the understory layer because the incident energy in the understory is affected by sky conditions and the overstory forest structure parameters. At the TMK site, the understory incident PAR was approximately $10 \%$ of the overstory incident PAR during the growing season when the LAI of the overstory was high $(\mathrm{LAI}=5)$. However, when the overstory LAI was low during the winter period (DOY 300 to 365), the understory incident PAR was $30 \%$ of the overstory incident energy (Figure 3A). However, in EBFs such as those at the DHS site, the overstory LAI did not intensely change throughout the year. Figure 3B shows that the incident PAR of the shrub layer at the DHS site was $40 \%$ that of the tree layer, and the incident PAR of the grass layer was $20 \%$ that of the tree layer. After DOY 250, the incident PAR of the overstory layer was steadier than that in the summer period (before DOY 250) because there were fewer cloudy days; thus, the incident PAR of the grass layer was approximately $15 \%$ that of the overstory layer.

The vertical forest structure parameters also influence the amount of understory incident PAR. Table 3 indicates that the total amount of incident PAR in the understory layer was underestimated by $2 \%$ to $3 \%$ at these two sites. 

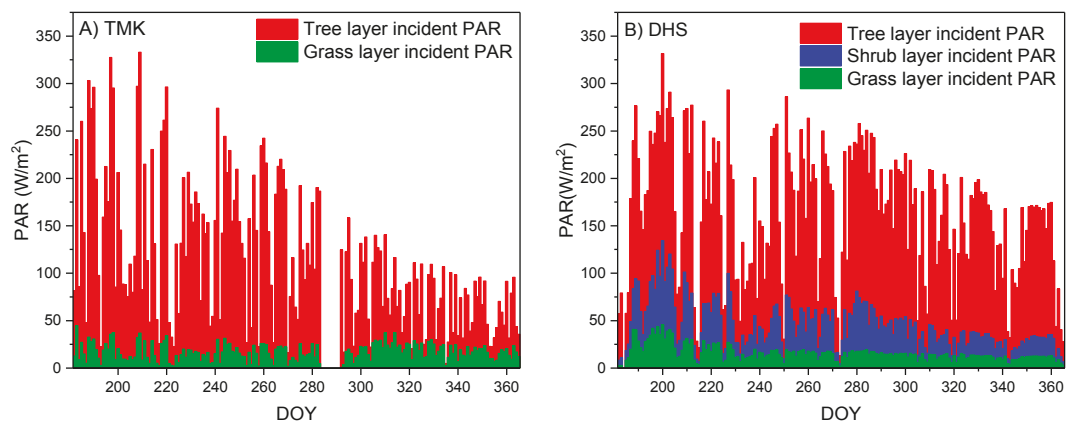

Figure 3. Bar chart for the daily average incident PAR in sub-ecosystems at the selected sites; each bar represents the daily mean incident PAR in each layer (A) TMK; (B) DHS.

\subsection{Influence of Vertical Stratification on GPP Estimation}

Two main factors determine the estimation of GPP in LUE models: (1) the influences of temperature, VPD, and LUEmax on LUE, and (2) the effects of PAR and LAI on the absorbed shortwave energy in different components of the whole ecosystem. In this study, the major difference between considering and not considering the vertical structure during the GPP estimation is the difference between the overstory and understory LUE and APAR.

4.2.1. LUE in Different Sub-Ecosystems Is Influenced by the Vertical Stratification of Temperature, VPD, and LUEmax

Vertical variations in temperature, VPD, and the LUEmax of sub-ecosystems led to differences in LUE at various forest canopy heights. Some studies have shown that the vegetation in the understory layer such as grass has a lower LUEmax. In addition, the vertical differences in temperature and VPD led to variations during different periods of the year. Figure 4 shows the modeled LUE, which combined the vertical variations in temperature and VPD in the sub-ecosystems. At the DHS site, high layers in the canopy, such as the tree and shrub layers, had LUEs that were $0.3 \mathrm{gC} / \mathrm{MJ}$ to $0.6 \mathrm{gC} / \mathrm{MJ}$ lower than those of understory layers, such as grass layers, throughout the study period. Prior to DOY 320 , the LUE of the shrub layer was $0.1 \mathrm{gC} / \mathrm{MJ}$ lower than that of the tree layer. After DOY 330, the LUEs of the shrub layer and the tree layer were almost the same. Similarly, the understory LUE at TMK was approximately $0.4 \mathrm{gC} / \mathrm{MJ}$ higher than that of the overstory layer throughout the growing season (DOY 210 to 300 ).
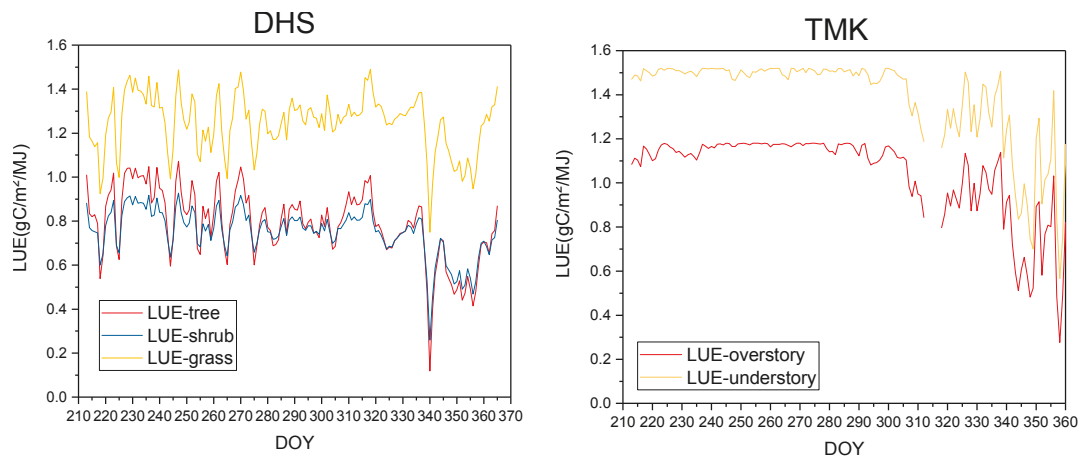

Figure 4. Modeled LUE values in the different sub-ecosystems. 
The mean canopy air temperature influenced the estimation of GPP. The trend in GPPdif in Figure 5 shows that with decreasing canopy mean temperature, the estimation based on GPPm becomes much lower than that based on GPPs, and with increasing mean temperature, the estimation based on GPPm becomes larger than that based on GPPs. The estimates of the three models are similar: GPPdif reaches 0 at approximately $16{ }^{\circ} \mathrm{C}$. The optimal growing temperatures of different species in one sub-ecosystem, such as the tree layer, would not exhibit much variance. When the mean canopy temperature reaches approximately $16^{\circ} \mathrm{C}$, the grass ecosystem reaches its optimal growing temperature, and the LUE of grass would be $10 \%$ higher than that at $10{ }^{\circ} \mathrm{C}$ or lower. At the same time, the LAI of the understory increases with increasing temperature, and the grass understory has a higher photosynthetic ability. This situation causes the value of GPPm to be higher than that of GPPs.

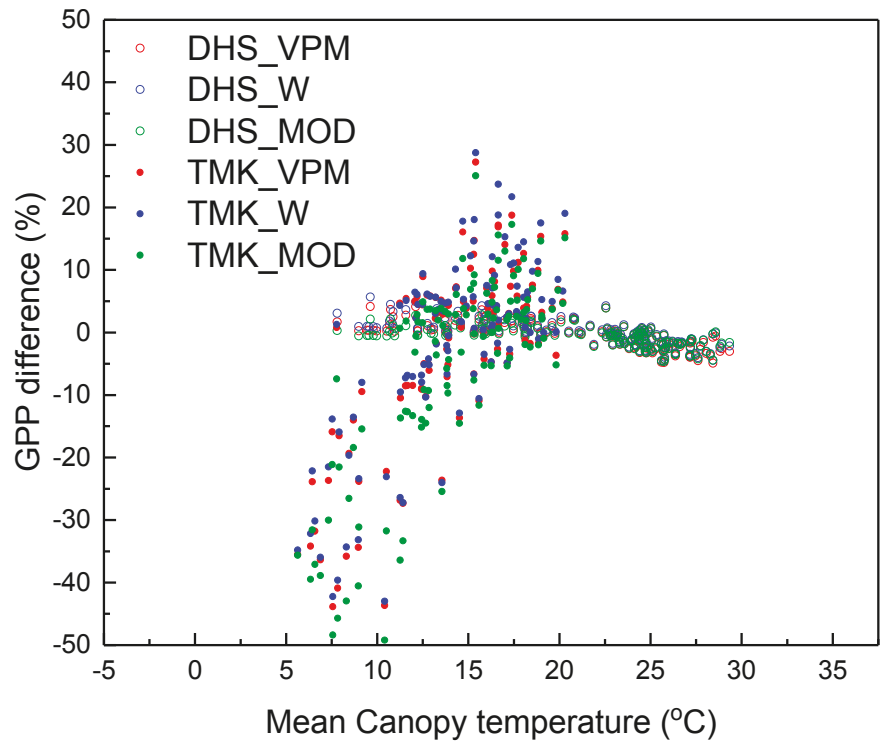

Figure 5. Differences in gross primary production (GPP) under different mean canopy temperatures. The GPP difference is calculated as (GPPm-GPPs)/GPPs. DHS_VPM, DHS_W, and DHS_MOD are the values calculated at the DHS site and by VPM, Wu's method, and the MOD17 model, respectively.

The canopy vertical VPD stratification has a pattern that is similar to that of the canopy vertical temperature stratification: there are variations in photosynthesis in the vertical layers that lead to GPP estimation bias. Figure 6 shows that the GPPdif values are completely different at the DHS and TMK sites. The trend of the change in GPPdif in Figure 6B shows that the estimation from GPPm becomes much lower than that from GPPs as the mean canopy VPD decreases, and with an increase in the mean VPD, the estimation from GPPm becomes larger than that from GPPs. At the DHS site, the GPPdif varied nonsignificantly from approximately $-5 \%$ to $5 \%$. GPPdif declines with increasing canopy mean VPD, which is opposite the pattern observed at the TMK site, where GPPdif increases with VPD, because the VPD increases from 0 to a higher value at the DHS site. When the VPD is greater than 0 , the photosynthetic capacities of different vegetation components, including the tree, shrub, and grass layers decrease, and the total GPP declines. GPPdif changes from $-50 \%$ to $30 \%$ when the mean canopy VPD increases from approximately $0 \mathrm{~Pa}$ to $500 \mathrm{~Pa}$ at the TMK site. 

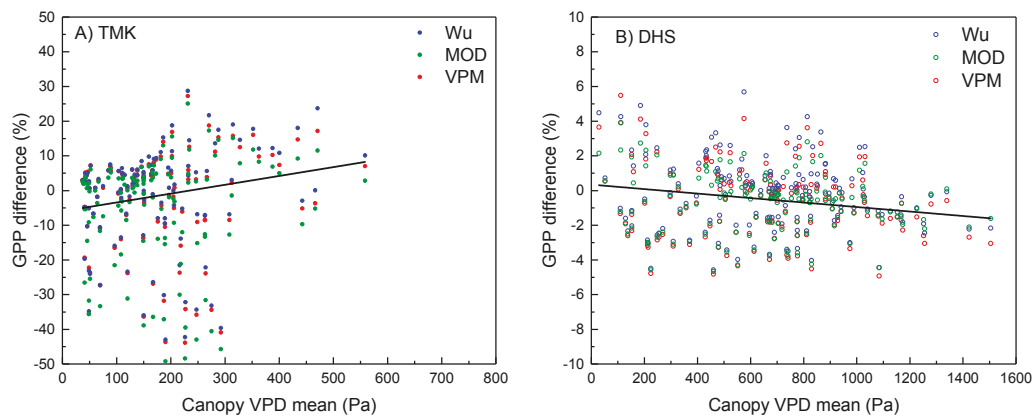

Figure 6. Effects of both considering and not considering the vertical differences in VPD on GPP. The black line is the fit line for the GPP difference scatterplot at different sites. Subfigures (A,B) are the GPP difference comparisons based on different canopy mean VPDs at TMK and DHS, respectively.

\subsubsection{Vertical Stratification of APAR Influenced GPP Estimation by the Multilayer Model}

Forest structure parameters such as LAI influenced the absorption of radiation by the forest canopy, so the total APAR of the forest canopy can be calculated using LAI and incident PAR. In this section, we analyze LAI and incident PAR as influenced by the APAR in different sub-ecosystems and the effect of separate APARs in the sub-ecosystems on GPP modeling.

In a natural environment, the variety of vertical LAI distributions leads to different absorbed PAR distributions in the tree, shrub, and grass layers, which have diverse photosynthetic capacities that lead to GPP modeling biases. Figure 7 shows the total GPP in different LAI distributions. We made two assumptions. The first assumption is that the LUE on a daily scale in different layers is the mean value, which is $1.05 \mathrm{gC} / \mathrm{m}^{2} / \mathrm{MJ}$ in the tree layer, $0.83 \mathrm{gC} / \mathrm{m}^{2} / \mathrm{MJ}$ in the shrub layer, and $1.47 \mathrm{gC} / \mathrm{m}^{2} / \mathrm{MJ}$ in the grass layer. These values are the average values of the results of three models considering optimal growing environments. The APAR for each layer is influenced by only the LAI and the total incident PAR in the top layer. The second assumption is that the LUEmax in the shrub layer is lower than that in the tree and grass layers [32]. Similar to the results at the DHS site, when the LAI ratio in the tree, shrub, and grass layers is 2.5:0.6:0.9 (at DOY 249), GPPm is 5\% higher than GPPs. At the TMK site during the summer, the LAI ratio in the tree and grass layers is 2:1, and GPPm is $15 \%$ higher than GPPs. As in the shrub and grass layers, the understory LAI would thus be the most important factor for GPPdif, and its ratio to total LAI leads to a range of $-40 \%$ to $25 \%$ for the GPP estimation uncertainties.

Incident PAR in the forest canopy also determined the GPP modeling difference whether or not vertical stratification was considered. Figure $8 \mathrm{~A}, \mathrm{C}$ show that considering the effect of vertical stratification on GPP leads to results that are similar to the GPP values that are measured by the flux towers because understory species such as shrubs and grasses have high photosynthetic capacities despite receiving little incident PAR [82]. When the incident PAR of the top of the canopy is lower than $175 \mathrm{~W} / \mathrm{m}^{2}$ at these two sites, the value of the flux tower-based GPP is $1-2 \mathrm{gC} / \mathrm{m}^{2}$ lower than GPP, and is closer to GPPm than GPPs. When the incident PAR is higher than $175 \mathrm{~W} / \mathrm{m}^{2}, \mathrm{GPPm}$ is $0.5-2 \mathrm{gC} / \mathrm{m}^{2}$ higher than the flux tower-based GPP, which has a lower bias than GPPs. 


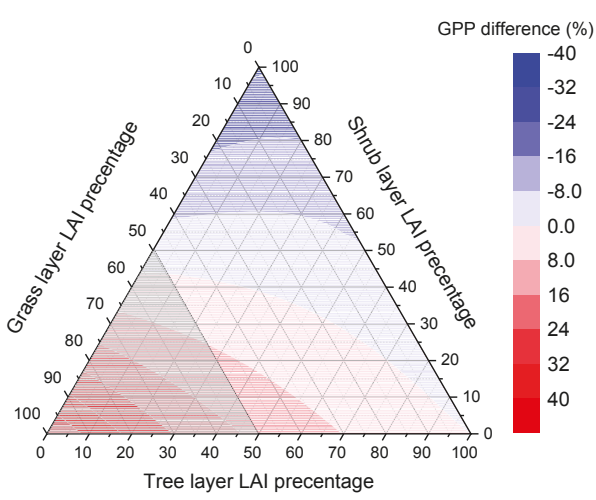

Figure 7. Ternary contour for the differences between considering and not considering the vertical structure during the GPP estimation with LAI separated into tree, shrub, and grass layers. This graph illustrates that when the total LAI of the canopy is four, there is little temperature and humidity stress on the LUE. We assumed that the LUE of the grass layer was $1.28 \mathrm{gC} / \mathrm{m}^{2} /$ day $/ \mathrm{MJ}$, that of the shrub layer was $0.85 \mathrm{gC} / \mathrm{m}^{2} /$ day $/ \mathrm{MJ}$, and that of the tree layer was $1.02 \mathrm{gC} / \mathrm{m}^{2} /$ day $/ \mathrm{MJ}$.
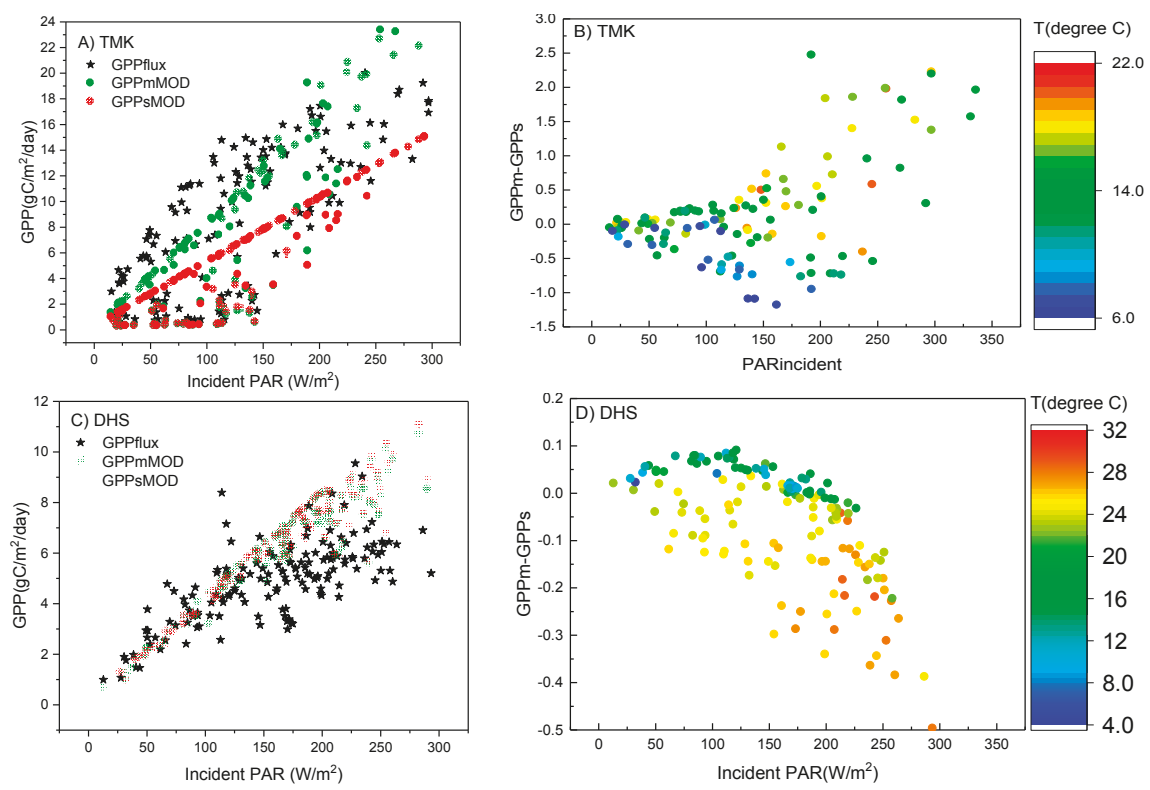

Figure 8. GPP differences considering multilayer and single-layer estimations of incident PAR. Subfigure $(\mathbf{A}, \mathbf{C})$ show the GPP simulation results with flux tower measurements of GPP. The green points represent multilayer results (GPPmMOD), and the red points represent single-layer results (GPPsMOD). The black points represent the flux tower-based GPP results (GPPactual). Subfigure (B,D) show GPP differences under various incident PAR.

Moreover, at high temperatures at the DHS site, the estimation of GPPm is lower than the estimation of GPPs, and the difference between GPPm and GPPs increases with increasing incident PAR (Figure 8B,D). At the TMK site, the difference between GPPm and GPPs increases with increasing PAR, and reaches a maximum of approximately 70\% of GPPs, which is much higher than that at the DHS site. 
When the incident PAR increases, the PAR reaching the understory also increases. Understory layers have higher LUE values, and GPPm is capable of considering the higher photosynthesis from understory components, which explains why GPPm is higher than GPPs. At the TMK site, GPPm is lower than GPPs when the temperature is low, which is the opposite pattern of that at the DHS site. The high temperatures at the DHS site and the low temperatures at the TMK site represent non-optimal growing temperature conditions, under which the biases between GPPm and flux tower-measured GPP are always lower than those between GPPs and flux tower-measured GPP with different degrees of variation at different sites. When the temperature enters the optimal growing temperature range, the estimation of GPPm is higher than that of GPPs, which considers the high photosynthesis capacity of the understory.

\subsection{Performance of GPP Estimation Results Based on Flux Tower-Based GPP}

Estimating GPP with stratification information in the multilayer GPP model results in higher estimation precision than with the single-layer method (Table 4). Table 4 illustrates that the GPPm estimates at the DHS and TMK sites had higher accuracies than the GPPs estimations, and generally had a lower RMSE (GPPm is $0.2 \mathrm{gC} / \mathrm{m}^{2}$ / day lower than GPPs). At the DHS site, the modeled GPP that considered vertical stratification showed a similar correlation when vertical layers were considered, but with the higher LAI of the understory, the $\mathrm{R}^{2}$ value was $2 \%$ to $5 \%$ higher with GPPm than with GPPs at the TMK site. The low mean bias in the multilayer model (DHS_m and TMK_m) indicates that the multilayer model performs better than the single-layer model.

Table 4. Comparison of flux tower-based GPP and different model output results when considering and not considering the vertical layer. Here, sitename_s is the single-layer GPP, sitename_m is the multilayer GPPm, and sitename_adjust uses the same temperature and VPD inputs (root mean square error (RMSE) and mean bias unit is $\mathrm{gC} / \mathrm{m}^{2} /$ day).

\begin{tabular}{cccccccc}
\hline & & DHS_s & DHS_m & DHS_adjust & TMK_s & TMK_m & TMK_adjust \\
\hline \multirow{3}{*}{ VPM } & $\mathrm{R}^{2}$ & 0.587 & 0.588 & 0.589 & 0.738 & 0.769 & 0.753 \\
& RMSE & 0.99 & 0.99 & 0.99 & 2.51 & 2.36 & 2.43 \\
& mean bias & -1.15 & -1.08 & -0.55 & 4.16 & 1.85 & 0.59 \\
\hline \multirow{3}{*}{$\mathrm{Wu}$} & $\mathrm{R}^{2}$ & 0.595 & 0.599 & 0.595 & 0.726 & 0.771 & 0.754 \\
& $\mathrm{RMSE}$ & 0.98 & 0.97 & 0.96 & 2.57 & 2.35 & 2.44 \\
& mean bias & 0.53 & 0.49 & 0.09 & 3.84 & 1.5 & 0.21 \\
\hline \multirow{3}{*}{ MOD } & $\mathrm{R}^{2}$ & 0.491 & 0.489 & 0.491 & 0.777 & 0.801 & 0.786 \\
& RMSE & 1.1 & 1.1 & 1.1 & 2.32 & 2.19 & 2.27 \\
& mean bias & -1.67 & -1.57 & -1.05 & 3.96 & 1.68 & 0.47 \\
\hline
\end{tabular}

These selected GPP estimation models, including VPM, Wu's model, and MOD, showed high correlations with the flux tower-based GPP. Wu's model and the VPM showed higher $\mathrm{R}^{2}$ values than the MOD model at DHS, because they consider scalar temperature at high temperatures. The MOD17 model showed lower RMSE and higher $\mathrm{R}^{2}$ values than both Wu's model and the VPM at the TMK site, indicating that the MOD model exhibits better performance at that site. The estimation results for the two sites were combined, which indicated that $\mathrm{Wu}^{\prime}$ 's model exhibited the best model performance with the highest mean $R^{2}\left(R^{2}=0.685\right)$ and the lowest mean RMSE at these two sites $\left(\mathrm{RMSE}=1.66 \mathrm{gC} / \mathrm{m}^{2} /\right.$ day) when the vertical stratification information was included in the GPP estimation. Figure 9 indicates that Wu's model can track the variations in growing season GPP when the vertical stratification information in the canopy is incorporated. 

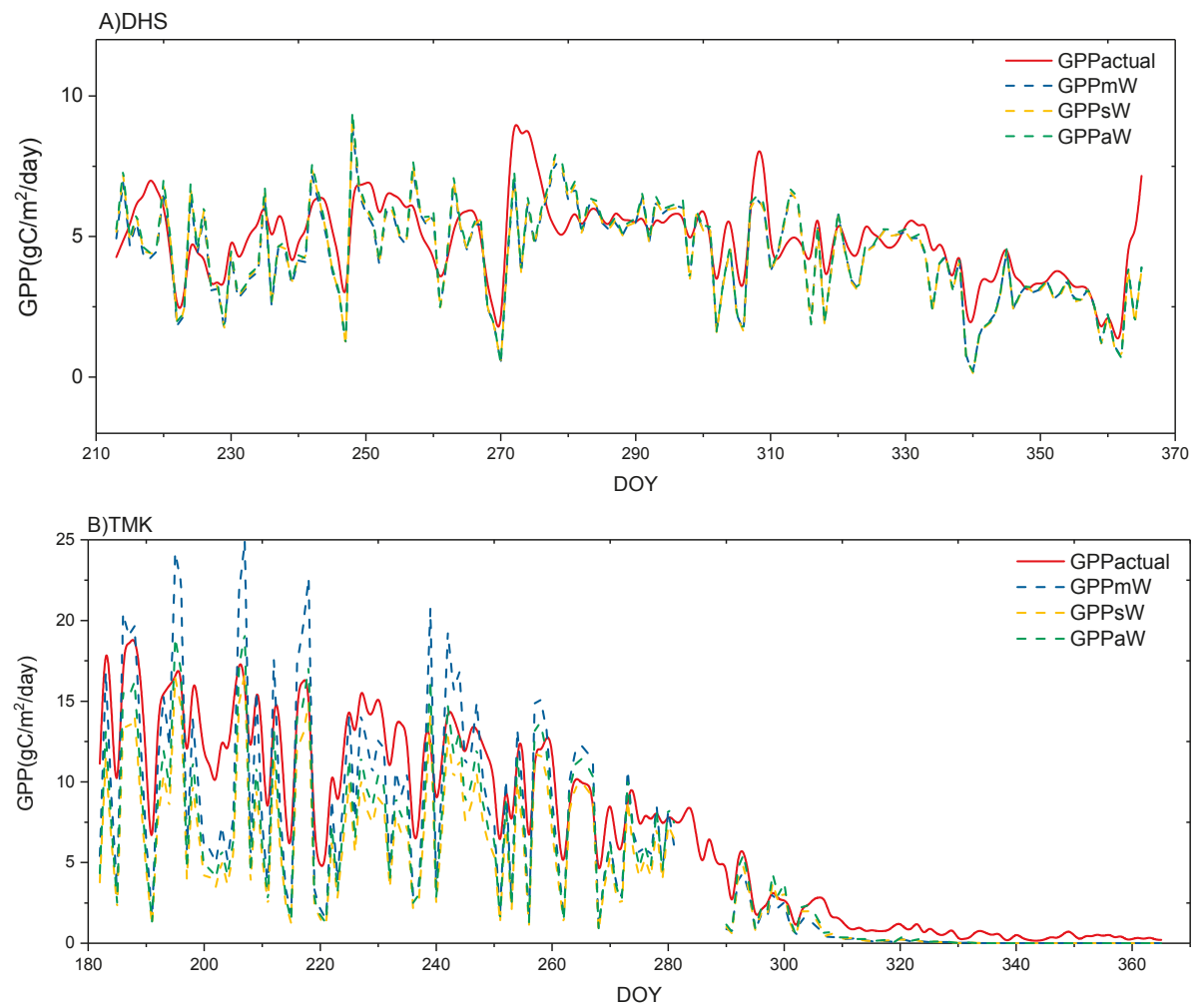

Figure 9. Differences in GPP estimations in different seasons. Subfigure (A) showed the seasonal GPP variance in DHS, while subfigure (B) showed the condition in TMK. Here, we used three different types of LUEmax parametric approaches to model GPP using Wu's method. GPPmW is the multilayer result using two or three different LUEmax inputs for GPP estimation. GPPsW is the single-layer result using an EBF-based LUEmax. GPPaW is the average LUEmax input in GPPmW, using the average LUEmax for the different components.

Simplifying the vertical stratification parameters in the GPP model will also yield high estimation accuracies. To simplify the parameters when considering vertical stratification during GPP estimation, the adjusted method in Table 4 used the same temperature and VPD for every layer as inputs, the LAI values in different sub-ecosystems were separated from that for the total ecosystem, and LUEmax was used for different sub-ecosystems. The results showed that among all the methods, the adjusted method had the smallest mean bias at the TMK and DHS sites. Additionally, the $\mathrm{R}^{2}$ value differed depending on whether or not multilayer models were considered. This method requires the canopy mean temperature and the VPD and LAI proportions in the understory and overstory, the LUEmax set for different sub-ecosystems, and the incident PAR at the top of the canopy. The data used here are easier to obtain than those required by the multilayer GPP estimation method, and the performance results were also better than those when not considering vertical stratification.

Modeled GPP and flux tower-based GPP values still differ to some degree, as illustrated in Figure 10. On a daily scale, the multilayer GPP model has a lower mean bias than the single-layer GPP model. Figure 10b demonstrates that the multilayer method performs well during the growing season (DOY 180 to 280) when the understory LAI is large. On some days when the shortwave incident radiation is high, GPPm was overestimated because the understory PAR was overestimated, but GPPm 
showed little variance when the understory LAI was low (Figure 10a,b DOY 290 to 360). Most of the results estimated by GPPs were lower than the GPP measured by the flux towers because the understory LUEmax was underestimated. Therefore, the GPP estimation method that considered vertical structure would be useful when the understory LAI is high $\left(>1 \mathrm{~m}^{2} / \mathrm{m}^{2}\right)$. Generally, adding the understory vegetation GPP to the total GPP reduces the underestimation when the understory LAI is high.

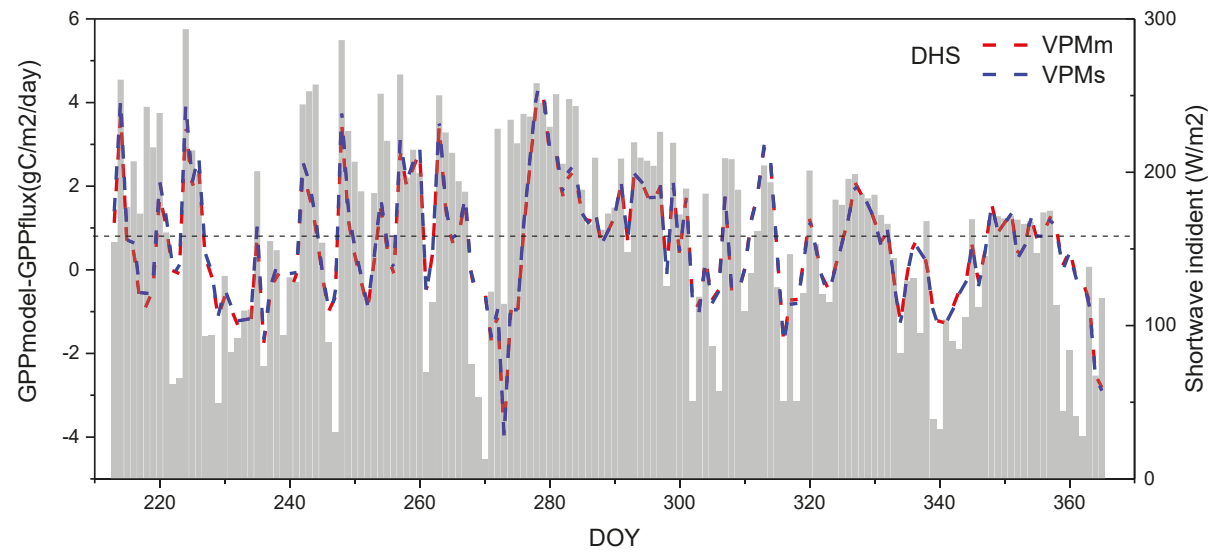

(a)

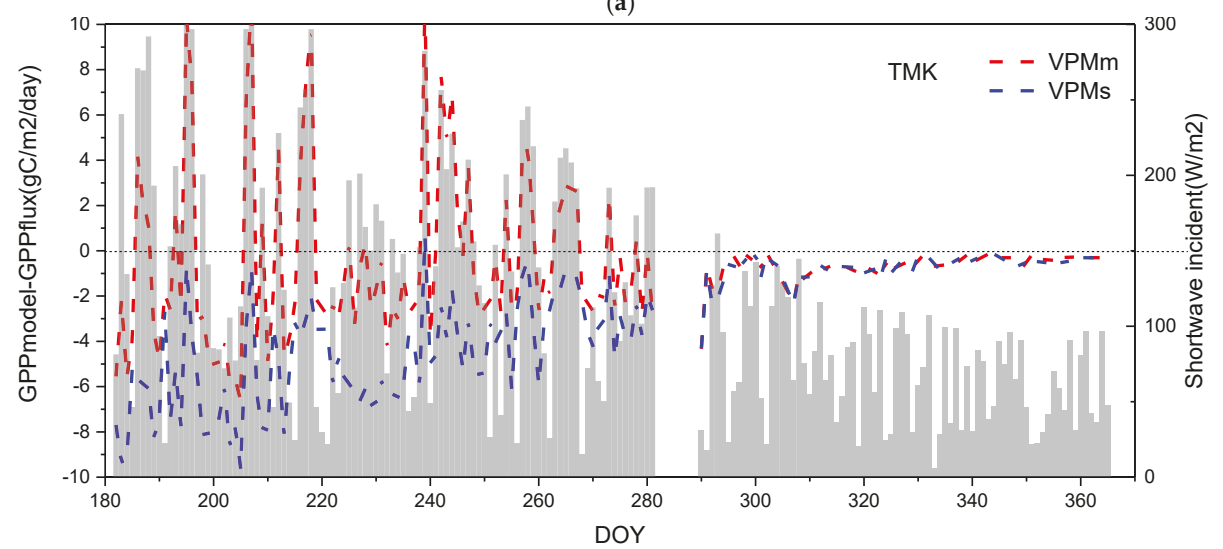

(b)

Figure 10. Differences in GPP estimation using different models with carbon flux tower measurements. Subfigure (a) showed the condition in DHS, and subfigure (b) showed it in TMK. VPMm results from using the multilayer VPM GPP minus the flux tower-based GPP. VPMs results from using the single-layer VPM GPP minus the flux tower-based GPP. The Y-axis on the right is the daily mean shortwave incident radiation.

\section{Discussion}

In this research, we examined the addition of vertical stratification parameters such as temperature, VPD, PAR, LAI, and LUEmax to support remote sensing GPP estimation at the site level. Temperature and VPD influenced the LUEmax at various canopy heights because the vertical meteorology environments have greater variations at forest sites than those in other vegetation 
types, such as shrub and grass sites. PAR and LAI were used to determine the APAR of different sub-ecosystems, which would lead to a great deal of uncertainty in energy allocation.

Vertical LUE is influenced by the vertical distributions of temperature, VPD, and LUEmax, but there are uncertainties when modeling the LUE in different sub-ecosystems. LUEmax values differ in sub-ecosystems, as explained in Section 4.2.1 of this article. At the TMK site, the GPPdif changes from $-50 \%$ to $30 \%$ when the air temperature at the canopy height increases from approximately $7{ }^{\circ} \mathrm{C}$ to $20^{\circ} \mathrm{C}$. The variation is more significant at the TMK site than that at the DHS site, and one reason for this difference is that the temperature differences among layers are more obvious at the TMK site than at the DHS site, as shown in Figure 2. The effect of temperature stress on LUE differs for tree, shrub, and grass layers under the same mean canopy weather conditions. As the optimal temperatures of the tree, shrub, and grass layers differ by $2-5^{\circ} \mathrm{C}$ at the same air temperatures in the forest canopy, the temperature stresses of the layers on LUEmax differ, varying by $5-20 \%$ around the optimal temperature $[78,83]$. However, when the temperature is above $23^{\circ} \mathrm{C}$, GPPdif changes only slightly because the canopy and the understory layers experience high temperature stress, and the LUE would decrease rapidly at this temperature range. On the other hand, the variation in GPPdif did not significantly change with the increase in VPD, as Figure 6B shows that GPPdif at the DHS site decreases only slightly when the VPD increased. Nepstad et al. [84] showed that the water contents of plants are more related to soil moisture than to the water content of the air in seasonally moist forests. The plants in the overstory and understory layers share the soil water content. Thus, the VPD does not have a significant effect on the GPP estimation in dense forests. All of these effects result in different LUE values at distinct canopy heights, as shown by previous research [85].

Uncertainties in the vertical LAI distribution lead to uncertainties in APAR in the sub-ecosystems. LAI, which is a key parameter for separating an ecosystem into different photosynthetic components, determines the contribution of different species to total ecosystem photosynthesis, the light transfer process in the canopy, and the micrometeorological environment inside the forest [58]. As many studies have shown that the distribution of PAR through the canopy structure is highly affected by LAD, LAI, and the characteristics of different species in forest canopies with high heterogeneity, the PAR distribution must consider the vertical structure $[39,58,82]$. The overstory tree layer determines the intercept ratio of the incident PAR, so if the LAI in the overstory is high, a large amount of PAR would be absorbed during the first turn of intercepted light. The vertical LAI distribution in different vegetation covers follows its own rules (Figure 11), and therefore, remote sensing-based understory and overstory LAI estimations are important for separating the APAR of sub-ecosystems and adding unique LUE values when mapping the global GPP [86-89]. For all of the cases in a natural environment, when the total LAI is four, the highest GPP appears when all of the leaves are grass species. As shown in Figure 7, the LAI of the grass layer would not be higher than $2 \mathrm{~m}^{2} / \mathrm{m}^{2}$, and the highest GPP would thus appear when the LAI of both the tree and grass layers is two. The highest GPP in a dense natural forest would have a ratio of 0.5:0:0.5 for the tree, shrub, and grass layers. Under such circumstances, GPPdif reaches its highest value, and increases in the LUE of both the tree and grass layers would produce a greater GPP. The greatest GPP difference is $25 \%$ higher than that estimated via the single-layer method, and if the canopy has a higher proportion of shrubs, the GPP difference is $20 \%$ lower. When the proportion of LAI is high in the shrub layer, the total GPP is lower than that estimated by the single-layer method because the shrub LUE is low. As discussed in Section 4.2.2, a high LAI in the understory produces a high GPP because the understory component absorbs diffuse light and provides a better growing environment than that of the overstory. Figure 10 shows that different ecosystems have different vertical LAI ratios. In forest sites such as EBF, DBF, ENF, and DNF, the tree LAI ratio can be $60 \%$ or more, and the understory LAI can be as a great as $20 \%$ during the growing season. With different LAI distributions in the forest ecosystem, the total APAR allocation of the canopy in a sub-ecosystem would lead to uncertainties in the GPP estimation. 


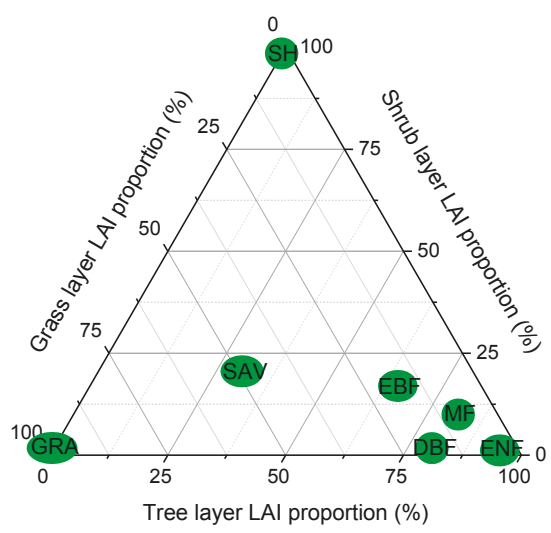

Figure 11. Vertical LAI ratios in different ecosystems (EBF, evergreen broadleaf forest [71,90]; DNF, deciduous needleleaf forest [72]; ENF, evergreen needleleaf forest [91,92]; DBF, deciduous broadleaf forest [26]; SAV, savanna [93]).

Wu's model exhibited the best performance of the three models. This model better considers the effect of temperature on GPP in overstory and understory layers. Wu's model and the VPM consider the temperature stresses when the temperature is high. Therefore, these models exhibit better variance when the canopy temperature is high in both the multilayer model and single-layer model. In the VPM, phenology was proven to be an important indicator. However, at the site level, the VPM cannot obtain accurate spatially matched enhanced vegetation index (EVI) values, and the LAI of the canopy changes only slightly during the growing season; thus, we omitted the effect of phenology. In future research on multilayer GPP models, the phenology variance between understory and overstory layers would be a potential way to improve the GPP estimation accuracy in the VPM and Wu's model.

The continuous vertical acquisition of LAI is also challenging. On the one hand, many researchers have shown that remote sensing methods underestimate understory LAI, indicating that other GPP components are also underestimated in dense forests $[39,94]$. On the other hand, most remote sensing-based LAI products currently do not separate the overstory from the understory in forest sites. Without the LAI of different layers, the GPP of distinct vertical layers cannot be modeled; thus, understory GPP is often underestimated from the high understory LUE.

Future versions of remote sensing-based GPP products should add forest vertical stratification information to GPP models. For example, the use of remote sensing-based understory and overstory LAI [90-93] to separate sub-ecosystems and the addition of prior LUE when combining the specific LUEmax from sub-ecosystem-based temperature and VPD response functions can improve GPP estimations compared to the performance of approaches that do not consider the vertical structure ("adjusted" method in Table 4). The 'adjusted' method in Table 4 showed lower systemic biases and a RMSE that was similar to that of GPPm, which indicated that this method has an estimation accuracy that is similar to that of the multilayer method. However, this method requires only the two main data sources as prior information, the LUEmax in height of the canopy, the canopy mean air temperature, VPD, and the LAI values of the sub-ecosystems, which are simple to obtain via remote sensing. This method can potentially be applied in remote sensing-based multilayer GPP models. Thus, a high GPP estimation accuracy could be obtained in ecosystems with high understory LAI values by considering the vertical structure (multilayer model).

The accuracy of GPP estimates in a complicated, vertically stratified forest canopy where the understory LAI is high (e.g., in a tropical rainforest or old-growth forest) can be improved by using multilayer methods. Additionally, Wu's model showed that a potential model could estimate the GPP with multilayer data inputs, but further global validation of the GPP model parameters is needed. 
Some articles have added vertical stratification data to GPP estimations in cropland $[95,96]$, but unlike the homogeneous landscape in croplands, a vertical structure would lead to variations in the physiological parameters of vegetation in forest sites. Thus, there are still some limitations to this study.

\section{Conclusions}

In this article, we used three different LUE-based GPP models to analyze the effect of vertical vegetation stratification on GPP calculations. Additionally, in situ temperature, VPD, and PAR data were used to analyze the environmental factors that control GPP modeling. The results showed the following. (1) Vertical temperature and VPD stratification at the selected sites varied with the forest type, time of day, and season, but the difference was not significant. Moreover, incident PAR followed the Beer-Lambert law; there was a significant difference between the overstory and understory layers, and this difference was affected by the distributions of LAI in different sub-ecosystems. (2) The stratifications of temperature, VPD, PAR, LAI, and LUEmax have an interactive influence on the improvement of GPPm. Due to the importance of PAR in the estimation of GPP, the stratification of PAR becomes obvious when the incident PAR increases and the difference between GPPm and GPPs exceeds $50 \%$ of GPPs. GPPdif is approximately $30 \%$ when temperature stratification is considered, and this value is $20 \%$ when LAI stratification is considered. Multilayer LUEmax inputs are important for accurate GPP estimations, and different inputs may lead to contrary results concerning the difference between GPPm and GPPs. Therefore, quantitatively corrected understory LUEmax reduces the bias in GPP estimations. (3) The accuracy of the GPP estimation can be improved by using multilayer models, but the improvement is related to the forest type, the stratification of the canopy, and the growing conditions. Furthermore, the improvement is more obvious when the multilayer model is applied to canopies with obvious vertical stratification under optimal growing conditions. In this article, GPPm was the most accurate at the TMK site from July to September. Since a multilayer GPP estimation model considers the high photosynthetic ability and LAI of grass, GPPm is always larger than GPPs. However, when the conditions are not optimal for growth-for example, if the temperature becomes very low or high-the GPPm will be smaller than the GPPs.

In future studies on GPP remote sensing products, vertical stratification should be considered in the models. Adding overstory and understory information such as LAI and LUEmax for diverse sub-ecosystems can improve the estimation accuracy of the GPP product.

Supplementary Materials: The following are available online at http://www.mdpi.com/2072-4292/10/9/1329/ s1. Models and input parameters. Table S1: Parameter inputs in different models.

Author Contributions: J.L., Q.L. and S.L. conceived and designed the experiments. S.L. and A.H. analyzed the data. S.L., J.L., Q.L., A.H. and L.L. wrote the original paper and completed the revisions.

Funding: This work was supported by the National Natural Science Foundation of China (No. 41671374) and the National Key R\&D Program of China (2017YFA0603001).

Acknowledgments: We would also like to acknowledge Guoyi Zhou and Junhua Yan from the South China Botanical Garden, CAS, for providing the experimental data for the DHS site. We thank Nobuko Saigusa, Ryuichi Hirata and Reiko Ide from the National Institute for Environmental Studies, who provided the in situ measurement data for the TMK site. We acknowledge Gerald Flerchinger from the USDA Agricultural Research Service, who offered us valuable suggestions concerning the modeling. And we thank the anonymous reviewers gave us valuable suggestions to improve this research.

Conflicts of Interest: The authors declare no conflicts of interest.

\section{References}

1. Litton, C.M.; Raich, J.W.; Ryan, M.G. Carbon allocation in forest ecosystems. Glob. Chang. Boil. 2007, 13, 2089-2109. [CrossRef]

2. Bonan, G.B. Forests and Climate Change: Forcings, Feedbacks, and the Climate Benefits of Forests. Science 2008, 320, 1444. [CrossRef] [PubMed] 
3. Beer, C.; Reichstein, M.; Tomelleri, E.; Ciais, P.; Jung, M.; Carvalhais, N.; Rödenbeck, C.; Arain, M.A.; Baldocchi, D.; Bonan, G.B. Terrestrial gross carbon dioxide uptake: Global distribution and covariation with climate. Science 2010, 329, 834-838. [CrossRef] [PubMed]

4. Running, S.W.; Baldocchi, D.D.; Turner, D.P.; Gower, S.T.; Bakwin, P.S.; Hibbard, K.A. A Global Terrestrial Monitoring Network Integrating Tower Fluxes, Flask Sampling, Ecosystem Modeling and EOS Satellite Data. Remote Sens. Environ. 1999, 70, 108-127. [CrossRef]

5. Andrewd, F.; Almut, A.; Nancyy, K.; Mark, L.; Jérôme, O.; Christian, R.; Stevenw, R.; Jeandiego, S.; Stephen, S.; Nicolas, V. FLUXNET and modelling the global carbon cycle. Glob. Chang. Boil. 2007, 13, 610-633.

6. Campbell, J.E.; Berry, J.A.; Seibt, U.; Smith, S.J.; Montzka, S.A.; Launois, T.; Belviso, S.; Bopp, L.; Laine, M. Large historical growth in global terrestrial gross primary production. Nature 2017, 544, 84. [CrossRef] [PubMed]

7. Vitousek, P.M.; Aber, J.D.; Howarth, R.W.; Likens, G.E.; Matson, P.A.; Schindler, D.W.; Schlesinger, W.H.; Tilman, D.G. Human Alteration of the global nitrogen cycle: sources and consequences. Ecol. Appl. 1997, 7, 737-750. [CrossRef]

8. Zhao, M.; Running, S.W. Drought-induced reduction in global terrestrial net primary production from 2000 through 2009. Science 2010, 329, 940. [CrossRef] [PubMed]

9. Jung, M.; Reichstein, M.; Margolis, H.A.; Cescatti, A.; Richardson, A.D.; Arain, M.A.; Arneth, A.; Bernhofer, C.; Bonal, D.; Chen, J. Global patterns of land-atmosphere fluxes of carbon dioxide, latent heat, and sensible heat derived from eddy covariance, satellite, and meteorological observations. J. Geophys. Res. Biogeosci. 2015, 116, 245-255.

10. Zhao, M.; Heinsch, F.A.; Nemani, R.R.; Running, S.W. Improvements of the MODIS terrestrial gross and net primary production global data set. Remote Sens. Environ. 2005, 95, 164-176. [CrossRef]

11. Alton, P.B.; North, P.R.; Los, S.O. The impact of diffuse sunlight on canopy light-use efficiency, gross photosynthetic product and net ecosystem exchange in three forest biomes. Glob. Chang. Boil. 2007, 13, 776-787. [CrossRef]

12. Harper, A.B.; Cox, P.M.; Friedlingstein, P.; Wiltshire, A.J.; Jones, C.D.; Sitch, S.; Mercado, L.M.; Groenendijk, M.; Robertson, E.; Kattge, J. Improved representation of plant functional types and physiology in the Joint UK Land Environment Simulator (JULES v4.2) using plant trait information. Geosci. Model Dev. 2016, 9, 1-64. [CrossRef]

13. Jiang, C.; Ryu, Y. Multi-scale evaluation of global gross primary productivity and evapotranspiration products derived from Breathing Earth System Simulator (BESS). Remote Sens. Environ. 2016, 186, 528-547. [CrossRef]

14. Chen, J.M.; Liu, J.; Cihlar, J.; Goulden, M.L. Daily canopy photosynthesis model through temporal and spatial scaling for remote sensing applications. Ecol. Model. 1999, 124, 99-119. [CrossRef]

15. Ju, W.; Chen, J.; Liu, R.; Liu, Y. Changes of global terrestrial carbon budget and major drivers in recent 30 years simulated using the remote sensing driven BEPS model. In AGU Fall Meeting Abstracts; American Geophysical Union: Washington, DC, USA, 2013; B23A-0535.

16. Keenan, R.J.; Reams, G.A.; Achard, F.; Freitas, J.V.D.; Grainger, A.; Lindquist, E. Dynamics of global forest area: Results from the FAO Global Forest Resources Assessment 2015. For. Ecol. Manag. 2015, 352, 9-20. [CrossRef]

17. Malhi, Y.; Baldocchi, D.D.; Jarvis, P.G. The carbon balance of tropical, temperate and boreal forests. Plant Cell Environ. 1999, 22, 715-740. [CrossRef]

18. Nilsson, M.C.; Wardle, D.A. Understory vegetation as a forest ecosystem driver: Evidence from the northern Swedish boreal forest. Front. Ecol. Environ. 2005, 3, 421-428. [CrossRef]

19. Thiffault, N.; Fenton, N.J.; Munson, A.D.; Hébert, F.; Fournier, R.A.; Valeria, O.; Bradley, R.L.; Bergeron, Y.; Grondin, P.; Paré, D. Managing Understory Vegetation for Maintaining Productivity in Black Spruce Forests: A Synthesis within a Multi-Scale Research Model. Forests 2013, 4, 613-631. [CrossRef]

20. Kolari, P.; Pumpanen, J.; Kulmala, L.; Ilvesniemi, H.; Nikinmaa, E.; Gronholm, T.; Hari, P. Forest floor vegetation plays an important role in photosynthetic production of boreal forests. For. Ecol. Manag. 2006, 221, 241-248. [CrossRef]

21. Kim, D.; Oren, R.; Qian, S.S. Response to $\mathrm{CO}_{2}$ enrichment of understory vegetation in the shade of forests. Glob. Chang. Boil. 2016, 22, 944. [CrossRef] [PubMed] 
22. Misson, L.; Baldocchi, D.D.; Black, T.A.; Blanken, P.D.; Brunet, Y.; Curiel, Y.J.; Dorsey, J.R.; Falk, M.; Granier, A.; Irvine, M.R. Partitioning forest carbon fluxes with overstory and understory eddy-covariance measurements: A synthesis based on FLUXNET data. Agric. For. Meteorol. 2007, 144, 14-31. [CrossRef]

23. Moore, C.E.; Beringer, J.; Evans, B.; Hutley, L.B.; Mchugh, I.; Tapper, N.J. The contribution of trees and grasses to productivity of an Australian tropical savanna. Biogeosci. Discuss. 2016, 12, 19307-19350. [CrossRef]

24. Sakai, T.; Akiyama, T.; Saigusa, N.; Yamamoto, S.; Yasuoka, Y. The contribution of gross primary production of understory dwarf bamboo, Sasa senanensis, in a cool-temperate deciduous broadleaved forest in central Japan. For. Ecol. Manag. 2007, 236, 259-267. [CrossRef]

25. He, L.; Chen, J.M.; Gonsamo, A.; Luo, X.; Wang, R.; Liu, Y.; Liu, R. Changes in the Shadow: The Shifting Role of Shaded Leaves in Global Carbon and Water Cycles under Climate Change. Geophys. Res. Lett. 2018, 45, 5052-5061. [CrossRef]

26. Black, T.A.; Hartog, G.D.; Neumann, H.H.; Blanken, P.D.; Yang, P.C.; Russell, C.; Nesic, Z.; Lee, X.; Chen, S.G.; Staebler, R. Annual cycles of water vapour and carbon dioxide fluxes in and above a boreal aspen forest. Glob. Chang. Boil. 1996, 2, 219-229. [CrossRef]

27. Landhäusser, S.M.; Lieffers, V.J. Photosynthesis and carbon allocation of six boreal tree species grown in understory and open conditions. Tree Physiol. 2001, 21, 243-250. [CrossRef] [PubMed]

28. Pons, T.L.; Westbeek, M.H.M. Analysis of differences in photosynthetic nitrogen-use efficiency between four contrasting species. Physiol. Plant. 2004, 122, 68-78. [CrossRef]

29. Mielke, M.S.; Almeida, A.A.F.D.; Gomes, F.P. Photosynthetic traits of five neotropical rainforest tree species: Interactions between light response curves and leaf-to-air vapour pressure deficit. Braz. Arch. Boil. Technol. 2005, 48, 815-824. [CrossRef]

30. Xue, B.L.; Kumagai, T.O.; Iida, S.I.; Nakai, T.; Matsumoto, K.; Komatsu, H.; Otsuki, K.; Ohta, T. Influences of canopy structure and physiological traits on flux partitioning between understory and overstory in an eastern Siberian boreal larch forest. Ecol. Model. 2011, 222, 1479-1490. [CrossRef]

31. Yuan, W.; Cai, W.; Xia, J.; Chen, J.; Liu, S.; Dong, W.; Merbold, L.; Law, B.; Arain, A.; Beringer, J. Global comparison of light use efficiency models for simulating terrestrial vegetation gross primary production based on the LaThuile database. Agric. For. Meteorol. 2014, 192-193, 108-120. [CrossRef]

32. Zhou, Y.; Wu, X.; Ju, W.; Chen, J.M.; Wang, S.; Wang, H.; Yuan, W.; Andrew Black, T.; Jassal, R.; Ibrom, A. Global parameterization and validation of a two-leaf light use efficiency model for predicting gross primary production across FLUXNET sites. J. Geophys. Res. 2016, 121, 1045-1072. [CrossRef]

33. Gebremichael, M.; Barros, A.P. Evaluation of MODIS Gross Primary Productivity (GPP) in tropical monsoon regions. Remote Sens. Environ. 2006, 100, 150-166. [CrossRef]

34. He, M.; Zhou, Y.; Ju, W.; Chen, J.; Li, Z.; Wang, S.; Saigusa, N.; Hirata, R.; Murayama, S.; Liu, Y. Evaluation and improvement of MODIS gross primary productivity in typical forest ecosystems of East Asia based on eddy covariance measurements. J. For. Res. 2013, 18, 31-40. [CrossRef]

35. Propastin, P.; Ibrom, A.; Knohl, A.; Erasmi, S. Effects of canopy photosynthesis saturation on the estimation of gross primary productivity from MODIS data in a tropical forest. Remote Sens. Environ. 2012, 121, 252-260. [CrossRef]

36. Wolf, A.; Akshalov, K.; Saliendra, N.; Johnson, D.A.; Laca, E.A. Inverse estimation of Vcmax, leaf area index, and the Ball-Berry parameter from carbon and energy fluxes. J. Geophys. Res. Atmos. 2006, 111. [CrossRef]

37. Bonan, G.B.; Oleson, K.W.; Fisher, R.A.; Lasslop, G.; Reichstein, M. Reconciling leaf physiological traits and canopy flux data: Use of the TRY and FLUXNET databases in the Community Land Model version 4. J. Geophys. Res. Biogeosci. 2015, 117, 313-325. [CrossRef]

38. He, L.; Chen, J.M.; Pisek, J.; Schaaf, C.B.; Strahler, A.H. Global clumping index map derived from the MODIS BRDF product. Remote Sens. Environ. 2012, 119, 118-130. [CrossRef]

39. Govind, A.; Guyon, D.; Roujean, J.L.; Yauschew-Raguenes, N.; Kumari, J.; Pisek, J.; Wigneron, J.P. Effects of canopy architectural parameterizations on the modeling of radiative transfer mechanism. Ecol. Model. 2013, 251, 114-126. [CrossRef]

40. Brooks, J.R.; Flanagan, L.B.; Varney, G.T.; Ehleringer, J.R. Vertical gradients in photosynthetic gas exchange characteristics and refixation of respired $\mathrm{CO}_{2}$ within boreal forest canopies. Tree Physiol. 1997, 17, 1-12. [CrossRef] [PubMed]

41. Beaumont, S.; Burns, K.C. Vertical gradients in leaf trait diversity in a New Zealand forest. Trees 2009, 23, 339-346. [CrossRef] 
42. Coble, A.P.; Vanderwall, B.; Mau, A.; Cavaleri, M.A. How vertical patterns in leaf traits shift seasonally and the implications for modeling canopy photosynthesis in a temperate deciduous forest. Tree Physiol. 2016, 36, 1077. [CrossRef] [PubMed]

43. Slot, M.; Winter, K. Photosynthetic acclimation to warming in tropical forest tree seedlings. J. Exp. Bot. 2017, 68, 2275-2284. [CrossRef] [PubMed]

44. Tan, Z.H.; Zeng, J.; Zhang, Y.J.; Slot, M.; Gamo, M.; Hirano, T.; Kosugi, Y.; Rocha, H.R.D.; Saleska, S.R.; Goulden, M.L. Optimum air temperature for tropical forest photosynthesis: Mechanisms involved and implications for climate warming. Environ. Res. Lett. 2017, 12, 054022. [CrossRef]

45. Man, R.; Yang, H.; Schnare, J.W. Data on overstory and understory trees in aspen-dominated boreal mixedwood stands over 20 years after partial harvesting. Data Brief 2018, 17, 284-287. [CrossRef] [PubMed]

46. Ames, G.M.; Anderson, S.M.; Ungberg, E.A.; Wright, J.P. Functional traits of the understory plant community of a pyrogenic longleaf pine forest across environmental gradients. Ecology 2017, 98, 2225. [CrossRef] [PubMed]

47. Jagodziński, A.M.; Horodecki, P.; Rawlik, K.; Dyderski, M.K. Do understory or overstory traits drive tree encroachment on a drained raised bog? Plant Boil. 2017, 19, 571-583. [CrossRef] [PubMed]

48. Sterck, F.; Schieving, F. Modelling functional trait acclimation for trees of different height in a forest light gradient: Emergent patterns driven by carbon gain maximization. Tree Physiol. 2011, 31, 1024-1037. [CrossRef] [PubMed]

49. Schimel, D.; Pavlick, R.; Fisher, J.B.; Asner, G.P.; Saatchi, S.; Townsend, P.; Miller, C.; Frankenberg, C.; Hibbard, K.; Cox, P. Observing terrestrial ecosystems and the carbon cycle from space. Glob. Chang. Boil. 2015, 21, 1762. [CrossRef] [PubMed]

50. Alsaidi, A.; Fukuzawa, Y.; Furukawa, N.; Ueno, M.; Baba, S.; Kawamitsu, Y. A system for the measurement of vertical gradients of $\mathrm{CO}_{2}, \mathrm{H}_{2} \mathrm{O}$ and air temperature within and above the canopy of plant. Plant Prod. Sci. 2009, 12, 139-149. [CrossRef]

51. Lantinga, E.A.; Nassiri, M.; Kropff, M.J. Modelling and measuring vertical light absorption within grass-clover mixtures. Agric. For. Meteorol. 1999, 96, 71-83. [CrossRef]

52. Ohkubo, S.; Kosugi, Y.; Takanashi, S.; Matsuo, N.; Tani, M.; Nik, A.R. Vertical profiles and storage fluxes of $\mathrm{CO}_{2}$, heat and water in a tropical rainforest at Pasoh, Peninsular Malaysia. Tellus 2008, 60, 569-582. [CrossRef]

53. Kenlonishida, N.; Hiroyuki, M.; Shin, N.; Hiroaki, M. Vertical integration of leaf area index in a Japanese deciduous broad-leaved forest. Agric. For. Meteorol. 2008, 148, 1136-1146.

54. Kobayashi, H.; Baldocchi, D.D.; Ryu, Y.; Chen, Q.; Ma, S.; Osuna, J.L.; Ustin, S.L. Modeling energy and carbon fluxes in a heterogeneous oak woodland: A three-dimensional approach. Agric. For. Meteorol. 2012, 152, 83-100. [CrossRef]

55. Poorter, L.; Oberbauer, S.F.; Clark, D.B. Leaf Optical Properties Along a Vertical Gradient in a Tropical Rain Forest Canopy in Costa Rica. Am. J. Bot. 1995, 82, 1257-1263. [CrossRef]

56. Gallego, H.A.; Rico, M.; Moreno, G.; Santa, R.I. Leaf water potential and stomatal conductance in Quercus pyrenaica Willd. forests: Vertical gradients and response to environmental factors. Tree Physiol. 1994, 14, 1039-1047. [CrossRef] [PubMed]

57. Flerchinger, G.N.; Reba, M.L.; Link, T.E.; Marks, D. Modeling temperature and humidity profiles within forest canopies. Agric. For. Meteorol. 2015, 213, 251-262. [CrossRef]

58. Kuusk, A. Absorption profiles of shortwave radiation in a vegetation canopy. Agric. For. Meteorol. 1992, 62, 191-204. [CrossRef]

59. Widlowski, J.L.; Mio, C.; Disney, M.; Adams, J.; Andredakis, I.; Atzberger, C.; Brennan, J.; Busetto, L.; Chelle, M.; Ceccherini, G. The fourth phase of the radiative transfer model intercomparison (RAMI) exercise: Actual canopy scenarios and conformity testing. Remote Sens. Environ. 2015, 169, 418-437. [CrossRef]

60. Widlowski, J.L.; Pinty, B.; Lopatka, M.; Atzberger, C.; Buzica, D.; Chelle, M.; Disney, M.; Gastellu-Etchegorry, J.P.; Gerboles, M.; Gobron, N. The fourth radiation transfer model intercomparison (RAMI-IV): Proficiency testing of canopy reflectance models with ISO-13528. J. Geophys. Res. Atmos. 2013, 118, 6869-6890. [CrossRef]

61. Tang, H.; Dubayah, R.; Swatantran, A.; Hofton, M.; Sheldon, S.; Clark, D.B.; Blair, B. Retrieval of vertical LAI profiles over tropical rain forests using waveform lidar at La Selva, Costa Rica. Remote Sens. Environ. 2012, 124, 242-250. [CrossRef] 
62. Coops, N.C.; Hermosilla, T.; Hilker, T.; Black, T.A. Linking stand architecture with canopy reflectance to estimate vertical patterns of light-use efficiency. Remote Sens. Environ. 2017, 194, 322-330. [CrossRef]

63. Wu, J.; Guan, K.; Hayek, M.; Restrepocoupe, N.; Wiedemann, K.T.; Xu, X.; Wehr, R.; Christoffersen, B.O.; Miao, G.; Da, S.R. Partitioning controls on Amazon forest photosynthesis between environmental and biotic factors at hourly to inter-annual time scales. Glob. Chang. Boil. 2017, 23, 1240. [CrossRef] [PubMed]

64. Kosugi, Y.; Takanashi, S.; Tani, M.; Ohkubo, S.; Matsuo, N.; Itoh, M.; Noguchi, S.; Nik, A.R. Effect of inter-annual climate variability on evapotranspiration and canopy $\mathrm{CO}_{2}$ exchange of a tropical rainforest in Peninsular Malaysia. J. For. Res. 2012, 17, 227-240. [CrossRef]

65. Monteith, J.L. Solar radiation and productivity in tropical ecosystems. J. Appl. Ecol. 1972, 9, 747-766. [CrossRef]

66. Xiao, X.; Zhang, Q.; Braswell, B.; Urbanski, S.; Boles, S.; Wofsy, S.; Iii, B.M.; Ojima, D. Modeling gross primary production of temperate deciduous broadleaf forest using satellite images and climate data. Remote Sens. Environ. 2004, 91, 256-270. [CrossRef]

67. Running, S.W.; Nemani, R.R.; Heinsch, F.A.; Zhao, M.S.; Reeves, M.; Hashimoto, H. A continuous satellite-derived measure of global terrestrial primary production. Bioscience 2004, 54, 547-560. [CrossRef]

68. Wang, C.; Zhou, G.; Tang, X.; Wang, X.; Zhou, C.; Yu, G.; Tang, L.; Meng, Z. Ecosystem respiration and its controlling factors in a coniferous and broad-leaved mixed forest in Dinghushan, China. Acta Ecol. Sin. 2007, 27, 2659-2668. [CrossRef]

69. Wang, C.; Zhou, G.; Wang, X.; Tang, X.; Zhou, C.; Yu, G. Below-canopy $\mathrm{CO}_{2}$ flux and its environmental response characteristics in a coniferous and broad-leaved mixed forest in Dinghushan, China. Acta Ecol. Sin. 2007, 27, 846-853. [CrossRef]

70. Zhang, L.M.; Yu, G.R.; Sun, X.M.; Wen, X.F.; Ren, C.Y.; Fu, Y.L.; Li, Q.K.; Li, Z.Q.; Liu, Y.F.; Guanc, D.X. Seasonal variations of ecosystem apparent quantum yield (alpha) and maximum photosynthesis rate (P-max) of different forest ecosystems in China. Agric. For. Meteorol. 2006, 137, 176-187. [CrossRef]

71. Yu, G.R.; Wen, X.F.; Sun, X.M.; Tanner, B.D.; Lee, X.; Chen, J.Y. Overview of ChinaFLUX and evaluation of its eddy covariance measurement. Agric. For. Meteorol. 2006, 137, 125-137. [CrossRef]

72. Hirata, R.; Hirano, T.; Saigusa, N.; Fujinuma, Y.; Inukai, K.; Kitamori, Y.; Takahashi, Y. Seasonal and interannual variations in carbon dioxide exchange of a temperate larch forest. Agric. For. Meteorol. 2007, 147, 110-124. [CrossRef]

73. Peel, M.C.; Finlayson, B.L.; McMahon, T.A. Updated world map of the köppen-geiger climate classification. Hydrol. Earth Sys. Sci. 2007, 11, 259-263. [CrossRef]

74. Ueyama, M.; Hirata, R.; Mano, M.; Hamotani, K. Influences of various calculation options on heat, water and carbon fluxes determined by open- and closed-path eddy covariance methods. Tellus Ser. B-Chem. Phys. Meteorol. 2011, 64, 91-102. [CrossRef]

75. Jakubauskas, M.E. Harmonic analysis of time-series avhrr ndvi data. Photogr. Eng. Remote Sens. 2001, 67, 461-470.

76. Madani, N.; Kimball, J.S.; Affleck, D.L.R.; Kattge, J.; Graham, J.; Bodegom, P.M.; Reich, P.B.; Running, S.W. Improving ecosystem productivity modeling through spatially explicit estimation of optimal light use efficiency. J. Geophys. Res. Biogeosci. 2015, 119, 1755-1769. [CrossRef]

77. Maselli, F.; Barbati, A.; Chiesi, M.; Chirici, G.; Corona, P. Use of remotely sensed and ancillary data for estimating forest gross primary productivity in Italy. Remote Sens. Environ. 2006, 100, 563-575. [CrossRef]

78. Maselli, F.; Papale, D.; Puletti, N.; Chirici, G.; Corona, P. Combining remote sensing and ancillary data to monitor the gross productivity of water-limited forest ecosystems. Remote. Sens. Environ. 2009, 113, 657-667. [CrossRef]

79. Campbell, G.S.; Norman, J.M. Radiation Fluxes in Natural Environments; Springer: New York, NY, USA, 1998; pp. 167-184.

80. Gastellu-Etchegorry, J.P.; Trichon, V. A modeling approach of PAR environment in a tropical rain forest in Sumatra: Application to remote sensing. Ecol. Model. 1998, 108, 237-264. [CrossRef]

81. Gastelluetchegorry, J.P.; Yin, T.; Lauret, N.; Cajgfinger, T.; Gregoire, T.; Grau, E.; Feret, J.B.; Lopes, M.; Guilleux, J.; Dedieu, G. Discrete Anisotropic Radiative Transfer (DART 5) for Modeling Airborne and Satellite Spectroradiometer and LIDAR Acquisitions of Natural and Urban Landscapes. Remote Sens. 2015, 7, 1667-1701. [CrossRef] 
82. Montgomery, R.A.; Chazdon, R.L. Forest structure, canopy architecture, and light transmittance in tropical wet forests. Ecology 2001, 82, 2707-2718. [CrossRef]

83. Pettigrew, C.A.; Breen, A.; Corcoran, C.; Sayler, G.S. Chlorinated biphenyl mineralization by individual populations and consortia of freshwater bacteria. Appl. Environ. Microbiol. 1990, 56, 2036-2045. [PubMed]

84. Nepstad, D.C.; Carvalho, C.R.D.; Davidson, E.A.; Jipp, P.H.; Lefebvre, P.A.; Negreiros, G.H.; Silva, E.D.D.; Stone, T.A.; Trumbore, S.E.; Vieira, S. The role of deep roots in the hydrological and carbon cycles of Amazonian forests and pastures. Nature 1994, 372, 666-669. [CrossRef]

85. Onoda, Y.; Saluñga, J.B.; Akutsu, K.; Aiba, S.I.; Yahara, T.; Anten, N.P.R. Trade-off between light interception efficiency and light use efficiency: Implications for species coexistence in one-sided light competition. J. Ecol. 2014, 102, 167-175. [CrossRef]

86. Liu, Y.; Liu, R.; Pisek, J.; Chen, J.M. Separating overstory and understory leaf area indices for global needleleaf and deciduous broadleaf forests by fusion of MODIS and MISR data. Biogeosciences 2017, 14, 1-32. [CrossRef]

87. Pisek, J.; Chen, J.; Kobayashi, H.; Rautiainen, M.; Schaepman, M.; Karnieli, A.; Sprintsin, M.; Ryu, Y.; Nikopensius, M.; Raabe, K. Retrieval of seasonal dynamics of forest understory reflectance from semi-arid to boreal forests using MODIS BRDF data. In Proceedings of the EGU General Assembly Conference, Vienna, Austria, 17-22 April 2016.

88. Pisek, J.; Rautiainen, M.; Heiskanen, J.; Mõttus, M. Retrieval of seasonal dynamics of forest understory reflectance in a Northern European boreal forest from MODIS BRDF data. Remote Sens. Environ. 2012, 117, 464-468. [CrossRef]

89. Pisek, J.; Rautiainen, M.; Nikopensius, M.; Raabe, K. Estimation of seasonal dynamics of understory NDVI in northern forests using MODIS BRDF data: Semi-empirical versus physically-based approach. Remote Sens. Environ. 2015, 163, 42-47. [CrossRef]

90. Hutyra, L.R.; Munger, J.W.; Saleska, S.R.; Gottlieb, E.; Daube, B.C.; Dunn, A.L.; Amaral, D.F.; De Camargo, P.B.; Wofsy, S.C. Seasonal controls on the exchange of carbon and water in an Amazonian rain forest. J. Geophys. Res. Biogeosci. 2015, 112, 488-497. [CrossRef]

91. Falk, M.; Kyaw, T.P.U.; Wharton, S.; Schroeder, M. Is soil respiration a major contributor to the carbon budget within a Pacific Northwest old-growth forest? Agric. For. Meteorol. 2005, 135, 269-283. [CrossRef]

92. Lamaud, E.; Ogee, J.; Brunet, Y.; Berbigier, P. Validation of eddy flux measurements above the understorey of a pine forest. Agric. For. Meteorol. 2001, 106, 187-203. [CrossRef]

93. Biudes, M.S.; Machado, N.G.; Danelichen, V.H.D.M.; Souza, M.C.; Vourlitis, G.L.; Nogueira, J.D.S. Ground and remote sensing-based measurements of leaf area index in a transitional forest and seasonal flooded forest in Brazil. Int. J. Biometeorol. 2014, 58, 1181-1193. [CrossRef] [PubMed]

94. Tang, H.; Ganguly, S.; Zhang, G.; Hofton, M.A.; Nelson, R.F.; Dubayah, R. Characterizing Leaf Area Index (LAI) and Vertical Foliage Profile (VFP) over the United States. Biogeosci. Discuss. 2016, 12, 13675-13710. [CrossRef]

95. Oppelt, N.M. Vertical profiling of chlorophyll within wheat canopies using multi-angular remote sensing data. Can. J. Remote Sens. 2008, 34, S314-S325. [CrossRef]

96. Verónica, C.; Anatoly, G.; James, S. Vertical Profile and Temporal Variation of Chlorophyll in Maize Canopy: Quantitative "Crop Vigor" Indicator by Means of Reflectance-Based Techniques. Agron. J. 2008, 100, 1409-1417.

(c) 2018 by the authors. Licensee MDPI, Basel, Switzerland. This article is an open access article distributed under the terms and conditions of the Creative Commons Attribution (CC BY) license (http:/ / creativecommons.org/licenses/by/4.0/). 
Article

\title{
Estimating Diurnal Courses of Gross Primary Production for Maize: A Comparison of Sun-Induced Chlorophyll Fluorescence, Light-Use Efficiency and Process-Based Models
}

\author{
Tianxiang Cui ${ }^{1,2}$, Rui Sun ${ }^{1,2}$, , Chen Qiao ${ }^{1,2}$, Qiang Zhang ${ }^{1,2}$, Tao Yu ${ }^{1,2}$, Gang Liu ${ }^{1,2}$ and \\ Zhigang Liu 1,2 \\ 1 State Key Laboratory of Remote Sensing Science, Jointly Sponsored by Beijing Normal University and \\ Institute of Remote Sensing and Digital Earth of Chinese Academy of Sciences, Beijing 100875, China; \\ txiang.c@gmail.com (T.C.); qiaochenbnu@gmail.com (C.Q.); zhangqiang1228@mail.bnu.edu.cn (Q.Z.); \\ yutaogis@163.com (T.Y.); gang4.liu@changhong.com (G.L.); zhigangliu@bnu.edu.cn (Z.L.) \\ 2 Beijing Engineering Research Center for Global Land Remote Sensing Products, Institute of Remote Sensing \\ Science and Engineering, Faculty of Geographical Science, Beijing Normal University, Beijing 100875, China \\ * Correspondence: sunrui@bnu.edu.cn; Tel.: +86-10-5880-5457
}

Received: 2 October 2017; Accepted: 5 December 2017; Published: 7 December 2017

\begin{abstract}
Accurately quantifying gross primary production (GPP) is of vital importance to understanding the global carbon cycle. Light-use efficiency (LUE) models and process-based models have been widely used to estimate GPP at different spatial and temporal scales. However, large uncertainties remain in quantifying GPP, especially for croplands. Recently, remote measurements of solar-induced chlorophyll fluorescence (SIF) have provided a new perspective to assess actual levels of plant photosynthesis. In the presented study, we evaluated the performance of three approaches, including the LUE-based multi-source data synergized quantitative (MuSyQ) GPP algorithm, the process-based boreal ecosystem productivity simulator (BEPS) model, and the SIF-based statistical model, in estimating the diurnal courses of GPP at a maize site in Zhangye, China. A field campaign was conducted to acquire synchronous far-red SIF $\left(\mathrm{SIF}_{760}\right)$ observations and flux tower-based GPP measurements. Our results showed that both $\mathrm{SIF}_{760}$ and GPP were linearly correlated with APAR, and the $\mathrm{SIF}_{760}$-GPP relationship was adequately characterized using a linear function. The evaluation of the modeled GPP against the GPP measured from the tower demonstrated that all three approaches provided reasonable estimates, with $R^{2}$ values of $0.702,0.867$, and 0.667 and RMSE values of $0.247,0.153$, and $0.236 \mathrm{mg} \mathrm{m}^{-2} \mathrm{~s}^{-1}$ for the MuSyQ-GPP, BEPS and SIF models, respectively. This study indicated that the BEPS model simulated the GPP best due to its efficiency in describing the underlying physiological processes of sunlit and shaded leaves. The MuSyQ-GPP model was limited by its simplification of some critical ecological processes and its weakness in characterizing the contribution of shaded leaves. The $\mathrm{SIF}_{760}$-based model demonstrated a relatively limited accuracy but showed its potential in modeling GPP without dependency on climate inputs in short-term studies.
\end{abstract}

Keywords: GPP; SIF; MuSyQ-GPP algorithm; BEPS

\section{Introduction}

As the underlying process for plant growth, photosynthesis serves as an essential indicator of plant efficiency and agricultural management practices. Spatially and temporally explicit estimations of photosynthesis at the ecosystem scale (Gross Primary Production, GPP) can provide important information used to study the terrestrial carbon budget $[1,2]$. 
Traditionally, GPP studies have focused on field-based observations of specific species at individual sites [3-5], which are usually time-consuming and laborious. In addition, these sparsely distributed measurements are difficult to extend over large scales due to the spatial heterogeneity of the land surface. At the landscape or regional scale, GPP is generally obtained using models and algorithms that integrate ground observations with remotely sensed data [6]. Over the past several decades, a variety of approaches have been developed to estimate the GPP of terrestrial ecosystems, and they can be grouped into four categories [7]: (1) light use efficiency (LUE) models; (2) process-based models; (3) data-driven models upscaled from eddy covariance (EC) data; and (4) models based on sun-induced chlorophyll fluorescence (SIF). In general, LUE models are well-known for their simplicity and efficiency when using remotely sensed data, but they lose the links to some critical ecological processes by using empirical relationships and constants. These models are represented by Carnegie, Ames, Stanford approach (CASA) [8], global production efficiency model (GLO-PEM) [9], vegetation photosynthesis model (VPM) [10], moderate resolution imaging spectroradiometer (MODIS) GPP [11], and multi-source data synergized quantitative (MuSyQ) GPP [12]. Process-based biogeochemical models usually employ the enzyme kinetics theory encapsulated by Farquhar et al. [13] for C3 plants and its modification for C4 plants [14]. However, these process-based models generally suffer from high complexity, large computational demands, and calibration difficulties. The boreal ecosystem productivity simulator (BEPS) $[15,16]$, terrestrial ecosystem model (TEM) [17], simple biosphere ( $\mathrm{SiB}$ ) model [18], and biome biogeochemical cycle (Biome-BGC) model [19] can be categorized as process-based models. By employing EC-derived GPP data, data-driven models use various algorithms, such as model tree ensembles (MTE) [20] or regression tree [21,22] approaches, and provide a practical way to upscale GPP from individual sites to regional scales. These data-driven methods establish a series of rules using data mining that relate in situ flux observations to satellite-based indices and climate data. SIF provides a new perspective to assess plant photosynthesis and estimate regional GPP due to its inherent link to the photosynthesis process [23-25]. However, the application of SIF is limited because of the difficulty in obtaining high quality SIF data, including proper spatial and temporal resolution, and because of the difficulty in quantitatively assessing the factors that affecting the relationship between SIF and GPP. Although various methods have been developed to model GPP, it should be noted that large uncertainties remain regarding the spatial distribution and seasonal dynamics of GPP [26,27], and model comparison is also necessary to address to what extent the GPP models can capture the spatial and temporal patterns of GPP.

Remote sensing has provided fundamental data to support the study and monitoring of GPP over regional and global scales in recent years. It usually provides reflectance-based spectral measurements of vegetation conditions. However, reflectance-based indices are proxies for vegetation "greenness" and photosynthetic capacity, they often have a relatively low sensitivity to short term variation in plant photosynthetic functioning and cannot be used to reflect the actual photosynthesis [28]. In addition, vegetation parameters derived from surface reflectance have little link to actual plant photosynthetic functions [29]. To our knowledge, only the photochemical reflectance index (PRI), which is based on the sensitivity of the reflectance at $531 \mathrm{~nm}$ to xanthophyll pigments, has been shown to be sensitive to tracking actual photosynthesis through its correlation with the non-photochemical quenching (NPQ) [30]. The PRI was designed to track NPQ that is related to the de-epoxidation cycle of xanthophyll at leaf level [30]. The potential of PRI as a remote sensing proxy of photosynthetic capacity has been demonstrated [31]. However, the PRI has also been proven to be very sensitive to soil background, canopy structure and viewing angle [32]. Additionally, PRI has been shown to be controlled by the changes in leaf pigments rather than NPQ over seasonal scales [33]. Unlike the PRI, the SIF signal is emitted by the photosynthetic machinery of vegetation and is thus linked to the actual photosynthesis. Although canopy SIF can also be affected by canopy structure [34], viewing angle [35] and leaf pigment contents [36], SIF observations can provide an additional way to track the functional status of plant photosynthesis. Recent field studies have demonstrated that 
SIF shows strong links to the GPP at the canopy level [25,37-39]. As SIF be successfully retrieved using orbiting platforms such as the Japanese Greenhouse gases Observing SATellite (GOSAT) [23,40], the SCanning Imaging Absorption spectroMeter for Atmospheric CHartographY (SCIAMACHY) [41], the Global Ozone Monitoring Experiment-2 (GOME-2) [42], and the Orbiting Carbon Observatory-2 (OCO-2) [43], high correlations between SIF and GPP at ecosystem, landscape and regional scales have been shown by Frankenberg et al. [23], Guanter et al. [24,44], Zhang et al. [7], and Cui et al. [45], and simple regression models between remotely sensed SIF and GPP have been established. On the other hand, SIF has also been considered to be more sensitive in tracking the GPP than the traditionally used normalized difference vegetation index (NDVI) and the enhanced vegetation index (EVI) [34,46]. In addition, some studies have also shown the potential use of SIF data to calibrate parameters (maximum carboxylation capacity, $V_{\text {cmax }}$ ) in the photosynthesis model and provide an accurate estimation of agricultural productivity [47,48].

Despite the experimental evidence of the direct and highly linear correlation between SIF and GPP, the applications of SIF-based GPP models are relatively rare when compared to other GPP models due to data availability and quality. Moreover, little research has been conducted to compare the performances of SIF-based models and other GPP models. To the best of our knowledge, there is only one study that has compared SIF-based, LUE-based, and process-based GPP models [49], in which they used satellite-derived data combined with field observations to derive the daily GPP of crops. However, some uncertainties arise due to spatial mismatches between satellite remote sensing data and EC footprints and temporal mismatches between instant SIF and daily GPP measurements. These mismatches should be reduced before assessing the performance of GPP models. In this context, the objective of this study is to compare the performance of three GPP modeling approaches, SIF-based, LUE-based MuSyQ-GPP and process-based BEPS models, in estimating the diurnal courses of GPP for cropland. We focus on field observations with relatively small spatial and temporal scales in this study, and data-driven models are beyond the scope of our research. This study lays the foundation for accurately estimating the GPP of croplands at larger spatial and temporal resolutions.

\section{Materials and Methods}

\subsection{Study Site Description}

A field experiment was conducted near an eddy flux tower in the Zhangye oasis irrigation area located in the middle of the Heihe watershed, northwestern China $\left(100.372^{\circ} \mathrm{E}, 38.856^{\circ} \mathrm{N}\right.$, altitude $\left.1556 \mathrm{~m}\right)$ (Figure 1). This station was funded in May 2012 with the support of the Heihe Watershed Allied Telemetry Experimental Research (HiWATER) (http://westdc.westgis.ac.cn/data) [50,51]. It was dominated by maize during our experimental period. The research area is characterized by a mean annual temperature of $6{ }^{\circ} \mathrm{C}$ and a mean annual precipitation of $114.9 \mathrm{~mm}$, which falls mostly from June to September. During our campaign, the average height of the canopy was approximately $1.8 \mathrm{~m}$. 


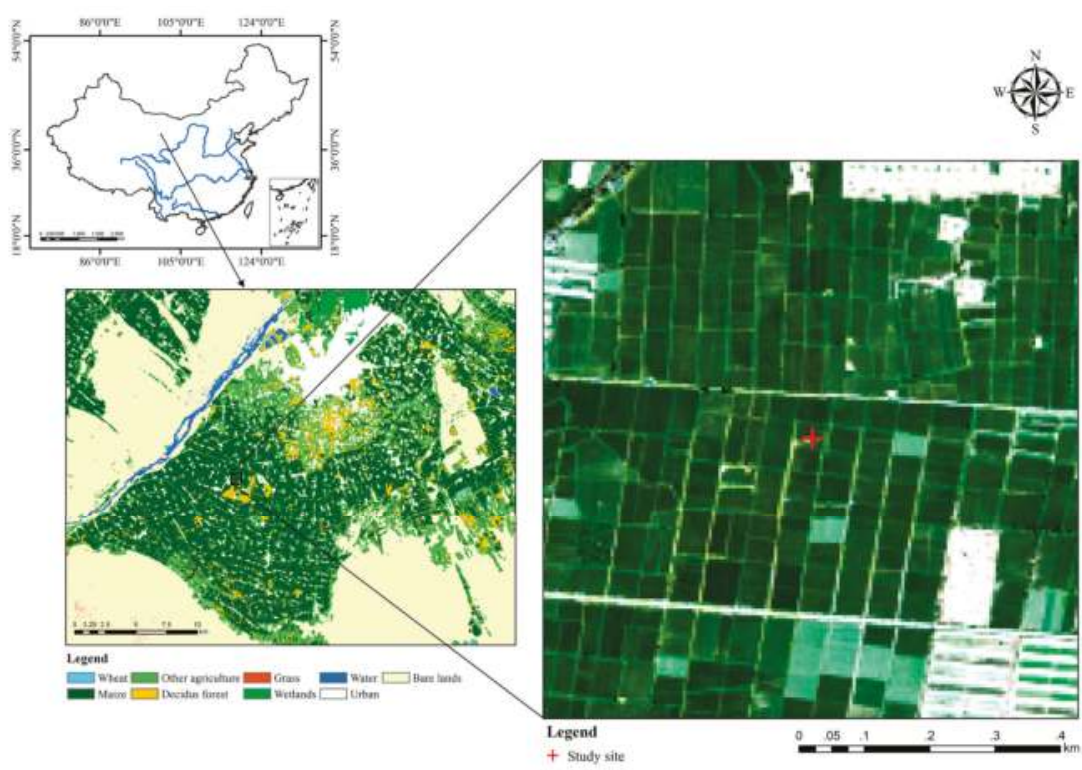

Figure 1. Location of the study site.

\subsection{Eddy Covariance Measurements}

The EC system that was installed within our study area collected continuous measurements beginning on 25 May 2012. It consisted of a three-dimensional sonic anemometer (CSAT3, Campbell Scientific Inc., Logan, UT, USA) and an open path $\mathrm{CO}_{2} / \mathrm{H}_{2} \mathrm{O}$ analyzer (LI7500A, LI-COR Biosciences, Lincoln, NE, USA). The anemometer measured the three-dimensional wind velocity and temperature, and the $\mathrm{CO}_{2} / \mathrm{H}_{2} \mathrm{O}$ analyzer monitored the $\mathrm{CO}_{2} / \mathrm{H}_{2} \mathrm{O}$ density. Both sensors were placed $4.5 \mathrm{~m}$ above the ground and separated $17 \mathrm{~cm}$ away from each other in the horizontal direction. They were connected to a data logger (CR1000, Campbell Scientific Inc., Logan, UT, USA) that recorded the measurements with a sampling frequency of $10 \mathrm{~Hz}$. The EC measurements were processed to half-hourly averages using the EddyPro software package [50,51].

Measurements of air temperature and relative humidity were taken close to the EC system at seven heights ( $3 \mathrm{~m}, 5 \mathrm{~m}, 10 \mathrm{~m}, 15 \mathrm{~m}, 20 \mathrm{~m}, 30 \mathrm{~m}$, and $40 \mathrm{~m})$. All data were processed to 10 min intervals [51]. To match the EC measurements, we used measurements at a height of $5 \mathrm{~m}$ in this research.

The diurnal courses of GPP $\left(\mathrm{mg} \mathrm{m}^{-2} \mathrm{~s}^{-1}\right)$ during our experiment period were obtained by partitioning the observed net ecosystem exchange (NEE) into GPP and ecosystem respiration $\left(\mathrm{R}_{\mathrm{e}}\right)$ according to Coops et al. [52] and Zhang et al. [53].

\subsection{Field Data Collection}

Spectral measurements at the canopy level were carried out under clear-sky conditions in July and August 2015, when the maize plants were at the late big trumpet period and the ripening stage, respectively. The vegetation fraction during our campaign is greater than 0.8 . A calibrated HR4000 high-resolution spectroradiometer (Ocean Optics Inc., Dunedin, FL, USA) was installed at approximately $20 \mathrm{~m}$ from the eddy flux tower to measure the diurnal cycles of the canopy radiometric response (Figure 2a). It records reflected radiation within the spectral domain of 647-1087 nm with a full width at half maximum (FWHM) bandwidth of $0.13 \mathrm{~nm}$. During our experiment, the spectroradiometer was housed in a thermally regulated box (SteadiQ, Ocean Optics Inc., Dunedin, FL, USA) and the internal temperature was maintained at $25^{\circ} \mathrm{C}$ to reduce dark current 
drift. Spectralon reflectance standards (WS-1-SL, Ocean Optics Inc., Dunedin, FL, USA) were used to measure the incident irradiance.

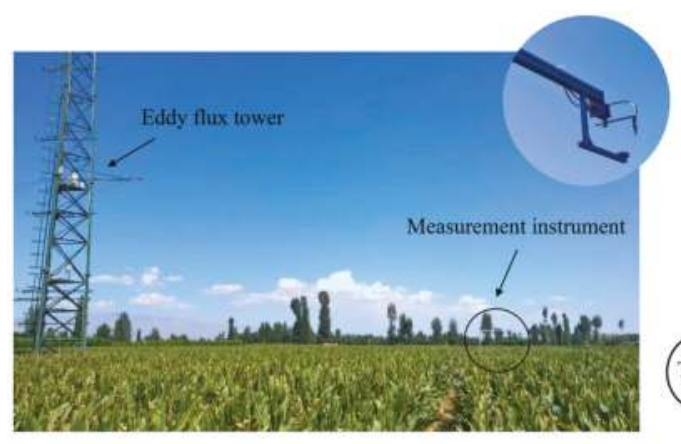

(a)

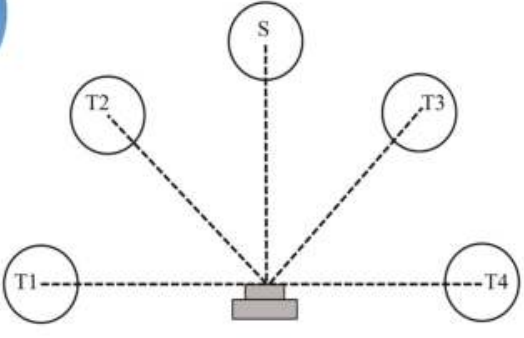

(b)

Figure 2. Overview of the spectral measurements: (a) spectral measurement system; and (b) positions of the standard reflectance panels (S) and the four canopy targets (T1-T4).

For the canopy measurements, the instrument's fiber optic was attached to a swiveling mount that was elevated approximately $1 \mathrm{~m}$ above the canopy and adjusted for nadir view. The daily cycles of the reflectance spectra under clear sky conditions at four different canopy locations were collected in our campaign. As the FOV (Field of View) of the instrument is $25^{\circ}$, each measurement can acquire a circle area of $0.5 \mathrm{~m}$ in diameter (Figure 2b). During the measurement, the swiveling mount was manually controlled to acquire sequential observations of the standard reference panel and the canopy targets. Specifically, each acquisition session consisted of the consecutive collection of spectra of instrument dark current, radiance of the standard reference panel, canopy radiance and radiance of the standard reference panel. After acquiring the radiance of the standard reference panel, the fiber optic was immediately switched to the canopy target to acquire canopy radiance. Then the fiber optic was switched to the standard reference panel to acquire its radiance again. The radiance of the standard reference panel at the time of the canopy measurement was determined using the averaged spectrum of the two measurements. To ensure high-quality spectral data, ten consecutive spectra were averaged as a single spectrum at each measurement. The integration time was automatically optimized during the day to maximize the signal-to-noise ratio (SNR). In the experiment, the spectral acquisitions were conducted at $0.5 \mathrm{~h}$ intervals. Eventually, five diurnal courses of reflectance spectra were acquired (Table 1).

Table 1. Summary of the available day courses of radiometric measurements.

\begin{tabular}{cccc}
\hline Period & Date & Time Window (hh:mm) & Growth Stage \\
\hline 1 & 10 July & $11: 30-19: 00$ & Late big trumpet period \\
2 & 17 July & $8: 00-18: 30$ & Late big trumpet period \\
3 & 18 July & $9: 00-19: 00$ & Late big trumpet period \\
4 & 21 August & $8: 00-18: 30$ & Ripening stage \\
5 & 22 August & $8: 00-18: 00$ & Ripening stage \\
\hline
\end{tabular}

In addition to the spectral measurements, the absorbed photosynthetically active radiation (APAR) was collected simultaneously within $10 \mathrm{~m}$ away the FOV of the spectrometer using an LI-190SA quantum sensor (LI-COR Biosciences, Lincoln, NE, USA) that measures PAR in the range of 400-700 nm. APAR acquisition is based on the measurements of four radiation components: PAR reaching the canopy, PAR reflected from the canopy, PAR transmitted through the canopy, and PAR reflected from the ground. APAR was determined by referring to Gallo and Daughtry [54]. 
During our campaign, LAI was measured twice at 18 July and 22 August, respectively. Each measurement was conducted shortly after sunset using an LI-2200 plant canopy analyzer (LI-COR Biosciences, Lincoln, NE, USA). The measurements were conducted within a $10 \mathrm{~m} \times 10 \mathrm{~m}$ area around the spectral measurement instrument. The measurement at 18 July with value of 3.97 was used for all the July studies, and the measurement at 22 August with value of 4.22 was used for all the August studies, respectively.

\subsection{SIF Retrival}

Chlorophyll fluorescence is the re-emission of solar radiation absorbed by leaf chlorophyll at longer wavelengths, and it has a distinct spectral shape with one peak at $685-690 \mathrm{~nm}$ (red fluorescence) that is mainly attributed to the fluorescence emission of photosystem II (PSII) and another at 730-740 nm (far-red fluorescence) that is attributed to both PSI and PSII [34]. The solar-induced fluorescence (SIF) adds a weak signal to the reflected solar radiation. Depending on the wavelength, the amount of SIF that is emitted by the canopy is approximately $8.7-21.9 \%$ and $2-5.2 \%$ of the total reflected light at $685 \mathrm{~nm}$ and $740 \mathrm{~nm}$, respectively [25,55,56].

The SIF signal is usually discriminated using the Fraunhofer lines in which irradiance is strongly reduced [57,58]. Three Fraunhofer lines are generally used in the visible and near-infrared region: $\mathrm{H} \alpha$ line (centered at $656.4 \mathrm{~nm}$ ) due to hydrogen absorption in the solar atmosphere, $\mathrm{O}_{2}-\mathrm{A}$ (centered at $760.4 \mathrm{~nm}$ ) and $\mathrm{O}_{2}-\mathrm{B}$ (centered at $687 \mathrm{~nm}$ ) due to telluric oxygen absorption in the atmosphere [59-61]. As PSI emits fluorescence only in the near-infrared part of the fluorescence spectrum and PSII emits over a wide spectrum and peaks in the red part, the two oxygen absorption lines that corresponded with the two fluorescence emission peaks can be used to obtain SIF signal emitted mainly by PSI and PSII, respectively [34]. Previous studies have demonstrated that red SIF is affected by the reabsorption of leaf chlorophyll and LAI variations, which causes a lower correlation between red SIF and GPP when compared with the far-red SIF [36,62]. Thus, we mainly consider the far-red SIF in this study.

Almost all SIF retrieval algorithms are based on the Fraunhofer line depth (FLD) principle that was originally proposed by Plascyk [57] and Plascyk and Gabriel [58]. The principle assumes that the reflectance signal and the SIF signal within and outside the dark line remain constant (see the review written by Meroni et al. [59]).

In this study, we used the $\mathrm{O}_{2}$-A line to obtain the far-red SIF $\left(\mathrm{SIF}_{760}\right)$ according to the modified FLD method proposed by Maier et al. [63]. This approach, which is referred to as 3FLD, assumes that the variations in the SIF and reflectance signals are linear. The signals at three bands, including one within the absorption valley and two outside the valley, are used to derive the SIF. The weights of the two outside channels are defined as

$$
w_{\text {left }}=\frac{\lambda_{\text {right }}-\lambda_{\text {in }}}{\lambda_{\text {right }}-\lambda_{\text {left }}}
$$

and

$$
w_{\text {right }}=\frac{\lambda_{\text {in }}-\lambda_{\text {left }}}{\lambda_{\text {right }}-\lambda_{\text {left }}}
$$

where $\lambda_{\text {left }}$ and $\lambda_{\text {right }}$ indicate the shorter and longer wavelengths adjacent to the absorption valley, respectively. The SIF can be generated using

$$
S I F=\frac{\left(E_{\text {left }} w_{\text {left }}+E_{\text {right }} w_{\text {right }}\right) L_{\text {in }}-E_{\text {in }}\left(L_{\text {left }} w_{\text {left }}+L_{\text {right }} w_{\text {right }}\right)}{\left(E_{\text {left }} w_{\text {left }}+E_{\text {right }} w_{\text {right }}\right)-E_{\text {in }}}
$$

where $E$ and $L$ represent the ground irradiance and the canopy radiance, respectively.

Former studies have indicated that the 3FLD algorithm can generate reliable SIF signals when used on datasets obtained using the aforementioned HR4000 ( $\mathrm{SR}=0.13 \mathrm{~nm}$, SNR > 300) $[64,65]$. Therefore, we considered the derived SIF to be suitable for our research. 


\subsection{Statistical Analysis of the SIF-GPP Relationship}

According to the LUE theory proposed by Monteith [66], the GPP can be obtained by

$$
G P P=A P A R \times L U E_{P}
$$

where APAR represents the absorbed photosynthetically active radiation and $L U E_{\mathrm{P}}$ refers to the efficiency in which APAR is used in photosynthesis.

As a by-product of photosynthesis, SIF can be similarly expressed as

$$
S I F=A P A R \times L U E_{\mathrm{F}} \times f_{\mathrm{esc}}
$$

where $L U E_{\mathrm{F}}$ is the light-use efficiency for SIF, which represents the fraction of APAR that is re-emitted from the canopy as SIF photons and $f_{\text {esc }}$ is the fraction of SIF photons escaping the canopy [24].

By combining Equations (4) and (5), SIF can be theoretically linked with GPP as

$$
G P P=S I F \times \frac{L U E_{\mathrm{P}}}{L U E_{\mathrm{F}} \times f_{\mathrm{esc}}} \approx S I F \times \frac{L U E_{\mathrm{P}}}{L U E_{\mathrm{F}}}
$$

where $f_{\text {esc }} \approx 1$ can be assumed to be due to the low absorptance of leaves in the far-red wavelengths and the relatively simple plant structure and high leaf area index of crops [24,67].

As shown in Equation (6), if the $L U E_{\mathrm{P}} / L U E_{\mathrm{F}}$ ratio is constant, the SIF would be a good constraint used to determine GPP regardless of changes in the APAR or stresses. Previous studies have demonstrated that these two terms $\left(L U E_{\mathrm{P}}\right.$ and $L U E_{\mathrm{F}}$ ) covary in most cases, and the ratio of one to the other tends to be constant under high light conditions, which makes it possible to establish a linear statistical model between SIF and GPP $[23,25,68]$. In this study, we generated a statistical model to assess the ability of SIF to estimate GPP.

\subsection{MuSyQ-GPP Algorithm}

The MuSyQ-GPP algorithm was designed as part of the multi-source data synergized quantitative (MuSyQ) remote sensing production system [12]. It presents an LUE approach to model the GPP using remotely sensed data and meteorological data with different spatial and temporal resolutions. The algorithm was adopted to generate the GPP at the site level in this research.

The MuSyQ-GPP algorithm estimates GPP using the LUE theory described in Equation (4). It employs down-regulating scalars to characterize the effects of temperature and water stress on the $L U E_{\mathrm{P}}$ as

$$
L U E_{\mathrm{P}}=L U E_{0} \times f_{1}(T) \times f_{2}(\beta)
$$

where $L U E_{0}$ is the potential or maximum $L U E_{\mathrm{P}}\left(\mathrm{gC} \mathrm{mol}{ }^{-1} \mathrm{PPFD}\right)$, and $f_{1}(T)$ and $f_{2}(\beta)$ represent the down-regulation effects of temperature and water conditions on $L U E_{0}$, respectively. A theoretical $L U E_{0}$ value of $0.60 \mathrm{gC} \mathrm{mol}^{-1}$ PPFD that was reported in a previous study [69] was used in this study.

The temperature-limited effect on $L U E_{0}$ is generated using two terms, $T_{1}$ and $T_{2}$

$$
f_{1}(T)=T_{1} \times T_{2}
$$

where

$$
\begin{gathered}
T_{1}=0.8+0.02 T_{\mathrm{opt}}-0.0005 T_{\mathrm{opt}^{2}} \\
T_{2}=1.1814 /\left\{\left[1+e^{0.2\left(T_{\mathrm{opt}}-10-T\right)}\right]\left[1+e^{0.3\left(-T_{\mathrm{opt}}-10+T\right)}\right]\right\}
\end{gathered}
$$

where $T_{\text {opt }}$ represents the optimum temperature, which is defined as $28{ }^{\circ} \mathrm{C}$ in our study as in Kalfas et al. [70] 
The limited effect of water conditions on plant photosynthesis, which ranges between 0.5 and 1 , is derived following the algorithm

$$
f_{2}(\beta)=0.5+0.5 E / E_{\mathrm{P}}
$$

where $E$ and $E_{\mathrm{P}}$ represent the actual and potential evapotranspiration, respectively.

In the MuSyQ-GPP algorithm, a modified Penman-Monteith (P-M) approach with biome-specific canopy conductance [71-74] is used to estimate the actual evapotranspiration (see Text S1 in Supplementary Materials). The potential evapotranspiration is calculated using the Priestley and Taylor (P-T) equation [75].

The original version of the MuSyQ-GPP model was established to estimate the daily GPP over regional and global scales [12]. In our study, we deployed its original algorithms to generate half-hourly averaged GPP at the site level to evaluate the performance of the LUE model.

\subsection{BEPS Approach}

The BEPS model used in our study is an hourly process-based diagnostic model $[13,14,76]$ that computes the canopy level GPP as the sum of the sunlit and shaded leaf groups using the Farquhar, von Caemmerer and Berry (FvCB) photosynthesis model [18]. Although the BEPS was initially developed for boreal ecosystems, it has been expanded and used for temperate and tropical ecosystems in Asia [77,78], which laid the foundation for its application in croplands.

The BEPS adopts a sunlit-shaded leaves separation modeling mechanism that upscales photosynthesis from the leaf level to the canopy level. This kind of "two-leaf" model has been proven to be more effective in modeling canopy photosynthesis than the conventional "big-leaf" model [13,79]. The photosynthetic rate of the canopy, $A_{c}$, is modeled as the sum of the sunlit and shaded leaf groups as

$$
\begin{gathered}
A_{\mathrm{c}}=A_{\text {sunlit }} L A I_{\text {sunlit }}+A_{\text {shaded }} L A I_{\text {shaded }} \\
L A I_{\text {sunlit }}=2 \cos \theta\left(1-e^{-0.5 \Omega L A I / \cos \theta}\right) \\
L A I_{\text {shaded }}=L A I-L A I_{\text {sunlit }}
\end{gathered}
$$

where sunlit and shaded denote the sunlit and shaded leaf groups, respectively; $\theta$ is the solar zenith angle; and $\Omega$ is the clumping index.

The photosynthesis model in the BEPS model is suitable for C3 carbon fixation pathways. However, maize is a C4 species according to its photosynthetic pathway. In this study, the BEPS model was modified to be suitable for $\mathrm{C} 4$ species estimation according to the theory of Collatz et al. [19]. The photosynthetic rate of the leaves for $C 4$ species, $A_{n}$, is assumed to be limited by the phosphoenolpyruvate carboxylase (PEP-carboxylase)-limited rate of assimilation $\left(W_{\mathrm{c}}\right)$, the light-limited rate of assimilation $\left(W_{\mathrm{e}}\right)$, and the $\mathrm{CO}_{2}$-limited rate of assimilation $\left(W_{\mathrm{s}}\right)$ [80]

$$
\begin{gathered}
A_{n}=\min \left(W_{\mathrm{c}}, W_{\mathrm{e}}, W_{\mathrm{s}}\right)-R_{\mathrm{d}} \\
W_{\mathrm{c}}=V_{\mathrm{cmax}} \\
W_{\mathrm{e}}=P A R \times(1-\omega) \times \varepsilon \\
W_{\mathrm{s}}=2 \times 10^{4} V_{\mathrm{cmax}} C_{\mathrm{i}} / p \\
R_{\mathrm{d}}=0.0025 V_{\mathrm{cmax}}
\end{gathered}
$$

where $V_{\mathrm{cmax}}$ is defined as the maximum carboxylation rate $\left(\mathrm{mol} \mathrm{m}^{-2} \mathrm{~s}^{-1}\right), \omega$ is the leaf-scattering coefficient for PAR, $\varepsilon$ represents the intrinsic quantum efficiency of $\mathrm{CO}_{2}$ uptake $\left(\mathrm{mol} \mathrm{mol}^{-1}\right)$, and $R_{\mathrm{d}}$ is the leaf dark respiration rate $\left(\mathrm{mol} \mathrm{m}^{-2} \mathrm{~s}^{-1}\right)$. 
The C4 photosynthesis model was integrated into the BEPS model to couple with the Ball-Berry stomatal conductance model to generate the photosynthesis rate using an iteration calculating procedure $[19,81]$.

\section{Results}

\subsection{Diurnal Patterns in GPP and SIF760}

The diurnal courses of GPP, $\mathrm{SIF}_{760}$, and PAR during our campaign are shown in Figure 3. Since the canopy spectra were collected at four different positions, four SIF $_{760}$ values together with one GPP value were obtained at a time. As shown in Figure 3, both $\mathrm{SIF}_{760}$ and GPP exhibit similar patterns to that of PAR with lower values in the early morning and late afternoon and higher values in the middle of the day. These values tend to exhibit a single peak curve that follows the variation of PAR. The distinct decrease in GPP at midday that is commonly found in C3 species, known as the "midday depression", was not observed in our experiment, which is consistent with the studies by Cheng et al. [82] and Liu et al. [25] For C3 species, higher temperatures can decrease the affinity of enzymes for $\mathrm{CO}_{2}$ and cause the carboxylation enzyme, Rubisco, to be less active, which leads to the midday reductions in carbon assimilation. In addition, the higher evaporative demands at midday reduces the stomatal conductance, which also affects the assimilation rate. In contrast, for C4 species, $\mathrm{CO}_{2}$ is delivered to Rubisco, which is localized in the bundle sheath chloroplasts, by a metabolic pump that concentrates $\mathrm{CO}_{2}$ [19]. This mechanism can inhibit photorespiration and promote carbon assimilation. Consequently, $\mathrm{C} 4$ species exhibit higher photosynthesis capacities at high temperatures where photorespiration is stimulated. In addition, $\mathrm{C} 4$ species generally have lower stomatal conductance than C3 species [19]. Lower conductance coupled with higher photosynthetic capacity in C4 species results in higher water use efficiencies in comparison to $\mathrm{C} 3$ species. The similar pattern between $\mathrm{SIF}_{760}$ and PAR indicates the capacity of SIF in protecting plant tissues from light energy that may be excessive for photosynthesis and could otherwise damage tissues. Although some research has demonstrated that SIF will increase with PAR at low light then decrease when NPQ increases and further increase when NPQ reaches saturation [83], we did not find this kind of variation clearly in our experiment. Actually, only one set of data on 17 July showed slight decreases at approximately 11:30 and 14:30, which might correspond to the aforementioned decreasing trend. However, we also noticed that the $\mathrm{SIF}_{760}$ values of the four canopy positions had relatively higher diversity on 17 July (as well as 10 July) than on the other days. It is not clear whether it can to be attributed to the NPQ increment or observation bias. Thus, further studies are needed, and both data quality and quantity should be considered before drawing such a conclusion. For our study, we found that $\operatorname{SIF}_{760}$ showed a pattern with a single peak that followed the variation of PAR as concluded by Liu et al. [25]. 


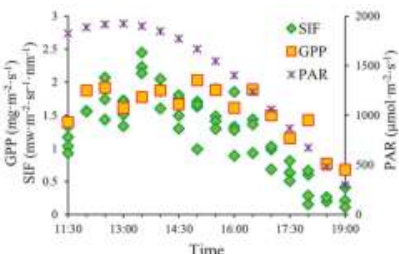

(a)

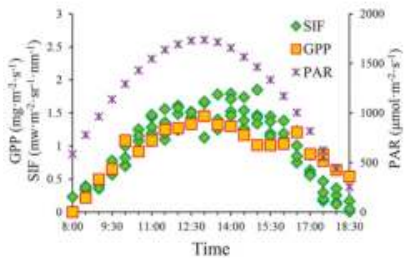

(d)

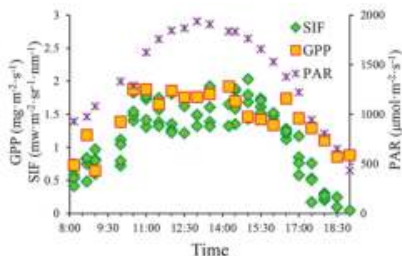

(b)

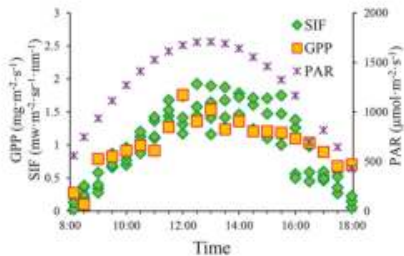

(e)

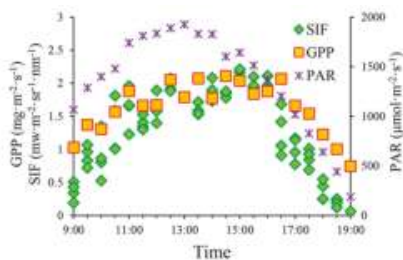

(c)

Figure 3. Diurnal patterns of PAR, GPP and $\mathrm{SIF}_{760}$ during the experiment: (a) 10 July; (b) 17 July; (c) 18 July; (d) 21 August; and (e) 22 August.

We also analyzed the relationships between APAR and both $\mathrm{SIF}_{760}$ and GPP. Both showed strong linear correlations, and an $\mathrm{R}^{2}$ of 0.713 (Figure $4 \mathrm{a}$ ) and 0.591 (Figure $4 \mathrm{~b}$ ) was achieved between APAR and $\mathrm{SIF}_{760}$ and APAR and GPP, respectively. The averaged $\mathrm{SIF}_{760}$ values were also generated to reduce some uncertainties introduced during our spectral acquisition. As our measurements were conducted at four different canopy positions, we assumed their mean values could adequately represent the situations of our study site. By analyzing the relationship between APAR and the averaged SIF 760 , the $\mathrm{R}^{2}$ increased to 0.798 (Figure $4 \mathrm{c}$ ). As illustrated in Figure 4, the slope of the $\mathrm{SIF}_{760}$-APAR relationship is approximately the same $L U E_{\mathrm{F}}$, and the slope of the GPP-APAR relationship can be considered as $L U E_{\mathrm{P}}$. Because both relations can be adequately expressed by linear equations, the ratio between $L U E_{\mathrm{P}}$ and $L U E_{\mathrm{F}}$, as described in Equation (6), can be considered nearly constant, which means that $\mathrm{SIF}_{760}$ and GPP can be directly linked.

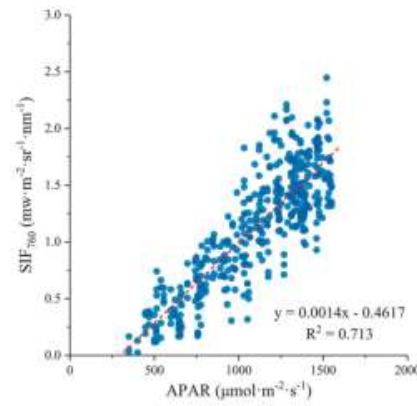

(a)

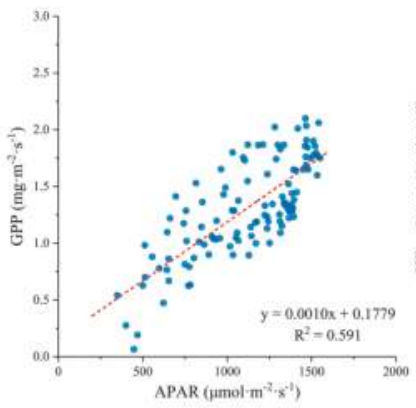

(b)

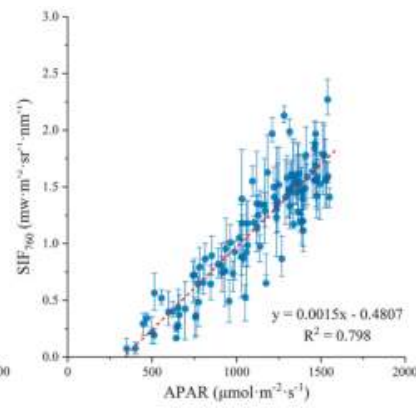

(c)

Figure 4. Relationship between APAR (absorbed photosynthetically active radiation) and: (a) individual $\mathrm{SIF}_{760}$; (b) GPP; and (c) averaged SIF. The error bar indicates the range of $\mathrm{SIF}_{760}$ values for four measurements. 


\subsection{Relationship between SIF and GPP}

Statistical analysis was conducted to reveal the relationship between SIF $_{760}$ and GPP. As shown in Figure 5, we found a significant correlation between $\mathrm{SIF}_{760}$ and GPP, and their relationship could be characterized by a linear function, regardless of using individual SIF $_{760}$ of the four group measurements $\left(\mathrm{GPP}=0.570 \times \mathrm{SIF}_{760}+0.666\right)$ or the averaged measurements $\left(\mathrm{GPP}=0.621 \times \mathrm{SIF}_{760}+0.614\right)$, which suggests that $\mathrm{SIF}_{760}$ can be used as a direct proxy for GPP.

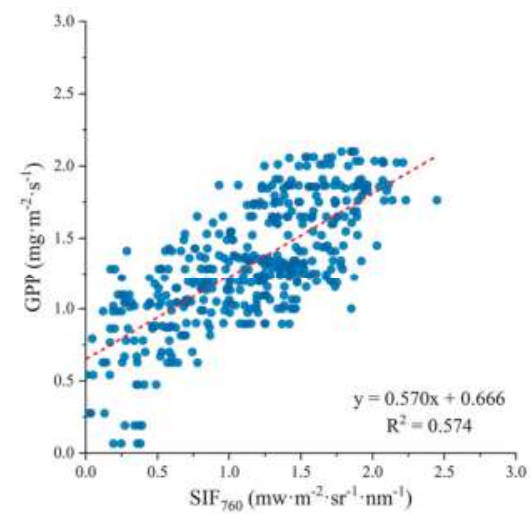

(a)

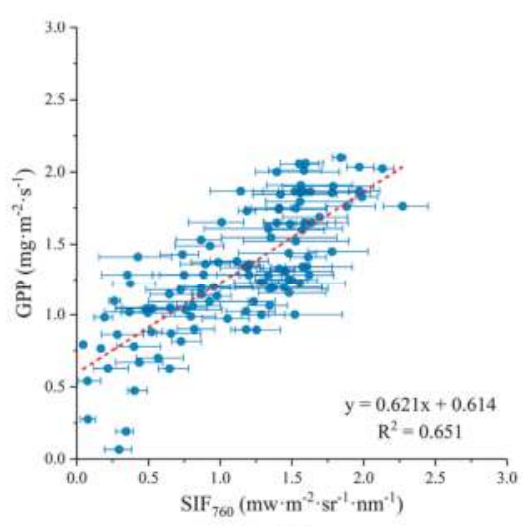

(b)

Figure 5. Relationship between: (a) individual $\mathrm{SIF}_{760}$ and GPP; and (b) averaged $\mathrm{SIF}_{760}$ and GPP. The error bars indicate the range of $\operatorname{SIF}_{760}$ values for the four measurements.

\subsection{Comparison of GPP Modeled by SIF, MuSyQ-GPP, and BEPS Models}

In addition to the SIF-based GPP estimation, we also generated GPP using both the LUE-based MuSyQ-GPP algorithm and the process-based BEPS model. As the BEPS model used in our study was initially designed to run at hourly intervals, we modified it to a half-hourly time step to match the observations in this study. Thus, all approaches were performed every $30 \mathrm{~min}$ in our analysis. As the average of the four $\mathrm{SIF}_{760}$ measurements may represent the situations of our study site better than the individual measurements, we only considered the statistical model generated using the averaged SIF $_{760}$ values $\left(\mathrm{GPP}=0.621 \times \mathrm{SIF}_{760}+0.614\right)$ in this study. As shown in Figure 6, all three approaches estimate the GPP well. Among which, the BEPS model $\left(\mathrm{R}^{2}=0.867\right.$, RMSE $\left.=0.153 \mathrm{mg} \mathrm{m}^{-2} \mathrm{~s}^{-1}\right)$ performs better than that of $\operatorname{SIF}_{760}\left(\mathrm{R}^{2}=0.624\right.$, RMSE $\left.=0.237 \mathrm{mg} \mathrm{m}^{-2} \mathrm{~s}^{-1}\right)$ and MuSyQ-GPP $\left(\mathrm{R}^{2}=0.702\right.$, RMSE $\left.=0.247 \mathrm{mg} \mathrm{m}^{-2} \mathrm{~s}^{-1}\right)$ models. We also modeled GPP using the statistical model generated with $\mathrm{SIF}_{686}\left(\mathrm{GPP}=0.682 \times \mathrm{SIF}_{686}+0.539\right)$ (see detailed results in Text S2, Figures S1-S4 in Supplementary Materials). Compared with the $\mathrm{SIF}_{760}$-based model, $\mathrm{SIF}_{686}$-based GPP model showed a limited accuracy with $\mathrm{R}^{2}=0.456$ and $\mathrm{RMSE}=0.363 \mathrm{mg} \mathrm{m}^{-2} \mathrm{~s}^{-1}$. 


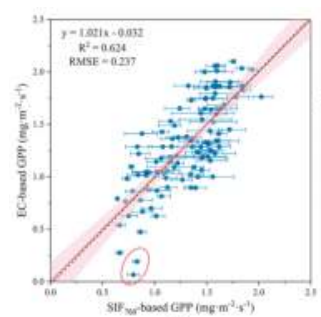

(a)

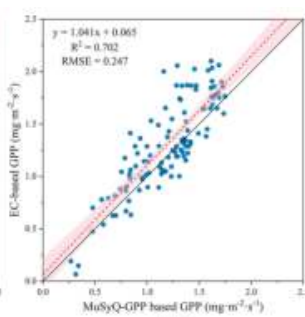

(b)

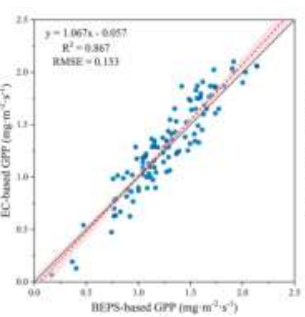

(c)

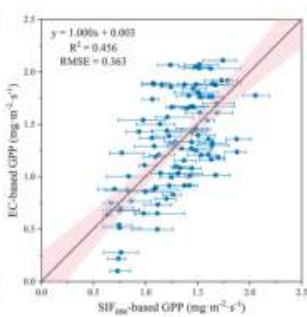

(d)

Figure 6. Relationships between modeled GPP and EC-based GPP by: (a) SIF 760 ; (b) MuSyQ-GPP; (c) BEPS; and (d) SIF $_{686}$ during the experiment period. The dots in the red circle are the values at 8:30 on 21 August, and 8:30 on 22 August. The error bars indicate the range of the four SIF-based GPP values, the red shades represent the $95 \%$ confidence bands for the regression functions.

We noticed that some of the SIF-based GPP estimates were higher than the EC-based estimates when GPP was relatively low, which corresponded to the observations at 8:30 on 21 August and 8:30 on 22 August (dots in the red circle in Figure 6a). When these two groups of data were excluded from the analysis, both SIF based GPP models performed better with $\mathrm{R}^{2}$ increased to 0.667 and RMSE decreased to $0.236 \mathrm{mg} \mathrm{m}^{-2} \mathrm{~s}^{-1}$ for the SIF $_{760}$-based model, and $\mathrm{R}^{2}$ increased to 0.459 and RMSE decreased to $0.352 \mathrm{mg} \mathrm{m}^{-2} \mathrm{~s}^{-1}$ for the $\mathrm{SIF}_{686}$-based model, respectively. The MuSyQ-GPP algorithm was found to underestimate the GPP values at high productivity, which could be attributed to the assignment of a constant $L U E_{0}$ value in the algorithm as it could not properly handle the contribution of both sunlit and shaded leaves to the total GPP. BEPS model was found to slightly underestimate GPP at high productivity and overestimate GPP at low productivity. Among these three approaches, BEPS model showed better performance than that of SIF-based or LUE-based MuSuQ-GPP models, with higher $\mathrm{R}^{2}$ and lower RMSE.

\section{Discussion}

\subsection{Uncertainties in the SIF Measurements}

The SIF retrieval accuracy is dependent on the spectral characteristics of the reflectance and irradiance spectra around the oxygen absorption lines. According to our validation based on EC-based data, the SIF 760 -based GPP at 8:30 on 21 August and 8:30 on 22 August deviate from the EC-based values significantly (dots in the red circles in Figure 6). During our experiment, we measured four canopy positions sequentially from $\mathrm{T} 1$ to $\mathrm{T} 4$ as illustrated in Figure 2. The spectra of the reference panel were collected eight times: once before and once after each of the four canopy measurements. Incident radiance at 8:30 on 21 August and 8:30 on 22 August was then obtained (Figure 7). The incident radiance showed significant variations during our measurement, especially at the left and right shoulders of the absorption line. Additionally, the measured irradiance did not present a gradual increase trend as expect. This may be attributed to the instability of irradiance in the morning. As light travels a longer path at high solar zenith angle (SZA) in the morning, the influence of atmospheric conditions would be larger than that at lower SZA. Hence, in future studies, it is necessary to consider the influence of air conditions and the affection of imaging geometrical conditions such as the SZA and relative azimuth angle in SIF retrieval. 


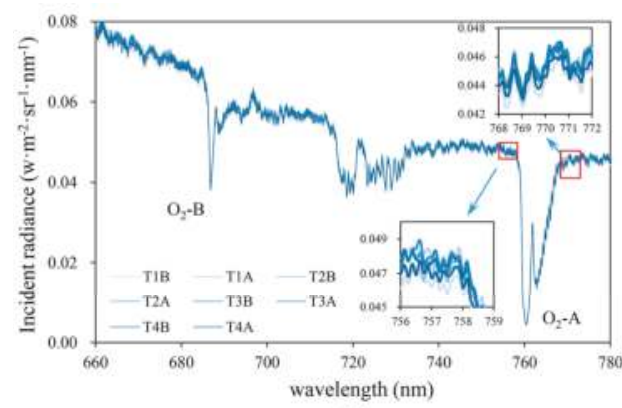

(a)

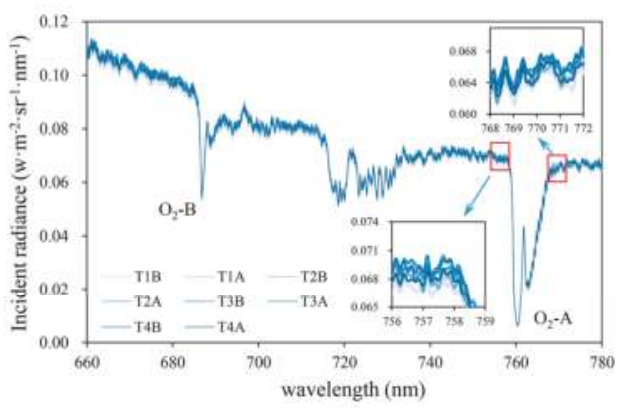

(b)

Figure 7. Incident radiance at: (a) 8:30 on 21 August; and (b) 8:30 on 22 August. T1 to T4 represent the four canopy targets, B and A denote measurements before and after canopy measurement, respectively.

The data quality (DQ) indicator $D Q_{\text {s }}$ presented by Cogliati et al. [84] was adopted to evaluate the stability of the incident radiance. $D Q_{\mathrm{s}}$ is defined as the percentage of variation between the first and the second irradiance measurement

$$
D Q_{\mathrm{s}}=\left|E_{\mathrm{B}}-E_{\mathrm{A}}\right| / E_{\mathrm{B}} \times 100
$$

where $E_{\mathrm{B}}$ and $E_{\mathrm{A}}$ represents the incident radiance before and after the canopy measurement, respectively.

In this study, the $D Q_{s}$ values of the three bands used in the $3 F L D$ algorithm were averaged to represent the irradiance stability. As shown in Figure 8, the $D Q_{\mathrm{s}}$ values were generally higher at morning and evening and lower at noon, the higher values also corresponded with the higher SZA and longer path. For the observations at 8:30 on 21 August and 8:30 on 22 August, the $D Q_{s}$ showed the largest values, which indicated that the irradiance changed significantly during these measurement sessions. Therefore, data quality check is needed especially when irradiance demonstrates significant variations, the acquisition that do not meet the quality check should be rejected and no longer considered in the analyses. 


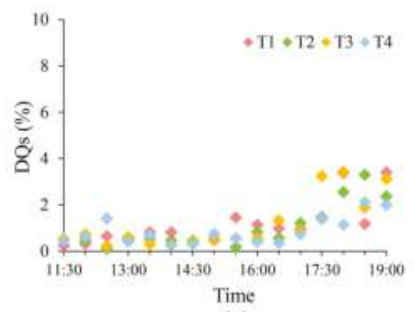

(a)

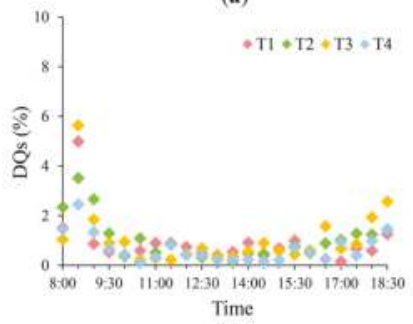

(d)

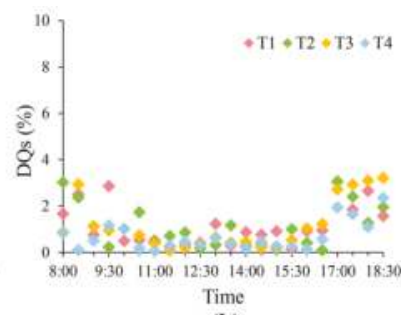

(b)

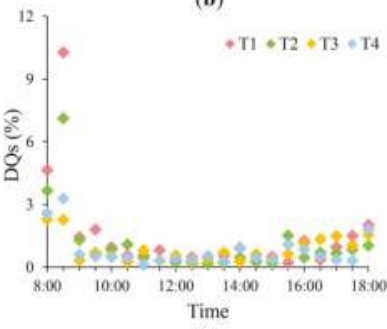

(e)

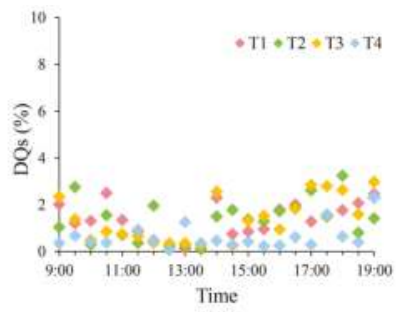

(c)

Figure 8. $D Q_{\mathrm{s}}$ values of the measurements: (a) 10 July; (b) 17 July; (c) 18 July; (d) 21 August; and (e) 22 August. T1 to T4 represent the measurements of the four canopy targets.

We used the 3FLD algorithm to generate SIF in this study. The 3FLD approach is based on the assumption that the reflectance signal and the SIF signal vary linearly within and outside the absorption band. However, the actual variations of reflectance and SIF signal are not being linear, which causes a violation of the underlying assumption of the 3FLD method and lead to some uncertainties in SIF retrieval. Additional studies are needed in generating SIF with more advanced SIF retrieval approaches such as the improved FLD (iFLD) method [85] or the Spectral Fitting Methods (SFM) [86].

\subsection{Uncertainties in the SIF-Based GPP Model}

Previous studies have demonstrated that the relationship between remotely sensed SIF and GPP can be characterized by a linear and ecosystem-specific function [23-25]. There is also evidence indicating that the SIF and GPP relationship can be affected by various factors such as the rate of NPQ [61,87], light conditions [46,87], nutrient availability [61], water availability [87], temperature [23,46,68], and canopy structure [34]. Recent studies have suggested that there is a curvilinear relationship between instantaneous SIF and GPP due to the rate of photosynthesis saturated under high illumination, and this relationship tends to become linear when both SIF and GPP are temporally aggregated [28,34]. However, the saturation of GPP with APAR were not found in our experiment (Figure 4) as $\mathrm{C} 4$ species exhibit higher photosynthesis capacities at higher temperatures than C3 species. Therefore, the relationship between SIF and GPP could be adequately characterized by a linear function in our research. However, the SIF-GPP relationship may not be constant in spatial and temporal modeling, which can be attributed to the ecosystem-specific structural and physiological characteristics as well as the environmental situations associated with each ecosystem [34]. Therefore, for accurate quantification and characterization of the SIF-GPP relationship, further experiments that overlap the entire growth periods in various ecosystems are needed, especially for C3 species.

Remote measurements of SIF are likely representative of the upper layer of the canopy, which is mainly characterized by sunlit leaves [34,59]. Although the upper layer substantially contributes to the total GPP, there are still many layers of leaves that contribute to the canopy photosynthesis at different rates depending on their sunlit or shaded exposure [59]. Thus, the heterogeneity of the vertical canopy may lead to a mismatch between the SIF-based estimates and the EC-based GPP. 
Additionally, under low light unstressed conditions, there exists a competitive relationship between leaf fluorescence and photosynthesis and a positive correlation between them under stressed and high light conditions $[87,88]$. The relationships between SIF and GPP for sunlit and shaded leaves are different as sunlit leaves receive more light and shaded leaves only receive diffuse light [88]. Therefore, additional studies are needed to address the contributions of the shaded leaf groups and the structural sensitivities of canopy-leaving SIF signals.

Light energy absorbed by leaf chlorophyll molecules has three different pathways: photochemistry, $\mathrm{NPQ}$, and SIF. Accurately estimating any one of the de-excitation pathways require measurement of the other two. Some researches indicated that most absorbed energy is used in photosynthesis and both fluorescence and NPQ are low under low light conditions. Under high light and stressed conditions, NPQ becomes dominant and the yields of fluorescence and photosynthesis decrease [87]. As a result, the relationship between SIF and GPP is influenced by NPQ. The mechanism of NPQ and its regulation should be considered in the further studies.

Plant photosynthesis starts with absorption of light, mainly by chlorophyll molecules. Accordingly, some mechanisms were used to modulate photosynthetic light. Former studies have indicated that chloroplasts can change their orientation within the cell in response to the intensity and direction of the incident light [89]. When light is limiting to photosynthesis, chloroplasts move to positions that yielding maximum light absorption, whereas in saturating light they hide themselves to make the light absorption minimized. The chloroplast avoidance movements can lead to decreased light absorption and then influence the observed SIF signal, and further studies are needed. Additionally, leaf movements and leaf angle adjustments can also significantly affect the fractions of sunlit and shaded leaves, which should be considered in future research.

The mismatches of the spatial and temporal scales between canopy SIF and EC-based GPP also lead to some uncertainties. The footprint of the EC tower covers $0.01 \mathrm{~km}^{2}$ to $10 \mathrm{~km}^{2}$ depending on the measurement height, wind speed and turbulent state of atmosphere [90], whereas the canopy SIF are instantaneously measured with a footprint less than one square meter.

The availability, quality, and spatiotemporal coverage of SIF data are expected to increase drastically over the next few years. SIF measurements have been conducted at ecosystem, landscape, and global scales using field spectroradiometers, airborne instruments, and satellite platforms, respectively. The NASA's OCO-2 [43], ESA's Sentinel-5 Precursor (TROPOMI) [91] and ESA's Earth Explorer Fluorescence Explorer (FLEX) [92,93] can provide SIF data at much higher spatial resolutions. In particular, the FLEX mission can provide auxiliary information such as NPQ and canopy temperature along with fluorescence information. These data would improve our estimation of the global carbon budget and our ability to track the health of terrestrial ecosystems. Additionally, the development of the SCOPE (Soil-Canopy Observation, Photosynthesis and Energy balance) model provides a theoretical way to model canopy SIF and photosynthesis by combing Soil-Vegetation-Atmosphere-transfer (SVAT) model with radiative transfer models [94]. Despite this promising scenario and the empirical evidence that SIF provides novel information to estimate photosynthesis, as found in our study, there are still many uncertainties in relating SIF and GPP, and further studies are needed.

\subsection{Limitations of the LUE-Based Model}

The basis of the LUE-based models is the initial linear relationship between the GPP and APAR of the canopy [63]. However, this relationship is not linear at the individual leaf level and is characterized by temporal heterogeneity associated with the variation of illumination levels [95]. Photosynthesis in sunlit leaves is often light-saturated and limited by the level of Rubisco activity related to leaf nutrient conditions and the ambient temperature [18], which results in a lower LUE, while photosynthesis in shaded leaves is often not light-saturated and essentially has a linear response to APAR, which results in a higher LUE [13,96-99]. In the LUE-based MuSyQ-GPP algorithm, LUE is determined by assigning a constant maximum value of $L U E_{0}$ and adjusting downward based on temperature and water 
conditions. Daily variability in the sunlit and shaded leaf fractions cannot be represented in this model. Consequently, the internal physiological processes of these two leaf groups are substantially different and the LUE of the shaded leaves is generally higher than that of the sunlit leaves [95]. As GPP can vary greatly in space and time as a result of the variability between sunlit and shaded leaf fractions, we argue that scaling the $L U E_{0}$ without considering the difference between the sunlit and shaded leaf groups can significantly limit the performances of the LUE-based models. There are some literature suggests that the LUE models underestimate GPP at high productivity and overestimate GPP at low productivity [96], and the underestimation is consistent with our research. However, the overestimation cannot be clearly shown in our analysis. These results are not contradictory since our data at low productivity is limited. In addition, the $L U E_{0}$ used in the LUE-based models is usually defined as a constant value for each broadly defined plant functional type (PFT). For our study, we used a constant value of $0.60 \mathrm{gC} \mathrm{mol}^{-1}$ PPFD from the study by Zhang [69], which was conducted at the same site as our study. However, other literature has suggested that $L U E_{0}$ for maize at different sites can be assigned as $1.50 \mathrm{gC} \mathrm{mol}^{-1}$ PPFD [67,100], $0.93 \mathrm{~g} \mathrm{C} \mathrm{mol}^{-1}$ PPFD [101], or $1.22 \mathrm{~g} \mathrm{C}$ $\mathrm{mol}^{-1}$ PPFD [101]. Therefore, $L U E_{0}$ varies among vegetation types and study sites and should be calibrated before using in the LUE-based approach. Moreover, the MuSyQ-GPP algorithm deploys two down-regulating factors to represent the effects of temperature and water conditions while scaling the $L U E_{0}$. Although various strategies have been developed to characterize the impacts of temperature and water stresses [8-11], the descriptions of their influences in the LUE-based approaches are still empirical, which may introduce some uncertainties, especially for the water stress scalar. For the MuSyQ-GPP algorithm, a calculation of evapotranspiration is conducted to represent water availability. Predictably, the uncertainties existing in the evapotranspiration estimation would lead to significant deviations in the final GPP estimation.

\subsection{Uncertainties in the BEPS Model}

The core idea of the BEPS model is that it adopts separate sunlit and shaded leaves modeling mechanisms. The two-leaf model has been proven to be more efficient in simulating the daily variation of canopy photosynthesis than the conventional "big-leaf" model [13]. The BEPS model employs the enzyme kinetics theory for C3 plants proposed by Farquhar et al. [18] to generate the photosynthesis rate. In this study, we adopted a modified version that was applicable for C4 species [19] and integrated it into the BEPS model to couple with the Ball-Berry stomatal conductance model [81] to generate GPP as well as the water and heat fluxes. Although it is designed in an explicit and tightly linked manner that simulates the coupled hydrological, ecophysiological, and biogeochemical processes and the associated feedback mechanisms, it should be noted that numerous parameters should be assigned before modeling. One of the most important parameters in modeling photosynthesis, the maximum carboxylation rate $\left(V_{\text {cmax }}\right.$ in Equations (16), (18), and (19)), has been proven to vary temporally [48,102-104], although it is usually assigned as a specific value for each PFT [105]. In this study, we adopted a constant value of $37 \mu \mathrm{mol} \mathrm{m}{ }^{-2} \mathrm{~s}^{-1}$ by referring to Zhang et al. [48]. Although BEPS model simulated GPP better than the SIF-based and LUE-based model in our study, there are still some underestimations at high productivity and overestimations at low productivity. Therefore, additional studies are needed in generating the seasonal variation of $V_{\mathrm{cmax}}$. Former studies have demonstrated that $V_{\mathrm{cmax}}$ can be related with reflectance [106] or leaf chlorophyll content [107], which made it retrievable using remotely sensed data. Recently, a study conducted by Zhang et al. [48] presented an SIF-based approach for modeling seasonal $V_{\mathrm{cmax}}$, which provided a new method to assess plant photosynthesis using SIF.

\section{Conclusions}

In this study, we evaluated the ability of three different approaches, SIF, LUE-based MuSyQ-GPP, and process-based BEPS, to estimate the diurnal courses of GPP at a maize site. In general, all three approaches adequately estimated the GPP, and the process-based BEPS model had the best performance, followed by the LUE-based MuSyQ-GPP algorithm and the SIF-based statistical 
model. The MuSyQ-GPP algorithm underestimated the GPP at high productivity, which can be attributed to its weakness in characterizing the underlying ecological processes of vegetation and the contribution of shaded leaves to total GPP. In contrast, the BEPS model that was established based on the two-leaf theory and physiological mechanisms can provide more accurate estimations. Our study indicated that the $\mathrm{SIF}_{760}$-based statistical model can provide reasonable estimates of GPP in the short term without additional information such as climate inputs, which are required by the process-based and LUE models.

The observational approach revealed that both $\mathrm{SIF}_{760}$ and GPP exhibited similar diurnal patterns as PAR, both $\mathrm{SIF}_{760}$ and GPP were linearly correlated with APAR, and a linear function could well characterize the relationship between $\operatorname{SIF}_{760}$ and GPP for maize over our study period. Despite its limitations in applying to large area and long-term studies, SIF provided a proxy for GPP not available from any other remote sensing measurements of vegetation.

Supplementary Materials: The following are available online at www.mdpi.com/2072-4292/9/12/1267/s1, Text S1: Estimation of water stress factor in the MuSyQ-GPP algorithm, Text S2: Performance of the SIF 686 -based GPP model, Figure S1: Diurnal patterns of PAR, GPP and SIF $_{686}$ during the experiment, Figure S2: Relationship between APAR and individual $\mathrm{SIF}_{686}$ and averaged $\mathrm{SIF}_{686}$, Figure S3: Relationship between individual SIF 686 and GPP, averaged SIF $_{686}$ and GPP, Figure S4: Relationships between SIF $_{686}$-based GPP and EC-based GPP during the experiment period.

Acknowledgments: This work was supported by the National Key R\&D Program of China (2017YFA0603002) and the National Natural Science Foundation of China (61661136006001, 41471349, and 41531174). We would like to thank Jingming Chen and Gang Mo from University of Toronto for providing the BEPS model.

Author Contributions: Tianxiang Cui, Rui Sun and Zhigang Liu conceived and designed the experiments; Tianxiang Cui, Chen Qiao, Qiang Zhang, Tao Yu and Gang Liu implemented the experiments; Tianxiang Cui preprocessed and analyzed the data; and Tianxiang Cui and Rui Sun prepared the paper.

Conflicts of Interest: The authors declare no conflict of interest.

\section{References}

1. Melillo, J.M.; Mcguire, A.D.; Kicklighter, D.W.; Moore, B.; Vorosmarty, C.J.; Schloss, A.L. Global Climate-Change and Terrestrial Net Primary Production. Nature 1993, 363, 234-240. [CrossRef]

2. Thurner, M.; Beer, C.; Santoro, M.; Carvalhais, N.; Wutzler, T.; Schepaschenko, D.; Shvidenko, A.; Kompter, E.; Ahrens, B.; Levick, S.R.; et al. Carbon stock and density of northern boreal and temperate forests. Glob. Ecol. Biogeogr. 2014, 23, 297-310. [CrossRef]

3. Baldocchi, D. Breathing of the terrestrial biosphere: Lessons learned from a global network of carbon dioxide flux measurement systems. Aust. J. Bot. 2008, 56, 1-26. [CrossRef]

4. Goulden, M.L.; Munger, J.W.; Fan, S.M.; Daube, B.C.; Wofsy, S.C. Measurements of carbon sequestration by long-term eddy covariance: Methods and a critical evaluation of accuracy. Glob. Chang. Biol. 1996, 2, 169-182. [CrossRef]

5. Long, S.P.; Bernacchi, C.J. Gas exchange measurements, what can they tell us about the underlying limitations to photosynthesis? Procedures and sources of error. J. Exp. Bot. 2003, 54, 2393-2401. [CrossRef] [PubMed]

6. Prieto-Blanco, A.; North, P.R.J.; Barnsley, M.J.; Fox, N. Satellite-driven modelling of net primary productivity (npp): Theoretical analysis. Remote Sens. Environ. 2009, 113, 137-147. [CrossRef]

7. Zhang, Y.; Xiao, X.M.; Jin, C.; Dong, J.W.; Zhou, S.; Wagle, P.; Joiner, J.; Guanter, L.; Zhang, Y.G.; Zhang, G.L.; et al. Consistency between sun-induced chlorophyll fluorescence and gross primary production of vegetation in North America. Remote Sens. Environ. 2016, 183, 154-169. [CrossRef]

8. Potter, C.S.; Randerson, J.T.; Field, C.B.; Matson, P.A.; Vitousek, P.M.; Mooney, H.A.; Klooster, S.A. Terrestrial ecosystem production-A process model-based on global satellite and surface data. Glob. Biogeochem. Cycles 1993, 7, 811-841. [CrossRef]

9. Prince, S.D.; Goward, S.N. Global primary production: A remote sensing approach. J. Biogeogr. 1995, 22, 815-835. [CrossRef]

10. Xiao, X.M.; Zhang, Q.Y.; Braswell, B.; Urbanski, S.; Boles, S.; Wofsy, S.; Berrien, M.; Ojima, D. Modeling gross primary production of temperate deciduous broadleaf forest using satellite images and climate data. Remote Sens. Environ. 2004, 91, 256-270. [CrossRef] 
11. Running, S.W.; Zhao, M.S. User's Guide. Daily GPP and Annual NPP (MOD17A2/A3) Products NASA Earth Observing System MODIS Land Algorithm; Version 3.0 for Collection 6. Available online: https://lpdaac.usgs.gov/sites/default/files/public/product_documentation/mod17_user_guide.pdf (accessed on 6 December 2017).

12. Cui, T.; Wang, Y.; Sun, R.; Qiao, C.; Fan, W.; Jiang, G.; Hao, L.; Zhang, L. Estimating vegetation primary production in the Heihe river basin of China with multi-source and multi-scale data. PLoS ONE 2016, 11, e0153971. [CrossRef] [PubMed]

13. Farquhar, G.D.; von Caemmerer, S.; Berry, J.A. A biochemical model of photosynthetic $\mathrm{CO}_{2}$ assimilation in leaves of c 3 species. Planta 1980, 149, 78-90. [CrossRef] [PubMed]

14. Collatz, G.J.; Ribas-Carbo, M.; Berry, J.A. Coupled photosynthesis-stomatal conductance model for leaves of C4 plants. Aust. J. Plant Physiol. 1992, 19, 519-538. [CrossRef]

15. Chen, J.M.; Liu, J.; Cihlar, J.; Goulden, M.L. Daily canopy photosynthesis model through temporal and spatial scaling for remote sensing applications. Ecol. Model. 1999, 124, 99-119. [CrossRef]

16. Liu, J.; Chen, J.M.; Cihlar, J.; Park, W.M. A process-based boreal ecosystem productivity simulator using remote sensing inputs. Remote Sens. Environ. 1997, 62, 158-175. [CrossRef]

17. McGuire, A.D.; Melillo, J.M.; Kicklighter, D.W.; Joyce, L.A. Equilibrium responses of soil carbon to climate change: Empirical and process-based estimates. J. Biogeogr. 1995, 22, 785-796. [CrossRef]

18. Sellers, P.J.; Mintz, Y.; Sud, Y.C.; Dalcher, A. A simple biosphere model (SiB) for use within general-circulation models. J. Atmos. Sci. 1986, 43, 505-531. [CrossRef]

19. Running, S.W.; Hunt, E.R., Jr. Generalization of a Forest Ecosystem Process Model for Other Biomes, Biome-BGC, and an Application for Global-Scale Models; Academic Press: San Diego, CA, USA, 1993.

20. Jung, M.; Reichstein, M.; Bondeau, A. Towards global empirical upscaling of fluxnet eddy covariance observations: Validation of a model tree ensemble approach using a biosphere model. Biogeosciences 2009, 6, 2001-2013. [CrossRef]

21. Xiao, J.F.; Ollinger, S.V.; Frolking, S.; Hurtt, G.C.; Hollinger, D.Y.; Davis, K.J.; Pan, Y.D.; Zhang, X.Y.; Deng, F.; Chen, J.Q.; et al. Data-driven diagnostics of terrestrial carbon dynamics over North America. Agric. For. Meteorol. 2014, 197, 142-157. [CrossRef]

22. Xiao, J.F.; Zhuang, Q.L.; Baldocchi, D.D.; Law, B.E.; Richardson, A.D.; Chen, J.Q.; Oren, R.; Starr, G.; Noormets, A.; Ma, S.Y.; et al. Estimation of net ecosystem carbon exchange for the conterminous united states by combining modis and ameriflux data. Agric. For. Meteorol. 2008, 148, 1827-1847. [CrossRef]

23. Frankenberg, C.; Fisher, J.B.; Worden, J.; Badgley, G.; Saatchi, S.S.; Lee, J.E.; Toon, G.C.; Butz, A.; Jung, M.; Kuze, A.; et al. New global observations of the terrestrial carbon cycle from gosat: Patterns of plant fluorescence with gross primary productivity. Geophys. Res. Lett. 2011, 38, 351-365. [CrossRef]

24. Guanter, L.; Zhang, Y.; Jung, M.; Joiner, J.; Voigt, M.; Berry, J.A.; Frankenberg, C.; Huete, A.R.; Zarco-Tejada, P.; Lee, J.E.; et al. Global and time-resolved monitoring of crop photosynthesis with chlorophyll fluorescence. Proc. Natl. Acad. Sci. USA 2014, 111, E1327-E1333. [CrossRef] [PubMed]

25. Liu, L.Y.; Guan, L.L.; Liu, X.J. Directly estimating diurnal changes in GPP for C3 and C4 crops using far-red sun-induced chlorophyll fluorescence. Agric. For. Meteorol. 2017, 232, 1-9. [CrossRef]

26. Wu, C.Y.; Munger, J.W.; Niu, Z.; Kuang, D. Comparison of multiple models for estimating gross primary production using MODIS and eddy covariance data in Harvard forest. Remote Sens. Environ. 2010, 114, 2925-2939. [CrossRef]

27. Campbell, J.E.; Berry, J.A.; Seibt, U.; Smith, S.J.; Montzka, S.A.; Launois, T.; Belviso, S.; Bopp, L.; Laine, M. Large historical growth in global terrestrial gross primary production. Nature 2017, 544, 84-87. [CrossRef] [PubMed]

28. Zhang, Y.G.; Guanter, L.; Berry, J.A.; van der Tol, C.; Yang, X.; Tang, J.W.; Zhang, F.M. Model-based analysis of the relationship between sun-induced chlorophyll fluorescence and gross primary production for remote sensing applications. Remote Sens. Environ. 2016, 187, 145-155. [CrossRef]

29. Grace, J.; Nichol, C.; Disney, M.; Lewis, P.; Quaife, T.; Bowyer, P. Can we measure terrestrial photosynthesis from space directly, using spectral reflectance and fluorescence? Glob. Chang. Biol. 2007, 13, 1484-1497. [CrossRef]

30. Gamon, J.A.; Penuelas, J.; Field, C.B. A narrow-waveband spectral index that tracks diurnal changes in photosynthetic efficiency. Remote Sens. Environ. 1992, 41, 35-44. [CrossRef] 
31. Evain, S.; Flexas, J.; Moya, I. A new instrument for passive remote sensing: 2. Measurement of leaf and canopy reflectance changes at $531 \mathrm{~nm}$ and their relationship with photosynthesis and chlorophyll fluorescence. Remote Sens. Environ. 2004, 91, 175-185. [CrossRef]

32. Barton, C.V.M.; North, P.R.J. Remote sensing of canopy light use efficiency using the photochemical reflectance index: Model and sensitivity analysis. Remote Sens. Environ. 2001, 78, 264-273. [CrossRef]

33. Rahimzadeh-Bajgiran, P.; Munehiro, M.; Omasa, K. Relationships between the photochemical reflectance index (PRI) and chlorophyll fluorescence parameters and plant pigment indices at different leaf growth stages. Photosynth. Res. 2012, 113, 261-271. [CrossRef] [PubMed]

34. Damm, A.; Guanter, L.; Paul-Limoges, E.; van der Tol, C.; Hueni, A.; Buchmann, N.; Eugster, W.; Ammann, C.; Schaepman, M.E. Far-red sun-induced chlorophyll fluorescence shows ecosystem-specific relationships to gross primary production: An assessment based on observational and modeling approaches. Remote Sens. Environ. 2015, 166, 91-105. [CrossRef]

35. Liu, L.; Liu, X.; Wang, Z.; Zhang, B. Measurement and analysis of bidirectional SIF emissions in wheat canopies. IEEE Trans. Geosci. Remote Sens. 2016, 54, 2640-2651. [CrossRef]

36. Cheng, Z.H.; Liu, L.Y. Estimating light-use efficiency by the separated solar-induced chlorophyll fluorescence from canopy spectral data. J. Remote Sens. 2010, 14, 356-371.

37. Liu, L.Y.; Zhang, Y.J.; Jiao, Q.J.; Peng, D.L. Assessing photosynthetic light-use efficiency using a solar-induced chlorophyll fluorescence and photochemical reflectance index. Int. J. Remote Sens. 2013, 34, 4264-4280. [CrossRef]

38. Rossini, M.; Nedbal, L.; Guanter, L.; Ac, A.; Alonso, L.; Burkart, A.; Cogliati, S.; Colombo, R.; Damm, A.; Drusch, M.; et al. Red and far red sun-induced chlorophyll fluorescence as a measure of plant photosynthesis. Geophys. Res. Lett. 2015, 42, 1632-1639. [CrossRef]

39. Liu, L.Y.; Cheng, Z.H. Detection of vegetation light-use efficiency based on solar-induced chlorophyll fluorescence separated from canopy radiance spectrum. IEEE J. Sel. Top. Appl. Earth Obs. Remote Sens. 2010, 3, 306-312. [CrossRef]

40. Joiner, J.; Yoshida, Y.; Vasilkov, A.P.; Yoshida, Y.; Corp, L.A.; Middleton, E.M. First observations of global and seasonal terrestrial chlorophyll fluorescence from space. Biogeosciences 2011, 8, 637-651. [CrossRef]

41. Joiner, J.; Yoshida, Y.; Vasilkov, A.P.; Middleton, E.M.; Campbell, P.K.E.; Yoshida, Y.; Kuze, A.; Corp, L.A. Filling-in of near-infrared solar lines by terrestrial fluorescence and other geophysical effects: Simulations and space-based observations from SCIAMACHY and GOSAT. Atmos. Meas. Tech. Discuss. 2012, 5, 809-829. [CrossRef]

42. Joiner, J.; Guanter, L.; Lindstrot, R.; Voigt, M.; Vasilkov, A.P.; Middleton, E.M.; Huemmrich, K.F.; Yoshida, Y.; Frankenberg, C. Global monitoring of terrestrial chlorophyll fluorescence from moderate-spectral-resolution near-infrared satellite measurements: Methodology, simulations, and application to GOME-2. Atmos. Meas. Tech. Discuss. 2013, 6, 2803-2823. [CrossRef]

43. Frankenberg, C.; O’Dell, C.; Berry, J.; Guanter, L.; Joiner, J.; Kohler, P.; Pollock, R.; Taylor, T.E. Prospects for chlorophyll fluorescence remote sensing from the Orbiting Carbon Observatory-2. Remote Sens. Environ. 2014, 147, 1-12. [CrossRef]

44. Guanter, L.; Frankenberg, C.; Dudhia, A.; Lewis, P.E.; Gomez-Dans, J.; Kuze, A.; Suto, H.; Grainger, R.G. Retrieval and global assessment of terrestrial chlorophyll fluorescence from GOSAT space measurements. Remote Sens. Environ. 2012, 121, 236-251. [CrossRef]

45. Cui, T.; Sun, R.; Qiao, C. Assessing the factors determining the relationship between solar-induced chlorophyll fluorescence and GPP. In Proceedings of the 2016 IEEE International Geoscience and Remote Sensing Symposium (IGARSS), Beijing, China, 10-15 July 2016; pp. 3520-3523.

46. Yang, X.; Tang, J.W.; Mustard, J.F.; Lee, J.E.; Rossini, M.; Joiner, J.; Munger, J.W.; Kornfeld, A.; Richardson, A.D. Solar-induced chlorophyll fluorescence that correlates with canopy photosynthesis on diurnal and seasonal scales in a temperate deciduous forest. Geophys. Res. Lett. 2015, 42, 2977-2987. [CrossRef]

47. Guan, K.Y.; Berry, J.A.; Zhang, Y.G.; Joiner, J.; Guanter, L.; Badgley, G.; Lobell, D.B. Improving the monitoring of crop productivity using spaceborne solar-induced fluorescence. Glob. Chang. Biol. 2016, 22, 716-726. [CrossRef] [PubMed] 
48. Zhang, Y.G.; Guanter, L.; Berry, J.A.; Joiner, J.; van der Tol, C.; Huete, A.; Gitelson, A.; Voigt, M.; Kohler, P. Estimation of vegetation photosynthetic capacity from space-based measurements of chlorophyll fluorescence for terrestrial biosphere models. Glob. Chang. Biol. 2014, 20, 3727-3742. [CrossRef] [PubMed]

49. Wagle, P.; Zhang, Y.; Jin, C.; Xiao, X. Comparison of solar-induced chlorophyll fluorescence, light-use efficiency, and process-based GPP models in maize. Ecol. Appl. 2016, 26, 1211-1222. [CrossRef] [PubMed]

50. Li, X.; Cheng, G.D.; Liu, S.M.; Xiao, Q.; Ma, M.G.; Jin, R.; Che, T.; Liu, Q.H.; Wang, W.Z.; Qi, Y.; et al. Heihe watershed allied telemetry experimental research (Hiwater): Scientific objectives and experimental design. Bull. Am. Meteorol. Soc. 2013, 94, 1145-1160. [CrossRef]

51. Liu, S.M.; Xu, Z.W.; Wang, W.Z.; Jia, Z.Z.; Zhu, M.J.; Bai, J.; Wang, J.M. A comparison of eddy-covariance and large aperture scintillometer measurements with respect to the energy balance closure problem. Hydrol. Earth Syst. Sci. 2011, 15, 1291-1306. [CrossRef]

52. Coops, N.C.; Black, T.A.; Jassal, R.P.S.; Trofymow, J.A.T.; Morgenstern, K. Comparison of MODIS, eddy covariance determined and physiologically modelled gross primary production (GPP) in a Douglas-fir forest stand. Remote Sens. Environ. 2007, 107, 385-401. [CrossRef]

53. Zhang, L.; Sun, R.; Xu, Z.W.; Qiao, C.; Jiang, G.Q. Diurnal and seasonal variations in carbon dioxide exchange in ecosystems in the Zhangye oasis area, Northwest China. PLoS ONE 2015, 10, e0120660. [CrossRef]

54. Gallo, K.P.; Daughtry, C.S.T. Techniques for measuring intercepted and absorbed photosynthetically active radiation in corn canopies. Agron. J. 1986, 78, 752-756. [CrossRef]

55. Campbell, P.K.; Middleton, E.M.; Corp, L.A.; Kim, M.S. Contribution of chlorophyll fluorescence to the apparent vegetation reflectance. Sci. Total Environ. 2008, 404, 433-439. [CrossRef] [PubMed]

56. Guanter, L.; Alonso, L.; Gomez-Chova, L.; Meroni, M.; Preusker, R.; Fischer, J.; Moreno, J. Developments for vegetation fluorescence retrieval from spaceborne high-resolution spectrometry in the $\mathrm{O}_{2} \mathrm{a}$ and $\mathrm{O}_{2} \mathrm{~b}$ absorption bands. J. Geophys. Res. Atmos. 2010, 115, 1485-1490. [CrossRef]

57. Plascyk, J.A. Mk II fraunhofer line discriminator (FLD-II) for airborne and orbital remote-sensing of solar-stimulated luminescence. Opt. Eng. 1975, 14, 339-346. [CrossRef]

58. Plascyk, J.A.; Gabriel, F.C. Fraunhofer line discriminator MKII-Airborne instrument for precise and standardized ecological luminescence measurement. IEEE Trans. Instrum. Meas. 1975, 24, 306-313. [CrossRef]

59. Damm, A.; Elbers, J.; Erler, A.; Gioli, B.; Hamdi, K.; Hutjes, R.; Kosvancova, M.; Meroni, M.; Miglietta, F.; Moersch, A.; et al. Remote sensing of sun-induced fluorescence to improve modeling of diurnal courses of gross primary production (GPP). Glob. Chang. Biol. 2010, 16, 171-186. [CrossRef]

60. Meroni, M.; Rossini, M.; Guanter, L.; Alonso, L.; Rascher, U.; Colombo, R.; Moreno, J. Remote sensing of solar-induced chlorophyll fluorescence: Review of methods and applications. Remote Sens Environ. 2009, 113, 2037-2051. [CrossRef]

61. Porcar-Castell, A.; Tyystjarvi, E.; Atherton, J.; van der Tol, C.; Flexas, J.; Pfundel, E.E.; Moreno, J.; Frankenberg, C.; Berry, J.A. Linking chlorophyll a fluorescence to photosynthesis for remote sensing applications: Mechanisms and challenges. J. Exp. Bot. 2014, 65, 4065-4095. [CrossRef] [PubMed]

62. Liu, L.Y.; Liu, X.J.; Guan, L.L. In Uncertainties in linking solar-induced chlorophyll fluorescence to plant photosynthetic activities. In Proceedings of the 2016 IEEE International Geoscience and Remote Sensing Symposium (IGARSS), Beijing, China, 10-15 July 2016; pp. 4414-4417.

63. Maier, S.W.; Günther, K.P.; Stellmes, M. Sun-induced fluorescence: a new tool for precision farming. In Digital Imaging and Spectral Techniques: Applications to Precision Agriculture and Crop Physiology; American Society of Agronomy: Madison, WI, USA, 2003; pp. 209-222.

64. Damm, A.; Erler, A.; Hillen, W.; Meroni, M.; Schaepman, M.E.; Verhoef, W.; Rascher, U. Modeling the impact of spectral sensor configurations on the FLD retrieval accuracy of sun-induced chlorophyll fluorescence. Remote Sens. Environ. 2011, 115, 1882-1892. [CrossRef]

65. Liu, X.J.; Liu, L.Y. Assessing band sensitivity to atmospheric radiation transfer for space-based retrieval of solar-induced chlorophyll fluorescence. Remote Sens. 2014, 6, 10656-10675. [CrossRef]

66. Monteith, J.L. Solar-radiation and productivity in tropical ecosystems. J. Appl. Ecol. 1972, 9, 747-766. [CrossRef]

67. Fournier, A.; Daumard, F.; Champagne, S.; Ounis, A.; Goulas, Y.; Moya, I. Effect of canopy structure on sun-induced chlorophyll fluorescence. ISPRS J. Photogramm. Remote Sens. 2012, 68, 112-120. [CrossRef] 
68. Cui, T.X.; Sun, R.; Qiao, C. Analyzing the Relationship between Solar-induced Chlorophyll Fluorescence and Gross Primary Production using Remotely Sensed Data and Model Simulation. Int. J. Earth Environ. Sci. 2017, 2, 129. [CrossRef]

69. Zhang, L. A Study on Carbon Fluxes of Different Underlying Surfaces in Zhangye Oasis Irrigation Area. Ph.D. Thesis, Beijing Normal University, Beijing, China, 2016.

70. Kalfas, J.L.; Xiao, X.M.; Vanegas, D.X.; Verma, S.B.; Suyker, A.E. Modeling gross primary production of irrigated and rain-fed maize using MODIS imagery and $\mathrm{CO}_{2}$ flux tower data. Agric. For. Meteorol. 2011, 151, 1514-1528. [CrossRef]

71. Qiao, C.; Sun, R.; Xu, Z.W.; Zhang, L.; Liu, L.Y.; Hao, L.Y.; Jiang, G.Q. A study of shelterbelt transpiration and cropland evapotranspiration in an irrigated area in the middle reaches of the Heihe River in northwestern China. IEEE Geosci. Remote Sens. Lett. 2015, 12, 369-373. [CrossRef]

72. Zhang, K.; Kimball, J.S.; Mu, Q.Z.; Jones, L.A.; Goetz, S.J.; Running, S.W. Satellite based analysis of northern et trends and associated changes in the regional water balance from 1983 to 2005. J. Hydrol. 2009, 379, 92-110. [CrossRef]

73. Zhang, Y.Q.; Chiew, F.H.S.; Zhang, L.; Leuning, R.; Cleugh, H.A. Estimating catchment evaporation and runoff using MODIS leaf area index and the Penman-Monteith equation. Water Resour. Res. 2008, 44, 2183-2188. [CrossRef]

74. Zhang, K.; Kimball, J.S.; Nemani, R.R.; Running, S.W. A continuous satellite-derived global record of land surface evapotranspiration from 1983 to 2006. Water Resour. Res. 2010, 46, 109-118. [CrossRef]

75. Priestley, C.H.B.; Taylor, R.J. On the assessment of surface heat flux and evaporation using large-scale parameters. Mon. Weather Rev. 1972, 100, 81-92. [CrossRef]

76. Ju, W.M.; Chen, J.M.; Black, T.A.; Barr, A.G.; Liu, J.; Chen, B.Z. Modelling multi-year coupled carbon and water fluxes in a boreal aspen forest. Agric. For. Meteorol. 2006, 140, 136-151. [CrossRef]

77. Matsushita, B.; Tamura, M. Integrating remotely sensed data with an ecosystem model to estimate net primary productivity in East Asia. Remote Sens. Environ. 2002, 81, 58-66. [CrossRef]

78. Matsushita, B.; Xu, M.; Chen, J.; Kameyama, S.; Tamura, M. Estimation of regional net primary productivity (NPP) using a process-based ecosystem model: How important is the accuracy of climate data? Ecol. Model. 2004, 178, 371-388. [CrossRef]

79. Leuning, R.; Kelliher, F.M.; Depury, D.G.G.; Schulze, E.D. Leaf nitrogen, photosynthesis, conductance and transpiration: Scaling from leaves to canopies. Plant Cell Environ. 1995, 18, 1183-1200. [CrossRef]

80. Sellers, P.J.; Randall, D.A.; Collatz, G.J.; Berry, J.A.; Field, C.B.; Dazlich, D.A.; Zhang, C.; Collelo, G.D.; Bounoua, L. A revised land surface parameterization (SiB2) for atmospheric GCMs.1. Model formulation. J. Clim. 1996, 9, 676-705. [CrossRef]

81. Ball, J.T.; Woodrow, I.E.; Berry, J.A. A model predicting stomatal conductance and its contribution to the control of photosynthesis under different environmental conditions. In Progress in Photosynthesis Research; Springer: Dordrecht, The Netherlands, 1987; pp. 221-224.

82. Cheng, Y.B.; Middleton, E.M.; Zhang, Q.Y.; Huemmrich, K.F.; Campbell, P.K.E.; Corp, L.A.; Cook, B.D.; Kustas, W.P.; Daughtry, C.S. Integrating solar induced fluorescence and the photochemical reflectance index for estimating gross primary production in a cornfield. Remote Sens. 2013, 5, 6857-6879. [CrossRef]

83. Cendrero-Mateo, M.P.; Carmo-Silva, A.E.; Porcar-Castell, A.; Hamerlynck, E.P.; Papuga, S.A.; Moran, M.S. Dynamic response of plant chlorophyll fluorescence to light, water and nutrient availability. Funct. Plant Biol. 2015, 42, 746-757. [CrossRef]

84. Cogliati, S.; Rossini, M.; Julitta, T.; Meroni, M.; Schickling, A.; Burkart, A.; Pinto, F.; Rascher, U.; Colombo, R. Continuous and long-term measurements of reflectance and sun-induced chlorophyll fluorescence by using novel automated field spectroscopy systems. Remote Sens. Environ. 2015, 164, 270-281. [CrossRef]

85. Alonso, L.; Gómez-Chova, L.; Vila-Francés, J.; Amorós-López, J.; Guanter, L.; Calpe, J. Improved Fraunhofer Line Discrimination method for vegetation fluorescence quantification. IEEE Geosci. Remote Sens. Lett. 2008, 5, 620-624. [CrossRef]

86. Meroni, M.; Colombo, R. Leaf level detection of solar induced chlorophyll fluorescence by means of a subnanometer resolution spectroradiometer. Remote Sens. Environ. 2006, 103, 438-448. [CrossRef] 
87. Van der Tol, C.; Berry, J.A.; Campbell, P.K.; Rascher, U. Models of fluorescence and photosynthesis for interpreting measurements of solar-induced chlorophyll fluorescence. J. Geophys. Res. Biogeosci. 2014, 119, 2312-2327. [CrossRef] [PubMed]

88. Wang, Z. Sunlit Leaf Photosynthesis Rate Correlates Best with Chlorophyll Fluorescence of Terrestrial Ecosystems. Master's Thesis, University of Toronto, Toronto, ON, Canadian, 2014.

89. Brugnoli, E.; Björkman, O. Chloroplast movements in leaves: influence on chlorophyll fluorescence and measurements of light-induced absorbance changes related to $\Delta \mathrm{pH}$ and zeaxanthin formation. Photosynth. Res. 1992, 32, 23-35. [CrossRef] [PubMed]

90. Chen, B.; Black, T.A.; Coops, N.C.; Hilker, T.; Trofymow, J.T.; Morgenstern, K. Assessing tower flux footprint climatology and scaling between remotely sensed and eddy covariance measurements. Bound Lay Meteorol. 2009, 130, 137-167. [CrossRef]

91. Veefkind, J.P.; Aben, I.; McMullan, K.; Forster, H.; de Vries, J.; Otter, G.; Claas, J.; Eskes, H.J.; de Haan, J.F.; Kleipool, Q.; et al. Tropomi on the ESA sentinel-5 precursor: A GMES mission for global observations of the atmospheric composition for climate, air quality and ozone layer applications. Remote Sens. Environ. 2012, 120, 70-83. [CrossRef]

92. Rascher, U. Flex-Fluorescence explorer: A remote sensing approach to quantify spatio-temporal variations of photosynthetic efficiency from space. Photosynth. Res. 2007, 91, 293-294. [CrossRef]

93. Drusch, M.; Moreno, J.; Bello, U.D.; Franco, R.; Goulas, Y.; Huth, A.; Kraft, S.; Middleton, E.M.; Miglietta, F.; Mohammed, G.; et al. The FLuorescence EXplorer Mission Concept-ESA's Earth Explorer 8. IEEE Trans. Geosci. Remote Sens. 2017, 55, 1273-1284. [CrossRef]

94. Van der Tol, C.; Verhoef, W.; Timmermans, J.; Verhoef, A.; Su, Z. An integrated model of soil-canopy spectral radiances, photosynthesis, fluorescence, temperature and energy balance. Biogeosciences 2009, 6, 3109-3129. [CrossRef]

95. Zhang, F.M.; Chen, J.M.; Chen, J.Q.; Gough, C.M.; Martin, T.A.; Dragoni, D. Evaluating spatial and temporal patterns of MODIS GPP over the conterminous us against flux measurements and a process model. Remote Sens. Environ. 2012, 124, 717-729. [CrossRef]

96. Dai, Y.J.; Dickinson, R.E.; Wang, Y.P. A two-big-leaf model for canopy temperature, photosynthesis, and stomatal conductance. J. Clim. 2004, 17, 2281-2299. [CrossRef]

97. De Pury, D.G.G.; Farquhar, G.D. Simple scaling of photosynthesis from leaves to canopies without the errors of big-leaf models. Plant Cell Environ. 1997, 20, 537-557. [CrossRef]

98. Leuning, R.; Dunin, F.X.; Wang, Y.P. A two-leaf model for canopy conductance, photosynthesis and partitioning of available energy. II. Comparison with measurements. Agric. For. Meteorol. 1998, 91, 113-125. [CrossRef]

99. Wang, Y.P.; Leuning, R. A two-leaf model for canopy conductance, photosynthesis and partitioning of available energy I: Model description and comparison with a multi-layered model. Agric. For. Meteorol. 1998, 91, 89-111. [CrossRef]

100. Emerson, R.; Lewis, C.M. Carbon dioxide exchange and the measurement of the quantum yield of photosynthesis. Am. J. Bot. 1941, 28, 789-804. [CrossRef]

101. Cheng, Y.B.; Zhang, Q.Y.; Lyapustin, A.I.; Wang, Y.J.; Middleton, E.M. Impacts of light use efficiency and fpar parameterization on gross primary production modeling. Agric. For. Meteorol. 2014, 189, 187-197. [CrossRef]

102. Kosugi, Y.; Shibata, S.; Kobashi, S. Parameterization of the $\mathrm{CO}_{2}$ and $\mathrm{H}_{2} \mathrm{O}$ gas exchange of several temperate deciduous broad-leaved trees at the leaf scale considering seasonal changes. Plant Cell Environ. 2003, 26, 285-301. [CrossRef]

103. Medvigy, D.; Wofsy, S.C.; Munger, J.W.; Hollinger, D.Y.; Moorcroft, P.R. Mechanistic scaling of ecosystem function and dynamics in space and time: Ecosystem demography model version 2. J. Geophys. Res. Biogeosci. 2009, 114, 270-271. [CrossRef]

104. Wilson, K.B.; Baldocchi, D.D.; Hanson, P.J. Leaf age affects the seasonal pattern of photosynthetic capacity and net ecosystem exchange of carbon in a deciduous forest. Plant Cell Environ. 2001, 24, 571-583. [CrossRef]

105. Koffi, E.N.; Rayner, P.J.; Norton, A.J.; Frankenberg, C.; Scholze, M. Investigating the usefulness of satellite-derived fluorescence data in inferring gross primary productivity within the carbon cycle data assimilation system. Biogeosciences 2015, 12, 4067-4084. [CrossRef] 
106. Serbin, S.P.; Singh, A.; Desai, A.R.; Dubois, S.G.; Jablonski, A.D.; Kingdon, C.C.; Kruger, E.L.; Townsend, P.A. Remotely estimating photosynthetic capacity, and its response to temperature, in vegetation canopies using imaging spectroscopy. Remote Sens. Environ. 2015, 167, 78-87. [CrossRef]

107. Houborg, R.; Cescatti, A.; Migliavacca, M.; Kustas, W.P. Satellite retrievals of leaf chlorophyll and photosynthetic capacity for improved modeling of GPP. Agric. For. Meteorol. 2013, 177, 10-23. [CrossRef]

(c) 2017 by the authors. Licensee MDPI, Basel, Switzerland. This article is an open access article distributed under the terms and conditions of the Creative Commons Attribution (CC BY) license (http:/ / creativecommons.org/licenses/by/4.0/). 
Article

\title{
Monitoring Rice Phenology Based on Backscattering Characteristics of Multi-Temporal RADARSAT-2 Datasets
}

\author{
Ze He ${ }^{1}$, Shihua $\mathrm{Li}^{1,2, *}$, Yong Wang ${ }^{3}$, Leiyu Dai ${ }^{1}$ and Sen $\operatorname{Lin}^{1}$ \\ 1 School of Resources and Environment, University of Electronic Science and Technology of China, No. 2006, \\ Xiyuan Ave, West Hi-Tech Zone, Chengdu 611731, China; 201622180134@std.uestc.edu.cn (Z.H.); \\ 201622180135@std.uestc.edu.cn (L.D.); 201622180136@std.uestc.edu.cn (S.L.) \\ 2 Center for Information Geoscience, University of Electronic Science and Technology of China, No. 2006, \\ Xiyuan Ave, West Hi-Tech Zone, Chengdu 611731, China \\ 3 Department of Geography, Planning, and Environment, East Carolina University, Greenville, NC 27858, \\ USA; wangy@ecu.edu \\ * Correspondence: lishihua@uestc.edu.cn; Tel.: +86-135-5126-5998
}

Received: 5 January 2018; Accepted: 18 February 2018; Published: 23 February 2018

\begin{abstract}
Accurate estimation and monitoring of rice phenology is necessary for the management and yield prediction of rice. The radar backscattering coefficient, one of the most direct and accessible parameters has been proved to be capable of retrieving rice growth parameters. This paper aims to investigate the possibility of monitoring the rice phenology (i.e., transplanting, vegetative, reproductive, and maturity) using the backscattering coefficients or their simple combinations of multi-temporal RADARSAT-2 datasets only. Four RADARSAT-2 datasets were analyzed at 30 sample plots in Meishan City, Sichuan Province, China. By exploiting the relationships of the backscattering coefficients and their combinations versus the phenology of rice, $\mathrm{HH} / \mathrm{VV}, \mathrm{VV} / \mathrm{VH}$, and $\mathrm{HH} / \mathrm{VH}$ ratios were found to have the greatest potential for phenology monitoring. A decision tree classifier was applied to distinguish the four phenological phases, and the classifier was effective. The validation of the classifier indicated an overall accuracy level of $86.2 \%$. Most of the errors occurred in the vegetative and reproductive phases. The corresponding errors were $21.4 \%$ and $16.7 \%$, respectively.
\end{abstract}

Keywords: phenology; RADARSAT-2; rice; Synthetic Aperture Radar (SAR); decision tree

\section{Introduction}

Rice is one of the most important cultivated grain crops and is the staple food of nearly half of the world's population [1]. Approximately 969 million metric tons of rice were produced worldwide in 2010 and about one-third of them were from China [2]. Effective management of rice cultivation that includes the prediction of the growth conditions and stages of rice accurately and timely is crucial to food security and social stability. Thus, the accurate acquisition of rice phenological information is an important component of the farming management system [3-5]. This type of information provides an accurate knowledge on the status of rice plants, leading to different cultivation practices (e.g., irrigation, fertilization, or harvest) [6-8]. Rice phenology is also used as an input in the rice growth and yield prediction models, ecosystem productivity models, and land surface process models $[6,9,10]$.

The phenological stages of crops can be measured using a field survey, simulation by bioclimatic models, or detection with remotely sensed data [7,11]. Conventional ground-based rice phenology monitoring provides accurate in situ information if properly designed and executed. The monitoring is, unfortunately, linked to enormous costs of time, money, and man-power [12,13], and is not practical at a large spatial extent and for long-term monitoring and analysis. During the past decades, a series 
of spaceborne optical and synthetic aperture radar (SAR) sensors with a high or moderate resolution have been launched, and new approaches for the phenology study have been conceived. Optical data such as the Moderate Resolution Imaging Spectroradiometer (MODIS) [3,14-17], and HJ-1 and Landsat data [18] are used to effectively estimate rice phenology. However, an optical sensor is vulnerable to the variation of atmospheric conditions and cloud covers. Compared to the optical sensor, SAR has an all-time and all-weather imaging capability. SAR data are sensitive to surface features [19] and inundated/un-inundated rice fields [20]. Thus, SAR plays an important role in the monitoring of rice in cloudy or foggy areas.

Many researchers have obtained successful results in rice planting monitoring using X-band sensors (e.g., COSMO-SkyMed and TerraSAR-X) [13,21-25] and C-band sensors (e.g., Envisat-ASAR, RADARSAT-2, and Sentinel-1) [19,20,26-28]. After the successful launch of spaceborne SARs with short revisit periods (compared to the main phenological phases of rice with a short duration, e.g., about 16 days of reproductive phase) or polarimetric mode, the acquisition of timely and accurate information about the rice condition during the growing period became readily possible. Lopez-Sanchez et al. [5] used TerraSAR- $X$ data to retrieve rice phenological phases with the correlation and phase difference between co-polar channels and parameters provided by polarimetric decomposition techniques. The potential of TerraSAR-X data for rice phenology monitoring has been well documented $[5,7,21-23]$. However, the penetration depth into the canopy is shallow for radar energy at a high frequency compared to that at a low frequency [29]. Therefore, the backscattering at X-band may provide less information on the structure of rice plants than that at C-band [29,30]. Tian et al. [28] have used C-band Sentinel-1A and Landsat-8 data to map multi-season paddy rice based on backscattering coefficients $\left(\sigma^{0}\right)$ and the normalized difference vegetation index (NDVI) with a K-Means unsupervised classifier. The high temporal resolution, six days using the two-satellite constellation of Sentinel-1, makes Sentinel-1 data suitable for rice monitoring. However, Sentinel-1 SAR can only provide single or dual-pol data. Since various polarized radar backscattering coefficients respond to rice plants differently $[6,25,31]$, quad-polarized data (e.g., RADARSAT-2) can supply more comprehensive information on rice growth status than single and dual-polarized data.

Although the revisit time of TerraSAR-X (11 days) and Sentinel-1 (six or 12 days) is shorter than that of RADARSAT-2, whose revisit period is 24 days, RADARSAT-2 can still observe a rice field at least four times during the typical cultivation cycle of rice [6,9]. Because of the different cultivation management (e.g., transplanting time and fertilization) and rice varieties in individual fields, the pace of rice phenology evolution varies at field-by-field levels [11]. Thus, it is acceptable to observe the fields in each of the four principle phenological phases (i.e., transplanting, vegetative, reproductive, and maturity) once in 24 days or longer. Besides, RADARSAT-2 provides quad-polarization (VH, $\mathrm{VV}, \mathrm{HH}, \mathrm{HV}$ ) data, and is very effective in retrieving rice canopy parameters and estimating biomass that are linked to the crop yield [30,32]. As a result, the sensor has become an ideal data source for rice phenology monitoring. Lopez-Sanchez et al. [6] analyzed RADARSAT-2 data and proposed a retrieval algorithm with radar target decomposition and compact polarimetric $(\mathrm{CP})$ variables. Francis et al. [27] found that the combination of the alpha angle and beta angle derived from the Cloude-Pottier decomposition of RADARSAT-2 data provided satisfactory estimations of the crop phenology. Yang et al. [9] used RADARSAT-2 data to simulate the CP SAR data for identifying seven phenological phases of rice fields. However, most of these studies require various indirect parameters like the $\mathrm{CP}$ and target decomposition parameters. The retrieval processes are generally complex including filtering and image classification that may create new uncertainties in data analysis.

Of the SAR polarimetric parameters (i.e., amplitude and phase data), the backscattering coefficient is proportional to the square of amplitude and is the most basic one [5]. Many researches have demonstrated that the rice backscattering coefficient alone is sensitive to plant height, biomass, and leaf area index (LAI) [19,20,33-37]. These plant parameters are highly correlated with rice phenology development, showing a great potential to monitor rice phenology. However, the observed correlation between rice biophysical variables and certain polarizations $[25,30]$ can be temporally unstable. This 
is caused by changes of the electromagnetic interactions between radar waves and rice scattering components as the plant grows and different polarization responses to the changes [20]. Li et al. [31] used multi-temporal, quad-polarization RADARSAT-2 data to establish empirical regression models to link rice biophysical parameters to backscattering coefficients. The optimal polarization to estimate biophysical variables was a function of different stages, which suggests a piecewise fitting strategy using different polarizations at different growing stages. As rice plants develop during a growing season and biophysical variables change during different phenological phases, $\sigma^{0}$ acquired at different phases should be clustered in the domains of polarizations and/or combinations of polarizations, making the decision tree strategy with particular thresholds theoretically feasible for the separation of phenological phases. Therefore, the objective of this study is to use only radar backscattering coefficients and their combinations derived from multi-temporal and full-polarized RADARSAT-2 datasets for rice phenology monitoring. The focus of the study is to utilize a simple decision tree model for the characterization of rice phenology in the feature space of backscattering coefficients of multi-temporal RADARSAT-2 datasets.

\section{Materials and Methods}

\subsection{Study Area and Field Observation}

Chengdu Basin, southwestern China, is a main rice production region in China, where rice is cultivated once per year. The growing season is typically from May to August. Under the influence of subtropical monsoon climate and basin terrain characteristics, the sky is constantly cloudy and rainy for most of the growing period. The study area, Meishan City, is located in the western part of Chengdu Basin. Field observations were conducted about every 12 days from 2 May to 8 August in 2016 at 30 sample sites (Figure 1). Each site was at least 50m wide and 50m long. A hand-held GPS unit was used to locate the sites and 11 ground control points (GCPs), which were used to geo-reference the radar data and sites. Plant height, stem diameter, length and width of leaves, and tiller and ear number were measured to identify rice phenology.

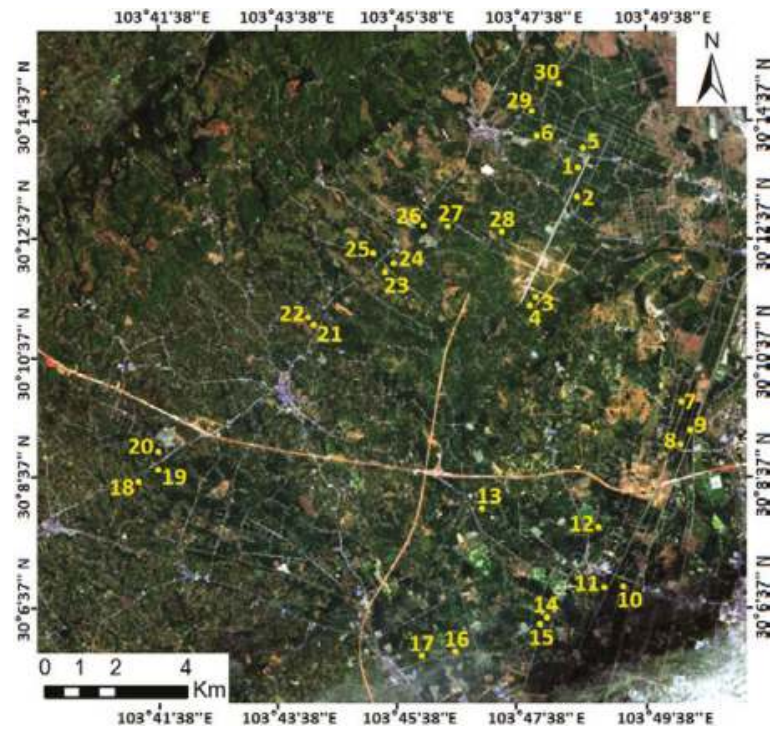

Figure 1. The study area imaged by the French SPOT-6 optical sensor on 15 July 2016. Thirty sample sites are located and numbered. 


\subsection{Rice Phenology}

Rice phenology can be divided into three main phases: vegetative, reproductive, and maturity, according to the widely-used Biologische Bundesanstalt, Bundessortenamt und $\mathrm{CHemische} \mathrm{scale}$ (a German scale used to identify the phenological development stages of cereals, BBCH) $[13,21,38]$. The principal phases and corresponding numerical ranges are shown in Table 1. Most of the Asian rice cultivars are largely grown under the transplanting procedure, which marks the beginning of a cultivation cycle in fields. Transplanting induces a development stoppage followed by a recovery period that can last about 19 days on average in this study area. Besides, the backscattering properties of a rice paddy during this period can be quite different from other stages in the vegetative phase, as shown later in this study. Therefore, the transplanting phase was extracted from the vegetative phase and specially analyzed.

Table 1. Description of the phenology of cereals using the BBCH Scale.

\begin{tabular}{ccc}
\hline Principle Phase & BBCH & Name \\
\hline Vegetative & $00-09$ & Germination \\
& $10-19$ & Leaf development \\
& $20-29$ & Tillering \\
& $30-39$ & Stem elongation \\
& $40-49$ & Booting \\
\hline Reproductive & $50-59$ & Heading \\
& $60-69$ & Flowering \\
\hline Maturity & $70-79$ & Development of fruit \\
& $80-89$ & Ripening \\
& $90-99$ & Senescence \\
\hline Transplanting & $00-19$ & Transplanting, recovery (rice only)
\end{tabular}

Four phenological phases, including the transplanting (from stage 0 to 19), vegetative (from stage 20 to 49 ), reproductive (from stage 50 to 69), and maturity (from stage 70 to 99) phases, are studied. They are distinguishable based on field observations (Figure 2) according to the $\mathrm{BBCH}$ scale. Fields at a sample site reach a particular BBCH stage when more than $50 \%$ of their plants reach that stage [6]. This criterion is considered in the analyses of the ground measurements and radar images.

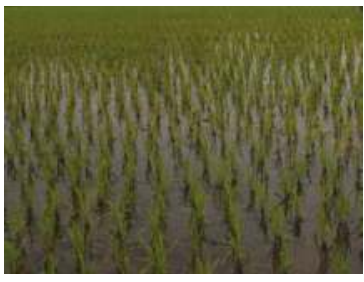

(a)

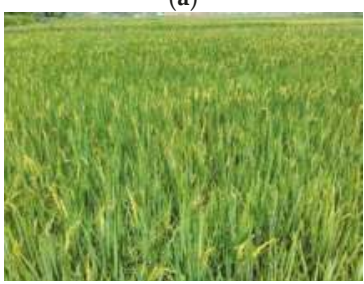

(c)

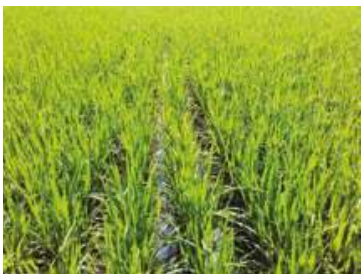

(b)

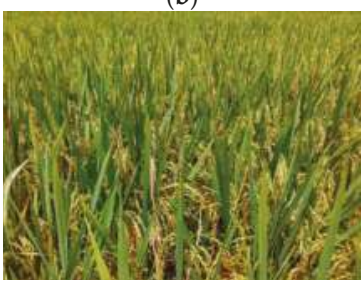

(d)

Figure 2. Four rice phenological phases in the study area in 2016. (a) transplanting, (b) vegetative, (c) reproductive, and (d) maturity. 
According to field observations, the average durations of the transplanting, vegetative, reproductive, and maturity phase were 19, 45, 16, and 30 days, respectively, for fields in 30 sample sites in 2016. The 24-day revisit period of RADARSAT-2 might be too coarse for the monitoring of the phase with 16 or 19 days. However, because of the different cultivation management (e.g., transplanting time and fertilization) and rice varieties, the evolution pace of rice phenology varied at different sites. Accordingly, each phenological phase had an extended span and the corresponding phase can be observed. For example, the reproductive phase lasted about 16 days for most of the fields, but field observations showed that the reproductive phase started from June 22 and ended on July 26 in 2016 (Figure 3).

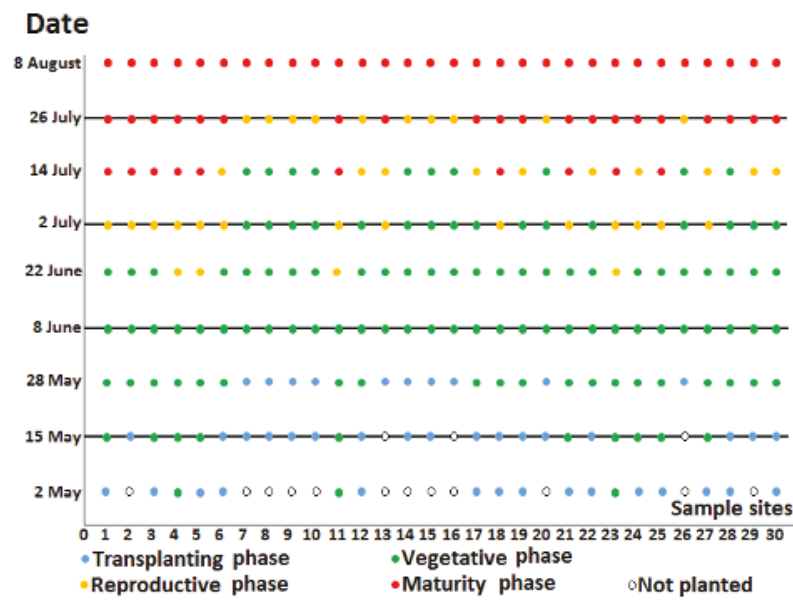

Figure 3. In situ rice phenology at 30 sample sites in 2016. Blue, green, yellow, and red dots, respectively, represent the transplanting, vegetative, reproductive, and maturity phase. Four horizontal lines denote the acquisition dates of four RADARSAT-2 datasets.

Table 2 shows the typical growth rhythm extracted from the field measurement. About $63 \%$, $100 \%, 47 \%$, and $67 \%$ of sample sites were, respectively, in transplanting, vegetative, reproductive, and maturity phases on the dates of RADARSAT-2 acquisitions in 2016 (Table 2). It should be noted that on 15 May 2016, fields where three sample sites were located were not planted yet.

Table 2. The number of sample sites in different phenological phases when SAR datasets were acquired. On 15 May 2016, fields where three sample sites were located were not planted yet.

\begin{tabular}{cccccc}
\hline & & \multicolumn{3}{c}{ Acquisition Dates of SAR Datasets } \\
\cline { 3 - 6 } & & 15 May & 8 June & 2 July & 26 July \\
\hline \multirow{3}{*}{ Fields hase } & Transplanting & 17 & 0 & 0 & 0 \\
& Vegetative & 10 & 30 & 16 & 0 \\
& Reproductive & 0 & 0 & 14 & 10 \\
& Maturity & 0 & 0 & 0 & 20 \\
\hline
\end{tabular}

\subsection{RADARSAT-2 Data Preprocessing}

Four fine quad-polarized single-look complex (SLC) RADARSAT-2 SAR images were acquired between May and July of 2016 (Table 2). The revisit period was 24 days. The images had a nominal spatial resolution of $5.4 \mathrm{~m}$ in the slant range direction and $7.9 \mathrm{~m}$ in the azimuth direction. The swath width of the image was about $25 \mathrm{~km}$. The incidence angle ranged from $31^{\circ}$ at near range to $33^{\circ}$ at far 
range. The Next ESA SAR Toolbox (NEST) software (https:/ / earth.esa.int/web/nest/downloads/) was utilized for data preprocessing including radiometric correction, multilooking, geometry reprojection, speckle filtering, and multi-temporal coregistration. A color composite is shown in Figure 4. Backscattering coefficients (linear scale) of all images on four polarizations ( $\mathrm{VH}, \mathrm{VV}, \mathrm{HH}$, and HV) were extracted for 30 sample sites. As described previously, each sample site is at least $50 \mathrm{~m}$ wide and $50 \mathrm{~m}$ long and the preprocessed images have a pixel size of $11.6 \mathrm{~m} \times 11.6 \mathrm{~m}$, which means that each sample site has at least 4 by 4 or 16 pixels. Therefore, the mean value of the backscattering coefficients within a sample site was calculated to represent the backscatter properties of that site.

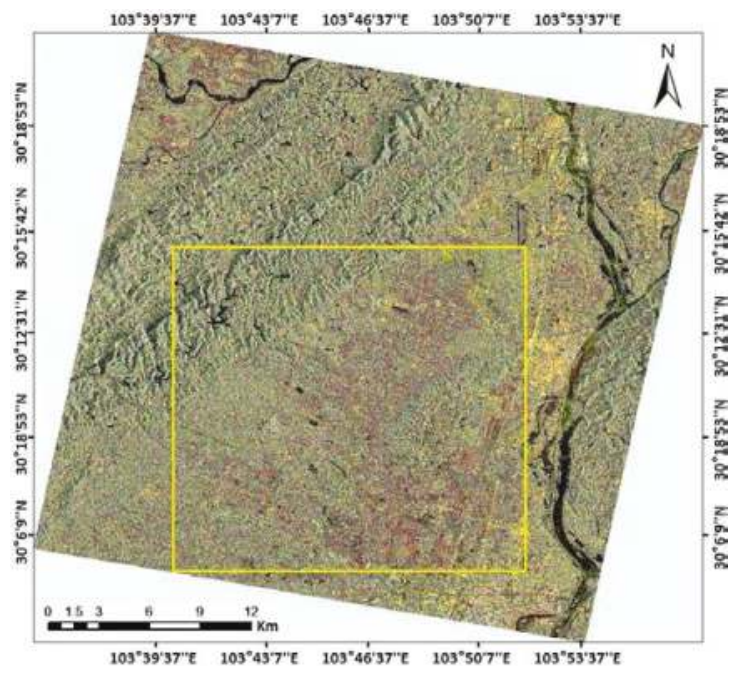

Figure 4. A RADARSAT-2 image acquired on 2 July 2016. VH, VV, and HH bands were assigned as red, green, and blue colors, respectively. Study area is within the yellow box.

Cross-polarization VH data and HV data were the same. Thus, only VH, VV, and $\mathrm{HH}$ data were used. Previous studies have found that combinations (e.g., addition, subtraction, multiplication, and ratioing) of different polarized backscattering coefficients were more sensitive to the morphological structures of rice plants $[20,31,39]$. These operations calculated in a linear scale first, and then the results expressed in a logarithmic scale were explored.

All backscattering coefficients and combinations, acquired at different sample sites and on different dates, were divided into four groups based on the four phenological phases identified from ground observations (Table 2) for further analyses. Three-quarters of each group (i.e., 13 of 17 transplanting datasets, 42 of 56 vegetative datasets, 18 of 24 reproductive datasets, and 15 of 20 maturity datasets) were randomly chosen for training. The rest in each group was used for validation, respectively.

\subsection{Decision Tree Method for Phenology Retrieval}

The decision tree method is a classification strategy to process non-linear relationships between features and classes without any assumptions about the data probability distributions and to handle data measured on different scales [40]. The decision tree or similar methods like the decision plane is effective in rice monitoring with compact polarimetric $(\mathrm{CP})$ parameters or target decomposition parameters $[5,6,9]$. The decision tree is of a treelike structure. The leaves are class labels, and intermediate nodes are criteria with several possible outcome branches. The classification processes begin at the root note and intermediate notes until encountering a leaf. A typical binary decision tree is shown in Figure 5. 


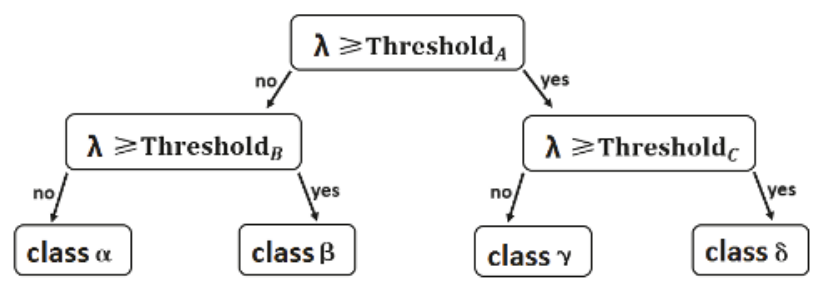

Figure 5. A simple decision tree of a variable, $\lambda$. Three thresholds divide $\lambda$ into four classes through two decision layers.

In this study, four concerned phenological phases (i.e., transplanting, vegetative, reproductive, and maturity) need to be separated. Therefore, at least three thresholds are required. In order to distinguish rice phenology with the thresholds, one needs to ensure that backscattering coefficients or their combinations must have reliable separability among four critical phases. Since rice plants develop rapidly during the growing season and biophysical variables vary with phenological phases, the development phases are separable from each other. For example, distributions of the phases vs. VH backscattering coefficients are shown in Figure 6 using a boxplot. A division line near $-20 \mathrm{~dB}$ can divide the transplanting phase from the other three. Similar division lines between other phenological phases were also observed on other polarizations (as shown in Figure 7). Once segmental thresholds to delineate four phenological phases were determined, a decision tree classifier could be developed to separate each development phase using backscattering coefficients.

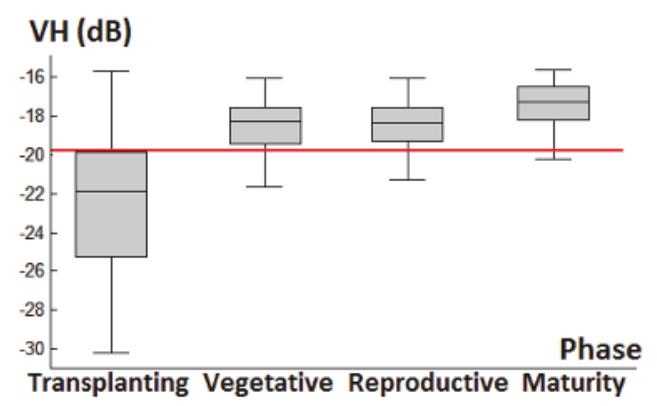

Figure 6. Distributions of development phases vs. VH backscattering coefficients. The data are training data. At about $-20 \mathrm{~dB}$, the transplanting phase is separated from other three phases.

\section{Results}

\subsection{Backscattering Coefficient Analysis and Decision Tree Development}

Distributions of backscattering coefficients of the training dataset, expressed in a logarithmic scale, and their combinations (calculated in linear scale then expressed in logarithmic scale) at each phase, are shown in boxplots (Figure 7). In the figure, $\mathrm{VH} /(2 \mathrm{VH}+\mathrm{VV}+\mathrm{HH})$ was also plotted because of its sensitivity to vegetation parameters [41]. In each subplot, four boxes were drawn to represent the distributions of four phases. The red line visually showed a feasible division value. For example, the $\mathrm{VH}$ backscattering coefficients increased in general from the transplanting phase to the maturity phase (Figure $7 \mathrm{a}$ ). The red line near $-20 \mathrm{~dB}$ divided the transplanting phase from later phases with few data points crossing the division line. Ranges of the later phases, especially the vegetative and reproductive phase, severely overlapped with each other. Thus, VH data could not discriminate the vegetative phase from the reproductive phase. 


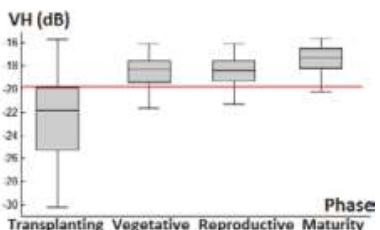

(a)

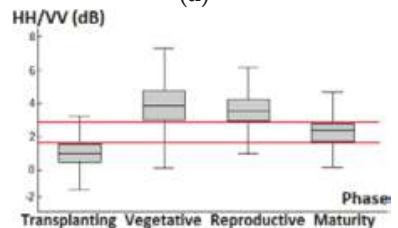

(d)

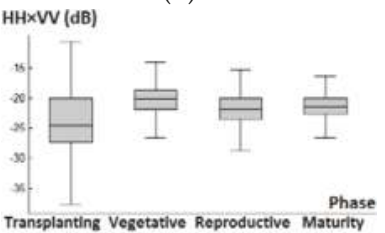

(g)

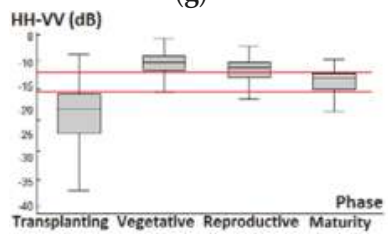

(j)

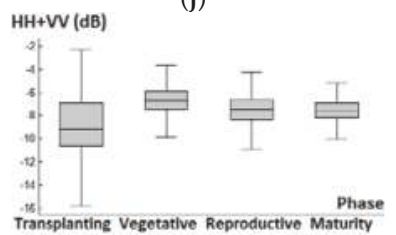

(m)

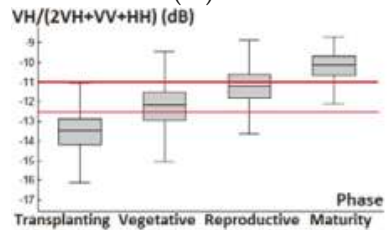

(p)

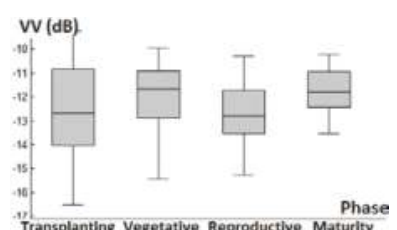

(b)

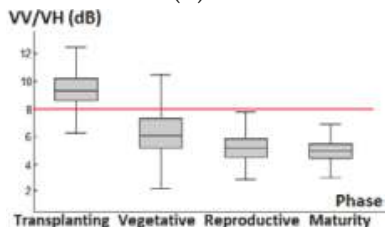

(e)

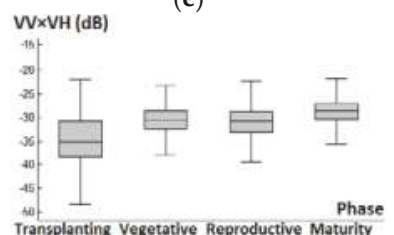

(h)

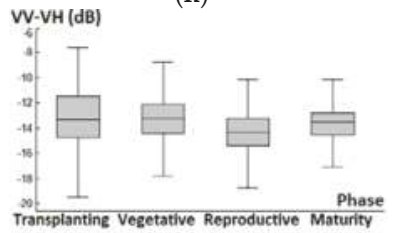

(k)

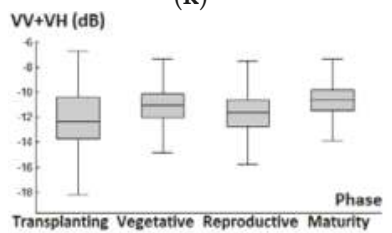

(n)

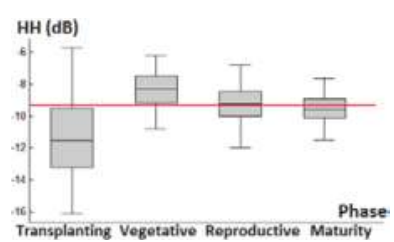

(c)

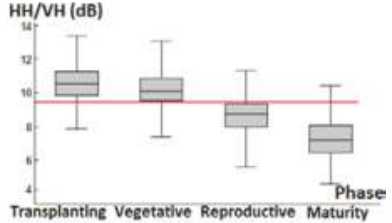

(f)

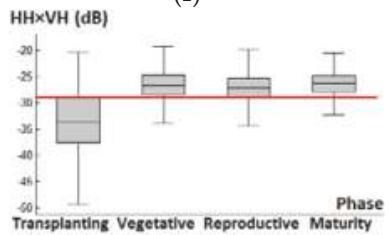

(i)

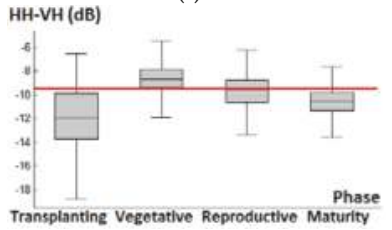

(1)

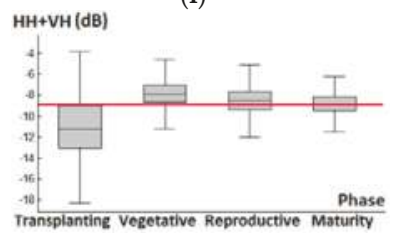

(o)

Figure 7. Boxplots of backscattering coefficients (training dataset, expressed in logarithmic scale) and their combinations (calculated in linear scale then expressed in logarithmic scale) at each phase, (a) $\mathrm{VH}$, (b) $\mathrm{VV}$, (c) $\mathrm{HH}$, (d) $\mathrm{HH} / \mathrm{VV}$, (e) $\mathrm{VV} / \mathrm{VH},($ f) $\mathrm{HH} / \mathrm{VH},(\mathrm{g}) \mathrm{HH} \times \mathrm{VV}$, (h) $\mathrm{VV} \times \mathrm{VH}$, (i) $\mathrm{HH} \times \mathrm{VH}$, (j) $\mathrm{HH}-\mathrm{VV},(\mathbf{k}) \mathrm{VV}-\mathrm{VH},(\mathbf{l}) \mathrm{HH}-\mathrm{VH},(\mathbf{m}) \mathrm{HH}+\mathrm{VV},(\mathbf{n}) \mathrm{VV}+\mathrm{VH},(\mathbf{o}) \mathrm{HH}+\mathrm{VH},(\mathbf{p}) \mathrm{VH} /(2 \mathrm{VH}+$ $\mathrm{VV}+\mathrm{HH})$. Red lines represent possible division values to separate at least two interquartile ranges (grey part of boxes). 
As shown in Figure 7, there are varying degrees of overlaps between different phases and for different polarization data. The single polarized $\mathrm{HH}$ or VV data can hardly differentiate all phenological phases, but the joint use of several polarized data can achieve the needed separation. Taking subplot (d), (e), and (f) of Figure 7 as an example, one can use HH/VV data to divide the maturity phase from the vegetative and reproductive phases or the transplanting phase from the other three phases. The VV/VH parameter can be used to distinguish the transplanting phase from later phases. The HH/VH parameter can be used to differentiate the transplanting and vegetative phase from the reproductive and maturity phase. Thus, a combination of three polarized data can be used to distinguish four phenological phases. Then, a decision tree can be constructed (Figure 8).

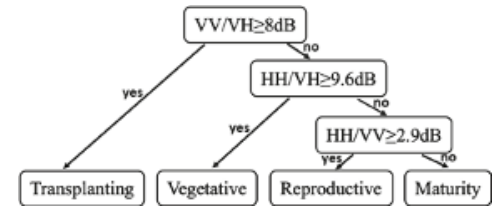

Figure 8. A decision tree classifier. Thresholds of $\mathrm{VV} / \mathrm{VH}, \mathrm{HH} / \mathrm{VV}$, and $\mathrm{HH} / \mathrm{VH}$ divide SAR data into four phenological phases.

Thresholds, $8 \mathrm{~dB}$ of $\mathrm{VV} / \mathrm{VH}, 9.6 \mathrm{~dB}$ of $\mathrm{HH} / \mathrm{VH}$, and $2.9 \mathrm{~dB}$ of $\mathrm{HH} / \mathrm{VV}$, are optimal division lines which can separate the phases with the fewest overlaps of backscattering coefficient data (Figure 7). For instance, when a $\mathrm{VV} / \mathrm{VH}$ threshold is set to $8 \mathrm{~dB}$ to divide the transplanting phase from the next three phases, the majority of backscattering coefficients are properly divided, and only $10.8 \%$ of backscattering coefficients overstep the threshold boundary, as shown in Table 3.

Table 3. Thresholds of selected parameters. T stands for transplanting phase, V vegetative phase, $\mathrm{R}$ reproductive phase, and $\mathrm{M}$ maturity phase. An error rate means the proportion of pixels whose backscattering coefficients cross the threshold line.

\begin{tabular}{cccc}
\hline & \multicolumn{3}{c}{ Decision Tree Parameters } \\
\cline { 2 - 4 } & VV/VH & HH/VH & HH/VV \\
\hline Divided Phase & T vs. V, R, or M & V vs. R or M & R vs. M \\
\hline Threshold & $8 \mathrm{~dB}$ & $9.6 \mathrm{~dB}$ & $2.9 \mathrm{~dB}$ \\
Error rate & $10.8 \%$ & $19.3 \%$ & $23.2 \%$ \\
\hline
\end{tabular}

\subsection{Validation}

The performance of the decision tree classifier is tested using the reserved validation data. A confusion table [42] is built (Table 4). The overall accuracy (OA) is $86.2 \%$. The producer's accuracy (PA) is between $78.6 \%$ and $100 \%$. The user's accuracy (UA) is between $71.4 \%$ and $100 \%$. The kappa coefficient is 0.802 .

Table 4. Confusion table derived by validation data. UA stands for user's accuracy, PA producer's accuracy, and OA overall accuracy. Kappa denotes kappa coefficient.

\begin{tabular}{ccccccc}
\hline & \multicolumn{5}{c}{ Ground Measured Phase } \\
\cline { 3 - 6 } & & Transplanting & Vegetative & Reproductive & Maturity & UA \\
\hline \multirow{2}{*}{ Extracted } & Transplanting & 4 & 1 & 0 & 0 & $80 \%$ \\
phase & Vegetative & 0 & 11 & 1 & 0 & $91.7 \%$ \\
& Reproductive & 0 & 2 & 5 & 0 & $71.4 \%$ \\
& Maturity & 0 & 0 & 0 & 5 & $100 \%$ \\
& PA & $100 \%$ & $78.6 \%$ & $83.3 \%$ & $100 \%$ & OA $=86.2 \%$ \\
& & & & & Kappa $=0.802$ \\
\hline
\end{tabular}


The backscattering properties of a rice paddy during the transplanting period were mainly affected by water since the plant was small and the plan coverage was sparse. Thus, backscattering at this phase was quite different from that during the later three phases. As a result, backscattering coefficients of the transplanting phase were more distinctive by many polarized data (Figure 7). This was attributed to the high level of the determination accuracy (table 4). On the other hand, most of the misclassification occurred in the vegetative and reproductive phases because the water was very shallow or the field was dry. The rice canopy almost covered the fields continuously. Backscattering coefficients exhibited no distinctive changes from the vegetative phase to the reproductive phase for most polarized data (Figure 7). This was especially true during days of phase transition in which the plant height and water content of the plants changed slowly. The backscattering was mainly influenced by the plant density because rice plants in some fields had similar growth statuses (e.g., the number, size, orientation and water content of stems, leaves and young heads). The statuses usually differed because of different rice varieties and plants health.

\subsection{Phenology Extraction and Mapping}

Based on the decision tree constructed in Figure 8, spatial distributions of rice phenology were mapped with multi-temporal SAR images (Figure 9). Pixels for the phase of transplanting, vegetative, reproductive, and maturity were colored blue, green, yellow, and red, respectively. Pixel statistics of each phase were annotated behind the legends, indicating a significant growth trend of rice paddies.

It is obvious that the spatial distribution of rice phenological phases varies on four dates, which shows a general trend of phenology evolution over time. On 15 May, almost all of the pixels are labeled as transplanting and vegetative phases, and transplanting pixels account for nearly $67 \%$, showing that in most fields at this time, rice seedlings were just transplanted to the water and some earlier transplanted rice plants entered the vegetative phase. On 8 June, vegetative plants continued with the vegetative growth and prepared for the coming reproductive phase. Late seedlings were also extensively vegetative. These can be seen in map (b), where the pixels of the vegetative phase increase to an overwhelming proportion of $74 \%$, while the transplanting ones sharply fall to $15 \%$. On 2 July, about $58 \%$ of rice plants were in the reproductive phase. This ratio dropped to $17 \%$ on 26 July. In the last period, harvest pixels became the majority, with a proportion of $67 \%$. All of these statistics are consistent with field survey observations, which demonstrates a feasibility of the decision tree classification.

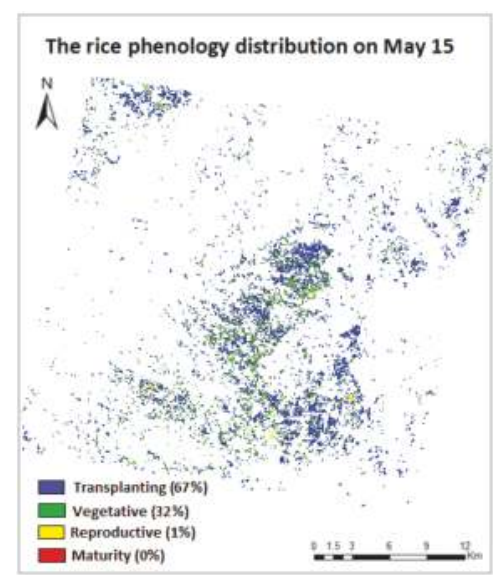

(a)

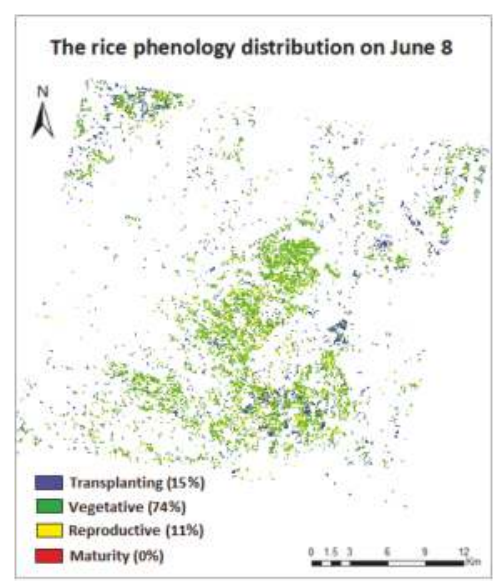

(b)

Figure 9. Cont. 


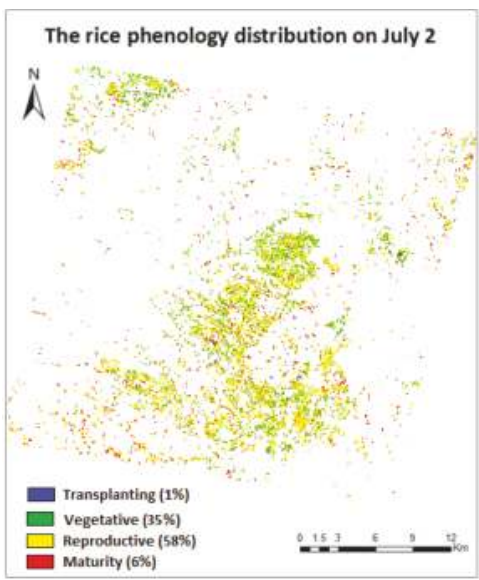

(c)

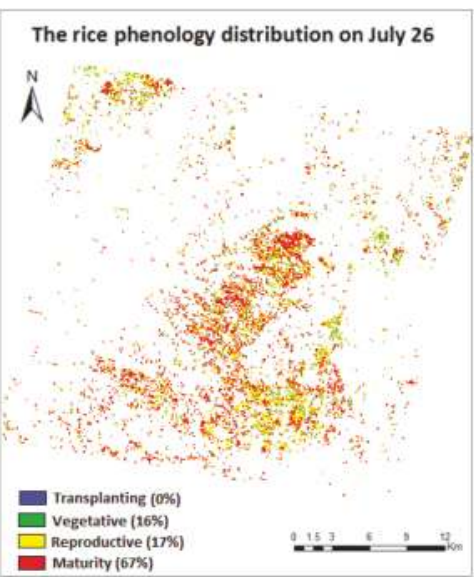

(d)

Figure 9. Map of rice phenological phases spatial distribution on (a) 15 May, (b) 8 June, (c) 2 July, and (d) 26 July. Blue, green, yellow, and red colors, respectively, represent transplanting, vegetative, reproductive, and maturity phases of rice plants in the study area.

\section{Discussion}

\subsection{Phenology Extraction Comparison between Backscattering Coefficients and Decomposition Parameters}

Compared with the most satisfactory retrieval accuracy given by previous studies, that is, Lopez-Sanchez et al. [6] used eigenvalue/vector decomposition parameters (entropy, anisotropy, and dominant alpha ( $\alpha_{1}$, alpha of the dominant scattering mechanism)) to achieve an overall success rate of $96 \%$, our study achieved a lower overall accuracy of $86.2 \%$. In order to investigate the retrieval accuracy of the method proposed by Lopez-Sanchez et al. [6] in this study area, three eigenvalue/vector decomposition parameters (entropy, anisotropy, and dominant alpha) were extracted using the Polarimetric SAR Data Processing and Educational Tool (PolSARpro) software (http://step.esa.int/main/download/). The entropy $(\mathrm{H})$ characterizes the randomness of the scattering processes. The anisotropy (A) characterizes the relative strengths of the second and third scattering mechanisms. The alpha angle $(\alpha)$ defines the scattering mechanism for a given eigenvector $[43,44]$. Dominant alpha $\left(\alpha_{1}\right)$ is the alpha of the dominant scattering mechanism [6].

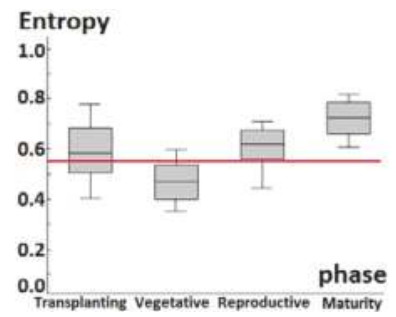

(a)

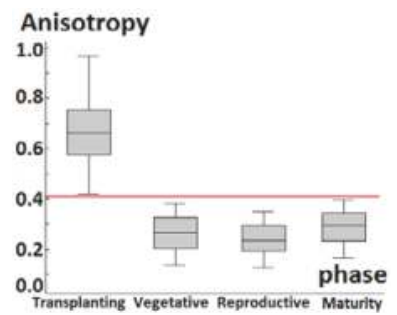

(b)

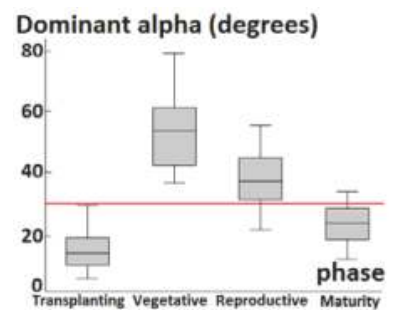

(c)

Figure 10. Evolution of observables (training dataset) provided by the eigenvalue/vector decomposition of the coherency matrix versus phenology, (a) Entropy, (b) Anisotropy, (c) Dominant alpha angle ( $\alpha_{1}$, alpha of the dominant scattering mechanism). Red lines represent possible division values to separate at least two interquartile ranges (grey part of boxes). 
For each extracted decomposition parameter, we used all pixels of a sample site for averaging. Again, all decomposition parameters were divided into four groups based on phenological phases identified in field observations (i.e., transplanting, vegetative, reproductive, and maturity). Three-quarters of each group were chosen for training. The rest in each group were used for validation, respectively. Distributions of decomposition parameters (training dataset) during each phenological phase are shown in the boxplots of Figure 10.

A decision tree can be constructed using entropy, anisotropy, and dominant alpha (Figure 11), which is similar to the phenology retrieval algorithm designed by Lopez-Sanchez et al. [6].

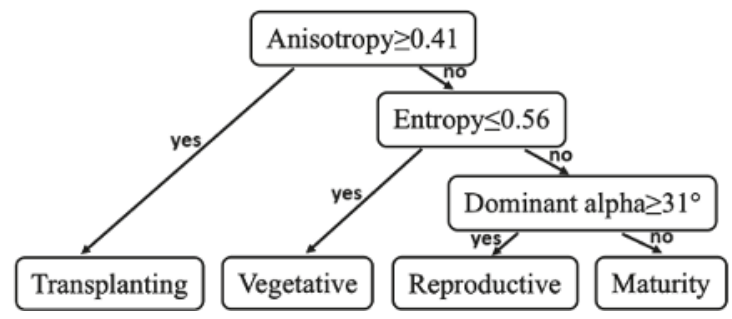

Figure 11. Phenology decision tree. Thresholds of anisotropy, entropy, and dominant alpha angle divide SAR data into four phenological phases.

The performance of the decision tree classifier is tested using the reserved validation data. A confusion table [42] is built (Table 5).

Table 5. Confusion table derived by validation data for the phenology retrieval algorithm using entropy, anisotropy, and dominant alpha. UA stands for user's accuracy, PA producer's accuracy, and OA overall accuracy. Kappa denotes kappa coefficient.

\begin{tabular}{ccccccc}
\hline & \multicolumn{5}{c}{ Ground Measured Phase } \\
\cline { 3 - 6 } & & Transplanting & Vegetative & Reproductive & Maturity & UA \\
\hline \multirow{3}{*}{ Extracted } & Transplanting & 4 & 0 & 0 & 0 & $100 \%$ \\
hase & Vegetative & 0 & 13 & 1 & 0 & $92.8 \%$ \\
& Reproductive & 0 & 1 & 5 & 0 & $83.3 \%$ \\
& Maturity & 0 & 0 & 0 & 5 & $100 \%$ \\
& PA & $100 \%$ & $92.8 \%$ & $83.3 \%$ & $100 \%$ & OA $=93.1 \%$ \\
\hline
\end{tabular}

The method using eigenvalue/vector decomposition parameters achieved a higher overall accuracy $(93.1 \%)$ than the method using backscattering coefficients $(86.2 \%)$. Compared with the backscattering coefficients method, the decomposition parameters method had two fewer wrong estimates (Tables 4 and 5). The first case corresponded to \#8 sample site on 8 June. The phenology identified in the ground observation was stage 32 (vegetative phase), but the backscattering coefficients method misclassified the rice plants at the transplanting phase. Plants at \#8 sample site were the last to enter the transplanted phase. On 8 June, tillers and leaves were only partially developed, which resulted in relatively low VH backscattering coefficients (about $-20 \mathrm{~dB}$ ). Scattering from the rough surface of the flooded ground made VV exhibit a relatively high value (about $-12 \mathrm{~dB}$ ). Thus, rice plants at \#8 sample site had similar VV/VH values with the transplanting ones. However, using decomposition parameters can correctly determine the phenology because low anisotropy (about 0.32) was observed due to the comparable contribution from surface scattering and double-bounce scattering, which were weaker than volume scattering during this time. The second case corresponded to \#24 sample site on 2 July. The ground campaign indicated that the rice plants were in stage 44 (vegetative 
phase), whereas the backscattering coefficients method misclassified their phase as the reproductive phase. One possible cause for the misclassification was that rice at the \#24 sample site had a denser canopy than other sites on 2 July. The attenuation by the denser canopy resulted in lower $\mathrm{HH}$ backscattering coefficients. Hence rice plants at the \#24 sample site had similar $\mathrm{HH} / \mathrm{VH}$ values with the plants in reproductive phase. However, the low entropy (about 0.5 ) of the \#24 sample site can be used to identify the vegetative phase. Since the ears had not appeared and the stems were mostly vertical, double-bounce scattering still contributed significantly to the radar backscattering mechanism. Another two wrong estimates of the backscattering coefficients method also appeared in the decomposition parameters method. Corresponding rice plants were in the transition period from the vegetative to the reproductive phase (stage 47 and 55). The gradual change of rice plants and the similar backscatter properties of fields during this period caused the misclassification.

In general, the phenology retrieval method using backscattering coefficients is less effective than the method using eigenvalue/vector decomposition parameters. Since additional data (phase data) are considered in the decomposition, eigenvalue/vector decomposition parameters can provide more information on the physical scattering mechanism. However, the result of the backscattering coefficients method is still acceptable because only amplitude information of backscattering coefficients is used.

\subsection{Response of Backscattering Coefficients in Phenology Retrieval}

Evolution of the backscattering coefficients during the growing season (Figure 7) reflected the characteristics of rice phenology evolution and growth status change. By taking advantage of responses of backscattering coefficients to different polarizations and in different phenological phases, the phenology retrieval method (Figure 8) achieved a high overall accuracy (86.2\%).

During the transplanting phase, rice plants were very small and short. The amount of scattering attenuation was small because of the low rice height and sparse rice canopy. The Bragg scattering from the rough surface of the flooded ground predicted that VV backscattering coefficients had moderate values ( $-12.5 \mathrm{~dB}$ on average) $[5,26]$. VH backscattering coefficients were low $(-22 \mathrm{~dB}$ on average) because of the sparse rice canopy since $\mathrm{VH}$ polarization was sensitive to volume scattering from the rice canopy $[25,26]$. HH backscattering coefficients were low (compared with later phases) due to the weak double-bounce scattering between the small rice plants and the underlying water surface, since $\mathrm{HH}$ polarization was sensitive to double-bounce scattering between rice plants and the ground surface [45]. The difference between the responses at $\mathrm{VV}$ and $\mathrm{VH}$ was clearly illustrated by the VV/VH ratio, which can be used to separate the transplanting phase from later phases.

During the vegetative phase, $\mathrm{VH}$ increased significantly with the development of the plants and hence with increasing canopy density. $\mathrm{HH}$ also increased significantly because of the larger stalks and increasing plants height. Double-bounce scattering between the rice plants and the underlying surface increased [26]. VV increased slightly because it was affected by an extinction due to the vertical preferred orientation of the plant elements (e.g., stems and leaves) [6].

During the reproductive phase, $\mathrm{VH}$ increased due to the denser plants canopy. Rice heads emerged as new scattering elements in the upper part of the canopy. HH decreased because the dense canopy reduced the microwave penetration to the surface and the double bounce off the rice plants [29]. VV decreased because the stems remained predominantly vertical and the amount of double-bounce and surface scattering was small [26]. Because of the different trends in the $\mathrm{HH}$ and VH channels, the $\mathrm{HH} / \mathrm{VH}$ ratio can be used to separate the vegetative phase from later phases.

During the maturity phase, $\mathrm{VH}$ increased because the amount and size of rice heads increased. $\mathrm{HH}$ decreased because of the large amount of attenuation by the rice canopy and the low volumetric moisture content. Stems became drier and the reduced water content caused less vertical extinction, especially for VV polarization [25]. The orientation of plant elements (i.e., stems, leaves, and heads) became random during this period. The attenuation of the $\mathrm{VV}$ polarization by the vertical orientation of the cylinders was reduced [25]. Therefore, VV stopped decreasing and the HH/VV ratio can be 
used to separate the reproductive phase from the maturity phase due to the different trends in $\mathrm{HH}$ and VV channels.

Most of the misclassification occurred in the transition from the vegetative to the reproductive phase, as shown in Table 4. In this period, backscattering properties of rice plants were mainly influenced by planting density and rice plants growth status, which were usually affected by rice varieties and plants health. Late vegetative (from stage 40 to 49 ) rice plants had similar backscatter properties with early reproductive (from stage 50 to 59) rice plants because of the gradual development of plants morphological structure. As shown in Figure 12, values of $\mathrm{HH} / \mathrm{VH}$ decreased monotonously from the early vegetative phase (from stage 20 to 39) to the late reproductive phase (from stage 60 to 69). The slow growth of rice plants and weak rice heads at the turn of the vegetative and reproductive phase resulted in no significant change of $\mathrm{HH} / \mathrm{VH}$ data. Range overlap appeared during the late vegetative and early reproductive phase. As the water content of rice plants continuously fell and rice heads gradually became important scattering elements in the late reproductive phase, backscatter properties exhibited a notable change.

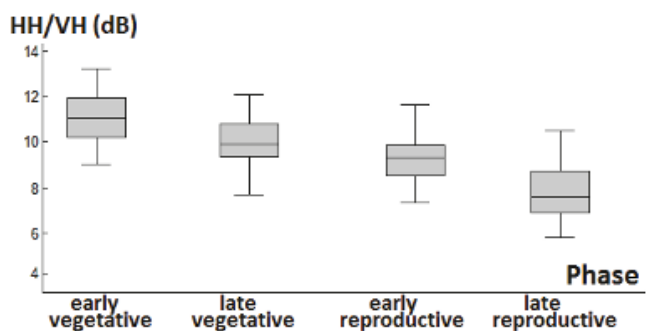

Figure 12. Gradual temporal change of $\mathrm{HH} / \mathrm{VH}$ data on subdivisions of vegetative and reproductive phase.

There are still several issues waiting to be addressed in further research and development. First, since phenology evolution is a continuous process, dividing phenology into discrete intervals makes it hard to decide whether the plants are in one phase or another during the transition between phases. For example, ground observation indicated that rice plants at \#19 sample site on 2 July were at the vegetative phase (stage 47), whereas the retrieval algorithm misclassified them as reproductive plants (stages 50-69). In the BBCH scale, an error between stage 47 and 50 is minimal (3 over 100), but in discrete intervals, it means an error between two phases (1 over 4). A possible alternative to avoid this problem consists of dividing phenology into more phases or retrieving phenology in a continuous range. This brings up another issue, that is, more ground observation and SAR data are needed. Rice plants grow rapidly during the bimestrial season. The phenological phase (e.g., transplanting and reproductive phase) lasts about two weeks on average and phase transition usually takes place in a short interval. The 24-day revisit period of RADARSAT-2 is relatively long for monitoring growth changes timely. To overcome this problem, different spaceborne C-band SAR sensors (e.g., Sentinel-1 and Gaofen-3) can be combined to increase the temporal resolution of SAR observations.

\section{Conclusions}

We analyzed statistical relationships between backscatter coefficients and rice phenology using multi-temporal quad-polarized RADARSAT-2 SAR datasets and rice biophysical data measured in the Meishan study area, Sichuan Province, China. Although the degree of the correlation is affected by uncertain factors such as diverse patterns of rice cultivation, there was a strong correlation between the RADARSAT-2 backscattering coefficients and rice phenology.

$\mathrm{VH}, \mathrm{VV}$, and $\mathrm{HH}$ backscattering coefficients were used to distinguish transplanting, vegetative, reproductive, and maturity phases. In particular, the $\mathrm{VV} / \mathrm{VH}$ threshold was used to separate plants in the transplanting phase from plants in the vegetative, reproductive, and maturity phases, the $\mathrm{HH} / \mathrm{VH}$ 
threshold to delineate plants in the vegetative phase from plants in the reproductive and maturity phases, and the HH/VV threshold to distinguish plants in the reproductive phase from plants in the maturity phase. Then, a decision tree classifier was developed. The accuracy level was $86.2 \%$ in the delineation of rice phenology. The analysis procedure was relatively simple, ensuring the reliability of the method. Therefore, there should be a great potential to use RADARSAT-2 SAR data to monitor rice phenology during its growth period and in large spatial extent. Finally, the concise decision logic should make this study easy understood and implemented in other studies related to local rice production.

Acknowledgments: This work was supported by the National Natural Science Foundation of China (41471294), and Fundamental Research Funds for the Central Universities (ZYGX2015J112).

Author Contributions: Shihua Li and Ze He conceived and designed the study, and developed the proposed methods; Ze He collated and analyzed the data; Ze He, Shihua Li, and Yong Wang wrote the paper; Leiyu Dai and Sen Lin participated in the fieldwork. All authors have read and approved the final version of the manuscript.

Conflicts of Interest: The authors declare no conflict of interest.

\section{References}

1. Chen, C.; Son, N.T.; Chang, L. Monitoring of rice cropping intensity in the upper Mekong Delta, Vietnam using time-series MODIS data. Adv. Space Res. 2012, 49, 292-301. [CrossRef]

2. McLean, J.; Hardy, B.; Hettel, G. Rice Almanac, 4th ed.; International Rice Research Institute: Los Banos, Philippines, 2013; ISBN 978-971-22-0300-8.

3. Sakamoto, T. A crop phenology detection method using time-series MODIS data. Remote Sens. Environ. 2005, 96, 366-374. [CrossRef]

4. Lausch, A.; Salbach, C.; Schmidt, A.; Doktor, D.; Merbach, I.; Pause, M. Deriving phenology of barley with imaging hyperspectral remote sensing. Ecol. Model. 2015, 295, 123-135. [CrossRef]

5. Lopez-Sanchez, J.M.; Cloude, S.R.; Ballester-Berman, J.D. Rice phenology monitoring by means of SAR polarimetry at X-band. IEEE Trans. Geosci. Remote Sens. 2012, 50, 2695-2709. [CrossRef]

6. Lopez-Sanchez, J.M.; Vicente-Guijalba, F.; Ballester-Berman, J.D.; Cloude, S.R. Polarimetric response of rice fields at C-band: Analysis and phenology retrieval. IEEE Trans. Geosci. Remote Sens. 2014, 52, 2977-2993. [CrossRef]

7. Vicente-Guijalba, F.; Martinez-Marin, T.; Lopez-Sanchez, J.M. Crop phenology estimation using a multitemporal model and a Kalman filtering strategy. IEEE Geosci. Remote Sens. Lett. 2014, 11, 1081-1085. [CrossRef]

8. $\mathrm{Xu}, \mathrm{D} . ; \mathrm{Fu}, \mathrm{M}$. Detection and modeling of vegetation phenology spatiotemporal characteristics in the middle part of the Huai river region in China. Sustainability 2015, 7, 2841-2857. [CrossRef]

9. Yang, Z.; Li, K.; Liu, L.; Shao, Y.; Brisco, B.; Li, W. Rice growth monitoring using simulated compact polarimetric C band SAR. Radio Sci. 2014, 49, 1300-1315. [CrossRef]

10. Zhang, Y.; Li, L.; Wang, H.; Zhang, Y.; Wang, N.; Chen, J. Land surface phenology of Northeast China during 2000-2015: temporal changes and relationships with climate changes. Environ Monit Assess. 2017, 189. [CrossRef] [PubMed]

11. Li, S.; Xiao, J.; Ni, P.; Zhang, J.; Wang, H.; Wang, J. Monitoring paddy rice phenology using time series MODIS data over Jiangxi Province, China. Int. J. Agric. \& Biol. Eng. 2014, 7, 28-36.

12. Dash, J.; Jeganathan, C.; Atkinson, P.M. The use of MERIS Terrestrial Chlorophyll Index to study spatio-temporal variation in vegetation phenology over India. Remote Sens. Environ. 2010, 114, 1388-1402. [CrossRef]

13. Corcione, V.; Nunziata, F.; Mascolo, L.; Migliaccio, M. A study of the use of COSMO-SkyMed SAR PingPong polarimetric mode for rice growth monitoring. Int. J. Remote Sens. 2016, 37, 633-647. [CrossRef]

14. Peng, D.; Huete, A.R.; Huang, J.; Wang, F.; Sun, H. Detection and estimation of mixed paddy rice cropping patterns with MODIS data. Int. J. Appl. Earth Obs. Geoinf. 2011, 13, 13-23. [CrossRef]

15. Motohka, T.; Nasahara, K.N.; Miyata, A.; Mano, M.; Tsuchida, S. Evaluation of optical satellite remote sensing for rice paddy phenology in monsoon Asia using a continuous in situ dataset. Int. J. Remote Sens. 2009, 30, 4343-4357. [CrossRef] 
16. Boschetti, L.; Roy, D.P. Strategies for the fusion of satellite fire radiative power with burned area data for fire radiative energy derivation. J. Geophys. Res. Atmos. 2009, 114, 215-216. [CrossRef]

17. Wang, H.; Chen, J.; Wu, Z.; Lin, H. Rice heading date retrieval based on multi-temporal MODIS data and polynomial fitting. Int. J. Remote Sens. 2012, 33, 1905-1916. [CrossRef]

18. Wang, J.; Huang, J.; Wang, X.; Jin, M.; Zhou, Z.; Guo, Q.; Zhao, Z.; Huang, W.; Zhang, Y.; Song, X. Estimation of rice phenology date using integrated HJ-1 CCD and Landsat-8 OLI vegetation indices time-series images. J. Zhejiang Univ. Sci. B 2015, 16, 832-844. [CrossRef] [PubMed]

19. Zhang, Y.; Liu, X.; Su, S.; Wang, C. Retrieving canopy height and density of paddy rice from Radarsat-2 images with a canopy scattering model. Int. J. Appl. Earth Obs. Geoinf. 2014, 28, 170-180. [CrossRef]

20. Bouvet, A.; Le Toan, T.; Lam-Dao, N. Monitoring of the rice cropping system in the Mekong delta using ENVISAT / ASAR dual polarization data. IEEE Trans. Geosci. Remote Sens. 2009, 47, 517-526. [CrossRef]

21. Yuzugullu, O.; Erten, E.; Hajnsek, I. Rice Growth monitoring by means of X-Band co-polar SAR: Feature clustering and BBCH scale. IEEE Geosci. Remote Sens. Lett. 2015, 12, 1218-1222. [CrossRef]

22. Çağlar, K.; Gülşen, T.; Erten, E. Paddy-rice phenology classification based on machine-learning methods using multi-temporal co-polar X-Band SAR images. IEEE J. Sel. Top. Appl. Earth Observ. Remote Sens. 2016, 9, 2509-2519.

23. De Bernardis, C.G.; Vicente-Guijalba, F.; Martinez-Marin, T.; Lopez-Sanchez, J.M. Estimation of key dates and stages in rice crops using dual-polarization SAR time series and a particle filtering approach. IEEE J. Sel. Top. Appl. Earth Observ. Remote Sens. 2015, 8, 1008-1018. [CrossRef]

24. Erten, E.; Lopez-Sanchez, J.M.; Yuzugullu, O.; Hajnsek, I. Retrieval of agricultural crop height from space: A comparison of SAR techniques. Remote Sens. Environ. 2017, 187, 130-144. [CrossRef]

25. Koppe, W.; Gnyp, M.L.; Hütt, C.; Yao, Y.; Miao, Y.; Chen, X.; Bareth, G. Rice monitoring with multi-temporal and dual-polarimetric TerraSAR-X data. Int. J. Appl. Earth Obs. Geoinf. 2013, 21, 568-576. [CrossRef]

26. Yang, Z.; Shao, Y.; Li, K.; Liu, Q.; Liu, L.; Brian, B. An improved scheme for rice phenology estimation based on time-series multispectral HJ-1A/B and polarimetric RADARSAT-2 data. Remote Sens. Environ. 2017, 195, 184-201. [CrossRef]

27. Francis, C.; Shang, J.; Liu, J.; Huang, X.; Ma, B.; Jiao, X.; Geng, X.; John, M.K.; Dan, W. Tracking crop phenological development using multi-temporal polarimetric Radarsat-2 data. Remote Sens. Environ. 2017. [CrossRef]

28. Tian, H.; Wu, M.; Wang, L.; Niu, Z. Mapping early, middle and late rice extent using sentinel-1A and Landsat-8 data in the poyang lake plain, China. Sensors 2018, 18, 185. [CrossRef] [PubMed]

29. Inoue, Y.; Kurosu, T.; Maeno, H.; Uratsuka, S.; Kozu, T.; Dabrowska-Zielinska, K.; Qi, J. Season-long daily measurements of multifrequency (Ka, Ku, X, C, and L) and full-polarization backscatter signatures over paddy rice field and their relationship with biological variables. Remote Sens. Environ. 2002, 81, 194-204. [CrossRef]

30. Wu, F.; Wang, C.; Zhang, H.; Zhang, B.; Tang, Y. Rice crop monitoring in South China with RADARSAT-2 quad-polarization SAR data. IEEE Geosci. Remote Sens. Lett. 2011, 8, 196-200. [CrossRef]

31. Li, S.; Ni, P.; Cui, G.; He, P.; Liu, H.; Li, L.; Liang, Z. Estimation of rice biophysical parameters using multitemporal RADARSAT-2 images. In Proceedings of the Symposium of the International Society for Digital Earth (ISDE), Halifax, NS, Canada, 5-9 October 2015; p. 012019.

32. Yang, S.; Zhao, X.; Li, B.; Hua, G. Interpreting RADARSAT-2 quad-polarization SAR signatures from rice paddy based on experiments. IEEE Geosci. Remote Sens. Lett. 2012, 9, 65-69. [CrossRef]

33. Ulaby, F.; Allen, C.; Eger, G.; Kanemasu, E. Relating the microwave backscattering coefficient to leaf area index. Remote Sens. Environ. 1984, 14, 113-133. [CrossRef]

34. Bouman, B. Crop parameter estimation from ground-based X-band (3-cm wave) radar backscattering data. Remote Sens. Environ. 1991, 37, 193-205. [CrossRef]

35. Inoue, Y.; Sakaiya, E. Relationship between X-band backscattering coefficients from high-resolution satellite SAR and biophysical variables in paddy rice. Remote Sens. Lett. 2013, 4, 288-295. [CrossRef]

36. Inoue, Y.; Sakaiya, E.; Wang, C. Capability of C-band backscattering coefficients from high-resolution satellite SAR sensors to assess biophysical variables in paddy rice. Remote Sens. Environ. 2014, 140, 257-266. [CrossRef]

37. Yuzugullu, O.; Erten, E.; Hajnsek, I. Estimation of rice crop height from X- and C-Band PolSAR by Metamodel-Based optimization. IEEE J. Sel. Top. Appl. Earth Observ. Remote Sens. 2017, 10, 194-204. [CrossRef] 
38. Zadoks, J.C.; Chang, T.T.; Konzak, C.F. A decimal code for the growth stages of cereals. Weed Res. 1974, 14, 415-421. [CrossRef]

39. Rossi, C.; Erten, E. Paddy-rice monitoring using TanDEM-X. IEEE Trans. Geosci. Remote Sens. 2015, 53, 900-910. [CrossRef]

40. Pal, M.; Mather, P.M. An assessment of the effectiveness of decision tree methods for land cover classification. Remote Sens. Environ. 2003, 86, 554-565. [CrossRef]

41. Francis, C.; Richard, F. ALOS PALSAR L-band polarimetric SAR data and in situ measurements for leaf area index assessment. Remote Sens. Lett. 2012, 3, 221-229.

42. Congalton, R.G. Accuracy assessment and validation of remotely sensed and other spatial information. Int. J. Wildland Fire. 2001, 10, 321-328. [CrossRef]

43. Cloude, S.R.; Pottier, E. An entropy based classification scheme for land applications of polarimetric SAR. IEEE Trans. Geosci. Remote Sens. 1997, 35, 68-78. [CrossRef]

44. Pacheco, A.; McNairn, H.; Li, Y.; Lampropoulos, G.; Powers, J. Using RADARSAT-2 and TerraSAR-X satellite data for the identification of canola crop phenology. SPIE Remote Sens. 2016, 9998. [CrossRef]

45. Wang, L.; Kong, J.; Ding, K.; Le Toan, T.; Ribbes-Baillarin, F.; Floury, N. Electromagetic scattering model for rice canopy based on Monte Carlo simulation. Prog. Electromagn. Res. 2005, 52, 153-171. [CrossRef]

(c) 2018 by the authors. Licensee MDPI, Basel, Switzerland. This article is an open access article distributed under the terms and conditions of the Creative Commons Attribution (CC BY) license (http:/ / creativecommons.org/licenses/by/4.0/). 
Article

\title{
Uncertainty of Remote Sensing Data in Monitoring Vegetation Phenology: A Comparison of MODIS C5 and C6 Vegetation Index Products on the Tibetan Plateau
}

\author{
Zhoutao Zheng ${ }^{1,2,3, *,+}$ and Wenquan Zhu ${ }^{1,2,3, *, \dagger}$ \\ 1 State Key Laboratory of Earth Surface Processes and Resource Ecology, Beijing Normal University, \\ Beijing 100875, China \\ 2 Joint Center for Global Change Studies (JCGCS), Beijing 100875, China \\ 3 Beijing Engineering Research Center for Global Land Remote Sensing Products, Institute of Remote Sensing \\ Science and Engineering, Faculty of Geographical Science, Beijing Normal University, Beijing 100875, China \\ * Correspondence: zhengzt90@mail.bnu.edu.cn (Z.Z.); zhuwq75@bnu.edu.cn (W.Z.); \\ Tel. /Fax: +86-10-5880-7053 (W.Z.) \\ + These authors contributed equally to this work.
}

Received: 29 September 2017; Accepted: 7 December 2017; Published: 11 December 2017

\begin{abstract}
Vegetation phenology is considered a sensitive indicator of climate change, which controls carbon, nitrogen, and water cycles within terrestrial ecosystems. The Moderate Resolution Imaging Spectroradiometer (MODIS) Normalized Difference Vegetation Index (NDVI) is an important moderate resolution remote sensing data for monitoring vegetation phenology. However, Terra MODIS Collection 5 (C5) vegetation index products were identified to be affected by sensor degradation, which has been addressed in the recently released MODIS Collection 6 (C6) vegetation index products. In order to compare the difference between MODIS C5 and C6 NDVI in monitoring vegetation phenology, the start and end of growing season (SOS and EOS) of the alpine grassland on the Tibetan Plateau (TP) were extracted using four common methods. Then, the C5 and C6 NDVI-derived $\mathrm{SOS}\left(\mathrm{SOS}_{\mathrm{C} 5}\right.$ and $\left.\mathrm{SOS}_{\mathrm{C} 6}\right)$ and $\mathrm{EOS}\left(\mathrm{EOS}_{\mathrm{C} 5}\right.$ and $\left.\mathrm{EOS}_{\mathrm{C} 6}\right)$ were compared with ground-observed phenology data. Results showed that the multi-year average growing season NDVIs of $\mathrm{C} 6$ were lower than those of $\mathrm{C} 5$ in most areas, while the inter-annual variation patterns of regional average $\mathrm{SOS}_{\mathrm{C} 5}$ and $\mathrm{SOS}_{\mathrm{C} 6}\left(\mathrm{EOS}_{\mathrm{C} 5}\right.$ and $\left.\mathrm{EOS}_{\mathrm{C} 6}\right)$ were consistent. However, large spatial differences in phenological trends were found between C5 and C6 NDVI products. From C5 to $\mathrm{C} 6$, pixels with a SOS (EOS) trend shifting from significant to insignificant or from insignificant to significant accounted for at least $14.58 \%(9.07 \%)$ of the total pixels. $\mathrm{SOS}_{\mathrm{C} 5}$ was more consistent than $\mathrm{SOS}_{\mathrm{C} 6}$ with the ground-observed green-up dates. C5 NDVI may be more appropriate for monitoring SOS than C6 NDVI in the study region, but more ground-observed phenology records are needed to confirm it due to only four observational sites in this study. However, large differences and poor correlations existed between $\mathrm{EOS}_{\mathrm{C} 5}\left(\mathrm{EOS}_{\mathrm{C} 6}\right)$ and the ground-observed beginning of leaf coloring. To further evaluate the uncertainty of MODIS C5 and C6 NDVI in monitoring vegetation phenology, higher resolution near-surface remote sensing data and corresponding validation methods should be applied.
\end{abstract}

Keywords: vegetation phenology; Tibetan Plateau; MODIS; NDVI; start of growing season (SOS); end of growing season (EOS) 


\section{Introduction}

Vegetation phenology dynamics can reflect the response of terrestrial ecosystem to climate change and play an important role in adjusting the cycling of carbon, nitrogen, and water [1-3]. Remote sensing data have been widely used to monitor vegetation phenology at large scales [4-6], because satellite-derived vegetation indices can measure vegetation canopy greenness and have the advantages of wide coverage, high revisiting frequency, and relatively low cost. The Normalized Difference Vegetation Index (NDVI) is one of the most commonly used vegetation indexes for monitoring vegetation phenology [7-9].

Moderate Resolution Imaging Spectroradiometer (MODIS) remote sensing data have been increasingly used for monitoring vegetation phenology. MODIS sensors aboard Terra and Aqua satellites have been in operation since 1999 and 2002, respectively, and can provide long-term remote sensing records of $>10$ years. However, the designed lifetimes of the sensors are only six years. In recent years, severe data problems were found to exist in MODIS Collection 5 (C5) ocean colors [10], aerosols [11], and NDVI products [12-14], mainly resulting from sensor degradation. Due to the increasing exposure of MODIS sensor to solar and cosmic radiation, severe degradation of Terra MODIS near-infrared, red and blue bands has been observed. The sensor degradation was the most pronounced in the Terra blue band and decreased with wavelength $[12,13]$. Though the blue band is not used directly to calculate NDVI, degradation of blue top-of-atmosphere reflectance over time will influence the calculation of surface reflectance in other spectral bands and NDVI $[13,15,16]$. Moreover, the sensor degradation was much faster for Terra than Aqua [13,17]. To remove the effects of sensor degradation, improved calibrated approaches were adopted to produce the recently released MODIS Collection 6 (C6) products [12,18]. When compared with C5, the C6 Level 1B data, including the top-of-atmosphere reflectance in the near-infrared, red and blue bands were calibrated [13]. In addition, the NDVI retrieval algorithms were also improved [19]. Unlike C5 NDVI, which uses daily reflectance data, C6 NDVI uses pre-composed (8-day) surface reflectance data that are atmospherically corrected with a modified compositing algorithm that aims to reduce the aerosol issues (minimizing the blue band) $[17,19]$.

The differences between MODIS C5 and C6 NDVI have been evaluated in some previous studies (e.g., $[17,20])$. However, no study has conducted a comparative analysis of the performance of MODIS C5 and C6 NDVI in monitoring vegetation phenology. Given that MODIS C5 NDVI has been extensively used for monitoring vegetation phenology [21-23], it is necessary to analyze the difference between vegetation phenology derived from C5 and C6 NDVI and consequently investigate the uncertainty in monitoring vegetation phenology due to sensor degradation. Due to the fact that Terra data is more affected by the sensor degradation than Aqua data [13,17], this study focused on the Terra MODIS NDVI products.

Four common methods were adopted to identify the start and end of growing season (SOS and EOS) of the alpine grassland on the Tibetan Plateau (TP) based on Terra MODIS C5 and C6 NDVI. Then, a comparative analysis of vegetation phenology derived from the two NDVI products was conducted for each phenology extraction method. Meanwhile, the performances of vegetation phenology derived from C5 and C6 NDVI in capturing ground-observed phenology were also evaluated.

\section{Data and Methods}

\subsection{Remote Sensing Data}

The Terra MODIS $250 \mathrm{~m}$ 16-day composited NDVI (MOD13Q1) products originating from C5 and C6 during 2001-2015 were acquired from National Aeronautics and Space Administration (NASA) Earth Observing System Data and Information System (EOSDIS). To reduce the effect of cloud and Nadir Bidirectional Reflectance Distribution Function (BRDF), the composite of NDVI was performed by the Constrained View Angle-Maximum Value Composite (CV-MVC) algorithm. The pixel reliability (PR) layer from MOD13Q1 products was used to determine the pixel quality and calibrate NDVI time 
series. A PR value of 0 or 1 represents good pixel or marginal pixel in the NDVI time series. A PR value of 2 or 3 represents pixel covered by ice/snow or cloud, which should be corrected.

\subsection{Ground-Observed Phenology Data}

The ground-observed phenology data were collected from the nation-wide phenological observation network that was established by the China Meteorological Administration [24]. As Kobresia humilis is the dominant species in the alpine grassland on the TP, the ground-observed phenology data of K. humilis, including green-up (GU) and beginning of leaf coloring (BLC), were collected at Haiyan, Gande, Henan, and Qumarleb sites from 2001-2012 (Table 1).

Table 1. Summary of ground-observed phenology data of Kobresia humilis.

\begin{tabular}{ccccc}
\hline Site Name & Longitude & Latitude & Altitude $(\mathrm{m})$ & Number of Years \\
\hline Haiyan & $100^{\circ} 51^{\prime} \mathrm{E}$ & $36^{\circ} 37^{\prime} \mathrm{N}$ & 3140 & 12 \\
Gande & $99^{\circ} 54^{\prime} \mathrm{E}$ & $33^{\circ} 58^{\prime} \mathrm{N}$ & 4050 & 12 \\
Henan & $101^{\circ} 36^{\prime} \mathrm{E}$ & $34^{\circ} 44^{\prime} \mathrm{N}$ & 3500 & 12 \\
Qumarleb & $95^{\circ} 47^{\prime} \mathrm{E}$ & $34^{\circ} 08^{\prime} \mathrm{N}$ & 4175 & 12 \\
\hline
\end{tabular}

\subsection{Data Pre-Processing}

The snow cover during the non-growing season on the TP will reduce NDVI values, resulting in retrieval errors for the phenology data. To reduce the effect of snow, snow-contaminated NDVI values (PR value equals 2 ) were replaced by the median value of the uncontaminated NDVI values (PR value equals 0 or 1) during the non-growing season (from November to the following March) for each pixel [25]. However, plenty of snow-contaminated pixels could not be flagged out by the PR values. Therefore, another way was applied to eliminate snow contamination. For each pixel, all of the NDVI values that were lower than the mean of the NDVI values during the non-growing season were replaced by the mean. After that, the Savitzky-Golay filter was used to reconstruct the NDVI time series to further remove cloud contamination [26]. In this study, all data were re-projected to the Albers conic equal area projection.

Only the vegetation phenology in the alpine grassland was analyzed in this study. To eliminate the effects of bare soil, sparse vegetation and evergreen forest, grass pixels were selected by the following criteria [25,27]: (1) the average NDVI for June-September should be greater than 0.1 ; (2) the annual maximum NDVI should exceed 0.15 and occur within July-September; (3) the average NDVI for July-September should be greater than 1.2 times of the average NDVI for November-March; and, (4) the average NDVI in winter (December-February) should be lower than 0.4.

\subsection{Phenology Extraction Methods}

Many methods have been used to extract vegetation phenology, but the vegetation phenology varied with extraction methods [28]. To avoid the effect of phenology extraction methods on the uncertainty analysis of remote sensing data in monitoring vegetation phenology, four commonly used methods were adopted to extract the SOS and EOS in the alpine grassland on the TP, i.e., the maximum curvature change method (MCC), dynamic threshold methods with a threshold of 0.2 and 0.5 (DT2 and DT5), and maximum slope method (MS).

\subsubsection{Method}

A four-parameter logistic function [29] was employed to fit each increasing or decreasing section of a NDVI time series and then the daily NDVI values were derived from the fitted function, as shown below:

$$
y(t)=\frac{c}{1+e^{a+b t}}+d
$$


where $t$ is the time (Julian day of year, DOY), $y(t)$ is the NDVI value at time $t, a$ and $b$ are fitting parameters, $c+d$ is the maximum NDVI value, and $d$ is the initial background NDVI value [29]. Then, the curvature-change rate (CCR) of the fitted logistic curve was used to extract phenological dates, according to Equation (2) [29]:

$$
C C R=b^{3} c z \times 3 z(1-z)(1+z)^{3} \frac{2(1+z)^{3}+b^{2} c^{2} z}{\left[(1+z)^{4}+(b c z)^{2}\right]^{2.5}}-b^{3} c z \times(1+z)^{2} \frac{1+2 z-5 z^{2}}{\left[(1+z)^{4}+(b c z)^{2}\right]^{1.5}}
$$

where $z=e^{a+b}$. SOS is defined as the DOY when CCR reaches its first local maximum value during the growth period, while EOS is defined as the DOY when CCR reaches its second minimum value during the senescence period.

\subsubsection{DT2 and DT5 Methods}

The phenological metrics were derived using the dynamic threshold method developed by White et al. [30]. In this method, the daily NDVI values were first generated using a linear interpolation approach from the original 16-day composites. SOS and EOS are defined as the DOY when the NDVI ratio reaches a certain threshold during the NDVI rising stage and decline stage, respectively. The NDVI ratio is defined as:

$$
N D V I_{\text {ratio }}=\frac{N D V I_{t}-N D V I_{\min }}{N D V I_{\max }-N D V I_{\min }}
$$

where $N D V I_{t}$ is the NDVI value at time $t, N D V I_{\max }$ is the annual maximum NDVI value, $N D V I_{\min }$ is the annual minimum NDVI value during the growth period for SOS or during the senescence period for EOS. In this study, the threshold was set to be 0.2 (0.5) for DT2 (DT5) method.

\subsubsection{MS Method}

In this method, SOS or EOS is defined as the DOY when NDVI begins to rapidly increase (SOS) or decrease (EOS) [31], which is identified based on the maximum absolute slope of the fitted NDVI curve in Equation (1) during the growth or senescence period.

\subsection{Data Analysis Methods}

The temporal change trends of regional average growing season (April-October) NDVI (GSNDVI) based on $\mathrm{C} 5$ and $\mathrm{C} 6$ products $\left(\mathrm{GSNDVI}_{\mathrm{C} 5}\right.$ and $\mathrm{GSNDVI}_{\mathrm{C} 6}$ ) during 2001-2015 were calculated and compared. The change trends in the regional average GSNDVI were computed as the slope of the linear regression of the regional average GSNDVI against year. To analyze the spatial differences between C5 and C6 GSNDVIs, the pixel-by-pixel multi-year average values and linear trends were further calculated. With the same methods being used for comparing GSNDVI, the C5 and C6 NDVI-derived $\mathrm{SOS}\left(\mathrm{SOS}_{\mathrm{C} 5}\right.$ and $\left.\mathrm{SOS}_{\mathrm{C} 6}\right)$ as well as $\mathrm{EOS}\left(\mathrm{EOS}_{\mathrm{C} 5}\right.$ and $\left.\mathrm{EOS}_{\mathrm{C} 6}\right)$ identified by each phenology extraction method were further compared. Paired-samples $t$ tests were conducted to compare the GSNDVI or phenological metrics between C5 and C6.

To validate the satellite-derived vegetation phenology, the average phenology of a $3 \times 3$ window centered at each site was extracted for comparison with the ground-observed phenology. The mean error (ME) and the mean absolute error (MAE) were used to estimate the difference between the satellite-derived phenology and the ground-observed phenology. They are calculated by the following formulas:

$$
\begin{aligned}
M E & =\frac{1}{n} \sum_{i}^{n}\left(P(r s)_{i}-P(\text { site })_{i}\right) \\
M A E & =\frac{1}{n} \sum_{i}^{n} \mid\left(P(r s)_{i}-P(\text { site })_{i}\right) \mid
\end{aligned}
$$


where $P(r s)_{i}$ and $P(s i t e)_{i}$ are the satellite-derived phenology and the ground-observed phenology at sample $i$, respectively; $n$ is the number of samples. In addition, the correlations between the satellite-derived phenology and the ground-observed phenology were also calculated to evaluate their consistency.

The statistical significance of all the regression coefficients and correlation coefficients was examined using the F-test. $P$ values less than 0.05 were considered significant.

\section{Results}

\subsection{Comparison Between $G S N D V I_{C 5}$ and $G S N D V I_{C 6}$}

The regional average GSNDVI C6 $_{\text {was significantly }}(p<0.001)$ lower than GSNDVI $_{\mathrm{C} 5}$ for the alpine grassland on the TP (Figure 1). The annual regional average GSNDVI showed no obvious trend for $\mathrm{C} 5(p=0.424)$, but a significant increasing trend for $\mathrm{C} 6\left(7.26 \times 10^{-4} \mathrm{yr}^{-1}, p<0.05\right)$ during 2001-2015 (Figure 1). At spatial scale, the multi-year average GSNDVI $\mathrm{C6}$ was lower than GSNDVI ${ }_{\mathrm{C} 5}$ over $85.8 \%$ of the total pixels (Figure 2). The multi-year average GSNDVI decreased more than $5 \%$ from $\mathrm{C} 5$ to $\mathrm{C} 6$ over $30.13 \%$ of the pixels, while it increased more than $5 \%$ over only $0.12 \%$ of the pixels (Figure 2a). Moreover, large spatial differences in trends between $\mathrm{GSNDVI}_{\mathrm{C} 5}$ and $\mathrm{GSNDVI}_{\mathrm{C} 6}$ were found (Figure 3a). From GSNDVI $\mathrm{C}_{5}$ to $\mathrm{GSNDVI}_{\mathrm{C} 6}$, significant trends $(p<0.05)$ became insignificant over $7.33 \%$ of pixels, while insignificant trends became significant over $9.98 \%$ of pixels. With regard to the pixels where both GSNDVI ${ }_{\mathrm{C} 5}$ and $\mathrm{GSNDVI}_{\mathrm{C} 6}$ indicated significant trends, GSNDVI trend became more negative over $9.03 \%$ of pixels and more positive over $4.10 \%$ of pixels from C5 to C6. A significant $(p<0.001)$ difference between GSNDVI ${ }_{\mathrm{C} 5}$ and $\mathrm{GSNDVI}_{\mathrm{C} 6}$ trends was observed. The mean difference in GSNDVI trend (C6-C5) was $-3.74 \times 10^{-4} \mathrm{yr}^{-1}$. Besides, GSNDVI $\mathrm{C6}$ showed increasing trends over more area and decreasing trends over less area ( $65.3 \%$ increasing, $18.6 \%$ significantly increasing; $34.7 \%$ decreasing, $4.5 \%$ significantly decreasing) when compared with GSNDVI $_{\mathrm{C} 5}(55.8 \%$ increasing, $12.9 \%$ significantly increasing; $44.2 \%$ decreasing, $7.6 \%$ significantly decreasing) (Figure $3 \mathrm{~b}$ ).

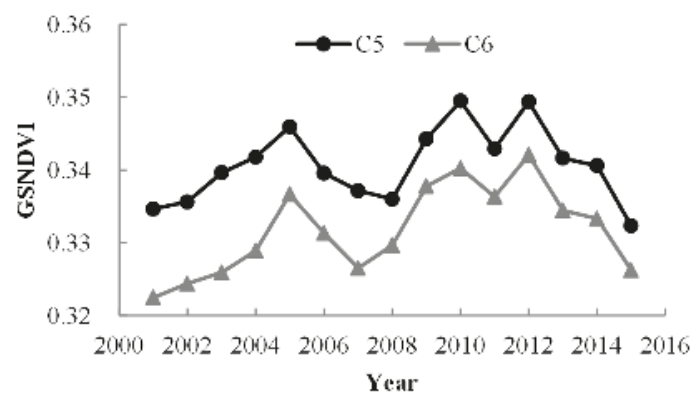

Figure 1. Comparison of annual regional average growing season Normalized Difference Vegetation Index derived from MODIS C5 (GSNDVI $\left.{ }_{\mathrm{C} 5}\right)$ and $\mathrm{C} 6\left(\mathrm{GSNDVI}_{\mathrm{C} 6}\right)$ for the alpine grassland on the Tibetan Plateau during 2001-2015. 

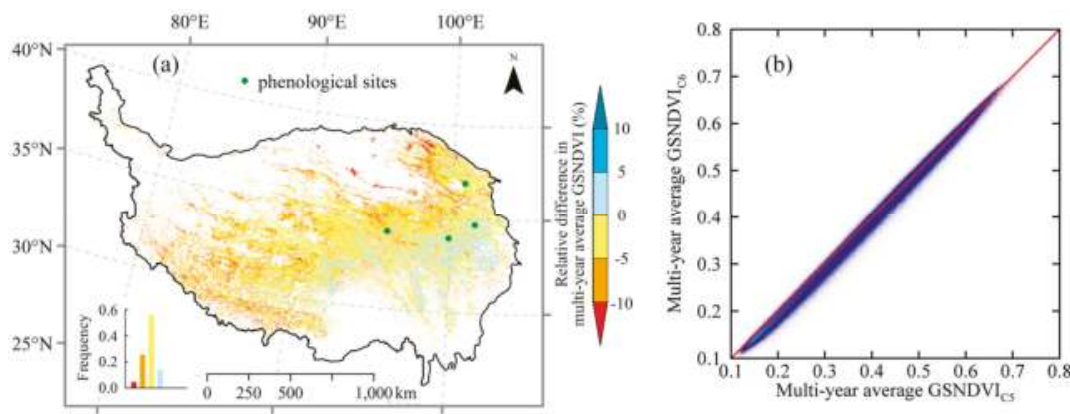

Figure 2. (a) The relative difference in the multi-year average growing season Normalized Difference Vegetation Index between C6 (GSNDVI $\left._{\mathrm{C} 6}\right)$ and $\mathrm{C} 5$ (GSNDVI $\left._{\mathrm{C} 5}\right)((\mathrm{C} 6-\mathrm{C} 5) / \mathrm{C} 5)$ during 2001-2015; (b) The scatter plot of the multi-year average GSNDVI $_{\mathrm{C} 6}$ against $\mathrm{GSNDVI}_{\mathrm{C} 5}$ during 2001-2015.
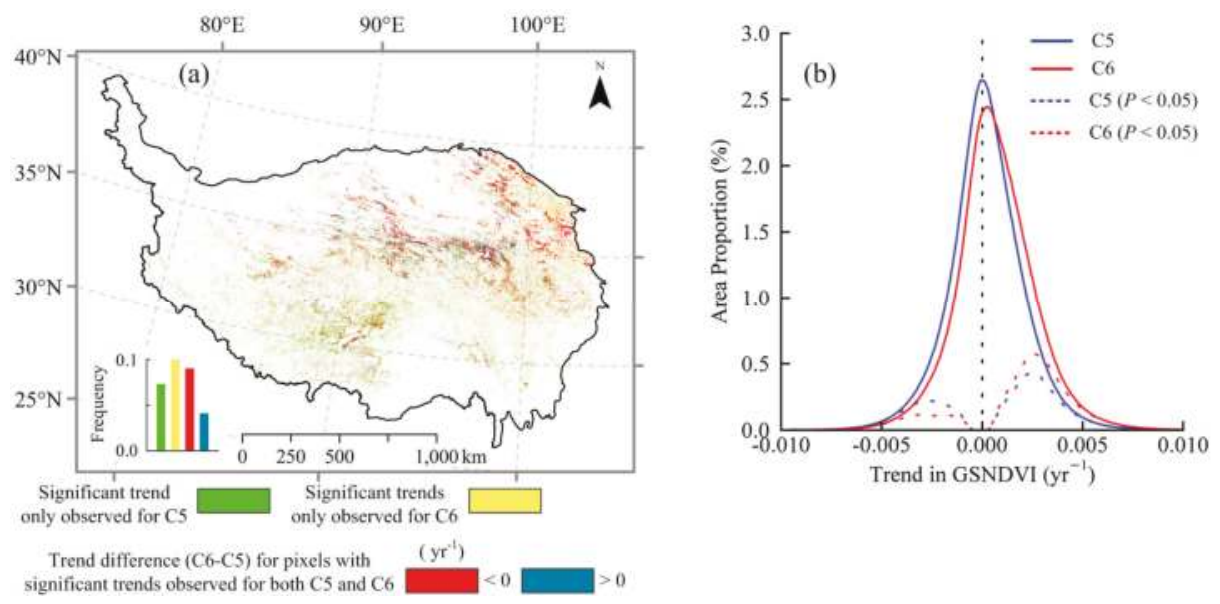

Figure 3. (a) Comparison of trends in growing season Normalized Difference Vegetation Index between C5 (GSNDVI (G5 $_{\text {) and C6 }\left(\text { GSNDVI }_{\mathrm{C} 6}\right) \text { during 2001-2015; and, (b) The histograms of trends in GSNDVI }}$ and GSNDVI $\mathrm{C}_{6}$ during 2001-2015.

\subsection{Temporal Differences in Regional Phenology}

For each method, similar advancing trends were found between regional average $\mathrm{SOS}_{\mathrm{C} 5}$ and SOS $_{\mathrm{C} 6}$ during 2001-2015, but the advancing trend of $\mathrm{SOS}_{\mathrm{C} 5}$ was slightly smaller than that of $\mathrm{SOS}_{\mathrm{C} 6}$ (Figure 4, Table 2). For each method, the multi-year average regional $\mathrm{SOS}_{\mathrm{C} 5}$ was significantly later than $\mathrm{SOS}_{\mathrm{C} 6}(p<0.001)$, but the difference was only about one day (Table 2$)$. 

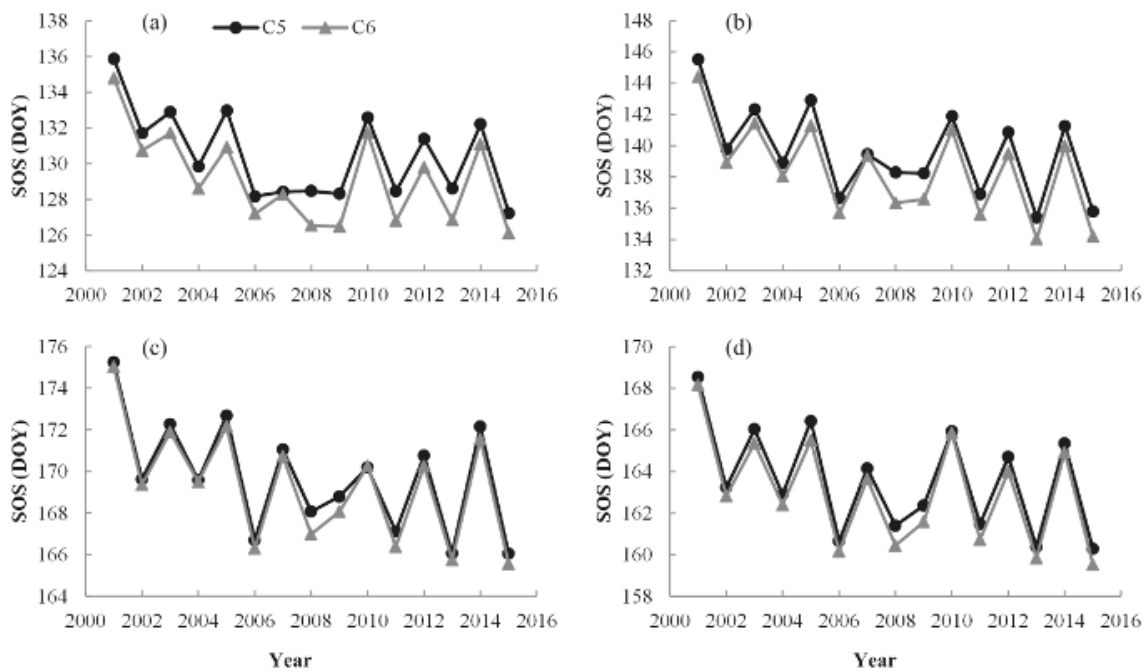

Figure 4. Comparisons of annual regional average start of growing season from MODIS C5 (SOS $\mathrm{C}_{5}$ ) and $\mathrm{C} 6$ (SOS $_{\mathrm{C} 6}$ ) for the alpine grassland on the Tibetan Plateau during 2001-2015 for different methods: (a) Maximum Curvature Change (MCC); (b) Dynamic Threshold 0.2 (DT2); (c) Dynamic Threshold 0.5 (DT5); and, (d) Maximum Slope (MS).

Table 2. Summary statistics for the regional average start of growing season derived from C5 and C6 NDVI products for the alpine grassland on the Tibetan Plateau during 2001-2015.

\begin{tabular}{ccccccccc}
\hline Method & \multicolumn{2}{c}{ MCC } & \multicolumn{2}{c}{ DT2 } & \multicolumn{2}{c}{ DT5 } & \multicolumn{2}{c}{ MS } \\
\hline Product & C5 & C6 & C5 & C6 & C5 & C6 & C5 & C6 \\
\hline Mean & 130.5 & 129.2 & 139.6 & 138.4 & 169.8 & 169.3 & 163.6 & 163.0 \\
Std & 2.5 & 2.6 & 2.9 & 3.0 & 2.7 & 2.8 & 2.5 & 2.6 \\
Trend & -0.278 & -0.298 & -0.339 & -0.378 & -0.312 & -0.332 & -0.258 & -0.264 \\
$p$ value & 0.060 & 0.050 & 0.043 & 0.028 & 0.047 & 0.040 & 0.088 & 0.091 \\
$D_{\text {all }}$ & $30.79 \%$ & $30.59 \%$ & $26.87 \%$ & $26.37 \%$ & $27.91 \%$ & $27.70 \%$ & $28.65 \%$ & $29.31 \%$ \\
$D_{\text {sig }}$ & $1.18 \%$ & $1.17 \%$ & $0.91 \%$ & $0.79 \%$ & $1.00 \%$ & $0.93 \%$ & $1.01 \%$ & $1.03 \%$ \\
$A_{\text {all }}$ & $69.2 \%$ & $69.4 \%$ & $73.13 \%$ & $73.63 \%$ & $72.09 \%$ & $72.30 \%$ & $71.35 \%$ & $70.69 \%$ \\
$A_{\text {sig }}$ & $11.39 \%$ & $12.03 \%$ & $14.95 \%$ & $16.31 \%$ & $12.80 \%$ & $13.52 \%$ & $13.08 \%$ & $12.75 \%$ \\
\hline
\end{tabular}

Mean: multi-year average regional SOS. Std: temporal standard deviation. Trend: slope of linear regression of regional average SOS against year $\left(\right.$ days $\mathrm{yr}^{-1}$ ). $p$ value: significance level for the trend. $A_{\text {all }}$ and $D_{\text {all }}$ : proportions of advancing and delaying trends, respectively. $A_{\text {sig }}$ and $D_{\text {sig }}$ : proportions of significantly $(p<0.05)$ advancing and delaying trends, respectively.

The regional average $\mathrm{EOS}_{\mathrm{C} 5}$ and $\mathrm{EOS}_{\mathrm{C} 6}$ also showed consistent inter-annual variations but no significant trends were found (Figure 5, Table 3). Different from SOS, the multi-year average regional $\mathrm{EOS}_{\mathrm{C} 5}$ was slightly earlier than $\mathrm{EOS}_{\mathrm{C} 6}$ for each method, with a difference of less than one day, but the difference was only significant $(p<0.05)$ for MCC and DT2 methods (Table 3$)$. 

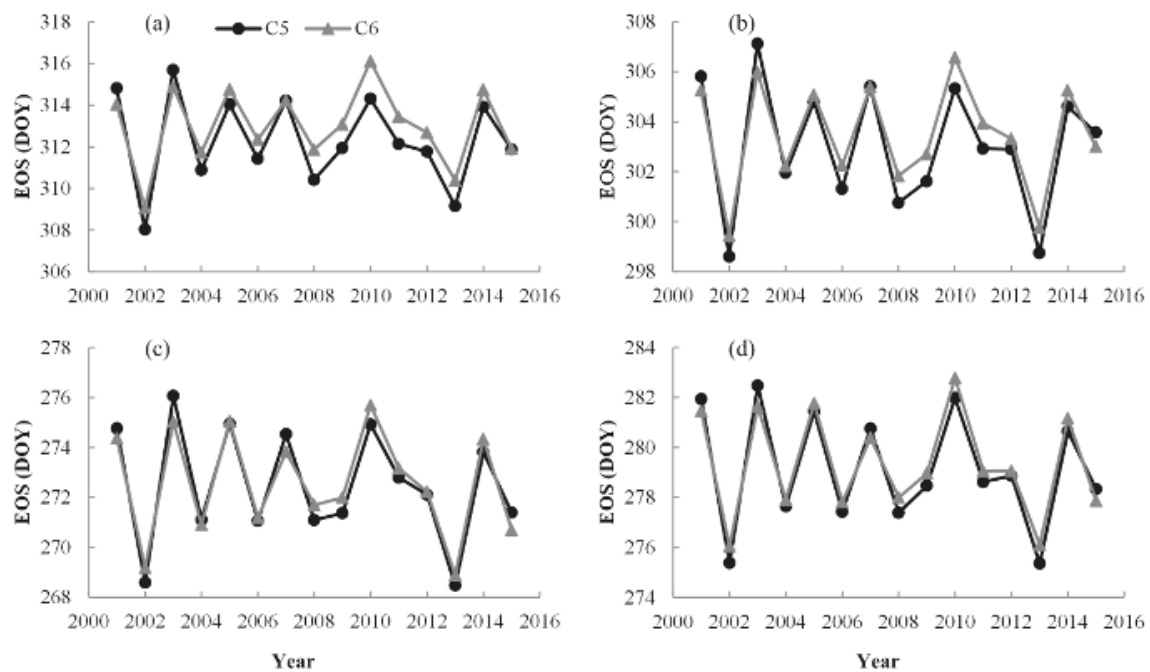

Figure 5. Comparisons of annual regional average end of growing season from MODIS C5 (SOS ${ }_{\mathrm{C} 5}$ ) and $\mathrm{C} 6\left(\mathrm{SOS}_{\mathrm{C} 6}\right)$ for the alpine grassland on the Tibetan Plateau during 2001-2015 for different methods: (a) Maximum Curvature Change (MCC); (b) Dynamic Threshold 0.2 (DT2); (c) Dynamic Threshold 0.5 (DT5); (d) Maximum Slope (MS).

Table 3. Summary statistics for the regional average end of growing season derived from C5 and C6 Normalized Difference Vegetation Index (NDVI) products for the alpine grassland on the Tibetan Plateau during 2001-2015.

\begin{tabular}{ccccccccc}
\hline Method & \multicolumn{2}{c}{ MCC } & \multicolumn{2}{c}{ DT2 } & \multicolumn{2}{c}{ DT5 } & \multicolumn{2}{c}{ MS } \\
\hline Product & C5 & C6 & C5 & C6 & C5 & C6 & C5 & C6 \\
\hline Mean & 312.3 & 313.0 & 303.0 & 303.5 & 272.5 & 272.6 & 279.1 & 279.3 \\
Std & 2.2 & 1.9 & 2.5 & 2.2 & 2.3 & 2.1 & 2.3 & 2.1 \\
Trend & -0.060 & 0.010 & -0.068 & -0.018 & -0.101 & -0.067 & -0.093 & -0.061 \\
$p$ value & 0.665 & 0.929 & 0.670 & 0.895 & 0.490 & 0.622 & 0.523 & 0.645 \\
$D_{\text {all }}$ & $43.75 \%$ & $49.75 \%$ & $42.53 \%$ & $47.09 \%$ & $39.66 \%$ & $42.38 \%$ & $39.64 \%$ & $42.83 \%$ \\
$D_{\text {sig }}$ & $1.96 \%$ & $2.80 \%$ & $1.90 \%$ & $2.54 \%$ & $1.66 \%$ & $2.00 \%$ & $1.49 \%$ & $1.85 \%$ \\
$A_{\text {all }}$ & $56.25 \%$ & $50.25 \%$ & $57.47 \%$ & $52.91 \%$ & $60.34 \%$ & $57.62 \%$ & $60.36 \%$ & $57.17 \%$ \\
$A_{\text {sig }}$ & $3.91 \%$ & $2.75 \%$ & $4.35 \%$ & $3.40 \%$ & $4.89 \%$ & $4.26 \%$ & $5.18 \%$ & $4.31 \%$ \\
\hline
\end{tabular}

Mean: multi-year average regional EOS. Std: temporal standard deviation. Trend: slope of linear regression of regional average EOS against year (days $\mathrm{yr}^{-1}$ ). $p$ value: significance level for the trend. $A_{\text {all }}$ and $D_{\text {all }}$ : proportions of advancing and delaying trends, respectively. $A_{\text {sig }}$ and $D_{\text {sig }}$ : proportions of significantly $(p<0.05)$ advancing and delaying trends, respectively.

\subsection{Spatial Differences in Multi-Year Average Phenology}

For each method, various degrees of differences between the multi-year average $\mathrm{SOS}_{\mathrm{C} 5}$ and $\mathrm{SOS}_{\mathrm{C} 6}$ were found (Figure 6). The multi-year average $\mathrm{SOS}_{\mathrm{C} 6}$ was earlier than the multi-year average $\mathrm{SOS}_{\mathrm{C} 5}$ for most of pixels (68.7\% for MCC, $66.2 \%$ for DT2, 57.6\% for DT5 and $61.6 \%$ for MS) (Figure 6 ). As for the pixels with differences of more than five days, SOS based on MCC and MS methods showed the largest $(14.4 \%)$ and smallest (4.9\%) proportions of the total area, respectively (Figure 6$)$. The corresponding proportions for DT2 and DT5 methods were $13.2 \%$ and $7.4 \%$, respectively. All four methods indicated that the pixels with $\mathrm{SOS}_{\mathrm{C} 6}$ later than $\mathrm{SOS}_{\mathrm{C} 5}$ were mainly distributed in the northwestern TP, while the differences between $\mathrm{SOS}_{\mathrm{C} 5}$ and $\mathrm{SOS}_{\mathrm{C} 6}$ varied with methods in the southern TP, with larger differences for MCC and DT2 methods and less for DT5 and MS methods (Figure 6). 

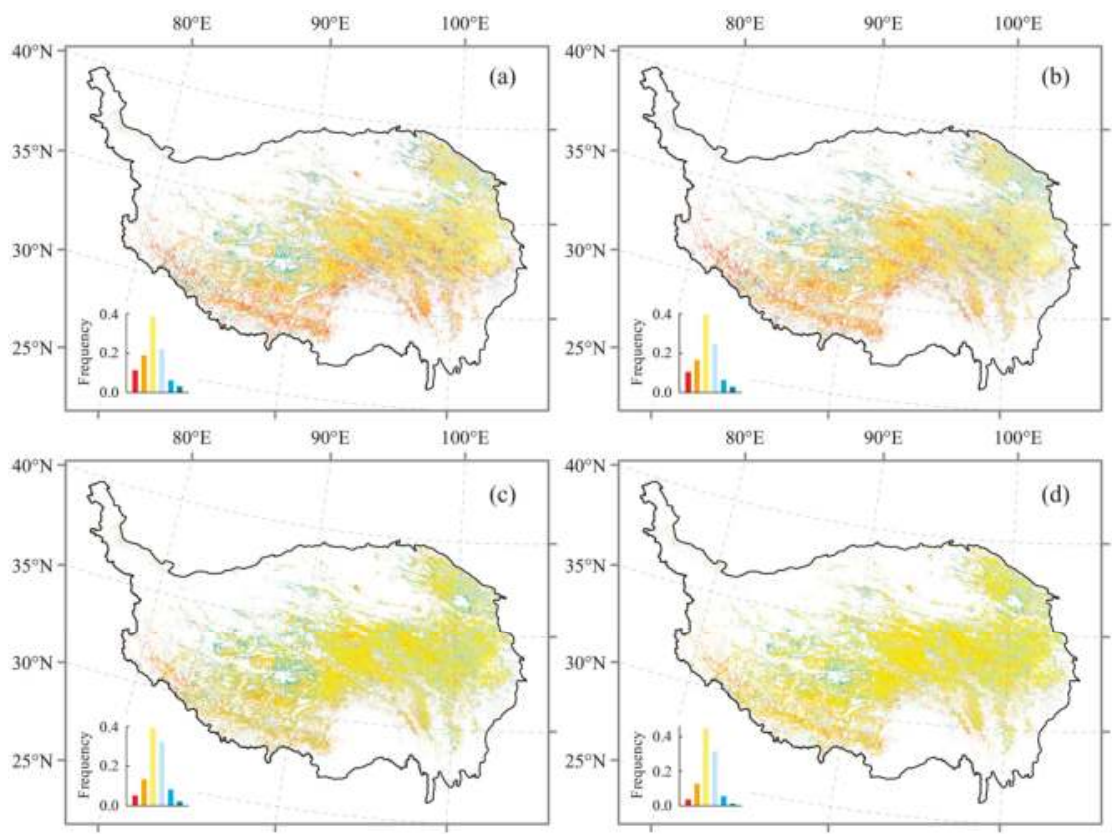

Difference in multi-year average SOS (C6-C5) (days)
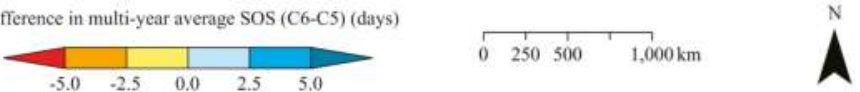

Figure 6. Spatial differences between the multi-year average $\mathrm{SOS}_{\mathrm{C} 6}$ and $\mathrm{SOS}_{\mathrm{C} 5}(\mathrm{C6} 6 \mathrm{C} 5)$ for different methods: (a) Maximum Curvature Change (MCC); (b) Dynamic Threshold 0.2 (DT2); (c) Dynamic Threshold 0.5 (DT5); (d) Maximum Slope (MS).

Similar to SOS, large differences were found for multi-year average $\mathrm{EOS}_{\mathrm{C} 5}$ and $\mathrm{EOS}_{\mathrm{C} 6}$ based on four methods (Figure 7). The EOS $\mathrm{C}_{6}$ was later than the $\mathrm{EOS}_{\mathrm{C} 5}$ over $58.5 \%$ of pixels for MCC method, while the proportions were $55.8 \%, 50.0 \%$, and $53.1 \%$ for DT2, DT5, and MS methods, respectively (Figure 7). The pixels with the differences of more than five days between $\mathrm{EOS}_{\mathrm{C} 5}$ and $\mathrm{EOS}_{\mathrm{C} 6}$ accounted for $11.7 \%, 7.1 \%, 6.4 \%$ and $4.1 \%$ of the total area for MCC, DT2, DT5 and MS methods, respectively. For each method, the pixels where $\mathrm{EOS}_{\mathrm{C} 6}$ was earlier than $\mathrm{EOS}_{\mathrm{C} 5}$ were mainly distributed in the eastern and southwestern TP. The differences of EOS in the central TP were larger for MCC method than the other methods. 

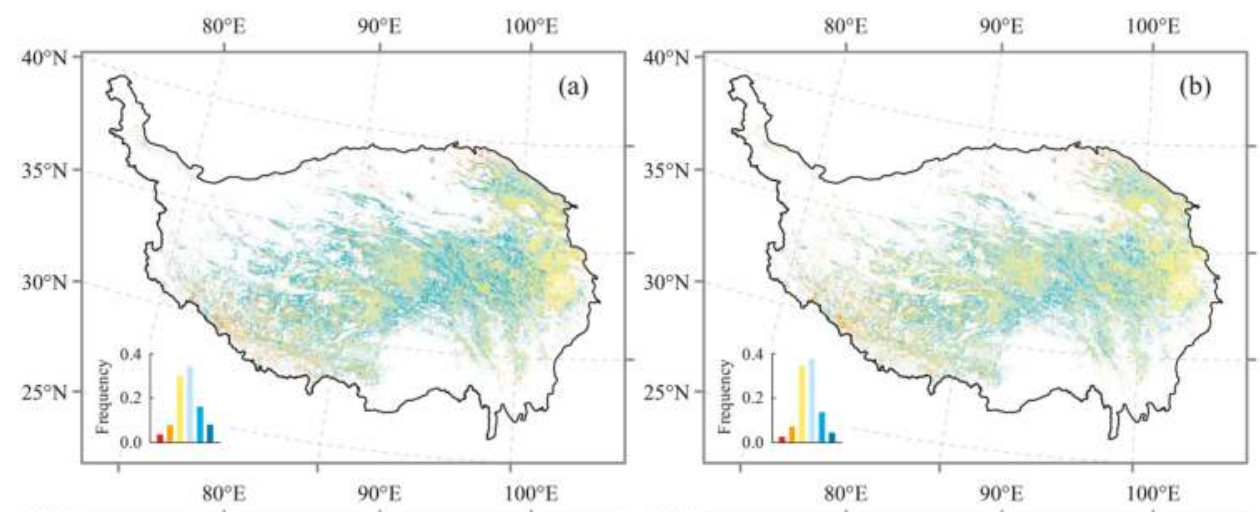

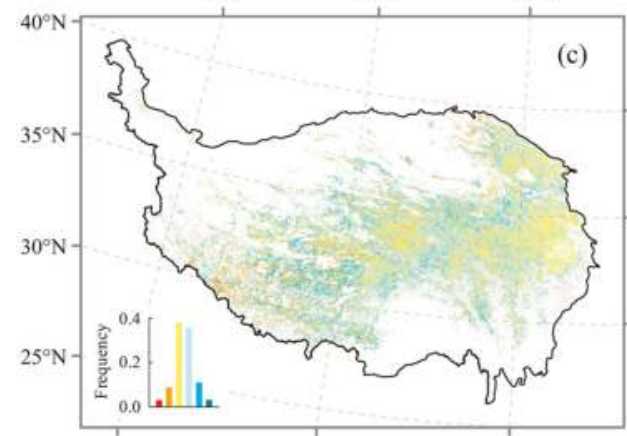

Difference in multi-year average EOS (C6-C5) (days)

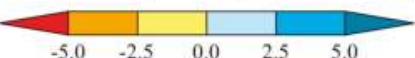

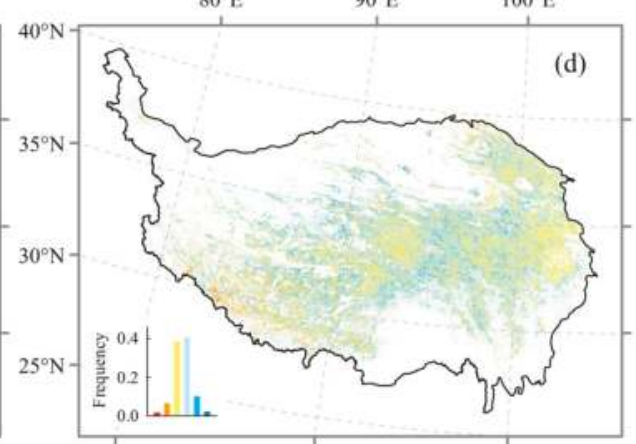

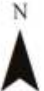

Figure 7. Spatial differences between the multi-year average $\mathrm{EOS}_{\mathrm{C} 6}$ and $\mathrm{EOS}_{\mathrm{C} 5}(\mathrm{C} 6-\mathrm{C} 5)$ for different methods: (a) Maximum Curvature Change (MCC); (b) Dynamic Threshold 0.2 (DT2); (c) Dynamic Threshold 0.5 (DT5); and, (d) Maximum Slope (MS).

\subsection{Spatial Differences in Phenological Trends}

For each method, the proportion of advancing trends was much higher than that of delaying trends for both $\mathrm{SOS}_{\mathrm{C} 5}$ and $\mathrm{SOS}_{\mathrm{C} 6}$ (Table 2). The proportions of advancing (delaying) trends, as well as significantly advancing (delaying) trends $(p<0.05)$ in $\mathrm{SOS}_{\mathrm{C} 5}$ and $\mathrm{SOS}_{\mathrm{C} 6}$ were similar based on the same method (Table 2). However, the trends in $\mathrm{SOS}_{\mathrm{C} 5}$ and $\mathrm{SOS}_{\mathrm{C} 6}$ showed obvious spatial inconsistencies (Figure 8). For all four methods, the significant levels of SOS trends shifted over a considerable proportion of the study region. Significant trends in $\mathrm{SOS}_{\mathrm{C} 5}$ became insignificant in $\mathrm{SOS}_{\mathrm{C} 6}$ over $6.98 \%$, $7.67 \%, 7.55 \%$, and $7.74 \%$ of pixels for MCC, DT2, DT5, and MS methods, respectively (Figure 8). Meanwhile, insignificant trends in $\mathrm{SOS}_{\mathrm{C} 5}$ became significant in $\mathrm{SOS}_{\mathrm{C} 6}$ over $7.60 \%, 8.92 \%, 8.20 \%$, and $7.42 \%$ of pixels for MCC, DT2, DT5, and MS methods, respectively (Figure 8 ). For the pixels with significant trends in both $\mathrm{SOS}_{\mathrm{C} 5}$ and $\mathrm{SOS}_{\mathrm{C} 6}$, $\mathrm{SOS}$ trends tended to be more negative from $\mathrm{C} 5$ to $\mathrm{C} 6$ for each method (Figure 8). Significant $(p<0.001)$ difference was observed between the SOS trends of C5 and $\mathrm{C} 6$ for each method, with mean differences between $\mathrm{C} 5$ and $\mathrm{C} 6$ (C6-C5) of $-0.05,-0.07,-0.05$, and -0.03 day $\mathrm{yr}^{-1}$ for MCC, DT2, DT5, and MS methods, respectively. 

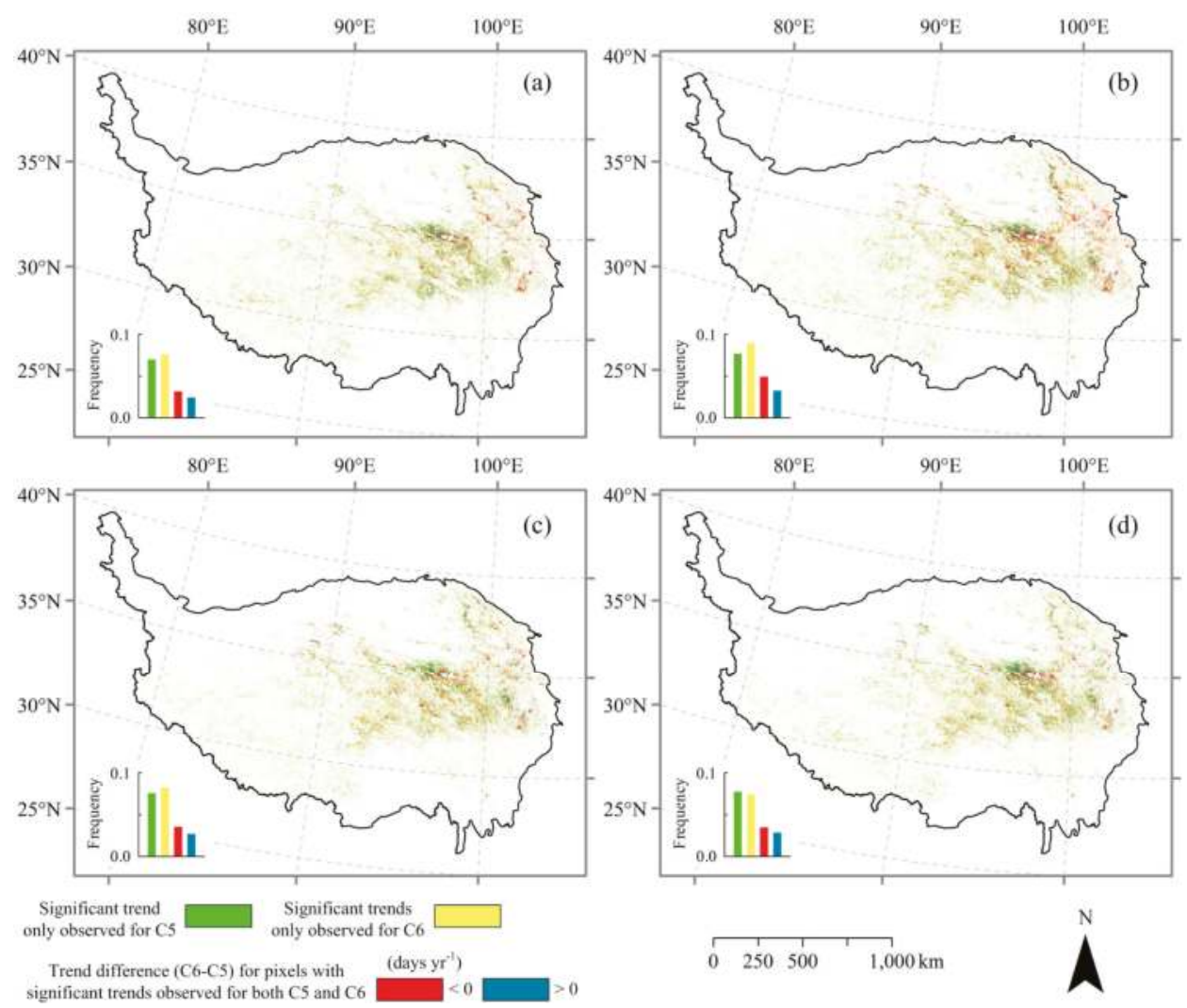

Figure 8. Comparison of trends in start of growing season between $\mathrm{C} 5\left(\mathrm{SOS}_{\mathrm{C} 5}\right)$ and $\mathrm{C6}\left(\mathrm{SOS}_{\mathrm{C} 6}\right)$ during 2001-2015 for different methods: (a) Maximum Curvature Change (MCC); (b) Dynamic Threshold 0.2 (DT2); (c) Dynamic Threshold 0.5 (DT5); and, (d) Maximum Slope (MS).

The proportion of advancing trends was higher than that of delaying trends for both $\mathrm{EOS}_{\mathrm{C} 5}$ and $\mathrm{EOS}_{\mathrm{C} 6}$ for each method (Table 3). Only a small proportion of significantly advancing or delaying trends was found for both $\mathrm{EOS}_{\mathrm{C} 5}$ and $\mathrm{EOS}_{\mathrm{C} 6}$ (Table 3). For each method, the advancing trends in $\mathrm{EOS}_{\mathrm{C} 6}$ accounted for a higher proportion of the total pixels than those in $\mathrm{EOS}_{\mathrm{C} 5}$, while the delaying trends in $\mathrm{EOS}_{\mathrm{C} 6}$ accounted for a lower proportion than those in $\mathrm{EOS}_{\mathrm{C} 5}$ (Table 3). Large spatial differences in trends between $\mathrm{EOS}_{\mathrm{C} 5}$ and $\mathrm{EOS}_{\mathrm{C} 6}$ were also found (Figure 9). From $\mathrm{C} 5$ to $\mathrm{C} 6$, pixels with a EOS trend shifting from significant to insignificant accounted for $4.70 \%, 4.71 \%, 4.80 \%$, and $4.90 \%$ of the total pixels for MCC, DT2, DT5, and MS methods, respectively. Meanwhile, insignificant trends in EOS $_{\text {C5 }}$ became significant in $\mathrm{EOS}_{\mathrm{C} 6}$ over $4.37 \%, 4.41 \%, 4.51 \%$, and $4.39 \%$ of pixels for MCC, DT2, DT5, and MS methods, respectively. Although a significant difference was also found between $\mathrm{EOS}_{\mathrm{C} 5}$ and EOS $_{\mathrm{C} 6}$ trends $(p<0.001)$ for each method, a very small proportion of pixels with significant trends for both $\mathrm{EOS}_{\mathrm{C} 5}$ and $\mathrm{EOS}_{\mathrm{C} 6}$ was found for each method (1.18\% for MCC, $1.54 \%$ for DT2, 1.75\% for DT5, and $1.77 \%$ for MS) (Figure 9). 

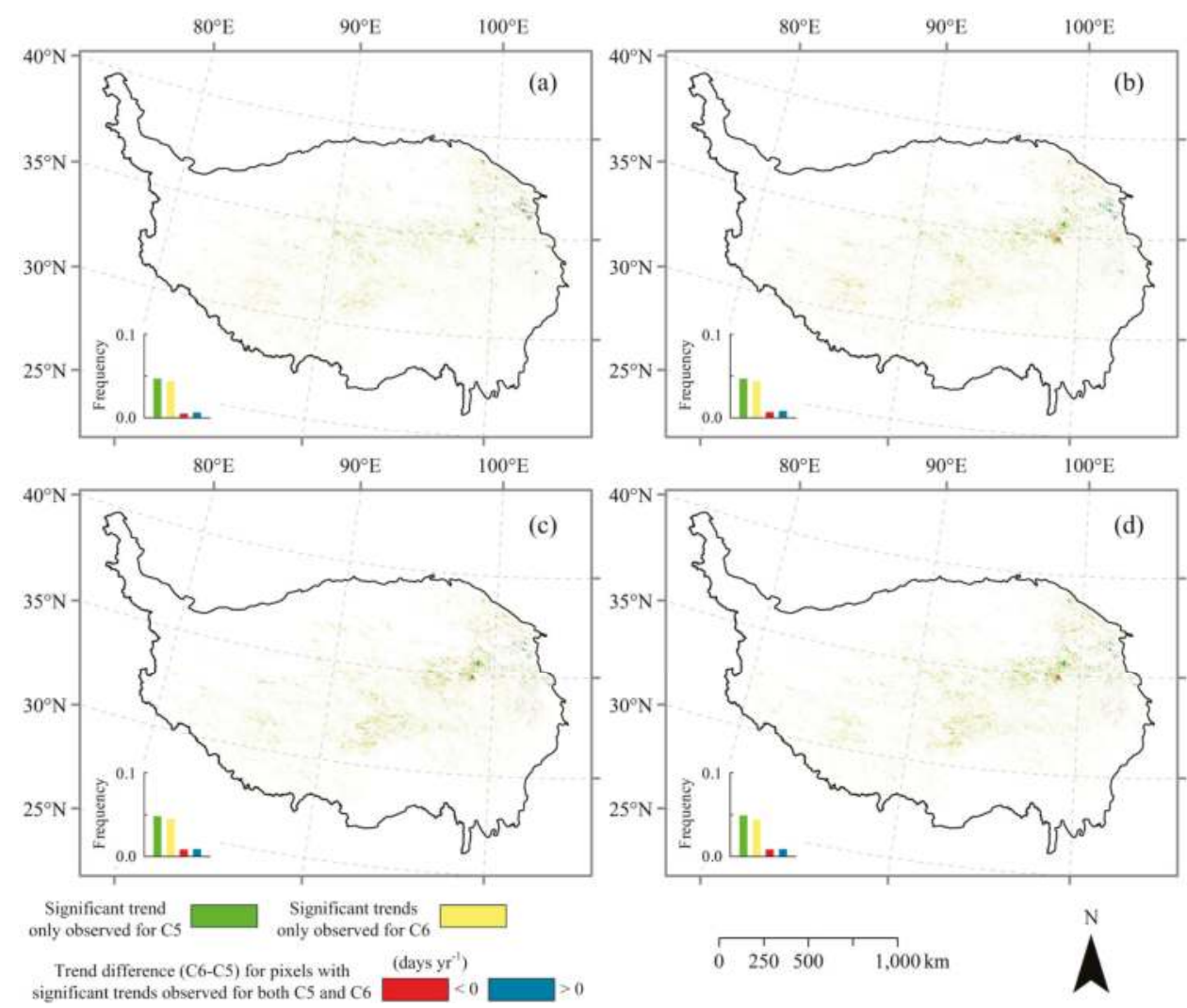

Figure 9. Comparison of trends in end of growing season between $\mathrm{C} 5\left(\mathrm{EOS}_{\mathrm{C} 5}\right)$ and $\mathrm{C} 6\left(\mathrm{EOS}_{\mathrm{C} 6}\right)$ during 2001-2015 for different methods: (a) Maximum Curvature Change (MCC); (b) Dynamic Threshold 0.2 (DT2); (c) Dynamic Threshold 0.5 (DT5); and, (d) Maximum Slope (MS).

\subsection{Comparison Between Satellite-Derived and Ground-Observed Phenology}

The differences between the satellite-derived $\mathrm{SOS}\left(\mathrm{SOS}_{\mathrm{C} 5}\right.$ and $\left.\mathrm{SOS}_{\mathrm{C} 6}\right)$ and the ground-observed GU were smaller for MCC and DT2 methods and larger for DT5 and MS methods (Table 4). For both $\mathrm{SOS}_{\mathrm{C} 5}$ and $\mathrm{SOS}_{\mathrm{C} 6}$, the MEs and MAEs were about 10 days based on MCC and DT2 methods, but more than 30 days based on DT5 and MS methods (Table 4). However, $\mathrm{SOS}_{\mathrm{C} 5}$ had a slightly smaller ME or MAE than $\mathrm{SOS}_{\mathrm{C} 6}$ for each method (Table 4). Besides, both $\mathrm{SOS}_{\mathrm{C} 5}$ and $\mathrm{SOS}_{\mathrm{C} 6}$ were significantly and positively correlated with GU for all of the methods $(p<0.01)$, but the correlation coefficient between $\mathrm{SOS}_{\mathrm{C} 5}$ and GU was larger than that between $\mathrm{SOS}_{\mathrm{C} 6}$ and GU for each method (Table 4). Results based on MCC method showed the highest correlation coefficients between SOS and GU (Table 4).

However, large differences between the satellite-derived $\mathrm{EOS}\left(\mathrm{EOS}_{\mathrm{C} 5}\right.$ and $\left.\mathrm{EOS}_{\mathrm{C} 6}\right)$ and the ground-observed BLC were found. The MEs and MAEs of EOS were more than 18 days for each method (Table 4). Moreover, both $\mathrm{EOS}_{\mathrm{C} 5}$ and $\mathrm{EOS}_{\mathrm{C} 6}$ showed very poor positive correlations with BLC for all of the methods (Table 4). Nevertheless, the correlation between EOS 5 and BLC was still slightly higher than that between $\mathrm{EOS}_{\mathrm{C} 6}$ and BLC for each method (Table 4). 
Table 4. Mean error (ME), mean absolute error (MAE) and correlation coefficient $(r)$ between the satellite-derived phenology and the ground-observed phenology.

\begin{tabular}{|c|c|c|c|c|}
\hline Method & Phenological Metric & ME & MAE & $r$ \\
\hline \multirow{4}{*}{ MCC } & $\mathrm{SOS}_{\mathrm{C} 5}$ & -7.375 & 9.542 & $0.675^{* *}$ \\
\hline & $\mathrm{SOS}_{\mathrm{C} 6}$ & -7.979 & 10.479 & $0.637 * *$ \\
\hline & $\mathrm{EOS}_{\mathrm{C} 5}$ & 60.354 & 60.354 & 0.073 \\
\hline & $\mathrm{EOS}_{\mathrm{C} 6}$ & 59.896 & 59.896 & 0.067 \\
\hline \multirow{4}{*}{ DT2 } & $\mathrm{SOS}_{\mathrm{C} 5}$ & 4.146 & 9.479 & 0.580 ** \\
\hline & $\mathrm{SOS}_{\mathrm{C} 6}$ & 4.271 & 9.688 & $0.538^{* *}$ \\
\hline & $\mathrm{EOS}_{\mathrm{C} 5}$ & 48.979 & 48.979 & 0.172 \\
\hline & $\mathrm{EOS}_{\mathrm{C} 6}$ & 48.396 & 48.396 & 0.164 \\
\hline \multirow{4}{*}{ DT5 } & $\mathrm{SOS}_{\mathrm{C} 5}$ & 35.125 & 35.125 & $0.532 * *$ \\
\hline & $\mathrm{SOS}_{\mathrm{C} 6}$ & 35.417 & 35.417 & $0.478^{* *}$ \\
\hline & $\mathrm{EOS}_{\mathrm{C5}}$ & 18.813 & 19.521 & 0.207 \\
\hline & $\mathrm{EOS}_{\mathrm{C} 6}$ & 18.292 & 18.750 & 0.180 \\
\hline \multirow{4}{*}{ MS } & $\mathrm{SOS}_{\mathrm{C} 5}$ & 30.771 & 30.771 & $0.526^{* *}$ \\
\hline & $\mathrm{SOS}_{\mathrm{C} 6}$ & 30.833 & 30.833 & $0.464 * *$ \\
\hline & $\mathrm{EOS}_{\mathrm{C} 5}$ & 22.563 & 22.729 & 0.178 \\
\hline & $\mathrm{EOS}_{\mathrm{C} 6}$ & 22.375 & 22.375 & 0.175 \\
\hline
\end{tabular}

\section{Discussion}

The annual regional average GSNDVI for the alpine grassland on the TP showed no obvious trend for C5 $(p=0.424)$, but a significant increasing trend for C6 ( $<00.05)$ (Figure 1). Moreover, GSNDVI ${ }_{\mathrm{C} 6}$ showed increasing trends over more areas than $\mathrm{GSNDVI}_{\mathrm{C} 5}$ (Figure 3). These greening trends may result from the removal of sensor degradation, which can lead to a decline in NDVI, as previous studies suggested [12,13]. Zhang et al. [17] also reported that the annual NDVI from Terra C6 showed larger greening vegetation area than Terra C 5 at global scale, which was consistent with the result of our study. Besides, in a comparative study of cross-product NDVI dynamics in a tropical region in Tanzania, significant increasing trends in NDVI also became more apparent in Terra C6 NDVI than C5 NDVI [20]. In this study, the regional average GSNDVI ${ }_{\mathrm{C} 6}$ was significantly lower than $\mathrm{GSNDVI}_{\mathrm{C} 5}$ (Figure 1). At spatial scale, the multi-year average GSNDVI ${ }_{\mathrm{C} 6}$ was lower than the GSNDVI $\mathrm{C}_{5}$ in most areas (Figure 2). These could cause the change of the NDVI curve shape, resulting in some differences between phenology derived from C5 and C6 NDVI [32].

The identified vegetation phenology can be affected by the phenology extraction methods [28]. To avoid the bias of a single phenology extraction method [21], this study applied four common methods to identify the vegetation phenological metrics. The identified SOSs from the earliest to the latest ranked by MCC, DT2, MS, and DT5 (Table 2), which was almost consistent with the study of Shen et al. [25]. Meanwhile, the order of the identified EOSs from the earliest to the latest was DT5, MS, DT2, and MCC (Table 3). The difference in average SOS and EOS could be more than one month among different methods. White et al. [28] found that individual methods differed in average SOS by \pm 60 days, and the spatial phenological patterns derived from different methods often differed among ecoregions by comparing 10 commonly used methods for estimating the SOS based on the Advanced Very High Resolution Radiometer (AVHRR) NDVI in North America. Such differences in phenology among methods were also found in other previous studies [33-35]. In our study, the differences between phenology derived from C5 and C6 NDVI varied among methods (Tables 2 and 3, Figures 6-9). Generally, most pixels showed an earlier multi-year average $\mathrm{SOS}_{\mathrm{C} 6}$ than $\mathrm{SOS}_{\mathrm{C} 5}$ and later EOS $_{\mathrm{C} 6}$ than $\mathrm{EOS}_{\mathrm{C} 5}$ (Figures 6 and 7). From $\mathrm{C} 5$ to $\mathrm{C} 6$, pixels with a SOS (EOS) trend changed from significant to insignificant, or from insignificant to significant accounted for at least $14.58 \%(9.07 \%)$ of the total pixels (Figures 8 and 9). This further confirmed the influence of the change in NDVI values originated from sensor degradation and calibration methods on vegetation phenology monitoring. 
By comparing the satellite-derived with the ground-observed phenology, SOS identified by MCC and DT2 methods was more consistent and correlated with the ground-observed GU than that by MS and DT5 methods for both C5 and C6 NDVI (Table 4), implying that MCC and DT2 methods were more suitable to monitor GU for the alpine grassland on the TP. Yu et al. [36] also found that SOS that was extracted by DT2 from AVHRR NDVI was close to the ground observations on the TP. Moreover, smaller differences and higher correlations were found between $\mathrm{SOS}_{\mathrm{C} 5}$ and $\mathrm{GU}$ than between $\mathrm{SOS}_{\mathrm{C} 6}$ and $\mathrm{GU}$ (Table 4), implying that $\mathrm{SOS}_{\mathrm{C} 5}$ might be more consistent than $\mathrm{SOS}_{\mathrm{C} 6}$ with the ground-observed GU. C5 NDVI may be more appropriate for monitoring SOS than C6 NDVI in this area, but more ground-observed phenology records are needed to confirm it due to only four available sites in our study. However, regarding EOS, large differences and poor correlations between $\mathrm{EOS}_{\mathrm{C} 5}$, as well as $\mathrm{EOS}_{\mathrm{C} 6}$ and $\mathrm{BLC}$, were found for all of the phenology extraction methods (Table 4). When compared with SOS, EOS is much difficult to be monitored [37-39]. Many factors can influence the evaluation of vegetation phenology shifts based on remote sensing data [38,40]. When comparing the satellite-derived with the ground-observed phenology, due to the difference in observation scale (pixel versus individual plant) and content (spectral response of plant versus specific phenological event of plant), the satellite-derived phenology (e.g., EOS) is considered to be related, but not identical, to the ground-observed phenology (e.g., BLC) [28,41,42]. Therefore, it brings much uncertainty to the validation of the satellite-derived phenology. Besides, plants often experience a longer and slower change in canopy greenness in autumn than in spring [38,39,43], which may result in a lower inter-annual variability of changes in EOS relative to SOS and consequently make it more difficult to detect EOS from remote sensing time-series data [38]. Therefore, there is a need to use near-surface remote sensing data with a higher resolution, including digital camera data [44-46] and corresponding validation methods, for further evaluating the uncertainty in monitoring vegetation phenology with MODIS C5 and C6 NDVI products.

This study aimed to compare the differences between MODIS C5 and C6 NDVI time series in monitoring vegetation phenology on the TP. Based on the same selected grassland pixels, the same data pre-processing methods and phenology extraction methods, the differences in phenology derived from the two products were found to be mainly due to the sensor degradation and different calibration methods. However, it should be noted that the selection criteria for grassland pixels and the data pre-processing methods might influence the values of the satellite-derived phenological metrics. Besides, the satellite-derived phenology is also influenced by the temporal [47,48], spatial [49], and spectral resolutions [21] of remote sensing data.

\section{Conclusions}

This study conducted a comparative analysis of MODIS C5 and C6 NDVI-derived phenology for the alpine grassland on the TP. Although the regional average $\mathrm{SOS}_{\mathrm{C} 5}$ and $\mathrm{SOS}_{\mathrm{C} 6}\left(\mathrm{EOS}_{\mathrm{C} 5}\right.$ and $\left.\mathrm{EOS}_{\mathrm{C} 6}\right)$ showed consistent inter-annual variations, large spatial differences in trends between $\mathrm{SOS}_{\mathrm{C} 5}$ and $\mathrm{SOS}_{\mathrm{C} 6}$ $\left(\mathrm{EOS}_{\mathrm{C} 5}\right.$ and $\mathrm{EOS}_{\mathrm{C} 6}$ ) were found. From $\mathrm{C} 5$ to $\mathrm{C} 6$, pixels with a SOS (EOS) trend changed from significant to insignificant, or from insignificant to significant, accounted for at least $14.58 \%(9.07 \%)$ of the total pixels. By comparing the satellite-derived phenology with the ground-observed phenology, $\mathrm{SOS}_{\mathrm{C} 5}$ was found to be more consistent than $\mathrm{SOS}_{\mathrm{C} 6}$ with the ground-observed green-up dates. C5 NDVI may be more appropriate for monitoring SOS than C6 NDVI in this area, but more ground-observed phenology records are needed to confirm it due to the only four available sites. However, both $\mathrm{EOS}_{\mathrm{C} 5}$ and $\mathrm{EOS}_{\mathrm{C} 6}$ showed large differences and poor correlations with the ground-observed beginning of leaf coloring. The accuracy of vegetation phenology derived from remote sensing data was impacted by many factors. Therefore, there is a need to use near-surface remote sensing data with a higher resolution and corresponding validation methods for further evaluating the uncertainty of MODIS C5 and C6 NDVI products in monitoring vegetation phenology.

Acknowledgments: The authors thank the editor and reviewers for helpful comments and suggestions. The authors also thank the Meteorological Information Center of the China Meteorological Administration 
for providing phenology data. This work was supported by National Natural Science Foundation of China (Grant No. 41371389, No. 41771047) and the project from State Key Laboratory of Earth Surface Processes and Resource Ecology (Grant No. 2017-FX-01(1)).

Author Contributions: Zhoutao Zheng and Wenquan Zhu designed this study and wrote the manuscript; Zhoutao Zheng performed the data pre-processing; Zhoutao Zheng and Wenquan Zhu conducted the analysis.

Conflicts of Interest: The authors declare no conflict of interest.

\section{References}

1. Cleland, E.E.; Chuine, I.; Menzel, A.; Mooney, H.A.; Schwartz, M.D. Shifting plant phenology in response to global change. Trends Ecol. Evol. 2007, 22, 357-365. [CrossRef] [PubMed]

2. Richardson, A.D.; Keenan, T.F.; Migliavacca, M.; Ryu, Y.; Sonnentag, O.; Toomey, M. Climate change, phenology, and phenological control of vegetation feedbacks to the climate system. Agric. For. Meteorol. 2013, 169, 156-173. [CrossRef]

3. Piao, S.L.; Friedlingstein, P.; Ciais, P.; Viovy, N.; Demarty, J. Growing season extension and its impact on terrestrial carbon cycle in the Northern Hemisphere over the past 2 decades. Glob. Biogeochem. Cycles 2007, 21, GB30183. [CrossRef]

4. Jeong, S.J.; Ho, C.H.; Gim, H.J.; Brown, M.E. Phenology shifts at start vs. end of growing season in temperate vegetation over the Northern Hemisphere for the period 1982-2008. Glob. Chang. Biol. 2011, 17, 2385-2399. [CrossRef]

5. Zhu, W.Q.; Tian, H.Q.; Xu, X.F.; Pan, Y.Z.; Chen, G.S.; Lin, W.P. Extension of the growing season due to delayed autumn over mid and high latitudes in North America during 1982-2006. Glob. Ecol. Biogeogr. 2012, 21, 260-271. [CrossRef]

6. Piao, S.; Wang, X.; Ciais, P.; Zhu, B.; Wang, T.; Liu, J. Changes in satellite-derived vegetation growth trend in temperate and boreal Eurasia from 1982 to 2006. Glob. Chang. Biol. 2011, 17, 3228-3239. [CrossRef]

7. Jönsson, P.; Eklundh, L. Seasonality extraction by function fitting to time-series of satellite sensor data. IEEE Trans. Geosci. Remote Sens. 2002, 40, 1824-1832. [CrossRef]

8. Stockli, R.; Vidale, P.L. European plant phenology and climate as seen in a 20-year AVHRR land-surface parameter dataset. Int. J. Remote Sens. 2004, 25, 3303-3330. [CrossRef]

9. Zhang, G.L.; Zhang, Y.J.; Dong, J.W.; Xiao, X.M. Green-up dates in the Tibetan Plateau have continuously advanced from 1982 to 2011. Proc. Natl. Acad. Sci. USA 2013, 110, 4309-4314. [CrossRef] [PubMed]

10. Djavidnia, S.; Melin, F.; Hoepffner, N. Comparison of global ocean colour data records. Ocean Sci. 2010, 6, 61-76. [CrossRef]

11. Levy, R.C.; Remer, L.A.; Kleidman, R.G.; Mattoo, S.; Ichoku, C.; Kahn, R.; Eck, T.F. Global evaluation of the Collection 5 MODIS dark-target aerosol products over land. Atmos. Chem. Phys. 2010, 10, 10399-10420. [CrossRef]

12. Lyapustin, A.; Wang, Y.; Xiong, X.; Meister, G.; Platnick, S.; Levy, R.; Franz, B.; Korkin, S.; Hilker, T.; Tucker, J.; et al. Scientific impact of MODIS C5 calibration degradation and C6+ improvements. Atmos. Meas. Tech. 2014, 7, 4353-4365. [CrossRef]

13. Wang, D.; Morton, D.; Masek, J.; Wu, A.; Nagol, J.; Xiong, X.; Levy, R.; Vermote, E.; Wolfe, R. Impact of sensor degradation on the MODIS NDVI time series. Remote Sens. Environ. 2012, 119, 55-61. [CrossRef]

14. Zhao, M.; Running, S.W. Response to comments on "Drought-induced reduction in global terrestrial net primary production from 2000 through 2009". Science 2011, 333, 1093. [CrossRef]

15. Vermote, E.F.; El Saleous, N.Z.; Justice, C.O. Atmospheric correction of MODIS data in the visible to middle infrared: First results. Remote Sens. Environ. 2002, 83, 97-111. [CrossRef]

16. Kotchenova, S.Y.; Vermote, E.F.; Levy, R.; Lyapustin, A. Radiative transfer codes for atmospheric correction and aerosol retrieval: Intercomparison study. Appl. Opt. 2008, 47, 2215-2226. [CrossRef] [PubMed]

17. Zhang, Y.; Song, C.; Band, L.E.; Sun, G.; Li, J. Reanalysis of global terrestrial vegetation trends from MODIS products: Browning or greening? Remote Sens. Environ. 2017, 191, 145-155. [CrossRef]

18. Toller, G.; Xiong, X.; Sun, J.; Wenny, B.N.; Geng, X.; Kuyper, J.; Angal, A.; Chen, H.; Madhavan, S.; Wua, A. Terra and Aqua moderate-resolution imaging spectroradiometer collection 6 level 1B algorithm. J. Appl. Remote Sens. 2013, 7, 073557. [CrossRef] 
19. Didan, K.; Munoz, A.B.; Solano, R.; Huete, A. MODIS Vegetation Index Users Guide (MOD13 Series); Version 3.00 (Collection 6); Vegetation Index and Phenology Lab, The University of Arizona: Tucson, AZ, USA, 2015.

20. Detsch, F.; Otte, I.; Appelhans, T.; Nauss, T. A comparative study of cross-product NDVI dynamics in the Kilimanjaro region a matter of sensor, degradation calibration, and significance. Remote Sens. 2016, 8, 159. [CrossRef]

21. Atzberger, C.; Klisch, A.; Mattiuzzi, M.; Vuolo, F. Phenological metrics derived over the European continent from NDVI3g data and MODIS time series. Remote Sens. 2014, 6, 257-284. [CrossRef]

22. Xin, Q.; Broich, M.; Zhu, P.; Gong, P. Modeling grassland spring onset across the Western United States using climate variables and MODIS-derived phenology metrics. Remote Sens. Environ. 2015, 161, 63-77. [CrossRef]

23. Zeng, H.; Jia, G.; Epstein, H. Recent changes in phenology over the northern high latitudes detected from multi-satellite data. Environ. Res. Lett. 2011, 6, 045508. [CrossRef]

24. Chen, X. East Asia. In Phenology: An Integrative Environmental Science, 2nd ed.; Schwartz, M.D., Ed.; Springer: Dordrecht, The Netherlands, 2013; pp. 9-22.

25. Shen, M.G.; Zhang, G.X.; Cong, N.; Wang, S.P.; Kong, W.D.; Piao, S.L. Increasing altitudinal gradient of spring vegetation phenology during the last decade on the Qinghai-Tibetan Plateau. Agric. For. Meteorol. 2014, 189-190, 71-80. [CrossRef]

26. Chen, J.; Jonsson, P.; Tamura, M.; Gu, Z.H.; Matsushita, B.; Eklundh, L. A simple method for reconstructing a high-quality NDVI time-series data set based on the Savitzky-Golay filter. Remote Sens. Environ. 2004, 91, 332-344. [CrossRef]

27. Ding, M.; Zhang, Y.; Sun, X.; Liu, L.; Wang, Z.; Bai, W. Spatiotemporal variation in alpine grassland phenology in the Qinghai-Tibetan Plateau from 1999 to 2009. Chin. Sci. Bull. 2013, 58, 396-405. [CrossRef]

28. White, M.A.; Beurs, D.; Kirsten, M.; Didan, K.; Inouye, D.W.; Richardson, A.D.; Jensen, O.P.; O’Keefe, J.; Zhang, G.; Nemani, R.R.; et al. Intercomparison, interpretation, and assessment of spring phenology in North America estimated from remote sensing for 1982-2006. Glob. Chang. Biol. 2009, 15, 2335-2359. [CrossRef]

29. Zhang, X.Y.; Friedl, M.A.; Schaaf, C.B.; Strahler, A.H.; Hodges, J.; Gao, F.; Reed, B.C.; Huete, A. Monitoring vegetation phenology using MODIS. Remote Sens. Environ. 2003, 84, 471-475. [CrossRef]

30. White, M.A.; Thornton, P.E.; Running, S.W. A continental phenology model for monitoring vegetation responses to interannual climatic variability. Glob. Biogeochem. Cycles 1997, 11, 217-234. [CrossRef]

31. Studer, S.; Stoeckli, R.; Appenzeller, C.; Vidale, P.L. A comparative study of satellite and ground-based phenology. Int. J. Biometeorol. 2007, 51, 405-414. [CrossRef] [PubMed]

32. Luo, X.; Chen, X.; Xu, L.; Myneni, R.; Zhu, Z. Assessing performance of NDVI and NDVI3g in monitoring leaf unfolding dates of the deciduous broadleaf forest in northern china. Remote Sens. 2013, 5, 845-861. [CrossRef]

33. Ding, M.; Li, L.; Zhang, Y.; Sun, X.; Liu, L.; Gao, J.; Wang, Z.; Li, Y. Start of vegetation growing season on the Tibetan Plateau inferred from multiple methods based on GIMMS and SPOT NDVI data. J. Geogr. Sci. 2015, 25, 131-148. [CrossRef]

34. Cong, N.; Piao, S.L.; Chen, A.P.; Wang, X.H.; Lin, X.; Chen, S.P.; Han, S.J.; Zhou, G.S.; Zhang, X.P. Spring vegetation green-up date in China inferred from SPOT NDVI data: A multiple model analysis. Agric. For. Meteorol. 2012, 165, 104-113. [CrossRef]

35. Chang, Q.; Zhang, J.; Jiao, W.; Yao, F. A comparative analysis of the NDVIg and NDVI3g in monitoring vegetation phenology changes in the Northern Hemisphere. Geocarto Int. 2016, 33, 1-20. [CrossRef]

36. Yu, H.Y.; Luedeling, E.; Xu, J.C. Winter and spring warming result in delayed spring phenology on the Tibetan Plateau. Proc. Natl. Acad. Sci. USA 2010, 107, 22151-22156. [CrossRef] [PubMed]

37. Jeong, S.; Schimel, D.; Frankenberg, C.; Drewry, D.T.; Fisher, J.B.; Verma, M.; Berry, J.A.; Lee, J.; Joiner, J. Application of satellite solar-induced chlorophyll fluorescence to understanding large-scale variations in vegetation phenology and function over northern high latitude forests. Remote Sens. Environ. 2017, 190, 178-187. [CrossRef]

38. Wu, C.; Peng, D.; Soudani, K.; Siebicke, L.; Gough, C.M.; Arain, M.A.; Bohrer, G.; Lafleur, P.M.; Peichl, M.; Gonsamo, A.; et al. Land surface phenology derived from normalized difference vegetation index (NDVI) at global FLUXNET sites. Agric. For. Meteorol. 2017, 233, 171-182. [CrossRef] 
39. Liu, Y.; Wu, C.; Peng, D.; Xu, S.; Gonsamo, A.; Jassal, R.S.; Arain, M.A.; Lu, L.; Fang, B.; Chen, J.M. Improved modeling of land surface phenology using MODIS land surface reflectance and temperature at evergreen needleleaf forests of central North America. Remote Sens. Environ. 2016, 176, 152-162. [CrossRef]

40. Peng, D.; Zhang, X.; Wu, C.; Huang, W.; Gonsamo, A.; Huete, A.R.; Didan, K.; Tan, B.; Liu, X.; Zhang, B. Intercomparison and evaluation of spring phenology products using National Phenology Network and AmeriFlux observations in the contiguous United States. Agric. For. Meteorol. 2017, 242, 33-46. [CrossRef]

41. Badeck, F.W.; Bondeau, A.; Bottcher, K.; Doktor, D.; Lucht, W.; Schaber, J.; Sitch, S. Responses of spring phenology to climate change. New Phytol. 2004, 162, 295-309. [CrossRef]

42. Xu, H.; Twine, T.E.; Yang, X. Evaluating remotely sensed phenological metrics in a dynamic ecosystem model. Remote Sens. 2014, 6, 4660-4686. [CrossRef]

43. Gallinat, A.S.; Primack, R.B.; Wagner, D.L. Autumn, the neglected season in climate change research. Trends Ecol. Evol. 2015, 30, 169-176. [CrossRef] [PubMed]

44. Migliavacca, M.; Galvagno, M.; Cremonese, E.; Rossini, M.; Meroni, M.; Sonnentag, O.; Cogliati, S.; Manca, G.; Diotri, F.; Busetto, L.; et al. Using digital repeat photography and eddy covariance data to model grassland phenology and photosynthetic $\mathrm{CO}_{2}$ uptake. Agric. For. Meteorol. 2011, 151, 1325-1337. [CrossRef]

45. Richardson, A.D.; Hollinger, D.Y.; Dail, D.B.; Lee, J.T.; Munger, J.W.; O'Keefe, J. Influence of spring phenology on seasonal and annual carbon balance in two contrasting New England forests. Tree Physiol. 2009, 29, 321-331. [CrossRef] [PubMed]

46. Sonnentag, O.; Hufkens, K.; Teshera-Sterne, C.; Young, A.M.; Friedl, M.; Braswell, B.H.; Milliman, T.; O'Keefe, J.; Richardson, A.D. Digital repeat photography for phenological research in forest ecosystems. Agric. For. Meteorol. 2012, 152, 159-177. [CrossRef]

47. Zhang, X.; Friedl, M.A.; Schaaf, C.B. Sensitivity of vegetation phenology detection to the temporal resolution of satellite data. Int. J. Remote Sens. 2009, 30, 2061-2074. [CrossRef]

48. Kross, A.; Fernandes, R.; Seaquist, J.; Beaubien, E. The effect of the temporal resolution of NDVI data on season onset dates and trends across Canadian broadleaf forests. Remote Sens. Environ. 2011, 115, 1564-1575. [CrossRef]

49. Klosterman, S.T.; Hufkens, K.; Gray, J.M.; Melaas, E.; Sonnentag, O.; Lavine, I.; Mitchell, L.; Norman, R.; Friedl, M.A.; Richardson, A.D. Evaluating remote sensing of deciduous forest phenology at multiple spatial scales using PhenoCam imagery. Biogeosciences 2014, 11, 4305-4320. [CrossRef]

(C) 2017 by the authors. Licensee MDPI, Basel, Switzerland. This article is an open access article distributed under the terms and conditions of the Creative Commons Attribution (CC BY) license (http:/ / creativecommons.org/licenses/by/4.0/). 
Article

\title{
Developing an Integrated Remote Sensing Based Biodiversity Index for Predicting Animal Species Richness
}

\author{
Jinhui $\mathrm{Wu}^{1}$ and Shunlin Liang ${ }^{1,2, *}$ \\ 1 State Key Laboratory of Remote Sensing Science, Faculty of Geography Science, Beijing Normal University, \\ Beijing 100875, China; jwu@mail.bnu.edu.cn \\ 2 Department of Geographical Sciences, University of Maryland, College Park, MD 20742, USA \\ * Correspondence: sliang@umd.edu; Tel.: +86-10-5880-9071; Fax: +86-10-5880-3002
}

Received: 2 March 2018; Accepted: 5 May 2018; Published: 10 May 2018

\begin{abstract}
Many remote sensing metrics have been applied in large-scale animal species monitoring and conservation. However, the capabilities of these metrics have not been well compared and assessed. In this study, we investigated the correlation of 21 remote sensing metrics in three categories with the global species richness of three different animal classes using several statistical methods. As a result, we developed a new index by integrating several highly correlated metrics. Of the 21 remote sensing metrics analyzed, evapotranspiration (ET) had the greatest impact on species richness on a global scale (explained variance: $52 \%$ ). The metrics with a high explained variance on the global scale were mainly in the energy/productivity category. The metrics in the texture category exhibited higher correlation with species richness at regional scales. We found that radiance and temperature had a larger impact on the distribution of bird richness, compared to their impacts on the distributions of both amphibians and mammals. Three machine learning models (i.e., support vector machine, random forests, and neural networks) were evaluated for metric integration, and the random forest model showed the best performance. Our newly developed index exhibited a 0.7 explained variance for the three animal classes' species richness on a global scale, with an explained variance that was $20 \%$ higher than any of the univariate metrics.
\end{abstract}

Keywords: biodiversity; remote sensing; species richness; metric comparison; metric integration

\section{Introduction}

Changes and losses in global biodiversity have been rapidly accelerating in recent years [1-6]. Understanding of both current patterns and change tendency is a key concern for scientists, ecologists, and policy-makers. Biodiversity has a multitude of facets that can be quantified, and the most commonly considered one is species richness $[7,8]$. Species richness is the number of species in a site, habitat, or clade [1,9]. Much research has focused on the distribution of animal species richness [10-15]. In addition, a growing amount of data infrastructure has been constructed for continental-to-global scale species monitoring and analysis $[16,17]$. The Global Biodiversity Information Facility (GBIF) and the International Union for Conservation of Nature (IUCN) provides access to millions of current global digitized species records [18-20]. However, the scale limitation of in situ data has hindered large-scale species monitoring. The collection of in situ data is too costly to be applied in long time-series species monitoring.

Remote sensing is a powerful tool in global biodiversity assessment because it enables consistent observations of species across time and space, as well as the tracking of climatic change and other drivers of species change [4]. Based on remote sensing technology, two common methods are used for the observation of species richness: direct species monitoring and indirect species monitoring. In direct species monitoring, the high spatial and spectral resolution of remote sensing data enables individual 
species to be distinguished from nearby pixels, and the associations of single species can be evaluated based on the individual species [21,22]. However, this method requires extremely high spatial and spectral resolution remote sensing data, which are generally not available to the general public. Considering the distribution differences between different species or classes, a comprehensive biodiversity assessment on a global scale is too costly to achieve by using direct species monitoring $[10,11,23,24]$. To increase the monitoring scale and evaluate more species or classes, indirect species monitoring mainly maps the key environmental factors which relate to species distribution using remote sensing data $[25,26]$. Compared with direct species monitoring, indirect species monitoring shows less requirement for high spatial and spectral resolution of remote sensing data, and can thus facilitate continuous large-scale species monitoring.

Previous studies have found that a variety of factors impact the distribution of animal species, mainly in four categories: energy/productivity [27-29]; climate [11,30-32]; ecosystem texture [33,34]; and evolutionary history [24]. Energy/productivity, climate, and ecosystem texture can be continuously monitored using remote sensing data. The species-energy hypothesis predicts that more species and higher abundances of individual species will occur where more energy in the form of food is consistently available [35-38]. Climate change can lead to systematic changes of species distribution [39,40]. Ecosystem texture determines the physical structure of the environment, and therefore, has a considerable influence on the distributions and interactions of animal species [41-43]. Based on these theories and hypotheses, recent studies have developed several frameworks, such as Essential Biodiversity Variables (EBVs) and Remote Sensing Essential Biodiversity Variables (RS-EBVs), which have been used to construct a global species-observing system $[2,44]$. Meanwhile, many new remote sensing metrics have been developed to monitor species on a global scale $[25,26,45-48]$. The most outstanding feature of these metrics is that they can be generated on a global scale. Assessment of different remote sensing metrics on a global scale and the development of a multivariate integration index is essential for global biodiversity assessment, but such efforts are missing from the literature. Available larger-scale species richness datasets offer a valuable database for the assessment of these remote sensing metrics. Instituto de Pesquisas Ecológicas (Brasil) offers a suite of large-scale species richness data [49], which has been validated in previous studies and exhibits high accuracy $[50,51]$.

Machine learning provides a great opportunity for the study of species richness, while construction of mechanistic models between species richness and remote sensing metrics is still challenging $[52,53]$. Compared with traditional mechanistic approaches, machine learning avoids the over-simplification during modelling. Machine learning models are as complex as real ecosystems, therefore, in most cases the results that come from machine learning are more valid for drawing any conclusions for real situations [54,55]. Moreover, machine learning has been widely used for species assessment and prediction [56-58].

In this study, to assess remote sensing metrics on a global scale, we selected in situ species richness data on three animal classes (mammals, birds, and amphibians) and 21 remote sensing metrics that all had global coverage and long-term availability (Table 1). We analyzed the correlations between animal species richness and remote sensing metrics, and developed a multivariate integration index based on a machine learning model. This study aimed to address the following questions:

(1) What are the differences between the distributions of various animal classes (mammals, birds, and amphibians) on a global scale?

(2) What are the correlations between remote sensing metrics and species richness distributions on a global scale?

(3) Given the major driving metrics, can we develop a multivariate integration index to map the global species richness continuously? 
Table 1. Remote sensing metrics.

\begin{tabular}{ccccc}
\hline \multirow{2}{*}{ Category } & Metrics & $\begin{array}{c}\text { Spatial } \\
\text { Resolution }\end{array}$ & $\begin{array}{c}\text { Temporal } \\
\text { Resolution }\end{array}$ & $\begin{array}{c}\text { Temporal } \\
\text { Coverage }\end{array}$ \\
\hline \multirow{5}{*}{ Energy/productivity } & Dynamic habitat index (DHI)-cum & $1 / 12^{\circ}$ & yearly & $2000-2011$ \\
& Dynamic habitat index (DHI)-min & $1 / 12^{\circ}$ & yearly & $2000-2011$ \\
& Dynamic habitat index (DHI)-sea & $1 / 12^{\circ}$ & yearly & $2000-2011$ \\
& Gross primary production (GPP) & $0.05^{\circ}$ & monthly & $2001-2015$ \\
& Potential Evapotranspiration (PET) & $0.05^{\circ}$ & monthly & $2001-2014$ \\
Climate & Land surface temperature (LST) & $0.05^{\circ}$ & monthly & $2000-2011$ \\
& Evapotranspiration (ET) & $0.05^{\circ}$ & monthly & $2000-2014$ \\
& Coefficient of variation (CV) & $1 \mathrm{Km}$ & 5 -year & $2001-2005$ \\
& Evenness & $1 \mathrm{Km}$ & 5 -year & $2001-2005$ \\
Contrast (CON) & $1 \mathrm{Km}$ & 5 -year & $2001-2005$ \\
& Dissimilarity (DIS) & $1 \mathrm{Km}$ & 5 -year & $2001-2005$ \\
& Entropy (ENT) & $1 \mathrm{Km}$ & 5 -year & $2001-2005$ \\
& Homogeneity (HOM) & $1 \mathrm{Km}$ & 5 -year & $2001-2005$ \\
& Range & $1 \mathrm{Km}$ & 5 -year & $2001-2005$ \\
& Shannon & $1 \mathrm{Km}$ & 5 -year & $2001-2005$ \\
& Simpson & $1 \mathrm{Km}$ & 5 -year & $2001-2005$ \\
& Standard deviation (SD) & 5-year & $2001-2005$ \\
& Correlation (COR) & $1 \mathrm{Km}$ & 5 -year & $2001-2005$ \\
& Maximum (MAX) & $1 \mathrm{Km}$ & 5 -year & $2001-2005$ \\
& Uniformity (UNI) & 5-year & $2001-2005$ \\
& Variance (VAR) & 5-year & $2001-2005$ \\
\hline
\end{tabular}

\section{Data and Methods}

\subsection{Data}

\subsubsection{Species Richness Data}

Considering coverage and taxonomy, we chose to use the species richness dataset from the Instituto de Pesquisas Ecológicas (Brasil) in this study. This dataset (grid format) consists of global total mammal richness, global total bird richness, and global total amphibian richness. In this dataset, the primary species range map data used to create the species richness maps are from the IUCN for mammal and amphibian species, and jointly from BirdLife International and NatureServe for bird species. During the data process, extinct species were removed, as were non-native distributions of extant species. For each grid cell, any species that overlapped any part of the cell were counted as a presence of that species. All species richness data exhibited a spatial resolution of $10 \mathrm{~km}$ and used the equal-area projection $[3,49,50,59]$.

\subsubsection{Remote Sensing Data}

Considering the availability of datasets, three categories of remote sensing metrics were collected: energy/productivity metrics, climate metrics, and ecosystem texture metrics. Because the species richness data was recorded and updated for decades, we selected the remote sensing data as long-term as possible. In addition, all remote sensing data had global coverage.

For the energy/productivity category, we selected gross primary production (GPP), dynamic habitat index (DHI), and potential evapotranspiration (PET). The GPP product was obtained from the global monthly MOD17A2 GPP product [60]. The DHI, including Cumulative Annual Productivity (DHI-cum), Minimum Annual Apparent Cover (DHI-min), and Seasonal Variation of Greenness (DHI-sea), is a composite vector deduced from the Fraction of Absorbed Photosynthetically Active Radiation (FAPAR) time-series, representing the vegetation dynamics. The monthly maximum FAPAR-value is the basic input dataset to compute the three relevant annual indices for the subsequent habitat analysis [51,61]. DHI-cum provides an indication of overall site vegetation productivity. DHI-min represents the lowest (minimum value) level of vegetative productivity in a year. DHI-sea refers to the variation of the vegetative productivity. Further details of the DHI can be found in previous publications [51,61-63]. In this study, DHI was calculated from the Global Inventory Modelling and 
Mapping Studies (GIMMS) AVHRR-FAPAR 3g dataset. The PET product was obtained from the MOD16A2 product. In our study, PET was taken as an energy/productivity metric, because it characterizes the features of the surface temperature and radiance.

For the climate category, we selected evapotranspiration (ET) and land surface temperature (LST). The ET product was obtained from the global monthly MOD16A2 ET product [64-68]. The LST product used in this study was obtained from the global monthly MOD11C3 LST product, which belonged to the temperature/surface emissivity global data sets (C5) [69].

For the texture category, we selected a suite of global terrestrial habitat heterogeneity data, which was developed by Haralick et al. and computed by Tuanmu and Jetz using the Moderate Resolution Imaging Spectroradiometer (MODIS) Enhanced Vegetation Index (EVI) product [25,70]. Heterogeneity is an important indicator of ecosystem texture, which has long been recognized as a key landscape characteristic with strong relevance for biodiversity [71,72]. These heterogeneity metrics consist of the coefficient of variation (CV), evenness, contrast (CON), dissimilarity (DIS), entropy (ENT), homogeneity (HOM), range, Shannon, Simpson, standard deviation (SD), correlation (COR), maximum (MAX), uniformity (UNI), and variance (VAR).

\subsubsection{Biome Data}

Biome data acquired from World Wildlife Fund (WWF) were used for this study. This data depicted the 14 terrestrial biomes of the globe, which include eight forest biomes, four grassland biomes, one tundra biome, and one desert biome [73]. Biomes are relatively large units of land containing distinct assemblages of natural communities and species, with boundaries that approximate the original extent of natural communities prior to major land-use change [74]. This comprehensive, global data provide a useful tool for identifying areas of outstanding biodiversity and conservation priority.

\subsection{Methods}

The overall procedure for the proposed method is briefly illustrated in Figure 1. Comparison of the remote sensing metrics and development of the multivariate integration index were achieved through four tasks.

\subsubsection{Data Integration and Standardization}

In data integration, to enable all data to have the same resolution, long time-series LST, ET, GPP, PET, DHI-cum, DHI-min, and DHI-sea data were averaged on time-scales. Mammal richness, bird richness, and amphibian richness were added together to generate a variate "Allclasses" as the surrogate for all three animal classes.

In data standardization, remote sensing metrics were normalized (0-1) using the feature scaling method, because absolute values had large differences in magnitude. All species richness data and remote sensing metrics were summed to a $0.1^{\circ}$ spatial resolution separately using the nearest-neighbor interpolation method, because the lowest spatial resolution of the datasets was $10 \mathrm{~km}$ (species richness data). In order to remove the impact of non-value area, we excluded the areas which had non-value pixels of species richness data or remote sensing metrics. 


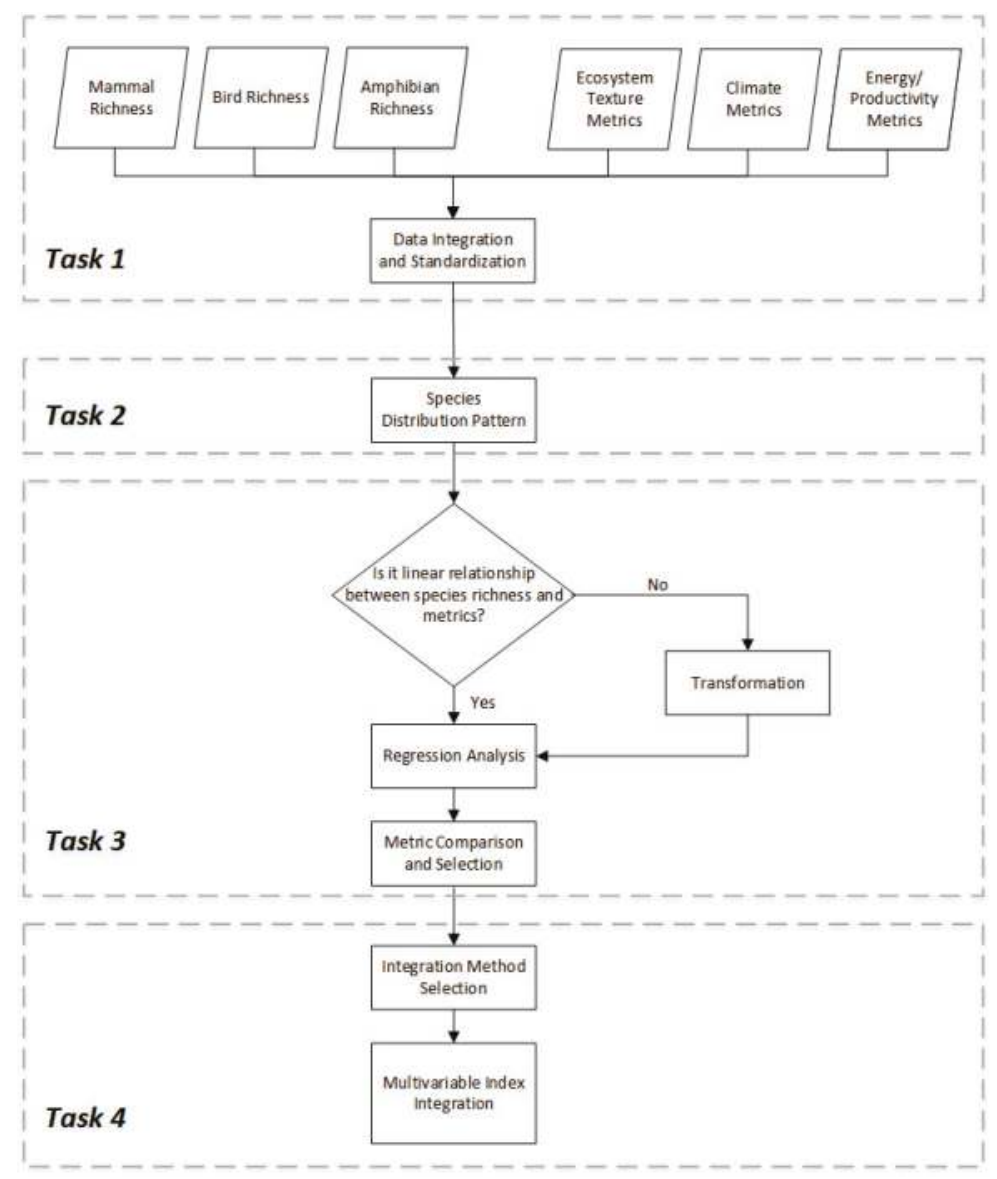

Figure 1. Flowchart of the comparison of the remote sensing metrics and development of a multivariate integration index.

\subsubsection{Regression Analysis}

Before the regression, we used the function randomu in Interface Description Language (IDL) to extract 10,000 pixels randomly from the global terrestrial areas of species richness data and each remote sensing metric. The univariate linear regression model was used to evaluate the correlation of these metrics with animal species richness. Since the relationships between species richness and some metrics were nonlinear, we used locally weighted regression (LOESS) to determine the pattern of relationships $[75,76]$. We took a logarithmic transform for $\mathrm{COR}, \mathrm{CV}$, and DHI-sea, and used polynomial regression for evenness, CON, DIS, ENT, HOM, range, Shannon, Simpson, SD, MAX, UNI, VAR, LST, ET, GPP, and PET. 


\subsubsection{The Akaike Information Criterion}

The Akaike information criterion (AIC) was used to evaluate the model quality. The AIC is a measure of the quality of each model, relative to each of the other models for a given set of data [77-79], and the AIC value of the model can be expressed as:

$$
A I C=-\ln L+2 c
$$

where $L$ is the maximum value of the likelihood function for the model, and $c$ is the number of free parameters in the model. The model with the smallest AIC is the best performance. Thus, the good performance of different models in this study was normally based on the low AIC values.

\subsubsection{Three Machine Learning Methods for Metrics Integration}

After determining the relationships between each remote sensing metric and species richness, we selected the high-explained-variance metrics to develop a multivariate integration index using machine learning models. In this study, we compared the capability of support vector machine (SVM), random forests $(\mathrm{RF})$, and neural networks (NN) to integrate various metrics. Species richness was trained on $50 \%$ of the randomly extracted pixels and cross-validated on the remaining $50 \%$. The same number of pixels was used for training and predicting in order to avoid under- or over-fitting due to an unbalanced dataset [80].

Support vector machine (SVM) is a supervised machine learning model with associated learning algorithms that analyze data used for classification and regression analysis [81]. SVM uses kernel functions implicitly to map training data into a higher-dimensional feature space. The maximum separation hyperplane is defined by a set of support vectors, which are a function of the training data that lie on/the closest to the separating margin [82]. In this study, the $e 1071$ library of the R statistical package was utilized to optimize the SVM parameters [83].

Random forests (RF) is a regression model that grows an ensemble of trees [84,85]. Each tree casts a unit vote for the most popular class according to the input variables. The growing process of RF is by bootstrap aggregating (or bagging), where a tree is randomly grown from the dataset, and this process can provide substantial gains in the accuracy of predicting models [86,87], and it requires no pruning. In this study, we used the RF algorithm implemented in the randomForest $\mathrm{R}$ package, with the parameter values for the algorithm, (i.e., number of trees to grow (ntree)) equaled to 500, and the number of descriptors randomly sampled at each split (mtry) equaled to the total number of descriptors in the dataset divided by three.

Neural networks (NN) is a non-linear statistical data modelling tool used for prediction and regression. We selected the multilayer feed-forward neural network, which is one of the most popular approaches to neural networks (NNs) [88-90]. The multilayer feed-forward NN is a backpropagation network that trains the data using a backpropagation algorithm $[84,91]$. This network comprises three layers: an input layer, one or more hidden layers, and an output layer. Neurons from one layer are in one direction linked to all neurons in the subsequent layers [92]. This study used the nnet library provided by the R package in order to develop the model [36]. The nnet package is the library for establishing multiple feed-forward NNs with more than one hidden layer [93]. This study constructed the model by carrying out the learning for a total of 30 times with the maximum number of iterations at 100 .

\section{Results}

\subsection{Richness Distribution of Three Animal Classes}

The distribution of mammal, bird, and amphibian species richness (normalized) was mapped on a global scale. Mammals, birds, and amphibians showed similar distribution patterns at low latitudes (Figure 2). In the Amazon, the species richness patterns of mammals, birds, and amphibians were almost equal. Distribution differences between mammals, birds, and amphibians were observed at 
mid-high latitudes. The species richness of birds and mammals was slightly higher than that of amphibians in both Central Africa and Southeast Asia. Overall, the distribution patterns of species richness for these three animal classes were not the same.
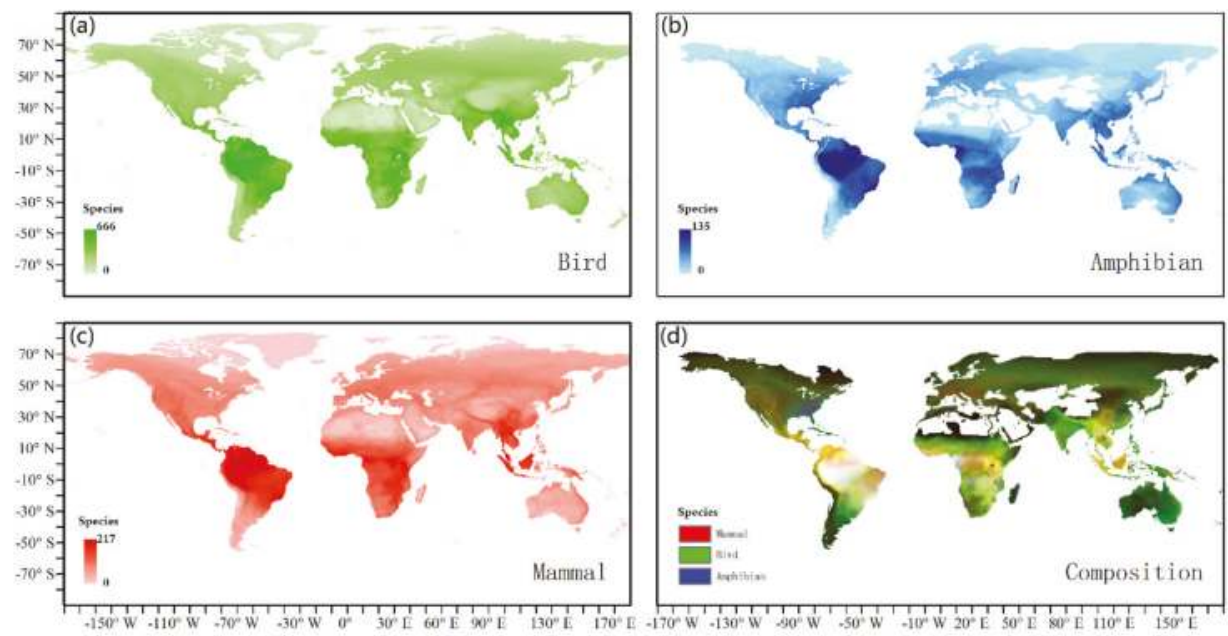

Figure 2. Geographic distribution of three animal classes. (a) bird richness, (b) amphibian richness, (c) mammal richness, and (d) combined species richness where we assigned mammal richness to the red band, bird richness to the green band, and amphibian richness to the blue band of the image. All other colors show transition zones of mixtures of the different animal classes.

\subsection{Characterization of Various Remote Sensing Metrics}

The scatter plots of various metrics versus species richness showed that most metrics exhibited nonlinear relationships with species richness, and one metric possessed the same type of relationship with various animal classes (Figure S1). DHI-cum and DHI-min showed clear linear positive correlations with species richness. Among the metrics which exhibited nonlinear relationships with species richness, COR, CV, and DHI-sea followed power laws, while the other metrics showed mildly or strongly unimodal relationships (Figure S2).

DHI-min, DHI-cum, ET, and GPP exhibited more than $40 \%$ explained variance with the distribution of the sum richness of three animal classes on a global scale (Figure 3). These metrics with high explained variance were mainly in the energy/productivity category and the climate category, while the texture category showed weak explanatory power with the distribution of sum richness on a global scale. Among the metrics in the energy/productivity category, DHI-min explained $50 \%$ of the sum richness distribution, GPP explained $48 \%$, and DHI-cum explained $46 \%$. DHI-sea exhibited lower than $10 \%$ explained variance of DHI-min. The low explained variance of DHI-sea in the southern hemisphere drew down its explained variance on a global scale (Figure 4). For DHI, we detected three dramatic declines of explained variance in $40^{\circ} \mathrm{N}$ zones, $10^{\circ} \mathrm{S}$ zones, and $40^{\circ} \mathrm{S}$ zones (Figure 5). In climate category, ET explained $51 \%$ of the sum richness of the three animal classes global distribution, and LST explained $16 \%$. We further observed a large data gap between PET and ET, and PET exhibited lower than $40 \%$ explained variance of ET, which indicated that vegetation played an important role in the distribution of animal species richness. The vegetation contributed less to the PET calculation compared to the ET calculation, which might be the primary cause of the difference between PET and ET in the explanation of species richness. Compared with the metrics in the energy/productivity and the climate categories, almost all texture metrics showed low explained variance. Among the 
texture category, CV exhibited the highest explained variance (22\%), while COR and evenness showed negligible explained variance.

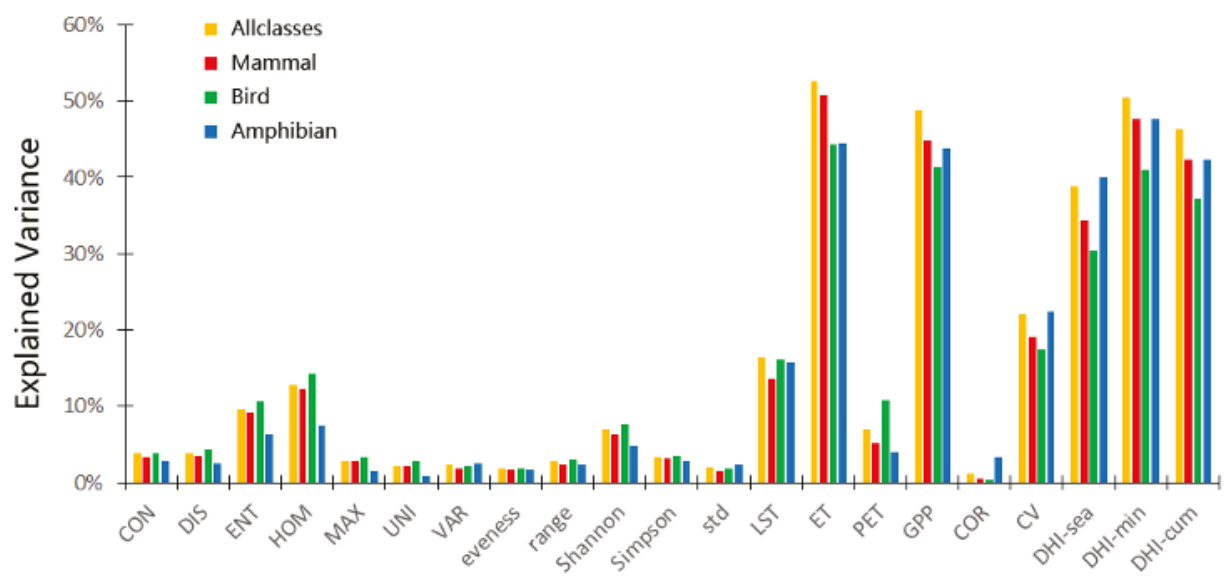

Figure 3. The explained variance of remote sensing metrics on species richness. Percentages of variance explained by the regression model was built with different remote sensing metrics. For DHI-min and $\mathrm{DHI}$-cum, univariate linear regression was used to calculate the explained variance. For COR, CV, and DHI-sea, linearization was achieved by taking the log of the data before using univariate linear regression to calculate the explained variance. For the other metrics, polynomial regression was used to calculate the explained variance. Allclasses is the sum of mammal richness, bird richness, and amphibian richness, which was normalized. The measured values were obtained for 10,000 0.1-degree pixels randomly selected from global terrestrial areas. The full names of abbreviations can be found in the Table 1 .

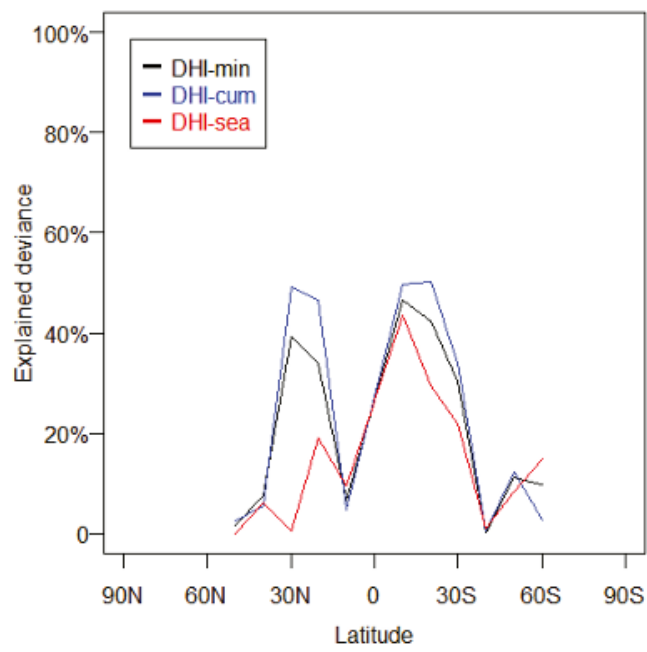

Figure 4. The explained variance of DHI metrics on mammal richness at latitudinal zones $\left(360^{\circ} \times 10^{\circ}\right)$. For DHI-min and DHI-cum, univariate linear regression was used to calculate the explained variance. For DHI-sea, linearization was achieved by taking the log of the data before using univariate linear regression to calculate the explained variance. The measured values were obtained for 10,000 0.1-degree pixels randomly selected from global terrestrial areas. 
(a)
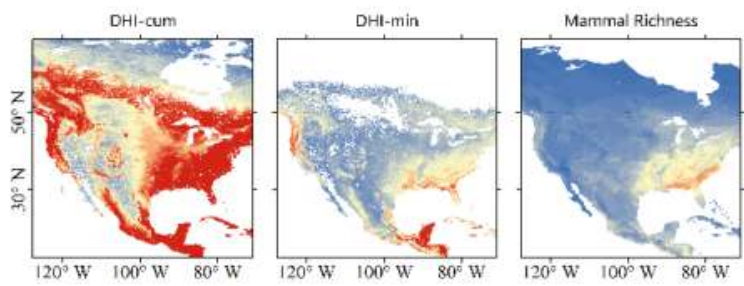

(b)
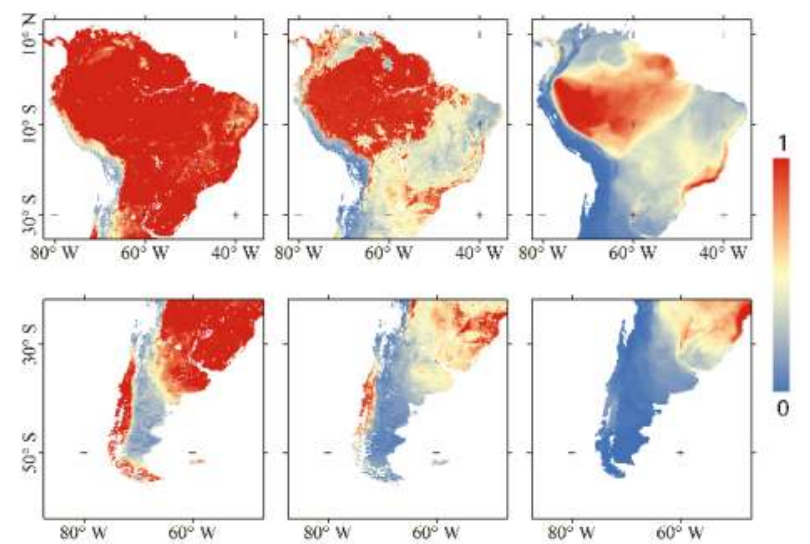

Figure 5. Geographic distribution of DHI-cum, DHI-min, mammal richness. (a) North America, (b) Argentina and Chile, (c) Amazon.

Some differences were observed when we examined the relationship between remote sensing metrics and individual animal class (Figure 3). Compared with mammals and amphibians, birds exhibited higher explained variance with PET, and exhibited lower explained variance with ET. Birds showed lower sensitivity than amphibians and mammals to the energy / productivity category metrics (e.g., GPP, DHI-sea, DHI-min, and DHI-cum). Mammals were more sensitive to ET, with an explained variance that was $6 \%$ higher than that of birds and amphibians. For ENT and HOM, the explained variance of amphibians was lower than that of mammals and birds.

\subsection{Integration of Various Remote Sensing Metrics}

Based on the characterization of various metrics and AIC values, DHI-min, DHI-cum, DHI-sea, GPP, CV, ET, and LST were selected as the major metrics for the development of an integrated biodiversity index. For the AIC values, the RF model gave the lowest AIC for species richness of the three animal classes when compared to SVM and NN (Figure 6). The species richness data of each animal class predicted by the same machine learning model were quite similar, and RF exhibited a higher r-square value than SVM and NN in species richness of all three animal classes (Figure 7). The r-square values of mammals, birds, and amphibians were $0.76,0.76,0.77$, respectively, according to the RF model; $0.7,0.71$, and 0.66 , respectively, according to the SVM model; and $0.67,0.67$, and 0.67 , respectively, according to the NN model. In terms of the root mean squared error (RMSE), the prediction of the RF model exhibited a lower RMSE than the other two models for all three animal classes. Overall, the RF model outperformed the other two models for species richness prediction. We used the RF model to integrate the seven metrics and develop a multivariate integration index.

The multivariate integration index exhibited a higher explained variance than the univariate metrics, and exhibited more than $76 \%$ explained variance for the species richness of the three animal classes on a global scale. For a single animal class, the gap between the multivariate integration 
index and the univariate metrics was more than 30\% (Figures 3 and 7). On the whole, the simulated species richness showed a consistent spatial pattern with in situ richness (Figure 8). At regional scales, the simulated species richness was in good agreement with in situ species richness in most regions. However, the differences between simulated species richness and in situ species richness were observed. For mammals, simulated species richness was higher than in situ species richness in $40^{\circ}-60^{\circ} \mathrm{S}$ zones and $120^{\circ}-140^{\circ} \mathrm{E}$, and simulated species richness was lower than in situ species richness in $0^{\circ}-10^{\circ} \mathrm{N}$ and $30^{\circ}-60^{\circ} \mathrm{W}$. For amphibians, simulated species richness was lower than in situ species richness in $30^{\circ}-60^{\circ} \mathrm{W}$. Although disagreements between simulated species richness and in situ species richness were found in some regions, the differences were relatively small in magnitude.

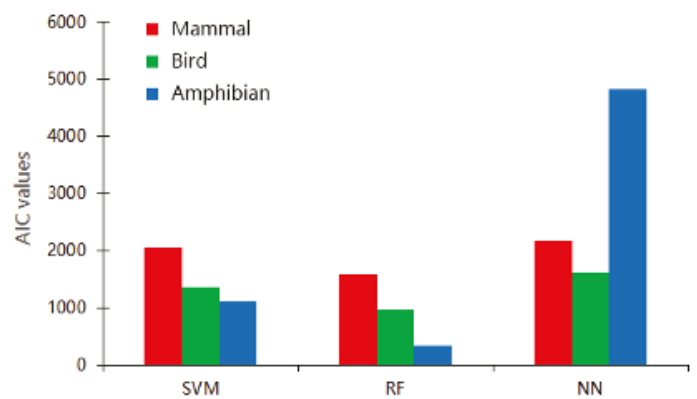

Figure 6. The Akaike information criterion (AIC) values of the three models.
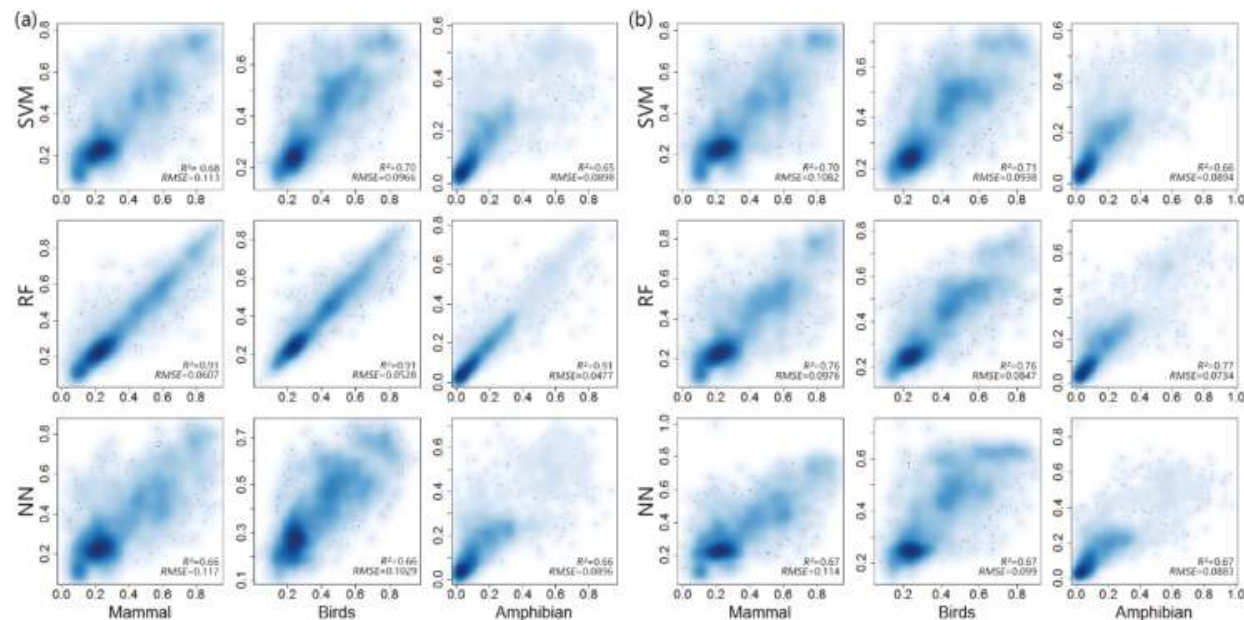

Figure 7. The correlation between predicted species richness and in situ species richness. (a) training dataset, (b) prediction dataset. The measured values were obtained for 10,000 0.1-degree pixels randomly selected from terrestrial areas of the globe, and 5000 pixels were randomly selected for training and prediction separately. 

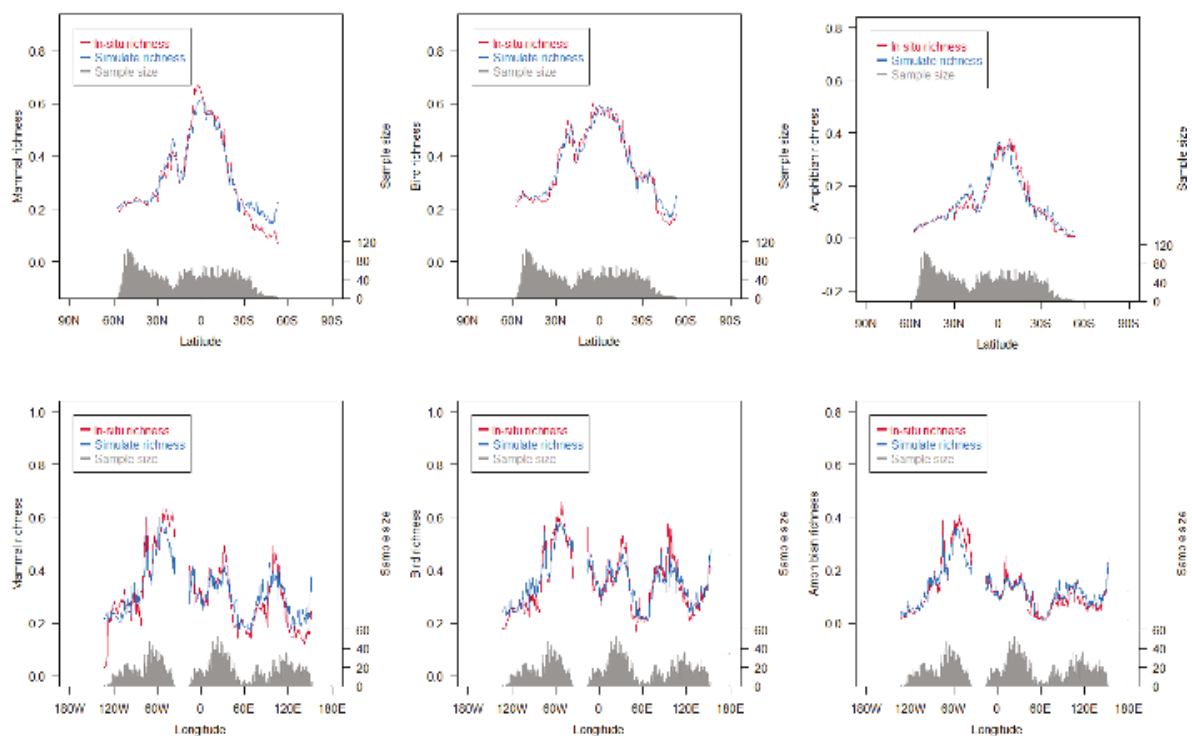

Figure 8. Distribution of simulated species richness and in situ species richness at latitudinal/longitudinal zones. The data are from a prediction dataset, which has 5000 samples.

\section{Discussion}

\subsection{Differences in Distribution of the Three Animal Classes}

Previous studies have found large mismatches between the priorities of biodiversity protection and protected lands in the United States, even though many biodiversity protected areas have been established since biodiversity loss was identified $[6,50,94,95]$. Beside the lack of biodiversity in situ datasets, this mismatch has likely arisen from differences in the distribution of various animal classes. Protected areas are generally established for the protection of specific species, while biodiversity includes many different taxa. In the United States, mammal richness was found to be very high in the west, and bird richness was high in coastal regions [49,50]. A similar result was also found in our comparison, in which we examined the distribution of mammals, birds, and amphibians, based on the latest global in situ data. In addition, the differences in the distribution of mammals, birds, and amphibians were mainly observed at mid-high latitudes. This is probably because the limitation of water or energy/productivity at mid-high latitudes leads the difference in species richness among the three animal classes [96]. In this study, biodiversity metrics did not exhibit a large gap in explained variance between various animal classes, although the global distribution of various animal classes were different. This may be due to the offset of regional differences, which led to a similar statistical result on a global scale.

\subsection{Attribution of Differences between Remote Sensing Metrics}

For energy/productivity category, a large number of studies have attempted to discover the relationship between energy/productivity and species richness in past decades $[29,97,98]$. The species-energy theory proposes that the supply of useable energy in the environment is very important to species richness $[96,99]$. Although this theory is based on studies which are mainly focused on the richness of plant species, for animals, there is a less dramatic shift in the relationship between productivity and species richness [100]. Animal richness is limited primarily by the production of plants at the base of the global food web $[35,99]$. That is probably the reason that the high explained 
variance metrics were mainly found in the energy/productivity category among the remote sensing metrics in this study. The relationship between energy/productivity metrics and the richness of animal species exhibits several patterns, namely linear pattern, unimodal pattern, exponential pattern, or no pattern at all [100-102]. Past researchers also found this relationship differs in different scales [100]. Our study showed, on a global scale, that DHI-min and DHI-cum exhibited a linear relationship with richness of the three animal classes; DHI-sea exhibited an exponential relationship with richness of the three animal classes; and GPP exhibited an unimodal relationship with richness of the three animal classes. In our study, DHI-sea exhibited a low explained variance of $38.9 \%$, which was different to DHI-min and DHI-cum. Species richness may be reduced through a decrease in either cumulative annual productivity or minimum annual productivity. However, the impact of DHI-sea on species richness can be different to the impacts of DHI-min and DHI-cum. The influence of annual variation on productivity will be limited when the cumulative annual productivity or minimum annual productivity is constant. Since the impact of short-term low productivity can be offset by subsequent high productivity, it has a less immediate impact than DHI-min and DHI-cum. That is probably why we observed a smaller impact of DHI-sea. Overall, DHI-min, DHI-cum, and GPP should be selected as main factors for predicting animal species richness on a global scale.

For climate category, past investigations showed that global patterns of species richness were widely correlated with climate [24,103]. Climate change can influence the animal distribution directly by itself, and also affects animal species richness through changing vegetation productivity. In this study, we evaluated the impacts of LST and ET on animal species richness. ET exhibited the highest explained variance among all remote sensing metrics, which indicated the great impact of the water component on species distribution. Moreover, the difference of explained variance between ET and PET indicated that vegetation productivity played an important role in the distribution of animal species richness, because vegetation contributed less to the PET calculation compared to the ET calculation. The explained variance of LST was lower than ET, DHI-min, DHI-cum, and GPP. This may be due to the time-lag effects in the response of species richness to climate change. Previous studies showed that the change in animal diversity lagged behind climate change [39]. Some research has revealed that a warmer winter will lead animal diversity to change by causing the long-distance migration of animals [40]. Moreover, climate can alter species richness distribution through changing the vegetation productivity [104], and many studies have found a time-lag in the global vegetation response to climate change [105-108]. Because the influence of climate factors on animal diversity varies with different regions which leads to an offset on global-scale analysis, we expect that explained variance of LST are higher in regional analysis.

For the ecosystem texture category, texture metrics have been proved to be important drivers of animal species richness $[11,25,48,71,72,109]$, but the relationship pattern of texture metrics with animal species richness is still controversial. Various studies have found that species richness exhibited positive relationships with environmental heterogeneity, while negative relationships between environmental heterogeneity and species richness have also been reported [27,43,110-113]. Tuanmu and Jetz [25] validated the performance of 14 heterogeneity metrics, using North American bird richness. They showed that the texture metrics combined with net primary productivity (NPP) can explain up to $35.8 \%$ of the variance. In addition, homogeneity could still explain about $8 \%$ of the variance by itself. In the ecosystem texture category, our results showed that the CV was the strongest metric on a global scale, with an explained variance of $22 \%$. Compared with metrics in energy/productivity and climate category, this study showed that texture metrics exhibited low explained variance on a global scale, while high correlations were observed at smaller scales. In fact, the pattern of the relationship between animal species richness and habitat heterogeneity was impacted by many factors. Previous studies have showed that the possible effects of heterogeneity may also vary relative to the structural variable measured, and the effect of habitat heterogeneity for one species group may differ in relation to the spatial scale [43,71]. In this study, the high-correlation areas were mainly covered shrub lands, grasslands, and savannas, while the explained variance of texture metrics were relatively low in forests (Table S1). This is probably because the texture 
dataset we used mainly focused on the horizontal structure of an ecosystem, while forest biome has more complex vertical structures than shrub lands, grasslands, and savannas. Moreover, different regions showed various major driving texture metrics. For example, DIS, ENT, and HOM exhibited high explained variance in grasslands and savannas. Moreover, previous studies found that the predictive performance of texture metrics would vary across different spatial scales [114]. Overall, texture metrics should be primarily considered when determining the species distribution at regional scales.

For different animal classes, we found a gap in the explained variance between bird species richness and the species richness of the other two animal classes, among the climate metrics. The explained variance of PET on bird richness was much higher than that of both amphibian and mammal richness, which indicated that radiance and temperature played a more important role in bird richness distribution than in the other two animal classes.

\subsection{Integration of Various Remote Sensing Metrics}

The effect mechanism of different environmental variables on species distribution is debatable [115], which limits the application of certain metrics to model the species distribution quantitatively. We applied three commonly used machine learning models in the integration of the remote sensing metrics. There were differences in the results generated from the three models; RF generally outperformed SVM and NN in our case study. In this study, SVM and NN exhibited high uncertainty in species richness modeling, with the estimated datasets showing higher r-square and lower RMSE than the training datasets. In consideration of the AIC values, the RF model provided a better representation of the metrics integration of remote sensing metrics in this study than the other two models. Previous studies have suggested that differences in the results of various machine learning models may be due to the sample size, or the ratio between the size of the training sample and the estimated sample. $[116,117]$. However, there was no improvement in the performance of SVM and NN in species richness modeling, when we increased the proportion of the training sample and decreased the proportion of the estimated sample (Figure S3). RF has broader scope than SVM and $\mathrm{NN}$, because it has no limitation on the distribution pattern of the training data. The complexity of the parameter setting may limit the performance of SVM and NN for model training [118]. Although, SVM has the advantage on solving non-linear problems, a major downside of SVM is that it can be painfully inefficient to train $[119,120]$. Another main advantage of RF is that it handles very well with a large number of training examples, and it is not recommended to use SVM to handle large training examples, which might lead to a better performance of the RF. In the training process, we observed a large gap between RF, SVM, and NN. In addition, the computation time required for modelling differed considerably among the three machine learning models (Table 2). The computational efficiency of the SVM and NN models were much higher than that of the RF model.

Table 2. The time statistics of the three machine learning models with 5000 training samples and 5000 prediction samples.

\begin{tabular}{cccc}
\hline Species & SVM & RF & NN \\
\hline Mammal & $4.514 \mathrm{~s}$ & $13.635 \mathrm{~s}$ & $2.639 \mathrm{~s}$ \\
Bird & $4.465 \mathrm{~s}$ & $14.252 \mathrm{~s}$ & $2.651 \mathrm{~s}$ \\
Amphibian & $3.497 \mathrm{~s}$ & $14.173 \mathrm{~s}$ & $2.421 \mathrm{~s}$ \\
\hline
\end{tabular}

In this study, the multivariate integration index based on the RF model exhibited $20 \%$ more explained variance than the univariate metrics. Many species richness monitoring and modelling studies have been carried out in previous decades [3,4]. Our result, using three categories of remote sensing metrics, exhibited twice the explained variance of previous studies, which used texture metrics and net primary productivity (NPP) [25]. Previous studies showed DHI components explained between $47 \%$ and $75 \%$ of total bird species richness (BBS) in Ontario (North America), and we extended the spatial scale from regional to global [61]. Although these studies selected different species richness 
datasets, a comparison of the correlations was still reasonable, and we asserted that the multivariate integration index had a better correlation with species distribution than univariate metrics on a global scale. Since the evolution of a species is a very slow process, the link between these remote sensing metrics and species characteristics is stable for decades [121]. Thus, the multivariate integration index we developed can be used for mapping the global species richness annually, which would provide great support for global biodiversity trend detection.

At latitudinal and longitudinal zones, some differences between simulated species richness and in situ species richness were observed (Figure 8). There were two possible reasons that led to this disagreement. Firstly, these zones (e.g., $40^{\circ}-60^{\circ} \mathrm{S}$ zones) where gaps of species richness were found mostly had large areas of water or deserts, which led to a small sample size for model training. This small sample size brought large uncertainty in simulated species richness. Secondly, in some regions $\left(0^{\circ}-10^{\circ} \mathrm{N}\right.$ zones), the limited in situ data constrained much detail of species richness, while the remote sensing metrics we used exhibited high spatial resolution. This difference limited the explained variance of some remote sensing metrics (e.g., DHI-min, DHI-cum, and DHI-sea) (Figures 3 and 7). Overall, the impact of these differences on the multivariate integration index was limited, since these differences were relatively small in magnitude.

\subsection{Limitation and Recommendation for Future Research}

Although the multivariate integration index showed a consistent pattern with in situ richness data, it faced two known limitations. Firstly, the limited availability of in situ richness data constrained the comparison and integration of remote sensing metrics. Three dramatic declines of DHI's explained variance in $40^{\circ} \mathrm{N}$ zones, $10^{\circ} \mathrm{S}$ zones, and $40^{\circ} \mathrm{S}$ zones were found. It appeared that the in situ data of mammal richness were insufficient, because of the detail loss in these regions (Figure 5). Once higher resolution species richness data are available, the multivariate integration index should exhibit higher accuracy. Secondly, relationship patterns between energy/productivity, climate and ecosystem texture metrics, and animal diversity were limited by many other factors, namely scale, resolution, latitude, and so on.

Future research will consider both scale and resolution when comparing remote sensing metrics. Because the focus is taking full advantage of long time-series remote sensing data for species monitoring, some important metrics (e.g., precipitation and air temperature) which are not available from remote sensing data globally, were excluded in this study. To make biodiversity monitoring and assessment more globally accurate, future research will consider more datasets from both remote sensing data and model simulation data in integration index development. In addition, with the collection of more species information of both plants and animals, future research will develop the integration index for predicting plant species richness, as well as animal species richness.

\section{Conclusions}

In this study, 21 remote sensing metrics were assessed to determine their relationships with the richness distribution of three animal classes. The correlation between the species richness of the three animal classes and 21 biodiversity metrics was evaluated based on regression models, and ET exhibited the strongest correlation with the global distribution of the three animal classes. The metrics with high explained variance were mainly in the energy/productivity category. The ecosystem texture category exhibited a higher correlation with species richness at regional scales. For a single class, we found radiance and temperature had a larger impact on the distribution of birds than the other two animal classes. Three commonly used machine learning models were evaluated to determine their capabilities in developing a multivariate integration index, and the RF model was selected as the optimal model for multivariate index integration. Having taken DHI-min, DHI-cum, DHI-sea, GPP, CV, ET, and LST as the major metrics, the multivariate integration index exhibited a $20 \%$ higher explained variance of the global distributions of mammal, bird, and amphibian richness, compared to the univariate metrics. However, the in situ biodiversity data were still insufficient. With improvements in the availability 
of these datasets, further studies can consider more factors and offer higher-accuracy metrics for the assessment of global biodiversity.

Supplementary Materials: Supplementary materials are available online at http:/ / www.mdpi.com/s1. Figure S1: Correlations of all remote sensing metrics; Figure S2: Scatterplots between species richness and power-law metrics; Figure S3: Correlation between predicted species richness and in situ species richness; Table S1: The explained variance of remote sensing metrics in different biomes.

Author Contributions: J.W. and S.L. conceived and designed the experiments; J.W. performed the experiments; J.W. analyzed the data; J.W. and S.L. contributed to the analysis of the results; all of the aforementioned contributed towards writing the manuscript.

Acknowledgments: This work was partially supported by the National Key Research and Development Plan of China (Grant No. 2016YFA0600102) and the Natural National Science Foundation of China (grant No. 41331173).

Conflicts of Interest: The authors declare no conflict of interest.

\section{References}

1. Gaston, K.J. Global patterns in biodiversity. Nature 2000, 405, 220-227. [CrossRef] [PubMed]

2. Pereira, H.M.; Ferrier, S.; Walters, M.; Geller, G.; Jongman, R.; Scholes, R.; Bruford, M.W.; Brummitt, N.; Butchart, S.; Cardoso, A. Essential biodiversity variables. Science 2013, 339, 277-278. [CrossRef] [PubMed]

3. Pimm, S.L.; Jenkins, C.N.; Abell, R.G.; Brooks, T.M.; Gittleman, J.L.; Joppa, L.N.; Raven, P.; Roberts, C.M.; Sexton, J.O. The biodiversity of species and their rates of extinction, distribution, and protection. Science 2014, 344, 1246752. [CrossRef] [PubMed]

4. Turner, W. Sensing biodiversity. Science 2014, 346, 301-302. [CrossRef] [PubMed]

5. Newbold, T.; Hudson, L.N.; Hill, S.L.L.; Contu, S.; Lysenko, I.; Senior, R.A.; Boerger, L.; Bennett, D.J.; Choimes, A.; Collen, B.; et al. Global effects of land use on local terrestrial biodiversity. Nature 2015, 520, 45-50. [CrossRef] [PubMed]

6. Myers, N.; Mittermeier, R.A.; Mittermeier, C.G.; Da Fonseca, G.A.; Kent, J. Biodiversity hotspots for conservation priorities. Nature 2000, 403, 853-858. [CrossRef] [PubMed]

7. Butchart, S.H.; Walpole, M.; Collen, B.; Van Strien, A.; Scharlemann, J.P.; Almond, R.E.; Baillie, J.E.; Bomhard, B.; Brown, C.; Bruno, J. Global biodiversity: Indicators of recent declines. Science 2010, 328, 1164-1168. [CrossRef] [PubMed]

8. Noss, R.F. Indicators for monitoring biodiversity: A hierarchical approach. Conserv. Biol. 1990, 4, 355-364. [CrossRef]

9. Purvis, A.; Hector, A. Getting the measure of biodiversity. Nature 2000, 405, 212-219. [CrossRef] [PubMed]

10. Jetz, W.; Rahbek, C. Geographic range size and determinants of avian species richness. Science 2002, 297, 1548-1551. [CrossRef] [PubMed]

11. Kreft, H.; Jetz, W. Global patterns and determinants of vascular plant diversity. Proc. Natl. Acad. Sci. USA 2007, 104, 5925-5930. [CrossRef] [PubMed]

12. Guenard, B.; Weiser, M.D.; Dunn, R.R. Global models of ant diversity suggest regions where new discoveries are most likely are under disproportionate deforestation threat. Proc. Natl. Acad. Sci. USA 2012, 109, 7368-7373. [CrossRef] [PubMed]

13. Jenkins, C.N.; Guenard, B.; Diamond, S.E.; Weiser, M.D.; Dunn, R.R. Conservation implications of divergent global patterns of ant and vertebrate diversity. Divers. Distrib. 2013, 19, 1084-1092. [CrossRef]

14. Costanza, R.; Fisher, B.; Mulder, K.; Liu, S.; Christopher, T. Biodiversity and ecosystem services: A multi-scale empirical study of the relationship between species richness and net primary production. Ecol. Econ. 2007, 61, 478-491. [CrossRef]

15. Hurlbert, A.H.; Jetz, W. Species richness, hotspots, and the scale dependence of range maps in ecology and conservation. Proc. Natl. Acad. Sci. USA 2007, 104, 13384-13389. [CrossRef] [PubMed]

16. Pardieck, K.L.; Ziolkowski, D.J., Jr.; Lutmerding, M.; Campbell, K.; Hudson, M.-A.R. North American Breeding Bird Survey Dataset 1966-2013, Version 2016.0; US Geological Survey: Reston, VA, USA; Patuxent Wildlife Research Center: Laurel, MD, USA, 2017; Available online: www.pwrc.usgs.gov/BBS/RawData/ (accessed on 8 May 2018).

17. Constable, H.; Guralnick, R.; Wieczorek, J.; Spencer, C.L.; Peterson, A.T. Vertnet: A new model for biodiversity data sharing. PLoS Biol. 2010, 8, e1000309. [CrossRef] [PubMed] 
18. Garciarosello, E.; Guisande, C.; Heine, J.; Pelayovillamil, P.; Manjarreshernandez, A.; Vilas, L.G.; Gonzalezdacosta, J.; Vaamonde, A.; Granadolorencio, C. Using modestr to download, import and clean species distribution records. Methods Ecol. Evol. 2014, 5, 708-713. [CrossRef]

19. IUCN. The Iucn Red List of Threatened Species. 2017. Available online: http://www.iucnredlist.org/ (accessed on 8 May 2018).

20. GBIF. Gbif Data Portal. 2017. Available online: http://www.gbif.org/ (accessed on 8 May 2018).

21. Nagendra, H. Using remote sensing to assess biodiversity. Int. J. Remote Sens. 2001, 22, 2377-2400. [CrossRef]

22. Gougeon, F.A. Comparison of possible multispectral classification schemes for tree crowns individually delineatedon high spatial resolution meis images. Can. J. Remote Sens. 1995, 21, 1-9. [CrossRef]

23. Kier, G.; Kreft, H.; Lee, T.M.; Jetz, W.; Ibisch, P.L.; Nowicki, C.; Mutke, J.; Barthlott, W. A global assessment of endemism and species richness across island and mainland regions. Proc. Natl. Acad. Sci. USA 2009, 106, 9322-9327. [CrossRef] [PubMed]

24. Rahbek, C.; Graves, G.R. Multiscale assessment of patterns of avian species richness. Proc. Natl. Acad. Sci. USA 2001, 98, 4534-4539. [CrossRef] [PubMed]

25. Tuanmu, M.N.; Jetz, W. A global, remote sensing-based characterization of terrestrial habitat heterogeneity for biodiversity and ecosystem modelling. Glob. Ecol. Biogeogr. 2015, 24, 1329-1339. [CrossRef]

26. Duro, D.C.; Coops, N.C.; Wulder, M.A.; Han, T. Development of a large area biodiversity monitoring system driven by remote sensing. Prog. Phys. Geogr. 2007, 31, 235-260. [CrossRef]

27. Currie, D.J. Energy and large-scale patterns of animal- and plant-species richness. Am. Nat. 1991, 137, $27-49$. [CrossRef]

28. Rosenzweig, M.L.; Abramsky, Z. Species Diversity in Ecological Communities; University of Chicago Press: Chicago, IL, USA, 1993.

29. Liang, J.; Crowther, T.W.; Picard, N.; Wiser, S.; Zhou, M.; Alberti, G.; Schulze, E.-D.; McGuire, A.D.; Bozzato, F.; Pretzsch, H.; et al. Positive biodiversity-productivity relationship predominant in global forests. Science 2016, 354, aaf8957. [CrossRef] [PubMed]

30. Nagalingum, N.S.; Knerr, N.; Laffan, S.W.; González-Orozco, C.E.; Thornhill, A.H.; Miller, J.T.; Mishler, B.D. Continental scale patterns and predictors of fern richness and phylogenetic diversity. Front. Genet. 2015, 6, 132. [CrossRef] [PubMed]

31. Wilson, A.M.; Jetz, W. Remotely sensed high-resolution global cloud dynamics for predicting ecosystem and biodiversity distributions. PLoS Biol. 2016, 14, e1002415. [CrossRef] [PubMed]

32. Currie, D.J.; Mittelbach, G.G.; Cornell, H.V.; Field, R.; Guégan, J.F.; Hawkins, B.A.; Kaufman, D.M.; Kerr, J.T.; Oberdorff, T.; O'Brien, E. Predictions and tests of climate-based hypotheses of broad-scale variation in taxonomic richness. Ecol. Lett. 2004, 7, 1121-1134. [CrossRef]

33. Hansson, L.; Fahrig, L.; Merriam, G. Mosaic Landscapes and Ecological Processes; Springer Science \& Business Media: Berlin, Germany, 2012.

34. Guegan, J.-F.; Lek, S.; Oberdorff, T. Energy availability and habitat heterogeneity predict global riverine fish diversity. Nature 1998, 391, 382-384. [CrossRef]

35. Wright, D.H. Species-energy theory: An extension of species-area theory. Oikos 1983, 41, 496-506. [CrossRef]

36. Brown, J.H. Two decades of homage to santa rosalia: Toward a general theory of diversity. Integr. Comp. Biol. 1981, 21, 877-888. [CrossRef]

37. Hutchinson, G.E. Homage to santa rosalia or why are there so many kinds of animals. Am. Nat. 1959, 93, 145-159. [CrossRef]

38. Hobi, M.L.; Dubinin, M.; Graham, C.H.; Coops, N.C.; Clayton, M.K.; Pidgeon, A.M.; Radeloff, V.C. A comparison of dynamic habitat indices derived from different modis products as predictors of avian species richness. Remote Sens. Environ. 2017, 195, 142-152. [CrossRef]

39. Menendez, R.; Megias, A.G.; Hill, J.K.; Braschler, B.; Willis, S.G.; Collingham, Y.C.; Fox, R.; Roy, D.B.; Thomas, C.D. Species richness changes lag behind climate change. Proc. R. Soc. B Biol. Sci. 2006, 273, 1465-1470. [CrossRef] [PubMed]

40. Lemoine, N.; Bohninggaese, K. Potential impact of global climate change on species richness of long-distance migrants. Conserv. Biol. 2003, 17, 577-586. [CrossRef]

41. Lawton, J.H. Plant architecture and the diversity of phytophagous insects. Annu. Rev. Entomol. 1983, 28, 23-39. [CrossRef] 
42. McCoy, E.D.; Bell, S.S. Habitat structure: The evolution and diversification of a complex topic. In Habitat Structure; Springer: Berlin, Germany, 1991; pp. 3-27.

43. Tews, J.; Brose, U.; Grimm, V.; Tielbörger, K.; Wichmann, M.; Schwager, M.; Jeltsch, F. Animal species diversity driven by habitat heterogeneity/diversity: The importance of keystone structures. J. Biogeogr. 2004, 31, 79-92. [CrossRef]

44. Skidmore, A.K.; Pettorelli, N. Agree on biodiversity metrics to track from space. Nature 2015, 523, $403-405$. [CrossRef] [PubMed]

45. Scholes, R.; Biggs, R. A biodiversity intactness index. Nature 2005, 434, 45-49. [CrossRef] [PubMed]

46. Petrou, Z.I.; Manakos, I.; Stathaki, T. Remote sensing for biodiversity monitoring: A review of methods for biodiversity indicator extraction and assessment of progress towards international targets. Biodivers. Conserv. 2015, 24, 2333-2363. [CrossRef]

47. Buchanan, G.M.; Nelson, A.; Mayaux, P.; Hartley, A.; Donald, P.F. Delivering a global, terrestrial, biodiversity observation system through remote sensing. Conserv. Biol. 2009, 23, 499-502. [CrossRef] [PubMed]

48. Rocchini, D.; Balkenhol, N.; Carter, G.A.; Foody, G.M.; Gillespie, T.W.; He, K.S.; Kark, S.; Levin, N.; Lucas, K.; Luoto, M. Remotely sensed spectral heterogeneity as a proxy of species diversity: Recent advances and open challenges. Ecol. Inform. 2010, 5, 318-329. [CrossRef]

49. Jenkins, C.N.; Pimm, S.L.; Joppa, L.N. Global patterns of terrestrial vertebrate diversity and conservation. Proc. Natl. Acad. Sci. USA 2013, 110, E2602-E2610. [CrossRef] [PubMed]

50. Jenkins, C.N.; Van Houtan, K.S.; Pimm, S.L.; Sexton, J.O. Us protected lands mismatch biodiversity priorities. Proc. Natl. Acad. Sci. USA 2015, 112, 5081-5086. [CrossRef] [PubMed]

51. Zhang, C.; Cai, D.; Guo, S.; Guan, Y.; Fraedrich, K.; Nie, Y.; Liu, X.; Bian, X. Spatial-temporal dynamics of china's terrestrial biodiversity: A dynamic habitat index diagnostic. Remote Sens. 2016, 8, 227. [CrossRef]

52. Golestani, A.; Gras, R. Using Machine Learning Techniques for Identifying Important Characteristics to Predict Changes in Species Richness in Ecosim, an Individual-Based Ecosystem Simulation. In Proceedings of the World Congress on Engineering and Computer Science, San Francisco, CA, USA, 24-26 October 2012.

53. Olaya-Marín, E.J.; Martínez-Capel, F.; Vezza, P. A comparison of artificial neural networks and random forests to predict native fish species richness in mediterranean rivers. Knowl. Manag. Aquat. Ecosyst. 2013. [CrossRef]

54. Golestani, A.; Gras, R. Regularity analysis of an individual-based ecosystem simulation. Chaos 2010, 20, 043120. [CrossRef] [PubMed]

55. Devaurs, D.; Gras, R. Species abundance patterns in an ecosystem simulation studied through fisher's logseries. Simul. Model. Pract. Theory 2010, 18, 100-123. [CrossRef]

56. Mouton, A.M.; Alcaraz-Hernández, J.D.; De Baets, B.; Goethals, P.L.; Martínez-Capel, F. Data-driven fuzzy habitat suitability models for brown trout in spanish mediterranean rivers. Environ. Model. Softw. 2011, 26, 615-622. [CrossRef]

57. Leclere, J.; Oberdorff, T.; Belliard, J.; Leprieur, F. A comparison of modeling techniques to predict juvenile 0+ fish species occurrences in a large river system. Ecol. Inform. 2011, 6, 276-285. [CrossRef]

58. Knudby, A.; LeDrew, E.; Brenning, A. Predictive mapping of reef fish species richness, diversity and biomass in zanzibar using ikonos imagery and machine-learning techniques. Remote Sens. Environ. 2010, 114, 1230-1241. [CrossRef]

59. IUCN. The Iucn Red List of Threatened Species. Version 2010-4. 2010, Volume 9. Available online: http: //www.iucnredlist.org/info/categories_criteria2001.html (accessed on 12 November 2017).

60. Zhao, M.; Heinsch, F.A.; Nemani, R.R.; Running, S.W. Improvements of the modis terrestrial gross and net primary production global data set. Remote Sens. Environ. 2005, 95, 164-176. [CrossRef]

61. Coops, N.C.; Wulder, M.A.; Iwanicka, D. Demonstration of a satellite-based index to monitor habitat at continental-scales. Ecol. Indic. 2009, 9, 948-958. [CrossRef]

62. Coops, N.C.; Wulder, M.A.; Duro, D.C.; Han, T.; Berry, S. The development of a canadian dynamic habitat index using multi-temporal satellite estimates of canopy light absorbance. Ecol. Indic. 2008, 8, 754-766. [CrossRef]

63. Perez, L.; Nelson, T.A.; Coops, N.C.; Fontana, F.; Drever, C.R. Characterization of spatial relationships between three remotely sensed indirect indicators of biodiversity and climate: A 21years' data series review across the canadian boreal forest. Int. J. Digit. Earth 2016, 9, 676-696. [CrossRef] 
64. Mu, Q.; Zhao, M.; Running, S.W. Improvements to a modis global terrestrial evapotranspiration algorithm. Remote Sens. Environ. 2011, 115, 1781-1800. [CrossRef]

65. Mu, Q.; Heinsch, F.A.; Zhao, M.; Running, S.W. Development of a global evapotranspiration algorithm based on modis and global meteorology data. Remote Sens. Environ. 2007, 111, 519-536. [CrossRef]

66. Monteith, J. Evaporation and environment. In The State and Movement of Water in Living Organisms. Symposium of the Society of Experimental Biology; Cambridge University Press: Cambridge, UK, 1965; Volume 19, pp. 205-234.

67. Tasumi, M.; Hirakawa, K.; Hasegawa, N.; Nishiwaki, A.; Kimura, R. Application of modis land products to assessment of land degradation of alpine rangeland in northern india with limited ground-based information. Remote Sens. 2014, 6, 9260-9276. [CrossRef]

68. Mu, Q.; Zhao, M.; Running, S.W. Modis Global Terrestrial Evapotranspiration (et) Product (Nasa Mod16a2/a3); Algorithm Theoretical Basis Document, Collection; NASA: Washington, DC, USA, 2013; Volume 5.

69. Wang, X.; Fan, J.; Xu, J.; Liu, F.; Gao, S.; Wei, X. Land surface emissivity change in china from 2001 to 2010. J. Geogr. Sci. 2012, 22, 407-415. [CrossRef]

70. Haralick, R.M.; Shanmugam, K.S.; Dinstein, I. Textural features for image classification. IEEE Trans. Syst. Man Cybern. 1973, 3, 610-621. [CrossRef]

71. Stein, A.; Gerstner, K.; Kreft, H. Environmental heterogeneity as a universal driver of species richness across taxa, biomes and spatial scales. Ecol. Lett. 2014, 17, 866-880. [CrossRef] [PubMed]

72. Kerr, J.T.; Packer, L. Habitat heterogeneity as a determinant of mammal species richness in high-energy regions. Nature 1997, 385, 252-254. [CrossRef]

73. Olson, D.M.; Dinerstein, E.; Wikramanayake, E.D.; Burgess, N.D.; Powell, G.V.N.; Underwood, E.C.; D'Amico, J.A.; Itoua, I.; Strand, H.E.; Morrison, J.C. Terrestrial ecoregions of the world: A new map of life on earth. Bioscience 2009, 51, 933-938. [CrossRef]

74. Olson, D.M.; Dinerstein, E. The Global 200: Priority ecoregions for global conservation. Ann. Mo. Bot. Gard. 2002, 89, 199-224. [CrossRef]

75. Nagendra, H.; Rocchini, D.; Ghate, R.; Sharma, B.; Pareeth, S. Assessing plant diversity in a dry tropical forest: Comparing the utility of landsat and ikonos satellite images. Remote Sens. 2010, 2, 478-496. [CrossRef]

76. Cleveland, W.S.; Devlin, S.J. Locally weighted regression: An approach to regression analysis by local fitting. J. Am. Stat. Assoc. 1988, 83, 596-610. [CrossRef]

77. Akaike, H. A new look at the statistical model identification. IEEE Trans. Autom. Control 1974, 19, 716-723. [CrossRef]

78. Loehlin, J.C. Latent Variable Models: An Introduction to Factor, Path, and Structural Analysis; Lawrence Erlbaum Associates Publishers: Mahwah, NJ, USA, 1998.

79. Yao, Y.; Liang, S.; Li, X.; Chen, J.; Liu, S.; Jia, K.; Zhang, X.; Xiao, Z.; Fisher, J.B.; Mu, Q. Improving global terrestrial evapotranspiration estimation using support vector machine by integrating three process-based algorithms. Agric. For. Meteorol. 2017, 242, 55-74. [CrossRef]

80. Eitrich, T.; Lang, B. Parallel Tuning of Support Vector Machine Learning Parameters for Large and Unbalanced Data Sets. In Proceedings of the International Symposium on Computational Life Science, Konstanz, Germany, 25-27 September 2005; Springer: Berlin, Germany, 2005; pp. 253-264.

81. Vapnik, V. The nature of statistical learning theory. Technometrics 1995, 38, 409.

82. Abdel-Rahman, E.M.; Mutanga, O.; Adam, E.; Ismail, R. Detecting sirex noctilio grey-attacked and lightning-struck pine trees using airborne hyperspectral data, random forest and support vector machines classifiers. ISPRS J. Photogramm. Remote Sens. 2014, 88, 48-59. [CrossRef]

83. Meyer, D.; Dimitriadou, E.; Hornik, K.; Weingessel, A.; Leisch, F.; Chang, C.-C.; Lin, C.-C.; Meyer, M.D. Package 'e1071'; R Package Version; 2017. Available online: https://cran.r-project.org/web/packages/ e1071/index.html (accessed on 12 November 2017).

84. Sor, R.; Park, Y.-S.; Boets, P.; Goethals, P.L.; Lek, S. Effects of species prevalence on the performance of predictive models. Ecol. Model. 2017, 354, 11-19. [CrossRef]

85. Cassano, A.; Marchese Robinson, R.L.; Palczewska, A.; Puzyn, T.; Gajewicz, A.; Tran, L.; Manganelli, S.; Cronin, M. Comparing the coral and random forest approaches for modelling the in vitro cytotoxicity of silica nanomaterials. Altern. Lab. Anim. 2016, 44, 533-556. [PubMed]

86. Breiman, L. Bagging predictors. Mach. Learn. 1996, 24, 123-140. [CrossRef]

87. Breiman, L. Random forests. Mach. Learn. 2001, 45, 5-32. [CrossRef] 
88. Goethals, P.L.; Dedecker, A.P.; Gabriels, W.; Lek, S.; De Pauw, N. Applications of artificial neural networks predicting macroinvertebrates in freshwaters. Aquat. Ecol. 2007, 41, 491-508. [CrossRef]

89. Lek, S.; Guégan, J.-F. Artificial neural networks as a tool in ecological modelling, an introduction. Ecol. Model. 1999, 120, 65-73. [CrossRef]

90. Lek, S.; Delacoste, M.; Baran, P.; Dimopoulos, I.; Lauga, J.; Aulagnier, S. Application of neural networks to modelling nonlinear relationships in ecology. Ecol. Model. 1996, 90, 39-52. [CrossRef]

91. Lee, Y.Y.; Kim, J.T.; Yun, G.Y. The neural network predictive model for heat island intensity in Seoul. Energy Build. 2016, 110, 353-361. [CrossRef]

92. Van Echelpoel, W.; Boets, P.; Landuyt, D.; Gobeyn, S.; Everaert, G.; Bennetsen, E.; Mouton, A.; Goethals, P.L. Species distribution models for sustainable ecosystem management. In Developments in Environmental Modelling; Elsevier: New York, NY, USA, 2015; Volume 27, pp. 115-134.

93. Ripley, B.; Venables, W.; Ripley, M.B. Package 'nnet'; R Package Version 7.3-12; 2016. Available online: https:/ / cran.r-project.org/web/packages/nnet/index.html (accessed on 12 November 2017).

94. Myers, N. The biodiversity challenge: Expanded hot-spots analysis. Environmentalist 1990, 10, $243-256$. [CrossRef] [PubMed]

95. Rodrigues, A.S.; Andelman, S.J.; Bakarr, M.I.; Boitani, L.; Brooks, T.M.; Cowling, R.M.; Fishpool, L.D.; da Fonseca, G.A.; Gaston, K.J.; Hoffmann, M. Effectiveness of the global protected area network in representing species diversity. Nature 2004, 428, 640. [CrossRef] [PubMed]

96. Hawkins, B.A.; Field, R.; Cornell, H.V.; Currie, D.J.; Guegan, J.; Kaufman, D.M.; Kerr, J.T.; Mittelbach, G.G.; Oberdorff, T.; Obrien, E.M. Energy, water, and broad-scale geographic patterns of species richness. Ecology 2003, 84, 3105-3117. [CrossRef]

97. Gillman, L.N.; Wright, S.D. The influence of productivity on the species richness of plants: A critical assessment. Ecology 2006, 87, 1234-1243. [CrossRef]

98. Isbell, F.; Craven, D.; Connolly, J.; Loreau, M.; Schmid, B.; Beierkuhnlein, C.; Bezemer, T.M.; Bonin, C.L.; Bruelheide, H.; De Luca, E. Biodiversity increases the resistance of ecosystem productivity to climate extremes. Nature 2015, 526, 574-577. [CrossRef] [PubMed]

99. Wright, D.H.; Currie, D.J.; Maurer, B.A. Energy supply and patterns of species richness on local and regional scales. In Species Diversity in Ecological Communities: Historical and Geographical Perspectives; University of Chicago Press: Chicago, IL, USA, 1993; pp. 66-74.

100. Waide, R.B.; Willig, M.R.; Steiner, C.F.; Mittelbach, G.G.; Gough, L.; Dodson, S.I.; Juday, G.P.; Parmenter, R. The relationship between productivity and species richness. Annu. Rev. Ecol. Evol. System. 2003, 30, 257-300. [CrossRef]

101. Pavlik, B.M. Species diversity in ecological communities: Historical and geographical perspectives. Madroño 1995, 42, 523-525.

102. Rosenzweig, M.L. Species Diversity in Space and Time; Cambridge University Press: Cambridge, UK, 1995.

103. Currie, D.J.; Francis, A.P.; Kerr, J.T. Some general propositions about the study of spatial patterns of species richness. Ecoscience 1999, 6, 392-399. [CrossRef]

104. Pacifici, M.; Visconti, P.; Butchart, S.H.; Watson, J.E.; Cassola, F.M.; Rondinini, C. Species/'traits influenced their response to recent climate change. Nat. Clim. Chang. 2017, 7, 205-208. [CrossRef]

105. Davis, M.B. Lags in vegetation response to greenhouse warming. Clim. Chang. 1989, 15, 75-82. [CrossRef]

106. Saatchi, S.; Asefi-Najafabady, S.; Malhi, Y.; Aragão, L.E.; Anderson, L.O.; Myneni, R.B.; Nemani, R. Persistent effects of a severe drought on amazonian forest canopy. Proc. Natl. Acad. Sci. USA 2013, 110, 565-570. [CrossRef] [PubMed]

107. Chen, T.; De Jeu, R.; Liu, Y.; Van der Werf, G.; Dolman, A. Using satellite based soil moisture to quantify the water driven variability in ndvi: A case study over mainland australia. Remote Sens. Environ. 2014, 140, 330-338. [CrossRef]

108. Wu, D.; Zhao, X.; Liang, S.; Zhou, T.; Huang, K.; Tang, B.; Zhao, W. Time-lag effects of global vegetation responses to climate change. Glob. Chang. Biol. 2015, 21, 3520-3531. [CrossRef] [PubMed]

109. MacArthur, R.H.; MacArthur, J.W. On bird species diversity. Ecology 1961, 42, 594-598. [CrossRef]

110. Stein, A.; Kreft, H. Terminology and quantification of environmental heterogeneity in species-richness research. Biol. Rev. 2015, 90, 815-836. [CrossRef] [PubMed]

111. Tamme, R.; Hiiesalu, I.; Laanisto, L.; Szava-Kovats, R.; Pärtel, M. Environmental heterogeneity, species diversity and co-existence at different spatial scales. J. Veg. Sci. 2010, 21, 796-801. [CrossRef] 
112. Gazol, A.; Tamme, R.; Price, J.N.; Hiiesalu, I.; Laanisto, L.; Pärtel, M. A negative heterogeneity-diversity relationship found in experimental grassland communities. Oecologia 2013, 173, 545-555. [CrossRef] [PubMed]

113. Laanisto, L.; Tamme, R.; Hiiesalu, I.; Szava-Kovats, R.; Gazol, A.; Pärtel, M. Microfragmentation concept explains non-positive environmental heterogeneity-diversity relationships. Oecologia 2013, 171, $217-226$. [CrossRef] [PubMed]

114. Belmaker, J.; Jetz, W. Cross-scale variation in species richness-environment associations. Glob. Ecol. Biogeogr. 2011, 20, 464-474. [CrossRef]

115. Pouteau, R.; Meyer, J.-Y.; Taputuarai, R.; Stoll, B. Support vector machines to map rare and endangered native plants in pacific islands forests. Ecol. Inform. 2012, 9, 37-46. [CrossRef]

116. Lee, Y.; Lin, Y.; Wahba, G. Multicategory support vector machines: Theory and application to the classification of microarray data and satellite radiance data. J. Am. Stat. Assoc. 2004, 99, 67-81. [CrossRef]

117. Jia, K.; Liang, S.; Liu, S.; Li, Y.; Xiao, Z.; Yao, Y.; Jiang, B.; Zhao, X.; Wang, X.; Xu, S. Global land surface fractional vegetation cover estimation using general regression neural networks from modis surface reflectance. IEEE Trans. Geosci. Remote Sens. 2015, 53, 4787-4796. [CrossRef]

118. Yang, L.; Jia, K.; Liang, S.; Liu, J.; Wang, X. Comparison of four machine learning methods for generating the glass fractional vegetation cover product from modis data. Remote Sens. 2016, 8, 682. [CrossRef]

119. Sun, C.; Vilalta, R. Data selection using sash trees for support vector machines. In Proceedings of the Machine Learning and Data Mining in Pattern Recognition, Leipzig, Germany, 18-20 July 2007; pp. 286-295.

120. Zhai, J.; Li, C.; Li, T. Sample selection based on kl divergence for effectively training svm. In Proceedings of the 2013 IEEE International Conference on Systems, Man, and Cybernetics (SMC), Washington, DC, USA, 13-16 October 2013; pp. 4837-4842.

121. Bellard, C.; Bertelsmeier, C.; Leadley, P.W.; Thuiller, W.; Courchamp, F. Impacts of climate change on the future of biodiversity. Ecol. Lett. 2012, 15, 365-377. [CrossRef] [PubMed]

(C) 2018 by the authors. Licensee MDPI, Basel, Switzerland. This article is an open access article distributed under the terms and conditions of the Creative Commons Attribution (CC BY) license (http:/ / creativecommons.org/licenses/by/4.0/). 


\title{
Article \\ SPI-Based Analyses of Drought Changes over the Past 60 Years in China's Major Crop-Growing Areas
}

\author{
Lang Xia ${ }^{1,3}$, Fen Zhao ${ }^{4}$, Kebiao Mao ${ }^{1,2,5, *}$, Zijin Yuan ${ }^{1}$, Zhiyuan Zuo ${ }^{1}$ and Tongren Xu ${ }^{2}$ \\ 1 National Hulunber Grassland Ecosystem Observation and Research Station, Institute of Agricultural \\ Resources and Regional Planning, Chinese Academy of Agricultural Sciences, Beijing 100081, China; \\ xialang2012@163.com (L.X.); yuanzhijin2004@126.com (Z.Y.); zuozhiyuan2004@126.com (Z.Z.) \\ 2 State Key Laboratory of Remote Sensing Science, Institute of Remote Sensing and Digital Earth Research, \\ Chinese Academy of Science and Beijing Normal University, Beijing 100086, China; 11112011088@bnu.edu.cn \\ 3 Beijing Research Center of Intelligent Equipment for Agriculture, Beijing Academy of Agriculture and \\ Forestry Sciences, Beijing 100081, China \\ 4 Key Laboratory of Ecosystem Network Observation and Modeling, Institute of Geographic Sciences and \\ Natural Resources Research, University of Chinese academy of Science, Beijing 100086, China; \\ zhaofen198931@163.com \\ 5 College of Resources and Environments, Hunan Agricultural University, Changsha 410128, China \\ * Correspondence: maokebiao@caas.cn; Tel.: +86-10-8210-8769
}

Received: 1 December 2017; Accepted: 23 January 2018; Published: 25 January 2018

\begin{abstract}
This study analyzes the changes in drought patterns in China's major crop-growing areas over the past 60 years. The analysis was done using both weather station data and Tropical Rainfall Measuring Mission (TRMM) Microwave Imager (TMI) rainfall data to calculate the Standardized Precipitation Index (SPI). The results showed that the occurrences of extreme drought were the most serious in recent years in the Southwest China and Sichuan crop-growing areas. The Yangtze River (MLRY) and South China crop-growing areas experienced extreme droughts during 1960-1980, whereas the Northeast China and Huang-Huai-Hai crop-growing areas experienced extreme droughts around 2003. The analysis showed that the SPIs calculated by TRMM data at time scales of one, three, and six months were reliable for monitoring drought in the study regions, but for 12 months, the SPIs calculated by gauge and TRMM data showed less consistency. The analysis of the spatial distribution of droughts over the past 15 years using TMI rainfall data revealed that more than $60 \%$ of the area experienced extreme drought in 2011 over the MLRY region and in 1998 over the Huang-Huai-Hai region. The frequency of different intensity droughts presented significant spatial heterogeneity in each crop-growing region.
\end{abstract}

Keywords: drought; SPI; TMI data; crop-growing regions

\section{Introduction}

Droughts caused by long-term shortages of precipitation are a global issue. Droughts generally occur randomly, last for long periods of time, and impact large areas [1-3]. Compared to the impacts of other natural disasters, the impacts of drought on agricultural production and food safety are more significant [4]. Droughts have long been monitored and analyzed, and with the evolving awareness of drought occurrences, drought monitoring methods are continuously improving. These improvements are mainly reflected in the methodologies and data sources used. In the mid-1900s, drought monitoring was primarily done through drought estimation using precipitation alone or combined with other meteorological elements [5]. For example, Thornthwaite [6] proposed using precipitation minus evapotranspiration as an index of drought, and Van et al. [7] proposed the concept of agricultural drought days. After the mid-1900s, the monitoring of meteorological droughts was 
more actively studied. Palmer [8] corrected deficiencies in previous drought monitoring indices and proposed the Palmer drought severity index in 1965. McKee et al. [9] proposed the Standardized Precipitation Index (SPI) for drought monitoring in Colorado, USA. In addition, several drought monitoring methods have been proposed and used in different areas, such as the meteorological drought composite index (CI) proposed by the China Meteorological Administration [10] and the Standardized Precipitation-Evapotranspiration Index (SPEI), which combines the features of the SPI and the Palmer drought index [11,12].

Compared to remote sensing data, meteorological data from weather stations generally cover longer periods of time and are more precise. Thus, meteorological drought monitoring methods are more advantageous for analyzing the periodicities of drought. Meanwhile, remote sensing data cover larger areas, are less constrained by geographic conditions, and thus are more suitable for large-scale drought monitoring. At present, drought monitoring methods based on remote sensing data, e.g., the Temperature Vegetation Drought Index (TVDI) [13,14], Vegetation Condition Index (VCI) [15], and Microwave Integrated Drought index (MIDI) [16], are mainly focused on using vegetation indices, land surface temperatures and microwave data. In addition, many methods were also proposed to monitor agricultural drought. For example, Lessel et al. presented a method based on quasi-climatological anomalies of remotely sensed data to monitor agricultural drought [17]. Tigkas et al. proposed an Effective Reconnaissance Drought Index (eRDI) to improve the ability to assess agricultural drought [18]. Li et al. developed a new agricultural drought index based on the Variable Infiltration Capacity (VIC) model coupled with an irrigation scheme and a reservoir module to monitor drought [19].

With global climate change, China, whose per capita cultivated land is only $32 \%$ that of the global average, has suffered severe droughts in recent decades $[4,20]$. The economic losses caused by droughts far exceed those caused by other natural disasters. Several studies have revealed changes in drought in China for different regions by using ground gauge data [4,21-27] and TRMM precipitation products. For example, Hui et al. evaluated drought in the Jiangsu Province by using TRMM 3B43 data [28], and Zeng et al. used TRMM data to monitor drought in the Lancang River Basin [29]. In general, such studies mainly covered individual watersheds or plains and rarely aimed at researching meteorological changes in drought across the major crop-growing areas. In this study, two data sources were used, including weather station data with longer monitoring and recording histories and the Tropical Rainfall Measuring Mission (TRMM) [30] Microwave Imager (TMI) precipitation data (3B43) with a better spatial distribution. SPI was adopted to analyze the changes in drought in the major crop-growing areas of China over the past 60 years using meteorological data, and the SPI calculated using the TRMM 3B43 product for the years of 1998 to 2013 was validated by the meteorological data. Finally, the spatial and temporal features of drought area were analyzed by the TRMM data in the major crop-growing areas of China.

\section{Study Area and Data}

The National Regionalization of Priority Development Areas (access at: http:/ /www.gov.cn/zwgk/ 2011-06/08/content_1879180.htm) was released on 21 December 2010 by the State Council of China. Based on the planning for China's agricultural strategy given in this notice, the research area of this study was selected, as shown in Figure 1. According to a previous study [31], major crop-growing areas in different geographic locations were subject to the influences of various types of monsoons. For example, Southwest China and the Sichuan area were mainly influenced by the combined effects of the Southwest monsoons from the Indian Ocean and the summer monsoons from the Pacific Ocean. Meanwhile, the South China area, the middle and lower reaches of the Yangtze River (MLRY), the Huang-Huai-Hai area, and Northeast China were mainly influenced by summer monsoons from the Pacific Ocean. Due to the different arrival times of the monsoons, the starting time of the rainy seasons varies from south to north, as shown in Table 1. 


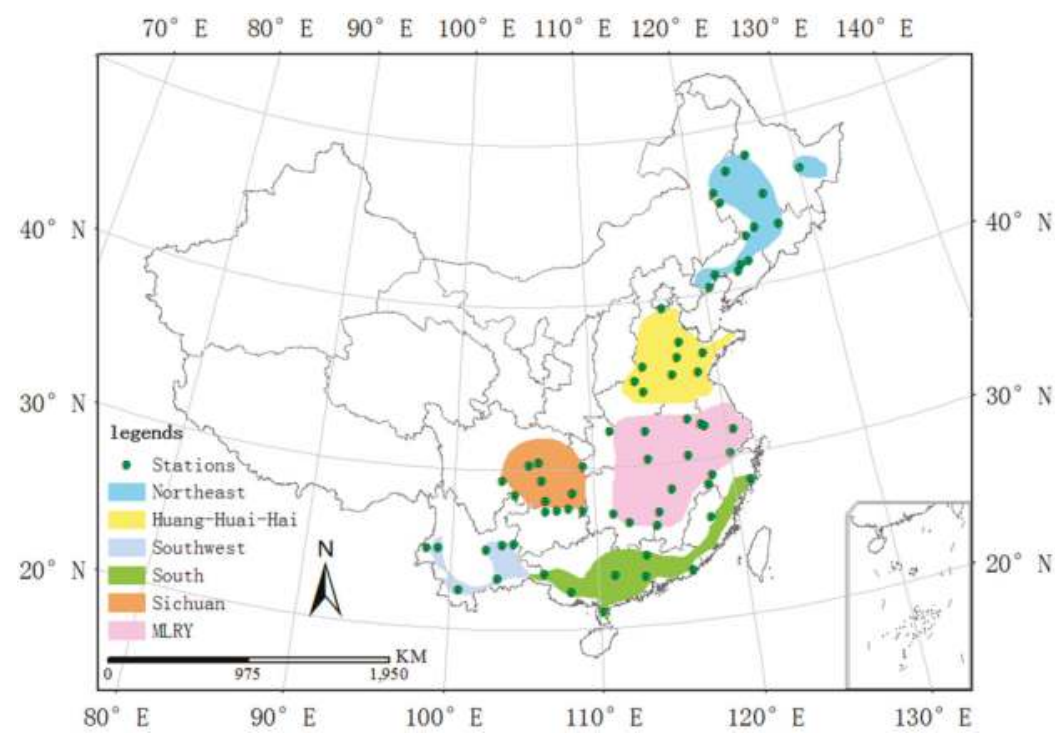

Figure 1. Study areas and meteorological station locations.

Table 1. Information on the study areas.

\begin{tabular}{cccc}
\hline Crop-Growing Area & Rainy Season & $\begin{array}{c}\text { Annual Average } \\
\text { Precipitation }(\mathbf{m m})\end{array}$ & Major Crops \\
\hline Northeast China & July-September & $500-750$ & Wheat, Corn, Rice \\
Huang-Huai-Hai & July-September & $400-800$ & Wheat, Corn, Rice \\
MLRY & May-August & $1000-1600$ & Rice, Wheat \\
South China & March-September & $1500-2000$ & Rice, Corn \\
Sichuan & April-August & $900-1200$ & Rice, Wheat \\
Southwest China & May-October & $1000-2000$ & Rice, Wheat \\
\hline
\end{tabular}

The data used in this study include weather station data and remote sensing rainfall data. The weather station data were obtained from meteorological data sharing websites (access at: http: / / data.cma.cn/). The daily data from 752 basic or standardized ground meteorological stations were collected from 1951 to 2013. Since the observed precipitation data were not continuous at some stations but the SPI computation requires complete data, the data were filtered using the following rule: the data from any station with more than one day of data missing or containing abnormal measurements were excluded. Hence, 64 stations in or near the research regions were obtained, as shown in Figure 1. In addition, the daily precipitation values were merged to obtain the monthly rainfall data to calculate the monthly SPI values.

The TRMM satellite was launched in 1997 for weather and climate research [32]. In 2014, it suffered some battery issues and did not have enough propellant to support the required orbit altitude, so it had to turn off the Visible and Infrared Scanner (VIRS) and slowly decayed in orbit while continuing to collect data. The satellite stopped gathering data on 15 June 2015. This study used the TRMM 3B43 monthly precipitation data with a resolution of 0.25 degrees from 1998 to 2013 as the satellite gauged data to explore a robust spatial characterization of drought.

\section{Methodology}

The SPI based on the best fit of the precipitation to a probability distribution was designed by McKee et al. [9] for drought monitoring in Colorado, USA. SPI at different time scales can be used 
to monitor the impacts of decreased rainfall on different habitats [5]. Shorter time scales (one to three months) can be used for drought monitoring and early warning signs, as well as drought severity estimations. Time scales of six to 24 months can be used to monitor the impacts of decreased rainfall on hydrology, reservoirs, and surface water. To analyze the periodicities and variations of droughts over a long time period, the time span of the SPI should be set to six to 24 months.

Compared to PDSI [8] and SPEI [11], SPI only requires precipitation for its estimation to measure droughts at different time scales, and it is less complex to calculate than others. However, the SPI does not account for factors that influence evapotranspiration (e.g., soil types and temperature) [33]. Although it is plausible to consider additional variables in drought monitoring, the TRMM data were used to monitor the drought, and there is no gauged temperature for the TRMM coverage region. Hence, the simple SPI was selected in the study to monitor the changes in drought.

The SPIs for short timescales can monitor drought well, but these data are not suitable to trace the potential impacts of long periods of dryness on agriculture and water supply [33]. However, the proper characterization of agricultural drought depends on many factors, such as crop type and cultivation period. Using the same SPI for different plant regions may cause different degrees of drought damage for the crops. Hence, this study mainly analyzed the long-term change in drought among the plant regions, and a timescale with a cumulative duration of 12 months was used to analyze the change in drought in the main crop-growing areas of China.

The mean SPI calculated by Equation (1) can be used to evaluate general drought intensity when the gauge points are almost evenly distributed spatially:

$$
S P I_{\text {mean }}=\sum_{0}^{n} S P I_{i, t}
$$

where SPI mean is the mean SPI, SPI $I_{i, t}$ is the SPI at time $t$ for site $i$, and $n$ is the number of total stations for the region. As shown in Figure 1, the gauge stations are almost evenly distributed in each crop-growing region, hence Equation (1) was used to present the general drought intensity for each crop-growing region.

The TRMM 3B43 data in the study regions were extracted, and the projection was converted to the Albers projection to obtain the equation area for each pixel. The rainfall of pixels among each region was extracted from the average monthly TRMM rainfall raster data from 1998 to 2013. In this way, time series of the spatial rainfall distribution were obtained. Then, time scales of one, three, six, and 12 months were used to calculate the SPI.

Dryness and wetness severity classifications according to the SPI values are listed in Table 2.

Table 2. Drought intensities according to SPI values.

\begin{tabular}{cc}
\hline SPI Value & Drought Level \\
\hline SPI $\geq 2.0$ & Extreme wet \\
$1.5 \leq$ SPI $<2.0$ & Very wet \\
$1.0 \leq$ SPI $<1.5$ & Moderate wet \\
$-1.0 \leq$ SPI $<1.0$ & Normal \\
$-1.5 \leq$ SPI $<-1.0$ & Moderate drought \\
$-2.0 \leq \mathrm{SPI}<-1.5$ & Severe drought \\
$-2.0 \leq \mathrm{SPI}$ & Extreme drought \\
\hline
\end{tabular}

\section{Results}

\subsection{Drought Results by Using Gauge Data}

The SPI at a time scale of 12 month was used here to analyze the changes in drought in the study regions, as shown by the gray filled areas in Figures $2-4$. The mean SPIs for each region calculated by equation (1) are presented as the blue filled areas shown in Figures 2-4. As shown in Figure 2a, the Northeast China crop-growing area experienced adequate precipitation before 1965, with rare 
drought occurrences. The end of the 1960s was a time of wet-to-dry transitions in the area, and then a 20-year dry period occurred in the area and reached its peak at the end of the 1970s. The precipitation increased in the 1980s, and the drought was alleviated to a certain extent. In 1990s, the droughts and wet periods changed every three years. After 2000, the subsequent droughts lasted for 10 years, and the intensity was more severe than the droughts of the 1970s. In 2010, the precipitation gradually increased, and the droughts were alleviated slightly.

The Huang-Huai-Hai crop-growing area also had low occurrences of drought before 1964, during which this area was moist, as shown in Figure 2b. At the beginning of 1965, the drought intensified, reaching its peak at the end of the 1960s. The 1970s was a relatively moist stage, and after this period, precipitation decreased and the drought intensified. The drought reached its peak at the end of the 1980s and gradually lessened over the following 10 years. Entering the 21st century, the drought situation intensified once again. However, the duration of this drought was less than those of the droughts in the 1980s, and after 2010, the occurrences of drought gradually lessened.

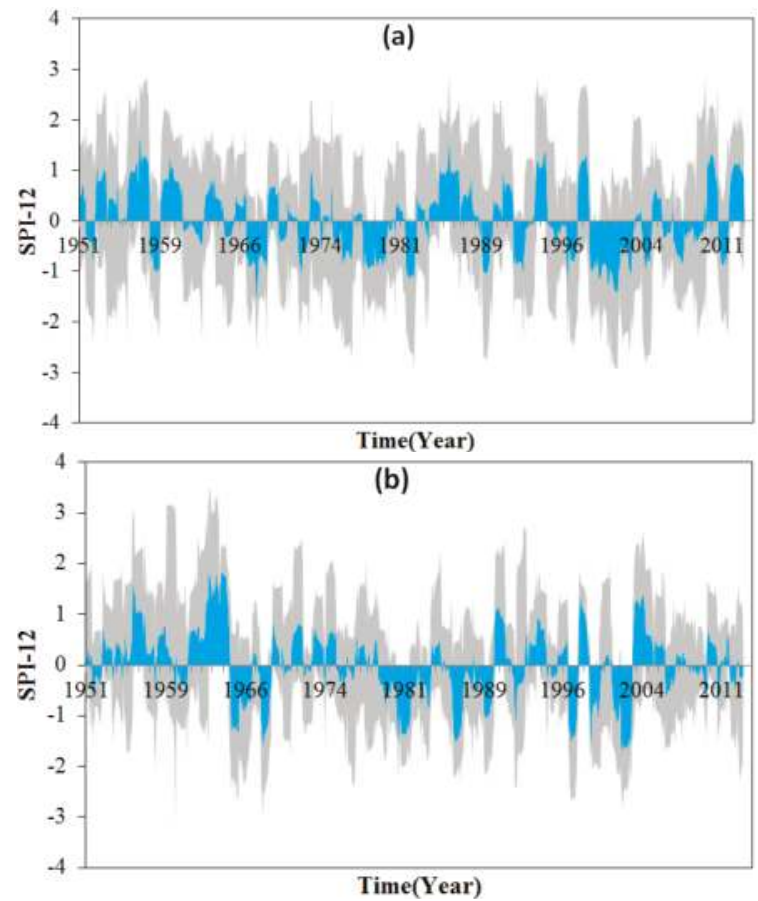

Figure 2. The SPI values for 1951-2013 for (a) Northeast China and (b) Huang-Huai-Hai.

In the 1950s, the MLRY crop-growing area was relatively wet (Figure 3a). However, it was extremely dry in the following 10 years, and the drought reached its peak in 1966. In the 1970s, the wet periods and droughts changed over time spans of three years, and the drought intensified at the end of the 1970s. This extreme drought was the driest period from 1951 to 2013. In the 1980s and 1990s, the area was quite wet. In the 21st century, the intensity of drought in this region was enhanced. In the year of 2004 and 2011, the area was impacted by an extreme drought.

Figure $3 \mathrm{~b}$ indicates that the most intense drought in the past 60 years occurred during the 1950 s to 1960s. At the end of the 1970s, the drought lessened slightly. The mid-1980s experienced relatively wet periods. In the following 10 years, the drought intensified again and reached its peak at the beginning of the 1990s. The lowest period of drought intensity occurred in the early 21st century. However, 
after 2003, the occurrences of drought became more frequent, and the area entered another relatively dry period.
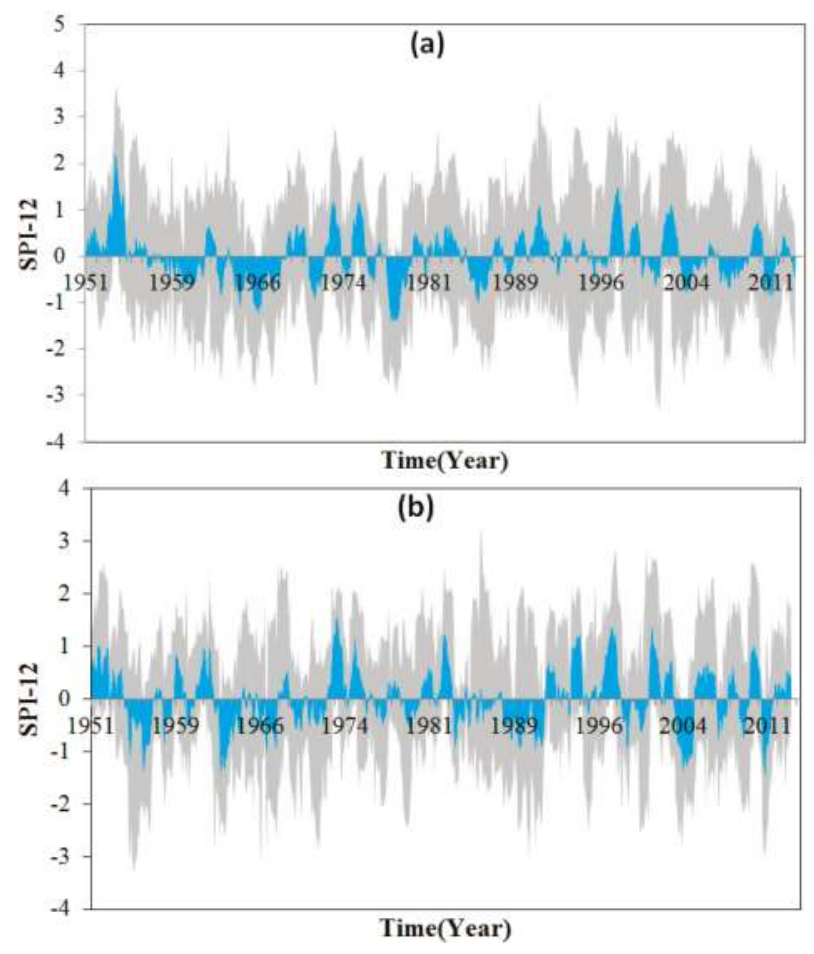

Figure 3. The SPI values for 1951-2013 for (a) MLRY and (b) South areas.

At the beginning of the 1950s, the Sichuan basin region experienced an extreme wet period, and at the end of the 1950s, a short, mild arid period occurred in the basin. In the next twenty years, except for the years around 1966 and 1970, this area experienced a wet period. At the end of the 1970s and at the beginning of the 1980s, the drought was intensified over this region. During the 1980s and 1990s, the drought gradually intensified and reached its peak in 1996. In the early 21st century, the drought in the area was alleviated. However, the drought gradually intensified until approximately 2004, when the most severe drought of the past 60 years occurred.

The variation of the drought in Southwest China was similar to that of the Sichuan basin, as shown in Figure 4b. A low-intensity drought occurred around 1960, and in the following 20 years, the occurrences of drought gradually decreased. Around 1980, this region experienced severe drought, similar to that in 1960. In the 1990s and the beginning of the 21st century, the drought intensity decreased, and this region experienced a wet period. However, after 2008, the drought intensified again and the most extreme drought of the past 60 years occurred. Overall, the trends on drought occurrences in the Sichuan area and Southwest China were similar around 1960 and 1980. After 2008, the droughts in both areas intensified significantly. 

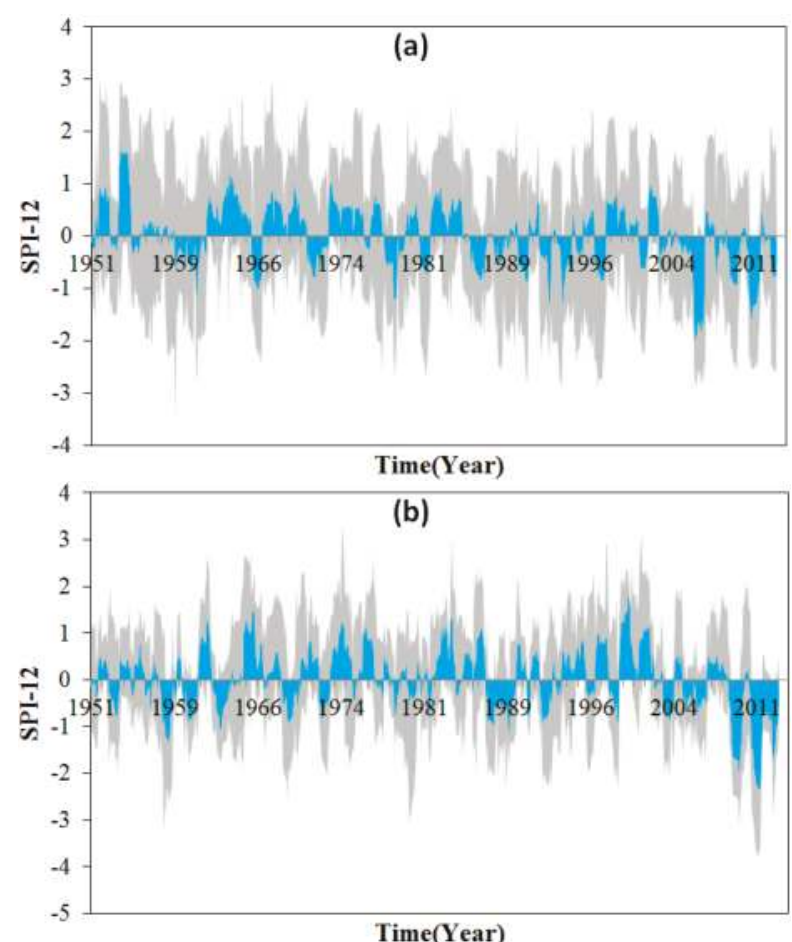

Figure 4. The SPI values for 1951-2013 for (a) Sichuan basin and (b) Southwest China.

\subsection{Drought Results by Using TRMM Data}

Although some spatial interpolations that use interpolation techniques (e.g., Inverse Distance Weighted (IDW), Kriging etc. [34],) may help to infer drought conditions at large scales, they still face high uncertainties [33]. For example, altitude differences in mountainous regions and the random property of precipitation makes it very difficult to predict the correct values near in the adjacent areas. Compared to ground station gauge rainfall data, the advantage of TRMM data is that TRMM data provide better spatial coverage. On the other hand, as shown in Sections 3 and 5.1, the time span of 6 to 24 months can be used to analyze the periodicities and variations in the drought over a long time period, and the SPI calculated by the TRMM data at s time scale of 12 months was not very accurate for evaluating the change in drought and. Hence, the SPI at a time scale of 6 months was selected to monitor drought in this section.

The frequencies of moderate, severe, and extreme drought are presented in Figure 5 to show the spatial distribution of different intensities of drought. Figure 5 shows that there are significant spatial differences in the probability of different intensity droughts among each crop-growing region. The northeast region suffered less extreme droughts than other regions but a high frequency of moderate droughts. The southeastern part of the Huang-Huai-Hai region suffered a high frequency of extreme droughts, but the north Huang-Huai-Hai region presented a high frequency of moderate and severe droughts. The north region of the MLRY region presented a high frequency of extreme droughts, whereas the frequency of moderate droughts was high in the south MLRY region. For the south region, the extreme droughts occurred less frequently in this region, but there was often a high frequency of moderate droughts in this region. Both the Sichuan and southwest crop-growing regions were less influenced by moderate droughts. The frequency of extreme droughts was quite high in the east part of the southwest region and the northeastern part of the Sichuan region. 


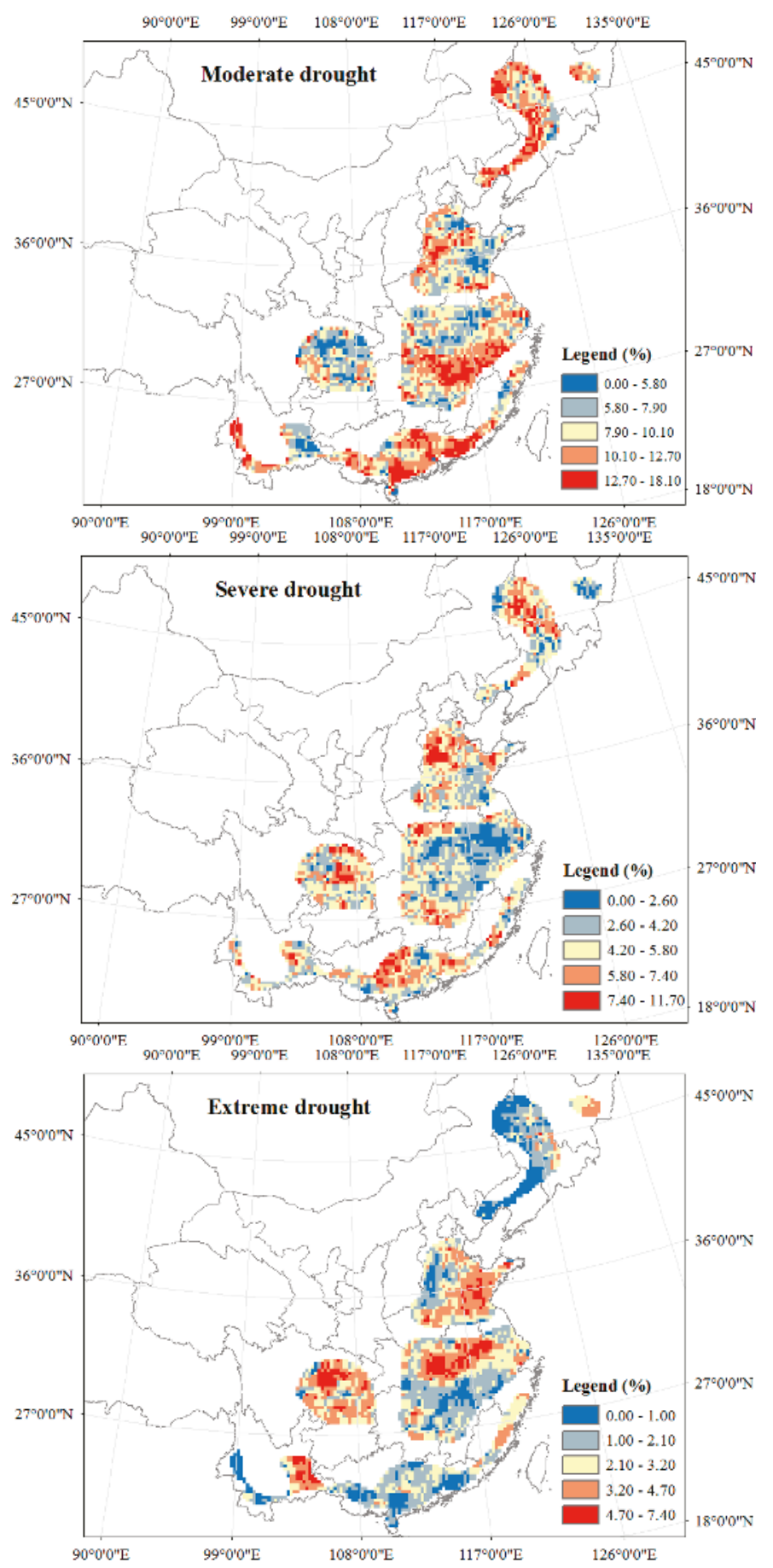

Figure 5. Frequencies of moderate droughts, severe droughts and extreme droughts for different regions from 1998 to 2013. 
In addition, the area percentages of the crop-growing regions under different drought intensities are presented in Figure 6 to further analyze the drought changes in spatial and temporal scales. In Figure 6, moderately dry regions are depicted in peach, severely dry regions are depicted in orange, and extremely dry regions are depicted in red.
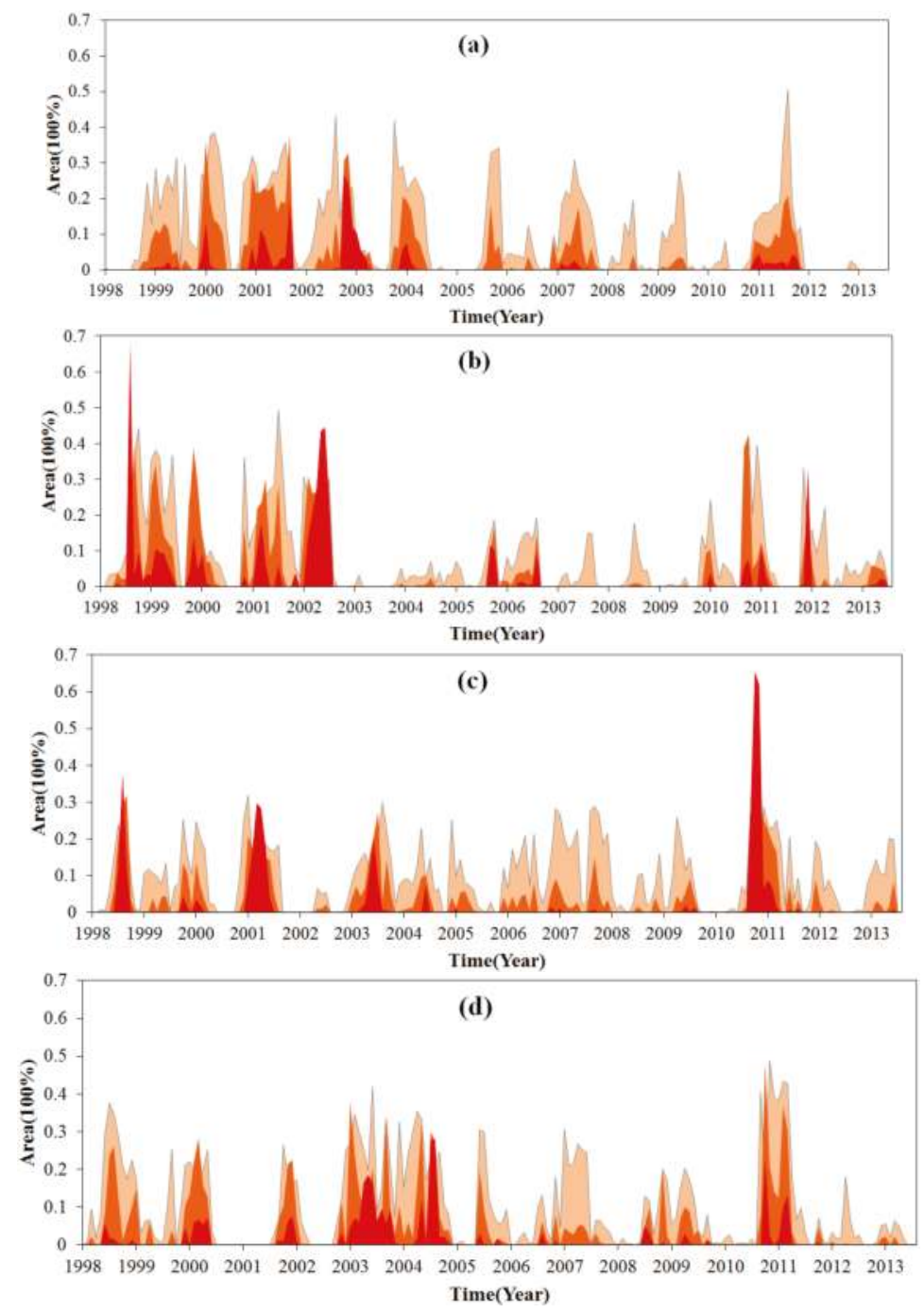

Figure 6. Cont. 

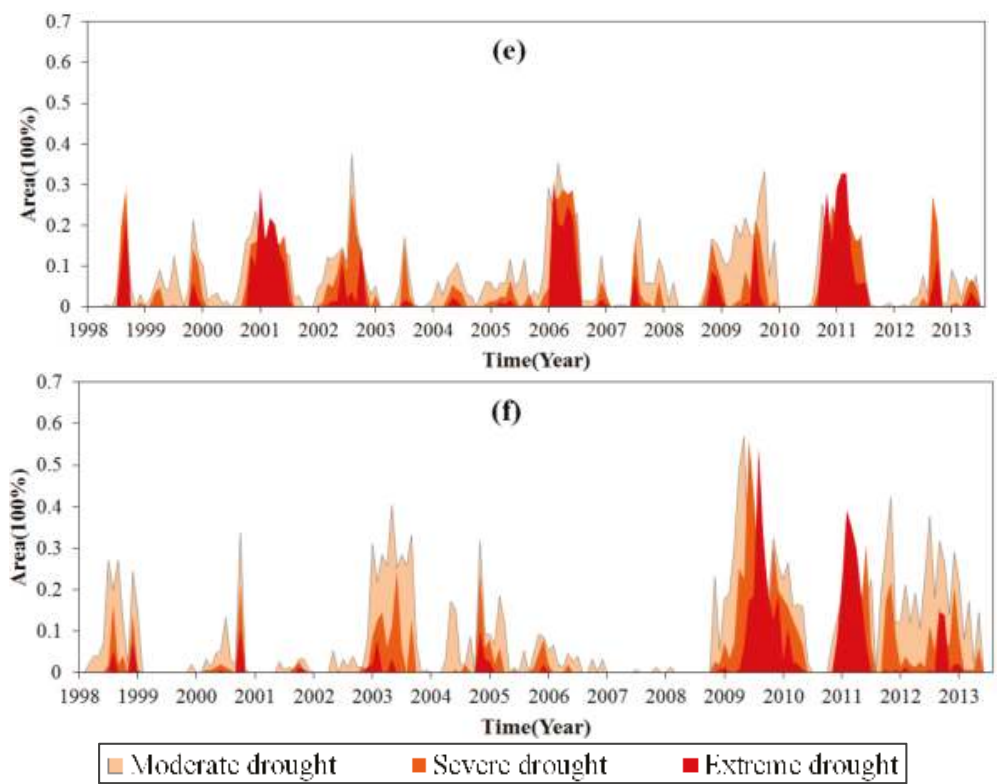

Figure 6. TRMM 3B43 for drought monitoring (SPI at time scale of six months) for different regions: (a) Northeast; (b) Huang-Huai-Hai; (c) MLRY; (d) South; (e) Sichuan basin; and (f) Southwest.

Figure 6 shows that the driest period in terms of drought severity was 2003 for the northeast region and that no severe drought occurred in this region from 1998 to 2010. In all, the area and frequency of extreme droughts in this region were lower than other regions. For the Huang-Huai-Hai region, the drought was severe from 1998 to 2002, but from 2003 to 2013, only a small percentage of this region suffered drought. The MLRY region suffered extreme drought in 2011, and more than $60 \%$ of the area experienced extreme drought. At the end of 1998 and 2001, this region also experienced extreme drought. The area and frequency of extreme drought in South China were quite low in 1998 to 2013, and this region experienced extreme drought in 2003, 2005, and 2011. The Sichuan basin suffered extreme drought in 2001,2006, and 2011, and the characteristics of droughts in this region were that the percentage of severe drought accounted for a larger proportion than droughts of other intensities. The Southwest region suffered quite a wet period before 2009, but this region suffered severe drought after 2009. In all, the North of China (the Huang-Huai-Hai and the Northeast regions) experienced severe drought from 1998 to 2003, whereas the MLRY, the South, the Southern, and the Sichuan regions experienced severe drought around 2011.

\section{Discussion}

\subsection{Reliability of Using the TRMM Data}

Since satellite-derived data estimates precipitation indirectly and depends on the properties of cloud tops and path-integrated hydrometeor content [34], the results of retrieval precipitation data must be evaluated. The validation of TRMM data has been widely conducted, and the study performed by Chen et al. [35] showed that the TRMM 3B43 achieved reasonable accuracy in most regions of China, which includes the study regions of the study.

Another problem of using TRMM data to calculate SPI is that SPI calculations often require more than 40 years of accumulated precipitation, but TRMM data only include rainfall for 15 years, which is a short length of time to calculate the SPI. There is no comparison between the SPI values 
calculated by TRMM data and gauge data in the study regions. Hence, it is a prerequisite to evaluate the reliability of using TRMM data to calculate the SPI before these data are used to monitor drought in the crop-growing regions.

The monthly gauge data with long records from 1951 to 2013, monthly TRMM 3B43 data were selected, and the TRMM data corresponding to the stations were extracted. The SPIs were calculated at time scales of one, three, six, and twelve months for each station and TRMM extracted data. Then, the SPIs for each station between 1998 and 2013 were selected to match the SPIs calculated by TRMM extracted data, and a multiple linear regression fit was used to compare the two SPIs. In general, the SPIs at time scales of one, three, and six months were significant with corresponding TRMM extracted data $(p<0.01)$, but the SPIs at a time scale of twelve months were not significant. Figure 7 presents the detailed coefficient of determination $\mathrm{R}^{2}$ for difference regions.
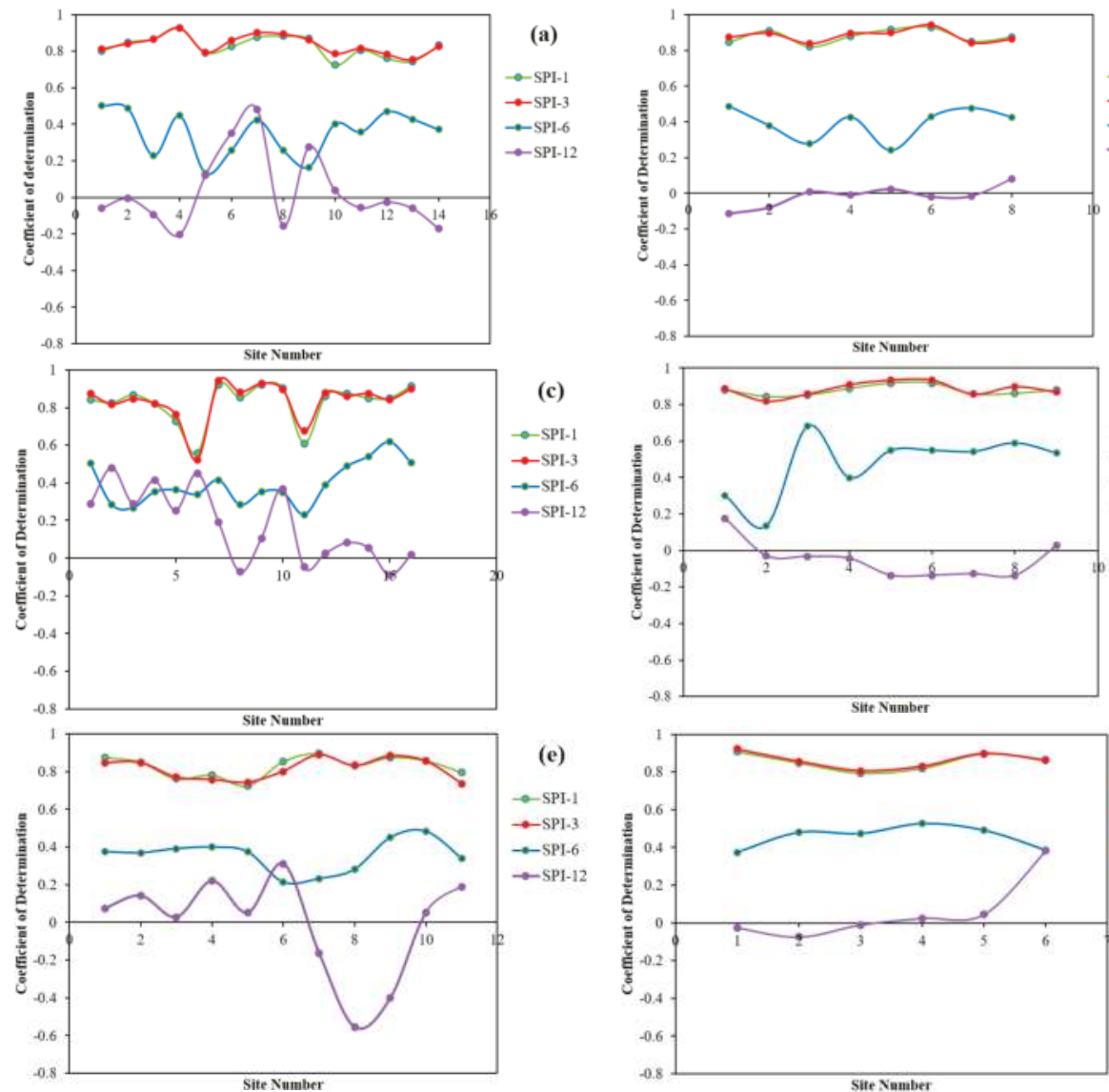

(d)

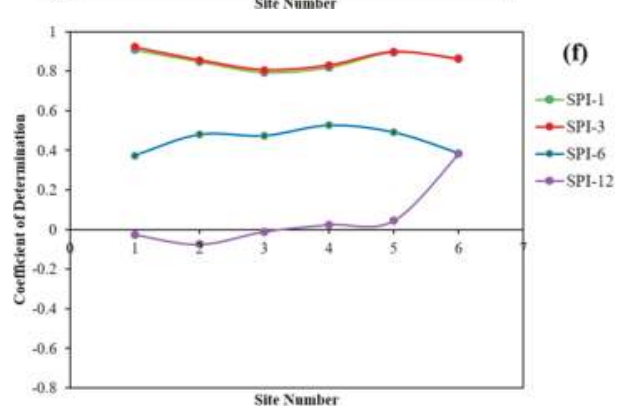

Figure 7. Coefficient of determination $\mathrm{R}^{2}$ between two datasets for difference regions: (a) Northeast; (b) Huang-Huai-Hai; (c) MLRY; (d) South; (e) Sichuan basin; (f) Southwest.

Figure 7 indicates that the correlations between the SPIs at time scales of 1, 3 months (station data and TRMM data) are quite high, as most of the correlations are greater than 0.8. More specifically, in addition to the Huang-Huai-Hai area, the other regions present high fitting coefficients, and the coefficients of the South of China, e.g., South China, Sichuan basin, Southwest China, are higher than those of North of China, e.g., the MLRY and Northeast. The reason for this may be due to 
the uncertainty of TRMM data to sense frozen precipitation in winter in the North of China [36]. For the SPIs at the time scale of 6 months, most of the coefficients of determination range from 0.3 to 0.5 for most regions. For the SPIs at the time scale of 12 months, the coefficients of determination are predominantly low, and this may be due to the short measurement series. As a whole, the SPIs calculated by using the TRMM data at time scales of 1,3 and 6 months are reliable for monitoring drought in the study regions.

\subsection{Comparison between TRMM and Station Data}

SPIs calculated at a time scale of 6 months by gauge data were selected, and are depicted as the gray filled areas shown in Figure 8. The mean SPIs for each region calculated by Equation (1) are presented as the blue filled areas. As can be seen in Figures 6-8, the changes in drought presented in Figure 8 from year of 1998 to 2013 were similar to the changes monitored by TRMM data shown in Figure 6. Most of the drought events detected by station gauge data were also detected by TRMM data; this means the TRMM data provide not only the spatial coverage of the drought, but also accurate drought intensities at specific time scale compared to station gauge data.
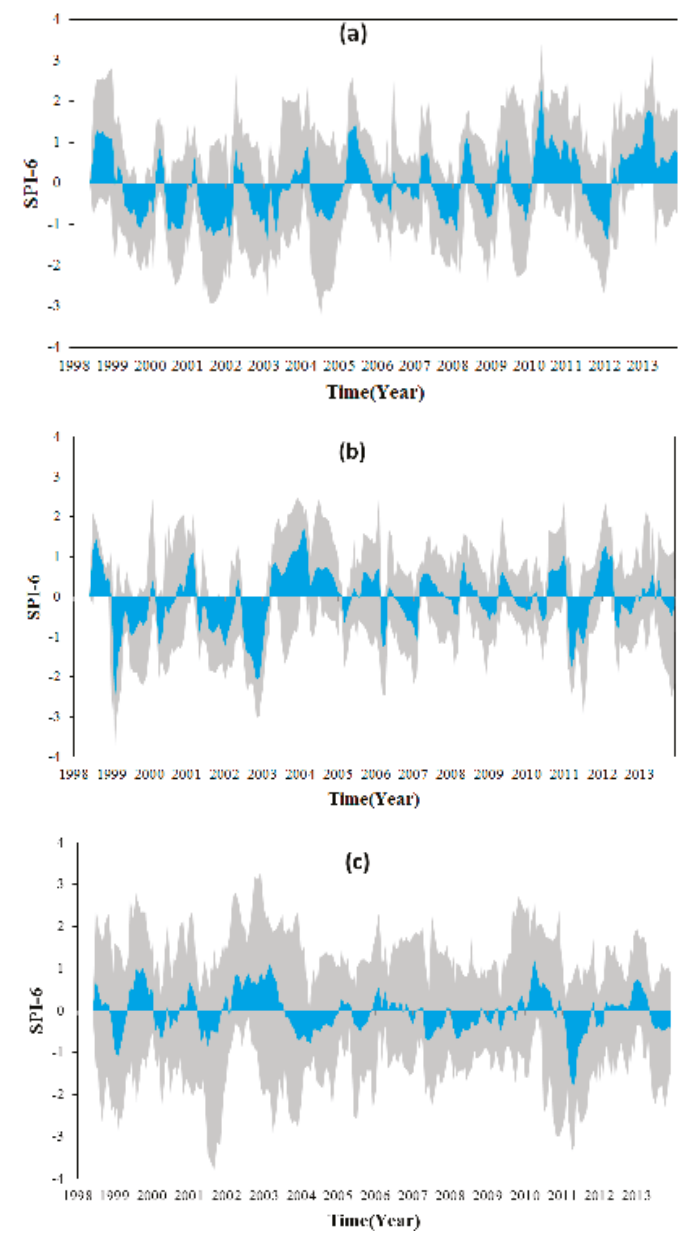

Figure 8. Cont. 

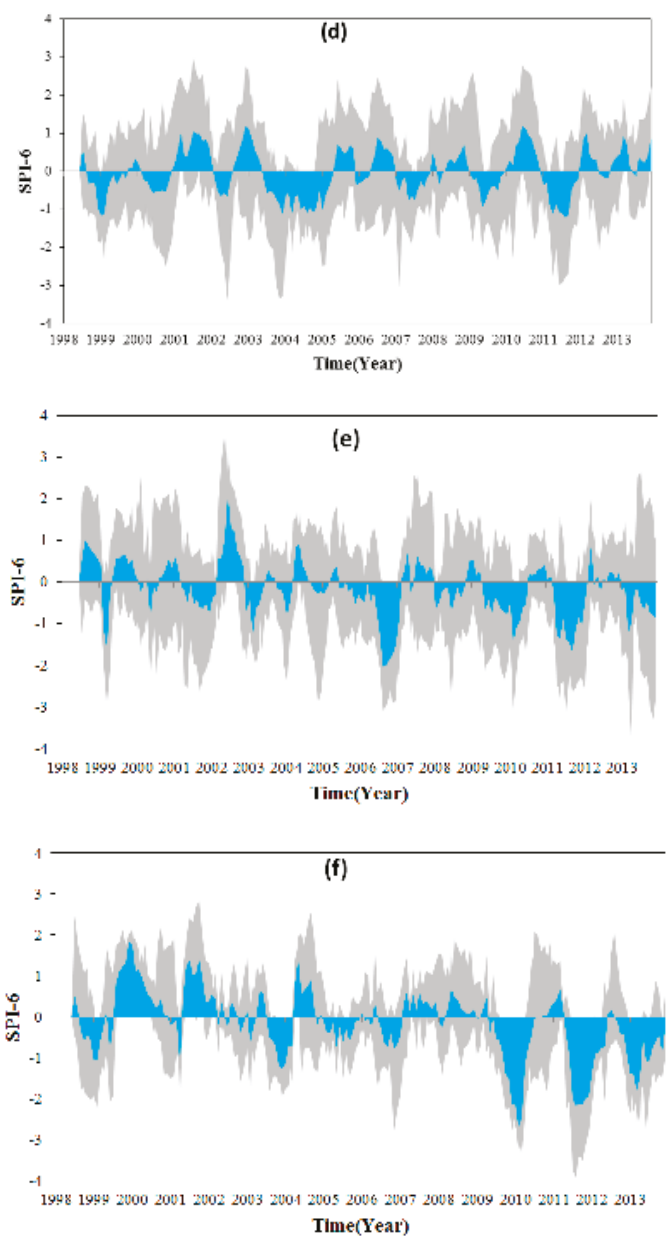

Figure 8. Gauge data for drought monitoring (SPI at time scale of 6 month) for difference regions: (a) Northeast, (b) Huang-Huai-Hai, (c) MLRY, (d) South, (e) Sichuan basin and (f) Southwest.

Droughts are climatic events caused by various factors. When the factors that influence precipitation do not satisfy the requirements for precipitation in an area, climatic abnormities occur, i.e., droughts or floods. In recent decades, the temperature across China has gradually increased. The increases in temperature inevitably accelerate surface evapotranspiration. In addition, industrialization in China is consuming more and more water resources. Thus, reasonable approaches to coping with severe drought include constructing additional water conservation facilities in areas with a high frequency of severe, extreme droughts and improving water use efficiency in agricultural production.

\section{Conclusions}

In this study, weather station data with longer recording histories and TMI precipitation data were used to calculate the SPI values for the major crop-growing areas of China. The analyses of drought based on weather station data showed that the driest periods in the past 60 years were at the end of the 1960s, the years around 1980 and the beginning of the 21st century in the Northeast 
and Huang-Huai-Hai areas; 1966 and 1980 for the MLRY region; and the middle of the 1950s for South China. For the Sichuan basin and Southwest China areas, after 2005, these regions suffered the most extreme drought in the past 60 years.

The validation based on station gauge rainfall data showed that the SPIs calculated using TRMM data at time scales of one, three, and six months were reliable for monitoring drought in crop-growing regions. The analyses of drought based on TMI data from 1989 to 2013 showed that the frequency of different intensity droughts presented significant spatial heterogeneity in each crop-growing region. The extreme droughts mainly occurred in the east part of the southwest region and the northeastern part of the Sichuan region, whereas the north part of the MLRY region, the southeastern part of the Huang-Huai-Hai region, and the Northeast region suffered less extreme drought than the other regions. The droughts monitored by TRMM data were almost coincident with gauged data.

Acknowledgments: This work was supported by the Natural Science Foundation of China (No. 41571427), Natural Key Project of China (No. 2016YFC0500203), Innovative group guide project (Grant No. Y2017JC33) and Open Fund of State Key Laboratory of Remote Sensing Science (Grant No. OFSLRSS201708).

Author Contributions: Lang Xia, Fen Zhao, and Kebiao Mao conceived the research. Lang Xia and Kebiao Mao wrote the manuscript. Lang Xia, Fen Zhao and Kebiao Mao edited the manuscript. Lang Xia, Fen Zhao, Kebiao Mao, Tongren $\mathrm{Xu}, \mathrm{Zijin}$ Yuan, and Zhiyuan Zuo contributed to the data analyses. All the authors reviewed the manuscript.

Conflicts of Interest: The authors declare no conflicts of interest.

\section{References}

1. Hyman, G.; Fujisaka, S.; Jones, P.G.; Wood, S.; De Vicente, M.C.; Dixon, J. Strategic approaches to targeting technology generation: Assessing the coincidence of poverty and drought-prone crop production. Agric. Syst. 2008, 98, 50-61. [CrossRef]

2. Dai, A. Drought under global warming: A review. Wiley Interdiscip. Rev. Clim. Chang. 2011, 2, 45-65. [CrossRef]

3. Dai, A. Increasing drought under global warming in observations and models. Nat. Clim. Chang. 2013, 3, 52-58. [CrossRef]

4. Chen, H.; Sun, J. Changes in Drought Characteristics over China Using the Standardized Precipitation Evapotranspiration Index. J. Clim. 2015, 28, 5430-5447. [CrossRef]

5. Heim, R.R., Jr. A review of twentieth-century drought indices used in the United States. Bull. Am. Meteorol. Soc. 2002, 83, 1149-1165.

6. Thornthwaite, C.W. An approach toward a rational classification of climate. Geogr. Rev. 1948, 38, 55-94. [CrossRef]

7. Van Bavel, C.H.M.; Verlinden, F.J. Agricultural Drought in North Carolina: Technicial Bulletin No. 122; Agriclultural Experiment Station, North Carolina State College: Raleigh, NC, USA, 1956.

8. Palmer, W.C. Meteorological Drought; US Department of Commerce, Weather Bureau: Washington, DC, USA, 1965.

9. McKee, T.B.; Doesken, N.J.; Kleist, J. The relationship of drought frequency and duration to time scales. In Proceedings of the Eighth Conference on Applied Climatology, Anaheim, CA, USA, 17-22 January 1993; pp. 179-184.

10. Zhang, Q.; Zou, X.K.; Xiao, F.J.; Lü, H.Q.; Liu, H.B.; Zhu, C.H.; Zhu, S.Q. An Classification of Meteorological Drought; China Meteorological Press: Beijing, China, 2008; pp. 33-53. (In Chinese)

11. Vicente-Serrano, S.M.; Beguería, S.; López-Moreno, J.I. A multiscalar drought index sensitive to global warming: The standardized precipitation evapotranspiration index. J. Clim. 2010, 23, 1696-1718. [CrossRef]

12. Beguería, S.; Vicente-Serrano, S.M.; Reig, F.; Latorre, B. Standardized precipitation evapotranspiration index (SPEI) revisited: Parameter fitting, evapotranspiration models, tools, datasets and drought monitoring. Int. J. Climatol. 2014, 34, 3001-3023. [CrossRef]

13. Sandholt, I.; Rasmussen, K.; Andersen, J. A simple interpretation of the surface temperature-vegetation index space for assessment of surface moisture status. Remote Sens. Environ. 2002, 79, 213-224. [CrossRef]

14. Yao, Y.; Liang, S.; Qin, Q.; Wang, K. Monitoring Drought over the Conterminous United States Using MODIS and NCEP Reanalysis-2 Data. J. Appl. Meteorol. Climatol. 2010, 49, 1665-1680. [CrossRef] 
15. Quiring, S.M.; Ganesh, S. Evaluating the utility of the Vegetation Condition Index (VCI) for monitoring meteorological drought in Texas. Agric. For. Meteorol. 2010, 150, 330-339. [CrossRef]

16. Zhang, A.; Jia, G. Monitoring meteorological drought in semiarid regions using multi-sensor microwave remote sensing data. Remote Sens. Environ. 2013, 134, 12-23. [CrossRef]

17. Jerrod, L.; Sweeney, A.; Ceccato, P. An agricultural drought severity index using quasi-climatological anomalies of remotely sensed data. Int. J. Remote Sens. 2016, 37, 913-925.

18. Tigkas, D.; Vangelis, H.; Tsakiris, G. An enhanced effective Reconnaissance drought index for the characterisation of agricultural drought. Environ. Process. 2017, 4, 1-12. [CrossRef]

19. Li, Z.; Hao, Z.; Shi, X.; Déry, S.J.; Li, J.; Chen, S.; Li, Y. An agricultural drought index to incorporate the irrigation process and reservoir operations: A case study in the Tarim River Basin. Glob. Planet. Chang. 2016, 143, 10-20. [CrossRef]

20. Barriopedro, D.; Gouveia, C.M.; Trigo, R.M.; Wang, L. The 2009/10 drought in China: Possible causes and impacts on vegetation. J. Hydrometeorol. 2012, 13, 1251-1267. [CrossRef]

21. Song, L.; Deng, Z.; Dong, A. Hot Topics of Global Change_Drought; China Meteorological Press: Beijing, China, 2003. (In Chinese)

22. Zou, X.; Zhai, P.; Zhang, Q. Variations in droughts over China: 1951-2003. Geophys. Res. Lett. 2005, 32, 4. [CrossRef]

23. Zhai, J.; Su, B.; Krysanova, V.; Vetter, T.; Gao, C.; Jiang, T. Spatial variation and trends in PDSI and SPI indices and their relation to streamflow in 10 large regions of China. J. Clim. 2010, 23, 649-663. [CrossRef]

24. Wang, A.; Lettenmaier, D.; Sheffield, J. Soil moisture drought in China, 1950-2006. J. Clim. 2011, 24, 3257-3271. [CrossRef]

25. Lin, W.; Wen, C. Characteristics of multi-timescale variabilities of the drought over last 100 years in Southwest China. Adv. Meteorol. Sci. Technol. 2012, 2, 21-26.

26. Zhang, M.; He, J.; Wang, B.; Wang, S.; Li, S.; Liu, W.; Ma, X. Extreme drought changes in Southwest China from 1960 to 2009. J. Geogr. Sci. 2013, 23, 3-16. [CrossRef]

27. Yu, M.; Li, Q.; Hayes, M.J.; Svoboda, M.D.; Heim, R.R. Are droughts becoming more frequent or severe in China based on the standardized precipitation evapotranspiration index: 1951-2010. Int. J. Climatol. 2014, 34, 545-558. [CrossRef]

28. Tao, H.; Fischer, T.; Zeng, Y.; Fraedrich, K. Evaluation of TRMM 3B43 precipitation data for drought monitoring in Jiangsu Province, China. Water 2016, 8, 221. [CrossRef]

29. Zeng, H.; Li, L.; Li, J. The evaluation of TRMM Multisatellite Precipitation Analysis (TMPA) in drought monitoring in the Lancang River Basin. J. Geogr. Sci. 2012, 22, 273-282. [CrossRef]

30. Kummerow, C.; Simpson, J.; Thiele, O.; Barnes, W.; Chang, A.T.C.; Stocker, E.; Ashcroft, P. The status of the Tropical Rainfall Measuring Mission (TRMM) after two years in orbit. J. Appl. Meteorol. 2000, 39, 1965-1982. [CrossRef]

31. Wang, B.; Wu, Z.; Li, J.; Liu, J.; Chang, C.P.; Ding, Y.; Wu, G. How to measure the strength of the East Asian summer monsoon. J. Clim. 2008, 21, 4449-4463. [CrossRef]

32. Nicholson, S.E.; Some, B.; McCollum, J.; Nelkin, E.; Klotter, D.; Berte, Y.; Noukpozounkou, J.N. Validation of TRMM and other rainfall estimates with a high-density gauge dataset for West Africa, Part II: Validation of TRMM rainfall products. J. Appl. Meteorol. 2003, 42, 1355-1368. [CrossRef]

33. De Jesús, A.; Breña-Naranjo, J.A.; Pedrozo-Acuña, A.; Alcocer Yamanaka, V.H. The use of TRMM 3B42 product for drought monitoring in Mexico. Water 2016, 8, 325. [CrossRef]

34. Cressie, N. The origins of kriging. Math. Geol. 1990, 22, 239-252. [CrossRef]

35. Smith, T.M.; Arkin, P.A.; Bates, J.J.; Huffman, G.J. Estimating Bias of Satellite-Based Precipitation Estimates. J. Hydrometeorol. 2006, 7, 841-856. [CrossRef]

36. Chen, F.; Li, X. Evaluation of IMERG and TRMM 3 B43 monthly precipitation products over mainland China. Remote Sens. 2016, 8, 472. [CrossRef]

(C) 2018 by the authors. Licensee MDPI, Basel, Switzerland. This article is an open access article distributed under the terms and conditions of the Creative Commons Attribution (CC BY) license (http:/ / creativecommons.org/licenses/by/4.0/). 
Article

\title{
Individual and Interactive Influences of Anthropogenic and Ecological Factors on Forest $\mathbf{P M}_{2.5}$ Concentrations at an Urban Scale
}

\author{
Guoliang Yun ${ }^{1,2}$, Shudi Zuo ${ }^{1,2,3}$, Shaoqing Dai ${ }^{1,2}$, Xiaodong Song ${ }^{4}$, Chengdong $\mathrm{Xu}^{5}$, \\ Yilan Liao ${ }^{5}$, Peiqiang Zhao ${ }^{1,3}$, Weiyin Chang ${ }^{6}$, Qi Chen ${ }^{7}$, Yaying Li ${ }^{1,3}$, Jianfeng Tang ${ }^{1,3}$, \\ Wang Man ${ }^{8}$ and Yin Ren ${ }^{1,3, *}$
}

1 Key Lab of Urban Environment and Health, Institute of Urban Environment, Chinese Academy of Sciences, Xiamen 361021, China; glyun@iue.ac.cn (G.Y.); sdzuo@iue.ac.cn (S.Z.); sqdai@iue.ac.cn (S.D.); pqzhao@iue.ac.cn (P.Z.); yyli@iue.ac.cn (Y.L.); jftang@iue.ac.cn (J.T.)

2 University of Chinese Academy of Sciences, Beijing 100049, China

3 Ningbo Urban Environment Observation and Research Station-NUEORS, Chinese Academy of Sciences, Ningbo 315800, China

4 College of Environment and Natural Resources, Zhejiang University, Hangzhou 310058, China; xdsongy@zju.edu.cn

5 State Key Laboratory of Resources and Environmental Information Systems, Institute of Geographic Sciences and Nature Resources Research, Chinese Academy of Sciences, Beijing 100101, China; xucd@Ireis.ac.cn (C.X.); liaoyl@Ireis.ac.cn (Y.L.)

6 College of Forestry, Fujian Agriculture and Forestry University, Fuzhou 350002, China; cwy.forest@gmail.com

7 Department of Geography, University of Hawai'i at Manoa, Honolulu, HI 96822, USA; qichen@hawaii.edu

8 Department of Spatial Information Science and Engineering, Xiamen University of Technology, Xiamen 361024, China; manwang@126.com

* Correspondence: yren@iue.ac.cn; Tel.: +86-592-619-0679

Received: 4 January 2018; Accepted: 24 March 2018; Published: 26 March 2018

\begin{abstract}
Integration of Landsat images and multisource data using spatial statistical analysis and geographical detector models can reveal the individual and interactive influences of anthropogenic activities and ecological factors on concentrations of atmospheric particulate matter less than 2.5 microns in diameter $\left(\mathrm{PM}_{2.5}\right)$. This approach has been used in many studies to estimate biomass and forest disturbance patterns and to monitor carbon sinks. However, the approach has rarely been used to comprehensively analyze the individual and interactive influences of anthropogenic factors (e.g., population density, impervious surface percentage) and ecological factors (e.g., canopy density, stand age, and elevation) on $\mathrm{PM}_{2.5}$ concentrations. To do this, we used Landsat- 8 images and meteorological data to retrieve quantitative data on the concentrations of particulates $\left(\mathrm{PM}_{2.5}\right)$, then integrated a forest management planning inventory (FMPI), population density distribution data, meteorological data, and topographic data in a Geographic Information System database, and applied a spatial statistical analysis model to identify aggregated areas (hot spots and cold spots) of particulates in the urban area of Jinjiang city, China. A geographical detector model was used to analyze the individual and interactive influences of anthropogenic and ecological factors on $\mathrm{PM}_{2.5}$ concentrations. We found that particulate concentration hot spots are mainly distributed in urban centers and suburbs, while cold spots are mainly distributed in the suburbs and exurban region. Elevation was the dominant individual factor affecting $\mathrm{PM}_{2.5}$ concentrations, followed by dominant tree species and meteorological factors. A combination of human activities (e.g., population density, impervious surface percentage) and multiple ecological factors caused the dominant interactive effects, resulting in increased $\mathrm{PM}_{2.5}$ concentrations. Our study suggests that human activities and multiple ecological factors effect $\mathrm{PM}_{2.5}$ concentrations both individually and interactively. We conclude that in order to reveal the direct and indirect effects of human activities
\end{abstract}


and multiple factors on $\mathrm{PM}_{2.5}$ concentrations in urban forests, quantification of fusion satellite data and spatial statistical methods should be conducted in urban areas.

Keywords: multisource data fusion; aerosol retrieval; urban scale; vegetation dust-retention; multiple ecological factors; geographical detector model

\section{Introduction}

As industrialization and urbanization have intensified, so has the concentration of fine particulates in the atmosphere. These particulates, known as $\mathrm{PM}_{2.5}$ (aerodynamic diameters $<2.5 \mu \mathrm{m}$ [1]), originate from vehicle exhaust, coal-fired power plants, building construction (dust), and domestic heating (coal). Fine particulates are not only detrimental to human health (respiratory problems, lung disease, etc. [2,3]), but they also cause global atmospheric changes [4].

At present, most studies of $\mathrm{PM}_{2.5}$ focus on their sources and methods for monitoring them [5]. However, studies examining the degree to which urban forests trap particulates, combined with data from forest management planning inventories (FMPI), remote sensing imagery, population density, and impervious surface percentages, are rare. These types of environmental data could be extremely helpful for managing urban forests and improving air quality [6]. Focusing on urban forests is important because urban forests help protect human health and improve environmental quality by improving air quality (e.g., forests absorb pollutants and reduce chemical reaction rates $[7,8]$ ). Tiwary [9] has linked urban forests with human health effects. In one study of a $10 \times 10 \mathrm{~km}$ grid in London with a tree coverage of $25 \%$, the urban forest was estimated to remove $900 \mathrm{t}$ of $\mathrm{PM}_{10}$ annually, which is the equivalent of preventing two deaths and two hospital admissions each year [10].

Most studies have investigated the effects of ecological factors (e.g., canopy density, leaf area index (LAI), and Normalized Difference Vegetation Index (NDVI)) on $\mathrm{PM}_{2.5}$ concentrations at ground level using a variety of instruments and statistical approaches. For instance, Jin [11] measured canopy density and LAI with a laser dust monitor and applied a mixed-effect model to quantitatively analyze the effect of particulates on vegetation. Similarly, Liu [12] used a TH-150C particulate sampler to obtain $\mathrm{PM}_{2.5}$ concentrations in sample plots and applied a multiple regression model to analyze the relationship between $\mathrm{PM}_{2.5}$ concentrations and NDVI and LAI. Although particle collectors can accurately capture $\mathrm{PM}_{2.5}$ concentrations in real time, these instruments are expensive to operate. Furthermore, because particle collectors require many sampling points to ensure accuracy, sampling is extremely labor intensive [13]. In addition, these ground-based studies used remote sensing indices and traditional statistical analyses, both of which have a number of limitations. First, single remote sensing indices (e.g., NDVI, Enhanced Vegetation Index (EVI), and LAI) are indicators of physiognomic parameters of vegetation, but cannot be used to quantify important site-specific parameters, such as soil depth, stand age, canopy density, and dominant tree species. Second, past studies have not considered that different tree species have significantly different PM-retaining capacities. Gao [14] pointed out that forest type and tree species differ significantly in their settlement rate of particulates. Finally, traditional statistical analyses only reveal the effects of single or multiple environmental factors and remote sensing indices on $\mathrm{PM}_{2.5}$ concentrations, ignoring anthropogenic factors. Past studies have also not considered whether multiple impact factors act individually or synergistically on $\mathrm{PM}_{2.5}$ concentrations [15].

Saebo [16] pointed out that in order to comprehensively understand the individual and interactive influences of anthropogenic and ecological factors on $\mathrm{PM}_{2.5}$ concentrations, Landsat images and multisource data must be integrated with a spatial statistical analysis that takes into account physical parameters of vegetation and underlying surface features. Therefore, in this paper, we use Landsat- 8 images and meteorological data to retrieve quantitative $\mathrm{PM}_{2.5}$ concentrations in an urban area. To do this, we created a GIS database that integrated multisource data (including Landsat-8 images, 
FMPI, population density distribution data, topographic data, and meteorological data) in a spatial statistical analysis model to identify specific areas of high concentration of $\mathrm{PM}_{2.5}$ (hot spots) and low concentration (cold spots) in various parts of the urban environment. A geographical detector model was used to analyze the independent and interactive influences of anthropogenic and ecological factors on urban forest $\mathrm{PM}_{2.5}$ concentrations.

The goal of this study was to analyze the independent and interactive influences of various factors on urban forest $\mathrm{PM}_{2.5}$ concentrations. The findings can be used to identify crucial impact factors of $\mathrm{PM}_{2.5}$ concentrations, which is an important first step for policymakers when managing air pollution, monitoring pollution, and estimating pollution exposure.

\section{Materials and Methods}

\subsection{Overview}

We approached this study in three steps. First, a GIS database was created that integrated multiple types of data (including Landsat- 8 images, FMPI, meteorological data, and population density distribution data). Second, areas with abnormally high concentrations of $\mathrm{PM}_{2.5}$ (hot spots) and abnormally low concentrations (cold spots) were identified using Global Moran's I and Getis-Ord $\mathrm{Gi}^{*}$ algorithms $[17,18]$. The optimal threshold distance was calculated using an incremental spatial autocorrelation module. Third, a geographical detector model was used to analyze the individual and interactive influences of anthropogenic and ecological factors on urban forest $\mathrm{PM}_{2.5}$ concentrations (these factors included human activities, topographic parameters, soil characteristics, meteorological factors, and vegetation characteristics).

\subsection{Developing a Multiple-Source Spatial Database}

The data used to model $\mathrm{PM}_{2.5}$ concentrations consisted of five distinct datasets. The first, obtained from the National Statistics Bureau, provided data on the population density distribution of Jinjiang, which was mapped using kernel density. The second dataset was composed of spatially explicit $\mathrm{PM}_{2.5}$ concentration data, which were retrieved from three Landsat- 8 images and verified using meteorological observation data. The three remote sensing images consisted of Row 119/Path 43 Landsat-8 images from 13 December 2014, 29 December 2014, and 14 January 2015, all acquired during winter under clear atmospheric conditions at approximately 10:29 a.m., local time. The third dataset comprised FMPI data obtained from the Jinjiang Forestry Bureau, which were collected every 10 years. The accuracy of the FMPI data was evaluated using stratified systematic sampling. The sampling accuracy of total stand volume was $90 \%$ and the reliability was $95 \%$ [19]. The FMPI of the attribute database included three parts: (1) forest characteristics (patch area, stand age, canopy density, and dominant tree species); (2) soil characteristics (soil depth, humus depth, and site index); and (3) topography (elevation, slope degree, slope position, and slope direction). The fourth dataset was meteorological data from the China Meteorological Forcing Dataset (doi:10.3972/westdc.0294.db) [20]. This dataset was produced by merging a variety of data sources. Its spatial resolution is 0.1 degree and its temporal resolution is $3 \mathrm{~h}$, and it can be used for hydrological modeling, land surface modeling, land data assimilation, and other terrestrial modeling. The dataset included seven parts (temperature, pressure, specific humidity, wind speed, downward shortwave radiation, downward longwave radiation, and precipitation rate). Four meteorological factors were studied in our research: temperature (TEM), pressure (PS), specific humidity (SH), and wind speed (WS). Our dataset can be obtained at http:/ / westdc.westgis.ac.cn/data/7a35329c-c53f-4267-aa07-e0037d913a21. The format of our meteorological data is a network common data format file (NetCDF) and we used ArcGIS10.3 (ArcGIS10.3.1) to convert the NetCDF file to a TIFF file. The fifth dataset is meteorological observation data obtained from the Jinjiang Meteorological Bureau. The $\mathrm{PM}_{2.5}$ spatial data and population density data were raster-based, while the FMPI data were vector-based. The vector and raster data differed in structure and form, so it was difficult to integrate these data. In order to solve the integration 
problem, we standardized the data by converting formats, transforming coordinates, and applying geometric corrections. We used forest patch size as the basic spatial unit and then calculated average population density and average $\mathrm{PM}_{2.5}$ concentrations for each patch using zonal, statistical tools.

\subsection{Calculation of $\mathrm{PM}_{2.5}$ Concentrations}

\subsubsection{Satellite-Derived Aerosol Optical Depth (AOD)}

We assumed that the land surface could be represented as a Lambert surface and that the atmospheric level was uniform. Apparent reflectance $\left(\rho_{t o a}\right)$ at the top of the atmosphere can be expressed as [21]

$$
\rho_{\text {toa }}\left(\theta_{s}, \theta_{v}, \phi\right)=\rho_{0}\left(\theta_{s}, \theta_{v}, \phi\right)+T\left(\theta_{s}\right) \cdot T\left(\theta_{v}\right) \cdot \frac{\rho_{s}\left(\theta_{s}, \theta_{v}, \phi\right)}{\left[1-\rho_{s}\left(\theta_{s}, \theta_{v}, \phi\right) \cdot S\right]}
$$

where $\theta_{S}$ is the solar zenith angle; $\theta_{v}$ is the satellite zenith angle; $\phi$ is the azimuth of the scattered radiation from the solar beam; $\rho_{0}$ is path radiance; $\rho_{S}$ is the angular surface reflectance; $S$ is the atmospheric backscattering ratio; $T\left(\theta_{S}\right)$ is the normalized downward flux for zero surface reflectance; and $T\left(\theta_{v}\right)$ represents upward total transmission into the satellite's field of view. We downloaded Landsat-8 data from the Geospatial Data Cloud (http:/ / www.gscloud.cn) and then used ENVI5.3 software to preprocess the imagery. The pretreatment process mainly included masking, radiation calibration, geometric correction, and calculating the apparent reflectance $\left(\rho_{\text {toa }}\right)$ of the atmosphere. The mid-infrared $(2.12 \mathrm{~mm}$ ) channel is less sensitive to aerosol scattering (because the wavelengths are larger than the size of most aerosol particles); however, the channels are sensitive to ground surface characteristics [22] and the apparent reflectance of mid-infrared at the top of the atmosphere, the angular surface reflectance of the red band, and the angular surface reflectance of the blue band have a fixed function, this function was modified using a Dark Dense Vegetation (DDV) algorithm [23]. Next, we used the DDV algorithm to calculate the surface reflectance of the red and blue bands. An aerosol lookup table (LUT) was constructed using the Second Simulation of the Satellite Signal in the Solar Spectrum (6S) atmosphere transmission model for the blue and red bands [21]. Finally, we obtained the atmospheric status of the computed reflectance which best matched the apparent reflectance of the atmosphere and the corresponding AOD value [22].

\subsubsection{Calculation of $\mathrm{PM}_{2.5}$ from AOD Data}

AOD values indicate the accumulation of the extinction coefficient in the entire atmospheric column. The $\mathrm{PM}_{2.5}$ concentrations only represent the near-surface "dry" aerosol extinction coefficient. An estimate of $\mathrm{PM}_{2.5}$ concentrations from AOD data alone would produce large uncertainties. Therefore, we had to account for changes in $\mathrm{PM}_{2.5}$ concentrations with elevation and correct for relative humidity in order to reduce these uncertainties [24]. Vertical and humidity corrections are needed to calculate $\mathrm{PM}_{2.5}$ concentrations in urban forests from AOD data. To do this, the Height of Planetary Boundary Layer (HPBL) was incorporated into the vertical correction, while the effects of hygroscopic growth were used to determine the humidity correction $[25,26]$. In this study, AOD data were converted to a near-surface "dry" aerosol extinction coefficient and their correlation relationships with ground $\mathrm{PM}_{2.5}$ concentrations $[27,28]$. The correlation between AOD and the surface aerosol extinction coefficient was affected by the vertical distribution of aerosols, so we determined the vertical correction for $\mathrm{PM}_{2.5}$ concentrations using horizontal visibility to calculate the surface aerosol extinction coefficient. We also needed to correct for humidity because the meteorological stations measured $\mathrm{PM}_{2.5}$ concentrations at a specific relative humidity, so we used relative humidity data to calculate the "dry" aerosol extinction coefficient. Next, we performed module estimations. Wang [29] found that it was possible to develop linear correlative models between a "dry" aerosol extinction coefficient and $\mathrm{PM}_{2.5}$ concentration. We used the "dry" aerosol extinction coefficient and $\mathrm{PM}_{2.5}$ concentrations collected in real time (at twelve ground monitoring sites) to establish models 
based on their correlation and estimate $\mathrm{PM}_{2.5}$ concentrations. Our results indicated that the Landsat estimation of $\mathrm{PM}_{2.5}$ correlates with ground-based measurements on 13 December 2014, 29 December 2014, and 14 January 2015, with $\mathrm{R}^{2}=0.68,0.66$, and 0.72 , respectively (Figure 1 ). For additional details, please see Supplementary Materials.
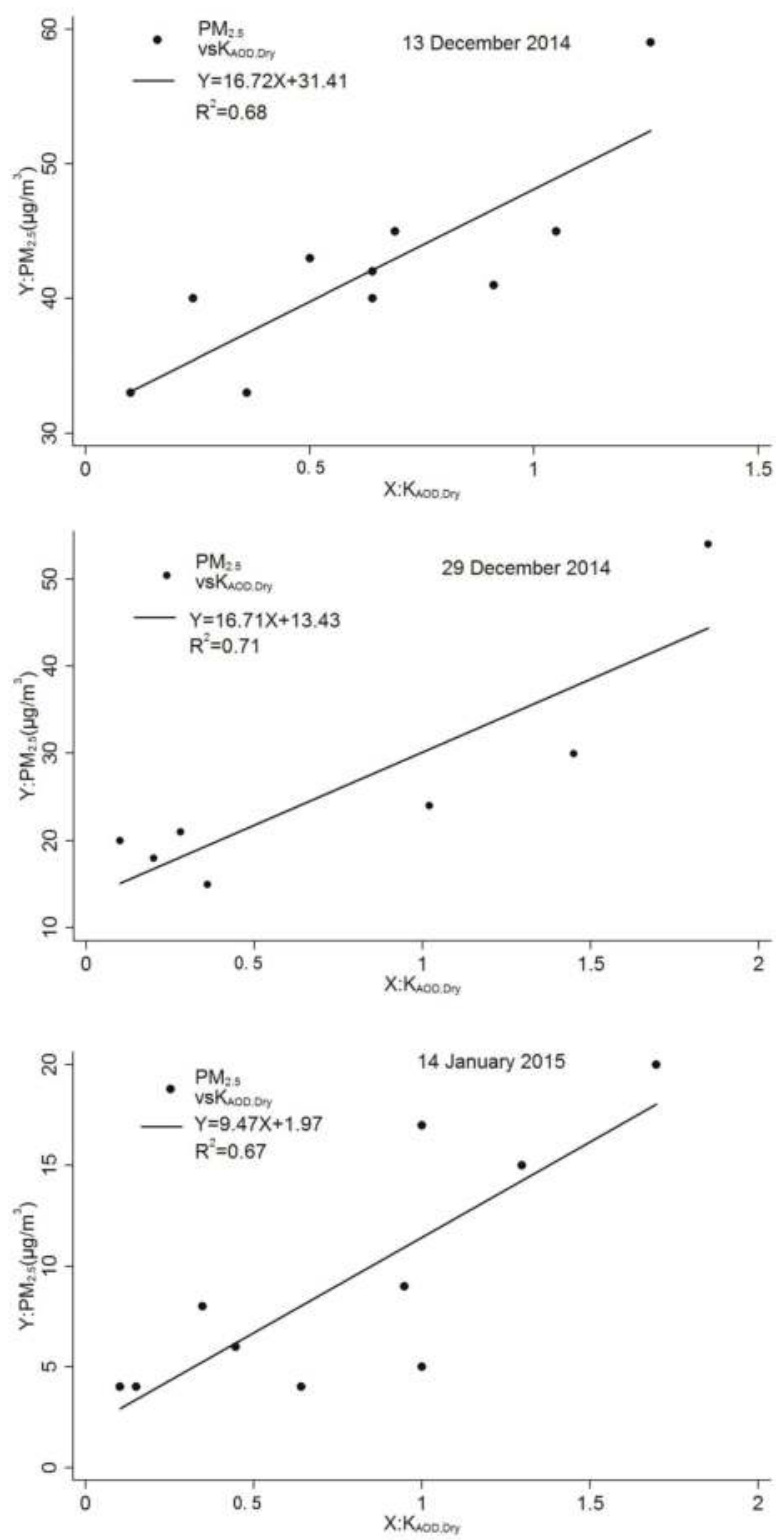

Figure 1. Correlation between $k_{A O D, D r y}$ and $\mathrm{PM}_{2.5}$ concentrations on 13 December 2014, 29 December 2014, and 14 January 2015 (both acquired from Environmental Protection of Jinjiang $k_{A O D, D r y}$ represents the aerosol extinction coefficient in dry conditions). 


\subsection{Spatial Statistical Analysis}

We used Global Moran's I spatial autocorrelation method to determine the value of the relationship between a feature's location and its attributes. We used the Getis-Ord Gi* spatial statistical method to provide us with a set of weighted values and identify statistically significant hot spots and cold spots on the ground. (Hot spots represent positions of statistically significant clustering of high $\mathrm{PM}_{2.5}$ concentrations $(p$-value $<0.05)$ and cold spots represent positions of statistically significant clustering of low $\mathrm{PM}_{2.5}$ concentrations ( $p$-value < 0.05) [30]). Both Moran's I and Getis-Ord Gi* have been used in a variety of ways, such as for helping to predict urban development patterns, analyzing pollution patterns, and examining traffic accident patterns [31,32]. We used the Moran's I index to first analyze the spatial autocorrelation of $\mathrm{PM}_{2.5}$. We then used the Getis-Ord $\mathrm{Gi}^{*}$ index to identify areas of aggregated $\mathrm{PM}_{2.5}$ (hot spots and cold spots). However, the Getis-Ord $\mathrm{Gi}^{*}$ module demands that optimal threshold distances (i.e., distances at which spatial processes that promote clustering are most obvious) be supplied before it can analyze spatial data. Therefore, we used the incremental spatial autocorrelation module to determine the optimal distance threshold for aggregated areas [33]. Measures of spatial autocorrelation for a range of distances and optimal distances establish a line graph for those distances and their corresponding z-scores. Z-scores demonstrate the intensity of spatial clustering. However, statistically significant peak z-scores reveal distances where the spatial processes that promote clustering are most obvious. We used the incremental spatial autocorrelation module to determine the optimal distance threshold by increasing the threshold distance from 500 to $7000 \mathrm{~m}$ at intervals of $500 \mathrm{~m}$ until it reached its maximum value $(3500 \mathrm{~m})$. Therefore, we defined $3500 \mathrm{~m}$ as the optimal distance threshold in our study.

\subsection{Geographical Detector Model Description}

The geographical detector is a statistical tool for detecting spatially stratified heterogeneity and revealing the factors responsible for the heterogeneity [34]. In our study, we used a range of detectors designed to assess ecological and anthropogenic factors associated with $\mathrm{PM}_{2.5}$ concentrations as determined by spatial variance analysis (SVA). The fundamental idea of SVA is to measure the spatial consistency of $\mathrm{PM}_{2.5}$ concentration distribution versus ecological factors (e.g., forest, soils, topography) and anthropogenic factors (e.g., population density). The power of determinant (PD) of different impact factors on $\mathrm{PM}_{2.5}$ concentration can be expressed using Equation (2):

$$
\mathrm{PD}=1-\frac{1}{N * \sigma^{2}} \sum_{i=1}^{L} N_{i} * \sigma_{i}^{2}
$$

A detailed explanation of Equation (2) is as follows:

$$
\mathrm{PD}=\mathrm{q}=1-\frac{\sum_{h=1}^{l} \sum_{i=1}^{N_{h}}\left(Y_{h i}-\bar{Y}_{h}\right)^{2}}{\sum_{i=1}^{N}\left(Y_{i}-\bar{Y}\right)^{2}}=1-\frac{\sum_{h=1}^{l} N_{h} \sigma_{h}^{2}}{N \sigma^{2}}=1-\frac{S S W}{S S T}
$$

where the total sum of squares is

$$
S S T=\sum_{i}^{N}\left(Y_{i}-\bar{Y}\right)^{2}=N \sigma^{2}
$$

and the sum of squares within is

$$
S S W=\sum_{h=1}^{l} \sum_{i}^{N_{h}}\left(Y_{h i}-\bar{Y}_{h}\right)^{2}=\sum_{h=1}^{l} N_{h} \sigma_{h}^{2}
$$


where $\mathrm{PD}$ is the power of determinant of impact factors on $\mathrm{PM}_{2.5}$ concentration; $N$ is the number of forest patches and is stratified into $h=1, \ldots, 2, L$ strata; stratum $h$ is composed of $N_{h}$ units; $Y_{i}$ and $Y_{h i}$ denote the value of unit $i$ in the population and in stratum $h$, respectively; the stratum mean is $\bar{Y}_{h}=\left(\frac{1}{N_{h}}\right) \sum_{i=1}^{N_{h}} Y_{h i}$; the stratum variance is $\sigma_{h}^{2}=\left(\frac{1}{N_{h}}\right) \sum_{i}^{N_{h}}\left(Y_{h i}-Y_{h}\right)^{2}$; the population mean is $Y=\left(\frac{1}{N_{h}}\right) \sum_{i=1}^{N} Y_{i}$; and the population variance of $\mathrm{PM}_{2.5}$ concentrations of the entire region is $\sigma^{2}=\left(\frac{1}{N_{h}}\right) \sum_{i}^{N_{h}}\left(Y_{i}-Y\right)^{2}$. PD $\in[0,1]$, where PD approaches 0 , implies that the determinant is completely unrelated to the $\mathrm{PM}_{2.5}$ concentrations. Where $\mathrm{PD}$ approaches 1 , the determinant completely controls $\mathrm{PM}_{2.5}$ concentration. PD has a corresponding $p$-value that can be used to evaluate uncertainty [35]. For additional details, please see Supplementary Materials. Based on this idea, we used geographical detector tools (factor detector and interaction detector) to detect various factors that may influence $\mathrm{PM}_{2.5}$ concentrations, the degree of influence of each factor, and the interactions between factors based on spatial analysis of variance [36]. The factor detector quantifies the impact of ecological and anthropogenic factors on an observed spatial $\mathrm{PM}_{2.5}$ pattern. The interaction detector probes whether two impact factors taken together enhance or weaken each other, or whether they affect $\mathrm{PM}_{2.5}$ concentrations independently [37].

$$
\begin{gathered}
\text { Enhance, nonlinear : } \operatorname{PD}\left(X_{1} \cap X_{2}=X 3\right)>\operatorname{PD}\left(X_{1}\right)+\operatorname{PD}\left(X_{2}\right), \\
\text { Independent : } \operatorname{PD}\left(X_{1} \cap X_{2}=X 3\right)=\operatorname{PD}\left(X_{1}\right)+\operatorname{PD}\left(X_{2}\right), \\
\text { Enhance, bi : } \operatorname{PD}\left(X_{1} \cap X_{2}=X 3\right)>\operatorname{Max}\left(\operatorname{PD}\left(X_{1}\right), \operatorname{PD}\left(X_{2}\right),\right.
\end{gathered}
$$

where $X_{1}, X_{2}$, and $X 3$ represent the impact factors. $\operatorname{PD}\left(X_{1}\right), \operatorname{PD}\left(X_{2}\right)$, and $\operatorname{PD}\left(X_{1} \cap X_{2}=X 3\right)$ are the power of determinants of the impact factors on $\operatorname{PM}_{2.5}$ concentrations. $\operatorname{Max}\left(\operatorname{PD}\left(X_{1}\right), \operatorname{PD}\left(X_{2}\right)\right)$ represents the maximum value of $\operatorname{PD}\left(X_{1}\right), \operatorname{PD}\left(X_{2}\right)$. So, the $\mathrm{PD}$ value of each impact factor and the $\mathrm{PD}$ value of their interactions are used to quantitatively evaluate the relationships between potential impacts and their determinants. The geographical detector models used in this study are freely available from http://www.sssampling.org/Excel-geodetector/. According to the geographical detector input rules, all independent variables should be discrete. Therefore, the rules in the FMPI instruction manual and the natural breaks method were chosen as the classification method. Multiple environmental factors and population density were applied as independent variables in the geographical detector model. Specifically, $\mathrm{PM}_{2.5}$ concentration is the dependent variable. We used geographical detector models to examine the individual and interactive effects of multiple ecological and anthropogenic factors on urban forest $\mathrm{PM}_{2.5}$ concentrations.

\section{Results}

\subsection{Spatial Distribution Pattern of Urban Forest $P M_{2.5}$ Concentrations}

The spatial distributions of $\mathrm{PM}_{2.5}$ concentrations on 13 and 29 December 2014 and 14 January 2015 showed significant spatial clustering (Global Moran's I = 0.192, z-score $=63.412$ on 13 December 2014; Global Moran's I $=0.336$, z-score $=110.860$ on 29 December 2014; Global Moran's $I=0.185$, z-score $=95.761$ on 14 January 2015) (Table 1). The aggregated areas (hot spots and cold spots) of $\mathrm{PM}_{2.5}$ identified by Getis-Ord Gi* showed that $\mathrm{PM}_{2.5}$ hot spots were concentrated in urban centers and suburbs ( $n=1917$ on 13 December 2014; $n=2283$ on 29 December 2014; $n=1887$ on 14 January 2015) and cold spots were mainly distributed in the suburbs and exurban regions ( $n=1082$ on 13 December 2014; $n=1212$ on 29 December 2014; $n=1337$ on 14 January 2015) (Figure 2). 

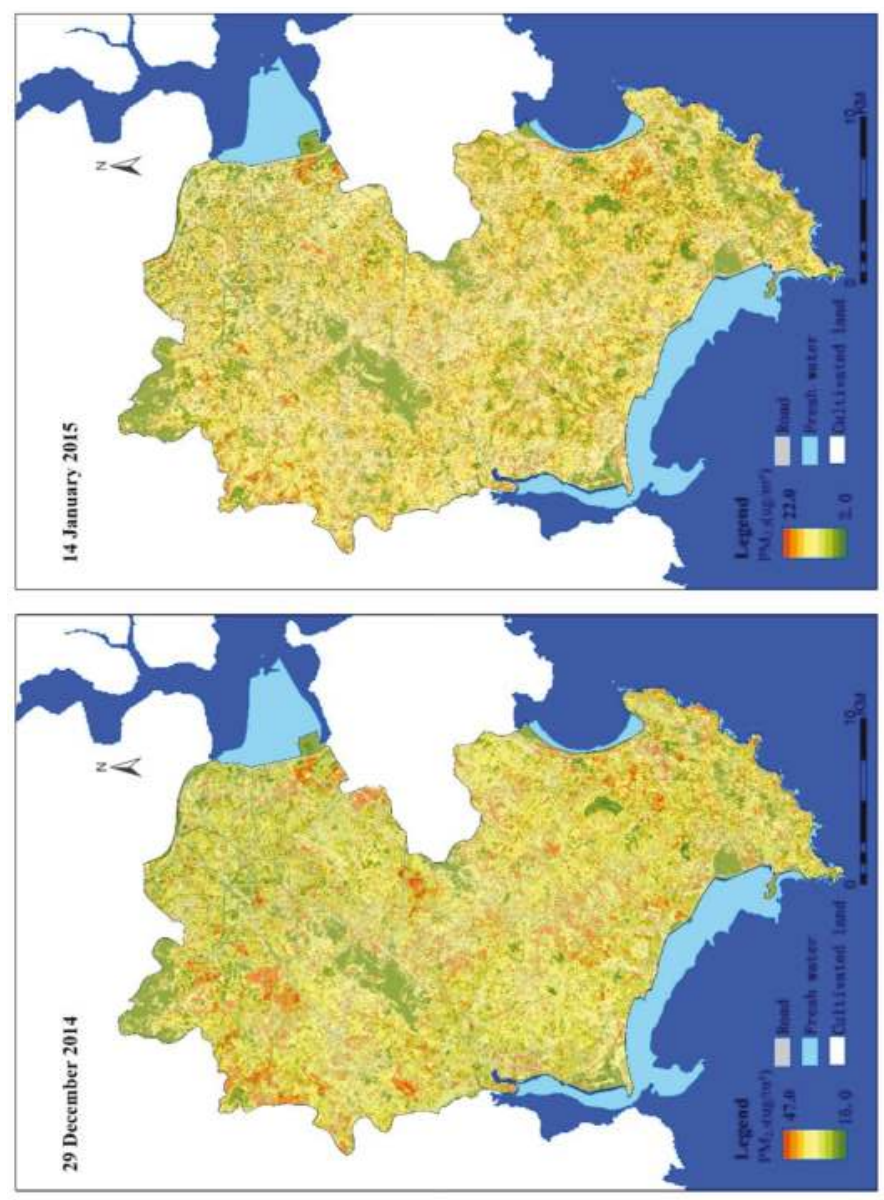

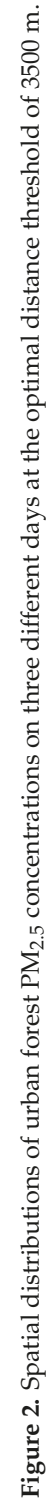

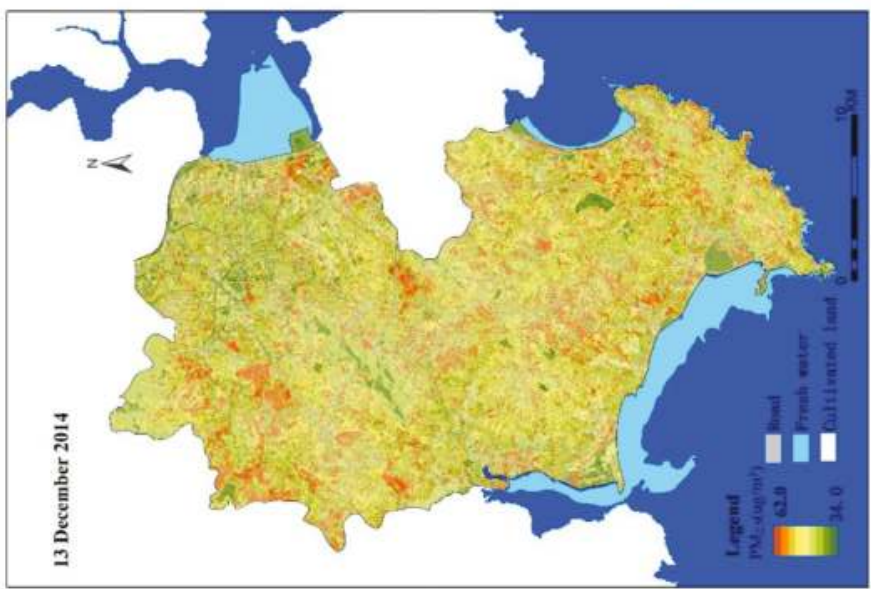


Table 1. The global spatial autocorrelation statistics (Moran's I) of $\mathrm{PM}_{2.5}$ concentrations on 13 December 2014, 29 December 2014, and 14 January 2015.

\begin{tabular}{cccc}
\hline Time & 13 December 2014 & 29 December 2014 & 14 January 2015 \\
\hline Moran's I Index & $0.192^{* *}$ & $0.336^{* *}$ & $0.185^{* *}$ \\
z-score & 63.412 & 110.860 & 95.761 \\
Pattern & Clustered & Clustered & Clustered \\
\hline
\end{tabular}

Note: $p<0.001$ in all regions in 2014 and 2015; ** represents significant values.

\subsection{Population Density and Stand Structure}

The human population density of Jinjiang was 1530 people $\mathrm{km}^{-2}$ and the density distribution demonstrated a significant spatial autocorrelation (Moran's I = 0.642, z-score $=269.293$ ) in 2014 (Figure 3). The Getis-Ord Gi* statistics revealed that the spatial distributions of the population and $\mathrm{PM}_{2.5}$ concentrations were similar in the northwest and on the central coast, but were different in the south. According to the FMPI data, the total area of forest in the study region was 9565.68 ha, average canopy density was 0.393 , and average tree age was $20.123 \pm 5.857$ years. The dominant tree species in the urban forests were Casuarina pusilla, Acacia crassicapa, Eucalyptus robusta Smith, Acacia confusa Merr, Cunninghamia lanceolate, and Pinus massoniana.

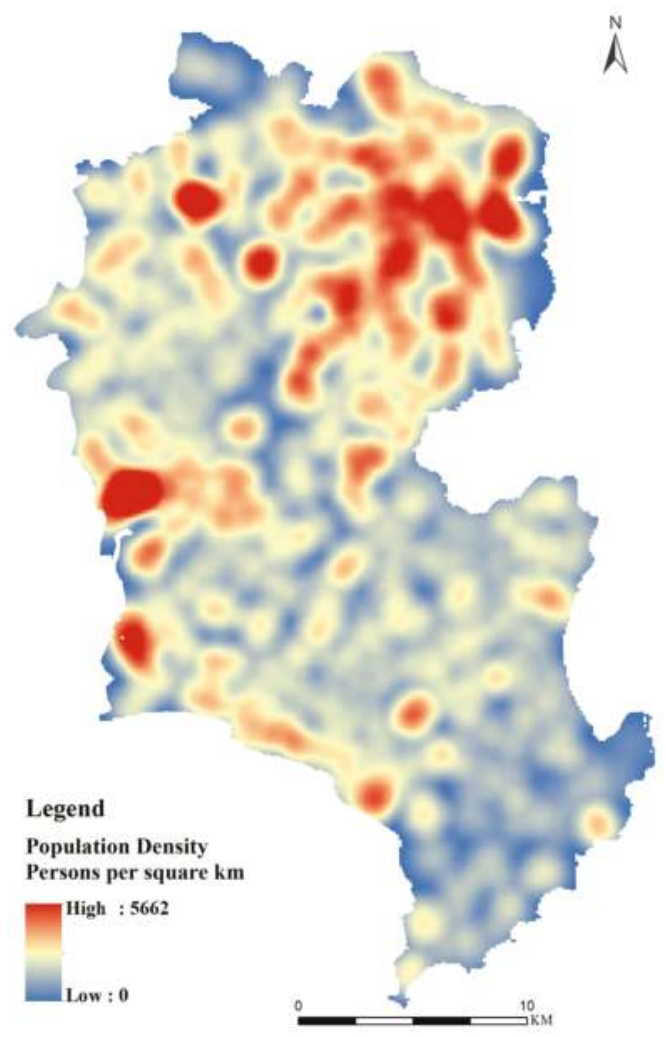

Figure 3. The spatial distribution of population density in 2014 (mapped using kernel density in Arc GIS 10.3). 


\subsection{Influences of Anthropogenic and Ecological Factors on Urban Forest $P M_{2.5}$ Concentrations}

We compared the locations of $\mathrm{PM}_{2.5}$ hot and cold spots on three dates. Our results showed that elevation (a topographic factor), dominant tree species (a forest characteristic factor), and wind speed (a meteorological factor) were the main factors related to urban forest $\mathrm{PM}_{2.5}$ concentrations. These three factors influenced the model more than population density (Figure 4, Table 2). In addition, the interaction between elevation and dominant tree species showed enhancement as did the interaction between elevation and impervious surface percentage. Dominant tree species and population density showed enhancement as did dominant tree species and impervious surface percentage. The influence of ecological factors on the model differed among the three dates. For example, on 13 December 2014, the main influential factors were dominant tree species $(\mathrm{PD}=0.044)$, wind speed $(\mathrm{PD}=0.038)$, and elevation ( $\mathrm{PD}=0.030)$. On 29 December 2014, the main influential factors were elevation $(\mathrm{PD}=0.108)$, wind speed $(P D=0.107)$, and dominant tree species $(P D=0.103)$. On 14 January 2015, the main influential factors were elevation $(\mathrm{PD}=0.077)$, dominant tree species $(\mathrm{PD}=0.075)$, and forest patch area $(\mathrm{PD}=0.063)$. Compared with the independent effect of population density, the interactive effect was significant. Although the independent effect of population density was weak, we cannot simply assume that population density was not crucial. Spatial heterogeneity is complex in urban environments and human activities have a significant impact on urban ecosystems. The interactive effect could be more than the sum of the independent effects of any two factors. For example, the interactive effect of dominant tree species and population density $(0.154)$ is greater than the sum of the individual effects of dominant tree species and population density (0.071). With the acceleration of urbanization, the interactive effects of population density, impervious surface percentage, and other factors could result in increased air pollution (Figure 5). The datasets in this study were limited by the availability of remote sensing image archives and the cloud-prone climate in southern China, which allowed us to select only three qualified scenes (i.e., 13 December 2014, 29 December 2014, and 14 January 2015) under relatively clear and stable atmospheric conditions.

Table 2. The power of determinant (PD) of forest attributes, soil, topography, meteorological factors, and population on $\mathrm{PM}_{2.5}$ concentrations on three different days.

\begin{tabular}{|c|c|c|c|c|}
\hline Factors & Factor Composition & 13 December 2014 & 29 December 2014 & 14 January 2015 \\
\hline \multirow{4}{*}{ Forest Characteristics } & PA & 0.013 & 0.063 & 0.063 \\
\hline & DS & 0.044 & 0.109 & 0.078 \\
\hline & $\mathrm{CD}$ & 0.016 & 0.019 & 0.009 \\
\hline & SA & 0.004 & 0.010 & 0.008 \\
\hline \multirow{3}{*}{ Soil } & SI & 0.005 & 0.011 & 0.006 \\
\hline & SD & 0.001 & 0.017 & 0.019 \\
\hline & HD & 0.001 & 0.001 & 0.001 \\
\hline \multirow{4}{*}{ Topography } & ELE & 0.030 & 0.117 & 0.086 \\
\hline & SDe & 0.029 & 0.077 & 0.058 \\
\hline & SPo & 0.018 & 0.061 & 0.050 \\
\hline & SDi & 0.011 & 0.018 & 0.020 \\
\hline \multirow{2}{*}{ Human Activity } & PopD & 0.029 & 0.042 & 0.029 \\
\hline & ISP & 0.024 & 0.044 & 0.035 \\
\hline \multirow{4}{*}{ Meteorological factors } & TEM & 0.012 & 0.100 & 0.057 \\
\hline & $\mathrm{SH}$ & 0.028 & 0.107 & 0.057 \\
\hline & PS & 0.038 & 0.093 & 0.049 \\
\hline & WS & 0.038 & 0.107 & 0.057 \\
\hline
\end{tabular}




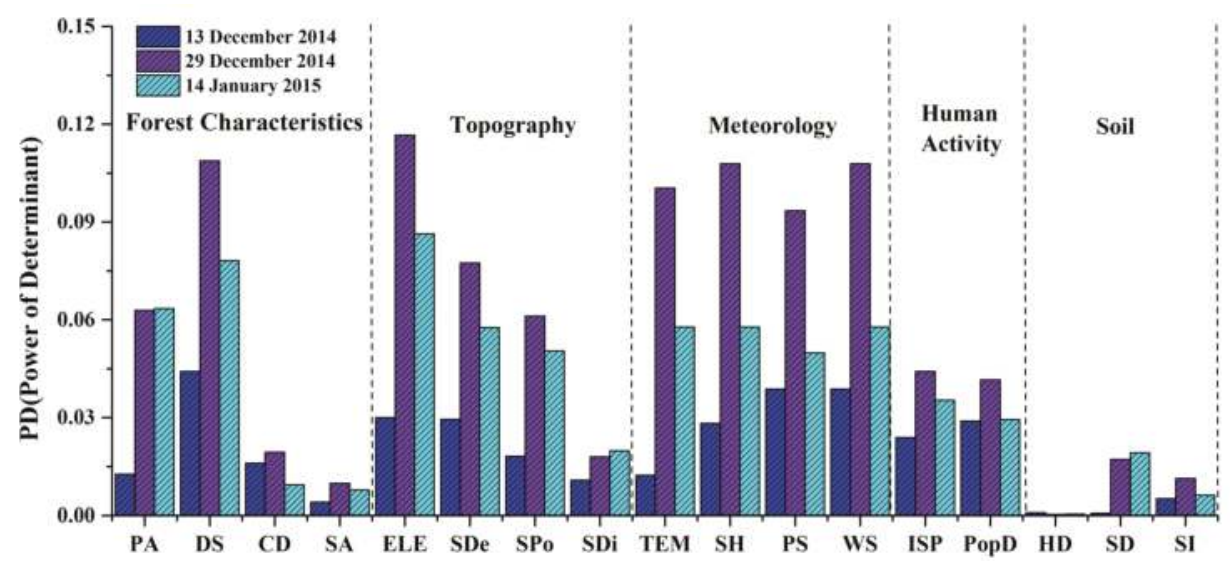

Figure 4. The power of determinants of different impact factors (forest, soils, topography, meteorological factors, and population) on $\mathrm{PM}_{2.5}$ concentrations in Jinjiang. ( $\mathrm{PA}=$ patch area, DS = dominant species, $\mathrm{CD}=$ canopy density, $\mathrm{SA}=$ stand age, $\mathrm{SI}=$ site index, $\mathrm{SD}=$ soil depth, $\mathrm{HD}=$ humus depth, ELE = elevation, $\mathrm{SDe}=$ degree of slope, $\mathrm{SPo}=$ slope of position, $\mathrm{SDi}=$ slope aspect, PopD = population density, ISP = impervious surface percentage, TEM = temperature, $\mathrm{SH}=$ specific humidity, PS = pressure, WS = wind speed $), p$-values $<0.1$ for all factors.

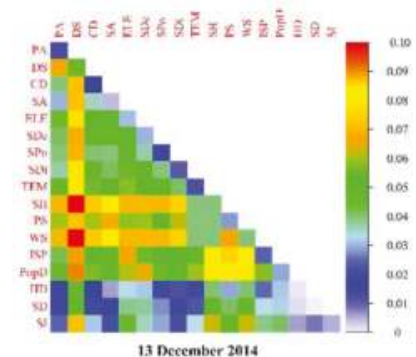

(A)

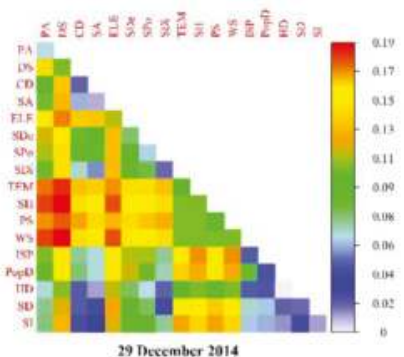

(B)

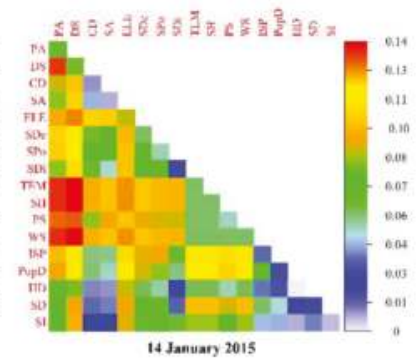

(C)

Figure 5. Scores for the interactive effects of impact factors sensitivity rankings at the three periods studied calculated based on simulations using multisource data ((A) 13 December 2014; (B) 29 December 2014; (C) 14 January 2015).

\section{Discussion}

\subsection{Significance}

Integration of Landsat images and multisource data using spatial statistical analysis and a geographical detector model can reveal the individual and interactive influences of anthropogenic and ecological factors on $\mathrm{PM}_{2.5}$ concentrations. The results of this study emphasize the importance of human activities and ecological factors in determining $\mathrm{PM}_{2.5}$ concentrations and the different strengths of these factors. Our study provides a more comprehensive analysis of human and ecological influencing factors through the use of remote sensing data, statistical analysis, and monitoring data at an urban scale. 


\subsection{Individual Functions}

The ecological factors of elevation, dominant tree species, and meteorological factors significantly influenced particulate concentrations in urban areas. Many studies have shown that, at small spatial scales, elevation affects particulate concentrations by influencing air flow, pressure, temperature, and precipitation [38,39]. There are several possible explanations for this finding: (1) Particulates are more buoyant in the air at low elevation (over time, $\mathrm{PM}_{2.5}$ at high elevations will sink down to low elevations, adding to the concentration of $\mathrm{PM}_{2.5}$ at the lower elevation); (2) Each $100 \mathrm{~m}$ increase in elevation coincides with a $0.6{ }^{\circ} \mathrm{C}$ drop in temperature, which affects particulate concentrations [40]. At higher elevations, the ground absorbs more radiation from the sun, which warms air near the ground and causes it to rise, creating convection currents in the upper atmosphere. This meteorological process encourages the proliferation of atmospheric pollutants (including particulates); (3) As atmospheric pressure decreases with increasing elevation [41,42], air volume expands and the atmosphere becomes less stable, thus leading to a widespread diffusion of air pollutants.

Tree species composition significantly affected particulate concentrations, presumably because the dust-removing ability of plants differs significantly by species $[43,44]$. The variations in particulate retention among tree species in this study were consistent with those found by Liu [12] and Yang [45] and may be explained by variations in morphological characteristics that enable plants to trap particulates (e.g., canopy structure, leaf density, leaf surface roughness, and wax) [16]. The three-dimensional structure of tree canopies encourages turbulent air movement and the more complex the canopy structure, the more particulates are deposited onto leaf surfaces [46]. Conifers retain more particulates than broadleaf trees because of their smaller, more densely packed leaves and more complex stem arrangements [47]. Rough leaf surfaces are also more effective than smooth leaf surfaces in accumulating particulates [48].

Wind speed is also an important factor affecting the diffusion of $\mathrm{PM}_{2.5}$ concentrations. In general, higher wind speeds contribute to $\mathrm{PM}_{2.5}$ diffusion. Under zero wind speed conditions, $\mathrm{PM}_{2.5}$ particulates aggregated at the surface layer [49].

\subsection{Interactive Functions}

Our study found that human activities significantly enhanced the effects of ecological factors on $\mathrm{PM}_{2.5}$ concentrations. This suggests that human activities might be the dominant factor affecting $\mathrm{PM}_{2.5}$ concentrations in Jinjiang, China, which is consistent with previous research [50]. Of the anthropogenic factors, we found that population density was most responsible for enhancing the effects of elevation and dominant tree species on $\mathrm{PM}_{2.5}$ concentrations. The increase in population density caused by increased residential areas and increased impervious surface percentage could significantly increase the $\mathrm{PM}_{2.5}$ concentrations in central urban areas [51]. Populations and industries tend to settle and build at low elevations. The resulting heavy human and industrial activity could therefore significantly increase $\mathrm{PM}_{2.5}$ concentrations in central urban areas [52]. The interactive effects between impervious surface percentage and other factors were similar to those just described for population density. These findings show that the combined influences of anthropogenic activities and multiple ecological factors on $\mathrm{PM}_{2.5}$ concentrations should be considered when developing pollution monitoring and control strategies in Jinjiang, China. Previous studies used multivariate statistics to study the individual impacts of multiple ecological factors (such as tree species composition, NDVI, etc.) on $\mathrm{PM}_{2.5}$ concentrations $[53,54]$. However, these studies ignored the interactions of these factors. Furthermore, multivariate statistical analyses have not considered the spatial heterogeneity of $\mathrm{PM}_{2.5}$ concentrations and have only focused on nonspatial features and attributes. Thus, we used geographical detector models to examine the individual and interactive influences of anthropogenic and ecological factors. This approach assesses the multiple ecological and anthropogenic factors associated with $\mathrm{PM}_{2.5}$ concentrations by means of spatial variance analysis (SVA). 


\subsection{Limitations and Advantages of the Study}

There are several limitations of the present study. First, the correlation between AOD and particulates was complex. We found that it varied at different temporal and spatial scales. In the future, we need to improve our understanding of the chemical properties of AOD and particulates to improve the model's accuracy. Second, because the urban ecosystem is complex and factors that affect pollution in cities differ spatially, we need to identify functional areas (e.g., clean areas, traffic areas, industrial areas) and study the different dust-retention mechanisms within them. Third, because of data limitations, the anthropogenic factors included only population density and impervious surface percentage, which cannot comprehensively express the characteristics of human activities. Fourth, future research should focus on integrating longer time series of remote sensing images to more accurately delineate the interactions between multiple ecological factors and their effects on urban forest $\mathrm{PM}_{2.5}$ concentrations, while also using remote sensing data fusion methods to achieve high temporal and spatial resolution simultaneously at the city scale. Finally, the process of comparing quantitative impact factors with qualitative impact factors is subjective, because arbitrary methods of discretization (e.g., standard deviation, equal interval, Jenks, and quantile) may not characterize the actual associations between impact factors and $\mathrm{PM}_{2.5}$ concentrations [55-57].

Despite these limitations, this study has several advantages. First, we integrated detailed datasets (including FMPI, Landsat-8 images, population density distribution data, topographic data, and meteorological data). Second, we analyzed the spatial distribution of $\mathrm{PM}_{2.5}$ concentrations and described their spatial heterogeneity. Finally, we revealed the individual and interactive influences of anthropogenic and ecological factors on $\mathrm{PM}_{2.5}$ concentrations, which improves our understanding of $\mathrm{PM}_{2.5}$ pollution distribution patterns in Jinjiang, China.

\section{Conclusions}

At present, most studies of $\mathrm{PM}_{2.5}$ concentrations are conducted at large spatial scales, have a broad research scope, and use remote sensing images with coarse image resolution. Here, we focus on urban ecosystems, using a case study to create a methodology for quantifying the interactions between human activities and multiple ecological factors at an urban scale. Our method reveals the individual and interactive influences of these factors on $\mathrm{PM}_{2.5}$ concentrations by integrating field surveys with satellite-derived $\mathrm{PM}_{2.5}$ data and population density data. We used a spatial statistical analysis model to identify aggregated areas (hot spots and cold spots) of particulates in an urban environment. A geographic detector model was used to quantify the impact of ecological and anthropogenic factors on observed spatial $\mathrm{PM}_{2.5}$ patterns and to probe whether two impact factors enhance, weaken, or remain independent of each other when considering their combined impacts on $\mathrm{PM}_{2.5}$ concentrations. FMPI can provide vegetation attribute information (e.g., forest, soil, topography, and other attribute information) and model input parameters. Spatial statistics describe the spatial heterogeneity of particulate concentrations. We found that fine particulate concentration hot spots are mainly distributed in urban centers and suburbs, while cold spots are mainly distributed in the suburbs and exurban regions. Elevation was the dominant individual factor affecting $\mathrm{PM}_{2.5}$ concentrations, followed by dominant tree species and meteorological factors. In terms of interactive effects, the combination of human activities (e.g., population density, impervious surface percentage) and multiple ecological factors led to the largest increases in $\mathrm{PM}_{2.5}$ concentrations in our study area. In conclusion, in order to reveal the direct and indirect effects of human activities and multiple factors on $\mathrm{PM}_{2.5}$ concentrations in urban forests, quantification of fusion satellite data and spatial statistical methods should be conducted in urban areas. These findings extend our understanding of the factors influencing the spatial distribution of $\mathrm{PM}_{2.5}$ concentrations and may help guide efforts to manage air pollution, monitor pollution, and estimate pollution exposure.

Supplementary Materials: The following are available online at http:/ /www.mdpi.com/2072-4292/10/4/521/s1, This section describes in detail the calculation of $\mathrm{PM}_{2.5}$ concentrations and Geographical detector model. 
Acknowledgments: This work was supported by National Science Foundation of China $(31670645,31470578$ and 31200363), the National Key Research Program of China (2016YFC0502704), National Social Science Fund (17ZDA058), Fujian Provincial Department of S\&T Project (2016T3032, 2016T3037, 2016Y0083, 2018T3018 and 2015Y0083), Xiamen Municipal Department of Science and Technology (3502Z20130037 and 3502Z20142016), Key Laboratory of Urban Environment and Health of CAS (KLUEH-C-201701), Key Program of the Chinese Academy of Sciences (KFZDSW-324), and Youth Innovation Promotion Association CAS (2014267). We are grateful to $\mathrm{Hu} \mathrm{Li}$ and Yuanrong He for their helpful suggestions.

Author Contributions: Guoliang Yun conceived and performed the experiments, and wrote the first draft; Shudi Zuo and Shaoqing Dai did part of the data processing and translation; Xiaodong Song, Chengdong Xu, Yilan Liao, Peiqiang Zhao, Weiyin Chang, Qi Chen, Yaying Li, Jianfeng Tang, Wang Man, and Yin Ren revised the paper and assumed the responsibility of foundation; all authors contributed to and approved the final manuscript.

Conflicts of Interest: The authors declare no conflict of interest.

\section{References}

1. Wu, J.S.; Yao, F.; Li, W.F.; Si, M.L. Viirs-based remote sensing estimation of ground-level $\mathrm{PM}_{2.5}$ concentrations in beijing-tianjin-hebei: A spatiotemporal statistical model. Remote Sens. Environ. 2016, 184, 316-328. [CrossRef]

2. Pope, C.A.; Burnett, R.T.; Thun, M.J.; Calle, E.E.; Krewski, D.; Ito, K.; Thurston, G.D. Lung cancer, cardiopulmonary mortality, and long-term exposure to fine particulate air pollution. J. Am. Med. Assoc. 2002, 287, 1132-1141. [CrossRef]

3. Kappos, A.D.; Bruckmann, P.; Eikmann, T.; Englert, N.; Heinrich, U.; Hoppe, P.; Koch, E.; Krause, G.H.M.; Kreyling, W.G.; Rauchfuss, K.; et al. Health effects of particles in ambient air. Int. J. Hyg. Environ. Health 2004, 207, 399-407. [CrossRef] [PubMed]

4. Solomon, S.; Qin, D.; Manning, M.; Chen, Z.; Marquis, M.; Averyt, K.B.; Tignor, M.; Miller, H.L. Contribution of Working Group I to the Fourth Assessment Report of the Intergovernmental Panel on Climate Change, 2007; Cambridge University Press: Cambridge, UK; New York, NY, USA, 2007; p. 976.

5. Liu, Z.; Sun, Y.; Li, L.; Wang, Y. Particle mass concentrations and size distribution during and after the Beijing Olympic games. Huan jing ke xue = Huanjing kexue 2011, 32, 913-923. [PubMed]

6. Chen, B.; Lu, S.W.; Zhao, Y.G.; Li, S.N.; Yang, X.B.; Wang, B.; Zhang, H.J. Pollution remediation by urban forests: $\mathrm{PM}_{2.5}$ reduction in Beijing, China. Pol. J. Environ. Stud. 2016, 25, 1873-1881. [CrossRef]

7. Rissanen, T.; Hyotylainen, T.; Kallio, M.; Kronholm, J.; Kulmala, M.; Rikkola, M.L. Characterization of organic compounds in aerosol particles from a coniferous forest by GC-MS. Chemosphere 2006, 64, 1185-1195. [CrossRef] [PubMed]

8. Pio, C.; Alves, C.; Duarte, A. Organic components of aerosols in a forested area of central Greece. Atmos. Environ. 2001, 35, 389-401. [CrossRef]

9. Tiwary, A.; Sinnett, D.; Peachey, C.; Chalabi, Z.; Vardoulakis, S.; Fletcher, T.; Leonardi, G.; Grundy, C.; Azapagic, A.; Hutchings, T.R. An integrated tool to assess the role of new planting in $\mathrm{PM}_{10}$ capture and the human health benefits: A case study in London. Environ. Pollut. 2009, 157, 2645-2653. [CrossRef] [PubMed]

10. Gagne, S.A.; Sherman, P.J.; Singh, K.K.; Meentemeyer, R.K. The effect of human population size on the breeding bird diversity of urban regions. Biodivers. Conserv. 2016, 25, 653-671. [CrossRef]

11. Jin, S.J.; Guo, J.K.; Wheeler, S.; Kan, L.Y.; Che, S.Q. Evaluation of impacts of trees on PM $_{2.5}$ dispersion in urban streets. Atmos. Environ. 2014, 99, 277-287. [CrossRef]

12. Liu, X.H.; Yu, X.X.; Zhang, Z.M. PM 2.5 concentration differences between various forest types and its correlation with forest structure. Atmosphere 2015, 6, 1801-1815. [CrossRef]

13. Shu, J.; Dearing, J.A.; Morse, A.P.; Yu, L.Z.; Yuan, N. Determining the sources of atmospheric particles in Shanghai, China, from magnetic and geochemical properties. Atmos. Environ. 2001, 35, 2615-2625. [CrossRef]

14. Gao, G.J.; Sun, F.B.; Thao, N.T.T.; Lun, X.X.; Yu, X.X. Different concentrations of TSP, $\mathrm{PM}_{10}, \mathrm{PM}_{2.5}$, and $\mathrm{PM}_{1}$ of several urban forest types in different seasons. Pol. J. Environ. Stud. 2015, 24, 2387-2395. [CrossRef]

15. Hu, Y.; Xia, C.; Li, S.; Ward, M.P.; Luo, C.; Gao, F.; Wang, Q.; Zhang, S.; Zhang, Z. Assessing environmental factors associated with regional schistosomiasis prevalence in Anhui province, Peoples' Republic of China using a geographical detector method. Infect. Dis. Poverty 2017, 6, 87. [CrossRef] [PubMed] 
16. Saebo, A.; Popek, R.; Nawrot, B.; Hanslin, H.M.; Gawronska, H.; Gawronski, S.W. Plant species differences in particulate matter accumulation on leaf surfaces. Sci. Total Environ. 2012, 427, 347-354. [CrossRef] [PubMed]

17. Ord, J.K.; Getis, A. Local spatial autocorrelation statistics: Distributional issues and an application. Geogr. Anal. 1995, 27, 286-306. [CrossRef]

18. Barrell, J.; Grant, J. Detecting hot and cold spots in a seagrass landscape using local indicators of spatial association. Landsc. Ecol. 2013, 28, 2005-2018. [CrossRef]

19. Lei, X.D.; Tang, M.P.; Lu, Y.C.; Hong, L.X.; Tian, D.L. Forest inventory in China: Status and challenges. Int. For. Rev. 2009, 11, 52-63. [CrossRef]

20. He, J.; Yang, K. China Meteorological Forcing Dataset; Cold and Arid Regions Science Data Center at Lanzhou: Lanzhou, China, 2011; Available online: http:/ / westdc.westgis.ac.cn/data/7a35329c-c53f-4267aa07-e0037d913a21 (accessed on 1 March 2018).

21. Vermote, E.F.; Tanre, D.; Deuze, J.L.; Herman, M.; Morcrette, J.J. Second simulation of the satellite signal in the solar spectrum, 6s: An overview. IEEE Trans. Geosci. Remote Sens. 1997, 35, 675-686. [CrossRef]

22. Chen, Y.P.; Han, W.H.; Chen, S.Z.; Tong, L. Estimating ground-level $\mathrm{PM}_{2.5}$ concentration using Landsat 8 in Chengdu, China. In Remote Sensing of the Atmosphere, Clouds, and Precipitation v; Im, E., Yang, S., Zhang, P., Eds.; SPIE: Bellingham, WA, USA, 2014; Volume 9259.

23. Levy, R.C.; Remer, L.A.; Mattoo, S.; Vermote, E.F.; Kaufman, Y.J. Second-generation operational algorithm: Retrieval of aerosol properties over land from inversion of moderate resolution imaging spectroradiometer spectral reflectance. J. Geophys. Res. Atmos. 2007, 112. [CrossRef]

24. Koelemeijer, R.B.A.; Homan, C.D.; Matthijsen, J. Comparison of spatial and temporal variations of aerosol optical thickness and particulate matter over Europe. Atmos. Environ. 2006, 40, 5304-5315. [CrossRef]

25. Lin, C.Q.; Li, Y.; Yuan, Z.B.; Lau, A.K.H.; Li, C.C.; Fung, J.C.H. Using satellite remote sensing data to estimate the high-resolution distribution of ground-level $\mathrm{PM}_{2.5}$. Remote Sens. Environ. 2015, 156, 117-128. [CrossRef]

26. He, Q.S.; Li, C.C.; Geng, F.H.; Zhou, G.Q.; Gao, W.; Yu, W.; Li, Z.K.; Du, M.B. A parameterization scheme of aerosol vertical distribution for surface-level visibility retrieval from satellite remote sensing. Remote Sens. Environ. 2016, 181, 1-13. [CrossRef]

27. Jung, J.; Lee, H.; Kim, Y.J.; Liu, X.; Zhang, Y.; Gu, J.; Fan, S. Aerosol chemistry and the effect of aerosol water content on visibility impairment and radiative forcing in Guangzhou during the 2006 Pearl River Delta campaign. J. Environ. Manag. 2009, 90, 3231-3244. [CrossRef] [PubMed]

28. Huang, W.X.; Cheng, X.W. Multiple regression method for estimating concentration of $\mathrm{PM}_{2.5}$ using remote sensing and meteorological data. J. Environ. Prot. Ecol. 2017, 18, 417-424.

29. Wang, Z.F.; Chen, L.F.; Tao, J.H.; Zhang, Y.; Su, L. Satellite-based estimation of regional particulate matter (PM) in Beijing using vertical-and-RH correcting method. Remote Sens. Environ. 2010, 114, 50-63. [CrossRef]

30. Eck, J.; Chainey, S.; Cameron, J.; Wilson, R. Mapping Crime: Understanding Hotspots; U.S. Department of Justice, Office of Justice Programs: Washington, DC, USA, 2005.

31. Kara, C.; Akcit, N. Traffic accident analysis using Gis: A case study of Kyrenia city. In Third International Conference on Remote Sensing and Geoinformation of the Environment; Hadjimitsis, D.G., Themistocleous, K., Michaelides, S., Papadavid, G., Eds.; SPIE: Bellingham, WA, USA, 2015; Volume 9535.

32. Mei, Z.X.; Xu, S.J.; Ouyang, J. Spatio-temporal association analysis of county potential in the Pearl River Delta during 1990-2009. J. Geogr. Sci. 2015, 25, 319-336. [CrossRef]

33. Stopka, T.J.; Krawczyk, C.; Gradziel, P.; Geraghty, E.M. Use of spatial epidemiology and hot spot analysis to target women eligible for prenatal women, infants, and children services. Am. J. Public Health 2014, 104, S183-S189. [CrossRef] [PubMed]

34. Hu, Y.; Wang, J.; Li, X.; Ren, D.; Zhu, J. Geographical detector-based risk assessment of the under-five mortality in the 2008 Wenchuan Earthquake, China. PLoS ONE 2011, 6, e21427. [CrossRef] [PubMed]

35. Du, Z.Q.; Xu, X.M.; Zhang, H.; Wu, Z.T.; Liu, Y. Geographical detector-based identification of the impact of major determinants on Aeolian Desertification Risk. PLoS ONE 2016, 11, e0151331. [CrossRef] [PubMed]

36. Wang, J.F.; Li, X.H.; Christakos, G.; Liao, Y.L.; Zhang, T.; Gu, X.; Zheng, X.Y. Geographical detectors-based health risk assessment and its application in the neural tube defects study of the Heshun region, China. Int. J. Geogr. Inf. Sci. 2010, 24, 107-127. [CrossRef] 
37. Cao, Z.; Liu, T.; Li, X.; Wang, J.; Lin, H.L.; Chen, L.L.; Wu, Z.F.; Ma, W.J. Individual and interactive effects of socio-ecological factors on dengue fever at fine spatial scale: A geographical detector-based analysis. Int. J. Environ. Res. Public Health 2017, 14, 795. [CrossRef] [PubMed]

38. Zhang, T.H.; Liu, G.; Zhu, Z.M.; Gong, W.; Ji, Y.X.; Huang, Y.S. Real-time estimation of satellite-derived $\mathrm{PM}_{2.5}$ based on a semi-physical geographically weighted regression model. Int. J. Environ. Res. Public Health 2016, 13, 974. [CrossRef] [PubMed]

39. Cisneros, R.; Schweizer, D.; Preisler, H.; Bennett, D.H.; Shaw, G.; Bytnerowicz, A. Spatial and seasonal patterns of particulate matter less than 2.5 microns in the sierra Nevada Mountains, California. Atmos. Pollut. Res. 2014, 5, 581-590. [CrossRef]

40. Halsey, L.A.; Vitt, D.H.; Zoltai, S.C. Disequilibrium response of permafrost in boreal continental Western Canada to climate change. Clim. Chang. 1995, 30, 57-73. [CrossRef]

41. Stone, J.O. Air pressure and cosmogenic isotope production. J. Geophys. Res.-Solid Earth 2000, 105, 23753-23759. [CrossRef]

42. Körner, C. The use of 'altitude' in ecological research. Trends Ecol. Evol. 2007, 22, 569-574. [CrossRef] [PubMed]

43. Zhang, Z.M.; Liu, J.K.; Wu, Y.N.; Yan, G.X.; Zhu, L.J.; Yu, X.X. Multi-scale comparison of the fine particle removal capacity of urban forests and wetlands. Sci. Rep. 2017, 7, 46214. [CrossRef] [PubMed]

44. Nguyen, T.; Yu, X.X.; Zhang, Z.M.; Liu, M.M.; Liu, X.H. Relationship between types of urban forest and $\mathrm{PM}_{2.5}$ capture at three growth stages of leaves. J. Environ. Sci. 2015, 27, 33-41. [CrossRef] [PubMed]

45. Yang, J.; Xie, B.Z.; Shi, H.; Wang, H.X.; Wang, Y.H. Study on capturing $\mathrm{PM}_{2.5}$ capability of tree species in different functional areas. In Proceedings of the 2015 International Conference on Industrial Technology and Management Science; Zhao, J., Ed.; Atlantis Press: Paris, France, 2015; Volume 34, pp. 585-588.

46. Fowler, D.; Cape, J.N.; Unsworth, M.H. Deposition of atmospheric pollutants on forests. Philos. Trans. R. Soc. Lond. Ser. B Biol. Sci. 1989, 324, 247-265. [CrossRef]

47. Beckett, K.P.; Freer-Smith, P.H.; Taylor, G. Urban woodlands: Their role in reducing the effects of particulate pollution. Environ. Pollut. 1998, 99, 347-360. [CrossRef]

48. Hwang, H.-J.; Yook, S.-J.; Ahn, K.-H. Experimental investigation of submicron and ultrafine soot particle removal by tree leaves. Atmos. Environ. 2011, 45, 6987-6994. [CrossRef]

49. Kim, K.H.; Kim, M.Y.; Hong, S.M.; Youn, Y.H.; Hwang, S.J. The effects of wind speed on the relative relationships between different sized-fractions of airborne particles. Chemosphere 2005, 59, 929-937. [CrossRef] [PubMed]

50. Lou, C.R.; Liu, H.Y.; Li, Y.F.; Li, Y.L. Socioeconomic drivers of $\mathrm{PM}_{2.5}$ in the accumulation phase of air pollution episodes in the Yangtze River Delta of China. Int. J. Environ. Res. Public Health 2016, 13, 928. [CrossRef] [PubMed]

51. Beelen, R.; Hoek, G.; Pebesma, E.; Vienneau, D.; de Hoogh, K.; Briggs, D.J. Mapping of background air pollution at a fine spatial scale across the European Union. Sci. Total Environ. 2009, 407, 1852-1867. [CrossRef] [PubMed]

52. Hoek, G.; Beelen, R.; de Hoogh, K.; Vienneau, D.; Gulliver, J.; Fischer, P.; Briggs, D. A review of land-use regression models to assess spatial variation of outdoor air pollution. Atmos. Environ. 2008, 42, 7561-7578. [CrossRef]

53. Beckett, K.P.; Freer-Smith, P.H.; Taylor, G. Particulate pollution capture by urban trees: Effect of species and windspeed. Glob. Chang. Biol. 2000, 6, 995-1003. [CrossRef]

54. Baxter, L.K.; Burke, J.; Lunden, M.; Turpin, B.J.; Rich, D.Q.; Thevenet-Morrison, K.; Hodas, N.; Ozkaynak, H. Influence of human activity patterns, particle composition, and residential air exchange rates on modeled distributions of $\mathrm{PM}_{2.5}$ exposure compared with central-site monitoring data. J. Expo. Sci. Environ. Epidemiol. 2013, 23, 241-247. [CrossRef] [PubMed]

55. Bai, H.; Li, D.; Ge, Y.; Wang, J. Detecting nominal variables' spatial associations using conditional probabilities of neighboring surface objects' categories. Inf. Sci. 2016, 329, 701-718. [CrossRef] 
56. Tang, L.N.; Shao, G.F.; Piao, Z.J.; Dai, L.M.; Jenkins, M.A.; Wang, S.X.; Wu, G.; Wu, J.G.; Zhao, J.Z. Forest degradation deepens around and within protected areas in east Asia. Biol. Conserv. 2010, 143, 1295-1298. [CrossRef]

57. Zhao, J.Z.; Liu, X.; Dong, R.C.; Shao, G.F. Landsenses ecology and ecological planning towards sustainable development. Int. J. Sustain. Dev. World Ecol. 2016, 23, 293-297. [CrossRef]

(C) 2018 by the authors. Licensee MDPI, Basel, Switzerland. This article is an open access article distributed under the terms and conditions of the Creative Commons Attribution (CC BY) license (http:/ / creativecommons.org/licenses/by/4.0/). 
Article

\title{
Design of a Novel Spectral Albedometer for Validating the MODerate Resolution Imaging Spectroradiometer Spectral Albedo Product
}

\author{
Hongmin Zhou ${ }^{1,2, *}$, Jindi Wang ${ }^{1,2}$ and Shunlin Liang ${ }^{3}$ \\ 1 The State Key Laboratory of Remote Sensing Science, Jointly Sponsored by Beijing Normal University and \\ Institute of Remote Sensing and Digital Earth of Chinese Academy of Sciences, Beijing 100875, China; \\ wangjd@bnu.edu.cn \\ 2 Beijing Engineering Research Center for Global Land Remote Sensing Products, Institute of Remote Sensing \\ Science and Engineering, Faculty of Geographical Science, Beijing Normal University, Beijing 100875, China \\ 3 Department of Geographical Sciences, University of Maryland, College Park, MD 20742, USA; \\ sliang@umd.edu \\ * Correspondence: zhouhm@bnu.edu.cn; Tel.: +86-10-5880-6011
}

Received: 27 November 2017; Accepted: 10 January 2018; Published: 12 January 2018

\begin{abstract}
Land surface shortwave broadband albedo is a key parameter in general circulation models and surface energy budget models. Multispectral satellite data are typically used to generate broadband albedo products in a three-step process: atmospheric correction, for converting the top-of-atmosphere observations to surface directional reflectance; angular modeling, for converting the surface directional reflectance to spectral albedo of each individual band; and finally, narrowband-to-broadband conversion, for transforming the spectral albedos to broadband albedos. Spectroradiometers can be used for validating surface directional reflectance products and pyranometers or broadband albedometers, for validating broadband albedo products, but spectral albedo products are rarely validated using ground measurements. In this study, we designed a new type of albedometer that can measure spectral albedos. It consists of multiple interference filters and a silicon detector, for measuring irradiance from $400-1100 \mathrm{~nm}$. The linearity of the sensors is $99 \%$, and the designed albedometer exhibits consistency up to 0.993 , with a widely-used commercial instrument. A field experiment for measuring spectral albedo of grassland using this new albedometer was conducted in Yudaokou, China and the measurements are used for validating the MODerate Resolution Imaging Spectroradiometer (MODIS) spectral albedos. The results show that the biases of the MODIS spectral albedos of the first four bands are $-0.0094,0.0065,0.0159$, and -0.0001 , respectively. This new instrument provides an effective technique for validating spectral albedos of any satellite sensor in this spectral range, which is critical for improving satellite broadband albedo products.
\end{abstract}

Keywords: spectral; albedometer; interference filter; photoelectric detector; validation

\section{Introduction}

The land surface albedo is a key input parameter required in current general circulation models. It is relevant to radiation balance, atmospheric dynamics, and climate characteristics [1]. Accurate surface albedo estimates are a prerequisite for the accurate retrieval of other surface parameters [2-4]; an absolute accuracy of $0.02-0.05$ is required for any effective climate modeling study [1]. Satellite remote sensing is the only feasible means to obtain reliable and accurate estimates of the global surface albedo [5,6]. An array of remote sensing surface albedo products exists [7-11]; the precise validation of these products is important because their accuracy is critical to the scientific 
community for various applications. Any feedback from the process of validation can also help improve the design, implementation, and very generation of these products [12].

The basic validation of remote sensing albedo products is primarily centered on the broadband products, which are compared directly against field measurements, using a combination of pyranometers or albedometers [2,13,14]. Consider the MODerate Resolution Imaging Spectroradiometer (MODIS) albedo product (MCD43) as an example-it includes three visible bands (460, 555, and $659 \mathrm{~nm}$ ), four near-infrared bands $(865,1240,1640$, and $2130 \mathrm{~nm}$ ), and three broadband albedos (visible, near-infrared, and shortwave). The broadband albedo products are linear combinations of the spectral albedos [15-17]. Most MODIS albedo product validation work involves comparing the three broadband albedos with field measurements, using broadband pyranometers and albedometers, but the spectral albedos are seldom validated. The band integration and band inconsistencies also create erroneous disparities between the remote sensing albedo products and field instruments, which are generally not taken into consideration, though they inevitably result in errors in the accuracy assessment.

In fact, in addition to broadband albedo products, spectral albedo products are also important for land energy budget research. There are many advantages to spectral albedo products, compared to broadband albedo products-they represent a more fine-tuned understanding of the land surface, for example, and they include more spectral information [18]. When they are retrieved directly from spectral observations, using a Bidirectional Reflectance Distribution Function (BRDF) model, the retrieval model can be accurately assessed by evaluating the spectral albedo product accuracy. Spectral albedos are also critical for the retrieval of other land surface parameters. Cheng and Liang [19] used the MODIS spectral albedo product to estimate thermal-infrared emissivity, where the accuracy of the spectral albedo is critical to the final estimation. Picard, Libois [18] also used spectral albedo measurements to accurately estimate a near-surface snow specific surface area.

However, the scarcity (or absence) of spectral albedometers means that spectral albedo products have not yet been effectively validated. In recent years, researchers have sought various methods to remedy this. Wuttke, Seckmeyer [20] used a scanning spectroradiometer with a shaped Teflon diffuser to conduct spectral albedo measurements, in order to describe Antarctic radiation conditions. Wright, Bergin [21] compared independent MODIS spectral to high resolution spectral measurements in Greenland with an Analytical Spectral Device (ASD) Fieldspec Pro spectroradiometer, using ASD Remote Cosine Receptor (RCR) foreoptics. Similar measurements were gathered in Antarctica [22-24]. The use of spectroradiometers with the Cosine Receptor allows scientists to obtain accurate narrow band albedos; however, this is time- and labor-consuming and does not yield the time series observations which are vital for long-term climate change research. Spectrometers can also be used to measure spectral albedo, by integration over the BRF. They may even more accurate than measuring with albedometers, but apply only to smaller areas.

To this effect, there is urgent demand for a new instrument, which can directly observe spectral albedo in supplying time series observations. Our goal in conducting the present study was to design and validate the performance of a set of automatic spectral albedometers. In the pursuit of this goal, we also established a novel method to directly obtain spectral albedo time series measurements. We used a customized interference filter and silicon detector to realize the spectral albedo detection of MODIS's visible bands (460, 555, and $659 \mathrm{~nm}$ ) and near-infrared band $(865 \mathrm{~nm})$. The system design and materials used are introduced in Section 2, and the experiments performed indoors and on a grassland site in Yudaokou, China, are discussed in Section 3. Section 4 reports our results, and Section 5 provides concluding remarks.

\section{Spectral Albedometer Design}

\subsection{Theoretical Foundation}

The broadband land surface albedo is the ratio of the amount of radiation traveling upwards and downwards from the Earth's surface [1]. It serves as an indicator of the energy capacity for 
surface reflection over the entire shortwave spectral range. In contrast, the spectral albedo depicts the capacity for reflection of the land surface over a smaller, specific spectral range [25]. To obtain the latter, it is necessary to measure the upward and downward irradiance over the desired spectral range. According to the definition of the surface albedo, the spectral albedo is obtained by Equation (1)

$$
\rho_{\lambda}=\frac{I_{\uparrow \lambda}}{I_{\downarrow \lambda}}
$$

where $\lambda$ is a typical spectra, from wavelength $\lambda_{1}$ to $\lambda_{1} ; I_{\downarrow \lambda}$ and $I_{\uparrow \lambda}$ are the hemispherical incidence and exitance in the $\lambda$ band, which are functions of radiation energy and the spectral response of the sensors.

$$
I_{\lambda}=\int_{\lambda_{1}}^{\lambda_{2}} f_{i} \times I_{i} d \lambda_{i}
$$

where $I_{\lambda}$ is the incident irradiance of the sensor and $f_{i}$ is the spectral response function. We designed a MODIS-corresponding spectral albedometer in this study, so is the spectral response function of the MODIS bands.

\subsection{Spectral Albedometer Design}

Two sensors were included in each spectral albedometer: one upwards and one downwards. The upwards sensor was used to measure downwelling radiation $\left(I_{\downarrow \lambda}\right.$ in Equation (1)), and the downwards sensor was used to measure the upwelling radiation $\left(I_{\uparrow \lambda}\right.$ in Equation (1)). The spectral albedometer structure is shown in Figure 1. The spectral albedometer for each band consists of a shell, mounting stem, two domes, and two sensors.

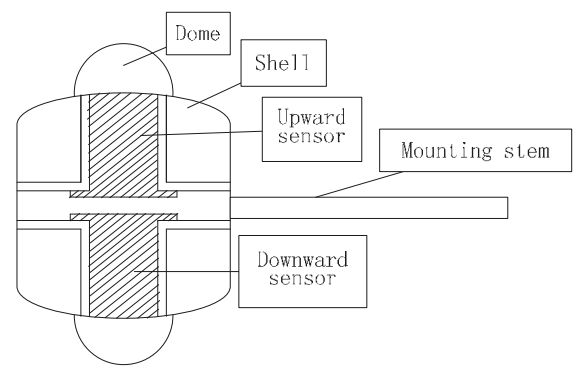

Figure 1. Spectral albedometer structure. For each band, upwards and downwards sensors are mounted back-to-back, to receive downwards and upwards radiation, respectively.

The upwards and downwards sensors are the same for each albedometer; each is composed of a cosine corrector, filter, photoelectric detector, and signal output port (Figure 2). 


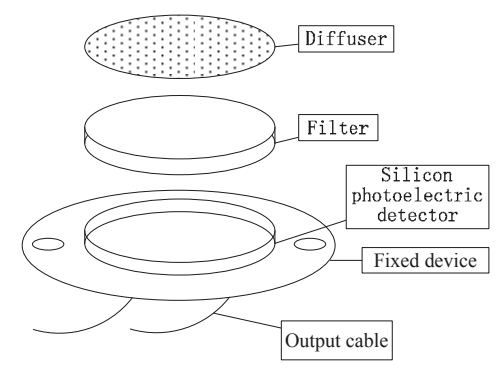

Figure 2. Sensor design. The first layer is a Teflon TM diffuser, the second is an interference filter, the third is a silicon photoelectric detector. Two sensors are included in each band's albedometer: one for $I_{\downarrow}$ and one for $I_{\uparrow}$.

\subsubsection{Dome and Cosine Corrector}

A customized quartz glass dome was designed, according to the sensor's size and installed, to protect the probe against intrusions from the external environment. The dome was fixed to the up and down surfaces of the sensor with O-rings to ensure it was waterproof and easy to replace. The dome mainly serves to isolate the radiometer from long wave radiation [26]. Here, all sensors take into account only the MODIS bands with wavelengths $<1100 \mathrm{~nm}$, so the glass cover in our design mainly protects the sensor [20].

The accuracy of the radiation detection sensor is affected by many factors, including the incidence angle, equipment temperature, equipment installation inclination, proportion of direct light, and degree of linearity in the sensor's response [27]. We used polytetrafluoroethylene (TeflonTM) as a cosine corrector, to minimize the effects of incidence angle on observation accuracy. Its optical properties remain constant over a wide range of wavelengths, from UV up to near-infrared, and it is widely used for radiation sensor cosine correction [28-30]. Light transmitted through the diffuser radiates, according to Lambert's cosine law. The cosine corrector, which has a thickness of $5 \mathrm{~mm}$ and diameter of $14 \mathrm{~mm}$, was manufactured at the photoelectric instrument factory of Beijing Normal University. The designed thickness was tested to ensure it had the optimal cosine response [30].

\subsubsection{Interference Filter}

We used an interference filter, customized to the MODIS band spectral response function, to guarantee the consistency in the detected wavelength range. This allowed us to obtain the upwards and downwards incident radiation in the same range as the MODIS band, and thus obtain the spectral albedo using Equation (1). The interference filter is a high-precision band-pass filter, commonly included in optical sensors [31,32]. It is possible to obtain the maximum transmittance of light in the required spectral bands by using the interference effect of dielectric and metallic multi-layers, while light beyond the band range is totally blocked. We used the first four bands of MODIS as an example and designed the interference filter according to the MODIS band by setting the filter transmittance to be identical to the MODIS spectral response function value. The central wavelength and full width at half maximum (FWHM) of the first four MODIS bands is provided in Table 1.

Table 1. Band information of first four MODerate Resolution Imaging Spectroradiometer (MODIS) bands.

\begin{tabular}{ccccc}
\hline & Band 1 & Band 2 & Band 3 & Band 4 \\
\hline Central wavelength (nm) & 645 & 858.5 & 469 & 555 \\
Full width at half maximum (FWHM) (nm) & 50 & 35 & 20 & 20 \\
\hline
\end{tabular}




\subsubsection{Silicon Photoelectric Detector}

Incident light passing through the interference filters was detected with a silicon photoelectric detector, installed directly behind the filter, which was then converted into a current signal that was proportional to the incident irradiance $\left(\mathrm{W} / \mathrm{m}^{2}\right)$. The silicon photoelectric detector has high sensitivity, a wide spectral response, and strong stability; it is widely used in optical instrumentation $[27,33]$. The 2DU10 photoelectric detector was purchased from Shenzhen Jeking Micro Electronic Technology Co., Ltd., (Shenzhen, China) and has a very large sensitivity area of $100 \mathrm{~mm}^{2}$ and a high photo-responsivity of $0.55 \mathrm{~A} / \mathrm{W}$; its operating temperature range is also very wide, from -20 to $+60^{\circ} \mathrm{C}$, and it can measure radiation in a wavelength range from 320 to $1100 \mathrm{~nm}$. The proposed spectral albedometer design is shown in Figure 3.

The output signal of the photoelectric detector is a current, which is directly proportional to the incident radiance determined by the sensitivity area and photo responsivity. Thus, for a downward radiance of $1000 \mathrm{~W} / \mathrm{m}^{2}$, the output current is:

$$
I_{0}=1000 \mathrm{~W} / \mathrm{m}^{2} \times 100 \mathrm{~mm}^{2} \times 0.55 \mathrm{~A} / \mathrm{W}=0.055 \mathrm{~A}
$$

We used a two-stage amplifying circuit to preserve the accuracy of the output signal. The output current was first amplified with a high precision amplifier (ICL7650SCPDZ) manufactured by Intersil Americas incorporated company (Milpitas, CA, USA). A $1.5 \mathrm{~K} \Omega$ resistor was connected to the ICL7650SCPDZ amplifier to reduce the DC error, and a $1000 \mathrm{pf}$ capacitor was connected parallel to the resistor as a filter condenser to stabilize the signal. The second-stage amplifier is a wide bandwidth single Junction Field-effect Transistor (JFET) operational amplifier (LF351) manufactured by Fairchild Semiconductor Corporation (Phoenix, AZ, USA), which we connected in its typical configuration. We used an isolation transmitter (ISO-U8-P3-O4) purchased from Anfu century electronics limited company (Shenzhen, China) to detect the sensor's signal transceiver and collected the output with a data logger. For ease of operation, we installed the amplifying circuit alongside the data logger and data transfer system with a single power supply. The amplifying circuit in this design is operational, but not unique. The data, as-collected, were transferred back to a local computer, via the General Packet Radio Service (GPRS) network.

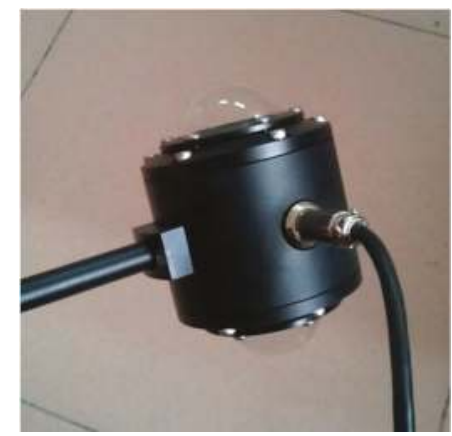

Figure 3. Developed spectral albedometer for one band.

\section{Results}

\subsection{Interference Filter Transmittance}

To ensure that the radiation obtained by the sensor is comparable to that measured by the MODIS sensor, the interference filters must have transmittance equal to the MODIS band spectral response function value. Filter transmittance characterization was carried out at the State Key Laboratory of 
Remote Sensing Science, Beijing Normal University. The field spectroradiometer SVC-HR1024 came from the Spectra Vista Corporation (New York, NY, USA) and the LI-COR1800 integrating sphere came from LI-COR (Lincoln, NE, USA). The spectral resolution of SVC-HR1024 exceeds $3.5 \mathrm{~nm}$ in the 300-1000 nm range, which can satisfactorily describe the spectral variation characteristics of surface features. The integrating sphere was equipped with a standard halogen lamp. To measure the filter transmittance, first, source light was injected directly into the integrating sphere and measured. Next, the filter was carefully placed onto a sample stage in the integrating sphere using clean tweezers and light was shined into the integrating sphere through the filter. We then measured the intensity of the transmitted light and calculated the filter transmittance, according to Equation (4):

$$
T_{\lambda}=\frac{I_{t \lambda}}{I_{\lambda}}
$$

where $T$ is the filter transmittance; $I$ and $I_{t}$ are the direct light intensity and light intensity through the filter, respectively (i.e., the digital number values in the experiment), and $\lambda$ is the band wavelength.

The band consistency of the proposed albedometer with the MODIS sensors is the premise of the direct validation of the spectral albedo product. Figure 4 shows a comparison of the filter transmittance and MODIS band response function value for each band. As shown in Figure 4, the customized interference filter transmittance has close consistency with the MODIS band response function. Table 2 shows the key indicators of center wavelength and FWHM of the customized interference. In comparison to the values shown in Table 1, the customized interference filter has the same center wavelength of each band as the MODIS band, and the FWHMs are nearly the same; deviations range from 1-5 nm, which is about $5.0-10.0 \%$ of the FWHM.
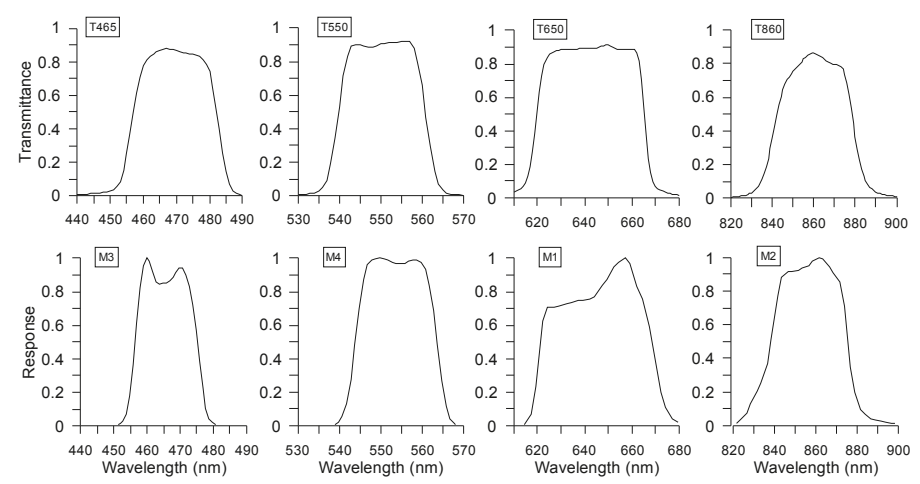

Figure 4. Customized interference filter transmittance versus MODIS band response functions. The top figure of each column is the transmittance of the customized interference filter; the bottom figure is the spectral response function of the MODIS band.

Table 2. Characteristics of the customized interference filter.

\begin{tabular}{ccccc}
\hline & Band 1 & Band 2 & Band 3 & Band 4 \\
\hline Central wavelength (nm) & 645 & 858.5 & 469 & 555 \\
FWHM (nm) & 45 & 38 & 22 & 21 \\
\hline
\end{tabular}

\subsection{Calibration}

Theoretically, it is only necessary to ensure the consistency of the upwards and downwards sensors to obtain an accurate albedo measurement. That is, it is only necessary to make relative radiometric calibrations of the sensor. In this study, we carried out absolute calibrations of the sensor, 
in order to verify the spectral net radiation measurement at the same time: (1) we ensured the upward and downward sensors were consistent with each other; and (2) we obtained the absolute radiation and net radiation corresponding to each band. The sensors were subjected to absolute radiation calibration at the photoelectric instrument factory in Beijing Normal University, where the light source system and standard radiometer used for calibration were a standard source transferred from the National Institute of Metrology (NIM).

A standard radiometer is often used as a reference for general broadband radiometer calibration. First, we put the standard radiometer under standard light and read the standard radiometer value $\left(E_{0}\right)$, then we removed the standard radiometer and put the detector to be calibrated at the same position. The distance between the detector and the standard light source was kept identical to the standard radiometer and light source. We recorded the current readings $\left(I_{1}\right)$ of the detector sensor to be calibrated, then turned the downward detector upwards and recorded the current readings $\left(I_{2}\right)$ of the other side of the detector sensor to be calibrated with the same method. We changed the light intensity several times and obtained the corresponding readings of the standard radiometer and the to-be-calibrated sensors $\left(E_{0 i}, I_{1 i}, I_{2 i}\right)$. We obtained the calibration coefficients of both sensors by respectively fitting $E_{0 i}, I_{1 i}$ and $E_{0 i}, I_{2 i}$.

A large disparity emerged in the band response range between the standard radiometer and the spectral albedometer when calibrating the spectral albedometer, so we used a spectral radiometer to calibrate the spectral albedometer instead of a broadband standard radiometer. The RAYSPHERE spectrum radiation meter (American Ocean Optics Company, Dunedin, FL, USA) can measure 350-1100 nm spectral irradiance with $1.9 \mathrm{~nm}$ spectral resolution. The total irradiance of a given band can be calculated using Equation (2), according to the band response functions of the sensors to be calibrated. The correction coefficient of the upwards and downwards sensors were obtained via the same stepwise process as the broadband sensor calibration. The calibration results are shown in Figure 5 .

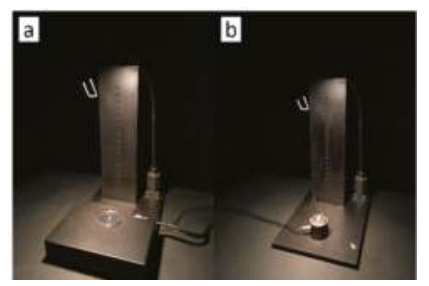

Figure 5. Spectral albedometer calibration. (a) Incident light is first measured by the spectral albedometer and the radiation of each band is integrated; (b) Incident light is then measured by the spectral albedometer sensor. The upward and downward sensors are calibrated independently.

The linearity of a given sensor is a key specification of the instrument. We calibrated a total of 80 sensors to quantify the linearity of the selected sensors with five different light intensities: 0,250 , 500,750 , and $1000 \mathrm{~W} / \mathrm{m}^{2}$ for the broadband range $(300-1100 \mathrm{~nm})$. The spectral light intensities of each band were measured with the RAYSPHERE spectrum radiation meter, described above, and integrated using Equation (2). The worst-fitting line of the sensor, corresponding to MODIS band 1, is shown in Figure 6.

The sensor's reading had a very close linear correlation with the incoming light intensity. The coefficient of determination $\left(R^{2}\right)$ was calculated to be 0.9996 , which indicates that the linearity of the sensor is very good. We used the non-linear error (Equation (5)) to quantify the linearity of all the sensors:

$$
\delta=\frac{\Delta_{\max }}{I_{2}-I_{1}}
$$


where $\delta$ is the nonlinearity of the sensor (non-dimensional), $\Delta_{\max }$ is the maximum deviation between the actual response curve and the fitting line, and $I_{1}$ and $I_{2}$ are the maximum and minimum response values, respectively.

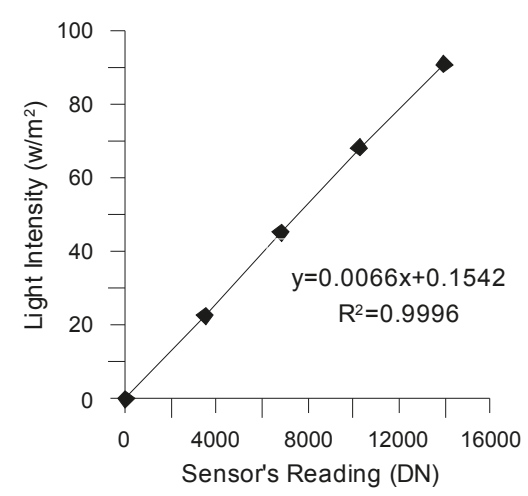

Figure 6. Calibration result of the worst-fitting sensor in the total of 80 calibrated sensors, with coefficient of determination of 0.9996 .

Again, we calibrated 80 sensors in total. Figure 7 shows a nonlinearity histogram of the sensor calibration results, where more than $40 \%$ of the sensors are absolutely linear and $78 \%$ of the sensors have a linearity better than $99.9 \%$ (non-linear error less than 0.001 ). All of the sensors have linearity exceeding $99 \%$, which fully satisfies the detection requirements.

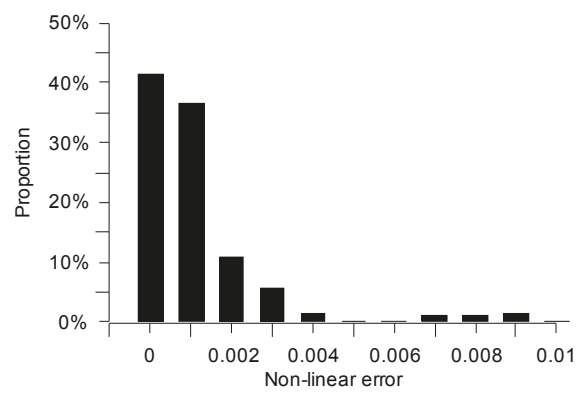

Figure 7. Non-linear error histogram of 80 calibrated sensors. More than $40 \%$ of the sensors are absolutely linear, with a non-linear error of 0 ; all sensors' non-linear error is below 0.01 .

Figure 8 shows the coefficients of determination of the linear fitting of the calibration results, which exceed 0.999 for all of the sensors we tested.

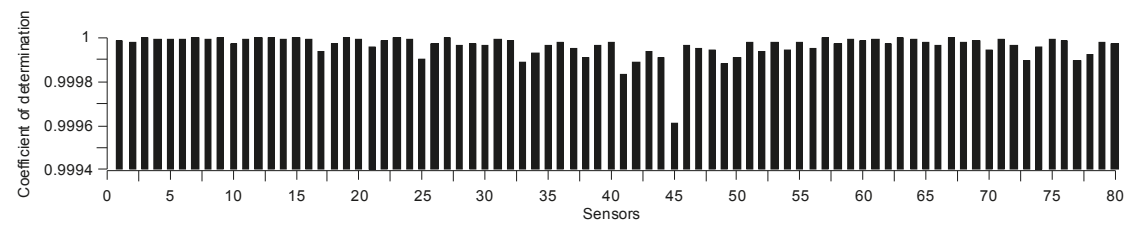

Figure 8. Coefficients of determination of linear fitting results. 


\subsection{Comparison with Commercial Instrument}

The designed albedometer was then compared with the widely-used commercial instrument CNR4 net radiometer from Kipp \& Zonen, Delft, The Netherlands [34]. The CNR4 net radiometer consists of two pyranometers for measuring downward and upward solar shortwave radiation, and two pyrgeometers for measuring upward and downward thermal longwave radiation. The designed albedometer was mounted alongside the CNR4 radiometer at the same height. Downward radiation was collected from 6:00 hr to 16:00 hr on 19 June 2014. Figure 9 shows a comparison of band radiation against the CNR4 pyranometers' measurements. The spectral albedometer measurement has close consistency with the CNR4 measurement, with coefficients of determination of 0.9966 for the $465 \mathrm{~nm}$ sensor, 0.9979 for the $550 \mathrm{~nm}$ sensor, 0.9984 for the $650 \mathrm{~nm}$ sensor, and 0.993 for the $860 \mathrm{~nm}$ sensor, respectively.

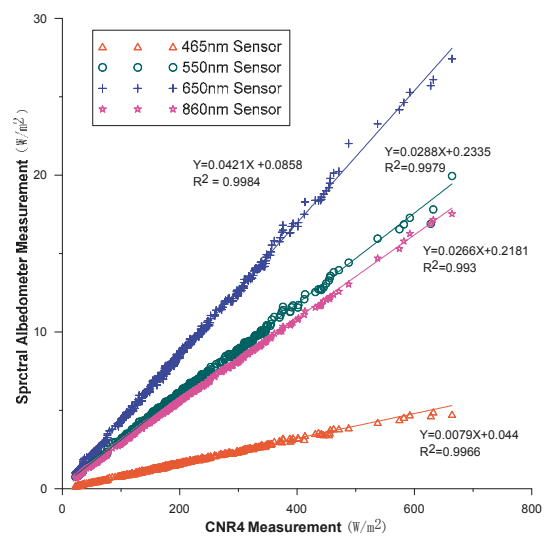

Figure 9. Spectral albedometer compared with CNR4 radiometer.

\subsection{MODIS Spectral Albedo Product Validation}

After the spectral albedometers were absolutely calibrated, we conducted a series of field experiments in Yudaokou, Hebei, China $\left(42.24^{\circ} \mathrm{N}, 117.07^{\circ} \mathrm{E}\right)$. The land cover type at the study area is entirely grassland. Spectral albedometers were set up atop a 20-m tower and covered an area of about $150 \times 150 \mathrm{~m}$ (Figure 10). The surface is uniform, so we took the tower observations as the true ground measurement values of a MODIS $500 \times 500 \mathrm{~m}$ image element, to directly validate the MODIS spectral albedo products.

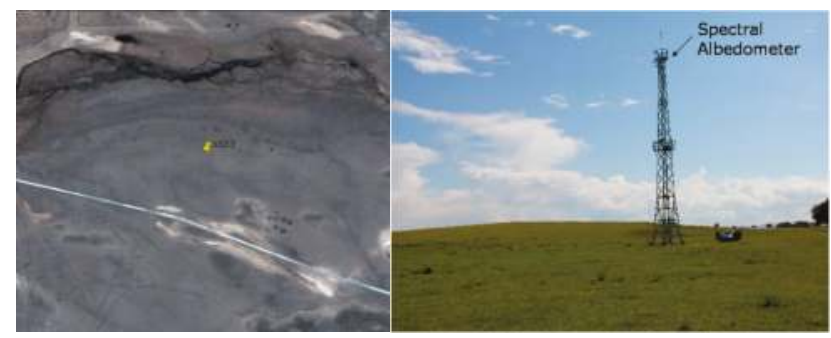

Figure 10. Field observation of the designed spectral albedometer. The left side shows the tower location from Google Earth on 30 April 2013. The site is homogeneous grassland, except for a road passing through the area. The right figure is the $20-\mathrm{m}$ tower. The designed spectral albedometers are mounted atop the tower. 
Field measurements were performed from October 2014, to May 2015. The continuous upwards and downwards spectral radiation intensities of the grassland were recorded and the spectral albedo was calculated with Equation (1) for each band. The standard 500-m gridded MODIS V006 BRDF/albedo product (MCD43A3) was then validated using the field observations. Black sky albedo and white sky albedo were generated by integrating the BRDF calculated from the three retrieved parameters $[3,7,35,36]$. Blue sky albedo, which encompasses both diffuse and direct radiation, was calculated, as follows [37]:

$$
a_{\text {blue-sky }}\left(\theta_{i}\right)=\operatorname{SKYL}\left(\theta_{i}\right) \times a_{\text {white-sky }}+\left(1-\operatorname{SKYL}\left(\theta_{i}\right)\right) \times a_{\text {black-sky }}\left(\theta_{i}\right)
$$

where SKYL is the proportion of diffuse irradiation at a certain solar zenith angle, $\theta_{i}$ [35]. The optical depth of the study area was extracted from the MODIS aerosol product (MOD08) [38], and SKYL was calculated with the Look Up Table (LUT), according to Remer, Tanre [39], with the optical depth of the area at 0.55 micron from MOD08 and the solar zenith angle calculated based on the site location and observation time.

The spectral albedo time series was directly compared against the MODIS spectral band albedo products, to validate the remote sensing spectral albedo products. Considering the homogeneity of the landscape, the field observations were compared directly with the $500 \mathrm{~m}$ MODIS albedo product (MCD43A3).

Figure 11 shows the direct validation of the MODIS spectral albedo product with the spectral albedometer measurements. The MODIS albedo product is a combination of a 16-day observation, with weight as a function of the quality; the observation coverage and temporal distance from the day of interest represents the daily value at noon [36]. The spectral albedometer measurements, from 11:45 hr to 12:15 hr of each day were averaged to generate the daily noon value.

Figure 11 shows where the MODIS spectral albedo was in close accordance with our field observations during snow-free periods; the MODIS product is highly continuous and stable. During snowy periods, there is some missing data in the MODIS product, due to retrieval failure (Figure 11), i.e., a failure to capture snow events, such as on day of year (DOY) 92, 2015. There were also some missing data in the field observations in late 2014, due to a low battery in the data transfer system. The statistical results of the direct comparison of the MODIS spectral albedo and spectral albedometer measurements during snow-free periods are listed in Table 3. 


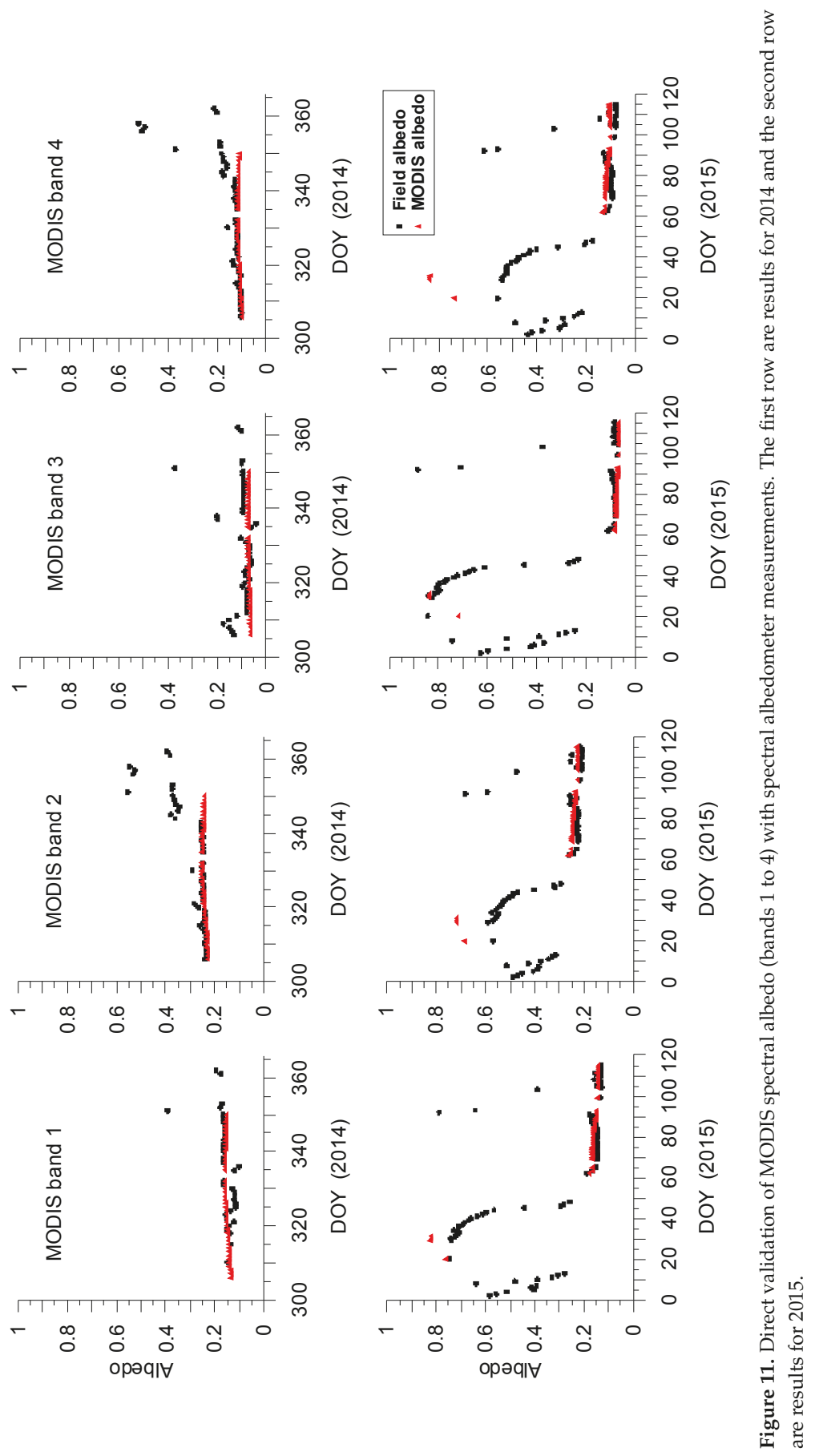


Table 3. Statistical results of direct comparison of MODIS spectral albedo and spectral albedometer measurements.

\begin{tabular}{ccc}
\hline & RMSE & BIAS \\
\hline Band 1 & 0.019 & -0.0094 \\
band 2 & 0.0368 & 0.0065 \\
band 3 & 0.0317 & 0.0159 \\
band 4 & 0.0244 & -0.0001 \\
\hline
\end{tabular}

Table 3 shows where the accuracy of the MODIS spectral albedo products at our experimental site is relatively high. Compared to the field measurements, the root mean square errors (RMSE) of the four bands are $0.019,0.0368,0.0317$, and 0.0244 and the biases are $-0.0094,0.0065,0.0159$, and -0.0001 , respectively.

\section{Discussion}

Calleja, Recondo [40] found that inaccuracy in the MODIS albedo product is partly due to narrow to broadband conversion. Thus, assessing the remote sensing spectral albedo product is the best way to verify the retrieval algorithm. The designed spectral albedometer provides a novel approach for obtaining field time series observations, which are critical for remote sensing spectral albedo product validation.

\subsection{Errors Induced by the Spectral Albedometer Design}

There are some error sources in the spectral albedometer design discussed here. The first is a discrepancy between the customized interference transmittance and the MODIS spectral response function. The deviations in FWHM range from 1-5 nm, which induce about $5.0-10.0 \%$ of the observation error. The MODIS spectral response also has a considerably different 'top' than the customized filter shown in Figure 4, which means that the weighted spectral average of the ground hyperspectral image could conceivably be different between the two systems and thus introduce error. Though accurate calibration can reduce some error, it is impossible to remove all of it.

The second source of error may have been induced by albedometer body temperature. The silicon photoelectric detector is temperature-dependent [41]: the output increases as the device's temperature goes up. The general means of accurate observation is temperature control [30]. In our design, the upward and downward radiation is divided, to yield a spectral albedo for each band. The temperature dependence is reduced when the division is performed, so no temperature control system needs to be added to the system.

\subsection{Land Surface Homogeneity}

Field observation was conducted at a Yudaokou grassland site and directly compared with the remote sensing product, under the assumption that the land surface was sufficiently homogeneous. In actuality, land surface homogeneity varies with time. We analyzed the land surface homogeniety of the research area with a Landsat 8 OLI data-derived albedo [42], according to the literature [3,43], during dormant and growing seasons. The fitted sill values were both less than 0.001 [44], which indicates that the land surface is indeed homogeneous enough for direct comparison.

\subsection{Capability of Simultaneous Multi-Parameter Observation}

We designed and tested albetometers for four bands in this study. It is possible to obtain different band combinations using different filters, such as visible band $(400-700 \mathrm{~nm})$ radiation detection. By mounting one albedometer under canopy and one over canopy, the visible band albedo and Fraction of Absorbed Photosynthetically Active Radiation (FAPAR) can be simultaneously obtained, 
fairly easily. The multi-parameter combined observation has significant value in regards to its efficiency and physically-consistent parameter estimations.

\section{Conclusions}

In this study, we designed a measuring system that allowed us to accurately obtain spectral albedos corresponding to satellite sensors. Our design effectively avoids errors associated with traditional broadband surface albedo product verification due to wavelength conversion and band mismatch; it also mitigates the defects in spectroradiometer measurements, which are labor- and time-consuming to gather and are difficult to use when conducting regional-scale time-series observations. We conducted a series of experiments in the Yudaokou grasslands to demonstrate that the proposed device can feasibly and effectively provide spectral albedo time series observations. Other important characteristics of the proposed device can be summarized as follows.

- Strong linear response of the sensor: The sensor utilizes an interference filter equipped with sophisticated technology to gather incident radiation measurements over specific wavelength ranges. We used a highly sensitive photoelectric detector in our design which is simple and reliable, with a $>99 \%$ linear response that fully satisfies the requirements for precise albedo detection, with an absolute accuracy of 0.02-0.05 [1].

- Flexibility in the sensor design: We ran a successful spectral albedo test using the MODIS band set as an example. Our design can be easily extended to other satellite sensors and other bands. A wide range of photodetectors can also be used for the direct measurement of broadband surface albedos with multi-band combinations.

- Applicability to multi-point observation layouts: Compared to other currently available radiometers, the materials necessary for our design are relatively inexpensive. To this effect, our sensor can realize multi-point layouts in a given heterogeneous area to resolve issues with single-point observations in representing remote sensing pixel values.

We designed our sensor to apply to spectral albedos over the 400-1000 nm range, but not the broadband shortwave range (300-2800 nm), as the silicon-sensitive photoelectric detector cannot detect incident light with wavelengths $>1100 \mathrm{~nm}$. The broadband albedo is significant in regards to scientific use [6], so it is necessary to design additional spectral albedometers that can operate at ranges between 1100 and $2800 \mathrm{~nm}$. The thermopile detector is often applied to broadband radiation detection, which may be useful in this regard $[45,46]$. We will focus on the design and performance of the spectral albedometer design at wavelengths greater than $1100 \mathrm{~nm}$, and will continue to carry out observation experiments tailored to heterogeneous surfaces.

Acknowledgments: This work was supported by the Key research and development program of China 2016YFB0501404, 2016YFB0501502, the Chinese 973 Program under grant 2013CB733403, and the National Natural Science Foundation of China under grant 41171263.

Author Contributions: Hongmin Zhou, Jindi Wang, and Shunlin Liang conceived and designed the experiments; Hongmin Zhou performed the experiments and analyzed the data; Hongmin Zhou wrote the paper; Jindi Wang and Shunlin Liang revised the paper.

Conflicts of Interest: The authors declare no conflict of interest.

\section{References}

1. Dickinson, R.E. Land surface processes and climate surface albedos and energy-balance. Adv. Geophys. 1983, 25, 305-353.

2. Wang, K.; Liang, S.; Schaaf, C.L.; Strahler, A.H. Evaluation of Moderate Resolution Imaging Spectroradiometer land surface visible and shortwave albedo products at FLUXNET sites. J. Geophys. Res. Atmos. 2010, 115. [CrossRef] 
3. Wang, Z.; Schaaf, C.B.; Strahler, A.H.; Chopping, M.J.; Román, M.O.; Shuai, Y.; Woodcock, C.E.; Hollinger, D.Y.; Fitzjarrald, D.R. Evaluation of MODIS albedo product (MCD43A) over grassland, agriculture and forest surface types during dormant and snow-covered periods. Remote Sens. Environ. 2014, 140, 60-77. [CrossRef]

4. Wang, Z.; Zeng, X. Evaluation of snow albedo in land models for weather and climate studies. J. Appl. Meteorol. Climatol. 2010, 49, 363-380. [CrossRef]

5. Lewis, P.; Disney, M.; Barnsley, M.; Muller, J.-P. Deriving albedo maps for HAPEX-Sahel from ASAS data using kernel-driven BRDF models. Hydrol. Earth Syst. Sci. Discuss. 1999, 3, 1-11. [CrossRef]

6. Liang, S. Narrowband to broadband conversions of land surface albedo I: Algorithms. Remote Sens. Environ. 2000, 76, 213-238. [CrossRef]

7. Schaaf, C.B.; Gao, F.; Strahler, A.H.; Lucht, W.; Li, X.; Tsang, T.; Strugnell, N.C.; Zhang, X.; Jin, Y.; Muller, J.-P.; et al. First operational BRDF, albedo nadir reflectance products from MODIS. Remote Sens. Environ. 2002, 83, 135-148. [CrossRef]

8. Pinty, B.; Roveda, F.; Verstraete, M.M.; Gobron, N.; Govaerts, Y.; Martonchik, J.V.; Diner, D.J.; Kahn, R.A. Surface albedo retrieval from Meteosat: 1. Theory. J. Geophys. Res. Atmos. 2000, 105, 18099-18112. [CrossRef]

9. Wang, D.; Liang, S.; He, T.; Yu, Y. Direct estimation of land surface albedo from VIIRS data: Algorithm improvement and preliminary validation. J. Geophys. Res. Atmos. 2013, 118, 577-586. [CrossRef]

10. Wang, D.; Liang, S.; He, T.; Yu, Y.; Schaaf, C.; Wang, Z. Estimating daily mean land surface albedo from MODIS data. J. Geophys. Res. Atmos. 2015, 120, 4825-4841. [CrossRef]

11. He, T.; Liang, S.; Wang, D. Direct Estimation of Land Surface Albedo From Simultaneous MISR Data. IEEE Trans. Geosci. Remote Sens. 2017, 55, 2605-2617. [CrossRef]

12. Liang, S.; Fang, H.; Chen, M.; Shuey, C.J.; Walthall, C.; Daughtry, C.; Morisette, J.; Schaaf, C.; Strahler, A. Validating MODIS land surface reflectance and albedo products: Methods and preliminary results. Remote Sens. Environ. 2002, 83, 149-162. [CrossRef]

13. Wang, K.; Liu, J.; Zhou, X.; Sparrow, M.; Ma, M.; Sun, Z.; Jiang, W. Validation of the MODIS global land surface albedo product using ground measurements in a semidesert region on the Tibetan Plateau. J. Geophys. Res. Atmos. 2004, 109. [CrossRef]

14. Cescatti, A.; Marcolla, B.; Santhana Vannan, S.K.; Pan, J.Y.; Román, M.O.; Yang, X.; Ciais, P.; Cook, R.B.; Law, B.E.; Matteucci, G.; et al. Intercomparison of MODIS albedo retrievals and in situ measurements across the global FLUXNET network. Remote Sens. Environ. 2012, 121, 323-334. [CrossRef]

15. Song, J.; Gao, W. An improved method to derive surface albedo from narrowband AVHRR satellite data: Narrowband to broadband conversion. J. Appl. Meteorol. 1999, 38, 239-249. [CrossRef]

16. Valiente, J.; Nunez, M.; Lopez-Baeza, E.; Moreno, J. Narrow-band to broad-band conversion for meteosat-visiible channel and broad-band albedo using both AVHRR-1 and-2 channels. Remote Sens. 1995, 16, 1147-1166. [CrossRef]

17. Lucht, W.; Hyman, A.H.; Strahler, A.H.; Barnsley, M.J.; Hobson, P.; Muller, J.-P. A comparison of satellite-derived spectral albedos to ground-based broadband albedo measurements modeled to satellite spatial scale for a semidesert landscape. Remote Sens. Environ. 2000, 74, 85-98. [CrossRef]

18. Picard, G.; Libois, Q.; Arnaud, L.; Verin, G.; Dumont, M. Development and calibration of an automatic spectral albedometer to estimate near-surface snow SSA time series. Cryosphere 2016, 10, 1297-1316. [CrossRef]

19. Cheng, J.; Liang, S. Estimating the broadband longwave emissivity of global bare soil from the MODIS shortwave albedo product. J. Geophys. Res. Atmos. 2014, 119, 614-634. [CrossRef]

20. Wuttke, S.; Seckmeyer, G.; König-Langlo, G. Measurements of spectral snow albedo at Neumayer, Antarctica. Ann. Geophys. 2006, 24, 7-21. [CrossRef]

21. Wright, P.; Bergin, M.; Dibb, J.; Lefer, B.; Domine, F.; Carman, T.; Carmagnola, C.; Dumont, M.; Courville, Z.; Schaaf, C.; et al. Comparing MODIS daily snow albedo to spectral albedo field measurements in Central Greenland. Remote Sens. Environ. 2014, 140, 118-129. [CrossRef]

22. Grenfell, T.C.; Warren, S.G.; Mullen, P.C. Reflection of solar radiation by the Antarctic snow surface at ultraviolet, visible, and near-infrared wavelengths. J. Geophys. Res. Atmos. 1994, 99, 18669-18684. [CrossRef] 
23. Hudson, S.R.; Warren, S.G.; Brandt, R.E.; Grenfell, T.C.; Six, D. Spectral bidirectional reflectance of Antarctic snow: Measurements and parameterization. J. Geophys. Res. Atmos. 2006, 111. [CrossRef]

24. Marks, A.; Fragiacomo, C.; Macarthur, A.; Zibordi, G.; Fox, N.; King, M.D. Characterisation of the HDRF (as a proxy for BRDF) of snow surfaces at Dome C, Antarctica, for the inter-calibration and inter-comparison of satellite optical data. Remote Sens. Environ. 2015, 158, 407-416. [CrossRef]

25. Feister, U.; Grewe, R. Spectral albedo measurements in the UV and visible region over different types of surfaces. Photochem. Photobiol. 1995, 62, 736-744. [CrossRef]

26. Angström, A. The albedo of various surfaces of ground. Geogr. Ann. 1925, 7, 323-342.

27. King, D.L.; Myers, D.R. Silicon-photodiode pyranometers: Operational characteristics, historical experiences, and new calibration procedures. In Proceedings of the Conference Record of the Twenty Sixth IEEE Photovoltaic Specialists Conference-1997, Anaheim, CA, USA, 29 Septemebr-3 October 1997.

28. Michalsky, J.; Harrison, L.; Berkheiser Iii, W. Cosine response characteristics of some radiometric and photometric sensors. Sol. Energy 1995, 54, 397-402. [CrossRef]

29. Lowry, J.H.; Mendlowitz, J.S. Optical characteristics of Teflon AF fluoroplastic materials. Opt. Eng. 1992, 31, 1982-1985. [CrossRef]

30. Martínez, M.A.; Andújar, J.M.; Enrique, J.M. A new and inexpensive pyranometer for the visible spectral range. Sensors 2009, 9, 4615-4634. [CrossRef] [PubMed]

31. Apfel, J.H. Infra-Red Interference Filter. U.S. Patent 3,682,528 A, 8 August 1972.

32. Yokota, S.; Sugiyama, M.; Kawagoe, N.; Miyaura, T. Spectral Sensor with Interference Filter. U.S. Patent 4,822,998A, 18 April 1989.

33. Ndukwe, I. Measurement of solar energy radiation at Okigwe using silicon solar cell. Energy Convers. Manag. 2000, 41, 189-197. [CrossRef]

34. Xu, Z.; Liu, S.; Li, X.; Shi, S.; Wang, J.; Zhu, Z.; Xu, T.; Wang, W.; Ma, M. Intercomparison of surface energy flux measurement systems used during the HiWATER-MUSOEXE. J. Geophys. Res. Atmos. 2013, 118. [CrossRef]

35. Lucht, W.; Schaaf, C.B.; Strahler, A.H. An algorithm for the retrieval of albedo from space using semiempirical BRDF models. IEEE Trans. Geosci. Remote Sens. 2000, 38, 977-998. [CrossRef]

36. Shuai, Y. Tracking Daily Land Surface Albedo and Reflectance Anisotropy with MODerate-Resolution Imaging Spectroradiometer (MODIS); Boston University: Boston, MA, USA, 2010; p. 118.

37. Lewis, P.; Barnsley, M.J. Influence of the sky radiance distribution on various formulations of the Earth surface albedo. In Proceedings of the International Symposium on Physical Measurements \& Signatures in Remote Sensing Isprs, Val d'Isere, France, 17-24 January 1994.

38. Hubaks, P.; Platnik, S.; King, M.; Ridgway, B. MODIS Atmosphere L3 Gridded Product Algorithm Theoretical Basis Document (ATBD) \& Users Guide; MODIS Algorithm Theoretical Basis Document No. ATBD-MOD-30. Available online: https://modis-atmos.gsfc.nasa.gov/_docs/L3_ATBD_C6_2015_05_06.pdf (accessed on 9 January 2018).

39. Remer, L.A.; Tanre, D.; Kaufman, Y.J.; Levy, R.; Mattoo, S. Algorithm for Remote Sensing of Tropospheric Aerosol from MODIS: Collection 005. Natl. Aeronaut. Space Admin. Available online: https://pdfs. semanticscholar.org/566d/19c074f199963abba848ad53b77062d1333d.pdf (accessed on 9 January 2018).

40. Calleja, J.F.; Recondo, C.; Peón, J.; Fernández, S.; De La Cruz, F.; González-Piqueras, J. A New Method for the Estimation of Broadband Apparent Albedo Using Hyperspectral Airborne Hemispherical Directional Reflectance Factor Values. Remote Sens. 2016, 8, 183. [CrossRef]

41. Degenhardt, C.; Prescher, G.; Frach, T.; Thon, A.; De Gruyter, R.; Schmitz, A.; Ballizany, R. The digital silicon photomultiplier-A novel sensor for the detection of scintillation light. In Proceedings of the 2009 IEEE Nuclear Science Symposium Conference Record (NSS/MIC), Orlando, FL, USA, 24 October-1 November 2009.

42. He, T.; Liang, S.; Wang, D.; Cao, Y.; Gao, F.; Yu, Y.; Feng, M. Evaluating land surface albedo estimation from Landsat MSS, TM, ETM+, and OLI data based on the unified direct estimation approach. Remote Sens. Environ. 2018, 204 (Suppl. C), 181-196. [CrossRef]

43. Wang, Z.; Schaaf, C.B.; Chopping, M.J.; Strahler, A.H.; Wang, J.; Román, M.O.; Rocha, A.V.; Woodcock, C.E.; Shuai, Y. Evaluation of Moderate-resolution Imaging Spectroradiometer (MODIS) snow albedo product (MCD43A) over tundra. Remote Sens. Environ. 2012, 117, 264-280. [CrossRef] 
44. Burakowski, E.A.; Ollinger, S.V.; Lepine, L.; Schaaf, C.B.; Wang, Z.; Dibb, J.E.; Hollinger, D.Y.; Kim, J.; Erb, A.; Martin, M. Spatial scaling of reflectance and surface albedo over a mixed-use, temperate forest landscape during snow-covered periods. Remote Sens. Environ. 2015, 158, 465-477. [CrossRef]

45. Proctor, D.; Trickett, E. An improved pyranometer. Sol. Energy 1982, 29, 189-194. [CrossRef]

46. Beaubien, D.; Bisberg, A.; Beaubien, A. Investigations in pyranometer design. J. Atmos. Ocean. Technol. 1998, 15, 677-686. [CrossRef]

(C) 2018 by the authors. Licensee MDPI, Basel, Switzerland. This article is an open access article distributed under the terms and conditions of the Creative Commons Attribution (CC BY) license (http://creativecommons.org/licenses/by/4.0/). 
Article

\title{
Spatiotemporally Representative and Cost-Efficient Sampling Design for Validation Activities in Wanglang Experimental Site
}

\author{
Gaofei Yin ${ }^{1}$, Ainong $\mathrm{Li}^{1}{ }^{1} *$ and Aleixandre Verger ${ }^{2}$ \\ 1 Research Center for Digital Mountain and Remote Sensing Application, Institute of Mountain Hazards \\ and Environment, Chinese Academy of Sciences, Chengdu 610041, China; gaofeiyin@imde.ac.cn \\ 2 CREAF, E08193 Bellaterra (Cerdanyola del Vallès), Catalonia, Spain; verger@creaf.uab.cat \\ * Correspondence: ainongli@imde.ac.cn; Tel.: +86-028-8535-3832
}

Received: 12 September 2017; Accepted: 23 November 2017; Published: 26 November 2017

\begin{abstract}
Spatiotemporally representative Elementary Sampling Units (ESUs) are required for capturing the temporal variations in surface spatial heterogeneity through field measurements. Since inaccessibility often coexists with heterogeneity, a cost-efficient sampling design is mandatory. We proposed a sampling strategy to generate spatiotemporally representative and cost-efficient ESUs based on the conditioned Latin hypercube sampling scheme. The proposed strategy was constrained by multi-temporal Normalized Difference Vegetation Index (NDVI) imagery, and the ESUs were limited within a sampling feasible region established based on accessibility criteria. A novel criterion based on the Overlapping Area (OA) between the NDVI frequency distribution histogram from the sampled ESUs and that from the entire study area was used to assess the sampling efficiency. A case study in Wanglang National Nature Reserve in China showed that the proposed strategy improves the spatiotemporally representativeness of sampling (mean annual $\mathrm{OA}=74.7 \%)$ compared to the single-temporally constrained $(\mathrm{OA}=68.7 \%)$ and the random sampling $(\mathrm{OA}=63.1 \%)$ strategies. The introduction of the feasible region constraint significantly reduces in-situ labour-intensive characterization necessities at expenses of about $9 \%$ loss in the spatiotemporal representativeness of the sampling. Our study will support the validation activities in Wanglang experimental site providing a benchmark for locating the nodes of automatic observation systems (e.g., LAINet) which need a spatially distributed and temporally fixed sampling design.
\end{abstract}

Keywords: spatiotemporal representative; cost-efficient, sampling design; heterogeneity; validation

\section{Introduction}

Accurate spatiotemporal characterization of land surface heterogeneity [1-3] is essential for remote sensing [4] and the land surface [5] modeling. The assumption of surface spatial homogeneity within the Elementary Modeling Unit (EMU) (e.g., a pixel for a remote sensing image or a grid for a land surface model) induces scaling errors $[6,7]$. This is especially the case for coarse spatial resolution EMU and satellite land surface products with resolutions ranging from $500 \mathrm{~m} \mathrm{[8]} \mathrm{to}$ $5 \mathrm{~km}$ [9]. The magnitude of the scaling error is determined by the nonlinearity of the process to be modeled and the surface heterogeneity within the EMU which is often ignored [10,11]. Quantifying the sub-pixel/-grid heterogeneity is the prerequisite for the parameterization, calibration and validation of the remote sensing and the land surface process models [1,12].

Spatiotemporal representative field measurements are required to reproduce the surface heterogeneity within a coarse spatial resolution EMU and to support validation of satellite surface products. However, labour-intensive field measurement collection is usually limited by budget and 
time constraints. In this sense, the design of efficient sampling strategies preserving the statistics of the population [13-15] within an affordable cost is urgently needed.

The Land Product Validation (LPV of the Committee Earth Observing Satellites' Working Group on Calibration and Validation (CEOS WGCV) recommended a two-stage nested sampling framework to account for the multi-scale nature of the heterogeneity [16]. Fine spatial resolution satellite imagery (about $30 \mathrm{~m}$ ) are used as a bridge to upscale field measurements to the EMU scale. One of the key points in this framework is the introduction of Elementary Sampling Unit (ESUs) that has approximately the same size as the fine resolution image pixel. Another key point in this framework is the establishment of a transfer function to relate the field measurements over the ESUs with the fine resolution image data. This transfer function can then be used to generate a fine resolution reference map which serves as the benchmark to characterize the surface heterogeneity within the EMU's coarse spatial resolution. The two-stage nested sampling refers to the sampling of field measurements within the ESU and the sampling of ESUs within the EMU [17]. At ESU scale, square, cross or transect sampling schemes are recommended depending on the measuring instrument and the characteristics of the surface [17]. The sampling scheme at the EMU scale is still far from mature.

The most commonly used sampling strategies at the EMU scale can be categorized into random, systematic, and stratified sampling [18]. Recent researches have increasingly relied on the stratified sampling based on a priori information. Stratified sampling strategies firstly select auxiliary variables which can be easily generated from remote sensing observations (vegetation indices, generally) to represent the target variables (e.g., leaf area index, fractional vegetation cover and chlorophyll content), then subdivide each auxiliary variable into several strata, and finally sample the plots randomly within each stratum. Stratified sampling strategies are assumed to be capable of optimally capturing the variability across the site extent [13,19-21]. The conditioned Latin hypercube (CLH) sampling is among the most appealing stratified sampling strategies [22]. CLH was proposed in the context of digital soil mapping, but has been used in many other fields because of its representativeness and extensibility [22-24]. Recently, Zeng et al. [25] and Yin et al. [14] introduced this method to the ESU sampling.

Existing researches on field sampling design generally focused on capturing the landscape spatial heterogeneity by distributing the ESUs across the entire study area [16,18-21]. Two key issues, which are the main scientific questions addressed in this paper, were often ignored in traditional ESU sampling schemes: First, the spatial heterogeneity changes over time. Second, some parts of the study area may be inaccessible because of the rugged terrain, lack of roads or barrier of river. Addressing the temporal dynamics of the surface spatial $[26,27]$ is key for the development of the near-surface remote sensing technologies which can provide long-term measurements automatically [28-30]. Zeng et al. [25] employed multi-temporal vegetation index maps as a priori information to constrain the sampling process and generate spatiotemporally representative ESUs. However, the accessibility of the sampling plots was neglected in this scheme [25] which may be critical in traffic inconvenient regions. In fact, the cost limitation in field campaign was already considered by few previous studies. Yin et al. [14] incorporated a cost-objective function to traditional CLH to define a cost-efficient sampling design. However, some inaccessible ESUs may remain in this sampling scheme, which is based on a global minimization of the cost-objective function. In addition, the temporal variation of the spatial heterogeneity was not considered. To summarize, the spatiotemporally heterogeneity and cost limitation were separately accounted for by [14] and [25], respectively. However, an integrated approach for defining an optimal sampling design capturing the land surface spatiotemporally heterogeneity in a cost-efficient way is still urgently needed. This sampling design is especially important for mountainous areas which are characterized both by extreme heterogeneity and inaccessibility.

The Wanglang Integrated Observation and Experiment Station for Mountain Ecological Remote Sensing was recently established in the Wanglang Nature Reserve, one of China's first nature reserves established to protect the giant panda in 1965. Temporally continuous field measurements of 
biophysical variables (including leaf area index, fractional vegetation cover, fraction of absorbed photosynthetically active radiation) are planned to be implemented in 2018 to support the parameterization, calibration and validation of the remote sensing and the land surface process models. Establishing an optimized sampling design to generate spatiotemporally representative and cost-efficient ESUs is the prerequisite for the planned measurements in Wanglang experimental site and the main objective of this paper.

\section{Study Site}

The Wanglang Nature Reserve (Figure 1) is located in the Hengduan Mountains, a global biodiversity hotspot. The reserve covers approximately $320 \mathrm{~km}^{2}$ with altitudes ranging between 2000 and $5000 \mathrm{~m}$ (Figure 1c). It receives $862.5 \mathrm{~mm}$ of rainfall annually, with the lowest mean air temperature of $-6.1{ }^{\circ} \mathrm{C}$ in January, and the highest at $12.7^{\circ} \mathrm{C}$ in July [31]. The major vegetation types include deciduous forest, conifer-deciduous mixed forest, and conifer forest. The transportation is inconvenient with the roads built along rivers (Figure 1b). Most of the parts in the reserve are difficult or even impossible to visit.

Based on the reserve, the Wanglang Integrated Observation and Experiment Station for Mountain Ecological Remote Sensing was established in 2017. One of the scientific objective of this station is to implement temporally continuous validation for existing remote sensing products. To complete this objective, temporally continuous field measurements are planned to be implemented in 2018 . Wireless sensor network system, which needs the support of permanent ESUs [25], is an efficient means to collect these temporally continuous field measurements [28,30].
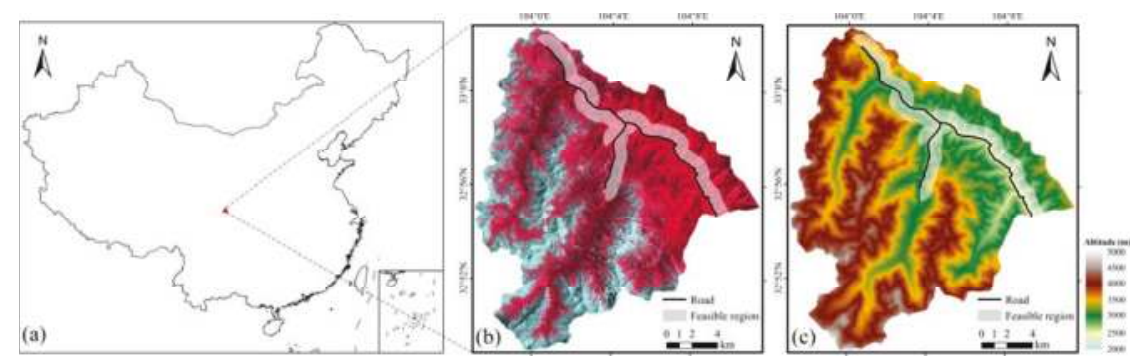

Figure 1. (a) Map of China with the location of Wanglang experimental site. (b) A Landsat 8 Operational Land Imager (OLI) image and (c) an elevation map of the study area. The roads and the feasible accessible region are shown.

\section{Materials and Methods}

\subsection{Auxiliary Satellite Imagery}

We used Normalized Difference Vegetation Index (NDVI) [32] as the auxiliary variable to represent the target biophysical properties. NDVI has been demonstrated to show a strong correlation with many biophysical variables including leaf area index (LAI) [11,33], fraction of absorbed photosynthetically active radiation (FAPAR) [34,35], and fraction of vegetation cover (FVC) [36,37]. In addition, the NDVI reduces the sensitive to topographic effects due to its ratio formulation [38,39].

NDVI maps of the study area were here computed from Landsat top of canopy reflectance data in the near infrared and red bands. Landsat data were downloaded from the United States Geological Survey (USGS) EarthExplorer (https:/ / earthexplorer.usgs.gov/) [40]. The Landsat reflectance data were atmospherically corrected using the Second Simulation of the Satellite Signal in the Solar Spectrum-Vector (6SV) model [41]. 
To capture the seasonal variations of the vegetation, we collected five scenes of images spanning nearly the whole growing season peak in our study area: Day of Year (DOY) 152, 197, 240, 261, 296. Because of the cloud contamination, no single year can provide cloud-free images covering the whole growing season peak. The five selected scenes were from different years and sensors (Table 1). This treatment neglected the inter-annual variation of vegetation and the difference in spectral response function between Landsat 8/OLI and Landsat 5/TM.

Table 1. Overview of Landsat data (path: 130, row: 37) used in this study.

\begin{tabular}{ccc}
\hline DOY & Year & Satellite/Sensor \\
\hline 152 & 2014 & Landsat 8/OLI \\
197 & 2013 & Landsat 8/OLI \\
240 & 2011 & Landsat 5/TM \\
261 & 2007 & Landsat 5/TM \\
296 & 2014 & Landsat 8/OLI \\
\hline
\end{tabular}

\subsection{Definition of the Sampling Feasible Region}

The study area is characterized by inaccessibility because of the rugged terrain, lack of roads and barrier of river. Therefore, we should consider the cost limitation when determining the spatial distribution of the ESUs. Different from Silva et al. [24] and Yin et al. [14], who calculated the visiting cost for each pixel and incorporated a cost-objective function to constrain the sampling process, in this paper, we first established the feasible region for the ESU sampling within an affordable cost. The establishment of feasible region avoid the generation of unaffordable ESUs located in inaccessible regions, which is a potential problem of the methods of Silva et al. [24] and Yin et al. [14].

The feasible region (Figure 1) is defined according to two practical criteria:

I. The distance between the ESUs and the roads should be less than $1000 \mathrm{~m}$.

II. The ESUs and the roads should be on the same side of the rivers.

The criterion I considers the influence of the rugged terrain and lack of roads. Note that the distance threshold of $1000 \mathrm{~m}$ was set according to expert experiences, and it directly influence the cost and the spatiotemporal representativeness of the sampled ESUs. The criterion II considers the block of rivers. The roads in our study area are often built along the rivers, so the ESUs and the roads should be on the same side of the rivers to avoid crossing rivers.

\subsection{Multi-Temporal Constraint Sampling Based on the Conditioned Latin Hypercube (CLH)}

The proposed sampling scheme aims to obtain representative samples and reproduce the probability distribution functions of the five NDVI maps (Table 1) using $n$ spatially-distributed and temporally-fixed ESUs. It is based on the CLH sampling procedure [22] and iteratively selects samples from the NDVI maps by using a stratified random sampling scheme based on the cumulative distributions of NDVI. CLH is implemented by the following steps:

I. Divide the probability distributions of NDVI for the five dates into $n$ equiprobable strata.

II. Randomly pick one sample (ESU) per stratum. The location of the ESUs is constrained within the feasible region (Figure 1).

III. The objective function is defined as follows:

$$
O=\sum_{j=1}^{k} \sum_{i=1}^{n} \frac{\left|\eta_{i j}-1\right|}{n}
$$

where $n$ is the number of ESUs, $k$ is the number of NDVI maps ( $k=5$, in this study), $\eta_{i j}$ is the times that a stratum $i$ on date $j$ is sampled. 
IV. Perform an annealing schedule [42] to minimize the objective function. To avoid being trapped in a local optimum, the simulated annealing algorithm accepts some of the changes that worsen the objective function, and the probability of accepting a worse sample is given by:

$$
p=\exp (-\Delta O / T)
$$

where $\Delta O$ is the change in the objective function and $T$ is a control parameter for optimizing the global minimization of the objective function $O$. For each $T$ value (between 0 and 1 ) there is a corresponding probability $p$ to get out of a local minimum in the minimization procedure. The greater of $T$, the higher probability to get out of a local minimum, but with more computation time, and vice versa. We fixed the initial value of $T$ to 1 and it was decreased by a factor 0.95 during each iteration.

V. Perform the replacement of an ESU in the selected sample with an ESU outside the current sample. The replacement can be random or systematic, according to a probability of $F$. Specifically, generate a random number $r a n d$, if rand $<F$, pick a ESU randomly from currently generated sample (random replacement) and swap it with a random ESU outside the current sample. Otherwise, remove the ESU from current sample which has the largest overall objective function value (systematic replacement), and replace it with a random ESU outside the current sample. The value of $F$ was fixed to 0.5 using a trial-and-error approach.

VI. Repeat steps III-V a number of 5000 iterations to converge to the final solution.

\subsection{Evaluation Approach}

Similar to the VALERI's sampling protocol [17], the size of the ESUs corresponds to the size of the pixel of the high spatial resolution satellite data, i.e., the $30 \mathrm{~m}$ of Landsat data in our case. The NDVI values for each ESU and for the different dates were extracted from the five Landsat NDVI maps (Table 1). To quantitatively evaluate the capacity of the multi-temporally constrained (MC) sampling strategy for reproducing the NDVI values across the study area and for the different dates, we computed the Overlapping Area (OA) between the NDVI frequency distribution histogram from the ESUs (denoted by $F_{s}$ ) and that from the entire study area (denoted by $F_{p}$ ) considering the NDVI values of all the different dates. The OA can be formulated as [43],

$$
O A=\frac{\operatorname{Area}_{F_{p} \cap F_{s}}}{\text { Area }_{F_{p}}}
$$

The numerator denotes the overlapping area of $F_{s}$ and $F_{p}$, and the denominator represents the area of $F_{p}$.

$\mathrm{R}^{2}$ and root mean square error (RMSE) between the mean NDVI values from the ESUs and the average values for the entire study area for each date were also computed to quantify if resulting ESUs can preserve the average state of the study area.

The proposed multi-temporally constrained (MC) sampling was compared with two alternative sampling schemes: the single-temporally constrained (SC) sampling that uses only one NDVI map (DOY 197) to construct the objective function (Equation (1)) and the random sampling (RS) without addition constraints. Similarly to MC, the distribution of samples for the SC and RS schemes were limited to the feasible region. Finally, a multi-temporally constrained sampling with ESUs located across the entire study area (MCE) was used for evaluating the theoretical impact in terms of spatiotemporal representativeness of introducing a feasible region for the cost limitation. Note however that the MCE sampling design is unaffordable in the practice due to the inaccessibility of some of the ESUs. 


\section{Results}

\subsection{Influence of the Number of Elementary Sampling Units in the Spatiotemporal Representativeness}

To determine the minimum number of ESUs required to capture the spatiotemporal heterogeneity of the study area, we analyzed the variations of the mean OA of the five dates as a function of the number of ESUs (Figure 2). The OA increases with the number of ESUs indicating, as expected, that the spatiotemporal heterogeneity of the study area can be better represented with a higher number of ESUs. The OA can be well fitted $\left(\mathrm{R}^{2}=0.99\right)$ by an exponential function:

$$
\mathrm{OA}=0.74-0.37 \exp (-0.11 n)
$$

where $n$ is the number of ESUs. For low $n$ values, the OA increased rapidly with $n$. For high $n$ values, the OA reaches an asymptotic value of 0.74 . For $n=20$ samples, $70 \%$ of the heterogeneity of the study area is well represented. This cut-off point of $n=20$ was fixed as the minimum required number of ESUs.

Note that about $25 \%$ of the spatiotemporal heterogeneity cannot be represented by the MC sampling scheme (Figure 2). This may be partially explained because the samples were located in a feasible region significantly smaller than the entire study area and may not completely cover all the range of vegetation conditions (Figure 3).

Figure 3 shows the spatial distribution of the 20 selected ESUs by the multi-temporal and cost constrained sampling strategy within the pre-defined sampling feasible region.

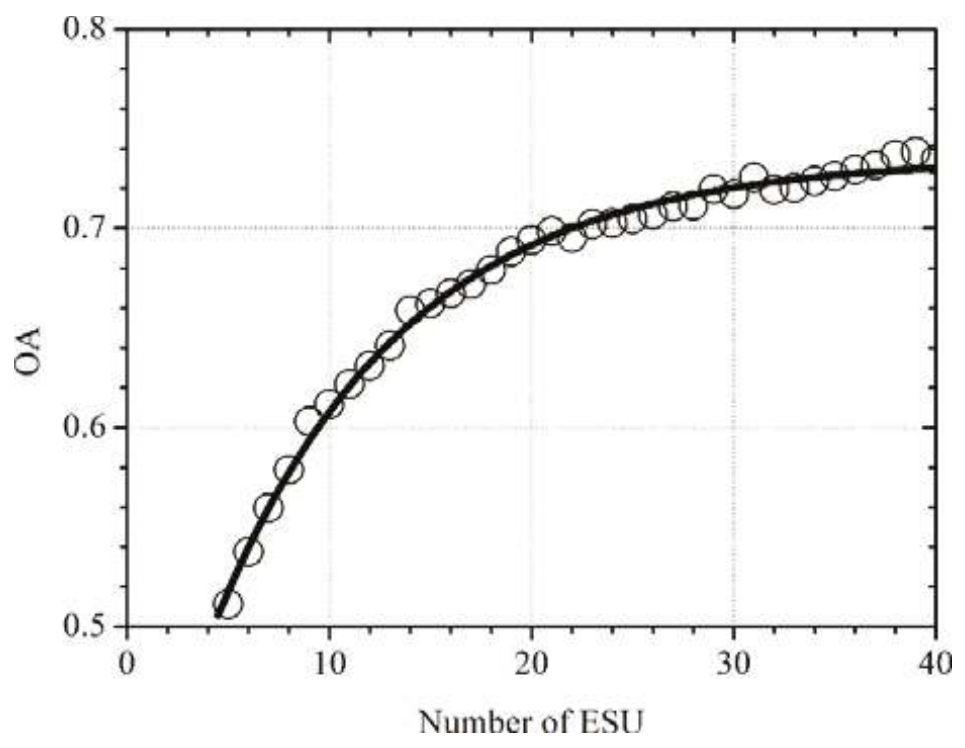

Figure 2. Overlapping area (OA) of the NDVI histogram distributions for the sampled and entire study area as a function of the number of Elementary Sampling Units (ESU). 


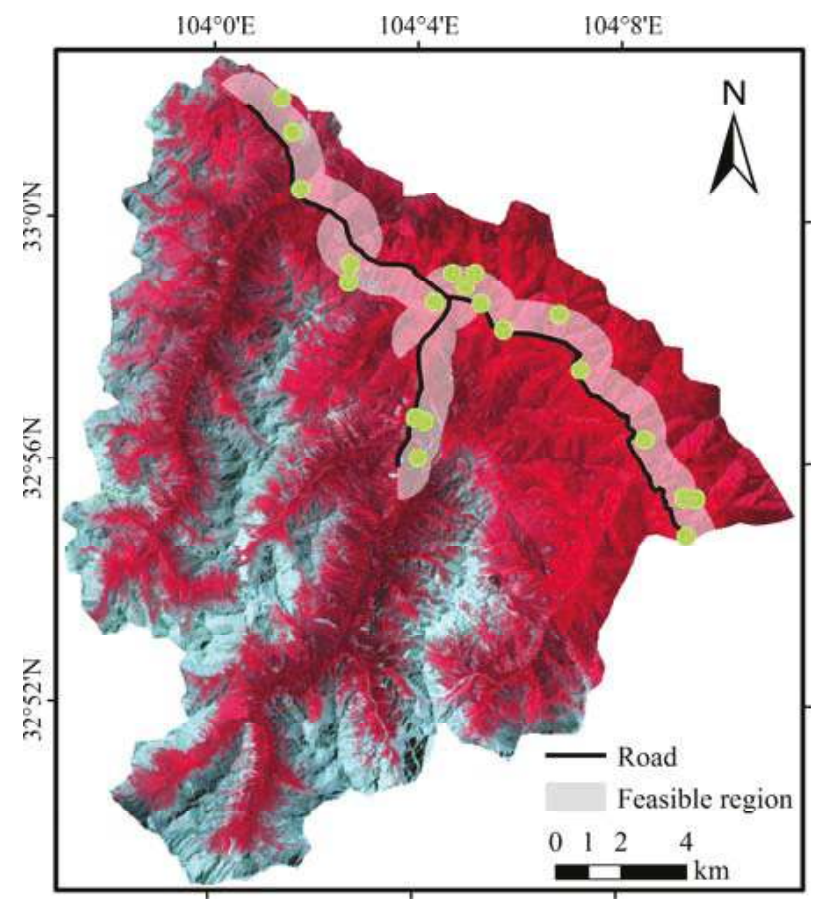

Figure 3. The spatial distribution of the 20 elementary sampling units for the multi-temporal constrained sampling design in the feasible region.

\subsection{Performance Evaluation: Comparison with Alternative Sampling Designs}

Figure 4 shows the NDVI frequency distribution histograms of the 20 selected ESUs for the four sampling strategies: random sampling (RS), single-temporally constrained sampling (SC), and multi-temporally constrained sampling both in the feasible region (MC) and in the entire study area without the sampling feasible constraint (MCE).

The MCE sampling strategy performed the best and reconstructed the NDVI frequency distribution histograms of the entire study area for the different dates more accurately than the feasible region constrained sampling designs. The introduction of sampling feasible region slightly degreased the spatiotemporal representativeness of sampling but, in general, the three methods RS, SC and MC applied for the feasible region can all preserve the overall shapes of the frequency distribution histograms of the entire study area (red lines in Figure 4), with the MC performing the best. On DOY 152, RS under-sampled the interval 0.4-0.65 and over-sampled the NDVI interval 0.8-0.85. On DOY 261, RS and SC both under-sampled the NDVI intervals less than 0.6. On DOY 197, all methods significantly over-sampled high NDVI values for the range 0.85-0.9.

The comparison of the OA for the four sampling strategies (Table 2) showed that MCE performed the best during the whole study period $(\mathrm{OA}=83.5 \%)$ followed by $\mathrm{MC}(\mathrm{OA}=74.7 \%)$. RS performed the worst $(\mathrm{OA}=63.1 \%)$ because it exploited no a priori information. The efficiency of SC was between RS and MC $(\mathrm{OA}=68.7 \%)$, but it improved the spatiotemporal representativeness for DOY $197(\mathrm{OA}=75.9 \%)$ because this specific date was used to constrain the SC sampling procedure. 


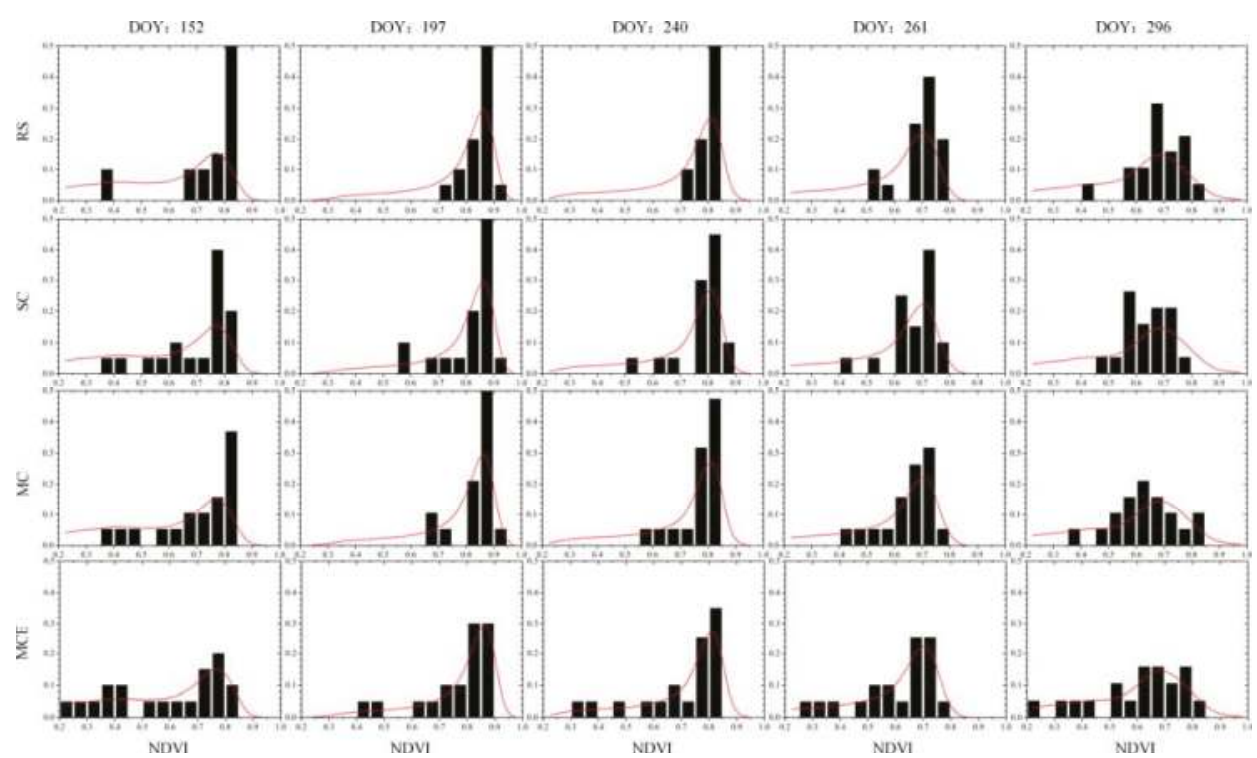

Figure 4. NDVI frequency distribution histograms of 20 selected elementary sampling units for the four sampling strategies (from top to bottom): random sampling (RS), single-temporally constrained sampling (SC), and multi-temporally constrained sampling (MC) in the feasible region (MC) and in the entire study area without the sampling feasible constraint (MCE). The red lines represent the NDVI frequency distribution histograms of the entire study area.

Table 2. Overlapping area (OA, \%) for random sampling (RS), single-temporally constrained sampling (SC), and multi-temporally constrained sampling applied to the feasible region (MC) and to the entire study area (MCE).

\begin{tabular}{ccccc}
\hline DOY & RS & SC & MC & MCE \\
\hline 152 & 50.3 & 64.2 & 72.2 & 85.8 \\
197 & 72.0 & 75.9 & 71.3 & 82.3 \\
240 & 62.2 & 70.7 & 74.1 & 83.2 \\
261 & 63.2 & 67.8 & 82.9 & 84.0 \\
296 & 68.0 & 65.0 & 72.8 & 82.0 \\
Annual mean & 63.1 & 68.7 & 74.7 & 83.5 \\
\hline
\end{tabular}

The scatterplots between the average of sampled NDVI and the average NDVI calculated from the whole study area were also analyzed (Figure 5). The MCE method was the most accurate (RMSE < 0.01) for NDVI sampling $\left(\mathrm{R}^{2}=0.99\right)$. The three sampling strategies constrained to the feasible region all overestimated the regional means. The feasible region is located in the lowland areas which generally have better hydrologic and thermal conditions than highland areas for vegetation growth. Compared to the RS and SC, MC sampling significantly alleviated the overestimation phenomenon.

The reduction of standard deviation from the ESUs compared to that from the entire study area indicates the information loss on spatiotemporal heterogeneity [12]. Figure 5 shows that the RS had the smallest standard deviation for each DOY, followed by SC and MC. MCE and MC show similar variability in terms of the standard deviation. This demonstrates the efficiency of CLH strategy for defining spatiotemporal representativeness sampling schemes. 

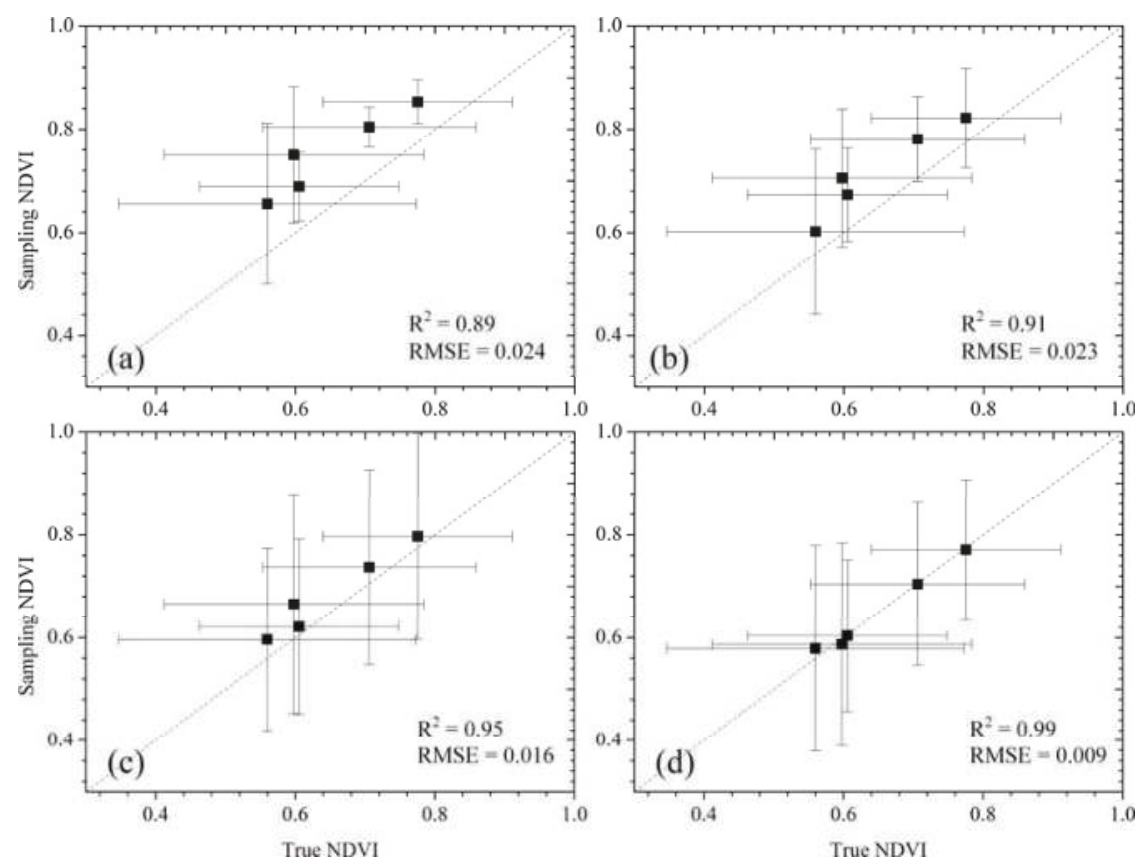

Figure 5. Scatterplots between the average sampled NDVI and the average true NDVI values for the entire study area and for the five different dates. (a) Random sampling in the feasible region. (b) Single-temporally constrained sampling in the feasible region. (c) Multi-temporally constrained sampling in the feasible region. (d) Multi-temporally constrained sampling in the entire region. The bars indicate the standard deviations.

\section{Discussion}

This study proposed a novel sampling strategy to generate spatiotemporally representative and cost-efficient ESUs. Comparing to existing study about ESU sampling methods $[14,20,25]$, our method can make an appropriate compromise between spatiotemporal representativeness and implementation cost, so it is particularly useful for heterogenous and traffic-inconvenient regions, e.g., mountainous regions.

The multi-temporal constrained sampling was demonstrated to better capture the spatiotemporal surface heterogeneity compared to random or single-temporal constrained methods. The introduction of the feasible region (Figure 1) reduced about $9 \%$ the spatiotemporal representativeness of the multi-temporal constrained sampling based on CLH (compare MC and MCE in Figure 4 and Table 2). The feasible region is located in lowland areas with high NDVI values and may not completely represent all the range of conditions of vegetation in the entire mountainous region, which is characterized by a vertical negative gradient in NDVI (Figure 5). However, the feasible region was established based on accessibility criteria and the spatial distribution of the available roads in the study area. The loss of information of the feasible constrained sampling (MC) is compensated by the significant reduction of the implementation cost.

A sensitivity analysis showed that 20 ESUs can reproduce $70 \%$ of heterogeneity of the study area. The distribution of the 20 selected ESUs within the pre-defined feasible region showed some spatial aggregation (Figure 3). This phenomenon was often criticized for information redundancy because of the spatial autocorrelation of the biophysical condition [44]. Although this aggregation of ESUs could be easily removed by introducing additional objective function in geographic space [14,21,25], 
the cluster of the ESUs would allow reducing the implementation cost of the field campaigns which is one of the key concerns of the present study.

A spatially-distributed and temporally-fixed strategy for the ESU sampling was adopted in this study. Alternatively, a temporally independent strategy could be adopted for defining the ESU sampling for multi-temporal field campaigns, i.e., different date uses different spatial distribution of ESUs. The temporally independent manner may show better capacity to capture the temporal variation of spatial heterogeneity. However, the temporally-fixed manner can provide additional information of the temporal variation of vegetation at each ESU. The temporally-fixed strategy was here selected because it allows locating the nodes of the wireless sensor network systems that will support the validation activities in Wanglang experimental site $[19,25,28]$.

To consider the rugged terrain, lack of roads and the block of rivers, we restricted the ESUs within the sampling feasible region. Currently, the feasible region was established in a semi-empirical manner based on our expert knowledge. Some more sophisticate algorithms (e.g., three-dimensional terrain modeling, 3D analyzing and the shortest path algorithm) would enhance the rationality in the definition of the feasible region.

In this study, NDVI was chosen to represent the biophysical properties of the study area. One reason to select NDVI is because of its strong correlation with many biophysical variables. Although other vegetation indices such as the soil adjusted vegetation index [45], the enhanced vegetation index [46], the normalized difference water index [47] or the normalized canopy index [48] were also recommended in some specified applications, they were often criticized for their vulnerability to topographic effects [39]. Figure 6 showed the density scatterplots between the NDVI and the cosine of the local solar incidence $(\cos (i))$ in our study area, which is the most widely used quantitative evaluation method for topographic effects [49]. It can be seen that NDVI was nearly independent from $\cos (i)$ with $\mathrm{R}^{2}$ between them ranging from 0.0002 to 0.0194 . NDVI was then selected as the auxiliary variable for our sampling procedure due to its correlation with many biophysical variables and its reduced sensibility to topographic effects.

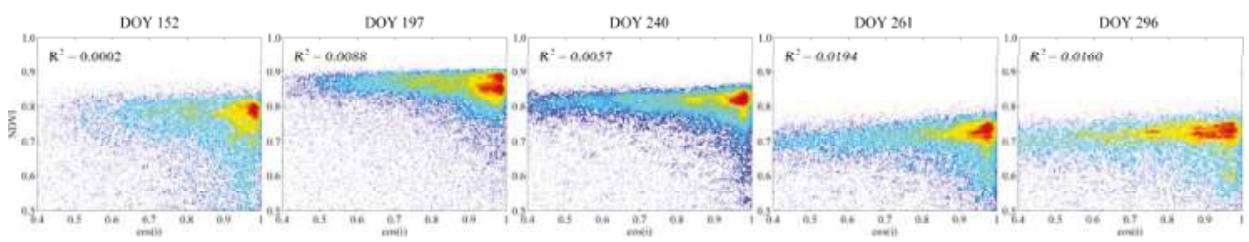

Figure 6. Density scatterplots between the NDVI and the cosine of the local solar incidence angle $(\cos (i))$ in Wanglang study area for the different acquisition dates.

The topography is well recognized to influence the vegetation biophysical properties $[50,51]$. Topographical factors (e.g., slope) were introduced as auxiliary variables in our previous study [14]. However, the NDVI relates to biophysical properties more explicitly. The exclusion of topography would not limit the representativeness of the sampled ESU considering the low sensibility of NDVI to topographic effects for the study area and considered period.

The use of NDVI data from different sensor and years may introduce uncertainties in the sampling procedure. However, our study area is a natural reserve where the anthropogenic activities (e.g., logging and mining) are very limited and, consequently, induced rapid changes in biophysical properties are not expected. Moreover, the study area is dominated by primeval forest with mean age around 200 years and significant inter-annual variations in vegetation are not expected for the period of the satellite acquisitions (Table 1). In our conditions, the NDVI data from different years is expected to successfully represent the seasonal variations in surface heterogeneity. In addition, it was demonstrated in a few studies that the Landsat data record shows satisfactory consistency, and can be 
safely used together for time series analysis $[52,53]$. Therefore, the combined use of Landsat 8/OLI and Landsat 5/TM is also acceptable in the sampling procedure. An alternative to capture the seasonal variations is the combination of Landsat 8 with Sentinel-2 images. However, the persistent cloud cover during the monsoon (from July to September) hampers the collection of clear Sentinel-2 images in our study area. Moreover, cross-calibration coefficients between Landsat 8 with Sentinel-2 retrieved from pseudo-invariant sites [54] may be not suitable for mountainous areas. The footprints of sensor observations vary considerably in size and center locations over time, which hamper the establishment of robust cross-calibration coefficients [55]. This also prevents the normalization between Landsat 8/OLI and Landsat 5/TM data over reference targets.

According to the protocols established by the LPV subgroup [16] and the VALERI project [17], each ESU corresponds to a pixel of the auxiliary high spatial resolution satellite data. In this study, the size of each ESU is $30 \mathrm{~m} \times 30 \mathrm{~m}$ corresponding to the spatial resolution of the OLI images. As recommended by the two-stage nested sampling framework [16], a sampling procedure would also be implement within each ESU by distributing the individual field measurements in squares, crosses or transects [17]. The specified sampling design within each ESU is out of the scope of this study.

Because of the lack of in-situ measurements, the sampling strategies were evaluated through the comparison of the sampled and true NDVI frequency distributions. The sampling strategies will be further evaluated using the in-situ measurements that will be collected in the Wanglang field campaigns planned for the year 2018.

\section{Conclusions}

We proposed a sampling strategy to generate spatiotemporally representative and cost-efficient ESUs based on the conditioned Latin hypercube methodology. The proposed sampling strategy was constrained by multi-temporal NDVI, and the ESUs were limited within a feasible region established based on accessibility criteria. A case study in Wanglang National Nature Reserve in China showed that the proposed strategy can obtain more spatiotemporally representative ESUs (mean annual Overlapping Area, $\mathrm{OA}=74.7 \%)$, compared to the single-temporally constrained $(\mathrm{OA}=68.7 \%)$ and random sampling $(\mathrm{OA}=63.1 \%)$ strategies. The minimum number of required ESUs was fixed to twenty as a compromise between the spatiotemporal representativeness and the implementation cost. The introduction of the feasible region constraint ensures an affordable cost for the field campaigns at expenses of a degradation of about $9 \%$ in the spatiotemporal representativeness of the sampling. The sampling design here proposed will support validation activities in Wanglang experimental site providing a benchmark of spatially distributed and temporally fixed ESUs for locating the nodes of wireless sensor network systems for the acquisition of temporally continuous field measurements.

Acknowledgments: This work was funded by the National Key Research and Development Program of China (2016YFA0600103), the National Natural Science Foundation of China (41601403, 41631180, 41571373, and 41531174), by the Youth Talent Team Program of Institute of Mountain Hazards and Environment, Chinese Academy of Sciences under Grant SDSQB-2015-02, and by the CAS "Light of West China" Program. A.V. acknowledges the support from the EC Copernicus Global Land Service (CGLOPS-1, 199494-JRC).

Author Contributions: Ainong Li conceived the experiments; Gaofei Yin designed and performed the experiments, and wrote the paper; Aleixandre Verger provided guidance and critically reviewed the manuscript.

Conflicts of Interest: The authors declare no conflict of interest.

\section{References}

1. Li, X. Characterization, controlling, and reduction of uncertainties in the modeling and observation of land-surface systems. Sci. China Earth Sci. 2014, 57, 80-87. [CrossRef]

2. Li, X.; Cheng, G.D.; Liu, S.M.; Xiao, Q.; Ma, M.G.; Jin, R.; Che, T.; Liu, Q.H.; Wang, W.Z.; Qi, Y.; et al. Heihe Watershed Allied Telemetry Experimental Research (HiWATER): Scientific objectives and experimental design. Bull. Am. Meteorol. Soc. 2013, 94, 1145-1160. [CrossRef] 
3. Xu, B.D.; Li, J.; Liu, Q.H.; Huete, A.R.; Yu, Q.; Zeng, Y.L.; Yin, G.F.; Zhao, J.; Yang, L. Evaluating spatial representativeness of station observations for remotely sensed leaf area index products. IEEE J. Sel. Top. App. Earth Obs. Remote Sens. 2016, 9, 3267-3282. [CrossRef]

4. Verhoef, W.; Bach, H. Coupled soil-leaf-canopy and atmosphere radiative transfier modeling to simulate hyperspectral multi-angular surface reflectance and TOA radiance data. Remote Sens. Environ. 2007, 109, 166-182. [CrossRef]

5. Kure, S.; Kavvas, M.L.; Ohara, N.; Jang, S. Upscaling of coupled land surface process modeling for heterogeneous landscapes: Stochastic approach. J. Hydrol. Eng. 2011, 16, 1017-1029. [CrossRef]

6. Li, X.; Wang, Y. Prospects on future developments of quantitative remote sensing. Acta Geogr. Sin. 2013, 68, 1163-1169.

7. Yan, G.J.; Hu, R.H.; Wang, Y.T.; Ren, H.Z.; Song, W.J.; Qi, J.B.; Chen, L. Scale effect in indirect measurement of leaf area index. IEEE Trans. Geosci. Remote Sens. 2016, 54, 3475-3484. [CrossRef]

8. Yan, K.; Park, T.; Yan, G.J.; Chen, C.; Yang, B.; Liu, Z.; Nemani, R.R.; Knyazikhin, Y.; Myneni, R.B. Evaluation of MODIS LAI/FPAR product collection 6. Part 1: Consistency and improvements. Remote Sens. 2016, 8, 359. [CrossRef]

9. Claverie, M.; Matthews, J.L.; Vermote, E.F.; Justice, C.O. A 30+ year AVHRR LAI and FAPAR climate data record: Algorithm description and validation. Remote Sens. 2016, 8, 263. [CrossRef]

10. Wu, H.; Li, Z.L. Scale issues in remote sensing: A review on analysis, processing and modeling. Sensors 2009, 9, 1768-1793. [CrossRef] [PubMed]

11. Yin, G.F.; Li, J.; Liu, Q.H.; Li, L.H.; Zeng, Y.L.; Xu, B.D.; Yang, L.; Zhao, J. Improving leaf area index retrieval over heterogeneous surface by integrating textural and contextual information: A case study in the Heihe River Basin. IEEE Geosci. Remote Sens. Lett. 2015, 12, 359-363.

12. Garrigues, S.; Allard, D.; Baret, F.; Weiss, M. Influence of landscape spatial heterogeneity on the non-linear estimation of leaf area index from moderate spatial resolution remote sensing data. Remote Sens. Environ. 2006, 105, 286-298. [CrossRef]

13. Grafstrom, A.; Schelin, L. How to select representative samples. Scand. J. Stat. 2014, 41, 277-290. [CrossRef]

14. Yin, G.F.; Li, A.N.; Zeng, Y.L.; Xu, B.D.; Zhao, W.; Nan, X.; Jin, H.A.; Bian, J.H. A cost-constrained sampling strategy in support of LAI product validation in mountainous areas. Remote Sens. 2016, 8, 704. [CrossRef]

15. Wang, J.F.; Stein, A.; Gao, B.B.; Ge, Y. A review of spatial sampling. Spat. Stat. 2012, 2, 1-14. [CrossRef]

16. Morisette, J.T.; Baret, F.; Privette, J.L.; Myneni, R.B.; Nickeson, J.E.; Garrigues, S.; Shabanov, N.V.; Weiss, M.; Fernandes, R.A.; Leblanc, S.G.; et al. Validation of global moderate-resolution LAI products: A framework proposed within the CEOS Land Product Validation subgroup. IEEE Trans. Geosci. Remote Sens. 2006, 44, 1804-1817. [CrossRef]

17. Baret, F.; Weiss, M.; Allard, D.; Garrigue, S.; Leroy, M.; Jeanjean, H.; Fernandes, R.; Myneni, R.; Privette, J.; Morisette, J.; et al. VALERI: A Network of Sites and a Methodology for the Validation of Medium Spatial Resolution Land Satellite Products. Available online: http://w3.avignon.inra.fr/valeri/ (accessed on 24 November 2017).

18. Martinez, B.; Garcia-Haro, F.J.; Camacho-de Coca, F. Derivation of high-resolution leaf area index maps in support of validation activities: Application to the cropland Barrax site. Agric. For. Meteorol. 2009, 149, 130-145. [CrossRef]

19. Ge, Y.; Wang, J.H.; Heuvelink, G.B.M.; Jin, R.; Li, X.; Wang, J.F. Sampling design optimization of a wireless sensor network for monitoring ecohydrological processes in the Babao River basin, China. Int. J. Geogr. Inf. Sci. 2015, 29, 92-110. [CrossRef]

20. Mu, X.; Hu, M.; Song, W.; Ruan, G.; Ge, Y.; Wang, J.; Huang, S.; Yan, G. Evaluation of sampling methods for validation of remotely sensed fractional vegetation cover. Remote Sens. 2015, 7, 16164-16182. [CrossRef]

21. Zeng, Y.L.; Li, J.; Liu, Q.H.; Li, L.H.; Xu, B.D.; Yin, G.F.; Peng, J.J. A sampling strategy for remotely sensed LAI product validation over heterogeneous land surfaces. IEEE J. Sel. Top. Appl. Earth Obs. Remote Sens. 2014, 7, 3128-3142. [CrossRef]

22. Minasny, B.; McBratney, A.B. A conditioned Latin hypercube method for sampling in the presence of ancillary information. Comput. Geosci. 2006, 32, 1378-1388. [CrossRef]

23. Mulder, V.L.; De Bruin, S.; Schaepman, M.E. Representing major soil variability at regional scale by constrained Latin Hypercube Sampling of remote sensing data. Int. J. Appl. Earth Obs. Geoinf. 2013, 21, 301-310. [CrossRef] 
24. Silva, S.H.G.; Owens, P.R.; Silva, B.M.; De Oliveira, G.C.; De Menezes, M.D.; Pinto, L.C.; Curi, N. Evaluation of conditioned Latin hypercube sampling as a support for soil mapping and spatial variability of soil properties. Soil Sci. Soc. Am. J. 2015, 79, 603-611. [CrossRef]

25. Zeng, Y.L.; Li, J.; Liu, Q.H.; Qu, Y.H.; Huete, A.R.; Xu, B.D.; Yin, G.F.; Zhao, J. An optimal sampling design for observing and validating long-term leaf area index with temporal variations in spatial heterogeneities. Remote Sens. 2015, 7, 1300-1319. [CrossRef]

26. Ding, Y.L.; Zhao, K.; Zheng, X.M.; Jiang, T. Temporal dynamics of spatial heterogeneity over cropland quantified by time-series NDVI, near infrared and red reflectance of Landsat 8 OLI imagery. Int. J. Appl. Earth Obs. Geoinf. 2014, 30, 139-145. [CrossRef]

27. Garrigues, S.; Allard, D.; Baret, F. Modeling temporal changes in surface spatial heterogeneity over an agricultural site. Remote Sens. Environ. 2008, 112, 588-602. [CrossRef]

28. Qu, Y.H.; Han, W.C.; Fu, L.Z.; Li, C.R.; Song, J.L.; Zhou, H.M.; Bo, Y.C.; Wang, J.D. LAINet-A wireless sensor network for coniferous forest leaf area index measurement: Design, algorithm and validation. Comput. Electron. Agric. 2014, 108, 200-208. [CrossRef]

29. Ryu, Y.; Lee, G.; Jeon, S.; Song, Y.; Kimm, H. Monitoring multi-layer canopy spring phenology of temperate deciduous and evergreen forests using low-cost spectral sensors. Remote Sens. Environ. 2014, 149, 227-238. [CrossRef]

30. Yin, G.F.; Li, A.N.; Jin, H.A.; Zhao, W.; Bian, J.H.; Qu, Y.H.; Zeng, Y.L.; Xu, B.D. Derivation of temporally continuous LAI reference maps through combining the LAINet observation system with CACAO. Agric. For. Meteorol. 2017, 233, 209-221. [CrossRef]

31. Kang, D.W.; Yang, H.W.; Li, J.Q.; Chen, Y.P.; Zhao, L.J. Habitat use by giant pandas Ailuropoda melanoleuca in the Wanglang Nature Reserve, Sichuan, China. Zool. Stud. 2013, 52, 6. [CrossRef]

32. Huete, A.; Didan, K.; Miura, T.; Rodriguez, E.P.; Gao, X.; Ferreira, L.G. Overview of the radiometric and biophysical performance of the MODIS vegetation indices. Remote Sens. Environ. 2002, 83, 195-213. [CrossRef]

33. Baret, F.; Guyot, G.; Major, D.J. Crop biomass evaluation using radiometric measurements. Photogrammetria 1989, 43, 241-256. [CrossRef]

34. Baret, F.; Guyot, G. Potentials and limits of vegetation indexes for LAI and APAR assessment. Remote Sens. Environ. 1991, 35, 161-173. [CrossRef]

35. Myneni, R.B.; Williams, D.L. On the relationship between FAPAR and NDVI. Remote Sens. Environ. 1994, 49, 200-211. [CrossRef]

36. Gutman, G.; Ignatov, A. The derivation of the green vegetation fraction from NOAA/AVHRR data for use in numerical weather prediction models. Int. J. Remote Sens. 1998, 19, 1533-1543. [CrossRef]

37. Yang, G.; Pu, R.; Zhang, J.; Zhao, C.; Feng, H.; Wang, J. Remote sensing of seasonal variability of fractional vegetation cover and its object-based spatial pattern analysis over mountain areas. ISPRS J. Photogramm. Remote Sens. 2013, 77, 79-93. [CrossRef]

38. Valeriano, M.D.M.; Sanches, I.D.A.; Formaggio, A.R. Topographic effect on spectral vegetation indices from Landsat TM data: Is topographic correction necessary? Bol. Ciências Geodésicas 2016, 22, 95-107.

39. Zhu, G.; Liu, Y.; Ju, W.; Chen, J. Evaluation of topographic effects on four commonly used vegetation indices. J. Remote Sens. 2013, 17, 210-234.

40. EarthExplorer. Available online: https:/ / earthexplorer.usgs.gov / (accessed on 24 November 2017).

41. Vermote, E.; Justice, C.; Claverie, M.; Franch, B. Preliminary analysis of the performance of the Landsat 8/OLI land surface reflectance product. Remote Sens. Environ. 2016, 185, 46-56. [CrossRef]

42. Press, W.H.; Teukolsky, S.A.; Flannery, B.P.; Vetterling, W.T. Numerical Recipes in FORTRAN: The Art of Scientific Computing; Cambridge University Press: Cambridge, UK, 1992.

43. Jin, H.A.; Li, A.N.; Wang, J.D.; Bo, Y.C. Improvement of spatially and temporally continuous crop leaf area index by integration of CERES-Maize model and MODIS data. Eur. J. Agron. 2016, 78, 1-12. [CrossRef]

44. Wang, J.F.; Christakos, G.; Hu, M.G. Modeling spatial means of surfaces with stratified nonhomogeneity. IEEE Trans. Geosci. Remote Sens. 2009, 47, 4167-4174. [CrossRef]

45. Qi, J.; Chehbouni, A.; Huete, A.R.; Kerr, Y.H.; Sorooshian, S. A modified soil adjusted vegetation index. Remote Sens. Environ. 1994, 48, 119-126. [CrossRef]

46. Jiang, Z.Y.; Huete, A.R.; Didan, K.; Miura, T. Development of a two-band enhanced vegetation index without a blue band. Remote Sens. Environ. 2008, 112, 3833-3845. [CrossRef] 
47. Gao, B.C. NDWI-A normalized difference water index for remote sensing of vegetation liquid water from space. Remote Sens. Environ. 1996, 58, 257-266. [CrossRef]

48. Vescovo, L.; Gianelle, D. Using the MIR bands in vegetation indices for the estimation of grassland biophysical parameters from satellite remote sensing in the Alps region of Trentino (Italy). Adv. Space Res. 2008, 41, 1764-1772. [CrossRef]

49. Sola, I.; Gonzalez-Audicana, M.; Alvarez-Mozos, J. Multi-criteria evaluation of topographic correction methods. Remote Sens. Environ. 2016, 184, 247-262. [CrossRef]

50. Davis, F.W.; Schimel, D.S.; Friedl, M.A.; Michaelsen, J.C.; Kittel, T.G.F.; Dubayah, R.; Dozier, J. Covariance of biophysical data with digital topographic and land-use maps over the FIFE site. J. Geophys. Res. Atmos. 1992, 97, 19009-19021. [CrossRef]

51. Shen, L.; Li, Z.; Guo, X. Remote sensing of leaf area index (LAI) and a spatiotemporally parameterized model for mixed grasslands. Int. J. Appl. Sci. Technol. 2014, 4, 46-61.

52. Lobo, F.L.; Costa, M.P.F.; Novo, E. Time-series analysis of Landsat-MSS/TM/OLI images over Amazonian waters impacted by gold mining activities. Remote Sens. Environ. 2015, 157, 170-184. [CrossRef]

53. Roy, D.P.; Kovalskyy, V.; Zhang, H.K.; Vermote, E.F.; Yan, L.; Kumar, S.S.; Egorov, A. Characterization of Landsat-7 to Landsat-8 reflective wavelength and normalized difference vegetation index continuity. Remote Sens. Environ. 2016, 185, 57-70. [CrossRef]

54. Li, S.; Ganguly, S.; Dungan, J.L.; Wang, W.; Nemani, R.R. Sentinel-2 MSI radiometric characterization and cross-calibration with Landsat-8 OLI. Adv. Remote Sens. 2017, 6, 147. [CrossRef]

55. Xin, Q.C.; Olofsson, P.; Zhu, Z.; Tan, B.; Woodcock, C.E. Toward near real-time monitoring of forest disturbance by fusion of MODIS and Landsat data. Remote Sens. Environ. 2013, 135, 234-247. [CrossRef]

(c) 2017 by the authors. Licensee MDPI, Basel, Switzerland. This article is an open access article distributed under the terms and conditions of the Creative Commons Attribution (CC BY) license (http:/ / creativecommons.org/licenses/by/4.0/). 
Article

\title{
Reconstruction of Single Tree with Leaves Based on Terrestrial LiDAR Point Cloud Data
}

\author{
Donghui Xie *, Xiangyu Wang, Jianbo Qi, Yiming Chen, Xihan Mu, Wuming Zhang and \\ Guangjian Yan \\ State Key Laboratory of Remote Sensing Science, Jointly Sponsored by Beijing Normal University and Institute \\ of Remote Sensing and Digital Earth of Chinese Academy of Sciences, Beijing Engineering Research Center for \\ Global Land Remote Sensing Products, Faculty of Geographical Science, Beijing Normal University, \\ Beijing 100875, China; wxy0103@126.com (X.W.); jianboqi@126.com (J.Q.); cym_bnu@mail.bnu.edu.cn (Y.C.); \\ muxihan@bnu.edu.cn (X.M.); wumingz@bnu.edu.cn (W.Z.); gjyan@bnu.edu.cn (G.Y.) \\ * Correspondence: xiedonghui@bnu.edu.cn; Tel.: +86-10-5880-5042
}

Received: 12 December 2017; Accepted: 25 April 2018; Published: 28 April 2018

\begin{abstract}
Many studies have been focusing on reconstructing the branch skeleton of a three-dimensional (3D) tree structure that is based on photos or point clouds scanned by a terrestrial laser scanner (TLS), but leaves, as the important component of a tree, are often ignored or simplified because of their complexity. Therefore, we develop a voxel-based method to add leaves to a reconstructed 3D branches structure based on TLS point clouds. The location and size of each leaf depend on the spatial distribution and density of leaves points in the voxel. We reconstruct a small 3D scene with four realistic 3D trees and a virtual tree (including trunk, branches, and leaves), and validate the structure of each tree through the directional gap fractions calculated based on simulated point clouds of this reconstructed scene by the ray-tracing algorithm. The results show good coherence with those from measured point clouds data. The relative errors of the directional gap fractions are no more than $4.1 \%$, though the method is limited by the effects of point occlusion. Therefore, this method is shown to give satisfactory consistency both visually and in the quantitative evaluation of the 3D structure.
\end{abstract}

Keywords: LiDAR; point cloud; leaf; gap fraction; 3D reconstruction

\section{Introduction}

The three-dimensional (3D) structure of trees, including trunk, branch, and foliage elements, is important and fundamental data for the study of forest management [1], forest ecosystems [2], and vegetation radiative transfer models [3,4].

The development of quantitative remote sensing has led to a greater focus on the real structure of ground objects, especially the vegetation structures in various radiative transfer models. Early one-dimensional (1D) radiative transfer (RT) models, such as SAIL [5] and Suits [6], were developed based on K-M theory [7], and assumed horizontally homogeneous canopies, an approach that ignored the 3D structural characteristics of the canopies. Later, Li et al. [8,9] developed geometric-optical (GO) models that simplified the crown structures to a spheroid for a broadleaf tree or a cone for a coniferous tree to capture the 3D characteristics of forests on a coarse scale. Thereafter, a four-scale bidirectional reflectance model took into account the influence of non-uniform distributions (such as clumping effect) of branches and shoots in a conifer forest, or the "leaves" in the crowns of a broadleaf forest on the bidirectional reflectance distribution function (BRDF) [10]. Meanwhile computer simulation models were developed based on explicit 3D virtual canopy scenes to simulate the canopy reflectance by using ray-tracing or radiosity theory [11-15]. 
Compared to the RT and GO models, computer simulation models take advantage of computer graphics algorithms to eliminate many hypotheses and statistical rules of canopy structure and, as a result, improve the simulation accuracy greatly. However, explicit 3D structural canopy scenes are often required as fundamental input parameters and are complex and difficult to be constructed. Therefore, some computer simulation models use simplified 3D canopy structures. For example, the scenes that are used in the DART model [16] comprise many small voxels with statistical foliage properties rather than a detailed leaf and branch structure. Huang et al. [17] simplified the detail structures of individual tree crowns to some porous thin objects in the RAPID model, which can simplify the representation of canopy structures and decrease the computing time. All of these simplifications assumed that the statistical canopy structure would provide the same scattering behavior as the explicit 3D situation. However, Disney et al. [18] had point out that simplified canopy scenes may cause an inherent loss of information. Widlowski et al. [19] found that architectural simplifications may have an impact on the fidelity of simulated satellite observations at the bottom of the atmosphere for a variety of spatial resolutions, spectral bands, and viewing and illumination geometries.

For these reasons, the simple statistical parameters for crown structure (such as LAI-leaf area index, LAD-leaf angle distribution) have been unable to satisfy the requirements of quantitative remote sensing research. The latest simulation from the Radiative Transfer Model Intercomparision Initiative (RAMI-http://rami-benchmark.jrc.ec.europa.eu/HTML/) increased the number of actual structural canopy scenes. This reflects the trend toward the development of remote sensing for vegetation. However, it is still difficult to acquire accurate 3D geometry and topology information for a real tree due to the complexity of the tree structure.

The development of terrestrial laser scanner (TLS) technology enables the use of point clouds that were scanned from trees to estimate the structural parameters for single trees or forests, including LAI, LAD [20], and leaf area density [21]. Additionally, point clouds are also used to reconstruct 3D trees. Most studies pay more attention to the reconstruction of branch skeletons [22-24]. An overview of this area can be found in a review from Huang et al. [25].

Only a few methods reconstructed a whole tree by adding leaves or needles to the reconstructed branches. For example, $\mathrm{Xu}$ et al. [26] assumed that the leaf density in a real tree was uniform and determined leaf locations using the number of feasible skeleton nodes that existed within a certain distance. This method requires that even small branches be reconstructed with high accuracy. Livny et al. [23] created leaves next to each leaf node in the branch-structure graph (BSG) and generated a random number between 20 and 50 leaves that were randomly placed within a sphere that was centered on the leaf node. They then added textures to the reconstructed geometry to enhance visual appeal. Côté et al. [27] added foliage to reconstruct conifer trees by repeatedly testing the radiation, reflectance characteristics, and gap fraction of canopy. Their method can reconstruct 3D trees with structural and radiative consistency.

These methods of adding leaves have not made full use of the spatial information obtained from foliage point cloud distributions. In this study, an easy method for reconstructing realistic 3D trees (including trunk, branches, and leaves) is developed. This method is based on aligned point clouds that were scanned from multiple sites and combined information regarding the spatial distribution of points with retrieved structural parameters. The reconstructed trees can meet the basic demands of computer simulation models and maintain a directional gap fraction that is consistent with that of the real ones.

\section{Experiment and Datasets}

\subsection{Study Area}

The experimental field located in an open and flat prairie in Chengde city, Hebei Province, China $\left(42^{\circ} 14^{\prime} 28.7^{\prime \prime} \mathrm{N}, 117^{\circ} 04^{\prime} 57.29^{\prime \prime} \mathrm{E}\right)$ is covered by the dominant grassland and scattered bushes and trees under the typical continental monsoon climate with an average annual temperature of $-1.4^{\circ} \mathrm{C}$. 
Four broadleaf, deciduous elm trees (Ulmaceae) with 6.0-7.5 m height and 15-25 cm diameters at breast (DBH) showed in the field center that was accompanied by approximately $3 \mathrm{~cm}$ understory sparse grasses. They had green leaves and a rough, dark, greyish-brown bark in summer. Some leaves were harvested and scanned with a laser scanner, and the average and maximum single-leaf areas were approximately $12 \mathrm{~cm}^{2}$ and $20 \mathrm{~cm}^{2}$, respectively.

\subsection{Data Acquisition}

\subsubsection{Point Clouds of TLS}

The experimental field was scanned by a TLS system, which included a laser scanner (Riegl VZ-1000 http:/ / www.riegl.com) and a high-quality digital camera (Nikon D300s, Nikon Inc., Tokyo, Japan), on the 25 July 2014. The digital camera and the laser scanner were mounted together on one tripod with a pan-and-tilt platform, and a series of high-resolution color digital photographs and their accompanying point clouds were collected synchronously.

The scanning angular step was set to $0.03^{\circ}$ and the laser scanner height was about $1 \mathrm{~m}$ above the ground. Six groups of point clouds were scanned from six sites around the field (Figure 1). All of the point cloud data were exported as ASCII files using the RiSCAN PRO software (http: / / www.riegl.com), a companion software package for the RIEGL Terrestrial 3D Laser Scanner Systems. The items in the file recorded each returned point's location (XYZ coordination), distance (range [m]), beam direction (zenith and azimuth angle), reflectance (intensity), etc.

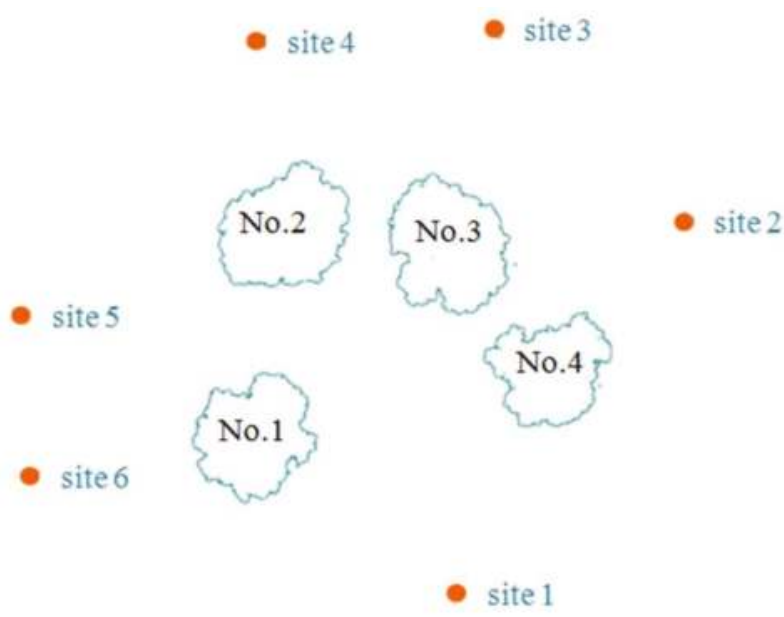

Figure 1. Relative positions of the four trees (No. 1, 2, 3, and 4) and six scanning sites (orange points), as seen from above. The outlines of the trees were drawn by projecting the scanned points in nadir.

\subsubsection{UAV Photographs}

A six-rotor unmanned aerial vehicle (UAV) carried on a small, color digital camera was used to take RGB photographs (1024 pixel * 1024 pixel) of the experimental field from multiple view directions. The flight height of the UAV was approximately $50 \mathrm{~m}$. One clear photograph taken in nadir was used in this study. 


\subsection{Point Cloud Data Processing}

\subsubsection{Elimination of Noisy Points.}

Generally, there are noise points in the point cloud data due to the environment and the scanner itself, which can affect the accuracy of the retrieved canopy structural parameters and the 3D tree reconstruction. For example, ghost points occur when the laser beam hits the edge of an object. Two or more returned points will be recorded by the scanner, but with the wrong distance or intensity information. Therefore, a pre-processing step was included to eliminate ghost points.

We assume that each laser pulse will be reflected once it hits an object. Later returned signals with the same or similar beam directions are taken as ghost points, and should be deleted. First, all points are grouped into many small rectangular pyramids based on the range of zenith and azimuth angles within the width of a scanning angular step $(\delta)$. For example, the $i$ th rectangular pyramid has the zenith range $\left[\theta_{i}-\delta / 2, \theta_{i}+\delta / 2\right)$ and azimuth range $\left[\varphi_{i}-\delta / 2, \varphi_{i}+\delta / 2\right)$. Where $\theta_{i}$ and $\varphi_{i}$ are the zenith and azimuth of the $i$ th scanning beam. The first data point in the pyramid is kept, but the others are deleted.

\subsubsection{Classification}

Point clouds can be classified using geometric information [28] or intensities [29]. In this study, all the point cloud data are classified into trunk/branch points and leaf points based on the digital photographs taken synchronously.

First, the high-resolution digital photographs are classified into leaves, trunk/branches, ground, and sky by the supervised classification method (maximum likelihood method supplied by the ENVI software). Only four classes of ROIs (region of interest), including leaves, trunk/branches, soil, and sky, are chosen manually as the training data. Therefore, parts of the ground covered with a few grasses are incorrectly classified as leaves, but these will be eliminated based on the position property of the points. Second, the photograph is registered with the point clouds using an inherent geometrical constraint [30,31]. Actually, the photos and points were obtained synchronously by the TLS system, therefore the registration parameters can be computed much more easily and accurately. Each point is assigned the property of leaf or branch based on the classification images. Finally, points are extracted based on the spatial scope of the tree, including leaf, trunk/branch, and soil. Soil and some misclassified leaf points on the ground are excluded according to elevation in the $\mathrm{z}$ direction. The height threshold $\mathrm{z}$ is set as $0.1 \mathrm{~m}$ based on the spatial characteristics of points. Then, all points with leaf and trunk/branch properties are extracted, separately.

\subsubsection{Registration}

A general process is carried out to register multi-station point clouds in a common coordinate system based on the RiSCAN PRO software.

It is difficult to find any natural tie points and control points in the field, so dozens of artificial spherical reflectors were pasted on the trunks or branches of the trees in the experimental field before scanning, and we made sure that no less than four reflectors can be "seen" in two neighboring scans. These reflectors can be automatically extracted from the point clouds based on their high reflectance. The same reflectors that were detected from multi-station scanned point clouds are identified as homonymy points and are registered with minimal errors. Transformation matrices are obtained for the point clouds that are based on the corresponding point pairs for the reflectors, which can be used to register the point clouds.

In this experiment, six-station scans are distributed in a ring around the experimental field, so a mode provided in the software called "Close gaps in ringed scan positions" is used to register multiple scans from a ring of positions simultaneously, which can minimize the cumulative error inherent in multiple registrations. The error of registration in all scans is less than $0.02 \mathrm{~m}$. 


\subsubsection{Terrain}

The terrain is also an important part in a 3D scene and it can be fitted to some complex curves, such as a surface or Triangulated Irregular Network (TIN) model [32,33]. In this study, because the field is flat in the field, all of the ground points are fitted to a plane function. Some of the grasses on the ground are ignored, and a flat terrain is composed of many triangle facets.

\subsubsection{Leaf Area Retrieval}

The modified gap fraction model integrating the path length distribution that was developed by Xie et al. [34] is applied to retrieve the leaf area of single trees based on single-site leaf-point cloud data. Leaf area (LA) is defined as total single-side leaf area of a single tree with the unit of square meters $\left(\mathrm{m}^{2}\right)$. Compared with the definition of LAI, total single-side leaf area per unit ground horizontal surface area $\left(\mathrm{m}^{2} / \mathrm{m}^{2}\right)$, LA is more suitable for quantifying the leaf area of single trees.

There are four single trees in the study area. In consideration of the effect of occlusion between crowns, only three groups of points for each tree are used to retrieve LAs (Table 1). They are scanned from three sites that covered the whole tree without any mask from the other trees. Because of the uneven distribution of leaves in the canopy and the effect of point noise (arose by sensors or environment, such as wind, etc.), it can be seen that the LAs of each tree retrieved based on the points from different scans are different. Therefore, LAs that were retrieved from different scans are averaged to decrease the effect of retrieval bias and the unevenness of crowns.

Table 1. The retrieval leaf areas and scanner sites for each tree.

\begin{tabular}{|c|c|c|c|}
\hline Tree ID & Averaged LA $\left(\mathrm{m}^{2}\right)$ & Site ID & Retrieval LA $\left(\mathrm{m}^{2}\right)$ \\
\hline \multirow{3}{*}{ No.1 } & \multirow{3}{*}{71.236} & Site 1 & 71.910 \\
\hline & & Site 3 & 71.350 \\
\hline & & Site 6 & 70.449 \\
\hline \multirow{3}{*}{ No.2 } & \multirow{3}{*}{75.895} & Site 1 & 80.548 \\
\hline & & Site 4 & 80.663 \\
\hline & & Site 5 & 66.474 \\
\hline \multirow{3}{*}{ No.3 } & \multirow{3}{*}{65.546} & Site 1 & 62.394 \\
\hline & & Site 2 & 71.946 \\
\hline & & Site 4 & 64.298 \\
\hline \multirow{3}{*}{ No.4 } & \multirow{3}{*}{48.075} & Site 1 & 47.825 \\
\hline & & Site 2 & 49.041 \\
\hline & & Site 5 & 47.359 \\
\hline
\end{tabular}

\subsection{Virtual Tree}

Some differences may arise in a real 3D scene due to data acquisition, such as the noise in the point clouds that arose from the TLS device and its environment during the measurement. There are also errors arose from the data processing, such as the retrieved LA of each tree and point classification and registration. To overcome these issues, a 3D virtual tree is generated and its LA can be calculated accurately. Accordingly, point clouds are simulated without noise.

\subsubsection{D Virtual Tree}

A 3D virtual tree with photorealistic structures is generated using OnyxTREE@ software (http:/ / www.onyxtree.com), a dedicated procedural creator and modeler of 3D broadleaf trees, shrubs, and bushes. Leaves, trunks, and branches in the crown are generated and arranged based on the plant growth rules and parametric topology structures. The components of $3 \mathrm{D}$ trees, including leaves, 
trunks, and branches, are all composed of triangular patches. The software had been used to simulate the structure of deciduous broadleaf canopies for simulation of various remote sensing signals [35]. According to the definition of LA, it can be calculated accurately using these triangular patches. $\mathrm{LAD}$ is another important canopy structural parameter, which describes the statistical distribution of the angular orientation of the leaves in the vegetation. In this study, we pay more attention to the orientation of each leaf normal (zenith and azimuth angles), instead of the mathematical description of total leaves angles.

In this case, the height of the virtually generated 3D tree is $8.53 \mathrm{~m}$ and its total leaf area is $20.32 \mathrm{~m}^{2}$. The average area of a single leaf is about $15 \mathrm{~cm}^{2}$.

\subsubsection{Simulation of Point Clouds}

Four groups of point clouds are simulated around the virtual tree based on the ray-tracing algorithm [36]. The sensor height is set to $1.5 \mathrm{~m}$ above the ground and the horizon distance for the tree was $6 \mathrm{~m}$. The scanning angle step is $0.03^{\circ}$.

Each simulated point is added property information (branch or leaf) in the end of the line in the file based on the intersected position of the beam and the virtual tree, which can help to classify the point clouds into two classes (branch- and leaf-point) accurately.

\section{Methodology}

Branches and leaves are the main components of trees, and can be reconstructed based on the classified branch- and leaf-point clouds. To reduce the effect of point cloud occlusion, the aligned point clouds from multiple scans are used here.

\subsection{Reconstruction of Branches}

Branch structures of single trees are constructed from the branch-point clouds using a structure-aware global optimization (SAGO) method [24]. This method obtains the tree skeleton from a minimum distance spanning tree and then defines the stretching directions for the branches on the skeleton to recover those missing or partly occluded points.

However, most of the small terminal branches with growing leaves cannot be reconstructed correctly, because the branch points are thoroughly occluded by leaves, or they cannot be classified accurately due to the low spatial resolution of the photographs. Some of the misclassified branch points may be marked as leaf type and later used in the process of adding leaves, which can maintain consistency between the reconstructed tree gap fraction and the real one.

\subsection{Adding Leaves}

Generally, there are a large number and variety of leaves in the crown, and their distribution is very complex. Therefore, it is difficult to accurately recover all of the information for each leaf, including its position, orientation, and size. We assume that the spatial distribution of leaf-point density can reflect the distribution of leaf characteristics in the crown.

\subsubsection{Leaf Structure Model}

A leaf is simplified to a quadrilateral, which is composed of a right triangle and an equilateral triangle. It is set based on the ratio of the length and width of a clipped leaf measured in the field (Figure 2a). This kind of leaf shape is also used by the generated virtual tree in this study. Its initial four vertex coordinates in the plane of XOY can be expressed as a four-order matrix P [37], with a normal $N(0,0,1)$. 


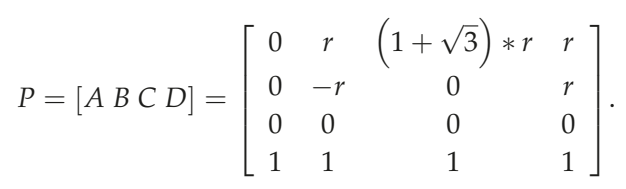

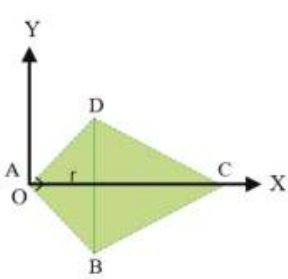

(a)

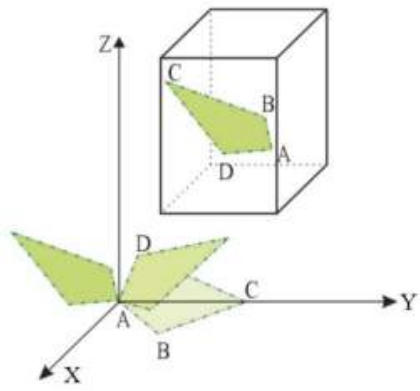

(b)

Figure 2. Diagram of leaf geometry (a) and its transform (b).

The lowest row represents homogeneous term in order to be convenient to transfer this polygon leaf in the space. In Equation (1), $r$ is a leaf shape parameter that controls the size of a leaf. The area of a leaf can be calculated as

$$
L A_{i}=(1+\sqrt{3}) r^{2}
$$

The leaf can then be rotated and translated to the right place in the tree crown by multiplying $\mathrm{P}$ with rotation and translation matrices [37], as shown in Figure $2 b$.

\subsubsection{Cell division and Leaf Placement}

Segmenting the canopy into cubic volumes/voxels based on point clouds can provide a convenient mean of describing the spatial distribution of foliage area in the tree crown [38] and of estimating the leaf area density [21], LAI and LAD [20], etc. Béland et al. [38] investigated the optimal voxel dimensions for estimating the spatial distribution of leaf area density within the crown. They found that the optimal voxel size is a function of leaf size, branching structure, and the predominance of occlusion effects, which provides important guiding principles for the use of voxel volume in the segmentation of point clouds.

In accordance with the research of Béland et al. [38], voxel size is set to $10 \mathrm{~cm}$ in this case, which is based on the averaged LA of each tree (Table 1) and branching structure is measured in the field. Assuming that the spatial distribution of leaf areas agrees with the aligned point-cloud density, each leaf point in the crown corresponds to a finite area of leaf LA/NUM, where NUM is the number of total leaf points. Therefore, based on the number of points in each voxel $\left(\right.$ num $\left._{i}\right)$, the area of one leaf in the voxel can be determined using $L A_{i}=n u m_{i} \times L A / N U M$. If the leaf area in the voxel $L A_{i}$ is larger than the maximum leaf area $\left(20 \mathrm{~cm}^{2}\right)$, it would be separated into a standard leaf with an area of $12 \mathrm{~cm}^{2}$ and a smaller leaf. The leaf shape parameter for each leaf, $r$, can be calculated using:

$$
r=\left[L A_{i} /(1+\sqrt{3})\right]^{1 / 2},
$$

where $L A_{i}$ is the leaf area in the voxel or the leaf area after division.

Leaf orientation in the voxel is also an important structural parameter. Generally, LAD can be measured in the field or estimated from point clouds using voxels [21]. To simply the procedure of 
the reconstruction and decrease the needed parameters, an averaged normal direction of all the leaf points in the voxel is used as the normal direction of the added leaf. Where the normal direction for each point is calculated based on the spatial distribution of neighbor points using the CloudCompare software (http://www.danielgm.net/cc/release/).

Leaves are placed in the appropriate cube by rotation and translation, according to their orientation and size. After the process of adding leaves is applied to all of the voxels in the scene, 3D trees with leaves are reconstructed.

\subsection{Assemble 3D Scene}

All single trees and terrain are reconstructed and assembled. In order to match the locations of the reconstructed trees with the real ones, the reconstructed trees are projected onto the plane to obtain the outlines of trees, and the aligned point clouds are used to outline the four trees (Figure 1). Then, the outlines from the reconstructed trees can be registered with the outlines from the real trees. In this study, the flat terrain is applied. Therefore, all reconstructed trees can be translated in the correct position of the terrain directly, and then the $3 \mathrm{D}$ scene is completed.

\section{Results and Discussion}

\subsection{Reconstruction of 3D Scene}

\subsubsection{Real Scene of the Study Area}

The 3D scene of the study area is composed of four single trees and flat ground. In consideration of the effect of occlusion between crowns, only three groups of points for each tree are used to reconstruct each single tree. First, the three sites scans for each tree in Table 1 are aligned, and then the averaged LA for the tree (Table 1) is applied to add leaves and to reconstruct the tree. At last, 3D scene that is similar to the real one is assembled by combining the reconstructed trees on a flat terrain.

A color photograph is simulated based on the reconstructed 3D scene using the physically based ray tracing (PBRT) algorithm [39] (Figure 3a). When compared with the corresponding photograph taken from UAV (Figure 3b), the structures are visually similar.

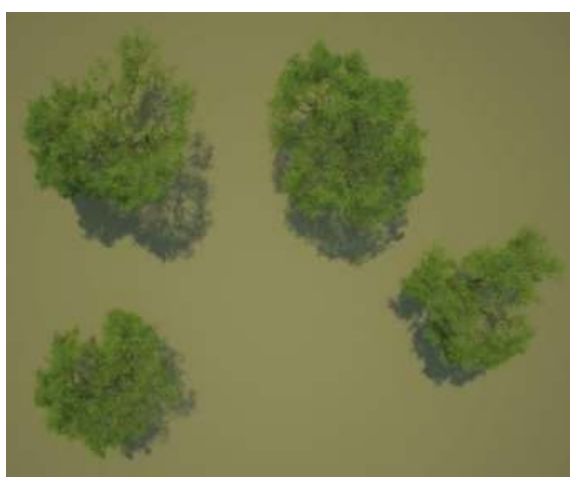

(a)

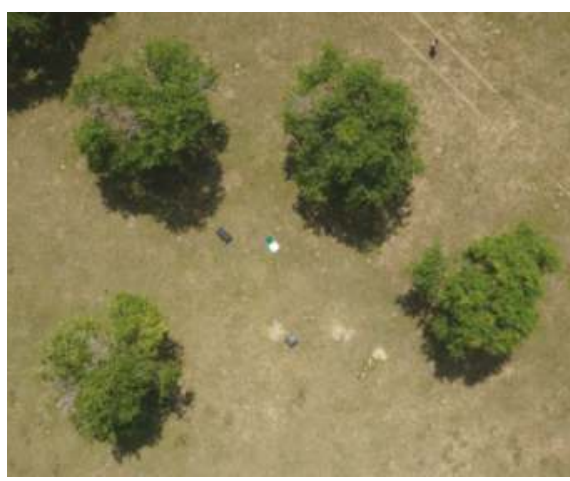

(b)

Figure 3. Comparison of simulated and real photos: (a) Simulated photograph based on the reconstructed scene; and, (b) Real photograph taken from unmanned aerial vehicle (UAV). 


\subsubsection{Virtual Tree}

A 3D tree is reconstructed based on the simulated points (Figure 4c). Its leaf area is $20.50 \mathrm{~m}^{2}$ and the relative error of the leaf areas between the reconstructed and the original virtual tree is less than $0.9 \%$. It looks similar to the original tree, including the spatial distribution of leaves.

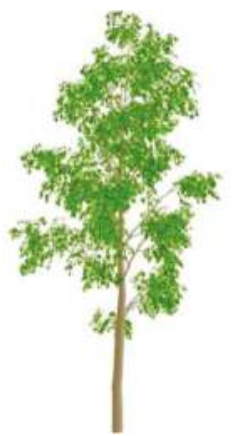

(a)

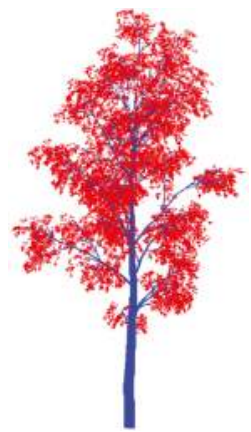

(b)

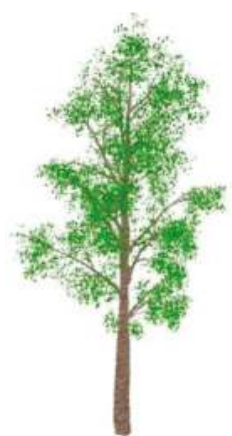

(c)

Figure 4. (a) A virtual tree generated with OnyxTREE software; (b) The simulated point clouds based on the virtual tree (Blue points represent trunks/branches and red points represent leaves); and, (c) The reconstructed $3 \mathrm{D}$ tree.

\subsection{Validation}

Quantitative validation of the structural characteristics of a rebuilt scene is not a trivial task [27]. The reconstructed scene should not only represent a good visual effect, but it must be structurally consistent with the real canopy. The directional gap fraction is used as an important standard to quantitatively evaluate the geometric accuracy of reconstructed 3D scenes or the 3D structures of trees.

\subsubsection{Validation with the Real Scene}

Point clouds are simulated based on the reconstructed 3D scene using the ray tracing algorithm [36]. The parameters are set according to the configuration in the field, including scanning position, scanning steps, beam diversity, etc. The measured and simulated point clouds are compared and are shown in Figure 5. It can be seen that they have good similarities in color and structure (the same color represents the same distance).

The directional gap fraction with a zenith angle slices $P_{g a p}(\theta)$ can be expressed as the ratio of the number of laser beams sent into the slice $P_{\text {send }}^{i}$ and the number of intercepted laser beams $P_{\text {receive }}^{i}$. The sending number is dependent on both the azimuth and zenith range in each slice, as well as the scanning step, and so the intercepted number can be calculated by counting the number of TLS points [40]. Therefore, the gap fraction can be expressed as follows:

$$
P_{\text {gap }}\left(\theta_{i}\right)=\left(P_{\text {send }}^{i}-P_{\text {receive }}^{i}\right) / P_{\text {send }}^{i} \quad i=1 \cdots \mathrm{N},
$$

where $N$ is the number of the zenith slices, and $\theta$ is a middle zenith direction of the layer.

The directional gap fractions for six-site point clouds are calculated based on the measured and simulated data, respectively, and compared in Figure 6. The two groups of directional gap fractions have similar tendencies: they are large in the upper and lower parts and small in the middle, which agree well with the characteristics of the vertical distribution of foliage in the crown. Because the distribution of branches and leaves are not uniform in all crowns, the gap fractions in some directions increase suddenly, such as at $70^{\circ}$ for site 3 and 4 , and at $75^{\circ}$ for site 6 (Figure 6 ). 


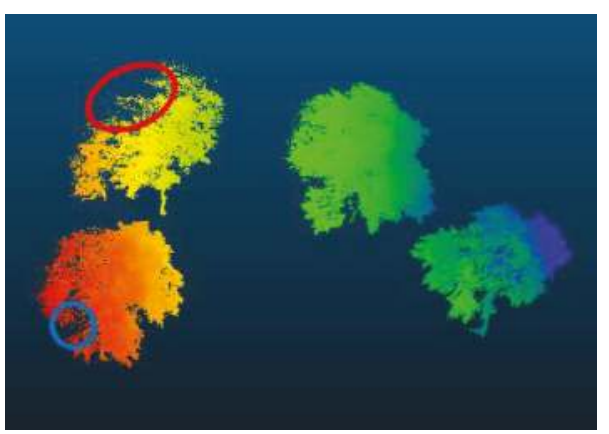

(a)

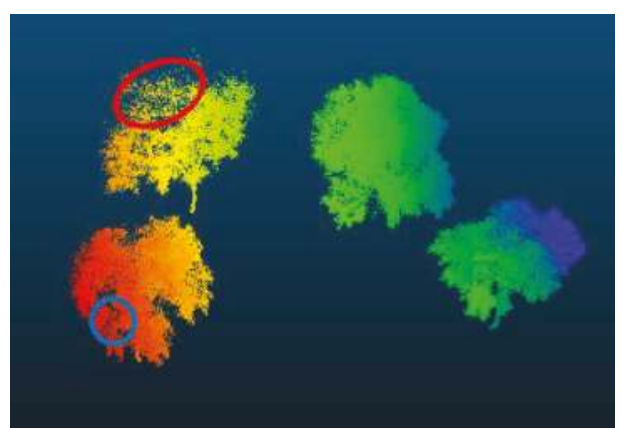

(b)

Figure 5. Comparison of the measured (a) and simulated (b) point clouds for one scan. The different colors represent the distances from scanner to objects. The red and blue circles highlight the difference between the measured and simulated point clouds.
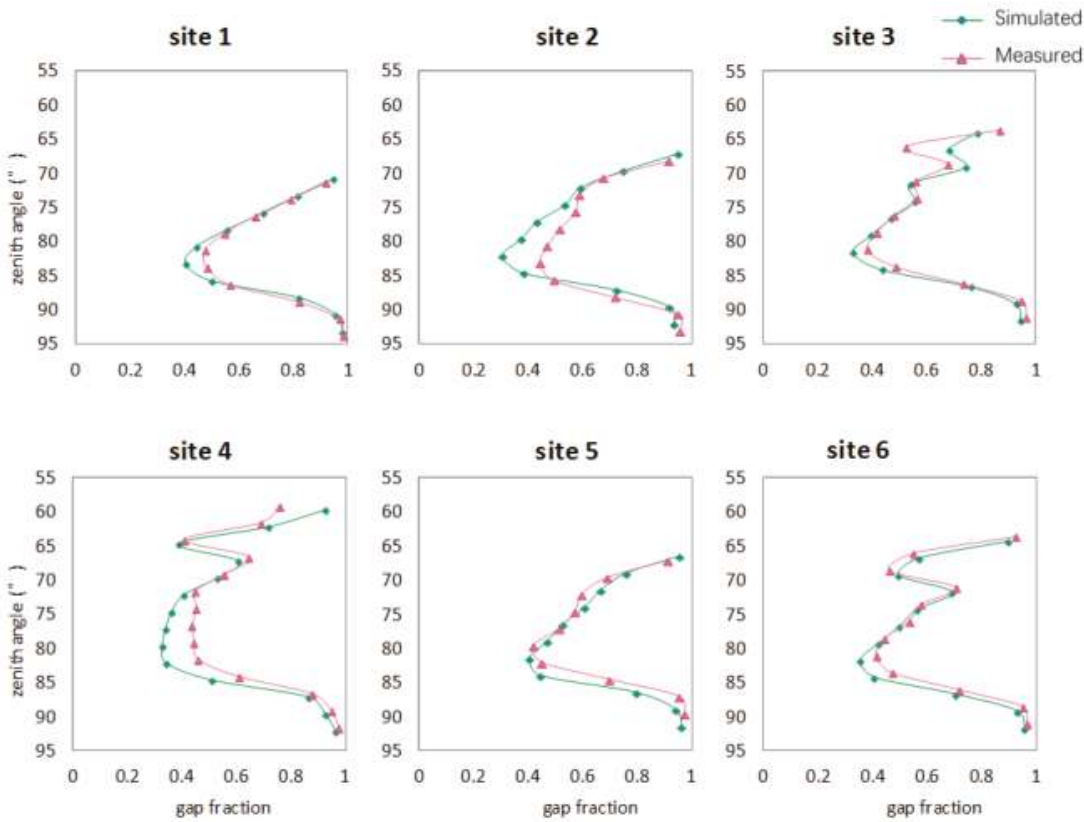

Figure 6. Comparison of the directional gap fractions from measured and simulated points for each site scan (zenith angle interval: $2.5^{\circ}$ ).

In general, all of the directional gap fractions from the reconstructed scene are in good agreement with those from the real one (Figure 7a). A high correlation coefficient $\left(R^{2}=0.912\right)$ and a low root mean square error $(\mathrm{RMSE}=0.065)$ between the directional gap fractions that were calculated based on the simulated and measured points quantitatively prove that the method is valid for reconstructing structurally coherent 3D trees. 


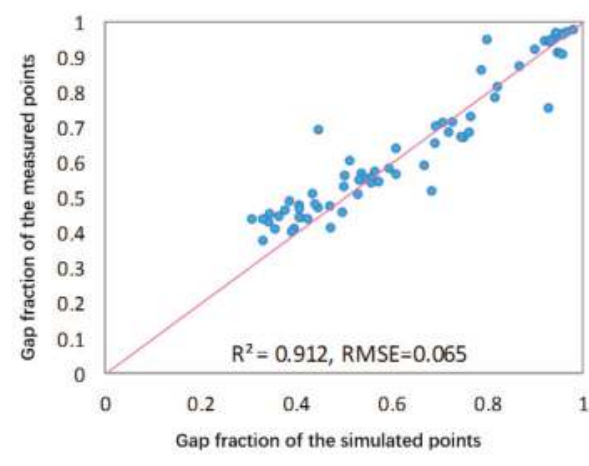

(a)

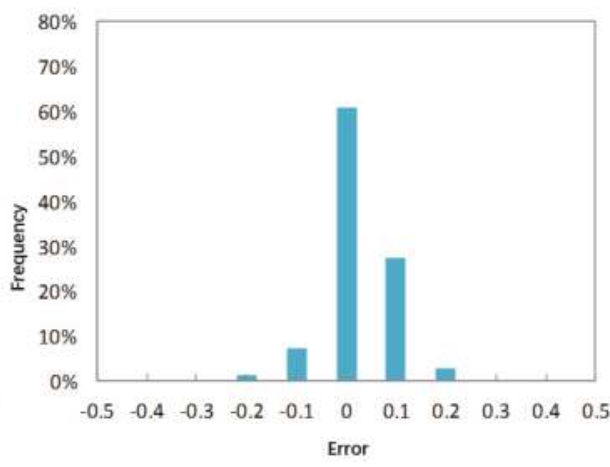

(b)

Figure 7. Relationship between the directional gap fractions of measured and simulated points (a) and the distribution of their errors (b).

Although the directional gap fraction is a popular standard to assess the canopy structure, it is affected by the parameters, such as azimuth ranges. It can be seen that there are still a little discrepancies of gap fractions between real and reconstructed trees, as shown in Figure 5. We found that the reason is from the difference of the scanning azimuth ranges in the same slices detected from the simulated and measured points. Generally, the voxel nearby the edge of the crown can reach out of its range, and the added leaves in these voxels often be placed in the centers of the voxels, so that the reconstructed crown is often a little wider than the real one. Accordingly, the azimuthal range of point clouds simulated based on the reconstructed tree is often bigger than the real one. To eliminate the influence of edge effect on the emitted beam numbers, the slicing point density is used to evaluate the structural accuracy of a reconstructed scene.

The slicing point density (PD) with the height is calculated based on all of the aligned points [41].

$$
P D\left(H_{i}\right)=\text { num }_{i} / N U M
$$

Here, $n u m_{i}$ is the number of points in the $i$ th layer with height $\left[H_{i}, H_{i}+\Delta H\right] ; \Delta H$ is the height interval; and, NUM is the total number of points intercepted by the tree.

In this study, we pay more attention to the effect of the reconstructed leaf, so the leaf-point density in each slice is calculated based on the separated leaf points. The horizontally slicing leaf-point densities based on the measured and simulated data are compared in Figure 8, and the vertical distribution of leaf-point densities from all four trees agree well. 
No.1

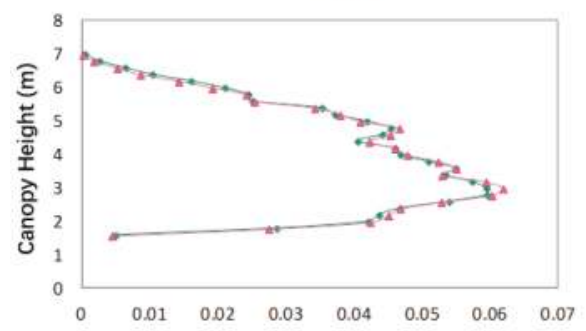

No.3

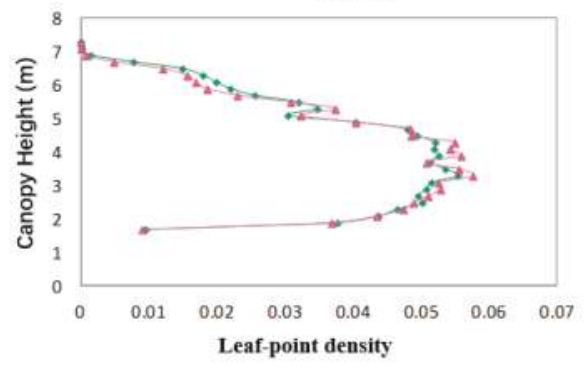

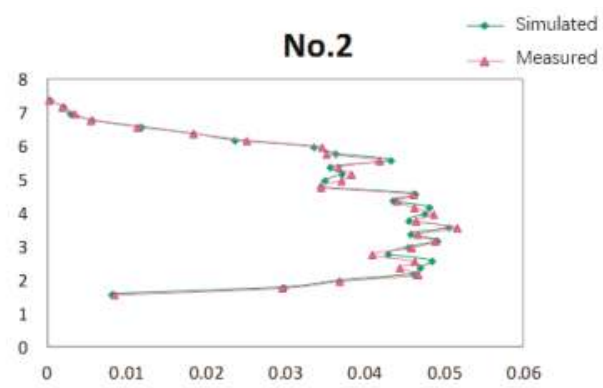

No.4

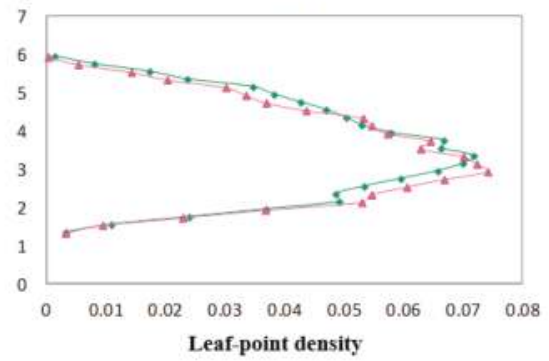

Figure 8. Comparison of the sliced leaf-point densities for each tree (No. 1-4), calculated based on measured and simulated point clouds (interval height: $0.2 \mathrm{~m}$ ).

\subsubsection{Validation with the Virtual Tree}

Because the 3D virtual tree in Figure $4 \mathrm{c}$ is reconstructed based on the simulated points, to improve the reliability of validation, we do not compare the gap fraction or point density calculated with the simulated points from the virtual tree and the reconstructed tree. In this study, some black-and-white images are simulated based on the original and reconstructed trees. These images are built by parallel projecting all triangle facets of the original or reconstructed tree along one view direction to a white plane with a high resolution. The pixel covered by the projected facet $(>50 \%)$ is black. Then, a gap fraction can be calculated from this image using the pixel ratio (a ratio of the white pixel numbers to the total number of pixels in the image).

The black-and-white images are simulated from several directions, including eight azimuth angles $\left(0^{\circ}, 45^{\circ}, 90^{\circ}, 135^{\circ}, 180^{\circ}, 225^{\circ}, 270^{\circ}, 315^{\circ}\right)$ and five zenith angles $\left(45^{\circ}, 63^{\circ}, 76^{\circ}, 90^{\circ}, 162^{\circ}\right)$. Images with the zenith directions of $\left(45^{\circ}, 63^{\circ}, 76^{\circ}, 90^{\circ}\right)$ are projected from bottom to top, and the image with $162^{\circ}$ zenith direction is projected from top to bottom. Then, the relative error of the directional gap fractions obtained using the simulated images from the original and reconstructed trees are shown in Figure 9. The relative errors of the directional gap fractions are not more than $4.1 \%$, which strongly supports the feasibility of our reconstruction method. The relative errors for different azimuth directions have no apparent difference because the structure of the generated virtual tree is basically azimuthally symmetrical. 


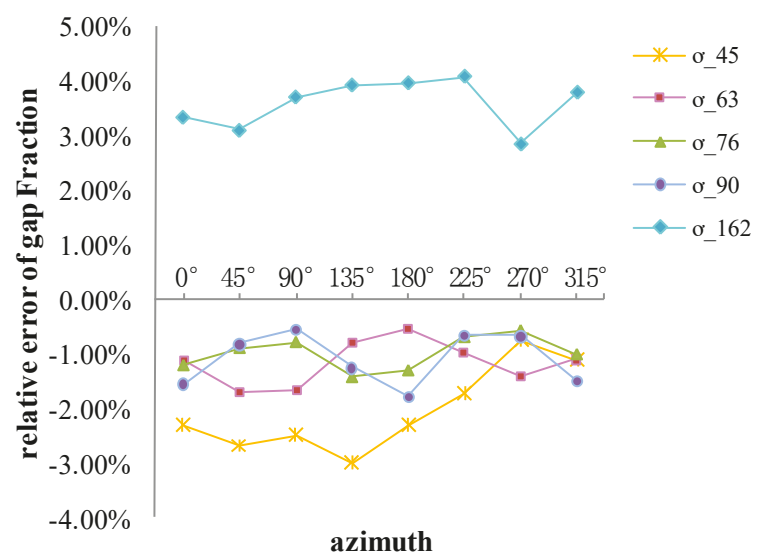

Figure 9. Relative errors of the gap fractions from the black-and-white images with different view directions simulated based on the original virtual tree and the reconstructed one.

\section{Discussion}

\subsection{Completeness and Accuracy of the Reconstructed Tree}

By applying our method to a real scene and a virtual tree, the reconstructed results show pretty well visual effects. However, after quantitatively comparing the directional gap fractions and point densities of the original and reconstructed ones, it is found that the occlusion and density distribution of points will affect the completeness and accuracy of the reconstructed tree.

As is known to all, the occlusion issue is inevitable for TLS to scan canopies. Although aligned multi-site scanned points can partly cover the shortage, the issue still cannot be solved completely for some dense crowns. For example, there are some small differences in Figure 5, highlighted using circles. One reason is that the scanner positions that were used in the simulation do not coincide with the one in the field. Therefore, some points occluded in the measured data (circle in Figure 5a) can be scanned in the simulation ones (Figure 5b).

Additionally, it can be seen from Figure 9 that the directional gap fractions are underestimated in the bottom-to-top zenith directions $\left(45^{\circ}, 63^{\circ}, 76^{\circ}, 90^{\circ}\right)$, but overestimated in the top-to-bottom zenith direction $\left(162^{\circ}\right)$. The major reason for this difference is the effect of point cloud occlusion. Because the TLS device is often used on the ground with a bottom-to-up scanning style, the upper crowns are inevitably occluded and only a few points from the upper parts of the tree can be obtained. Another reason is that the point density distribution is not uniform: the point density is larger in the lower part of the tree but is smaller than normal in the upper part of the tree. Because the tree reconstruction depends entirely on the spatial distribution of points, more leaves are added to the lower crown. This means that the relative errors for directional gap fractions that are close to the vertical directions (such as $45^{\circ}$ and $162^{\circ}$, in this case) will be enlarged.

A feasible method for addressing these issues would be to add some supplementary data, such as Airborne Laser Radar (ALS) data, which can make up for the TLS points missing from the upper crowns [31,42].

\subsection{Sensitivity of the Leaf Shape}

The leaf shape is various in the real world. It can be represented as some simple shapes. In this study, we use a quadrilateral, simplified based on the measured structure to reconstruct the trees. 
Similarly, the leaf can also be simplified as other shapes, such as a square or a rhombus (Figure 10). Then, the initial four vertex coordinates can be expressed as

$$
P=[A B C D]=\left[\begin{array}{cccc}
0 & r & 2 r & r \\
0 & -r & 0 & r \\
0 & 0 & 0 & 0 \\
1 & 1 & 1 & 1
\end{array}\right] \text { and }\left[\begin{array}{cccc}
0 & 4 r & 8 r & 4 r \\
0 & -1.5 r & 0 & 1.5 r \\
0 & 0 & 0 & 0 \\
1 & 1 & 1 & 1
\end{array}\right]
$$

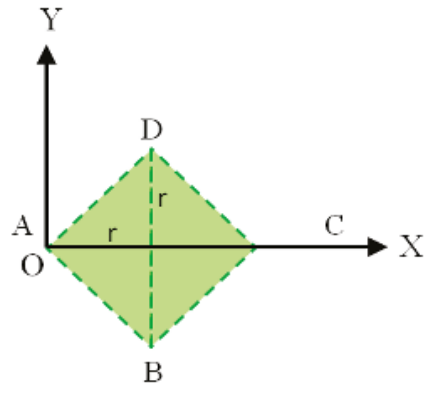

(a)

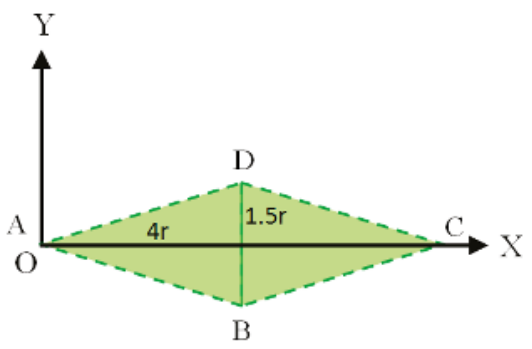

(b)

Figure 10. Diagram of leaf shape: (a) Square, (b) Rhombus.

The leaf area can be calculated as

$$
L A_{i}=4 r^{2} \text { and } L A_{i}=24 r^{2}
$$

Then, the leaf shape parameter, $r$, can be obtained by $r=\sqrt{L A_{i} / 4}$ and $r=\sqrt{L A_{i} / 24}$.

The virtual trees (Figure $4 a$ ) are reconstructed using the new leaf shapes, respectively (Figure 11). All reconstructed trees with different leaf shapes look similar.

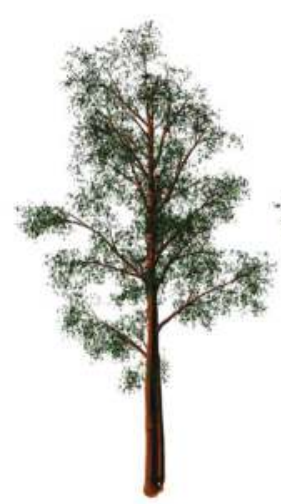

(a)

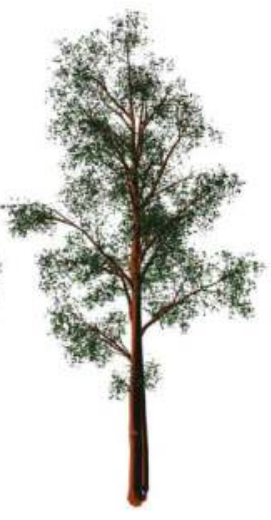

(b)

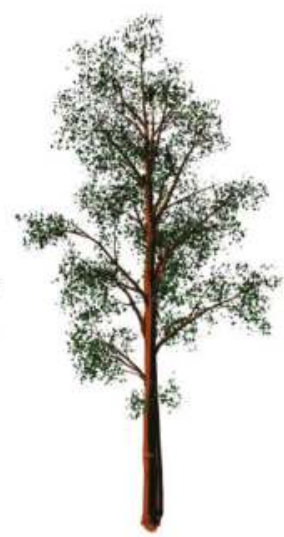

(c)

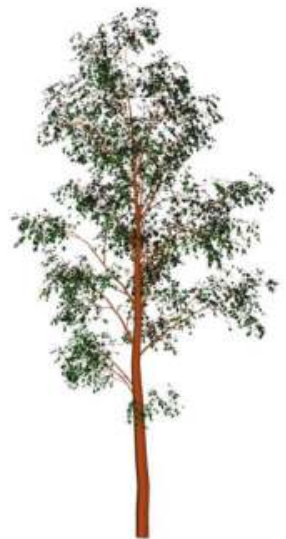

(d)

Figure 11. Comparison of the reconstructed trees using the different leaf shapes, including (a) rhombus, (b) square and (c) typical quadrilateral, with (d) the original virtual tree. 
Then point clouds of these three reconstructed virtual trees (Figure 11a-c) are simulated based on the same parameters (the sensor height, scanning distance and step). The slicing point density of each set of point clouds is calculated and compared in Figure 12.

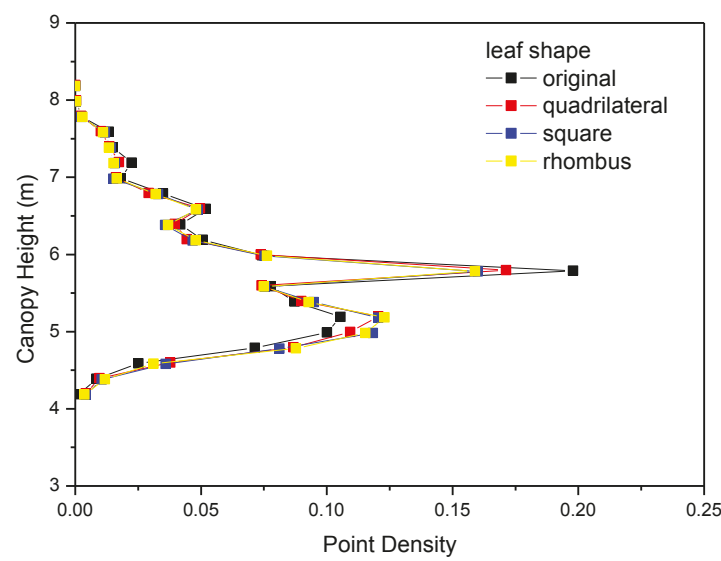

Figure 12. Comparison of point densities based on the simulated point clouds of the reconstructed trees with different leaf shapes and the original virtual tree (interval height: $0.2 \mathrm{~m}$ ).

These point densities agree well with the original ones. Specially, the point densities from the reconstructed trees are closer than that from the original virtual tree. RMSEs of the vertical point densities between the reconstructed trees and the original one are 1.10\% (square leaf shape), $1.13 \%$ (rhombus leaf shape), and $0.88 \%$ (quadrilateral leaf shape), separately. The reconstructed tree with the typical leaf shape (quadrilateral) shows better similarity with the original one. When comparing within these reconstructed trees, RMSEs of the point densities are no more than $0.4 \%$. It means that the reconstructed trees are more similar to each other.

Overall, our method is not sensitive to the leaf shape.

\section{Conclusions}

Based on TLS point cloud data, we developed the method for reconstructing entire 3D trees in this study. This method can effectively add leaves that are based on voxels and is insensitive to the leaf shape. Its performance is evaluated over a reconstructed small 3D scene and a virtual tree using the directional gap fraction and point density distribution, and it shows good consistency in leaf area and directional gap fraction of crowns when compared with the point cloud from the original ones. The relative errors of the gap fractions (the virtual tree) are less than $4.1 \%$.

While analyzing the directional gap fraction that is based on a virtual 3D tree, it is found that the reconstructed 3D trees are influenced by the effect of point cloud occlusion because the method is completely dependent on the spatial distribution of points. Though this method may slightly underestimate the leaf area for the upper crowns, and overestimate for the lower crowns, it is still feasible and has the potential to contribute the quantitative remote sensing in canopy radiative transfer models.

Several factors that impact the reconstruction of trees and some future possible improvements are summarized, including the quality of point clouds, the point classification, and LA retrieval accuracy. First, the quality of point clouds is critical, and factors, such as occlusion and ghost points, greatly complicate the data preprocessing and decrease the structural accuracy of reconstructed trees. In the future, it will be necessary to study how TLS data can be combined with supplementary data (such as ALS-aircraft laser scanner data or UAV data) to reconstruct 3D trees and reduce the 
effect of occlusions. Another option would be to apply the growing rules of plants to overcome the disadvantages of point clouds. Second, point classification accuracy is dependent of the quality of photographs, the classification algorithm and the registration between points and photographs. Bad classification results not only affect the LA retrieval, but also decrease the accuracy of reconstructed branches and leaves. Dual-wavelength [43] or multiple spectra laser sensors can help to improve the classification accuracy and to simplify the classifying process. Third, the LA of a real tree is determined using a retrieved value, and its accuracy will directly affect the reconstructed tree structure. Therefore, it is necessary to improve the retrieval algorithm for LA in the future.

In summary, the method that is presented for reconstructing 3D trees shows its potential in reconstructing complex forest scene and in evaluating the parameters of land surface ecosystem.

Author Contributions: Donghui Xie and Xiangyu Wang developed the method of reconstructing 3D trees; Jianbo Qi simulated point cloud data; Yiming Chen, Xihan Mu, Wuming Zhang and Guangjian Yan designed and performed the experiment; Yiming Chen help process the point clouds and all authors contributed in writing the paper.

Acknowledgments: This work is supported by National Natural Science Foundation of China (Grant No. 41571341, 41331171). Thanks the colleagues who helped collect field data and its processing.

Conflicts of Interest: The authors declare no conflict of interest.

\section{References}

1. Morsdorf, F.; Meier, E.; Kötz, B.; Itten, K.I.; Dobbertin, M.; Allgöwer, B. LIDAR-based geometric reconstruction of boreal type forest stands at single tree level for forest and wildland fire management. Remote Sens. Environ. 2004, 92, 353-362. [CrossRef]

2. Seidl, R.; Rammer, W.; Scheller, R.M.; Spies, T.A. An individual-based process model to simulate landscape-scale forest ecosystem dynamics. Ecol. Model. 2012, 231, 87-100. [CrossRef]

3. Disney, M.; Lewis, P.; Saich, P. 3D modelling of forest canopy structure for remote sensing simulations in the optical and microwave domains. Remote Sens. Environ. 2006, 100, 114-132. [CrossRef]

4. Fleck, S.; van der Zande, D.; Schmidt, M.; Coppin, P. Reconstructions of tree structure from laser-scans and their use to predict physiological properties and processes in canopies. Int. Arch. Photogramm. Remote Sens. Spat. Inf. Sci. 2004, 36, 119-123.

5. Verhoef, W. Light scattering by leaf layers with application to canopy reflectance modeling: The SAIL model. Remote Sens. Environ. 1984, 16, 125-141. [CrossRef]

6. Suits, G.H. The calculation of the directional reflectance of a vegetative canopy. Remote Sens. Environ. 1973, 2, 117-125. [CrossRef]

7. Kubelka, P.; Munk, F. Ein beitrag zur optik der farbanstriche. Zeitschrift fur Technische Physik 1931, 12, 501-593.

8. Li, X.; Strahler, A.H. Geometric-optical modeling of a conifer forest canopy. IEEE Trans. Geosci. Remote Sens. 1985, 5, 705-721. [CrossRef]

9. Li, X.; Strahler, A.H. Geometric-optical bidirectional reflectance modeling of the discrete crown vegetation canopy: Effect of crown shape and mutual shadowing. IEEE Trans. Geosci. Remote Sens. 1992, 30, $276-292$. [CrossRef]

10. Chen, J.M.; Leblanc, S.G. A four-scale bidirectional reflectance model based on canopy architecture. IEEE Trans. Geosci. Remote Sens. 1997, 35, 1316-1337. [CrossRef]

11. España, M.L.; Baret, F.; Aries, F.; Chelle, M.; Andrieu, B.; Prévot, L. Modeling maize canopy 3D architecture: Application to reflectance simulation. Ecol. Model. 1999, 122, 25-43. [CrossRef]

12. Goel, N.S.; Qin, W. Influences of canopy architecture on relationships between various vegetation indices and LAI and FPAR: A computer simulation. Remote Sens. Rev. 1994, 10, 309-347. [CrossRef]

13. Goel, N.S.; Rozehnal, I.; Thompson, R.L. A computer graphics based model for scattering from objects of arbitrary shapes in the optical region. Remote Sens. Environ. 1991, 36, 73-104. [CrossRef]

14. Govaerts, Y.M.; Verstraete, M.M. Raytran: A Monte Carlo ray-tracing model to compute light scattering in three-dimensional heterogeneous media. IEEE Trans. Geosci. Remote Sens. 1998, 36, 493-505. [CrossRef]

15. Qin, W.; Gerstl, S.A. 3-D scene modeling of semidesert vegetation cover and its radiation regime. Remote Sens. Environ. 2000, 74, 145-162. [CrossRef] 
16. Gastellu-Etchegorry, J.-P.; Demarez, V.; Pinel, V.; Zagolski, F. Modeling radiative transfer in heterogeneous 3-D vegetation canopies. Remote Sens. Environ. 1996, 58, 131-156. [CrossRef]

17. Huang, H.; Qin, W.; Liu, Q. RAPID: A Radiosity Applicable to Porous IndiviDual Objects for directional reflectance over complex vegetated scenes. Remote Sens. Environ. 2013, 132, 221-237. [CrossRef]

18. Disney, M.; Lewis, P.; North, P. Monte Carlo ray tracing in optical canopy reflectance modelling. Remote Sens. Rev. 2000, 18, 163-196. [CrossRef]

19. Widlowski, J.-L.; Côté, J.-F.; Béland, M. Abstract tree crowns in 3D radiative transfer models: Impact on simulated open-canopy reflectances. Remote Sens. Environ. 2014, 142, 155-175. [CrossRef]

20. Zheng, G.; Moskal, L.M. Leaf orientation retrieval from terrestrial laser scanning (TLS) data. IEEE Trans. Geosci. Remote Sens. 2012, 50, 3970-3979. [CrossRef]

21. Hosoi, F.; Nakai, Y.; Omasa, K. 3-D voxel-based solid modeling of a broad-leaved tree for accurate volume estimation using portable scanning lidar. ISPRS J. Photogramm. Remote Sens. 2013, 82, 41-48. [CrossRef]

22. Bucksch, A.; Lindenbergh, R.C.; Menenti, M. Skeltre-fast skeletonisation for imperfect point cloud data of botanic trees. In Proceedings of the 2nd Eurographics Conference on 3D Object Retrieval, Eurographics, Munich, Germany, 29 March-3 April 2009; pp. 13-20.

23. Livny, Y.; Yan, F.; Olson, M.; Chen, B.; Zhang, H. Automatic reconstruction of tree skeletal structures from point clouds. In Proceedings of the ACM Transactions on Graphics (TOG), Seoul, Korea, 15-18 December 2010.

24. Wang, Z.; Zhang, L.; Fang, T.; Mathiopoulos, P.T.; Qu, H.; Chen, D.; Wang, Y. A structure-aware global optimization method for reconstructing 3-D tree models from terrestrial laser scanning data. IEEE Trans. Geosci. Remote Sens. 2014, 52, 5653-5669. [CrossRef]

25. Huang, H.; Chen, C.; Zou, J.; Lin, D. Tree geometrical 3D modeling from terrestrial laser scanned point clouds: A review. Sci. Silvae Sin. 2013, 49, 123-130.

26. Xu, H.; Gossett, N.; Chen, B. Knowledge and heuristic-based modeling of laser-scanned trees. ACM Trans. Graph. 2007, 26, 19. [CrossRef]

27. Côté, J.-F.; Widlowski, J.L.; Fournie, R.A.; Verstraete, M.M. The structural and radiative consistency of three-dimensional tree reconstructions from terrestrial Lidar. Remote Sens. Environ. 2009, 113, 1067-1081. [CrossRef]

28. Tao, S.; Guo, Q.; Xu, S.; Su, Y.; Li, Y.; Wu, F. A geometric method for wood-leaf separation using terrestrial and simulated Lidar data. Photogramm. Eng. Remote Sens. 2015, 81, 767-776. [CrossRef]

29. Reymann, C.; Lacroix, S. Improving LiDAR point cloud classification using intensities and multiple echoes. In Proceedings of the 2015 IEEE/RSJ International Conference on Intelligent Robots and Systems (IROS), Hamburg, Germany, 28 September-2 October 2015; pp. 5122-5128.

30. Zhu, Z.; Zhang, W.; Zhu, L.; Zhao, J. Research on different slicing methods of acquiring LAI from terrestrial laser scanner data. In Proceedings of the 2011 IEEE International Conference on Spatial Data Mining and Geographical Knowledge Services (ICSDM), Fuzhou, China, 29 June-1 July 2011; pp. 295-299.

31. Zhang, W.; Zhao, J.; Chen, M.; Chen, Y.; Yan, K.; Li, L.; Qi, J.; Wang, X.; Luo, J.; Chu, Q. Registration of optical imagery and LiDAR data using an inherent geometrical constraint. Opt. Express 2015, 23, 7694-7702. [CrossRef] [PubMed]

32. Axelsson, P. DEM generation from laser scanner data using adaptive TIN models. Int. Arch. Photogramm. Remote Sens. 2000, 33, 111-118.

33. Zhang, W.; Qi, J.; Wan, P.; Wang, H.; Xie, D.; Wang, X.; Yan, G. An easy-to-use airborne LiDAR data filtering method based on cloth simulation. Remote Sens. 2016, 8, 501. [CrossRef]

34. Xie, D.; Wang, Y.; Hu, R.; Chen, Y.; Yan, G.; Zhang, W.; Wang, P. A modified gap fraction model of individual trees for estimating leaf area index using terrestrial laser scanner. J. Appl. Remote Sens. 2017, 11, 035012. [CrossRef]

35. Disney, M.I.; Lewis, P.E.; Bouvet, M.; Prieto-Blanco, A.; Hancock, S. Quantifying surface reflectivity for spaceborne LiDAR via two independent methods. IEEE Trans. Geosci. Remote Sens. 2009, 47, 3262-3271. [CrossRef]

36. Wang, Y. Simulation and analysis of point clouds from a terrestrial laser scanner. J. Remote Sens. 2015, 19, 391-399.

37. Donald, H.; Pauline, B.; Warren, R. Computer Graphics with OpenGL, 4th ed.; Prentice Hall PTR: Upper Saddle River, NJ, USA, 2010 
38. Béland, M.; Baldocchi, D.D.; Widlowski, J.L.; Fournier, R.A.; Verstraete, M.M. On seeing the wood from the leaves and the role of voxel size in determining leaf area distribution of forests with terrestrial LiDAR. Agric. For. Meteorol. 2014, 184, 82-97. [CrossRef]

39. Matt, P.; Wenzel, J.; Greg, H. Physically Based Rendering: From Theory to Implementation; Elsevier Science \& Technology Publication: San Francisco, CA, USA, 2016.

40. Danson, F.M.; Hetherington, D.; Morsdorf, F.; Koetz, B.; Allgower, B. Forest canopy gap fraction from terrestrial laser scanning. IEEE Trans. Geosci. Remote Sens. 2007, 4, 157-160. [CrossRef]

41. Zhu, Z.; Zhang, W. Estimating the LAI of a single tree from terrestrial laser scanner data. In Proceedings of the 2011 IEEE International Conference on Remote Sensing, Environment and Transportation Engineering (RSETE), Nanjing, China, 24-26 June 2011; pp. 4947-4951.

42. Paris, C.; Kelbe, D.; van Aardt, J.; Bruzzone, L. A novel automatic method for the fusion of ALS and TLS LiDAR data for robust assessment of tree crown structure. IEEE Trans. Geosci. Remote Sens. 2017, 55, 3679-3693. [CrossRef]

43. Douglas, E.S.; Strahler, A.; Martel, J.; Cook, T.; Mendillo, C.; Marshall, R.; Chakraba, S.; Woodcock, C.; Li, Z.; Yang, X.; et al. DWEL: A dual-wavelength echidna lidar for ground-based forest scanning. In Proceedings of the 2012 IEEE International Geoscience and Remote Sensing Symposium (IGARSS 2012), Munich, Germany, 22-27 July 2012; pp. 4998-5001.

(C) 2018 by the authors. Licensee MDPI, Basel, Switzerland. This article is an open access article distributed under the terms and conditions of the Creative Commons Attribution (CC BY) license (http:/ / creativecommons.org/licenses/by/4.0/). 
Article

\title{
From Geometric-Optical Remote Sensing Modeling to Quantitative Remote Sensing Science-In Memory of Academician Xiaowen Li
}

\author{
Qinhuo Liu ${ }^{1,2, *}$, Guangjian Yan $^{3}$, Ziti Jiao ${ }^{3}$, Qing Xiao ${ }^{1,2}$, Jianguang Wen ${ }^{1,2}$, \\ Shunlin Liang ${ }^{3,4, *}$, Jindi Wang ${ }^{3}$, Crystal Schaaf ${ }^{5}$ and Alan Strahler ${ }^{6}$ \\ 1 State Key Laboratory of Remote Sensing Science, Institute of Remote Sensing and Digital Earth, \\ Beijing 100101, China; xiaoqing@radi.ac.cn (Q.X.); wenjg@radi.ac.cn (J.W.) \\ 2 College of Resources and Environment, University of Chinese Academy of Sciences, Beijing 100190, China \\ 3 State Key Laboratory of Remote Sensing Science, Faculty of Geographical Science, Beijing Normal University, \\ Beijing 100101, China; gjyan@bnu.edu.cn (G.Y.); jiaozt@bnu.edu.cn (Z.J.); wangjd@bnu.edu.cn (J.W.) \\ 4 Department of Geography, University of Maryland, College Park, MD 20740, USA \\ 5 School for the Environment, University of Massachusetts, Boston, MA 02125, USA; Crystal.Schaaf@umb.edu \\ 6 Department of Earth and Environment, Boston University, Boston, MA 02215, USA; alan@bu.edu \\ * Correspondence: liuqh@radi.ac.cn (Q.L.); sliang@bnu.edu.cn (S.L.)
}

Received: 2 August 2018; Accepted: 5 November 2018; Published: 8 November 2018

\begin{abstract}
The academician Xiaowen Li devoted much of his life to pursuing fundamental research in remote sensing. A pioneer in the geometric-optical modeling of vegetation canopies, his work is held in high regard by the international remote sensing community. He codeveloped the Li-Strahler geometric-optic model, and this paper was selected by a member of the International Society for Optical Engineering (SPIE) milestone series. As a chief scientist, Xiaowen Li led a scientific team that made outstanding advances in bidirectional reflectance distribution modeling, directional thermal emission modeling, comprehensive experiments, and the understanding of spatial and temporal scale effects in remote sensing information, and of quantitative inversions utilizing remote sensing data. In addition to his broad research activities, he was noted for his humility and his dedication in making science more accessible for the general public. Here, the life and academic contributions of Xiaowen $\mathrm{Li}$ to the field of quantitative remote sensing science are briefly reviewed.
\end{abstract}

Keywords: geometric-optical model; thermal radiation directionality; quantitative remote sensing inversion; scale effects; comprehensive field experiment

\section{Introduction}

Remote sensing is an essential tool in ensuring the successful implementation of strategies for the sustainable development of nature and society. High-precision remote sensing models, parameter inversion, and the validation of remote sensing products are among the important frontier scientific issues [1]. A pioneer in the geometric-optical modeling of vegetation, the late Xiaowen Li, working with his mentor Prof Alan Strahler and numerous colleagues, developed a series of geometric-optical models for vegetation canopies and hybrid models of geometric optical-radiative transfer that are widely used in operational remote sensing algorithms for the retrieval of global surface albedo products. As a chief scientist, he led several research efforts that focused on quantitative remote sensing modeling, inversion, and the applications of retrieved data. His outstanding achievements include contributions to bidirectional reflectance distribution modeling, directional thermal emission modeling, an understanding of the effects of different spatial and temporal scales in remote sensing data, the development of the theory underlying quantitative inversion theory using prior knowledge, and the completion of comprehensive field experiments in remote sensing. Xiaowen Li was not only 
an outstanding scientist inthe field of remote sensing and geography, but was also an eminent writer of popular science. He was known as a teacher, researcher, scholar, and mentor who was always ready to help others. Herein is a brief summary of his life and his major contributions to remote sensing science.

\section{Biography of Xiaowen Li}

Xiaowen Li (Figure 1) graduated from the Chengdu Telecommunication Engineering Institute (now the University of Electronic Science and Technology of China, UESTC) in 1968 and was admitted to graduate studies at the Institute of Geography, Chinese Academy of Sciences, in 1978. In 1979, supported by government grants for study abroad programs, he enrolled at the University of California, Santa Barbara, and earned a Master of Science degree in geography in 1981, another Master of Science degree in electronics and computer engineering in 1985, and a doctoral degree in geography in 1985 while under the mentorship of Alan Strahler.

In 1986, he joined Prof. Strahler at the Department of Geography, Boston University (BU), as a researcher and research professor. For over a decade at BU, he served on numerous doctoral committees and published some of his seminal work on geometric optics. During this period, Xiaowen Li regularly traveled to China, holding positions as an associate researcher at the Institute of Remote Sensing Applications, Chinese Academy of Sciences, and as a researcher at the Key Laboratory of Remote Sensing Information Science of the Chinese Academy of Sciences. Beginning in 1999, he became a senior faculty member at Beijing Normal University, where he founded the Research Center for Remote Sensing and Geographic Information Systems (GIS). His eminence was recognized by the Ministry of Education and he was made a Distinguished Professor of the Chang Jiang Scholars Program. In 2001, he was elected as an Academician to the Chinese Academy of Sciences. From 2002-2007, he was the director of the Institute of Remote Sensing Applications of the Chinese Academy of Sciences. In 2003, he facilitated cooperative efforts between the Key Laboratory of Remote Sensing Information Science of the Chinese Academy of Sciences and the Research Center for Remote Sensing and Geographic Information System of Beijing Normal University, establishing the State Key Laboratory of Remote Sensing Science, and in 2005, he founded an innovation team for the Ministry of Education. A devoted alumnus of UESTC, he actively promoted its development in geoscience by founding the Institute of Land Surface Space Information Research in 2004 and serving as its director. In 2012, he became the first dean of the School of Resources and Environment at UESTC.

Xiaowen Li was enthusiastic about making science more accessible to the public. On 29 July 2007, he created his blog on ScienceNet.cn. He called himself "Huang Laoxie", a fictional character in the Legends of the Condor Heroes authored by Jin Yong, a renowned Chinese martial arts novelist whom he admired. Under the cyber-name of "Lix", Xiaowen Li authored almost 2000 articles covering a wide range of areas, including popular science, hot-button issues in science, and comments on scientific programs, as well as views on social issues and current affairs. He divided his blog into six categories: Daily LifeBits \& Pieces, A Look at History, Make ScienceNet Widely Known, Letters from Abroad, Explanatory Notes of Lectures, and Quirky Ideas. Through these channels, he exchanged views with netizens of ScienceNet. He showed great care and attention to young researchers and actively participated in various activities organized by ScienceNet [2].

In 2014, he delivered a lecture at the University of Chinese Academy of Sciences, where he was bestowed the nickname of the "Cloth Shoes Academician" because of his unpretentious look-cloth shoes and simple clothes. He sent a clear message that scientists should be dedicated to their own work with no interest in seeking fame and fortune. This view was highly praised by his fellow netizens in particular. Following his death in 2015, numerous netizens on ScienceNet wrote memorial blogs expressing their condolences and reverence for him [3]. 


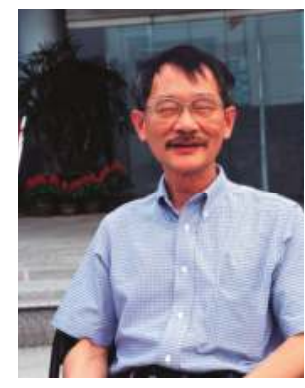

Figure 1. Academician Xiaowen Li.

\section{Geometric-Optical Bidirectional Reflectance Modeling of Vegetation Canopies}

Because of the three-dimensional structure of vegetation, anisotropic canopy reflectance in remote sensing observations is characterized by the bidirectional reflectance distribution function (BRDF) [4]. Multi-angle remote sensing plays an important role in the development of algorithms for the retrieval of various surface biophysical parameters that are influenced by reflectance anisotropy. Although radiative transfer theory was introduced into the field of land surface remote sensing in the 1960s, the basic assumption that underlies it—of volumetric scattering into and within a horizontally homogeneous medium - had greatly limited its scope of application. During his study in the United States, Xiaowen Li took part in numerous National Aeronautics and Space Administration (NASA) projects which greatly contributed to the generation of the Moderate Resolution Imaging Spectroradiometer (MODIS) albedo products [5]. He also conducted in-depth field and theoretical research on the geometric-optical bidirectional reflectance modeling of vegetation. He believed that at the remote sensing pixel scale, the key to the directional reflectance of vegetation lay in its surface structure.

The Li-Strahler geometric-optical (GO) model [6] was created by defining the geometric structure of tree crowns, individually and in stands, to describe the radiative interaction of vegetation surfaces. This model successfully described the anisotropic reflectance characteristics of vegetation surfaces. The paper describing the GO model was selected for the milestone series by the International Society for Optical Engineering (SPIE) because of the model's ability to capture the angular and spatial variabilities of reflectance using simple geometries defined by canopy architecture 6 .

An early mathematical model of the reflectance of a discontinuous forest canopy was proposed that treated trees as randomly distributed green cones on a contrasting background [7]. It was derived directly from the canopy scale, rather than on the scale of crop leaves and stems as defined by earlier efforts such as the Egbert model [8]. Inversion of this model allowed the direct calculation of the height and spacing of trees from remotely sensed reflectance values. Subsequently, this application of GO modelling methodology was adopted by $\mathrm{Li}$ and Strahler [6,9] for remote sensing image analysis. The models initially used cone geometry to simplify conifer tree crowns; however, the models were sufficiently general and robust for application to other geometric shapes and mixtures of simple shapes. The models simulated the angular distribution patterns of reflected solar radiance from forests and explained the major portions of the variance in a remotely sensed image of a forest stand.

Li et al. [10] further modeled the tree crown as an ellipsoid, which simplified the geometric projection and complexity of computation of the vegetation canopy. Furthermore, by taking into account mutual shadowing and the shadow overlap between incident and reflected directions, he developed the geometric-optical mutual shadowing model (GOMS). This work led to the derivation of several geometric-optical scattering kernels [11] and the development of the operational algorithm of the MODIS BRDF/Albedo [5]. This product has been widely utilized, especially in modeling terrestrial carbon and energy cycle dynamics and in characterizing the functioning of various ecosystems. 
The GOMS model has been further refined in order to describe the directional reflectance signatures of mountainous areas [12,13] and to contribute to the inversion of forest structure parameters such as the leaf area index (LAI) [14].

Li's recognition that the geometry of individual plants and their spacing is essential to the modeling of the gap frequency, which in turn is a key element in modeling the response of plant canopies to radiation, represented a major breakthrough in canopy remote sensing. Thus, Li et al. [15] proposed a method of modeling the probability of gaps occurring in a discontinuous vegetation canopy. This provides a quantitative understanding of how the gap probability of a generally discontinuous canopy differs from that of a generally continuous canopy. It also demonstrated that it was crucial to link geometrical optics with radiative transfer theory and led to the foundation of the later geometric optical radiative transfer (GORT) model [16], which described the effects of three-dimensional canopy structure on the radiation environment and allowed for the characterization of the heterogeneous radiation environment of natural vegetation at the forest stand scale. The GORT model treats vegetation canopies as assemblages of randomly distributed tree crowns that are ellipsoidal in shape, wherein the tree crowns are filled with leaves that absorb and scatter radiation passing through the crown. Principles of radiative transfer are used in describing the multiple scattering of leaves inside the crowns and the multiple scattering among crowns and the ground surface. The GORT model was extended to include the vertical probability distribution profile of the canopy, greatly improving the remote sensing inversion of forest structure parameters [17,18].

\section{Thermal Infrared Emission Directionality Modeling}

Research on the directional signatures of thermal infrared emission remote sensing has been relatively less developed compared to that of visible and near-infrared remote sensing. Li et al. [19] noted that when the accuracy required of land surface temperature retrieval from thermal remote sensing is not high, the concept of composite emissivity or equivalent emissivity could be employed by researchers, and the ambiguity in the definition of emissivity may be reasonable. Otherwise, the applicability of Planck's Law at the pixel scale must be taken into account when remote sensing errors of land surface temperature of less than $1 \mathrm{~K}$ are needed.

The pixel size in thermal infrared remote sensing is typically tens of meters to several kilometers. When large areas of water, desert, snow, and lush grass land are taken into consideration, the problem of mixed pixels is impossible to avoid. As for the effects of scale in Planck's Law, Li et al. [20] noted that under non-isothermal conditions, Planck's Law is not applicable to the equivalent emissivity; otherwise, the average pixel temperature will change abnormally with wavelength and viewing angle. Li was convinced that the definition of effective or equivalent emissivity at non-isothermal surface temperature was fundamental in exploring the directional mechanism of surface thermal emission [21]. Based on both multiple scattering and the temperature differences in the system, he proposed the concept of an apparent emissivity increment and then introduced a non-isothermal surface emissivity model, which combined multiple scattering and non-isothermal conditions for the first time [19,21]. Li et al. [22] demonstrated that the apparent emissivity increment is caused by two factors: the temperature differences of mixed pixels and the multiple scattering of three-dimensional (3D) structures captured in the pixels. Without a loss in generality, the equivalent emissivity of non-isothermal surface pixels can then be defined as the emissivity under the same temperature assumption of pixels, plus the apparent emissivity increment due to temperature differences under particular conditions (such as the materials, structures, and temperature distributions).The introduction of this increment term clearly explains the reason why the radiation of non-isothermal surface pixels is different from those of isothermal ones. It retains the universality of Planck's Law, while also maintaining the independence of material emissivity. The apparent increment in emissivity due to temperature differences is then used to cope with the spectral and directional variations of thermal radiation caused by non-isothermal surfaces. 
Li et al. [20] investigated the effects of scale for Planck's Law on non-isothermal blackbody surface and proposed a scale correction formula for real-surface thermal radiation [23]. Subsequently, Liu et al. [24] extracted the directional signatures of thermal infrared radiation of typical ground objects on the basis of experimental observations from an airborne camera. Chen et al. [25] and Yan et al. [26] made substantial improvements to the thermal radiation model to accommodate crown row structure. By considering crop gap probability both in the direction of sunlight and of the observation, they developed the bidirectional gap probability thermal radiation model, based on the model of discrete vegetation gap probability [15], and the cross-correlation probability of continuous vegetation, which has been demonstrated to better explain vegetation hotspots $[25,26]$. Recent research on the directional mechanism of thermal infrared radiation and the thermal infrared radiation directionality model on heterogeneous surfaces holds great promise [27].

\section{Quantitative Remote Sensing Inversion Theory}

The accumulation of multi-angle remote sensing data creates an opportunity for researchers to retrieve various biophysical parameters of the land surface through the inversion of bidirectional reflectance models. However, the implementation of quantitative methods in remote sensing remains difficult. Some theoretical guidance must to be obtained from the perspective of information theory, and a great deal of research has been focused on the operational practice of inversions [28-30].

In general, it is extremely difficult to obtain sufficiently distributed multi-angle data from single orbital overpasses of one of the modern operational satellites. Furthermore, such overpasses are rarely coincident with the main solar principal plane [31] and in the vicinity of the hotspot [32]. Even when sequential overpasses are utilized to improve multi-angle sampling, it is difficult to accurately estimate these information-rich attributes of the surface anisotropy [9]. It is worth noting that the accuracy of hotspot reflection often determines the precision of ground clumping parameter inversions [33,34]. In addition, even when orbital mechanics cooperate, the effects of clouds and aerosols make the acquisition of sufficient multi-angle data difficult $[29,35,36]$.

Therefore, in order to solve these practical problems in remote sensing inversion, Xiaowen $\mathrm{Li}$ and a close colleague, Jindi Wang, proposed a fusion scheme involving multi-angle and multispectral remote sensing information [37]. For quantitative remote sensing inversion, Li et al. [38] and Gao et al. [39] summarized a data-enabled decision-making process to meet an inversion target, and implemented a multistage inversion strategy, featuring the division of the dataset and parameter set, based on the parameter uncertainty and its sensitivity matrix. In response to these theoretical problems in remote sensing inversion, Li et al. [30] further summarized the major difficulties in this field, and noted the importance of introducing prior knowledge and its role in infinite-solution inversion. According to the concept of a "hard boundary" applied to prior knowledge, the "soft boundary" of parameters is constructed by the average expectations and uncertainties of parameters. This theoretically provided a method of introducing prior knowledge into the task of remote sensing inversion, enriching the inversion scheme with accumulated knowledge and enabling the reasonable and effective use of limited remote sensing information. Xiaowen Li believed that it is extremely important to measure the uncertainty of inversion parameters for any remote sensing inversion scheme, and explained that such uncertainty depends not only on the confidence in the prior knowledge, but also on the amount of available inversion data. He also explored the applicability of such a model, the influence of signal noise in the data, ways of acquiring appropriate prior knowledge, and the sensitivity of to-be-inverted parameters of the model to the data. Based on a form of joint probability distribution of data space and parameter space, he outlined an idea for describing this overall uncertainty, which has facilitated next-stage inversion strategies based on the stage inversion results and the uncertainty of inversion parameters.

Li et al. [40] proposed a new method of accumulating prior knowledge for BRDF retrieval. Using the example of a kernel-driven model inversion, prior knowledge was applied to the inversion of the surface spectral albedo based on Bayesian theory so as to improve the inversion robustness and 
its accuracy in the case of limited direct acquisition. By employing this prior knowledge, Gao et al. [41] used information inversion theory and proposed a "minimum variance" inversion principle by considering the errors of factors derived from models and measurements, in order to address problems of unstable inversion results when small samples were selected for the kernel-driven model inversion based only on the least squares method.

Yan et al. [42] noted that the relative positional relationship of the vegetation reflection spectrum curve can be used as prior knowledge in vegetation structure inversion. In the same year, they analyzed the applicability of existing constrained optimization methods in remote sensing inversion and proposed the concept of a penalty matrix from two perspectives [43]: an increase in inversion speed and a reduction of the ill-posed characteristics of the optimization method. Qin et al. [44] introduced the ensemble Kalman filter to achieve the inversion of surface parameters, which obtained effective inversion results while providing the posterior distribution of prior knowledge. Wang et al. [45] proposed a complete regularization theory for ill-posed inversions of surface parameters based on a regularization strategy. Zhao et al. [46] studied the role of regularization parameters in determining linear regularization inversion, and proposed a regularization parameter determination method based on the maximization reduction of Shannon entropy. Zhao et al. [47] also developed a quantitative method of determining the regularization parameter (the prior information ratio) in an iterative process for nonlinear, ill-posed problems, which was based on information utilization in the inversion process expressed by the Shannon's entropy reduction method.

Inversion is the essential characteristic of quantitative remote sensing [48]. How to quantitatively estimate the biophysical parameters of land surfaces with limited remotely sensed observation of the surface and under unfavorable observation sensing conditions is the primary task of quantitative remote sensing. Therefore, the application of prior knowledge to the inversion process can provide important and necessary step information. Quoting Confucius, Xiaowen Li stated [35]: “When you know a thing, to hold that you know it; and when you do not know a thing, to allow that you do not know it—this is knowledge." Based on multiyear data derived through satellite, airborne, and ground-based experiments, prior knowledge of ground objects can be accumulated to provide important support for remote sensing inversions.

\section{Spatiotemporal Scale Effects in Remote Sensing Data}

One of the essential problems in remote sensing research is related to scale: Do the laws of physics and the principles at the pixel scale need to be corrected, and if so, how? The Li-Strahler geometric-optical model explains non-Lambertian signatures on the pixel scale by using the four-component variance in different directions, based on the Lambertian assumption of crown and above-ground surfaces [5]. This model emphasizes that the Lambertian condition may prevail within the pixel, while non-Lambertian signatures will exist at the pixel scale overall.

In order to clarify the generality of scale effects in remote sensing science, Li et al. $[19,23]$ described the effects of scale caused by the three-dimensional structure inside a pixel by using a cross-valley example, which imagines a remote sensing pixel at the top of a $90^{\circ}$ north-south valley, containing both east- and west-facing slopes, and with both the sun and the sensor in the vertical plane of the valley. Although both the slope surfaces are Lambertian in reflectance, the surface reflection of the entire pixel no longer has a Lambertian property as a whole.

Xiaowen Li believed that many laws and principles of physics have been generalized, and deduced under specific experimental conditions. Therefore, they may only be applicable in homogeneous media or surfaces [23]. Given the complexity of terrestrial surfaces at the pixel scale of remote sensing, these laws and principles may not be still applicable to this scale; and if not, how might they be corrected? This remains a fundamental challenge that quantitative remote sensing must confront. An example of a scale-dependent physical law is Beer's Law. While Beer's Law works well in the atmosphere and in the oceans, there is a clear scale problem when it is applied to vegetation $[49,50]$; especially when the pixel spatial resolution is coarser than that of plant structures, Beer's Law must be scaled 
up [5]. Taking another example, the reciprocity principle is the cornerstone of radiative transfer theory. Xiaowen Li and Zhengming Wan demonstrated that the thermodynamic proof of Siegel and Howell was incorrect [51]. Snyder also proposed light reversal proof of the reciprocity principle, attempting to strictly extend the principle to the pixel scale [52]. Li et al. [20] noted that light reversal is in conflict with the law of the conservation of energy when homogeneous illumination produces spatially inhomogeneous reflections due to multiple scattering on the pixel.

The key issue of scale restricts the development of quantitative remote sensing. Li et al. [23] explained the change of quantity connotation, the change of quantity nature, and the applicability of physical laws at different scales through the geometric-optical model. He made preliminary efforts in establishing a scale-related and quantitative theoretical system for remote sensing, explaining the spatiotemporal scale effects of variable surface elements from point to surface, and studying the scaling model and information conversion model, all of which represent substantial progress [21].

The second issue in quantitative remote sensing is scale transfer. Xiaowen Li summarized the main problems of globally oriented quantitative remote sensing research as follows [53]: (1) The quantitative remote sensing products generated at different resolutions and from different remote sensing data are inconsistent with each other and with most traditional point observations. (2) Although remote sensing finds increasingly wide application, it is difficult to meet different spatiotemporal resolution and span requirements of users of different remote sensing products. This leads to: (a) Compared with the ability of remote sensing satellites to acquire data, the automation and quantification of remote sensing data and even the ability to understand remote sensing data and its effective use are far from sufficient. (b) Quantitative remote sensing research is not systematic and universally applicable as researchers often do their own work without interacting with their colleagues. Such fragmentation makes it difficult to make systematic and major achievements.

Xiaowen Li believed that the key to solve the afore mentioned problems relied on reliable, convenient, and self-consistent scale transfer. He further clarified that the scientific task of remote sensing is to achieve an understanding and transformation between the given spatiotemporal scale of high technology and that of geoscience applications. The remote sensing scale is physical and geometric in nature. Its physical nature can be interpreted as the phenomenon that shows differences in geophysical properties inverted from the remote sensing data of different resolutions. Its geometric nature presents different geometric signatures (e.g., the extent of cultivated land area) measured by pixels of different sizes, which is solved by surface descriptions, such as fractal dimensions and interdisciplinary geosciences. The accuracy of remote sensing inversion is determined by the physical nature of the remote sensing scale, making it a unique scale issue inherent to remote sensing itself.

Xiaowen Li tried to put forward a basic concept of a remote sensing trend surface in order to solve the physical scale effects and develop a framework for remote sensing geoscience cognition [54]. By drawing inspiration from the idea of a trend surface in geography, the remote sensing trend surface recognizes the uniqueness of the remote sensing scale issue and aims to construct a set of prior knowledge data with high spatiotemporal resolution without scale error. The trend is then obtained by summarizing and understanding various data, products, and models through geoscience, and remote sensing principles and methods. Therefore, the remote sensing trend surface gives priority to the construction, utilization, and accumulation of prior knowledge, while emphasizing interdisciplinary research within the geosciences. These efforts are conducive to solving the scaling issue in remote sensing.

\section{Quantitative Remote Sensing Experiments}

Remote sensing experiments can provide satellite, airborne, and ground observation data for the construction of remote sensing models suitable for ecological environmental applications, the evaluation of inversion algorithms, scale transfer mechanism analysis, and calibration and product validation. Xiaowen $\mathrm{Li}$ attached great importance to the role of remote sensing experiments in 
quantitative remote sensing research and repeatedly emphasized that "researchers must bear models in mind before an experiment is conducted".

For example, during the process of constructing the geometric-optical model, Li's core research achievement, the importance of observations stands out. After years of accumulating observation data and conducting scientific analyses, Xiaowen $\mathrm{Li}$ and Alan Strahler developed a series of geometric-optical models. Therefore, it can be concluded that remote sensing research should always undergo two crucial phases: first, gaining an objective understanding of the real world through observation, and then constructing models using appropriate mathematical and physical methods.

For the third phase of remote sensing research, namely the evaluation of models, observations are also indispensable. In the early 1990s, Xiaowen Li and his team collaborated with the Changchun Institute of Optics and Fine Mechanics of the Chinese Academy of Sciences to establish an indoor bidirectional reflectance observation laboratory at the Jingyuetan Experimental Station of Changchun, China. Dedicated to studying the distribution signatures of discontinuous canopy reflectance and its relationship with canopy geometry, their joint efforts also enabled the first international multi-angle remote sensing seminar to be held in Beijing. From May to October of 1995 and in August of 1996, Xiaowen Li and his team designed a simultaneous airborne and ground-based measurements experiment at the apple orchards of Xishatun Village, Changping District, Beijing, in China. The observation data were then used to validate the GOMS and GORT models $[9,16]$. The accumulation of sufficient multi-angle remote sensing data created numerous possibilities for scientists to retrieve various biophysical parameters of land surfaces through the use of bidirectional reflectance models [55].

Xiaowen $\mathrm{Li}$ and colleagues paid great attention to the role of quantitative remote sensing experiments. In China, Li was involved in organizing a series of simultaneous airborne, satellite-borne, and ground-based remote sensing experiments at places such as the Shunyi district of Beijing and the Poyang Lake Basin in Jiangxi Province [56], accumulating substantial and valuable data for the development of remote sensing models and the evaluation of algorithms. To further combine quantitative remote sensing with other disciplines, such as ecohydrology, two-phase experiments of the Heihe River Basin were conducted. By employing ground-based and airborne platforms, as well as active and passive remote sensing instruments, the researchers made a number of findings during this comprehensive experiment. Furthermore, they created a platform for theoretical validation, established scientific data sets, and enhanced quantitative remote sensing research and its applications in ecohydrology through information sharing $[57,58]$.

\section{Conclusions}

Remote sensing radiative transfer modeling, quantitative inversion, spatiotemporal scale effects in remote sensing data, comprehensive field experiments, and product validation lie at the forefront of remote sensing science. Among the most important and lasting contributions Xiaowen Li made to the field are:

(1) The construction of a series of geometric-optical models and hybrid models of geometric optical and radiative transfer, which led to kernel-driven bidirectional reflectance models and the foundations of global bidirectional reflectance distribution functions and albedo products.

(2) The definition of thermal infrared emissivity for inhomogeneous surfaces by the establishment of a conceptual model of thermal infrared radiation directivity for inhomogeneous surfaces, the development of an airborne multi-angle multispectral imaging system, and work in new fields of study and ways of examining thermal infrared radiation directionality mechanisms and multi-angle thermal infrared remote sensing.

(3) Noting the importance of prior knowledge and its role in inversion; then, developing the theory and method of quantitative remote sensing inversion based on prior knowledge, proposing a multi-stage inversion strategy featuring the division of datasets and parameter sets (based on 
parameter uncertainty and sensitivity matrices), and presiding over the development of the spectrum knowledge database of China for typical ground objects.

(4) Promoting research into the effects of scale and scale transfer in remote sensing, inducing the spatiotemporal scale issue of quantitative remote sensing and focusing on two aspects: the scale and scale transfer of remote sensing physical models, and the establishment of a universal theoretical framework for remote sensing scale transfer.

(5) Organizing and conducting comprehensive experiments of quantitative remote sensing in the Shunyi district of Beijing and Poyang Lake of Jiangxi, as well as comprehensive experiments in the ecohydrology of the Heihe River Basin. All of these efforts have resulted in substantial amounts of valuable data for the development of remote sensing models and algorithm evaluation and have fostered the further development of remote sensing science in ecohydrology.

The quantification of remote sensing applications is an important component of science and technology and will continue to play an increasingly important role in the study of Earth system science and global change. The contributions and achievements of Xiaowen Li and his fellow researchers in remote sensing mechanism modeling, quantitative inversion, the development of an understanding of the effects of different SPATIOTEMPORAL scales in remote sensing data, the design of remote sensing experiments, and product validation have all strengthened and deepened our understanding of remote sensing and its application to the global sustainable development of humankind.

Author Contributions: Q.L. and S.L. conceived the structure of this paper. Q.L. contributed the biography of X.L. Z.J. and J.W. (Jianguang Wen) conducted the geometric-optical bidirectional reflectance modeling of vegetation canopy. Q.L. conducted the thermal infrared emission directionality modeling. G.Y. conducted the spatiotemporal scale effect of remote sensing data. J.W. (Jindi Wang) conducted the quantitative inversion theory based on prior knowledge. Q.X. conducted the quantitative remote sensing experiments. C.S. and A.S. provided historical perspectives. All the authors contributed to the manuscript's revision.

Acknowledgments: This research was funded by the National Key Research and Development Program (grant number: 2018YFA0605503) and the National Basic Research Program (grant number: 2013CB733400).

Conflicts of Interest: The authors declare no conflict of interest.

\section{References}

1. Xu, G.; Liu, Q.; Chen, L.; Liu, L. Remote sensing for China's sustainable development: Opportunities and challenges. J. Remote Sens. 2016, 20, 679-688.

2. Li, X.; Chen, A. Master Xiaowen: Selection of Academician Xiaowen Li Blog Articles; China Science \& Technology Press: Beijing, China, 2015.

3. Wu, Y. Friends of Blog: In Memory of Xiaowen Li and His Blog Set; Science Press: Beijing, China, 2015.

4. Nicodemus, F.F.; Richmond, J.C.; Hsia, J.J.; Ginsberg, I.W.; Limperis, T.L. Geometrical Considerations and Nomenclature for Reflectance; US Department of Commerce, National Bureau of Standards: Washington, DC, USA, 1977; Volume 160.

5. Schaaf, C.B.; Gao, F.; Strahler, A.H.; Lucht, W.; Li, X.; Tsang, T.; Strugnell, N.C.; Zhang, X.; Jin, Y.; Muller, J.P. First operational brdf, albedo nadir reflectance products from modis. Remote Sens. Environ. 2002, 83, 135-148. [CrossRef]

6. Li, X.; Strahler, A.H. Geometric-optical modeling of a conifer forest canopy. IEEE Trans. Geosci. Remote Sens. 1985, GE-23, 705-721. [CrossRef]

7. Li, X. An Invertible Coniferous Forest Canopy Reflectance Model; University of California: Santa Barbara, CA, USA, 1981.

8. Egbert, D.D. A practical method for correcting bidirectional reflectance variations. In Proceedings of the Symposium on Machine Processing of Remotely Sensed Data, West Lafayette, Indiana, 21-23 June 1977.

9. Li, X.; Strahler, A.H. Geometric-optical bidirectional reflectance modeling of a conifer forest canopy. IEEE Trans. Geosci. Remote Sens. 1986, GE-24, 906-919. [CrossRef]

10. Li, X.; Strahler, A.H. Geometric-optical bidirectional reflectance modeling of the discrete crown vegetation canopy: Effect of crown shape and mutual shadowing. IEEE Trans. Geosci. Remote Sens. 1992, 30, 276-292. [CrossRef] 
11. Wanner, W.; Li, X.; Strahler, A.H. On the derivation of kernels for kernel-driven models of bidirectional reflectance. J. Geophys. Res. Atmos. 1995, 100, 21077-21089. [CrossRef]

12. Schaaf, C.; Li, X.; Strahler, A. Topographic effects on bidirectional and hemispherical reflectances calculated with a geometric-optical canopy model. IEEE Trans. Geosci. Remote Sens. 1994, 32, 1186-1193. [CrossRef]

13. Wu, S.; Wen, J.; Lin, X.; Hao, D.; You, D.; Xiao, Q.; Liu, Q.; Tang, Y. Modeling Discrete Forest Anisotropic Reflectance Over a Sloped Surface With an Extended GOMS and SAIL Model. IEEE Trans. Geosci. Remote Sens. 2018. [CrossRef]

14. Li, C.; Song, J.; Wang, J. Modifying geometric-optical bidirectional reflectance model for direct inversion of forest canopy leaf area index. Remote Sens. 2015, 7, 11083-11104. [CrossRef]

15. Li, X.; Strahler, A.H. Modeling the gap probability of a discontinuous vegetation canopy. IEEE Trans. Geosci. Remote Sens. 1988, 26, 161-170. [CrossRef]

16. Li, X.; Strahler, A.H.; Woodcock, C.E. A hybrid geometric optical-radiative transfer approach for modeling albedo and directional reflectance of discontinuous canopies. IEEE Trans. Geosci. Remote Sens. 1995, 33, 466-480.

17. Ni, W.; Li, X.; Woodcock, C.E.; Caetano, M.R.; Strahler, A.H. An analytical hybrid gort model for bidirectional reflectance over discontinuous plant canopies. IEEE Trans. Geosci. Remote Sens. 1999, 37, 987-999.

18. Woodcock, C.E.; Collins, J.B.; Jakabhazy, V.D.; Li, X.; Macomber, S.A.; Wu, Y. Inversion of the li-strahler canopy reflectance model for mapping forest structure. IEEE Trans. Geosci. Remote Sens. 1997, 35, 405-414. [CrossRef]

19. Li, X.; Strahler, A.H.; Friedl, M.A. A conceptual model for effective directional emissivity from nonisothermal surfaces. IEEE Trans. Geosci. Remote Sens. 1999, 37, 2508-2517.

20. Li, X.; Wang, J.; Strahler, A.H. Scale effect of Planck's law over nonisothermal blackbody surface. Sci. China 1999, 42, 652-656. [CrossRef]

21. Li, X.; Wang, J. The definition of effective emissivity of land surface at the scale of remote sensing pixels. Chin. Sci. Bull. 1999, 44, 2154-2158. [CrossRef]

22. Li, X.; Wang, J. The definition on thermal emissivity of non-isothermal surface. Chin. Sci. Bull. 1999, 44, 1612.

23. Li, X.; Wang, J.; Strahler, A.H. Scale effects and scaling-up by geometric-optical model. Sci. China Technol. Sci. 2000, 43, 17-22. [CrossRef]

24. Liu, Q.; Gu, X.; Li, X.; Jacob, F.; Hanocq, J.F.; Friedl, M.; Strahler, A.H.; Tao, Y.U.; Tian, G. Study on thermal infrared emission directionality over crop canopies with tir camera imagery. Sci. China Technol. Sci. 2000, 43, 95-103. [CrossRef]

25. Chen, L.F.; Liu, Q.; Fan, W.; Li, X.; Xiao, Q.; Yan, G.; Tian, G. A bi-directional gap model for simulating the directional thermal radiance of row crops. Sci. China 2002, 45, 1087-1098. [CrossRef]

26. Yan, G.; Jiang, L.; Wang, J.; Chen, L.F.; Li, X. Thermal bidirectional gap probability model for row crop canopies and validation. Sci. China Ser. D 2003, 46, 1241-1249. [CrossRef]

27. Liu, Q.; Cao, B.; Zeng, Y.; Li, J.; Du, Y.; Wen, J.; Fan, W.; Zhao, J.; Yang, L. Recent progresses on the remote sensing radiative transfer modeling over heterogeneous vegetation canopy. Yaogan Xuebao/J. Remote Sens. 2016, 20. [CrossRef]

28. Li, X. Bidirectional reflectance and angular signature of objects. Remote Sens. Environ. China. 1989, 4, 67-72.

29. Li, X.; Wang, J. Vegetation Optical Remote Sensing Model and Parameterized Vegetation Structure; Science Press: Beijing, China, 1995.

30. Li, X.; Wang, J.; Hu, B.; Strahler, A.H. The application of prion knowledge in remote sensing retrieval. Sci. China Ser. D 1998, 28, 67-72.

31. Wanner, W.; Strahler, A.H.; Hu, B.; Lewis, P.; Muller, J.P.; Li, X.; Schaaf, C.L.B.; Barnsley, M.J. Global retrieval of bidirectional reflectance and albedo over land from eosmodis and misr data: Theory and algorithm. J. Geophys. Res. Atmos. 1997, 102, 17143-17161. [CrossRef]

32. Jupp, D.; Walker, J.; Harrison, B.; Strahler, A.; Li, X. Using local spatial variance and directional radiance to monitor the structure of woodlands and forests. In Proceedings of the 12th Asian Conference on Remote Sensing, Singapore, 30 October-5 November 1991; p. 8.

33. Chen, J.M.; Menges, C.H.; Leblanc, S.G. Global mapping of foliage clumping index using multi-angular satellite data. Remote Sens. Environ. 2005, 97, 447-457. [CrossRef]

34. Jiao, Z.; Schaaf, C.B.; Dong, Y.; Román, M.; Hill, M.; Chen, J.; Wang, Z.; Zhang, H.; Saenz, E.; Poudyal, R.; et al. A method for improving hotspot directional signatures in BRDF models used for modis. Remote Sens. Environ. 2016, 186, 135-151. [CrossRef] 
35. Li, X.; Wang, J.; Wang, J.; Liu, Q. Multi-Angle and Thermal Infrared Remote Sensing of the Earth; Science Press: Beijing, China, 2001.

36. Li, X.; Gao, F.; Wang, J.; Strahler, A.; Lucht, W.; Schaaf, C. Estimation of the parameter error propagation in inversion based brdf observations at single sun position. Sci. China Ser. E 2000, 43, 9-16. [CrossRef]

37. Li, X.; Wang, J. Multiangular Remote Sensing and Applications, Remote Sensing in China; Survey and Mapping Press: Beijing, China, 1996.

38. Li, X.; Gao, F.; Wang, J.; Zhu, Q. Uncertainty and sensitivity matrix of parameters in inversion of physical brdf model. J. Remote Sens. 1997, 1, 5-14.

39. Gao, F.; Li, X.; Xia, Z.; Zhu, Q.; Strahler, A.H. Multi-angle inversion with multistage based on acknowledge. Sci. China Ser. D 1998, 28, 346-350.

40. Li, X.; Gao, F.; Wang, J.; Strahler, A. A priori knowledge accumulation and its application to linear brdf model inversion. J. Geophys. Res. Atmos. 2001, 106, 11925-11935. [CrossRef]

41. Gao, F.; Strahler, A.H.; Xia, Z.; Zhu, Q.; Li, X. New approach for remote sensing inversion using kernel-driven brdf model. Chin. Sci. Bull. 1998, 43, 1315-1318.

42. Yan, G.; Jun, W.U.; Wang, J.; Zhu, C.; Xiao, L.I. Spectral prior knowledge and its use in the remote sensing based inversion of vegetation structure. J. Remote Sens. 2002, 6, 1-6.

43. Yan, G.; Zhu, C.; Wang, J.; Li, X. An extension of augmented Lagrange multiplier method for remote sensing inversion. J. Remote Sens. China 2002, 6, 81-87.

44. Qin, J.; Yan, G.; Liu, S.; Liang, S.; Hao, Z.; Wang, J.; Li, X. Application of ensemble kalman filter to geophysical parameters retrieval in remote sensing: A case study of kernel-driven brdf model inversion. Sci. China 2006, 49, 632-640. [CrossRef]

45. Wang, Y.; Li, X.; Nashed, Z.; Zhao, F.; Yang, H.; Guan, Y.; Zhang, H. Regularized kernel-based brdf model inversion method for ill-posed land surface parameter retrieval. Remote Sens. Environ. 2007, 111, 36-50. [CrossRef]

46. Zhao, H.; Tang, Z.; Li, X. A regularization parameter choice method on linear quantitative remote sensing inversion. Geomat. Inf. Sci. Wuhan Univ. 2007, 32, 531-535.

47. Zhao, H.; Tang, Z.; Li, X. A regularization parameter choice method on nonlinear III-posed quantitative remote sensing inversion. Geomat. Inf. Sci. Wuhan Univ. 2008, 32, 531-535.

48. Li, X. Retrospect, prospect and innovation in quantitative remote sensing. J. Henan Univ. 2005, 5, 49-56.

49. Albers, B.J.; Strahler, A.H.; Li, X.W.; Liang, S.L.; Clarke, K.C. Radiometric measurements of gap probability in conifer tree canopies. Remote Sens. Environ. 1990, 34, 179-192. [CrossRef]

50. Yan, G.; Hu, R.; Wang, Y.; Ren, H.; Song, W.; Qi, J.; Chen, L. Scale effect in indirect measurement of leaf area index. IEEE Trans. Geosci. Remote Sens. 2016, 54, 3475-3484. [CrossRef]

51. Li, X.; Wan, Z. Comments on reciprocity in the directional reflectance modeling. Prog. Nat. Sci. China 1998, 8, 354-358.

52. Snyder, W.C. Reciprocity of the bidirectional reflectance distribution function (BRDF) in measurements and models of structured surfaces. IEEE Trans. Geosci. Remote Sens. 1998, 36, 685-691. [CrossRef]

53. Li, X.; Wang, Y. Prospects on future developments of quantitative remote sensing. Acta Geogr. Sin. 2013, 68, 1163-1169.

54. Wang, Y.; Xie, D.; Li, X. Universal scaling methodology in remote sensing science by constructing geographic trend surface. J. Remote Sens. 2014, 18, 1139-1146.

55. Li, X.; Strahler, A.; Zhu, Q.; Liu, G.; Zhang, R.; Liu, Y.; Yu, X.; Li, J. Geometric-optical bidirectional reflectance modelling of ground objects and its progress in measurement. Remote Sens. Land Resour. 1991, 3, 9-19.

56. Liu, Q.H.; Li, X.; Chen, L.F. Field campaign for quantitative remote sensing in Beijing. In Proceedings of the IEEE International Geoscience and Remote Sensing Symposium, Toronto, ON, Canada, 24-28 June 2002; Volume 3136, pp. 3133-3135. 
57. Li, X.; Cheng, G.; Liu, S.; Xiao, Q.; Ma, M.; Jin, R.; Che, T.; Liu, Q.; Wang, W.; Qi, Y. Heihe watershed allied telemetry experimental research (hiwater): Scientific objectives and experimental design. Bull. Am. Meteorol. Soc. 2013, 94, 1145-1160. [CrossRef]

58. Li, X.; Li, X.; Li, Z.; Ma, M.; Wang, J.; Xiao, Q.; Liu, Q.; Che, T.; Chen, E.; Yan, G. Watershed allied telemetry experimental research. J. Geophys. Res. Atmos. 2009, 114, 2191-2196. [CrossRef]

(C) 2018 by the authors. Licensee MDPI, Basel, Switzerland. This article is an open access article distributed under the terms and conditions of the Creative Commons Attribution (CC BY) license (http:/ / creativecommons.org/licenses/by/4.0/). 
MDPI

St. Alban-Anlage 66

4052 Basel

Switzerland

Tel. +41616837734

Fax +41 613028918

www.mdpi.com

Remote Sensing Editorial Office

E-mail: remotesens@mdpi.com www.mdpi.com/journal/remotesens

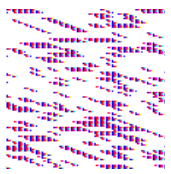




\section{MDPI}

St. Alban-Anlage 66

4052 Basel

Switzerland

Tel: +41 616837734

Fax: +41 613028918 